\title{
Seismicity of the United States, 1568-1989 (Revised)
}

U.S. GEOLOGICAL SURVEY PROFESSIONAL PAPER 1527

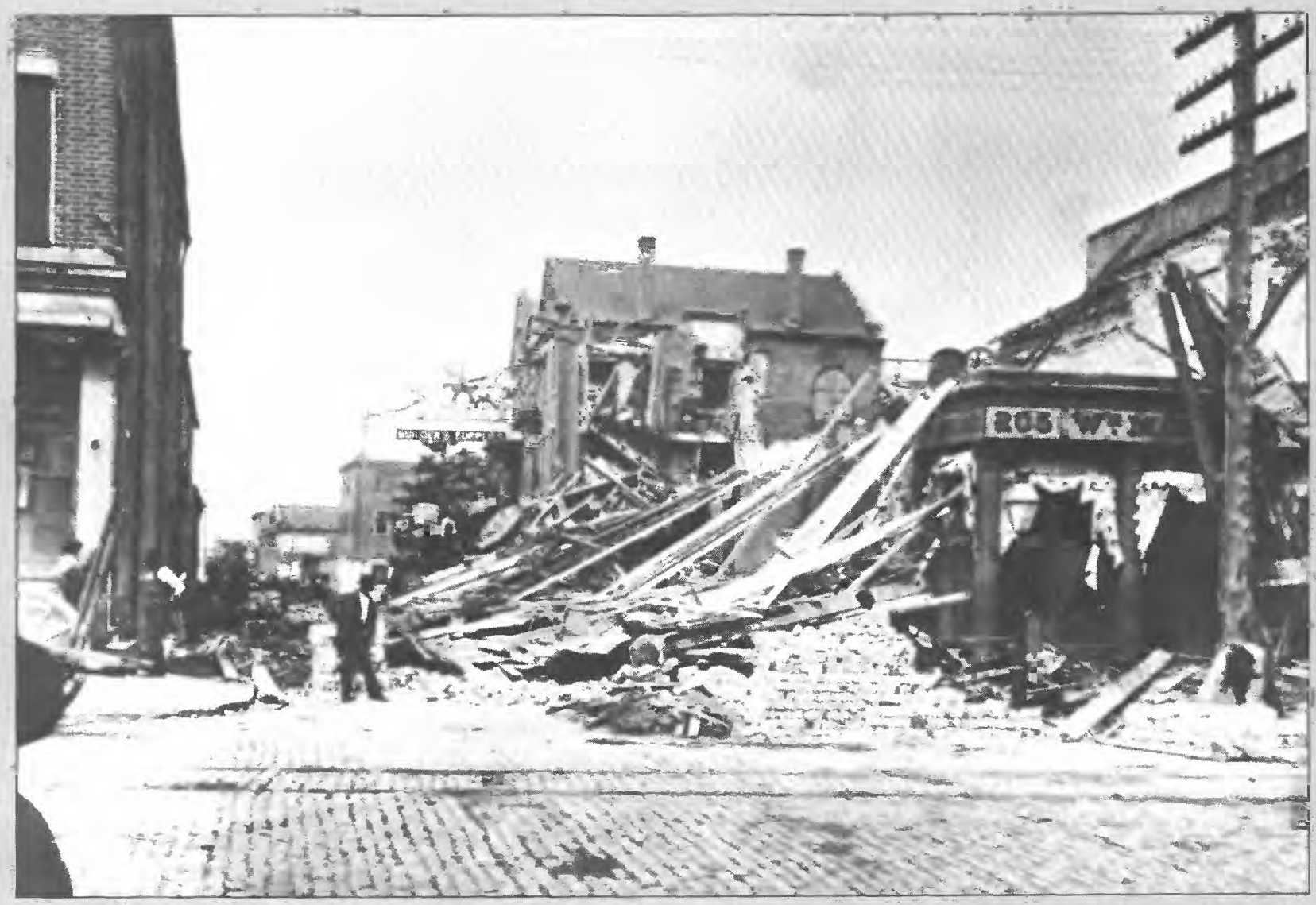


TABIE 3.-Deaths from earthquakes in the United States

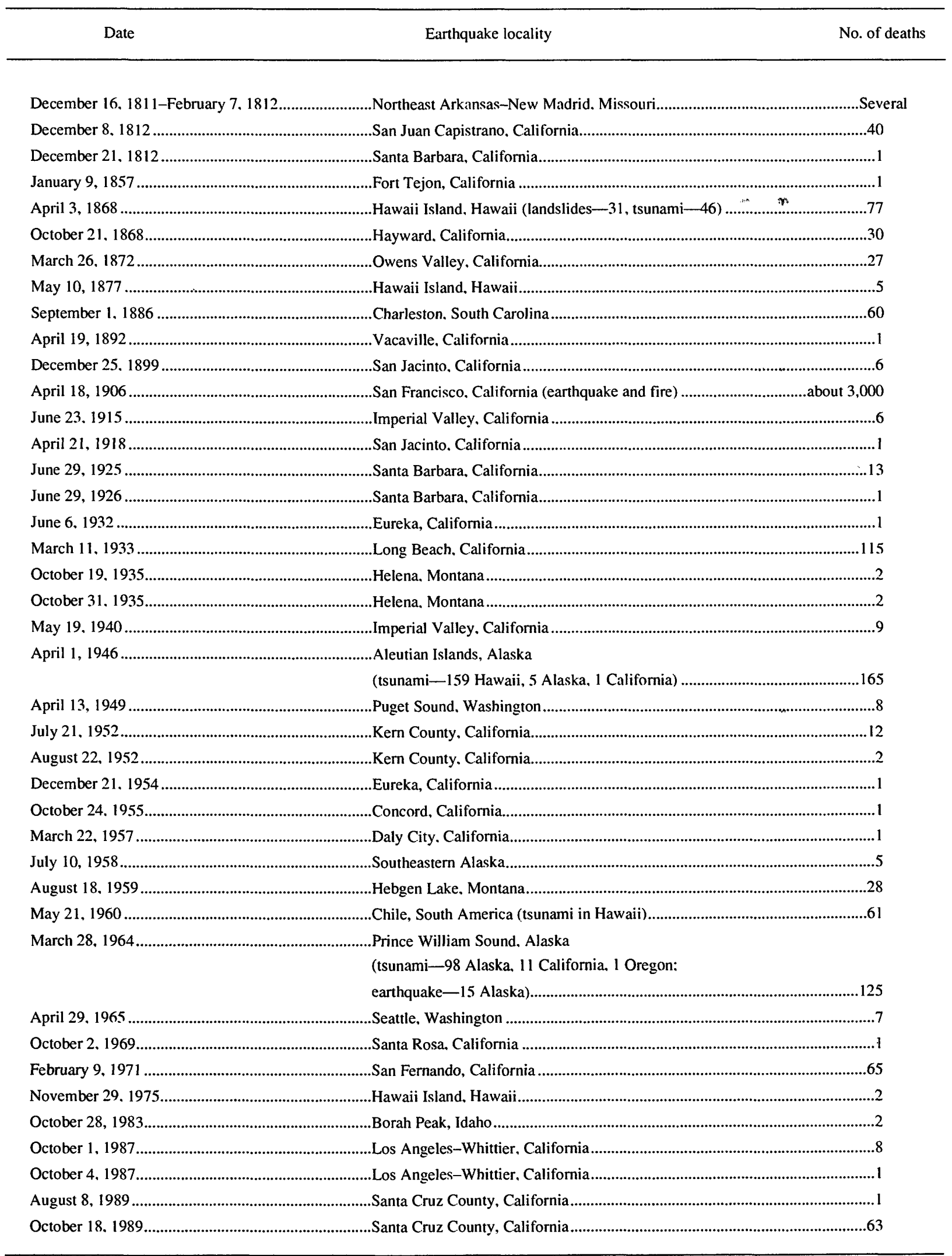


COVER-Photograph of damage on East Bay Street, Charleston, South Carolina, after the earthquake of September 1, 1886 (Aug, 31 EST). 


\section{Seismicity of the United States, 1568-1989 (Revised)}

By CARL W. STOVER and JERRY L. COFFMAN

U.S. GEOLOGICAL SURVEY PROFESSIONAL PAPER 1527

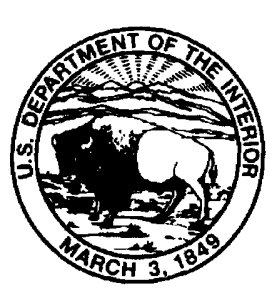

UNITED STATES GOVERNMENT PRINTING OFFICE, WASHINGTON ： 1993 


\title{
U.S. DEPARTMENT OF THE INTERIOR
}

MANUEL LUJAN, JR., Secretary

\section{U.S. GEOLOGICAL SURVEY}

\author{
Dallas L. Peck, Director
}

Published in the Central Region, Denver, Colorado

Manuscript approved for publication February 20, 1992

Edited by Richard W. Scott, Jr.

Graphics prepared by Wayne Hawkins; extensive use made of author-drafted material

Type composed by Shelly A. Fields

\begin{abstract}
Any use of trade, product, or firm names in this publication is for descriptive purposes only and does not imply endorsement by the U.S. Government
\end{abstract}

\section{Library of Congress Cataloging-in-Publication Data}

Stover, Carl W.

Seismicity of the United States, 1568-1989 / by Carl W. Stover and Jerry L. Coffman. - Rev. p. $\quad$ cm. - (U.S. Geological Survey professional paper ; 1527)

Includes bibliographical references.

Supt. of Docs. no.: I19.16: P1527

1. Earthquakes-United States. I. Coffman, Jerry L. II. Title. III. Series QE535.2.U6S77 1993

92-26509

$551.2^{\prime} 2^{\prime} 0973-\mathrm{dc} 20$

CIP

For sale by the Book and Open-File Report Sales

U.S. Geological Survey

Federal Center, Box 25286

Denver, CO 80225 
8. Yakutat Bay, Alaska, earthquake of September 10, 1899

9. Southeast Alaska earthquake of July 10, 1958

10. Prince William Sound, Alaska, earthquake of March 28, 1964.

11. Sonora, Mexico, earthquake of May 3, 1887

12. Arkansas earthquake of December 16, 1811

13. Fort Tejon, California, earthquake of January 9,1857 .

14. Owens Valley, California, earthquake of March 26, 1872

15. San Francisco, California, earthquake of April 18, 1906

16. Long Beach, California, earthquake of March 11, 1933

17. Kern County, California, earthquake of March 15, 1946

18. San Bernardino County, California, earthquake of April 10, 1947 ............................................................... 140

19. Riverside County, California, earthquake of December 4, 1948.................................................................... 142

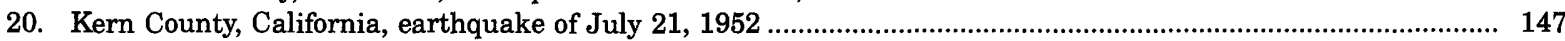

21. Borrego Mountain, California, earthquake of April 9, 1968 ............................................................................ 155

22. San Fernando, California, earthquake of February 9, 1971 .......................................................................... 160

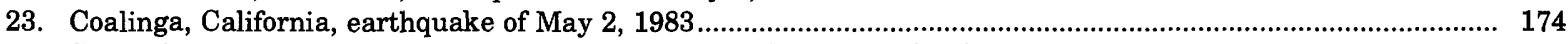

24. Santa Cruz Mountains (Loma Prieta), California, earthquake of October 18, 1989 ...................................... 186

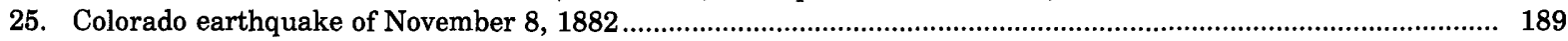

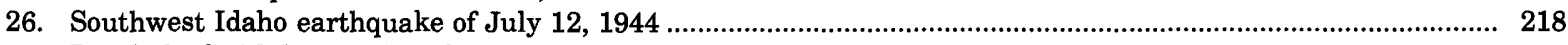

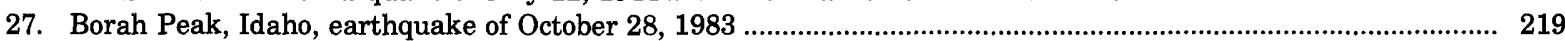

28. Southern Illinois earthquake of September 27, 1891........................................................................... 226

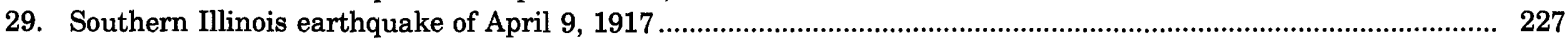

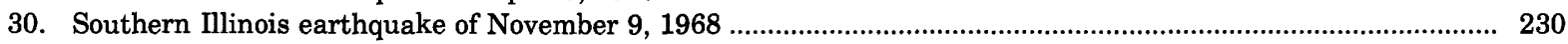

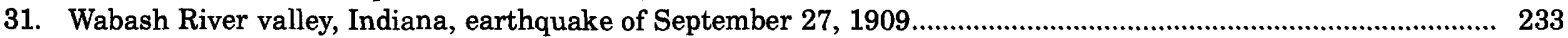

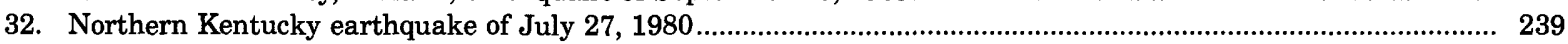

33. Southern Michigan earthquake of August 10, 1947 .................................................................................... 255

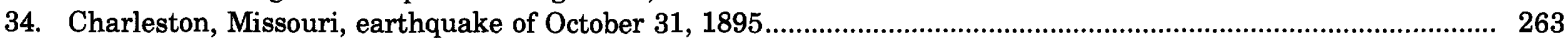

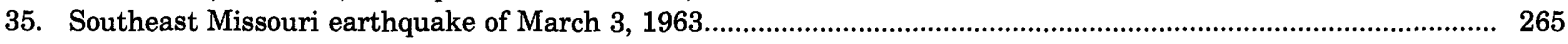

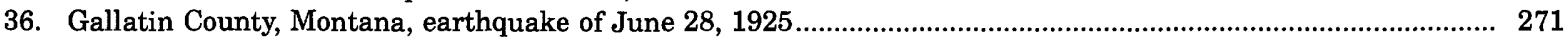

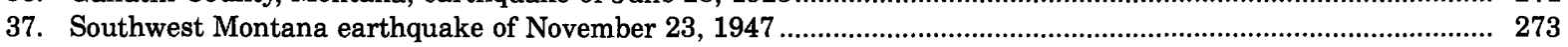

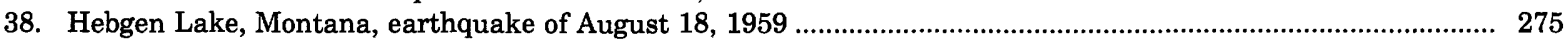

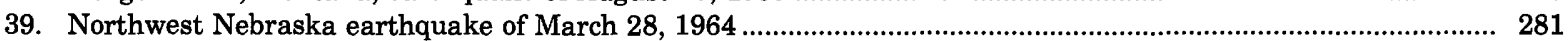

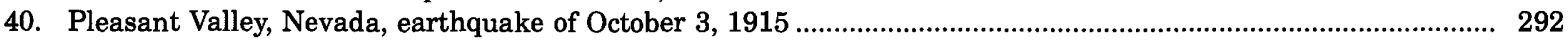

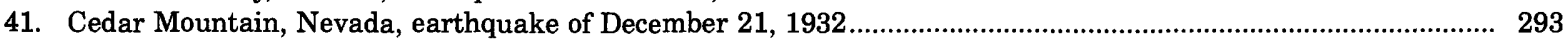

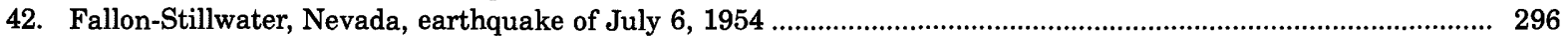

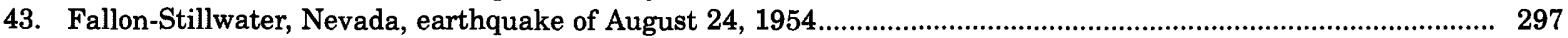

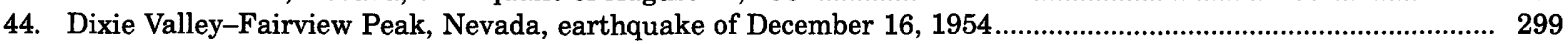

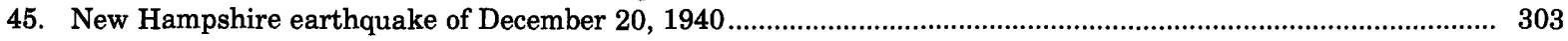

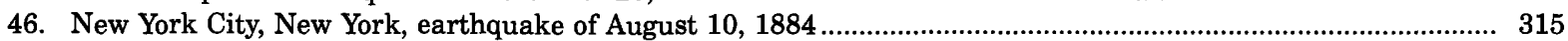

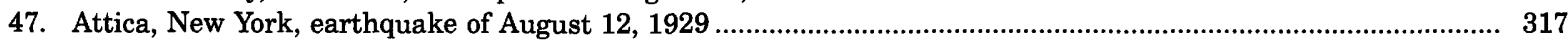

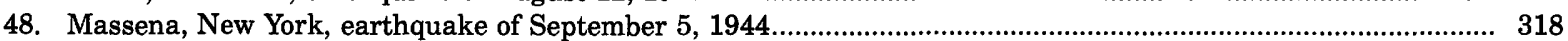

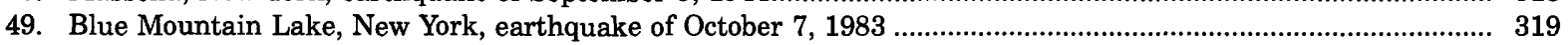

50. Waynesville, North Carolina, earthquake of February 21, 1916 .................................................................. 323

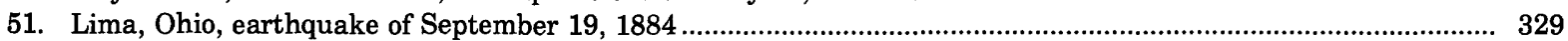

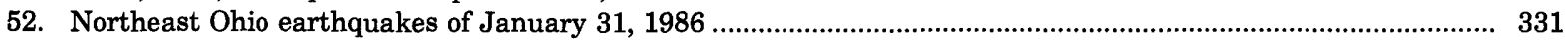

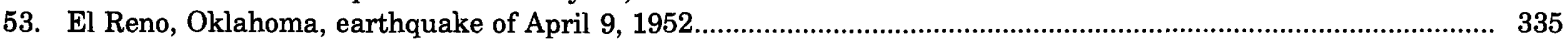

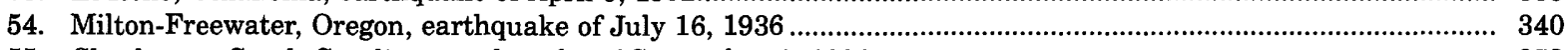

55. Charleston, South Carolina, earthquake of September 1, 1886 .................................................................. 352

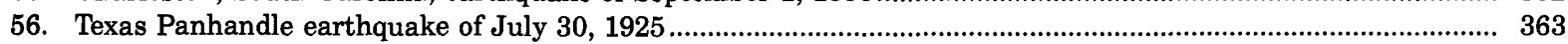

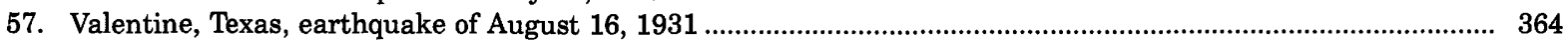

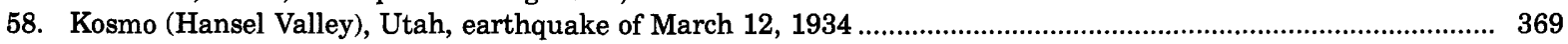

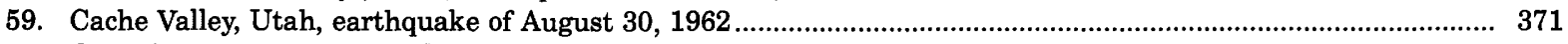

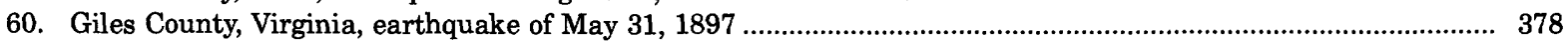

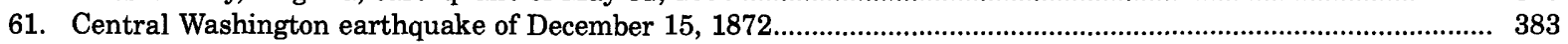

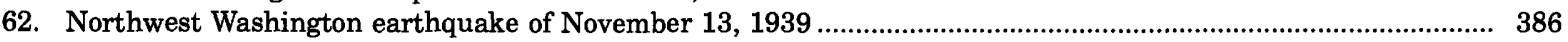

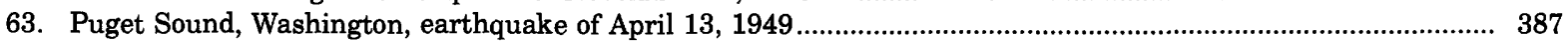

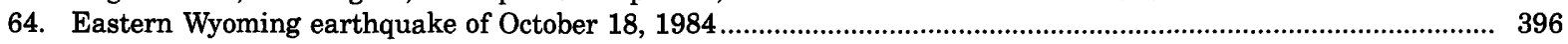




\section{TABLES}

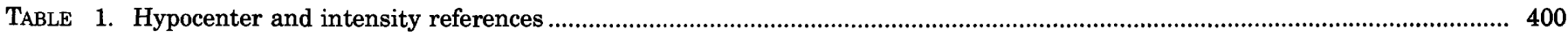

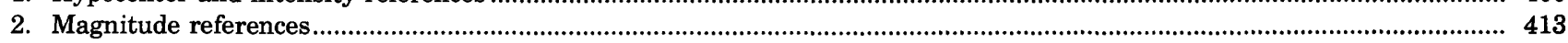

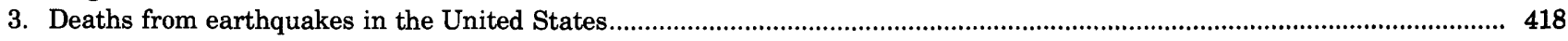





\title{
SEISMICITY OF THE UNITED STATES, 1568-1989 (REVISED)
}

\author{
By CARL W. STOVER and JERRY L. COFFMAN
}

\begin{abstract}
Macroseismic effects of the principal earthquakes occurring in the United States from 1568 through 1989 are described. Principal earthquakes are defined as those of Modified Mercalli intensity $\geq$ VI or Richter magnitude $\geq 4.5$. Exceptions are the State of Alaska and the offshore areas of California, Oregon, and Washington, where the magnitude cutoff is $\geq 5.5$. A tabular list of earthquake data giving date, location, magnitude, intensity, and reference information for each earthquake is provided for 47 States (earthquakes in the categories described above were not reported in Iowa, Maryland, Wisconsin, or the District of Columbia). Following each table is a brief narrative of the damaging effects of each earthquake of intensity $\geq$ VI. The narrative includes, where reported, a description of property damage and geologic effects as well as an estimate of the total area over which the shaking was sensibly felt by humans. Isoseismal maps, depicting the areal distribution of effects, and photographs of property damage and geologic effects complement the narratives of selected earthquakes.
\end{abstract}

\section{INTRODUCTION}

This publication is a history of the principal earthquakes in the United States from 1568 through 1989. It contains all pertinent information on the date, location, size, and effects of these historical events. We have included only those earthquakes of Modified Mercalli intensity ("MM intensity" or "MMI") $\geq \mathrm{VI}$ and magnitude $\geq 4.5$. Exceptions are the State of Alaska and offshore areas of California, Oregon, and Washington, where the magnitude range was increased to $\geq 5.5$ because of the high seismicity of those regions. This publication supersedes the compilation by Coffman and others (1982), published jointly by the U.S. Geological Survey (USGS) and the National Oceanic and Atmospheric Administration (NOAA).

This publication differs from the publication by Coffman and others (1982) "Earthquake History of the United States," as listed below:

1. It is organized by State and has many new or revised isoseismal maps.
2. Epicentral locations were evaluated, and some were revised.

3. Many more earthquakes were added, making the data more complete.

4. All maximum intensities were reevaluated, and a uniform criteria for intensity assignment was used, thereby changing some of the intensities previously published.

5. Magnitudes are listed by type and source and are completely referenced.

The summaries of earthquake damage and other effects enumerated in this publication have been compiled from many sources (see table 1). Selection of information to be included for each earthquake was based on the authors' experience in evaluating and publishing earthquake data as well as on a review of the type of data most often requested from the USGS National Earthquake Information Center by scientists and laymen.

The earthquakes described herein are listed alphabetically by State name and chronologically by date of occurrence (1568 through 1989). Further, earthquakes in Canada and Mexico that caused damage in the United States are included in the chapter for the State in which the damage occurred. Each State chapter includes the following: a map showing the distribution of earthquakes; a tabular listing of location, magnitude, maximum intensity, and references; brief summaries of damage caused by earthquakes of MMI $\geq \mathrm{VI}$; isoseismal maps showing felt areas for selected earthquakes; and photograph's of damage and geologic changes induced by destructive earthquakes.

Intensity and damage summaries for earthquakes that occurred in 1987-89 have been compiled from unpublished USGS data and are supplemented by data published in journal articles for the most damaging events. Thus, the summaries for 1987-89 reflect the data available at the time of compilation. 


\section{ACKNOWLEDGMENTS}

The isoseismal maps were drafted by F.W. Baldwin; L.R. Brewer prepared the tables and text for publication; and B.G. Reagor did the programming to format the tables. G.A. Bollinger, Virginia Polytechnic and State University, provided many discussions on the data and critically reviewed the final manuscript. T.R. Toppazada, California Division of Mines and Geology, reviewed the data for California and Nevada. R.L. Street, University of Kentucky, provided data for historical earthquakes in the Eastern and Central United States. Many of the photographs in this report were provided by the NOAA National Geophysical Data Center, Earthquake Hazards Photograph Library, Boulder, Colorado. After 32 years of providing earthquake information to the public, Carl W. Stover retired from government service on January 3, 1993.

\section{MAGNITUDES}

Magnitude, a logarithmic measure of the "size" of an earthquake, is related to the energy released as seismic waves at the focus of an earthquake. Although the magnitude scale has neither "top" nor "bottom" values, the highest magnitude known to have been calculated was about 9.5 , the lowest about -3.0 . On this logarithmic scale, a magnitude 6.0 shallow-focus earthquake represents elastic-wave energy about 30 times larger than that generated by a magnitude 5.0 earthquake, 900 times $(30 \times 30)$ larger than that of a magnitude 4.0 shock, and so forth. Many factors influence the determination of earthquake magnitude, including focal depth, distance between earthquake focus and observing station, frequency content of the sampled energy, and earthquake radiation pattern (variation of vibrational amplitude with azimuth). Magnitude values calculated by the USGS are based on the following six formulas:

Surface-Wave Magnitude:

$$
\mathrm{M}_{\mathrm{S}}=\log (A / T)+1.66(\log D)+3.3,
$$

as adopted by the International Association of Seismology and Physics of the Earth's Interior (Båth, 1966 , p. 153), where $A$ is the maximum vertical surface-wave ground amplitude in micrometers; $T$ is the period in seconds, and $18 \leq T \leq 22$; and $D$ is the distance in geocentric degrees (station to hypocenter), and $20 \leq D \leq 160^{\circ}$. No depth correction is made for depths less than $50 \mathrm{~km}$, and, generally, $\mathrm{M}_{\mathrm{S}}$ magnitudes are not computed for depths greater than 50 $\mathrm{km}$.

Body-Wave Magnitude:

$$
\mathrm{m}_{\mathrm{b}}=\log (A / T)+Q(D, h)
$$

as defined by Gutenberg and Richter (1956), except that $T$, the period in seconds, is restricted to $0.1 \leq T \leq 3.0$, and $A$, the ground amplitude in micrometers, is not necessarily the maximum of the $\mathrm{P}$-wave group. $Q$ is a function of distance $(D)$ and depth $(h)$, as published by Gutenberg and Richter (1956), where $D \geq 5^{\circ}$.

Local Magnitude (Western United States):

$$
\mathrm{M}_{\mathrm{L}}=\log A-\log A_{o}
$$

as defined by Richter (1958, p. 340), where $A$ is the maximum trace amplitude in millimeters, written by a Wood-Anderson torsion seismometer, and $\log A_{o}$ is a standard value as a function of distance, where the distance is $<600 \mathrm{~km}$. Values of $\mathrm{M}_{\mathrm{L}}$ are also calculated from other seismometers by converting recorded ground motion to the expected response of the torsion seismometer. $\mathrm{M}_{\mathrm{L}}$ magnitudes are listed for events with depths less than $70 \mathrm{~km} . \mathrm{M}_{\mathrm{L}}$ is the only true "Richter magnitude" (originally defined for California only), but general usage has extended that description to include other areas and types of magnitude as well. There is a potential for misuse in this practice, however. When technical or scientific applications are to be made, it is essential to specify the type of magnitude being used and not to resort to a generic "Richter magnitude."

Local and Regional Magnitude (Eastern United

States):

$$
\mathrm{M}_{\mathrm{n}}=3.75+0.90(\log D)+\log (A / T)
$$

$0.5 \leq D \leq 4.0^{\circ}$

$$
\mathrm{M}_{\mathrm{n}}=3.30+1.66(\log D)+\log (A / T)
$$

$4.0^{\circ} \leq D \leq 30.0^{\circ}$,

as proposed by Nuttli (1973) for North America east of the Rocky Mountains, where $A / T$ is expressed in micrometers per second, calculated from the verticalcomponent 1 -second $L_{g}$ waves, and $D$ is the distance in geocentric degrees. The designator $\mathrm{M}_{\mathrm{bLg}}$ often is used in place of $\mathrm{M}_{\mathrm{n}}$.

Moment Magnitude:

$$
\mathrm{M}=2 / 3\left(\log M_{o}\right)-10.7,
$$

as defined by Hanks and Kanamori (1979), where the seismic moment ( $M_{o}$-commonly expressed in dynecm but may be expressed in Newton-meters; 1 Newton-meter $=10^{7}$ dyne-cm) is equal to the product of the area of the earthquake fault, multiplied by the average fault slip over that area and by the shear modulus of the fault rocks; seismic moment may also be determined from the long-period body- and mantlewave moment tensor inversion method of Dziewonski and others (1981); or, in California, seismic moment is estimated from measurements on Wood-Anderson seismograms (Bolt and Herraiz, 1983). 
Other Magnitudes:

Variations of $\mathrm{M}_{\mathrm{S}}, \mathrm{m}_{\mathrm{b}}$, and $\mathrm{M}_{\mathrm{n}}$ magnitudes have been designed by seismologists for local seismographic networks or for particular geographic regions so that the results are compatible with the magnitude values derived from the standard formulas above. These variations are defined below.

$\mathrm{M}_{\mathrm{D}}$ designates magnitude estimates derived from the duration or coda length of earthquake vibrations. $\mathrm{M}_{\mathrm{D}}$ is commonly computed from the difference, in seconds, between $P_{n^{-}}$or $P_{g}$-wave arrival times and the time the final coda amplitude decreases to the pre-event background-noise amplitude. Duration or coda-length magnitude scales normally are adjusted to agree with $\mathrm{M}_{\mathrm{L}}$ or $\mathrm{M}_{\mathrm{n}}$ estimates, so that resulting magnitudes can be accepted as compatible. Thus, the $M_{D}$ formulas vary for different geographic regions and for different seismograph systems.

$\mathrm{M}_{\mathrm{fa}}$ is a body-wave $\left(\mathrm{m}_{\mathrm{b}}\right)$ magnitude commonly computed from the felt area for earthquakes occurring before seismic instruments were in general use. The computations are based on isoseismal maps or defined felt areas using either the intensity-attenuation method of Nuttli (1973), the magnitude-felt area relation of Nuttli and Zollweg (1974), or the intensity-felt area relation of Sibol and others (1987).

$\mathrm{M}_{\mathrm{La}}$ is a local magnitude (comparable to $\mathrm{M}_{\mathrm{L}}$ ) computed for earthquakes in California and adjacent areas and Hawaii. The computation is based on the area of perceptibility shown on isoseismal maps or on the area enclosed within a particular intensity level as defined by Toppozada (1975).

$\mathrm{M}_{\mathrm{Sn}}$ is a surface-wave $\left(\mathrm{M}_{\mathrm{S}}\right)$ magnitude that is based on the source parameters for mid-plate earthquakes as defined by Nuttli (1983).

Some seismograph network operators determine a magnitude formula for their specific network by comparing their computed magnitude values with magnitudes published by other sources, such as the USGS $\mathrm{m}_{\mathrm{b}}, \mathrm{M}_{\mathrm{L}}$, or $\mathrm{M}_{\mathrm{n}}$ magnitudes. In this publication, those types of magnitudes will be designated $\mathrm{m}_{\mathrm{x}}$ for bodywave magnitudes $\left(m_{b}\right)$ and $M_{x}$ for local magnitudes $\left(M_{L}\right.$ or $\left.M_{n}\right)$. $M_{L}$ applies west of the Rocky Mountains, $M_{n}$ east of the Rocky Mountains.

$M_{R}$ magnitude represents the average of $M_{S}$ magnitudes computed by different seismograph stations or taken from other seismic networks after standardization as defined and published by Rothé (1969).

A magnitude labeled as "Ukn" means that the computational method was unknown and could not be determined from the published sources.

The published sources for the "Other" and Moment (M) magnitudes are shown by an alphabetic code. These codes are defined in table 2.

\section{INTENSITY, FELT AREA, AND ISOSEISMAL MAPS}

The term "intensity," as applied to earthquakes, represents a number assigned to the effects on people (number affected, frightened, etc.), manmade structures (toppled chimneys, collapsed walls, etc.), and the Earth's surface (landslides, faulting, etc.). The intensities listed in this publication were assigned according to the effects outlined in the Modified Mercalli intensity scale of 1931 (Wood and Neumann, 1931), which has 12 discrete steps (see scale on p. 6). Modifications applied by the authors to this scale are described below. The single intensity value listed as a size parameter for each earthquake is the value assigned to the maximum reported effects of that particular earthquake. The maximum intensity is based on documented effects or damage at a place (usually a city or town) and are not necessarily the same as epicentral intensity $\left(I_{o}\right)$, which is the estimated intensity at the location of the earthquake.

Some of the maximum intensities in this publication have been changed from previously published values (namely in Coffman and others, 1982), mainly owing to new sources of information, or reevaluation of existing information, or both. Interestingly, many of those changes have resulted in the downgrading of intensities from MM intensity VI to V. This action has eliminated many earthquakes from this compilation if their associated magnitude was less than 4.5 ( $<5.5$ for Alaska and offshore California, Oregon, and Washington). All Rossi-Forel intensities gleaned from earlier publications were converted to MMI values using table 4 from Barosh (1969).

The Modified Mercalli intensity scale of 1931 has been further modified by the authors for assigning intensity values. These modifications were made for several reasons: The recent changes the USGS has made in the format of the questionnaire used to collect earthquake information have improved both the qualitative and quantitative data on the effects of shaking; the field experience of the authors (including personal observation of damage) has provided insight for more accurate intensity evaluation; and the subjective effects on people described in the MM intensity scale are not reliable considerations for assigning values above the intensity IV level. These modifications to the MM intensity scale of 1931 are described in more detail below:

IV-Felt by many to all. Trees and bushes were shaken slightly. Buildings shook moderately to strongly. Walls creaked loudly. Observer described the shaking as "strong."

$\mathrm{V}$-Felt, frightened, and awakened effects were not used at this or higher intensity levels. Hanging 
pictures fell. Spilled liquid effects were not used to assign any intensity. Trees and bushes were shaken moderately to strongly. People had difficulty standing or walking. Felt moderately by people in moving vehicles.

VI-At this level, there must be reports of physical damage to man-made structures as described in the MM intensity scale. The only exception is that intensity VI is still assigned if many small objects fell from shelves and (or) many glassware items or dishes were broken.

VII-Only damage to buildings or other man-made structures (as described in the MMI scale) is considered.

VIII-XII-The MM intensity scale is used as written, except that neither landslides nor effects on people were used for assigning an intensity. Geologic effects were used only if the earthquake occurred in an isolated area having no nearby structures that could be damaged.

The "felt areas" listed in the tables were taken from different published sources or were estimated by the authors from previously published or newly drawn isoseismal or intensity maps; the felt areas are listed to the nearest $1,000 \mathrm{~km}^{2}$. When an earthquake occurred in a State bordering an ocean, Canada, or Mexico, the total felt area sometimes could not be estimated precisely. In those instances, the felt area was estimated only for the land area in the United States.

Isoseismal maps depict the extent of the felt area of an earthquake and separate the effects into areas of different levels of intensity. The outer isoseismal line defines the total, or projected, contiguous geographical area over which the earthquake was sensibly felt by people. The isoseismal maps that illustrate this compilation either are modified versions of previously published maps or are new maps prepared by the authors (see figs. 7-64).

New isoseismal maps also have been compiled from MMI values assigned by the authors after reevaluating original sources of data. These intensity values were assigned only to the effects of shaking; geologic effects alone were not considered unless the area was uninhabited and there were no structures to damage. However, maximum MM intensities assigned by other researchers to geologic effects in remote areas (mainly in Nevada) have not been changed:

The isoseismal maps contained in this publication were mostly selected to represent geographic coverage. If States had a number of damaging earthquakes, only the isoseismal maps for the most damaging events were included.

\section{SEISMICITY MAPS}

The seismicity and intensity maps for the United States, Alaska, Hawaii (figs. 1-6), and those at the beginning of each State chapter, show the areal distribution of earthquakes of magnitude 4.5 or larger. Those earthquakes are depicted by open symbols that increase in size with increase in magnitude or intensity.

To depict the earthquakes by magnitude on the maps, we assigned arbitrary magnitudes to events that had no computed magnitudes. This was accomplished by correlating the maximum intensity with the average magnitude of earthquakes in four geographical areas (Western U.S., Eastern U.S., Hawaii, and Alaska) that had both magnitudes and intensities. The results for four areas are listed below:

\begin{tabular}{|c|c|}
\hline \multicolumn{2}{|c|}{ Western United States } \\
\hline $\mathrm{MMI}$ & Magnitude \\
\hline $\mathrm{V}$ & $<5.0$ \\
\hline VI & 5.0 \\
\hline VII & 5.5 \\
\hline VIII & 6.0 \\
\hline IX & 6.5 \\
\hline $\mathrm{X}-\mathrm{XII}$ & 7.0 \\
\hline \multicolumn{2}{|c|}{ Eastern United States } \\
\hline $\mathrm{MMI}$ & Magnitude \\
\hline VI & $<5.0$ \\
\hline VII & 5.0 \\
\hline VIII & 5.5 \\
\hline IX & 6.0 \\
\hline $\mathrm{X}-\mathrm{XII}$ & 6.5 \\
\hline \multicolumn{2}{|c|}{ Hawaii } \\
\hline $\mathrm{MMI}$ & Magnitude \\
\hline $\mathrm{V}$ & $<5.5$ \\
\hline VI & 5.5 \\
\hline VII & 6.0 \\
\hline VIII & 6.5 \\
\hline IX & 7.0 \\
\hline $\mathrm{X}-\mathrm{XII}$ & 7.5 \\
\hline \multicolumn{2}{|c|}{ Alaska } \\
\hline $\mathrm{MMI}$ & Magnitude \\
\hline $\mathrm{V}$ & $<6.0$ \\
\hline VI & 6.0 \\
\hline VII & 6.5 \\
\hline VIII & 7.0 \\
\hline IX & 7.5 \\
\hline X-XII & 8.0 \\
\hline
\end{tabular}

\section{DAMAGE SUMMARIES}

The effects of all earthquakes of MMI VI or higher are summarized for each State, and descriptive 
information that substantiates the maximum intensity assigned to each earthquake is listed. The data sources used to compile the effects (see table 1) are listed by source-reference numbers in parentheses at the end of each earthquake description. For example, "(Ref. 1, 2, 250)" means that those three references were used to compile that particular summary, including the epicenter.

Earthquakes are listed chronologically by date and time of occurrence (in Universal Coordinated TimeUTC). When the UTC date differs from the local date (i.e., the date/time of the earthquake at its epicenter), the local date is given in parentheses following the UTC date. When more than one earthquake occurred on the same date, origin times are repeated after the date to prevent ambiguity (HST, HawaiiAleutian standard time; AST, Alaska standard time; PST, Pacific standard time; MST, Mountain standard time; CST, Central standard time; EST; Eastern standard time).

All published magnitude values cannot be listed in the tables because of lack of space or other reasons. However, those available values that are not listed are included at the end of the earthquake summaries, e.g., Magnitude 7.0 $\mathrm{M}_{\mathrm{S}} \mathrm{GR}-\mathrm{GR}$ refers to a magnitude reference code; these codes are listed in table 2.

Dollar estimates of property damage are available for most destructive earthquakes. These estimates, where quoted, use the dollar value for the year in which the earthquake occurred. Table 3 lists the numbers of deaths caused by destructive U.S. earthquakes.

Information on the effects of many large earthquakes in Alaska's Aleutian Islands is not commonly available because of the remoteness of that region and the sparseness of its population. Therefore, the summaries for many large-magnitude earthquakes in Alaska give only date, region of occurrence, and magnitude.

\section{EXPLANATION OF TABLES}

Each State chapter includes a table that lists the basic parameters of the principal earthquakes in that State. Dates and origin times for each earthquake have been converted to the date and time of the Greenwich meridian and labeled as Universal Coordinated Time (UTC). The origin times for some earthquakes may differ from that given in the source reference. If so, the listed origin time was estimated using more accurate sources of data, such as seismograms, earthquake phase data, U.S. Weather Service reports, or other data sources.

Latitude and longitude values are listed in decimal degrees. The number of digits following the decimal point does not indicate the level of accuracy, however: it only duplicates the value published in the original source reference.

More than one location has been published for many earthquakes in the United States. We, therefore, have selected for this publication the epicenter/ hypocenter that, in our judgment, most accurately represents the location of each earthquake. Some of these epicenters either have been revised from those previously published or have been assigned new, previously unpublished epicenters, based on felt-area descriptions and locations of damage. All epicenters/ hypocenters included in the tables have been given a reference number (see table 1).

Only in recent years has the depth of an earthquake in the United States been reliably computed. Therefore, the State tables do not give depth values for most pre-1930 earthquakes.

Magnitudes are listed for all earthquakes that had a published value. Some erroneous $m_{b}$ magnitudes computed and published in the Preliminary Determination of Epicenters during the 1960's have been eliminated. Published magnitudes that were based on maximum MM intensity, however, are not included. Many original magnitudes have been recomputed on the basis of recent research and new computational techniques. Consequently, magnitudes selected to be listed in the State chapters are values that the authors believe most accurately represent the "size" of the earthquake. The $\mathrm{M}_{\mathrm{fa}}$ magnitudes, identified by the code "SC" in the tables for States east of the Rocky Mountains, were computed by the authors using the intensity-felt area relation of Sibol and others (1987). When magnitudes were published as a range (i.e., $6-61 / 4$ ), the average of this range is the value listed in the tables. Additional magnitudes are listed in parentheses at the end of the summary of effects for many earthquakes.

The alphabetic codes associated with the magnitudes represent the source reference for that magnitude. These alphabetic codes are defined in table 2 . West of longitude $126 \mathrm{~W}$. (offshore from California, Oregon, and Washington) only earthquakes with magnitudes $\geq 5.5$ were included.

The MMI values given in the State tables represent the maximum intensity observed for those earthquakes listed. However, for a few earthquakes in Canada or Mexico that caused damage in the United States, the maximum intensity given is that observed nearest the epicenter. If that maximum intensity is not known, the maximum intensity observed in the United States is then listed (see the State chapter for details). A "Felt" in the MM intensity column indicates that insufficient information was available to assign an intensity value. The 
source references for intensity are listed by number in the State tables and defined in table 1.

The felt area given in the tables should be considered only as an estimate because the limit of perceptibility of shaking requires detailed reports that are unavailable for many earthquakes.

\section{MODIFIED MERCALLI INTENSITY SCALE OF 1931}

(From Wood and Neumann, 1931)

I Not felt-or, except rarely under especially favorable circumstances.

Under certain conditions, at and outside the boundary of the area in which a great shock is felt: sometimes birds, animals, reported uneasy or disturbed; sometimes dizziness or nausea experienced; sometimes trees, structures, liquids, bodies of water, may sway-doors may swing very slowly.

II Felt indoors by few, especially on upper floors, or by sensitive, or nervous persons.

Also, as in grade I, but often more noticeably: sometimes hanging objects may swing, especially when delicately suspended; sometimes trees, structures, liquids, bodies of water may sway; doors may swing very slowly; sometimes birds, animals reported uneasy or disturbed; sometimes dizziness or nausea experienced.

III Felt indoors by several, motion usually rapid vibration.

Sometimes not recognized to be an earthquake at first. Duration estimated in some cases. Vibration like that due to passing of light, or lightly loaded trucks, or heavy trucks some distance away. Hanging objects may swing slightly. Movements may be appreciable on upper levels of tall structures. Rocked standing motor cars slightly.

IV Felt indoors by many, outdoors by few.

Awakened few, especially light sleepers. Frightened no one, unless apprehensive from previous experience. Vibration like that due to passing of heavy or heavily loaded trucks. Sensation like heavy body striking building or falling of heavy objects inside. Rattling of dishes, windows, doors; glassware and crockery clink and clash. Creaking of walls, frame, especially in the upper range of this grade. Hanging objects swung, in numerous instances. Disturbed liquids in open vessels slightly. Rocked standing motor cars noticeably.

V Felt indoors by practically all, outdoors by many or most: outdoors direction estimated.

Awakened many, or most. Frightened few-slight excitement, a few ran outdoors. Buildings trembled throughout. Broke dishes, glassware, to some extent. Cracked windows-in some cases, but not generally. Overturned vases, small or unstable objects, in many instances, with occasional fall. Hanging objects, doors, swing generally or considerably. Knocked pictures against walls, or swung them out of place. Opened, or closed, doors, shutters, abruptly. Pendulum clocks stopped, started or ran fast, or slow. Moved small objects, furnishings, the latter to slight extent. Spilled liquids in small amounts from well-filled open containers. Trees, bushes, shaken slightly.

VI Felt by all, indoors and outdoors.

Frightened many, excitement general, some alarm, many ran outdoors. Awakened all. Persons made to move unsteadily. Trees, bushes, shaken slightly to moderately. Liquid set in strong motion. Small bells rang-church, chapel, school, etc. Damage slight in poorly built buildings. Fall of plaster in small amount. Cracked plaster somewhat, especially fine cracks in chimneys in some instances. Broke dishes, glassware, in considerable quantity, also some windows. Fall of knickknacks, books, pictures. Overturned furniture in many instances. Moved furnishings of moderately heavy kind.

VII Frightened all-general alarm, all ran outdoors.

Some, or many, found it difficult to stand. Noticed by persons driving motor cars. Trees and bushes shaken moderately to strongly. Waves on ponds, lakes, and running water. Water turbid from mud stirred up. Incaving to some extent of sand or gravel stream banks. Rang large church 
bells, etc. Suspended objects made to quiver. Damage negligible in buildings of good design and construction, slight to moderate in well-built ordinary buildings, considerable in poorly built or badly designed buildings, adobe houses, old walls (especially where laid up without mortar), spires, etc. Cracked chimneys to considerable extent, walls to some extent. Fall of plaster in considerable to large amount, also some stucco. Broke numerous windows, furniture to some extent. Shook down loosened brickwork and tiles. Broke weak chimneys at the roofline (sometimes damaging roofs). Fall of cornices from towers and high buildings. Dislodged bricks and stones. Overturned heavy furniture, with damage from breaking. Damage considerable to concrete irrigation ditches.

VIII Fright general-alarm approaches panic.

Disturbed persons driving motor cars. Trees shaken strongly-branches, trunks, broken off, especially palm trees. Ejected sand and mud in small amounts. Changes: temporary, permanent; in flow of springs and wells; dry wells renewed flow; in temperature of spring and well waters. Damage slight in structures (brick) built especially to withstand earthquakes. Considerable in ordinary substantial buildings, partial collapse: racked, tumbled down, wooden houses in some cases; threw out panel walls in frame structures, broke off decayed piling. Fall of walls. Cracked, broke, solid stone walls seriously. Wet ground to some extent, also ground on steep slopes. Twisting, fall, of chimneys, columns, monuments, also factory stacks, towers. Moved conspicuously, overturned, very heavy furniture.

IX Panic general.

Cracked ground conspicuously. Damage considerable in (masonry) structures built especially to withstand earthquakes: threw out of plumb some wood-frame houses built especially to withstand earthquakes; great in substantial (masonry) buildings, some collapse in large part; or wholly shifted frame buildings off foundations, racked frames; serious to reservoirs; underground pipes sometimes broken.
Cracked ground, especially where loose and wet, up to widths of several inches; fissures up to a yard in width ran parallel to canal and stream banks.

Landslides considerable from river banks and steep coasts. Shifted sand and mud horizontally on beaches and flat land. Changed level of water in wells. Threw water on banks of canals, lakes, rivers, etc. Damage serious to dams, dikes, embankments. Severe to well-built wooden structures and bridges, some destroyed. Developed dangerous cracks in excellent brick walls. Destroyed most masonry and frame structures, also their foundations. Bent railroad rails slightly. Tore apart, or crushed endwise, pipelines buried in earth. Open cracks and broad wavy folds in cement pavements and asphalt road surfaces.

XI Disturbances in ground many and widespread varying with ground material.

Broad fissures, earth slumps, and land slips in soft, wet ground. Ejected water in large amounts charged with sand and mud. Caused sea waves ("tidal" waves) of significant magnitude. Damage severe to woodframe structures, especially near shock centers. Great to dams, dikes, embankments often for long distances. Few, if any (masonry) structures remained standing. Destroyed large well-built bridges by the wrecking of supporting piers, or pillars. Affected yielding wooden bridges less. Bent railroad rails greatly, and thrust them endwise. Put pipelines buried in earth completely out of service.

XII Damage total-practically all works of construction damaged greatly or destroyed.

Disturbances in ground great and varied, numerous shearing cracks. Landslides, falls of rock of significant character, slumping of river banks, etc., numerous and extensive. Wrenched loose, tore off, large rock masses. Fault slips in firm rock, with notable horizontal and vertical offset displacements. Water channels, surface and underground, disturbed and modified greatly. Dammed lakes, produced waterfalls, deflected rivers, etc. Waves seen on ground surfaces (actually seen, probably, in some cases). Distorted lines of sight. Threw objects upward into the air. 


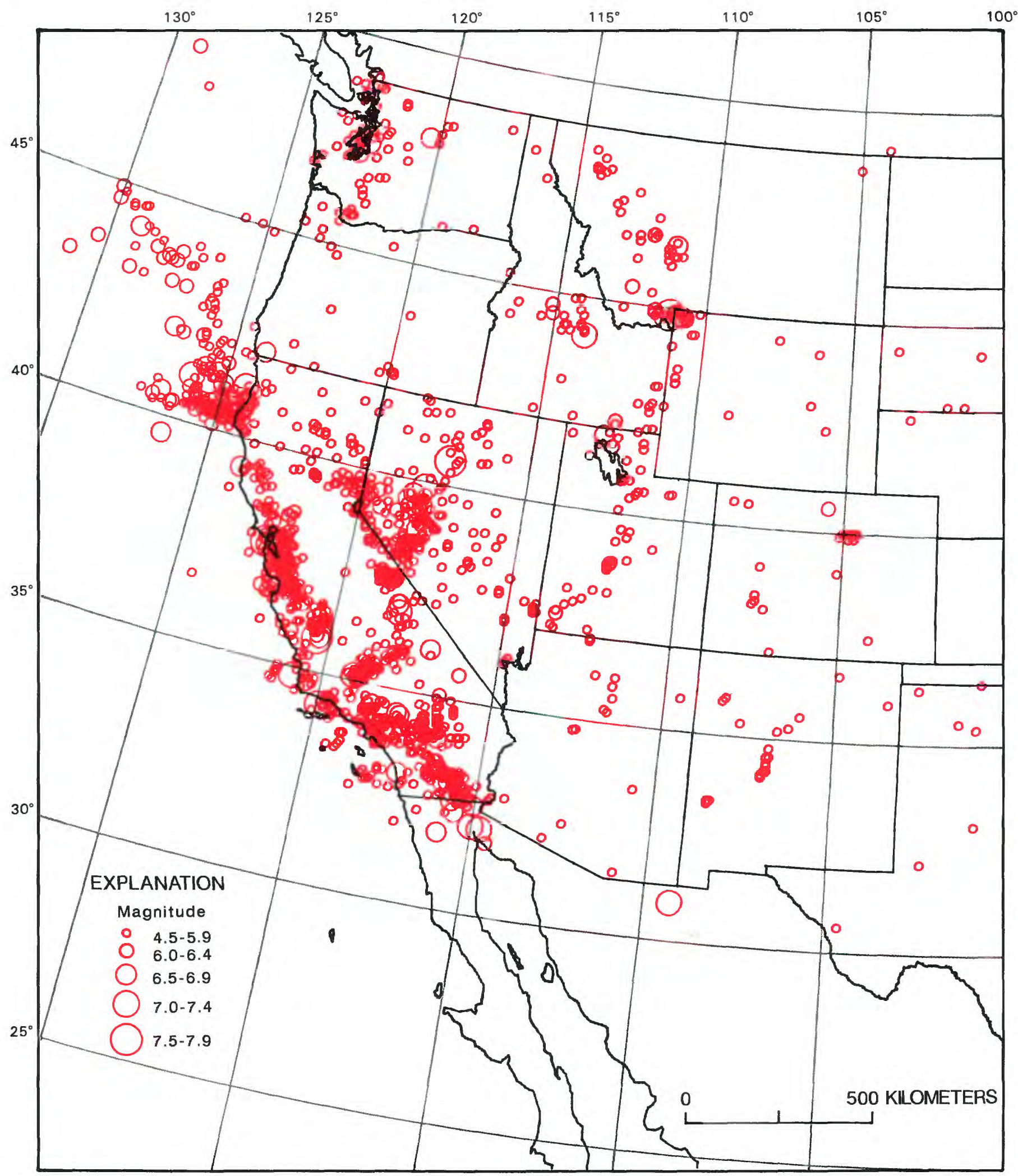

FIGURE 1.-Locations of magnitude $\geq 4.5$ or damaging earthquakes in the conterminous Western United States, 1769-1989. 


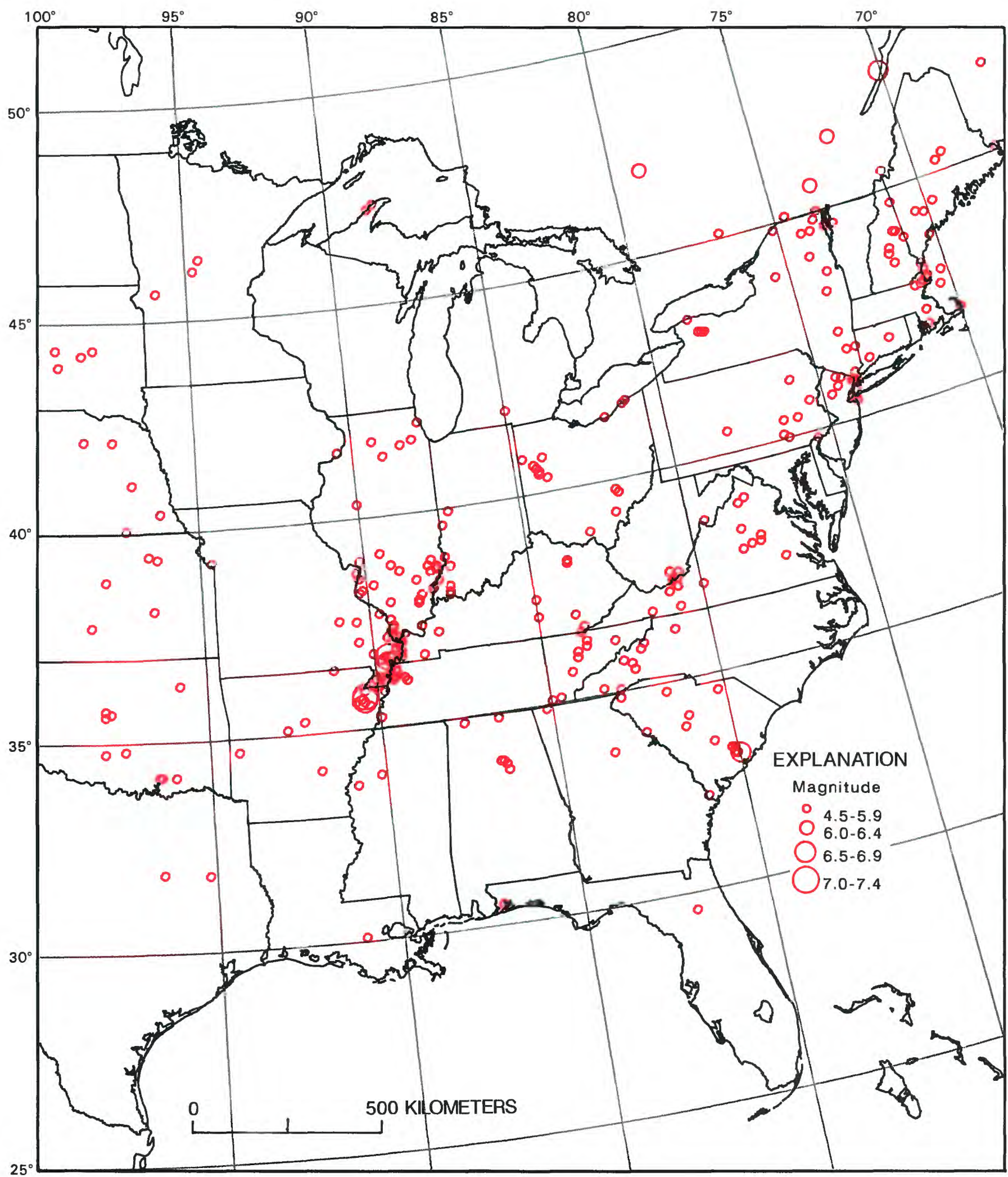

FIGURE 2.-Locations of magnitude $\geq 4.5$ or damaging earthquakes in the conterminous Eastern United States, 1568-1989. 


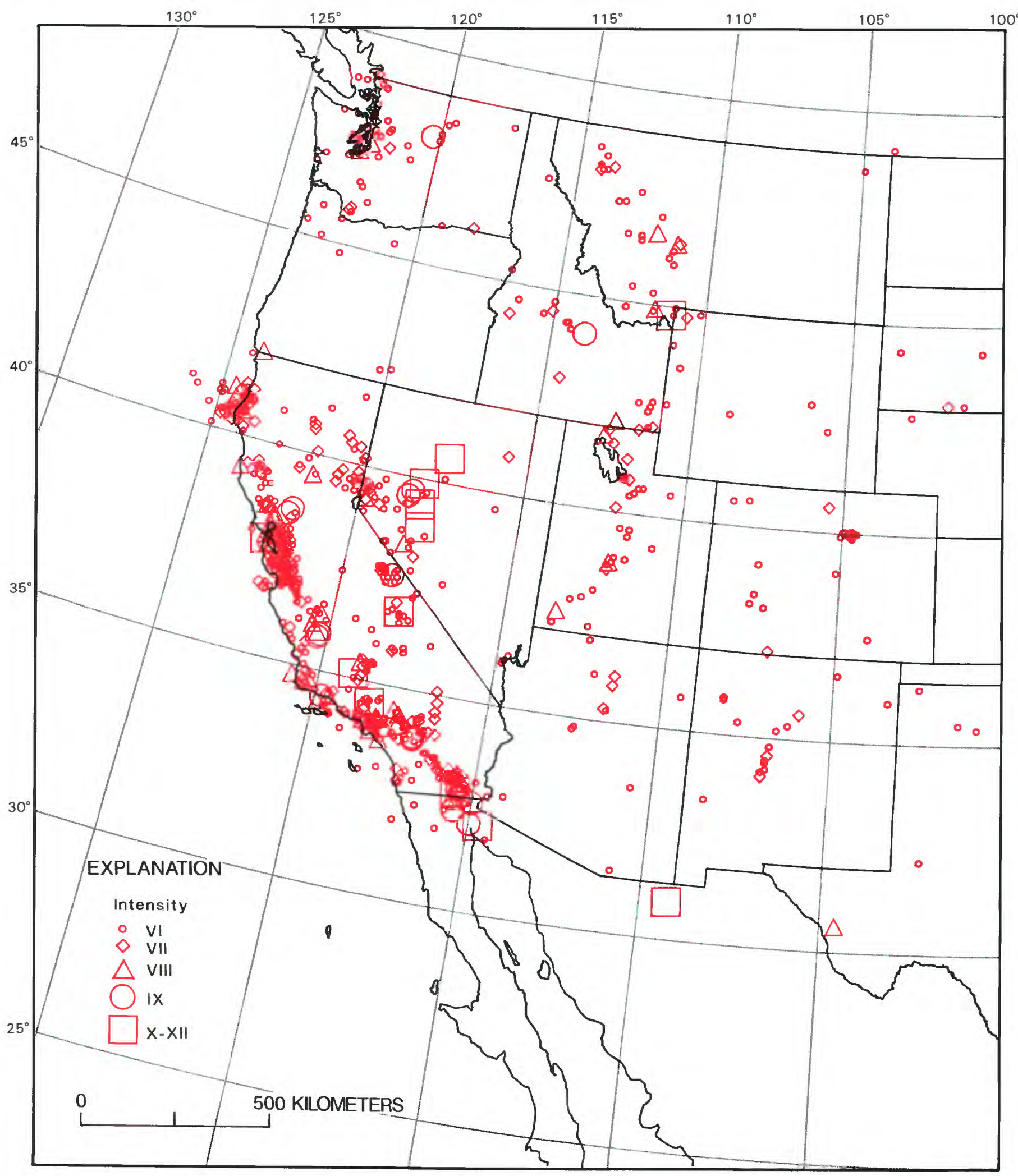

FIGURE 3.-Locations of earthquakes causing damage (MMI $\geq$ VI) in the conterminous Western United States, $1769-1989$. 


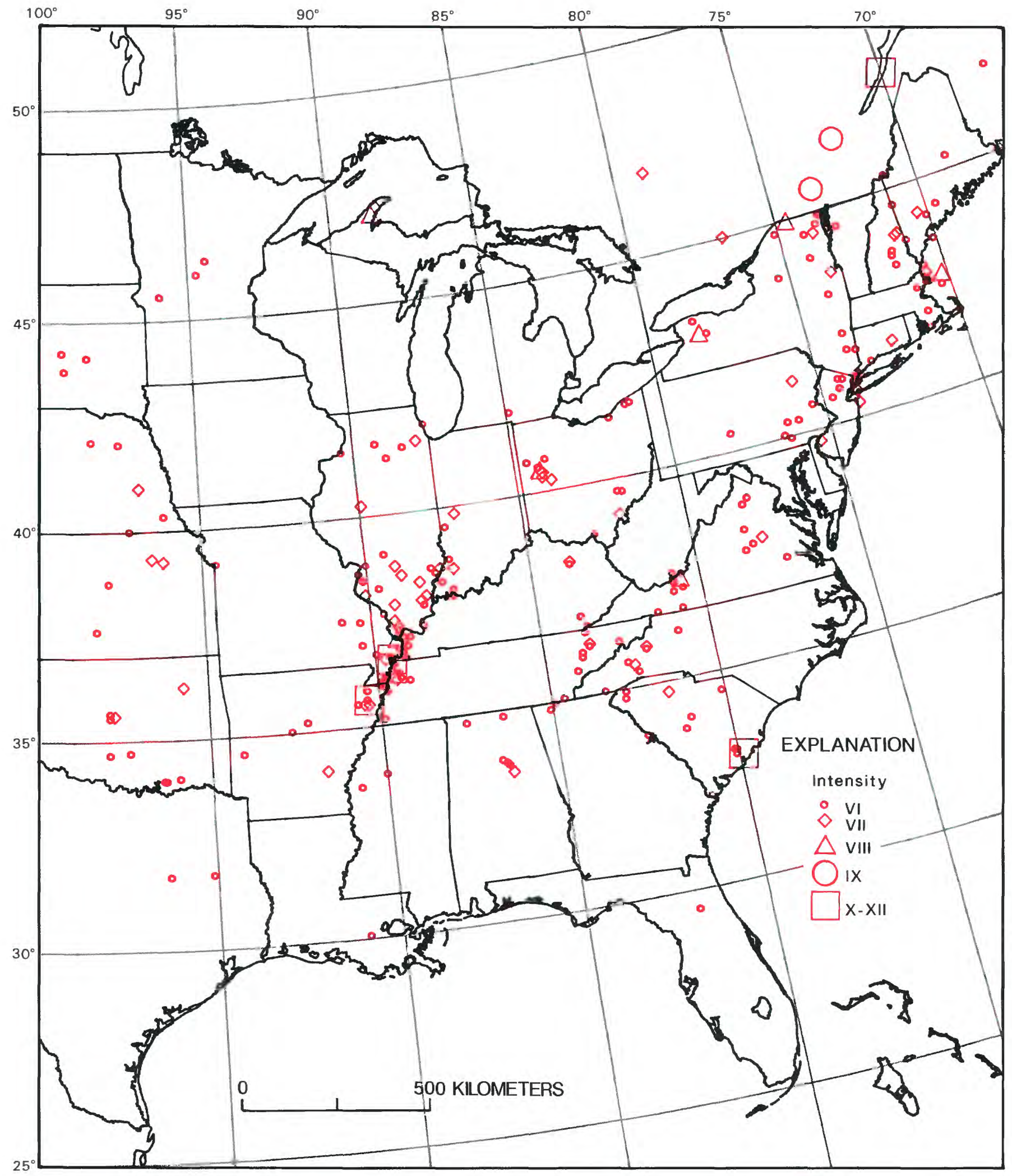

FIGURE 4.-Locations of earthquakes causing damage (MMI $\geq$ VI) in the conterminous Eastern United States, $1568-1989$. 


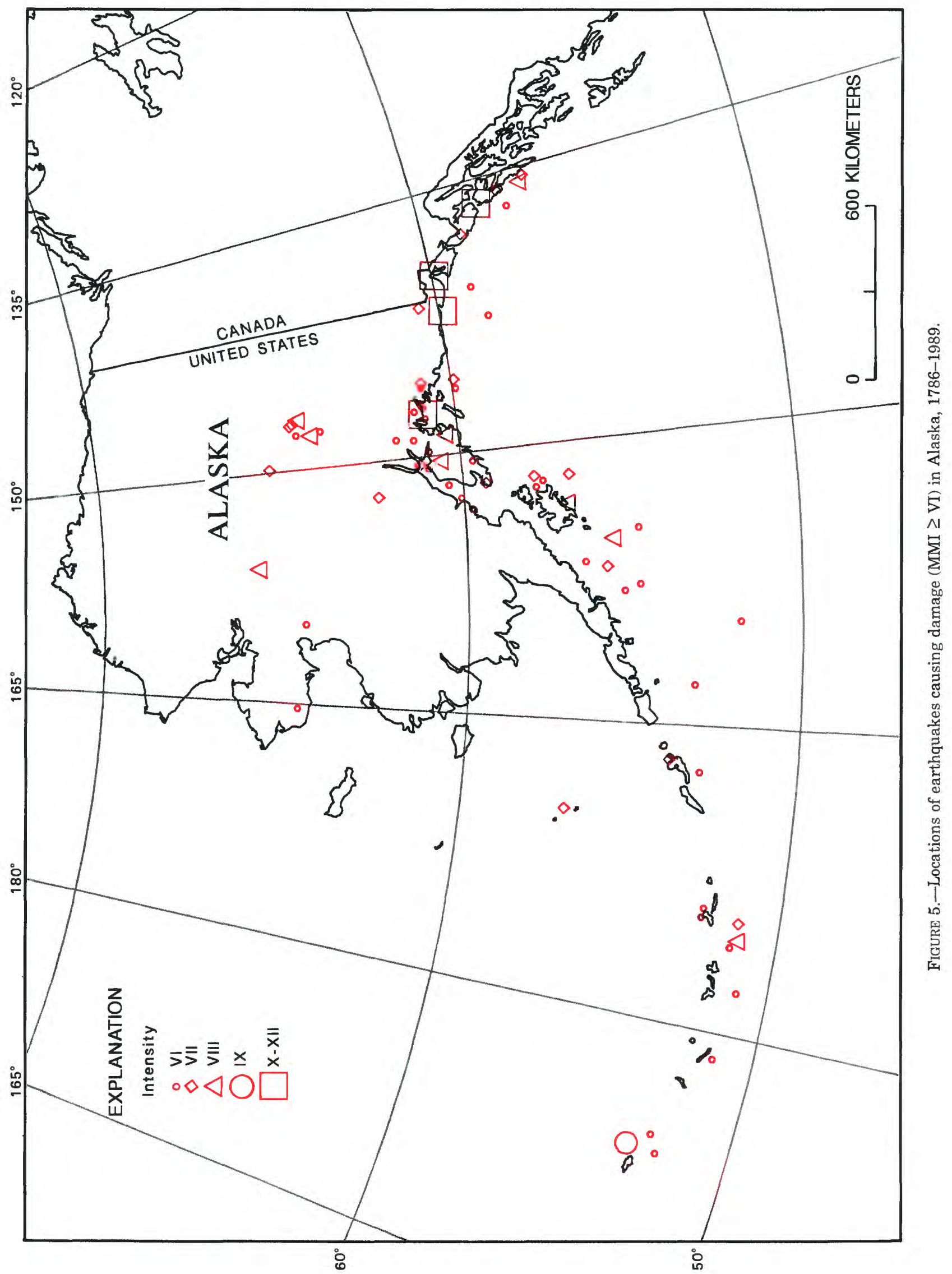




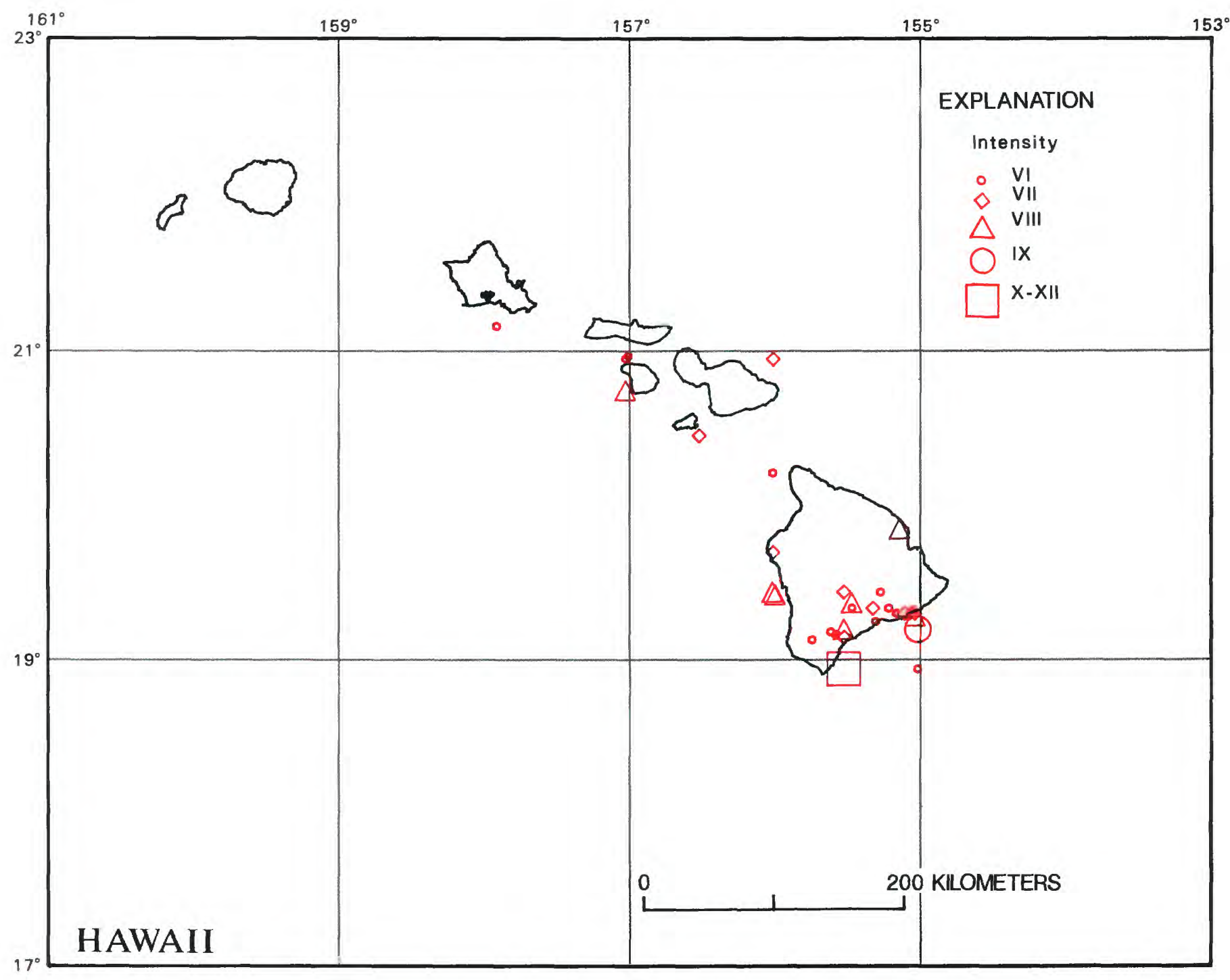

FIGURE 6.-Locations of earthquakes causing damage (MMI $\geq$ VI) in Hawaii, 1834-1989. 


\section{REFERENCES CITED}

Barosh, P.J., 1969, Use of seismic intensity data to predict the effects of earthquakes and underground nuclear explosions in various geologic settings: U.S. Geological Survey Bulletin $1279,93 \mathrm{p}$.

Ba:th, Markus, 1966, Earthquake energy and magnitude, in Physiss and Chemistry of the Earth, v. 7, New York, Pergamon Press, p. 115-165.

Bolt, B.A., and Herraiz, Miguel, 1983, Simplified estimation of seismic moment from seismograms: Seismological Society of America Bulletin, v. 73, no. 3, p. 735-748.

Coffman, J.L., von Hake, C.A., and Stover, C.W., 1982, Earthquake history of the United States: U.S. Department of Commerce,National Oceanic and Atmospheric Administration, and U.S. Department of the Interior, Geological Survey, Publication 411, revised edition [through 1980], $258 \mathrm{p}$.

Dziewonski, A.M., Chou, T.A., and Woodhouse, J.H., 1981, Determination of earthquake parameters from waveform data for studies of global and regional seismicity: Journal of Geophysical Research, v. 86, no. B4, p. 2825-2852.

Gutenberg, Beno, and Richter, C.F., 1956, Magnitude and energy of earthquakes: Annali di Geofisica, v. 9, no. 1, p. 1-15.

Hanks, T.C., and Kanamori, Hiroo, 1979, A moment magnitude scale: Journal of Geophysical Research, v. 84, no. B5, p. 2348-2350.
Nuttli, O.W., 1973, Seismic-wave attenuation and magnitude relations for eastern North America: Journal of Geophysical Research, v. 78, no. 5, p. 876-885.

1983, Average seismic source-parameter relations for midplate earthquakes: Seismological Society of America Bulletin, v. 73 , no. 2 , p. $519-535$.

Nuttli, O.W., and Zollweg, J.E., 1974, The relation between felt area and magnitude for Central United States earthquakes: Seismological Society of America Bulletin, v. 64, no. 1, p. 73-85.

Rothé, J.P., 1969, The Seismicity of the Earth, 1953-1965: Paris, France, United Nations Educational, Scientific, and Cultural Organization [UNESCO], $336 \mathrm{p}$.

Richter, C.F., 1958, Elementary Seismology, San Francisco and London, W.H. Freeman and Company, 768 p.

Sibol, M.S., Bollinger, G.A., and Birch, J.B., 1987, Estimation of magnitudes in central and eastern North America using intensity and felt area: Seismological Society of America Bulletin, v. 77 , no. 5 , p. $1635-1654$.

Toppozada, T.R., 1975, Earthquake magnitude as a function of intensity data in California and western Nevada: Seismological Society of America Bulletin, v. 65, no. 5, p. 1223-1238.

Wood, H.O., and Neumann, Frank, 1931, Modified Mercalli intensity scale of 1931: Seismological Society of America Bulletin, v. 21 , no. 4 , p. $277-283$. 


\section{ALABAMA}

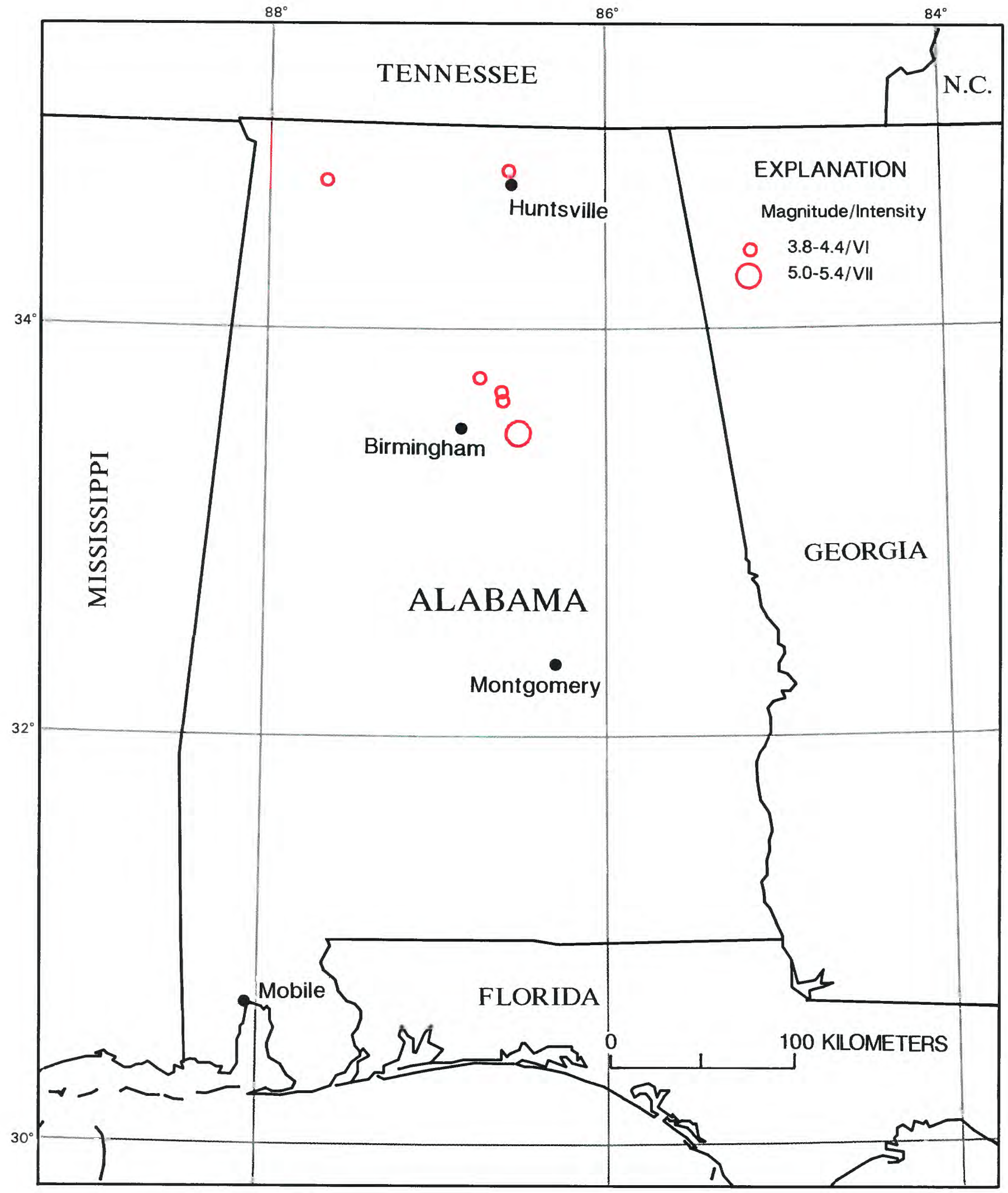

Earthquakes in Alabama with magnitudes $\geq 4.5$ or intensity $\geq V$ V. 
ALABAMA

[See table 1 for hypocenter and intensity references and table 2 for definitions of magnitude source codes. Leader ( - ) indicates information is not available]

\begin{tabular}{|c|c|c|c|c|c|c|c|c|c|c|c|c|c|}
\hline \multirow{2}{*}{\multicolumn{2}{|c|}{ Date }} & \multirow{2}{*}{$\begin{array}{c}\text { Origin } \\
\text { time (UTC) }\end{array}$} & \multicolumn{4}{|c|}{ Hypocenter } & \multicolumn{4}{|c|}{ Magnitude } & \multicolumn{2}{|c|}{ Intensity } & \multirow{3}{*}{$\begin{array}{l}\text { Felt area } \\
\left(1,000 \mathrm{~km}^{2}\right)\end{array}$} \\
\hline & & & \multirow{2}{*}{$\begin{array}{l}\text { Latitude } \\
\left({ }^{\circ}\right)\end{array}$} & \multirow{2}{*}{$\begin{array}{l}\text { Longitude } \\
\left(^{\circ}\right)\end{array}$} & \multirow{2}{*}{$\begin{array}{c}\text { Depth } \\
(\mathrm{km})\end{array}$} & \multirow[t]{2}{*}{ Ref } & \multicolumn{2}{|c|}{ USGS } & \multirow[t]{2}{*}{ Other } & \multirow{2}{*}{$\begin{array}{c}\text { Moment } \\
\text { M }\end{array}$} & \multirow{2}{*}{ MM } & \multirow{2}{*}{ Ref } & \\
\hline $\mathrm{Yr}$ & Mo Da & $\mathrm{h} \mathrm{m} s$ & & & & & $m_{b}$ & $M_{s}$ & & & & & \\
\hline 1916 & 1018 & 2204 & $33.5 \mathrm{~N}$ & $86.5 \mathrm{~W}$ & - & 272 & - & - & $5.10 \mathrm{M}_{\mathrm{fa}} \mathrm{SC}$ & - & VII & 38 & 384 \\
\hline 1931 & 0505 & 1218 & $33.7 \mathrm{~N}$ & $86.6 \mathrm{~W}$ & - & 38 & - & - & $4.00 \mathrm{M}_{\mathrm{fa}} \mathrm{SC}$ & - & VI & 38 & 17 \\
\hline 1957 & 0423 & 092339.0 & $33.770 \mathrm{~N}$ & $86.723 W$ & 005 & 349 & - & - & $4.10 \mathrm{M}_{\mathrm{fa}} \mathrm{SC}$ & - & VI & 30 & 28 \\
\hline 1959 & $\begin{array}{ll}08 & 12\end{array}$ & 180601.4 & $34.789 \mathrm{~N}$ & $86.562 \mathrm{~W}$ & 005 & 349 & - & - & $3.80 \mathrm{M}_{\mathrm{fa}} \mathrm{SC}$ & - & VI & 32 & 7 \\
\hline 1975 & 0829 & 042252.1 & $33.659 \mathrm{~N}$ & $86.588 \mathrm{~W}$ & 004 & 349 & 3.5 & - & $4.40 \mathrm{M}_{\mathrm{X}} \mathrm{JLM}$ & - & VI & 48 & 25 \\
\hline 1989 & 0820 & 000317.8 & $34.736 \mathrm{~N}$ & $87.645 \mathrm{~W}$ & 010 & 74 & - & - & $3.90 \mathrm{M}_{\mathrm{n}}$ GS & - & VI & 579 & 6 \\
\hline
\end{tabular}

[Reference (Ref.) numbers given in parentheses at the end of each description refer to sources of data in table 1. Magnitude values are described in the Introduction, and codes are defined in table 2.]

1916. Oct. 18. Irondale, Jefferson County, Ala. On the basis of the number of chimneys destroyed, this earthquake was more severe in Irondale than in any other town between Easonville and Birmingham. At Irondale, about $5 \mathrm{~km}$ north of Birmingham, 14 chimneys in a two-block area were partly destroyed, and six chimneys on a brick store were leveled almost to the roof. Many other chimneys either were leveled to the roofs or were cracked so badly that they had to be rebuilt. At Pell City, a few bricks were dislocated from one of the courthouse chimneys, and near Easonville, a few chimneys were damaged lightly. Poorly built chimneys on the eastern edge of Birmingham were damaged heavily.

A careful study of the Red Gap fault, which extends from near Gate City to beyond Irondale, did not reveal direct evidence of recent earth movement. The most significant geologic result was the effect of the earthquake on underground water, particularly in Irondale. Five wells in a one-block area of Irondale went dry immediately after the shock, and the water level in many others was lowered. At Pell City, the shock lowered the water level in one well about 50 $\mathrm{cm}$. Several small aftershocks occurred through Oct. 28. Also felt in Georgia, Indiana, Kentucky, Mississippi, North Carolina, South Carolina, and Tennessee (see fig. 7). (Ref. 38, 105, 272, 508.)

1931. May 5. Near Birmingham, Jefferson County, Ala. This earthquake knocked bricks from a chimney at Birmingham, and shook objects from walls of a blacksmith shop at Cullman. Also felt in
Georgia and possibly in South Carolina. (Ref. 38, 105, 508.)

1957. Apr. 23. Near Birmingham, Jefferson County, Ala. Several chimneys sustained minor damage at Birmingham; concrete steps were cracked and several small cracks formed on interior walls. Items on tables tumbled to the floor. Also felt in Georgia and Tennessee. Magnitude 4.2 $\mathrm{M}_{\mathrm{fa}} \mathrm{BAR}, 4.2$ $\mathrm{M}_{\mathrm{fa}}$ DG. (Ref. 30, 349, 508.)

1959. Aug. 12. Hazel Green, Madison County, Ala. This earthquake was strongest in Madison County in northern Alabama. North of Huntsville, at Hazel Green, bricks toppled from chimneys and alarmed residents ran from their houses. One chimney and a new concrete-block building were damaged at nearby Meridianville. Plaster cracked slightly at Huntsville, and merchandise was thrown from shelves. Also felt at several towns in Tennessee. Magnitude $3.8 \mathrm{M}_{\mathrm{n}} \mathrm{BAR}, 3.8 \mathrm{M}_{\mathrm{fa}}$ DG. (Ref. 32, 349, 508.)

1975. Aug. 29 (Aug. 28). Palmerdale, Jefferson County, Ala. The earthquake cracked a sheetrock ceiling and shifted lamps on tables at Palmerdale, north of Birmingham. It caused slight damage at Watson, where furniture was displaced slightly. Also felt in southern Tennessee. Magnitude 4.4 $\mathrm{M}_{\mathrm{n}}$ SLM. (Ref. 48, 349.)

1989. Aug. 20 (Aug. 19). Near Littleville, Colbert County, Ala. A Colbert County official reported that, south of Florence between Littleville and Russellville, a basement wall collapsed beneath a house. Only slight damage was reported north of the epicenter at Florence, where windows were cracked and hairline cracks formed in plaster. Also felt in Lauderdale, Lawrence, and Morgan Counties in northwest Alabama and Lawrence County in south-central Tennessee. (Ref. 74, 579.) 


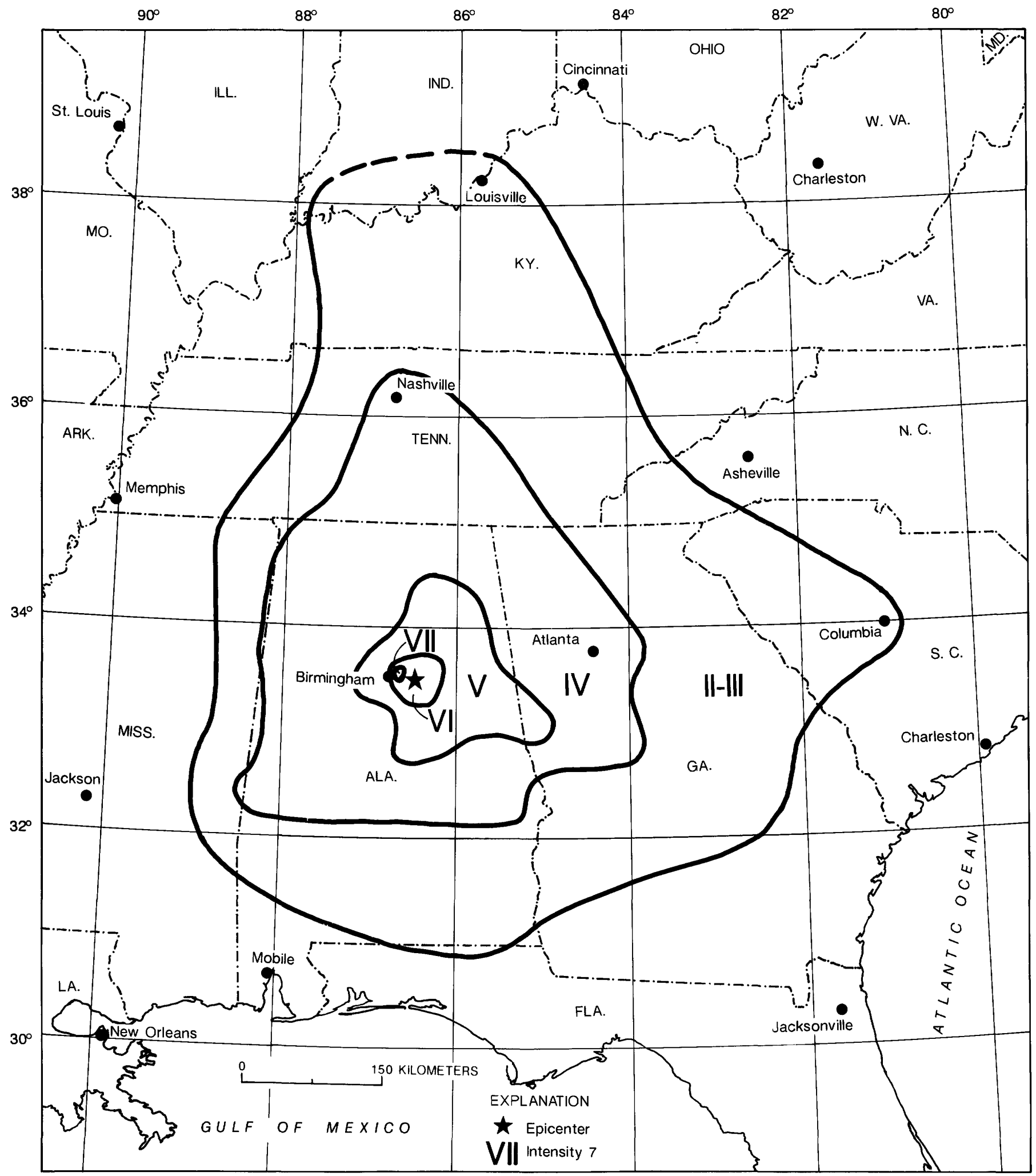

FIGURE 7.-Isoseismal map for the Alabama earthquake of October 18, 1916. Isoseismals are based on intensity estimates from data listed in reference 272 of table 1. 



\section{ALASKA}

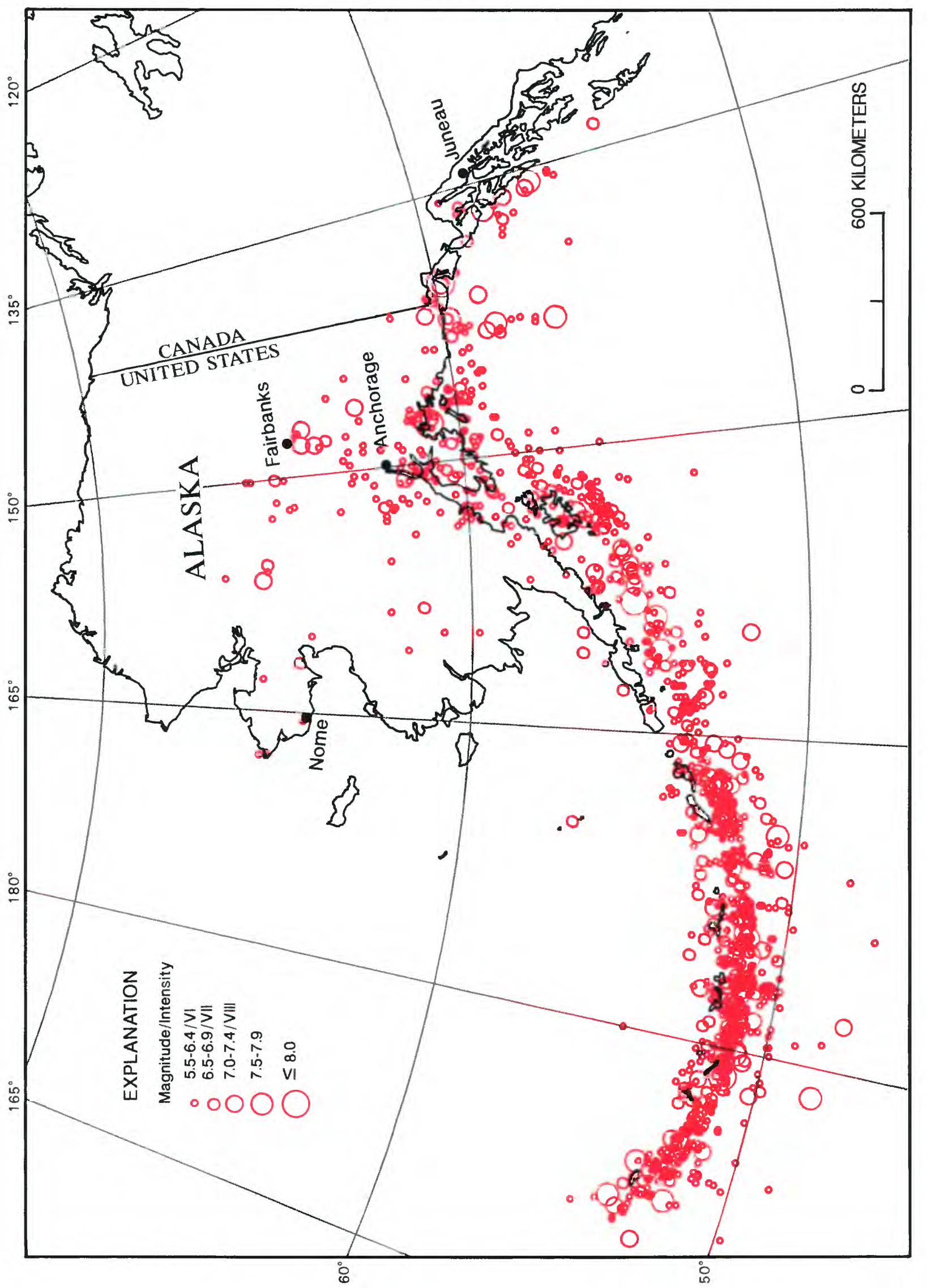

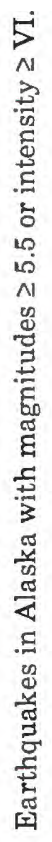


ALASKA

[See table 1 for hypocenter and intensity references and table 2 for definitions of magnitude source codes. \&, land area only. Leader (--) indicates information is not available]

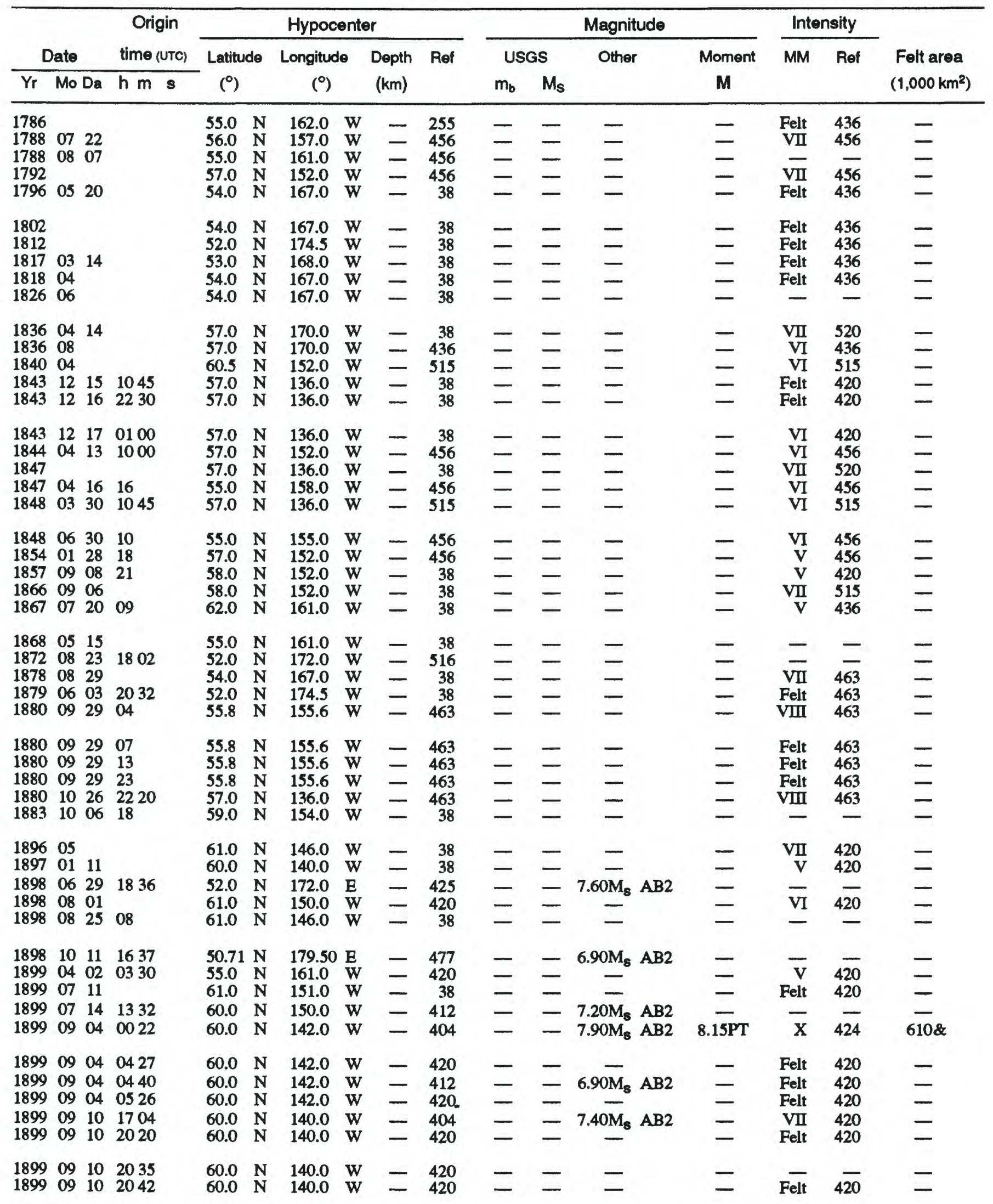


ALASKA-Continued

[See table 1 for hypocenter and intensity references and table 2 for definitions of magnitude source codes. \&, land area only. Leader (-.) indicates information is not available]

\begin{tabular}{|c|c|c|c|c|c|c|c|c|c|c|c|c|c|c|c|c|}
\hline & & & & & & Hypoce & enter & & & & & Magnitude & & Inte & sity & \\
\hline & late & & time (UTC) & Latitud & & Longitude & & Depth & Ref & US & & Other & Moment & MM & Ref & Felt area \\
\hline Yr & Mo D & & $\mathrm{h} m \mathrm{~s}$ & $\left({ }^{\circ}\right)$ & & ( $\left.{ }^{\circ}\right)$ & & (11) & & $m_{b}$ & $\mathbf{M}_{\mathbf{s}}$ & & $M$ & & &, $\left.000 \mathrm{~km}^{2}\right)$ \\
\hline $\begin{array}{l}1899 \\
1899 \\
1899\end{array}$ & $\begin{array}{l}09 \\
09 \\
09\end{array}$ & $\begin{array}{l}10 \\
10 \\
10\end{array}$ & $\begin{array}{l}2047 \\
2141 \\
2149\end{array}$ & $\begin{array}{l}60.0 \\
60.0 \\
60.0\end{array}$ & $\begin{array}{l}\mathbf{N} \\
\mathbf{N} \\
\mathbf{N}\end{array}$ & $\begin{array}{l}140.0 \\
140.0 \\
140.0\end{array}$ & $\begin{array}{l}\mathbf{W} \\
\mathbf{W} \\
\mathbf{W}\end{array}$ & - & $\begin{array}{l}420 \\
404 \\
420\end{array}$ & - & - & $\underset{8.00 \overline{\mathrm{M}}_{\mathrm{s}} \mathrm{AB2}}{-}$ & ${ }^{8.15 \mathrm{PT}}$ & $\underset{\text { Felt }}{\overline{X I}}$ & $\begin{array}{l}-\overline{420} \\
420\end{array}$ & $\overline{700 \&}$ \\
\hline $\begin{array}{l}1899 \\
1899 \\
1899 \\
1899 \\
1899\end{array}$ & $\begin{array}{l}09 \\
09 \\
09 \\
09 \\
09\end{array}$ & $\begin{array}{l}11 \\
11 \\
11 \\
16 \\
17\end{array}$ & $\begin{array}{ll}03 & 18 \\
0326 \\
0334 \\
0433 \\
1250\end{array}$ & $\begin{array}{l}60.0 \\
60.0 \\
60.0 \\
59.0 \\
59.0\end{array}$ & $\begin{array}{l}\mathbf{N} \\
\mathbf{N} \\
\mathbf{N} \\
\mathbf{N} \\
\mathbf{N}\end{array}$ & $\begin{array}{l}140.0 \\
140.0 \\
140.0 \\
138.0 \\
136.0\end{array}$ & $\begin{array}{l}\mathbf{W} \\
\mathbf{w} \\
\mathbf{W} \\
\mathbf{W} \\
\mathbf{w}\end{array}$ & $\begin{array}{l}- \\
- \\
-\end{array}$ & $\begin{array}{l}420 \\
420 \\
420 \\
420 \\
412\end{array}$ & $\begin{array}{l}- \\
- \\
-\end{array}$ & $\bar{z}$ & $\begin{array}{l}\bar{z} \\
\bar{Z} \\
6.90 \mathrm{M}_{\mathrm{s}} \mathrm{AB} 2\end{array}$ & $\begin{array}{l}- \\
- \\
-\end{array}$ & $\begin{array}{l}\text { Felt } \\
\text { Felt } \\
\text { Felt } \\
\text { VII } \\
\text { Felt }\end{array}$ & $\begin{array}{l}420 \\
420 \\
420 \\
420 \\
420\end{array}$ & $\begin{array}{l}- \\
- \\
-\end{array}$ \\
\hline $\begin{array}{l}1899 \\
1899 \\
1899 \\
1899 \\
1899\end{array}$ & $\begin{array}{l}092 \\
092 \\
09 \\
09 \\
09\end{array}$ & $\begin{array}{l}23 \\
23 \\
23 \\
26 \\
27\end{array}$ & $\begin{array}{ll}08 & 13 \\
1104 \\
1250 \\
1231 \\
0028\end{array}$ & $\begin{array}{l}55.0 \\
60.0 \\
59.0 \\
60.0 \\
60.0\end{array}$ & $\begin{array}{l}\mathbf{N} \\
\mathbf{N} \\
\mathbf{N} \\
\mathbf{N} \\
\mathbf{N}\end{array}$ & $\begin{array}{l}161.0 \\
143.0 \\
143.0 \\
143.0 \\
143.0\end{array}$ & $\begin{array}{l}\mathbf{w} \\
\mathbf{w} \\
\mathbf{w} \\
\mathbf{W} \\
\mathbf{w}\end{array}$ & $\begin{array}{l}- \\
- \\
-\end{array}$ & $\begin{array}{l}420 \\
412 \\
412 \\
420 \\
420\end{array}$ & $\begin{array}{l}- \\
- \\
-\end{array}$ & $\frac{-}{-}$ & $\begin{array}{c}-\overline{\mathrm{M}}_{\mathrm{s}} \mathrm{AB2} \\
7.00 \mathrm{M}_{\mathrm{s}} \mathrm{AB2} \\
-\end{array}$ & $\frac{-}{-}$ & $\begin{array}{c}\text { Felt } \\
\overline{-} \\
\text { Felt } \\
\text { Felt }\end{array}$ & $\begin{array}{l}420 \\
\overline{-} \\
420 \\
420\end{array}$ & $\begin{array}{l}- \\
- \\
-\end{array}$ \\
\hline $\begin{array}{l}1899 \\
1899 \\
1899 \\
1899 \\
1900\end{array}$ & $\begin{array}{l}12 \\
12 \\
12 \\
12 \\
02\end{array}$ & $\begin{array}{l}14 \\
20 \\
20 \\
21 \\
16\end{array}$ & $\begin{array}{l}0930 \\
1030 \\
1530 \\
0515 \\
2210\end{array}$ & $\begin{array}{l}60.0 \\
60.0 \\
60.0 \\
60.0 \\
60.0\end{array}$ & $\begin{array}{l}\mathbf{N} \\
\mathbf{N} \\
\mathbf{N} \\
\mathbf{N} \\
\mathbf{N}\end{array}$ & $\begin{array}{l}140.0 \\
140.0 \\
140.0 \\
140.0 \\
140.0\end{array}$ & $\begin{array}{l}\mathbf{w} \\
\mathbf{w} \\
\mathbf{w} \\
\mathbf{w} \\
\mathbf{w}\end{array}$ & $\begin{array}{l}- \\
- \\
-\end{array}$ & $\begin{array}{l}420 \\
420 \\
420 \\
420 \\
420\end{array}$ & $\begin{array}{l}- \\
-\end{array}$ & $\overline{-}$ & $\frac{-}{E}$ & $\begin{array}{l}- \\
- \\
-\end{array}$ & $\begin{array}{l}\text { Felt } \\
\text { Felt } \\
\text { Felt } \\
\text { Felt } \\
\text { Felt }\end{array}$ & $\begin{array}{l}420 \\
420 \\
420 \\
420 \\
420\end{array}$ & $\begin{array}{l}- \\
- \\
-\end{array}$ \\
\hline $\begin{array}{l}1900 \\
1900 \\
1901 \\
1901 \\
1901\end{array}$ & $\begin{array}{l}10 \\
12 \\
03 \\
12 \\
12\end{array}$ & $\begin{array}{l}09 \\
27 \\
30 \\
30\end{array}$ & $\begin{array}{l}1225 \\
1015\end{array}$ & $\begin{array}{l}57.09 \\
58.0 \\
65.0 \\
59.4 \\
52.0\end{array}$ & $\begin{array}{l}\mathbf{N} \\
\mathbf{N} \\
\mathbf{N} \\
\mathbf{N} \\
\mathbf{N}\end{array}$ & $\begin{array}{l}153.48 \\
150.0 \\
152.0 \\
153.5 \\
160.0\end{array}$ & $\begin{array}{l}\mathbf{W} \\
\mathbf{w} \\
\mathbf{W} \\
\mathbf{W} \\
\mathbf{w}\end{array}$ & $\overline{-}$ & $\begin{array}{r}477 \\
420 \\
38 \\
403 \\
426\end{array}$ & $\overline{-}$ & $\frac{-}{-}$ & $\begin{array}{c}7.70 \mathrm{M}_{\mathrm{s}} \mathrm{AB2} \\
\overline{-} \\
\overline{\mathrm{M}}_{\mathrm{s}} \mathrm{AB} 1\end{array}$ & $\begin{array}{c}7.85 \mathrm{PT} \\
- \\
- \\
-\end{array}$ & $\begin{array}{l}\text { VIII } \\
\text { Felt } \\
\text { Felt } \\
\text { Felt } \\
\text { VI }\end{array}$ & $\begin{array}{l}424 \\
420 \\
420 \\
403 \\
426\end{array}$ & $\begin{array}{l}310 \& \\
- \\
-\end{array}$ \\
\hline $\begin{array}{l}1901 \\
1902 \\
1903 \\
1903 \\
1903\end{array}$ & $\begin{array}{l}12 \\
01 \\
01 \\
02 \\
03\end{array}$ & $\begin{array}{l}31 \\
01 \\
17 \\
05\end{array}$ & $\begin{array}{l}090230.0 \\
052030.0 \\
1605 \\
1826\end{array}$ & $\begin{array}{l}51.45 \\
52.38 \\
50.85 \\
52.0 \\
61.0\end{array}$ & $\begin{array}{l}\mathbf{N} \\
\mathbf{N} \\
\mathbf{N} \\
\mathbf{N} \\
\mathbf{N}\end{array}$ & $\begin{array}{l}171.02 \\
167.45 \\
175.16 \\
175.0 \\
146.0\end{array}$ & $\begin{array}{l}\mathbf{W} \\
\mathbf{W} \\
\mathbf{W} \\
\mathbf{E} \\
\mathbf{W}\end{array}$ & $\begin{array}{l}- \\
z \\
-\end{array}$ & $\begin{array}{r}477 \\
404 \\
477 \\
425 \\
38\end{array}$ & $\begin{array}{l}- \\
- \\
-\end{array}$ & $\begin{array}{l}- \\
- \\
-\end{array}$ & $\begin{array}{cc}7.10 \mathrm{M}_{8} & \mathrm{AB2} \\
7.00 \mathrm{M}_{8} & \mathrm{AB2} \\
7.00 \mathrm{M}_{8} & \mathrm{AB2} \\
6.80 \mathrm{M}_{\mathrm{s}} & \mathrm{AB2} \\
- & \end{array}$ & $\begin{array}{l}- \\
- \\
-\end{array}$ & $\frac{-}{-}$ & $\frac{-}{\overline{-}}$ & $\begin{array}{l}- \\
- \\
-\end{array}$ \\
\hline $\begin{array}{l}1903 \\
1903 \\
1903 \\
1904 \\
1905\end{array}$ & $\begin{array}{l}06 \\
06 \\
07 \\
08 \\
02\end{array}$ & $\begin{array}{l}02 \\
03 \\
26 \\
27 \\
14\end{array}$ & $\begin{array}{l}1317 \\
0330 \\
\\
2156 \\
0846\end{array}$ & $\begin{array}{l}61.56 \\
61.0 \\
59.0 \\
64.66 \\
50.73\end{array}$ & $\begin{array}{l}\mathbf{N} \\
\mathbf{N} \\
\mathbf{N} \\
\mathbf{N} \\
\mathbf{N}\end{array}$ & $\begin{array}{l}158.54 \\
146.0 \\
138.0 \\
148.08 \\
178.55\end{array}$ & $\begin{array}{l}\mathbf{W} \\
\mathbf{W} \\
\mathbf{W} \\
\mathbf{W} \\
\mathbf{W}\end{array}$ & $\frac{-}{-}$ & $\begin{array}{r}477 \\
38 \\
38 \\
477 \\
477\end{array}$ & $\overline{-}$ & $\begin{array}{l}- \\
- \\
-\end{array}$ & $\begin{array}{cc}6.90 \mathrm{M}_{\mathrm{s}} & \mathrm{AB} 2 \\
- & \\
\overline{-} & \\
7.30 \mathrm{M}_{\mathrm{s}} & \mathrm{AB} 2 \\
7.30 \mathrm{M}_{\mathrm{s}} & \mathrm{AB} 2\end{array}$ & $\begin{array}{l}- \\
- \\
-\end{array}$ & $\frac{\overline{\text { Felt }}}{\overline{\mathrm{VI}}}$ & $\frac{\overline{38}}{38}$ & $\begin{array}{c}- \\
- \\
-\end{array}$ \\
\hline $\begin{array}{l}1905 \\
1905 \\
1905 \\
1905 \\
1906\end{array}$ & $\begin{array}{l}03 \\
09 \\
12 \\
12 \\
08\end{array}$ & $\begin{array}{l}22 \\
15 \\
08 \\
10 \\
17\end{array}$ & $\begin{array}{l}0338 \\
0602 \\
1236 \\
001042\end{array}$ & $\begin{array}{l}51.28 \\
52.06 \\
61.0 \\
53.88 \\
51.05\end{array}$ & $\begin{array}{l}\mathbf{N} \\
\mathbf{N} \\
\mathbf{N} \\
\mathbf{N} \\
\mathbf{N}\end{array}$ & $\begin{array}{l}174.83 \\
171.45 \\
160.0 \\
161.66 \\
179.69\end{array}$ & $\begin{array}{l}\mathbf{E} \\
\mathbf{W} \\
\mathbf{W} \\
\mathbf{W} \\
\mathbf{E}\end{array}$ & $\begin{array}{l}- \\
- \\
-\end{array}$ & $\begin{array}{l}477 \\
477 \\
427 \\
477 \\
477\end{array}$ & $\overline{-}$ & $\begin{array}{l}- \\
- \\
-\end{array}$ & $\begin{array}{cc}7.00 \mathrm{M}_{\mathrm{s}} & \mathrm{AB2} \\
7.40 \mathrm{M}_{\mathrm{s}} & \mathrm{AB} 2 \\
- & \\
6.90 \mathrm{M}_{\mathrm{s}} & \mathrm{AB2} \\
7.80 \mathrm{M}_{\mathrm{s}} & \mathrm{AB2}\end{array}$ & $\frac{-}{-}$ & $\begin{array}{l}- \\
\bar{v} \\
-\end{array}$ & $\begin{array}{l}\overline{-} \\
\frac{420}{-} \\
-\end{array}$ & $\begin{array}{c}- \\
- \\
-\end{array}$ \\
\hline $\begin{array}{l}1906 \\
1907 \\
1907 \\
1907 \\
1907\end{array}$ & $\begin{array}{l}12 \\
08 \\
09 \\
09 \\
12\end{array}$ & $\begin{array}{l}23 \\
22 \\
02 \\
24 \\
29\end{array}$ & $\begin{array}{l}1722 \\
222400 \\
1601 \\
1259\end{array}$ & $\begin{array}{l}56.85 \\
57.0 \\
52.59 \\
59.5 \\
66.0\end{array}$ & $\begin{array}{l}\mathbf{N} \\
\mathbf{N} \\
\mathbf{N} \\
\mathbf{N} \\
\mathbf{N}\end{array}$ & $\begin{array}{l}153.90 \\
161.0 \\
169.73 \\
135.5 \\
168.0\end{array}$ & $\begin{array}{l}\mathbf{W} \\
\mathbf{W} \\
\mathbf{E} \\
\mathbf{W} \\
\mathbf{W}\end{array}$ & $\begin{array}{l}\overline{120} \\
- \\
-\end{array}$ & $\begin{array}{r}477 \\
258 \\
477 \\
420 \\
38\end{array}$ & - & $\begin{array}{l}- \\
- \\
-\end{array}$ & $\begin{array}{cl}7.30 \mathrm{M}_{\mathrm{s}} & \mathrm{AB2} \\
6.50 \mathrm{~m}_{\mathrm{b}} \mathrm{GR} \\
7.40 \mathrm{M}_{\mathrm{s}} \mathrm{AB2} \\
- & \\
- & \end{array}$ & $\begin{array}{l}- \\
z \\
-\end{array}$ & $\frac{\overline{\text { Felt }}}{\overline{\mathrm{V}}}$ & $\frac{\overline{420}}{\overline{420}}$ & $\frac{-}{-}$ \\
\hline $\begin{array}{l}1908 \\
1908 \\
1909 \\
1909 \\
1909\end{array}$ & $\begin{array}{l}02 \\
05 \\
02 \\
04 \\
05\end{array}$ & $\begin{array}{l}14 \\
15 \\
16 \\
10 \\
06\end{array}$ & $\begin{array}{l}1125 \\
083136 \\
1650 \\
1936\end{array}$ & $\begin{array}{l}61.0 \\
59.0 \\
60.0 \\
52.0 \\
59.5\end{array}$ & $\begin{array}{l}\mathbf{N} \\
\mathbf{N} \\
\mathbf{N} \\
\mathbf{N} \\
\mathbf{N}\end{array}$ & $\begin{array}{l}146.25 \\
141.0 \\
140.0 \\
175.0 \\
139.5\end{array}$ & $\begin{array}{l}\mathbf{W} \\
\mathbf{W} \\
\mathbf{W} \\
\mathbf{E} \\
\mathbf{W}\end{array}$ & $\begin{array}{l}- \\
- \\
-\end{array}$ & $\begin{array}{r}38 \\
258 \\
420 \\
412 \\
38\end{array}$ & $\overline{-}$ & $\begin{array}{l}- \\
- \\
-\end{array}$ & $\begin{array}{c}7.00 \bar{M}_{s} \text { AB2 } \\
\overline{-}_{\text {7.00M }} \text { AB2 } \\
-\end{array}$ & $\begin{array}{l}- \\
- \\
-\end{array}$ & $\begin{array}{l}\text { VI } \\
\text { VI } \\
\text { VI } \\
\bar{V}\end{array}$ & $\begin{array}{r}420 \\
38 \\
420 \\
-\overline{420}\end{array}$ & $\frac{-}{-}$ \\
\hline $\begin{array}{l}1909 \\
1910\end{array}$ & $\begin{array}{l}09 \\
05\end{array}$ & $\begin{array}{l}08 \\
13\end{array}$ & $\begin{array}{l}164948 \\
075806\end{array}$ & $\begin{array}{l}52.5 \\
57.0\end{array}$ & $\begin{array}{l}\mathbf{N} \\
\mathbf{N}\end{array}$ & $\begin{array}{l}169.0 \\
160.0\end{array}$ & $\begin{array}{l}\text { w } \\
\text { w }\end{array}$ & $\begin{array}{l}090 \\
100\end{array}$ & $\begin{array}{l}258 \\
258\end{array}$ & - & - & $\begin{array}{ll}7.40 \mathrm{~m}_{\mathrm{b}} & G R \\
6.75 \mathrm{~m}_{\mathrm{b}} & G R\end{array}$ & - & $\underline{v}$ & $\begin{array}{r}420 \\
-\end{array}$ & - \\
\hline
\end{tabular}


ALASKA-Continued

[See table 1 for hypocenter and intensity references and table 2 for definitions of magnitude source codes. \&, land area only. Leader (-) indicates information is not available]

\begin{tabular}{|c|c|c|c|c|c|c|c|c|c|c|c|c|c|c|c|c|c|}
\hline \multirow{3}{*}{\multicolumn{4}{|c|}{$\begin{array}{cc} & \text { Origin } \\
\text { Date } & \text { t/me (uTc) } \\
\text { Yr Mo Da } h \text { m s }\end{array}$}} & \multicolumn{6}{|c|}{ Hypocenter } & \multicolumn{5}{|c|}{ Magnitude } & \multicolumn{2}{|c|}{ Intensity } & \multirow{3}{*}{$\begin{array}{l}\text { Felt area } \\
\left(1,000 \mathrm{~km}^{2}\right)\end{array}$} \\
\hline & & & & \multirow{2}{*}{\multicolumn{2}{|c|}{$\begin{array}{l}\text { Latitude } \\
\left({ }^{\circ}\right)\end{array}$}} & \multirow{2}{*}{\multicolumn{2}{|c|}{$\begin{array}{l}\text { Longitude } \\
\qquad\left(^{\circ}\right)\end{array}$}} & \multirow{2}{*}{$\begin{array}{l}\text { Depth } \\
(\mathrm{km})\end{array}$} & \multirow[t]{2}{*}{ Ref } & \multicolumn{2}{|c|}{ usas } & \multirow{2}{*}{\multicolumn{2}{|c|}{ Other }} & \multirow{2}{*}{$\begin{array}{c}\text { Moment } \\
\mathbf{M}\end{array}$} & \multirow[t]{2}{*}{ MMI } & \multirow[t]{2}{*}{ Ref } & \\
\hline & & & & & & & & & & $m_{b}$ & $M_{s}$ & & & & & & \\
\hline $\begin{array}{l}1910 \\
1910 \\
1910\end{array}$ & $\begin{array}{l}08 \\
09 \\
09\end{array}$ & $\begin{array}{l}05 \\
01 \\
09\end{array}$ & 011318 & $\begin{array}{l}60.0 \\
54.0 \\
50.75\end{array}$ & $\begin{array}{l}\mathbf{N} \\
\mathbf{N} \\
\mathbf{N}\end{array}$ & $\begin{array}{l}160.0 \\
168.0 \\
179.38\end{array}$ & $\begin{array}{l}\mathbf{W} \\
\mathbf{W} \\
\mathbf{E}\end{array}$ & $\overline{-}$ & $\begin{array}{l}420 \\
420 \\
477\end{array}$ & - & $\overline{-}$ & $\overline{7.00 \mathrm{M}_{s}}$ & $\mathrm{AB2}$ & $\overline{-}$ & $\begin{array}{r}\mathrm{V} \\
\text { Felt } \\
\mathrm{V}\end{array}$ & $\begin{array}{r}420 \\
420 \\
38\end{array}$ & $\overline{-}$ \\
\hline $\begin{array}{l}1911 \\
1911 \\
1911 \\
1911 \\
1912\end{array}$ & $\begin{array}{l}09 \\
09 \\
09 \\
11 \\
01\end{array}$ & $\begin{array}{l}17 \\
22 \\
13 \\
04\end{array}$ & $\begin{array}{l}0326 \\
050124 \\
161312 \\
154654\end{array}$ & $\begin{array}{l}60.0 \\
51.0 \\
60.5 \\
52.0 \\
52.0\end{array}$ & $\begin{array}{l}\mathbf{N} \\
\mathbf{N} \\
\mathbf{N} \\
\mathbf{N} \\
\mathbf{N}\end{array}$ & $\begin{array}{l}140.0 \\
180.0 \\
149.0 \\
173.0 \\
179.0\end{array}$ & $\begin{array}{l}\mathbf{W} \\
\mathbf{E} \\
\mathbf{W} \\
\mathbf{E} \\
\mathbf{W}\end{array}$ & $\begin{array}{l}- \\
\overline{0} \\
-\end{array}$ & $\begin{array}{l}420 \\
412 \\
258 \\
428 \\
258\end{array}$ & $\begin{array}{l}- \\
-\end{array}$ & $\frac{-}{-}$ & $\begin{array}{l}-\overline{7_{10}} \overline{\mathrm{M}}_{\mathrm{s}} \\
6.90 \mathrm{M}_{\mathrm{s}} \\
6.90 \mathrm{M}_{\mathrm{s}} \\
6.70 \mathrm{M}_{\mathrm{s}}\end{array}$ & $\begin{array}{l}\mathrm{AB2} \\
\mathrm{GR} \\
\mathrm{AB2} \\
\mathrm{ABE}\end{array}$ & $\begin{array}{l}- \\
- \\
-\end{array}$ & $\begin{array}{c}\text { Felt } \\
\text { viI } \\
- \\
-\end{array}$ & $\frac{420}{38}-$ & $\begin{array}{l}- \\
\overline{310} \& \\
-\end{array}$ \\
\hline $\begin{array}{l}1912 \\
1912 \\
1912 \\
1912 \\
1912\end{array}$ & $\begin{array}{l}01 \\
06 \\
06 \\
06 \\
06\end{array}$ & $\begin{array}{l}31 \\
04 \\
05 \\
07 \\
10\end{array}$ & $\begin{array}{l}095554 \\
160606\end{array}$ & $\begin{array}{l}61.0 \\
57.0 \\
57.0 \\
59.0 \\
59.0\end{array}$ & $\begin{array}{l}\mathbf{N} \\
\mathbf{N} \\
\mathbf{N} \\
\mathbf{N} \\
\mathbf{N}\end{array}$ & $\begin{array}{l}147.5 \\
156.0 \\
156.0 \\
153.0 \\
153.0\end{array}$ & $\begin{array}{l}\mathbf{w} \\
\mathbf{W} \\
\mathbf{W} \\
\mathbf{W} \\
\mathbf{W}\end{array}$ & $\frac{080}{-}$ & $\begin{array}{l}258 \\
427 \\
437 \\
258 \\
258\end{array}$ & $\frac{-}{-}$ & $\frac{-}{-}$ & $\begin{array}{c}7.00 \mathrm{~m}_{\mathrm{b}} \\
\frac{-}{-} \\
6.40 \mathrm{M}_{\mathrm{s}} \\
6.90 \mathrm{M}_{\mathrm{s}}\end{array}$ & $\begin{array}{l}\text { GR } \\
\text { AB2 }\end{array}$ & $\frac{-}{-}$ & $\begin{array}{c}\text { Felt } \\
\text { Felt } \\
\text { Felt } \\
\text { Felt } \\
-\end{array}$ & $\begin{array}{r}420 \\
437 \\
437 \\
437 \\
-\end{array}$ & $\begin{array}{l}388 \& \\
- \\
-\end{array}$ \\
\hline $\begin{array}{l}1912 \\
1912 \\
1912 \\
1912 \\
1913\end{array}$ & $\begin{array}{l}07 \\
08 \\
11 \\
12 \\
03\end{array}$ & $\begin{array}{l}07 \\
17 \\
07 \\
05 \\
31\end{array}$ & $\begin{array}{l}0757 \\
074024 \\
122736 \\
034106\end{array}$ & $\begin{array}{l}63.07 \\
58.5 \\
57.5 \\
57.5 \\
51.0\end{array}$ & $\begin{array}{l}\mathbf{N} \\
\mathbf{N} \\
\mathbf{N} \\
\mathbf{N} \\
\mathbf{N}\end{array}$ & $\begin{array}{l}146.14 \\
157.0 \\
155.0 \\
154.0 \\
179.0\end{array}$ & $\begin{array}{l}\mathbf{W} \\
\mathbf{W} \\
\mathbf{W} \\
\mathbf{W} \\
\mathbf{W}\end{array}$ & $\begin{array}{l}- \\
\overline{0} \\
090 \\
060\end{array}$ & $\begin{array}{r}477 \\
38 \\
258 \\
258 \\
258\end{array}$ & $\frac{-}{-}$ & $\frac{-}{-}$ & $\begin{array}{c}7.20 \mathrm{M}_{\mathrm{s}} \\
-1- \\
7.30 \mathrm{mb}_{\mathrm{b}} \\
6.90 \mathrm{mb}_{\mathrm{b}} \\
6.90 \mathrm{M}_{\mathrm{s}}\end{array}$ & $\begin{array}{l}\text { AB2 } \\
\text { ABE } \\
\text { ABE } \\
\text { GR }\end{array}$ & $\begin{array}{l}- \\
- \\
-\end{array}$ & $\begin{array}{r}\text { Felt } \\
\text { v } \\
\text { Felt } \\
- \\
-\end{array}$ & $\begin{array}{r}437 \\
38 \\
38 \\
- \\
-\end{array}$ & $\frac{518}{-}$ \\
\hline $\begin{array}{l}1913 \\
1913 \\
1913 \\
1914 \\
1914\end{array}$ & $\begin{array}{l}04 \\
04 \\
06 \\
01 \\
07\end{array}$ & $\begin{array}{l}29 \\
30 \\
22 \\
30 \\
17\end{array}$ & $\begin{array}{l}2330 \\
1135 \\
134952 \\
034232 \\
070625\end{array}$ & $\begin{array}{l}50.0 \\
50.0 \\
48.0 \\
50.0 \\
47.5\end{array}$ & $\begin{array}{l}\mathbf{N} \\
\mathbf{N} \\
\mathbf{N} \\
\mathbf{N} \\
\mathbf{N}\end{array}$ & $\begin{array}{l}171.0 \\
174.0 \\
178.0 \\
170.0 \\
174.0\end{array}$ & $\begin{array}{l}\mathbf{E} \\
\mathbf{W} \\
\mathbf{W} \\
\mathbf{W} \\
\mathbf{W}\end{array}$ & $\bar{z}$ & $\begin{array}{l}265 \\
265 \\
265 \\
265 \\
265\end{array}$ & $\bar{z}$ & $\begin{array}{l}z \\
z\end{array}$ & $\begin{array}{c}\overline{-} \\
7.20 \mathrm{Ukn} \\
-\end{array}$ & $\frac{\mathrm{nDDA}}{-}$ & $\begin{array}{l}- \\
z \\
-\end{array}$ & $\frac{-}{-}$ & $\begin{array}{l}- \\
- \\
-\end{array}$ & $\overline{-}$ \\
\hline $\begin{array}{l}1915 \\
1915 \\
1916 \\
1916 \\
1916\end{array}$ & $\begin{array}{l}04 \\
08 \\
02 \\
02 \\
02\end{array}$ & $\begin{array}{l}03 \\
16 \\
06 \\
15 \\
20\end{array}$ & $\begin{array}{lll}20 & 29 & 36 \\
00 & 56 & 22 \\
21 & 51 & 19 \\
11 & 35 & 52 \\
17 & 47 & 35\end{array}$ & $\begin{array}{l}48.5 \\
48.5 \\
48.5 \\
61.5 \\
51.0\end{array}$ & $\begin{array}{l}\mathbf{N} \\
\mathbf{N} \\
\mathbf{N} \\
\mathbf{N} \\
\mathbf{N}\end{array}$ & $\begin{array}{l}171.5 \\
179.0 \\
178.5 \\
145.0 \\
169.0\end{array}$ & $\begin{array}{l}\mathbf{W} \\
\mathbf{W} \\
\mathbf{E} \\
\mathbf{W} \\
\mathbf{W}\end{array}$ & $\begin{array}{l}- \\
- \\
-\end{array}$ & $\begin{array}{l}265 \\
265 \\
265 \\
265 \\
265\end{array}$ & $\begin{array}{l}z \\
z\end{array}$ & $\begin{array}{l}- \\
z\end{array}$ & $\begin{array}{c}\overline{-} \\
7.70 \overline{\mathrm{Ukn}} \\
-\end{array}$ & $\begin{array}{l}- \\
\overline{\mathrm{DDA}} \\
- \\
-\end{array}$ & $\begin{array}{l}z \\
z\end{array}$ & $\begin{array}{c}- \\
-\end{array}$ & $\frac{-}{-}$ & - \\
\hline $\begin{array}{l}1916 \\
1916 \\
1917 \\
1917 \\
1917\end{array}$ & $\begin{array}{l}04 \\
12 \\
05 \\
06 \\
06\end{array}$ & $\begin{array}{l}18 \\
14 \\
31 \\
04 \\
07\end{array}$ & $\begin{array}{lll}04 & 01 & 48 \\
16 & 52 & 16 \\
08 & 47 \\
01 & 2918 \\
02 & 47 & 43\end{array}$ & $\begin{array}{l}53.25 \\
49.0 \\
54.79 \\
53.5 \\
54.5\end{array}$ & $\begin{array}{l}\mathbf{N} \\
\mathbf{N} \\
\mathbf{N} \\
\mathbf{N} \\
\mathbf{N}\end{array}$ & $\begin{array}{l}170.0 \\
174.0 \\
159.12 \\
159.0 \\
160.0\end{array}$ & $\begin{array}{l}\mathbf{W} \\
\mathbf{E} \\
\mathbf{W} \\
\mathbf{W} \\
\mathbf{W}\end{array}$ & $\frac{170}{-}$ & $\begin{array}{l}258 \\
265 \\
477 \\
265 \\
265\end{array}$ & $=$ & $\begin{array}{l}- \\
- \\
-\end{array}$ & $\begin{array}{c}7.40 \mathrm{~m}_{\mathrm{b}} \\
7.90 \mathrm{M}_{\mathrm{s}} \\
-\end{array}$ & $\begin{array}{l}\text { ABE } \\
\text { AB1 }\end{array}$ & $\begin{array}{l}- \\
- \\
-\end{array}$ & $\frac{-}{\bar{v}}$ & $\frac{\bar{Z}}{272}$ & $\frac{-}{-}$ \\
\hline $\begin{array}{l}1917 \\
1917 \\
1917 \\
1917 \\
1918\end{array}$ & $\begin{array}{l}07 \\
07 \\
12 \\
12 \\
04\end{array}$ & $\begin{array}{l}25 \\
25 \\
21 \\
28 \\
15\end{array}$ & $\begin{array}{lll}03 & 19 & 00 \\
22 & 32 & 43 \\
17 & 54 & 16 \\
21 & 14 & 30 \\
08 & 27 & 40\end{array}$ & $\begin{array}{l}53.5 \\
53.5 \\
53.5 \\
55.5 \\
59.2\end{array}$ & $\begin{array}{l}\mathbf{N} \\
\mathbf{N} \\
\mathbf{N} \\
\mathbf{N} \\
\mathbf{N}\end{array}$ & $\begin{array}{l}159.0 \\
159.0 \\
152.0 \\
152.0 \\
151.0\end{array}$ & $\begin{array}{l}\mathbf{W} \\
\mathbf{W} \\
\mathbf{W} \\
\mathbf{W} \\
\mathbf{w}\end{array}$ & $\frac{-}{-}$ & $\begin{array}{l}265 \\
265 \\
265 \\
265 \\
265\end{array}$ & $\begin{array}{l}z \\
z\end{array}$ & $\frac{-}{-}$ & $\begin{array}{l}- \\
-\end{array}$ & & $\begin{array}{l}z \\
z\end{array}$ & $\begin{array}{l}- \\
-\end{array}$ & $\begin{array}{l}- \\
- \\
-\end{array}$ & $\begin{array}{l}- \\
-\end{array}$ \\
\hline $\begin{array}{l}1918 \\
1918 \\
1919 \\
1919 \\
1920\end{array}$ & $\begin{array}{l}09 \\
12 \\
05 \\
12 \\
08\end{array}$ & $\begin{array}{l}30 \\
09 \\
22 \\
15 \\
26\end{array}$ & $\begin{array}{lll}13 & 34 & 20 \\
18 & 03 & 45 \\
11 & 52 & 36 \\
01 & 10 \\
22 & 59 & 54\end{array}$ & $\begin{array}{l}51.0 \\
52.0 \\
52.0 \\
57.5 \\
52.5\end{array}$ & $\begin{array}{l}\mathbf{N} \\
\mathbf{N} \\
\mathbf{N} \\
\mathbf{N} \\
\mathbf{N}\end{array}$ & $\begin{array}{l}179.5 \\
178.0 \\
178.0 \\
137.0 \\
170.0\end{array}$ & $\begin{array}{l}\mathbf{W} \\
\mathbf{W} \\
\mathbf{W} \\
\mathbf{W} \\
\mathbf{W}\end{array}$ & $\begin{array}{l}- \\
- \\
-\end{array}$ & $\begin{array}{l}265 \\
265 \\
265 \\
272 \\
265\end{array}$ & $\begin{array}{l}\overline{-} \\
\overline{-}\end{array}$ & $\begin{array}{l}- \\
-\end{array}$ & $\overline{-}$ & & $\begin{array}{l}\bar{z} \\
\overline{-}\end{array}$ & $\frac{-}{\bar{v}}$ & $\frac{-}{272}$ & $\bar{z}$ \\
\hline $\begin{array}{l}1920 \\
1920 \\
1922 \\
1922 \\
1923\end{array}$ & $\begin{array}{l}10 \\
11 \\
04 \\
07 \\
04\end{array}$ & $\begin{array}{l}28 \\
29 \\
02 \\
02 \\
25\end{array}$ & $\begin{array}{l}072340 \\
080230 \\
191742 \\
133548 \\
193153\end{array}$ & $\begin{array}{l}51.0 \\
60.5 \\
53.3 \\
54.0 \\
59.0\end{array}$ & $\begin{array}{l}\mathbf{N} \\
\mathbf{N} \\
\mathbf{N} \\
\mathbf{N} \\
\mathbf{N}\end{array}$ & $\begin{array}{l}179.5 \\
147.0 \\
164.5 \\
160.5 \\
138.0\end{array}$ & $\begin{array}{l}\mathbf{W} \\
\mathbf{W} \\
\mathbf{W} \\
\mathbf{W} \\
\mathbf{W}\end{array}$ & $\overline{-}$ & $\begin{array}{l}265 \\
272 \\
265 \\
265 \\
258\end{array}$ & $\begin{array}{l}z \\
z\end{array}$ & $\frac{-}{-}$ & ${ }^{\bar{Z}} \overline{\bar{Z}}$ & GR & $\frac{-}{-}$ & $\bar{z}$ & $\frac{-}{\bar{E}}$ & - \\
\hline $\begin{array}{l}1923 \\
1923 \\
1923\end{array}$ & $\begin{array}{l}05 \\
06 \\
07\end{array}$ & $\begin{array}{l}04 \\
19 \\
17\end{array}$ & $\begin{array}{l}162639.3 \\
224330 \\
010211\end{array}$ & $\begin{array}{l}55.55 \\
61.8 \\
63.0\end{array}$ & $\begin{array}{l}\mathbf{N} \\
\mathbf{N} \\
\mathbf{N}\end{array}$ & $\begin{array}{l}156.75 \\
151.0 \\
147.0\end{array}$ & $\begin{array}{l}\mathbf{w} \\
\mathbf{w} \\
\mathbf{w}\end{array}$ & $\frac{000}{-}$ & $\begin{array}{l}432 \\
265 \\
258\end{array}$ & - & $\overline{-}$ & $\frac{7.10 \mathrm{M}_{\mathrm{s}}}{5.60 \mathrm{M}_{\mathrm{s}}}$ & $\begin{array}{l}\text { GR } \\
\text { GR }\end{array}$ & - & Felt & $\begin{array}{l}\overline{434} \\
-\end{array}$ & - \\
\hline
\end{tabular}


EARTHQUAKES IN ALASKA

ALASKA-Continued

[See table 1 for hypocenter and intensity references and table 2 for definitions of magnitude source codes. \&, land area only. Leader (--) indicates information is not available]

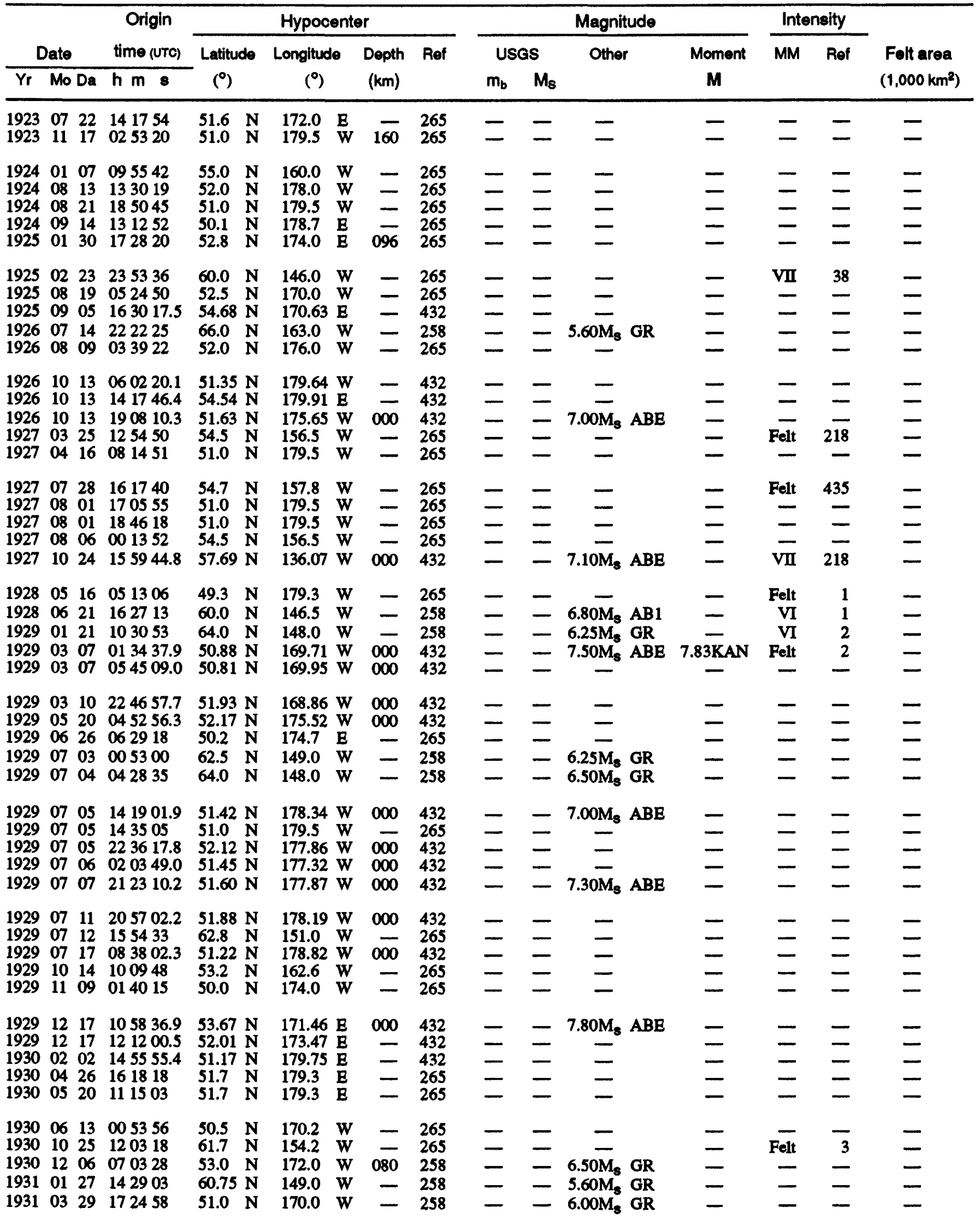


ALASKA-Continued

[See table 1 for hypocenter and intensity references and table 2 for definitions of magnitude source codes. \&, land area only. Leader (--) indicates information is not available]

\begin{tabular}{|c|c|c|c|c|c|c|c|c|c|c|c|c|c|c|c|c|}
\hline & & & Origin & & & Hypoce & entel & & & & & Magnitude & & Inte & sity & \\
\hline & ate & & time (UTC) & Latituc & & Longitude & & Depth & Ref & US & & Other & Moment & MM & Ref & Felt area \\
\hline $\mathrm{Yr}$ & Mo D & $\mathrm{Da}$ & $h \mathrm{~m} s$ & $\left({ }^{\circ}\right)$ & & $\left({ }^{\circ}\right)$ & & $(\mathrm{km})$ & & $m_{b}$ & $M_{\mathbf{s}}$ & & $\mathbf{M}$ & & & $\left(1,000 \mathrm{~km}^{2}\right)$ \\
\hline $\begin{array}{l}1931 \\
1931\end{array}$ & $\begin{array}{l}05 ? \\
05 ?\end{array}$ & $\begin{array}{l}29 \\
30\end{array}$ & $\begin{array}{l}051632 \\
113416\end{array}$ & $\begin{array}{l}63.0 \\
52.2\end{array}$ & $\stackrel{\mathbf{N}}{\mathbf{N}}$ & $\begin{array}{l}149.0 \\
173.3\end{array}$ & $\begin{array}{l}\mathbf{W} \\
\mathbf{E}\end{array}$ & - & $\begin{array}{l}258 \\
265\end{array}$ & - & - & $\begin{array}{l}5.60 \mathrm{M}_{\mathrm{S}} \mathrm{GR} \\
6.00 \mathrm{Ukn} \text { PAS }\end{array}$ & - & $\begin{array}{l}\text { IV } \\
\text { VI }\end{array}$ & $\begin{array}{r}4 \\
38\end{array}$ & - \\
\hline 1931 & 08 & 14 & 161203 & 52.5 & $\mathbf{N}$ & 168.0 & $\mathbf{w}$ & - & 258 & - & - & $6.00 \mathrm{M}_{\mathrm{s}} \mathrm{GR}$ & - & - & - & - \\
\hline 1931 & 101 & 17 & 123450 & 63.0 & $\mathbf{N}$ & 147.0 & $\mathbf{w}$ & - & 258 & - & - & $5.60 \mathrm{M}_{\mathrm{s}} \mathrm{GR}$ & - & V & 38 & - \\
\hline 1931 & 122 & 24 & 034040 & 60.0 & $\mathbf{N}$ & 152.0 & W & 100 & 258 & - & - & $6.25 \mathrm{M}_{\mathrm{s}} \mathrm{GR}$ & - & IV & 4 & 一 \\
\hline 1932 & 01 & 13 & 161727 & 52.0 & $\mathbf{N}$ & 179.0 & w & - & 258 & - & - & $6.00 \mathrm{M}_{\mathrm{s}} \mathrm{GR}$ & - & - & - & - \\
\hline 1932 & $03 c$ & 08 & 042930 & 51.5 & $\mathbf{N}$ & 178.0 & $\mathbf{w}$ & - & 258 & - & - & $6.00 \mathrm{M}_{\mathrm{s}} \mathrm{GR}$ & - & - & - & - \\
\hline 1932 & 032 & 25 & 235451 & 62.5 & $\mathbf{N}$ & 153.0 & $\mathbf{w}$ & - & 258 & - & - & $6.00 \mathrm{M}_{\mathrm{s}} \mathrm{GR}$ & - & - & - & - \\
\hline 1932 & 032 & 25 & 235831 & 62.5 & $\mathbf{N}$ & 152.5 & W & - & 258 & - & - & $6.90 \mathrm{M}_{\mathrm{s}} \mathrm{GR}$ & - & VII & 38 & - \\
\hline 1932 & 042 & 29 & 181823 & 51.5 & $\mathbf{N}$ & 178.0 & w & - & 258 & - & - & $6.25 \mathrm{M}_{\mathrm{s}} \mathrm{GR}$ & - & - & - & - \\
\hline 1932 & $06 \mathrm{c}$ & 08 & 075247 & 62.5 & $\mathbf{N}$ & 153.3 & w & - & 265 & - & - & $6.00 \mathrm{M}_{\mathrm{s}}$ PAS & - & III & 5 & - \\
\hline 1932 & 08 & 12 & 032357 & 52.25 & $\mathbf{N}$ & 169.0 & $\mathbf{w}$ & - & 258 & - & - & $6.75 M_{s}$ GR & - & - & - & - \\
\hline 1932 & 091 & 14 & 084323 & 61.0 & $\mathbf{N}$ & 148.0 & W & 050 & 258 & - & - & $6.25 \mathrm{M}_{\mathrm{s}} \mathrm{GR}$ & - & V & 5 & - \\
\hline 1932 & 10 & 16 & 120801 & 54.25 & $\mathbf{N}$ & 160.0 & W & 050 & 258 & - & - & $6.75 \mathrm{M}_{\mathrm{s}} \mathrm{GR}$ & - & - & - & - \\
\hline 1932 & 103 & 30 & 204656 & 55.0 & $\mathbf{N}$ & 159.75 & w & - & 258 & - & - & $6.75 \mathrm{M}_{\mathrm{S}} \mathrm{GR}$ & 一 & 一 & - & - \\
\hline 1933 & 01 & 04 & 035928 & 61.0 & $\mathbf{N}$ & 148.0 & W & - & 258 & - & - & $6.25 \mathrm{M}_{\mathrm{s}}$ PAS & - & VI & 38 & - \\
\hline 1933 & 032 & 28 & 042026 & 58.25 & $\mathbf{N}$ & 149.0 & W & - & 258 & - & - & $5.60 \mathrm{M}_{\mathrm{s}} \mathrm{GR}$ & - & III & 6 & - \\
\hline 1933 & 042 & 27 & 023604 & 61.25 & $\mathbf{N}$ & 150.75 & W & - & 258 & - & - & $6.90 \mathrm{M}_{\mathrm{S}} \mathrm{ABE}$ & - & VII & 38 & - \\
\hline 1933 & 042 & 27 & 115538 & 52.5 & $\mathbf{N}$ & 167.0 & W & - & 258 & - & - & $6.00 \mathrm{M}_{\mathrm{s}} \mathrm{GR}$ & - & - & - & 一 \\
\hline 1933 & 050 & 01 & 184947 & 51.75 & $\mathbf{N}$ & 173.0 & W & - & 258 & - & - & $6.50 \mathrm{M}_{\mathrm{s}} \mathrm{GR}$ & - & - & - & - \\
\hline 1933 & 06 & 12 & 152338 & 61.5 & $\mathbf{N}$ & 150.5 & W & - & 258 & - & - & $5.60 \mathrm{M}_{\mathrm{S}} \mathrm{GR}$ & - & Felt & 6 & - \\
\hline 1933 & 061 & 13 & 221947 & 61.0 & $\mathbf{N}$ & 151.0 & w & - & 258 & - & - & $6.25 \mathrm{M}_{\mathrm{s}} \mathrm{GR}$ & - & IV & 6 & - \\
\hline 1933 & 06 & 19 & 184743 & 61.25 & $\mathbf{N}$ & 150.5 & W & - & 258 & - & - & $6.00 \mathrm{M}_{\mathrm{s}} \mathrm{GR}$ & - & Felt & 6 & - \\
\hline 1933 & 062 & 28 & 233458 & 53.5 & $\mathbf{N}$ & 165.0 & W & - & 258 & - & - & $6.00 \mathrm{M}_{\mathrm{s}} \mathrm{GR}$ & - & Felt & 6 & - \\
\hline 1933 & 07 & 19 & 104529 & 51.75 & $\mathbf{N}$ & 174.0 & W & - & 258 & - & - & $6.00 \mathrm{M}_{\mathrm{s}} \mathrm{GR}$ & - & - & - & - \\
\hline 1933 & 07 . & 19 & 105553 & 51.75 & $\mathbf{N}$ & 174.0 & W & - & 258 & - & - & $6.00 \mathrm{M}_{\mathrm{s}} \mathrm{GR}$ & - & - & - & 一 \\
\hline 1933 & 07 & 19 & 133221 & 51.75 & $\mathbf{N}$ & 174.0 & W & - & 258 & - & - & $6.25 \mathrm{M}_{\mathrm{s}} \mathrm{GR}$ & - & - & - & - \\
\hline 1933 & 07 . & 19 & 145952 & 51.75 & $\mathbf{N}$ & 174.0 & W & - & 258 & - & - & $6.25 \mathrm{M}_{\mathrm{s}} \mathrm{GR}$ & - & - & - & - \\
\hline 1933 & 072 & 22 & 205513 & 53.0 & $\mathbf{N}$ & 169.5 & W & - & 258 & - & - & $6.75 \mathrm{M}_{\mathrm{S}} \mathrm{GR}$ & - & - & - & - \\
\hline 1933 & 072 & 26 & 045726 & 63.0 & $\mathbf{N}$ & 147.0 & W & - & 258 & - & - & $5.60 \mathrm{M}_{\mathrm{s}} \mathrm{GR}$ & - & - & - & 一 \\
\hline 1933 & 072 & 28 & 114808 & 52.6 & $\mathbf{N}$ & 168.7 & W & - & 265 & - & - & - & - & V & 6 & - \\
\hline 1933 & 092 & 24 & 151941 & 51.75 & $\mathbf{N}$ & 177.0 & w & 070 & 258 & - & - & $6.75 \mathrm{M}_{\mathrm{s}} \mathrm{GR}$ & - & - & - & - \\
\hline 1933 & 10 & 14 & 221901 & 53.75 & $\mathbf{N}$ & 164.0 & W & - & 258 & - & - & $6.25 \mathrm{M}_{\mathrm{s}} \mathrm{GR}$ & - & - & - & - \\
\hline 1933 & 11 & 02 & 122654 & 52.0 & $\mathbf{N}$ & 176.0 & W & - & 258 & - & - & $6.50 \mathrm{M}_{\mathrm{s}} \mathrm{GR}$ & - & - & - & - \\
\hline 1934 & 01 & 11 & 102155 & 50.5 & $\mathbf{N}$ & 177.5 & W & - & 265 & - & - & - & - & - & - & - \\
\hline 1934 & 05 & 04 & 043607 & 61.25 & $\mathbf{N}$ & 147.5 & W & 080 & 258 & - & - & $7.10 \mathrm{mb}_{\mathrm{b}} \mathrm{ABE}$ & - & VI & 7 & - \\
\hline 1934 & 05 & 14 & 221246 & 57.75 & $\mathbf{N}$ & 152.25 & $\mathbf{w}$ & 060 & 258 & - & - & $6.50 \mathrm{M}_{\mathrm{s}} \mathrm{GR}$ & - & VI & 7 & - \\
\hline 1934 & 06 & 02 & 164529 & 61.25 & $\mathbf{N}$ & 147.0 & W & - & 258 & - & - & $6.25 \mathrm{M}_{\mathrm{s}} \mathrm{GR}$ & - & IV & 7 & - \\
\hline 1934 & 06 & 18 & 091350 & 60.50 & $\mathbf{N}$ & 151.0 & W & 080 & 258 & - & - & $6.75 \mathrm{M}_{\mathrm{S}} \mathrm{GR}$ & - & V & 7 & - \\
\hline 1934 & 072 & 20 & 021044 & 52.0 & $\mathbf{N}$ & 173.0 & W & - & 258 & - & - & $6.00 \mathrm{M}_{\mathrm{s}} \mathrm{GR}$ & - & - & - & - \\
\hline 1934 & 072 & 28 & 213657 & 55.5 & $\mathbf{N}$ & 156.75 & $\mathbf{w}$ & - & 258 & - & - & $6.75 \mathrm{M}_{\mathrm{S}} \mathrm{GR}$ & - & - & - & - \\
\hline 1934 & 08 & 02 & 071308 & 61.5 & $\mathbf{N}$ & 147.5 & w & - & 258 & - & - & $6.00 \mathrm{M}_{\mathrm{s}} \mathrm{GR}$ & - & V & 7 & - \\
\hline 1934 & 110 & 05 & 230220 & 52.0 & $\mathbf{N}$ & 175.0 & $\mathbf{w}$ & - & 258 & - & - & $6.50 \mathrm{M}_{\mathrm{s}}^{\circ} \mathrm{GR}$ & - & - & - & - \\
\hline 1935 & 012 & 23 & 072400 & 52.25 & $\mathbf{N}$ & 169.5 & W & - & $258^{\circ}$ & - & - & $6.75 \mathrm{M}_{\mathrm{S}} \mathrm{GR}$ & - & Felt & 8 & - \\
\hline 1935 & 022 & 22 & 170554 & 52.25 & $\mathbf{N}$ & 175.0 & $\mathbf{E}$ & - & 258 & - & - & $7.10 \mathrm{M}_{S} \mathrm{ABE}$ & - & - & - & - \\
\hline 1935 & 09 & 04 & 012739 & 63.75 & $\mathbf{N}$ & 152.5 & W & - & 258 & - & - & $6.25 \mathrm{M}_{\mathrm{s}} \mathrm{GR}$ & - & III & 8 & - \\
\hline $\begin{array}{l}1936 \\
1936\end{array}$ & $\begin{array}{l}01 \\
03\end{array}$ & $\begin{array}{l}18 \\
10\end{array}$ & $\begin{array}{l}012000 \\
120508\end{array}$ & $\begin{array}{l}62.0 \\
51.8\end{array}$ & $\begin{array}{l}\mathbf{N} \\
\mathbf{N}\end{array}$ & $\begin{array}{l}152.0 \\
171.0\end{array}$ & $\begin{array}{l}W \\
W\end{array}$ & - & $\begin{array}{l}258 \\
265\end{array}$ & - & $\overline{-}$ & ${ }^{5.60 \mathrm{M}_{\mathrm{s}} \mathrm{GR}}$ & - & - & - & - \\
\hline
\end{tabular}


ALASKA-Continued

[See table 1 for hypocenter and intensity references and table 2 for definitions of magnitude source codes. \&, land area only. Leader (--) indicates information is not available]

\begin{tabular}{|c|c|c|c|c|c|c|c|c|c|c|c|c|c|c|c|c|c|}
\hline \multirow{3}{*}{\multicolumn{4}{|c|}{\begin{tabular}{cc} 
& \multicolumn{2}{c}{ Origin } \\
Date & time (UTC) \\
Yr Mo Da h m 8
\end{tabular}}} & \multicolumn{6}{|c|}{ Hypocenter } & \multicolumn{5}{|c|}{ Magnitude } & \multicolumn{2}{|c|}{ Intensity } & \multirow{3}{*}{$\begin{array}{l}\text { Felt area } \\
\left(1,000 \mathrm{~km}^{2}\right)\end{array}$} \\
\hline & & & & \multirow{2}{*}{\multicolumn{2}{|c|}{$\begin{array}{l}\text { Latitude } \\
\left(^{\circ}\right)\end{array}$}} & \multirow{2}{*}{\multicolumn{2}{|c|}{$\begin{array}{l}\text { Longitude } \\
\left({ }^{\circ}\right)\end{array}$}} & \multirow{2}{*}{$\begin{array}{c}\text { Depth } \\
(\mathrm{km})\end{array}$} & \multirow[t]{2}{*}{ Ref } & \multicolumn{2}{|c|}{ USGS } & \multirow{2}{*}{\multicolumn{2}{|c|}{ Other }} & \multirow{2}{*}{$\begin{array}{c}\text { Moment } \\
\text { M }\end{array}$} & \multirow[t]{2}{*}{ MM } & \multirow[t]{2}{*}{ Ref } & \\
\hline & & & & & & & & & & $m_{b}$ & $\mathbf{M}_{\mathbf{s}}$ & & & & & & \\
\hline $\begin{array}{l}1936 \\
1936\end{array}$ & $\begin{array}{l}04 \\
05\end{array}$ & $\begin{array}{l}23 \\
08\end{array}$ & $\begin{array}{lll}23 & 14 & 21 \\
17 & 22 & 18\end{array}$ & $\begin{array}{l}50.25 \\
61.0\end{array}$ & $\begin{array}{l}\mathbf{N} \\
\mathbf{N}\end{array}$ & $\begin{array}{l}179.0 \\
153.0\end{array}$ & $\begin{array}{l}\mathbf{E} \\
\mathbf{W}\end{array}$ & $\overline{170}$ & $\begin{array}{l}258 \\
258\end{array}$ & - & - & $\begin{array}{l}6.25 \mathrm{M}_{\mathrm{s}} \\
5.75 \mathrm{~m}_{\mathrm{b}}\end{array}$ & $\begin{array}{l}\text { GR } \\
\text { GR }\end{array}$ & - & $\overline{\text { II }}$ & $\overline{9}$ & $\overline{-}$ \\
\hline $\begin{array}{l}1936 \\
1937 \\
1937 \\
1937 \\
1937\end{array}$ & $\begin{array}{l}10 \\
04 \\
05 \\
07 \\
07\end{array}$ & $\begin{array}{l}23 \\
29 \\
04 \\
18 \\
22\end{array}$ & $\begin{array}{lll}06 & 24 & 25 \\
18 & 52 & 40 \\
05 & 08 & 41 \\
01 & 01 & 15 \\
17 & 09 & 28.0\end{array}$ & $\begin{array}{l}61.3 \\
54.3 \\
59.2 \\
54.0 \\
64.6\end{array}$ & $\begin{array}{l}\mathbf{N} \\
\mathbf{N} \\
\mathbf{N} \\
\mathbf{N} \\
\mathbf{N}\end{array}$ & $\begin{array}{l}150.7 \\
161.5 \\
153.8 \\
166.5 \\
147.1\end{array}$ & $\begin{array}{l}\mathbf{W} \\
\mathbf{W} \\
\mathbf{W} \\
\mathbf{W} \\
\mathbf{W}\end{array}$ & $\frac{-}{\overline{0}}$ & $\begin{array}{l}265 \\
265 \\
265 \\
258 \\
265\end{array}$ & $\begin{array}{l}z \\
- \\
-\end{array}$ & $\begin{array}{l}- \\
- \\
-\end{array}$ & 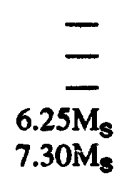 & $\begin{array}{l}\text { GR } \\
\text { ABE }\end{array}$ & $\begin{array}{l}- \\
- \\
-\end{array}$ & $\frac{\text { VI }}{\overline{\text { VII }}}$ & $\frac{-}{-}$ & $\frac{-}{-}$ \\
\hline $\begin{array}{l}1937 \\
1937 \\
1937 \\
1938 \\
1938\end{array}$ & $\begin{array}{l}07 \\
09 \\
10 \\
07 \\
11\end{array}$ & $\begin{array}{l}25 \\
03 \\
24 \\
24 \\
10\end{array}$ & $\begin{array}{lll}13 & 13 & 01 \\
18 & 48 & 12 \\
11 & 35 & 57 \\
13 & 12 & 13 \\
20 & 18 & 41.2\end{array}$ & $\begin{array}{l}60.2 \\
52.5 \\
60.0 \\
53.5 \\
55.48\end{array}$ & $\begin{array}{l}\mathbf{N} \\
\mathbf{N} \\
\mathbf{N} \\
\mathbf{N} \\
\mathbf{N}\end{array}$ & $\begin{array}{l}148.9 \\
177.5 \\
150.5 \\
167.0 \\
158.37\end{array}$ & $\begin{array}{l}\text { W } \\
\mathbf{W} \\
\mathbf{W} \\
\mathbf{W} \\
\mathbf{W}\end{array}$ & $\begin{array}{l}-\overline{080} \\
\overline{-} \\
050 \\
000\end{array}$ & $\begin{array}{l}265 \\
258 \\
265 \\
258 \\
432\end{array}$ & $\begin{array}{l}- \\
-\end{array}$ & $\begin{array}{l}- \\
z \\
-\end{array}$ & $\begin{array}{c}-2 \overline{\mathrm{mb}}_{\mathrm{b}} \\
-\overline{\mathrm{M}}_{\mathrm{s}} \\
6.25 \mathrm{M}_{\mathrm{s}}\end{array}$ & $\begin{array}{l}\text { ABE } \\
\text { GR } \\
\text { ABE }\end{array}$ & $\begin{array}{l}\bar{Z} \\
\overline{{ }_{8.25 \mathrm{KAN}}}\end{array}$ & $\begin{array}{c}\overline{\text { Felt }} \\
\frac{\mathrm{V}}{\mathrm{VI}}\end{array}$ & $\begin{array}{l}- \\
10 \\
38 \\
\overline{11}\end{array}$ & $\frac{-}{-}$ \\
\hline $\begin{array}{l}1938 \\
1938 \\
1938 \\
1938 \\
1938\end{array}$ & $\begin{array}{l}11 \\
11 \\
11 \\
11 \\
11\end{array}$ & $\begin{array}{l}11 \\
11 \\
15 \\
16 \\
17\end{array}$ & $\begin{array}{l}0057 \quad 41.1 \\
083050.9 \\
095201.8 \\
053609.0 \\
035434.0\end{array}$ & $\begin{array}{l}55.07 \\
55.98 \\
54.97 \\
55.02 \\
55.45\end{array}$ & $\begin{array}{l}\mathbf{N} \\
\mathbf{N} \\
\mathbf{N} \\
\mathbf{N} \\
\mathbf{N}\end{array}$ & $\begin{array}{l}158.84 \\
154.72 \\
160.91 \\
156.83 \\
157.55\end{array}$ & $\begin{array}{l}\mathbf{W} \\
\mathbf{W} \\
\mathbf{w} \\
\mathbf{W} \\
\mathbf{W}\end{array}$ & $\begin{array}{l}000 \\
000 \\
057 \\
000 \\
000\end{array}$ & $\begin{array}{l}432 \\
432 \\
432 \\
432 \\
432\end{array}$ & $\begin{array}{l}z \\
z\end{array}$ & $=$ & $\frac{\bar{Z}}{\bar{Z}}{ }_{7.30 \mathrm{M}_{\mathrm{s}}}$ & ABE & $\frac{-}{-}$ & - & $\begin{array}{l}- \\
-\end{array}$ & $\overline{-}$ \\
\hline $\begin{array}{l}1938 \\
1938 \\
1938 \\
1938 \\
1939\end{array}$ & $\begin{array}{l}11 \\
12 \\
12 \\
12 \\
02\end{array}$ & $\begin{array}{l}18 \\
09 \\
23 \\
30 \\
24\end{array}$ & $\begin{array}{lll}23 & 24 & 50.4 \\
03 & 55 & 24.4 \\
18 & 14 & 44.5 \\
12 & 10 & 48 \\
14 & 15 & 55.7\end{array}$ & $\begin{array}{l}56.10 \\
57.74 \\
55.86 \\
59.0 \\
54.05\end{array}$ & $\begin{array}{l}\mathbf{N} \\
\mathbf{N} \\
\mathbf{N} \\
\mathbf{N} \\
\mathbf{N}\end{array}$ & $\begin{array}{l}155.95 \\
153.03 \\
157.28 \\
153.0 \\
162.01\end{array}$ & $\begin{array}{l}\mathbf{W} \\
\mathbf{W} \\
\mathbf{W} \\
\mathbf{W} \\
\mathbf{W}\end{array}$ & $\begin{array}{l}000 \\
000 \\
000 \\
100 \\
079\end{array}$ & $\begin{array}{l}432 \\
432 \\
432 \\
258 \\
432\end{array}$ & $\begin{array}{l}- \\
- \\
-\end{array}$ & $\frac{-}{-}$ & 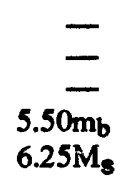 & $\begin{array}{l}\text { GR } \\
\text { GR }\end{array}$ & $\begin{array}{l}- \\
- \\
-\end{array}$ & $\overline{-}$ & $\frac{-}{-}$ & $\frac{-}{-}$ \\
\hline $\begin{array}{l}1939 \\
1939 \\
1939 \\
1939 \\
1939\end{array}$ & $\begin{array}{l}05 \\
05 \\
07 \\
08 \\
08\end{array}$ & $\begin{array}{l}09 \\
10 \\
02 \\
20 \\
21\end{array}$ & $\begin{array}{l}072828.0 \\
074416 \\
194252 \\
071726 \\
151903\end{array}$ & $\begin{array}{l}56.24 \\
51.7 \\
51.7 \\
54.0 \\
51.5\end{array}$ & $\begin{array}{l}\mathbf{N} \\
\mathbf{N} \\
\mathbf{N} \\
\mathbf{N} \\
\mathbf{N}\end{array}$ & $\begin{array}{l}155.17 \\
178.5 \\
178.5 \\
164.0 \\
177.0\end{array}$ & $\begin{array}{l}\mathbf{W} \\
\mathbf{W} \\
\mathbf{W} \\
\mathbf{W} \\
\mathbf{E}\end{array}$ & $\frac{026}{-}$ & $\begin{array}{l}432 \\
265 \\
265 \\
258 \\
258\end{array}$ & $\begin{array}{l}- \\
- \\
-\end{array}$ & $\begin{array}{l}z \\
-\end{array}$ & 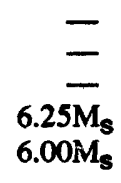 & $\begin{array}{l}\text { GR } \\
\text { GR }\end{array}$ & $\begin{array}{l}- \\
- \\
-\end{array}$ & $\frac{-}{v}$ & $\frac{-}{38}$ & $\begin{array}{c}- \\
- \\
-\end{array}$ \\
\hline $\begin{array}{l}1939 \\
1939 \\
1939 \\
1940 \\
1940\end{array}$ & $\begin{array}{l}09 \\
09 \\
12 \\
02 \\
02\end{array}$ & $\begin{array}{l}11 \\
15 \\
07 \\
07 \\
12\end{array}$ & $\begin{array}{lll}07 & 53 & 26 \\
21 & 48 & 58 \\
11 & 16 & 19 \\
17 & 1602 \\
09 & 1746\end{array}$ & $\begin{array}{l}53.4 \\
51.3 \\
51.7 \\
51.5 \\
55.0\end{array}$ & $\begin{array}{l}\mathbf{N} \\
\mathbf{N} \\
\mathbf{N} \\
\mathbf{N} \\
\mathbf{N}\end{array}$ & $\begin{array}{l}168.7 \\
175.1 \\
178.5 \\
175.0 \\
161.5\end{array}$ & $\begin{array}{l}\mathbf{W} \\
\mathbf{E} \\
\mathbf{W} \\
\mathbf{E} \\
\mathbf{W}\end{array}$ & $\frac{-}{\overline{070}}$ & $\begin{array}{l}265 \\
265 \\
265 \\
258 \\
258\end{array}$ & $\begin{array}{l}- \\
- \\
-\end{array}$ & $\begin{array}{l}- \\
- \\
-\end{array}$ & 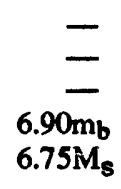 & $\begin{array}{l}\text { ABE } \\
\text { GR }\end{array}$ & $\begin{array}{l}- \\
- \\
-\end{array}$ & $\frac{-}{\bar{v}}$ & $\frac{-}{-}$ & $\begin{array}{l}- \\
- \\
-\end{array}$ \\
\hline $\begin{array}{l}1940 \\
1940 \\
1940 \\
1940 \\
1940\end{array}$ & $\begin{array}{l}04 \\
04 \\
04 \\
05 \\
05\end{array}$ & $\begin{array}{l}16 \\
16 \\
19 \\
04 \\
11\end{array}$ & $\begin{array}{l}060747.6 \\
064302.4 \\
000645 \\
072403.4 \\
135437.1\end{array}$ & $\begin{array}{l}52.30 \\
52.69 \\
52.4 \\
52.25 \\
52.15\end{array}$ & $\begin{array}{l}\mathbf{N} \\
\mathbf{N} \\
\mathbf{N} \\
\mathbf{N} \\
\mathbf{N}\end{array}$ & $\begin{array}{l}173.56 \\
173.25 \\
173.5 \\
172.94 \\
173.30\end{array}$ & $\begin{array}{l}\mathbf{E} \\
\mathbf{E} \\
\mathbf{E} \\
\mathbf{W} \\
\mathbf{W}\end{array}$ & $\begin{array}{l}000 \\
000 \\
- \\
000 \\
000\end{array}$ & $\begin{array}{l}432 \\
432 \\
265 \\
432 \\
432\end{array}$ & $\begin{array}{l}- \\
z \\
-\end{array}$ & $\begin{array}{l}\overline{-} \\
\bar{z}\end{array}$ & $\begin{array}{c}6.80 \mathrm{M}_{\mathrm{s}} \\
7.10 \mathrm{M}_{\mathrm{s}} \\
- \\
-\end{array}$ & $\begin{array}{l}\text { ABE } \\
\text { ABE } \\
- \\
-\end{array}$ & $\begin{array}{l}- \\
z \\
-\end{array}$ & - & $\frac{-}{-}$ & - \\
\hline $\begin{array}{l}1940 \\
1940 \\
1940 \\
1940 \\
1940\end{array}$ & $\begin{array}{l}05 \\
06 \\
07 \\
07 \\
07\end{array}$ & $\begin{array}{l}23 \\
18 \\
14 \\
15 \\
19\end{array}$ & $\begin{array}{ll}06 & 0124.4 \\
18 & 3859.6 \\
05 & 5253.5 \\
23 & 5614 \\
04 & 4728\end{array}$ & $\begin{array}{l}51.21 \\
52.52 \\
52.0 \\
52.0 \\
52.29\end{array}$ & $\begin{array}{l}\mathbf{N} \\
\mathbf{N} \\
\mathbf{N} \\
\mathbf{N} \\
\mathbf{N}\end{array}$ & $\begin{array}{l}173.31 \\
173.26 \\
178.2 \\
178.0 \\
174.01\end{array}$ & $\begin{array}{l}\mathbf{E} \\
\mathbf{E} \\
\mathbf{E} \\
\mathbf{E} \\
\mathbf{E}\end{array}$ & $\begin{array}{l}000 \\
000 \\
065 \\
065 \\
000\end{array}$ & $\begin{array}{l}432 \\
432 \\
265 \\
265 \\
432\end{array}$ & $\begin{array}{l}- \\
- \\
-\end{array}$ & $\begin{array}{l}- \\
- \\
-\end{array}$ & $\begin{array}{c}\text { - } \\
7.40 \mathrm{mb}_{\mathrm{b}} \\
5.75 \mathrm{M}_{\mathrm{s}}\end{array}$ & $\begin{array}{l}\mathrm{ABE} \\
\mathrm{GR}\end{array}$ & $\begin{array}{l}- \\
z \\
-\end{array}$ & $\begin{array}{l}- \\
- \\
-\end{array}$ & $\frac{-}{-}$ & $\begin{array}{l}- \\
- \\
-\end{array}$ \\
\hline $\begin{array}{l}1940 \\
1940 \\
1940 \\
1940 \\
1940\end{array}$ & $\begin{array}{l}07 \\
08 \\
09 \\
10 \\
11\end{array}$ & $\begin{array}{l}19 \\
22 \\
08 \\
11 \\
16\end{array}$ & $\begin{array}{l}1627 \\
032718 \\
101508 \\
075309 \\
022707.7\end{array}$ & $\begin{array}{l}61.0 \\
53.0 \\
53.3 \\
60.0 \\
52.14\end{array}$ & $\begin{array}{l}\mathbf{N} \\
\mathbf{N} \\
\mathbf{N} \\
\mathbf{N} \\
\mathbf{N}\end{array}$ & $\begin{array}{l}150.0 \\
165.5 \\
170.5 \\
150.5 \\
174.28\end{array}$ & $\begin{array}{l}\mathbf{W} \\
\mathbf{W} \\
\mathbf{E} \\
\mathbf{W} \\
\mathbf{E}\end{array}$ & $\frac{-}{\overline{0}}$ & $\begin{array}{r}38 \\
258 \\
265 \\
265 \\
432\end{array}$ & $\begin{array}{l}- \\
-\end{array}$ & $\begin{array}{l}- \\
z\end{array}$ & $\begin{array}{c}\frac{-00 \mathrm{M}_{\mathrm{s}}}{2.00 \mathrm{M}_{\mathrm{s}}} \\
-\end{array}$ & $\begin{array}{l}\text { ABE } \\
\text { GR }\end{array}$ & $\frac{-}{-}$ & $\begin{array}{l}\mathrm{VI} \\
\mathrm{V} \\
\overline{\mathrm{IV}} \\
-\end{array}$ & $\begin{array}{l}13 \\
13 \\
\overline{13} \\
-\end{array}$ & $\frac{-}{-}$ \\
\hline $\begin{array}{l}1941 \\
1941 \\
1941\end{array}$ & $\begin{array}{l}04 \\
04 \\
04\end{array}$ & $\begin{array}{l}01 \\
21 \\
21\end{array}$ & $\begin{array}{l}104059 \\
025404 \\
183205\end{array}$ & $\begin{array}{l}56.0 \\
53.6 \\
53.6\end{array}$ & $\begin{array}{l}\mathbf{N} \\
\mathbf{N} \\
\mathbf{N}\end{array}$ & $\begin{array}{l}153.5 \\
166.6 \\
166.6\end{array}$ & $\begin{array}{l}\mathbf{w} \\
\mathbf{W} \\
\mathbf{W}\end{array}$ & $\overline{-}$ & $\begin{array}{l}258 \\
265 \\
265\end{array}$ & - & $\overline{-}$ & $\frac{6.50 \mathrm{M}_{\mathrm{s}}}{-}$ & GR & $\overline{-}$ & $\bar{z}$ & $\overline{14}$ & $\overline{-}$ \\
\hline
\end{tabular}


ALASKA-Continued

[See table 1 for hypocenter and intensity references and table 2 for definitions of magnitude source codes. \&, land area only. Leader (-) indicates information is not available]

\begin{tabular}{|c|c|c|c|c|c|c|c|c|c|c|c|c|c|c|c|c|c|}
\hline \multirow{3}{*}{\multicolumn{4}{|c|}{$\begin{array}{cc} & \text { Origin } \\
\text { Date } & \text { time (UTC) }\end{array}$}} & \multicolumn{6}{|c|}{ Hypocenter } & \multicolumn{5}{|c|}{ Magnitude } & \multicolumn{2}{|c|}{ Intensity } & \multirow{3}{*}{$\begin{array}{l}\text { Folt area } \\
\left(1,000 \mathrm{~km}^{2}\right)\end{array}$} \\
\hline & & & & \multirow{2}{*}{\multicolumn{2}{|c|}{$\begin{array}{l}\text { Latitude } \\
\left(^{\circ}\right)\end{array}$}} & \multirow{2}{*}{\multicolumn{2}{|c|}{$\begin{array}{l}\text { Longitude } \\
\left.\text { ( }^{\circ}\right)\end{array}$}} & \multirow{2}{*}{$\begin{array}{l}\text { Depth } \\
(\mathrm{km})\end{array}$} & \multirow[t]{2}{*}{ Ref } & \multicolumn{2}{|c|}{ Usas } & \multirow{2}{*}{\multicolumn{2}{|c|}{ Other }} & \multirow{2}{*}{$\begin{array}{c}\text { Moment } \\
\mathbf{M}\end{array}$} & \multirow[t]{2}{*}{ MM } & \multirow[t]{2}{*}{ Ref } & \\
\hline & & & & & & & & & & $m_{b}$ & $\mathbf{M}_{\mathbf{s}}$ & & & & & & \\
\hline $\begin{array}{l}\overline{1941} \\
1941\end{array}$ & $\begin{array}{l}07 \\
08\end{array}$ & $\begin{array}{l}30 \\
04\end{array}$ & $\begin{array}{lll}015121 \\
1053 & 12.4\end{array}$ & $\begin{array}{l}61.0 \\
51.44\end{array}$ & $\begin{array}{l}\mathbf{N} \\
\mathbf{N}\end{array}$ & $\begin{array}{l}151.0 \\
178.59\end{array}$ & $\begin{array}{l}\mathbf{W} \\
\mathbf{E}\end{array}$ & $\overline{090}$ & $\begin{array}{l}258 \\
260\end{array}$ & - & - & $\begin{array}{l}6.25 \mathrm{M}_{\mathrm{s}} \\
6.75 \mathrm{M}_{\mathrm{s}}\end{array}$ & $\begin{array}{l}\text { GR } \\
\text { GR }\end{array}$ & - & VI & 38 & - \\
\hline $\begin{array}{l}1941 \\
1941 \\
1941 \\
1942 \\
1942\end{array}$ & $\begin{array}{l}08 \\
09 \\
11 \\
03 \\
09\end{array}$ & $\begin{array}{l}06 \\
28 \\
06 \\
20 \\
02\end{array}$ & $\begin{array}{llll}06 & 15 & 06 \\
05 & 34 & 12 \\
12 & 29 & 46 \\
01 & 12 & 59 \\
03 & 17 & 17\end{array}$ & $\begin{array}{l}55.75 \\
56.5 \\
54.0 \\
52.8 \\
53.4\end{array}$ & $\begin{array}{l}\mathbf{N} \\
\mathbf{N} \\
\mathbf{N} \\
\mathbf{N} \\
\mathbf{N}\end{array}$ & $\begin{array}{l}163.0 \\
157.5 \\
161.5 \\
168.2 \\
168.7\end{array}$ & $\begin{array}{l}\mathbf{W} \\
\mathbf{W} \\
\mathbf{W} \\
\mathbf{W} \\
\mathbf{W}\end{array}$ & $\begin{array}{l}150 \\
100 \\
- \\
-\end{array}$ & $\begin{array}{l}258 \\
258 \\
258 \\
265 \\
265\end{array}$ & $\begin{array}{l}- \\
z\end{array}$ & $\begin{array}{l}- \\
- \\
-\end{array}$ & $\begin{array}{c}6.75 \mathrm{~m}_{\mathrm{b}} \\
6.50 \mathrm{mb} \\
6.00 \mathrm{M}_{\mathrm{s}} \\
-\end{array}$ & $\begin{array}{l}\text { GR } \\
\text { GR } \\
\text { GR } \\
- \\
-\end{array}$ & $\frac{-}{-}$ & $\begin{array}{l}- \\
\overline{-}\end{array}$ & - & - \\
\hline $\begin{array}{l}1942 \\
1942 \\
1942 \\
1942 \\
1943\end{array}$ & $\begin{array}{l}09 \\
09 \\
12 \\
12 \\
01\end{array}$ & $\begin{array}{l}04 \\
09 \\
05 \\
09 \\
27\end{array}$ & $\begin{array}{lll}17 & 46 & 16 \\
01 & 25 & 17 \\
14 & 28 & 40 \\
22 & 18 & 59 \\
02 & 45 & 12\end{array}$ & $\begin{array}{l}53.4 \\
53.5 \\
59.5 \\
53.6 \\
51.4\end{array}$ & $\begin{array}{l}\mathbf{N} \\
\mathbf{N} \\
\mathbf{N} \\
\mathbf{N} \\
\mathbf{N}\end{array}$ & $\begin{array}{l}168.7 \\
165.9 \\
152.0 \\
166.6 \\
179.2\end{array}$ & $\begin{array}{l}\mathbf{W} \\
\mathbf{W} \\
\mathbf{W} \\
\mathbf{W} \\
\mathbf{W}\end{array}$ & $\begin{array}{l}- \\
080 \\
100 \\
- \\
-\end{array}$ & $\begin{array}{l}265 \\
265 \\
258 \\
265 \\
265\end{array}$ & $\begin{array}{l}- \\
z \\
-\end{array}$ & $\begin{array}{l}- \\
- \\
-\end{array}$ & $\begin{array}{c}- \\
6.90 \mathrm{mb} \\
6.50 \mathrm{mb} \\
- \\
-\end{array}$ & $\begin{array}{l}\text { ABE } \\
\text { GR }\end{array}$ & $\frac{-}{-}$ & $\begin{array}{l}- \\
\overline{-} \\
-\end{array}$ & - & $\begin{array}{l}- \\
- \\
-\end{array}$ \\
\hline $\begin{array}{l}1943 \\
1943 \\
1943 \\
1944 \\
1944\end{array}$ & $\begin{array}{l}06 \\
07 \\
11 \\
07 \\
08\end{array}$ & $\begin{array}{l}28 \\
09 \\
03 \\
27 \\
14\end{array}$ & $\begin{array}{lll}15 & 05 & 21 \\
2328 & 29 \\
14 & 3217.5 \\
000423 \\
110723\end{array}$ & $\begin{array}{l}51.7 \\
52.0 \\
61.90 \\
54.0 \\
59.0\end{array}$ & $\begin{array}{l}\mathbf{N} \\
\mathbf{N} \\
\mathbf{N} \\
\mathbf{N} \\
\mathbf{N}\end{array}$ & $\begin{array}{l}178.5 \\
166.9 \\
150.84 \\
165.5 \\
155.0\end{array}$ & $\begin{array}{l}\mathbf{W} \\
\mathbf{W} \\
\mathbf{W} \\
\mathbf{W} \\
\mathbf{W}\end{array}$ & $\begin{array}{l}- \\
\overline{000} \\
070 \\
100\end{array}$ & $\begin{array}{l}265 \\
265 \\
432 \\
258 \\
258\end{array}$ & $\begin{array}{l}- \\
- \\
-\end{array}$ & $\begin{array}{l}- \\
- \\
-\end{array}$ & $\begin{array}{c}-\overline{-} \\
7.40 \mathrm{M}_{\mathrm{s}} \\
7.10 \mathrm{~m}_{\mathrm{b}} \\
6.25 \mathrm{~m}_{\mathrm{b}}\end{array}$ & $\begin{array}{l}\text { ABE } \\
\text { ABE } \\
\text { GR }\end{array}$ & $\frac{-}{-}$ & $\begin{array}{l}\bar{z} \\
\bar{v} \\
-\end{array}$ & $\begin{array}{l}- \\
- \\
-\end{array}$ & $\begin{array}{l}- \\
- \\
-\end{array}$ \\
\hline $\begin{array}{l}1944 \\
1945 \\
1945 \\
1945 \\
1945\end{array}$ & $\begin{array}{l}12 \\
03 \\
06 \\
07 \\
11\end{array}$ & $\begin{array}{l}12 \\
18 \\
01 \\
11 \\
03\end{array}$ & $\begin{array}{l}041710 \\
185441 \\
151340 \\
003034 \\
220903\end{array}$ & $\begin{array}{l}51.5 \\
55.0 \\
53.4 \\
59.2 \\
58.5\end{array}$ & $\begin{array}{l}\mathbf{N} \\
\mathbf{N} \\
\mathbf{N} \\
\mathbf{N} \\
\mathbf{N}\end{array}$ & $\begin{array}{l}179.5 \\
156.5 \\
168.7 \\
152.4 \\
151.0\end{array}$ & $\begin{array}{l}\mathbf{E} \\
\mathbf{W} \\
\mathbf{W} \\
\mathbf{W} \\
\mathbf{W}\end{array}$ & $\frac{-}{0 \overline{00}} \frac{-}{050}$ & $\begin{array}{l}258 \\
265 \\
265 \\
265 \\
258\end{array}$ & $\bar{z}$ & $\begin{array}{l}z \\
-\end{array}$ & $\frac{-}{6.90 \mathrm{M}_{\mathrm{s}}}+$ & $\mathrm{ABE}$ & $\overline{-}$ & $\overline{-}$ & - & $\begin{array}{c}- \\
- \\
-\end{array}$ \\
\hline $\begin{array}{l}1945 \\
1945 \\
1946 \\
1946 \\
1946\end{array}$ & $\begin{array}{l}11 \\
12 \\
01 \\
02 \\
04\end{array}$ & $\begin{array}{l}16 \\
25 \\
12 \\
04 \\
01\end{array}$ & $\begin{array}{llll}18 & 02 & 22 \\
01 & 25 & 45 \\
20 & 25 & 40.2 \\
03 & 44 & 48 \\
12 & 28 & 56.0\end{array}$ & $\begin{array}{l}58.0 \\
52.2 \\
59.11 \\
53.0 \\
53.32\end{array}$ & $\begin{array}{l}\mathbf{N} \\
\mathbf{N} \\
\mathbf{N} \\
\mathbf{N} \\
\mathbf{N}\end{array}$ & $\begin{array}{l}136.5 \\
173.9 \\
148.94 \\
176.0 \\
163.19\end{array}$ & $\begin{array}{l}\mathbf{W} \\
\mathbf{E} \\
\mathbf{W} \\
\mathbf{W} \\
\mathbf{W}\end{array}$ & $\begin{array}{l}- \\
056 \\
160 \\
000\end{array}$ & $\begin{array}{l}258 \\
265 \\
432 \\
258 \\
432\end{array}$ & $\begin{array}{l}- \\
- \\
-\end{array}$ & $\begin{array}{l}- \\
-\end{array}$ & $\begin{array}{c}5.60 \mathrm{M}_{\mathrm{s}} \\
\overline{-70 \mathrm{M}_{\mathrm{s}}} \\
6.75 \mathrm{~m}_{\mathrm{b}} \\
7.30 \mathrm{M}_{\mathrm{s}}\end{array}$ & $\begin{array}{l}\text { GR } \\
\text { ABE } \\
\text { GR } \\
\text { ABE }\end{array}$ & $\begin{array}{l}- \\
- \\
-\end{array}$ & $\frac{\text { IV }}{\overline{I V}}$ & $\frac{\frac{18}{19}}{38}$ & $\begin{array}{l}- \\
- \\
-\end{array}$ \\
\hline $\begin{array}{l}1946 \\
1946 \\
1946 \\
1946 \\
1946\end{array}$ & $\begin{array}{l}04 \\
04 \\
04 \\
04 \\
04\end{array}$ & $\begin{array}{l}01 \\
01 \\
01 \\
01 \\
01\end{array}$ & $\begin{array}{l}125243.0 \\
125547.0 \\
132850 \\
152022 \\
155034\end{array}$ & $\begin{array}{l}\mathbf{5 4 . 0 8} \\
\mathbf{5 4 . 1 7} \\
\mathbf{5 3 . 4} \\
\mathbf{5 3 . 4} \\
\mathbf{5 3 . 4}\end{array}$ & $\begin{array}{l}\mathbf{N} \\
\mathbf{N} \\
\mathbf{N} \\
\mathbf{N} \\
\mathbf{N}\end{array}$ & $\begin{array}{l}162.61 \\
163.22 \\
163.1 \\
163.1 \\
163.1\end{array}$ & $\begin{array}{l}\mathbf{W} \\
\mathbf{W} \\
\mathbf{w} \\
\mathbf{W} \\
\mathbf{W}\end{array}$ & $\begin{array}{l}000 \\
000 \\
- \\
-\end{array}$ & $\begin{array}{l}432 \\
432 \\
265 \\
265 \\
265\end{array}$ & $\begin{array}{l}z \\
z\end{array}$ & $\begin{array}{l}- \\
-\end{array}$ & - & & $\begin{array}{l}- \\
- \\
-\end{array}$ & $\frac{-}{-}$ & $\frac{-}{-}$ & $\begin{array}{l}- \\
- \\
-\end{array}$ \\
\hline $\begin{array}{l}1946 \\
1946 \\
1946 \\
1946 \\
1946\end{array}$ & $\begin{array}{l}04 \\
04 \\
04 \\
04 \\
04\end{array}$ & $\begin{array}{l}01 \\
01 \\
02 \\
02 \\
02\end{array}$ & $\begin{array}{lll}16 & 59 & 14.0 \\
18 & 5735.4 \\
04 & 1338.1 \\
05 & 38 & 15.7 \\
05 & 57 & 12.4\end{array}$ & $\begin{array}{l}53.88 \\
53.95 \\
53.63 \\
53.98 \\
54.12\end{array}$ & $\begin{array}{l}\mathbf{N} \\
\mathbf{N} \\
\mathbf{N} \\
\mathbf{N} \\
\mathbf{N}\end{array}$ & $\begin{array}{l}163.48 \\
163.50 \\
163.73 \\
162.69 \\
162.06\end{array}$ & $\begin{array}{l}\mathbf{W} \\
\mathbf{W} \\
\mathbf{W} \\
\mathbf{w} \\
\mathbf{W}\end{array}$ & $\begin{array}{l}000 \\
000 \\
000 \\
000 \\
000\end{array}$ & $\begin{array}{l}432 \\
432 \\
432 \\
432 \\
432\end{array}$ & $\begin{array}{l}z \\
z\end{array}$ & $=$ & 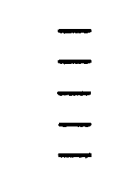 & & $\begin{array}{l}- \\
z\end{array}$ & $\begin{array}{l}- \\
-\end{array}$ & - & $\frac{z}{E}$ \\
\hline $\begin{array}{l}1946 \\
1946 \\
1946 \\
1946 \\
1946\end{array}$ & $\begin{array}{l}04 \\
04 \\
04 \\
04 \\
04\end{array}$ & $\begin{array}{l}02 \\
02 \\
03 \\
04 \\
04\end{array}$ & $\begin{array}{lll}14 & 27 & 27.4 \\
16 & 30 & 25.2 \\
08 & 58 & 33.3 \\
16 & 31 & 07.7 \\
21 & 25 & 41.8\end{array}$ & $\begin{array}{l}53.96 \\
53.96 \\
53.85 \\
53.56 \\
53.93\end{array}$ & $\begin{array}{l}\mathbf{N} \\
\mathbf{N} \\
\mathbf{N} \\
\mathbf{N} \\
\mathbf{N}\end{array}$ & $\begin{array}{l}163.18 \\
163.59 \\
163.42 \\
163.15 \\
163.19\end{array}$ & $\begin{array}{l}\mathbf{W} \\
\mathbf{W} \\
\mathbf{W} \\
\mathbf{W} \\
\mathbf{W}\end{array}$ & $\begin{array}{l}000 \\
000 \\
000 \\
000 \\
000\end{array}$ & $\begin{array}{l}432 \\
432 \\
432 \\
432 \\
432\end{array}$ & $\begin{array}{l}z \\
z\end{array}$ & $=$ & - & & $\begin{array}{l}- \\
-\end{array}$ & $\frac{-}{-}$ & $\frac{-}{-}$ & $\frac{-}{E}$ \\
\hline $\begin{array}{l}1946 \\
1946 \\
1946 \\
1946 \\
1946\end{array}$ & $\begin{array}{l}04 \\
06 \\
07 \\
07 \\
08\end{array}$ & $\begin{array}{l}06 \\
03 \\
12 \\
25 \\
02\end{array}$ & $\begin{array}{l}045234.1 \\
134408 \\
215627 \\
164207 \\
013756\end{array}$ & $\begin{array}{l}53.42 \\
52.1 \\
53.5 \\
51.4 \\
53.4\end{array}$ & $\begin{array}{l}\mathbf{N} \\
\mathbf{N} \\
\mathbf{N} \\
\mathbf{N} \\
\mathbf{N}\end{array}$ & $\begin{array}{l}163.59 \\
171.2 \\
169.0 \\
179.2 \\
163.1\end{array}$ & $\begin{array}{l}\mathbf{w} \\
\mathbf{W} \\
\mathbf{W} \\
\mathbf{W} \\
\mathbf{W}\end{array}$ & $\begin{array}{c}000 \\
\overline{100} \\
-\end{array}$ & $\begin{array}{l}432 \\
265 \\
258 \\
265 \\
265\end{array}$ & $\begin{array}{l}- \\
z\end{array}$ & $\begin{array}{l}- \\
z\end{array}$ & $\frac{-}{6.75 \mathrm{mb}_{\mathrm{b}}}+$ & GR & $\begin{array}{l}- \\
-\end{array}$ & $\frac{-}{-}$ & $\overline{-}$ & $\begin{array}{l}- \\
- \\
-\end{array}$ \\
\hline $\begin{array}{l}1946 \\
1946 \\
1946\end{array}$ & $\begin{array}{l}08 \\
10 \\
11\end{array}$ & $\begin{array}{l}07 \\
30 \\
01\end{array}$ & $\begin{array}{l}193129 \\
074734 \\
111424\end{array}$ & $\begin{array}{l}51.5 \\
54.25 \\
51.5\end{array}$ & $\begin{array}{l}\mathbf{N} \\
\mathbf{N} \\
\mathbf{N}\end{array}$ & $\begin{array}{l}173.5 \\
164.0 \\
174.5\end{array}$ & $\begin{array}{l}\mathbf{W} \\
\mathbf{W} \\
\mathbf{W}\end{array}$ & $\overline{-}$ & $\begin{array}{l}265 \\
258 \\
258\end{array}$ & $\overline{-}$ & $\overline{-}$ & $\begin{array}{l}6.90 \mathrm{M}_{s} \\
7.00 \mathrm{M}_{s}\end{array}$ & $\begin{array}{l}\text { GR } \\
\text { GR }\end{array}$ & - & - & - & $\overline{-}$ \\
\hline
\end{tabular}


EARTHQUAKES IN ALASKA

ALASKA-Continued

[See table 1 for hypocenter and intensity references and table 2 for definitions of magnitude source codes. \&, land area only. Leader (--) indicates information is not available]

\begin{tabular}{|c|c|c|c|c|c|c|c|c|c|c|c|c|c|c|c|c|}
\hline \multirow{2}{*}{\multicolumn{4}{|c|}{$\begin{array}{cc} & \text { Origin } \\
\text { Date } & \text { time (UTC) } \\
\end{array}$}} & \multicolumn{6}{|c|}{ Hypocenter } & \multicolumn{4}{|c|}{ Magnitude } & \multicolumn{2}{|c|}{ Intensity } & \multirow{3}{*}{$\begin{array}{l}\text { Felt area } \\
\left(1,000 \mathrm{~km}^{2}\right)\end{array}$} \\
\hline & & & & \multirow{2}{*}{\multicolumn{2}{|c|}{$\begin{array}{l}\text { Latitude } \\
\left(^{\circ}\right)\end{array}$}} & \multirow{2}{*}{\multicolumn{2}{|c|}{$\begin{array}{c}\text { Longitude } \\
\left.\text { ( }{ }^{\circ}\right)\end{array}$}} & \multirow{2}{*}{$\begin{array}{c}\text { Depth } \\
(\mathrm{km})\end{array}$} & \multirow[t]{2}{*}{ Ref } & \multicolumn{2}{|c|}{ USGS } & \multirow[t]{2}{*}{ Other } & \multirow{2}{*}{$\begin{array}{c}\text { Moment } \\
\mathbf{M}\end{array}$} & \multirow[t]{2}{*}{ MM } & \multirow[t]{2}{*}{ Ref } & \\
\hline & & & & & & & & & & $m_{b}$ & $\mathbf{M}_{\mathbf{s}}$ & & & & & \\
\hline $\begin{array}{l}1946 \\
1946\end{array}$ & $\begin{array}{l}11 \\
12\end{array}$ & $\begin{array}{l}12 \\
25\end{array}$ & $\begin{array}{l}055620 \\
111310\end{array}$ & $\begin{array}{l}53.6 \\
51.5\end{array}$ & $\begin{array}{l}\mathbf{N} \\
\mathbf{N}\end{array}$ & $\begin{array}{l}164.4 \\
180.0\end{array}$ & $\begin{array}{l}\mathbf{W} \\
\mathbf{E}\end{array}$ & $\overline{090}$ & $\begin{array}{l}265 \\
258\end{array}$ & - & - & $6.50 \mathrm{~m}_{b} G R$ & - & - & - & - \\
\hline $\begin{array}{l}1947 \\
1947 \\
1947 \\
1947\end{array}$ & $\begin{array}{l}01 \\
07 \\
10 \\
10\end{array}$ & $\begin{array}{l}23 \\
28 \\
07 \\
15\end{array}$ & $\begin{array}{l}155740 \\
034852 \\
015321 \\
193437\end{array}$ & $\begin{array}{l}53.3 \\
63.4 \\
64.2 \\
64.2\end{array}$ & $\begin{array}{l}\mathbf{N} \\
\mathbf{N} \\
\mathbf{N} \\
\mathbf{N}\end{array}$ & $\begin{array}{l}162.5 \\
147.9 \\
148.3 \\
148.3\end{array}$ & $\begin{array}{l}\mathbf{W} \\
\mathbf{W} \\
\mathbf{W} \\
\mathbf{W}\end{array}$ & $\bar{z}$ & $\begin{array}{l}265 \\
265 \\
265 \\
265\end{array}$ & $\bar{z}$ & Z & $\bar{z}$ & E & $\frac{\overline{\mathbf{I V}}}{\overline{-}}$ & $\begin{array}{l}\overline{20} \\
-\end{array}$ & - \\
\hline 1947 & 10 & 16 & 020952.5 & 64.28 & $\mathbf{N}$ & 148.23 & $w$ & 066 & 260 & - & - & $7.20 \mathrm{M}_{s} \mathrm{ABE}$ & - & VII & 20 & - \\
\hline $\begin{array}{l}1947 \\
1948 \\
1948 \\
1948 \\
1948\end{array}$ & $\begin{array}{l}10 \\
01 \\
02 \\
05 \\
05\end{array}$ & $\begin{array}{l}20 \\
16 \\
11 \\
14 \\
15\end{array}$ & $\begin{array}{lll}01 & 43 & 16 \\
11 & 08 & 38.2 \\
15 & 41 & 56 \\
22 & 31 & 43.4 \\
02 & 41 & 50.7\end{array}$ & $\begin{array}{l}64.2 \\
52.09 \\
63.8 \\
54.71 \\
54.81\end{array}$ & $\begin{array}{l}\mathbf{N} \\
\mathbf{N} \\
\mathbf{N} \\
\mathbf{N} \\
\mathbf{N}\end{array}$ & $\begin{array}{l}148.3 \\
174.73 \\
145.4 \\
160.88 \\
161.61\end{array}$ & $\begin{array}{l}\mathbf{W} \\
\mathbf{E} \\
\mathbf{W} \\
\mathbf{W} \\
\mathbf{W}\end{array}$ & $\begin{array}{l}\frac{\overline{033}}{\overline{000}} \\
048\end{array}$ & $\begin{array}{l}265 \\
260 \\
265 \\
432 \\
432\end{array}$ & $\begin{array}{l}\overline{-} \\
\overline{-}\end{array}$ & $\begin{array}{l}- \\
- \\
-\end{array}$ & $\begin{array}{l}6.70 \overline{\mathrm{Ukn}}_{\mathrm{kAS}} \\
\overline{-}_{\mathrm{T}} \mathrm{ABE}\end{array}$ & $\overline{-}$ & $\begin{array}{l}\overline{-} \\
\overline{I V} \\
-\end{array}$ & $\begin{array}{l}- \\
- \\
-\end{array}$ & $\begin{array}{l}- \\
- \\
-\end{array}$ \\
\hline $\begin{array}{l}1948 \\
1948 \\
1948 \\
1948 \\
1948\end{array}$ & $\begin{array}{l}05 \\
05 \\
08 \\
09 \\
12\end{array}$ & $\begin{array}{l}17 \\
26 \\
19 \\
19 \\
12\end{array}$ & $\begin{array}{l}174838.6 \\
091653 \\
135046 \\
061404 \\
131718\end{array}$ & $\begin{array}{l}55.08 \\
56.3 \\
63.0 \\
51.6 \\
51.6\end{array}$ & $\begin{array}{l}\mathbf{N} \\
\mathbf{N} \\
\mathbf{N} \\
\mathbf{N} \\
\mathbf{N}\end{array}$ & $\begin{array}{l}160.99 \\
153.8 \\
150.5 \\
177.8 \\
177.2\end{array}$ & $\begin{array}{l}\mathbf{W} \\
\mathbf{W} \\
\mathbf{W} \\
\mathbf{W} \\
\mathbf{E}\end{array}$ & $\frac{044}{100}$ & $\begin{array}{l}432 \\
265 \\
258 \\
265 \\
265\end{array}$ & $\begin{array}{l}z \\
\bar{z}\end{array}$ & $\begin{array}{l}- \\
- \\
-\end{array}$ & $\begin{array}{l}6.00 \overline{\mathrm{Ukn}}_{\mathrm{kAS}} \\
6.25 \mathrm{~m}_{\mathrm{b}} \mathrm{GR} \\
\overline{\mathrm{U}}_{\mathrm{U}} \mathrm{PAS}\end{array}$ & $\overline{-}$ & $\begin{array}{l}- \\
\bar{v} \\
-\end{array}$ & $\begin{array}{l}- \\
\bar{Z} \\
-\end{array}$ & $\begin{array}{l}- \\
-\end{array}$ \\
\hline $\begin{array}{l}1949 \\
1949 \\
1949 \\
1949 \\
1949\end{array}$ & $\begin{array}{l}02 \\
06 \\
08 \\
09 \\
10\end{array}$ & $\begin{array}{l}02 \\
15 \\
25 \\
27 \\
31\end{array}$ & $\begin{array}{lll}174129 \\
014717 \\
041421 \\
153045 \\
013929.5\end{array}$ & $\begin{array}{l}53.0 \\
51.4 \\
52.2 \\
59.75 \\
56.05\end{array}$ & $\begin{array}{l}\mathbf{N} \\
\mathbf{N} \\
\mathbf{N} \\
\mathbf{N} \\
\mathbf{N}\end{array}$ & $\begin{array}{l}173.0 \\
179.2 \\
179.3 \\
149.0 \\
135.69\end{array}$ & $\begin{array}{l}\mathbf{W} \\
\mathbf{W} \\
\mathbf{W} \\
\mathbf{W} \\
\mathbf{W}\end{array}$ & $\frac{220}{-}$ & $\begin{array}{l}258 \\
265 \\
265 \\
258 \\
448\end{array}$ & $\begin{array}{l}- \\
- \\
-\end{array}$ & $\begin{array}{l}- \\
- \\
-\end{array}$ & $\begin{array}{l}6.80 \mathrm{~m}_{\mathrm{b}} \mathrm{ABE} \\
6.75 \overline{\mathrm{U}}_{\mathrm{kn}} \mathrm{PAS} \\
6.70 \mathrm{M}_{\mathrm{s}} \mathrm{ABE} \\
6.25 \mathrm{M}_{\mathrm{s}} \mathrm{GR}\end{array}$ & $\frac{-}{-}$ & $\frac{-}{\bar{v}}$ & $\begin{array}{l}- \\
\overline{38} \\
-\end{array}$ & - \\
\hline $\begin{array}{l}1950 \\
1950 \\
1950 \\
1950 \\
1950\end{array}$ & $\begin{array}{l}03 \\
04 \\
04 \\
04 \\
05\end{array}$ & $\begin{array}{l}27 \\
04 \\
04 \\
05 \\
25\end{array}$ & $\begin{array}{l}130402 \\
022107 \\
022445 \\
011713 \\
083437\end{array}$ & $\begin{array}{l}53.3 \\
51.5 \\
51.5 \\
52.1 \\
65.5\end{array}$ & $\begin{array}{l}\mathbf{N} \\
\mathbf{N} \\
\mathbf{N} \\
\mathbf{N} \\
\mathbf{N}\end{array}$ & $\begin{array}{l}172.2 \\
173.5 \\
173.5 \\
177.5 \\
151.5\end{array}$ & $\begin{array}{l}\mathbf{E} \\
\mathbf{W} \\
\mathbf{W} \\
\mathbf{W} \\
\mathbf{W}\end{array}$ & $\begin{array}{l}- \\
-\end{array}$ & $\begin{array}{l}265 \\
265 \\
265 \\
265 \\
265\end{array}$ & $\begin{array}{l}z \\
z\end{array}$ & $\overline{-}$ & $\begin{array}{c}\text { 6.70Ukn PAS } \\
\bar{Z} \\
\text { 6.00Ukn PAS }\end{array}$ & $\bar{z}$ & $\begin{array}{l}z \\
z\end{array}$ & $\begin{array}{l}- \\
- \\
-\end{array}$ & $\begin{array}{l}- \\
-\end{array}$ \\
\hline $\begin{array}{l}1950 \\
1950 \\
1950 \\
1950 \\
1950\end{array}$ & $\begin{array}{l}07 \\
07 \\
08 \\
09 \\
09\end{array}$ & $\begin{array}{l}12 \\
19 \\
26 \\
02 \\
16\end{array}$ & $\begin{array}{lll}1109 & 10 \\
105157 \\
043927 \\
024713 \\
215815\end{array}$ & $\begin{array}{l}52.5 \\
51.5 \\
65.0 \\
52.5 \\
52.0\end{array}$ & $\begin{array}{l}\mathbf{N} \\
\mathbf{N} \\
\mathbf{N} \\
\mathbf{N} \\
\mathbf{N}\end{array}$ & $\begin{array}{l}167.5 \\
179.5 \\
162.0 \\
170.0 \\
177.0\end{array}$ & $\begin{array}{l}\mathbf{W} \\
\mathbf{E} \\
\mathbf{W} \\
\mathbf{W} \\
\mathbf{E}\end{array}$ & $\frac{-}{\overline{1}}$ & $\begin{array}{l}265 \\
265 \\
266 \\
265 \\
266\end{array}$ & $\begin{array}{l}z \\
z\end{array}$ & $\overline{-}$ & $\begin{array}{c}\text { 6.25Ukn PAS } \\
\text { 6.50Ukn PAS } \\
\text { 6.40Ukn PAS } \\
\text { 6.60Ukn PAS }\end{array}$ & $\begin{array}{l}- \\
- \\
-\end{array}$ & $\frac{\overline{-}}{\overline{I V}}$ & $\begin{array}{l}- \\
23 \\
-\end{array}$ & $\begin{array}{l}- \\
-\end{array}$ \\
\hline $\begin{array}{l}1950 \\
1951 \\
1951 \\
1951 \\
1951\end{array}$ & $\begin{array}{l}11 \\
01 \\
02 \\
02 \\
03\end{array}$ & $\begin{array}{l}22 \\
18 \\
12 \\
13 \\
31\end{array}$ & $\begin{array}{lll}10 & 16 & 28 \\
21 & 15 & 45 \\
03 & 31 & 40 \\
22 & 1253.8 \\
09 & 2034\end{array}$ & $\begin{array}{l}51.5 \\
52.1 \\
51.9 \\
55.55 \\
60.4\end{array}$ & $\begin{array}{l}\mathbf{N} \\
\mathbf{N} \\
\mathbf{N} \\
\mathbf{N} \\
\mathbf{N}\end{array}$ & $\begin{array}{l}176.5 \\
177.5 \\
179.4 \\
156.35 \\
153.6\end{array}$ & $\begin{array}{l}\mathbf{W} \\
\mathbf{W} \\
\mathbf{E} \\
\mathbf{W} \\
\mathbf{W}\end{array}$ & $\begin{array}{c}060 \\
- \\
- \\
000 \\
223\end{array}$ & $\begin{array}{l}266 \\
265 \\
265 \\
432 \\
265\end{array}$ & $\begin{array}{l}= \\
z \\
-\end{array}$ & $\begin{array}{l}- \\
- \\
-\end{array}$ & $\begin{array}{c}\text { 6.75Ukn PAS } \\
6.38 \mathrm{Ukn} \text { PAS } \\
\text { 7.10 } \overline{\mathrm{M}}_{\mathrm{S}} \mathrm{ABE} \\
-\end{array}$ & $\begin{array}{l}- \\
-\end{array}$ & $\begin{array}{c}\text { Felt } \\
- \\
-\end{array}$ & $\begin{array}{l}24 \\
- \\
-\end{array}$ & $\begin{array}{l}- \\
- \\
-\end{array}$ \\
\hline $\begin{array}{l}1951 \\
1951 \\
1951 \\
1951 \\
1951\end{array}$ & $\begin{array}{l}05 \\
06 \\
06 \\
07 \\
10\end{array}$ & $\begin{array}{l}10 \\
01 \\
25 \\
19 \\
01\end{array}$ & $\begin{array}{lll}19 & 44 & 47 \\
20 & 02 & 13 \\
16 & 1237 \\
20 & 41 & 28 \\
10 & 1150\end{array}$ & $\begin{array}{l}51.5 \\
53.1 \\
61.1 \\
51.6 \\
55.0\end{array}$ & $\begin{array}{l}\mathbf{N} \\
\mathbf{N} \\
\mathbf{N} \\
\mathbf{N} \\
\mathbf{N}\end{array}$ & $\begin{array}{l}179.5 \\
172.5 \\
150.1 \\
177.8 \\
164.0\end{array}$ & $\begin{array}{l}\mathbf{E} \\
\mathbf{W} \\
\mathbf{W} \\
\mathbf{W} \\
\mathbf{W}\end{array}$ & $\begin{array}{l}\overline{065} \\
128 \\
065 \\
-\end{array}$ & $\begin{array}{l}265 \\
265 \\
265 \\
265 \\
265\end{array}$ & $\begin{array}{l}\overline{-} \\
\overline{-}\end{array}$ & $\begin{array}{l}- \\
- \\
-\end{array}$ & $\begin{array}{c}-\overline{-} \\
\text { 6.25Ukn PAS } \\
\text { 5.88Ukn PAS } \\
-\end{array}$ & - & $\begin{array}{r}\bar{Z} \\
\text { Felt } \\
-\end{array}$ & $\begin{array}{l}\bar{Z} \\
24 \\
294 \\
-\end{array}$ & z \\
\hline $\begin{array}{l}1951 \\
1951 \\
1952 \\
1952 \\
1952\end{array}$ & $\begin{array}{l}11 \\
12 \\
01 \\
01 \\
01\end{array}$ & $\begin{array}{l}08 \\
30 \\
12 \\
19 \\
21\end{array}$ & $\begin{array}{llll}13 & 45 & 07.3 \\
17 & 42 & 14 \\
20 & 11 & 37 \\
07 & 15 & 31 \\
03 & 42 & 55\end{array}$ & $\begin{array}{l}54.33 \\
62.0 \\
52.5 \\
52.5 \\
52.5\end{array}$ & $\begin{array}{l}\mathbf{N} \\
\mathbf{N} \\
\mathbf{N} \\
\mathbf{N} \\
\mathbf{N}\end{array}$ & $\begin{array}{l}160.69 \\
148.8 \\
167.5 \\
167.5 \\
167.5\end{array}$ & $\begin{array}{l}\mathbf{W} \\
\mathbf{W} \\
\mathbf{W} \\
\mathbf{W} \\
\mathbf{W}\end{array}$ & $\frac{033}{E}$ & $\begin{array}{l}260 \\
265 \\
265 \\
265 \\
265\end{array}$ & $\begin{array}{l}= \\
\overline{-}\end{array}$ & $\begin{array}{l}- \\
- \\
-\end{array}$ & $\begin{array}{l}6.25 \mathrm{Ukn} \text { PAS } \\
\text { 6.50Ukn PAS } \\
6.7 \overline{\mathrm{U} k n} \text { PAS }\end{array}$ & $\begin{array}{l}z \\
z\end{array}$ & $\begin{array}{l}\overline{I V} \\
- \\
-\end{array}$ & $\begin{array}{l}\overline{24} \\
- \\
-\end{array}$ & - \\
\hline $\begin{array}{l}1952 \\
1952 \\
1952\end{array}$ & $\begin{array}{l}01 \\
02 \\
03\end{array}$ & $\begin{array}{l}24 \\
02 \\
22\end{array}$ & $\begin{array}{l}091404 \\
102007 \\
181542\end{array}$ & $\begin{array}{l}52.5 \\
51.4 \\
51.5\end{array}$ & $\begin{array}{l}\mathbf{N} \\
\mathbf{N} \\
\mathbf{N}\end{array}$ & $\begin{array}{l}167.5 \\
179.2 \\
173.5\end{array}$ & $\begin{array}{l}\mathbf{w} \\
\mathbf{w} \\
\mathbf{W}\end{array}$ & $\overline{096}$ & $\begin{array}{l}265 \\
265 \\
265\end{array}$ & E & - & $\overline{6.38 \mathrm{Ukn} \text { PAS }}^{-}$ & - & Felt & $\overline{25}$ & - \\
\hline
\end{tabular}


ALASKA-Continued

[See table 1 for hypocenter and intensity references and table 2 for definitions of magnitude source codes. \&, land area only. Leader (-) indicates information is not available]

\begin{tabular}{|c|c|c|c|c|c|c|c|c|c|c|c|c|c|c|c|c|}
\hline & & & Origin & & & Hypoce & sente & & & & & Magnitude & & Int & 1sity & \\
\hline & Date & & time (UTC) & Latitud & & Longitud & & Depth & Ref & US & & Other & Moment & MM & Ref & Felt area \\
\hline $\mathrm{Yr}$ & Mo & & h m 8 & $\left({ }^{\circ}\right)$ & & $\left({ }^{\circ}\right)$ & & $(\mathrm{km})$ & & $m_{b}$ & $M_{s}$ & & 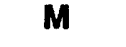 & & & $\left.1,000 \mathrm{~km}^{2}\right)$ \\
\hline $\begin{array}{l}1952 \\
1952\end{array}$ & $\begin{array}{l}05 \\
06\end{array}$ & $\begin{array}{l}01 \\
14\end{array}$ & $\begin{array}{l}150414 \\
020538\end{array}$ & $\begin{array}{l}51.3 \\
59.2\end{array}$ & $\stackrel{\mathbf{N}}{\mathbf{N}}$ & $\begin{array}{l}175.1 \\
152.4\end{array}$ & $\stackrel{\mathbf{E}}{\mathbf{W}}$ & $\overline{065}$ & $\begin{array}{l}265 \\
265\end{array}$ & - & $=$ & - & - & $\overline{\text { Felt }}$ & $\overline{25}$ & $\overline{-}$ \\
\hline $\begin{array}{l}1952 \\
1952 \\
1952 \\
1952 \\
1952\end{array}$ & $\begin{array}{l}07 \\
07 \\
08 \\
08 \\
09\end{array}$ & $\begin{array}{l}07 \\
29 \\
27 \\
28 \\
07\end{array}$ & $\begin{array}{l}025259 \\
195428 \\
112750 \\
105242 \\
043014\end{array}$ & $\begin{array}{l}54.2 \\
53.0 \\
55.6 \\
55.6 \\
51.5\end{array}$ & $\begin{array}{l}\mathbf{N} \\
\mathbf{N} \\
\mathbf{N} \\
\mathbf{N} \\
\mathbf{N}\end{array}$ & $\begin{array}{l}164.5 \\
175.0 \\
160.4 \\
160.4 \\
173.5\end{array}$ & $\begin{array}{l}\mathbf{w} \\
\mathbf{w} \\
\mathbf{w} \\
\mathbf{w} \\
\mathbf{w}\end{array}$ & $\begin{array}{l}- \\
\overline{033} \\
033 \\
033\end{array}$ & $\begin{array}{l}265 \\
265 \\
265 \\
265 \\
265\end{array}$ & $\overline{-}$ & $\overline{-}$ & $\begin{array}{c}\text { 6.25Ukn PAS } \\
- \\
=\end{array}$ & $\bar{z}$ & $\begin{array}{l}\text { Felt } \\
\overline{-}\end{array}$ & $\begin{array}{l}\overline{25} \\
\overline{-}\end{array}$ & $\overline{-}$ \\
\hline $\begin{array}{l}1952 \\
1952 \\
1952 \\
1952 \\
1952\end{array}$ & $\begin{array}{l}09 \\
11 \\
11 \\
12 \\
12\end{array}$ & $\begin{array}{l}24 \\
15 \\
29 \\
04 \\
07\end{array}$ & $\begin{array}{llll}20 & 29 & 24 \\
05 & 01 & 12 \\
23 & 46 & 27 \\
03 & 51 & 40 \\
00 & 50 & 17\end{array}$ & $\begin{array}{l}56.3 \\
52.5 \\
56.3 \\
52.0 \\
52.5\end{array}$ & $\begin{array}{l}\mathbf{N} \\
\mathbf{N} \\
\mathbf{N} \\
\mathbf{N} \\
\mathbf{N}\end{array}$ & $\begin{array}{l}158.6 \\
171.3 \\
153.8 \\
178.2 \\
174.2\end{array}$ & $\begin{array}{l}\mathbf{W} \\
\mathbf{W} \\
\mathbf{W} \\
\mathbf{E} \\
\mathbf{E}\end{array}$ & $\frac{096}{\overline{128}}$ & $\begin{array}{l}265 \\
265 \\
265 \\
265 \\
265\end{array}$ & $\begin{array}{l}- \\
- \\
-\end{array}$ & $\overline{-}$ & $\begin{array}{l}\text { - } \\
\text { 6.75Ukn PAS } \\
\text { 6.00Ukn BRK } \\
\text { 6.25Ukn PAS }\end{array}$ & $\begin{array}{l}- \\
-\end{array}$ & $\frac{\overline{\text { Felt }}}{\overline{\mathrm{VI}}}$ & $\frac{\bar{z}}{\frac{\overline{25}}{38}}$ & $\overline{-}$ \\
\hline $\begin{array}{l}1952 \\
1952 \\
1953 \\
1953 \\
1953\end{array}$ & $\begin{array}{l}12 \\
12 \\
01 \\
02 \\
02\end{array}$ & $\begin{array}{l}12 \\
28 \\
05 \\
09 \\
10\end{array}$ & $\begin{array}{l}004755 \\
045507 \\
074821.6 \\
213237 \\
100758\end{array}$ & $\begin{array}{l}56.3 \\
65.8 \\
53.32 \\
52.6 \\
52.6\end{array}$ & $\begin{array}{l}\mathbf{N} \\
\mathbf{N} \\
\mathbf{N} \\
\mathbf{N} \\
\mathbf{N}\end{array}$ & $\begin{array}{l}153.8 \\
167.8 \\
171.04 \\
169.4 \\
169.4\end{array}$ & $\begin{array}{l}\mathbf{W} \\
\mathbf{W} \\
\mathbf{E} \\
\mathbf{W} \\
\mathbf{W}\end{array}$ & $\begin{array}{l}\overline{-} \\
\overline{0} \\
-\end{array}$ & $\begin{array}{l}265 \\
265 \\
432 \\
265 \\
265\end{array}$ & $\begin{array}{l}- \\
- \\
-\end{array}$ & - & $\begin{array}{c}\overline{-} \\
7.10 \overline{\mathrm{M}}_{\mathrm{s}} \\
\overline{-}\end{array}$ & $\begin{array}{l}- \\
-\end{array}$ & $\begin{array}{c}\overline{\text { IV }} \\
\text { Felt } \\
\overline{-}\end{array}$ & $\begin{array}{l}-\overline{25} \\
26 \\
- \\
-\end{array}$ & $\begin{array}{l}- \\
-\end{array}$ \\
\hline $\begin{array}{l}1953 \\
1953 \\
1953 \\
1953 \\
1953\end{array}$ & $\begin{array}{l}02 \\
03 \\
03 \\
04 \\
05\end{array}$ & $\begin{array}{l}25 \\
23 \\
25 \\
19 \\
12\end{array}$ & $\begin{array}{llll}21 & 16 & 12 \\
12 & 36 & 13 \\
05 & 51 & 21 \\
22 & 47 & 39 \\
12 & 39 & 06\end{array}$ & $\begin{array}{l}56.0 \\
52.6 \\
52.6 \\
50.5 \\
52.3\end{array}$ & $\begin{array}{l}\mathbf{N} \\
\mathbf{N} \\
\mathbf{N} \\
\mathbf{N} \\
\mathbf{N}\end{array}$ & $\begin{array}{l}156.2 \\
169.4 \\
169.4 \\
179.0 \\
177.3\end{array}$ & $\begin{array}{l}\text { w } \\
\text { w } \\
\mathbf{w} \\
\mathbf{w} \\
\mathbf{w}\end{array}$ & $\frac{-}{\bar{z}}$ & $\begin{array}{r}265 \\
265 \\
265 \\
26 \\
265\end{array}$ & $\begin{array}{l}- \\
-\end{array}$ & $\bar{z}$ & $\begin{array}{c}\text { 6.75Ukn PAS } \\
\overline{-} \\
5.50 \overline{\mathrm{M}}_{\mathrm{R}} \\
-\end{array}$ & $\begin{array}{l}- \\
z\end{array}$ & $\bar{z}$ & $\begin{array}{l}\overline{-} \\
\overline{26} \\
26\end{array}$ & $\overline{-}$ \\
\hline $\begin{array}{l}1953 \\
1953 \\
1953 \\
1954 \\
1954\end{array}$ & $\begin{array}{l}05 \\
06 \\
06 \\
03 \\
03\end{array}$ & $\begin{array}{l}13 \\
15 \\
16 \\
03 \\
28\end{array}$ & $\begin{array}{l}041629 \\
174714 \\
194825 \\
204608.2 \\
171040\end{array}$ & $\begin{array}{l}52.5 \\
56.3 \\
55.6 \\
61.54 \\
52.8\end{array}$ & $\begin{array}{l}\mathbf{N} \\
\mathbf{N} \\
\mathbf{N} \\
\mathbf{N} \\
\mathbf{N}\end{array}$ & $\begin{array}{l}174.2 \\
153.8 \\
160.4 \\
146.78 \\
168.2\end{array}$ & $\begin{array}{l}\mathbf{E} \\
\mathbf{W} \\
\mathbf{W} \\
\mathbf{W} \\
\mathbf{W}\end{array}$ & $\begin{array}{l}065 \\
033 \\
056 \\
-\end{array}$ & $\begin{array}{l}265 \\
265 \\
265 \\
447 \\
265\end{array}$ & $\frac{-}{-}$ & $\bar{z}$ & $\begin{array}{c}5.50 \mathrm{M}_{\mathrm{R}} \text { ROT } \\
\text { 6.50Ukn PAS } \\
\text { 6.20Ukn PAS } \\
\text { 6.25Ukn KIR } \\
-\end{array}$ & $\bar{z}$ & $\frac{-}{\bar{v}}$ & $\frac{z}{27}$ & - \\
\hline $\begin{array}{l}1954 \\
1954 \\
1954 \\
1954 \\
1954\end{array}$ & $\begin{array}{l}03 \\
03 \\
04 \\
04 \\
04\end{array}$ & $\begin{array}{l}28 \\
28 \\
17 \\
24 \\
28\end{array}$ & $\begin{array}{l}203621 \\
205810 \\
201037 \\
083304.1 \\
045052\end{array}$ & $\begin{array}{l}51.6 \\
51.6 \\
51.5 \\
62.99 \\
51.6\end{array}$ & $\begin{array}{l}\mathbf{N} \\
\mathbf{N} \\
\mathbf{N} \\
\mathbf{N} \\
\mathbf{N}\end{array}$ & $\begin{array}{l}175.8 \\
175.8 \\
179.0 \\
148.54 \\
175.8\end{array}$ & $\begin{array}{l}\mathbf{E} \\
\mathbf{E} \\
\mathbf{W} \\
\mathbf{W} \\
\mathbf{E}\end{array}$ & $\begin{array}{l}\overline{065} \\
\overline{090} \\
-\end{array}$ & $\begin{array}{l}265 \\
265 \\
265 \\
447 \\
265\end{array}$ & $\frac{-}{-}$ & $\bar{z}$ & $\begin{array}{c}\text { 6.50Ukn BRK } \\
5.50 \mathrm{M}_{\mathrm{R}} \text { ROT } \\
6.75 \mathrm{Ukn} \text { BRK } \\
- \\
-\end{array}$ & $\begin{array}{l}- \\
-\end{array}$ & $\begin{array}{l}\text { - } \\
\text { Felt } \\
\text { III }\end{array}$ & $\begin{array}{l}- \\
\overline{27} \\
27\end{array}$ & $\overline{-}$ \\
\hline $\begin{array}{l}1954 \\
1954 \\
1954 \\
1954 \\
1955\end{array}$ & $\begin{array}{l}06 \\
08 \\
10 \\
12 \\
01\end{array}$ & $\begin{array}{l}17 \\
05 \\
03 \\
30 \\
13\end{array}$ & $\begin{array}{lll}01 & 42 & 23.2 \\
08 & 49 & 53 \\
11 & 18 & 45.9 \\
11 & 32 & 30 \\
02 & 03 & 43\end{array}$ & $\begin{array}{l}56.29 \\
51.7 \\
60.71 \\
52.5 \\
53.2\end{array}$ & $\begin{array}{l}\mathbf{N} \\
\mathbf{N} \\
\mathbf{N} \\
\mathbf{N} \\
\mathbf{N}\end{array}$ & $\begin{array}{l}154.09 \\
175.8 \\
150.52 \\
168.4 \\
167.4\end{array}$ & $\begin{array}{l}\mathbf{W} \\
\mathbf{E} \\
\mathbf{W} \\
\mathbf{W} \\
\mathbf{W}\end{array}$ & $\begin{array}{l}000 \\
065 \\
073 \\
065 \\
-\end{array}$ & $\begin{array}{l}447 \\
265 \\
447 \\
265 \\
265\end{array}$ & $\begin{array}{l}- \\
-\end{array}$ & $\bar{z}$ & $\begin{array}{l}\text { 6.50Ukn PAS } \\
\text { 6.00Ukn PAS } \\
\text { 6.75Ukn PAS } \\
\text { 6.63Ukn PAS } \\
\text { 6.88Ukn PAS }\end{array}$ & $\bar{z}$ & $\begin{array}{l}\text { - } \\
\text { VIII } \\
\text { Felt } \\
\text { Felt }\end{array}$ & $\begin{array}{r}\overline{-} \\
27 \\
266\end{array}$ & $\begin{array}{l}- \\
\overline{32} \& \\
-\end{array}$ \\
\hline $\begin{array}{l}1955 \\
1955 \\
1955 \\
1955 \\
1955\end{array}$ & $\begin{array}{l}01 \\
01 \\
01 \\
03 \\
04\end{array}$ & $\begin{array}{l}13 \\
13 \\
21 \\
14 \\
28\end{array}$ & $\begin{array}{l}023546 \\
024446 \\
141835 \\
131204.0 \\
190502\end{array}$ & $\begin{array}{l}53.2 \\
53.2 \\
53.2 \\
52.5 \\
51.8\end{array}$ & $\begin{array}{l}\mathbf{N} \\
\mathbf{N} \\
\mathbf{N} \\
\mathbf{N} \\
\mathbf{N}\end{array}$ & $\begin{array}{l}167.4 \\
167.4 \\
167.9 \\
173.5 \\
178.1\end{array}$ & $\begin{array}{l}\text { w } \\
\mathbf{w} \\
\mathbf{w} \\
\mathbf{w} \\
\mathbf{w}\end{array}$ & $\frac{-}{\overline{100}}$ & $\begin{array}{l}265 \\
265 \\
265 \\
266 \\
265\end{array}$ & $\begin{array}{l}- \\
- \\
-\end{array}$ & E & $\begin{array}{c}\text { 6.50Ukn PAS } \\
- \\
\text { 7.00Ukn PAS } \\
\text { 6.50Ukn PAS }\end{array}$ & $\overline{-}$ & $\frac{\text { Felt }}{\frac{\text { Felt }}{\text { IV }}}$ & $\frac{\frac{28}{28}}{28}$ & - \\
\hline $\begin{array}{l}1955 \\
1955 \\
1955 \\
1955 \\
1955\end{array}$ & $\begin{array}{l}05 \\
05 \\
06 \\
06 \\
06\end{array}$ & $\begin{array}{l}29 \\
29 \\
02 \\
02 \\
05\end{array}$ & $\begin{array}{lll}13 & 31 & 24.8 \\
21 & 03 & 09.3 \\
00 & 18 & 57 \\
02 & 02 & 11 \\
01 & 53 & 17\end{array}$ & $\begin{array}{l}55.66 \\
55.84 \\
51.4 \\
51.4 \\
51.5\end{array}$ & $\begin{array}{l}\mathbf{N} \\
\mathbf{N} \\
\mathbf{N} \\
\mathbf{N} \\
\mathbf{N}\end{array}$ & $\begin{array}{l}154.37 \\
154.27 \\
179.8 \\
179.8 \\
179.8\end{array}$ & $\begin{array}{l}\mathbf{w} \\
\mathbf{w} \\
\mathbf{w} \\
\mathbf{w} \\
\mathbf{w}\end{array}$ & $\begin{array}{l}000 \\
000 \\
- \\
-\end{array}$ & $\begin{array}{l}447 \\
447 \\
265 \\
265 \\
265\end{array}$ & $\begin{array}{l}- \\
- \\
-\end{array}$ & $\bar{z}$ & $\begin{array}{l}\text { 5.75Ukn PAS } \\
\text { 5.50Ukn PAS } \\
6.75 \mathrm{Ukn} \mathrm{PAS} \\
6.00 \mathrm{M}_{\mathrm{R}} \text { ROT } \\
6.38 \mathrm{Ukn} \text { PAS }\end{array}$ & $\bar{z}$ & E & $\begin{array}{l}z \\
z \\
-\end{array}$ & $\overline{-}$ \\
\hline $\begin{array}{l}1955 \\
1955 \\
1955 \\
1955 \\
1955\end{array}$ & $\begin{array}{l}06 \\
07 \\
07 \\
07 \\
07\end{array}$ & $\begin{array}{l}20 \\
03 \\
03 \\
04 \\
17\end{array}$ & $\begin{array}{l}120731 \\
080059 \\
142634 \\
141952 \\
215823\end{array}$ & $\begin{array}{l}51.4 \\
51.5 \\
51.6 \\
51.4 \\
54.4\end{array}$ & $\begin{array}{l}\mathbf{N} \\
\mathbf{N} \\
\mathbf{N} \\
\mathbf{N} \\
\mathbf{N}\end{array}$ & $\begin{array}{l}179.5 \\
177.2 \\
177.6 \\
177.4 \\
168.3\end{array}$ & $\begin{array}{l}\mathbf{W} \\
\mathbf{E} \\
\mathbf{E} \\
\mathbf{E} \\
\mathbf{W}\end{array}$ & $\begin{array}{r}033 \\
033 \\
033 \\
065 \\
-\end{array}$ & $\begin{array}{l}265 \\
265 \\
265 \\
265 \\
265\end{array}$ & $\begin{array}{l}- \\
-\end{array}$ & $\bar{z}$ & $\begin{array}{l}\text { 6.75Ukn PAS } \\
\text { 5.75Ukn KIR } \\
\text { 6.50Ukn PAS } \\
\text { 6.63Ukn PAS } \\
\text { 5.88Ukn PAS }\end{array}$ & $\bar{z}$ & $\bar{z}$ & $\frac{\bar{z}}{\overline{28}}$ & $=$ \\
\hline
\end{tabular}


ALASKA-Continued

\begin{tabular}{|c|c|c|c|c|c|c|c|c|c|c|c|c|c|c|}
\hline \multirow{2}{*}{\multicolumn{3}{|c|}{ Date }} & \multirow{2}{*}{$\begin{array}{c}\text { Origin } \\
\text { time (UTC) } \\
\end{array}$} & \multicolumn{4}{|c|}{ Hypocenter } & \multicolumn{4}{|c|}{ Magnitude } & \multicolumn{2}{|c|}{ Intensity } & \multirow{3}{*}{$\begin{array}{l}\text { Felt area } \\
\left(1,000 \mathrm{~km}^{2}\right)\end{array}$} \\
\hline & & & & \multirow{2}{*}{$\begin{array}{l}\text { Latitude } \\
\left({ }^{\circ}\right)\end{array}$} & \multirow{2}{*}{$\begin{array}{l}\text { Longitude } \\
\left({ }^{\circ}\right)\end{array}$} & \multirow{2}{*}{$\begin{array}{l}\text { Depth } \\
(\mathrm{km})\end{array}$} & \multirow[t]{2}{*}{ Ref } & \multicolumn{2}{|c|}{ USGS } & \multirow[t]{2}{*}{ Other } & \multirow{2}{*}{$\begin{array}{c}\text { Moment } \\
\text { M }\end{array}$} & \multirow[t]{2}{*}{ MM } & \multirow[t]{2}{*}{ Ref } & \\
\hline Yr & Mo [ & $\mathrm{Da}$ & $h \mathbf{m} s$ & & & & & $m_{b}$ & $\mathbf{M}_{\mathbf{s}}$ & & & & & \\
\hline $\begin{array}{l}1955 \\
1955 \\
1955 \\
1955 \\
1955\end{array}$ & $\begin{array}{l}07 \\
07 \\
07 \\
08 \\
08\end{array}$ & $\begin{array}{l}19 \\
26 \\
27 \\
02 \\
29\end{array}$ & $\begin{array}{lll}23 & 52 & 23.1 \\
04 & 04 & 19.4 \\
18 & 19 & 10.1 \\
22 & 12 & 47 \\
15 & 34 & 00\end{array}$ & $\begin{array}{ll}56.33 & \mathrm{~N} \\
56.51 & \mathrm{~N} \\
56.59 & \mathrm{~N} \\
51.7 & \mathrm{~N} \\
51.8 & \mathrm{~N}\end{array}$ & $\begin{array}{l}153.16 \mathrm{~W} \\
153.25 \mathrm{~W} \\
152.78 \mathrm{~W} \\
175.9 \mathrm{E} \\
178.1 \mathrm{~W}\end{array}$ & $\begin{array}{l}000 \\
000 \\
000 \\
065 \\
-\end{array}$ & $\begin{array}{l}447 \\
447 \\
447 \\
265 \\
265\end{array}$ & $\begin{array}{l}- \\
- \\
-\end{array}$ & $\frac{-}{-}$ & $\begin{array}{c}\text { 6.00Ukn PAS } \\
\text { 6.00Ukn PAS } \\
\text { 6.25Ukn PAS } \\
\text { 5.75Ukn UPP }\end{array}$ & $\begin{array}{l}- \\
- \\
-\end{array}$ & $\frac{-}{-}$ & $\frac{-}{-}$ & $\begin{array}{l}- \\
- \\
-\end{array}$ \\
\hline $\begin{array}{l}1955 \\
1955 \\
1955 \\
1955 \\
1955\end{array}$ & $\begin{array}{l}09 \\
09 \\
10 \\
10 \\
11\end{array}$ & $\begin{array}{l}13 \\
30 \\
09 \\
31 \\
15\end{array}$ & $\begin{array}{l}020040 \\
194424 \\
231349 \\
010552 \\
100647.2\end{array}$ & $\begin{array}{ll}52.0 & \mathbf{N} \\
51.5 & \mathrm{~N} \\
51.3 & \mathrm{~N} \\
51.5 & \mathrm{~N} \\
55.27 & \mathrm{~N}\end{array}$ & $\begin{array}{ll}176.0 & W \\
176.5 & W \\
177.1 & E \\
175.2 & W \\
155.64 & W\end{array}$ & $\frac{-}{065}$ & $\begin{array}{l}266 \\
266 \\
265 \\
265 \\
447\end{array}$ & $\begin{array}{l}- \\
I\end{array}$ & $\frac{-}{-}$ & $\begin{array}{l}\text { 5.88Ukn PAS } \\
\text { 6.00Ukn KIR } \\
\text { 5.88Ukn PAS } \\
\text { 6.38UknPAS }\end{array}$ & $\frac{-}{-}$ & $\begin{array}{l}- \\
- \\
-\end{array}$ & $\begin{array}{l}- \\
- \\
-\end{array}$ & $\begin{array}{l}- \\
- \\
-\end{array}$ \\
\hline $\begin{array}{l}1956 \\
1956 \\
1956 \\
1956 \\
1956\end{array}$ & $\begin{array}{l}01 \\
02 \\
03 \\
04 \\
04\end{array}$ & $\begin{array}{l}14 \\
19 \\
02 \\
18 \\
22\end{array}$ & $\begin{array}{lll}14 & 0852 \\
04 & 13 & 14.5 \\
115622.7 \\
110022 \\
172152.5\end{array}$ & $\begin{array}{ll}51.8 & \mathrm{~N} \\
58.52 & \mathrm{~N} \\
63.57 & \mathrm{~N} \\
51.8 & \mathrm{~N} \\
53.79 & \mathrm{~N}\end{array}$ & $\begin{array}{ll}172.9 & W \\
153.74 & W \\
149.33 & W \\
177.7 & W \\
161.48 & W\end{array}$ & $\begin{array}{l}065 \\
000 \\
079 \\
033 \\
000\end{array}$ & $\begin{array}{l}265 \\
447 \\
447 \\
265 \\
447\end{array}$ & $\begin{array}{l}- \\
z\end{array}$ & $\begin{array}{l}- \\
- \\
-\end{array}$ & $\begin{array}{l}\text { 6.00Ukn PAS } \\
\text { 5.60Ukn KIR } \\
\text { 5.50M ROT } \\
\text { 6.75Ukn PAS } \\
\text { 6.00Ukn PAS }\end{array}$ & $\begin{array}{l}- \\
z \\
-\end{array}$ & $\frac{-}{\overline{I V}}$ & $\begin{array}{l}- \\
- \\
-\end{array}$ & $\begin{array}{l}- \\
- \\
-\end{array}$ \\
\hline $\begin{array}{l}1956 \\
1956 \\
1956 \\
1956 \\
1956\end{array}$ & $\begin{array}{l}05 \\
06 \\
08 \\
08 \\
10\end{array}$ & $\begin{array}{l}06 \\
04 \\
24 \\
30 \\
19\end{array}$ & $\begin{array}{l}205718.1 \\
070919 \\
042734 \\
042421.5 \\
204731\end{array}$ & $\begin{array}{ll}54.50 & \mathrm{~N} \\
52.1 & \mathrm{~N} \\
52.74 & \mathrm{~N} \\
53.69 & \mathrm{~N} \\
52.27 & \mathrm{~N}\end{array}$ & $\begin{array}{ll}162.47 & W \\
170.6 & W \\
172.6 & E \\
163.88 & W \\
177.40 & E\end{array}$ & $\frac{000}{\overline{-}}$ & $\begin{array}{l}447 \\
265 \\
265 \\
447 \\
265\end{array}$ & $\bar{z}$ & $\begin{array}{l}- \\
- \\
-\end{array}$ & $\begin{array}{l}\text { 5.75Ukn PAS } \\
\text { 6.25Ukn PAS } \\
\text { 6.50Ukn PAS } \\
\text { 6.00Ukn PAS } \\
\text { 6.75Ukn PAS }\end{array}$ & $\begin{array}{l}- \\
-\end{array}$ & $\begin{array}{l}- \\
- \\
-\end{array}$ & $\begin{array}{l}- \\
- \\
-\end{array}$ & $\frac{-}{-}$ \\
\hline $\begin{array}{l}1956 \\
1956 \\
1956 \\
1956 \\
1956\end{array}$ & $\begin{array}{l}11 \\
12 \\
12 \\
12 \\
12\end{array}$ & $\begin{array}{l}17 \\
02 \\
03 \\
04 \\
08\end{array}$ & $\begin{array}{l}202717.2 \\
025955 \\
072006 \\
104207 \\
161025\end{array}$ & $\begin{array}{ll}54.55 & \mathrm{~N} \\
52.51 & \mathrm{~N} \\
52.64 & \mathrm{~N} \\
52.55 & \mathrm{~N} \\
51.37 & \mathrm{~N}\end{array}$ & $\begin{array}{l}133.67 \mathrm{~W} \\
169.05 \mathrm{~W} \\
168.61 \mathrm{~W} \\
169.15 \mathrm{~W} \\
179.17 \mathrm{~W}\end{array}$ & $\begin{array}{l}000 \\
= \\
-\end{array}$ & $\begin{array}{l}448 \\
265 \\
265 \\
265 \\
265\end{array}$ & $\begin{array}{l}- \\
z \\
-\end{array}$ & $\begin{array}{l}- \\
- \\
-\end{array}$ & $\begin{array}{l}\text { 6.50Ukn PAS } \\
\text { 5.50Ukn MOS } \\
\text { 6.63Ukn PAS } \\
\text { 6.50̄ }\end{array}$ & $\begin{array}{l}- \\
z \\
-\end{array}$ & $\frac{\text { IV }}{-}$ & $\begin{array}{l}29 \\
- \\
-\end{array}$ & $\begin{array}{c}- \\
- \\
-\end{array}$ \\
\hline $\begin{array}{l}1957 \\
1957 \\
1957 \\
1957 \\
1957\end{array}$ & $\begin{array}{l}01 \\
01 \\
01 \\
01 \\
01\end{array}$ & $\begin{array}{l}02 \\
02 \\
02 \\
02 \\
02\end{array}$ & $\begin{array}{l}003922.9 \\
021737.0 \\
031252.8 \\
033034.2 \\
034847.0\end{array}$ & $\begin{array}{ll}52.36 & \mathrm{~N} \\
52.41 & \mathrm{~N} \\
52.51 & \mathrm{~N} \\
52.72 & \mathrm{~N} \\
52.51 & \mathrm{~N}\end{array}$ & $\begin{array}{l}168.36 \mathrm{~W} \\
168.39 \mathrm{~W} \\
168.10 \mathrm{~W} \\
168.00 \mathrm{~W} \\
168.03 \mathrm{~W}\end{array}$ & $\begin{array}{l}000 \\
000 \\
000 \\
000 \\
000\end{array}$ & $\begin{array}{l}432 \\
432 \\
432 \\
432 \\
432\end{array}$ & $\begin{array}{l}- \\
z \\
-\end{array}$ & $\begin{array}{l}- \\
- \\
-\end{array}$ & $\begin{array}{l}\text { 6.50Ukn PAS } \\
\text { 6.75Ukn PAS } \\
6.63 \mathrm{Unn} \text { PAS } \\
6.00 \mathrm{M}_{\mathrm{R}} \text { ROT } \\
6.70 \mathrm{M}_{S} \text { AB3 }\end{array}$ & $\begin{array}{l}- \\
- \\
-\end{array}$ & $\frac{-}{-}$ & $\begin{array}{c}- \\
- \\
-\end{array}$ & $\begin{array}{c}- \\
- \\
-\end{array}$ \\
\hline $\begin{array}{l}1957 \\
1957 \\
1957\end{array}$ & $\begin{array}{l}01 \\
01 \\
01\end{array}$ & $\begin{array}{l}02 \\
02 \\
02\end{array}$ & $\begin{array}{l}040330.0 \\
104932.8 \\
124707.2\end{array}$ & $\begin{array}{l}52.65 \mathrm{~N} \\
52.66 \mathrm{~N} \\
52.57 \mathrm{~N}\end{array}$ & $\begin{array}{l}168.61 \mathrm{~W} \\
168.00 \mathrm{~W} \\
168.09 \mathrm{~W}\end{array}$ & $\begin{array}{l}000 \\
000 \\
000\end{array}$ & $\begin{array}{l}432 \\
432 \\
432\end{array}$ & $=$ & - & $\begin{array}{l}\text { 6.70Ukn UPP } \\
\text { 6.50Ukn PAS }\end{array}$ & E & $\overline{-}$ & - & - \\
\hline $\begin{array}{l}1957 \\
1957\end{array}$ & $\begin{array}{l}01 \\
01\end{array}$ & $\begin{array}{l}03 \\
09\end{array}$ & $\begin{array}{l}004103.0 \\
075256.4\end{array}$ & $\begin{array}{l}52.91 \mathrm{~N} \\
52.80 \mathrm{~N}\end{array}$ & $\begin{array}{l}167.88 \mathrm{~W} \\
167.44 \mathrm{~W}\end{array}$ & $\begin{array}{l}000 \\
000\end{array}$ & $\begin{array}{l}432 \\
432\end{array}$ & - & - & $\begin{array}{l}6.40 \mathrm{M}_{\mathrm{R}} \text { ROT } \\
\text { 6.50UknPAS }\end{array}$ & - & $\overline{-}$ & - & - \\
\hline $\begin{array}{l}1957 \\
1957\end{array}$ & $\begin{array}{l}01 \\
02\end{array}$ & $\begin{array}{l}25 \\
21\end{array}$ & $\begin{array}{l}033655.0 \\
143011.0\end{array}$ & $\begin{array}{l}51.76 \mathrm{~N} \\
53.02 \mathrm{~N}\end{array}$ & $\begin{array}{l}177.03 \mathrm{~W} \\
171.27 \mathrm{~W}\end{array}$ & $\begin{array}{l}047 \\
126\end{array}$ & $\begin{array}{l}265 \\
265\end{array}$ & - & - & $\begin{array}{l}\text { 6.50Ukn PAS } \\
\text { 6.75Ukn PAS }\end{array}$ & $\overline{-}$ & - & - & - \\
\hline $\begin{array}{l}1957 \\
1957 \\
1957\end{array}$ & $\begin{array}{l}03 \\
03 \\
03\end{array}$ & $\begin{array}{l}09 \\
09 \\
09\end{array}$ & $\begin{array}{l}142231.9 \\
154151.5 \\
193737.0\end{array}$ & $\begin{array}{l}51.292 \mathrm{~N} \\
50.74 \mathrm{~N} \\
51.546 \mathrm{~N}\end{array}$ & $\begin{array}{l}175.629 \mathrm{~W} \\
176.16 \mathrm{~W} \\
173.759 \mathrm{~W}\end{array}$ & $\begin{array}{l}033 \\
000 \\
033\end{array}$ & $\begin{array}{l}479 \\
432 \\
479\end{array}$ & E & $\overline{-}$ & $\begin{array}{c}\text { 8.10M } \mathrm{M}_{\mathrm{s}} \mathrm{ABE} \\
\text { 6.00Ukn ROT } \\
-\end{array}$ & $\begin{array}{c}\text { 8.82RK } \\
- \\
-\end{array}$ & $\begin{array}{l}\text { VIII } \\
\text { Felt } \\
\text { Felt }\end{array}$ & $\begin{array}{l}38 \\
30 \\
30\end{array}$ & - \\
\hline $\begin{array}{l}1957 \\
1957 \\
1957 \\
1957 \\
1957\end{array}$ & $\begin{array}{l}03 \\
03 \\
03 \\
03 \\
03\end{array}$ & $\begin{array}{l}09 \\
09 \\
10 \\
10 \\
10\end{array}$ & $\begin{array}{l}202203.3 \\
203916.5 \\
030618.8 \\
030905.6 \\
072324.2\end{array}$ & $\begin{array}{l}52.12 \mathrm{~N} \\
52.43 \mathrm{~N} \\
51.698 \mathrm{~N} \\
51.396 \mathrm{~N} \\
51.117 \mathrm{~N}\end{array}$ & $\begin{array}{l}169.55 \mathrm{~W} \\
169.58 \mathrm{~W} \\
173.981 \mathrm{~W} \\
174.289 \mathrm{~W} \\
175.944 \mathrm{~W}\end{array}$ & $\begin{array}{l}000 \\
000 \\
033 \\
033 \\
033\end{array}$ & $\begin{array}{l}432 \\
432 \\
479 \\
479 \\
479\end{array}$ & $\begin{array}{l}z \\
z\end{array}$ & $\begin{array}{l}- \\
- \\
-\end{array}$ & $\begin{array}{l}-\overline{\mathrm{M}}_{\mathrm{s}} \mathrm{ABE} \\
\text { 6.63Ukn PAS } \\
\text { 6.60Ukn UPP } \\
-\end{array}$ & $\begin{array}{l}- \\
z \\
-\end{array}$ & $\begin{array}{l}- \\
\text { Felt } \\
\text { Felt } \\
\text { Felt }\end{array}$ & $\begin{array}{l}- \\
\overline{30} \\
30 \\
30\end{array}$ & $\begin{array}{c}- \\
- \\
-\end{array}$ \\
\hline $\begin{array}{l}1957 \\
1957 \\
1957 \\
1957 \\
1957\end{array}$ & $\begin{array}{l}03 \\
03 \\
03 \\
03 \\
03\end{array}$ & $\begin{array}{l}10 \\
10 \\
10 \\
10 \\
10\end{array}$ & $\begin{array}{lll}11 & 2045.6 \\
12 & 3605.8 \\
12 & 4537.4 \\
13 & 1013.4 \\
1328 & 36.4\end{array}$ & $\begin{array}{l}51.88 \mathrm{~N} \\
51.18 \mathrm{~N} \\
50.566 \mathrm{~N} \\
51.28 \mathrm{~N} \\
51.083 \mathrm{~N}\end{array}$ & $\begin{array}{l}170.91 \mathrm{~W} \\
170.84 \mathrm{~W} \\
176.831 \mathrm{~W} \\
179.99 \mathrm{~W} \\
178.626 \mathrm{~W}\end{array}$ & $\begin{array}{l}000 \\
000 \\
033 \\
000 \\
030\end{array}$ & $\begin{array}{l}432 \\
432 \\
479 \\
432 \\
479\end{array}$ & $\begin{array}{l}z \\
z\end{array}$ & $\frac{-}{-}$ & $\begin{array}{l}\text { 6.50Ukn UPP } \\
6.00 \mathrm{M}_{\mathrm{R}} \text { ROT } \\
6.40 \mathrm{Ukn} \text { UPP } \\
6.10 \mathrm{M}_{\mathrm{R}} \text { ROT } \\
6.00 \mathrm{M}_{\mathrm{R}} \text { ROT }\end{array}$ & $\begin{array}{l}z \\
z \\
-\end{array}$ & $\begin{array}{c}\overline{-} \\
\overline{\text { Felt }} \\
-\end{array}$ & $\frac{-}{30}$ & $\frac{-}{-}$ \\
\hline $\begin{array}{l}1957 \\
1957 \\
1957\end{array}$ & $\begin{array}{l}03 \\
03 \\
03\end{array}$ & $\begin{array}{l}10 \\
10 \\
10\end{array}$ & $\begin{array}{l}152629.1 \\
163753.2 \\
194059.8\end{array}$ & $\begin{array}{l}51.575 \mathrm{~N} \\
51.568 \mathrm{~N} \\
51.348 \mathrm{~N}\end{array}$ & $\begin{array}{l}172.990 \mathrm{~W} \\
173.166 \mathrm{~W} \\
173.335 \mathrm{~W}\end{array}$ & $\begin{array}{l}033 \\
033 \\
033\end{array}$ & $\begin{array}{l}479 \\
479 \\
479\end{array}$ & E & $\overline{-}$ & $\begin{array}{c}\text { 6.75Ukn BRK } \\
6.00 \mathrm{M}_{R} \text { ROT } \\
-\end{array}$ & E & $\begin{array}{l}\text { Felt } \\
\text { Felt } \\
\text { Felt }\end{array}$ & $\begin{array}{l}30 \\
30 \\
30\end{array}$ & - \\
\hline
\end{tabular}


ALASKA-Continued

[See table 1 for hypocenter and intensity references and table 2 for definitions of magnitude source codes. \&, land area only. Leader (--) indicates information is not available]

\begin{tabular}{|c|c|c|c|c|c|c|c|c|c|c|c|c|c|c|}
\hline \multirow{2}{*}{\multicolumn{3}{|c|}{ Date }} & \multirow{2}{*}{$\begin{array}{c}\text { Origin } \\
\text { time (UTC) }\end{array}$} & \multicolumn{4}{|c|}{ Hypocenter } & \multicolumn{4}{|c|}{ Magnitude } & \multicolumn{2}{|c|}{ Intensity } & \multirow{3}{*}{$\begin{array}{c}\text { Felt area } \\
\left(1,000 \mathrm{~km}^{2}\right)\end{array}$} \\
\hline & & & & \multirow{2}{*}{$\begin{array}{c}\text { Latitude } \\
\left({ }^{\circ}\right)\end{array}$} & \multirow{2}{*}{$\begin{array}{c}\text { Longitude } \\
\left(^{\circ}\right)\end{array}$} & \multirow{2}{*}{$\begin{array}{l}\text { Depth } \\
(\mathrm{km})\end{array}$} & \multirow[t]{2}{*}{ Ref } & \multicolumn{2}{|c|}{ USGS } & \multirow[t]{2}{*}{ Other } & \multirow{2}{*}{$\begin{array}{c}\text { Moment } \\
\mathbf{M}\end{array}$} & \multirow[t]{2}{*}{ MM } & \multirow[t]{2}{*}{ Ref } & \\
\hline $\mathrm{Yr}$ & Mo & $\mathrm{Da}$ & $h \mathrm{~m} s$ & & & & & $m_{b}$ & $\mathbf{M}_{\mathbf{s}}$ & & & & & \\
\hline $\begin{array}{l}1957 \\
1957\end{array}$ & $\begin{array}{l}03 \\
03\end{array}$ & $\begin{array}{l}10 \\
11\end{array}$ & $\begin{array}{l}235649.5 \\
000811.5\end{array}$ & $\begin{array}{l}52.70 \mathrm{~N} \\
52.50 \mathrm{~N}\end{array}$ & $\begin{array}{l}168.40 \mathrm{~W} \\
169.50 \mathrm{~W}\end{array}$ & $\begin{array}{l}000 \\
000\end{array}$ & $\begin{array}{l}432 \\
432\end{array}$ & - & & $=-$ & Z & - & 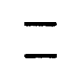 & - \\
\hline $\begin{array}{l}1957 \\
1957 \\
1957 \\
1957 \\
1957\end{array}$ & $\begin{array}{l}03 \\
03 \\
03 \\
03 \\
03\end{array}$ & $\begin{array}{l}11 \\
11 \\
11 \\
11 \\
11\end{array}$ & $\begin{array}{lll}03 & 12 & 47.0 \\
03 & 35 & 05.5 \\
04 & 05 & 18.2 \\
07 & 08 & 05.4 \\
09 & 58 & 44.4\end{array}$ & $\begin{array}{l}50.826 \mathrm{~N} \\
50.660 \mathrm{~N} \\
50.948 \mathrm{~N} \\
50.968 \mathrm{~N} \\
52.66 \mathrm{~N}\end{array}$ & $\begin{array}{l}177.250 \mathrm{~W} \\
176.960 \mathrm{~W} \\
176.915 \mathrm{~W} \\
177.222 \mathrm{~W} \\
169.02 \mathrm{~W}\end{array}$ & $\begin{array}{l}033 \\
033 \\
033 \\
033 \\
000\end{array}$ & $\begin{array}{l}479 \\
479 \\
479 \\
479 \\
432\end{array}$ & $\overline{-}$ & $\begin{array}{l}- \\
- \\
-\end{array}$ & $\begin{array}{c}6.88 \mathrm{Ukn} \mathrm{PAS} \\
6.00 \mathrm{M}_{\mathrm{R}} \mathrm{ROT} \\
- \\
- \\
7.00 \mathrm{M}_{\mathrm{S}} \mathrm{ABE}\end{array}$ & $\begin{array}{l}z \\
z\end{array}$ & $\begin{array}{l}\text { Felt } \\
\text { Felt } \\
\text { Felt } \\
\text { Felt } \\
-\end{array}$ & $\begin{array}{l}30 \\
30 \\
30 \\
30 \\
-\end{array}$ & $\begin{array}{l}- \\
-\end{array}$ \\
\hline $\begin{array}{l}1957 \\
1957 \\
1957 \\
1957 \\
1957\end{array}$ & $\begin{array}{l}03 \\
03 \\
03 \\
03 \\
03\end{array}$ & $\begin{array}{l}11 \\
11 \\
12 \\
12 \\
12\end{array}$ & $\begin{array}{l}145525.1 \\
153559.8 \\
072853.7 \\
073922.7 \\
080318.8\end{array}$ & $\begin{array}{l}51.094 \mathrm{~N} \\
51.013 \mathrm{~N} \\
51.526 \mathrm{~N} \\
51.014 \mathrm{~N} \\
50.992 \mathrm{~N}\end{array}$ & $\begin{array}{l}178.535 \mathrm{~W} \\
178.972 \mathrm{~W} \\
173.505 \mathrm{~W} \\
178.246 \mathrm{~W} \\
178.027 \mathrm{~W}\end{array}$ & $\begin{array}{l}033 \\
030 \\
033 \\
030 \\
033\end{array}$ & $\begin{array}{l}479 \\
479 \\
479 \\
479 \\
479\end{array}$ & $\overline{-}$ & $\bar{z}$ & $\begin{array}{c}6.90 \mathrm{M}_{\mathrm{s}} \mathrm{ABE} \\
6.50 \mathrm{Un}_{\mathrm{kAS}} \\
6.38 \mathrm{Ukn} \mathrm{PAS} \\
6.38 \mathrm{Ukn} \mathrm{PAS} \\
-\end{array}$ & $\begin{array}{l}z \\
z\end{array}$ & $\begin{array}{l}\text { Felt } \\
\text { Felt } \\
\text { Felt } \\
\text { Felt } \\
\text { Felt }\end{array}$ & $\begin{array}{l}30 \\
30 \\
30 \\
30 \\
30\end{array}$ & $\overline{-}$ \\
\hline $\begin{array}{l}1957 \\
1957 \\
1957 \\
1957 \\
1957\end{array}$ & $\begin{array}{l}03 \\
03 \\
03 \\
03 \\
03\end{array}$ & $\begin{array}{l}12 \\
12 \\
12 \\
12 \\
13\end{array}$ & $\begin{array}{lll}11 & 44 & 56.3 \\
12 & 46 & 12.1 \\
17 & 0029.3 \\
23 & 4532.8 \\
02 & 48 & 20.8\end{array}$ & $\begin{array}{l}51.187 \mathrm{~N} \\
52.95 \mathrm{~N} \\
51.447 \mathrm{~N} \\
51.675 \mathrm{~N} \\
51.70 \mathrm{~N}\end{array}$ & $\begin{array}{l}177.388 \mathrm{~W} \\
168.42 \mathrm{~W} \\
174.890 \mathrm{~W} \\
173.327 \mathrm{~W} \\
171.17 \mathrm{~W}\end{array}$ & $\begin{array}{l}033 \\
000 \\
033 \\
033 \\
000\end{array}$ & $\begin{array}{l}479 \\
432 \\
479 \\
479 \\
432\end{array}$ & $\frac{-}{-}$ & $\begin{array}{l}z \\
z\end{array}$ & $\begin{array}{c}7.00 \mathrm{M}_{\mathrm{S}} \text { ABE } \\
6.00 \mathrm{M}_{\mathrm{R}} \mathrm{ROT} \\
- \\
\overline{-} \\
6.10 \mathrm{M}_{\mathrm{R}} \mathrm{ROT}\end{array}$ & $\bar{z}$ & $\begin{array}{l}\text { Felt } \\
\text { Felt } \\
\text { Felt } \\
\text { Felt }\end{array}$ & $\begin{array}{l}\frac{30}{30} \\
30 \\
30\end{array}$ & $\begin{array}{l}- \\
-\end{array}$ \\
\hline $\begin{array}{l}1957 \\
1957 \\
1957 \\
1957 \\
1957\end{array}$ & $\begin{array}{l}03 \\
03 \\
03 \\
03 \\
03\end{array}$ & $\begin{array}{l}13 \\
13 \\
13 \\
13 \\
14\end{array}$ & $\begin{array}{lll}03 & 33 & 03.4 \\
09 & 09 & 34.9 \\
15 & 42 & 12.8 \\
19 & 59 & 24.1 \\
01 & 52 & 19.4\end{array}$ & $\begin{array}{l}51.362 \mathrm{~N} \\
52.51 \mathrm{~N} \\
51.131 \mathrm{~N} \\
53.96 \mathrm{~N} \\
52.59 \mathrm{~N}\end{array}$ & $\begin{array}{l}174.793 \mathrm{~W} \\
170.06 \mathrm{~W} \\
178.681 \mathrm{~W} \\
165.44 \mathrm{~W} \\
168.48 \mathrm{~W}\end{array}$ & $\begin{array}{l}033 \\
000 \\
033 \\
000 \\
000\end{array}$ & $\begin{array}{l}479 \\
432 \\
479 \\
432 \\
432\end{array}$ & $\begin{array}{l}- \\
-\end{array}$ & $\begin{array}{l}z \\
z\end{array}$ & $\begin{array}{l}\overline{-} \\
\text { 6.75Ukn PAS } \\
6.20 \mathrm{M}_{\mathrm{R}} \text { ROT } \\
\text { 5.70Ukn UPP }\end{array}$ & $\bar{z}$ & $\begin{array}{c}\text { Felt } \\
\text { Felt } \\
-\end{array}$ & $\frac{30}{30}$ & - \\
\hline $\begin{array}{l}1957 \\
1957 \\
1957 \\
1957 \\
1957\end{array}$ & $\begin{array}{l}03 \\
03 \\
03 \\
03 \\
03\end{array}$ & $\begin{array}{l}14 \\
14 \\
15 \\
15 \\
16\end{array}$ & $\begin{array}{lll}14 & 47 & 50.6 \\
15 & 51 & 06.4 \\
02 & 52 & 09.0 \\
04 & 13 & 02.4 \\
02 & 34 & 17.7\end{array}$ & $\begin{array}{l}51.098 \mathrm{~N} \\
50.991 \mathrm{~N} \\
52.73 \mathrm{~N} \\
51.114 \mathrm{~N} \\
51.159 \mathrm{~N}\end{array}$ & $\begin{array}{l}176.824 \mathrm{~W} \\
177.187 \mathrm{~W} \\
166.94 \mathrm{~W} \\
176.031 \mathrm{~W} \\
178.797 \mathrm{~W}\end{array}$ & $\begin{array}{l}033 \\
033 \\
000 \\
033 \\
033\end{array}$ & $\begin{array}{l}479 \\
479 \\
432 \\
479 \\
479\end{array}$ & $\begin{array}{l}- \\
- \\
-\end{array}$ & $\bar{z}$ & $\begin{array}{c}7.10 \mathrm{M}_{\mathrm{s}} \mathrm{ABE} \\
6.75 \overline{\mathrm{U}}_{\mathrm{kn}} \mathrm{PAS} \\
\overline{\mathrm{M}}_{\mathrm{S}} \mathrm{ABE}\end{array}$ & $\bar{z}$ & $\begin{array}{l}\text { Felt } \\
\text { Felt } \\
\text { Felt } \\
\text { Felt }\end{array}$ & $\begin{array}{l}30 \\
30 \\
30 \\
30\end{array}$ & $\begin{array}{l}- \\
- \\
-\end{array}$ \\
\hline $\begin{array}{l}1957 \\
1957 \\
1957 \\
1957 \\
1957\end{array}$ & $\begin{array}{l}03 \\
03 \\
03 \\
03 \\
03\end{array}$ & $\begin{array}{l}16 \\
17 \\
17 \\
17 \\
18\end{array}$ & $\begin{array}{lll}03 & 34 & 05.9 \\
07 & 53 & 52.2 \\
16 & 17 & 18.2 \\
22 & 44 & 44.8 \\
02 & 25 & 33.0\end{array}$ & $\begin{array}{l}51.861 \mathrm{~N} \\
51.57 \mathrm{~N} \\
52.02 \mathrm{~N} \\
53.79 \mathrm{~N} \\
52.19 \mathrm{~N}\end{array}$ & $\begin{array}{l}173.734 \mathrm{~W} \\
179.49 \mathrm{~W} \\
166.03 \mathrm{~W} \\
165.29 \mathrm{~W} \\
171.05 \mathrm{~W}\end{array}$ & $\begin{array}{l}033 \\
000 \\
042 \\
000 \\
051\end{array}$ & $\begin{array}{l}479 \\
432 \\
432 \\
432 \\
432\end{array}$ & $\begin{array}{l}- \\
-\end{array}$ & $\begin{array}{l}z \\
z\end{array}$ & $\begin{array}{l}6.10 \bar{M}_{R} \text { ROT } \\
6.40 \mathrm{M}_{\mathrm{R}} \text { ROT } \\
6.50 \mathrm{Un}_{\mathrm{RAS}} \\
6.20 \mathrm{M}_{\mathrm{R}} \text { ROT }\end{array}$ & $\bar{z}$ & $\begin{array}{c}\text { Felt } \\
\text { Felt } \\
= \\
=\end{array}$ & $\begin{array}{l}30 \\
30 \\
- \\
-\end{array}$ & $\begin{array}{l}- \\
-\end{array}$ \\
\hline $\begin{array}{l}1957 \\
1957 \\
1957 \\
1957 \\
1957\end{array}$ & $\begin{array}{l}03 \\
03 \\
03 \\
03 \\
03\end{array}$ & $\begin{array}{l}18 \\
19 \\
19 \\
19 \\
19\end{array}$ & $\begin{array}{lll}05 & 08 & 33.1 \\
08 & 14 & 09.8 \\
11 & 28 & 56.0 \\
12 & 50 & 57.2 \\
15 & 47 & 31.7\end{array}$ & $\begin{array}{l}50.75 \mathrm{~N} \\
52.65 \mathrm{~N} \\
51.070 \mathrm{~N} \\
51.249 \mathrm{~N} \\
51.582 \mathrm{~N}\end{array}$ & $\begin{array}{l}179.06 \mathrm{~W} \\
167.76 \mathrm{~W} \\
176.835 \mathrm{~W} \\
175.326 \mathrm{~W} \\
172.162 \mathrm{~W}\end{array}$ & $\begin{array}{l}000 \\
000 \\
033 \\
033 \\
033\end{array}$ & $\begin{array}{l}432 \\
432 \\
479 \\
479 \\
479\end{array}$ & $\begin{array}{l}- \\
- \\
-\end{array}$ & $\begin{array}{l}z \\
z\end{array}$ & $\begin{array}{l}\overline{-} \\
\text { 6.00M } \\
\text { 6.75UknPAS } \\
-\end{array}$ & $\bar{z}$ & $\begin{array}{l}\text { Felt } \\
\text { Felt } \\
\text { Felt } \\
-\end{array}$ & $\begin{array}{l}\frac{30}{30} \\
30 \\
-\end{array}$ & $\begin{array}{l}- \\
-\end{array}$ \\
\hline $\begin{array}{l}1957 \\
1957 \\
1957 \\
1957 \\
1957\end{array}$ & $\begin{array}{l}03 \\
03 \\
03 \\
03 \\
03\end{array}$ & $\begin{array}{l}19 \\
20 \\
20 \\
21 \\
22\end{array}$ & $\begin{array}{lll}17 & 04 & 25.4 \\
0000 & 57.8 \\
0022 & 22.8 \\
12 & 31 & 33.3 \\
14 & 21 & 05.5\end{array}$ & $\begin{array}{l}52.13 \mathrm{~N} \\
51.605 \mathrm{~N} \\
52.40 \mathrm{~N} \\
52.31 \mathrm{~N} \\
52.61 \mathrm{~N}\end{array}$ & $\begin{array}{l}170.74 \mathrm{~W} \\
172.710 \mathrm{~W} \\
168.66 \mathrm{~W} \\
170.87 \mathrm{~W} \\
165.76 \mathrm{~W}\end{array}$ & $\begin{array}{l}000 \\
033 \\
000 \\
000 \\
000\end{array}$ & $\begin{array}{l}432 \\
479 \\
432 \\
432 \\
432\end{array}$ & $\overline{-}$ & $\begin{array}{l}z \\
-\end{array}$ & $\begin{array}{l}\bar{z} \\
\bar{z} \\
{ }_{7.00 \mathrm{M}_{\mathrm{S}} \mathrm{ABE}}\end{array}$ & $\begin{array}{l}z \\
-\end{array}$ & $\begin{array}{c}\overline{-} \\
\text { Felt } \\
-\end{array}$ & $\frac{-}{30}-$ & $\begin{array}{l}- \\
- \\
-\end{array}$ \\
\hline $\begin{array}{l}1957 \\
1957 \\
1957 \\
1957 \\
1957\end{array}$ & $\begin{array}{l}03 \\
03 \\
03 \\
03 \\
03\end{array}$ & $\begin{array}{l}22 \\
24 \\
24 \\
25 \\
28\end{array}$ & $\begin{array}{l}170949.3 \\
110610.0 \\
113649.6 \\
003927.6 \\
200820.0\end{array}$ & $\begin{array}{ll}52.11 & \mathrm{~N} \\
52.29 & \mathrm{~N} \\
52.27 & \mathrm{~N} \\
52.79 & \mathrm{~N} \\
51.62 & \mathrm{~N}\end{array}$ & $\begin{array}{l}171.03 \mathrm{~W} \\
169.53 \mathrm{~W} \\
171.53 \mathrm{~W} \\
167.02 \mathrm{~W} \\
171.26 \mathrm{~W}\end{array}$ & $\begin{array}{l}000 \\
000 \\
000 \\
000 \\
000\end{array}$ & $\begin{array}{l}432 \\
432 \\
432 \\
432 \\
432\end{array}$ & $\begin{array}{l}- \\
- \\
-\end{array}$ & $\frac{-}{-}$ & $\begin{array}{ll}6 . \bar{M}_{\mathrm{R}} & \mathrm{ROT} \\
6.00 \mathrm{M}_{\mathrm{R}} & \mathrm{ROT} \\
6.00 \mathrm{M}_{\mathrm{R}} & \mathrm{ROT} \\
5.75 \mathrm{Ukn} \mathrm{MOS}\end{array}$ & $\begin{array}{l}- \\
- \\
-\end{array}$ & $\begin{array}{l}- \\
\overline{-} \\
-\end{array}$ & $\begin{array}{l}- \\
- \\
-\end{array}$ & $\begin{array}{l}- \\
- \\
-\end{array}$ \\
\hline $\begin{array}{l}1957 \\
1957 \\
1957 \\
1957 \\
1957\end{array}$ & $\begin{array}{l}03 \\
03 \\
03 \\
03 \\
03\end{array}$ & $\begin{array}{l}29 \\
29 \\
30 \\
30 \\
31\end{array}$ & $\begin{array}{l}051028.7 \\
224950.8 \\
004240.4 \\
091708.0 \\
100834.3\end{array}$ & $\begin{array}{l}53.40 \mathrm{~N} \\
52.65 \mathrm{~N} \\
51.47 \mathrm{~N} \\
51.817 \mathrm{~N} \\
51.181 \mathrm{~N}\end{array}$ & $\begin{array}{l}166.84 \mathrm{~W} \\
168.38 \mathrm{~W} \\
179.57 \mathrm{~W} \\
175.208 \mathrm{~W} \\
178.478 \mathrm{~W}\end{array}$ & $\begin{array}{l}000 \\
000 \\
000 \\
033 \\
033\end{array}$ & $\begin{array}{l}432 \\
432 \\
432 \\
479 \\
479\end{array}$ & - & $\begin{array}{l}E \\
-\end{array}$ & $\begin{array}{c}\text { 6.50Ukn PAS } \\
6.13 \mathrm{Ukn} \text { PAS } \\
-\overline{\mathrm{Ukn}}_{\mathrm{UPP}} \\
6.20 \mathrm{M} \mathrm{M} \text { ROT }\end{array}$ & $\begin{array}{l}- \\
-\end{array}$ & $\begin{array}{l}\overline{-} \\
\text { Felt } \\
\text { Felt }\end{array}$ & $\begin{array}{l}- \\
\overline{30} \\
30\end{array}$ & - \\
\hline
\end{tabular}


ALASKA-Continued

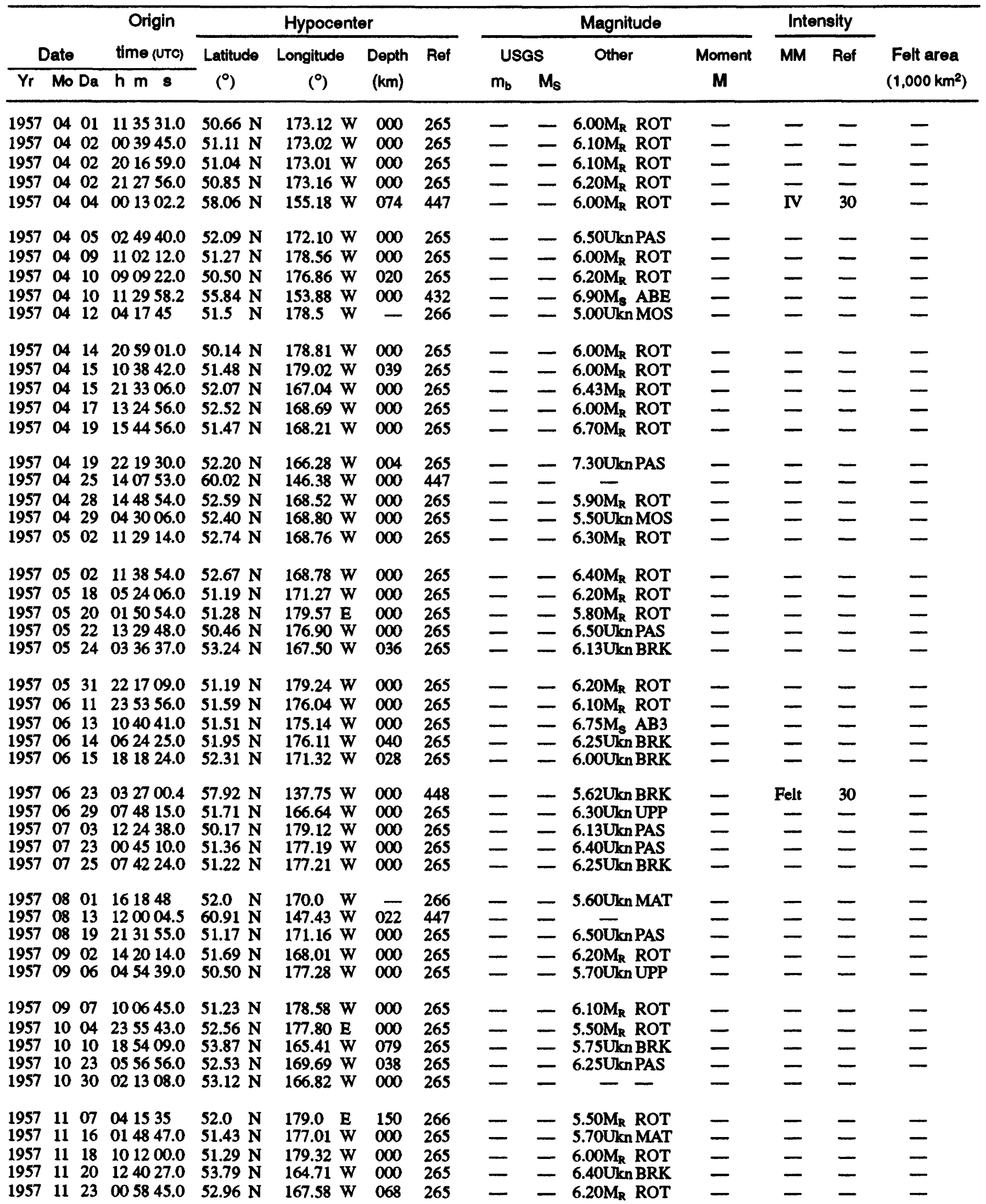


ALASKA-Continued

[See table 1 for hypocenter and intensity references and table 2 for definitions of magnitude source codes. \&, land area only. Leader (--) indicates information is not available]

\begin{tabular}{|c|c|c|c|c|c|c|c|c|c|c|c|c|c|c|}
\hline & & & Origin & & Hypocente & & & & & Magnitude & & Inte & isity & \\
\hline & Date & & time (UTC) & Latitude & Longitude & Depth & Ref & US & & Other & Moment & MM & Ref & \\
\hline$Y_{r}$ & Mo & $\mathrm{Da}$ & $\mathrm{h} \mathrm{m} s$ & $\left(^{\circ}\right)$ & $\left({ }^{\circ}\right)$ & $(\mathbf{k m})$ & & $m_{b}$ & $\mathbf{M}_{\mathbf{s}}$ & & M & & & $\left(1,000 \mathrm{~km}^{2}\right)$ \\
\hline $\begin{array}{l}1957 \\
1957 \\
1957 \\
1957 \\
1958\end{array}$ & $\begin{array}{l}11 \\
12 \\
12 \\
12 \\
01\end{array}$ & $\begin{array}{l}26 \\
03 \\
03 \\
13 \\
13\end{array}$ & $\begin{array}{l}113544.0 \\
014605 \\
214618.0 \\
202620.0 \\
000227.0\end{array}$ & $\begin{array}{ll}51.18 & \mathrm{~N} \\
51.5 & \mathrm{~N} \\
52.64 & \mathrm{~N} \\
52.12 & \mathrm{~N} \\
52.37 & \mathrm{~N}\end{array}$ & $\begin{array}{l}176.38 \mathrm{~W} \\
178.0 \mathrm{~W} \\
169.80 \mathrm{~W} \\
169.67 \mathrm{~W} \\
176.73 \mathrm{E}\end{array}$ & $\begin{array}{l}000 \\
000 \\
000 \\
121\end{array}$ & $\begin{array}{l}265 \\
266 \\
265 \\
265 \\
265\end{array}$ & $\begin{array}{l}- \\
- \\
-\end{array}$ & $\begin{array}{l}- \\
- \\
-\end{array}$ & $\begin{array}{l}6.20 \mathrm{M}_{\mathrm{R}} \text { ROT } \\
6.20 \mathrm{M}_{\mathrm{R}} \text { ROT } \\
5.60 \mathrm{Un}_{\mathrm{kn}} \mathrm{MAT} \\
6.00 \mathrm{M}_{\mathrm{R}} \text { ROT } \\
6.50 \mathrm{Un}_{\mathrm{kPP}}\end{array}$ & $\begin{array}{l}- \\
z \\
-\end{array}$ & $\begin{array}{l}- \\
- \\
-\end{array}$ & $\begin{array}{l}- \\
- \\
-\end{array}$ & $\begin{array}{l}- \\
- \\
-\end{array}$ \\
\hline $\begin{array}{l}1958 \\
1958 \\
1958 \\
1958 \\
1958\end{array}$ & $\begin{array}{l}01 \\
02 \\
02 \\
02 \\
02\end{array}$ & $\begin{array}{l}24 \\
12 \\
22 \\
22 \\
22\end{array}$ & $\begin{array}{lll}23 & 17 & 30.5 \\
23 & 43 & 52.0 \\
10 & 50 & 26.0 \\
13 & 21 & 50.0 \\
17 & 05 & 03.0\end{array}$ & $\begin{array}{l}60.16 \mathrm{~N} \\
51.79 \mathrm{~N} \\
50.32 \mathrm{~N} \\
50.39 \mathrm{~N} \\
52.15 \mathrm{~N}\end{array}$ & $\begin{array}{l}151.76 \mathrm{~W} \\
175.20 \mathrm{~W} \\
175.49 \mathrm{~W} \\
175.40 \mathrm{~W} \\
174.91 \mathrm{~W}\end{array}$ & $\begin{array}{l}052 \\
050 \\
000 \\
000 \\
000\end{array}$ & $\begin{array}{l}447 \\
265 \\
265 \\
265 \\
265\end{array}$ & $\overline{-}$ & $\overline{-}$ & $\begin{array}{c}\text { 6.40Ukn PAS } \\
\text { 6.00Ukn PAS } \\
\text { 6.75Ukn PAS } \\
\text { 5.50Ukn MAT }\end{array}$ & $\frac{-}{-}$ & $\begin{array}{l}\text { IV } \\
- \\
-\end{array}$ & $\begin{array}{l}31 \\
- \\
-\end{array}$ & $\bar{z}$ \\
\hline $\begin{array}{l}1958 \\
1958 \\
1958 \\
1958 \\
1958\end{array}$ & $\begin{array}{l}02 \\
03 \\
03 \\
04 \\
04\end{array}$ & $\begin{array}{l}25 \\
18 \\
20 \\
07 \\
08\end{array}$ & $\begin{array}{lll}01 & 56 & 39.0 \\
22 & 2000.0 \\
01 & 38 & 05.0 \\
15 & 30 & 40.3 \\
00 & 14 & 16.0\end{array}$ & $\begin{array}{l}51.42 \mathrm{~N} \\
50.24 \mathrm{~N} \\
50.49 \mathrm{~N} \\
65.99 \mathrm{~N} \\
65.87 \mathrm{~N}\end{array}$ & $\begin{array}{l}179.42 \mathrm{E} \\
172.95 \mathrm{~W} \\
172.84 \mathrm{~W} \\
156.55 \mathrm{~W} \\
155.95 \mathrm{~W}\end{array}$ & $\begin{array}{l}000 \\
000 \\
000 \\
000 \\
000\end{array}$ & $\begin{array}{l}265 \\
265 \\
265 \\
447 \\
447\end{array}$ & $\overline{-}$ & $\overline{-}$ & $\begin{array}{l}\text { 5.75Ukn MOS } \\
\text { 6.20Ukn UPP } \\
6.50 \mathrm{Un}_{\mathrm{kAS}} \\
7.30 \mathrm{M}_{\mathrm{s}} \text { ABE } \\
6.10 \mathrm{M}_{\mathrm{R}} \text { ROT }\end{array}$ & $\frac{-}{-}$ & $\underset{\text { Felt }}{\overline{-}}$ & $\begin{array}{l}- \\
\overline{31} \\
31\end{array}$ & $\frac{-}{-}$ \\
\hline $\begin{array}{l}1958 \\
1958 \\
1958 \\
1958 \\
1958\end{array}$ & $\begin{array}{l}04 \\
04 \\
04 \\
05 \\
05\end{array}$ & $\begin{array}{l}09 \\
13 \\
27 \\
10 \\
11\end{array}$ & $\begin{array}{l}061511.6 \\
090724.6 \\
190353.0 \\
225439.5 \\
052355.6\end{array}$ & $\begin{array}{l}56.14 \mathrm{~N} \\
65.83 \mathrm{~N} \\
52.94 \mathrm{~N} \\
65.12 \mathrm{~N} \\
65.01 \mathrm{~N}\end{array}$ & $\begin{array}{l}139.23 \mathrm{~W} \\
155.55 \mathrm{~W} \\
169.50 \mathrm{~W} \\
152.09 \mathrm{~W} \\
151.97 \mathrm{~W}\end{array}$ & $\begin{array}{l}000 \\
000 \\
000 \\
000 \\
000\end{array}$ & $\begin{array}{l}448 \\
447 \\
265 \\
447 \\
447\end{array}$ & $\overline{-}$ & $\begin{array}{l}- \\
- \\
-\end{array}$ & $\begin{array}{l}5.50 \mathrm{M}_{R} \text { ROT } \\
\text { 6.75Ukn PAS } \\
\text { 5.90M } \mathrm{M}_{R} \text { ROT } \\
\text { 6.38Ukn PAS } \\
\text { 6.38Ukn PAS }\end{array}$ & $\begin{array}{l}- \\
- \\
-\end{array}$ & $\begin{array}{l}\mathbf{V} \\
\mathbf{V} \\
\mathrm{V} \\
\mathrm{V}\end{array}$ & $\begin{array}{l}31 \\
31 \\
31 \\
31\end{array}$ & $\begin{array}{c}- \\
- \\
-\end{array}$ \\
\hline $\begin{array}{l}1958 \\
1958 \\
1958 \\
1958 \\
1958\end{array}$ & $\begin{array}{l}05 \\
05 \\
05 \\
05 \\
05\end{array}$ & $\begin{array}{l}12 \\
15 \\
17 \\
22 \\
25\end{array}$ & $\begin{array}{l}053818.0 \\
042450.0 \\
153822.0 \\
113251.0 \\
003523.0\end{array}$ & $\begin{array}{l}52.21 \mathrm{~N} \\
51.86 \mathrm{~N} \\
51.59 \mathrm{~N} \\
50.69 \mathrm{~N} \\
51.48 \mathrm{~N}\end{array}$ & $\begin{array}{l}169.54 \mathrm{~W} \\
173.64 \mathrm{~W} \\
179.29 \mathrm{~W} \\
175.02 \mathrm{~W} \\
177.42 \mathrm{~W}\end{array}$ & $\begin{array}{l}-\overline{000} \\
000 \\
000 \\
000\end{array}$ & $\begin{array}{l}265 \\
265 \\
265 \\
265 \\
265\end{array}$ & $\bar{z}$ & $\begin{array}{l}- \\
-\end{array}$ & $\frac{\bar{Z}}{\bar{Z}}$ & $\frac{-}{z}$ & $\begin{array}{l}- \\
-\end{array}$ & $\begin{array}{l}- \\
- \\
-\end{array}$ & $\begin{array}{l}- \\
- \\
-\end{array}$ \\
\hline $\begin{array}{l}1958 \\
1958 \\
1958 \\
1958 \\
1958\end{array}$ & $\begin{array}{l}05 \\
05 \\
05 \\
06 \\
06\end{array}$ & $\begin{array}{l}25 \\
26 \\
30 \\
01 \\
04\end{array}$ & $\begin{array}{l}145430.0 \\
105645.0 \\
180453.0 \\
182117.5 \\
142954.0\end{array}$ & $\begin{array}{l}51.27 \mathrm{~N} \\
53.26 \mathrm{~N} \\
52.73 \mathrm{~N} \\
60.57 \mathrm{~N} \\
52.69 \mathrm{~N}\end{array}$ & $\begin{array}{l}177.11 \mathrm{~W} \\
169.61 \mathrm{~W} \\
168.62 \mathrm{~W} \\
143.56 \mathrm{~W} \\
167.22 \mathrm{~W}\end{array}$ & $\begin{array}{l}000 \\
130 \\
000 \\
000 \\
019\end{array}$ & $\begin{array}{l}265 \\
265 \\
265 \\
447 \\
265\end{array}$ & $\overline{-}$ & $\bar{z}$ & $\begin{array}{c}\text { 5.63Ukn PAS } \\
\text { 6.13Ukn PAS } \\
\text { 6.13Ukn PAS } \\
\text { 6.13Ukn PAS }\end{array}$ & $\begin{array}{l}z \\
z\end{array}$ & $\frac{-}{-}$ & $\frac{-}{-}$ & $\begin{array}{l}- \\
- \\
-\end{array}$ \\
\hline $\begin{array}{l}1958 \\
1958 \\
1958 \\
1958 \\
1958\end{array}$ & $\begin{array}{l}06 \\
06 \\
06 \\
06 \\
06\end{array}$ & $\begin{array}{l}08 \\
09 \\
10 \\
12 \\
12\end{array}$ & $\begin{array}{l}003853.0 \\
155906.0 \\
001030 \\
205301.0 \\
213412\end{array}$ & 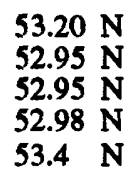 & $\begin{array}{l}166.79 \mathrm{~W} \\
167.22 \mathrm{~W} \\
167.25 \mathrm{~W} \\
166.97 \mathrm{~W} \\
166.3 \mathrm{~W}\end{array}$ & $\frac{\overline{1}}{\frac{000}{200}}$ & $\begin{array}{l}265 \\
265 \\
265 \\
265 \\
449\end{array}$ & $\bar{z}$ & $\overline{-}$ & $\begin{array}{c}6.00 \mathrm{M}_{\mathrm{R}} \mathrm{ROT} \\
- \\
\text { 6.50Ukn PAS } \\
5.50 \mathrm{M}_{\mathrm{R}} \mathrm{ROT}\end{array}$ & $\begin{array}{l}z \\
z \\
-\end{array}$ & $\begin{array}{l}- \\
- \\
-\end{array}$ & $\begin{array}{l}z \\
z\end{array}$ & $\begin{array}{l}- \\
- \\
-\end{array}$ \\
\hline $\begin{array}{l}1958 \\
1958 \\
1958 \\
1958 \\
1958\end{array}$ & $\begin{array}{l}07 \\
07 \\
07 \\
07 \\
07\end{array}$ & $\begin{array}{l}01 \\
07 \\
10 \\
13 \\
17\end{array}$ & $\begin{array}{l}055314.0 \\
0516 \quad 02.0 \\
061553.6 \\
081001.7 \\
1902 \quad 13.0\end{array}$ & $\begin{array}{l}51.59 \mathrm{~N} \\
50.06 \mathrm{~N} \\
58.34 \mathrm{~N} \\
57.91 \mathrm{~N} \\
51.54 \mathrm{~N}\end{array}$ & $\begin{array}{l}176.87 \mathrm{~W} \\
179.94 \mathrm{E} \\
136.52 \mathrm{~W} \\
136.95 \mathrm{~W} \\
176.68 \mathrm{~W}\end{array}$ & $\begin{array}{l}067 \\
000 \\
000 \\
000 \\
000\end{array}$ & $\begin{array}{l}265 \\
265 \\
448 \\
448 \\
265\end{array}$ & $\begin{array}{l}- \\
- \\
-\end{array}$ & $\begin{array}{l}- \\
- \\
-\end{array}$ & $\begin{array}{l}6.00 \mathrm{Ukn} \text { PAS } \\
5.50 \mathrm{M}_{\mathrm{R}} \text { ROT } \\
7.90 \mathrm{M}_{\mathrm{s}} \text { ABE } \\
5.63 \mathrm{Ukn}_{\mathrm{BRK}} \\
5.75 \mathrm{Ukn} \text { BRK }\end{array}$ & $\begin{array}{c}\overline{-} \\
8.26 \mathrm{KAN} \\
- \\
-\end{array}$ & $\begin{array}{r}\overline{-} \\
\text { Felt } \\
-\end{array}$ & $\begin{array}{l}- \\
\overline{31} \\
-\end{array}$ & $\begin{array}{l}- \\
\overline{570 \&} \\
-\end{array}$ \\
\hline $\begin{array}{l}1958 \\
1958 \\
1958 \\
1958 \\
1958\end{array}$ & $\begin{array}{l}07 \\
07 \\
07 \\
07 \\
08\end{array}$ & $\begin{array}{l}17 \\
18 \\
21 \\
24 \\
13\end{array}$ & $\begin{array}{l}205924.0 \\
003921.0 \\
143724.0 \\
130805.0 \\
201259.0\end{array}$ & $\begin{array}{l}51.45 \mathrm{~N} \\
51.45 \mathrm{~N} \\
51.50 \mathrm{~N} \\
52.74 \mathrm{~N} \\
50.59 \mathrm{~N}\end{array}$ & $\begin{array}{l}176.69 \mathrm{~W} \\
176.60 \mathrm{~W} \\
178.43 \mathrm{~W} \\
169.77 \mathrm{~W} \\
177.75 \mathrm{~W}\end{array}$ & $\begin{array}{l}000 \\
057 \\
000 \\
000\end{array}$ & $\begin{array}{l}265 \\
265 \\
265 \\
265 \\
265\end{array}$ & $\begin{array}{l}- \\
-\end{array}$ & $\bar{z}$ & $\begin{array}{l}\text { 6.00Ukn BRK } \\
\text { 5.75Ukn BRK } \\
\text { 6.25Ukn BRK } \\
\text { 5.75Ukn MAT } \\
\text { 6.40Ukn PAS }\end{array}$ & $\bar{z}$ & $\frac{-}{-}$ & $\begin{array}{l}z \\
z \\
-\end{array}$ & $\begin{array}{l}- \\
- \\
-\end{array}$ \\
\hline $\begin{array}{l}1958 \\
1958 \\
1958 \\
1958 \\
1958\end{array}$ & $\begin{array}{l}08 \\
08 \\
08 \\
08 \\
09\end{array}$ & $\begin{array}{l}14 \\
16 \\
17 \\
31 \\
09\end{array}$ & $\begin{array}{l}145512.0 \\
131754.0 \\
090835.0 \\
230017.0 \\
222336\end{array}$ & $\begin{array}{l}51.59 \mathrm{~N} \\
51.43 \mathrm{~N} \\
51.38 \mathrm{~N} \\
63.22 \mathrm{~N} \\
53.97 \mathrm{~N}\end{array}$ & $\begin{array}{l}175.39 \mathrm{~W} \\
176.11 \mathrm{~W} \\
176.23 \mathrm{~W} \\
144.32 \mathrm{~W} \\
171.14 \mathrm{E}\end{array}$ & $\begin{array}{l}000 \\
000 \\
000 \\
017 \\
000\end{array}$ & $\begin{array}{l}265 \\
265 \\
265 \\
447 \\
265\end{array}$ & $\overline{-}$ & $\bar{z}$ & $\begin{array}{c}\text { 6.50Ukn PAS } \\
6.13 \mathrm{Ukn} \text { PAS } \\
6.00 \mathrm{M}_{\mathrm{R}} \text { ROT } \\
5.90 \mathrm{Un} \text { BRK } \\
-\end{array}$ & $\begin{array}{l}- \\
z \\
-\end{array}$ & $\begin{array}{c}\text { - } \\
\text { Felt } \\
\text { V }\end{array}$ & $\begin{array}{r}- \\
266 \\
31 \\
-\end{array}$ & $\begin{array}{l}- \\
- \\
-\end{array}$ \\
\hline $\begin{array}{l}1958 \\
1958 \\
1958\end{array}$ & $\begin{array}{l}09 \\
10 \\
10\end{array}$ & $\begin{array}{l}24 \\
01 \\
20\end{array}$ & $\begin{array}{l}034417.6 \\
174717.0 \\
005532.0\end{array}$ & $\begin{array}{l}59.63 \mathrm{~N} \\
52.67 \mathrm{~N} \\
51.90 \mathrm{~N}\end{array}$ & $\begin{array}{l}142.93 \mathrm{~W} \\
165.40 \mathrm{~W} \\
175.15 \mathrm{~W}\end{array}$ & $\begin{array}{l}000 \\
000 \\
000\end{array}$ & $\begin{array}{l}447 \\
265 \\
265\end{array}$ & - & - & $\begin{array}{l}\text { 6.25Ukn PAS } \\
\text { 6.25Ukn PAS } \\
\text { 5.70Ukn UPP }\end{array}$ & - & $\overline{-}$ & $\overline{31}$ & - \\
\hline
\end{tabular}


EARTHQUAKES IN ALASKA

ALASKA-Continued

[See table 1 for hypocenter and intensity references and table 2 for definitions of magnitude source codes. \&, land area only. Leader (--) indicates information is not available]

\begin{tabular}{|c|c|c|c|c|c|c|c|c|c|c|c|c|c|}
\hline \multirow{2}{*}{\multicolumn{2}{|c|}{ Date }} & \multirow{2}{*}{$\begin{array}{c}\text { Origin } \\
\text { time (UTC) }\end{array}$} & \multicolumn{4}{|c|}{ Hypocenter } & \multicolumn{4}{|c|}{ Magnitude } & \multicolumn{2}{|c|}{ Intensity } & \multirow{3}{*}{$\begin{array}{l}\text { Felt area } \\
\left(1,000 \mathrm{~km}^{2}\right)\end{array}$} \\
\hline & & & \multirow{2}{*}{$\begin{array}{l}\text { Latitude } \\
\left({ }^{\circ}\right)\end{array}$} & \multirow{2}{*}{$\begin{array}{c}\text { Longitude } \\
\text { ( })\end{array}$} & \multirow{2}{*}{$\begin{array}{l}\text { Depth } \\
(\mathbf{k m})\end{array}$} & \multirow[t]{2}{*}{ Ref } & \multicolumn{2}{|c|}{ USGS } & \multirow{2}{*}{ Other } & \multirow{2}{*}{$\begin{array}{c}\text { Moment } \\
\text { M }\end{array}$} & \multirow[t]{2}{*}{$M M$} & \multirow[t]{2}{*}{ Ref } & \\
\hline$\overline{Y_{r}}$ & Mo Da & $\mathrm{h} m \mathrm{~s}$ & & & & & $m_{b}$ & $\mathbf{M}_{\mathbf{s}}$ & & & & & \\
\hline $\begin{array}{l}1958 \\
1958\end{array}$ & $\begin{array}{ll}10 & 28 \\
10 & 29\end{array}$ & $\begin{array}{l}235007.0 \\
074410.0\end{array}$ & $\begin{array}{l}51.53 \mathrm{~N} \\
51.49 \mathrm{~N}\end{array}$ & $\begin{array}{l}179.30 \mathrm{E} \\
179.41 \mathrm{E}\end{array}$ & $\begin{array}{l}000 \\
000\end{array}$ & $\begin{array}{l}265 \\
265\end{array}$ & $=$ & $\overline{-}$ & $\begin{array}{l}\text { 5.75Ukn MAT } \\
\text { 6.25Ukn PAS }\end{array}$ & - & $=$ & $=$ & $\bar{z}$ \\
\hline $\begin{array}{l}1958 \\
1958 \\
1958 \\
1958 \\
1958\end{array}$ & $\begin{array}{ll}10 & 29 \\
11 & 02 \\
11 & 18 \\
11 & 19 \\
12 & 19\end{array}$ & $\begin{array}{l}075513.0 \\
104447.0 \\
074522.0 \\
150215.7 \\
183624.0\end{array}$ & $\begin{array}{l}51.37 \mathrm{~N} \\
51.57 \mathrm{~N} \\
51.66 \mathrm{~N} \\
60.46 \mathrm{~N} \\
51.46 \mathrm{~N}\end{array}$ & $\begin{array}{l}179.15 \mathrm{E} \\
175.19 \mathrm{~W} \\
179.37 \mathrm{E} \\
150.91 \mathrm{~W} \\
177.68 \mathrm{~W}\end{array}$ & $\begin{array}{l}000 \\
000 \\
000 \\
046 \\
000\end{array}$ & $\begin{array}{l}265 \\
265 \\
265 \\
447 \\
265\end{array}$ & $\begin{array}{l}\bar{z} \\
\bar{z}\end{array}$ & $\begin{array}{l}\bar{z} \\
\bar{z}\end{array}$ & $\begin{array}{l}5.63 \overline{U k n}_{\mathrm{MAT}} \\
5.75 \mathrm{Ukn} \text { MAT } \\
5.90 \mathrm{M}_{\mathrm{R}} \text { ROT } \\
5.60 \mathrm{kn} \text { MAT }\end{array}$ & $\begin{array}{l}\bar{z} \\
\bar{z}\end{array}$ & $\bar{z}$ & $\bar{z}$ & $\begin{array}{l}\bar{z} \\
\bar{z}\end{array}$ \\
\hline $\begin{array}{l}1959 \\
1959 \\
1959 \\
1959 \\
1959\end{array}$ & $\begin{array}{ll}01 & 16 \\
01 & 29 \\
01 & 29 \\
02 & 05 \\
02 & 06\end{array}$ & $\begin{array}{lll}01 & 31 & 26 \\
20 & 21 & 27.0 \\
20 & 58 & 20.0 \\
01 & 04 & 50.5 \\
14 & 33 & 05.0\end{array}$ & $\begin{array}{l}52.34 \mathrm{~N} \\
51.88 \mathrm{~N} \\
52.14 \mathrm{~N} \\
57.43 \mathrm{~N} \\
51.72 \mathrm{~N}\end{array}$ & $\begin{array}{l}177.11 \mathrm{~W} \\
173.79 \mathrm{~W} \\
173.86 \mathrm{~W} \\
156.88 \mathrm{~W} \\
176.02 \mathrm{~W}\end{array}$ & $\begin{array}{l}041 \\
000 \\
000 \\
068 \\
044\end{array}$ & $\begin{array}{l}265 \\
265 \\
265 \\
447 \\
265\end{array}$ & $\begin{array}{l}\bar{z} \\
\bar{z}\end{array}$ & $\bar{z}$ & $\begin{array}{l}6.20 \mathrm{M}_{\mathrm{R}} \text { ROT } \\
5.88 \mathrm{U} \mathrm{kn} \text { PAS } \\
5.50 \mathrm{U} \mathrm{kn} \text { PAS } \\
6.0 \overline{\mathrm{U}}_{\mathrm{kn}} \mathrm{PAS}\end{array}$ & $\begin{array}{l}\bar{z} \\
\bar{z}\end{array}$ & $\begin{array}{l}\bar{z} \\
\bar{z}\end{array}$ & $\bar{z}$ & $\begin{array}{l}\bar{z} \\
\bar{z}\end{array}$ \\
\hline $\begin{array}{l}1959 \\
1959 \\
1959 \\
1959 \\
1959\end{array}$ & $\begin{array}{ll}02 & 09 \\
02 & 17 \\
02 & 28 \\
04 & 14 \\
04 & 19\end{array}$ & $\begin{array}{lll}04 & 42 & 35.0 \\
12 & 03 & 04.0 \\
01 & 32 & 24.0 \\
07 & 20 & 27.3 \\
15 & 03 & 30.7\end{array}$ & $\begin{array}{l}50.00 \mathrm{~N} \\
51.10 \mathrm{~N} \\
53.02 \mathrm{~N} \\
57.95 \mathrm{~N} \\
58.20 \mathrm{~N}\end{array}$ & $\begin{array}{l}177.64 \mathrm{~W} \\
171.23 \mathrm{~W} \\
168.06 \mathrm{~W} \\
155.01 \mathrm{~W} \\
151.74 \mathrm{~W}\end{array}$ & $\begin{array}{l}000 \\
000 \\
067 \\
037\end{array}$ & $\begin{array}{l}265 \\
265 \\
265 \\
447 \\
477\end{array}$ & $\begin{array}{l}\bar{z} \\
\bar{z}\end{array}$ & $\begin{array}{l}\bar{z} \\
\bar{z}\end{array}$ & $\begin{array}{l}6.20 \mathrm{M}_{R} \text { ROT } \\
6.13 \mathrm{Ukn}_{\mathrm{kAS}} \\
5.50 \mathrm{Un}_{\mathrm{kAT}} \mathrm{MAT} \\
6.00 \mathrm{M}_{\mathrm{R}} \mathrm{ROT} \\
6.25 \mathrm{k} \text {. PAS }\end{array}$ & $\bar{z}$ & $\begin{array}{l}z \\
\bar{z}\end{array}$ & $\begin{array}{l}\bar{z} \\
\bar{z}\end{array}$ & $\begin{array}{l}z \\
=\end{array}$ \\
\hline $\begin{array}{l}1959 \\
1959 \\
1959 \\
1959 \\
1959\end{array}$ & $\begin{array}{ll}04 & 22 \\
05 & 12 \\
05 & 12 \\
05 & 18 \\
06 & 04\end{array}$ & $\begin{array}{l}105511.0 \\
214023.0 \\
215956.0 \\
072408.0 \\
123156.4\end{array}$ & $\begin{array}{l}53.80 \mathrm{~N} \\
51.54 \mathrm{~N} \\
51.21 \mathrm{~N} \\
52.51 \mathrm{~N} \\
59.98 \mathrm{~N}\end{array}$ & $\begin{array}{l}166.87 \mathrm{~W} \\
177.14 \mathrm{~W} \\
176.95 \mathrm{~W} \\
173.69 \mathrm{E} \\
152.70 \mathrm{~W}\end{array}$ & $\begin{array}{l}051 \\
000 \\
000 \\
000 \\
099\end{array}$ & $\begin{array}{l}265 \\
265 \\
265 \\
265 \\
447\end{array}$ & $\begin{array}{l}\bar{z} \\
\overline{-}\end{array}$ & $\bar{z}$ & $\begin{array}{l}6.00 \mathrm{Ukn}_{\mathrm{kAS}} \\
5.80 \mathrm{M}_{\mathrm{R}} \text { ROT } \\
6.00 \mathrm{Ukn}_{\mathrm{kAS}} \\
6.10 \mathrm{M}_{\mathrm{R}} \text { ROT } \\
5.50 \mathrm{kn} \text { PAS }\end{array}$ & $\begin{array}{l}\bar{z} \\
\bar{z}\end{array}$ & $\begin{array}{l}\bar{z} \\
\overline{\text { III }}\end{array}$ & $\begin{array}{l}\bar{z} \\
\overline{32}\end{array}$ & $\begin{array}{l}\bar{z} \\
\bar{z}\end{array}$ \\
\hline $\begin{array}{l}1959 \\
1959 \\
1959 \\
1959 \\
1959\end{array}$ & $\begin{array}{ll}07 & 13 \\
07 & 14 \\
07 & 16 \\
08 & 07 \\
08 & 07\end{array}$ & 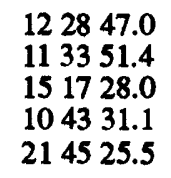 & $\begin{array}{ll}52.01 & N \\
56.83 & N \\
50.47 & N \\
56.41 & N \\
56.45 & N\end{array}$ & $\begin{array}{l}172.00 \mathrm{~W} \\
157.73 \mathrm{~W} \\
177.32 \mathrm{~W} \\
153.58 \mathrm{~W} \\
153.51 \mathrm{~W}\end{array}$ & $\begin{array}{l}000 \\
043 \\
000 \\
000 \\
000\end{array}$ & $\begin{array}{l}265 \\
447 \\
265 \\
447 \\
447\end{array}$ & $\begin{array}{l}z \\
z\end{array}$ & $\bar{z}$ & $\begin{array}{l}\text { 6.50UknPAS } \\
\bar{Z} \\
5.75 \overline{\mathrm{U}}_{\mathrm{knPAS}} \\
6.00 \mathrm{M}_{\mathrm{R}} \mathrm{ROT}\end{array}$ & $\begin{array}{l}\bar{z} \\
\bar{z}\end{array}$ & $\begin{array}{l}z \\
z\end{array}$ & $\begin{array}{l}\bar{z} \\
\bar{z}\end{array}$ & $\begin{array}{l}\bar{z} \\
\bar{z}\end{array}$ \\
\hline $\begin{array}{l}1959 \\
1959 \\
1959 \\
1959 \\
1959\end{array}$ & $\begin{array}{ll}08 & 28 \\
09 & 05 \\
09 & 21 \\
10 & 08 \\
11 & 30\end{array}$ & $\begin{array}{l}120747.4 \\
212841.0 \\
161619.9 \\
023521.0 \\
151836.2\end{array}$ & $\begin{array}{l}63.42 \mathrm{~N} \\
51.30 \mathrm{~N} \\
62.47 \mathrm{~N} \\
52.35 \mathrm{~N} \\
59.73 \mathrm{~N}\end{array}$ & $\begin{array}{l}148.85 \mathrm{~W} \\
179.36 \mathrm{E} \\
158.68 \mathrm{~W} \\
170.81 \mathrm{~W} \\
151.29 \mathrm{~W}\end{array}$ & $\begin{array}{l}044 \\
000 \\
000 \\
000 \\
000\end{array}$ & $\begin{array}{l}447 \\
265 \\
447 \\
265 \\
447\end{array}$ & $\begin{array}{l}\bar{z} \\
\overline{-}\end{array}$ & $\bar{z}$ & $\begin{array}{c}6.00 \mathrm{M}_{\mathrm{R}} \text { ROT } \\
5.63 \mathrm{U} \text { kn MAT } \\
5.50 \mathrm{M}_{\mathrm{R}} \text { ROT } \\
\overline{-}_{\mathrm{R}} \text { ROT }\end{array}$ & $\begin{array}{l}\bar{z} \\
\bar{z}\end{array}$ & $\begin{array}{l}\text { Felt } \\
\overline{-} \\
\text { Felt }\end{array}$ & $\frac{32}{\bar{z}}$ & $\begin{array}{l}\bar{z} \\
\bar{z}\end{array}$ \\
\hline $\begin{array}{l}1959 \\
1959 \\
1959 \\
1959 \\
1960\end{array}$ & $\begin{array}{ll}12 & 14 \\
12 & 18 \\
12 & 23 \\
12 & 26 \\
01 & 13\end{array}$ & $\begin{array}{l}220051.0 \\
162450.0 \\
034907.5 \\
181907.8 \\
162942.0\end{array}$ & $\begin{array}{l}52.45 \mathrm{~N} \\
52.56 \mathrm{~N} \\
56.45 \mathrm{~N} \\
59.67 \mathrm{~N} \\
51.73 \mathrm{~N}\end{array}$ & $\begin{array}{l}168.21 \mathrm{~W} \\
168.39 \mathrm{~W} \\
157.64 \mathrm{~W} \\
151.26 \mathrm{~W} \\
179.93 \mathrm{E}\end{array}$ & $\begin{array}{l}000 \\
000 \\
055 \\
000 \\
000\end{array}$ & $\begin{array}{l}265 \\
265 \\
447 \\
447 \\
265\end{array}$ & $\begin{array}{l}= \\
\bar{z}\end{array}$ & $\begin{array}{l}\bar{z} \\
\bar{z}\end{array}$ & $\begin{array}{l}6.40 \mathrm{M}_{\mathrm{R}} \text { ROT } \\
6.50 \mathrm{U} \mathrm{kn} \text { PAS } \\
{ }^{6.25 \mathrm{U}} \mathrm{kn} \mathrm{PAS} \\
-\end{array}$ & $\begin{array}{l}\bar{z} \\
\bar{z}\end{array}$ & $\frac{z}{I}$ & $\frac{-}{32}$ & $\begin{array}{l}\bar{z} \\
\bar{z}\end{array}$ \\
\hline $\begin{array}{l}1960 \\
1960 \\
1960 \\
1960 \\
1960\end{array}$ & $\begin{array}{ll}01 & 16 \\
02 & 19 \\
02 & 26 \\
02 & 27 \\
03 & 04\end{array}$ & $\begin{array}{l}204931.1 \\
050928.1 \\
232924.0 \\
081004.0 \\
021558.0\end{array}$ & $\begin{array}{l}63.29 \mathrm{~N} \\
60.87 \mathrm{~N} \\
51.32 \mathrm{~N} \\
51.53 \mathrm{~N} \\
50.60 \mathrm{~N}\end{array}$ & $\begin{array}{l}150.41 \mathrm{~W} \\
150.36 \mathrm{~W} \\
177.97 \mathrm{~W} \\
177.96 \mathrm{~W} \\
176.85 \mathrm{~W}\end{array}$ & $\begin{array}{l}125 \\
044 \\
000 \\
000 \\
000\end{array}$ & $\begin{array}{l}447 \\
447 \\
265 \\
265 \\
265\end{array}$ & $\begin{array}{l}\bar{z} \\
\bar{z}\end{array}$ & $\begin{array}{l}\bar{z} \\
\bar{z}\end{array}$ & 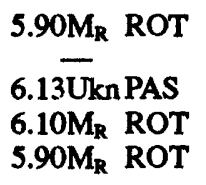 & $\begin{array}{l}\bar{z} \\
\bar{z}\end{array}$ & $\begin{array}{l}\text { III } \\
\text { V } \\
\text { Felt } \\
\text { Felt } \\
-\end{array}$ & $\begin{array}{l}33 \\
33 \\
33 \\
33 \\
-\end{array}$ & $\begin{array}{l}\bar{z} \\
\bar{z}\end{array}$ \\
\hline $\begin{array}{l}1960 \\
1960 \\
1960 \\
1960 \\
1960\end{array}$ & $\begin{array}{ll}03 & 15 \\
04 & 10 \\
05 & 13 \\
06 & 17 \\
06 & 22\end{array}$ & $\begin{array}{l}092056.0 \\
202611.0 \\
160711.2 \\
163533.0 \\
232847.0\end{array}$ & $\begin{array}{l}50.64 \mathrm{~N} \\
52.88 \mathrm{~N} \\
54.81 \mathrm{~N} \\
51.90 \mathrm{~N} \\
51.68 \mathrm{~N}\end{array}$ & $\begin{array}{l}174.55 \mathrm{~W} \\
167.05 \mathrm{~W} \\
161.35 \mathrm{~W} \\
173.15 \mathrm{~W} \\
173.43 \mathrm{~W}\end{array}$ & $\begin{array}{l}000 \\
000 \\
000 \\
000 \\
000\end{array}$ & $\begin{array}{l}265 \\
265 \\
447 \\
265 \\
265\end{array}$ & $\begin{array}{l}= \\
=\end{array}$ & $\begin{array}{l}\bar{z} \\
=\end{array}$ & $\begin{array}{c}\overline{-} \\
\text { 6.25Ukn PAS } \\
\text { 6.13UknPAS } \\
\text { 6.13Ukn PAS }\end{array}$ & $\begin{array}{l}\bar{z} \\
\bar{z}\end{array}$ & $\begin{array}{c}\overline{\overline{I V}} \\
\text { Felt } \\
-\end{array}$ & $\begin{array}{l}\overline{-} \\
33 \\
33 \\
-\end{array}$ & $\begin{array}{l}\bar{z} \\
\bar{z}\end{array}$ \\
\hline $\begin{array}{l}1960 \\
1960 \\
1960 \\
1960 \\
1960\end{array}$ & $\begin{array}{ll}06 & 29 \\
06 & 30 \\
07 & 03 \\
07 & 03 \\
07 & 05\end{array}$ & $\begin{array}{l}170657.0 \\
195837.7 \\
202046.0 \\
225224.0 \\
050756.0\end{array}$ & $\begin{array}{l}52.69 \mathrm{~N} \\
60.30 \mathrm{~N} \\
50.28 \mathrm{~N} \\
50.28 \mathrm{~N} \\
51.50 \mathrm{~N}\end{array}$ & $\begin{array}{l}168.04 \mathrm{~W} \\
150.90 \mathrm{~W} \\
177.10 \mathrm{~W} \\
177.19 \mathrm{~W} \\
178.27 \mathrm{~W}\end{array}$ & $\begin{array}{l}000 \\
055 \\
000 \\
000 \\
000\end{array}$ & $\begin{array}{l}265 \\
447 \\
265 \\
265 \\
265\end{array}$ & $\begin{array}{l}\bar{z} \\
\overline{-}\end{array}$ & $\begin{array}{l}\bar{z} \\
\bar{z}\end{array}$ & 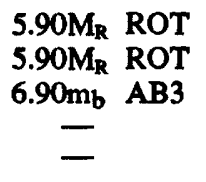 & $\begin{array}{l}\bar{z} \\
\bar{z}\end{array}$ & $\begin{array}{l}\overline{\text { Felt }} \\
\text { Felt } \\
\overline{-}\end{array}$ & $\begin{array}{l}\overline{33} \\
33 \\
-\end{array}$ & $\begin{array}{l}\bar{z} \\
\bar{z}\end{array}$ \\
\hline
\end{tabular}




\begin{tabular}{|c|c|c|c|c|c|c|c|c|c|c|c|c|c|c|c|}
\hline \multirow{2}{*}{\multicolumn{4}{|c|}{$\begin{array}{cc} & \text { Origin } \\
\text { Date } & \text { time (UTC) }\end{array}$}} & \multicolumn{5}{|c|}{ Hypocenter } & \multicolumn{4}{|c|}{ Magnitude } & \multicolumn{2}{|c|}{ Intensity } & \multirow{3}{*}{$\begin{array}{l}\text { Felt area } \\
\left(1,000 \mathrm{~km}^{2}\right)\end{array}$} \\
\hline & & & & \multirow{2}{*}{$\begin{array}{l}\text { Latitude } \\
\left({ }^{\circ}\right)\end{array}$} & \multirow{2}{*}{\multicolumn{2}{|c|}{$\begin{array}{l}\text { Longitude } \\
\left(^{\circ}\right)\end{array}$}} & \multirow{2}{*}{$\begin{array}{l}\text { Depth } \\
\text { (km) }\end{array}$} & \multirow[t]{2}{*}{ Ref } & \multicolumn{2}{|c|}{ USGS } & \multirow[t]{2}{*}{ Other } & \multirow{2}{*}{$\begin{array}{c}\text { Moment } \\
\mathbf{M}\end{array}$} & \multirow[t]{2}{*}{ MM } & \multirow[t]{2}{*}{ Ref } & \\
\hline$Y_{r}$ & & & $h \mathrm{~m} s$ & & & & & & $m_{b}$ & $\mathbf{M}_{\mathbf{s}}$ & & & & & \\
\hline $\begin{array}{l}1960 \\
1960 \\
1960 \\
1960 \\
1960\end{array}$ & $\begin{array}{l}08 \\
08 \\
08 \\
08 \\
08\end{array}$ & $\begin{array}{l}02 \\
04 \\
04 \\
05 \\
25\end{array}$ & $\begin{array}{lll}06 & 14 & 45.0 \\
07 & 34 & 48.0 \\
14 & 05 & 28.7 \\
22 & 27 & 41.0 \\
17 & 41 & 54.0\end{array}$ & $\begin{array}{ll}51.61 & \mathrm{~N} \\
51.41 & \mathrm{~N} \\
51.4 & \mathrm{~N} \\
51.34 & \mathrm{~N} \\
52.52 & \mathrm{~N}\end{array}$ & $\begin{array}{l}178.30 \mathrm{~V} \\
179.04 \mathrm{E} \\
178.9 \\
178.83 \\
169.67\end{array}$ & $\begin{array}{l}\mathbf{W} \\
\mathbf{E} \\
\mathbf{E} \\
\mathbf{E} \\
\mathbf{W}\end{array}$ & $\begin{array}{l}000 \\
000 \\
034 \\
052 \\
000\end{array}$ & $\begin{array}{l}265 \\
265 \\
266 \\
265 \\
265\end{array}$ & 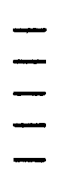 & $\begin{array}{l}z \\
z\end{array}$ & $\begin{array}{l}6.13 \overline{\mathrm{U}} \mathrm{kn} \text { PAS } \\
\text { 5.70Ukn MAT } \\
6.00 \mathrm{M}_{\mathrm{R}} \mathrm{ROT}\end{array}$ & $\begin{array}{l}z \\
- \\
-\end{array}$ & $\begin{array}{c}\text { Felt } \\
\text { Felt } \\
\overline{-} \\
-\end{array}$ & $\begin{array}{l}33 \\
33 \\
- \\
-\end{array}$ & $\begin{array}{l}- \\
-\end{array}$ \\
\hline $\begin{array}{l}1960 \\
1960 \\
1960 \\
1960 \\
1960\end{array}$ & $\begin{array}{l}09 \\
09 \\
09 \\
10 \\
10\end{array}$ & $\begin{array}{l}01 \\
02 \\
12 \\
01 \\
14\end{array}$ & $\begin{array}{lll}15 & 37 & 13.0 \\
22 & 02 & 44.0 \\
02 & 44 & 39.7 \\
16 & 10 & 52.0 \\
21 & 19 & 13.0\end{array}$ & $\begin{array}{ll}56.25 & \mathrm{~N} \\
52.21 & \mathrm{~N} \\
60.5 & \mathrm{~N} \\
51.79 & \mathrm{~N} \\
51.83 & \mathrm{~N}\end{array}$ & $\begin{array}{l}153.59 \\
171.51 \\
153.8 \\
172.40 \\
172.25\end{array}$ & $\begin{array}{l}\mathbf{W} \\
\mathbf{W} \\
\mathbf{W} \\
\mathbf{W} \\
\mathbf{W}\end{array}$ & $\begin{array}{l}000 \\
000 \\
195 \\
000 \\
041\end{array}$ & $\begin{array}{l}447 \\
265 \\
266 \\
265 \\
265\end{array}$ & $\begin{array}{l}- \\
- \\
-\end{array}$ & $\begin{array}{l}z \\
z \\
-\end{array}$ & $\begin{array}{l}\text { 6.13Ukn PAS } \\
\text { 5.88Ukn PAS } \\
\text { 5.50M R ROT } \\
\text { 6.50Ukn PAS } \\
\text { 6.50Ukn PAS }\end{array}$ & $\begin{array}{l}z \\
z \\
-\end{array}$ & $\begin{array}{l}\bar{Z} \\
\overline{I V} \\
-\end{array}$ & $\frac{-}{33}$ & $\begin{array}{l}- \\
z \\
-\end{array}$ \\
\hline $\begin{array}{l}1960 \\
1960 \\
1960 \\
1960 \\
1961\end{array}$ & $\begin{array}{l}11 \\
11 \\
12 \\
12 \\
01\end{array}$ & $\begin{array}{l}06 \\
13 \\
03 \\
21 \\
05\end{array}$ & $\begin{array}{l}221003.0 \\
092030.9 \\
070742.6 \\
143958.9 \\
140632.0\end{array}$ & $\begin{array}{l}52.69 \mathrm{~N} \\
51.23 \mathrm{~N} \\
52.7 \mathrm{~N} \\
61.81 \mathrm{~N} \\
51.83 \mathrm{~N}\end{array}$ & $\begin{array}{l}168.07 \\
168.86 \\
177.4 \\
152.35 \\
175.94\end{array}$ & $\begin{array}{l}\mathbf{W} \\
\mathbf{w} \\
\mathbf{W} \\
\mathbf{W} \\
\mathbf{W}\end{array}$ & $\begin{array}{l}000 \\
000 \\
160 \\
100 \\
051\end{array}$ & $\begin{array}{l}265 \\
432 \\
266 \\
447 \\
265\end{array}$ & $\begin{array}{l}- \\
- \\
-\end{array}$ & $\begin{array}{l}- \\
z \\
-\end{array}$ & $\begin{array}{l}6.20 \mathrm{M}_{\mathrm{R}} \text { ROT } \\
6.70 \mathrm{M}_{\mathrm{S}} \text { ABE } \\
5.50 \mathrm{M}_{\mathrm{R}} \text { ROT } \\
5.75 \mathrm{Un} \mathrm{PAS} \\
6.75 \mathrm{Unn} \text { PAS }\end{array}$ & $\begin{array}{l}- \\
z \\
-\end{array}$ & $\begin{array}{c}\overline{-} \\
\text { III } \\
\text { Felt } \\
\text { Felt }\end{array}$ & $\begin{array}{l}- \\
\overline{33} \\
33 \\
34\end{array}$ & $\begin{array}{l}- \\
- \\
-\end{array}$ \\
\hline $\begin{array}{l}1961 \\
1961 \\
1961 \\
1961 \\
1961\end{array}$ & $\begin{array}{l}01 \\
01 \\
01 \\
01 \\
01\end{array}$ & $\begin{array}{l}11 \\
12 \\
14 \\
20 \\
29\end{array}$ & $\begin{array}{lll}11 & 59 & 52.0 \\
14 & 13 & 30.9 \\
16 & 38 & 54.8 \\
17 & 09 & 12.3 \\
13 & 23 & 54.7\end{array}$ & $\begin{array}{ll}51.98 & \mathrm{~N} \\
57.81 & \mathrm{~N} \\
53.9 & \mathrm{~N} \\
56.52 & \mathrm{~N} \\
52.0 & \mathrm{~N}\end{array}$ & $\begin{array}{l}170.87 \\
155.47 \\
163.4 \\
152.42 \\
175.9\end{array}$ & $\begin{array}{l}\mathbf{w} \\
\mathbf{W} \\
\mathbf{W} \\
\mathbf{W} \\
\mathbf{W}\end{array}$ & $\begin{array}{l}000 \\
075 \\
038 \\
000 \\
041\end{array}$ & $\begin{array}{l}265 \\
447 \\
266 \\
447 \\
266\end{array}$ & $\overline{-}$ & $\begin{array}{l}z \\
z\end{array}$ & $\begin{array}{l}6.20 \mathrm{M}_{\mathrm{R}} \text { ROT } \\
\text { 5.75Ukn PAL } \\
\text { 6.38Ukn PAS } \\
-\end{array}$ & $\begin{array}{l}z \\
z\end{array}$ & $\begin{array}{l}z \\
z\end{array}$ & $\begin{array}{l}- \\
z \\
-\end{array}$ & $\begin{array}{l}- \\
z \\
-\end{array}$ \\
\hline $\begin{array}{l}1961 \\
1961 \\
1961 \\
1961 \\
1961\end{array}$ & $\begin{array}{l}01 \\
01 \\
02 \\
03 \\
04\end{array}$ & $\begin{array}{l}30 \\
31 \\
27 \\
28 \\
21\end{array}$ & $\begin{array}{lll}12 & 12 & 36.3 \\
00 & 48 & 35.5 \\
13 & 06 & 35.8 \\
12 & 29 & 15.0 \\
21 & 26 & 42.1\end{array}$ & $\begin{array}{ll}65.23 & \mathrm{~N} \\
56.17 & \mathrm{~N} \\
52.7 & \mathrm{~N} \\
51.90 & \mathrm{~N} \\
51.9 & \mathrm{~N}\end{array}$ & $\begin{array}{l}150.25 \\
153.77 \\
168.8 \\
176.15 \\
173.9\end{array}$ & $\begin{array}{l}\mathbf{W} \\
\mathbf{W} \\
\mathbf{W} \\
\mathbf{W} \\
\mathbf{W}\end{array}$ & $\begin{array}{l}000 \\
000 \\
056 \\
062 \\
036\end{array}$ & $\begin{array}{l}447 \\
447 \\
266 \\
265 \\
266\end{array}$ & $\bar{z}$ & $\begin{array}{l}z \\
z\end{array}$ & $\begin{array}{l}\text { 5.50Ukn PAL } \\
\text { 6.25Ukn PAS } \\
\text { 6.25Ukn PAS } \\
\text { 5.63Ukn PAL }\end{array}$ & $\bar{z}$ & $\frac{\mathrm{V}}{\overline{\text { Felt }}}$ & $\frac{34}{34}$ & $\begin{array}{l}z \\
z \\
-\end{array}$ \\
\hline $\begin{array}{l}1961 \\
1961 \\
1961 \\
1961 \\
1961\end{array}$ & $\begin{array}{l}05 \\
06 \\
06 \\
08 \\
08\end{array}$ & $\begin{array}{l}17 \\
26 \\
29 \\
08 \\
25\end{array}$ & $\begin{array}{l}192919.3 \\
144721.0 \\
140242.5 \\
121819.0 \\
065927.6\end{array}$ & $\begin{array}{ll}52.2 & \mathrm{~N} \\
52.08 & \mathrm{~N} \\
52.4 & \mathrm{~N} \\
51.15 & \mathrm{~N} \\
53.52 & \mathrm{~N}\end{array}$ & $\begin{array}{l}173.9 \\
174.62 \\
173.4 \\
170.63 \\
161.26\end{array}$ & $\begin{array}{l}\mathbf{E} \\
\mathbf{E} \\
\mathbf{W} \\
\mathbf{W} \\
\mathbf{W}\end{array}$ & $\begin{array}{l}021 \\
000 \\
076 \\
000 \\
000\end{array}$ & $\begin{array}{l}266 \\
265 \\
266 \\
265 \\
447\end{array}$ & $\begin{array}{l}- \\
- \\
-\end{array}$ & $\begin{array}{l}- \\
z \\
-\end{array}$ & $\begin{array}{c}\text { 6.00Ukn PAS } \\
6.20 \mathrm{M}_{\mathrm{R}} \text { ROT } \\
6.13 \overline{\mathrm{Ukn}}_{\mathrm{KAS}} \\
--\end{array}$ & $\begin{array}{l}- \\
z \\
-\end{array}$ & $\begin{array}{l}\text { Felt } \\
= \\
=\end{array}$ & $\frac{34}{-}$ & $\bar{z}$ \\
\hline $\begin{array}{l}1961 \\
1961 \\
1961 \\
1961 \\
1961\end{array}$ & $\begin{array}{l}09 \\
09 \\
09 \\
09 \\
09\end{array}$ & $\begin{array}{l}02 \\
04 \\
05 \\
11 \\
25\end{array}$ & $\begin{array}{l}002603.0 \\
094915.0 \\
113431.4 \\
024650.3 \\
022713.5\end{array}$ & $\begin{array}{ll}52.18 & \mathrm{~N} \\
51.58 & \mathrm{~N} \\
59.77 & \mathrm{~N} \\
51.4 & \mathrm{~N} \\
60.36 & \mathrm{~N}\end{array}$ & $\begin{array}{l}171.05 \\
178.25 \\
150.80 \\
180.0 \\
152.88\end{array}$ & $\begin{array}{l}\mathbf{W} \\
\mathbf{W} \\
\mathbf{W} \\
\mathbf{E} \\
\mathbf{W}\end{array}$ & $\begin{array}{l}000 \\
052 \\
000 \\
060 \\
117\end{array}$ & $\begin{array}{l}265 \\
265 \\
447 \\
266 \\
447\end{array}$ & $\begin{array}{l}- \\
- \\
-\end{array}$ & $\begin{array}{l}z \\
z\end{array}$ & $\begin{array}{l}\text { 5.50Ukn MAT } \\
\text { 6.25Ukn BRK } \\
6.13 \mathrm{Ukn} \text { PAS } \\
5.90 \mathrm{M}_{\mathrm{R}} \text { ROT } \\
5.88 \mathrm{kn} \text { PAS }\end{array}$ & $\begin{array}{l}z \\
z\end{array}$ & $\frac{\bar{Z}}{\overline{\text { VI }}}$ & $\frac{\bar{z}}{34}$ & $\begin{array}{l}- \\
z \\
-\end{array}$ \\
\hline $\begin{array}{l}1961 \\
1961 \\
1961 \\
1961 \\
1961\end{array}$ & $\begin{array}{l}09 \\
09 \\
09 \\
10 \\
12\end{array}$ & $\begin{array}{l}27 \\
27 \\
27 \\
31 \\
09\end{array}$ & $\begin{array}{l}112045.0 \\
192044.0 \\
192700.0 \\
014354.0 \\
021520.8\end{array}$ & $\begin{array}{ll}52.40 & \mathrm{~N} \\
52.46 & \mathrm{~N} \\
52.35 & \mathrm{~N} \\
51.83 & \mathrm{~N} \\
56.35 & \mathrm{~N}\end{array}$ & $\begin{array}{l}168.68 \\
168.79 \\
168.75 \\
175.82 \\
153.49\end{array}$ & $\begin{array}{l}\mathbf{W} \\
\mathbf{W} \\
\mathbf{W} \\
\mathbf{E} \\
\mathbf{W}\end{array}$ & $\begin{array}{l}000 \\
000 \\
000 \\
022 \\
000\end{array}$ & $\begin{array}{l}265 \\
265 \\
265 \\
265 \\
447\end{array}$ & $\begin{array}{l}- \\
- \\
-\end{array}$ & $\begin{array}{l}- \\
- \\
-\end{array}$ & 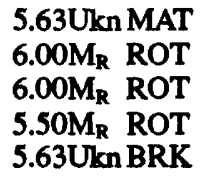 & $\begin{array}{l}- \\
- \\
-\end{array}$ & $\begin{array}{l}- \\
- \\
-\end{array}$ & $\begin{array}{l}- \\
- \\
-\end{array}$ & $\begin{array}{l}- \\
- \\
-\end{array}$ \\
\hline $\begin{array}{l}1961 \\
1962 \\
1962 \\
1962 \\
1962\end{array}$ & $\begin{array}{l}12 \\
01 \\
01 \\
01 \\
03\end{array}$ & $\begin{array}{l}30 \\
01 \\
03 \\
23 \\
11\end{array}$ & 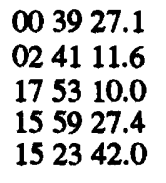 & $\begin{array}{ll}52.3 & \mathrm{~N} \\
52.2 & \mathrm{~N} \\
52.31 & \mathrm{~N} \\
52.8 & \mathrm{~N} \\
52.29 & \mathrm{~N}\end{array}$ & $\begin{array}{l}177.6 \\
177.7 \\
177.48 \\
169.0 \\
178.46\end{array}$ & $\begin{array}{l}\mathbf{E} \\
\mathbf{E} \\
\mathbf{E} \\
\mathbf{W} \\
\mathbf{E}\end{array}$ & $\begin{array}{l}056 \\
048 \\
077 \\
065 \\
110\end{array}$ & $\begin{array}{l}266 \\
266 \\
265 \\
266 \\
265\end{array}$ & $\begin{array}{l}- \\
-\end{array}$ & $\begin{array}{l}- \\
z \\
-\end{array}$ & $\begin{array}{c}\text { 6.75Ukn PAS } \\
\overline{-} \\
\text { 6.10Ukn UPP }\end{array}$ & $\begin{array}{l}- \\
z \\
-\end{array}$ & $\begin{array}{l}- \\
z \\
-\end{array}$ & $\begin{array}{l}- \\
z \\
z\end{array}$ & $\begin{array}{l}- \\
- \\
-\end{array}$ \\
\hline $\begin{array}{l}1962 \\
1962 \\
1962 \\
1962 \\
1962\end{array}$ & $\begin{array}{l}04 \\
04 \\
05 \\
05 \\
06\end{array}$ & $\begin{array}{l}01 \\
05 \\
10 \\
10 \\
29\end{array}$ & $\begin{array}{lll}12 & 1159.5 \\
03 & 4008.9 \\
00 & 03 & 39.9 \\
05 & 12 & 12.0 \\
16 & 28 & 03.8\end{array}$ & $\begin{array}{ll}63.4 & \mathrm{~N} \\
53.7 & \mathrm{~N} \\
61.96 \mathrm{~N} \\
52.39 \mathrm{~N} \\
62.40 \mathrm{~N}\end{array}$ & $\begin{array}{l}150.7 \\
163.6 \\
150.11 \\
171.03 \\
152.17\end{array}$ & $\begin{array}{l}\mathbf{W} \\
\mathbf{W} \\
\mathbf{W} \\
\mathbf{W} \\
\mathbf{W}\end{array}$ & $\begin{array}{l}108 \\
065 \\
082 \\
000 \\
023\end{array}$ & $\begin{array}{l}449 \\
266 \\
447 \\
265 \\
447\end{array}$ & $\begin{array}{l}- \\
- \\
-\end{array}$ & $\begin{array}{l}\overline{-} \\
\overline{-}\end{array}$ & $\begin{array}{l}5.50 \mathrm{M}_{\mathrm{R}} \text { ROT } \\
5.50 \mathrm{Ukn} \text { MOS } \\
6.00 \mathrm{Un} \text { BRK } \\
6.00 \mathrm{Un} \text { BRK } \\
6.00 \mathrm{M}_{\mathrm{R}} \text { ROT }\end{array}$ & $\begin{array}{l}z \\
z\end{array}$ & $\frac{\text { Felt }}{\bar{V}}$ & $\frac{449}{35}$ & $\bar{z}$ \\
\hline $\begin{array}{l}1962 \\
1962 \\
1962\end{array}$ & $\begin{array}{l}07 \\
07 \\
08\end{array}$ & $\begin{array}{l}07 \\
16 \\
18\end{array}$ & $\begin{array}{l}061248.9 \\
125443.1 \\
164355.9\end{array}$ & $\begin{array}{l}51.43 \mathrm{~N} \\
62.27 \mathrm{~N} \\
62.26 \mathrm{~N}\end{array}$ & $\begin{array}{l}178.76 \\
152.58 \\
152.54\end{array}$ & $\begin{array}{l}\mathbf{E} \\
\mathbf{W} \\
\mathbf{W}\end{array}$ & $\begin{array}{l}060 \\
050 \\
046\end{array}$ & $\begin{array}{l}266 \\
447 \\
447\end{array}$ & E & E & $\begin{array}{l}\text { 5.88Ukn PAS } \\
\text { 6.00Ukn PAL } \\
\text { 6.13Ukn PAS }\end{array}$ & $\overline{-}$ & $\begin{array}{l}\bar{v} \\
v\end{array}$ & $\begin{array}{l}\overline{35} \\
35\end{array}$ & $=$ \\
\hline
\end{tabular}


ALASKA-Continued

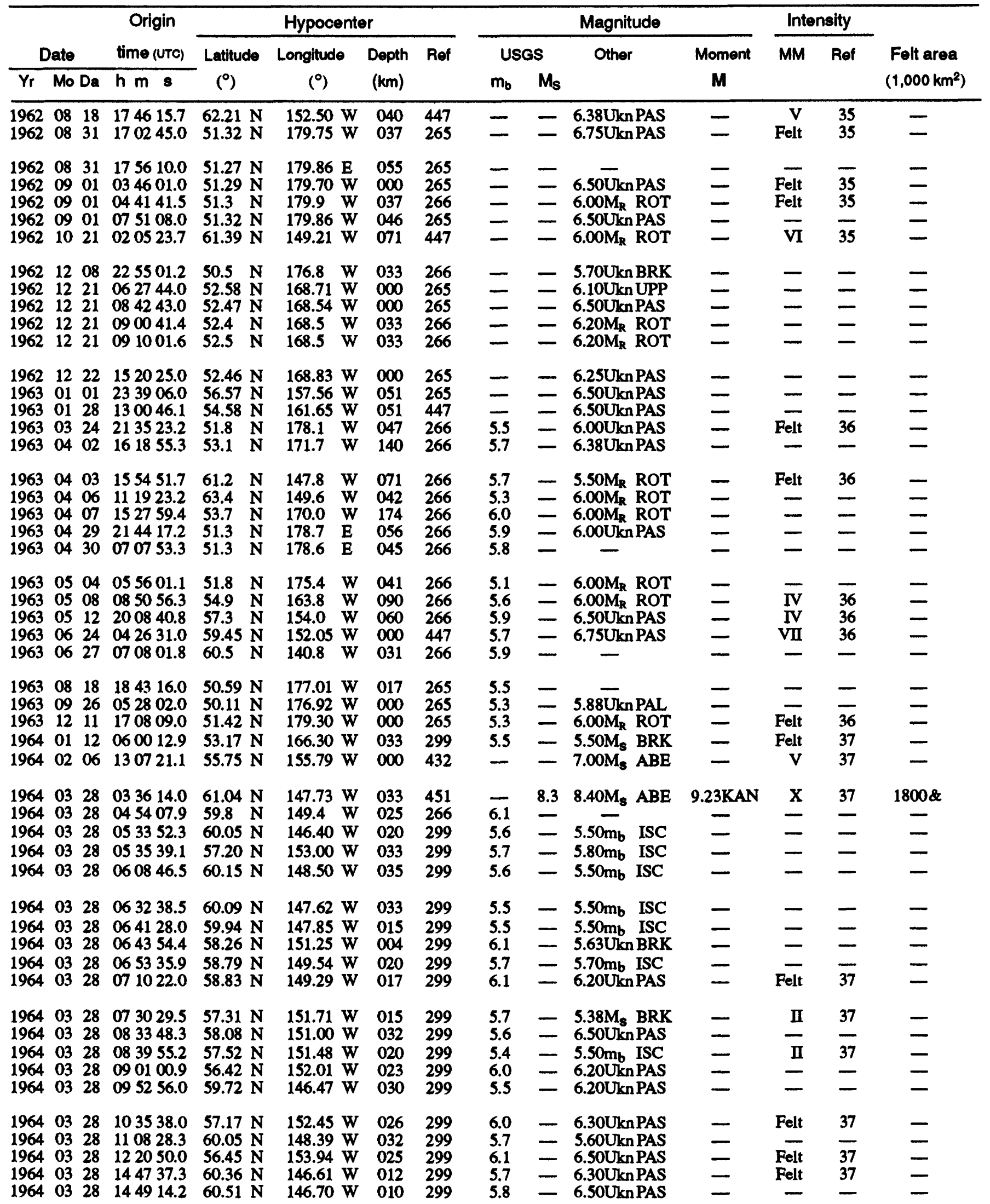




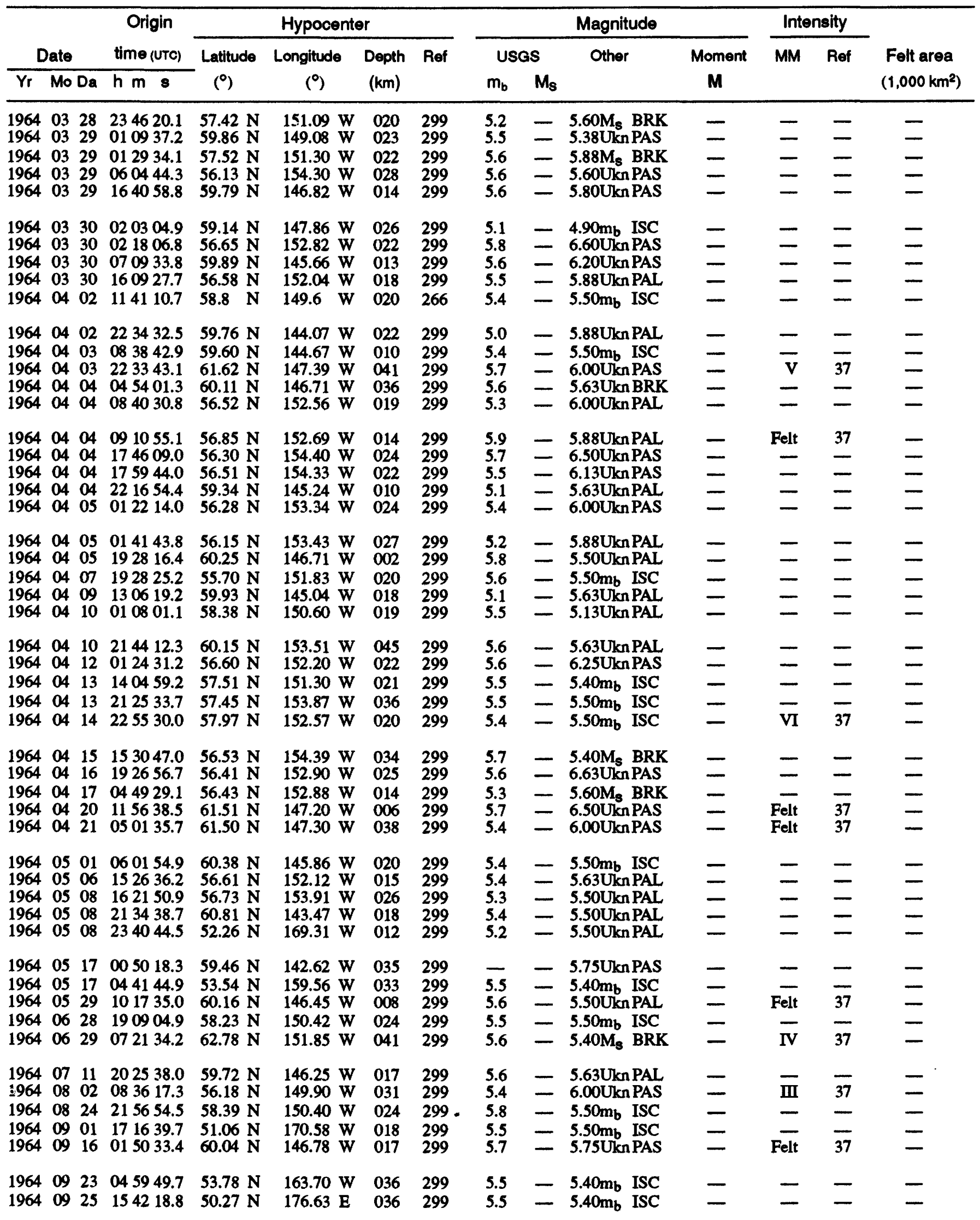


ALASKA-Continued

[See table 1 for hypocenter and intensity references and table 2 for definitions of magnitude source codes. \&, land area only. Leader (--) indicates information is not available]

\begin{tabular}{|c|c|c|c|c|c|c|c|c|c|c|c|c|c|c|}
\hline \multirow{2}{*}{\multicolumn{3}{|c|}{ Date }} & \multirow{3}{*}{$\begin{array}{c}\text { Origin } \\
\text { time (UTC) } \\
h \mathrm{~m} \mathrm{~s}\end{array}$} & \multicolumn{4}{|c|}{ Hypocenter } & \multicolumn{4}{|c|}{ Magnitude } & \multicolumn{2}{|c|}{ Intensity } & \multirow{3}{*}{$\begin{array}{l}\text { Felt area } \\
\left(1,000 \mathrm{~km}^{2}\right)\end{array}$} \\
\hline & & & & \multirow{2}{*}{$\begin{array}{l}\text { Latitude } \\
\text { ( })\end{array}$} & \multirow{2}{*}{$\begin{array}{l}\text { Longitude } \\
\left(^{\circ}\right)\end{array}$} & \multirow{2}{*}{$\begin{array}{c}\text { Depth } \\
(\mathrm{km})\end{array}$} & \multirow[t]{2}{*}{ Ref } & \multicolumn{2}{|c|}{ USGS } & \multirow[t]{2}{*}{ Other } & \multirow{2}{*}{$\begin{array}{c}\text { Moment } \\
\mathbf{M}\end{array}$} & \multirow[t]{2}{*}{ MM } & \multirow[t]{2}{*}{ Rof } & \\
\hline $\mathrm{Yr}$ & Mo L & $\mathrm{Da}$ & & & & & & $m_{b}$ & $\mathbf{M}_{\mathbf{s}}$ & & & & & \\
\hline $\begin{array}{l}1964 \\
1964 \\
1964\end{array}$ & $\begin{array}{l}10 \\
12 \\
12\end{array}$ & $\begin{array}{l}10 \\
13 \\
17\end{array}$ & $\begin{array}{l}200634.7 \\
003326.9 \\
234445.4\end{array}$ & $\begin{array}{l}60.38 \mathrm{~N} \\
64.878 \mathrm{~N} \\
51.211 \mathrm{~N}\end{array}$ & $\begin{array}{l}145.97 \mathrm{~W} \\
165.570 W \\
177.813 W\end{array}$ & $\begin{array}{l}001 \\
028 \\
030\end{array}$ & $\begin{array}{l}299 \\
299 \\
479\end{array}$ & $\begin{array}{l}5.4 \\
5.4 \\
5.5\end{array}$ & $\overline{-}$ & $\begin{array}{l}5.60 \mathrm{M}_{\mathrm{S}} \text { BRK } \\
6.00 \mathrm{Un}^{\mathrm{PAL}} \\
5.50 \mathrm{~m}_{\mathrm{b}} \text { ISC }\end{array}$ & - & $\begin{array}{r}\overline{\text { VI }} \\
\text { Felt }\end{array}$ & $\begin{array}{l}\overline{37} \\
37\end{array}$ & - \\
\hline $\begin{array}{l}1965 \\
1965 \\
1965 \\
1965 \\
1965\end{array}$ & $\begin{array}{l}01 \\
02 \\
02 \\
02 \\
02\end{array}$ & $\begin{array}{l}30 \\
04 \\
04 \\
04 \\
04\end{array}$ & $\begin{array}{l}043721.4 \\
045357.2 \\
050121.6 \\
051918.4 \\
054850.5\end{array}$ & $\begin{array}{ll}51.68 & \mathrm{~N} \\
51.18 & \mathrm{~N} \\
51.29 & \mathrm{~N} \\
50.4 & \mathrm{~N} \\
51.40 & \mathrm{~N}\end{array}$ & $\begin{array}{ll}179.67 & W \\
178.49 & E \\
178.55 & E \\
173.2 & E \\
174.84 & E\end{array}$ & $\begin{array}{l}088 \\
030 \\
036 \\
035 \\
033\end{array}$ & $\begin{array}{l}299 \\
299 \\
299 \\
299 \\
299\end{array}$ & $\begin{array}{l}5.6 \\
5.8 \\
6.0 \\
5.7 \\
-\end{array}$ & $\begin{array}{l}- \\
- \\
-\end{array}$ & $\begin{array}{ll}5.10 \mathrm{~m}_{\mathrm{b}} & \text { ISC } \\
5.30 \mathrm{~m}_{\mathrm{b}} & \text { ISC } \\
8.20 \mathrm{M}_{\mathrm{S}} & \text { ABE } \\
5.50 \mathrm{~m}_{\mathrm{b}} & \text { ISC } \\
5.75 \mathrm{M}_{\mathrm{s}} & \text { BRK }\end{array}$ & $\begin{array}{c}- \\
\overline{8.68 \mathrm{KAN}} \\
- \\
-\end{array}$ & $\begin{array}{l}- \\
\bar{v} \\
-\end{array}$ & $\begin{array}{l}\overline{7} \\
\overline{-}\end{array}$ & $\begin{array}{l}- \\
- \\
-\end{array}$ \\
\hline $\begin{array}{l}1965 \\
1965 \\
1965 \\
1965 \\
1965\end{array}$ & $\begin{array}{l}02 \\
02 \\
02 \\
02 \\
02\end{array}$ & $\begin{array}{l}04 \\
04 \\
04 \\
04 \\
04\end{array}$ & $\begin{array}{l}060458.2 \\
063705.5 \\
063930.1 \\
065252.4 \\
071121.4\end{array}$ & $\begin{array}{l}51.74 \mathrm{~N} \\
52.57 \mathrm{~N} \\
51.64 \mathrm{~N} \\
52.20 \mathrm{~N} \\
51.03 \mathrm{~N}\end{array}$ & $\begin{array}{ll}175.02 & \mathrm{E} \\
172.05 & \mathrm{E} \\
175.83 & \mathrm{E} \\
173.17 & \mathrm{E} \\
177.86 & \mathrm{E}\end{array}$ & $\begin{array}{l}037 \\
035 \\
030 \\
033 \\
024\end{array}$ & $\begin{array}{l}299 \\
299 \\
299 \\
299 \\
299\end{array}$ & $\begin{array}{l}6.1 \\
5.9 \\
5.5 \\
5.9\end{array}$ & $\begin{array}{l}- \\
- \\
-\end{array}$ & $\begin{array}{l}\text { 6.13Ukn BRK } \\
5.50 \mathrm{~m}_{\mathrm{b}} \text { ISC } \\
5.88 \mathrm{Ukn} \text { BRK } \\
5.40 \mathrm{~m}_{\mathrm{b}} \text { ISC } \\
5.50 \mathrm{~m}_{\mathrm{b}} \text { ISC }\end{array}$ & $\begin{array}{l}- \\
- \\
-\end{array}$ & $\overline{-}$ & $\frac{-}{-}$ & $\begin{array}{l}- \\
- \\
-\end{array}$ \\
\hline $\begin{array}{l}1965 \\
1965 \\
1965 \\
1965 \\
1965\end{array}$ & $\begin{array}{l}02 \\
02 \\
02 \\
02 \\
02\end{array}$ & $\begin{array}{l}04 \\
04 \\
04 \\
04 \\
04\end{array}$ & $\begin{array}{lll}07 & 1458.8 \\
07 & 23 & 12.9 \\
07 & 43 & 41.0 \\
08 & 04 & 10.3 \\
08 & 06 & 17.2\end{array}$ & $\begin{array}{ll}51.88 & \mathrm{~N} \\
51.8 & \mathrm{~N} \\
52.64 & \mathrm{~N} \\
52.18 & \mathrm{~N} \\
51.92 & \mathrm{~N}\end{array}$ & $\begin{array}{ll}173.94 & \mathrm{E} \\
173.4 & \mathrm{E} \\
172.79 & \mathrm{E} \\
172.87 & \mathrm{E} \\
174.30 & \mathrm{E}\end{array}$ & $\begin{array}{l}025 \\
021 \\
015 \\
030 \\
044\end{array}$ & $\begin{array}{l}299 \\
299 \\
299 \\
299 \\
299\end{array}$ & $\begin{array}{l}5.8 \\
5.5 \\
5.5 \\
5.9 \\
5.6\end{array}$ & $\begin{array}{l}- \\
- \\
-\end{array}$ & $\begin{array}{ll}5.70 \mathrm{~m}_{\mathrm{b}} & \text { ISC } \\
5.60 \mathrm{~m}_{\mathrm{b}} & \text { ISC } \\
5.40 \mathrm{mb}_{\mathrm{b}} & \text { ISC } \\
5.30 \mathrm{mb}_{\mathrm{b}} & \text { ISC } \\
5.50 \mathrm{mb}_{\mathrm{b}} & \text { ISC }\end{array}$ & $\begin{array}{l}- \\
- \\
-\end{array}$ & $\begin{array}{l}- \\
- \\
-\end{array}$ & $\begin{array}{l}- \\
- \\
-\end{array}$ & $\frac{-}{-}$ \\
\hline $\begin{array}{l}1965 \\
1965 \\
1965 \\
1965 \\
1965\end{array}$ & $\begin{array}{l}02 \\
02 \\
02 \\
02 \\
02\end{array}$ & $\begin{array}{l}04 \\
04 \\
04 \\
04 \\
04\end{array}$ & $\begin{array}{l}080629.0 \\
083341.3 \\
084042.1 \\
085920.0 \\
095159.2\end{array}$ & $\begin{array}{l}51.67 \mathrm{~N} \\
51.93 \mathrm{~N} \\
51.39 \mathrm{~N} \\
52.55 \mathrm{~N} \\
51.56 \mathrm{~N}\end{array}$ & $\begin{array}{ll}174.80 & \mathrm{E} \\
174.03 & \mathrm{E} \\
179.59 & \mathrm{E} \\
173.71 & \mathrm{E} \\
175.72 & \mathrm{E}\end{array}$ & $\begin{array}{l}040 \\
031 \\
040 \\
034 \\
004\end{array}$ & $\begin{array}{l}299 \\
299 \\
299 \\
299 \\
299\end{array}$ & $\begin{array}{l}-5.7 \\
6.4 \\
5.5 \\
5.6\end{array}$ & $\begin{array}{l}- \\
- \\
-\end{array}$ & $\begin{array}{ll}5.50 \mathrm{~m}_{\mathrm{b}} & \text { ISC } \\
5.60 \mathrm{~m}_{\mathrm{b}} & \text { ISC } \\
7.00 \mathrm{M}_{\mathrm{s}} & \text { ABE } \\
5.40 \mathrm{~m}_{\mathrm{b}} & \text { ISC } \\
5.30 \mathrm{~m}_{\mathrm{b}} & \text { ISC }\end{array}$ & $\begin{array}{l}- \\
- \\
-\end{array}$ & $\frac{\overline{-}}{\bar{F}}$ & $\bar{z}$ & $\begin{array}{l}- \\
- \\
-\end{array}$ \\
\hline $\begin{array}{l}1965 \\
1965 \\
1965 \\
1965 \\
1965\end{array}$ & $\begin{array}{l}02 \\
02 \\
02 \\
02 \\
02\end{array}$ & $\begin{array}{l}04 \\
04 \\
04 \\
04 \\
05\end{array}$ & $\begin{array}{lll}12 & 06 & 05.7 \\
14 & 18 & 26.5 \\
15 & 51 & 25.8 \\
22 & 30 & 05.6 \\
06 & 25 & 23.1\end{array}$ & $\begin{array}{ll}52.74 & \mathrm{~N} \\
53.03 & \mathrm{~N} \\
53.05 & \mathrm{~N} \\
51.82 & \mathrm{~N} \\
51.8 & \mathrm{~N}\end{array}$ & $\begin{array}{ll}172.05 & E \\
171.08 & E \\
170.83 & E \\
174.32 & E \\
177.0 & E\end{array}$ & $\begin{array}{l}030 \\
016 \\
040 \\
031 \\
040\end{array}$ & $\begin{array}{l}299 \\
299 \\
299 \\
299 \\
299\end{array}$ & $\begin{array}{l}5.8 \\
5.7 \\
5.7 \\
5.4 \\
5.5\end{array}$ & $\begin{array}{l}- \\
- \\
-\end{array}$ & $\begin{array}{l}\text { 6.50Ukn PAS } \\
6.25 \mathrm{Unn} \text { PAS } \\
6.25 \mathrm{Ukn} \text { PAS } \\
5.50 \mathrm{mb}_{\mathrm{b}} \text { ISC } \\
5.10 \mathrm{mb}_{\mathrm{b}} \text { ISC }\end{array}$ & $\begin{array}{l}- \\
- \\
-\end{array}$ & $\begin{array}{l}\text { Felt } \\
\text { Felt } \\
\text { Felt } \\
- \\
-\end{array}$ & $\begin{array}{l}75 \\
75 \\
75 \\
- \\
-\end{array}$ & $\begin{array}{l}- \\
-\end{array}$ \\
\hline $\begin{array}{l}1965 \\
1965 \\
1965 \\
1965 \\
1965\end{array}$ & $\begin{array}{l}02 \\
02 \\
02 \\
02 \\
02\end{array}$ & $\begin{array}{l}05 \\
05 \\
05 \\
05 \\
05\end{array}$ & $\begin{array}{l}063949.2 \\
093206.3 \\
133846.9 \\
140823.2 \\
190041.9\end{array}$ & $\begin{array}{ll}51.77 & \mathrm{~N} \\
52.37 & \mathrm{~N} \\
51.99 & \mathrm{~N} \\
51.74 & \mathrm{~N} \\
52.0 & \mathrm{~N}\end{array}$ & $\begin{array}{ll}174.84 & \mathrm{E} \\
174.33 & \mathrm{E} \\
173.96 & \mathrm{E} \\
174.38 & \mathrm{E} \\
173.2 & \mathrm{E}\end{array}$ & $\begin{array}{l}025 \\
016 \\
037 \\
035 \\
027\end{array}$ & $\begin{array}{l}299 \\
299 \\
299 \\
299 \\
299\end{array}$ & $\begin{array}{l}5.7 \\
5.9 \\
5.8 \\
5.5\end{array}$ & $\begin{array}{l}- \\
- \\
-\end{array}$ & $\begin{array}{ll}6.38 \mathrm{Ukn}^{2} & \text { PAS } \\
6.50 \mathrm{Un}^{\mathrm{N}} \text { PAS } \\
5.60 \mathrm{~m}_{\mathrm{b}} & \text { ISC } \\
5.40 \mathrm{~m}_{\mathrm{b}} & \text { ISC } \\
5.40 \mathrm{~m}_{\mathrm{b}} & \text { ISC }\end{array}$ & $\begin{array}{l}- \\
- \\
-\end{array}$ & $\begin{array}{l}- \\
- \\
-\end{array}$ & $\begin{array}{l}- \\
-\end{array}$ & $\begin{array}{l}- \\
- \\
-\end{array}$ \\
\hline $\begin{array}{l}1965 \\
1965 \\
1965 \\
1965 \\
1965\end{array}$ & $\begin{array}{l}02 \\
02 \\
02 \\
02 \\
02\end{array}$ & $\begin{array}{l}05 \\
05 \\
06 \\
06 \\
06\end{array}$ & $\begin{array}{l}204712.4 \\
22 \quad 1601.2 \\
014034.6 \\
040252.2 \\
165028.9\end{array}$ & $\begin{array}{l}51.83 \mathrm{~N} \\
51.53 \mathrm{~N} \\
53.14 \mathrm{~N} \\
52.05 \mathrm{~N} \\
53.26 \mathrm{~N}\end{array}$ & $\begin{array}{l}174.41 \\
176.64 \\
\mathrm{E} \\
161.85 \mathrm{~W} \\
175.60 \mathrm{E} \\
161.74 \mathrm{~W}\end{array}$ & $\begin{array}{l}030 \\
036 \\
043 \\
032 \\
033\end{array}$ & $\begin{array}{l}299 \\
299 \\
299 \\
299 \\
299\end{array}$ & $\begin{array}{l}5.7 \\
5.6 \\
6.4 \\
5.9 \\
6.1\end{array}$ & $\frac{-}{-}$ & $\begin{array}{l}5.70 \mathrm{mb}_{\mathrm{b}} \text { ISC } \\
5.30 \mathrm{mb} \text { ISC } \\
6.63 \mathrm{Ukn} \text { PAS } \\
\text { 6.00Ukn PAL } \\
\text { 6.50Ukn PAS }\end{array}$ & $\begin{array}{l}- \\
z \\
-\end{array}$ & $\frac{\bar{I}}{\frac{\overline{I V}}{\mathrm{IV}}}$ & $\frac{-}{75}$ & $\begin{array}{l}- \\
- \\
-\end{array}$ \\
\hline $\begin{array}{l}1965 \\
1965 \\
1965 \\
1965 \\
1965\end{array}$ & $\begin{array}{l}02 \\
02 \\
02 \\
02 \\
02\end{array}$ & $\begin{array}{l}06 \\
07 \\
07 \\
07 \\
09\end{array}$ & $\begin{array}{lll}21 & 02 & 58.5 \\
02 & 17 & 10.1 \\
04 & 11 & 20.2 \\
09 & 25 & 52.1 \\
04 & 34 & 59.7\end{array}$ & $\begin{array}{l}52.81 \mathrm{~N} \\
51.34 \mathrm{~N} \\
52.03 \mathrm{~N} \\
51.37 \mathrm{~N} \\
52.27 \mathrm{~N}\end{array}$ & $\begin{array}{ll}171.98 & E \\
173.44 & E \\
175.48 & E \\
179.20 & E \\
179.64 & E\end{array}$ & $\begin{array}{l}010 \\
045 \\
025 \\
038 \\
040\end{array}$ & $\begin{array}{l}299 \\
299 \\
299 \\
299 \\
299\end{array}$ & $\begin{array}{l}5.6 \\
6.0 \\
5.5 \\
5.3 \\
5.5\end{array}$ & $\begin{array}{l}- \\
- \\
-\end{array}$ & $\begin{array}{ll}5.00 \mathrm{mb}_{\mathrm{b}} & \text { ISC } \\
5.80 \mathrm{~m}_{\mathrm{b}} & \text { ISC } \\
5.40 \mathrm{mb}_{\mathrm{b}} & \text { ISC } \\
6.25 \mathrm{U} n & \text { PAS } \\
5.00 \mathrm{mb}_{\mathrm{b}} & \text { ISC }\end{array}$ & $\frac{-}{-}$ & $\frac{-}{-}$ & $\bar{z}$ & - \\
\hline $\begin{array}{l}1965 \\
1965 \\
1965 \\
1965 \\
1965\end{array}$ & $\begin{array}{l}02 \\
02 \\
02 \\
02 \\
02\end{array}$ & $\begin{array}{l}09 \\
12 \\
12 \\
15 \\
17\end{array}$ & $\begin{array}{l}173714.4 \\
004317.8 \\
005509.5 \\
012508.0 \\
101850.0\end{array}$ & $\begin{array}{l}52.73 \mathrm{~N} \\
51.46 \mathrm{~N} \\
52.32 \mathrm{~N} \\
51.11 \mathrm{~N} \\
51.64 \mathrm{~N}\end{array}$ & $\begin{array}{ll}171.99 & \mathrm{E} \\
175.84 & \mathrm{E} \\
172.84 & \mathrm{E} \\
179.39 & \mathrm{E} \\
176.63 & \mathrm{E}\end{array}$ & $\begin{array}{l}028 \\
034 \\
039 \\
042 \\
034\end{array}$ & $\begin{array}{l}299 \\
299 \\
299 \\
299 \\
299\end{array}$ & $\begin{array}{l}5.7 \\
5.4 \\
5.5 \\
5.8 \\
5.6\end{array}$ & $\overline{-}$ & $\begin{array}{l}5.80 \mathrm{mb} \text { ISC } \\
5.75 \mathrm{Ukn} \text { PAS } \\
\text { 6.00Ukn PAS } \\
\text { 6.00Ukn PAS } \\
\text { 6.50Ukn PAS }\end{array}$ & $\begin{array}{l}- \\
z\end{array}$ & $\begin{array}{l}- \\
z \\
-\end{array}$ & $\begin{array}{l}\overline{-} \\
\bar{z}\end{array}$ & $\begin{array}{l}z \\
z \\
-\end{array}$ \\
\hline $\begin{array}{l}1965 \\
1965\end{array}$ & $\begin{array}{l}02 \\
02\end{array}$ & $\begin{array}{l}18 \\
19\end{array}$ & $\begin{array}{l}231339.5 \\
185239.4\end{array}$ & $\begin{array}{l}51.45 \mathrm{~N} \\
51.13 \mathrm{~N}\end{array}$ & $\begin{array}{l}179.28 \mathrm{E} \\
178.47 \mathrm{E}\end{array}$ & $\begin{array}{l}048 \\
012\end{array}$ & $\begin{array}{l}299 \\
299\end{array}$ & $\begin{array}{l}5.4 \\
5.6\end{array}$ & - & $\begin{array}{l}6.00 \mathrm{Ukn} \text { PAS } \\
5.50 \mathrm{~m}_{\mathrm{b}} \text { ISC }\end{array}$ & - & Felt & $\frac{75}{-}$ & - \\
\hline
\end{tabular}


ALASKA-Continued

[See table 1 for hypocenter and intensity references and table 2 for definitions of magnitude source codes. \&, land area only. Leader (--) indicates information is not available]

\begin{tabular}{|c|c|c|c|c|c|c|c|c|c|c|c|c|c|c|}
\hline \multirow{2}{*}{\multicolumn{3}{|c|}{ Date }} & \multirow{2}{*}{$\begin{array}{c}\text { Origin } \\
\text { time (UTC) }\end{array}$} & \multicolumn{4}{|c|}{ Hypocenter } & \multicolumn{4}{|c|}{ Magnitude } & \multicolumn{2}{|c|}{ Intensity } & \multirow{3}{*}{$\begin{array}{l}\text { Felt area } \\
\left(1,000 \mathrm{~km}^{2}\right)\end{array}$} \\
\hline & & & & \multirow{2}{*}{$\begin{array}{l}\text { Latitude } \\
\left(^{\circ}\right)\end{array}$} & \multirow{2}{*}{$\begin{array}{c}\text { Longitude } \\
\left(^{\circ}\right)\end{array}$} & \multirow{2}{*}{$\begin{array}{l}\text { Depth } \\
(\mathbf{k m})\end{array}$} & \multirow[t]{2}{*}{ Ref } & \multicolumn{2}{|c|}{ USGS } & \multirow[t]{2}{*}{ Other } & \multirow{2}{*}{$\begin{array}{c}\text { Moment } \\
\mathbf{M}\end{array}$} & \multirow[t]{2}{*}{ MM } & \multirow[t]{2}{*}{ Ref } & \\
\hline $\mathrm{Yr}_{\mathrm{r}}$ & Mo & $\mathrm{Da}$ & $\mathrm{h} \mathrm{m} s$ & & & & & $m_{b}$ & $M_{s}$ & & & & & \\
\hline $\begin{array}{l}1965 \\
1965 \\
1965\end{array}$ & $\begin{array}{l}02 \\
02 \\
03\end{array}$ & $\begin{array}{l}22 \\
25 \\
01\end{array}$ & $\begin{array}{l}091451.7 \\
052214.5 \\
192159.9\end{array}$ & $\begin{array}{l}51.91 \mathrm{~N} \\
52.11 \mathrm{~N} \\
52.17 \mathrm{~N}\end{array}$ & $\begin{array}{l}173.49 \mathrm{E} \\
173.16 \mathrm{E} \\
174.05 \mathrm{E}\end{array}$ & $\begin{array}{l}032 \\
033 \\
013\end{array}$ & $\begin{array}{l}299 \\
299 \\
299\end{array}$ & $\begin{array}{l}5.5 \\
5.6 \\
5.5\end{array}$ & - & $\begin{array}{l}5.38 \mathrm{Ukn} \text { PAL } \\
5.70 \mathrm{~m}_{\mathrm{b}} \text { ISC } \\
5.50 \mathrm{~m}_{\mathrm{b}} \text { ISC }\end{array}$ & $\frac{-}{-}$ & - & - & - \\
\hline $\begin{array}{l}1965 \\
1965 \\
1965 \\
1965 \\
1965\end{array}$ & $\begin{array}{l}03 \\
03 \\
03 \\
03 \\
03\end{array}$ & $\begin{array}{l}03 \\
04 \\
05 \\
05 \\
05\end{array}$ & $\begin{array}{l}164725.0 \\
063018.8 \\
061459.8 \\
134244.5 \\
175913.6\end{array}$ & $\begin{array}{l}53.02 \mathrm{~N} \\
52.04 \mathrm{~N} \\
51.25 \mathrm{~N} \\
52.22 \mathrm{~N} \\
52.29 \mathrm{~N}\end{array}$ & $\begin{array}{ll}171.35 & E \\
175.14 & E \\
179.58 & E \\
174.98 & E \\
174.33 & E\end{array}$ & $\begin{array}{l}014 \\
057 \\
005 \\
033 \\
031\end{array}$ & $\begin{array}{l}299 \\
299 \\
299 \\
299 \\
299\end{array}$ & $\begin{array}{l}5.6 \\
5.5 \\
5.6 \\
5.3 \\
5.7\end{array}$ & $\begin{array}{l}- \\
- \\
-\end{array}$ & $\begin{array}{ll}5.90 \mathrm{~m}_{\mathrm{b}} & \text { ISC } \\
5.30 \mathrm{~m}_{\mathrm{b}} & \text { ISC } \\
5.50 \mathrm{~m}_{\mathrm{b}} & \text { ISC } \\
5.50 \mathrm{~m}_{\mathrm{b}} & \text { ISC } \\
5.60 \mathrm{~m}_{\mathrm{b}} & \text { ISC }\end{array}$ & $\begin{array}{l}- \\
- \\
-\end{array}$ & $\begin{array}{l}- \\
- \\
-\end{array}$ & $\frac{-}{-}$ & $\begin{array}{l}- \\
- \\
-\end{array}$ \\
\hline $\begin{array}{l}1965 \\
1965 \\
1965 \\
1965 \\
1965\end{array}$ & $\begin{array}{l}03 \\
03 \\
03 \\
03 \\
04\end{array}$ & $\begin{array}{l}13 \\
17 \\
30 \\
31 \\
04\end{array}$ & $\begin{array}{l}073323.5 \\
142711.7 \\
022703.4 \\
104610.9 \\
133037.4\end{array}$ & $\begin{array}{l}53.17 \mathrm{~N} \\
52.80 \mathrm{~N} \\
50.32 \mathrm{~N} \\
50.29 \mathrm{~N} \\
51.83 \mathrm{~N}\end{array}$ & $\begin{array}{l}162.07 \mathrm{~W} \\
171.97 \mathrm{E} \\
177.93 \mathrm{E} \\
178.35 \mathrm{E} \\
175.38 \mathrm{E}\end{array}$ & $\begin{array}{l}036 \\
014 \\
020 \\
048 \\
032\end{array}$ & $\begin{array}{l}299 \\
299 \\
299 \\
299 \\
299\end{array}$ & $\begin{array}{l}5.5 \\
6.0 \\
5.6 \\
5.7\end{array}$ & $\begin{array}{l}- \\
- \\
-\end{array}$ & $\begin{array}{l}5.50 \mathrm{~m}_{\mathrm{b}} \text { ISC } \\
5.70 \mathrm{mb} \text { ISC } \\
7.40 \mathrm{M}_{\mathrm{s}} \text { ABE } \\
5.30 \mathrm{mb}_{\mathrm{b}} \text { ISC } \\
6.00 \mathrm{Ukn}^{2} \text { PAS }\end{array}$ & $\begin{array}{c}- \\
7.64 \mathrm{KA} \\
- \\
-\end{array}$ & $\begin{array}{l}- \\
\text { Felt } \\
\text { Felt } \\
- \\
-\end{array}$ & $\begin{array}{l}-75 \\
75 \\
- \\
-\end{array}$ & $\begin{array}{l}- \\
- \\
-\end{array}$ \\
\hline $\begin{array}{l}1965 \\
1965 \\
1965 \\
1965 \\
1965\end{array}$ & $\begin{array}{l}04 \\
04 \\
04 \\
04 \\
04\end{array}$ & $\begin{array}{l}08 \\
10 \\
16 \\
20 \\
22\end{array}$ & $\begin{array}{l}134352.6 \\
165456.1 \\
232218.6 \\
064306.6 \\
183559.0\end{array}$ & $\begin{array}{ll}52.22 & \mathrm{~N} \\
53.11 & \mathrm{~N} \\
64.69 & \mathrm{~N} \\
52.42 & \mathrm{~N} \\
51.85 & \mathrm{~N}\end{array}$ & $\begin{array}{l}173.45 \mathrm{E} \\
170.95 \mathrm{E} \\
160.23 \mathrm{~W} \\
172.04 \mathrm{E} \\
176.16 \mathrm{E}\end{array}$ & $\begin{array}{l}037 \\
008 \\
005 \\
016 \\
015\end{array}$ & $\begin{array}{l}299 \\
299 \\
299 \\
299 \\
299\end{array}$ & $\begin{array}{l}5.4 \\
5.8 \\
5.8 \\
5.5 \\
5.1\end{array}$ & $\begin{array}{l}- \\
- \\
-\end{array}$ & $\begin{array}{l}\text { 5.70Ukn PAS } \\
\text { 5.38Ukn PAL } \\
\text { 5.88Ukn PAS } \\
\text { 5.10Ukn PAL } \\
\text { 5.60Ukn PAL }\end{array}$ & $\frac{-}{-}$ & $\begin{array}{l}- \\
\text { VI } \\
\text { Felt }\end{array}$ & $\begin{array}{l}- \\
\overline{75} \\
75 \\
-\end{array}$ & $\begin{array}{l}- \\
- \\
-\end{array}$ \\
\hline $\begin{array}{l}1965 \\
1965 \\
1965 \\
1965 \\
1965\end{array}$ & $\begin{array}{l}04 \\
05 \\
05 \\
05 \\
05\end{array}$ & $\begin{array}{l}26 \\
05 \\
11 \\
23 \\
25\end{array}$ & $\begin{array}{lll}20 & 29 & 07.5 \\
23 & 02 & 02.1 \\
17 & 37 & 39.1 \\
23 & 46 & 14.3 \\
1307 & 49.3\end{array}$ & $\begin{array}{ll}54.25 & \mathrm{~N} \\
52.56 & \mathrm{~N} \\
61.33 & \mathrm{~N} \\
52.17 & \mathrm{~N} \\
51.23 & \mathrm{~N}\end{array}$ & $\begin{array}{l}162.51 \mathrm{~W} \\
173.65 \mathrm{E} \\
149.52 \mathrm{~W} \\
175.17 \mathrm{E} \\
178.76 \mathrm{E}\end{array}$ & $\begin{array}{l}051 \\
031 \\
061 \\
031 \\
035\end{array}$ & $\begin{array}{l}299 \\
299 \\
299 \\
299 \\
299\end{array}$ & $\begin{array}{l}5.9 \\
5.6 \\
5.5 \\
6.1 \\
5.5\end{array}$ & $\frac{-}{-}$ & $\begin{array}{l}\text { 5.13Ukn PAL } \\
5.30 \mathrm{mb}_{\mathrm{b}} \text { ISC } \\
5.75 \mathrm{kn} \text { PAS } \\
\text { 6.00Ukn PAS } \\
\text { 5.88Ukn PAL }\end{array}$ & $\begin{array}{l}- \\
- \\
-\end{array}$ & $\frac{\text { IV }}{\text { IV }}$ & $\frac{75}{75}$ & $\begin{array}{l}- \\
- \\
-\end{array}$ \\
\hline $\begin{array}{l}1965 \\
1965 \\
1965 \\
1965 \\
1965\end{array}$ & $\begin{array}{l}06 \\
06 \\
06 \\
06 \\
06\end{array}$ & $\begin{array}{l}03 \\
09 \\
11 \\
15 \\
19\end{array}$ & $\begin{array}{l}074338.1 \\
132651.6 \\
023735.0 \\
044613.8 \\
063811.8\end{array}$ & $\begin{array}{l}51.91 \mathrm{~N} \\
52.55 \mathrm{~N} \\
51.80 \mathrm{~N} \\
50.07 \mathrm{~N} \\
52.39 \mathrm{~N}\end{array}$ & 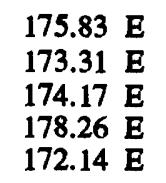 & $\begin{array}{l}046 \\
017 \\
032 \\
026 \\
038\end{array}$ & $\begin{array}{l}299 \\
299 \\
299 \\
299 \\
299\end{array}$ & $\begin{array}{l}5.5 \\
5.6 \\
5.5 \\
5.5 \\
5.5\end{array}$ & $\frac{-}{-}$ & $\begin{array}{l}5.30 \mathrm{mb} \text { ISC } \\
5.10 \mathrm{mb}^{\mathrm{b}} \text { ISC } \\
5.60 \mathrm{mb}_{\mathrm{b}} \text { ISC } \\
5.13 \mathrm{Ukn} \text { PAL } \\
5.38 \mathrm{Ukn} \text { PAL }\end{array}$ & $\begin{array}{l}- \\
- \\
-\end{array}$ & $\begin{array}{l}- \\
- \\
-\end{array}$ & $\frac{-}{-}$ & $\frac{-}{-}$ \\
\hline $\begin{array}{l}1965 \\
1965 \\
1965 \\
1965 \\
1965\end{array}$ & $\begin{array}{l}06 \\
06 \\
07 \\
07 \\
07\end{array}$ & $\begin{array}{l}23 \\
30 \\
02 \\
21 \\
22\end{array}$ & $\begin{array}{lll}11 & 09 & 16.5 \\
08 & 33 & 27.5 \\
20 & 58 & 38.1 \\
17 & 52 & 27.0 \\
01 & 18 & 52.2\end{array}$ & $\begin{array}{ll}56.61 & \mathrm{~N} \\
51.81 & \mathrm{~N} \\
53.03 & \mathrm{~N} \\
53.31 & \mathrm{~N} \\
50.96 & \mathrm{~N}\end{array}$ & $\begin{array}{l}152.68 \mathrm{~W} \\
176.62 \mathrm{E} \\
167.55 \mathrm{~W} \\
170.38 \mathrm{E} \\
175.95 \mathrm{E}\end{array}$ & $\begin{array}{l}031 \\
015 \\
040 \\
011 \\
044\end{array}$ & $\begin{array}{l}299 \\
299 \\
299 \\
299 \\
299\end{array}$ & $\begin{array}{l}5.7 \\
5.7 \\
6.7 \\
5.7 \\
5.6\end{array}$ & $\begin{array}{l}- \\
- \\
-\end{array}$ & $\begin{array}{l}\text { 6.38Ukn PAS } \\
5.63 \mathrm{Ukn} \text { PAS } \\
6.50 \mathrm{M}_{\mathrm{s}} \text { ABE } \\
5.70 \mathrm{~m}_{\mathrm{b}} \text { ISC } \\
5.30 \mathrm{~m}_{\mathrm{b}} \text { ISC }\end{array}$ & $\frac{-}{-}$ & $\frac{\bar{z}}{\overline{\mathrm{v}}}$ & $\begin{array}{l}- \\
\overline{75} \\
-\end{array}$ & $\begin{array}{l}- \\
- \\
-\end{array}$ \\
\hline $\begin{array}{l}1965 \\
1965 \\
1965 \\
1965 \\
1965\end{array}$ & $\begin{array}{l}07 \\
07 \\
07 \\
07 \\
08\end{array}$ & $\begin{array}{l}25 \\
29 \\
29 \\
29 \\
11\end{array}$ & $\begin{array}{lll}21 & 46 & 46.1 \\
08 & 29 & 22.0 \\
12 & 2023.2 \\
1508 & 32.7 \\
18 & 2938.5\end{array}$ & $\begin{array}{ll}51.52 & \mathrm{~N} \\
51.11 & \mathrm{~N} \\
50.88 & \mathrm{~N} \\
51.00 & \mathrm{~N} \\
59.36 & \mathrm{~N}\end{array}$ & $\begin{array}{l}175.95 \mathrm{E} \\
171.30 \mathrm{~W} \\
171.57 \mathrm{~W} \\
171.30 \mathrm{~W} \\
146.08 \mathrm{~W}\end{array}$ & $\begin{array}{l}037 \\
018 \\
037 \\
003 \\
015\end{array}$ & $\begin{array}{l}299 \\
299 \\
299 \\
299 \\
299\end{array}$ & $\begin{array}{l}5.5 \\
6.3 \\
5.5 \\
5.5 \\
5.5\end{array}$ & $\begin{array}{l}- \\
- \\
-\end{array}$ & $\begin{array}{ll}5.75 \mathrm{Ukn}^{2} & \text { PAS } \\
6.70 \mathrm{M}_{\mathrm{s}} & \text { ABE } \\
5.50 \mathrm{~m}_{\mathrm{b}} & \text { ISC } \\
5.40 \mathrm{~m}_{\mathrm{b}} & \text { ISC } \\
5.30 \mathrm{~m}_{\mathrm{b}} & \text { ISC }\end{array}$ & $\frac{-}{-}$ & $\begin{array}{c}\overline{\text { Felt }} \\
\overline{-} \\
-\end{array}$ & $\begin{array}{l}\overline{75} \\
- \\
-\end{array}$ & $\begin{array}{l}- \\
- \\
-\end{array}$ \\
\hline $\begin{array}{l}1965 \\
1965 \\
1965 \\
1965 \\
1965\end{array}$ & $\begin{array}{l}09 \\
09 \\
09 \\
09 \\
09\end{array}$ & $\begin{array}{l}02 \\
04 \\
08 \\
08 \\
18\end{array}$ & $\begin{array}{lll}04 & 26 & 37.8 \\
14 & 32 & 50.2 \\
03 & 2621.0 \\
11 & 16 & 33.6 \\
20 & 46 & 36.7\end{array}$ & $\begin{array}{ll}51.91 & \mathrm{~N} \\
58.29 & \mathrm{~N} \\
57.48 & \mathrm{~N} \\
55.71 & \mathrm{~N} \\
59.38 & \mathrm{~N}\end{array}$ & $\begin{array}{l}175.51 \mathrm{E} \\
152.50 \mathrm{~W} \\
152.10 \mathrm{~W} \\
155.30 \mathrm{~W} \\
145.18 \mathrm{~W}\end{array}$ & $\begin{array}{l}030 \\
030 \\
025 \\
024 \\
005\end{array}$ & $\begin{array}{l}299 \\
299 \\
299 \\
299 \\
299\end{array}$ & $\begin{array}{l}5.7 \\
6.2 \\
5.6 \\
5.5 \\
5.3\end{array}$ & $\frac{-}{-}$ & $\begin{array}{l}\text { 5.38Ukn PAL } \\
6.80 \mathrm{M}_{\mathrm{s}} \text { ABE } \\
\text { 5.38Ukn PAL } \\
\text { 5.50Ukn PAL } \\
\text { 6.00Ukn PAL }\end{array}$ & $\begin{array}{l}- \\
- \\
-\end{array}$ & $\begin{array}{l}- \\
\text { Felt } \\
\text { Felt } \\
-\end{array}$ & $\begin{array}{l}- \\
75 \\
- \\
-\end{array}$ & $\begin{array}{l}- \\
- \\
-\end{array}$ \\
\hline $\begin{array}{l}1965 \\
1965 \\
1965 \\
1965 \\
1965\end{array}$ & $\begin{array}{l}09 \\
10 \\
10 \\
10 \\
10\end{array}$ & $\begin{array}{l}27 \\
01 \\
12 \\
19 \\
20\end{array}$ & $\begin{array}{l}050907.5 \\
085201.9 \\
134059.4 \\
204845.9 \\
110810.3\end{array}$ & $\begin{array}{l}51.86 \mathrm{~N} \\
50.02 \mathrm{~N} \\
56.1 \quad \mathrm{~N} \\
52.37 \mathrm{~N} \\
51.254 \mathrm{~N}\end{array}$ & $\begin{array}{l}175.60 \mathrm{E} \\
178.28 \mathrm{E} \\
153.6 \mathrm{~W} \\
174.33 \mathrm{E} \\
173.762 \mathrm{~W}\end{array}$ & $\begin{array}{l}000 \\
005 \\
029 \\
029 \\
020\end{array}$ & $\begin{array}{l}299 \\
299 \\
299 \\
299 \\
479\end{array}$ & $\begin{array}{l}5.5 \\
6.3 \\
5.5 \\
5.6 \\
5.6\end{array}$ & $\begin{array}{l}- \\
- \\
-\end{array}$ & $\begin{array}{l}\text { 5.38Ukn PAL } \\
6.50 \mathrm{Ukn} \text { PAS } \\
5.40 \mathrm{~m}_{\mathrm{b}} \text { ISC } \\
5.88 \mathrm{Ukn} \text { PAL } \\
5.25 \mathrm{Ukn} \text { PAL }\end{array}$ & $\begin{array}{l}- \\
- \\
-\end{array}$ & $\begin{array}{c}\bar{E} \\
\overline{-} \\
-\end{array}$ & $\begin{array}{l}\overline{75} \\
- \\
-\end{array}$ & $\begin{array}{l}- \\
- \\
-\end{array}$ \\
\hline $\begin{array}{l}1965 \\
1965 \\
1965\end{array}$ & $\begin{array}{l}10 \\
11 \\
11\end{array}$ & $\begin{array}{l}23 \\
22 \\
22\end{array}$ & $\begin{array}{l}060052.5 \\
140029.3 \\
202531.4\end{array}$ & $\begin{array}{l}53.85 \mathrm{~N} \\
51.576 \mathrm{~N} \\
51.32 \mathrm{~N}\end{array}$ & $\begin{array}{l}165.30 \mathrm{~W} \\
175.948 \mathrm{~W} \\
179.67 \mathrm{~W}\end{array}$ & $\begin{array}{l}039 \\
046 \\
041\end{array}$ & $\begin{array}{l}299 \\
479 \\
299\end{array}$ & $\begin{array}{l}5.5 \\
5.6 \\
5.9\end{array}$ & - & $\begin{array}{ll}5.38 \mathrm{mb}_{\mathrm{b}} & \text { ISC } \\
5.50 \mathrm{~m}_{\mathrm{b}} & \text { ISC } \\
5.80 \mathrm{~m}_{\mathrm{b}} & \text { ISC }\end{array}$ & - & $\overline{\text { Felt }}$ & $\begin{array}{l}\overline{75} \\
-\end{array}$ & - \\
\hline
\end{tabular}


ALASKA-Continued

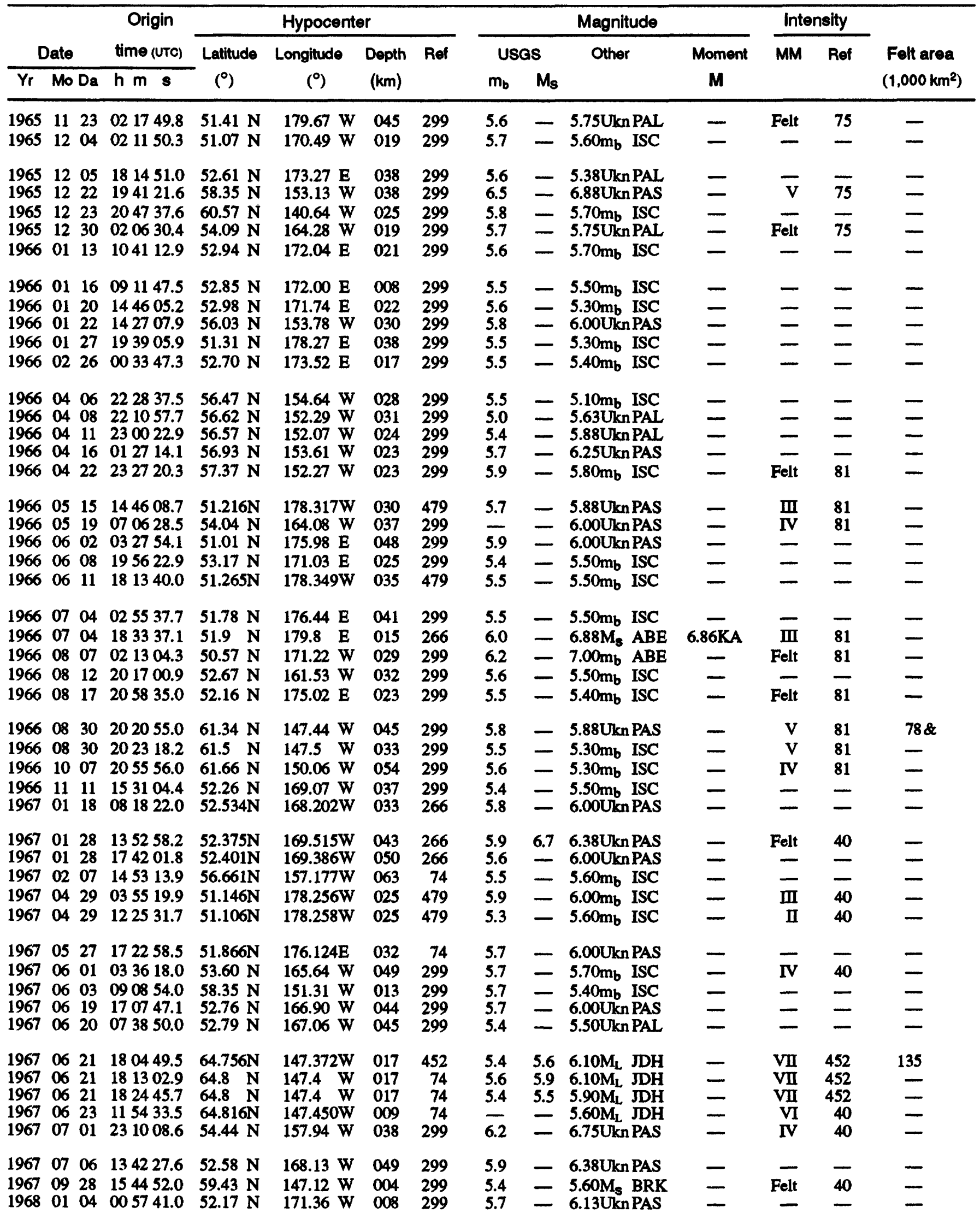


ALASKA-Continued

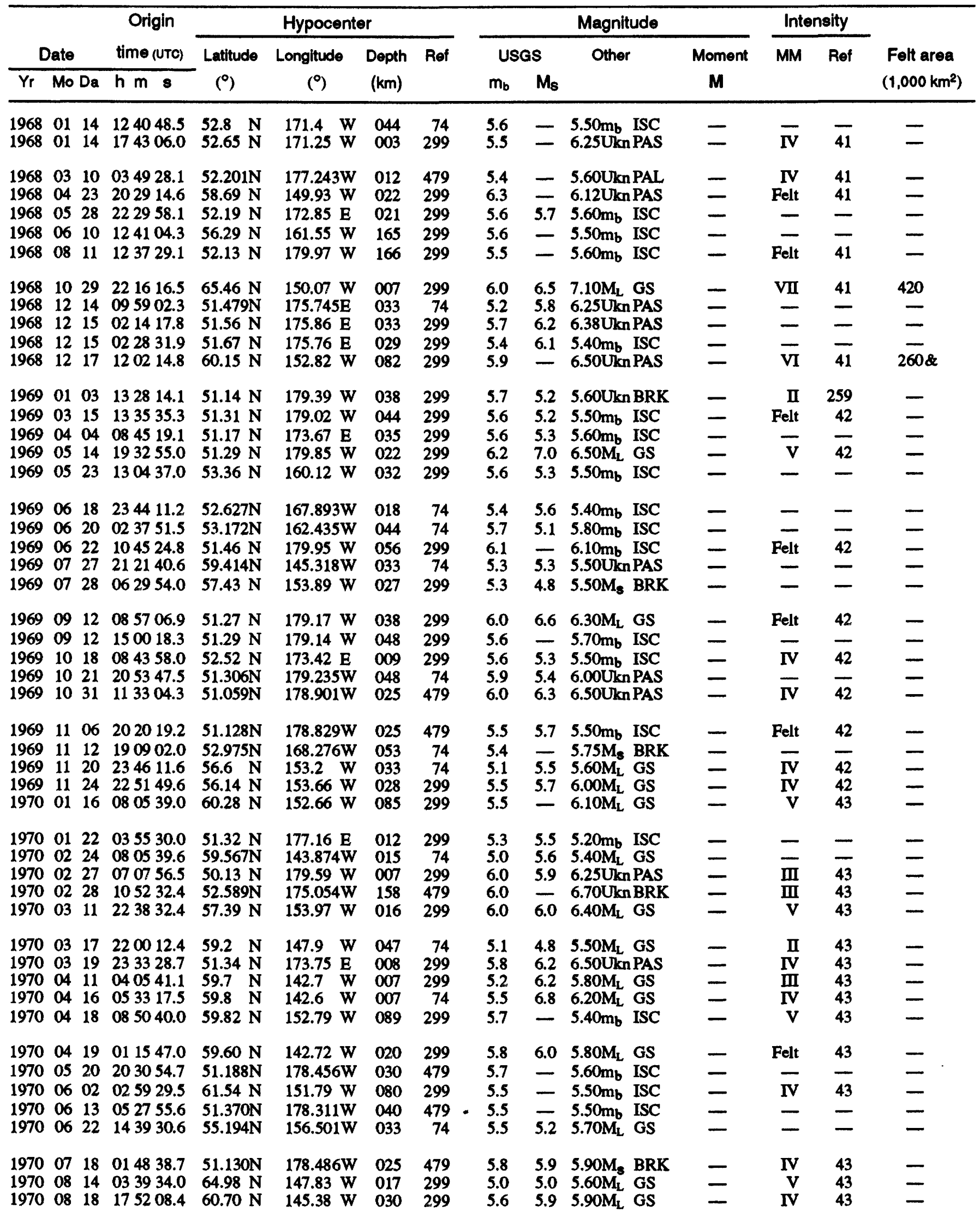


EARTHQUAKES IN ALASKA

ALASKA-Continued

[See table 1 for hypocenter and intensity references and table 2 for definitions of magnitude source codes. \&, land area only. Leader (--) indicates information is not available]

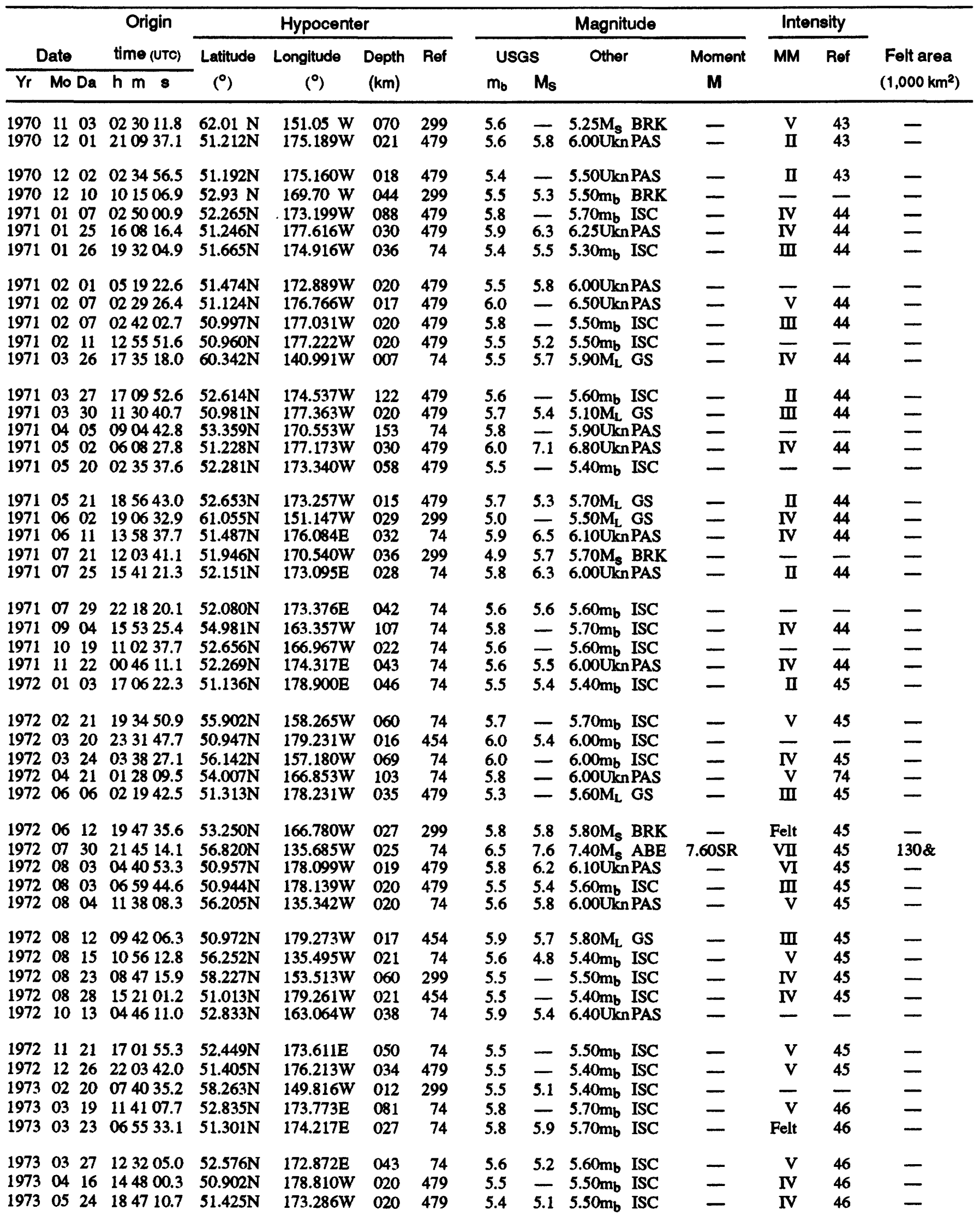


ALASKA-Continued

[See table 1 for hypocenter and intensity references and table 2 for definitions of magnitude source codes. \&, land area only. Leader (--) indicates information is not available]

\begin{tabular}{|c|c|c|c|c|c|c|c|c|c|c|c|c|c|c|}
\hline & & & & & Hypocente & & & & & Magnitude & & Inte & sity & \\
\hline & Date & & time (UTC) & Latitude & Longitude & Depth & Ref & & & Other & Moment & MM & Ref & Felt area \\
\hline $\mathrm{Yr}$ & Mo & $\mathrm{Da}$ & $\mathrm{h} \mathrm{m} s$ & $\left({ }^{\circ}\right)$ & $\left({ }^{\circ}\right)$ & (km) & & $m_{b}$ & $M_{s}$ & & $M$ & & & $\left.1,000 \mathrm{~km}^{2}\right)$ \\
\hline $\begin{array}{l}1973 \\
1973\end{array}$ & $\begin{array}{l}05 \\
05\end{array}$ & $\begin{array}{l}26 \\
29\end{array}$ & $\begin{array}{l}121934.2 \\
014644.9\end{array}$ & $\begin{array}{l}51.287 \mathrm{~N} \\
51.685 \mathrm{~N}\end{array}$ & $\begin{array}{l}179.708 \mathrm{~W} \\
176.239 \mathrm{E}\end{array}$ & $\begin{array}{l}035 \\
046\end{array}$ & $\begin{array}{r}299 \\
74\end{array}$ & $\begin{array}{l}5.8 \\
5.2\end{array}$ & $\overline{5.7}$ & $\begin{array}{ll}5.70 \mathrm{~m}_{\mathrm{b}} & \text { ISC } \\
5.50 \mathrm{M}_{\mathrm{s}} & \text { BRK }\end{array}$ & - & $\underline{\mathbf{v}}$ & 46 & - \\
\hline $\begin{array}{l}1973 \\
1973 \\
1973 \\
1973 \\
1973\end{array}$ & $\begin{array}{l}05 \\
06 \\
06 \\
07 \\
07\end{array}$ & $\begin{array}{l}29 \\
15 \\
23 \\
01 \\
03\end{array}$ & $\begin{array}{lll}06 & 14 & 22.3 \\
12 & 11 & 02.3 \\
05 & 26 & 48.5 \\
13 & 33 & 34.6 \\
16 & 59 & 35.1\end{array}$ & $\begin{array}{l}54.011 \mathrm{~N} \\
51.304 \mathrm{~N} \\
51.402 \mathrm{~N} \\
57.840 \mathrm{~N} \\
57.980 \mathrm{~N}\end{array}$ & $\begin{array}{l}163.760 \mathrm{~W} \\
179.392 \mathrm{~W} \\
176.673 \mathrm{~W} \\
137.330 \mathrm{~W} \\
138.021 \mathrm{~W}\end{array}$ & $\begin{array}{l}030 \\
048 \\
039 \\
033 \\
033\end{array}$ & $\begin{array}{r}74 \\
74 \\
479 \\
74 \\
74\end{array}$ & $\begin{array}{l}6.0 \\
5.8 \\
5.5 \\
6.1 \\
6.0\end{array}$ & $\begin{array}{l}5.5 \\
\frac{-}{6.7} \\
6.0\end{array}$ & $\begin{array}{ll}5.30 \mathrm{M}_{8} & \text { BRK } \\
5.80 \mathrm{~m}_{\mathrm{b}} & \text { ISC } \\
5.40 \mathrm{~m}_{\mathrm{b}} & \text { ISC } \\
6.70 \mathrm{Ukn} \text { PAS } \\
6.40 \mathrm{Ukn} \text { PAS }\end{array}$ & $\begin{array}{l}- \\
- \\
-\end{array}$ & $\begin{array}{l}\text { V } \\
\text { IV } \\
\text { V } \\
\text { V } \\
\text { V }\end{array}$ & $\begin{array}{l}46 \\
46 \\
46 \\
46 \\
46\end{array}$ & $\begin{array}{c}- \\
- \\
-\end{array}$ \\
\hline $\begin{array}{l}1973 \\
1973 \\
1973 \\
1973 \\
1973\end{array}$ & $\begin{array}{l}08 \\
08 \\
08 \\
09 \\
11\end{array}$ & $\begin{array}{l}16 \\
16 \\
22 \\
06 \\
06\end{array}$ & $\begin{array}{lll}12 & 1659.0 \\
14 & 2531.4 \\
18 & 1437.2 \\
10 & 5936.7 \\
09 & 36 & 07.4\end{array}$ & $\begin{array}{l}51.149 \mathrm{~N} \\
51.134 \mathrm{~N} \\
57.067 \mathrm{~N} \\
61.039 \mathrm{~N} \\
51.428 \mathrm{~N}\end{array}$ & $\begin{array}{l}176.608 W \\
176.535 W \\
154.101 W \\
146.828 W \\
175.356 \mathrm{~W}\end{array}$ & $\begin{array}{l}020 \\
020 \\
038 \\
029 \\
037\end{array}$ & $\begin{array}{r}479 \\
479 \\
74 \\
74 \\
479\end{array}$ & $\begin{array}{l}5.6 \\
5.6 \\
5.9 \\
5.5 \\
5.8\end{array}$ & $\begin{array}{l}5.8 \\
5.6 \\
5.3 \\
6.4\end{array}$ & $\begin{array}{ll}5.80 \mathrm{M}_{\mathrm{s}} & \text { BRK } \\
5.30 \mathrm{~m}_{\mathrm{b}} & \text { ISC } \\
5.50 \mathrm{M}_{\mathrm{s}} & \text { BRK } \\
5.50 \mathrm{M}_{\mathrm{L}} \text { PMR } \\
6.20 \mathrm{Ukn} \text { PAS }\end{array}$ & $\frac{-}{-}$ & $\begin{array}{r}\text { IV } \\
\text { III } \\
\text { Felt } \\
\text { III } \\
\text { IV }\end{array}$ & $\begin{array}{l}46 \\
46 \\
46 \\
46 \\
46\end{array}$ & $\begin{array}{l}- \\
- \\
-\end{array}$ \\
\hline $\begin{array}{l}1973 \\
1973 \\
1973 \\
1973 \\
1974\end{array}$ & $\begin{array}{l}11 \\
11 \\
12 \\
12 \\
01\end{array}$ & $\begin{array}{l}06 \\
09 \\
02 \\
14 \\
31\end{array}$ & $\begin{array}{lll}18 & 26 & 35.1 \\
14 & 13 & 03.5 \\
22 & 09 & 54.5 \\
17 & 37 & 34.7 \\
19 & 55 & 26.2\end{array}$ & $\begin{array}{l}51.331 \mathrm{~N} \\
52.444 \mathrm{~N} \\
52.283 \mathrm{~N} \\
51.172 \mathrm{~N} \\
52.357 \mathrm{~N}\end{array}$ & $\begin{array}{l}175.201 W \\
178.353 \mathrm{E} \\
168.735 \mathrm{~W} \\
177.827 \mathrm{~W} \\
168.740 \mathrm{~W}\end{array}$ & $\begin{array}{l}027 \\
182 \\
040 \\
030 \\
036\end{array}$ & $\begin{array}{r}479 \\
74 \\
74 \\
479 \\
74\end{array}$ & $\begin{array}{l}5.9 \\
5.6 \\
5.6 \\
5.8 \\
5.6\end{array}$ & $\frac{6.3}{\frac{5.0}{5.0}}$ & $\begin{array}{ll}6.20 U \mathrm{kn} & \text { PAS } \\
5.50 \mathrm{~m}_{\mathrm{b}} & \text { ISC } \\
5.60 \mathrm{~m}_{\mathrm{b}} & \text { ISC } \\
5.80 \mathrm{M}_{\mathrm{L}} & \text { ADK } \\
5.60 \mathrm{~m}_{\mathrm{b}} & \text { ISC }\end{array}$ & $\begin{array}{c}- \\
- \\
-\end{array}$ & $\frac{\text { IV }}{-}$ & $\frac{46}{\frac{46}{46}}$ & $\frac{-}{-}$ \\
\hline $\begin{array}{l}1974 \\
1974 \\
1974 \\
1974 \\
1974\end{array}$ & $\begin{array}{l}02 \\
03 \\
03 \\
04 \\
04\end{array}$ & $\begin{array}{l}06 \\
27 \\
29 \\
06 \\
06\end{array}$ & $\begin{array}{lll}04 & 04 & 07.2 \\
16 & 28 & 47.3 \\
21 & 50 & 35.3 \\
01 & 53 & 47.3 \\
03 & 56 & 01.8\end{array}$ & $\begin{array}{l}53.799 \mathrm{~N} \\
50.109 \mathrm{~N} \\
57.585 \mathrm{~N} \\
55.102 \mathrm{~N} \\
55.120 \mathrm{~N}\end{array}$ & $\begin{array}{l}164.672 W \\
179.657 \mathrm{~W} \\
153.922 \mathrm{~W} \\
160.440 \mathrm{~W} \\
160.443 \mathrm{~W}\end{array}$ & $\begin{array}{l}002 \\
037 \\
044 \\
027 \\
040\end{array}$ & $\begin{array}{l}74 \\
74 \\
74 \\
74 \\
74\end{array}$ & $\begin{array}{l}5.9 \\
5.6 \\
5.7 \\
5.7 \\
6.0\end{array}$ & $\begin{array}{l}6.5 \\
4.8 \\
5.2 \\
5.1 \\
5.3\end{array}$ & $\begin{array}{ll}6.30 \mathrm{Un}^{2} \text { PAS } \\
5.60 \mathrm{mb}_{\mathrm{b}} \text { ISC } \\
5.50 \mathrm{M}_{\mathrm{L}} \text { PMR } \\
5.80 \mathrm{~m}_{\mathrm{b}} & \text { ISC } \\
6.00 \mathrm{~m}_{\mathrm{b}} & \text { ISC }\end{array}$ & $\begin{array}{l}- \\
- \\
-\end{array}$ & $\begin{array}{c}\mathrm{V} \\
\mathrm{IV} \\
\mathrm{V} \\
\mathrm{V}\end{array}$ & $\begin{array}{l}\frac{47}{47} \\
47 \\
47\end{array}$ & $\frac{-}{-}$ \\
\hline $\begin{array}{l}1974 \\
1974 \\
1974 \\
1974 \\
1974\end{array}$ & $\begin{array}{l}05 \\
06 \\
08 \\
08 \\
08\end{array}$ & $\begin{array}{l}27 \\
15 \\
01 \\
01 \\
01\end{array}$ & $\begin{array}{l}140143.5 \\
023713.8 \\
050759.0 \\
055538.2 \\
075955.4\end{array}$ & $\begin{array}{l}60.328 \mathrm{~N} \\
52.262 \mathrm{~N} \\
56.516 \mathrm{~N} \\
56.670 \mathrm{~N} \\
56.515 \mathrm{~N}\end{array}$ & $\begin{array}{l}146.016 \mathrm{~W} \\
178.791 \mathrm{E} \\
152.315 \mathrm{~W} \\
152.105 \mathrm{~W} \\
152.431 \mathrm{~W}\end{array}$ & $\begin{array}{l}021 \\
157 \\
010 \\
033 \\
021\end{array}$ & $\begin{array}{r}74 \\
74 \\
74 \\
74 \\
299\end{array}$ & $\begin{array}{l}5.5 \\
5.7 \\
5.2 \\
5.7 \\
5.2\end{array}$ & $\begin{array}{l}5.7 \\
6.1 \\
6.3 \\
6.0\end{array}$ & $\begin{array}{ll}5.40 \mathrm{M}_{\mathrm{L}} & \text { PMR } \\
5.50 \mathrm{~m}_{\mathrm{b}} & \text { ISC } \\
5.30 \mathrm{~m}_{\mathrm{b}} & \text { ISC } \\
5.70 \mathrm{~m}_{\mathrm{b}} & \text { ISC } \\
5.1 \mathrm{~m}_{\mathrm{b}} & \text { ISC }\end{array}$ & $\frac{-}{-}$ & $\begin{array}{l}\text { III } \\
- \\
-\end{array}$ & $\frac{47}{-}$ & $\frac{-}{-}$ \\
\hline $\begin{array}{l}1974 \\
1974 \\
1974 \\
1974 \\
1974\end{array}$ & $\begin{array}{l}08 \\
08 \\
08 \\
08 \\
08\end{array}$ & $\begin{array}{l}13 \\
14 \\
16 \\
20 \\
24\end{array}$ & $\begin{array}{l}034620.6 \\
053454.3 \\
094131.9 \\
204501.4 \\
104111.2\end{array}$ & $\begin{array}{l}51.293 \mathrm{~N} \\
51.281 \mathrm{~N} \\
51.228 \mathrm{~N} \\
52.243 \mathrm{~N} \\
52.407 \mathrm{~N}\end{array}$ & $\begin{array}{l}178.068 W \\
178.118 W \\
177.791 W \\
174.972 E \\
168.273 W\end{array}$ & $\begin{array}{l}033 \\
034 \\
031 \\
058 \\
041\end{array}$ & $\begin{array}{r}479 \\
479 \\
479 \\
74 \\
74\end{array}$ & $\begin{array}{l}5.8 \\
5.7 \\
5.7 \\
5.6 \\
5.7\end{array}$ & $\frac{-}{5.8}$ & $\begin{array}{ll}5.90 \mathrm{M}_{8} & \text { BRK } \\
5.20 \mathrm{M}_{\mathrm{S}} & \text { BRK } \\
5.90 \mathrm{M}_{\mathrm{L}} & \text { ADK } \\
5.10 \mathrm{M}_{\mathrm{s}} & \text { BRK } \\
5.50 \mathrm{M}_{\mathrm{L}} & \text { ADK }\end{array}$ & $\begin{array}{l}- \\
- \\
-\end{array}$ & $\begin{array}{l}\text { V } \\
\text { II } \\
\text { IV } \\
\text { III } \\
\text { - }\end{array}$ & $\begin{array}{l}47 \\
47 \\
47 \\
47 \\
-\end{array}$ & $\frac{-}{-}$ \\
\hline $\begin{array}{l}1974 \\
1974 \\
1974 \\
1974 \\
1974\end{array}$ & $\begin{array}{l}11 \\
11 \\
12 \\
12 \\
12\end{array}$ & $\begin{array}{l}11 \\
14 \\
07 \\
25 \\
29\end{array}$ & $\begin{array}{l}051751.4 \\
044854.7 \\
073411.0 \\
024913.0 \\
182500.7\end{array}$ & $\begin{array}{l}51.454 \mathrm{~N} \\
58.797 \mathrm{~N} \\
51.857 \mathrm{~N} \\
51.697 \mathrm{~N} \\
61.597 \mathrm{~N}\end{array}$ & $\begin{array}{l}178.079 \mathrm{~W} \\
154.620 \mathrm{~W} \\
170.795 \mathrm{~W} \\
174.635 \mathrm{E} \\
150.511 \mathrm{~W}\end{array}$ & $\begin{array}{l}047 \\
037 \\
033 \\
040 \\
067\end{array}$ & $\begin{array}{r}479 \\
74 \\
74 \\
74 \\
74\end{array}$ & $\begin{array}{l}5.8 \\
5.5 \\
5.5 \\
5.7 \\
5.6\end{array}$ & $\begin{array}{l}-5.6 \\
5.8 \\
5.8 \\
-\end{array}$ & $\begin{array}{ll}5.20 \mathrm{M}_{\mathrm{S}} & \text { BRK } \\
5.40 \mathrm{M}_{\mathrm{L}} & \text { PMR } \\
5.10 \mathrm{M}_{\mathrm{S}} & \text { BRK } \\
5.90 \mathrm{M}_{\mathrm{L}} & \text { ADK } \\
5.60 \mathrm{~m}_{\mathrm{b}} & \text { ISC }\end{array}$ & $\overline{-}$ & $\begin{array}{c}\text { V } \\
\text { IV } \\
\text { IV } \\
\text { V }\end{array}$ & $\begin{array}{l}47 \\
47 \\
47 \\
47\end{array}$ & $\begin{array}{c}- \\
- \\
-\end{array}$ \\
\hline $\begin{array}{l}1975 \\
1975 \\
1975 \\
1975 \\
1975\end{array}$ & $\begin{array}{l}01 \\
01 \\
02 \\
02 \\
02\end{array}$ & $\begin{array}{l}01 \\
13 \\
02 \\
02 \\
22\end{array}$ & $\begin{array}{lll}03 & 55 & 12.0 \\
09 & 19 & 10.3 \\
07 & 24 & 53.3 \\
08 & 43 & 39.1 \\
08 & 36 & 07.4\end{array}$ & $\begin{array}{l}61.909 \mathrm{~N} \\
52.220 \mathrm{~N} \\
53.053 \mathrm{~N} \\
53.113 \mathrm{~N} \\
51.377 \mathrm{~N}\end{array}$ & $\begin{array}{l}149.738 W \\
171.142 W \\
173.446 \mathrm{E} \\
173.497 \mathrm{E} \\
179.419 \mathrm{~W}\end{array}$ & $\begin{array}{l}066 \\
042 \\
025 \\
010 \\
048\end{array}$ & $\begin{array}{l}74 \\
74 \\
74 \\
74 \\
74\end{array}$ & $\begin{array}{l}5.9 \\
5.7 \\
5.9 \\
6.1 \\
6.3\end{array}$ & $\begin{array}{l}- \\
5.6 \\
5.5 \\
7.6 \\
6.5\end{array}$ & $\begin{array}{ll}5.90 \mathrm{mb}_{\mathrm{b}} & \text { ISC } \\
5.50 \mathrm{M}_{8} & \text { BRK } \\
5.90 \mathrm{mb}^{\mathrm{b}} & \text { ISC } \\
7.40 \mathrm{M}_{8} & \text { ABE } \\
6.00 \mathrm{Un}^{2} & \text { PAS }\end{array}$ & $\begin{array}{l}- \\
- \\
-\end{array}$ & $\underset{\mathbf{V}}{\mathbf{I I}}$ & $\begin{array}{l}48 \\
48 \\
48 \\
48\end{array}$ & $\frac{-}{-}$ \\
\hline $\begin{array}{l}1975 \\
1975 \\
1975 \\
1975 \\
1975\end{array}$ & $\begin{array}{l}04 \\
05 \\
07 \\
08 \\
10\end{array}$ & $\begin{array}{l}11 \\
25 \\
25 \\
02 \\
17\end{array}$ & $\begin{array}{l}104715.3 \\
190434.4 \\
104025.0 \\
101817.9 \\
193912.5\end{array}$ & $\begin{array}{l}54.097 \mathrm{~N} \\
57.375 \mathrm{~N} \\
55.055 \mathrm{~N} \\
53.387 \mathrm{~N} \\
57.446 \mathrm{~N}\end{array}$ & $\begin{array}{l}163.248 W \\
150.119 W \\
160.377 W \\
161.485 W \\
149.008 W\end{array}$ & $\begin{array}{l}020 \\
033 \\
017 \\
033 \\
033\end{array}$ & $\begin{array}{l}74 \\
74 \\
74 \\
74 \\
74\end{array}$ & $\begin{array}{l}5.5 \\
5.6 \\
5.8 \\
6.2 \\
5.7\end{array}$ & $\begin{array}{l}5.2 \\
5.4 \\
5.2 \\
6.0 \\
5.5\end{array}$ & $\begin{array}{ll}5.70 \mathrm{M}_{8} & \text { BRK } \\
5.70 \mathrm{M}_{\mathrm{L}} & \text { PMR } \\
5.60 \mathrm{~m}_{\mathrm{b}} & \text { ISC } \\
5.70 \mathrm{M}_{\mathrm{s}} & \text { BRK } \\
5.50 \mathrm{~m}_{\mathrm{b}} & \text { ISC }\end{array}$ & $\begin{array}{l}- \\
- \\
-\end{array}$ & $\begin{array}{l}\text { IV } \\
\text { IV } \\
\text { V } \\
-\end{array}$ & $\begin{array}{l}\frac{48}{48} \\
\frac{48}{-}\end{array}$ & $\begin{array}{l}- \\
- \\
-\end{array}$ \\
\hline $\begin{array}{l}1975 \\
1975 \\
1976\end{array}$ & $\begin{array}{l}11 \\
11 \\
08\end{array}$ & $\begin{array}{l}01 \\
30 \\
22\end{array}$ & $\begin{array}{l}004823.4 \\
203017.0 \\
020147.4\end{array}$ & $\begin{array}{l}53.655 \mathrm{~N} \\
52.599 \mathrm{~N} \\
60.220 \mathrm{~N}\end{array}$ & $\begin{array}{l}163.366 \mathrm{~W} \\
167.184 \mathrm{~W} \\
153.304 \mathrm{~W}\end{array}$ & $\begin{array}{l}025 \\
024 \\
144\end{array}$ & $\begin{array}{l}74 \\
74 \\
74\end{array}$ & $\begin{array}{l}5.7 \\
5.7 \\
5.5\end{array}$ & $\begin{array}{l}5.7 \\
6.3 \\
-\end{array}$ & $\begin{array}{l}5.70 \mathrm{mb}_{\mathrm{b}} \text { ISC } \\
6.60 \mathrm{Ukn} \text { PAS } \\
5.50 \mathrm{~m}_{\mathrm{b}} \text { ISC }\end{array}$ & - & $\frac{-}{v}$ & $\frac{-}{49}$ & - \\
\hline
\end{tabular}


ALASKA-Continued

[See table 1 for hypocenter and intensity references and table 2 for definitions of magnitude source codes. \&, land area only. Leader (--) indicates information is not available]

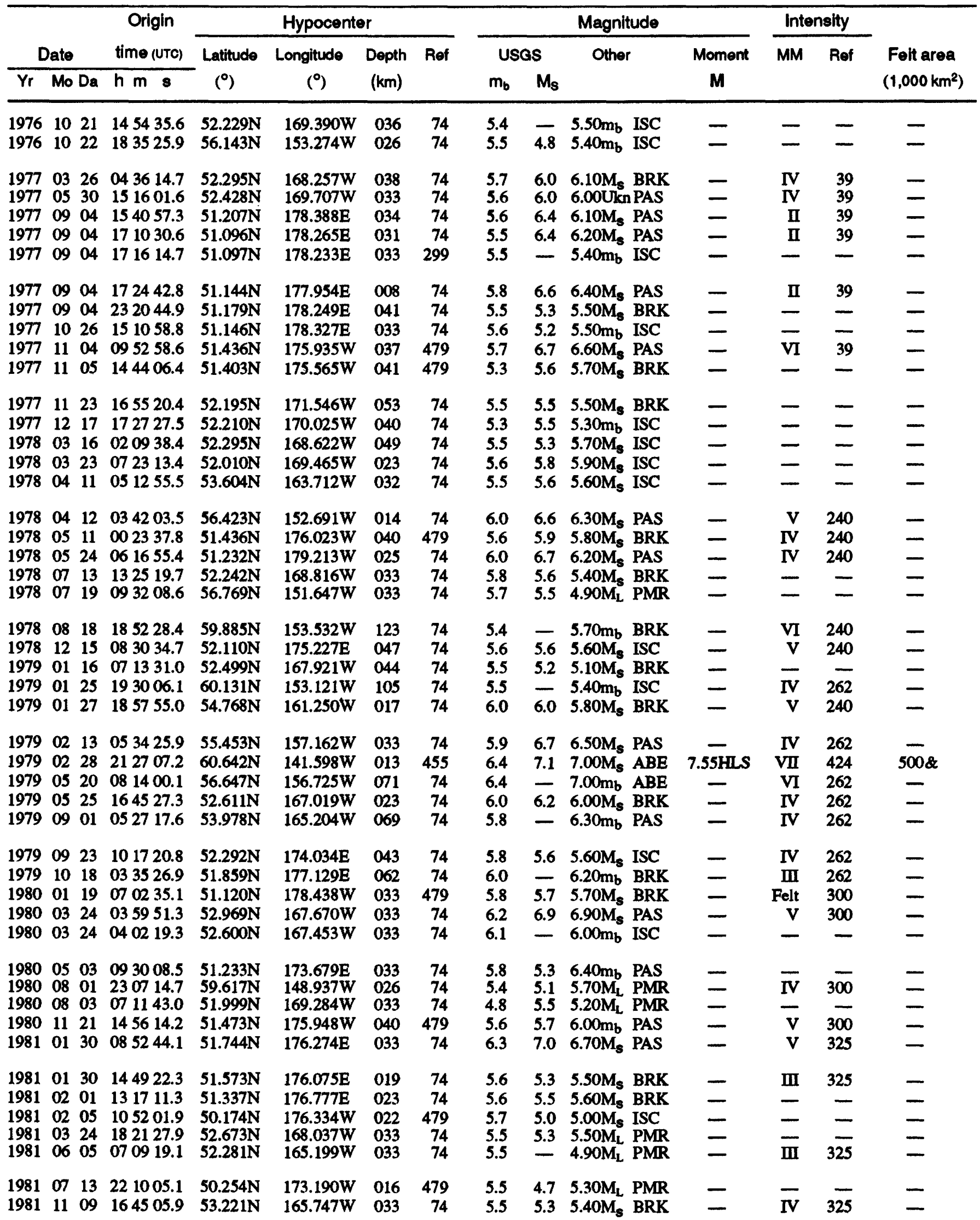


ALASKA-Continued

\begin{tabular}{|c|c|c|c|c|c|c|c|c|c|c|c|c|c|c|c|}
\hline & & & Origin & & Hypocente & & & & & Magnit & tude & & Inte & nsity & \\
\hline & Date & & time (UTC) & Latitude & Longitude & Depth & Ref & & & Other & & Moment & MM & Ref & Felt area \\
\hline Yr & Mo & $\mathrm{Da}$ & $h \mathrm{~m} s$ & & & (m) & & $m_{b}$ & $\mathbf{M}_{\mathbf{S}}$ & & & $\mathbf{M}$ & & & $\left.1000 \mathrm{~km}^{2}\right)$ \\
\hline $\begin{array}{l}1982 \\
1982 \\
1982\end{array}$ & $\begin{array}{l}01 \\
04 \\
06\end{array}$ & $\begin{array}{l}25 \\
23 \\
04\end{array}$ & $\begin{array}{lll}05 & 29 & 33.5 \\
23 & 18 & 23.5 \\
03 & 01 & 04.2\end{array}$ & $\begin{array}{l}53.222 \mathrm{~N} \\
51.178 \mathrm{~N} \\
51.280 \mathrm{~N}\end{array}$ & $\begin{array}{l}165.719 \mathrm{~W} \\
179.844 \mathrm{~W} \\
177.154 \mathrm{~W}\end{array}$ & $\begin{array}{l}060 \\
043 \\
039\end{array}$ & $\begin{array}{r}74 \\
74 \\
479\end{array}$ & $\begin{array}{l}6.1 \\
5.6 \\
5.8\end{array}$ & $\begin{array}{l}\overline{5.1} \\
-\end{array}$ & $\begin{array}{l}6.40 \mathrm{mb}_{\mathrm{b}} \\
5.20 \mathrm{M}_{3} \\
5.70 \mathrm{M}_{3}\end{array}$ & $\begin{array}{l}\text { PAS } \\
\text { BRK } \\
\text { BRK }\end{array}$ & $\begin{array}{l}\text { 5.93HAV } \\
5.64 \mathrm{HAV} \\
6.00 \mathrm{HAV}\end{array}$ & $\begin{array}{c}\text { IV } \\
\text { III } \\
\text { V }\end{array}$ & $\begin{array}{l}350 \\
350 \\
350\end{array}$ & - \\
\hline $\begin{array}{l}1982 \\
1982 \\
1982 \\
1982 \\
1982\end{array}$ & $\begin{array}{l}07 \\
07 \\
08 \\
09 \\
09\end{array}$ & $\begin{array}{l}01 \\
31 \\
06 \\
06 \\
12\end{array}$ & $\begin{array}{l}074153.2 \\
062915.5 \\
045359.2 \\
074854.9 \\
092223.1\end{array}$ & $\begin{array}{l}51.426 \mathrm{~N} \\
51.755 \mathrm{~N} \\
51.600 \mathrm{~N} \\
56.844 \mathrm{~N} \\
52.640 \mathrm{~N}\end{array}$ & $\begin{array}{l}179.943 \mathrm{~W} \\
176.137 \mathrm{E} \\
175.921 \mathrm{~W} \\
151.588 \mathrm{~W} \\
166.941 \mathrm{~W}\end{array}$ & $\begin{array}{l}047 \\
038 \\
043 \\
033 \\
033\end{array}$ & $\begin{array}{r}74 \\
74 \\
479 \\
74 \\
74\end{array}$ & $\begin{array}{l}6.3 \\
6.2 \\
5.4 \\
5.7 \\
5.7\end{array}$ & $\begin{array}{l}5.5 \\
6.0 \\
5.6 \\
5.9\end{array}$ & $\begin{array}{l}5.40 \mathrm{M}_{\mathrm{L}} \\
6.10 \mathrm{M}_{\mathrm{S}} \\
5.50 \mathrm{~m}_{\mathrm{b}} \\
5.40 \mathrm{M}_{\mathrm{s}} \\
5.60 \mathrm{M}_{\mathrm{s}}\end{array}$ & $\begin{array}{l}\text { PMR } \\
\text { BRK } \\
\text { ISC } \\
\text { BRK } \\
\text { PAS }\end{array}$ & $\begin{array}{l}\text { 5.84HAV } \\
\text { 6.20HAV } \\
5.48 \mathrm{HAV} \\
5.77 \mathrm{HAV} \\
6.23 \mathrm{HAV}\end{array}$ & $\begin{array}{l}\text { IV } \\
\text { III } \\
\text { IV } \\
\text { III } \\
\text { - }\end{array}$ & $\begin{array}{c}350 \\
350 \\
350 \\
350 \\
-\end{array}$ & $\begin{array}{l}- \\
- \\
-\end{array}$ \\
\hline $\begin{array}{l}1982 \\
1982 \\
1982 \\
1983 \\
1983\end{array}$ & $\begin{array}{l}09 \\
10 \\
12 \\
01 \\
01\end{array}$ & $\begin{array}{l}12 \\
04 \\
02 \\
14 \\
24\end{array}$ & $\begin{array}{l}165037.7 \\
074653.1 \\
094353.4 \\
182052.6 \\
130237.2\end{array}$ & $\begin{array}{l}52.819 \mathrm{~N} \\
51.421 \mathrm{~N} \\
51.883 \mathrm{~N} \\
55.911 \mathrm{~N} \\
51.381 \mathrm{~N}\end{array}$ & $\begin{array}{l}167.053 \mathrm{~W} \\
176.615 \mathrm{~W} \\
170.447 \mathrm{~W} \\
154.154 \mathrm{~W} \\
176.251 \mathrm{E}\end{array}$ & $\begin{array}{l}033 \\
036 \\
033 \\
033 \\
033\end{array}$ & $\begin{array}{r}74 \\
479 \\
74 \\
360 \\
360\end{array}$ & $\begin{array}{l}5.5 \\
5.5 \\
5.5 \\
5.6 \\
5.4\end{array}$ & $\begin{array}{l}5.1 \\
5.0 \\
4.8 \\
5.8 \\
5.7\end{array}$ & $\begin{array}{c}5.30 \mathrm{M}_{\mathrm{L}} \\
5.20 \mathrm{M}_{\mathrm{L}} \\
5.50 \mathrm{mb}_{\mathrm{b}} \\
- \\
-\end{array}$ & $\begin{array}{l}\text { PMR } \\
\text { PMR } \\
\text { ISC }\end{array}$ & $\begin{array}{c}\text { 5.64HAV } \\
5.42 \mathrm{HAV} \\
- \\
6.05 \mathrm{HAV} \\
5.84 \mathrm{HAV}\end{array}$ & $\begin{array}{c}\overline{\text { Felt }} \\
\overline{-} \\
-\end{array}$ & $\begin{array}{l}350 \\
= \\
=\end{array}$ & $\begin{array}{l}- \\
- \\
-\end{array}$ \\
\hline $\begin{array}{l}1983 \\
1983 \\
1983 \\
1983 \\
1983\end{array}$ & $\begin{array}{l}02 \\
02 \\
04 \\
06 \\
06\end{array}$ & $\begin{array}{l}14 \\
14 \\
03 \\
09 \\
28\end{array}$ & $\begin{array}{lll}03 & 20 & 03.7 \\
08 & 10 & 02.7 \\
19 & 14 & 05.0 \\
18 & 46 & 02.7 \\
03 & 25 & 17.6\end{array}$ & $\begin{array}{l}54.809 \mathrm{~N} \\
54.862 \mathrm{~N} \\
51.976 \mathrm{~N} \\
51.249 \mathrm{~N} \\
60.182 \mathrm{~N}\end{array}$ & $\begin{array}{l}159.108 \mathrm{~W} \\
158.875 \mathrm{~W} \\
179.259 \mathrm{E} \\
174.056 \mathrm{~W} \\
141.253 \mathrm{~W}\end{array}$ & $\begin{array}{l}016 \\
014 \\
116 \\
023 \\
012\end{array}$ & $\begin{array}{l}360 \\
360 \\
360 \\
479 \\
360\end{array}$ & $\begin{array}{l}5.9 \\
6.0 \\
5.6 \\
6.2 \\
6.0\end{array}$ & $\begin{array}{l}6.3 \\
5.6 \\
5.8 \\
5.4\end{array}$ & $\begin{array}{l}6.50 \mathrm{M}_{3} \\
6.00 \mathrm{M}_{\mathrm{L}} \\
5.60 \mathrm{mb}_{\mathrm{b}} \\
5.80 \mathrm{M}_{\mathrm{s}} \\
5.90 \mathrm{M}_{\mathrm{L}}\end{array}$ & $\begin{array}{l}\text { BRK } \\
\text { PAL } \\
\text { ISC } \\
\text { BRK } \\
\text { PMR }\end{array}$ & $\begin{array}{l}\text { 6.52HAV } \\
5.95 \mathrm{HAV} \\
5.54 \mathrm{HAV} \\
5.56 \mathrm{HAV} \\
5.86 \mathrm{HAV}\end{array}$ & $\begin{array}{l}\text { V } \\
\text { V } \\
\text { III } \\
\text { IV }\end{array}$ & $\begin{array}{l}360 \\
360 \\
- \\
360 \\
360\end{array}$ & $\begin{array}{l}- \\
-\end{array}$ \\
\hline $\begin{array}{l}1983 \\
1983 \\
1983 \\
1984 \\
1984\end{array}$ & $\begin{array}{l}07 \\
09 \\
12 \\
05 \\
07\end{array}$ & $\begin{array}{l}12 \\
07 \\
27 \\
06 \\
27\end{array}$ & $\begin{array}{l}151003.7 \\
192205.0 \\
230552.9 \\
1954 \quad 49.3 \\
155750.9\end{array}$ & $\begin{array}{l}61.035 \mathrm{~N} \\
60.978 \mathrm{~N} \\
53.586 \mathrm{~N} \\
51.389 \mathrm{~N} \\
50.324 \mathrm{~N}\end{array}$ & $\begin{array}{l}147.185 \mathrm{~W} \\
147.320 \mathrm{~W} \\
164.376 \mathrm{~W} \\
176.621 \mathrm{~W} \\
176.870 \mathrm{~W}\end{array}$ & $\begin{array}{l}030 \\
030 \\
040 \\
037 \\
021\end{array}$ & $\begin{array}{l}360 \\
360 \\
360 \\
479 \\
479\end{array}$ & $\begin{array}{l}6.2 \\
6.2 \\
5.6 \\
5.6 \\
5.8\end{array}$ & $\begin{array}{l}6.4 \\
6.2 \\
5.3 \\
5.0\end{array}$ & $\begin{array}{l}6.20 \mathrm{M}_{\mathrm{S}} \\
6.20 \mathrm{M}_{\mathrm{S}} \\
5.80 \mathrm{M}_{\mathrm{L}} \\
5.60 \mathrm{M}_{\mathrm{L}} \\
5.70 \mathrm{M}_{\mathrm{L}}\end{array}$ & $\begin{array}{l}\text { PAS } \\
\text { BRK } \\
\text { PAL } \\
\text { PMR } \\
\text { PMR }\end{array}$ & $\begin{array}{c}6.46 \mathrm{HAV} \\
6.34 \mathrm{HAV} \\
- \\
5.58 \mathrm{HAV} \\
5.57 \mathrm{HAV}\end{array}$ & $\begin{array}{l}\text { VI } \\
\text { VI } \\
\text { V } \\
\text { V } \\
\text { IV }\end{array}$ & $\begin{array}{l}360 \\
360 \\
360 \\
370 \\
370\end{array}$ & $\begin{array}{r}675 \\
230 \\
- \\
-\end{array}$ \\
\hline $\begin{array}{l}1984 \\
1984 \\
1984 \\
1984 \\
1984\end{array}$ & $\begin{array}{l}08 \\
09 \\
09 \\
11 \\
11\end{array}$ & $\begin{array}{l}14 \\
20 \\
23 \\
08 \\
19\end{array}$ & $\begin{array}{lll}01 & 02 & 08.4 \\
04 & 17 & 24.4 \\
17 & 06 & 36.3 \\
13 & 02 & 00.1 \\
04 & 10 & 42.4\end{array}$ & $\begin{array}{l}61.857 \mathrm{~N} \\
60.322 \mathrm{~N} \\
53.577 \mathrm{~N} \\
52.181 \mathrm{~N} \\
51.170 \mathrm{~N}\end{array}$ & $\begin{array}{l}149.104 \mathrm{~W} \\
146.001 \mathrm{~W} \\
165.424 \mathrm{~W} \\
170.999 \mathrm{~W} \\
179.096 \mathrm{E}\end{array}$ & $\begin{array}{l}019 \\
018 \\
033 \\
033 \\
038\end{array}$ & $\begin{array}{l}74 \\
74 \\
74 \\
74 \\
74\end{array}$ & $\begin{array}{l}5.7 \\
5.5 \\
5.7 \\
5.4 \\
5.6\end{array}$ & $\begin{array}{l}5.2 \\
5.2 \\
5.5 \\
5.3 \\
5.5\end{array}$ & $\begin{array}{l}5.70 \mathrm{M}_{\mathrm{L}} \\
5.20 \mathrm{M}_{\mathrm{L}} \\
5.90 \mathrm{M}_{\mathrm{L}} \\
5.50 \mathrm{M}_{\mathrm{L}} \\
5.70 \mathrm{M}_{\mathrm{L}}\end{array}$ & $\begin{array}{l}\text { PMR } \\
\text { PMR } \\
\text { PMR } \\
\text { PMR } \\
\text { PMR }\end{array}$ & $\begin{array}{l}\text { 5.82HAV } \\
\text { 5.47HAV } \\
\text { 5.89HAV } \\
5.90 \mathrm{HAV} \\
5.87 \mathrm{HAV}\end{array}$ & $\begin{array}{l}\text { VI } \\
\text { IV } \\
\text { IV } \\
\text { II }\end{array}$ & $\begin{array}{r}370 \\
370 \\
370 \\
-\overline{370}\end{array}$ & $\begin{array}{l}74 \& \\
- \\
- \\
-\end{array}$ \\
\hline $\begin{array}{l}1984 \\
1985 \\
1985 \\
1985 \\
1985\end{array}$ & $\begin{array}{l}11 \\
01 \\
01 \\
03 \\
03\end{array}$ & $\begin{array}{l}19 \\
02 \\
09 \\
09 \\
10\end{array}$ & $\begin{array}{l}120637.9 \\
053249.1 \\
192821.2 \\
140804.3 \\
133029.5\end{array}$ & $\begin{array}{l}51.580 \mathrm{~N} \\
55.428 \mathrm{~N} \\
60.289 \mathrm{~N} \\
66.239 \mathrm{~N} \\
66.136 \mathrm{~N}\end{array}$ & $\begin{array}{l}175.243 \mathrm{~W} \\
157.835 \mathrm{~W} \\
140.744 \mathrm{~W} \\
150.029 \mathrm{~W} \\
150.148 \mathrm{~W}\end{array}$ & $\begin{array}{l}039 \\
033 \\
014 \\
011 \\
010\end{array}$ & $\begin{array}{r}479 \\
74 \\
74 \\
74 \\
74\end{array}$ & $\begin{array}{l}5.6 \\
5.6 \\
5.7 \\
5.9 \\
5.2\end{array}$ & $\begin{array}{l}- \\
5.6 \\
5.1 \\
6.0 \\
4.9\end{array}$ & $\begin{array}{l}5.50 \mathrm{M}_{\mathrm{L}} \\
6.10 \mathrm{M}_{\mathrm{L}} \\
5.40 \mathrm{M}_{\mathrm{L}} \\
6.00 \mathrm{M}_{\mathrm{L}} \\
5.60 \mathrm{M}_{\mathrm{L}}\end{array}$ & $\begin{array}{l}\text { PMR } \\
\text { PMR } \\
\text { PMR } \\
\text { PMR } \\
\text { PMR }\end{array}$ & $\begin{array}{c}\text { 5.66HAV } \\
5.95 \mathrm{HAV} \\
- \\
6.14 \mathrm{HAV} \\
5.45 \mathrm{HAV}\end{array}$ & $\begin{array}{r}\text { IV } \\
\text { III } \\
\text { IV } \\
\text { V } \\
\text { Felt }\end{array}$ & $\begin{array}{l}370 \\
371 \\
371 \\
371 \\
371\end{array}$ & $\begin{array}{l}- \\
- \\
-\end{array}$ \\
\hline $\begin{array}{l}1985 \\
1985 \\
1985 \\
1985 \\
1985\end{array}$ & $\begin{array}{l}05 \\
05 \\
05 \\
07 \\
07\end{array}$ & $\begin{array}{l}09 \\
09 \\
24 \\
17 \\
31\end{array}$ & $\begin{array}{l}190521.5 \\
191407.7 \\
2204 \quad 45.4 \\
193129.5 \\
073754.6\end{array}$ & $\begin{array}{l}51.465 \mathrm{~N} \\
51.302 \mathrm{~N} \\
51.193 \mathrm{~N} \\
51.443 \mathrm{~N} \\
52.404 \mathrm{~N}\end{array}$ & $\begin{array}{l}177.913 \mathrm{E} \\
178.024 \mathrm{E} \\
178.367 \mathrm{~W} \\
172.883 \mathrm{~W} \\
173.487 \mathrm{E}\end{array}$ & $\begin{array}{l}033 \\
033 \\
033 \\
016 \\
045\end{array}$ & $\begin{array}{r}74 \\
74 \\
479 \\
479 \\
74\end{array}$ & $\begin{array}{l}5.7 \\
5.4 \\
5.8 \\
5.5 \\
5.7\end{array}$ & $\begin{array}{l}6.0 \\
6.0 \\
5.8 \\
5.9 \\
5.0\end{array}$ & $\begin{array}{l}6.20 \mathrm{M}_{3} \\
5.90 \mathrm{M}_{\mathrm{s}} \\
5.80 \mathrm{M}_{\mathrm{L}} \\
5.70 \mathrm{M}_{\mathrm{s}} \\
5.70 \mathrm{~m}_{\mathrm{b}}\end{array}$ & $\begin{array}{l}\text { BRK } \\
\text { BRK } \\
\text { PMR } \\
\text { BRK } \\
\text { ISC }\end{array}$ & $\begin{array}{c}6.25 \mathrm{HAV} \\
6 . \overline{4 H A V} \\
6.05 \mathrm{HAV} \\
5.61 \mathrm{HAV}\end{array}$ & $\begin{array}{l}\text { IV } \\
\text { III } \\
\text { III } \\
\text { III } \\
\text { IV }\end{array}$ & $\begin{array}{l}371 \\
371 \\
371 \\
371 \\
371\end{array}$ & $\begin{array}{l}- \\
- \\
-\end{array}$ \\
\hline $\begin{array}{l}1985 \\
1985 \\
1985 \\
1985 \\
1985\end{array}$ & $\begin{array}{l}08 \\
08 \\
09 \\
10 \\
10\end{array}$ & $\begin{array}{l}09 \\
30 \\
15 \\
01 \\
09\end{array}$ & $\begin{array}{lll}13 & 03 & 10.6 \\
17 & 31 & 11.9 \\
01 & 28 & 16.7 \\
15 & 54 & 51.1 \\
09 & 33 & 32.4\end{array}$ & $\begin{array}{l}52.424 \mathrm{~N} \\
53.097 \mathrm{~N} \\
59.102 \mathrm{~N} \\
52.296 \mathrm{~N} \\
54.765 \mathrm{~N}\end{array}$ & $\begin{array}{l}173.648 \mathrm{E} \\
172.629 \mathrm{E} \\
136.423 \mathrm{~W} \\
168.856 \mathrm{~W} \\
159.613 \mathrm{~W}\end{array}$ & $\begin{array}{l}037 \\
033 \\
001 \\
033 \\
030\end{array}$ & $\begin{array}{l}74 \\
74 \\
74 \\
74 \\
74\end{array}$ & $\begin{array}{l}5.5 \\
5.1 \\
5.4 \\
5.7 \\
6.2\end{array}$ & $\begin{array}{l}4.9 \\
5.0 \\
5.9 \\
5.4 \\
6.6\end{array}$ & $\begin{array}{l}5.40 M_{L} \\
5.60 M_{L} \\
5.10 M_{L} \\
5.30 M_{L} \\
6.60 M_{3}\end{array}$ & $\begin{array}{l}\text { PMR } \\
\text { PMR } \\
\text { PMR } \\
\text { PMR } \\
\text { BRK }\end{array}$ & $\begin{array}{l}5.38 \mathrm{HAV} \\
-\overline{0 H A V} \\
5.30 \mathrm{HAV} \\
5.67 \mathrm{HAV} \\
6.58 \mathrm{HAV}\end{array}$ & $\frac{\text { IV }}{\text { V }}$ & $\begin{array}{l}371 \\
371 \\
371 \\
371\end{array}$ & $\begin{array}{l}- \\
-\end{array}$ \\
\hline $\begin{array}{l}1985 \\
1985 \\
1985 \\
1985 \\
1985\end{array}$ & $\begin{array}{l}10 \\
10 \\
10 \\
10 \\
11\end{array}$ & $\begin{array}{l}25 \\
26 \\
30 \\
31 \\
14\end{array}$ & $\begin{array}{l}020904.3 \\
155936.0 \\
1905 \quad 37.5 \\
193306.5 \\
22 \quad 17 \quad 44.5\end{array}$ & $\begin{array}{l}52.072 \mathrm{~N} \\
54.838 \mathrm{~N} \\
51.801 \mathrm{~N} \\
53.249 \mathrm{~N} \\
54.756 \mathrm{~N}\end{array}$ & $\begin{array}{l}171.350 \mathrm{~W} \\
159.534 \mathrm{~W} \\
175.533 \mathrm{E} \\
166.936 \mathrm{~W} \\
159.787 \mathrm{~W}\end{array}$ & $\begin{array}{l}033 \\
033 \\
033 \\
030 \\
033\end{array}$ & $\begin{array}{l}74 \\
74 \\
74 \\
74 \\
74\end{array}$ & $\begin{array}{l}5.6 \\
5.6 \\
5.6 \\
5.8 \\
5.5\end{array}$ & $\begin{array}{l}5.5 \\
5.4 \\
5.7 \\
5.7\end{array}$ & $\begin{array}{l}5.60 \mathrm{M}_{\mathrm{L}} \\
5.30 \mathrm{M}_{\mathrm{L}} \\
5.50 \mathrm{M}_{\mathrm{L}} \\
5.80 \mathrm{M}_{3} \\
5.70 \mathrm{M}_{\mathrm{L}}\end{array}$ & $\begin{array}{l}\text { PMR } \\
\text { PMR } \\
\text { PMR } \\
\text { PAS } \\
\text { PMR }\end{array}$ & $\begin{array}{l}5.95 \mathrm{HAV} \\
5.36 \mathrm{HAV} \\
5.82 \mathrm{HAV} \\
6.14 \mathrm{HAV} \\
6.05 \mathrm{HAV}\end{array}$ & $\begin{array}{c}\text { II } \\
\text { V } \\
\text { II } \\
\text { IV } \\
\text { V }\end{array}$ & $\begin{array}{l}371 \\
371 \\
371 \\
371 \\
371\end{array}$ & $\begin{array}{l}- \\
-\end{array}$ \\
\hline $\begin{array}{l}1985 \\
1985\end{array}$ & $\begin{array}{l}12 \\
12\end{array}$ & $\begin{array}{l}28 \\
30\end{array}$ & $\begin{array}{l}074438.2 \\
124102.7\end{array}$ & $\begin{array}{l}56.580 \mathrm{~N} \\
61.541 \mathrm{~N}\end{array}$ & $\begin{array}{l}156.509 \mathrm{~W} \\
150.340 \mathrm{~W}\end{array}$ & $\begin{array}{l}058 \\
062\end{array}$ & $\begin{array}{l}74 \\
74\end{array}$ & $\begin{array}{l}5.3 \\
5.5\end{array}$ & - & $\begin{array}{l}5.70 \mathrm{mb}_{\mathrm{b}} \\
5.20 \mathrm{M}_{\mathrm{L}}\end{array}$ & $\begin{array}{l}\text { PAL } \\
\text { PMR }\end{array}$ & - & $\bar{v}$ & $3 \overline{71}$ & - \\
\hline
\end{tabular}


ALASKA-Continued

[See table 1 for hypocenter and intensity references and table 2 for definitions of magnitude source codes. \&, land area only. Leader (--) indicates information is not available]

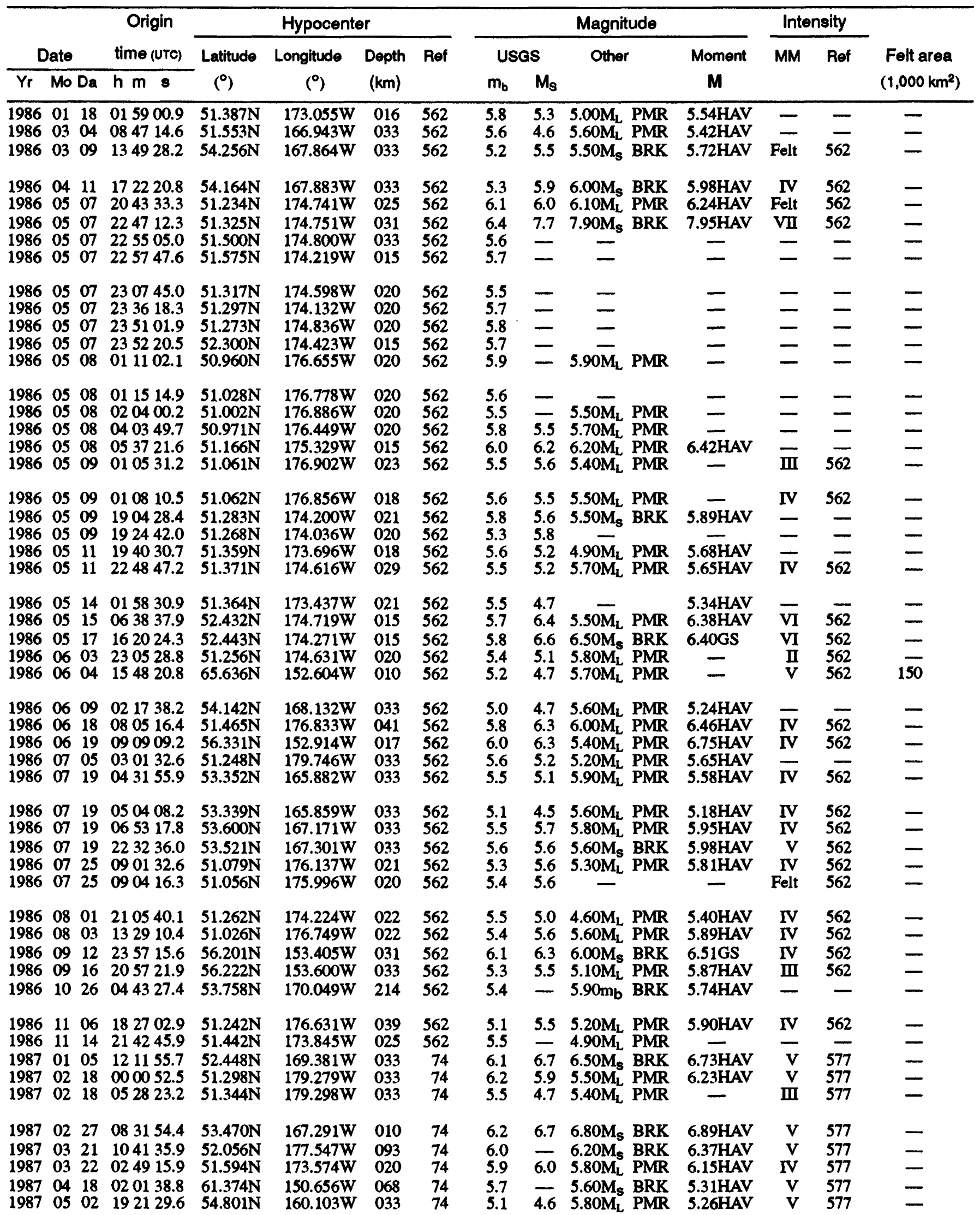


ALASKA-Continued

[See table 1 for hypocenter and intensity references and table 2 for definitions of magnitude source codes. \&, land area only. Leader (--) indicates information is not available]

\begin{tabular}{|c|c|c|c|c|c|c|c|c|c|c|c|c|c|c|}
\hline \multirow{2}{*}{\multicolumn{3}{|c|}{ Date }} & \multirow{2}{*}{$\begin{array}{c}\text { Origin } \\
\text { time (UTC) }\end{array}$} & \multicolumn{4}{|c|}{ Hypocenter } & \multicolumn{4}{|c|}{ Magnitude } & \multicolumn{2}{|c|}{ Intensity } & \multirow{3}{*}{$\begin{array}{l}\text { Felt area } \\
\left(1,000 \mathrm{~km}^{2}\right)\end{array}$} \\
\hline & & & & \multirow{2}{*}{$\begin{array}{l}\text { Latitude } \\
\left(^{\circ}\right)\end{array}$} & \multirow{2}{*}{$\begin{array}{l}\text { Longitude } \\
\left({ }^{\circ}\right)\end{array}$} & \multirow{2}{*}{$\begin{array}{l}\text { Depth } \\
(\mathbf{k m})\end{array}$} & \multirow[t]{2}{*}{ Ref } & \multicolumn{2}{|c|}{ USGS } & \multirow[t]{2}{*}{ Other } & \multirow{2}{*}{$\begin{array}{c}\text { Moment } \\
\text { M }\end{array}$} & \multirow[t]{2}{*}{ MM } & \multirow[t]{2}{*}{ Ref } & \\
\hline $\mathrm{Yr}$ & Mo & $\mathrm{Da}$ & $h \mathrm{~m} s$ & & & & & $m_{b}$ & $\mathbf{M}_{\mathbf{s}}$ & & & & & \\
\hline $\begin{array}{l}1987 \\
1987 \\
1987 \\
1987 \\
1987\end{array}$ & $\begin{array}{l}05 \\
05 \\
06 \\
06 \\
07\end{array}$ & $\begin{array}{l}06 \\
10 \\
21 \\
21 \\
05\end{array}$ & $\begin{array}{l}040614.1 \\
093906.6 \\
054610.0 \\
055526.9 \\
092300.0\end{array}$ & $\begin{array}{l}51.272 \mathrm{~N} \\
51.394 \mathrm{~N} \\
54.211 \mathrm{~N} \\
54.285 \mathrm{~N} \\
51.486 \mathrm{~N}\end{array}$ & $\begin{array}{l}179.898 \mathrm{~W} \\
179.820 \mathrm{~W} \\
162.601 \mathrm{~W} \\
162.597 \mathrm{~W} \\
174.660 \mathrm{~W}\end{array}$ & $\begin{array}{l}020 \\
033 \\
034 \\
033 \\
033\end{array}$ & $\begin{array}{l}74 \\
74 \\
74 \\
74 \\
74\end{array}$ & $\begin{array}{l}6.3 \\
5.0 \\
6.2 \\
5.5 \\
5.5\end{array}$ & $\frac{\frac{6.4}{6.2}}{5.2}$ & $\begin{array}{ll}6.10 M_{L} & P M R \\
5.50 M_{L} & P M R \\
6.60 M_{L} & P M R \\
5.40 M_{L} & P M R \\
5.40 M_{L} & P M R\end{array}$ & $\begin{array}{l}6.57 \mathrm{HAV} \\
6.50 \mathrm{HAV} \\
5 . \overline{\mathrm{HHAV}}\end{array}$ & $\frac{\mathrm{v}}{\mathrm{V}}$ & $\begin{array}{r}577 \\
577 \\
577 \\
74\end{array}$ & $\begin{array}{l}E \\
z\end{array}$ \\
\hline $\begin{array}{l}1987 \\
1987 \\
1987 \\
1987 \\
1987\end{array}$ & $\begin{array}{l}07 \\
07 \\
08 \\
08 \\
09\end{array}$ & $\begin{array}{l}06 \\
24 \\
14 \\
22 \\
10\end{array}$ & $\begin{array}{ll}002325.6 \\
052510.5 \\
173932.2 \\
050914.4 \\
034844.8\end{array}$ & $\begin{array}{l}51.508 \mathrm{~N} \\
56.231 \mathrm{~N} \\
53.416 \mathrm{~N} \\
52.164 \mathrm{~N} \\
51.931 \mathrm{~N}\end{array}$ & $\begin{array}{l}174.721 \mathrm{~W} \\
153.650 \mathrm{~W} \\
169.113 \mathrm{~W} \\
174.066 \mathrm{E} \\
176.006 \mathrm{~W}\end{array}$ & $\begin{array}{l}033 \\
033 \\
118 \\
033 \\
049\end{array}$ & $\begin{array}{l}74 \\
74 \\
74 \\
74 \\
74\end{array}$ & $\begin{array}{l}5.8 \\
5.5 \\
5.7 \\
5.5 \\
5.1\end{array}$ & $\begin{array}{l}5.5 \\
5.3 \\
\overline{4.8} \\
-\end{array}$ & $\begin{array}{rl}5.80 \mathrm{M}_{\mathrm{L}} & \text { PMR } \\
5.70 \mathrm{M}_{\mathrm{L}} & \mathrm{PMR} \\
-\overline{\mathrm{M}}_{\mathrm{L}} & \\
5.20 \mathrm{M}_{\mathrm{L}} & \mathrm{PMR} \\
5.50 \mathrm{M}_{\mathrm{L}} & \mathrm{PMR}\end{array}$ & $\begin{array}{c}\text { 5.89HAV } \\
\text { 5.65HAV } \\
\text { 5.98HAV } \\
\text { 5.21HAV } \\
-\end{array}$ & $\begin{array}{l}\text { IV } \\
\text { III } \\
\text { IV } \\
\text { IV } \\
\text { IV }\end{array}$ & $\begin{array}{r}577 \\
577 \\
74 \\
577 \\
74\end{array}$ & $\begin{array}{l}\overline{-} \\
\overline{-}\end{array}$ \\
\hline $\begin{array}{l}1987 \\
1987 \\
1987 \\
1987 \\
1987\end{array}$ & $\begin{array}{l}10 \\
11 \\
11 \\
11 \\
11\end{array}$ & $\begin{array}{l}20 \\
17 \\
17 \\
18 \\
23\end{array}$ & $\begin{array}{lll}09 & 23 & 36.2 \\
08 & 46 & 53.3 \\
09 & 38 & 12.3 \\
13 & 01 & 55.2 \\
07 & 18 & 20.5\end{array}$ & $\begin{array}{l}52.577 \mathrm{~N} \\
58.586 \mathrm{~N} \\
58.608 \mathrm{~N} \\
58.642 \mathrm{~N} \\
61.616 \mathrm{~N}\end{array}$ & $\begin{array}{l}172.320 \mathrm{E} \\
143.270 \mathrm{~W} \\
143.096 \mathrm{~W} \\
143.190 \mathrm{~W} \\
141.323 \mathrm{~W}\end{array}$ & $\begin{array}{l}033 \\
010 \\
010 \\
010 \\
005\end{array}$ & $\begin{array}{l}74 \\
74 \\
74 \\
74 \\
74\end{array}$ & $\begin{array}{l}5.5 \\
6.6 \\
5.5 \\
5.2 \\
5.7\end{array}$ & $\begin{array}{l}5.6 \\
6.9 \\
5.6 \\
5.0\end{array}$ & $\begin{array}{ll}5.50 M_{L} & \text { PMR } \\
7.00 M_{L} & \text { PMR } \\
5.20 M_{L} & \text { PMR } \\
5.60 M_{L} & \text { PMR } \\
5.40 M_{L} & \text { PMR }\end{array}$ & $\begin{array}{c}\text { 5.95HAV } \\
7.16 \mathrm{HAV} \\
-\overline{78 H A V} \\
5.50 \mathrm{HAV}\end{array}$ & $\frac{\bar{v}}{\overline{I V}}$ & $\frac{577}{5 \overline{77}}$ & $\begin{array}{l}\overline{375 \&} \\
- \\
-\end{array}$ \\
\hline $\begin{array}{l}1987 \\
1987 \\
1987 \\
1988 \\
1988\end{array}$ & $\begin{array}{l}11 \\
11 \\
12 \\
01 \\
01\end{array}$ & $\begin{array}{l}30 \\
30 \\
01 \\
13 \\
23\end{array}$ & $\begin{array}{lll}19 & 23 & 19.5 \\
19 & 48 & 26.0 \\
12 & 03 & 59.7 \\
01 & 01 & 50.2 \\
02 & 45 & 35.7\end{array}$ & $\begin{array}{l}58.679 \mathrm{~N} \\
58.239 \mathrm{~N} \\
57.953 \mathrm{~N} \\
51.309 \mathrm{~N} \\
51.400 \mathrm{~N}\end{array}$ & $\begin{array}{l}142.786 \mathrm{~W} \\
142.742 \mathrm{~W} \\
142.611 \mathrm{~W} \\
174.654 \mathrm{~W} \\
174.268 \mathrm{~W}\end{array}$ & $\begin{array}{l}010 \\
010 \\
010 \\
033 \\
048\end{array}$ & $\begin{array}{l}74 \\
74 \\
74 \\
74 \\
74\end{array}$ & $\begin{array}{l}6.7 \\
5.9 \\
5.4 \\
5.6 \\
5.5\end{array}$ & $\begin{array}{l}7.6 \\
5.7 \\
5.2 \\
4.7\end{array}$ & $\begin{array}{cc}7.10 \mathrm{M}_{\mathrm{L}} & \text { PMR } \\
5.60 \mathrm{M}_{\mathrm{L}} & \mathrm{PMR} \\
5.60 \mathrm{M}_{\mathrm{L}} & \mathrm{PMR} \\
- & \\
- & \end{array}$ & $\begin{array}{c}\text { 7.86HAV } \\
\text { 5.77HAV } \\
\text { 5.51HAV } \\
\text { 5.02HAV }\end{array}$ & $\begin{array}{c}\text { VI } \\
\text { Felt } \\
= \\
-\end{array}$ & $\begin{array}{r}577 \\
74 \\
= \\
-\end{array}$ & $\begin{array}{l}470 \& \\
- \\
-\end{array}$ \\
\hline $\begin{array}{l}1988 \\
1988 \\
1988 \\
1988 \\
1988\end{array}$ & $\begin{array}{l}02 \\
02 \\
02 \\
02 \\
02\end{array}$ & $\begin{array}{l}07 \\
07 \\
13 \\
13 \\
16\end{array}$ & $\begin{array}{lll}08 & 46 & 58.6 \\
18 & 15 & 05.6 \\
23 & 56 & 58.7 \\
23 & 57 & 45.6 \\
04 & 22 & 36.1\end{array}$ & $\begin{array}{l}60.296 \mathrm{~N} \\
50.785 \mathrm{~N} \\
50.636 \mathrm{~N} \\
52.296 \mathrm{~N} \\
51.564 \mathrm{~N}\end{array}$ & $\begin{array}{l}152.972 \mathrm{~W} \\
173.465 \mathrm{E} \\
173.410 \mathrm{E} \\
173.379 \mathrm{~W} \\
175.041 \mathrm{E}\end{array}$ & $\begin{array}{l}138 \\
033 \\
033 \\
054 \\
033\end{array}$ & $\begin{array}{l}74 \\
74 \\
74 \\
74 \\
74\end{array}$ & $\begin{array}{l}5.6 \\
6.2 \\
5.2 \\
5.7 \\
5.9\end{array}$ & $\begin{array}{l}\overline{6.0} \\
5.5 \\
\overline{5.7}\end{array}$ & $\begin{array}{cl}5.60 \mathrm{~m}_{\mathrm{b}} & \text { BRK } \\
5.90 \mathrm{M}_{\mathrm{S}} & \text { PAS } \\
- & \\
5.60 \overline{\mathrm{M}}_{\mathrm{L}} & \text { PMR }\end{array}$ & $\begin{array}{c}\text { 6.46HAV } \\
6.30 \mathrm{HAV} \\
5.21 \mathrm{HAV} \\
5.98 \mathrm{HAV}\end{array}$ & $\frac{\text { V }}{\text { III }}$ & $\begin{array}{l}578 \\
578 \\
\frac{74}{-}\end{array}$ & $\frac{175}{-}$ \\
\hline $\begin{array}{l}1988 \\
1988 \\
1988 \\
1988 \\
1988\end{array}$ & $\begin{array}{l}02 \\
02 \\
03 \\
03 \\
03\end{array}$ & $\begin{array}{l}16 \\
24 \\
06 \\
06 \\
08\end{array}$ & $\begin{array}{lll}05 & 44 & 38.6 \\
02 & 54 & 22.6 \\
22 & 35 & 38.1 \\
23 & 14 & 38.4 \\
16 & 27 & 18.8\end{array}$ & $\begin{array}{l}51.495 \mathrm{~N} \\
51.723 \mathrm{~N} \\
56.953 \mathrm{~N} \\
57.499 \mathrm{~N} \\
51.340 \mathrm{~N}\end{array}$ & $\begin{array}{l}175.054 \mathrm{E} \\
176.797 \mathrm{~W} \\
143.032 \mathrm{~W} \\
142.803 \mathrm{~W} \\
176.862 \mathrm{E}\end{array}$ & $\begin{array}{l}033 \\
060 \\
010 \\
010 \\
033\end{array}$ & $\begin{array}{l}74 \\
74 \\
74 \\
74 \\
74\end{array}$ & $\begin{array}{l}5.5 \\
5.5 \\
6.8 \\
6.2 \\
5.5\end{array}$ & $\frac{5.0}{\overline{7.6}}$ & $\begin{array}{cl}5.00 \mathrm{M}_{\mathrm{L}} & \text { PMR } \\
\overline{\mathrm{N}} & \\
7.40 \mathrm{M}_{\mathrm{L}} & \mathrm{PMR} \\
6.30 \mathrm{M}_{\mathrm{L}} & \mathrm{PMR} \\
5.50 \mathrm{M}_{\mathrm{L}} & \mathrm{PMR}\end{array}$ & $\begin{array}{c}\text { 5.54HAV } \\
5.34 \mathrm{HAV} \\
7.74 \mathrm{HAV} \\
5 . \overline{1 \mathrm{HAV}}\end{array}$ & $\begin{array}{l}\overline{\text { III }} \\
\text { v } \\
-\end{array}$ & $\begin{array}{r}\overline{74} \\
578 \\
- \\
-\end{array}$ & $\begin{array}{l}\overline{-} \\
580 \& \\
-\end{array}$ \\
\hline $\begin{array}{l}1988 \\
1988 \\
1988 \\
1988 \\
1988\end{array}$ & $\begin{array}{l}03 \\
03 \\
04 \\
05 \\
05\end{array}$ & $\begin{array}{l}25 \\
29 \\
26 \\
22 \\
25\end{array}$ & $\begin{array}{lll}21 & 58 & 20.5 \\
08 & 31 & 31.9 \\
01 & 47 & 35.0 \\
09 & 39 & 55.9 \\
14 & 05 & 17.6\end{array}$ & $\begin{array}{l}54.776 \mathrm{~N} \\
52.278 \mathrm{~N} \\
57.534 \mathrm{~N} \\
53.619 \mathrm{~N} \\
50.549 \mathrm{~N}\end{array}$ & $\begin{array}{l}159.840 \mathrm{~W} \\
168.182 \mathrm{~W} \\
143.073 \mathrm{~W} \\
163.267 \mathrm{~W} \\
174.571 \mathrm{~W}\end{array}$ & $\begin{array}{l}033 \\
033 \\
010 \\
033 \\
040\end{array}$ & $\begin{array}{l}74 \\
74 \\
74 \\
74 \\
74\end{array}$ & $\begin{array}{l}5.4 \\
5.4 \\
5.4 \\
5.7 \\
5.7\end{array}$ & $\begin{array}{l}4.6 \\
5.5 \\
5.6 \\
5.7 \\
4.9\end{array}$ & $\begin{array}{ll}5.90 \mathrm{M}_{\mathrm{L}} & \text { PMR } \\
5.10 \mathrm{M}_{\mathrm{L}} & \text { PMR } \\
5.90 \mathrm{M}_{\mathrm{L}} & \mathrm{PMR} \\
5.70 \mathrm{M}_{\mathrm{L}} & \text { PMR } \\
5.30 \mathrm{M}_{\mathrm{S}} & \mathrm{BRK}\end{array}$ & $\begin{array}{l}\text { 5.79HAV } \\
5.83 \mathrm{HAV} \\
5.95 \mathrm{HAV} \\
5.58 \mathrm{HAV}\end{array}$ & $\frac{\text { IV }}{\text { Felt }}$ & $\frac{74}{578}$ & $\frac{-}{-}$ \\
\hline $\begin{array}{l}1988 \\
1988 \\
1988 \\
1989 \\
1989\end{array}$ & $\begin{array}{l}11 \\
11 \\
11 \\
01 \\
01\end{array}$ & $\begin{array}{l}06 \\
15 \\
30 \\
08 \\
08\end{array}$ & $\begin{array}{lll}08 & 2057.0 \\
08 & 41 & 42.3 \\
08 & 5530.6 \\
19 & 57 & 06.0 \\
20 & 2625.1\end{array}$ & $\begin{array}{l}51.311 \mathrm{~N} \\
52.109 \mathrm{~N} \\
61.348 \mathrm{~N} \\
51.435 \mathrm{~N} \\
51.432 \mathrm{~N}\end{array}$ & $\begin{array}{l}178.148 \mathrm{~W} \\
171.103 \mathrm{~W} \\
152.270 \mathrm{~W} \\
174.880 \mathrm{~W} \\
174.799 \mathrm{~W}\end{array}$ & $\begin{array}{l}033 \\
023 \\
144 \\
033 \\
033\end{array}$ & $\begin{array}{l}74 \\
74 \\
74 \\
74 \\
74\end{array}$ & $\begin{array}{l}5.5 \\
5.9 \\
5.5 \\
5.7 \\
5.1\end{array}$ & $\begin{array}{l}4.8 \\
5.4 \\
5.5 \\
5.6\end{array}$ & $\begin{array}{cc}\overline{5}_{5.50 \mathrm{M}_{S}} & \text { BRK } \\
-\overline{\mathrm{M}}_{\mathrm{S}} & \mathrm{BRK} \\
5.70 \mathrm{M}_{\mathrm{S}} & \mathrm{BR} \\
5.70 \mathrm{M}_{\mathrm{L}} & \text { PMR }\end{array}$ & $\begin{array}{l}\text { 5.91HAV } \\
5.73 \mathrm{HAV} \\
6.00 \mathrm{HAV} \\
5.44 \mathrm{HAV}\end{array}$ & $\begin{array}{r}\text { III } \\
\text { Felt } \\
\text { V } \\
\text { III } \\
\text { III }\end{array}$ & $\begin{array}{r}578 \\
74 \\
578 \\
579 \\
579\end{array}$ & $\begin{array}{l}- \\
\overline{90} \\
-\end{array}$ \\
\hline $\begin{array}{l}1989 \\
1989 \\
1989 \\
1989 \\
1989\end{array}$ & $\begin{array}{l}01 \\
02 \\
04 \\
04 \\
05\end{array}$ & $\begin{array}{l}08 \\
22 \\
09 \\
23 \\
19\end{array}$ & $\begin{array}{lll}22 & 3730.9 \\
1025 & 45.2 \\
050750.6 \\
192106.4 \\
022156.3\end{array}$ & $\begin{array}{l}51.393 \mathrm{~N} \\
56.152 \mathrm{~N} \\
51.510 \mathrm{~N} \\
66.960 \mathrm{~N} \\
54.305 \mathrm{~N}\end{array}$ & $\begin{array}{l}174.758 \mathrm{~W} \\
153.642 \mathrm{~W} \\
178.386 \mathrm{~W} \\
156.289 \mathrm{~W} \\
165.574 \mathrm{~W}\end{array}$ & $\begin{array}{l}033 \\
033 \\
033 \\
006 \\
104\end{array}$ & $\begin{array}{l}74 \\
74 \\
74 \\
74 \\
74\end{array}$ & $\begin{array}{l}5.6 \\
5.7 \\
5.2 \\
5.7 \\
6.1\end{array}$ & $\begin{array}{l}5.4 \\
5.8 \\
4.7 \\
5.1 \\
-\end{array}$ & $\begin{array}{ll}5.80 M_{L} & \text { PMR } \\
5.80 M_{L} & \text { PMR } \\
5.70 M_{L} & \text { PMR } \\
5.30 M_{L} & \text { PMR } \\
5.40 M_{S} & \text { BRK }\end{array}$ & $\begin{array}{l}\text { 5.76HAV } \\
5.95 \mathrm{HAV} \\
5.25 \mathrm{HAV} \\
5.45 \mathrm{HAV} \\
6.15 \mathrm{HAV}\end{array}$ & $\underset{\text { V III }}{\frac{\text { III }}{\text { IV }}}$ & $\begin{array}{l}579 \\
579 \\
579 \\
579\end{array}$ & $\begin{array}{l}- \\
- \\
-\end{array}$ \\
\hline $\begin{array}{l}1989 \\
1989 \\
1989 \\
1989 \\
1989\end{array}$ & $\begin{array}{l}06 \\
07 \\
09 \\
09 \\
10\end{array}$ & $\begin{array}{l}16 \\
03 \\
04 \\
20 \\
07\end{array}$ & $\begin{array}{l}105121.5 \\
170955.8 \\
131458.2 \\
131931.9 \\
154829.0\end{array}$ & $\begin{array}{l}57.755 \mathrm{~N} \\
51.617 \mathrm{~N} \\
55.543 \mathrm{~N} \\
51.184 \mathrm{~N} \\
51.314 \mathrm{~N}\end{array}$ & $\begin{array}{l}153.992 \mathrm{~W} \\
175.208 \mathrm{~W} \\
156.835 \mathrm{~W} \\
178.821 \mathrm{E} \\
179.028 \mathrm{~W}\end{array}$ & $\begin{array}{l}058 \\
033 \\
011 \\
033 \\
020\end{array}$ & $\begin{array}{l}74 \\
74 \\
74 \\
74 \\
74\end{array}$ & $\begin{array}{l}5.8 \\
5.7 \\
6.5 \\
5.5 \\
6.1\end{array}$ & $\begin{array}{l}-5.7 \\
6.9 \\
5.8 \\
6.7\end{array}$ & 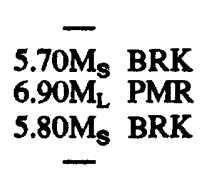 & $\begin{array}{l}\text { 5.67HAV } \\
\text { 6.05HAV } \\
\text { 7.06HAV } \\
\text { 6.22HAV } \\
6.79 \mathrm{HAV}\end{array}$ & $\begin{array}{r}V \\
\text { IV } \\
V \\
\text { Felt } \\
\text { IV }\end{array}$ & $\begin{array}{r}579 \\
579 \\
579 \\
74 \\
579\end{array}$ & $\begin{array}{l}- \\
- \\
-\end{array}$ \\
\hline $\begin{array}{l}1989 \\
1989 \\
1989\end{array}$ & $\begin{array}{l}10 \\
10 \\
10\end{array}$ & $\begin{array}{l}07 \\
07 \\
07\end{array}$ & $\begin{array}{lll}16 & 42 & 30.7 \\
1742 & 36.4 \\
1752 & 47.3\end{array}$ & $\begin{array}{l}51.188 \mathrm{~N} \\
51.137 \mathrm{~N} \\
51.115 \mathrm{~N}\end{array}$ & $\begin{array}{l}179.234 \mathrm{~W} \\
179.221 \mathrm{~W} \\
179.241 \mathrm{~W}\end{array}$ & $\begin{array}{l}033 \\
033 \\
033\end{array}$ & $\begin{array}{l}74 \\
74 \\
74\end{array}$ & $\begin{array}{l}5.7 \\
5.6 \\
5.5\end{array}$ & $\begin{array}{l}5.9 \\
5.7 \\
5.6\end{array}$ & $\begin{array}{c}5.70 \mathrm{M}_{\mathrm{L}} \mathrm{PMR} \\
-\end{array}$ & $\begin{array}{c}\text { 6.19HAV } \\
5.44 \mathrm{HAV} \\
-\end{array}$ & III & $\frac{74}{-}$ & I \\
\hline
\end{tabular}


ALASKA-Continued

[See table 1 for hypocenter and intensity references and table 2 for definitions of magnitude source codes. \&, land area only. Leader (-) indicates information is not available]

\begin{tabular}{|c|c|c|c|c|c|c|c|c|c|c|c|c|c|}
\hline \multirow{2}{*}{\multicolumn{2}{|c|}{ Date }} & \multirow{2}{*}{$\begin{array}{c}\text { Origin } \\
\text { time (UTC) }\end{array}$} & \multicolumn{4}{|c|}{ Hypocenter } & \multicolumn{4}{|c|}{ Magnitude } & \multicolumn{2}{|c|}{ Intensity } & \multirow{3}{*}{$\begin{array}{l}\text { Felt area } \\
\left(1,000 \mathrm{~km}^{2}\right)\end{array}$} \\
\hline & & & \multirow{2}{*}{$\begin{array}{l}\text { Latitude } \\
\left(^{\circ}\right)\end{array}$} & \multirow{2}{*}{$\begin{array}{l}\text { Longitude } \\
\left({ }^{\circ}\right)\end{array}$} & \multirow{2}{*}{$\begin{array}{l}\text { Depth } \\
(\mathrm{km})\end{array}$} & \multirow[t]{2}{*}{ Ref } & \multicolumn{2}{|c|}{ USGS } & \multirow[t]{2}{*}{ Other } & \multirow{2}{*}{$\begin{array}{c}\text { Moment } \\
\mathbf{M}\end{array}$} & \multirow[t]{2}{*}{ MM } & \multirow[t]{2}{*}{ Ref } & \\
\hline$\overline{Y r}$ & Mo Da & $\mathrm{h} m \mathrm{~s}$ & & & & & $m_{b}$ & $\mathbf{M}_{\mathbf{S}}$ & & & & & \\
\hline $\begin{array}{l}1989 \\
1989\end{array}$ & $\begin{array}{ll}10 & 07 \\
10 & 07\end{array}$ & $\begin{array}{l}185040.8 \\
190634.7\end{array}$ & $\begin{array}{l}51.076 \mathrm{~N} \\
51.173 \mathrm{~N}\end{array}$ & $\begin{array}{l}179.306 \mathrm{~W} \\
179.412 \mathrm{~W}\end{array}$ & $\begin{array}{l}033 \\
033\end{array}$ & $\begin{array}{l}74 \\
74\end{array}$ & $\begin{array}{l}5.5 \\
4.9\end{array}$ & 5.3 & $\begin{array}{ll}5.40 \mathrm{M}_{\mathrm{L}} & \text { PMR } \\
5.50 \mathrm{M}_{\mathrm{L}} & \mathrm{PMR}\end{array}$ & $\begin{array}{c}5.68 \mathrm{HAV} \\
-\end{array}$ & III & $\frac{74}{-}$ & - \\
\hline $\begin{array}{l}1989 \\
1989 \\
1989\end{array}$ & $\begin{array}{ll}10 & 09 \\
12 & 01 \\
12 & 21\end{array}$ & $\begin{array}{lll}18 & 01 & 07.8 \\
05 & 06 & 12.1 \\
16 & 53 & 19.3\end{array}$ & $\begin{array}{l}51.780 \mathrm{~N} \\
51.631 \mathrm{~N} \\
62.421 \mathrm{~N}\end{array}$ & $\begin{array}{l}171.869 \mathrm{E} \\
178.102 \mathrm{~W} \\
155.620 \mathrm{~W}\end{array}$ & $\begin{array}{l}026 \\
043 \\
000\end{array}$ & $\begin{array}{l}74 \\
74 \\
74\end{array}$ & $\begin{array}{l}6.0 \\
5.6 \\
5.4\end{array}$ & $\begin{array}{l}5.3 \\
5.0 \\
5.5\end{array}$ & $\begin{array}{l}5.40 M_{L} \text { PMR } \\
5.70 M_{L} \text { PMR } \\
5.50 M_{L} \text { PMR }\end{array}$ & $\begin{array}{c}\text { 5.73HAV } \\
\text { 5.59HAV } \\
-\end{array}$ & $\begin{array}{l}\overline{I V} \\
\text { IV }\end{array}$ & $\begin{array}{r}\overline{74} \\
579\end{array}$ & $\overline{-}$ \\
\hline
\end{tabular}

\section{INTRODUCTION}

Only those earthquakes of Modified Mercalli intensity $\geq$ VI or magnitude $\geq 5.5$ are listed in the Alaska table. Earthquakes without published magnitude or intensity values and those having intensities < VI were estimated to fall within the magnitude $\geq 5.5$ range if they met the following criteria:

1. Pre-1913 earthquakes (the year instrumental epicenters were first published) had to be well documented. It is believed that most shocks before 1913 would have to have a magnitude larger than 5.0 to generate sufficient interest for detailed information to be published;

2. The hypocenter had to be computed using a set of phase data comparable (in terms of reporting seismograph stations and distances recorded) to one published in the International Seismological Summary (ISS) or International Seismological Centre (ISC) bulletins with a computed magnitude $\geq 5.5$. The earthquake must have occurred within 3 years of the ISS or ISC event.

The origin times in the Alaska table may not be identical to those shown in the source reference. If not, the listed origin times were estimated from more accurate sources, such as seismograms, earthquake phase data, U.S. Weather Service reports, and others. Some corrections in dates also were necessary because all sources did not list the same date.

Many of the published origin times were given in "local time" (i.e., the time on the clock at the location reporting the earthquake). Most of these local times in the 1800's were local solar meridian time. We, therefore, converted all the local times to UTC. Other origin-time corrections resulted from differences between local time and the time that the phase data were recorded on seismograms. In those instances, the arrival times published from phase data were used to compute new origin times.
The Alaska table cannot be considered to be a complete listing of magnitude $\geq 5.5$ earthquakes. However, it is as complete as possible on the basis of the selection criteria listed above and on the data available to us. In the early days of seismology, the only earthquakes recorded were those having large magnitudes or those located near populated centers. Because of this, the list of earthquakes can be considered complete only after about 1964, when the installation of worldwide seismograph stations and the use of computers allowed the routine computation of hypocenters and magnitudes for smaller earthquakes.

[Reference (Ref.) numbers given in parentheses at the end of each description refer to sources of data in table 1. Magnitude values are described in the Introduction, and codes are defined in table 2.]

1786. Date unknown. Alaska Peninsula. Following an eruption of Pavlof Volcano, and during a severe earthquake, the north crater of the volcano collapsed with a tremendous noise. (Ref. $38,255,426$, 436,520 .)

1788. July 22. Shumagin Islands-Kodiak Island region, Alaska. Strong ground motion and landslides occurred on both Kodiak and Unga Islands. Land subsidence also was reported on Kodiak near the harbor. The earthquake is described as "a great shock that ruptured at least a $600-\mathrm{km}$ segment of the plate boundary" (extending from Kodiak Island perhaps to Unga Island or beyond). A tsunami perhaps as high as $10 \mathrm{~m}$ inundated Three Saints Bay and caused extensive loss on Kodiak Island. Aftershocks on Kodiak Island lasted a month or longer. (Ref. $38,426,456,520,610$.)

1788. Aug. 7. Unga Island, Alaska. A strong earthquake may have ruptured the plate boundary "from Unga to Sanak on Aug. 7. There was such a terrible flood on Unga Island that many Aleuts were 
killed, and the water rose to 50 sazhens (about 91 m)." The tsunami was much smaller at Pavlof Bay (on the south coast of the Alaska Peninsula) and did not affect the north side of Unimak Island. There is some doubt that this is a second earthquake in 1788 (Ref. 456, 520.)

1792. Date unknown. Kodiak Island, Alaska. A strong earthquake occurred on Kodiak Island, "continuing for 18 hours." All cabins collapsed, and rocks slid down the hillsides. A vessel that was entering Three Saints Bay (Kodiak Island) at the time of the earthquake "encountered a strong agitation and suffered greatly from the unusual and highly anomalous wave patterns." (Ref. 456.)

1796. May 20. Umnak Island, Alaska. An earthquake shook Umnak, and a new island (St. John Bogoslov) appeared in the Unalaska district. A rumble resembling shots from a cannon came from the mountains off the northeast point of Umnak. Rocks were thrown from the new island as far as Umnak Island. (Ref. 38, 436, 520.)

1802. Date unknown. Unalaska Island, Alaska. Makushin Volcano erupted "with great violence," and an earthquake destroyed "a considerable number of the earth huts." (Ref. $38,436,520$.)

1812. Date unknown. Atka Island, Alaska. Sarychef Volcano erupted, and a violent earthquake terrified residents on Atka. (Ref. 38, 255, 426, 436, 520.)

1817. Mar. 14. Umnak Island, Alaska. A violent earthquake occurred. Yunaska Volcano erupted, sending ash as far as Unimak Island. (Ref. 38, 255, 436, 520.)

1818. Apr. Unalaska Island, Alaska. An earthquake occurred following the strong rumbling of Makushin Volcano. To the residents on Unalaska, it seemed that the nearby island of Amakhnak had collapsed. (Ref. 38, 436, 520.)

1826. June. Unalaska Island, Alaska. Two violent earthquakes occurred, during which flames shot out of Makushin Volcano. (Ref. 38, 255, 436, 520.)

1836. Apr. 14. Pribilof Islands, Alaska. Shocks were so strong on St. Paul and St. George Islands that people could not remain standing. The first shocks collapsed many cliffs on the coast of St. George and caused others to settle. A barn collapsed on St. Paul. (Ref. 38, 255, 426, 520.)

1836. Aug. Pribilof Islands, Alaska. The earthquake was less violent and the rumblings were more muted than the shocks and noises experienced in April 1836. (Ref. 38, 255, 426, 436, 520.)

1840. Apr. Alaska Peninsula. A strong earthquake occurred at Nikolaev Redoubt in Kenai Bay
(Kenai Village, Cook Inlet). Stoves fell to pieces and chimneys collapsed. (Ref. 515,520 .)

1843. Dec. 17 (Dec. 16). Sitka, Alaska. At 4 p.m. local time, a strong 3-second earthquake "rent houses" and moved trees back and forth. Cracking noises were heard. Two light foreshocks occurred on Dec. 15 and 16. (Ref. 38, 420, 515, 520.)

1844. Apr. 13. Kodiak Island, Alaska. A strong earthquake accompanied by a loud noise occurred at Pavlof (St. Paul) Harbor. The shock continued for more than 2 minutes and sent residents fleeing from their homes. Older residents reported this shock was the strongest in memory. The epicenter is uncertain. (Ref. 456, 515, 520.)

1847. Date unknown. Sitka, Alaska. This earthquake generally was felt along the Alaska coast and was severe at Sitka. The Governor's mansion was destroyed. Flame and ashes arose from the summit of Mount St. Elias. The epicenter is uncertain (Ref. 38, 436, 520.)

1847. Apr. 16, 16 UTC. Chirikof Island, Alaska. A large earthquake on Ukamok (Chirikof Island) cracked the ground in many places and collapsed cliffs. On Unga Island, Alaska, a "fairly large" earthquake occurred early in the morning. At 10 a.m. (20 UTC), it "grew" to the point that it was impossible to remain standing. At about the same time, a large earthquake was felt on the Alaska Peninsula. These two events, 4 hours and $320 \mathrm{~km}$ apart in time and space, are considered to describe one earthquake; however, the possibility exists that two earthquakes occurred. (Ref. $515,456,520$.)

1848. Mar. 30. Sitka, Alaska. A large earthquake knocked down chimneys, damaged stoves, and cracked stones on many houses. The 15-second shock, which was accompanied by a muffled underground noise, terrified residents. The trembling continued almost without interruption until Apr. 27. (Ref. 515, 520.)

1848. June 30. Chirikof Island, Alaska. An earthquake on Ukamok (Chirikof Island) began about midnight. Several minutes later, the earth shook so hard that it was impossible to remain standing. It was accompanied by a wind-like noise and a rumbling. (Ref. $456,515,520$.)

1866. Sept. 6. Kodiak Island, Alaska. A violent earthquake struck Pavlof (St. Paul) Harbor. It "half destroyed" the landing on Woody Island and damaged many buildings, stoves, and chimneys. Huge rocks were thrown down the mountains. (Ref. 38, $426,515,520$.)

1868. May 15. Shumagin Islands, Alaska. "During a slight earthquake the elevation is said to have amounted locally at Unga to over 20 feet." (Ref. $38,420,610$.) 
1872. Aug. 23. Aleutian Islands region, between the Andreanof and Fox Islands, Alaska. It is suggested that, on Aug. 23, a tectonic displacement of the Aleutian continental shelf or slope was accompanied by an earthquake of large magnitude. A tsunami of $1.3 \mathrm{~m}$ was observed in Hilo harbor, lesser heights were observed at Hanalei, Honolulu, and Nawiliwili, Hawaii. It was also recorded at Honolulu, Hawaii; Astoria, Oreg.; and San Francisco and San Diego, Calif. From the reports of the arrival of the tsunami in Hawaii and marigraphic evidence of its arrival in Oregon and California, its source has been determined to be off the Aleutian Islands. (Ref. 516, 610.)

1878. Aug. 29. Unalaska Island, Alaska. The village of Makushin was destroyed by an earthquake. Major volcanic eruptions and earthquakes occurred on several Aleutian islands in late August and early September 1878. (Ref. 38, 426, 463, 520.)

1880. Sept. 29, 04 UTC (Sept. 28). Chirikof Island, Alaska. A major earthquake occurred, followed by three severe aftershocks at 07,13 , and 23 UTC. Many deep fissures $38-51 \mathrm{~cm}$ in width were observed on Chirikof after the shocks. In a one-story log cabin, shelves were thrown from walls, a brick stove was upset, flooring was twisted out of shape, and heavy barrels were pitched from one side of the room to the other. Outside, no one was able to remain standing owing to a violent jerking and rotary motion of the earth, which continued for at least 20 minutes.

At low tide, the sea rose several times and traveled onshore about $55 \mathrm{~m}$. On the south side of the island, a small, shallow creek widened about $2 \mathrm{~m}$, and its depth increased. On the southwest shore of the island, heavy breakers were observed where they had not been seen previously. On the west side of the island, the tide did not rise as high as before the shocks. From the beginning of the earthquake series through Oct. 16, 1880, an uninterrupted trembling motion, interspersed with heavy subterranean rumbling sounds, was observed.

During these earthquakes, the northwest part of the island, bounded by a northeast-trending fault, tilted to the southeast. The fault on the southeast boundary displaced vertically about $1.8 \mathrm{~m}$. Evidence of this faulting is still preserved in dammed streams and uplifted, wave-cut terraces. (Ref. 426, 463, 520.)

1880. Oct. 26. Sitka, Alaska. A severe earthquake along with cracking and splitting earth noises occurred at Sitka. At the warm springs $32 \mathrm{~km}$ southeast of Sitka, the springs spouted like geysers. The shocks were severe at Whale Bay, about $58 \mathrm{~km}$ southeast, and a "tidal wave of huge dimensions ran into the bay." The shock was violent on Tihiagreff Island (north of Baranof Island). At Hoonah village, people were thrown around "like chips in an eddy." Aftershocks continued through Nov. 14. (Ref. 426, 463.)

1883. Oct. 6. Alaska Peninsula. Mount St. Augustine burst into volcanic activity. It was accompanied by a severe earthquake and tsunami. A wave estimated at 7.6-9.1 m flooded Port Graham, followed by 2 waves of lesser height (Ref. 38, 426.)

1896. Late May. Prince William Sound region, Alaska. About $12 \mathrm{~km}$ north of Orca, this severe earthquake bent trees almost to the breaking point and made it difficult to remain standing. Waves appeared in the ground. Water in the creek splashed from one side to the other. (Ref. 38, 420, 426.)

1898. Aug. 1. Susitna Station, Alaska. Trees swayed violently. (Ref. 420.)

1899. July 11 or July 14. Cook Inlet, Alaska. A "severe" earthquake occurred at Tyonek. Possibly, there is a misprint in the date in ref. 420 (the table in ref. 420 lists earthquakes in chronological order, but the July 11 event is listed between two events on July 14 ), and the date should be July 14, 1899 (magnitude 7.2 , epicenter at lat $60^{\circ} \mathrm{N}$., long $150^{\circ} \mathrm{W}$.). Tyonek is located at lat $61^{\circ} \mathrm{N}$., long $151^{\circ} \mathrm{W}$. (Ref. 38, 412, 420.)

1899. Sept. 4, 0022 UTC (Sept. 3). Near Cape Yakataga, Alaska. During September, the Yakutat Bay region was shaken by a series of severe earthquakes. The first earthquake at 0022 UTC was moderately strong at Yakutat but was extremely violent at Cape Yakataga, about $160 \mathrm{~km}$ west. The shock broke off the tops of trees, generated landslides, and raised the ocean beach about $1 \mathrm{~m}$. Although no loss of life or property occurred in the region, reported effects include uplift of the coast, landslides, difficulty in standing upright, water waves on the bay, and shaking of houses. Faulting probably occurred at Cape Yakataga. The shock was felt at about 30 known locations, the most distant on the lower Yukon River, about 1,100 km from Yakutat Bay. Strong aftershocks were observed on Sept. 4. Magnitude of first shock, $8.3 \mathrm{M}_{\mathrm{S}}$ CFR. (Ref. 38, 404, $412,420,424$.)

1899. Sept. 10, 1704 and 2141 UTC. Yakutat Bay, Alaska. During September, the Yakutat Bay region was shaken by a series of major earthquakes, the most violent of which were felt at all settlements within a radius of $400 \mathrm{~km}$. Several heavy shocks occurred on Sept. 4 and 10, but the main earthquake that caused great topographic changes occurred at 21 41 UTC, Sept. 10 (see fig. 8.)

A U.S. Geological Survey team did not study the region until 6 years after the shocks, but the topographic changes were obvious. Dead barnacles and 
other shellfish were found everywhere, and several uplifted beaches were observed. A maximum uplift of $14.5 \mathrm{~m}$ occurred on the west coast of Disenchantment Bay, and changes of $5 \mathrm{~m}$ or more affected a large area. Subsidence of as much as $2 \mathrm{~m}$ was observed in a few areas. Phenomena observed included surface faulting, avalanches, fissures, spouting from sand craterlets, and slight damage to buildings. A destructive tsunami $10.6 \mathrm{~m}$ in height occurred in Yakutat Bay, and tsunamis also were observed at other places along the Alaskan coast.

The earthquake altered the regimen of glaciers in the area. The shattering of Muir Glacier started the rapid discharge of icebergs and the later retreat of this and other ice tongues in Glacier Bay. Avalanching resulted in the later advance of at least nine glaciers in Yakutat Bay and perhaps many others in more remote regions. Some severely crevassed glacier fronts, which were found 6 years later, had taken several years for the fractured parts to reach the sea.

The first earthquake on Sept. 10 lasted 90 seconds and was heavier at Yakutat than that of Sept. $4(00$ 22 UTC). It was strong enough to throw people off their feet at Disenchantment Bay. The main earthquake on Sept. 10 was felt over a largely unsettled region, and so the total felt area is unknown. Prospectors camped on Disenchantment Bay felt over 50 shocks on Sept. 10, two of which were strong. Residents at Yakutat village also described as severe two of the many shocks observed on that day. Ten or more earthquakes were felt in the Coast and Geodetic Survey camp near the Copper River delta, and several of them were violent. Several shocks were also felt on Sept. 10 in the Chugach Mountains near Prince William Sound; five were reported about 300 $\mathrm{km}$ to the northeast on the Yukon River; and several were felt to the southeast at Juneau and Skagway. Many large aftershocks occurred in September and the following months. Magnitude $7.8 \mathrm{M}_{\mathrm{S}}$ CFR (first shock), 8.6 $\mathrm{M}_{\mathrm{S}} \mathrm{CFR}$ (second shock). (Ref. 38, 404, 420,426 .)

1899. Sept. 16 (Sept. 15). Yakutat Bay, Alaska. Two strong earthquakes were felt at Yakutat village, each lasting as long as it took to run outdoors. At Skagway, this shock was more pronounced than those of Sept. 4 and 10. A section of one of the long piers at Skagway sank into the water. Several buildings moved "a foot or two" on their foundations, and two small buildings toppled. (Ref. 420.)

1899. Sept. 23, 1104 and 1250 UTC. Near the Copper River delta, Alaska. Eight shocks were noted in the Coast and Geodetic Survey camp, one of which was strong enough to awaken everyone in the camp. A plumb bob vibrated through $25 \mathrm{~cm}$ from northwest to southeast; the vibrations were distinct and slow. Possibly felt at Sitka. (Ref. 412, 420.)

1900. Oct. 9. Kodiak Island region, Alaska. The wharf at Woody Island was partly destroyed; chimneys, windows, and crockery were destroyed in Kodiak. About 50 slight aftershocks continued through the next day. Felt along all of southern Alaska and probably to the west of Kodiak. Magnitude 8.3 $\mathrm{M}_{\mathrm{S}}$ CFR. (Ref. 420, 424, 477.)

1901. Dec. 30. Kenai, Alaska. A strong volcanic eruption occurred. The accompanying earthquake caused several tsunamis. This event is listed as "destructive" in Ref. 426. (Ref. 38, 426.)

1901. Dec. 31 (Dec. 30). Cook Inlet, Alaska. A strong volcanic eruption occurred. The accompanying earthquake caused several tsunamis. Magnitude 7.8 $\mathrm{M}_{\mathrm{S}}$ CFR. (Ref. 38, 477.)

1902. Jan. 1 (Dec. 31, 1901). Fox Islands, Aleutian Islands, Alaska. Magnitude $7.8 \mathrm{M}_{\mathrm{S}}$ CFR. (Ref. 404.)

1903. June 2. Southwest Alaska. Magnitude 8.3 $\mathrm{M}_{\mathrm{S}}$ CFR. (Ref. 38, 477.)

1904. Aug. 27. Rampart, Alaska. Buildings swayed and cracked. Magnitude 8.3 $\mathrm{M}_{\mathrm{S}} \mathrm{CFR}, 7.7 \mathrm{M}_{\mathrm{S}}$ GR, $7.8 \mathrm{~m}_{\mathrm{b}} \mathrm{ABE}$. (Ref. 38, 477.)

1905. Feb. 14 (Feb. 13). Andreanof Islands, Aleutian Islands, Alaska. Magnitude $7.9 \mathrm{M}_{\mathrm{S}} \mathrm{CFR}$, 7.7 $\mathrm{M}_{\mathrm{S}}$ GR, $7.5 \mathrm{~m}_{\mathrm{b}}$ ABE. (Ref. 477.)

1906. Aug. 17 (Aug. 16). Rat Islands, Aleutian Islands, Alaska. Magnitude $8.3 \mathrm{M}_{\mathrm{S}} \mathrm{CFR}, 8.0 \mathrm{M}_{\mathrm{S}}$ GR. (Ref. 477.)

1907. Sept. 2. Aleutian Islands, Alaska. Magnitude 7.4 $\mathrm{M}_{\mathrm{S}} \mathrm{GR}, 7.3 \mathrm{~m}_{\mathrm{b}} \mathrm{ABE}$. (Ref. 477.)

1908. Feb. 14. Prince William Sound, Alaska. This earthquake was strong at Valdez, where it upset bottles and vases and threw objects from shelves in all the stores. The Valdez-Sitka and Valdez-Seward submarine cables were broken close to Valdez, well inside Valdez Narrows. A ship approaching the Valdez dock reported it "felt as though the ship struck on bottom." Also felt at Cordova, Ellamar, Katalla, Landlock, and Latouche. (Ref. 38, 420.)

1908. May 15 (May 14). Southeast Alaska. At Katalla, the ceiling in one house was cracked, furniture was displaced, and dishes were knocked from shelves. Rockslides were reported at Cape Yakataga. Felt from Sitka to Seward. Magnitude 7.0 $\mathrm{M}_{\mathrm{S}}$ GR, 7.1 $\mathrm{m}_{\mathrm{b}} \mathrm{ABE}$. (Ref. 38, 258, 420.)

1909. Feb. 16. Near Yakutat, Alaska. This earthquake was felt more strongly at Skagway and Yakutat than at Juneau, Seward, and Sitka. (Ref. 420.)

1910. Sept. 9 (Sept. 8). Rat Islands, Aleutian Islands, Alaska. The earthquake displaced furniture 


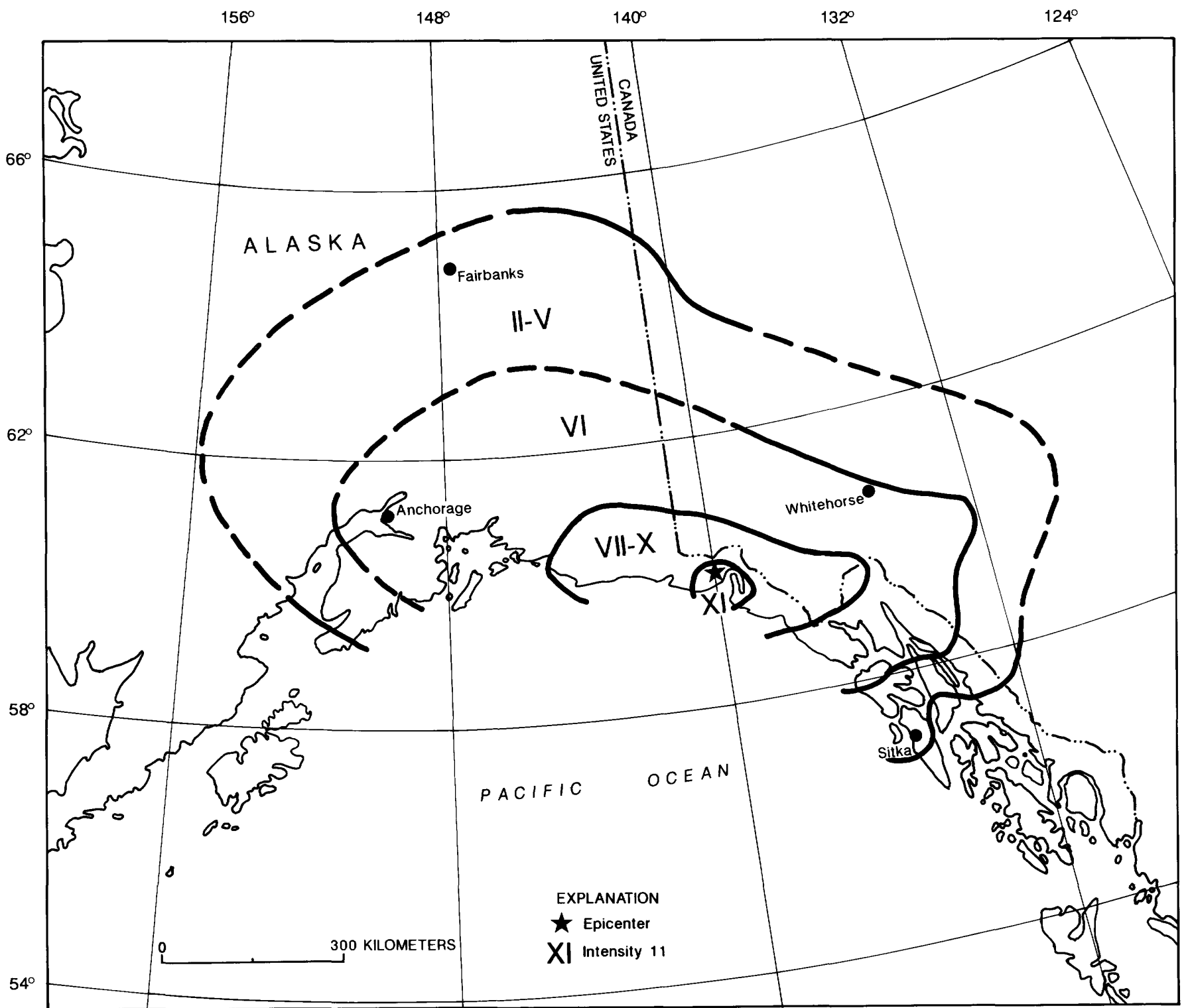

FIGURE 8.-Isoseismal map for the Yakutat Bay, Alaska, earthquake of September 10, 1899. This map is a simplified version of figure 4 in reference 424 of table 1.

in some houses. Magnitude 7.1 $\mathrm{M}_{\mathrm{S}} \mathrm{GR}, 7.1 \mathrm{~m}_{\mathrm{b}} \mathrm{ABE}$. (Ref. 38, 477.)

1911. Sept. 17 (Sept. 16). Rat Islands, Aleutian Islands, Alaska. Magnitude $7.0 \mathrm{mb}_{\mathrm{b}} \mathrm{ABE}$. (Ref. 412.)

1911. Sept. 22 (Sept. 21). Prince William Sound region, Alaska. This severe earthquake broke a submarine cable near Valdez, close to a point where the cable was broken in the earthquake of Feb. 14, 1908. Objects fell from shelves at Valdez. Large rockslides occurred at the head of the sound. At Golden, on the shores of Wells Bay in northern Prince William Sound, rockslides buried a gulch. One huge slide, carrying with it a part of a small glacier, "passed within a few hundred feet of the town." The shock also was "heavy" in the mountains of Kenai Peninsula, which borders the western shore of Prince William Sound. An observer north of Kenai Lake reported that it was difficult to remain upright, trees swayed, and rocks rolled down the mountain slopes. Several aftershocks were observed in the area. (Ref. $38,258,420$.)

1911. Nov. 13. Near Islands, Aleutian Islands, Alaska. Magnitude $7.0 \mathrm{~m}_{\mathrm{b}} \mathrm{ABE}$. (Ref. 428.)

1912. Jan. 4. Andreanof Islands, Aleutian Islands, Alaska. Magnitude $7.0 \mathrm{M}_{\mathrm{S}} \mathrm{GR}, 7.0 \mathrm{~m}_{\mathrm{b}}$ ABE. (Ref. 258.) 
1912. June 10. Cook Inlet, Alaska. Magnitude 7.0 $\mathrm{M}_{\mathrm{S}} \mathrm{GR}, 7.3 \mathrm{~m}_{\mathrm{b}} \mathrm{ABE}$. (Ref. 258.)

1912. July 7 (July 6). Central Alaska. This earthquake was violent at Fairbanks and strong at Kennicott. The earth "heaved and rolled" at the north base of Mount McKinley, and the country was scarred with landslides. Magnitude 7.4 $\mathrm{M}_{\mathrm{S}}$ GR, 7.3 $\mathrm{m}_{\mathrm{b}}$ ABE. (Ref. 38, 437.)

1919. Dec. 15 (Dec. 14). Juneau area, Alaska. Buildings were badly shaken by "one of the heaviest earthquakes experienced here in years." (Ref. 38, 272.)

1923. May 4. Alaska Peninsula area, Alaska. Magnitude $7.0 \mathrm{~m}_{\mathrm{b}}$ ABE. (Ref. 432.)

1925. Feb. 23. Gulf of Alaska. Several chimneys were destroyed and walls were cracked at Anchorage and Seward. One bridgehead was cracked, and the submarine cable between Seward and Valdez was severed. Part of a dock collapsed at Valdez. Two shocks, the second of which was stronger, followed the first within a few seconds. Several aftershocks were observed to Feb. 28. (Ref. 38, 218, 265, 610.)

1926. Oct. 13, 1908 UTC. Andreanof Islands, Aleutian Islands, Alaska. Magnitude 7.1 $\mathrm{M}_{\mathrm{S}}$ GR, $7.1 \mathrm{~m}_{\mathrm{b}}$ ABE. (Ref. 432.)

1927. Oct. 24. Southeast Alaska. Submarine cables were broken between Petersburg and Wrangell and between Juneau and Skagway. At Sitka, cracks formed in buildings and people were thrown from their feet. Near Hoonah, water in Icy Straits was "churned and muddy," and strong "tide rips" were reported on Cross Sound. A few windows were broken at Juneau, and several hundred were broken at Petersburg. At Seattle, Wash., small waves were observed on water in swimming pools. Felt throughout southeast Alaska, west to Cordova, and to the north of Fairbanks. Magnitude $7.1 \mathrm{M}_{\mathrm{S}}$ GR, $7.1 \mathrm{~m}_{\mathrm{b}}$ ABE. (Ref. 38, 218, 432, 477.)

1928. June 21. Gulf of Alaska. Three distinct shocks were felt in the Cordova area, the second of which was the strongest. Men were thrown from their bunks $48 \mathrm{~km}$ north of Cordova; plaster cracked at Cordova; and landslides occurred at several places in the mountains. The earthquake was "heavy" at Valdez. Also felt at Anchorage, Chickaloon, Matanuska, and Seward. Magnitude $7.0 \mathrm{M}_{\mathrm{S}} \mathrm{GR}, 7.3 \mathrm{~m}_{\mathrm{b}}$ ABE. (Ref. 1, 258, 477.)

1929. Jan. 21. Near Fairbanks, Alaska. The first earthquake was the most severe at Fairbanks, where it lasted several seconds. Windows were broken, furniture was displaced, and many residents rushed into the streets. Heavy rumbling was heard. The shocks lasted about 4 hours and continued into the next day. (Ref. 2, 258.)
1929. Mar. 7, 0134 UTC (Mar. 6). Fox Islands, Aleutian Islands, Alaska. This earthquake was felt onboard several ships at sea and severely at Dutch Harbor, Alaska. A small tsunami was recorded at Hilo, Hawaii. Magnitude 8.3 $\mathrm{M}_{\mathrm{S}} \mathrm{CFR}, 8.1 \mathrm{M}_{\mathrm{S}} \mathrm{GR}, 7.9$ $\mathrm{m}_{\mathrm{b}} \mathrm{ABE}$. (Ref. $2,432,610$.)

1929. July 5, 1419 UTC. Andreanof Islands, Aleutian Islands, Alaska. Magnitude $7.0 \mathrm{M}_{\mathrm{S}} \mathrm{GR}$, $6.9 \mathrm{~m}_{\mathrm{b}} \mathrm{ABE}$. (Ref. 432.)

1929. July 7. Andreanof Islands, Aleutian Islands, Alaska. Magnitude $7.3 \mathrm{M}_{\mathrm{S}} \mathrm{GR}, 7.4 \mathrm{mb}$ ABE. (Ref. 432.)

1929. Dec. 17, 1058 UTC. Near Islands area, Aleutian Islands, Alaska. Magnitude $7.6 \mathrm{M}_{\mathrm{S}} \mathrm{GR}$, $7.4 \mathrm{~m}_{\mathrm{b}}$ ABE. (Ref. 432.)

1931. May 30, 11 34 UTC. Attu Island, Alaska. The earthquake broke dishes and overturned small objects on Attu. Almost all canned goods in a store were thrown to the floor. (Ref. 38, 265.)

1932. Mar. 25, 2358 UTC. South-central Alaska. This earthquake was strongest at Seward, where it broke a water main; landslides occurred on the railroad to the north. (Ref. 5, 38, 258.)

1933. Jan. 4 (Jan. 3). Southern Alaska. Ground cracks formed in the streets of Seward and at several other points in Alaska. The valley leading north from Seward was noticeably disturbed; cracks formed in the Forestry Bureau road for a distance of about $32 \mathrm{~km}$ to Kenai Lake. Felt at several towns in the region. Magnitude $7.0 \mathrm{M}_{\mathrm{S}} \mathrm{GR}, 7.1 \mathrm{~m}_{\mathrm{b}} \mathrm{ABE}$. (Ref. $6,38,258,477$.

1933. Apr. 27 (Apr. 26). Near Anchorage, Alaska. Houses were shaken from their foundations at Old Tyonek, west of Anchorage across Cook Inlet. Plate-glass windows were broken in several stores in Anchorage, and merchandise tumbled from shelves. Telegraph lines were down for a distance of $80 \mathrm{~km}$ from Anchorage. The shock was felt strongly on Kodiak Island and along the Aleutian Islands. Many aftershocks occurred. Magnitude $7.0 \mathrm{M}_{\mathrm{S}} \mathrm{GR}, 7.1 \mathrm{~m}_{\mathrm{b}}$ ABE. (Ref. 6, 38, 258.)

1934. May 4 (May 3). Southern Alaska. At Anchorage, windows were broken, stock in stores was jarred from shelves, and telephone lines were "knocked out of commission." Felt at several towns in the region. (Ref. 7, 258.)

1934. May 14. Kodiak Island region, Alaska. Kodiak and Whale Islands were shaken severely. Plaster cracked at Kodiak and stock fell from shelves. A landslide occurred near Kodiak. The shock "probably was almost as severe" on Afognak and Whale Islands. (Ref. 7, 258.)

1935. Feb. 22. Near Islands, Aleutian Islands, Alaska. Magnitude $6.9 \mathrm{M}_{\mathrm{S}} \mathrm{GR}, 6.7 \mathrm{~m}_{\mathrm{b}}$ ABE. (Ref. 258.) 
1936. Oct. 23 (Oct. 22). Near Anchorage, Alaska. The earthquake was described as the strongest shock in three years at Anchorage, where property loss was estimated at $\$ 500$. The shock was described as "heavy" at Seward and "strong" at Susitna. Many aftershocks occurred. (Ref. 9, 265.)

1937. July 22. Central Alaska. Only slight damage was caused by this major earthquake because the epicentral area was sparsely populated. Fairbanks sustained considerable minor damage, consisting mostly of broken windows and loss of merchandise in stores. Slight damage also was reported at Anchorage, about $580 \mathrm{~km}$ south of Fairbanks. At Salcha Bluff, southeast of Fairbanks, the highway was blocked for several meters by a landslide. Near there, mud boils appeared and cracks as wide as 38 $\mathrm{cm}$ formed. Water in the nearby slough rose considerably above its normal level and did not subside for several days.

At mile 33 station of the Alaska Road Commission, a two-story $\log$ structure was knocked askew and several windows were broken. About $22 \mathrm{~km}$ from Fairbanks, small cracks formed in the road, and near the mile 18 roadhouse, silt and sand from many cracks covered the highway. The main earthquake was felt over most of central Alaska. Aftershocks occurred for several months. Magnitude $7.3 \mathrm{M}_{\mathrm{S}}$ GR. (Ref. 10, 265.)

1938. Nov. 10. Shumagin Islands region, Alaska. A major submarine earthquake, centered in a sparsely populated area, was felt strongly at False Pass, Unimak Island. It also was reported at Port Moller and Anchorage. The earthquake generated a small tsunami, which was recorded at Dutch Harbor, Seward, and Sitka, Alaska, and at Hilo and Honolulu, Hawaii. Magnitude $8.7 \mathrm{M}_{\mathrm{S}} \mathrm{CFR}, 8.3 \mathrm{M}_{\mathrm{S}} \mathrm{GR}, 8.2$ $\mathrm{m}_{\mathrm{b}}$ ABE. (Ref. 11, 38, 432, 610.)

1938. Nov. 17 (Nov. 16). Alaska Peninsula area, Alaska. Magnitude $7.2 \mathrm{M}_{\mathrm{S}} \mathrm{GR}, 7.2 \mathrm{~m}_{\mathrm{b}} \mathrm{ABE}$. (Ref. 432.)

1940. Apr. 16, 0607 UTC (Apr. 15). Near Islands, Aleutian Islands, Alaska. Magnitude 7.1 $\mathrm{M}_{\mathrm{S}} \mathrm{GR}, 7.1 \mathrm{~m}_{\mathrm{b}} \mathrm{ABE}$. (Ref. 432.)

1940. Apr. 16, 0643 UTC (Apr. 15). Near Islands, Aleutian Islands, Alaska. Magnitude 7.2 $\mathrm{M}_{\mathrm{S}}$ GR. (Ref. 432.)

1940. July 19, 1627 UTC. Kenai Peninsula area, Alaska. Slight damage occurred at Anchorage, where walls were cracked and small objects were displaced. (Ref. 13, 38.)

1940. Aug. 22 (Aug. 21). Near Unalaska Island, Alaska. Magnitude $7.1 \mathrm{M}_{\mathrm{S}} \mathrm{GR}, 7.2 \mathrm{~m}_{\mathrm{b}} \mathrm{ABE}$. (Ref. 258.)

1941. July 30 (July 29). Kenai Peninsula area, Alaska. At Anchorage, one building was shaken from its foundation and four "pipes" (probably water pipes) were broken. Plaster fell, dishes broke, windows cracked, and stock toppled to the floor. Three aftershocks were observed. (Ref. 14, $38,258$.

1943. Nov. 3. South-central Alaska. This earthquake was felt at Anchorage, where an abrupt, heaving motion swung doors and rattled windows. Magnitude 7.3 $\mathrm{M}_{\mathrm{S}} \mathrm{GR}, 7.2 \mathrm{~m}_{\mathrm{b}} \mathrm{ABE}$. (Ref. 38, 432.)

1944. Dec. 12 (Dec. 11). Rat Islands, Aleutian Islands, Alaska. Magnitude $7.0 \mathrm{M}_{\mathrm{S}} \mathrm{GR}, 7.1 \mathrm{~m}_{\mathrm{b}}$ ABE. (Ref. 258.)

1946. Jan. 12. Gulf of Alaska. Magnitude 7.2 $\mathrm{M}_{\mathrm{S}} \mathrm{GR}, 7.2 \mathrm{~m}_{\mathrm{b}} \mathrm{ABE}$. (Ref. 432.)

1946. Apr. 1, 1228 UTC. Unimak Island region, Alaska, southeast of Scotch Cap Lighthouse. This major earthquake caused only minor damage to buildings on Unimak Island, but it generated a tsunami that devastated the lighthouse and swept away its five occupants. The height of the wave at the lighthouse was estimated at about $35 \mathrm{~m}$. Tsunami damage also occurred at Dutch Harbor and Ikatan Island in the Aleutian Islands, on the west coasts of North and South America, and in Hawaii. At Hilo, Hawaii, the tsunami took 159 lives and caused $\$ 26$ million loss to property. The tsunami caused one death in California. Magnitude $7.4 \mathrm{M}_{\mathrm{S}}$ GR, 7.2 $\mathrm{m}_{\mathrm{b}}$ ABE. (Ref. 19, 38, 432, 533, 610.)

1946. Nov. 1 (Oct. 31). Andreanof Islands, Aleutian Islands, Alaska. Magnitude $7.0 \mathrm{M}_{\mathrm{S}} \mathrm{ABE}$, $6.9 \mathrm{~m}_{\mathrm{b}} \mathrm{ABE}$. (Ref. 258.)

1947. Oct. 16 (Oct. 15). Fairbanks area, Alaska. This major earthquake centered southeast of Nenana on the Salcha River fault. Small fissures formed in the ground near the Nenana Airport, southwest of Fairbanks. Streets "upheaved" at Nenana in several places, and several long cracks formed in the ground. Cracks in river mud and ice occurred from Shaw Creek on Richardson Highway to the headwaters of the Kantishna and Tolavana Rivers. Cracks $56 \mathrm{~cm}$ wide, as much as $30 \mathrm{~cm}$ deep, and several meters long were reported about $24 \mathrm{~km}$ below Chena Bluffs on the Tanana River. A few pressure ridges were observed where large, frozen blocks came together.

Alaska Railroad officials reported that rails were bent between Julius, Nenana, and Browne, and that some changes were observed in the elevation of the roadbed. Landslides occurred on the Richardson Highway, and rockslides were observed between Fairbanks and Nenana on the Tanana River.

In Fairbanks, merchandise in stores was damaged heavily, many windows were broken, and a powerline short-circuit occurred near the University of Alaska. Changes in the flow of water in several wells were reported both at Fairbanks and Nenana. Trees and 
poles were shaken violently. At the town of Clear, south of Nenana, some prefabricated buildings were damaged.

The felt area is rather well defined to the south and west but is uncertain to the north and east because of the sparse population. The earthquake was felt over most of central and southern Alaska and at two places in the Yukon Territory of Canada, an area within a radius of about $500 \mathrm{~km}$ of the epicenter. This earthquake series consisted of more than 200 foreshocks and aftershocks. The strongest aftershock occurred on Oct. 20. Magnitude 7.0 $\mathrm{M}_{\mathrm{S}}$ GR, 6.9 $\mathrm{m}_{\mathrm{b}}$ ABE. (Ref. 20, 38, 260.)

1948. May 14. Alaska Peninsula area, Alaska. Magnitude 7.5 $\mathrm{M}_{\mathrm{S}} \mathrm{GR}, 7.3 \mathrm{mb}_{\mathrm{b}} \mathrm{ABE}$. (Ref. 432.)

1949. Sept. 27. Gulf of Alaska. Magnitude 7.0 $\mathrm{m}_{\mathrm{b}}$ ABE. (Ref. 258.)

1951. Feb. 13. Alaska Peninsula area, Alaska. Magnitude 7.1 $\mathrm{M}_{\mathrm{S}}$ GR, 7.1 $\mathrm{m}_{\mathrm{b}}$ ABE. (Ref. 432.)

1952. Dec. 7 (Dec. 6). Shemya Island, Alaska. This earthquake disconnected a water main and electrical cables and cracked a cement floor at Shemya Air Force Base. (Ref. 25, 38, 265.)

1953. Jan. 5 (Jan. 4). Near Islands area, Aleutian Islands, Alaska. Felt on Attu Island, Alaska. Magnitude $7.0 \mathrm{mb}_{\mathrm{b}}$ ABE. (Ref. 26, 432.)

1954. Oct. 3. Kenai Peninsula, Alaska. At Anchorage, the violent shaking broke water connections in two 14-story buildings. North of Potter, more than $42 \mathrm{~m}$ of railroad track was "knocked out of commission." Minor landslides spilled down on the Seward-Anchorage Highway. Cracks in concrete walls, fallen plaster, and shattered plate-glass windows were reported at Anchorage, Homer, Kenai, Seward, Sterling, and Valdez. Magnitude 6.5 Ukn BRK. (Ref. 27, 38, 447.)

1957. Mar. 9, 1422 UTC. Andreanof Islands, Aleutian Islands, Alaska. This great earthquake destroyed two bridges on Adak Island, damaged houses, and left a $4.5-\mathrm{m}$ crack in a road. On Umnak Island, part of a dock was destroyed, and Mount Vsevidof erupted after being dormant for 200 years. Further, this shock generated a $15-\mathrm{m}$ tsunami that smashed into the coastline at Scotch Cap and an 8-m tsunami that washed away many buildings and damaged oil lines extensively at Sand Bay. This tsunami continued to Hawaii, where it destroyed two villages and inflicted about $\$ 5$ million in property damage on Oahu and Kauai Islands. The tsunami also caused minor damage in San Diego Bay, Calif., before traveling to such distant countries as Chile, El Salvador, Japan, and other areas in the Pacific region. More than 300 aftershocks were reported along the southern edge of the Aleutians, from Unimak Island to
Amchitka Pass. Magnitude $7.7 \mathrm{~m}_{\mathrm{b}} \mathrm{ABE}, 8.3 \mathrm{Ukn}$ PAS. (Ref. 30, 38, 479, 610.)

1957. Mar. 9, 2039 UTC. Fox Islands, Andreanof Islands, Alaska. Felt on Adak Island. Magnitude 7.1 Ukn PAS, $7.0 \mathrm{~m}_{\mathrm{b}}$ ABE. (Ref. 30, 432, 479.)

1957. Mar. 11, 0958 UTC (Mar. 10). Fox Islands, Aleutian Islands, Alaska. Magnitude 6.9 Ukn PAS, 7.1 Ukn BRK, $6.9 \mathrm{mb}_{\mathrm{b}}$ ABE. (Ref. 432.)

1957. Mar. 11, 1455 UTC. Andreanof Islands, Aleutian Islands, Alaska. Felt on Adak and Umnak Islands. Magnitude $7.0 \mathrm{mb}_{\mathrm{b}} \mathrm{ABE}, 6.7 \mathrm{Ukn}$ PAS, 7.1 Ukn BRK. (Ref. 30, 479.)

1957. Mar. 12, 1144 UTC. Andreanof Islands, Aleutian Islands, Alaska. Felt on Adak and Umnak Islands. Magnitude $7.1 \mathrm{~m}_{\mathrm{b}} \mathrm{ABE}, 7.3$ Ukn PAS. (Ref. 30, 479.)

1957. Mar. 14, 1447 UTC. Andreanof Islands, Aleutian Islands, Alaska. Felt on Adak Island. Magnitude $7.0 \mathrm{~m}_{\mathrm{b}}$ ABE, 7.2 Ukn PAS, 7.4 Ukn BRK. (Ref. 30, 479.)

1957. Mar. 16, $02 \quad 34$ UTC (Mar. 15). Andreanof Islands, Aleutian Islands, Alaska. Felt on Adak Island. Magnitude $7.0 \mathrm{~m}_{\mathrm{b}} \mathrm{ABE}, 6.7$ Ukn PAS. (Ref. 30, 479.)

1957. Mar. 22, 1421 UTC. Fox Islands, Aleutian Islands, Alaska. Magnitude $6.9 \mathrm{mb}_{\mathrm{b}} \mathrm{ABE}, 7.0$ Ukn PAS, 7.0 Ukn BRK. (Ref. 432.)

1957. Apr. 10, 1129 UTC. Kodiak Island region, Alaska. Magnitude $7.0 \mathrm{~m}_{\mathrm{b}} \mathrm{ABE}, 7.1 \mathrm{Ukn}$ PAS, 7.2 Ukn BRK. (Ref. 432.)

1957. Apr. 19, 2219 UTC. Fox Islands, Aleutian Islands, Alaska. Magnitude 6.5 $\mathrm{M}_{\mathrm{S}} \mathrm{ABE}, 7.1$ $\mathrm{m}_{\mathrm{b}}$ ABE. (Ref. 265.)

1957. June 13. Andreanof Islands, Aleutian Islands, Alaska. Magnitude $6.7 \mathrm{~m}_{\mathrm{b}} \mathrm{ABE}$. (Ref. 265.)

1958. Apr. 7. Central Alaska. A major earthquake on this date caused mud flows, widespread breakage of lake and river ice, and formation of many ground cracks within a $65-$ to $80-\mathrm{km}$ radius of Huslia (west of Fairbanks). Observers also reported pressure ridges, thawing of ice on lakes, and craters $6 \mathrm{~m}$ across and about $2 \mathrm{~m}$ deep in the ground. Ground cracks occurred at Tanana. Minor damage to roofs and foundations of buildings was reported at Huslia. Slight damage also was observed at several central Alaska towns. Magnitude 7.3 Ukn PAS, 7.1 $\mathrm{m}_{\mathrm{b}} \mathrm{ABE}$. (Ref. 31, 447.)

1958. July 10, 0615 UTC (July 9). Southeast Alaska. This was the largest earthquake in southeast Alaska since the Yakutat shocks of 1899. The only permanent settlement in the epicentral region was Yakutat; therefore, effects on man-made works were moderate for such a large earthquake. On Khantaak Island (in Yakutat Bay), three persons 
were killed when the north end of the island slumped into the sea, and two people were missing and presumed dead in Lituya Bay from a wave generated by the collapse of 300 million $\mathrm{m}^{3}$ of rock into Gilbert Bay. At Yakutat, bridges, docks, and oil lines were damaged, a water tower fell, and a few cabins were destroyed. Many sand blows and ground fissures were observed on the low coastal plain southeast of Yakutat, and large landslides were reported in the mountains. A cabin collapsed and the ground was fissured at Dry Bay (East River); many sand blows and ground cracks occurred at Dry Bay (Akwe River); and submarine cables were severed in the Haines-Skagway area and at Lena Point (north of Juneau). Slight damage also occurred at Auke Bay, Baranof, Juneau, Pelican, and Sitka.

A massive rockslide at the head of Lituya Bay caused water to surge about $530 \mathrm{~m}$, generating a "gravity wave" that swept out of the bay. A fishing boat anchored in Anchorage Cove was carried in front of the largest wave crest, and those onboard estimated they cleared La Chaussee Spit (at the mouth of Lituya Bay) by $30 \mathrm{~m}$ or more. Two people on another fishing boat disappeared after being caught in the huge wave. This major earthquake was felt over a large area of southeast Alaska, as far south as Seattle, Wash., and east to Whitehorse, Y.T., Canada (see fig. 9). Magnitude 7.1 $\mathrm{m}_{\mathrm{b}} \mathrm{ABE}, 7.9 \mathrm{Ukn}$ PAS, 8.0 Ukn BRK. (Ref. 31, 38, 448, 533, 610.)

1960. Nov. 13 (Nov. 12). Fox Islands, Aleutian Islands, Alaska. Magnitude $7.1 \mathrm{~m}_{\mathrm{b}} \mathrm{ABE}, 7.0 \mathrm{Ukn}$ PAS, 7.2 Ukn BRK. (Ref. 432.)

1961. Sept. 5. Kenai Peninsula, Alaska. Slight damage in the form of cracked plaster and broken dishes and mirrors occurred at Seward. The shock also was felt at several other towns in the region. Magnitude 5.75 Ukn BRK. (Ref. 34, 447.)

1962. Oct. 21 (Oct. 20). Anchorage area, Alaska. Slight damage to telephone lines, merchandise in stores and houses, and plaster was observed in the Anchorage-Girdwood-Wasilla area. Also felt at Kenai, Soldotna, and Susitna Station. (Ref. 35, 447.)

1963. June 24 (June 23). Cook Inlet, Alaska. At Seldovia, a brick chimney toppled; at Homer, a ceiling beam burst and posts were torn from the ground; and at Barbara Point $(8 \mathrm{~km}$ north of Seldovia), a heavy fireplace slab was displaced about 15 $\mathrm{cm}$, and a concrete foundation was cracked. Rockslides were observed in the area. The shock also was felt at Anchorage, Cordova, and Homer area. Magnitude 6.5-6.75 Ukn BRK. (Ref. 36, 447.)

1964. Feb. 6, 1307 UTC. Near Chirikof Island, Alaska. Magnitude $7.1 \mathrm{~m}_{\mathrm{b}} \mathrm{ABE}, 6.9 \mathrm{Ukn}$ PAS, 6.9 Ukn PAL, 6.6 Ukn BRK. (Ref. 37, 432.)
1964. Mar. 28 (Mar. 27). Prince William Sound, Alaska. This great earthquake and ensuing tsunami took 125 lives (tsunami 110, earthquake 15), and caused about $\$ 311$ million in property loss. Earthquake effects were heavy in many towns, including Anchorage, Chitina, Glennallen, Homer, Hope, Kasilof, Kenai, Kodiak, Moose Pass, Portage, Seldovia, Seward, Sterling, Valdez, Wasilla, and Whittier.

Anchorage, about $120 \mathrm{~km}$ northwest of the epicenter, sustained the most severe damage to property. About 30 blocks of dwellings and commercial buildings were damaged or destroyed in the downtown area. The J.C. Penney Company building was damaged beyond repair; the Four Seasons apartment building, a new six-story structure, collapsed; and many other multistory buildings were damaged heavily. The schools in Anchorage were almost devastated. The Government Hill Grade School, sitting astride a huge landslide, was almost a total loss. Anchorage High School and Denali Grade School were damaged severely. Duration of the shock was estimated at 3 minutes.

Landslides in Anchorage caused heavy damage. Huge slides occurred in the downtown business section, at Government Hill, and at Turnagain Heights. The largest and most devastating landslide occurred at Turnagain Heights. An area of about 130 acres was devastated by displacements that broke the ground into many deranged blocks that were collapsed and tilted at all angles. This slide destroyed about 75 private houses. Water mains and gas, sewer, telephone, and electrical systems were disrupted throughout the area.

The earthquake was accompanied by vertical displacement over an area of about $520,000 \mathrm{~km}^{2}$. The major area of uplift trended northeast from southern Kodiak Island to Prince William Sound and trended east-west to the east of the sound. Vertical displacements ranged from about $11.5 \mathrm{~m}$ of uplift to $2.3 \mathrm{~m}$ of subsidence relative to sea level. Off the southwest end of Montague Island, there was absolute vertical displacement of about 13-15 m. Uplift also occurred along the extreme southeast coast of Kodiak Island, Sitkalidak Island, and over part or all of Sitkinak Island. This zone of subsidence covered about $285,000 \mathrm{~km}^{2}$, including the north and west parts of Prince William Sound, the west part of the Chugach Mountains and a part of the lowlands north of the mountains, most of Kenai Peninsula, and almost all the Kodiak Island group.

This shock generated a tsunami that devastated many towns along the Gulf of Alaska and left serious damage at Alberni and Port Alberni, Canada, along 


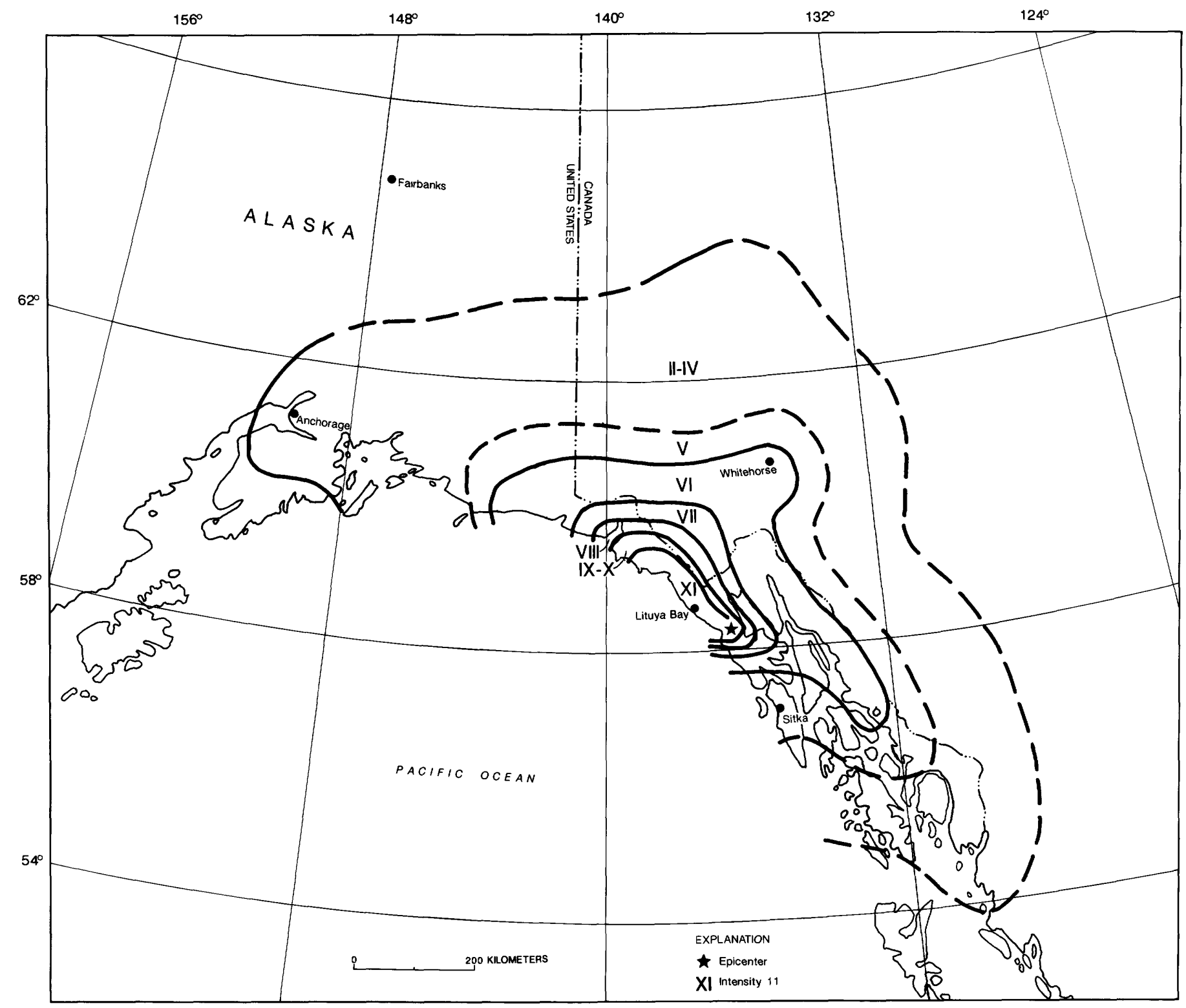

FIGURE 9.-Isoseismal map for the southeast Alaska earthquake of July 10, 1958. This map is a simplified version of figure 6 in reference 424 of table 1.

the West Coast of the United States (15 killed), and in Hawaii. The maximum wave height recorded was $67 \mathrm{~m}$ at Valdez Inlet. Seiche action in rivers, lakes, bayous, and protected harbors and waterways along the Gulf Coast of Louisiana and Texas caused minor damage. It was also recorded on tide gages in Cuba and Puerto Rico.

This great earthquake was felt over a large area of Alaska and in parts of western Yukon Territory and British Columbia, Canada (see fig. 10). Magnitude $7.9 \mathrm{~m}_{\mathrm{b}} \mathrm{ABE}, 8.4 \mathrm{Ukn}$ PAS, 8.6 Ukn BRK, 8.6 Ukn PAL. (Ref. 37, 38, 451, 523, 533, 611.)

1964. Apr. 14. Kodiak Island region, Alaska. At Kodiak, light fixtures broke and several cracks formed in one building. The City Hall, fire station, and library building were "further weakened." (Ref. 37, 299.)

1964. Dec. 13 (Dec. 12). Nome area, Alaska. At Nome, a water pipe broke; concrete floor, walls, and plaster cracked; and crusted snow broke into chunks. Also felt strongly at Teller. Several aftershocks were felt in the Nome area. (Ref. 37, 299.)

1965. Feb. 4, 0501 and 0840 UTC (Feb. 3). Rat Islands, Aleutian Islands, Alaska. On Adak Island, cracks occurred in prefabricated wood buildings; on Shemya Island, cracks were observed in an asphalt runway. Hairline cracks also formed in the runways at the U.S. Coast Guard Loran Station on 


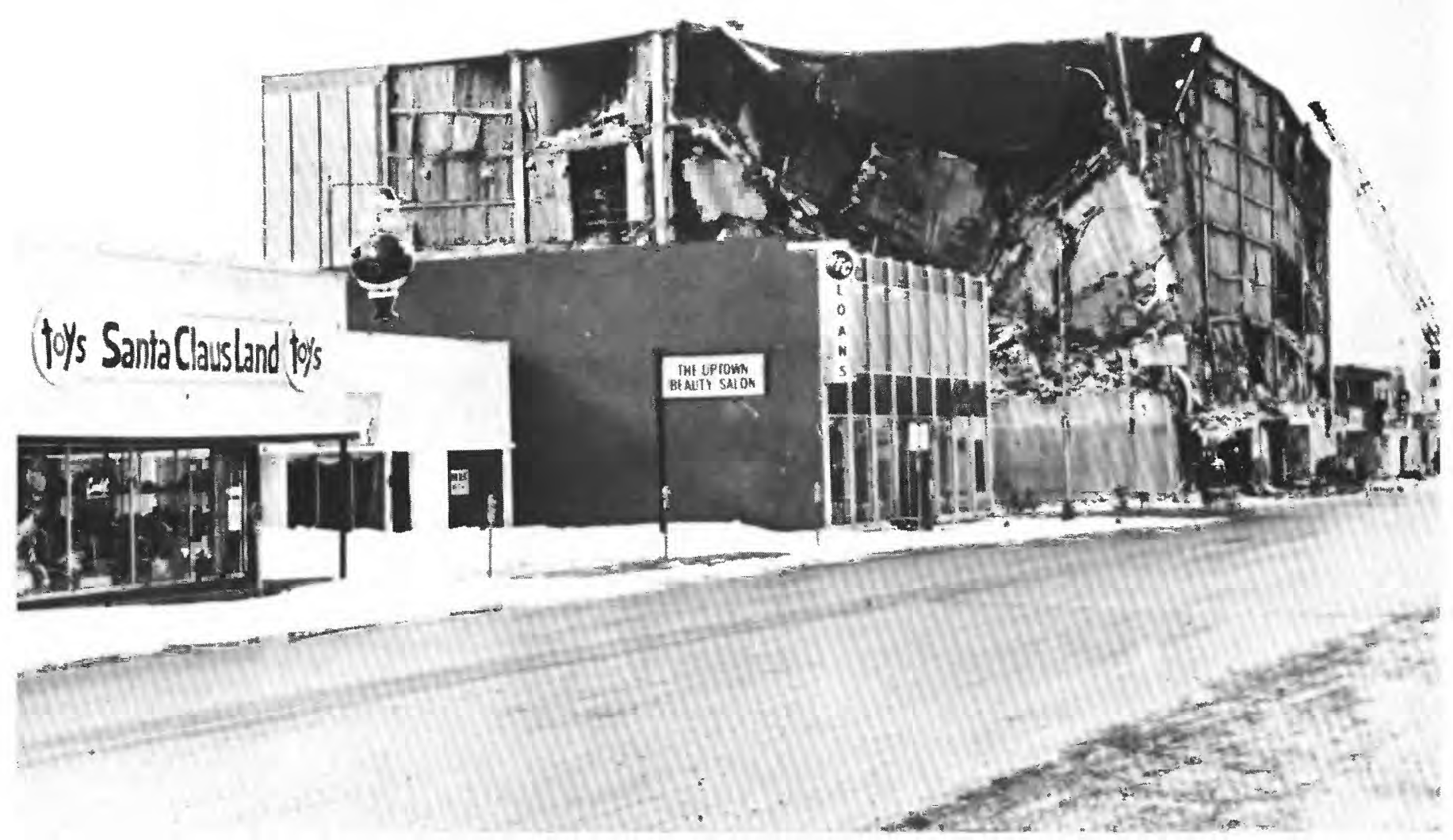

Five-story J.C. Penney Building, 5th Avenue and Downing Street, Anchorage, Alaska, partly collapsed by the March 28,1964 (Mar. 27 AST), earthquake. Note undamaged buildings nearby.

Attu Island. This earthquake generated a tsunami reported to be about $10.7 \mathrm{~m}$ high on Shemya Island. Loss caused by flooding on Amchitka Island was estimated at about $\$ 10,000$. An aftershock at 0740 UTC was assigned MMI VI. Magnitude $7.7 \mathrm{~m}_{\mathrm{b}} \mathrm{ABE}, 7.7$ Ukn PAS, 7.7 Ukn BRK (first shock); $7.0 \mathrm{~m}_{\mathrm{b}} \mathrm{ABE}$, 6.9 Ukn PAS (second shock). (Ref. 75, 299, 610.)

1965. Mar. 30, 0227 UTC (Mar. 29). Rat Islands, Aleutian Islands, Alaska. Felt on Adak and Amchitka Islands. Magnitude $7.4 \mathrm{~m}_{\mathrm{b}} \mathrm{ABE}, 7.2$ Ukn PAS, 6.9 Ukn BRK, 7.7 Ukn PAL. (Ref. 75, 299.)

1965. Apr. 16. Central Alaska. Slight damage to walls and dishes occurred at Elim. River ice was cracked at Kaltag and Unalakleet. Thunderous earth noises were reported. Felt over a small area of central Alaska. (Ref. 75, 299.)

1965. July 2. Fox Islands, Aleutian Islands, Alaska. In one house on Umnak Island, dishes were broken and books were thrown over all the room. A minor tsunami having a 9-cm amplitude was registered on Unalaska Island. Also observed at Cold Bay. Magnitude 7.0 mb ABE, 6.9 Ukn PAS, 6.8 Ukn BRK, 6.7 Ukn PAL. (Ref. 75, 299.)
1966. Aug. 7 (Aug. 6). Andreanof Islands, Aleutian Islands, Alaska. Felt on Adak Island. Magnitude 6.4 $\mathrm{M}_{\mathrm{S}} \mathrm{ABE}, 6.7 \mathrm{Ukn}$ PAS, 7.0 Ukn BRK. (Ref. 81, 299.)

1967. June 21, 18 04, 1813 , and 1824 UTC. Fairbanks area, Alaska. Three moderate earthquakes caused minor damage to property in the Fairbanks area. The most extensive loss occurred at the State Court and Office Building in Fairbanks. Both interior and exterior walls cracked, parts of hanging ceilings and light fixtures fell, plumbing broke, and cabinets and shelves overturned. Several chimneys fell in the area, a tall chimney stack buckled, windows broke, and considerable loss of merchandise was reported in stores.

Three ground cracks about $2 \mathrm{~m}$ apart were observed at Badger Pass and Peede Road, and blue mud seeped through the cracks. On Richardson Highway, water spouted from ground cracks, a sewer line broke, and chimneys were offset. Small landslides occurred. About 6,000 aftershocks were recorded through June 28. About 60 aftershocks were observed by residents, and some caused additional 


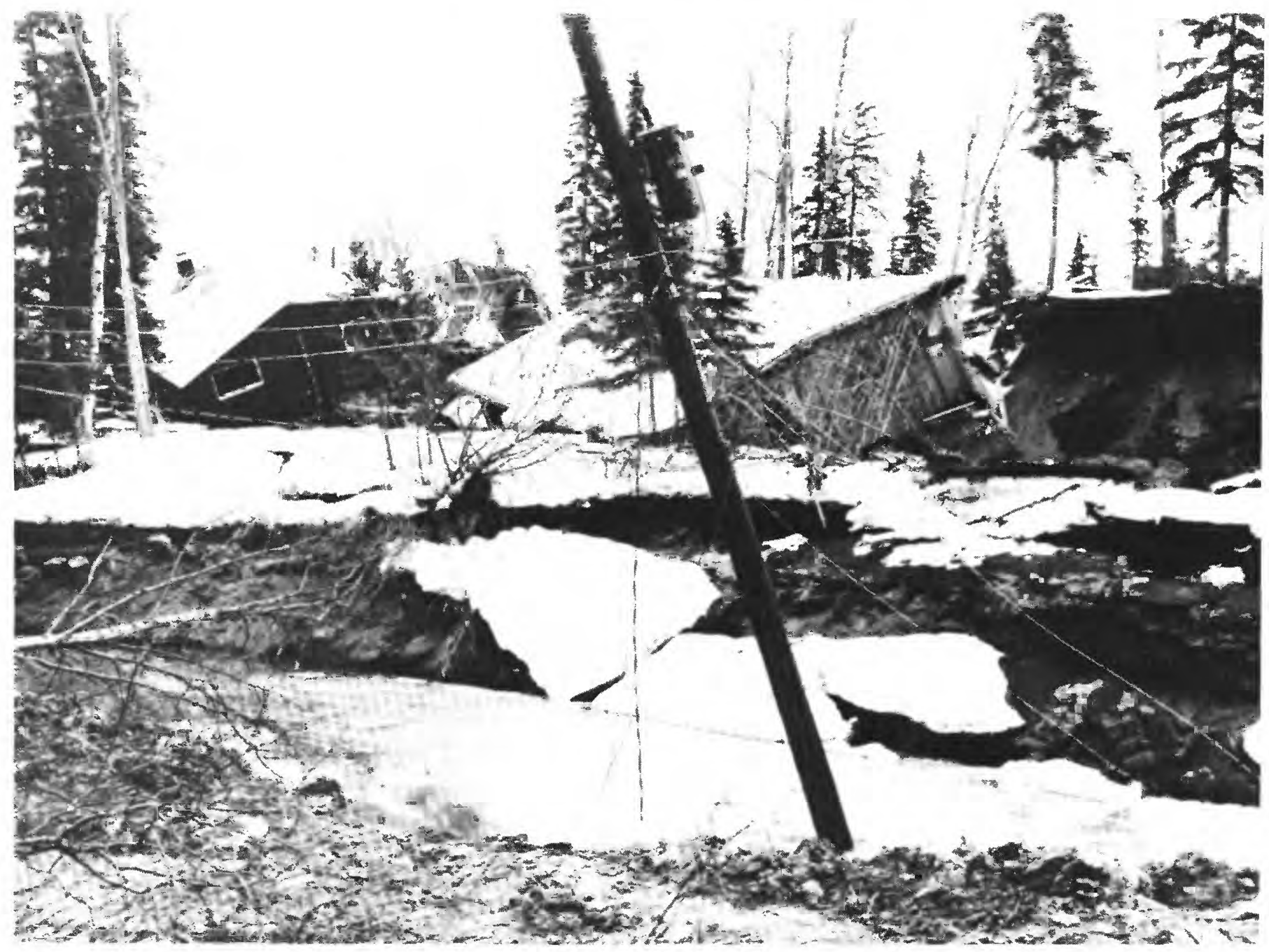

Landslide and slumping effects in the Turnagain Heights area, Anchorage, Alaska, caused by the March 28, 1964 (Mar. 27 AST), earthquake.

damage. Magnitude 5.5 Ukn PAS (first shock). (Ref. $40,74,452$.

1967. June 23. Fairbanks area, Alaska. The State Court and Office Building in Fairbanks sustained additional damage. Merchandise fell in stores. (Ref. 40, 74.)

1968. Oct. 29. Central Alaska. The epicentral area, southeast of Rampart on the Yukon River, was shaken badly but sustained no serious damage because most of the buildings were constructed of logs. Stock tumbled from shelves, residents ran from buildings, and equipment not bolted down was displaced. In the Hunter Creek area, about $16 \mathrm{~km}$ from Rampart, many landslides were observed on the south-facing slopes. Lake ice was cracked extensively in places. At Nenana, about $80 \mathrm{~km}$ southeast of Rampart, cracks formed in the ground and plaster was broken. More than 2,000 aftershocks were recorded through Nov. 12. Magnitude $6.4 \mathrm{M}_{\mathrm{S}} \mathrm{BRK}$, 6.9 Ukn PAS, 7.0 Ukn GOL. (Ref. 41, 299.)

1968. Dec. 17. Southern Alaska. Slight damage occurred at Kenai. Felt from Cantwell southeast to Kodiak. Magnitude 6.4 $\mathrm{M}_{\mathrm{S}}$ BRK. (Ref. 38, 41, 299.)

1972. July 30. Southeast Alaska. At Sitka, a few chimneys fell and some minor landslides were reported. Slight damage also was sustained at Hoonah, Juneau, Pelican, and Yakutat. The Fairweather fault ruptured over a length of $75 \mathrm{~km}$. Seiches were observed in swimming pools as far south as Seattle, Wash. At least 19 aftershocks were felt at Sitka through Aug. 29. A tsunami of $10 \mathrm{~cm}$ was recorded at Juneau and $8 \mathrm{~cm}$ at Sitka. The main shock was felt over a large area of southeast Alaska and British Columbia, Canada. Magnitude $7.4 \mathrm{mb}_{\mathrm{b}}$ AB3, 7.2 Ukn PAS, 7.1 Ukn BRK. (Ref. 45, 74, 610.) 


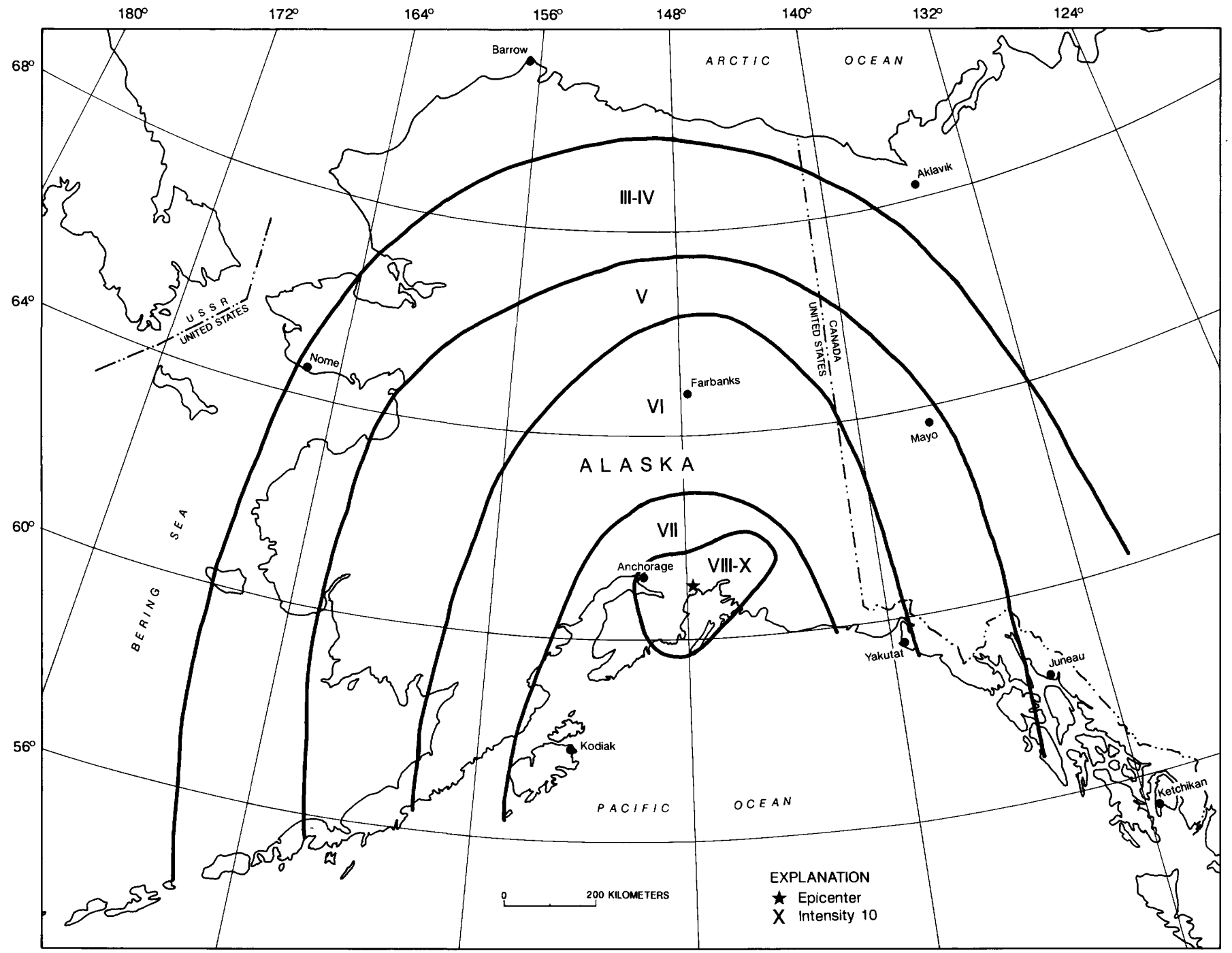

Figure 10.-Isoseismal map for Prince William Sound, Alaska, earthquake of March 28, 1964. This map is a simplified version of the map in reference 528 of table 1.

1972. Aug. 3 (Aug. 2), 0440 UTC. Andreanof Islands, Aleutian Islands, Alaska. Minor damage occurred on Adak Island. Magnitude 6.4 $\mathrm{M}_{\mathrm{S}}$ BRK. (Ref. 45, 479.)

1975. Feb. 2, 0843 UTC (Feb. 1). Near Islands, Aleutian Islands, Alaska. A major earthquake injured several residents and caused severe damage on Shemya Island. The Shemya Air Force Base runway sustained cracks. Crevasses having as much as $16.5 \mathrm{~m}$ of vertical displacement were observed on Shemya. Landslides occurred, water tanks twisted, and underground water pipes broke. Many aftershocks were felt during the weeks following the main shock. Also felt on Attu Island. Magnitude $7.0 \mathrm{~m}_{\mathrm{b}} \mathrm{AB} 3,7.5 \mathrm{Ukn}$ PAS, 7.4 Ukn BRK. (Ref. 48, 74.)
1977. Nov. 4 (Nov. 3). Andreanof Islands, Aleutian Islands, Alaska. This earthquake downed plaster and moved heavy furniture on Adak Island and cracked chimneys on Atka Island. Magnitude 7.0 $\mathrm{M}_{\mathrm{S}}$ BRK. (Ref. 39, 479.)

1978. Aug. 18. Southern Alaska. At Clam Gulch, southwest of Anchorage on the Kenai Peninsula, hairline cracks formed in cinder-block walls, and plasterboard was cracked. Felt over a small area of south-central Alaska. (Ref. 74, 240.)

1979. Feb. 28. Southeast Alaska. This major earthquake was located about $50 \mathrm{~km}$ northwest of Mt. St. Elias, near the east end of the Chugach Mountains. It affected the area only slightly because it centered in an unpopulated area of ice fields. Seven major earthquakes have been located in the 
region between Controller Bay and northern Chichagof Island in southeast Alaska from 1899 to 1979, three of which were of magnitude 8 or larger. This earthquake was strongest at the Icy Bay Lumber Camp, about $76 \mathrm{~km}$ south of the epicenter, where a parked logging truck was bounced sideways across the road, trees and bushes were shaken strongly, and people had difficulty in standing. Minor damage occurred in Alaska at Border City, Cape Yakataga, Juneau area, Valdez, and Yakutat; minor damage occurred in Yukon Territory, Canada, at Beaver Creek, Burwash Landing, Destruction Bay, and Kluane Lake Fishing Camp. A 15-cm tsunami was recorded at Yakutat. Magnitude $7.3 \mathrm{M}_{\mathrm{S}}$ BRK, $7.1 \mathrm{~m}_{\mathrm{b}}$ AB3. (Ref. 424, 455, 610.)

1979. May 20 (May 19). Alaska Peninsula area. Minor damage at Larsen Bay included cracks in plaster and drywall and hairline cracks in exterior walls. Also felt at a few other towns in the area. Magnitude $6.5 \mathrm{~m}_{\mathrm{b}}$ PAS, $6.2 \mathrm{~m}_{\mathrm{b}}$ BRK. (Ref. 74, 262.)

1983. July 12, 1510 UTC. Prince William Sound, Alaska. The epicenter of this earthquake is near that of the great 1964 earthquake (which had a magnitude of 8.3). At Valdez, an estimated $\$ 1$ million in damage occurred at the airport terminal building. Its exterior concrete-block walls cracked from roof to ground, and its inside walls also sustained cracks. Ceiling tiles fell in the high school and elementary school buildings; cracks formed in streets and sidewalks. Minor damage also was observed at Girdwood. This shock was felt from Yakutat on the south to Fairbanks on the north and east to Whitehorse, Y.T., Canada. Magnitude 6.3 $\mathrm{M}_{\mathrm{S}} \mathrm{BRK}, 6.3 \mathrm{~m}_{\mathrm{b}} \mathrm{BRK}, 6.4 \mathrm{~m}_{\mathrm{b}}$ PAS. (Ref. 360.)

1983. Sept. 7. Prince William Sound, Alaska. This moderate earthquake, whose epicenter is near that of the great 1964 earthquake, caused property damage at Gakona and Valdez. Damage was most severe at Valdez, where chimneys and foundations cracked and hairline cracks formed in interior walls. The flow of water in wells was disturbed at Gakona, and small landslides and slumping of roadfill occurred. Magnitude $6.3 \mathrm{~m}_{\mathrm{b}} \mathrm{BRK}, 6.4 \mathrm{~m}_{\mathrm{b}}$ PAS. (Ref. 360.)

1984. Aug. 14 (Aug. 13). Southern Alaska. Slight damage in the form of shattered windows and broken dishes occurred at Palmer, Sutton, and Willow. Buildings were shaken strongly, and people had difficulty standing. Small landslides were observed at Palmer and on Glenn Highway, near Sutton. The earthquake shook objects from walls and knocked merchandise from store shelves throughout the area. Residents observed several slight aftershocks during the week. (Ref. 74, 370.)

1985. Oct. 9 (Oct. 8). Shumagin Islands area, Alaska. Magnitude 6.6 $\mathrm{M}_{\mathrm{S}} \mathrm{PAL}, 6.0 \mathrm{M}_{\mathrm{S}}$ PAS. (Ref. 74, 371.)

1986. May 7, 2247 UTC. Andreanof Islands, Aleutian Islands, Alaska. This earthquake caused moderate damage to structures on Adak Island and slight damage on Atka Island. Damage to buildings on Adak Island consisted of cracked masonry and concrete walls, failure of partitions and suspended ceilings, spalling on concrete beams and piers, and differential settlement of house foundations. Soil liquefaction was observed in localized areas of backfilled soil, and sand boils were observed. Laterally spreading cracks and differential ground settlement occurred along a small wharf. This earthquake caused a small tsunami that was recorded throughout the Pacific Ocean. Magnitude 7.9 $\mathrm{M}_{\mathrm{S}}$ BRK. (Ref. 562,610 .)

1986. May 15 (May 14). Andreanof Islands, Aleutian Islands, Alaska. On Atka Island, underground pipes were broken, bridges were slightly damaged, and hairline cracks formed in interior walls. Magnitude 6.4 $\mathrm{M}_{\mathrm{S}}$ BRK. (Ref. 562.)

1986. May 17. Andreanof Islands, Aleutian Islands, Alaska. An airstrip on Atka Island was damaged and officially closed. Underground pipes were broken, furniture was overturned, and landslides were reported. Magnitude $6.5 \mathrm{M}_{\mathrm{S}}$ BRK. (Ref. 562.)

1987. Nov. 30, 1923 UTC. Gulf of Alaska. Damage at Yakutat, northwest of Juneau, consisted mainly of broken glassware and cracks in plaster, drywall, windows, and a foundation. Light damage occurred at several other towns. Also, cracks in wet ground were reported at Yakutat. Strong building vibrations made it difficult to stand or walk. Two ships in the epicentral area were damaged, and three others in the area felt the shaking strongly. A small tsunami was recorded at Seward, Sitka, and Yakutat. This earthquake had the largest magnitude of any event in the region since that on Oct. 9, 1900. Magnitude $7.7 \mathrm{M}_{\mathrm{S}}$ BRK. (Ref. 74, 577, 610.) 


\section{ARIZONA}

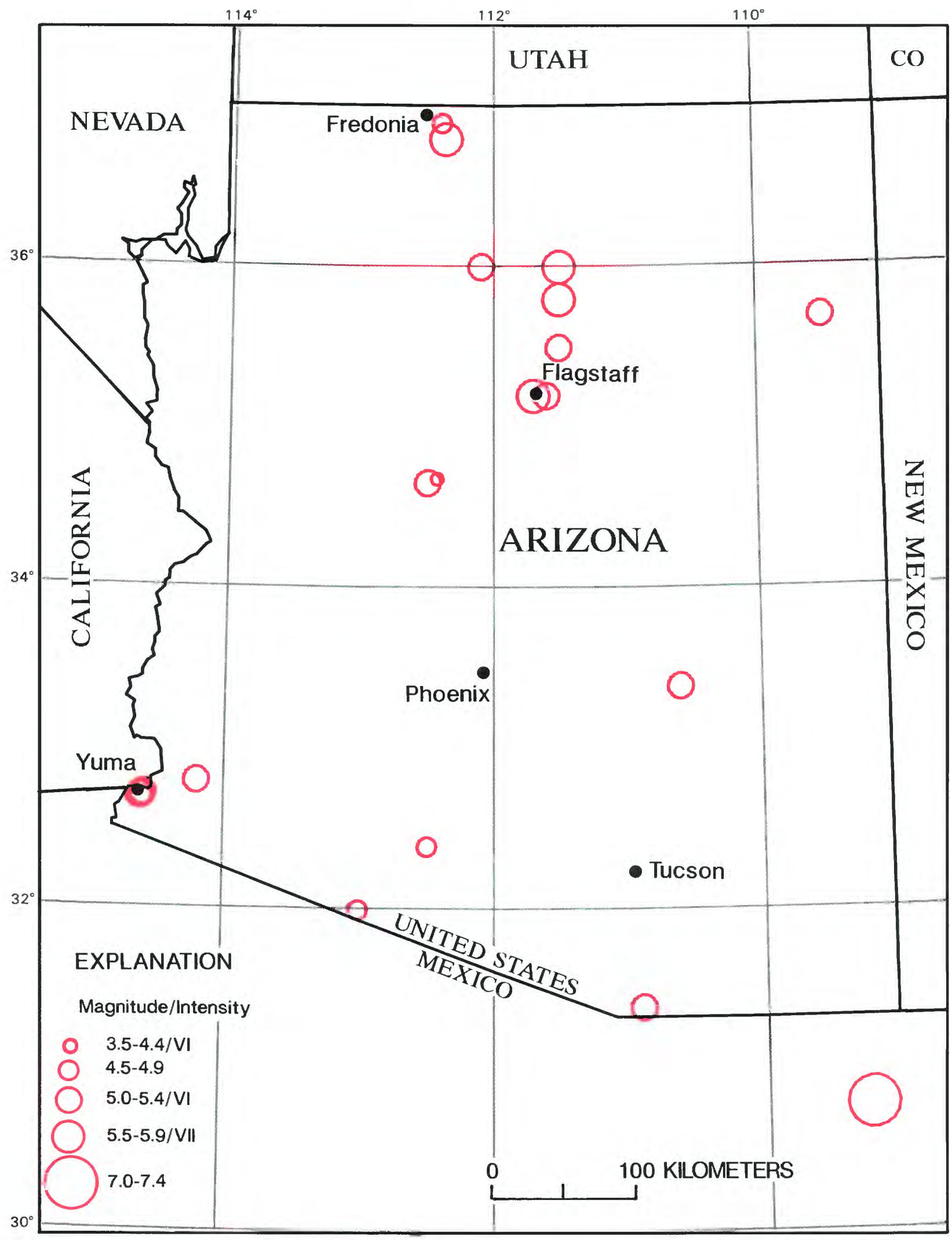

Earthquakes in Arizona with magnitudes $\geq 4.5$ or intensity $\geq$ VI. 
ARIZONA

[See table 1 for hypocenter and intensity references and table 2 for definitions of magnitude source codes. Leader (--) indicates information is not available]

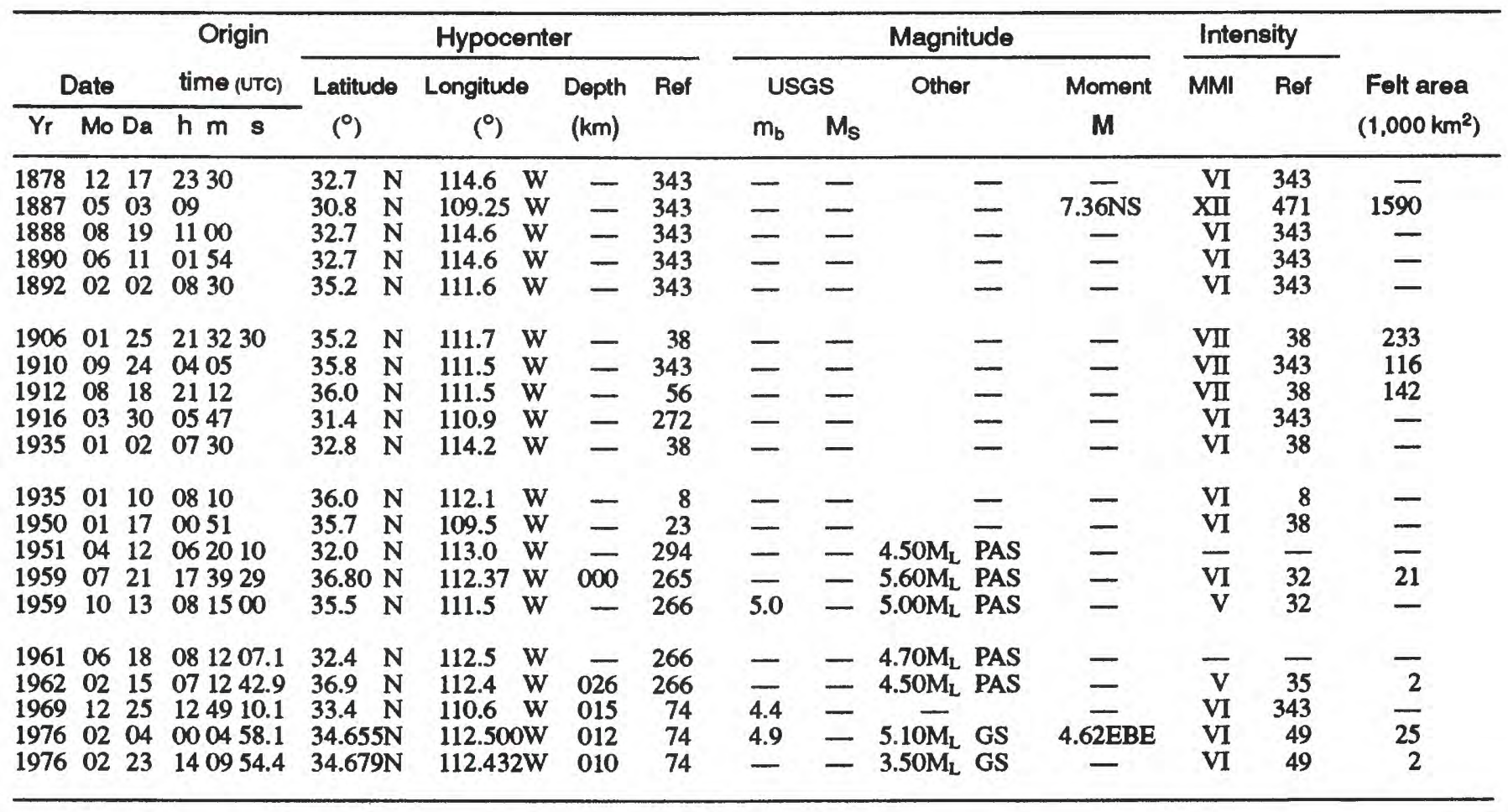

[Reference (Ref.) numbers given in parentheses at the end of each description refer to sources of data in table 1. Magnitude values are described in the Introduction, and codes are defined in table 2.]

1878. Dec. 17. Yuma, Ariz. This shock cracked walls in several houses in Yuma. Almost all the buildings in town were shaken. Also felt in Calif. The epicenter of this event probably was in Baja California, Mexico. (Ref. 343.)

1887. May 3. Northern Sonora, Mexico. This earthquake occurred in a sparsely settled region of Northern Sonora, Mexico. It caused widespread damage to property, 51 deaths (ref. 494 reports 150 deaths at Bavispe, Sonora), and many injuries. From Guaymas, Sonora, Mexico to Nogales, Ariz.; Benson and Tucson, Ariz.; El Paso, Tex.; and at towns as far away as Albuquerque, N. Mex., water in tanks slopped over, railroad cars were set in motion on tracks, chimneys were thrown down, and buildings were cracked. Other U.S. cities that sustained moderate to heavy damage include: Bisbee, Fairbank, Fort Huachuca, Saint David, San Simon, Solomonville, Tombstone, Tres Alamos, and Willcox, Ariz.; and Deming, Sabinal, and Silver City, N. Mex. Near the epicenter, liquefaction effects induced significant ground failure that led to the collapse of buildings and other structures.
At Tepic, Sonora, a town about $190 \mathrm{~km}$ south of Tombstone, Ariz., the walls and roofs of every house were shattered-many of the walls had fallen out, and the roofs had collapsed. The plaza and streets at Tepic were "ripped up" by fissures, some as wide as $15 \mathrm{~cm}$, and irrigation ditches around the town were broken. At Moctezuma, about $32 \mathrm{~km}$ south of Tepic, the houses were wrecked, and all inhabitants were living outside. At Oputo, about $56 \mathrm{~km}$ northeast of Tepic, a church collapsed and killed 40 people who had run there for shelter from the earthquake. American prospectors in that area reported that a ground fissure about $0.8 \mathrm{~m}$ wide was created by the earthquake.

The 76-km-long fault scarp produced by this earthquake is clearly exposed on the east side of the San Bernardino Valley of Northern Sonora, southeast of Douglas, Ariz. The maximum displacement on the Pitaycachi fault is 4.5 to $5.1 \mathrm{~m}$, and evidence exists for previous ruptures on the fault. A significant region of liquefaction was reported as far as $100 \mathrm{~km}$ from the fault, and landslides were observed at farther distances. In late 1972, the 1887 scarp was observed from the air along its total length. This study revealed many additional scarps, previously unmapped, paralleling the main fault trace. These 
scarps appear to represent active faulting over the previous several thousand years.

Seismic motion was felt from Toluca, Mexico (near Mexico City) on the south to Albuquerque and Santa Fe, N. Mex., on the north; and from Baja California, Mexico, and Yuma, Ariz., on the west to a point 100 $\mathrm{km}$ east of El Paso, Tex., on the east (see fig. 11). There also was a report that the earthquake was felt in California. Many aftershocks were observed. (Ref. $38,343,471,494,497$.

1888. Aug. 19. Yuma, Ariz. This earthquake shook down a few old sheds at Yuma. Everyone awoke and ran outside. (Ref. 343.)

1890. June 11 (June 10). Yuma, Ariz. A sharp earthquake that broke several windows at Yuma probably centered in Baja California, Mexico. (Ref. 343.)

1892. Feb. 2. Flagstaff, Ariz. At Flagstaff, the earthquake awakened many people who ran into the street. At Two Guns, a land bridge collapsed; a huge rock toppled onto the entrance of a cave; and stones shook loose from the walls inside the caverns. (Ref. 343.)

1906. Jan. 25. Flagstaff, Ariz. Chimneys were thrown down and walls were cracked at Flagstaff. At the courthouse and the Coconino County Hospital, plaster fell from the ceilings. At the school, plaster cracked in every room. Stones and dead trees were shaken down the San Francisco Peaks. Also felt in southern Utah and northwestern $\mathrm{N}$. Mex. (Ref. 38, 343.)

1910. Sept. 24 (Sept. 23). Coconino National Forest, near Flagstaff, Ariz. At Cedar Wash, a house was moved off its foundation, its chimney was shaken down, and one corner of the house was cracked severely. Huge lava stones weighing many tons were torn from the old lava beds and thrown down the mountainside. About $80 \mathrm{~km}$ north of Flagstaff, in the Coconino Forest, boulders rolled down a mountain into the camp of a construction crew. Fiftytwo shocks were felt by the construction crew between Sept. 10 and 23, 1910 (local time). The strongest shock was so violent that the construction crew temporarily abandoned the work site. Plaster cracked and fell at Flagstaff. (Ref. 343.)

1912. Aug. 18. Northern Arizona. Rockslides roared down the mountain in Lockett Tanks country, southeast of the Grand Canyon. Boulders were shaken from cliffs, and rocks were reportedly lifted free from the ground. An unconfirmed report stated that an earth fracture extended from Lockett Tanks to Coconino Mountain, a distance of more than 48 $\mathrm{km}$. Also felt in N. Mex. and Utah. (Ref. 38, 56, 343.)

1916. Mar. 30 (Mar. 29). Nogales, Santa Cruz County, Ariz. The earthquake cracked walls at Nogales and knocked down plaster at the courthouse. People ran from the Nogales theater. (Ref. 272, 343.) 1935. Jan. 2 (Jan. 1). Wellton, Yuma County, Ariz. Walls and plaster cracked at Wellton, east of Yuma. Everyone felt the ground quiver and their houses shake. (Ref. 38, 343.)

1935. Jan. 10. Grand Canyon, Ariz. Walls or plaster cracked and windows broke at Grand Canyon. Minor rockslides were reported. (Ref. 8, 343.)

1950. Jan. 17 (Jan. 16). Apache County, Ariz. South of the Ganado Trading Post, several cracks formed in the ground. A 6.5-m-high offset fissure occurred in the creek bed beneath a timber bridge (near lat $35.55^{\circ} \mathrm{N}$., long $109.10^{\circ} \mathrm{W}$.). (Ref. $23,38,343$.)

1959. July 21. Arizona-Utah border. Minor damage to chimneys and walls was reported at Fredonia, Ariz., and Kanab, Utah, about $15 \mathrm{~km}$ north of Fredonia. In addition, windows broke in houses and stores and dishes fell from shelves at Fredonia. Almost all merchandise was shaken from shelves in stores. A rockslide at Mather Point in the Grand Canyon was attributed to the shock. (Ref. 32, 265.)

1969. Dec. 25. Southern Arizona. The tremor broke dishes and windows in the Globe-Miami area, east of Phoenix in Gila County. Hundreds of residents were awakened by the strong shaking. Walls were cracked on the San Carlos Reservation. (Ref. $74,343$.

1976. Feb. 4 (Feb. 3). Western Arizona. Slight damage occurred in Chino Valley, Cottonwood, and Miller Valley (a suburb of Prescott). At Chino Valley, one ceiling beam loosened slightly at the Buckaroo Shopping Center, and plaster separated from the ceiling. Waves on the ground were reported by one observer in the area. Water became muddy in a well. At Miller Valley, small cracks formed in the west wall at a supermarket. Magnitude 5.2 $\mathrm{M}_{\mathrm{L}}$ PAS. (Ref. $49,74$.

1976. Feb. 23. Western Arizona. Plaster was cracked at Chino Valley, about $25 \mathrm{~km}$ north of Prescott. (Ref. 49, 74.) 


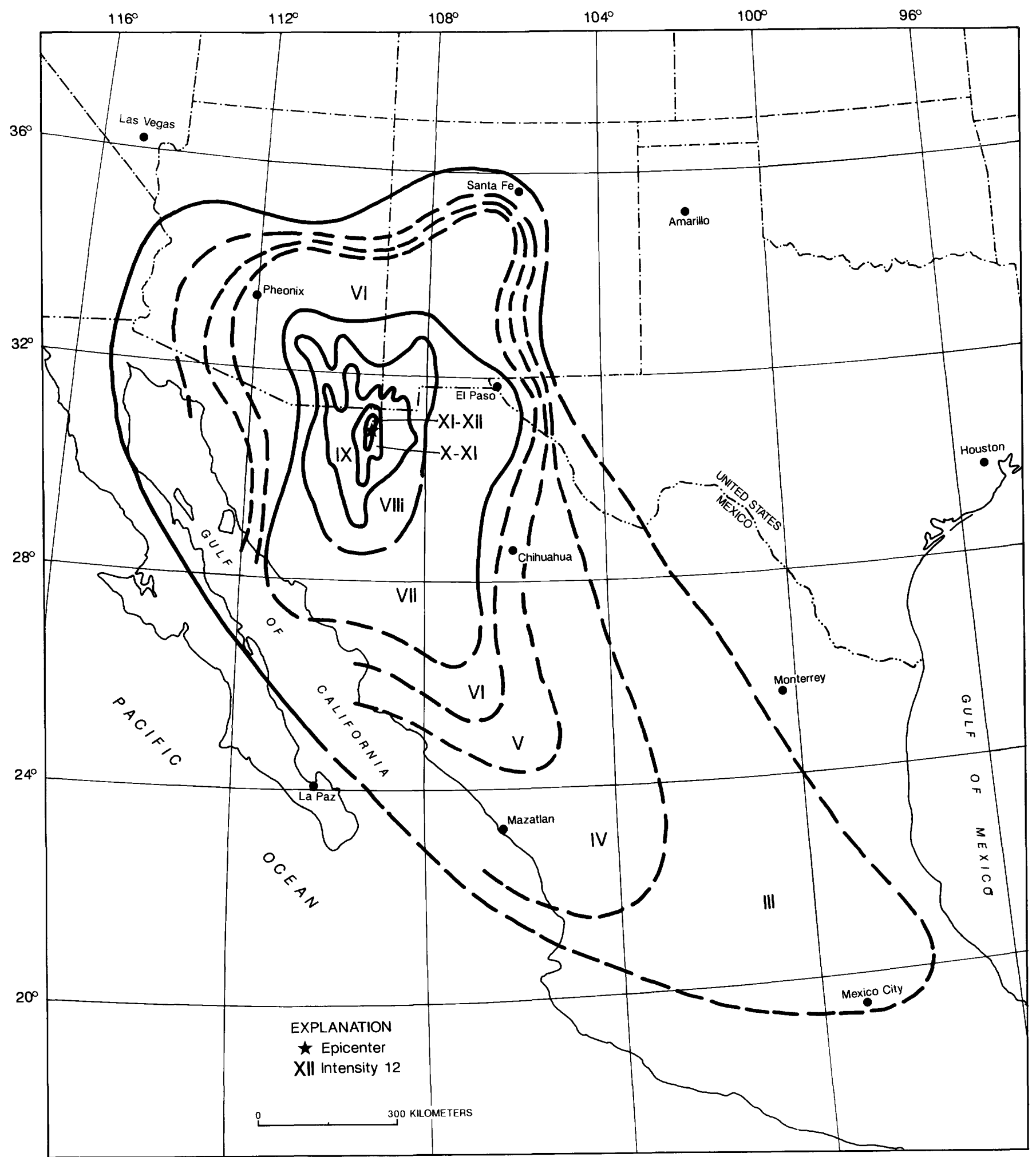

FIGURE 11.-Isoseismal map for the Sonora, Mexico, earthquake of May 3, 1887, which caused damage in the United States. This map is a simplified version of the map in reference 343 of table 1 . 


\section{ARKANSAS}

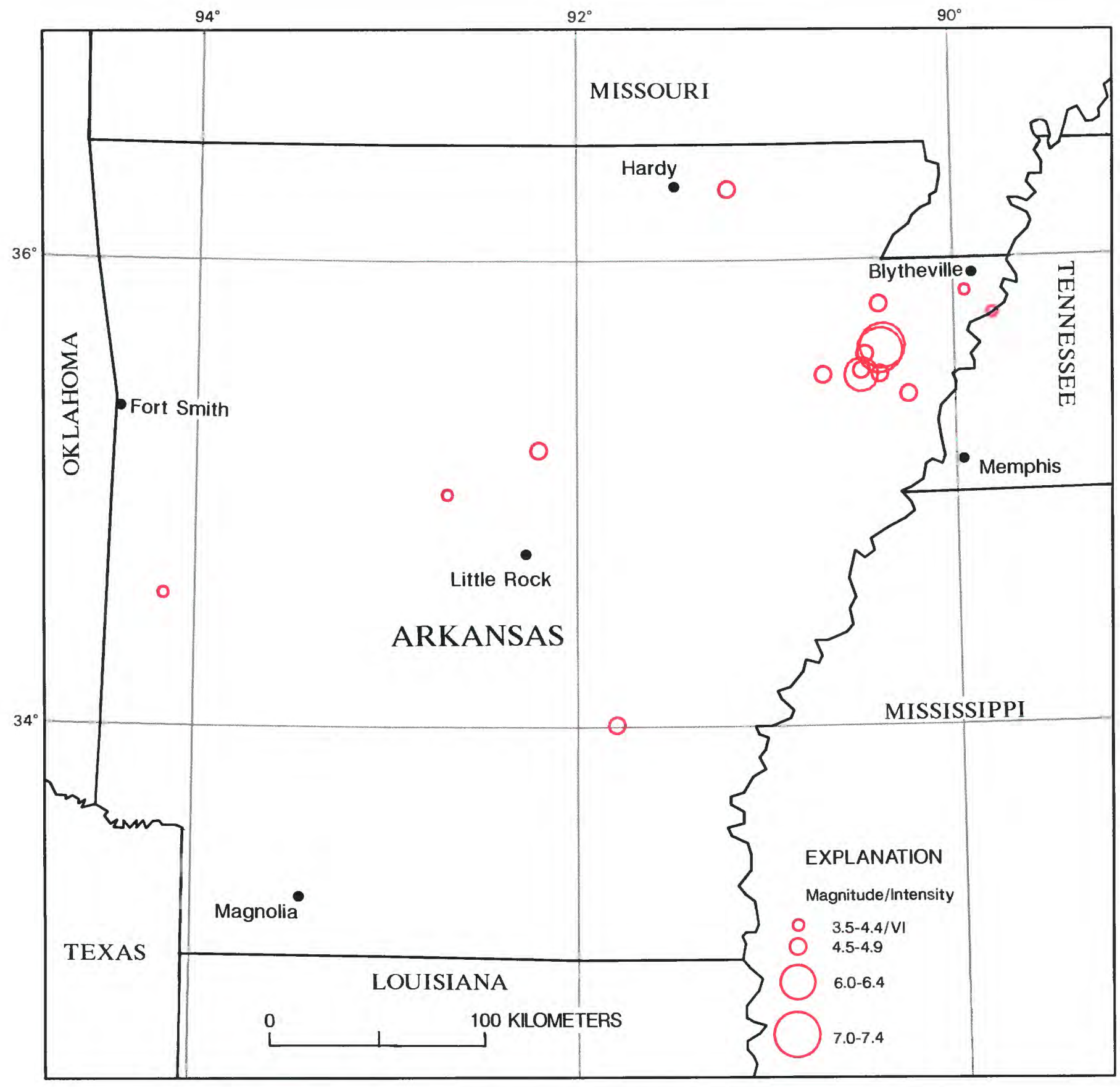

Earthquakes in Arkansas with magnitudes $\geq 4.5$ or intensity $\geq$ VI. 
ARKANSAS

[See table 1 for hypocenter and intensity references and table 2 for definitions of magnitude source codes. Leader (--) indicates information is not available]

\begin{tabular}{|c|c|c|c|c|c|c|c|c|c|c|c|c|c|}
\hline & & Origin & & Hypocent & & & & & Magnitude & & Inter & sity & \\
\hline & Date & time (UTC) & Latitude & Longitude & Depth & Ref & & & Other & Moment & MMI & Ref & Felt area \\
\hline $\mathrm{Yr}_{\mathrm{r}}$ & Mo Da & $\mathrm{h} m \mathrm{~s}$ & $\left({ }^{\circ}\right)$ & $\left({ }^{\circ}\right)$ & $(\mathrm{km})$ & & $m_{b}$ & $M_{s}$ & & $\mathbf{M}$ & & & $\left(1,000 \mathrm{~km}^{2}\right)$ \\
\hline 1811 & 1216 & 0815 & $35.6 \mathrm{~N}$ & $90.4 \mathrm{~W}$ & - & 114 & - & - & $7.20 \mathrm{M}_{\mathrm{fa}} \mathrm{NU}$ & $7.68 \mathrm{NLI}$ & XI & 114 & 5000 \\
\hline 1811 & 1216 & 1415 & $35.6 \mathrm{~N}$ & $90.4 \mathrm{~W}$ & - & 345 & - & - & $7.00 \mathrm{M}_{\mathrm{fa}}$ SEE & - & $\mathbf{X}$ & 529 & - \\
\hline 1843 & 0105 & 0245 & $35.5 \mathrm{~N}$ & $90.5 \mathrm{~W}$ & - & 113 & - & - & $6.00 \mathrm{M}_{\mathrm{fa}} \mathrm{NTI}$ & - & VII & 113 & 1500 \\
\hline 1843 & $02 \quad 17$ & 05 & $35.5 \mathrm{~N}$ & $90.5 \mathrm{~W}$ & - & 113 & - & - & $4.80 \mathrm{M}_{\mathrm{fa}} \mathrm{NTI}$ & - & $\mathbf{V}$ & 113 & 250 \\
\hline 1878 & 1119 & 0552 & $35.5 \mathrm{~N}$ & $90.7 \mathrm{~W}$ & - & 529 & - & - & $4.90 \mathrm{M}_{\mathrm{fa}} \mathrm{NTI}$ & - & VI & 529 & 350 \\
\hline 1883 & 1205 & 1520 & $36.3 \mathrm{~N}$ & $91.2 \mathrm{~W}$ & - & 105 & - & - & $4.80 \mathrm{M}_{\mathrm{fa}} \mathrm{BAR}$ & - & V & 105 & 250 \\
\hline 1911 & $03 \quad 31$ & 1657 & $34.0 \mathrm{~N}$ & $91.8 \mathrm{~W}$ & - & 529 & - & - & $4.70 \mathrm{M}_{\mathrm{fa}} \mathrm{SC}$ & - & VII & 529 & 99 \\
\hline 1923 & 1028 & 1710 & $35.5 \mathrm{~N}$ & $90.4 \mathrm{~W}$ & - & 105 & - & - & $4.80 \mathrm{M}_{\mathrm{fa}} \mathrm{SC}$ & - & VII & 105 & 120 \\
\hline 1927 & 0507 & 0828 & $35.8 \mathrm{~N}$ & $90.4 \mathrm{~W}$ & - & 218 & - & - & $4.80 \mathrm{M}_{\mathrm{fa}} \mathrm{BAR}$ & - & VI & 529 & 100 \\
\hline 1938 & $\begin{array}{ll}09 & 17\end{array}$ & 033428.3 & $35.413 \mathrm{~N}$ & $90.254 \mathrm{~W}$ & 001 & 349 & - & - & $4.80 \mathrm{M}_{\mathrm{fa}} \mathrm{DG}$ & 一 & V & 105 & 250 \\
\hline 1956 & 0129 & 044415.5 & $35.756 \mathrm{~N}$ & $89.803 w$ & 016 & 349 & - & - & $4.00 \mathrm{M}_{\mathrm{fa}} \mathrm{DG}$ & - & VI & 29 & 13 \\
\hline 1969 & 0101 & 233538.7 & $34.991 \mathrm{~N}$ & $92.688 W$ & 007 & 349 & 4.2 & - & $4.40 M_{n}$ DG & 4.33STT & VI & 42 & 62 \\
\hline 1970 & 1117 & 021354.1 & $35.856 \mathrm{~N}$ & $89.947 W$ & 014 & 349 & 3.6 & - & $4.30 \mathrm{M}_{\mathrm{n}} \mathrm{DG}$ & 4.00STT & VI & 43 & 92 \\
\hline 1976 & 0325 & 004120.8 & $35.585 \mathrm{~N}$ & $90.478 \mathrm{~W}$ & 017 & 349 & 4.9 & - & $4.90 \mathrm{M}_{\mathrm{n}} \mathrm{DG}$ & 4.61HRN & VI & 49 & 280 \\
\hline 1977 & 0602 & 232910.6 & $34.560 \mathrm{~N}$ & $94.172 W$ & 010 & 349 & 4.3 & - & $3.60 \mathrm{M}_{\mathrm{n}} \mathrm{DG}$ & - & VI & 39 & 4 \\
\hline 1982 & 0121 & 003354.8 & $35.18 \mathrm{~N}$ & $92.21 \mathrm{~W}$ & 003 & 350 & 4.5 & - & $4.70 \mathrm{M}_{\mathrm{n}}$ TUL & 3.86SRT & VI & 350 & 31 \\
\hline
\end{tabular}

[Reference (Ref.) numbers given in parentheses at the end of each description refer to sources of data in table 1. Magnitude values are described in the Introduction, and codes are defined in table 2.]

1811. Dec. 16, 0815 UTC. Northeast Arkansas. On the basis of the large area of damage $\left(600,000 \mathrm{~km}^{2}\right)$, the widespread area of perceptibility $\left(5,000,000 \mathrm{~km}^{2}\right)$, and the complex physiographic changes that occurred, the Mississippi River valley earthquakes of 1811-12 rank as some of the largest in the United States since its settlement by Europeans. The area of strong shaking associated with these shocks is two to three times larger than that of the 1964 Alaska earthquake and 10 times larger than that of the 1906 San Francisco earthquake.

The magnitudes of these series of earthquakes, usually named the New Madrid, Mo., earthquakes, vary considerably between the $m_{b}$ and $M_{S}$ values estimated by Nuttli (ref. 114, 569). The $\mathrm{m}_{\mathrm{b}}$ (or $\mathrm{M}_{\mathrm{fa}}$ as listed above) was estimated from isoseismal maps, and the $\mathrm{M}_{\mathrm{S}}$ (or $\mathrm{M}_{\mathrm{Sn}}$ as listed below) was estimated from a spectral scaling relation by Nuttli (ref. 569) for mid-plate earthquakes. The value of $\mathrm{M}_{\mathrm{S}}$ (or $\mathrm{M}_{\mathrm{Sn}}$ ) magnitude has a functional relationship to the $m_{b}$ (or $\mathrm{M}_{\mathrm{fa}}$ ). The authors have chosen to include the $\mathrm{M}_{\mathrm{fa}}$ magnitude in the above list because it was estimated from isoseismal maps, as were most of the historical earthquakes.
The first and second earthquakes occurred in Arkansas (Dec. 16, 1811-two shocks- $-\mathrm{M}_{\mathrm{fa}} 7.2, \mathrm{M}_{\mathrm{Sn}}$ 8.5 and $\mathrm{M}_{\mathrm{fa}} 7.0, \mathrm{M}_{\mathrm{Sn}} 8.0$ ) and the third and fourth in Missouri (Jan. 23, 1812, $\mathrm{M}_{\mathrm{fa}} 7.1, \mathrm{M}_{\mathrm{Sn}}$ 8.4; and Feb. 7, $1812, \mathrm{M}_{\mathrm{fa}}$ 7.4, $\mathrm{M}_{\mathrm{Sn}}$ 8.8). Otto Nuttli (ref. 330), however, has postulated another strong earthquake in Arkansas on Dec. 16 at 1800 UTC ( $\mathrm{M}_{\text {Sn }}$ 8.0). This would make a total of five earthquakes of magnitude $\mathrm{M}_{\mathrm{Sn}} 8.0$ or higher occurring in the period Dec. 16, 1811 through Feb. 7, 1812. Ref. 330 was published shortly after the death of Otto Nuttli, but his sources of data were never published; therefore, source data on the fifth earthquake are not included in the Arkansas table.

The first earthquake caused only slight damage to man-made structures, mainly because of the sparse population in the epicentral area. The extent of the area that experienced damaging earth motion (MMI $\geq \mathrm{VII}$ ) is estimated to be $600,000 \mathrm{~km}^{2}$. However, shaking strong enough to alarm the general population (MM intensity $\geq \mathrm{V}$ ) occurred over an area of 2.5 million $\mathrm{km}^{2}$ (see generalized isoseismal map, fig. 12). This map covers an area from Canada to New Orleans, La., and from the headwaters of the Mississippi River to the Atlantic Ocean.

At the onset of the earthquake the ground rose and fell-bending the trees until their branches intertwined and opening deep cracks in the ground. 


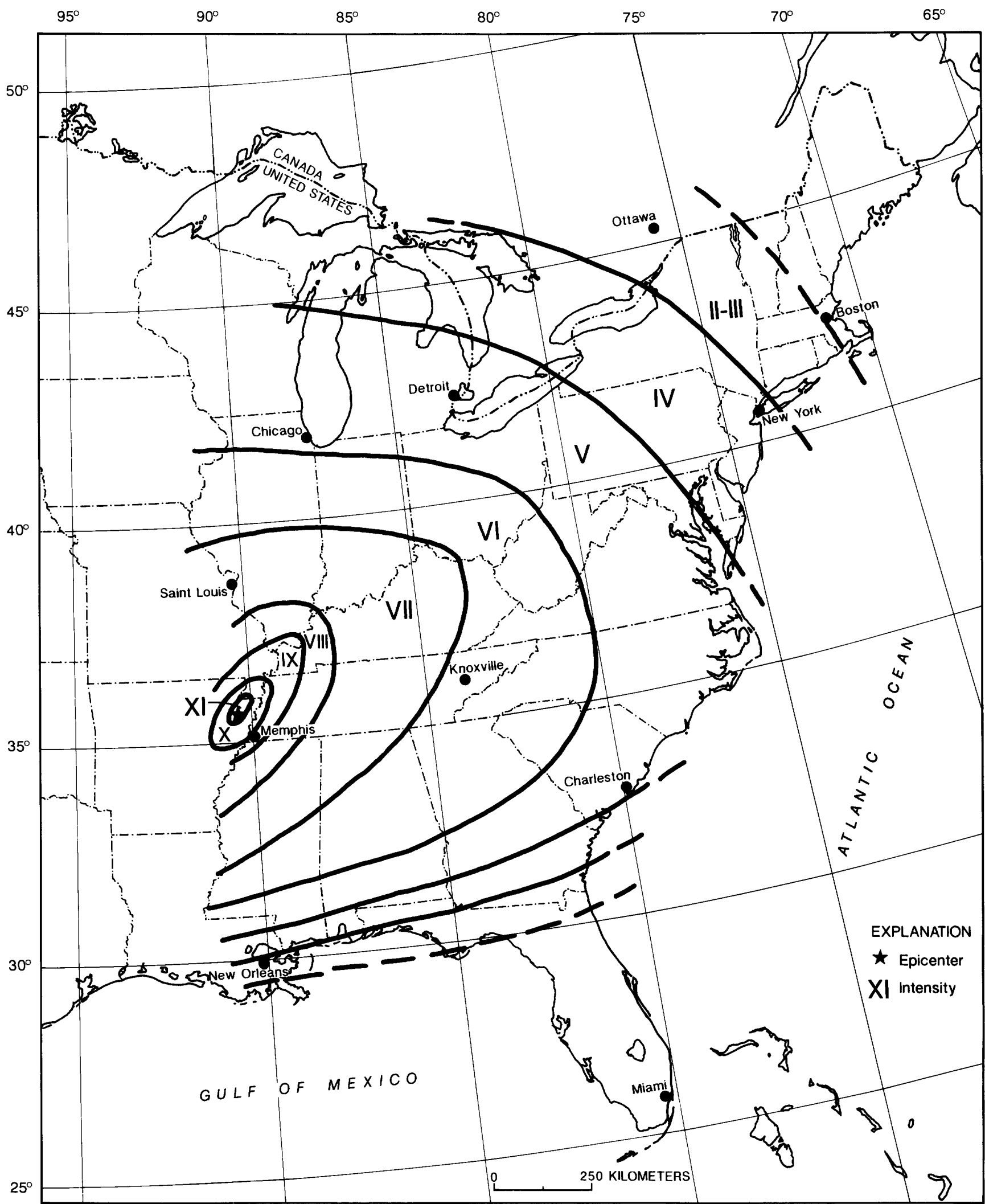

FIGURE 12.-Isoseismal map for the Arkansas earthquake of December 16, 1811, 08:15 UTC (first of the 1811-1812 New Madrid series). This map is a simplified version of figure 1 in reference 114 and figure 2 in reference 557 of table 1. 
Landslides swept down the steeper bluffs and hillsides; large areas of land were uplifted; and still larger areas sank and were covered with water that emerged through fissures or craterlets. Huge waves on the Mississippi River overwhelmed many boats and washed others high on the shore. High banks caved and collapsed into the river; sand bars and points of islands gave way; whole islands disappeared. Surface rupturing did not occur, however. The region most seriously affected was characterized by raised or sunken lands, fissures, sinks, sand blows, and large landslides that covered an area of $78,000-129,000 \mathrm{~km}^{2}$, extending from Cairo, Ill., to Memphis, Tenn., and from Crowleys Ridge to Chickasaw Bluffs, Tenn.

Although the motion during the first shock was violent at New Madrid, Mo., it was not as heavy and destructive as that caused by two aftershocks about 6 hours later. Only one life was lost in falling buildings at New Madrid, but chimneys were toppled and log cabins were thrown down or were heavily damaged. Chimneys also were downed as far distant as Cincinnati, Ohio; St. Louis, Mo.; and in many places in Kentucky, Missouri, and Tennessee.

The Lake County uplift, about $50 \mathrm{~km}$ long and 23 $\mathrm{km}$ wide, upwarps the Mississippi River valley as much as $10 \mathrm{~m}$ in parts of southwest Kentucky, southeast Missouri, and northwest Tennessee. The uplift apparently resulted from vertical movement along several, ancient, subsurface structures; most of this uplift has occurred during earthquakes. The Lake County uplift can be subdivided into several topographic bulges, including Tiptonville dome, Ridgely Ridge, and the south end of Sikeston Ridge. A strong correlation exists between modern seismicity and the uplift, indicating that stresses that produced the uplift still exist today.

Tiptonville dome, which is $14 \mathrm{~km}$ in width and about $11 \mathrm{~km}$ in length, shows the largest upwarping and the highest topographic relief on the uplift. It is bounded on the east by Reelfoot scarp, which has a zone of normal faults (displacement about $3 \mathrm{~m}$ ) at its base. Although most of Tiptonville dome formed between 200 and 2,000 years ago, additional uplifting deformed the northwest and southeast parts of the dome during the earthquakes of 1811-12.

A notable area of subsidence is Reelfoot Lake in Tennessee, just east of Tiptonville dome. Subsidence there ranged from 1.5 to $6 \mathrm{~m}$, although larger amounts were reported. It may be that the lake was enlarged by compaction, upwarping, and subsidence occurring simultaneously during the New Madrid earthquakes.
Other areas subsided by as much as $5 \mathrm{~m}$, although 1.5 to $2.5 \mathrm{~m}$ was more common. Lake St. Francis, in eastern Arkansas, which was formed by subsidence, is $64 \mathrm{~km}$ long by $1 \mathrm{~km}$ wide. Coal and sand were ejected from fissures in the swamp land adjacent to the St. Francis River, and the water level is reported to have risen there by 8 to $9 \mathrm{~m}$.

Large waves were generated on the Mississippi River by fissures opening and closing below the surface. Local uplifts of the ground and water waves moving upstream gave the illusion that the river was flowing upstream. Ponds of water also were agitated noticeably.

Otto Nuttli (ref. 330) reported that more than 200 moderate to large earthquakes occurred on the New Madrid fault between Dec. 16, 1811, and Mar. 15, 1812 ( 5 of $\mathrm{M}_{\mathrm{S}}$ about $7.7 ; 10$ of $\mathrm{M}_{\mathrm{S}}$ about $6.7 ; 35$ of $\mathrm{M}_{\mathrm{S}}$ about 5.9; 65 of $\mathrm{M}_{\mathrm{S}}$ about 5.3 ; and 89 of $\mathrm{M}_{\mathrm{S}}$ about 4.3). Nuttli also noted that about 1,800 earthquakes of $m_{b}$ about 3.0 to 4.5 occurred in that same period. Magnitude of main shock $8.5 \mathrm{M}_{\mathrm{Sn}}$ NLI. (Ref. 38, 114, $301,330,529,558$.

1811. Dec. 16, 1415 UTC. Northeast Arkansas. On the basis of the effects reported at the same locations, the MM intensity of this earthquake has been inferred to be similar to that of the earlier shock at 0815 UTC (see description above). Thus, the inference is that, if the documented intensities are the same or are similar at identical locations, then the maximum intensities at the epicenter must be about the same; therefore, the intensity at the epicenter of this earthquake must be at the MM intensity X-XI level. The maximum documented intensity for both earthquakes on Dec. 16, 1811, is MM intensity VIII at Richmond, Ky. Magnitude $8.0 \mathrm{M}_{\mathrm{Sn}}$ STT. (Ref. 345, 529.)

1843. Jan. 5 (Jan. 4). Northeast Arkansas. This earthquake is the strongest to occur in this region since the 1811-12 sequence. Damage was severe at Memphis, Tenn., where walls cracked, windows broke, and one building collapsed. The earth sank in places near New Madrid, Mo., and unconfirmed reports state that a lake was formed and several hunters were drowned. Chimneys were thrown down at Helena, Ark., and Hickman, Ky. The earthquake was felt on the seacoast of Georgia and the Carolinas and northeastward to Providence, R.I., a distance of $1,400 \mathrm{~km}$. The southern limit of the felt area appears to have included Natchez, Miss.; the western limit passed beyond the frontier military posts; and the northern limit reached Iowa and Indiana. Magnitude 6.3 $\mathrm{M}_{\mathrm{S}}$ NTL, 6.0 $\mathrm{M}_{\mathrm{fa}}$ NTI. (Ref. 38, $109,113,529$. 

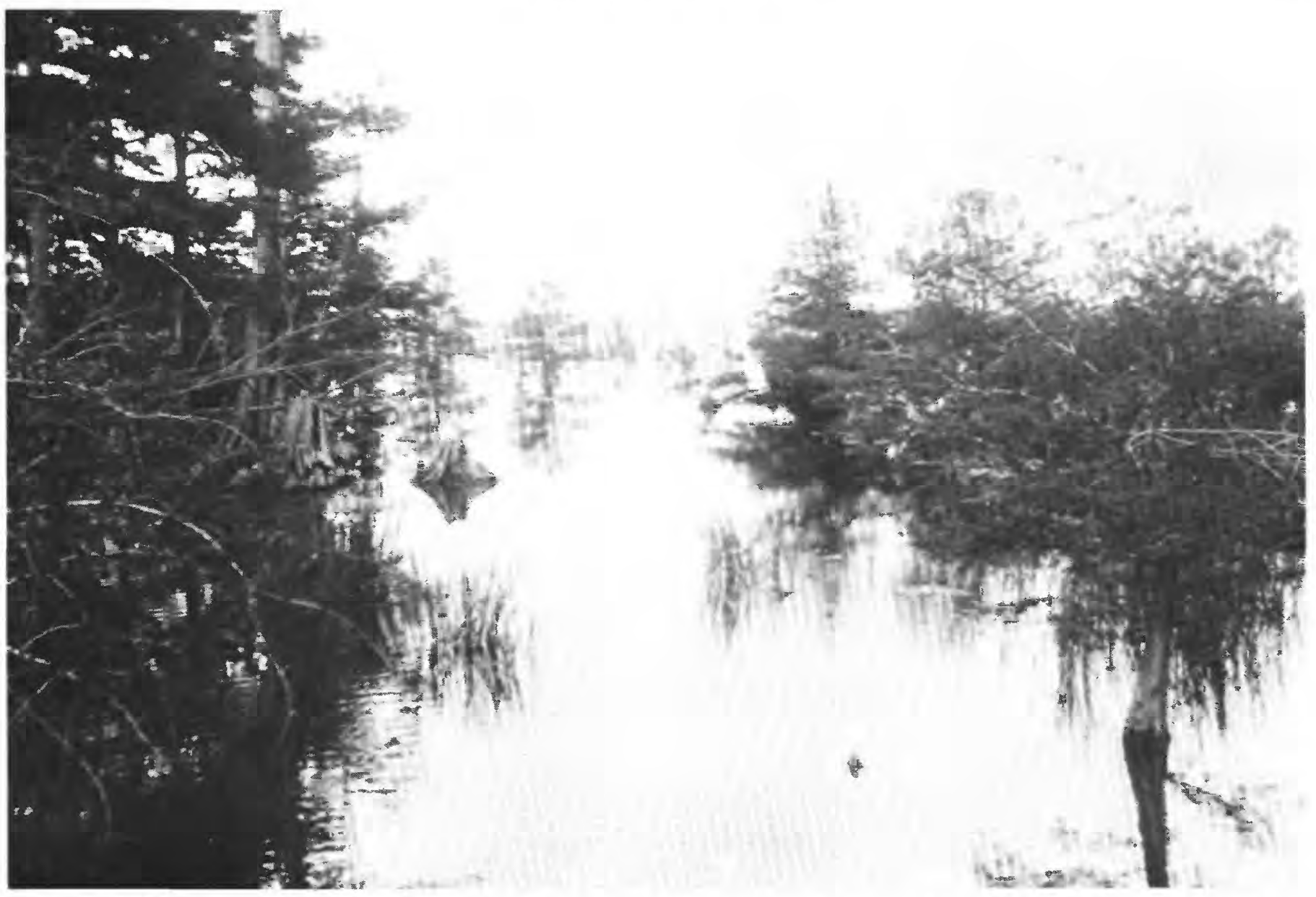

Lower end of Reelfoot Lake, Tennessee, showing trunks of trees killed when the land was submerged by the December 16, 1811, northeast Arkansas earthquake. (Photograph by M.L. Fuller taken about 100 years after the earthquake.)

1878. Nov. 19 (Nov. 18). Arkansas. The earthquake was severe east of Kansas City along the Missouri River from Lexington to Glasgow, Mo., but the maximum disturbance occurred along the Mississippi River valley between Cairo, Ill., and Memphis, Tenn. Northwest of Memphis, several stone buildings were damaged in Stone County at Batesville, Ark., and the tops of chimneys were toppled in Poinsett County at Harrisburg, Ark.; loose bricks fell from chimneys at Cairo, Ill. Felt in Alabama, Arkansas, Illinois, Kansas, Kentucky, Missouri, and Tennessee. Magnitude $4.9 \mathrm{M}_{\mathrm{fa}}$ NTI. (Ref. 38, 105, 109, 529.)

1911. Mar. 31. Near Rison and Warren, Ark. The earthquake cracked walls, broke windows and dishes, and sent people rushing into the streets at Pine Bluff, Ark., south of Little Rock in Jefferson County. Walls cracked at the 14th Avenue school at Pine Bluff, and plaster fell on the pupils. Minor damage also occurred at Argenta, Dumas, Monticello, Rison, and Warren, Ark. The shock was felt throughout southeast Arkansas, northeast Louisiana, and along the Mississippi River from Memphis, Tenn., to Vicksburg, Miss. The felt area generally was confined to the soft sediments of the Mississippi River valley. Magnitude $4.3 \mathrm{M}_{\mathrm{fa}} \mathrm{BAR}, 4.2 \mathrm{M}_{\mathrm{fa}} \mathrm{SG}$. (Ref. 38, 105, 109, 529.)

1923. Oct. 28. Marked Tree, Ark. This shock downed several old chimneys, shattered windows, and cracked walls at Marked Tree (Poinsett County), about $70 \mathrm{~km}$ northwest of Memphis, Tenn. It reportedly disturbed the surface of the St. Francis River. Also felt in Illinois, Kentucky, Mississippi, Missouri, and Tennessee. Magnitude 4.5 $\mathrm{M}_{\mathrm{fa}}$ BAR. (Ref. 38, 105, 529.)

1927. May 7. Between Jonesboro and Marked Tree, Ark. At least one chimney was damaged at Jonesboro, and windowpanes and dishes were broken in the Memphis, Tenn., area. In the area of Reelfoot Lake, Tenn., the earthquake left "gravel pilings." Felt from Decatur, Ala., to Carbondale, Ill., and from Pocahontas, Ark., to Jackson, Tenn. Also reported in 
Mississippi and Missouri. Magnitude $4.8 \mathrm{M}_{\mathrm{fa}}$ BAR. (Ref. 38, 105, 109, 218, 529.)

1956. Jan. 29 (Jan. 28). Arkansas-Tennessee border. A few chimneys and walls were cracked at Covington, Tenn., northeast of Memphis. Also felt in Arkansas. Magnitude $4.1 \mathrm{M}_{\mathrm{fa}} \mathrm{BAR}, 4.0 \mathrm{M}_{\mathrm{fa}} \mathrm{DG}$. (Ref. 29, 349.)

1969. Jan. 1. Central Arkansas. Walls and floors cracked and dishes broke at Little Rock, Ark. Felt in northern and central Arkansas, southern Missouri, and at Memphis, Tenn. Magnitude $4.5 \mathrm{M}_{\mathrm{n}}$ BAR, 3.3 $\mathrm{MS}_{\mathrm{S}}$ BAR. (Ref. 42, 349.)

1970. Nov. 17 (Nov. 16). Northeast Arkansas. Plaster cracked and fell at Manila (Mississippi County), about $28 \mathrm{~km}$ west of the epicenter. At Keiser, about $27 \mathrm{~km}$ south of Manila, the shock shifted furniture, cracked plaster, and caused three electrical wall outlets to burn their wires. Also felt in Illinois, Kentucky, Mississippi, Missouri, and Tennessee. Magnitude $4.4 \mathrm{M}_{\mathrm{n}}$ BAR, $2.9 \mathrm{M}_{\mathrm{S}}$ BAR, $4.4 \mathrm{~m}_{\mathrm{b}}$ NUT, $2.9 \mathrm{M}_{\mathrm{S}}$ NUT. (Ref. 43, 263, 349.)

1976. March 25 (Mar. 24). Northeast Arkansas. Slight damage characterized by cracks in plaster and drywall, downed ceiling tiles, and broken windows occurred in several towns in Arkansas, Mississippi, Missouri, and Tennessee. Also felt in Alabama, Illinois, Indiana, and Kentucky. Magnitude 5.0 $\mathrm{M}_{\mathrm{n}}$ BAR. (Ref. 49,349 .)

1977. June 2. Western Arkansas. Chimneys and exterior walls were cracked at Board Camp; foundations and sidewalks were cracked at Hatfield. Both towns are in Polk County, near the Oklahoma border. Magnitude 4.0 $\mathrm{M}_{\mathrm{n}} \mathrm{SLM}, 4.63 \mathrm{M}$ JOH. (Ref. 39, 349.)

1982. Jan. 21 (Jan. 20). Area of Enola-Naylor, Faulkner County, Ark. This earthquake is the strongest of a swarm of more than 30,000 shocks that began in the area on Jan. 12, 1982. Several of the shocks were felt in the region. Leveling lines run by the Arkansas State Surveyor show an uplift of about $0.2 \mathrm{~m}$ in the area of the epicenters. On Highway 36, west of Naylor, hairline cracks formed in a concrete cellar, and tiles fell off a tile-lined well. In addition, a fireplace and sheetrock walls sustained cracks. Also felt in Mississippi and Missouri. Magnitude $4.5 \mathrm{M}_{\mathrm{n}}$ TEC. (Ref. 350, 511.) 


\section{CALIFORNIA}

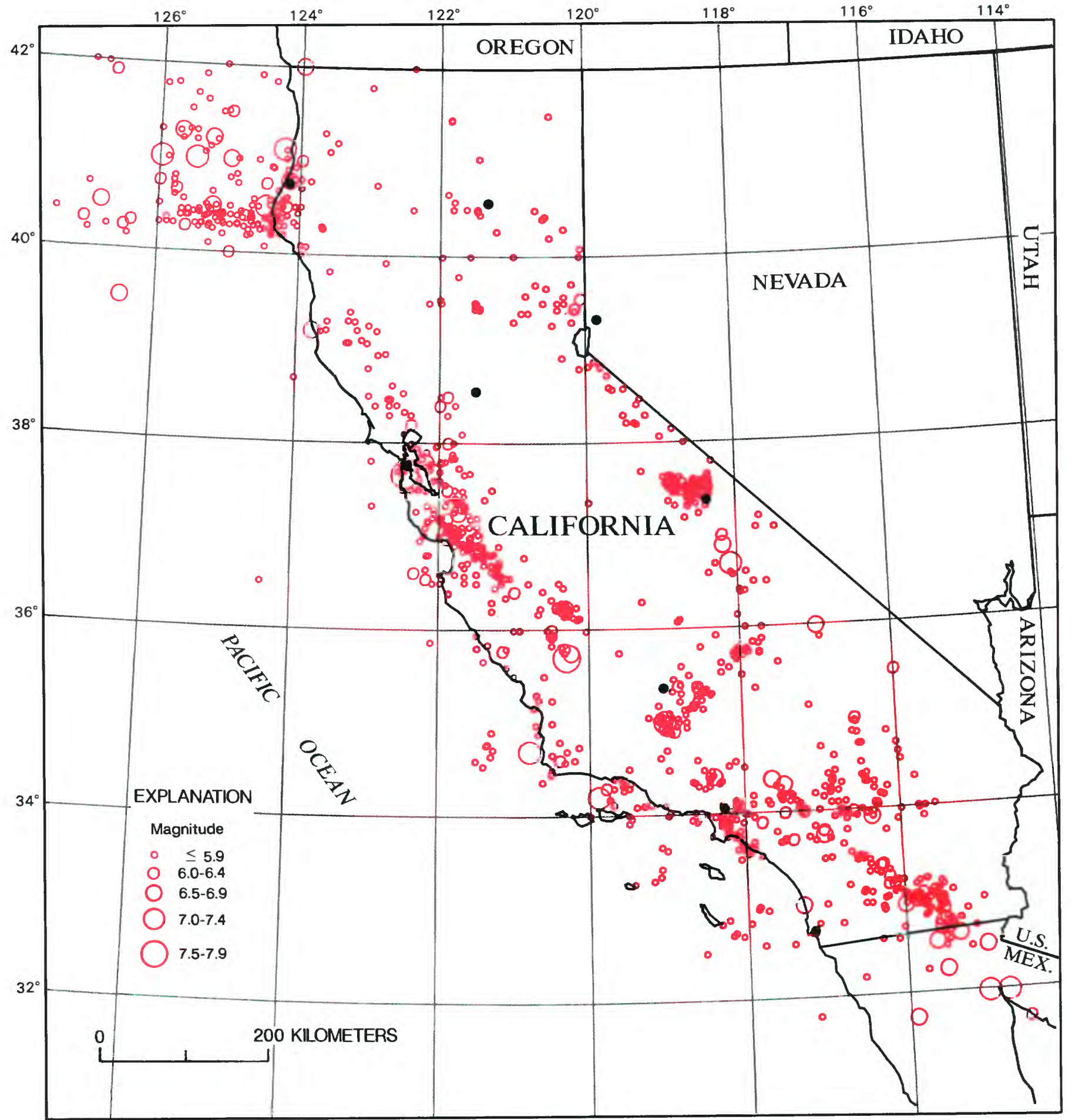

Earthquakes in California with magnitudes $\geq 4.5$ or intensity $\geq$ VI. 
CALIFORNIA

[See table 1 for hypocenter and intensity references and table 2 for definitions of magnitude source codes. \&, land area only; \#, land area only in the United States for an earthquake near a coastline; +, land area in the United States when the felt area did not extend to the coast; @, felt area is less than $1,000 \mathrm{~km}^{2}$. Leader (-) indicates information is not available]

\begin{tabular}{|c|c|c|c|c|c|c|c|c|c|c|c|c|c|c|c|c|}
\hline \multirow{3}{*}{\multicolumn{4}{|c|}{$\begin{array}{cc} & \text { Origin } \\
\text { Date } & \text { time (urc) }\end{array}$}} & \multicolumn{6}{|c|}{ Hypocenter } & \multicolumn{4}{|c|}{ Magnitude } & \multicolumn{2}{|c|}{ Intensity } & \multirow{3}{*}{$\begin{array}{l}\text { Felt area } \\
\left(1,000 \mathrm{~km}^{2}\right)\end{array}$} \\
\hline & & & & \multirow{2}{*}{\multicolumn{2}{|c|}{$\begin{array}{l}\text { Latitudo } \\
\left.{ }^{\circ}\right)\end{array}$}} & \multirow{2}{*}{\multicolumn{2}{|c|}{$\begin{array}{l}\text { Longitude } \\
\left({ }^{\circ}\right)\end{array}$}} & \multirow{2}{*}{$\begin{array}{l}\text { Depth } \\
(\mathrm{km})\end{array}$} & \multirow[t]{2}{*}{ Ref } & \multicolumn{2}{|c|}{ USGS } & \multirow[t]{2}{*}{ Other } & \multirow{2}{*}{$\begin{array}{c}\text { Moment } \\
\text { M }\end{array}$} & \multirow[t]{2}{*}{ MMI } & \multirow[t]{2}{*}{ Ref } & \\
\hline & & & & & & & & & & $m_{b}$ & $\mathbf{M}_{\mathbf{S}}$ & & & & & \\
\hline 1769 & 07 & 28 & & 33.9 & $\mathbf{N}$ & 117.8 & W & - & 368 & - & - & $6.00 \mathrm{M}_{\mathrm{La}} \mathrm{ELL}$ & - & VIII & 493 & - \\
\hline 1770 & & & & 34.5 & $\mathrm{~N}$ & 118.0 & W & - & 493 & - & - & - & - & Felt & 493 & - \\
\hline 1790 & & & & 37.5 & $\mathbf{N}$ & 118.8 & w & - & 368 & - & - & - & - & Felt & 493 & - \\
\hline 1800 & 10 & 11 & & 36.9 & $\mathbf{N}$ & 121.6 & W & - & 368 & - & - & - & - & VII & 368 & - \\
\hline 1800 & 11 & 22 & 2130 & 33.0 & $\mathbf{N}$ & 117.3 & W & - & 368 & - & - & $6.50 \mathrm{M}_{\mathrm{La}} \mathrm{DMG}$ & - & VII & 38 & - \\
\hline 1803 & 04 & & & 34.5 & $\mathbf{N}$ & 118.0 & w & - & 493 & - & - & - & - & Felt & 493 & - \\
\hline 1803 & 05 & 25 & & 32.8 & $\mathbf{N}$ & 117.1 & w & - & 368 & - & - & - & - & VI & 56 & - \\
\hline 1806 & 03 & 25 & 0800 & 34.4 & $\mathbf{N}$ & 119.7 & W & - & 368 & - & - & - & - & VI & 368 & - \\
\hline 1808 & 06 & 21 & & 37.8 & $\mathbf{N}$ & 122.5 & W & - & 368 & - & - & $6.00 \mathrm{M}_{\mathrm{La}}$ ELL & - & VIII & 368 & - \\
\hline 1812 & & & & 37.5 & $\mathrm{~N}$ & 122.5 & W & - & 493 & - & - & - & - & Felt & 493 & - \\
\hline 1812 & 05 & & & 33.7 & $\mathbf{N}$ & 117.9 & W & - & 493 & - & - & - & - & Felt & 493 & - \\
\hline 1812 & 12 & 08 & 1500 & 34.37 & $\mathbf{N}$ & 117.65 & W & - & 521 & - & - & $6.90 \mathrm{M}_{\mathrm{La}} \mathrm{DMG}$ & - & VIII & 368 & - \\
\hline 1812 & 12 & 21 & 1900 & 34.2 & $\mathbf{N}$ & 119.9 & W & - & 368 & - & - & $7.10 \mathrm{M}_{\mathrm{La}} \mathrm{DMG}$ & - & VIII & 368 & - \\
\hline 1821 & 01 & 01 & & 32.8 & $\mathbf{N}$ & 117.1 & W & - & 493 & - & - & - & - & Felt & 493 & - \\
\hline 1827 & 09 & 24 & 0400 & 34.0 & $\mathbf{N}$ & 119.0 & W & - & 368 & - & - & $5.50 \mathrm{M}_{\mathrm{La}} \mathrm{DMG}$ & - & VI & 368 & - \\
\hline 1829 & 09 & & & 37.5 & $\mathbf{N}$ & 122.5 & W & - & 56 & - & - & - & - & VII & 56 & - \\
\hline 1830 & & & & 35.5 & $\mathbf{N}$ & 120.6 & W & - & 368 & - & - & - & - & VII & 368 & - \\
\hline 1836 & 04 & 25 & 13 & 36.5 & $\mathbf{N}$ & 122.0 & W & - & 56 & - & - & - & - & Felt & 493 & - \\
\hline 1836 & 06 & 10 & 1530 & 37.8 & $\mathbf{N}$ & 122.2 & W & - & 368 & - & - & $6.80 \mathrm{M}_{\mathrm{La}} \mathrm{DMG}$ & - & VIII & 368 & - \\
\hline 1838 & 06 & & & 37.6 & $\mathbf{N}$ & 122.4 & W & - & 368 & - & - & $7.00 \mathrm{M}_{\mathrm{La}} \mathrm{DMG}$ & 一 & VIII & 368 & - \\
\hline 1840 & 01 & 16 & & 36.5 & $\mathbf{N}$ & 122.1 & $\mathbf{w}$ & - & 56 & - & - & - & - & VI & 56 & - \\
\hline 1841 & 07 & 03 & 2207 & 36.6 & $\mathbf{N}$ & 122.0 & w & - & 368 & - & - & - & - & VI & 368 & - \\
\hline 1849 & 09 & 17 & & 33.0 & $\mathbf{N}$ & 116.5 & W & - & 56 & - & - & - & - & Felt & 493 & - \\
\hline 1849 & 09 & 22 & 23 & 33.0 & $\mathbf{N}$ & 116.5 & W & - & 56 & - & - & - & - & Felt & 493 & - \\
\hline 1851 & 05 & 15 & 1610 & 37.8 & $\mathbf{N}$ & 122.4 & W & - & 368 & - & - & - & - & VI & 38 & - \\
\hline 1852 & 11 & 23 & 07 & 37.5 & $\mathbf{N}$ & 122.4 & W & - & 56 & - & - & - & - & Felt & 493 & - \\
\hline 1852 & 11 & 29 & 2000 & 32.5 & $\mathbf{N}$ & 115.0 & w & - & 521 & - & - & $6.50 \mathrm{M}_{\mathrm{La}} \mathrm{DMG}$ & - & IX & 368 & 260 \\
\hline 1852 & 12 & 17 & & 35.5 & $\mathbf{N}$ & 121.0 & w & - & 56 & - & - & - & - & VI & 56 & - \\
\hline 1853 & 02 & 01 & 2100 & 35.6 & $\mathbf{N}$ & 121.1 & W & - & 368 & - & - & - & - & VI & 368 & - \\
\hline 1853 & 10 & 23 & 1100 & 40.8 & $\mathbf{N}$ & 124.2 & w & - & 368 & - & - & - & - & VI & 368 & - \\
\hline 1855 & 01 & 25 & 0600 & 39.5 & $\mathbf{N}$ & 120.3 & w & - & 368 & - & - & $5.50 \mathrm{M}_{\mathrm{La}} \mathrm{DMG}$ & - & VI & 56 & - \\
\hline 1855 & 03 & 20 & 0030 & 40.75 & $\mathbf{N}$ & 124.20 & $\mathbf{w}$ & - & 56 & - & - & - & - & $\mathbf{V}$ & 56 & - \\
\hline 1855 & 07 & 11 & 0415 & 34.1 & $\mathbf{N}$ & 118.1 & W & - & 368 & - & - & $6.00 \mathrm{M}_{\mathrm{La}} \mathrm{ELL}$ & - & VIII & 38 & - \\
\hline 1855 & 08 & 27 & 1100 & 38.1 & $\mathbf{N}$ & 122.5 & W & - & 368 & - & - & $4.90 \mathrm{M}_{\mathrm{La}} \mathrm{DMG}$ & - & VI & 368 & - \\
\hline 1856 & 01 & 02 & 1815 & 37.5 & $\mathbf{N}$ & 122.5 & w & - & 368 & - & - & $5.30 \mathrm{M}_{\mathrm{La}} \mathrm{DMG}$ & - & VI & 368 & - \\
\hline 1856 & 02 & 15 & 1325 & 37.5 & $\mathbf{N}$ & 122.3 & W & - & 368 & - & - & $5.50 \mathrm{M}_{\mathrm{La}} \mathrm{DMG}$ & - & VII & 368 & - \\
\hline 1856 & 09 & 21 & 0730 & 33.0 & $\mathbf{N}$ & 117.0 & w & - & 368 & - & - & - & - & VI & 368 & - \\
\hline 1857 & 01 & 09 & 1624 & 35.7 & $\mathbf{N}$ & 120.3 & w & - & 521 & - & - & $7.60 \mathrm{M}_{\mathrm{La}} \mathrm{DMG}$ & $7.92 \mathrm{HK}$ & IX & 379 & $290 \#$ \\
\hline 1858 & 11 & 26 & 0835 & 37.5 & $\mathbf{N}$ & 121.9 & W & - & 368 & - & - & $6.10 \mathrm{M}_{\mathrm{La}} \mathrm{DMG}$ & - & VII & 38 & - \\
\hline 1858 & 12 & 16 & 0230 & 34.0 & $\mathrm{~N}$ & 117.5 & $\mathbf{w}$ & - & 368 & - & - & - & - & $\overline{V I}$ & 368 & - \\
\hline 1858 & 12 & 16 & 1000 & 34.0 & $\mathbf{N}$ & 117.5 & W & - & 521 & - & - & $6.00 \mathrm{M}_{\mathrm{La}} \mathrm{ELL}$ & - & VIII & 368 & - \\
\hline 1859 & 10 & 05 & 2016 & 37.8 & $\mathbf{N}$ & 122.4 & W & - & 368 & - & - & - & - & VI & 368 & - \\
\hline 1860 & 11 & 13 & 0000 & 40.8 & $\mathbf{N}$ & 124.2 & w & - & 368 & - & - & - & - & VII & 368 & - \\
\hline 1861 & 07 & 04 & 0011 & 37.8 & $\mathbf{N}$ & 122.0 & w & - & 368 & - & - & $5.60 \mathrm{M}_{\mathrm{La}} \mathrm{DMG}$ & - & VIII & 38 & - \\
\hline 1862 & 05 & 27 & 2000 & 32.7 & $\mathbf{N}$ & 117.2 & W & - & 368 & - & - & $5.90 \mathrm{M}_{\mathrm{La}} \mathrm{DMG}$ & - & VII & 368 & $45 \&$ \\
\hline 1863 & 12 & 19 & 2238 & 37.5 & $\mathbf{N}$ & 122.2 & w & - & 368 & - & - & $4.80 \mathrm{M}_{\mathrm{La}} \mathrm{DMG}$ & - & V & 368 & 23 \\
\hline 1864 & 02 & 26 & 1347 & 37.1 & $\mathbf{N}$ & 121.7 & $\mathbf{W}$ & - & 368 & - & - & $5.90 \mathrm{M}_{\mathrm{La}} \mathrm{DMG}$ & - & VI & 368 & - \\
\hline 1864 & 03 & 05 & 1649 & 37.7 & $\mathbf{N}$ & 122.0 & $\mathbf{W}$ & - & 368 & - & - & $5.70 \mathrm{M}_{\mathrm{La}} \mathrm{DMG}$ & - & VI & 38 & - \\
\hline
\end{tabular}


CALIFORNIA-Continued

[See table 1 for hypocenter and intensity references and table 2 for definitions of magnitude source codes. \&, land area only; \#, land area only in the United States for an earthquake near a coastline; +, land area in the United States when the felt area did not extend to the coast; @, felt area is less than $1,000 \mathrm{~km}^{2}$. Leader (--) indicates information is not available]

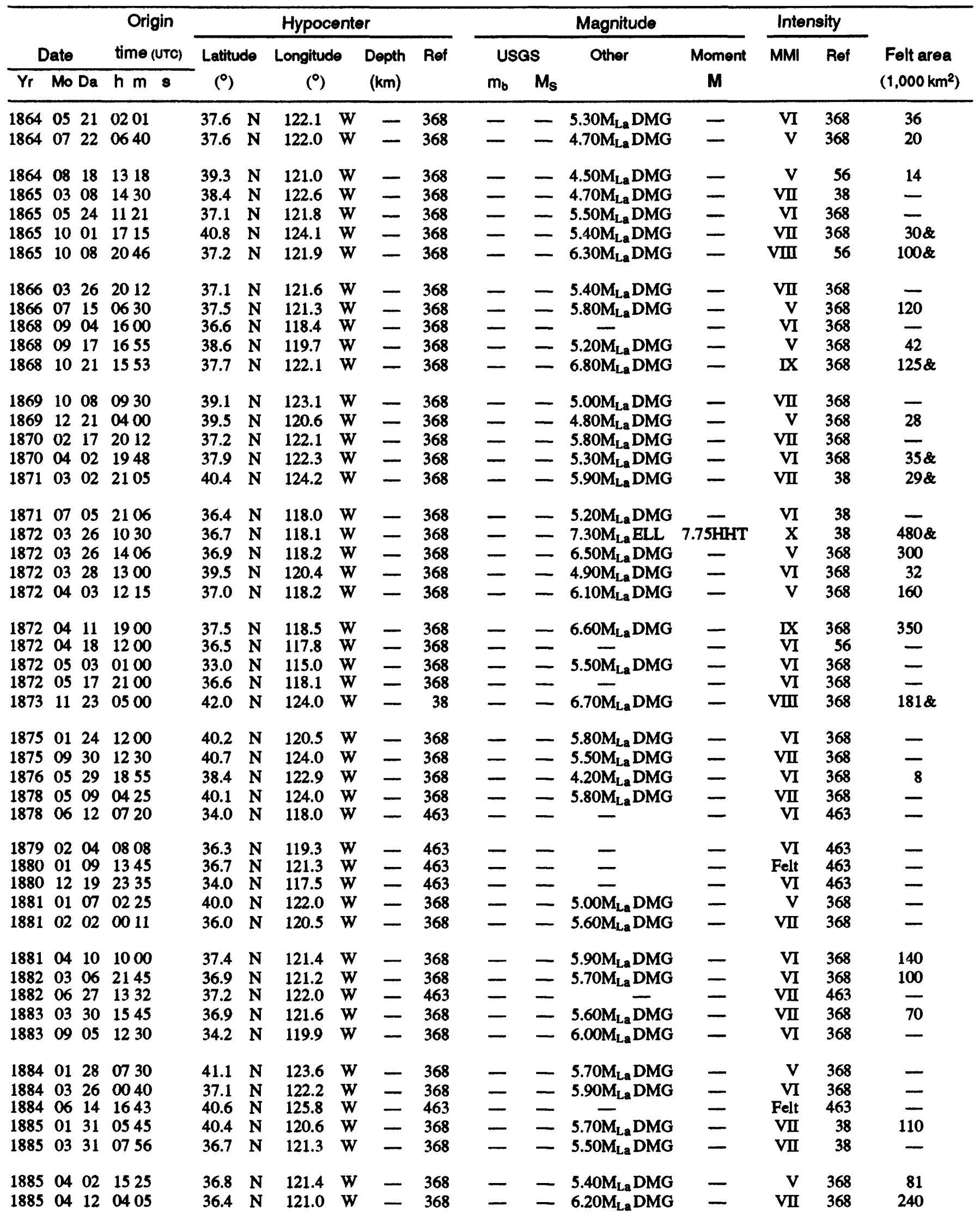


CALIFORNLA-Continued

[See table 1 for hypocenter and intensity references and table 2 for definitions of magnitude source codes. \&, land area only; \#, land area only in the United States for an earthquake near a coastline; + , land area in the United States when the felt area did not extend to the coast; $\Theta$, felt area is less than $1,000 \mathrm{~km}^{2}$. Leader (--) indicates information is not available]

\begin{tabular}{|c|c|c|c|c|c|c|c|c|c|c|c|c|c|c|c|c|}
\hline & & & & & & Hypoc & ente & & & & & Magnitude & & Inte & sity & \\
\hline & Date & & time (UTC) & Latituc & & Longitud & & Depth & Ref & & & Other & Moment & MMI & Ref & Felt area \\
\hline Yr & Mo & $\mathrm{Da}$ & $h \mathrm{~m} s$ & $\left(^{\circ}\right)$ & & $\left({ }^{\circ}\right)$ & & $(\mathrm{km})$ & & $m_{b}$ & $M_{s}$ & & $\mathbf{M}$ & & & $\left.1,000 \mathrm{~km}^{2}\right)$ \\
\hline $\begin{array}{l}1885 \\
1886\end{array}$ & $\begin{array}{l}08 \\
04\end{array}$ & $\begin{array}{l}01 \\
14\end{array}$ & $\begin{array}{l}0010 \\
0320\end{array}$ & $\begin{array}{l}38.8 \\
37.2\end{array}$ & $\begin{array}{l}\mathbf{N} \\
\mathbf{N}\end{array}$ & $\begin{array}{l}123.0 \\
117.7\end{array}$ & $\begin{array}{l}\text { W } \\
\text { W }\end{array}$ & - & $\begin{array}{r}368 \\
54\end{array}$ & $=$ & - & - & - & $\begin{array}{l}\text { VI } \\
\text { VI }\end{array}$ & $\begin{array}{r}368 \\
54\end{array}$ & - \\
\hline 1887 & 12 & 03 & 1855 & 39.3 & $\mathbf{N}$ & 123.6 & $\mathbf{W}$ & - & 368 & - & - & $4.90 \mathrm{M}_{\mathrm{La}} \mathrm{DMG}$ & 一 & VII & 368 & - \\
\hline 1888 & 02 & 18 & 1050 & 39.2 & $\mathbf{N}$ & 123.6 & $\mathbf{w}$ & - & 368 & - & - & $4.90 \mathrm{M}_{\mathrm{La}} \mathrm{DMG}$ & - & V & 368 & - \\
\hline 1888 & 02 & 29 & 2250 & 38.3 & $\mathrm{~N}$ & 122.7 & $\mathbf{W}$ & 一 & 368 & - & - & $4.20 \mathrm{M}_{\mathrm{La}} \mathrm{DMG}$ & - & $\mathbf{V I}$ & 368 & 9 \\
\hline 1888 & 04 & 14 & 0330 & 41.5 & $\mathbf{N}$ & 120.5 & W & - & 368 & - & - & $4.60 \mathrm{M}_{\mathrm{La}} \mathrm{DMG}$ & - & VI & 368 & - \\
\hline 1888 & 04 & 29 & 0448 & 39.7 & $\mathbf{N}$ & 120.7 & $\mathbf{W}$ & - & 368 & - & - & $5.90 \mathrm{M}_{\mathrm{La}} \mathrm{DMG}$ & - & VII & 38 & - \\
\hline 1888 & 09 & 17 & 1151 & 37.1 & $\mathbf{N}$ & 121.8 & W & - & 368 & - & - & $4.50 \mathrm{M}_{\mathrm{La}} \mathrm{DMG}$ & - & V & 368 & 15 \\
\hline 1888 & 11 & 18 & 2228 & 37.9 & $\mathbf{N}$ & 122.3 & W & - & 368 & - & - & $4.30 \mathrm{M}_{\mathrm{La}} \mathrm{DMG}$ & - & VII & 38 & 11 \\
\hline 1889 & 02 & 07 & 0520 & 34.1 & $\mathbf{N}$ & 116.7 & W & - & 368 & - & - & $5.30 \mathrm{M}_{\mathrm{La}} \mathrm{DMG}$ & - & $\vec{v}$ & 56 & 69 \\
\hline 1889 & 04 & 15 & 0328 & 37.1 & $\mathbf{N}$ & 121.9 & $\mathbf{W}$ & - & 368 & - & - & $4.80 \mathrm{M}_{\mathrm{La}} \mathrm{DMG}$ & 一 & VI & 368 & 38 \\
\hline 1889 & 05 & 19 & 1110 & 38.0 & $\mathbf{N}$ & 121.9 & W & - & 368 & - & - & $6.00 \mathrm{M}_{\mathrm{La}} \mathrm{DMG}$ & - & VII & 38 & - \\
\hline 1889 & 06 & 20 & 0600 & 40.5 & $\mathbf{N}$ & 120.7 & W & - & 368 & - & - & $5.90 \mathrm{M}_{\mathrm{La}} \mathrm{DMG}$ & - & VII & 38 & 120 \\
\hline 1889 & 07 & 31 & 1247 & 37.8 & $\mathbf{N}$ & 122.2 & W & - & 368 & - & - & $5.20 \mathrm{M}_{\mathrm{La}} \mathrm{DMG}$ & - & VII & 38 & 53 \\
\hline 1889 & 08 & 28 & 0215 & 34.1 & $\mathbf{N}$ & 117.9 & $\mathbf{W}$ & - & 368 & - & - & $5.20 \mathrm{M}_{\mathrm{La}} \mathrm{DMG}$ & - & VI & 38 & 52 \\
\hline 1889 & 09 & 30 & 0520 & 37.2 & $\mathbf{N}$ & 118.7 & $\mathbf{w}$ & - & 368 & - & - & $5.60 \mathrm{M}_{\mathrm{La}} \mathrm{DMG}$ & - & V & 56 & 92 \\
\hline 1890 & 02 & 09 & 1206 & 33.4 & $\mathbf{N}$ & 116.3 & $\mathbf{W}$ & - & 368 & - & - & $6.30 \mathrm{M}_{\mathrm{La}} \mathrm{DMG}$ & $6.73 \mathrm{HK}$ & $\mathbf{V I}$ & 38 & - \\
\hline 1890 & 04 & 24 & 1136 & 36.9 & $\mathbf{N}$ & 121.6 & W & - & 368 & - & - & $6.00 \mathrm{M}_{\mathrm{La}} \mathrm{DMG}$ & - & VIII & 368 & - \\
\hline 1890 & 07 & 26 & 0940 & 40.5 & $\mathbf{N}$ & 124.2 & W & - & 368 & - & - & $6.00 \mathrm{M}_{\mathrm{La}} \mathrm{DMG}$ & - & VII & 38 & $100 \&$ \\
\hline 1891 & 01 & 02 & 2000 & 37.3 & $\mathbf{N}$ & 121.8 & $\mathbf{w}$ & - & 368 & - & - & $5.50 \mathrm{M}_{\mathrm{La}} \mathrm{DMG}$ & - & $\overline{V I}$ & 38 & $50 \&$ \\
\hline 1891 & 10 & 12 & 0628 & 38.3 & $\mathbf{N}$ & 122.4 & W & - & 368 & - & - & $5.50 \mathrm{M}_{\mathrm{La}} \mathrm{DMG}$ & - & VIII & 368 & 54 \\
\hline 1892 & 02 & 24 & 0720 & 32.55 & $\mathbf{N}$ & 115.63 & $\mathbf{W}$ & - & 521 & - & - & $6.70 \mathrm{M}_{\mathrm{La}} \mathrm{DMG}$ & 7.75HHT & VII & 368 & - \\
\hline 1892 & 04 & 19 & 1050 & 38.4 & $\mathbf{N}$ & 122.0 & W & - & 368 & - & - & $6.40 \mathrm{M}_{\mathrm{La}} \mathrm{DMG}$ & - & IX & 38 & - \\
\hline 1892 & 04 & 21 & 1743 & 38.5 & $\mathbf{N}$ & 121.9 & $\mathbf{W}$ & - & 368 & - & - & $6.20 \mathrm{M}_{\mathrm{La}} \mathrm{DMG}$ & - & $\mathbf{I X}$ & 38 & $170 \&$ \\
\hline 1892 & 04 & 30 & 0009 & 38.4 & $\mathbf{N}$ & 121.8 & $\mathbf{W}$ & - & 368 & - & - & $5.50 \mathrm{M}_{\mathrm{La}} \mathrm{DMG}$ & - & VI & 368 & - \\
\hline 1892 & 05 & 28 & 1115 & 33.2 & $\mathbf{N}$ & 116.2 & $\mathbf{W}$ & - & 368 & - & - & $6.30 \mathrm{M}_{\mathrm{La}} \mathrm{DMG}$ & - & $\mathbf{V}$ & 56 & - \\
\hline 1892 & 06 & 14 & 1325 & 34.2 & $\mathrm{~N}$ & 117.5 & $\mathbf{w}$ & - & 368 & - & - & $4.90 \mathrm{M}_{\mathrm{La}} \mathrm{DMG}$ & 一 & V & 368 & - \\
\hline 1892 & 11 & 13 & 1245 & 36.8 & $\mathbf{N}$ & 121.5 & W & - & 368 & - & - & $5.60 \mathrm{M}_{\mathrm{La}} \mathrm{DMG}$ & 一 & VI & 38 & 一 \\
\hline 1893 & 04 & 04 & 1940 & 34.3 & $\mathbf{N}$ & 118.6 & $\mathbf{w}$ & - & 368 & - & - & $5.40 \mathrm{M}_{\mathrm{La}} \mathrm{DMG}$ & - & VIII & 368 & 71 \\
\hline 1893 & 05 & 19 & 0035 & 34.1 & $\mathbf{N}$ & 119.4 & $\mathbf{W}$ & - & 368 & - & - & $5.50 \mathrm{M}_{\mathrm{La}} \mathrm{DMG}$ & - & $\mathbf{V}$ & 368 & $39 \&$ \\
\hline 1893 & 06 & 30 & 1330 & 38.0 & $\mathbf{N}$ & 122.4 & $\mathbf{W}$ & - & 368 & - & - & $4.60 \mathrm{M}_{\mathrm{La}} \mathrm{DMG}$ & 一 & V & 56 & 19 \\
\hline 1893 & 08 & 09 & 0915 & 38.4 & $\mathbf{N}$ & 122.6 & $\mathbf{W}$ & - & 368 & - & - & $5.10 \mathrm{M}_{\mathrm{La}} \mathrm{DMG}$ & - & VII & 38 & - \\
\hline 1894 & 07 & 30 & 0512 & 34.3 & $\mathbf{N}$ & 117.6 & W & - & 368 & - & - & $5.90 \mathrm{M}_{\mathrm{La}} \mathrm{DMG}$ & - & $\mathbf{V I}$ & 368 & - \\
\hline 1894 & 09 & 30 & 1736 & 40.3 & $\mathbf{N}$ & 123.7 & $\mathbf{W}$ & - & 368 & - & - & $5.80 \mathrm{M}_{\mathrm{La}} \mathrm{DMG}$ & 一 & VII & 368 & $80 \&$ \\
\hline 1894 & 10 & 23 & 2303 & 32.8 & $\mathbf{N}$ & 116.8 & $\mathbf{W}$ & - & 368 & - & - & $5.70 \mathrm{M}_{\mathrm{La}} \mathrm{DMG}$ & - & $\overline{\mathbf{V I}}$ & 368 & - \\
\hline 1896 & 08 & 17 & 1130 & 36.7 & $\mathbf{N}$ & 118.3 & $\mathbf{W}$ & - & 368 & - & - & $5.90 \mathrm{M}_{\mathrm{La}} \mathrm{DMG}$ & - & VI & 56 & - \\
\hline 1897 & 06 & 20 & 2014 & 37.0 & $\mathbf{N}$ & 121.5 & $\mathbf{W}$ & - & 38 & - & - & $6.20 \mathrm{M}_{\mathrm{La}} \mathrm{DMG}$ & 一 & VII & 38 & $130 \&$ \\
\hline 1898 & 03 & 31 & 0743 & 38.2 & $\mathbf{N}$ & 122.4 & $\mathbf{W}$ & - & 368 & - & - & $6.20 \mathrm{M}_{\mathrm{La}} \mathrm{DMG}$ & - & VIII & 368 & $120 \&$ \\
\hline 1898 & 04 & 15 & 0707 & 39.2 & $\mathbf{N}$ & 123.8 & $\mathbf{W}$ & - & 368 & - & - & $6.90 \mathrm{M}_{\mathrm{s}} \mathrm{AB2}$ & - & VIII & 38 & - \\
\hline 1899 & 04 & 16 & 1340 & 41.0 & $\mathbf{N}$ & 126.0 & $\mathbf{W}$ & - & 521 & - & - & $7.00 \mathrm{M}_{\mathrm{s}} \mathrm{ELL}$ & - & $\mathbf{V I}$ & 368 & - \\
\hline 1899 & 04 & 30 & 2241 & 36.9 & $\mathbf{N}$ & 121.7 & $\mathbf{W}$ & - & 368 & - & - & $5.60 \mathrm{M}_{\mathrm{La}}^{\circ} \mathrm{DMG}$ & - & VII & 38 & $52 \&$ \\
\hline 1899 & 06 & 02 & 0719 & 37.7 & $\mathbf{N}$ & 122.5 & $\mathbf{W}$ & - & 368 & - & - & $5.40 \mathrm{M}_{\mathrm{La}} \mathrm{DMG}$ & - & VII & 368 & - \\
\hline 1899 & 07 & 06 & 2010 & 37.2 & $\mathbf{N}$ & 121.5 & $\mathbf{W}$ & - & 368 & 一 & - & $5.80 \mathrm{M}_{\mathrm{La}} \mathrm{DMG}$ & - & VII & 56 & 98 \\
\hline 1899 & 07 & 22 & 0046 & 34.2 & $\mathbf{N}$ & 117.4 & W & - & 368 & - & - & $5.50 \mathrm{M}_{\mathrm{La}} \mathrm{DMG}$ & - & VI & 368 & 70 \\
\hline 1899 & 07 & 22 & 2032 & 34.3 & $\mathbf{N}$ & 117.5 & W & - & 368 & - & - & $6.50 \mathrm{M}_{\mathrm{La}} \mathrm{DMG}$ & 6.35HHT & VIII & 38 & - \\
\hline 1899 & 10 & 13 & 0500 & 38.4 & $\mathbf{N}$ & 122.7 & $\mathbf{W}$ & - & 368 & - & - & - & - & VII & 38 & 2 \\
\hline 1899 & 12 & 25 & 1225 & 33.8 & $\mathbf{N}$ & 117.0 & W & - & 368 & - & - & $6.40 \mathrm{M}_{\mathrm{s}}$ ELL & $6.73 \mathrm{HK}$ & $\mathbf{I X}$ & 38 & - \\
\hline 1900 & 04 & 30 & 224114 & 36.9 & $\mathbf{N}$ & 121.6 & $\mathbf{W}$ & - & 3 & - & - & $4.50 \mathrm{M}_{\mathrm{La}} \mathrm{DMG}$ & - & $\mathbf{V}$ & 56 & 30 \\
\hline
\end{tabular}


CALIFORNIA-Continued

[See table 1 for hypocenter and intensity references and table 2 for definitions of magnitude source codes. \&, land area only; \#, land area only in the United States for an earthquake near a coastline; +, land area in the United States when the felt area did not extend to the coast; @, felt area is less than $1,000 \mathrm{~km}^{2}$. Leader (-) indicates information is not available]

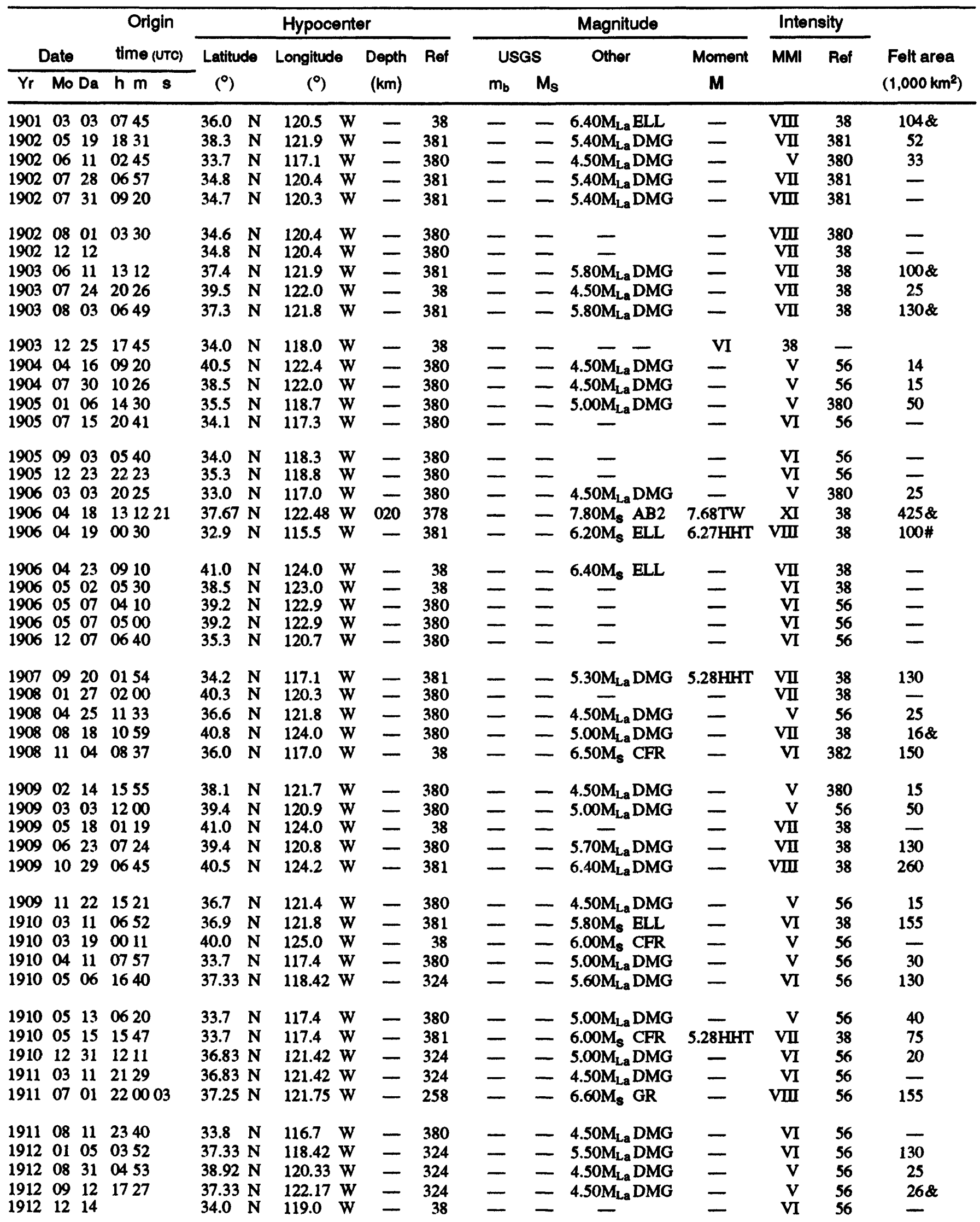


CALIFORNIA-Continued

[See table 1 for hypocenter and intensity references and table 2 for definitions of magnitude source codes. \&, land area only; \#, land area only in the United States for an earthquake near a coastline; +, land area in the United States when the felt area did not extend to the coast; @, felt area is less than $1,000 \mathrm{~km}^{2}$. Leader (--) indicates information is not available]

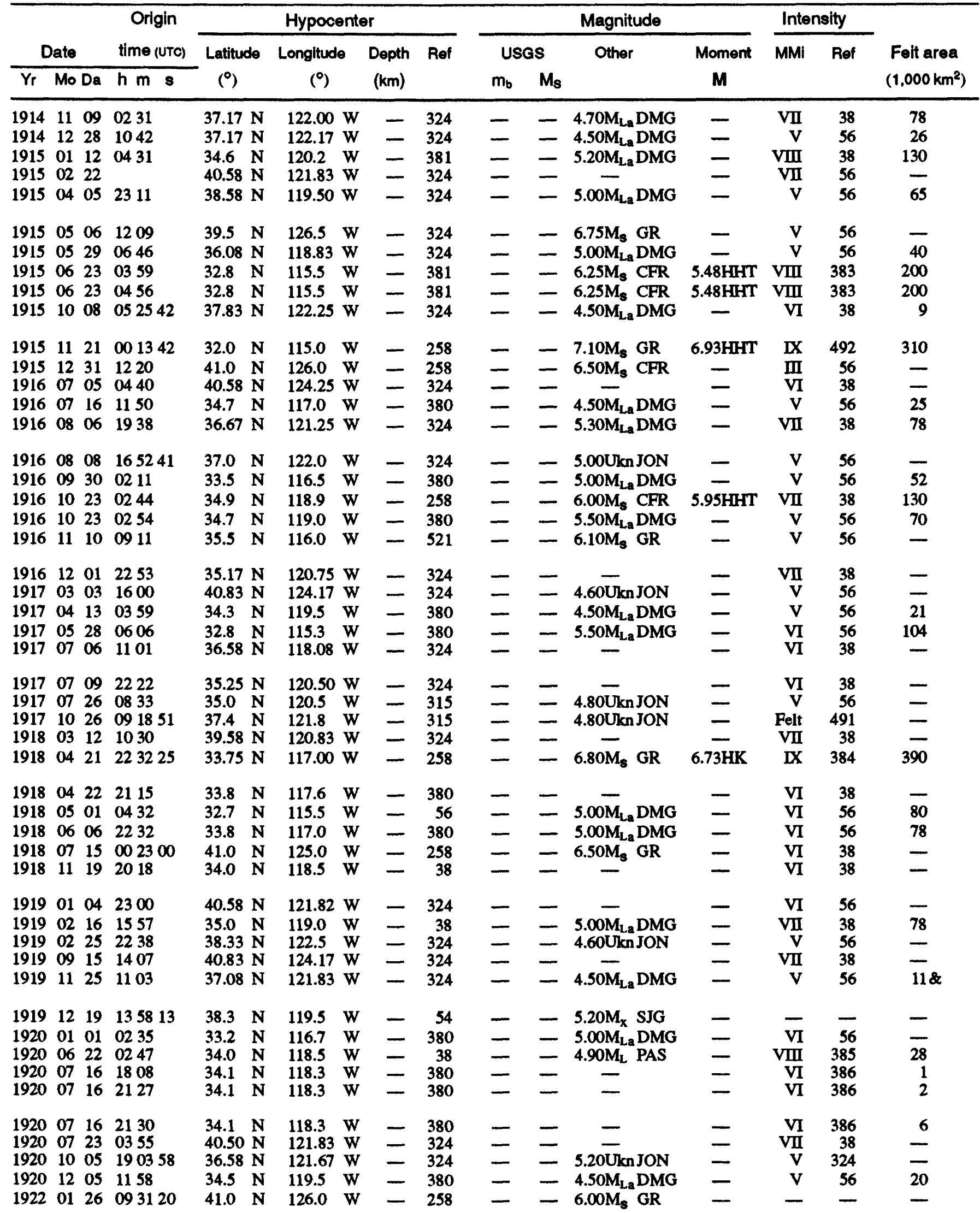


EARTHQUAKES IN CALIFORNIA

CALIFORNIA-Continued

[See table 1 for hypocenter and intensity references and table 2 for definitions of magnitude source codes. \&, land area only; \#, land area only in the United States for an earthquake near a coastline; +, land area in the United States when the felt area did not extend to the coast; @, felt area is less than $1,000 \mathrm{~km}^{2}$. Leader (--) indicates information is not available]

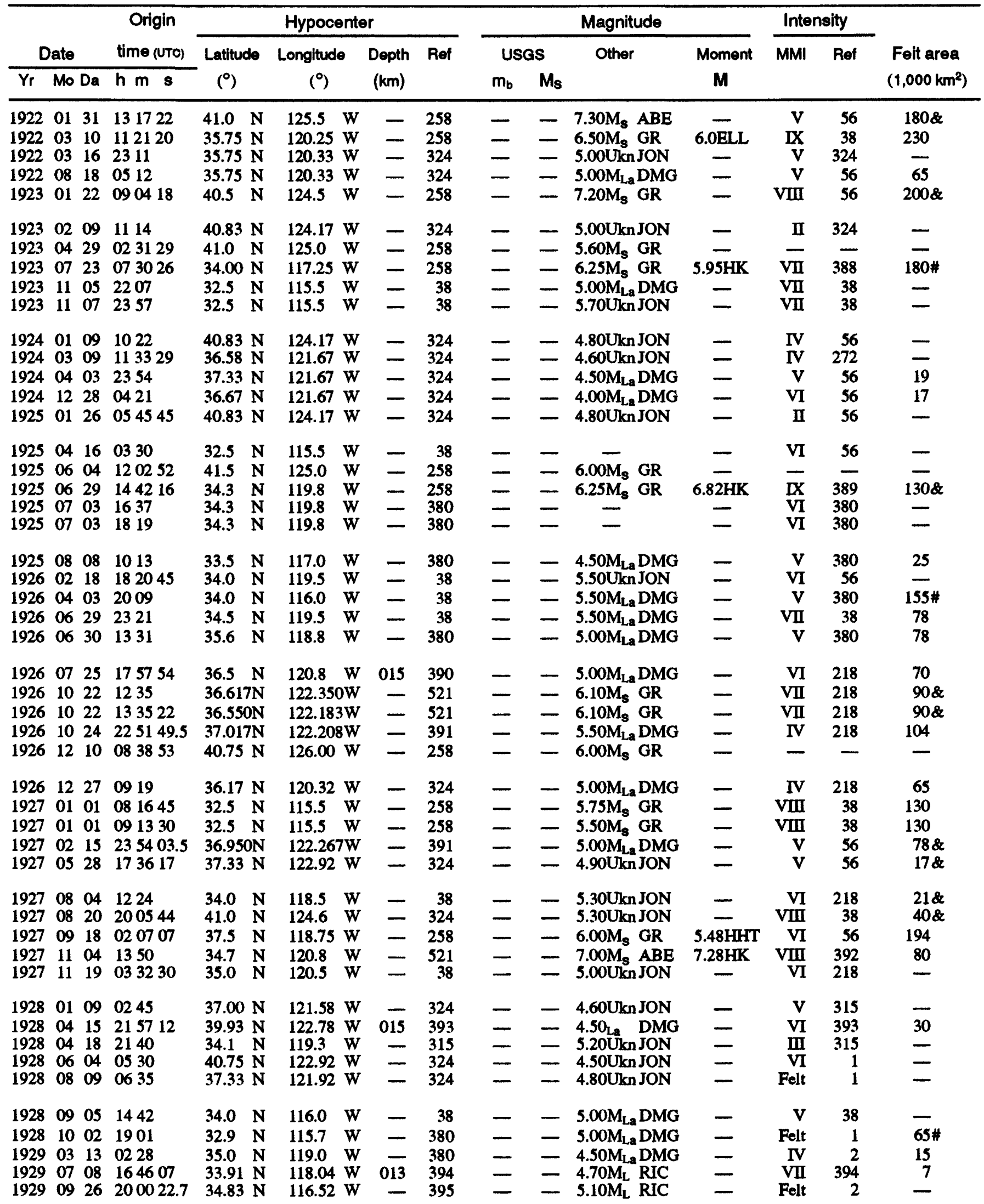


CALIFORNIA-Continued

[See table 1 for hypocenter and intensity references and table 2 for definitions of magnitude source codes. \&, land area only; \#, land area only in the United States for an earthquake near a coastline; +, land area in the United States when the felt area did not extend to the coast; @, felt area is less than $1,000 \mathrm{~km}^{2}$. Leader (--) indicates information is not available]

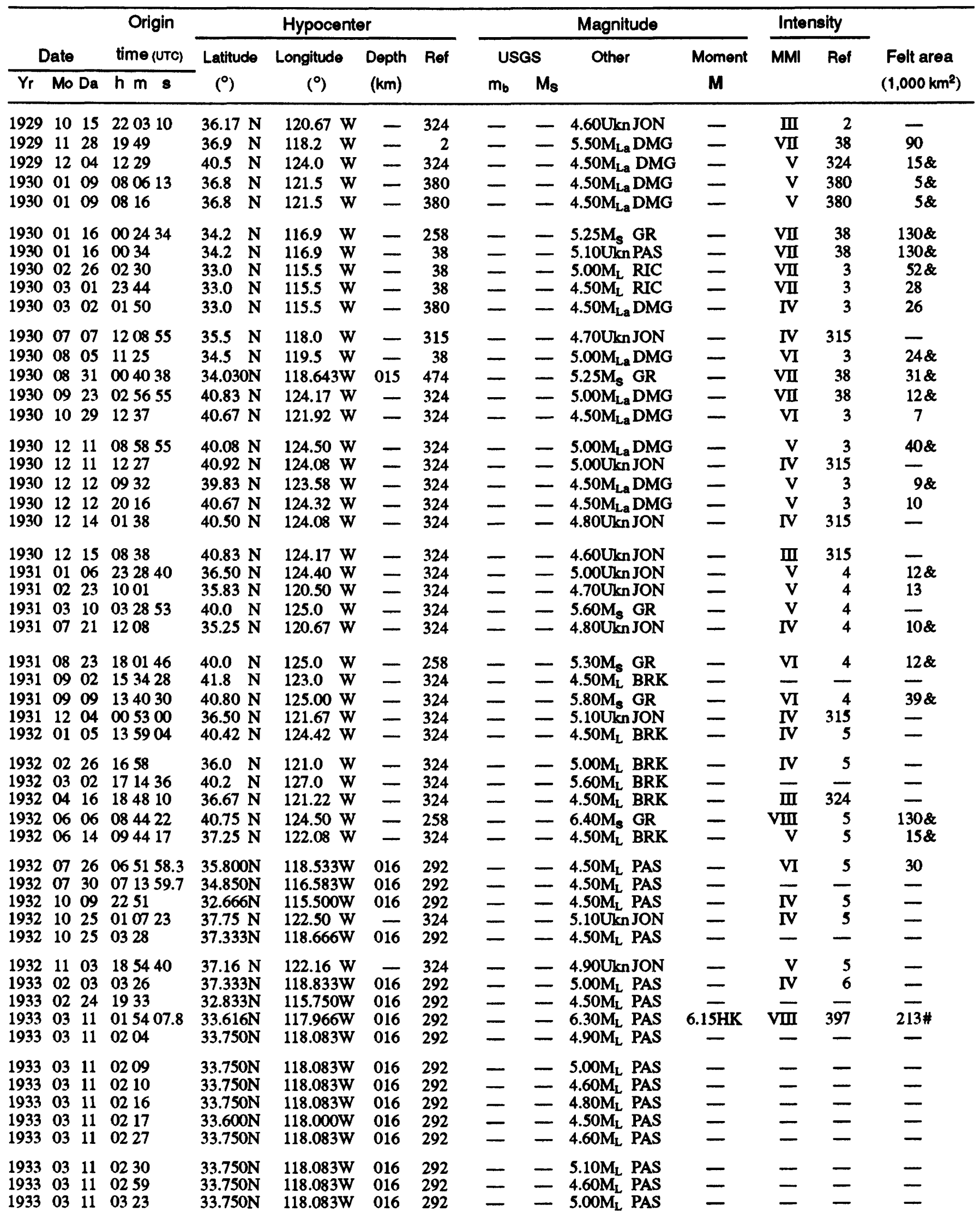


CALIFORNLA-Continued

[See table 1 for hypocenter and intensity references and table 2 for definitions of magnitude source codes. \&, land area only; \#, land area only in the United States for an earthquake near a coastline; +, land area in the United States when the felt area did not extend to the coast; @, felt area is less than $1,000 \mathrm{~km}^{2}$. Leader (-) indicates information is not available]

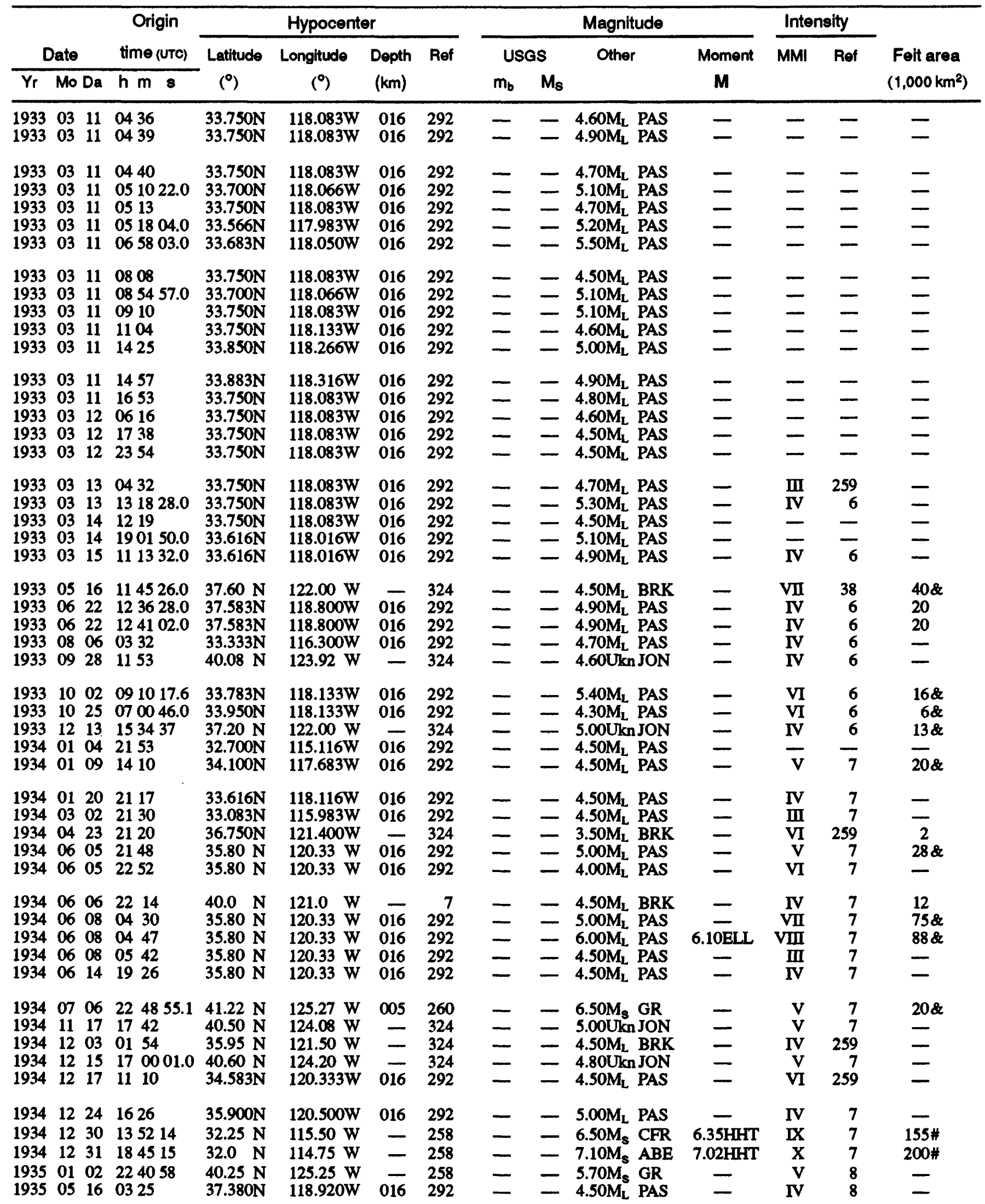


CALIFORNIA-Continued

[See table 1 for hypocenter and intensity references and table 2 for definitions of magnitude source codes. \&, land area only; \#, land area only in the United States for an earthquake near a coastline; +, land area in the United States when the felt area did not extend to the coast; @, felt area is less than 1,000 km². Leader (-) indicates information is not available]

\begin{tabular}{|c|c|c|c|c|c|c|c|c|c|c|c|c|c|c|}
\hline \multirow{2}{*}{\multicolumn{3}{|c|}{ Date }} & \multirow{2}{*}{$\begin{array}{c}\text { Origin } \\
\text { time (UTC) }\end{array}$} & \multicolumn{4}{|c|}{ Hypocenter } & \multicolumn{4}{|c|}{ Magnitude } & \multicolumn{2}{|c|}{ Intensity } & \multirow{3}{*}{$\begin{array}{l}\text { Felt area } \\
\left(1,000 \mathrm{~km}^{2}\right)\end{array}$} \\
\hline & & & & \multirow{2}{*}{$\begin{array}{l}\text { Latitude } \\
\left(^{\circ}\right)\end{array}$} & \multirow{2}{*}{$\begin{array}{l}\text { Longitude } \\
\left({ }^{\circ}\right)\end{array}$} & \multirow{2}{*}{$\begin{array}{l}\text { Depth } \\
(\mathrm{km})\end{array}$} & \multirow[t]{2}{*}{ Ref } & \multicolumn{2}{|c|}{ USGS } & \multirow[t]{2}{*}{ Other } & \multirow{2}{*}{$\begin{array}{c}\text { Moment } \\
\mathbf{M}\end{array}$} & \multirow[t]{2}{*}{ MMI } & \multirow[t]{2}{*}{ Ref } & \\
\hline $\mathrm{Yr}$ & Mol & $\mathrm{Da}$ & $h \mathrm{~m} s$ & & & & & $m_{b}$ & $\mathbf{M}_{\mathbf{s}}$ & & & & & \\
\hline $\begin{array}{l}1935 \\
1935 \\
1935 \\
1935 \\
1935\end{array}$ & $\begin{array}{l}06 \\
06 \\
07 \\
09 \\
09\end{array}$ & $\begin{array}{l}03 \\
19 \\
13 \\
03 \\
08\end{array}$ & $\begin{array}{l}1708 \\
0955 \\
105416.5 \\
0647 \\
1440\end{array}$ & $\begin{array}{l}41.00 \mathrm{~N} \\
37.250 \mathrm{~N} \\
34.200 \mathrm{~N} \\
34.033 \mathrm{~N} \\
32.900 \mathrm{~N}\end{array}$ & $\begin{array}{l}124.00 \mathrm{~W} \\
118.500 \mathrm{~W} \\
117.900 \mathrm{~W} \\
117.316 \mathrm{~W} \\
115.216 \mathrm{~W}\end{array}$ & $\begin{array}{l}-\overline{016} \\
016 \\
016 \\
016\end{array}$ & $\begin{array}{l}324 \\
292 \\
292 \\
292 \\
292\end{array}$ & $\overline{-}$ & $\bar{z}$ & $\begin{array}{ll}5.00 \mathrm{M}_{\mathrm{L}} & \text { BRK } \\
4.50 \mathrm{M}_{\mathrm{L}} & \text { PAS } \\
4.70 \mathrm{M}_{\mathrm{L}} & \text { PAS } \\
4.50 \mathrm{M}_{\mathrm{L}} & \text { PAS } \\
4.50 \mathrm{M}_{\mathrm{L}} & \text { PAS }\end{array}$ & $\bar{z}$ & $\begin{array}{l}\bar{V} \\
\mathbf{V} \\
\mathbf{V} \\
\mathbf{I V}\end{array}$ & $\begin{array}{l}- \\
8 \\
8 \\
8 \\
8\end{array}$ & $\begin{array}{l}\overline{33} \\
25 \& \\
- \\
-\end{array}$ \\
\hline $\begin{array}{l}1935 \\
1935 \\
1935 \\
1935 \\
1935\end{array}$ & $\begin{array}{l}09 \\
10 \\
10 \\
10 \\
10\end{array}$ & $\begin{array}{l}08 \\
11 \\
24 \\
24 \\
24\end{array}$ & $\begin{array}{l}1703 \\
1406 \\
144807.6 \\
1451 \\
1452\end{array}$ & $\begin{array}{l}32.900 \mathrm{~N} \\
32.900 \mathrm{~N} \\
34.100 \mathrm{~N} \\
34.100 \mathrm{~N} \\
34.100 \mathrm{~N}\end{array}$ & $\begin{array}{l}115.216 \mathrm{~W} \\
115.216 \mathrm{~W} \\
116.800 \mathrm{~W} \\
116.883 \mathrm{~W} \\
116.883 \mathrm{~W}\end{array}$ & $\begin{array}{l}016 \\
016 \\
016 \\
016 \\
016\end{array}$ & $\begin{array}{l}292 \\
292 \\
292 \\
292 \\
292\end{array}$ & $\begin{array}{l}- \\
- \\
-\end{array}$ & $\bar{z}$ & $\begin{array}{ll}5.00 \mathrm{M}_{\mathrm{L}} & \text { PAS } \\
5.00 \mathrm{M}_{\mathrm{L}} & \text { PAS } \\
5.10 \mathrm{M}_{\mathrm{L}} & \text { PAS } \\
4.50 \mathrm{M}_{\mathrm{L}} & \text { PAS } \\
4.50 \mathrm{M}_{\mathrm{L}} & \text { PAS }\end{array}$ & $\frac{-}{-}$ & $\begin{array}{l}\text { IV } \\
\text { III } \\
\text { V } \\
\text { IV } \\
\text { IV }\end{array}$ & $\begin{array}{r}8 \\
259 \\
8 \\
8 \\
259\end{array}$ & $\begin{array}{l}\overline{\overline{4}} \\
\overline{-}\end{array}$ \\
\hline $\begin{array}{l}1935 \\
1935 \\
1935 \\
1936 \\
1936\end{array}$ & $\begin{array}{l}11 \\
12 \\
12 \\
02 \\
04\end{array}$ & $\begin{array}{l}04 \\
20 \\
25 \\
23 \\
07\end{array}$ & $\begin{array}{l}0355 \\
0745 \\
1715 \\
222042.7 \\
2253\end{array}$ & $\begin{array}{l}33.500 \mathrm{~N} \\
33.166 \mathrm{~N} \\
33.600 \mathrm{~N} \\
34.127 \mathrm{~N} \\
32.900 \mathrm{~N}\end{array}$ & $\begin{array}{l}116.916 \mathrm{~W} \\
115.500 \mathrm{~W} \\
118.016 \mathrm{~W} \\
117.338 \mathrm{~W} \\
115.216 \mathrm{~W}\end{array}$ & $\begin{array}{l}016 \\
016 \\
016 \\
010 \\
016\end{array}$ & $\begin{array}{l}292 \\
292 \\
292 \\
292 \\
292\end{array}$ & $\bar{z}$ & $\bar{z}$ & $\begin{array}{ll}4.50 \mathrm{M}_{\mathrm{L}} & \text { PAS } \\
5.00 \mathrm{M}_{\mathrm{L}} & \text { PAS } \\
4.50 \mathrm{M}_{\mathrm{L}} & \text { PAS } \\
4.50 \mathrm{M}_{\mathrm{L}} & \text { PAS } \\
4.50 \mathrm{M}_{\mathrm{L}} & \text { PAS }\end{array}$ & $\frac{-}{-}$ & $\begin{array}{l}\text { IV } \\
\text { IV } \\
\text { V } \\
\text { V } \\
\text { V }\end{array}$ & $\begin{array}{r}259 \\
8 \\
8 \\
9 \\
9\end{array}$ & $\frac{22 \&}{\frac{-}{28}}$ \\
\hline $\begin{array}{l}1936 \\
1936 \\
1936 \\
1936 \\
1936\end{array}$ & $\begin{array}{l}05 \\
05 \\
05 \\
05 \\
06\end{array}$ & $\begin{array}{l}07 \\
09 \\
10 \\
27 \\
03\end{array}$ & $\begin{array}{l}1147 \\
202040 \\
174013.2 \\
1955 \\
0915\end{array}$ & $\begin{array}{l}33.133 \mathrm{~N} \\
40.50 \mathrm{~N} \\
37.610 \mathrm{~N} \\
36.50 \mathrm{~N} \\
40.16 \mathrm{~N}\end{array}$ & $\begin{array}{l}116.083 \mathrm{~W} \\
121.65 \mathrm{~W} \\
118.368 \mathrm{~W} \\
121.17 \mathrm{~W} \\
126.45 \mathrm{~W}\end{array}$ & $\frac{016}{010}$ & $\begin{array}{l}292 \\
324 \\
292 \\
324 \\
324\end{array}$ & $\overline{-}$ & $\bar{z}$ & $\begin{array}{l}\text { 4.50M }, \text { PAS } \\
\text { 4.50Ukn JON } \\
5.00 \mathrm{M}_{\mathrm{L}} \text { PAS } \\
4.50 \mathrm{M}_{\mathrm{L}} \text { BRK } \\
5.80 \mathrm{M}_{\mathrm{L}} \text { BRK }\end{array}$ & $\frac{-}{-}$ & $\begin{array}{l}\mathrm{V} \\
\mathrm{V} \\
\mathrm{V} \\
\mathrm{V}\end{array}$ & $\begin{array}{l}9 \\
9 \\
9 \\
9\end{array}$ & $\frac{\overline{4}}{\overline{45} \&}$ \\
\hline $\begin{array}{l}1936 \\
1936 \\
1936 \\
1937 \\
1937\end{array}$ & $\begin{array}{l}09 \\
10 \\
11 \\
02 \\
02\end{array}$ & $\begin{array}{l}18 \\
10 \\
18 \\
07 \\
17\end{array}$ & $\begin{array}{l}144032.1 \\
012532.0 \\
180218.5 \\
044134 \\
0333\end{array}$ & $\begin{array}{l}32.856 \mathrm{~N} \\
40.3 \mathrm{~N} \\
34.460 \mathrm{~N} \\
40.50 \mathrm{~N} \\
36.70 \mathrm{~N}\end{array}$ & $\begin{array}{l}115.710 \mathrm{~W} \\
126.0 \mathrm{~W} \\
120.522 \mathrm{~W} \\
125.25 \mathrm{~W} \\
121.20 \mathrm{~W}\end{array}$ & $\frac{010}{010}$ & $\begin{array}{l}292 \\
265 \\
292 \\
258 \\
324\end{array}$ & $\begin{array}{l}- \\
- \\
-\end{array}$ & $\bar{z}$ & $\begin{array}{ll}4.50 \mathrm{M}_{\mathrm{L}} & \text { PAS } \\
5.00 \mathrm{M}_{\mathrm{L}} & \text { BRK } \\
4.50 \mathrm{M}_{\mathrm{L}} & \text { PAS } \\
5.75 \mathrm{M}_{\mathrm{S}} & \mathrm{GR} \\
4.50 \mathrm{M}_{\mathrm{L}} & \text { BRK }\end{array}$ & $\frac{-}{-}$ & $\begin{array}{c}\bar{Z} \\
\text { IV } \\
\text { IV }\end{array}$ & $\begin{array}{r}- \\
9 \\
10 \\
259\end{array}$ & $\frac{\bar{z}}{\overline{26 \&}}$ \\
\hline $\begin{array}{l}1937 \\
1937 \\
1937 \\
1937 \\
1937\end{array}$ & $\begin{array}{l}03 \\
03 \\
03 \\
03 \\
03\end{array}$ & $\begin{array}{l}05 \\
08 \\
25 \\
26 \\
27\end{array}$ & $\begin{array}{l}1247 \\
103112 \\
164901.8 \\
210906 \\
0742\end{array}$ & $\begin{array}{l}36.70 \mathrm{~N} \\
37.80 \mathrm{~N} \\
33.408 \mathrm{~N} \\
40.25 \mathrm{~N} \\
33.466 \mathrm{~N}\end{array}$ & $\begin{array}{l}121.70 \mathrm{~W} \\
122.20 \mathrm{~W} \\
116.261 \mathrm{~W} \\
126.75 \mathrm{~W} \\
116.583 \mathrm{~W}\end{array}$ & $\frac{\overline{-}}{\overline{010}} \frac{}{016}$ & $\begin{array}{l}324 \\
324 \\
292 \\
258 \\
292\end{array}$ & $\overline{-}$ & $\bar{z}$ & $\begin{array}{ll}4.50 \mathrm{M}_{\mathrm{L}} & \text { BRK } \\
4.50 \mathrm{M}_{\mathrm{L}} & \text { BRK } \\
6.00 \mathrm{M}_{\mathrm{L}} & \text { PAS } \\
5.50 \mathrm{M}_{\mathrm{S}} & \mathrm{GR} \\
4.50 \mathrm{M}_{\mathrm{L}} & \text { PAS }\end{array}$ & $\begin{array}{l}\overline{-} \\
5.60 \mathrm{HHT} \\
-\end{array}$ & $\begin{array}{l}\text { V } \\
\text { VII } \\
\text { VI } \\
\frac{\text { IV }}{}\end{array}$ & $\begin{array}{r}10 \\
10 \\
10 \\
259\end{array}$ & $\begin{array}{l}5 \& \\
20 \& \\
80 \# \\
- \\
-\end{array}$ \\
\hline $\begin{array}{l}1937 \\
1937 \\
1937 \\
1937 \\
1937\end{array}$ & $\begin{array}{l}08 \\
09 \\
09 \\
10 \\
11\end{array}$ & $\begin{array}{l}06 \\
01 \\
01 \\
27 \\
22\end{array}$ & $\begin{array}{l}0324 \\
134808.2 \\
163533.5 \\
1553 \\
041253.8\end{array}$ & $\begin{array}{l}38.8 \mathrm{~N} \\
34.210 \mathrm{~N} \\
34.183 \mathrm{~N} \\
36.60 \mathrm{~N} \\
34.370 \mathrm{~N}\end{array}$ & $\begin{array}{l}120.1 \mathrm{~W} \\
117.530 \mathrm{~W} \\
117.548 \mathrm{~W} \\
121.50 \mathrm{~W} \\
120.623 \mathrm{~W}\end{array}$ & $\begin{array}{l}\overline{010} \\
010 \\
\overline{010}\end{array}$ & $\begin{array}{r}10 \\
292 \\
292 \\
324 \\
292\end{array}$ & $\overline{-}$ & $\overline{-}$ & $\begin{array}{ll}4.50 \mathrm{M}_{\mathrm{L}} & \text { BRK } \\
4.50 \mathrm{M}_{\mathrm{L}} & \text { PAS } \\
4.50 \mathrm{M}_{\mathrm{L}} & \text { PAS } \\
4.50 \mathrm{M}_{\mathrm{L}} & \text { BRK } \\
4.50 \mathrm{M}_{\mathrm{L}} & \text { PAS }\end{array}$ & $\bar{z}$ & $\begin{array}{l}\text { V } \\
\text { V } \\
\text { V } \\
\text { IV } \\
\text { V }\end{array}$ & $\begin{array}{r}259 \\
10 \\
10 \\
10 \\
10\end{array}$ & $\frac{17}{-}$ \\
\hline $\begin{array}{l}1938 \\
1938 \\
1938 \\
1938 \\
1938\end{array}$ & $\begin{array}{l}01 \\
02 \\
02 \\
04 \\
04\end{array}$ & $\begin{array}{l}04 \\
12 \\
15 \\
13 \\
28\end{array}$ & $\begin{array}{l}0029 \\
200014.0 \\
074539.8 \\
1929 \\
060728.0\end{array}$ & $\begin{array}{l}33.466 \mathrm{~N} \\
37.000 \mathrm{~N} \\
34.173 \mathrm{~N} \\
32.883 \mathrm{~N} \\
32.717 \mathrm{~N}\end{array}$ & $\begin{array}{l}116.583 W \\
122.000 \mathrm{~W} \\
116.257 \mathrm{~W} \\
115.583 \mathrm{~W} \\
118.172 \mathrm{~W}\end{array}$ & $\begin{array}{l}016 \\
010 \\
016 \\
010\end{array}$ & $\begin{array}{l}292 \\
324 \\
292 \\
292 \\
292\end{array}$ & $\bar{z}$ & $\bar{z}$ & $\begin{array}{ll}4.50 \mathrm{M}_{\mathrm{L}} & \text { PAS } \\
4.50 \mathrm{M}_{\mathrm{L}} & \text { BRK } \\
4.50 \mathrm{M}_{\mathrm{L}} & \text { PAS } \\
4.50 \mathrm{M}_{\mathrm{L}} & \text { PAS } \\
4.50 \mathrm{M}_{\mathrm{L}} & \text { PAS }\end{array}$ & $\bar{z}$ & $\begin{array}{l}\text { IV } \\
\text { VI } \\
\text { V } \\
\text { V } \\
\text { IV }\end{array}$ & $\begin{array}{r}259 \\
11 \\
259 \\
259 \\
259\end{array}$ & $\begin{array}{l}-8 \& \\
-\end{array}$ \\
\hline $\begin{array}{l}1938 \\
1938 \\
1938 \\
1938 \\
1938\end{array}$ & $\begin{array}{l}05 \\
05 \\
06 \\
07 \\
07\end{array}$ & $\begin{array}{l}10 \\
31 \\
06 \\
01 \\
05\end{array}$ & $\begin{array}{l}1032 \\
083455.4 \\
0242 \\
1813 \\
180655.8\end{array}$ & $\begin{array}{l}36.200 \mathrm{~N} \\
33.699 \mathrm{~N} \\
32.900 \mathrm{~N} \\
41.00 \mathrm{~N} \\
33.682 \mathrm{~N}\end{array}$ & $\begin{array}{l}121.300 \mathrm{~W} \\
117.510 \mathrm{~W} \\
115.216 \mathrm{~W} \\
124.00 \mathrm{~W} \\
117.553 \mathrm{~W}\end{array}$ & $\begin{array}{l}\overline{010} \\
016 \\
\overline{010}\end{array}$ & $\begin{array}{l}324 \\
292 \\
292 \\
324 \\
292\end{array}$ & $\begin{array}{l}- \\
- \\
-\end{array}$ & $\bar{z}$ & $\begin{array}{ll}4.50 \mathrm{M}_{\mathrm{L}} & \text { BRK } \\
5.50 \mathrm{M}_{\mathrm{L}} & \text { PAS } \\
5.00 \mathrm{M}_{\mathrm{L}} & \text { PAS } \\
5.00 \mathrm{M}_{\mathrm{L}} & \text { BRK } \\
4.50 \mathrm{M}_{\mathrm{L}} & \text { PAS }\end{array}$ & $\bar{z}$ & 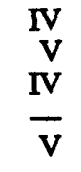 & $\begin{array}{r}259 \\
11 \\
259 \\
259\end{array}$ & $\begin{array}{l}\overline{50 \#} \\
\overline{-}\end{array}$ \\
\hline $\begin{array}{l}1938 \\
1938 \\
1938 \\
1938 \\
1938\end{array}$ & $\begin{array}{l}08 \\
08 \\
09 \\
09 \\
09\end{array}$ & $\begin{array}{l}18 \\
31 \\
12 \\
17 \\
27\end{array}$ & $\begin{array}{l}073945.4 \\
031814.3 \\
0610 \\
142304.1 \\
122348\end{array}$ & $\begin{array}{l}34.847 \mathrm{~N} \\
33.759 \mathrm{~N} \\
40.00 \mathrm{~N} \\
35.630 \mathrm{~N} \\
36.45 \mathrm{~N}\end{array}$ & $\begin{array}{l}116.143 \mathrm{~W} \\
118.253 \mathrm{~W} \\
124.00 \mathrm{~W} \\
117.513 \mathrm{~W} \\
121.25 \mathrm{~W}\end{array}$ & $\begin{array}{l}010 \\
010 \\
- \\
-\end{array}$ & $\begin{array}{l}292 \\
292 \\
324 \\
292 \\
324\end{array}$ & $\begin{array}{l}- \\
- \\
-\end{array}$ & $\begin{array}{l}- \\
- \\
z\end{array}$ & $\begin{array}{ll}4.50 \mathrm{M}_{\mathrm{L}} & \text { PAS } \\
4.50 \mathrm{M}_{\mathrm{L}} & \text { PAS } \\
5.50 \mathrm{M}_{\mathrm{a}} & \text { GR } \\
5.00 \mathrm{M}_{\mathrm{L}} & \text { PAS } \\
5.00 \mathrm{M}_{\mathrm{L}} & \text { PAS }\end{array}$ & $\begin{array}{l}z \\
z\end{array}$ & $\begin{array}{l}\text { IV } \\
\text { VI } \\
\text { VI } \\
\text { IV } \\
\text { V }\end{array}$ & $\begin{array}{r}259 \\
11 \\
11 \\
259 \\
11\end{array}$ & $\begin{array}{l}\overline{5} \& \\
35 \& \\
\overline{23} \&\end{array}$ \\
\hline $\begin{array}{l}1938 \\
1938 \\
1938 \\
1938 \\
1938\end{array}$ & $\begin{array}{l}10 \\
11 \\
11 \\
12 \\
12\end{array}$ & $\begin{array}{l}18 \\
15 \\
22 \\
01 \\
03\end{array}$ & $\begin{array}{l}0505 \\
1348 \\
1530 \\
1617 \\
174252.6\end{array}$ & $\begin{array}{l}40.00 \mathrm{~N} \\
39.25 \mathrm{~N} \\
35.93 \mathrm{~N} \\
37.50 \mathrm{~N} \\
37.453 \mathrm{~N}\end{array}$ & $\begin{array}{l}124.00 \mathrm{~W} \\
123.00 \mathrm{~W} \\
120.48 \mathrm{~W} \\
121.80 \mathrm{~W} \\
118.603 \mathrm{~W}\end{array}$ & $\frac{-}{\bar{z}}$ & $\begin{array}{l}324 \\
324 \\
324 \\
324 \\
292\end{array}$ & $\bar{z}$ & $\bar{z}$ & $\begin{array}{cl}4.50 \mathrm{M}_{\mathrm{L}} & \text { BRK } \\
-\overline{\mathrm{M}}_{\mathrm{L}} & \text { PAS } \\
4.50 \mathrm{M}^{2} & \text { BRK } \\
4.50 \mathrm{M}_{\mathrm{L}} & \text { PAS } \\
5.70 \mathrm{M}_{\mathrm{L}} & \text { PAS }\end{array}$ & $\bar{z}$ & $\begin{array}{l}\text { III } \\
\text { VI } \\
\text { IV } \\
\text { VI } \\
\text { VI }\end{array}$ & $\begin{array}{r}259 \\
11 \\
259 \\
11 \\
11\end{array}$ & $\frac{\overline{7}}{\overline{62}}$ \\
\hline
\end{tabular}


CALIFORNIA-Continued

[See table 1 for hypocenter and intensity references and table 2 for definitions of magnitude source codes. \&, land area only; \#, land area only in the United States for an earthquake near a coastline; +, land area in the United States when the felt area did not extend to the coast; $\left(0\right.$, felt area is less than $1,000 \mathrm{~km}^{2}$. Leader (--) indicates information is not available]

\begin{tabular}{|c|c|c|c|c|c|c|c|c|c|c|c|c|c|c|}
\hline \multirow{2}{*}{\multicolumn{3}{|c|}{ Date }} & \multirow{3}{*}{$\begin{array}{c}\text { Origin } \\
\text { time (UTC) } \\
\mathrm{h} m \mathrm{~s}\end{array}$} & \multicolumn{4}{|c|}{ Hypocenter } & \multicolumn{4}{|c|}{ Magnitude } & \multicolumn{2}{|c|}{ Intensity } & \multirow{3}{*}{$\begin{array}{l}\text { Felt area } \\
\left(1,000 \mathrm{~km}^{2}\right)\end{array}$} \\
\hline & & & & \multirow{2}{*}{$\begin{array}{l}\text { Latitude } \\
\left(^{\circ}\right)\end{array}$} & \multirow{2}{*}{$\begin{array}{l}\text { Longitude } \\
\left(^{\circ}\right)\end{array}$} & \multirow{2}{*}{$\begin{array}{l}\text { Depth } \\
(\mathrm{km})\end{array}$} & \multirow[t]{2}{*}{ Ref } & \multicolumn{2}{|c|}{ USGS } & \multirow[t]{2}{*}{ Other } & \multirow{2}{*}{$\begin{array}{c}\text { Moment } \\
\mathbf{M}\end{array}$} & \multirow[t]{2}{*}{ MMi } & \multirow[t]{2}{*}{ Ref } & \\
\hline Yr & Mo & Da & & & & & & $m_{b}$ & $M_{s}$ & & & & & \\
\hline $\begin{array}{l}1938 \\
1939 \\
1939 \\
1939 \\
1939\end{array}$ & $\begin{array}{l}12 \\
01 \\
02 \\
02 \\
03\end{array}$ & $\begin{array}{l}03 \\
07 \\
23 \\
23 \\
12\end{array}$ & $\begin{array}{l}1841 \quad 16.4 \\
202150.2 \\
0845 \quad 51.7 \\
091846.7 \\
2204\end{array}$ & $\begin{array}{l}37.808 \mathrm{~N} \\
36.007 \mathrm{~N} \\
34.912 \mathrm{~N} \\
34.885 \mathrm{~N} \\
41.0 \mathrm{~N}\end{array}$ & $\begin{array}{l}118.323 \mathrm{~W} \\
117.720 \mathrm{~W} \\
118.973 \mathrm{~W} \\
119.002 \mathrm{~W} \\
125.9 \mathrm{~W}\end{array}$ & $\begin{array}{l}010 \\
010 \\
010 \\
010 \\
-\end{array}$ & $\begin{array}{l}292 \\
292 \\
292 \\
292 \\
324\end{array}$ & $\begin{array}{l}- \\
- \\
-\end{array}$ & $\overline{-}$ & $\begin{array}{l}4.50 \mathrm{M}_{\mathrm{L}} \text { PAS } \\
4.50 \mathrm{M}_{\mathrm{L}} \text { PAS } \\
4.50 \mathrm{M}_{\mathrm{L}} \text { PAS } \\
4.50 \mathrm{M}_{\mathrm{L}} \text { PAS } \\
4.90 \mathrm{Un}_{\mathrm{JON}}\end{array}$ & - & $\begin{array}{c}\text { IV } \\
\text { IV } \\
\text { IV } \\
\text { II }\end{array}$ & $\begin{array}{r}-759 \\
259 \\
259 \\
11\end{array}$ & - \\
\hline $\begin{array}{l}1939 \\
1939 \\
1939 \\
1939 \\
1939\end{array}$ & $\begin{array}{l}03 \\
04 \\
05 \\
05 \\
05\end{array}$ & $\begin{array}{l}17 \\
19 \\
01 \\
08 \\
12\end{array}$ & $\begin{array}{l}1309 \\
0741 \\
215318.0 \\
024805.3 \\
192502.3\end{array}$ & $\begin{array}{l}40.75 \mathrm{~N} \\
32.583 \mathrm{~N} \\
40.20 \mathrm{~N} \\
34.903 \mathrm{~N} \\
33.466 \mathrm{~N}\end{array}$ & $\begin{array}{l}126.00 \mathrm{~W} \\
117.800 \mathrm{~W} \\
124.70 \mathrm{~W} \\
119.038 \mathrm{~W} \\
116.433 \mathrm{~W}\end{array}$ & $\begin{array}{l}\overline{016} \\
\overline{010} \\
016\end{array}$ & $\begin{array}{r}324 \\
292 \\
12 \\
292 \\
292\end{array}$ & $\overline{-}$ & $\overline{-}$ & 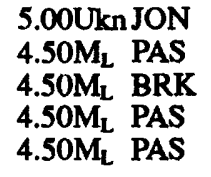 & $\begin{array}{l}- \\
- \\
-\end{array}$ & $\begin{array}{l}\text { III } \\
\text { IV } \\
\text { V } \\
\text { IV } \\
\text { IV }\end{array}$ & $\begin{array}{r}11 \\
259 \\
259 \\
259 \\
259\end{array}$ & $\begin{array}{l}- \\
- \\
-\end{array}$ \\
\hline $\begin{array}{l}1939 \\
1939 \\
1939 \\
1939 \\
1939\end{array}$ & $\begin{array}{l}06 \\
06 \\
07 \\
11 \\
12\end{array}$ & $\begin{array}{l}24 \\
25 \\
17 \\
07 \\
27\end{array}$ & $\begin{array}{l}130206 \\
0149 \\
092454 \\
185208.4 \\
192849.0\end{array}$ & $\begin{array}{l}36.80 \mathrm{~N} \\
32.700 \mathrm{~N} \\
36.85 \mathrm{~N} \\
34.000 \mathrm{~N} \\
33.783 \mathrm{~N}\end{array}$ & $\begin{array}{l}121.45 \mathrm{~W} \\
118.200 \mathrm{~W} \\
121.68 \mathrm{~W} \\
117.283 \mathrm{~W} \\
118.200 \mathrm{~W}\end{array}$ & $\begin{array}{l}- \\
\overline{016} \\
016\end{array}$ & $\begin{array}{l}324 \\
292 \\
324 \\
292 \\
292\end{array}$ & $\begin{array}{l}- \\
- \\
-\end{array}$ & $\overline{-}$ & $\begin{array}{ll}5.50 \mathrm{M}_{\mathrm{L}} & \text { BRK } \\
4.50 \mathrm{M}_{\mathrm{L}} & \text { PAS } \\
4.50 \mathrm{M}_{\mathrm{L}} & \text { BRK } \\
4.70 \mathrm{M}_{\mathrm{L}} & \text { PAS } \\
4.70 \mathrm{M}_{\mathrm{L}} & \text { PAS }\end{array}$ & $\begin{array}{l}- \\
-\end{array}$ & $\begin{array}{l}\text { VII } \\
\text { III } \\
\text { IV } \\
\text { VI }\end{array}$ & $\begin{array}{r}259 \\
259 \\
12 \\
259 \\
12\end{array}$ & $\begin{array}{l}26 \& \\
\overline{15 \&}\end{array}$ \\
\hline $\begin{array}{l}1939 \\
1940 \\
1940 \\
1940 \\
1940\end{array}$ & $\begin{array}{l}12 \\
02 \\
02 \\
02 \\
02\end{array}$ & $\begin{array}{l}28 \\
08 \\
13 \\
19 \\
24\end{array}$ & $\begin{array}{l}121538.0 \\
080559.0 \\
2353 \\
120655.7 \\
0938\end{array}$ & $\begin{array}{l}35.80 \mathrm{~N} \\
40.00 \mathrm{~N} \\
40.0 \mathrm{~N} \\
34.017 \mathrm{~N} \\
37.500 \mathrm{~N}\end{array}$ & $\begin{array}{l}120.33 \mathrm{~W} \\
121.60 \mathrm{~W} \\
124.0 \mathrm{~W} \\
117.050 \mathrm{~W} \\
118.533 \mathrm{~W}\end{array}$ & $\overline{-}$ & $\begin{array}{l}324 \\
324 \\
324 \\
292 \\
292\end{array}$ & $\begin{array}{l}- \\
- \\
-\end{array}$ & $\frac{-}{-}$ & $\begin{array}{cl}5.00 \mathrm{M}_{\mathrm{L}} & \text { BRK } \\
5.70 \mathrm{M}_{\mathrm{L}} & \text { BRK } \\
-\overline{\mathrm{M}}_{\mathrm{L}} & \\
4.60 \mathrm{M} & \mathrm{PAS} \\
4.50 \mathrm{M}_{\mathrm{L}} & \mathrm{PAS}\end{array}$ & $\begin{array}{l}- \\
-\end{array}$ & $\begin{array}{l}\text { VI } \\
\text { VII } \\
\text { VI } \\
\text { V } \\
\text { IV }\end{array}$ & $\begin{array}{r}12 \\
13 \\
259 \\
259 \\
259\end{array}$ & $\begin{array}{l}42 \& \\
72 \\
25 \& \\
- \\
-\end{array}$ \\
\hline $\begin{array}{l}1940 \\
1940 \\
1940 \\
1940 \\
1940\end{array}$ & $\begin{array}{l}02 \\
05 \\
05 \\
05 \\
05\end{array}$ & $\begin{array}{l}28 \\
18 \\
18 \\
18 \\
18\end{array}$ & $\begin{array}{l}172807.0 \\
050358.5 \\
055120.3 \\
060430.6 \\
072132.7\end{array}$ & $\begin{array}{l}33.133 \mathrm{~N} \\
34.083 \mathrm{~N} \\
34.067 \mathrm{~N} \\
34.067 \mathrm{~N} \\
34.067 \mathrm{~N}\end{array}$ & $\begin{array}{l}116.083 \mathrm{~W} \\
116.300 \mathrm{~W} \\
116.333 \mathrm{~W} \\
116.317 \mathrm{~W} \\
116.333 \mathrm{~W}\end{array}$ & $\begin{array}{l}016 \\
016 \\
016 \\
016 \\
016\end{array}$ & $\begin{array}{l}292 \\
292 \\
292 \\
292 \\
292\end{array}$ & $\begin{array}{l}- \\
- \\
-\end{array}$ & $\overline{-}$ & $\begin{array}{ll}4.50 \mathrm{M}_{\mathrm{L}} & \text { PAS } \\
5.40 \mathrm{M}_{\mathrm{L}} & \text { PAS } \\
5.20 \mathrm{M}_{\mathrm{L}} & \text { PAS } \\
4.60 \mathrm{M}_{\mathrm{L}} & \text { PAS } \\
5.00 \mathrm{M}_{\mathrm{L}} & \text { PAS }\end{array}$ & $\begin{array}{l}- \\
z\end{array}$ & $\begin{array}{l}\text { IV } \\
\text { V } \\
\text { IV } \\
- \\
-\end{array}$ & $\begin{array}{l}259 \\
259 \\
259 \\
- \\
-\end{array}$ & $\begin{array}{l}\overline{65} \\
- \\
-\end{array}$ \\
\hline $\begin{array}{l}1940 \\
1940 \\
1940 \\
1940 \\
1940\end{array}$ & $\begin{array}{l}05 \\
05 \\
05 \\
05 \\
05\end{array}$ & $\begin{array}{l}18 \\
19 \\
19 \\
19 \\
19\end{array}$ & $\begin{array}{l}134719.0 \\
022602.0 \\
022730.0 \\
043640.9 \\
044847.0\end{array}$ & $\begin{array}{l}34.050 \mathrm{~N} \\
34.050 \mathrm{~N} \\
34.050 \mathrm{~N} \\
32.733 \mathrm{~N} \\
32.767 \mathrm{~N}\end{array}$ & $\begin{array}{l}116.283 W \\
116.283 W \\
116.283 W \\
115.500 W \\
115.483 W\end{array}$ & $\begin{array}{l}016 \\
016 \\
016 \\
016 \\
016\end{array}$ & $\begin{array}{l}292 \\
292 \\
292 \\
292 \\
292\end{array}$ & $\begin{array}{l}- \\
- \\
-\end{array}$ & $\begin{array}{l}- \\
- \\
-\end{array}$ & $\begin{array}{ll}4.50 M_{L} & \text { PAS } \\
4.50 M_{L} & \text { PAS } \\
4.50 M_{L} & \text { PAS } \\
7.10 M_{S} & \text { ABE } \\
4.50 M_{L} & \text { PAS }\end{array}$ & $\frac{\bar{Z}}{{ }_{6.93 \mathrm{HHT}}}$ & $\overline{-}$ & $\begin{array}{l}- \\
\overline{13} \\
13\end{array}$ & $\frac{-}{\overline{170}} \overline{-}$ \\
\hline $\begin{array}{l}1940 \\
1940 \\
1940 \\
1940 \\
1940\end{array}$ & $\begin{array}{l}05 \\
05 \\
05 \\
05 \\
05\end{array}$ & $\begin{array}{l}19 \\
19 \\
19 \\
19 \\
19\end{array}$ & $\begin{array}{l}045500.0 \\
0504 \\
054437.0 \\
055134.0 \\
055717.0\end{array}$ & $\begin{array}{l}32.767 \mathrm{~N} \\
32.767 \mathrm{~N} \\
32.766 \mathrm{~N} \\
32.767 \mathrm{~N} \\
32.767 \mathrm{~N}\end{array}$ & $\begin{array}{l}115.483 W \\
115.483 W \\
115.483 W \\
115.483 W \\
115.483 W\end{array}$ & $\begin{array}{l}016 \\
016 \\
016 \\
016 \\
016\end{array}$ & $\begin{array}{l}292 \\
292 \\
292 \\
292 \\
292\end{array}$ & $\overline{-}$ & $\frac{-}{-}$ & $\begin{array}{ll}5.50 M_{L} & \text { PAS } \\
4.50 M_{L} & \text { PAS } \\
4.50 M_{L} & \text { PAS } \\
5.50 M_{L} & \text { PAS } \\
4.50 M_{L} & \text { PAS }\end{array}$ & $\begin{array}{l}- \\
-\end{array}$ & $\begin{array}{r}\text { Felt } \\
\text { Felt } \\
\text { Felt } \\
\text { DX } \\
\text { Felt }\end{array}$ & $\begin{array}{l}13 \\
13 \\
13 \\
13 \\
13\end{array}$ & $\begin{array}{l}- \\
-\end{array}$ \\
\hline $\begin{array}{l}1940 \\
1940 \\
1940 \\
1940 \\
1940\end{array}$ & $\begin{array}{l}05 \\
05 \\
05 \\
05 \\
05\end{array}$ & $\begin{array}{l}19 \\
19 \\
19 \\
19 \\
19\end{array}$ & $\begin{array}{l}061742.0 \\
063320.0 \\
063540.0 \\
0701 \\
153033.0\end{array}$ & $\begin{array}{l}32.767 \mathrm{~N} \\
32.767 \mathrm{~N} \\
32.767 \mathrm{~N} \\
32.767 \mathrm{~N} \\
32.767 \mathrm{~N}\end{array}$ & $\begin{array}{l}115.483 W \\
115.483 W \\
115.483 W \\
115.483 W \\
115.483 W\end{array}$ & $\begin{array}{l}016 \\
016 \\
016 \\
016 \\
016\end{array}$ & $\begin{array}{l}292 \\
292 \\
292 \\
292 \\
292\end{array}$ & $\overline{-}$ & $\frac{-}{-}$ & $\begin{array}{ll}4.50 M_{L} & \text { PAS } \\
5.00 M_{L} & \text { PAS } \\
5.50 M_{L} & \text { PAS } \\
4.50 M_{L} & \text { PAS } \\
4.50 M_{L} & \text { PAS }\end{array}$ & $\begin{array}{l}- \\
-\end{array}$ & $\begin{array}{c}\text { Felt } \\
\text { Felt } \\
\text { Felt } \\
- \\
-\end{array}$ & $\begin{array}{l}13 \\
13 \\
13 \\
- \\
-\end{array}$ & $\frac{-}{-}$ \\
\hline $\begin{array}{l}1940 \\
1940 \\
1940 \\
1940 \\
1940\end{array}$ & $\begin{array}{l}05 \\
05 \\
05 \\
05 \\
06\end{array}$ & $\begin{array}{l}22 \\
23 \\
23 \\
23 \\
01\end{array}$ & $\begin{array}{l}105831.0 \\
1100 \\
1730 \\
1845 \\
052701.2\end{array}$ & $\begin{array}{l}32.767 \mathrm{~N} \\
32.767 \mathrm{~N} \\
32.769 \mathrm{~N} \\
32.767 \mathrm{~N} \\
34.083 \mathrm{~N}\end{array}$ & $\begin{array}{l}115.483 W \\
115.483 W \\
115.483 W \\
115.483 W \\
116.333 W\end{array}$ & $\frac{016}{\overline{-}}$ & $\begin{array}{l}292 \\
259 \\
259 \\
259 \\
292\end{array}$ & $\overline{-}$ & $\overline{-}$ & $\begin{array}{c}4.50 \mathrm{M}_{\mathrm{L}} \text { PAS } \\
\overline{-} \\
\overline{-} \\
4.70 \mathrm{M}_{\mathrm{L}} \text { PAS }\end{array}$ & $\begin{array}{l}- \\
-\end{array}$ & $\begin{array}{l}\overline{\text { VI }} \\
\text { VI } \\
\text { VI } \\
\text { IV }\end{array}$ & $\begin{array}{l}259 \\
259 \\
259 \\
259\end{array}$ & $\begin{array}{l}- \\
z \\
-\end{array}$ \\
\hline $\begin{array}{l}1940 \\
1940 \\
1940 \\
1940 \\
1940\end{array}$ & $\begin{array}{l}06 \\
06 \\
06 \\
07 \\
07\end{array}$ & $\begin{array}{l}01 \\
02 \\
04 \\
08 \\
22\end{array}$ & $\begin{array}{lll}23 & 5936.0 \\
06 & 13 & 10.2 \\
10 & 35 & 08.3 \\
10 & 5736.5 \\
23 & 0032.9\end{array}$ & $\begin{array}{l}32.767 \mathrm{~N} \\
34.083 \mathrm{~N} \\
33.000 \mathrm{~N} \\
37.616 \mathrm{~N} \\
37.633 \mathrm{~N}\end{array}$ & $\begin{array}{l}115.483 W \\
116.333 W \\
116.433 W \\
118.800 W \\
118.767 W\end{array}$ & $\begin{array}{l}016 \\
016 \\
016 \\
016 \\
016\end{array}$ & $\begin{array}{l}292 \\
292 \\
292 \\
292 \\
292\end{array}$ & $\frac{-}{-}$ & $\frac{-}{-}$ & $\begin{array}{l}4.50 \mathrm{M}_{\mathrm{L}} \text { PAS } \\
4.50 \mathrm{M}_{\mathrm{L}} \text { PAS } \\
5.10 \mathrm{M}_{\mathrm{L}} \text { PAS } \\
4.80 \mathrm{M}_{\mathrm{L}} \text { PAS } \\
4.60 \mathrm{M}_{\mathrm{L}} \text { PAS }\end{array}$ & $\begin{array}{l}- \\
-\end{array}$ & $\begin{array}{l}- \\
\text { V } \\
\text { IV } \\
\text { IV }\end{array}$ & $\begin{array}{l}- \\
259 \\
259 \\
259\end{array}$ & $\begin{array}{l}- \\
-\end{array}$ \\
\hline $\begin{array}{l}1940 \\
1940 \\
1940 \\
1940 \\
1940\end{array}$ & $\begin{array}{l}09 \\
09 \\
10 \\
10 \\
10\end{array}$ & $\begin{array}{l}07 \\
27 \\
11 \\
21 \\
22\end{array}$ & $\begin{array}{l}130206.0 \\
170336 \\
055712.3 \\
064933.0 \\
1101\end{array}$ & $\begin{array}{l}36.500 \mathrm{~N} \\
40.5 \mathrm{~N} \\
33.767 \mathrm{~N} \\
33.117 \mathrm{~N} \\
40.5 \mathrm{~N}\end{array}$ & $\begin{array}{l}121.500 \mathrm{~W} \\
125.0 \mathrm{~W} \\
118.450 \mathrm{~W} \\
116.417 \mathrm{~W} \\
124.1 \mathrm{~W}\end{array}$ & $\begin{array}{l}- \\
016 \\
016 \\
-\end{array}$ & $\begin{array}{l}324 \\
324 \\
292 \\
292 \\
324\end{array}$ & $\frac{-}{-}$ & - & $\begin{array}{ll}4.50 M_{L} & \text { BRK } \\
4.50 M_{L} & \text { BRK } \\
4.75 M_{L} & \text { PAS } \\
4.50 M_{L} & \text { PAS } \\
4.50 M_{x} & \text { JON }\end{array}$ & $\begin{array}{l}- \\
-\end{array}$ & $\begin{array}{l}\text { III } \\
\text { IV } \\
\text { VI } \\
\text { IV } \\
\text { VI }\end{array}$ & $\begin{array}{r}259 \\
259 \\
13 \\
259 \\
13\end{array}$ & $\frac{-}{18 \&}$ \\
\hline
\end{tabular}


CALIFORNIA-Continued

[See table 1 for hypocenter and intensity references and table 2 for definitions of magnitude source codes. \&, land area on]y; \#, land area only in the United States for an earthquake near a coastline; +, land area in the United States when the felt area did not extend to the coast; @, felt area is less than $1,000 \mathrm{~km}^{2}$. Leader (--) indicates information is not available]

\begin{tabular}{|c|c|c|c|c|c|c|c|c|c|c|c|c|c|c|}
\hline & & & & & Hypocente & & & & & Magnitude & & Inte & sity & \\
\hline & Date & & time (UTC) & Latitude & Longitude & Depth & Ref & Us & & Other & Moment & MMI & Ref & Felt area \\
\hline $\mathrm{Yr}$ & Mo & $\overline{D a}$ & $h \mathrm{~ms}$ & $\left({ }^{\circ}\right)$ & (0) & km) & & $m_{b}$ & $M_{s}$ & & $M$ & & & $\left..000 \mathrm{~km}^{2}\right)$ \\
\hline $\begin{array}{l}1940 \\
1940 \\
1941 \\
1941 \\
1941\end{array}$ & $\begin{array}{l}11 \\
12 \\
01 \\
02 \\
02\end{array}$ & $\begin{array}{l}19 \\
20 \\
23 \\
09 \\
11\end{array}$ & $\begin{array}{l}1835 \\
234042.0 \\
1203 \\
094404.0 \\
0451\end{array}$ & $\begin{array}{l}40.75 \mathrm{~N} \\
40.00 \mathrm{~N} \\
40.5 \mathrm{~N} \\
40.50 \mathrm{~N} \\
40.00 \mathrm{~N}\end{array}$ & $\begin{array}{l}124.90 \mathrm{~W} \\
124.00 \mathrm{~W} \\
125.0 \mathrm{~W} \\
125.25 \mathrm{~W} \\
125.00 \mathrm{~W}\end{array}$ & $\begin{array}{l}- \\
- \\
-\end{array}$ & $\begin{array}{l}324 \\
324 \\
324 \\
258 \\
324\end{array}$ & $\begin{array}{l}- \\
- \\
-\end{array}$ & $\frac{-}{-}$ & $\begin{array}{ll}4.70 \mathrm{M}_{\mathrm{L}} & \mathrm{BRK} \\
5.50 \mathrm{M}_{\mathrm{S}} & \mathrm{GR} \\
4.70 \mathrm{M}_{\mathrm{L}} & \mathrm{BRK} \\
6.60 \mathrm{M}_{\mathrm{s}} & \mathrm{GR} \\
4.50 \mathrm{M}_{\mathrm{S}} & \mathrm{GR}\end{array}$ & $\begin{array}{l}- \\
- \\
-\end{array}$ & $\begin{array}{l}\text { VI } \\
\text { VI } \\
\text { V } \\
\text { VI } \\
-\end{array}$ & $\begin{array}{l}13 \\
13 \\
14 \\
14 \\
-\end{array}$ & $\begin{array}{r}21 \& \\
23 \& \\
5 \& \\
50 \& \\
-\end{array}$ \\
\hline $\begin{array}{l}1941 \\
1941 \\
1941 \\
1941 \\
1941\end{array}$ & $\begin{array}{l}02 \\
05 \\
05 \\
05 \\
05\end{array}$ & $\begin{array}{l}23 \\
01 \\
13 \\
16 \\
28\end{array}$ & $\begin{array}{l}183614.0 \\
1329 \\
160154.0 \\
023642.0 \\
062318.0\end{array}$ & $\begin{array}{l}33.500 \mathrm{~N} \\
40.00 \mathrm{~N} \\
40.30 \mathrm{~N} \\
40.30 \mathrm{~N} \\
37.08 \mathrm{~N}\end{array}$ & $\begin{array}{l}116.483 \mathrm{~W} \\
121.00 \mathrm{~W} \\
126.40 \mathrm{~W} \\
125.00 \mathrm{~W} \\
121.75 \mathrm{~W}\end{array}$ & $\frac{016}{-}$ & $\begin{array}{l}292 \\
324 \\
324 \\
324 \\
324\end{array}$ & $\begin{array}{l}- \\
- \\
-\end{array}$ & $\bar{z}$ & $\begin{array}{ll}4.50 \mathrm{M}_{\mathrm{L}} & \text { PAS } \\
4.50 \mathrm{M}_{\mathrm{L}} & \mathrm{BRK} \\
6.00 \mathrm{M}_{\mathrm{S}} & \mathrm{GR} \\
4.50 \mathrm{M}_{\mathrm{L}} & \mathrm{BRK} \\
4.50 \mathrm{M}_{\mathrm{L}} & \mathrm{BRK}\end{array}$ & $\begin{array}{l}- \\
- \\
-\end{array}$ & $\begin{array}{l}\text { III } \\
\text { IV } \\
\text { V } \\
\text { V }\end{array}$ & $\begin{array}{r}259 \\
14 \\
14 \\
14\end{array}$ & $\begin{array}{l}- \\
\overline{12} \&\end{array}$ \\
\hline $\begin{array}{l}1941 \\
1941 \\
1941 \\
1941 \\
1941\end{array}$ & $\begin{array}{l}07 \\
07 \\
07 \\
07 \\
09\end{array}$ & $\begin{array}{l}01 \\
01 \\
12 \\
22 \\
08\end{array}$ & $\begin{array}{l}075054.8 \\
2354 \\
1618 \\
185205.0 \\
031245.0\end{array}$ & $\begin{array}{l}34.367 \mathrm{~N} \\
34.333 \mathrm{~N} \\
34.333 \mathrm{~N} \\
32.733 \mathrm{~N} \\
34.333 \mathrm{~N}\end{array}$ & $\begin{array}{l}119.583 W \\
119.583 W \\
119.583 W \\
115.450 W \\
119.583 W\end{array}$ & $\begin{array}{l}016 \\
016 \\
016 \\
016 \\
016\end{array}$ & $\begin{array}{l}292 \\
292 \\
292 \\
292 \\
292\end{array}$ & $\begin{array}{l}- \\
- \\
-\end{array}$ & $\bar{z}$ & $\begin{array}{ll}5.90 \mathrm{M}_{\mathrm{L}} & \text { PAS } \\
4.50 \mathrm{M}_{\mathrm{L}} & \text { PAS } \\
4.50 \mathrm{M}_{\mathrm{L}} & \text { PAS } \\
4.50 \mathrm{M}_{\mathrm{L}} & \text { PAS } \\
4.50 \mathrm{M}_{\mathrm{L}} & \text { PAS }\end{array}$ & $\begin{array}{c}\text { 5.92HHT } \\
= \\
= \\
-\end{array}$ & $\begin{array}{c}\text { VII } \\
\text { IV } \\
\text { IV } \\
\text { V } \\
\text { V }\end{array}$ & $\begin{array}{r}14 \\
259 \\
259 \\
259 \\
14\end{array}$ & $\begin{array}{l}52 \# \\
- \\
-\end{array}$ \\
\hline $\begin{array}{l}1941 \\
1941 \\
1941 \\
1941 \\
1941\end{array}$ & $\begin{array}{l}09 \\
09 \\
09 \\
09 \\
09\end{array}$ & $\begin{array}{l}14 \\
14 \\
14 \\
14 \\
14\end{array}$ & $\begin{array}{l}164331.8 \\
165458.0 \\
182118.7 \\
183911.9 \\
211601.0\end{array}$ & $\begin{array}{l}37.567 \mathrm{~N} \\
37.567 \mathrm{~N} \\
37.567 \mathrm{~N} \\
37.567 \mathrm{~N} \\
37.570 \mathrm{~N}\end{array}$ & $\begin{array}{l}118.733 W \\
118.733 W \\
118.733 W \\
118.733 W \\
118.730 W\end{array}$ & $\begin{array}{l}016 \\
016 \\
016 \\
016 \\
016\end{array}$ & $\begin{array}{l}292 \\
292 \\
292 \\
292 \\
292\end{array}$ & $\begin{array}{l}- \\
- \\
-\end{array}$ & $\overline{-}$ & $\begin{array}{ll}5.80 \mathrm{M}_{\mathrm{L}} & \text { PAS } \\
4.50 \mathrm{M}_{\mathrm{L}} & \text { PAS } \\
5.50 \mathrm{M}_{\mathrm{L}} & \text { PAS } \\
6.00 \mathrm{M}_{\mathrm{L}} & \text { PAS } \\
5.00 \mathrm{M}_{\mathrm{L}} & \text { BRK }\end{array}$ & $\begin{array}{c}\overline{-} \\
\overline{5.48 \mathrm{HHT}} \\
-\end{array}$ & $\begin{array}{l}\text { VI } \\
\text { VI } \\
\text { VI } \\
\text { III }\end{array}$ & $\begin{array}{l}259 \\
259 \\
259 \\
259\end{array}$ & $\frac{90}{\frac{-}{90}}$ \\
\hline $\begin{array}{l}1941 \\
1941 \\
1941 \\
1941 \\
1941\end{array}$ & $\begin{array}{l}09 \\
10 \\
10 \\
10 \\
10\end{array}$ & $\begin{array}{l}21 \\
03 \\
06 \\
22 \\
22\end{array}$ & $\begin{array}{l}195307.2 \\
161308.0 \\
0659 \\
065718.5 \\
103221.8\end{array}$ & $\begin{array}{l}34.867 \mathrm{~N} \\
40.40 \mathrm{~N} \\
40.40 \mathrm{~N} \\
33.817 \mathrm{~N} \\
33.867 \mathrm{~N}\end{array}$ & $\begin{array}{l}118.933 \mathrm{~W} \\
124.80 \mathrm{~W} \\
125.00 \mathrm{~W} \\
118.217 \mathrm{~W} \\
118.217 \mathrm{~W}\end{array}$ & $\begin{array}{l}016 \\
- \\
016 \\
016\end{array}$ & $\begin{array}{l}292 \\
324 \\
324 \\
292 \\
292\end{array}$ & $\begin{array}{l}- \\
- \\
-\end{array}$ & $\begin{array}{l}- \\
- \\
-\end{array}$ & $\begin{array}{ll}5.20 \mathrm{M}_{\mathrm{L}} & \text { PAS } \\
6.40 \mathrm{M}_{\mathrm{S}} & \text { GR } \\
5.00 \mathrm{M}_{\mathrm{L}} & \text { BRK } \\
4.90 \mathrm{M}_{\mathrm{L}} & \text { PAS } \\
3.80 \mathrm{M}_{\mathrm{L}} & \text { PAS }\end{array}$ & $\begin{array}{l}- \\
- \\
-\end{array}$ & $\frac{\mathrm{V}}{\mathrm{VI}}$ & $\begin{array}{r}14 \\
259 \\
14 \\
14\end{array}$ & $\begin{array}{l}60 \& \\
30 \& \\
- \\
5 \&\end{array}$ \\
\hline $\begin{array}{l}1941 \\
1941 \\
1941 \\
1941 \\
1941\end{array}$ & $\begin{array}{l}10 \\
10 \\
11 \\
12 \\
12\end{array}$ & $\begin{array}{l}23 \\
25 \\
14 \\
24 \\
31\end{array}$ & $\begin{array}{l}204431.0 \\
0709 \\
084136.3 \\
073012.0 \\
064844.0\end{array}$ & $\begin{array}{l}37.567 \mathrm{~N} \\
39.50 \mathrm{~N} \\
33.783 \mathrm{~N} \\
32.60 \mathrm{~N} \\
37.567 \mathrm{~N}\end{array}$ & $\begin{array}{l}118.733 \mathrm{~W} \\
122.17 \mathrm{~W} \\
118.250 \mathrm{~W} \\
116.10 \mathrm{~W} \\
118.733 \mathrm{~W}\end{array}$ & $\begin{array}{l}016 \\
- \\
016 \\
016 \\
016\end{array}$ & $\begin{array}{l}292 \\
324 \\
292 \\
292 \\
292\end{array}$ & $\begin{array}{l}- \\
- \\
-\end{array}$ & $\begin{array}{l}- \\
- \\
-\end{array}$ & $\begin{array}{ll}4.50 \mathrm{M}_{\mathrm{L}} & \text { PAS } \\
4.60 \mathrm{M}_{\mathrm{x}} & \text { JON } \\
5.40 \mathrm{M}_{\mathrm{S}} & \text { GR } \\
4.50 \mathrm{M}_{\mathrm{L}} & \text { PAS } \\
5.40 \mathrm{M}_{\mathrm{L}} & \text { PAS }\end{array}$ & $\begin{array}{l}- \\
- \\
-\end{array}$ & $\begin{array}{r}\text { IV } \\
\text { V } \\
\text { VIII } \\
\text { IV } \\
\text { VI }\end{array}$ & $\begin{array}{r}259 \\
14 \\
259 \\
259 \\
14\end{array}$ & $\begin{array}{l}\frac{-}{9 \&} \\
\frac{72 \&}{4}\end{array}$ \\
\hline $\begin{array}{l}1941 \\
1942 \\
1942 \\
1942 \\
1942\end{array}$ & $\begin{array}{l}12 \\
01 \\
02 \\
02 \\
02\end{array}$ & $\begin{array}{l}31 \\
01 \\
01 \\
01 \\
04\end{array}$ & $\begin{array}{l}180544.0 \\
034101.0 \\
151828.0 \\
160334.0 \\
033203.0\end{array}$ & $\begin{array}{l}37.567 \mathrm{~N} \\
37.567 \mathrm{~N} \\
34.400 \mathrm{~N} \\
34.400 \mathrm{~N} \\
37.567 \mathrm{~N}\end{array}$ & $\begin{array}{l}118.733 \mathrm{~W} \\
118.733 \mathrm{~W} \\
116.917 \mathrm{~W} \\
116.917 \mathrm{~W} \\
118.733 \mathrm{~W}\end{array}$ & $\begin{array}{l}016 \\
016 \\
016 \\
016 \\
016\end{array}$ & $\begin{array}{l}292 \\
292 \\
292 \\
292 \\
292\end{array}$ & $\begin{array}{l}- \\
- \\
-\end{array}$ & $\bar{z}$ & $\begin{array}{ll}4.50 \mathrm{M}_{\mathrm{L}} & \text { PAS } \\
4.50 \mathrm{M}_{\mathrm{L}} & \text { PAS } \\
4.50 \mathrm{M}_{\mathrm{L}} & \text { PAS } \\
4.50 \mathrm{M}_{\mathrm{L}} & \text { PAS } \\
4.50 \mathrm{M}_{\mathrm{L}} & \text { PAS }\end{array}$ & $\begin{array}{l}- \\
z\end{array}$ & $\begin{array}{l}\overline{I V} \\
\text { IV } \\
\text { IV }\end{array}$ & $\begin{array}{l}\overline{259} \\
259 \\
259\end{array}$ & $\begin{array}{l}- \\
- \\
-\end{array}$ \\
\hline $\begin{array}{l}1942 \\
1942 \\
1942 \\
1942 \\
1942\end{array}$ & $\begin{array}{l}03 \\
05 \\
07 \\
08 \\
09\end{array}$ & $\begin{array}{l}03 \\
23 \\
06 \\
07 \\
03\end{array}$ & 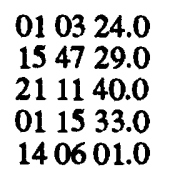 & $\begin{array}{l}34.000 \mathrm{~N} \\
32.983 \mathrm{~N} \\
37.567 \mathrm{~N} \\
34.300 \mathrm{~N} \\
34.483 \mathrm{~N}\end{array}$ & $\begin{array}{l}115.750 \mathrm{~W} \\
115.983 \mathrm{~W} \\
118.733 \mathrm{~W} \\
116.417 \mathrm{~W} \\
118.983 \mathrm{~W}\end{array}$ & $\begin{array}{l}016 \\
016 \\
016 \\
016 \\
016\end{array}$ & $\begin{array}{l}292 \\
292 \\
292 \\
292 \\
292\end{array}$ & $\begin{array}{l}- \\
- \\
-\end{array}$ & $\bar{z}$ & $\begin{array}{ll}5.00 \mathrm{M}_{\mathrm{L}} & \text { PAS } \\
5.00 \mathrm{M}_{\mathrm{L}} & \text { PAS } \\
4.50 \mathrm{M}_{\mathrm{L}} & \text { PAS } \\
4.50 \mathrm{M}_{\mathrm{L}} & \text { PAS } \\
4.50 \mathrm{M}_{\mathrm{L}} & \text { PAS }\end{array}$ & $\frac{-}{z}$ & $\begin{array}{l}\text { IV } \\
\text { V } \\
\text { IV } \\
\text { V }\end{array}$ & $\begin{array}{r}259 \\
15 \\
259 \\
-15\end{array}$ & $\begin{array}{l}\overline{16+} \\
- \\
-\end{array}$ \\
\hline $\begin{array}{l}1942 \\
1942 \\
1942 \\
1942 \\
1942\end{array}$ & $\begin{array}{l}09 \\
10 \\
10 \\
10 \\
10\end{array}$ & $\begin{array}{l}04 \\
21 \\
21 \\
21 \\
21\end{array}$ & $\begin{array}{l}063433.0 \\
162213.0 \\
162519.0 \\
162654.0 \\
163439.0\end{array}$ & $\begin{array}{l}34.483 \mathrm{~N} \\
32.966 \mathrm{~N} \\
32.967 \mathrm{~N} \\
32.967 \mathrm{~N} \\
32.967 \mathrm{~N}\end{array}$ & $\begin{array}{l}118.983 \mathrm{~W} \\
116.000 \mathrm{~W} \\
116.000 \mathrm{~W} \\
116.000 \mathrm{~W} \\
116.000 \mathrm{~W}\end{array}$ & $\begin{array}{l}016 \\
016 \\
016 \\
016 \\
016\end{array}$ & $\begin{array}{l}292 \\
292 \\
292 \\
292 \\
292\end{array}$ & $\overline{-}$ & $\frac{-}{-}$ & $\begin{array}{ll}4.50 \mathrm{M}_{\mathrm{L}} & \text { PAS } \\
6.50 \mathrm{M}_{\mathrm{S}} & \mathrm{GR} \\
5.00 \mathrm{M}_{\mathrm{L}} & \mathrm{PAS} \\
5.00 \mathrm{M}_{\mathrm{L}} & \text { PAS } \\
4.50 \mathrm{M}_{\mathrm{L}} & \text { PAS }\end{array}$ & $\begin{array}{c}\text { 6.59HHT } \\
= \\
-\end{array}$ & $\begin{array}{l}v \\
\text { VI } \\
- \\
-\end{array}$ & $\begin{array}{l}15 \\
15 \\
- \\
-\end{array}$ & $\begin{array}{l}\overline{90 \#} \\
- \\
-\end{array}$ \\
\hline $\begin{array}{l}1942 \\
1942 \\
1942 \\
1942 \\
1942\end{array}$ & $\begin{array}{l}10 \\
10 \\
10 \\
10 \\
10\end{array}$ & $\begin{array}{l}21 \\
21 \\
21 \\
22 \\
22\end{array}$ & $\begin{array}{l}163806.0 \\
191028.0 \\
214928.0 \\
015038.0 \\
181326.0\end{array}$ & $\begin{array}{l}32.967 \mathrm{~N} \\
32.967 \mathrm{~N} \\
32.967 \mathrm{~N} \\
33.233 \mathrm{~N} \\
32.967 \mathrm{~N}\end{array}$ & $\begin{array}{l}116.000 \mathrm{~W} \\
116.000 \mathrm{~W} \\
116.000 \mathrm{~W} \\
115.717 \mathrm{~W} \\
116.000 \mathrm{~W}\end{array}$ & $\begin{array}{l}016 \\
016 \\
016 \\
016 \\
016\end{array}$ & $\begin{array}{l}292 \\
292 \\
292 \\
292 \\
292\end{array}$ & $\begin{array}{l}- \\
-\end{array}$ & $\bar{z}$ & $\begin{array}{ll}4.50 \mathrm{M}_{\mathrm{L}} & \text { PAS } \\
4.50 \mathrm{M}_{\mathrm{L}} & \text { PAS } \\
4.50 \mathrm{M}_{\mathrm{L}} & \text { PAS } \\
5.50 \mathrm{M}_{\mathrm{L}} & \text { PAS } \\
5.00 \mathrm{M}_{\mathrm{L}} & \text { PAS }\end{array}$ & $\overline{-}$ & $\frac{-}{\bar{v}}$ & $\frac{\bar{Z}}{259}$ & - \\
\hline $\begin{array}{l}1942 \\
1942 \\
1942 \\
1942 \\
1942\end{array}$ & $\begin{array}{l}10 \\
10 \\
10 \\
10 \\
10\end{array}$ & $\begin{array}{l}26 \\
26 \\
29 \\
29 \\
30\end{array}$ & $\begin{array}{l}030215.0 \\
061504.0 \\
155600.0 \\
162157.0 \\
053545.0\end{array}$ & $\begin{array}{l}33.233 \mathrm{~N} \\
33.233 \mathrm{~N} \\
32.967 \mathrm{~N} \\
32.967 \mathrm{~N} \\
32.967 \mathrm{~N}\end{array}$ & $\begin{array}{l}115.717 \mathrm{~W} \\
115.717 \mathrm{~W} \\
116.000 \mathrm{~W} \\
116.000 \mathrm{~W} \\
116.000 \mathrm{~W}\end{array}$ & $\begin{array}{l}016 \\
016 \\
016 \\
016 \\
016\end{array}$ & $\begin{array}{l}292 \\
292 \\
292 \\
292 \\
292\end{array}$ & $\begin{array}{l}- \\
- \\
-\end{array}$ & $\bar{z}$ & $\begin{array}{ll}\text { 4.50M } & \text { PAS } \\
4.50 \mathrm{M}_{\mathrm{L}} & \text { PAS } \\
4.50 \mathrm{M}_{\mathrm{L}} & \text { PAS } \\
4.50 \mathrm{M}_{\mathrm{L}} & \text { PAS } \\
4.50 \mathrm{M}_{\mathrm{L}} & \text { PAS }\end{array}$ & $\begin{array}{l}z \\
z\end{array}$ & $\begin{array}{l}\text { III } \\
\text { IV } \\
\text { IV } \\
\text { IV }\end{array}$ & $\begin{array}{l}259 \\
259 \\
259 \\
259\end{array}$ & $\frac{-}{-}$ \\
\hline
\end{tabular}


EARTHQUAKES IN CALIFORNIA

CALIFORNIA-Continued

[See table 1 for hypocenter and intensity references and table 2 for definitions of magnitude source codes. \&, land area only; \#, land area only in the United States for an earthquake near a coastline; +, land area in the United States when the felt area did not extend to the coast; @, felt area is less than $1,000 \mathrm{~km}{ }^{2}$. Leader (--) indicates information is not available]

\begin{tabular}{|c|c|c|c|c|c|c|c|c|c|c|c|c|c|c|}
\hline \multirow{2}{*}{\multicolumn{3}{|c|}{ Date }} & \multirow{2}{*}{$\begin{array}{c}\text { Origin } \\
\text { time (UTC) }\end{array}$} & \multicolumn{4}{|c|}{ Hypocenter } & \multicolumn{4}{|c|}{ Magnitude } & \multicolumn{2}{|c|}{ Intensity } & \multirow{3}{*}{$\begin{array}{l}\text { Felt area } \\
\left(1,000 \mathrm{~km}^{2}\right)\end{array}$} \\
\hline & & & & \multirow{2}{*}{$\begin{array}{l}\text { Latitude } \\
\left(^{\circ}\right)\end{array}$} & \multirow{2}{*}{$\begin{array}{l}\text { Longitude } \\
\text { ( })\end{array}$} & \multirow{2}{*}{$\begin{array}{c}\text { Depth } \\
(\mathrm{km})\end{array}$} & \multirow[t]{2}{*}{ Ref } & \multicolumn{2}{|c|}{ USGS } & \multirow[t]{2}{*}{ Other } & \multirow{2}{*}{$\begin{array}{c}\text { Moment } \\
\mathbf{M}\end{array}$} & \multirow[t]{2}{*}{ MMI } & \multirow[t]{2}{*}{ Rof } & \\
\hline $\mathrm{Yr}$ & Mo & $\overline{\mathrm{Da}}$ & $\mathrm{h} m \mathrm{~s}$ & & & & & $m_{b}$ & $M_{s}$ & & & & & \\
\hline $\begin{array}{l}1942 \\
1942 \\
1942 \\
1942 \\
1942\end{array}$ & $\begin{array}{l}11 \\
11 \\
11 \\
12 \\
12\end{array}$ & $\begin{array}{l}02 \\
03 \\
09 \\
05 \\
17\end{array}$ & $\begin{array}{l}125942.0 \\
050629.0 \\
203425.0 \\
185207.0 \\
150743.0\end{array}$ & $\begin{array}{l}32.967 \mathrm{~N} \\
32.967 \mathrm{~N} \\
34.617 \mathrm{~N} \\
37.567 \mathrm{~N} \\
38.87 \mathrm{~N}\end{array}$ & $\begin{array}{l}116.000 \mathrm{~W} \\
116.000 \mathrm{~W} \\
116.000 \mathrm{~W} \\
118.733 \mathrm{~W} \\
119.90 \mathrm{~W}\end{array}$ & $\begin{array}{l}016 \\
016 \\
016 \\
016 \\
-\end{array}$ & $\begin{array}{l}292 \\
292 \\
292 \\
292 \\
324\end{array}$ & $\begin{array}{l}z \\
z\end{array}$ & $\bar{z}$ & $\begin{array}{ll}4.50 \mathrm{M}_{\mathrm{L}} & \text { PAS } \\
4.50 \mathrm{M}_{\mathrm{L}} & \text { PAS } \\
4.50 \mathrm{M}_{\mathrm{L}} & \text { PAS } \\
4.50 \mathrm{M}_{\mathrm{L}} & \text { PAS } \\
5.10 \mathrm{M}_{\mathrm{L}} & \text { BRK }\end{array}$ & $\begin{array}{l}- \\
-\end{array}$ & $\bar{z}$ & $\frac{-}{\overline{15}}$ & $\begin{array}{l}- \\
- \\
-\end{array}$ \\
\hline $\begin{array}{l}1942 \\
1943 \\
1943 \\
1943 \\
1943\end{array}$ & $\begin{array}{l}12 \\
01 \\
03 \\
03 \\
05\end{array}$ & $\begin{array}{l}20 \\
02 \\
17 \\
30 \\
31\end{array}$ & $\begin{array}{lll}05 & 4739.0 \\
14 & 11 & 18.0 \\
00 & 40 & 44.0 \\
21 & 07 & 28.0 \\
20 & 1653.0\end{array}$ & $\begin{array}{l}38.72 \mathrm{~N} \\
33.417 \mathrm{~N} \\
32.733 \mathrm{~N} \\
39.430 \mathrm{~N} \\
37.383 \mathrm{~N}\end{array}$ & $\begin{array}{l}119.73 \mathrm{~W} \\
116.417 \mathrm{~W} \\
115.433 \mathrm{~W} \\
120.400 \mathrm{~W} \\
118.600 \mathrm{~W}\end{array}$ & $\begin{array}{l}\overline{016} \\
016 \\
\overline{016}\end{array}$ & $\begin{array}{l}324 \\
292 \\
292 \\
324 \\
292\end{array}$ & $\begin{array}{l}- \\
-\end{array}$ & $\bar{z}$ & $\begin{array}{ll}4.50 \mathrm{M}_{\mathrm{L}} & \text { BRK } \\
4.50 \mathrm{M}_{\mathrm{L}} & \text { PAS } \\
4.50 \mathrm{M}_{\mathrm{L}} & \text { PAS } \\
5.30 \mathrm{M}_{\mathrm{L}} & \text { BRK } \\
4.50 \mathrm{M}_{\mathrm{L}} & \text { PAS }\end{array}$ & $\begin{array}{l}z \\
z\end{array}$ & $\begin{array}{c}\text { Felt } \\
\text { IV } \\
\text { V } \\
\text { V } \\
\text { V }\end{array}$ & $\begin{array}{r}15 \\
259 \\
16 \\
259 \\
16\end{array}$ & $\begin{array}{l}- \\
\overline{39} \\
24\end{array}$ \\
\hline $\begin{array}{l}1943 \\
1943 \\
1943 \\
1943 \\
1943\end{array}$ & $\begin{array}{l}06 \\
08 \\
09 \\
10 \\
10\end{array}$ & $\begin{array}{l}18 \\
29 \\
16 \\
02 \\
14\end{array}$ & $\begin{array}{l}161546.0 \\
034513.0 \\
075222.0 \\
065641.0 \\
142844.0\end{array}$ & $\begin{array}{l}33.117 \mathrm{~N} \\
34.267 \mathrm{~N} \\
36.017 \mathrm{~N} \\
40.50 \mathrm{~N} \\
34.333 \mathrm{~N}\end{array}$ & $\begin{array}{l}116.117 \mathrm{~W} \\
116.967 \mathrm{~W} \\
117.933 \mathrm{~W} \\
124.60 \mathrm{~W} \\
116.883 \mathrm{~W}\end{array}$ & $\begin{array}{l}016 \\
016 \\
016 \\
\overline{016}\end{array}$ & $\begin{array}{l}292 \\
292 \\
292 \\
324 \\
292\end{array}$ & $\begin{array}{l}- \\
-\end{array}$ & $\bar{z}$ & $\begin{array}{ll}4.50 \mathrm{M}_{\mathrm{L}} & \text { PAS } \\
5.50 \mathrm{M}_{\mathrm{L}} & \text { PAS } \\
4.50 \mathrm{M}_{\mathrm{L}} & \text { PAS } \\
4.60 \mathrm{M}_{\mathrm{L}} & \text { BRK } \\
4.50 \mathrm{M}_{\mathrm{L}} & \text { PAS }\end{array}$ & $\begin{array}{l}z \\
z\end{array}$ & $\begin{array}{l}\text { IV } \\
\text { VI } \\
\text { IV } \\
\text { IV } \\
\text { III }\end{array}$ & $\begin{array}{r}16 \\
259 \\
259 \\
259 \\
259\end{array}$ & $\begin{array}{l}\overline{37} \& \\
= \\
-\end{array}$ \\
\hline $\begin{array}{l}1943 \\
1943 \\
1943 \\
1943 \\
1943\end{array}$ & $\begin{array}{l}10 \\
10 \\
10 \\
11 \\
11\end{array}$ & $\begin{array}{l}15 \\
26 \\
31 \\
02 \\
02\end{array}$ & $\begin{array}{l}165001.0 \\
045033.0 \\
131210.0 \\
164759.0 \\
175041.0\end{array}$ & $\begin{array}{l}34.350 \mathrm{~N} \\
37.43 \mathrm{~N} \\
33.783 \mathrm{~N} \\
32.967 \mathrm{~N} \\
32.967 \mathrm{~N}\end{array}$ & $\begin{array}{l}116.867 \mathrm{~W} \\
121.68 \mathrm{~W} \\
116.200 \mathrm{~W} \\
116.000 \mathrm{~W} \\
116.000 \mathrm{~W}\end{array}$ & $\begin{array}{l}016 \\
016 \\
016 \\
016\end{array}$ & $\begin{array}{l}292 \\
324 \\
292 \\
292 \\
292\end{array}$ & $\overline{-}$ & $\bar{z}$ & $\begin{array}{ll}4.50 \mathrm{M}_{\mathrm{L}} & \text { PAS } \\
4.90 \mathrm{M}_{\mathrm{L}} & \text { BRK } \\
4.50 \mathrm{M}_{\mathrm{L}} & \text { PAS } \\
4.50 \mathrm{M}_{\mathrm{L}} & \text { PAS } \\
4.50 \mathrm{M}_{\mathrm{L}} & \text { PAS }\end{array}$ & $\bar{z}$ & $\begin{array}{l}\text { IV } \\
\text { VI } \\
\text { IV } \\
\text { IV } \\
\text { IV }\end{array}$ & $\begin{array}{r}259 \\
16 \\
259 \\
259 \\
259\end{array}$ & $\begin{array}{l}\overline{65} \& \\
= \\
-\end{array}$ \\
\hline $\begin{array}{l}1943 \\
1943 \\
1943 \\
1944 \\
1944\end{array}$ & $\begin{array}{l}11 \\
11 \\
12 \\
01 \\
01\end{array}$ & $\begin{array}{l}16 \\
17 \\
22 \\
12 \\
16\end{array}$ & $\begin{array}{l}213847.0 \\
112841.0 \\
155028.0 \\
150240.0 \\
022529.0\end{array}$ & $\begin{array}{l}37.78 \mathrm{~N} \\
33.917 \mathrm{~N} \\
34.333 \mathrm{~N} \\
40.30 \mathrm{~N} \\
40.30 \mathrm{~N}\end{array}$ & $\begin{array}{l}122.12 \mathrm{~W} \\
116.700 \mathrm{~W} \\
115.800 \mathrm{~W} \\
124.90 \mathrm{~W} \\
125.10 \mathrm{~W}\end{array}$ & $\begin{array}{l}\overline{016} \\
016 \\
- \\
-\end{array}$ & $\begin{array}{l}324 \\
292 \\
292 \\
324 \\
324\end{array}$ & $\begin{array}{l}- \\
-\end{array}$ & $\bar{z}$ & $\begin{array}{ll}3.60 \mathrm{M}_{\mathrm{L}} & \text { BRK } \\
4.50 \mathrm{M}_{\mathrm{L}} & \text { PAS } \\
5.50 \mathrm{M}_{\mathrm{L}} & \text { PAS } \\
5.10 \mathrm{M}_{\mathrm{L}} & \text { BRK } \\
5.10 \mathrm{M}_{\mathrm{L}} & \text { BRK }\end{array}$ & $\begin{array}{l}- \\
-\end{array}$ & $\begin{array}{l}\text { VI } \\
\text { IV } \\
\text { IV } \\
\text { V } \\
\text { IV }\end{array}$ & $\begin{array}{l}16 \\
16 \\
16 \\
17 \\
17\end{array}$ & $\frac{-}{-}$ \\
\hline $\begin{array}{l}1944 \\
1944 \\
1944 \\
1944 \\
1944\end{array}$ & $\begin{array}{l}06 \\
06 \\
06 \\
06 \\
06\end{array}$ & $\begin{array}{l}10 \\
12 \\
12 \\
13 \\
19\end{array}$ & $\begin{array}{lll}11 & 11 & 50.5 \\
10 & 4534.7 \\
11 & 1636.0 \\
082732.0 \\
000333.0\end{array}$ & $\begin{array}{l}34.013 \mathrm{~N} \\
33.977 \mathrm{~N} \\
33.995 \mathrm{~N} \\
34.667 \mathrm{~N} \\
33.867 \mathrm{~N}\end{array}$ & $\begin{array}{l}116.772 \mathrm{~W} \\
116.720 \mathrm{~W} \\
116.712 \mathrm{~W} \\
120.500 \mathrm{~W} \\
118.217 \mathrm{~W}\end{array}$ & $\begin{array}{l}010 \\
010 \\
010 \\
016 \\
016\end{array}$ & $\begin{array}{l}292 \\
292 \\
292 \\
292 \\
292\end{array}$ & $\begin{array}{l}z \\
z\end{array}$ & $\bar{z}$ & $\begin{array}{ll}4.50 \mathrm{M}_{\mathrm{L}} & \text { PAS } \\
5.10 \mathrm{M}_{\mathrm{L}} & \text { PAS } \\
5.30 \mathrm{M}_{\mathrm{L}} & \text { PAS } \\
4.60 \mathrm{M}_{\mathrm{L}} & \text { PAS } \\
4.50 \mathrm{M}_{\mathrm{L}} & \text { PAS }\end{array}$ & $\begin{array}{c}3.38 \mathrm{TH} \\
- \\
- \\
-\end{array}$ & $\begin{array}{l}\text { V } \\
\text { VI } \\
\text { VI } \\
\text { V } \\
\text { VI }\end{array}$ & $\begin{array}{l}17 \\
17 \\
17 \\
17 \\
17\end{array}$ & $\begin{array}{l}\overline{41 \&} \\
41 \& \\
\overline{16} \&\end{array}$ \\
\hline $\begin{array}{l}1944 \\
1944 \\
1944 \\
1944 \\
1944\end{array}$ & $\begin{array}{l}06 \\
07 \\
07 \\
11 \\
12\end{array}$ & $\begin{array}{l}19 \\
03 \\
29 \\
08 \\
23\end{array}$ & $\begin{array}{lll}03 & 06 & 07.0 \\
05 & 38 & 23.5 \\
11 & 37 & 15.0 \\
11 & 08 & 44.0 \\
08 & 16 & 22.0\end{array}$ & $\begin{array}{l}33.867 \mathrm{~N} \\
35.350 \mathrm{~N} \\
40.30 \mathrm{~N} \\
41.50 \mathrm{~N} \\
36.400 \mathrm{~N}\end{array}$ & $\begin{array}{l}118.217 \mathrm{~W} \\
117.827 \mathrm{~W} \\
125.80 \mathrm{~W} \\
125.00 \mathrm{~W} \\
117.917 \mathrm{~W}\end{array}$ & $\frac{016}{\overline{-}}$ & $\begin{array}{l}292 \\
292 \\
324 \\
324 \\
292\end{array}$ & $\begin{array}{l}z \\
z\end{array}$ & $\bar{z}$ & $\begin{array}{ll}4.40 \mathrm{M}_{\mathrm{L}} & \text { PAS } \\
4.70 \mathrm{M}_{\mathrm{L}} & \text { PAS } \\
4.50 \mathrm{M}_{\mathrm{L}} & \text { BRK } \\
4.70 \mathrm{M}_{\mathrm{L}} & \text { BRK } \\
4.70 \mathrm{M}_{\mathrm{L}} & \text { PAS }\end{array}$ & $\frac{-}{z}$ & $\begin{array}{l}\text { VI } \\
\text { V } \\
\frac{-}{I V}\end{array}$ & $\begin{array}{l}17 \\
17 \\
- \\
17\end{array}$ & $\begin{array}{l}13 \& \\
= \\
-\end{array}$ \\
\hline $\begin{array}{l}1945 \\
1945 \\
1945 \\
1945 \\
1945\end{array}$ & $\begin{array}{l}01 \\
03 \\
04 \\
05 \\
05\end{array}$ & $\begin{array}{l}07 \\
20 \\
01 \\
02 \\
17\end{array}$ & $\begin{array}{l}222533.0 \\
215507.0 \\
234342.0 \\
194754.0 \\
150647.0\end{array}$ & $\begin{array}{l}36.730 \mathrm{~N} \\
34.250 \mathrm{~N} \\
34.000 \mathrm{~N} \\
41.200 \mathrm{~N} \\
36.82 \mathrm{~N}\end{array}$ & $\begin{array}{l}121.200 \mathrm{~W} \\
116.167 \mathrm{~W} \\
120.017 \mathrm{~W} \\
123.500 \mathrm{~W} \\
121.37 \mathrm{~W}\end{array}$ & $\begin{array}{l}-\overline{016} \\
016 \\
-\end{array}$ & $\begin{array}{l}324 \\
292 \\
292 \\
324 \\
324\end{array}$ & $\begin{array}{l}- \\
z\end{array}$ & $\bar{E}$ & $\begin{array}{ll}4.70 \mathrm{M}_{\mathrm{L}} & \text { BRK } \\
5.00 \mathrm{M}_{\mathrm{L}} & \text { PAS } \\
5.40 \mathrm{M}_{\mathrm{L}} & \text { PAS } \\
5.00 \mathrm{M}_{\mathrm{L}} & \text { BRK } \\
4.60 \mathrm{M}_{\mathrm{L}} & \text { BRK }\end{array}$ & $\frac{\overline{-}}{5.14 \mathrm{TH}}$ & $\begin{array}{l}\text { VI } \\
\text { IV } \\
\text { IV } \\
\text { V } \\
\text { VI }\end{array}$ & $\begin{array}{r}18 \\
259 \\
18 \\
18 \\
18\end{array}$ & $\begin{array}{l}34 \& \\
\frac{2}{9} \\
15\end{array}$ \\
\hline $\begin{array}{l}1945 \\
1945 \\
1945 \\
1945\end{array}$ & $\begin{array}{l}05 \\
06 \\
08 \\
08\end{array}$ & $\begin{array}{l}19 \\
14 \\
15 \\
27\end{array}$ & $\begin{array}{l}150704.0 \\
033013.0 \\
175624.0 \\
091304.0\end{array}$ & $\begin{array}{l}40.25 \mathrm{~N} \\
37.083 \mathrm{~N} \\
33.217 \mathrm{~N} \\
37.27 \mathrm{~N}\end{array}$ & $\begin{array}{l}126.50 \mathrm{~W} \\
117.500 \mathrm{~W} \\
116.133 \mathrm{~W} \\
121.80 \mathrm{~W}\end{array}$ & $\begin{array}{l}-\overline{016} \\
016 \\
-\end{array}$ & $\begin{array}{l}258 \\
292 \\
292 \\
324\end{array}$ & E & $\bar{z}$ & $\begin{array}{ll}6.20 \mathrm{M}_{\mathrm{S}} & \mathrm{GR} \\
5.00 \mathrm{M}_{\mathrm{L}} & \mathrm{PAS} \\
5.70 \mathrm{M}_{\mathrm{L}} & \text { PAS } \\
4.50 \mathrm{M}_{\mathrm{L}} & \mathrm{BRK}\end{array}$ & $\frac{-}{-}$ & $\frac{\mathrm{V}}{\mathrm{VI}}$ & $\frac{18}{18}$ & $\begin{array}{l}4 \& \\
\frac{39 \#}{34 \&}\end{array}$ \\
\hline 1945 & 09 & 28 & 222405.0 & $41.90 \mathrm{~N}$ & $126.70 \mathrm{~W}$ & - & 324 & - & - & $6.12 \mathrm{M}_{s} \mathrm{CFR}$ & - & - & - & - \\
\hline $\begin{array}{l}1946 \\
1946 \\
1946 \\
1946\end{array}$ & $\begin{array}{l}01 \\
01 \\
03 \\
03\end{array}$ & $\begin{array}{l}08 \\
13 \\
15 \\
15\end{array}$ & $\begin{array}{l}185418.0 \\
163115.0 \\
132100.9 \\
134935.9\end{array}$ & $\begin{array}{l}33.000 \mathrm{~N} \\
37.317 \mathrm{~N} \\
35.753 \mathrm{~N} \\
35.725 \mathrm{~N}\end{array}$ & $\begin{array}{l}115.833 \mathrm{~W} \\
118.650 \mathrm{~W} \\
117.987 \mathrm{~W} \\
118.055 \mathrm{~W}\end{array}$ & $\begin{array}{l}016 \\
016 \\
016 \\
022\end{array}$ & $\begin{array}{l}292 \\
292 \\
292 \\
292\end{array}$ & E & $\overline{-}$ & $\begin{array}{ll}5.40 \mathrm{M}_{\mathrm{L}} & \text { PAS } \\
4.70 \mathrm{M}_{\mathrm{L}} & \text { PAS } \\
5.20 \mathrm{M}_{\mathrm{L}} & \text { PAS } \\
6.30 \mathrm{M}_{\mathrm{L}} & \text { PAS }\end{array}$ & $\frac{\bar{Z}}{6.06 \mathrm{TH}}$ & $\begin{array}{l}\text { V } \\
\text { VI } \\
\text { VI } \\
\text { VII }\end{array}$ & $\begin{array}{r}19 \\
259 \\
259 \\
19\end{array}$ & $\frac{31 \#}{\overline{222} \&}$ \\
\hline 1946 & 03 & 15 & 140035.4 & $35.715 \mathrm{~N}$ & $118.073 W$ & 016 & 292 & - & - & $5.30 \mathrm{M}_{\mathrm{L}}$ PAS & - & VI & 259 & - \\
\hline $\begin{array}{l}1946 \\
1946 \\
1946 \\
1946 \\
1946\end{array}$ & $\begin{array}{l}03 \\
03 \\
03 \\
03 \\
03\end{array}$ & $\begin{array}{l}15 \\
15 \\
16 \\
17 \\
18\end{array}$ & $\begin{array}{l}191853.6 \\
215433.4 \\
094617.9 \\
081636.0 \\
100555.1\end{array}$ & $\begin{array}{l}35.715 \mathrm{~N} \\
35.752 \mathrm{~N} \\
35.745 \mathrm{~N} \\
35.632 \mathrm{~N} \\
35.723 \mathrm{~N}\end{array}$ & $\begin{array}{l}117.977 \mathrm{~W} \\
118.029 \mathrm{~W} \\
118.038 \mathrm{~W} \\
118.268 \mathrm{~W} \\
118.035 \mathrm{~W}\end{array}$ & $\begin{array}{l}016 \\
016 \\
016 \\
\overline{016}\end{array}$ & $\begin{array}{l}292 \\
292 \\
292 \\
292 \\
292\end{array}$ & $\begin{array}{l}- \\
-\end{array}$ & $\frac{-}{E}$ & $\begin{array}{ll}5.40 \mathrm{M}_{\mathrm{L}} & \text { PAS } \\
5.20 \mathrm{M}_{\mathrm{L}} & \text { PAS } \\
5.10 \mathrm{M}_{\mathrm{L}} & \text { PAS } \\
4.60 \mathrm{M}_{\mathrm{L}} & \text { PAS } \\
4.90 \mathrm{M}_{\mathrm{L}} & \text { PAS }\end{array}$ & $\begin{array}{l}-\overline{-} \\
4.54 \mathrm{TH} \\
4.10 \mathrm{TH} \\
4.35 \mathrm{TH}\end{array}$ & $\begin{array}{l}\text { Felt } \\
\text { Felt } \\
\text { Felt } \\
\text { Felt } \\
\text { IV }\end{array}$ & $\begin{array}{r}259 \\
259 \\
19 \\
19 \\
259\end{array}$ & $\begin{array}{l}- \\
-\end{array}$ \\
\hline
\end{tabular}


CALIFORNIA-Continued

[See table 1 for hypocenter and intensity references and table 2 for definitions of magnitude source codes. \&, land area only; \#, land area only in the United States for an earthquake near a coastline; +, land area in the United States when the felt area did not extend to the coast; @, felt area is less than $1,000 \mathrm{~km}^{2}$. Leader (--) indicates information is not available]

\begin{tabular}{|c|c|c|c|c|c|c|c|c|c|c|c|c|c|c|}
\hline & & & Origin & & Hypocente & & & & & Magnitude & & Inte & sity & \\
\hline & Date & & time (UTC) & Latitude & Longitude & Depth & Ref & US & & Other & Moment & MMI & Ref & Felt area \\
\hline $\mathrm{Yr}$ & Mo & $\mathrm{Da}$ & $\mathrm{h} \mathrm{m}$ s & 0, & ?) & $(\mathrm{km})$ & & $m_{b}$ & $M_{s}$ & & 0 & & &, $\left.000 \mathrm{~km}^{2}\right)$ \\
\hline $\begin{array}{l}1946 \\
1946 \\
1946 \\
1946 \\
1946\end{array}$ & $\begin{array}{l}03 \\
03 \\
03 \\
05 \\
05\end{array}$ & $\begin{array}{l}18 \\
18 \\
24 \\
02 \\
29\end{array}$ & $\begin{array}{lll}15 & 49 & 25.7 \\
15 & 50 & 42.6 \\
20 & 00 & 03.0 \\
01 & 26 & 12.0 \\
17 & 51 & 03.0\end{array}$ & $\begin{array}{l}35.780 \mathrm{~N} \\
35.747 \mathrm{~N} \\
35.728 \mathrm{~N} \\
37.68 \mathrm{~N} \\
36.77 \mathrm{~N}\end{array}$ & $\begin{array}{l}117.922 \mathrm{~W} \\
117.908 \mathrm{~W} \\
118.098 \mathrm{~W} \\
121.60 \mathrm{~W} \\
121.42 \mathrm{~W}\end{array}$ & $\begin{array}{l}016 \\
004 \\
016 \\
- \\
-\end{array}$ & $\begin{array}{l}292 \\
292 \\
292 \\
324 \\
324\end{array}$ & I & $\begin{array}{l}z \\
z\end{array}$ & $\begin{array}{ll}4.80 \mathrm{M}_{\mathrm{L}} & \text { PAS } \\
5.30 \mathrm{M}_{\mathrm{L}} & \text { PAS } \\
4.50 \mathrm{M}_{\mathrm{L}} & \text { PAS } \\
4.60 \mathrm{M}_{\mathrm{L}} & \text { BRK } \\
4.50 \mathrm{M}_{\mathrm{L}} & \text { BRK }\end{array}$ & $\begin{array}{c}4.30 \mathrm{TH} \\
4.99 \mathrm{TH} \\
- \\
-\end{array}$ & $\begin{array}{l}\mathrm{V} \\
\mathrm{V} \\
\mathrm{V} \\
\mathrm{V}\end{array}$ & $\begin{array}{r}259 \\
259 \\
19 \\
259\end{array}$ & $\frac{-}{8}$ \\
\hline $\begin{array}{l}1946 \\
1946 \\
1946 \\
1946 \\
1946\end{array}$ & $\begin{array}{l}06 \\
06 \\
07 \\
07 \\
08\end{array}$ & $\begin{array}{l}04 \\
15 \\
07 \\
18 \\
30\end{array}$ & $\begin{array}{l}120524.0 \\
194653.0 \\
065515.0 \\
142758.0 \\
11 \quad 1645.0\end{array}$ & $\begin{array}{l}33.917 \mathrm{~N} \\
32.600 \mathrm{~N} \\
40.500 \mathrm{~N} \\
34.533 \mathrm{~N} \\
33.233 \mathrm{~N}\end{array}$ & $\begin{array}{l}115.700 \mathrm{~W} \\
116.317 \mathrm{~W} \\
121.500 \mathrm{~W} \\
115.983 \mathrm{~W} \\
115.700 \mathrm{~W}\end{array}$ & $\begin{array}{l}016 \\
016 \\
016 \\
016\end{array}$ & $\begin{array}{l}292 \\
292 \\
324 \\
292 \\
292\end{array}$ & $\begin{array}{l}- \\
-\end{array}$ & $\begin{array}{l}z \\
-\end{array}$ & $\begin{array}{ll}4.80 \mathrm{M}_{\mathrm{L}} & \text { PAS } \\
4.80 \mathrm{M}_{\mathrm{L}} & \text { PAS } \\
5.00 \mathrm{M}_{\mathrm{L}} & \text { BRK } \\
5.60 \mathrm{M}_{\mathrm{L}} & \text { PAS } \\
4.60 \mathrm{M}_{\mathrm{L}} & \text { PAS }\end{array}$ & $\frac{\bar{Z}}{5.39 \mathrm{TH}}$ & $\begin{array}{l}\text { IV } \\
\text { V } \\
\text { V } \\
\text { V } \\
-\end{array}$ & $\begin{array}{r}259 \\
19 \\
19 \\
259 \\
-\end{array}$ & $\begin{array}{l}- \\
\overline{18} \& \\
70 \\
-\end{array}$ \\
\hline $\begin{array}{l}1946 \\
1946 \\
1947 \\
1947 \\
1947\end{array}$ & $\begin{array}{l}09 \\
12 \\
02 \\
02 \\
03\end{array}$ & $\begin{array}{l}28 \\
18 \\
05 \\
06 \\
30\end{array}$ & $\begin{array}{l}071909.0 \\
142028.0 \\
061423 \\
172040.1 \\
0744\end{array}$ & $\begin{array}{l}33.950 \mathrm{~N} \\
40.30 \mathrm{~N} \\
36.23 \mathrm{~N} \\
35.677 \mathrm{~N} \\
40.38 \mathrm{~N}\end{array}$ & $\begin{array}{l}116.850 \mathrm{~W} \\
124.60 \mathrm{~W} \\
120.65 \mathrm{~W} \\
118.067 \mathrm{~W} \\
124.68 \mathrm{~W}\end{array}$ & $\frac{016}{\frac{-}{016}}$ & $\begin{array}{l}292 \\
324 \\
324 \\
292 \\
324\end{array}$ & $\bar{z}$ & $\begin{array}{l}z \\
z\end{array}$ & $\begin{array}{ll}5.00 \mathrm{M}_{\mathrm{L}} & \text { PAS } \\
4.70 \mathrm{M}_{\mathrm{L}} & \text { BRK } \\
5.00 \mathrm{M}_{\mathrm{L}} & \text { BRK } \\
4.60 \mathrm{M}_{\mathrm{L}} & \text { PAS } \\
4.60 \mathrm{M}_{\mathrm{L}} & \mathrm{BRK}\end{array}$ & $\begin{array}{l}- \\
\overline{-}\end{array}$ & $\begin{array}{l}\text { VI } \\
\text { V } \\
\text { VI } \\
\text { V } \\
\text { V }\end{array}$ & $\begin{array}{r}19 \\
259 \\
20 \\
259 \\
259\end{array}$ & $\begin{array}{c}23 \& \\
9 \& \\
- \\
5 \&\end{array}$ \\
\hline $\begin{array}{l}1947 \\
1947 \\
1947 \\
1947 \\
1947\end{array}$ & $\begin{array}{l}04 \\
04 \\
04 \\
04 \\
04\end{array}$ & $\begin{array}{l}10 \\
10 \\
10 \\
11 \\
19\end{array}$ & $\begin{array}{l}155806.0 \\
1603 \\
171822.0 \\
0747 \\
022909.0\end{array}$ & $\begin{array}{l}34.983 \mathrm{~N} \\
34.967 \mathrm{~N} \\
34.950 \mathrm{~N} \\
34.967 \mathrm{~N} \\
34.967 \mathrm{~N}\end{array}$ & $\begin{array}{l}116.550 \mathrm{~W} \\
116.550 \mathrm{~W} \\
116.533 \mathrm{~W} \\
116.550 \mathrm{~W} \\
116.550 \mathrm{~W}\end{array}$ & $\begin{array}{l}016 \\
016 \\
016 \\
016 \\
016\end{array}$ & $\begin{array}{l}292 \\
292 \\
292 \\
292 \\
292\end{array}$ & $\bar{z}$ & $\begin{array}{l}- \\
z\end{array}$ & $\begin{array}{ll}6.20 \mathrm{M}_{\mathrm{L}} & \text { PAS } \\
5.10 \mathrm{M}_{\mathrm{L}} & \text { PAS } \\
5.00 \mathrm{M}_{\mathrm{L}} & \text { PAS } \\
5.00 \mathrm{M}_{\mathrm{L}} & \text { PAS } \\
4.70 \mathrm{M}_{\mathrm{L}} & \text { PAS }\end{array}$ & $\begin{array}{c}6.51 \mathrm{HK} \\
- \\
4.76 \mathrm{TH} \\
4.38 \mathrm{TH}\end{array}$ & $\begin{array}{l}\text { VII } \\
\text { Felt } \\
\text { Felt } \\
-\end{array}$ & $\begin{array}{r}20 \\
259 \\
\frac{20}{-}\end{array}$ & $\frac{1518}{-}$ \\
\hline $\begin{array}{l}1947 \\
1947 \\
1947 \\
1947 \\
1947\end{array}$ & $\begin{array}{l}05 \\
05 \\
06 \\
07 \\
07\end{array}$ & $\begin{array}{l}11 \\
27 \\
22 \\
24 \\
24\end{array}$ & $\begin{array}{l}050620.0 \\
205942 \\
232933 \\
221046.0 \\
225426.0\end{array}$ & $\begin{array}{l}34.233 \mathrm{~N} \\
40.40 \mathrm{~N} \\
37.00 \mathrm{~N} \\
34.017 \mathrm{~N} \\
34.017 \mathrm{~N}\end{array}$ & $\begin{array}{l}116.333 \mathrm{~W} \\
124.70 \mathrm{~W} \\
121.77 \mathrm{~W} \\
116.500 \mathrm{~W} \\
116.500 \mathrm{~W}\end{array}$ & $\frac{016}{-}$ & $\begin{array}{l}292 \\
324 \\
324 \\
292 \\
292\end{array}$ & $\bar{z}$ & $\begin{array}{l}z \\
z\end{array}$ & $\begin{array}{ll}4.90 \mathrm{M}_{\mathrm{L}} & \text { PAS } \\
5.20 \mathrm{M}_{\mathrm{L}} & \text { BRK } \\
4.70 \mathrm{M}_{\mathrm{L}} & \text { BRK } \\
5.50 \mathrm{M}_{\mathrm{L}} & \text { PAS } \\
4.90 \mathrm{M}_{\mathrm{L}} & \text { PAS }\end{array}$ & $\begin{array}{l}- \\
-\end{array}$ & $\begin{array}{l}\text { Felt } \\
\text { VI } \\
\text { VI } \\
\text { V } \\
\text { IV }\end{array}$ & $\begin{array}{r}20 \\
20 \\
20 \\
20 \\
259\end{array}$ & $\begin{array}{l}-68 \\
35 \& \\
60 \# \\
-\end{array}$ \\
\hline $\begin{array}{l}1947 \\
1947 \\
1947 \\
1947 \\
1947\end{array}$ & $\begin{array}{l}07 \\
07 \\
07 \\
07 \\
07\end{array}$ & $\begin{array}{l}25 \\
25 \\
25 \\
25 \\
26\end{array}$ & $\begin{array}{l}004631.0 \\
015647.0 \\
061949.0 \\
161453.0 \\
024941.0\end{array}$ & $\begin{array}{l}34.017 \mathrm{~N} \\
34.017 \mathrm{~N} \\
34.017 \mathrm{~N} \\
34.017 \mathrm{~N} \\
34.017 \mathrm{~N}\end{array}$ & $\begin{array}{l}116.500 \mathrm{~W} \\
116.500 \mathrm{~W} \\
116.500 \mathrm{~W} \\
116.500 \mathrm{~W} \\
116.500 \mathrm{~W}\end{array}$ & $\begin{array}{l}016 \\
016 \\
016 \\
016 \\
016\end{array}$ & $\begin{array}{l}292 \\
292 \\
292 \\
292 \\
292\end{array}$ & $\bar{z}$ & $\begin{array}{l}z \\
-\end{array}$ & $\begin{array}{ll}5.00 \mathrm{M}_{\mathrm{L}} & \text { PAS } \\
4.60 \mathrm{M}_{\mathrm{L}} & \text { PAS } \\
5.20 \mathrm{M}_{\mathrm{L}} & \text { PAS } \\
4.50 \mathrm{M}_{\mathrm{L}} & \text { PAS } \\
5.10 \mathrm{M}_{\mathrm{L}} & \text { PAS }\end{array}$ & $\begin{array}{c}4.64 \mathrm{TH} \\
4.15 \mathrm{TH} \\
5.15 \mathrm{TH} \\
4.26 \mathrm{TH} \\
-\end{array}$ & $\begin{array}{c}\overline{-} \\
\text { IV } \\
\text { IV } \\
\text { Felt }\end{array}$ & $\begin{array}{l}- \\
259 \\
259 \\
259\end{array}$ & - \\
\hline $\begin{array}{l}1947 \\
1947 \\
1947 \\
1947 \\
1947\end{array}$ & $\begin{array}{l}07 \\
08 \\
09 \\
09 \\
09\end{array}$ & $\begin{array}{l}26 \\
10 \\
08 \\
08 \\
23\end{array}$ & $\begin{array}{l}230425.0 \\
215824 \\
0552 \\
0713 \\
135255.0\end{array}$ & $\begin{array}{l}34.017 \mathrm{~N} \\
36.88 \mathrm{~N} \\
39.30 \mathrm{~N} \\
39.30 \mathrm{~N} \\
40.40 \mathrm{~N}\end{array}$ & $\begin{array}{l}116.500 \mathrm{~W} \\
121.42 \mathrm{~W} \\
120.20 \mathrm{~W} \\
120.20 \mathrm{~W} \\
125.20 \mathrm{~W}\end{array}$ & $\begin{array}{l}016 \\
= \\
-\end{array}$ & $\begin{array}{l}292 \\
324 \\
324 \\
324 \\
324\end{array}$ & $\bar{z}$ & $\begin{array}{l}z \\
z\end{array}$ & $\begin{array}{ll}4.50 \mathrm{M}_{\mathrm{L}} & \text { PAS } \\
4.40 \mathrm{M}_{\mathrm{L}} & \text { BRK } \\
4.50 \mathrm{M}_{\mathrm{L}} & \text { BRK } \\
4.70 \mathrm{M}_{\mathrm{L}} & \text { BRK } \\
5.30 \mathrm{M}_{\mathrm{L}} & \text { BRK }\end{array}$ & $\overline{-}$ & $\begin{array}{l}\overrightarrow{V I} \\
\text { V } \\
\text { VI } \\
\text { V }\end{array}$ & $\begin{array}{r}\overline{20} \\
20 \\
20 \\
259\end{array}$ & $\begin{array}{l}\overline{10 \&} \\
\overline{19 \&}\end{array}$ \\
\hline $\begin{array}{l}1947 \\
1947 \\
1947 \\
1948 \\
1948\end{array}$ & $\begin{array}{l}11 \\
11 \\
11 \\
01 \\
02\end{array}$ & $\begin{array}{l}02 \\
10 \\
18 \\
10 \\
11\end{array}$ & $\begin{array}{l}0701 \\
022255.0 \\
215903.0 \\
004550 \\
032928.0\end{array}$ & $\begin{array}{l}40.10 \mathrm{~N} \\
34.400 \mathrm{~N} \\
33.267 \mathrm{~N} \\
42.0 \mathrm{~N} \\
36.10 \mathrm{~N}\end{array}$ & $\begin{array}{l}125.30 \mathrm{~W} \\
116.417 \mathrm{~W} \\
119.450 \mathrm{~W} \\
127.0 \mathrm{~W} \\
118.80 \mathrm{~W}\end{array}$ & $\begin{array}{l}-\overline{016} \\
016 \\
- \\
-\end{array}$ & $\begin{array}{l}324 \\
292 \\
292 \\
324 \\
324\end{array}$ & $\bar{z}$ & $\bar{z}$ & $\begin{array}{ll}4.80 \mathrm{M}_{\mathrm{L}} & \text { BRK } \\
4.50 \mathrm{M}_{\mathrm{L}} & \text { PAS } \\
5.00 \mathrm{M}_{\mathrm{L}} & \text { PAS } \\
5.30 \mathrm{M}_{\mathrm{L}} & \text { BRK } \\
4.90 \mathrm{M}_{\mathrm{L}} & \text { BRK }\end{array}$ & $\begin{array}{c}\bar{Z} \\
4.78 \mathrm{TH} \\
-\end{array}$ & $\frac{\bar{v}}{\frac{\bar{v}}{\mathrm{VI}}}$ & $\begin{array}{r}259 \\
\frac{20}{21}\end{array}$ & $\overline{-}$ \\
\hline $\begin{array}{l}1948 \\
1948 \\
1948 \\
1948 \\
1948\end{array}$ & $\begin{array}{l}02 \\
02 \\
02 \\
03 \\
03\end{array}$ & $\begin{array}{l}19 \\
20 \\
24 \\
01 \\
28\end{array}$ & $\begin{array}{lll}08 & 25 & 09.0 \\
04 & 21 & 24.0 \\
08 & 15 & 10.0 \\
08 & 12 & 13.0 \\
22 & 38 & 03.0\end{array}$ & $\begin{array}{l}41.00 \mathrm{~N} \\
33.917 \mathrm{~N} \\
32.500 \mathrm{~N} \\
34.167 \mathrm{~N} \\
36.850 \mathrm{~N}\end{array}$ & $\begin{array}{l}124.90 \mathrm{~W} \\
118.217 \mathrm{~W} \\
118.550 \mathrm{~W} \\
117.533 \mathrm{~W} \\
121.570 \mathrm{~W}\end{array}$ & $\begin{array}{l}- \\
016 \\
016 \\
016 \\
-\end{array}$ & $\begin{array}{l}324 \\
292 \\
292 \\
292 \\
324\end{array}$ & $\bar{z}$ & $\begin{array}{l}\overline{-} \\
-\end{array}$ & $\begin{array}{ll}4.80 \mathrm{M}_{\mathrm{L}} & \text { BRK } \\
3.60 \mathrm{M}_{\mathrm{L}} & \text { PAS } \\
5.30 \mathrm{M}_{\mathrm{L}} & \text { PAS } \\
4.70 \mathrm{M}_{\mathrm{L}} & \text { PAS } \\
4.60 \mathrm{M}_{\mathrm{L}} & \text { BRK }\end{array}$ & $\begin{array}{c}\overline{-} \\
\overline{-} \\
-\end{array}$ & $\begin{array}{l}\text { III } \\
\text { VI } \\
\text { IV } \\
\text { VI } \\
\text { V }\end{array}$ & $\begin{array}{r}259 \\
21 \\
21 \\
21 \\
21\end{array}$ & $\begin{array}{l}\overline{2} \\
\overline{31} \& \\
10 \&\end{array}$ \\
\hline $\begin{array}{l}1948 \\
1948 \\
1948 \\
1948 \\
1948\end{array}$ & $\begin{array}{l}03 \\
04 \\
06 \\
07 \\
08\end{array}$ & $\begin{array}{l}28 \\
16 \\
18 \\
26 \\
18\end{array}$ & $\begin{array}{lll}22 & 4500.0 \\
22 & 2624.0 \\
10 & 3500.0 \\
17 & 5001.4 \\
19 & 1157.0\end{array}$ & $\begin{array}{l}36.850 \mathrm{~N} \\
34.017 \mathrm{~N} \\
39.07 \mathrm{~N} \\
35.582 \mathrm{~N} \\
40.50 \mathrm{~N}\end{array}$ & $\begin{array}{l}121.570 \mathrm{~W} \\
118.967 \mathrm{~W} \\
123.28 \mathrm{~W} \\
118.158 \mathrm{~W} \\
124.70 \mathrm{~W}\end{array}$ & $\frac{\overline{016}}{\overline{005}}$ & $\begin{array}{l}324 \\
292 \\
324 \\
292 \\
324\end{array}$ & - & $\bar{z}$ & $\begin{array}{ll}4.50 \mathrm{M}_{\mathrm{L}} & \text { BRK } \\
4.70 \mathrm{M}_{\mathrm{L}} & \text { PAS } \\
3.80 \mathrm{M}_{\mathrm{L}} & \text { BRK } \\
4.50 \mathrm{M}_{\mathrm{L}} & \text { PAS } \\
5.00 \mathrm{M}_{\mathrm{L}} & \text { BRK }\end{array}$ & - & $\begin{array}{l}\text { V } \\
\text { VI } \\
\text { VI } \\
\text { V } \\
\text { V }\end{array}$ & $\begin{array}{r}21 \\
21 \\
21 \\
259 \\
259\end{array}$ & $\begin{array}{c}10 \& \\
48 \\
1 \\
-\end{array}$ \\
\hline $\begin{array}{l}1948 \\
1948 \\
1948 \\
1948 \\
1948\end{array}$ & $\begin{array}{l}11 \\
12 \\
12 \\
12 \\
12\end{array}$ & $\begin{array}{l}12 \\
04 \\
05 \\
05 \\
11\end{array}$ & $\begin{array}{lll}23 & 10 & 25.0 \\
23 & 43 & 17.0 \\
0007 & 21.0 \\
00 & 42 & 35.0 \\
16 & 12 & 20.0\end{array}$ & $\begin{array}{l}40.40 \mathrm{~N} \\
33.933 \mathrm{~N} \\
33.933 \mathrm{~N} \\
33.967 \mathrm{~N} \\
33.967 \mathrm{~N}\end{array}$ & $\begin{array}{l}124.32 \mathrm{~W} \\
116.383 \mathrm{~W} \\
116.367 \mathrm{~W} \\
116.433 \mathrm{~W} \\
116.450 \mathrm{~W}\end{array}$ & $\begin{array}{l}-\overline{016} \\
016 \\
016 \\
016\end{array}$ & $\begin{array}{l}324 \\
292 \\
292 \\
292 \\
292\end{array}$ & $\overline{-}$ & $=$ & $\begin{array}{ll}4.50 \mathrm{M}_{\mathrm{L}} & \text { BRK } \\
6.50 \mathrm{M}_{\mathrm{L}} & \text { PAS } \\
4.90 \mathrm{M}_{\mathrm{L}} & \text { PAS } \\
4.60 \mathrm{M}_{\mathrm{L}} & \text { PAS } \\
4.50 \mathrm{M}_{\mathrm{L}} & \text { PAS }\end{array}$ & $\begin{array}{c}\overline{5.95 \mathrm{HK}} \\
4.44 \mathrm{TH} \\
4.48 \mathrm{TH} \\
-\end{array}$ & $\begin{array}{l}\text { Felt } \\
\text { VII } \\
\text { Felt } \\
\overline{\text { IV }}\end{array}$ & $\begin{array}{r}324 \\
21 \\
259 \\
259\end{array}$ & $\begin{array}{l}\overline{180 \#} \\
- \\
-\end{array}$ \\
\hline
\end{tabular}


CALIFORNIA-Continued

[See table 1 for hypocenter and intensity references and table 2 for definitions of magnitude source codes. \&, land area only; \#, land area only in the United States for an earthquake near a coastline; +, land area in the United States when the felt area did not extend to the coast; 0 , felt area is less than $1,000 \mathrm{~km}^{2}$. Leader (--) indicates information is not available]

\begin{tabular}{|c|c|c|c|c|c|c|c|c|c|c|c|c|c|}
\hline \multirow{2}{*}{\multicolumn{2}{|c|}{ Date }} & \multirow{2}{*}{$\begin{array}{c}\text { Origin } \\
\text { time (UTC) }\end{array}$} & \multicolumn{4}{|c|}{ Hypocenter } & \multicolumn{4}{|c|}{ Magnitude } & \multicolumn{2}{|c|}{ Intensity } & \multirow{3}{*}{$\begin{array}{c}\text { Felt area } \\
\left(1,000 \mathrm{~km}^{2}\right)\end{array}$} \\
\hline & & & \multirow{2}{*}{$\begin{array}{l}\text { Latitude } \\
\left(^{\circ}\right)\end{array}$} & \multirow{2}{*}{$\begin{array}{l}\text { Longitude } \\
\left(^{\circ}\right)\end{array}$} & \multirow{2}{*}{$\begin{array}{l}\text { Depth } \\
\text { (km) }\end{array}$} & \multirow[t]{2}{*}{ Ref } & \multicolumn{2}{|c|}{ usas } & \multirow[t]{2}{*}{ Other } & \multirow{2}{*}{$\begin{array}{c}\text { Moment } \\
\text { M }\end{array}$} & \multirow[t]{2}{*}{ MMI } & \multirow[t]{2}{*}{ Ref } & \\
\hline $\mathrm{Yr}$ & Mo Da & $h \mathrm{~m} s$ & & & & & $m_{b}$ & $\mathbf{M}_{\mathbf{s}}$ & & & & & \\
\hline $\begin{array}{l}1948 \\
1948 \\
1948 \\
1949 \\
1949\end{array}$ & $\begin{array}{ll}12 & 20 \\
12 & 29 \\
12 & 31 \\
01 & 01 \\
01 & 03\end{array}$ & $\begin{array}{lll}04 & 42 & 46.0 \\
1253 & 28.0 \\
14 & 3546.0 \\
01 & 1754.0 \\
134340.0\end{array}$ & $\begin{array}{l}35.80 \mathrm{~N} \\
39.550 \mathrm{~N} \\
35.67 \mathrm{~N} \\
36.90 \mathrm{~N} \\
34.967 \mathrm{~N}\end{array}$ & $\begin{array}{l}121.50 \mathrm{~W} \\
120.080 \mathrm{~W} \\
121.40 \mathrm{~W} \\
121.62 \mathrm{~W} \\
116.550 \mathrm{~W}\end{array}$ & $\begin{array}{l}E \\
z\end{array}$ & $\begin{array}{l}324 \\
324 \\
324 \\
324 \\
292\end{array}$ & $\begin{array}{l}- \\
- \\
-\end{array}$ & $\begin{array}{l}z \\
z \\
-\end{array}$ & $\begin{array}{ll}4.50 \mathrm{M}_{\mathrm{L}} & \text { BRK } \\
6.00 \mathrm{M}_{\mathrm{L}} & \text { BRK } \\
4.60 \mathrm{M}_{\mathrm{L}} & \text { BRK } \\
4.50 \mathrm{M}_{\mathrm{L}} & \text { BRK } \\
4.80 \mathrm{M}_{\mathrm{L}} & \text { PAS }\end{array}$ & $\begin{array}{l}z \\
z\end{array}$ & $\begin{array}{c}\text { III } \\
\text { VII } \\
\text { VII } \\
\text { IV }\end{array}$ & $\begin{array}{r}259 \\
21 \\
21 \\
21 \\
259\end{array}$ & $\frac{\overline{104}}{\overline{9} \&}$ \\
\hline $\begin{array}{l}1949 \\
1949 \\
1949 \\
1949 \\
1949\end{array}$ & $\begin{array}{ll}01 & 20 \\
02 & 11 \\
02 & 27 \\
03 & 09 \\
03 & 24\end{array}$ & $\begin{array}{l}075923.0 \\
210524.0 \\
133547.0 \\
122839.0 \\
205656.0\end{array}$ & $\begin{array}{l}39.55 \mathrm{~N} \\
37.083 \mathrm{~N} \\
41.20 \mathrm{~N} \\
37.02 \mathrm{~N} \\
41.30 \mathrm{~N}\end{array}$ & $\begin{array}{l}120.08 \mathrm{~W} \\
117.750 \mathrm{~W} \\
125.20 \mathrm{~W} \\
121.48 \mathrm{~W} \\
126.00 \mathrm{~W}\end{array}$ & $\frac{\overline{016}}{-}$ & $\begin{array}{l}324 \\
292 \\
324 \\
324 \\
324\end{array}$ & $\begin{array}{c}- \\
- \\
-\end{array}$ & $\begin{array}{l}z \\
z\end{array}$ & $\begin{array}{ll}4.80 \mathrm{M}_{\mathrm{L}} & \text { BRK } \\
5.60 \mathrm{M}_{\mathrm{L}} & \text { PAS } \\
4.80 \mathrm{M}_{\mathrm{L}} & \text { BRK } \\
5.20 \mathrm{M}_{\mathrm{L}} & \text { BRK } \\
5.90 \mathrm{M}_{\mathrm{L}} & \text { BRK }\end{array}$ & $\begin{array}{c}\overline{5.25 \mathrm{TH}} \\
- \\
- \\
-\end{array}$ & $\begin{array}{l}\text { IV } \\
\text { VI } \\
\overline{\text { VII }} \\
\text { Felt }\end{array}$ & $\begin{array}{r}259 \\
22 \\
\overline{22} \\
324\end{array}$ & $\begin{array}{l}\overline{77} \\
\overline{52} \& \\
-\end{array}$ \\
\hline $\begin{array}{l}1949 \\
1949 \\
1949 \\
1949 \\
1949\end{array}$ & $\begin{array}{ll}04 & 13 \\
05 & 02 \\
05 & 02 \\
05 & 10 \\
05 & 25\end{array}$ & $\begin{array}{l}075826.0 \\
112458.0 \\
112547.0 \\
040633.0 \\
173146.0\end{array}$ & $\begin{array}{l}37.667 \mathrm{~N} \\
34.017 \mathrm{~N} \\
34.017 \mathrm{~N} \\
34.017 \mathrm{~N} \\
34.017 \mathrm{~N}\end{array}$ & $\begin{array}{l}118.383 \mathrm{~W} \\
115.767 \mathrm{~W} \\
115.683 \mathrm{~W} \\
115.683 \mathrm{~W} \\
115.683 \mathrm{~W}\end{array}$ & $\begin{array}{l}016 \\
016 \\
016 \\
016 \\
016\end{array}$ & $\begin{array}{l}292 \\
292 \\
292 \\
292 \\
292\end{array}$ & $\begin{array}{l}- \\
- \\
-\end{array}$ & $\begin{array}{l}- \\
z \\
-\end{array}$ & $\begin{array}{ll}4.50 \mathrm{M}_{\mathrm{L}} & \text { PAS } \\
4.60 \mathrm{M}_{\mathrm{L}} & \text { PAS } \\
5.90 \mathrm{M}_{\mathrm{L}} & \text { PAS } \\
4.70 \mathrm{M}_{\mathrm{L}} & \text { PAS } \\
4.50 \mathrm{M}_{\mathrm{L}} & \text { PAS }\end{array}$ & $\begin{array}{l}- \\
- \\
-\end{array}$ & $\begin{array}{c}\text { IV } \\
\text { Felt } \\
\text { V } \\
- \\
-\end{array}$ & $\begin{array}{r}259 \\
259 \\
22 \\
-\end{array}$ & $\begin{array}{l}\overline{-} \\
\overline{82 \#} \\
-\end{array}$ \\
\hline $\begin{array}{l}1949 \\
1949 \\
1949 \\
1949 \\
1949\end{array}$ & $\begin{array}{ll}06 & 10 \\
06 & 27 \\
08 & 08 \\
08 & 21 \\
08 & 27\end{array}$ & $\begin{array}{l}030640.0 \\
103531.0 \\
110003.0 \\
204816.0 \\
145146.0\end{array}$ & $\begin{array}{l}37.30 \mathrm{~N} \\
35.80 \mathrm{~N} \\
37.95 \mathrm{~N} \\
40.27 \mathrm{~N} \\
34.500 \mathrm{~N}\end{array}$ & $\begin{array}{l}121.67 \mathrm{~W} \\
121.10 \mathrm{~W} \\
122.32 \mathrm{~W} \\
121.23 \mathrm{~W} \\
120.500 \mathrm{~W}\end{array}$ & $\bar{z}$ & $\begin{array}{l}324 \\
324 \\
324 \\
324 \\
292\end{array}$ & $\overline{-}$ & $\begin{array}{l}\overline{-} \\
z\end{array}$ & $\begin{array}{ll}4.60 \mathrm{M}_{\mathrm{L}} & \text { BRK } \\
4.50 \mathrm{M}_{\mathrm{L}} & \text { BRK } \\
3.30 \mathrm{M}_{\mathrm{L}} & \text { BRK } \\
4.50 \mathrm{M}_{\mathrm{L}} & \text { BRK } \\
4.90 \mathrm{M}_{\mathrm{L}} & \text { PAS }\end{array}$ & $\frac{\bar{Z}}{\overline{-}}$ & $\begin{array}{l}\text { VI } \\
\text { IV } \\
\text { VI } \\
\text { IV } \\
\text { VI }\end{array}$ & $\begin{array}{r}22 \\
259 \\
22 \\
259 \\
22\end{array}$ & $\frac{21 \&}{18}$ \\
\hline $\begin{array}{l}1949 \\
1949 \\
1949 \\
1949 \\
1949\end{array}$ & $\begin{array}{ll}09 & 19 \\
10 & 22 \\
10 & 28 \\
11 & 04 \\
11 & 05\end{array}$ & $\begin{array}{l}050813.5 \\
214520.0 \\
022916.0 \\
204238.0 \\
043524.0\end{array}$ & $\begin{array}{l}33.960 \mathrm{~N} \\
36.58 \mathrm{~N} \\
40.90 \mathrm{~N} \\
32.200 \mathrm{~N} \\
32.200 \mathrm{~N}\end{array}$ & $\begin{array}{l}118.187 \mathrm{~W} \\
121.17 \mathrm{~W} \\
124.20 \mathrm{~W} \\
116.550 \mathrm{~W} \\
116.550 \mathrm{~W}\end{array}$ & $\frac{000}{\overline{0}}$ & $\begin{array}{l}292 \\
324 \\
324 \\
292 \\
292\end{array}$ & $\begin{array}{l}- \\
- \\
-\end{array}$ & $\begin{array}{l}z \\
z\end{array}$ & $\begin{array}{ll}3.10 \mathrm{M}_{\mathrm{L}} & \text { PAS } \\
4.70 \mathrm{M}_{\mathrm{L}} & \text { BRK } \\
4.50 \mathrm{M}_{\mathrm{L}} & \text { BRK } \\
5.70 \mathrm{M}_{\mathrm{L}} & \text { PAS } \\
5.10 \mathrm{M}_{\mathrm{L}} & \text { PAS }\end{array}$ & $\bar{F} \overline{-}$ & $\begin{array}{l}\text { VI } \\
\text { V } \\
\text { V } \\
\text { VI } \\
\text { VI }\end{array}$ & $\begin{array}{r}22 \\
259 \\
259 \\
22 \\
22\end{array}$ & $\frac{2}{\overline{3} \# \#}$ \\
\hline $\begin{array}{l}1949 \\
1950 \\
1950 \\
1950 \\
1950\end{array}$ & $\begin{array}{ll}12 & 09 \\
01 & 14 \\
01 & 27 \\
02 & 26 \\
03 & 20\end{array}$ & $\begin{array}{ll}123902.0 \\
195230 \\
104720 \\
000622.0 \\
152217.0\end{array}$ & $\begin{array}{l}37.467 \mathrm{~N} \\
40.217 \mathrm{~N} \\
42.0 \mathrm{~N} \\
34.617 \mathrm{~N} \\
40.450 \mathrm{~N}\end{array}$ & $\begin{array}{l}118.367 \mathrm{~W} \\
124.417 \mathrm{~W} \\
125.1 \mathrm{~W} \\
119.083 \mathrm{~W} \\
121.467 \mathrm{~W}\end{array}$ & $\frac{016}{016}$ & $\begin{array}{l}292 \\
324 \\
324 \\
292 \\
324\end{array}$ & $\begin{array}{l}- \\
- \\
-\end{array}$ & $\begin{array}{l}z \\
z\end{array}$ & $\begin{array}{ll}4.60 \mathrm{M}_{\mathrm{L}} & \text { PAS } \\
4.60 \mathrm{M}_{\mathrm{L}} & \text { BRK } \\
4.70 \mathrm{M}_{\mathrm{L}} & \text { BRK } \\
4.70 \mathrm{M}_{\mathrm{L}} & \text { PAS } \\
5.50 \mathrm{M}_{\mathrm{L}} & \mathrm{BRK}\end{array}$ & $\frac{-}{-}$ & $\begin{array}{l}\text { IV } \\
\text { VI } \\
\text { VI } \\
\text { V }\end{array}$ & $\begin{array}{l}22 \\
23 \\
23 \\
23\end{array}$ & $\begin{array}{c}- \\
-68 \\
10\end{array}$ \\
\hline $\begin{array}{l}1950 \\
1950 \\
1950 \\
1950 \\
1950\end{array}$ & $\begin{array}{ll}03 & 23 \\
04 & 15 \\
06 & 09 \\
07 & 27 \\
07 & 27\end{array}$ & $\begin{array}{l}041650.0 \\
115632.0 \\
130744 \\
112926.0 \\
2251\end{array}$ & $\begin{array}{l}40.500 \mathrm{~N} \\
35.750 \mathrm{~N} \\
41.283 \mathrm{~N} \\
33.117 \mathrm{~N} \\
33.117 \mathrm{~N}\end{array}$ & $\begin{array}{l}121.500 \mathrm{~W} \\
119.617 \mathrm{~W} \\
125.733 \mathrm{~W} \\
115.567 \mathrm{~W} \\
115.567 \mathrm{~W}\end{array}$ & $\frac{\overline{016}}{0 \overline{016}}$ & $\begin{array}{l}266 \\
292 \\
324 \\
292 \\
292\end{array}$ & $\begin{array}{l}- \\
- \\
-\end{array}$ & $\begin{array}{l}- \\
z\end{array}$ & $\begin{array}{ll}4.60 \mathrm{M}_{\mathrm{L}} & \text { BRK } \\
4.60 \mathrm{M}_{\mathrm{L}} & \text { PAS } \\
4.80 \mathrm{M}_{\mathrm{L}} & \text { BRK } \\
4.80 \mathrm{M}_{\mathrm{L}} & \text { PAS } \\
4.50 \mathrm{M}_{\mathrm{L}} & \text { PAS }\end{array}$ & $\frac{-}{-}$ & $\frac{\overline{I V}}{\overline{V I}}$ & $\frac{259}{23}$ & $\begin{array}{l}- \\
- \\
-\end{array}$ \\
\hline $\begin{array}{l}1950 \\
1950 \\
1950 \\
1950 \\
1950\end{array}$ & $\begin{array}{ll}07 & 28 \\
07 & 28 \\
07 & 28 \\
07 & 28 \\
07 & 29\end{array}$ & $\begin{array}{l}0325 \\
1727 \\
175048.0 \\
175812.0 \\
0017\end{array}$ & $\begin{array}{l}33.117 \mathrm{~N} \\
33.117 \mathrm{~N} \\
33.117 \mathrm{~N} \\
33.117 \mathrm{~N} \\
33.117 \mathrm{~N}\end{array}$ & $\begin{array}{l}115.567 \mathrm{~W} \\
115.567 \mathrm{~W} \\
115.567 \mathrm{~W} \\
115.567 \mathrm{~W} \\
115.567 \mathrm{~W}\end{array}$ & $\begin{array}{l}016 \\
016 \\
016 \\
016 \\
016\end{array}$ & $\begin{array}{l}292 \\
292 \\
292 \\
292 \\
292\end{array}$ & $\begin{array}{l}- \\
- \\
-\end{array}$ & $\begin{array}{l}- \\
z\end{array}$ & $\begin{array}{ll}4.70 \mathrm{M}_{\mathrm{L}} & \text { PAS } \\
4.70 \mathrm{M}_{\mathrm{L}} & \text { PAS } \\
5.40 \mathrm{M}_{\mathrm{L}} & \text { PAS } \\
4.80 \mathrm{M}_{\mathrm{L}} & \text { PAS } \\
4.50 \mathrm{M}_{\mathrm{L}} & \text { PAS }\end{array}$ & $\begin{array}{l}\overline{ } \\
4.77 \mathrm{TH} \\
5.22 \mathrm{TH} \\
\overline{4.63 \mathrm{TH}}\end{array}$ & $\begin{array}{r}\text { V } \\
\text { Felt } \\
\text { VI } \\
\text { Felt }\end{array}$ & $\begin{array}{r}259 \\
23 \\
23 \\
23\end{array}$ & $\begin{array}{l}- \\
\overline{32 \#} \\
-\end{array}$ \\
\hline $\begin{array}{l}1950 \\
1950 \\
1950 \\
1950 \\
1950\end{array}$ & $\begin{array}{ll}07 & 29 \\
07 & 29 \\
07 & 29 \\
08 & 01 \\
08 & 14\end{array}$ & $\begin{array}{l}143632.0 \\
1509 \\
184300.0 \\
083720.0 \\
191600.0\end{array}$ & $\begin{array}{l}33.117 \mathrm{~N} \\
33.117 \mathrm{~N} \\
33.117 \mathrm{~N} \\
33.117 \mathrm{~N} \\
33.117 \mathrm{~N}\end{array}$ & $\begin{array}{l}115.567 \mathrm{~W} \\
115.567 \mathrm{~W} \\
115.567 \mathrm{~W} \\
115.567 \mathrm{~W} \\
115.567 \mathrm{~W}\end{array}$ & $\begin{array}{l}016 \\
016 \\
016 \\
016 \\
016\end{array}$ & $\begin{array}{l}292 \\
292 \\
292 \\
292 \\
292\end{array}$ & $\overline{-}$ & $\bar{z}$ & $\begin{array}{ll}5.50 \mathrm{M}_{\mathrm{L}} & \text { PAS } \\
4.50 \mathrm{M}_{\mathrm{L}} & \text { PAS } \\
4.70 \mathrm{M}_{\mathrm{L}} & \text { PAS } \\
4.70 \mathrm{M}_{\mathrm{L}} & \text { PAS } \\
4.70 \mathrm{M}_{\mathrm{L}} & \text { PAS }\end{array}$ & $\frac{-}{-}$ & $\begin{array}{l}\text { VIII } \\
\text { IV } \\
\text { IV } \\
\text { VI } \\
-\end{array}$ & $\begin{array}{r}23 \\
259 \\
259 \\
259 \\
-\end{array}$ & $\begin{array}{l}\text { 44\# } \\
- \\
-\end{array}$ \\
\hline $\begin{array}{l}1950 \\
1950 \\
1950 \\
1950 \\
1950\end{array}$ & $\begin{array}{ll}09 & 05 \\
10 & 08 \\
11 & 14 \\
11 & 14 \\
11 & 17\end{array}$ & $\begin{array}{l}191956.0 \\
122419 \\
023550 \\
063432 \\
034651.0\end{array}$ & $\begin{array}{l}33.650 \mathrm{~N} \\
40.283 \mathrm{~N} \\
40.483 \mathrm{~N} \\
40.483 \mathrm{~N} \\
33.917 \mathrm{~N}\end{array}$ & $\begin{array}{l}116.750 \mathrm{~W} \\
124.800 \mathrm{~W} \\
121.500 \mathrm{~W} \\
121.500 \mathrm{~W} \\
118.317 \mathrm{~W}\end{array}$ & $\frac{016}{-}$ & $\begin{array}{l}292 \\
324 \\
324 \\
324 \\
292\end{array}$ & $\frac{-}{-}$ & $\begin{array}{l}- \\
-\end{array}$ & $\begin{array}{ll}4.80 \mathrm{M}_{\mathrm{L}} & \text { PAS } \\
4.60 \mathrm{M}_{\mathrm{L}} & \text { BRK } \\
4.60 \mathrm{M}_{\mathrm{L}} & \text { BRK } \\
4.50 \mathrm{M}_{\mathrm{L}} & \text { BRK } \\
3.80 \mathrm{M}_{\mathrm{L}} & \text { PAS }\end{array}$ & $\frac{-}{-}$ & $\begin{array}{c}\mathrm{VI} \\
\mathrm{V} \\
\text { Felt } \\
\mathrm{VI}\end{array}$ & $\begin{array}{l}\frac{23}{23} \\
23 \\
23\end{array}$ & $\begin{array}{l}26 \& \\
= \\
-\end{array}$ \\
\hline $\begin{array}{l}1950 \\
1950 \\
1951\end{array}$ & $\begin{array}{ll}12 & 14 \\
12 & 14 \\
01 & 13\end{array}$ & $\begin{array}{l}085934.0 \\
132419 \\
203132\end{array}$ & $\begin{array}{l}40.083 \mathrm{~N} \\
40.083 \mathrm{~N} \\
40.4 \mathrm{~N}\end{array}$ & $\begin{array}{l}120.067 \mathrm{~W} \\
120.067 \mathrm{~W} \\
125.0 \mathrm{~W}\end{array}$ & - & $\begin{array}{l}324 \\
324 \\
324\end{array}$ & - & E & $\begin{array}{ll}4.50 \mathrm{M}_{\mathrm{L}} & \text { BRK } \\
5.60 \mathrm{M}_{\mathrm{L}} & \text { BRK } \\
4.80 \mathrm{M}_{\mathrm{L}} & \text { BRK }\end{array}$ & - & $\begin{array}{c}\text { Felt } \\
\text { VII } \\
\text { IV }\end{array}$ & $\begin{array}{l}23 \\
23 \\
24\end{array}$ & $\overline{80}$ \\
\hline
\end{tabular}


CALIFORNIA-Continued

[See table 1 for hypocenter and intensity references and table 2 for definitions of magnitude source codes. \&, land area only; \#, land area only in the United States for an earthquake near a coastline; +, land area in the United States when the felt area did not extend to the coast; @, felt area is less than $1,000 \mathrm{~km}^{2}$. Leader (-) indicates information is not available ]

\begin{tabular}{|c|c|c|c|c|c|c|c|c|c|c|c|c|c|c|}
\hline & & & Origin & & Hypocente & & & & & Magnitude & & Inte & sity & \\
\hline & Date & & time (UTC) & Latitude & Longitude & Depth & Ref & US & & Other & Moment & MMI & Ref & Felt area \\
\hline$\overline{Y r}$ & Mo & $\overline{D a}$ & h m s & $\left({ }^{\circ}\right)$ & $\left(^{\circ}\right)$ & $(\mathrm{km})$ & & $m_{b}$ & $M_{s}$ & & נח & & &, $\left.000 \mathrm{~km}^{2}\right)$ \\
\hline $\begin{array}{l}1951 \\
1951\end{array}$ & $\begin{array}{l}01 \\
01\end{array}$ & $\begin{array}{l}24 \\
25\end{array}$ & $\begin{array}{l}071702.6 \\
210018\end{array}$ & $\begin{array}{l}32.983 \mathrm{~N} \\
37.750 \mathrm{~N}\end{array}$ & $\begin{array}{l}115.733 \mathrm{~W} \\
122.183 \mathrm{~W}\end{array}$ & $\underline{016}$ & $\begin{array}{l}292 \\
324\end{array}$ & - & - & $\begin{array}{l}5.60 \mathrm{M}_{\mathrm{L}} \text { PAS } \\
2.90 \mathrm{M}_{\mathrm{L}} \text { BRK }\end{array}$ & - & VII & $\begin{array}{l}24 \\
24\end{array}$ & $\underline{56 \#}$ \\
\hline $\begin{array}{l}1951 \\
1951 \\
1951 \\
1951 \\
1951\end{array}$ & $\begin{array}{l}02 \\
02 \\
04 \\
06 \\
07\end{array}$ & $\begin{array}{l}15 \\
15 \\
01 \\
25 \\
29\end{array}$ & $\begin{array}{l}104759.0 \\
104957.0 \\
192108 \\
194541.7 \\
105345.0\end{array}$ & $\begin{array}{l}33.483 \mathrm{~N} \\
33.483 \mathrm{~N} \\
40.467 \mathrm{~N} \\
35.772 \mathrm{~N} \\
36.583 \mathrm{~N}\end{array}$ & $\begin{array}{l}116.500 \mathrm{~W} \\
116.500 \mathrm{~W} \\
125.300 \mathrm{~W} \\
117.948 \mathrm{~W} \\
121.183 \mathrm{~W}\end{array}$ & $\begin{array}{l}016 \\
016 \\
\overline{0} \\
-\end{array}$ & $\begin{array}{l}292 \\
292 \\
324 \\
292 \\
324\end{array}$ & $\frac{-}{-}$ & - & $\begin{array}{ll}4.80 \mathrm{M}_{\mathrm{L}} & \text { PAS } \\
4.80 \mathrm{M}_{\mathrm{L}} & \text { PAS } \\
5.00 \mathrm{M}_{\mathrm{L}} & \text { BRK } \\
4.60 \mathrm{M}_{\mathrm{L}} & \text { PAS } \\
5.00 \mathrm{M}_{\mathrm{L}} & \text { BRK }\end{array}$ & $\bar{z}$ & $\begin{array}{c}\text { v } \\
\text { v } \\
\text { III } \\
\text { V } \\
\text { VI }\end{array}$ & $\begin{array}{l}24 \\
24 \\
24 \\
24 \\
24\end{array}$ & $\begin{array}{l}18 \& \\
18 \& \\
- \\
-\end{array}$ \\
\hline $\begin{array}{l}1951 \\
1951 \\
1951 \\
1951 \\
1951\end{array}$ & $\begin{array}{l}08 \\
10 \\
10 \\
11 \\
11\end{array}$ & $\begin{array}{l}06 \\
08 \\
31 \\
13 \\
14\end{array}$ & $\begin{array}{l}090502 \\
041035.0 \\
205819 \\
1124 \quad 42 \\
08 \quad 3953\end{array}$ & $\begin{array}{l}36.617 \mathrm{~N} \\
40.283 \mathrm{~N} \\
36.900 \mathrm{~N} \\
40.4 \mathrm{~N} \\
40.433 \mathrm{~N}\end{array}$ & $\begin{array}{l}121.217 \mathrm{~W} \\
124.800 \mathrm{~W} \\
121.417 \mathrm{~W} \\
125.3 \mathrm{~W} \\
124.050 \mathrm{~W}\end{array}$ & $\begin{array}{l}z \\
z\end{array}$ & $\begin{array}{l}324 \\
324 \\
324 \\
324 \\
324\end{array}$ & $\frac{-}{-}$ & $\bar{z}$ & $\begin{array}{ll}4.90 \mathrm{M}_{\mathrm{L}} & \text { BRK } \\
5.80 \mathrm{M}_{\mathrm{L}} & \text { BRK } \\
4.80 \mathrm{M}_{\mathrm{L}} & \text { BRK } \\
4.80 \mathrm{M}_{\mathrm{L}} & \text { BRK } \\
4.70 \mathrm{M}_{\mathrm{L}} & \text { BRK }\end{array}$ & $\bar{z}$ & $\begin{array}{c}\text { VI } \\
\text { VII } \\
\text { V } \\
\text { V } \\
\text { VI }\end{array}$ & $\begin{array}{l}24 \\
24 \\
24 \\
24 \\
24\end{array}$ & $\begin{array}{r}6 \& \\
28 \& \\
6 \& \\
-8 \&\end{array}$ \\
\hline $\begin{array}{l}1951 \\
1951 \\
1951 \\
1952 \\
1952\end{array}$ & $\begin{array}{l}12 \\
12 \\
12 \\
02 \\
02\end{array}$ & $\begin{array}{l}05 \\
26 \\
28 \\
09 \\
13\end{array}$ & $\begin{array}{l}155314.0 \\
004654.0 \\
024927.0 \\
084330.9 \\
151337.0\end{array}$ & $\begin{array}{l}33.100 \mathrm{~N} \\
32.817 \mathrm{~N} \\
37.567 \mathrm{~N} \\
36.607 \mathrm{~N} \\
32.867 \mathrm{~N}\end{array}$ & $\begin{array}{l}115.400 \mathrm{~W} \\
118.350 \mathrm{~W} \\
118.583 \mathrm{~W} \\
117.905 \mathrm{~W} \\
118.250 \mathrm{~W}\end{array}$ & $\begin{array}{l}016 \\
016 \\
016 \\
008 \\
016\end{array}$ & $\begin{array}{l}292 \\
292 \\
292 \\
292 \\
292\end{array}$ & $\begin{array}{l}- \\
- \\
-\end{array}$ & $\bar{z}$ & $\begin{array}{ll}4.50 \mathrm{M}_{\mathrm{L}} & \text { PAS } \\
5.90 \mathrm{M}_{\mathrm{L}} & \text { PAS } \\
5.20 \mathrm{M}_{\mathrm{L}} & \text { PAS } \\
4.10 \mathrm{M}_{\mathrm{L}} & \text { PAS } \\
4.70 \mathrm{M}_{\mathrm{L}} & \text { PAS }\end{array}$ & 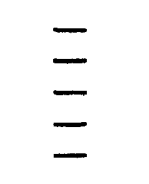 & $\begin{array}{l}\text { VII } \\
\text { VI } \\
\text { V } \\
\text { VI } \\
\text { IV }\end{array}$ & $\begin{array}{l}24 \\
24 \\
24 \\
25 \\
25\end{array}$ & $\begin{array}{l}15+ \\
35 \& \\
33 \\
- \\
-\end{array}$ \\
\hline $\begin{array}{l}1952 \\
1952 \\
1952 \\
1952 \\
1952\end{array}$ & $\begin{array}{l}02 \\
05 \\
07 \\
07 \\
07\end{array}$ & $\begin{array}{l}17 \\
06 \\
21 \\
21 \\
21\end{array}$ & $\begin{array}{lll}12 & 36 & 58.3 \\
17 & 21 & 10 \\
11 & 52 & 14.0 \\
11 & 54 \\
11 & 55\end{array}$ & $\begin{array}{l}33.997 \mathrm{~N} \\
41.9 \mathrm{~N} \\
35.000 \mathrm{~N} \\
35.000 \mathrm{~N} \\
35.000 \mathrm{~N}\end{array}$ & $\begin{array}{l}117.270 \mathrm{~W} \\
124.6 \mathrm{~W} \\
119.017 \mathrm{~W} \\
119.033 \mathrm{~W} \\
119.033 \mathrm{~W}\end{array}$ & $\begin{array}{l}016 \\
016 \\
016 \\
016\end{array}$ & $\begin{array}{l}292 \\
324 \\
292 \\
292 \\
292\end{array}$ & $\begin{array}{l}- \\
- \\
-\end{array}$ & $\bar{z}$ & $\begin{array}{ll}4.50 \mathrm{M}_{\mathrm{L}} & \text { PAS } \\
4.70 \mathrm{M}_{\mathrm{L}} & \text { BRK } \\
7.20 \mathrm{M}_{\mathrm{L}} & \text { BLT } \\
4.50 \mathrm{M}_{\mathrm{L}} & \text { PAS } \\
4.50 \mathrm{M}_{\mathrm{L}} & \text { PAS }\end{array}$ & $\frac{\overline{ }}{7.48 \mathrm{HK}}$ & $\begin{array}{l}\text { IV } \\
\text { IV } \\
\text { XI } \\
- \\
-\end{array}$ & $\begin{array}{r}25 \\
259 \\
25 \\
- \\
-\end{array}$ & $\begin{array}{l}- \\
\overline{353} \& \\
-\end{array}$ \\
\hline $\begin{array}{l}1952 \\
1952 \\
1952 \\
1952 \\
1952\end{array}$ & $\begin{array}{l}07 \\
07 \\
07 \\
07 \\
07\end{array}$ & $\begin{array}{l}21 \\
21 \\
21 \\
21 \\
21\end{array}$ & $\begin{array}{ll}11 & 57 \\
11 & 58 \\
1159 \\
1202 \\
120531.0\end{array}$ & $\begin{array}{l}35.000 \mathrm{~N} \\
35.000 \mathrm{~N} \\
35.000 \mathrm{~N} \\
35.000 \mathrm{~N} \\
35.000 \mathrm{~N}\end{array}$ & $\begin{array}{l}119.033 \mathrm{~W} \\
119.033 \mathrm{~W} \\
119.033 \mathrm{~W} \\
119.033 \mathrm{~W} \\
119.000 \mathrm{~W}\end{array}$ & $\begin{array}{l}016 \\
016 \\
016 \\
016 \\
016\end{array}$ & $\begin{array}{l}292 \\
292 \\
292 \\
292 \\
292\end{array}$ & $\frac{-}{-}$ & $\bar{z}$ & $\begin{array}{ll}4.50 \mathrm{M}_{\mathrm{L}} & \text { PAS } \\
4.60 \mathrm{M}_{\mathrm{L}} & \text { PAS } \\
4.50 \mathrm{M}_{\mathrm{L}} & \text { PAS } \\
5.60 \mathrm{M}_{\mathrm{L}} & \text { PAS } \\
6.40 \mathrm{M}_{\mathrm{L}} & \text { PAS }\end{array}$ & $\frac{\bar{Z}}{\bar{Z}} \overline{ }$ & $\begin{array}{l}\text { Felt } \\
\text { Felt } \\
\underset{V}{\text { Felt }}\end{array}$ & $\begin{array}{l}25 \\
25 \\
-25 \\
25\end{array}$ & $=$ \\
\hline $\begin{array}{l}1952 \\
1952 \\
1952 \\
1952 \\
1952\end{array}$ & $\begin{array}{l}07 \\
07 \\
07 \\
07 \\
07\end{array}$ & $\begin{array}{l}21 \\
21 \\
21 \\
21 \\
21\end{array}$ & $\begin{array}{ll}12 & 06 \\
12 & 07 \\
12 & 10 \\
12 & 12 \\
12 & 1936.0\end{array}$ & $\begin{array}{l}35.000 \mathrm{~N} \\
35.000 \mathrm{~N} \\
35.000 \mathrm{~N} \\
35.000 \mathrm{~N} \\
34.950 \mathrm{~N}\end{array}$ & $\begin{array}{l}119.000 \mathrm{~W} \\
119.000 \mathrm{~W} \\
119.000 \mathrm{~W} \\
119.000 \mathrm{~W} \\
118.867 \mathrm{~W}\end{array}$ & $\begin{array}{l}016 \\
016 \\
016 \\
016 \\
016\end{array}$ & $\begin{array}{l}292 \\
292 \\
292 \\
292 \\
292\end{array}$ & $\begin{array}{l}- \\
- \\
-\end{array}$ & $\bar{z}$ & $\begin{array}{ll}4.80 \mathrm{M}_{\mathrm{L}} & \text { PAS } \\
4.70 \mathrm{M}_{\mathrm{L}} & \text { PAS } \\
4.50 \mathrm{M}_{\mathrm{L}} & \text { PAS } \\
4.60 \mathrm{M}_{\mathrm{L}} & \text { PAS } \\
5.30 \mathrm{M}_{\mathrm{L}} & \text { PAS }\end{array}$ & - & $\begin{array}{l}\text { Felt } \\
\text { Felt } \\
\text { Felt } \\
\text { Felt }\end{array}$ & $\begin{array}{l}- \\
25 \\
25 \\
25 \\
25\end{array}$ & $\overline{-}$ \\
\hline $\begin{array}{l}1952 \\
1952 \\
1952 \\
1952 \\
1952\end{array}$ & $\begin{array}{l}07 \\
07 \\
07 \\
07 \\
07\end{array}$ & $\begin{array}{l}21 \\
21 \\
21 \\
21 \\
21\end{array}$ & $\begin{array}{ll}12 & 22 \\
12 & 25 \\
12 & 40 \\
13 & 08 \\
13 & 13\end{array}$ & $\begin{array}{l}35.000 \mathrm{~N} \\
35.000 \mathrm{~N} \\
35.000 \mathrm{~N} \\
35.000 \mathrm{~N} \\
35.000 \mathrm{~N}\end{array}$ & $\begin{array}{l}119.000 \mathrm{~W} \\
119.000 \mathrm{~W} \\
119.000 \mathrm{~W} \\
119.000 \mathrm{~W} \\
119.000 \mathrm{~W}\end{array}$ & $\begin{array}{l}016 \\
016 \\
016 \\
016 \\
016\end{array}$ & $\begin{array}{l}292 \\
292 \\
292 \\
292 \\
292\end{array}$ & $\begin{array}{l}- \\
- \\
-\end{array}$ & $\bar{z}$ & $\begin{array}{ll}4.90 \mathrm{M}_{\mathrm{L}} & \text { PAS } \\
4.70 \mathrm{M}_{\mathrm{L}} & \text { PAS } \\
4.90 \mathrm{M}_{\mathrm{L}} & \text { PAS } \\
4.50 \mathrm{M}_{\mathrm{L}} & \text { PAS } \\
4.50 \mathrm{M}_{\mathrm{L}} & \text { PAS }\end{array}$ & $\begin{array}{l}z \\
z\end{array}$ & $\begin{array}{l}\text { Felt } \\
\text { Felt } \\
- \\
-\end{array}$ & $\begin{array}{l}\overline{25} \\
25 \\
- \\
-\end{array}$ & $\bar{z}$ \\
\hline $\begin{array}{l}1952 \\
1952 \\
1952 \\
1952 \\
1952\end{array}$ & $\begin{array}{l}07 \\
07 \\
07 \\
07 \\
07\end{array}$ & $\begin{array}{l}21 \\
21 \\
21 \\
21 \\
21\end{array}$ & $\begin{array}{l}132512.0 \\
1359 \\
151358.0 \\
1553 \\
1638\end{array}$ & $\begin{array}{l}35.000 \mathrm{~N} \\
35.000 \mathrm{~N} \\
35.183 \mathrm{~N} \\
35.000 \mathrm{~N} \\
35.000 \mathrm{~N}\end{array}$ & $\begin{array}{l}119.000 \mathrm{~W} \\
119.000 \mathrm{~W} \\
118.650 \mathrm{~W} \\
119.000 \mathrm{~W} \\
119.000 \mathrm{~W}\end{array}$ & $\begin{array}{l}-\overline{-} \\
016 \\
016 \\
016\end{array}$ & $\begin{array}{l}292 \\
292 \\
292 \\
292 \\
292\end{array}$ & $\begin{array}{l}- \\
- \\
-\end{array}$ & $\bar{z}$ & $\begin{array}{ll}4.50 \mathrm{M}_{\mathrm{L}} & \text { PAS } \\
4.60 \mathrm{M}_{\mathrm{L}} & \mathrm{PAS} \\
5.10 \mathrm{M}_{\mathrm{L}} & \text { PAS } \\
4.50 \mathrm{M}_{\mathrm{L}} & \text { PAS } \\
4.50 \mathrm{M}_{\mathrm{L}} & \text { PAS }\end{array}$ & $\bar{z}$ & $\begin{array}{l}\overline{-} \\
\overline{\text { VI }} \\
\mathbf{v} \\
-\end{array}$ & $\begin{array}{l}- \\
\overline{25} \\
25 \\
-\end{array}$ & $\bar{z}$ \\
\hline $\begin{array}{l}1952 \\
1952 \\
1952 \\
1952 \\
1952\end{array}$ & $\begin{array}{l}07 \\
07 \\
07 \\
07 \\
07\end{array}$ & $\begin{array}{l}21 \\
21 \\
21 \\
21 \\
21\end{array}$ & $\begin{array}{l}174244.0 \\
1800 \\
182338.0 \\
194122.0 \\
235328.0\end{array}$ & $\begin{array}{l}35.233 \mathrm{~N} \\
35.000 \mathrm{~N} \\
35.300 \mathrm{~N} \\
35.133 \mathrm{~N} \\
34.983 \mathrm{~N}\end{array}$ & $\begin{array}{l}118.533 W \\
119.000 W \\
118.533 W \\
118.767 W \\
119.033 W\end{array}$ & $\begin{array}{l}016 \\
016 \\
016 \\
016 \\
016\end{array}$ & $\begin{array}{l}292 \\
292 \\
292 \\
292 \\
292\end{array}$ & $\overline{-}$ & $\bar{z}$ & $\begin{array}{ll}5.10 \mathrm{M}_{\mathrm{L}} & \text { PAS } \\
4.50 \mathrm{M}_{\mathrm{L}} & \text { PAS } \\
4.50 \mathrm{M}_{\mathrm{L}} & \text { PAS } \\
5.50 \mathrm{M}_{\mathrm{L}} & \text { PAS } \\
4.50 \mathrm{M}_{\mathrm{L}} & \text { PAS }\end{array}$ & $\frac{\overline{-}}{4.3 \overline{\mathrm{TH}}}$ & $\frac{-}{v}$ & $\frac{-}{25}$ & 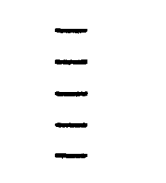 \\
\hline $\begin{array}{l}1952 \\
1952 \\
1952 \\
1952 \\
1952\end{array}$ & $\begin{array}{l}07 \\
07 \\
07 \\
07 \\
07\end{array}$ & $\begin{array}{l}22 \\
22 \\
22 \\
22 \\
22\end{array}$ & $\begin{array}{lll}01 & 41 & 02.0 \\
08 & 47 & 34.0 \\
09 & 10 & 25.0 \\
13 & 31 & 43.0 \\
22 & 31 & 33.0\end{array}$ & $\begin{array}{l}35.133 \mathrm{~N} \\
35.083 \mathrm{~N} \\
35.233 \mathrm{~N} \\
35.000 \mathrm{~N} \\
35.033 \mathrm{~N}\end{array}$ & $\begin{array}{l}118.517 \mathrm{~W} \\
118.750 \mathrm{~W} \\
118.600 \mathrm{~W} \\
119.000 \mathrm{~W} \\
118.933 \mathrm{~W}\end{array}$ & $\begin{array}{l}016 \\
016 \\
016 \\
016 \\
016\end{array}$ & $\begin{array}{l}292 \\
292 \\
292 \\
292 \\
292\end{array}$ & $\begin{array}{l}- \\
- \\
-\end{array}$ & $\begin{array}{l}- \\
- \\
-\end{array}$ & $\begin{array}{ll}4.50 \mathrm{M}_{\mathrm{L}} & \text { PAS } \\
4.70 \mathrm{M}_{\mathrm{L}} & \text { PAS } \\
4.50 \mathrm{M}_{\mathrm{L}} & \text { PAS } \\
4.80 \mathrm{M}_{\mathrm{L}} & \text { PAS } \\
4.70 \mathrm{M}_{\mathrm{L}} & \text { PAS }\end{array}$ & $\begin{array}{c}4.70 \mathrm{TH} \\
4.56 \mathrm{TH} \\
- \\
4.62 \mathrm{TH} \\
4.69 \mathrm{TH}\end{array}$ & $\frac{\overline{I V}}{\mathrm{~V}}$ & $\begin{array}{l}\frac{}{25} \\
\frac{25}{-}\end{array}$ & - \\
\hline $\begin{array}{l}1952 \\
1952\end{array}$ & $\begin{array}{l}07 \\
07\end{array}$ & $\begin{array}{l}23 \\
23\end{array}$ & $\begin{array}{l}003832.0 \\
004738.0\end{array}$ & $\begin{array}{l}35.366 \mathrm{~N} \\
35.367 \mathrm{~N}\end{array}$ & $\begin{array}{l}118.583 W \\
118.583 W\end{array}$ & $\begin{array}{l}016 \\
016\end{array}$ & $\begin{array}{l}292 \\
292\end{array}$ & - & - & $\begin{array}{l}6.10 \mathrm{M}_{\mathrm{L}} \text { PAS } \\
4.60 \mathrm{M}_{\mathrm{L}} \text { PAS }\end{array}$ & 5.70HHT & $\underline{\text { VI }}$ & 25 & - \\
\hline
\end{tabular}


CALIFORNIA-Continued

[See table 1 for hypocenter and intensity references and table 2 for definitions of magnitude source codes. \&, land area only; \#, land area only in the United States for an earthquake near a coastline; +, land area in the United States when the felt area did not extend to the coast; @, felt area is less than $1,000 \mathrm{~km}^{2}$. Leader (--) indicates information is not available]

\begin{tabular}{|c|c|c|c|c|c|c|c|c|c|c|c|c|c|c|}
\hline & & & & & Hypocente & & & & & Magnitude & & Inter & sity & \\
\hline & Date & & time (UTC) & Latitude & Longitude & Depth & Ref & US & & Other & Moment & $\mathrm{MMi}$ & Ref & \\
\hline Yr & Mo & $\mathrm{Da}$ & $\mathrm{h} \mathrm{m} s$ & $\left({ }^{\circ}\right)$ & $\left({ }^{\circ}\right)$ & $(\mathrm{km})$ & & $m_{b}$ & $M_{\mathbf{s}}$ & & $\mathbf{M}$ & & & $\left(1,000 \mathrm{~km}^{2}\right)$ \\
\hline $\begin{array}{l}1952 \\
1952 \\
1952\end{array}$ & $\begin{array}{l}07 \\
07 \\
07\end{array}$ & $\begin{array}{l}23 \\
23 \\
23\end{array}$ & $\begin{array}{l}031923.0 \\
034928.0 \\
040140.0\end{array}$ & $\begin{array}{l}35.367 \mathrm{~N} \\
35.283 \mathrm{~N} \\
35.367 \mathrm{~N}\end{array}$ & $\begin{array}{l}118.583 W \\
118.550 W \\
118.583 W\end{array}$ & $\begin{array}{l}016 \\
016 \\
016\end{array}$ & $\begin{array}{l}292 \\
292 \\
292\end{array}$ & $=$ & $\overline{-}$ & $\begin{array}{ll}5.00 \mathrm{M}_{\mathrm{L}} & \text { PAS } \\
4.70 \mathrm{M}_{\mathrm{L}} & \text { PAS } \\
4.70 \mathrm{M}_{\mathrm{L}} & \text { PAS }\end{array}$ & $\overline{-}$ & $\frac{v}{-}$ & $\frac{25}{-}$ & $\overline{-}$ \\
\hline $\begin{array}{l}1952 \\
1952 \\
1952 \\
1952 \\
1952\end{array}$ & $\begin{array}{l}07 \\
07 \\
07 \\
07 \\
07\end{array}$ & $\begin{array}{l}23 \\
23 \\
23 \\
23 \\
23\end{array}$ & $\begin{array}{l}054603.0 \\
073700.0 \\
075319.0 \\
131705.0 \\
161838.0\end{array}$ & $\begin{array}{l}35.383 \mathrm{~N} \\
35.283 \mathrm{~N} \\
35.000 \mathrm{~N} \\
35.217 \mathrm{~N} \\
35.333 \mathrm{~N}\end{array}$ & $\begin{array}{l}118.567 \mathrm{~W} \\
118.550 \mathrm{~W} \\
118.833 \mathrm{~W} \\
118.817 \mathrm{~W} \\
118.600 \mathrm{~W}\end{array}$ & $\begin{array}{l}016 \\
016 \\
016 \\
016 \\
016\end{array}$ & $\begin{array}{l}292 \\
292 \\
292 \\
292 \\
292\end{array}$ & $\begin{array}{l}- \\
z\end{array}$ & $\bar{z}$ & $\begin{array}{ll}4.70 \mathrm{M}_{\mathrm{L}} & \text { PAS } \\
4.80 \mathrm{M}_{\mathrm{L}} & \text { PAS } \\
5.40 \mathrm{M}_{\mathrm{L}} & \text { PAS } \\
5.70 \mathrm{M}_{\mathrm{L}} & \text { PAS } \\
4.50 \mathrm{M}_{\mathrm{L}} & \text { PAS }\end{array}$ & $\begin{array}{c}-\overline{\mathrm{T}} \\
4.42 \mathrm{TH} \\
5.23 \mathrm{TH} \\
5.76 \mathrm{TH} \\
-\end{array}$ & $\begin{array}{l}- \\
\text { VII } \\
\text { VII }\end{array}$ & $\begin{array}{l}\overline{-} \\
\overline{25} \\
\frac{25}{-}\end{array}$ & $\begin{array}{l}- \\
\overline{-}\end{array}$ \\
\hline $\begin{array}{l}1952 \\
1952 \\
1952 \\
1952 \\
1952\end{array}$ & $\begin{array}{l}07 \\
07 \\
07 \\
07 \\
07\end{array}$ & $\begin{array}{l}23 \\
23 \\
23 \\
24 \\
25\end{array}$ & 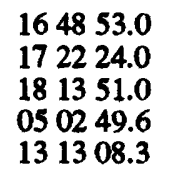 & $\begin{array}{l}35.333 \mathrm{~N} \\
35.335 \mathrm{~N} \\
35.000 \mathrm{~N} \\
35.340 \mathrm{~N} \\
35.310 \mathrm{~N}\end{array}$ & $\begin{array}{l}118.600 W \\
118.475 W \\
118.833 W \\
118.473 W \\
118.498 W\end{array}$ & $\begin{array}{l}016 \\
007 \\
016 \\
002 \\
003\end{array}$ & $\begin{array}{l}292 \\
292 \\
292 \\
292 \\
292\end{array}$ & $\bar{z}$ & $\frac{-}{-}$ & $\begin{array}{ll}4.50 \mathrm{M}_{\mathrm{L}} & \text { PAS } \\
4.50 \mathrm{M}_{\mathrm{L}} & \text { PAS } \\
5.20 \mathrm{M}_{\mathrm{L}} & \text { PAS } \\
4.50 \mathrm{M}_{\mathrm{L}} & \text { PAS } \\
5.00 \mathrm{M}_{\mathrm{L}} & \text { PAS }\end{array}$ & $\begin{array}{l}z \\
z\end{array}$ & $\frac{\overline{\text { III }}}{\text { VI }}$ & $\begin{array}{l}\overline{25} \\
\frac{25}{25}\end{array}$ & $\frac{-}{-}$ \\
\hline $\begin{array}{l}1952 \\
1952 \\
1952 \\
1952 \\
1952\end{array}$ & $\begin{array}{l}07 \\
07 \\
07 \\
07 \\
07\end{array}$ & $\begin{array}{l}25 \\
25 \\
25 \\
26 \\
29\end{array}$ & $\begin{array}{l}190944.6 \\
194323.7 \\
200606.1 \\
224103.0 \\
070347.0\end{array}$ & $\begin{array}{l}35.317 \mathrm{~N} \\
35.315 \mathrm{~N} \\
35.298 \mathrm{~N} \\
35.183 \mathrm{~N} \\
35.383 \mathrm{~N}\end{array}$ & $\begin{array}{l}118.495 \mathrm{~W} \\
118.515 \mathrm{~W} \\
118.435 \mathrm{~W} \\
118.600 \mathrm{~W} \\
118.850 \mathrm{~W}\end{array}$ & $\begin{array}{l}006 \\
011 \\
\overline{016} \\
016\end{array}$ & $\begin{array}{l}292 \\
292 \\
292 \\
292 \\
292\end{array}$ & $=$ & $\bar{z}$ & $\begin{array}{ll}5.70 \mathrm{M}_{\mathrm{L}} & \text { PAS } \\
5.70 \mathrm{M}_{\mathrm{L}} & \text { PAS } \\
4.80 \mathrm{M}_{\mathrm{L}} & \text { PAS } \\
4.60 \mathrm{M}_{\mathrm{L}} & \text { PAS } \\
6.10 \mathrm{M}_{\mathrm{L}} & \text { PAS }\end{array}$ & $\begin{array}{c}5.76 \mathrm{TH} \\
5.94 \mathrm{TH} \\
- \\
\overline{-} \\
6.27 \mathrm{HK}\end{array}$ & $\begin{array}{l}\text { VI } \\
\text { VI } \\
\text { IV } \\
\overline{\text { VII }}\end{array}$ & $\begin{array}{l}25 \\
25 \\
25 \\
\frac{25}{25}\end{array}$ & $\begin{array}{l}- \\
-\end{array}$ \\
\hline $\begin{array}{l}1952 \\
1952 \\
1952 \\
1952 \\
1952\end{array}$ & $\begin{array}{l}07 \\
07 \\
07 \\
07 \\
07\end{array}$ & $\begin{array}{l}29 \\
29 \\
29 \\
31 \\
31\end{array}$ & $\begin{array}{l}080146.0 \\
154950.0 \\
195132.0 \\
120909.0 \\
171908.0\end{array}$ & $\begin{array}{l}35.400 \mathrm{~N} \\
35.183 \mathrm{~N} \\
35.333 \mathrm{~N} \\
35.333 \mathrm{~N} \\
35.283 \mathrm{~N}\end{array}$ & $\begin{array}{l}118.817 \mathrm{~W} \\
118.600 \mathrm{~W} \\
118.917 \mathrm{~W} \\
118.600 \mathrm{~W} \\
118.583 \mathrm{~W}\end{array}$ & $\begin{array}{l}016 \\
016 \\
016 \\
016 \\
016\end{array}$ & $\begin{array}{l}292 \\
292 \\
292 \\
292 \\
292\end{array}$ & $\begin{array}{l}- \\
z\end{array}$ & $\bar{z}$ & $\begin{array}{ll}5.10 \mathrm{M}_{\mathrm{L}} & \text { PAS } \\
4.90 \mathrm{M}_{\mathrm{L}} & \text { PAS } \\
4.50 \mathrm{M}_{\mathrm{L}} & \text { PAS } \\
5.80 \mathrm{M}_{\mathrm{L}} & \text { PAS } \\
4.50 \mathrm{M}_{\mathrm{L}} & \text { PAS }\end{array}$ & $\begin{array}{c}- \\
5.48 \mathrm{TH} \\
-\end{array}$ & $\begin{array}{c}\mathrm{V} \\
\text { Felt } \\
\overline{\mathrm{VI}} \\
-\end{array}$ & $\begin{array}{l}25 \\
\frac{25}{25} \\
-\end{array}$ & $\begin{array}{l}- \\
- \\
-\end{array}$ \\
\hline $\begin{array}{l}1952 \\
1952 \\
1952 \\
1952 \\
1952\end{array}$ & $\begin{array}{l}07 \\
08 \\
08 \\
08 \\
08\end{array}$ & $\begin{array}{l}31 \\
01 \\
01 \\
07 \\
10\end{array}$ & $\begin{array}{l}195314.0 \\
0316 \quad 11.6 \\
130430.0 \\
163151.0 \\
122318.0\end{array}$ & $\begin{array}{l}35.333 \mathrm{~N} \\
35.283 \mathrm{~N} \\
34.900 \mathrm{~N} \\
35.033 \mathrm{~N} \\
35.288 \mathrm{~N}\end{array}$ & $\begin{array}{l}118.917 \mathrm{~W} \\
118.550 \mathrm{~W} \\
118.950 \mathrm{~W} \\
119.050 \mathrm{~W} \\
118.412 \mathrm{~W}\end{array}$ & $\begin{array}{l}016 \\
016 \\
016 \\
016 \\
004\end{array}$ & $\begin{array}{l}292 \\
292 \\
292 \\
292 \\
292\end{array}$ & $\frac{-}{E}$ & $\bar{z}$ & $\begin{array}{ll}\text { 4.50M } & \text { PAS } \\
4.50 \mathrm{M}_{\mathrm{L}} & \text { PAS } \\
5.10 \mathrm{M}_{\mathrm{L}} & \text { PAS } \\
4.90 \mathrm{M}_{\mathrm{L}} & \text { PAS } \\
4.60 \mathrm{M}_{\mathrm{L}} & \text { PAS }\end{array}$ & $\begin{array}{l}- \\
z\end{array}$ & $\begin{array}{l}\text { IV } \\
v \\
\text { V } \\
-\end{array}$ & $\begin{array}{l}\frac{25}{25} \\
25 \\
-\end{array}$ & $\overline{-}$ \\
\hline $\begin{array}{l}1952 \\
1952 \\
1952 \\
1952 \\
1952\end{array}$ & $\begin{array}{l}08 \\
08 \\
08 \\
08 \\
08\end{array}$ & $\begin{array}{l}13 \\
13 \\
18 \\
19 \\
22\end{array}$ & $\begin{array}{l}042940.6 \\
173925.0 \\
044010.0 \\
191226.0 \\
224124.0\end{array}$ & $\begin{array}{l}35.293 \mathrm{~N} \\
35.150 \mathrm{~N} \\
35.033 \mathrm{~N} \\
35.050 \mathrm{~N} \\
35.333 \mathrm{~N}\end{array}$ & $\begin{array}{l}118.400 \mathrm{~W} \\
118.683 \mathrm{~W} \\
119.050 \mathrm{~W} \\
119.233 \mathrm{~W} \\
118.917 \mathrm{~W}\end{array}$ & $\begin{array}{l}014 \\
016 \\
016 \\
016 \\
016\end{array}$ & $\begin{array}{l}292 \\
292 \\
292 \\
292 \\
292\end{array}$ & $=$ & $\bar{z}$ & $\begin{array}{ll}4.60 \mathrm{M}_{\mathrm{L}} & \text { PAS } \\
4.70 \mathrm{M}_{\mathrm{L}} & \mathrm{PAS} \\
4.70 \mathrm{M}_{\mathrm{L}} & \mathrm{PAS} \\
4.50 \mathrm{M}_{\mathrm{L}} & \mathrm{PAS} \\
5.80 \mathrm{M}_{\mathrm{L}} & \mathrm{PAS}\end{array}$ & $\frac{\bar{Z}}{\bar{Z}}$ & $\begin{array}{c}\text { Felt } \\
\text { VI } \\
\text { IV } \\
\text { V } \\
\text { VIII }\end{array}$ & $\begin{array}{l}25 \\
25 \\
25 \\
25 \\
25\end{array}$ & $\frac{\bar{z}}{\overline{120} \&}$ \\
\hline $\begin{array}{l}1952 \\
1952 \\
1952 \\
1952 \\
1952\end{array}$ & $\begin{array}{l}08 \\
08 \\
08 \\
09 \\
09\end{array}$ & $\begin{array}{l}23 \\
25 \\
30 \\
02 \\
02\end{array}$ & $\begin{array}{l}100907.1 \\
062026.0 \\
045559.8 \\
124132.0 \\
204556.0\end{array}$ & $\begin{array}{l}34.520 \mathrm{~N} \\
35.100 \mathrm{~N} \\
35.315 \mathrm{~N} \\
35.133 \mathrm{~N} \\
34.967 \mathrm{~N}\end{array}$ & $\begin{array}{l}118.198 \mathrm{~W} \\
118.967 \mathrm{~W} \\
118.482 \mathrm{~W} \\
118.700 \mathrm{~W} \\
119.000 \mathrm{~W}\end{array}$ & $\begin{array}{l}013 \\
016 \\
005 \\
016 \\
016\end{array}$ & $\begin{array}{l}292 \\
292 \\
292 \\
292 \\
292\end{array}$ & $\begin{array}{l}\overline{-} \\
\overline{-}\end{array}$ & $\overline{-}$ & $\begin{array}{ll}5.00 \mathrm{M}_{\mathrm{L}} & \text { PAS } \\
4.70 \mathrm{M}_{\mathrm{L}} & \text { PAS } \\
4.70 \mathrm{M}_{\mathrm{L}} & \text { PAS } \\
4.60 \mathrm{M}_{\mathrm{L}} & \text { PAS } \\
4.70 \mathrm{M}_{\mathrm{L}} & \text { PAS }\end{array}$ & $\begin{array}{c}4.85 \mathrm{TH} \\
- \\
-\end{array}$ & $\begin{array}{l}\text { VI } \\
\text { V } \\
\mathbf{V} \\
\mathbf{V} \\
\mathbf{V}\end{array}$ & $\begin{array}{l}25 \\
25 \\
25 \\
25 \\
25\end{array}$ & $\begin{array}{l}60 \& \\
- \\
-\end{array}$ \\
\hline $\begin{array}{l}1952 \\
1952 \\
1952 \\
1952 \\
1952\end{array}$ & $\begin{array}{l}09 \\
09 \\
09 \\
11 \\
11\end{array}$ & $\begin{array}{l}12 \\
15 \\
22 \\
07 \\
22\end{array}$ & $\begin{array}{l}103525.0 \\
044013.2 \\
114125 \\
085535.0 \\
074636.0\end{array}$ & $\begin{array}{l}35.000 \mathrm{~N} \\
35.317 \mathrm{~N} \\
40.200 \mathrm{~N} \\
35.000 \mathrm{~N} \\
35.768 \mathrm{~N}\end{array}$ & $\begin{array}{l}119.050 \mathrm{~W} \\
118.487 \mathrm{~W} \\
124.417 \mathrm{~W} \\
119.083 \mathrm{~W} \\
121.145 \mathrm{~W}\end{array}$ & $\begin{array}{l}016 \\
004 \\
\overline{016} \\
010\end{array}$ & $\begin{array}{l}292 \\
292 \\
324 \\
292 \\
476\end{array}$ & $\begin{array}{l}\overline{-} \\
\overline{-}\end{array}$ & $\overline{-}$ & $\begin{array}{ll}4.50 \mathrm{M}_{\mathrm{L}} & \text { PAS } \\
4.90 \mathrm{M}_{\mathrm{L}} & \text { PAS } \\
5.20 \mathrm{M}_{\mathrm{L}} & \text { BRK } \\
4.60 \mathrm{M}_{\mathrm{L}} & \text { PAS } \\
6.00 \mathrm{M}_{\mathrm{L}} & \text { BRK }\end{array}$ & $\frac{-}{E}$ & $\frac{\mathrm{V}}{\mathrm{VII}}$ & $\begin{array}{l}\frac{25}{25} \\
25 \\
25\end{array}$ & $\begin{array}{l}- \\
\overline{10} \& \\
\overline{82} \&\end{array}$ \\
\hline $\begin{array}{l}1953 \\
1953 \\
1953 \\
1953 \\
1953\end{array}$ & $\begin{array}{l}02 \\
03 \\
04 \\
05 \\
05\end{array}$ & $\begin{array}{l}23 \\
22 \\
29 \\
25 \\
25\end{array}$ & $\begin{array}{l}074251 \\
051900 \\
124745.0 \\
032401.0 \\
040759\end{array}$ & $\begin{array}{l}41.500 \mathrm{~N} \\
38.817 \mathrm{~N} \\
35.000 \mathrm{~N} \\
35.000 \mathrm{~N} \\
39.3 \mathrm{~N}\end{array}$ & $\begin{array}{l}125.133 \mathrm{~W} \\
119.983 \mathrm{~W} \\
118.733 \mathrm{~W} \\
119.017 \mathrm{~W} \\
123.3 \mathrm{~W}\end{array}$ & $\begin{array}{l}- \\
\overline{016} \\
016 \\
-\end{array}$ & $\begin{array}{l}324 \\
324 \\
292 \\
292 \\
324\end{array}$ & $\begin{array}{l}z \\
z\end{array}$ & $\bar{z}$ & $\begin{array}{ll}4.70 \mathrm{M}_{\mathrm{L}} & \text { BRK } \\
5.00 \mathrm{M}_{\mathrm{L}} & \text { BRK } \\
4.70 \mathrm{M}_{\mathrm{L}} & \text { PAS } \\
4.80 \mathrm{M}_{\mathrm{L}} & \text { PAS } \\
3.20 \mathrm{M}_{\mathrm{L}} & \text { BRK }\end{array}$ & $\begin{array}{l}= \\
z\end{array}$ & $\begin{array}{c}\bar{V} \\
\text { IV } \\
\text { V } \\
\text { VI }\end{array}$ & $\begin{array}{l}\overline{26} \\
26 \\
26 \\
26\end{array}$ & $\begin{array}{l}- \\
-\end{array}$ \\
\hline $\begin{array}{l}1953 \\
1953 \\
1953 \\
1953 \\
1953\end{array}$ & $\begin{array}{l}06 \\
06 \\
10 \\
11 \\
12\end{array}$ & $\begin{array}{l}14 \\
14 \\
07 \\
24 \\
15\end{array}$ & $\begin{array}{l}041729.9 \\
042958.0 \\
145921.0 \\
054606.0 \\
124436.0\end{array}$ & $\begin{array}{l}32.950 \mathrm{~N} \\
32.950 \mathrm{~N} \\
35.033 \mathrm{~N} \\
35.883 \mathrm{~N} \\
35.217 \mathrm{~N}\end{array}$ & $\begin{array}{l}115.717 \mathrm{~W} \\
115.717 \mathrm{~W} \\
118.850 \mathrm{~W} \\
116.967 \mathrm{~W} \\
118.817 \mathrm{~W}\end{array}$ & $\begin{array}{l}016 \\
016 \\
016 \\
016 \\
016\end{array}$ & $\begin{array}{l}292 \\
292 \\
292 \\
292 \\
292\end{array}$ & $\begin{array}{l}- \\
-\end{array}$ & $\overline{-}$ & $\begin{array}{ll}5.50 \mathrm{M}_{\mathrm{L}} & \text { PAS } \\
4.80 \mathrm{M}_{\mathrm{L}} & \text { PAS } \\
4.90 \mathrm{M}_{\mathrm{L}} & \text { PAS } \\
4.90 \mathrm{M}_{\mathrm{L}} & \text { PAS } \\
4.60 \mathrm{M}_{\mathrm{L}} & \text { PAS }\end{array}$ & $\begin{array}{l}z \\
-\end{array}$ & $\begin{array}{l}\text { VII } \\
\mathbf{V} \\
\mathbf{V} \\
\mathbf{I V} \\
\mathbf{V}\end{array}$ & $\begin{array}{l}26 \\
26 \\
26 \\
26 \\
26\end{array}$ & $\begin{array}{l}30 \# \\
= \\
-\end{array}$ \\
\hline
\end{tabular}


CALIFORNIA-Continued

[See table 1 for hypocenter and intensity references and table 2 for definitions of magnitude source codes. \&, land area only; \#, land area only in the United States for an earthquake near a coastline; +, land area in the United States when the felt area did not extend to the coast; $@$, felt area is less than $1,000 \mathrm{~km}^{2}$. Leader (-) indicates information is not available]

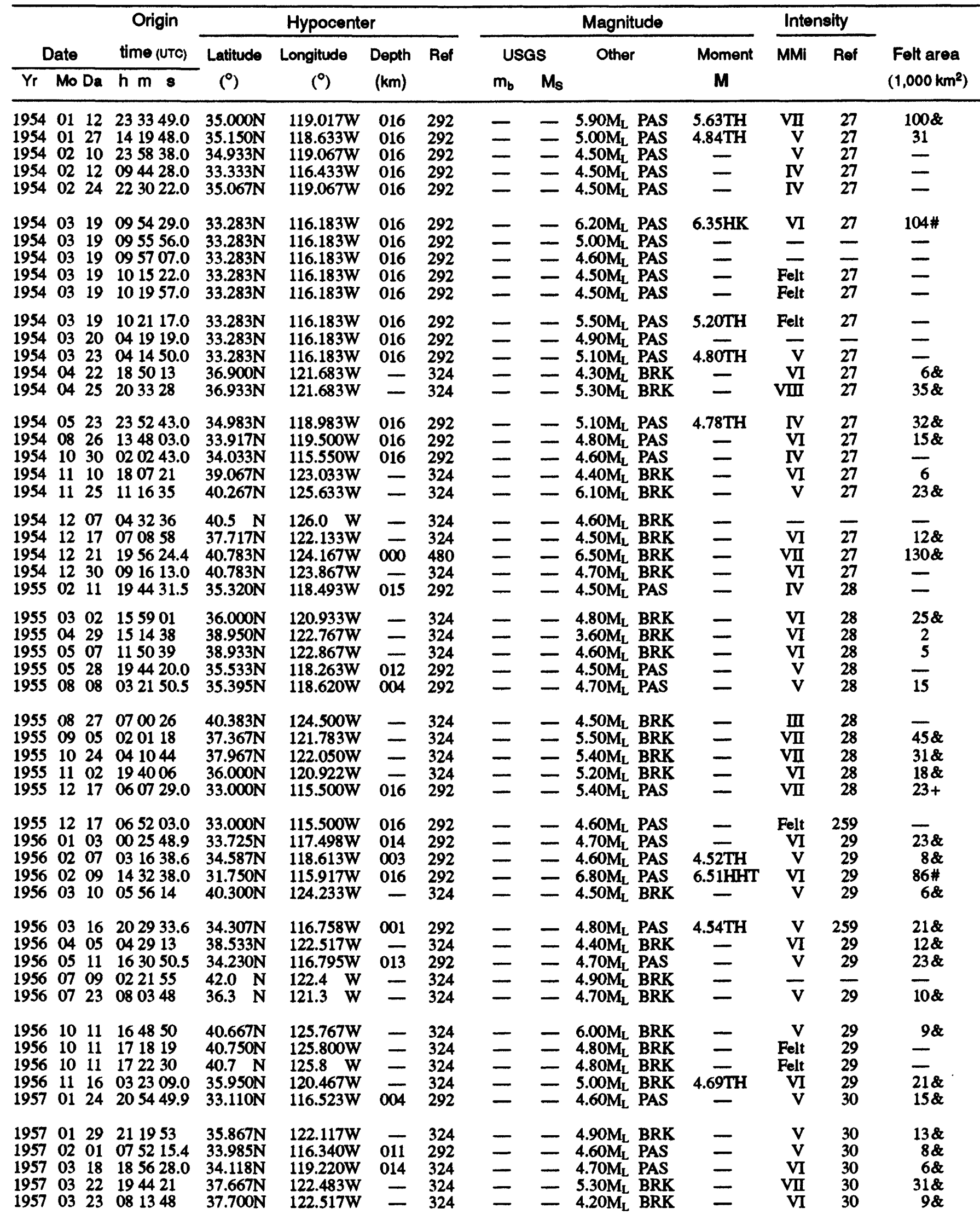


EARTHQUAKES IN CALIFORNIA

CALIFORNIA-Continued

[See table 1 for hypocenter and intensity references and table 2 for definitions of magnitude source codes. \&, land area only; \#, land area only in the United States for an earthquake near a coastline; + , land area in the United States when the felt area did not extend to the coast; @, felt area is less than $1,000 \mathrm{~km}{ }^{2}$. Leader (--) indicates information is not available]

\begin{tabular}{|c|c|c|c|c|c|c|c|c|c|c|c|c|c|c|}
\hline \multirow{2}{*}{\multicolumn{3}{|c|}{ Date }} & \multirow{2}{*}{$\begin{array}{c}\text { Origin } \\
\text { time (UTC) }\end{array}$} & \multicolumn{4}{|c|}{ Hypocenter } & \multicolumn{4}{|c|}{ Magnitude } & \multicolumn{2}{|c|}{ Intensity } & \multirow{3}{*}{$\begin{array}{l}\text { Felt area } \\
\left(1,000 \mathrm{~km}^{2}\right)\end{array}$} \\
\hline & & & & \multirow{2}{*}{$\begin{array}{l}\text { Latitude } \\
\left(^{\circ}\right)\end{array}$} & \multirow{2}{*}{$\begin{array}{l}\text { Longitude } \\
\left({ }^{\circ}\right)\end{array}$} & \multirow{2}{*}{$\begin{array}{l}\text { Depth } \\
(\mathbf{k m})\end{array}$} & \multirow[t]{2}{*}{ Ref } & \multicolumn{2}{|c|}{ USGS } & \multirow[t]{2}{*}{ Other } & \multirow{2}{*}{$\begin{array}{c}\text { Moment } \\
\mathbf{M}\end{array}$} & \multirow[t]{2}{*}{ MMI } & \multirow[t]{2}{*}{ Ref } & \\
\hline Yr & Mo & $\mathrm{Da}$ & $h \mathbf{m} s$ & & & & & $m_{b}$ & $M_{\mathbf{s}}$ & & & & & \\
\hline $\begin{array}{l}1957 \\
1957 \\
1957 \\
1957 \\
1957\end{array}$ & $\begin{array}{l}04 \\
04 \\
05 \\
06 \\
09\end{array}$ & & $\begin{array}{l}215738.7 \\
222412.0 \\
155933.6 \\
004125 \\
163841\end{array}$ & $\begin{array}{l}33.217 \mathrm{~N} \\
33.183 \mathrm{~N} \\
33.232 \mathrm{~N} \\
37.700 \mathrm{~N} \\
41.533 \mathrm{~N}\end{array}$ & $\begin{array}{l}115.808 \mathrm{~W} \\
115.850 \mathrm{~W} \\
116.005 \mathrm{~W} \\
119.283 \mathrm{~W} \\
125.583 \mathrm{~W}\end{array}$ & $\begin{array}{l}000 \\
016 \\
015 \\
- \\
-\end{array}$ & $\begin{array}{l}292 \\
292 \\
292 \\
324 \\
324\end{array}$ & $\frac{-}{E}$ & $\bar{z}$ & $\begin{array}{ll}5.20 \mathrm{M}_{\mathrm{L}} & \text { PAS } \\
5.10 \mathrm{M}_{\mathrm{L}} & \text { PAS } \\
5.00 \mathrm{M}_{\mathrm{L}} & \text { PAS } \\
4.60 \mathrm{M}_{\mathrm{L}} & \text { BRK } \\
4.50 \mathrm{M}_{\mathrm{L}} & \text { BRK }\end{array}$ & $\frac{-}{z}$ & $\begin{array}{c}\text { VII } \\
\text { Felt } \\
\text { IV } \\
\text { V } \\
-\end{array}$ & $\begin{array}{r}30 \\
30 \\
259 \\
30 \\
-\end{array}$ & $\begin{array}{l}\frac{31 \&}{18 \#} \\
-\end{array}$ \\
\hline $\begin{array}{l}1957 \\
1957 \\
1958 \\
1958 \\
1958\end{array}$ & $\begin{array}{l}09 \\
10 \\
05 \\
07 \\
09\end{array}$ & $\begin{array}{l}28 \\
31 \\
24 \\
14 \\
21\end{array}$ & $\begin{array}{l}210439 \\
024746 \\
230447 \\
052555.3 \\
072455\end{array}$ & $\begin{array}{l}36.600 \mathrm{~N} \\
39.183 \mathrm{~N} \\
40.300 \mathrm{~N} \\
34.348 \mathrm{~N} \\
36.350 \mathrm{~N}\end{array}$ & $\begin{array}{l}121.233 \mathrm{~W} \\
123.683 \mathrm{~W} \\
124.050 \mathrm{~W} \\
119.492 \mathrm{~W} \\
121.117 \mathrm{~W}\end{array}$ & $\overline{-}$ & $\begin{array}{l}324 \\
324 \\
324 \\
292 \\
324\end{array}$ & $\begin{array}{l}- \\
- \\
-\end{array}$ & $\bar{z}$ & $\begin{array}{ll}4.50 \mathrm{M}_{\mathrm{L}} & \text { BRK } \\
4.70 \mathrm{M}_{\mathrm{L}} & \text { BRK } \\
4.90 \mathrm{M}_{\mathrm{L}} & \text { BRK } \\
4.70 \mathrm{M}_{\mathrm{L}} & \text { PAS } \\
4.60 \mathrm{M}_{\mathrm{L}} & \text { BRK }\end{array}$ & $\begin{array}{l}z \\
z\end{array}$ & $\begin{array}{l}\text { IV } \\
\text { V } \\
\text { V } \\
\text { V } \\
\text { VI }\end{array}$ & $\begin{array}{r}30 \\
30 \\
259 \\
31 \\
31\end{array}$ & $\begin{array}{l}- \\
6 \& \\
98 \\
138 \\
-\end{array}$ \\
\hline $\begin{array}{l}1958 \\
1958 \\
1958 \\
1958 \\
1959\end{array}$ & $\begin{array}{l}10 \\
10 \\
12 \\
12 \\
01\end{array}$ & $\begin{array}{l}01 \\
10 \\
01 \\
11 \\
05\end{array}$ & $\begin{array}{lll}21 & 42 & 11 \\
13 & 05 & 16 \\
03 & 21 & 18.0 \\
09 & 52 & 27 \\
12 & 36 & 03.3\end{array}$ & $\begin{array}{l}39.567 \mathrm{~N} \\
35.933 \mathrm{~N} \\
32.250 \mathrm{~N} \\
37.700 \mathrm{~N} \\
36.148 \mathrm{~N}\end{array}$ & $\begin{array}{l}120.300 \mathrm{~W} \\
120.500 \mathrm{~W} \\
115.750 \mathrm{~W} \\
122.567 \mathrm{~W} \\
118.025 \mathrm{~W}\end{array}$ & $\frac{-}{0 \overline{16}}$ & $\begin{array}{l}324 \\
324 \\
292 \\
324 \\
292\end{array}$ & $\bar{z}$ & $\bar{z}$ & $\begin{array}{ll}4.60 \mathrm{M}_{\mathrm{L}} & \text { BRK } \\
4.50 \mathrm{M}_{\mathrm{L}} & \text { BRK } \\
5.80 \mathrm{M}_{\mathrm{L}} & \text { PAS } \\
4.70 \mathrm{M}_{\mathrm{L}} & \text { BRK } \\
4.70 \mathrm{M}_{\mathrm{L}} & \text { PAS }\end{array}$ & $\frac{\bar{Z}}{\bar{Z}}$ & $\begin{array}{l}\text { VI } \\
\text { V } \\
\text { VI } \\
\text { VI } \\
\text { V }\end{array}$ & $\begin{array}{l}31 \\
31 \\
31 \\
31 \\
32\end{array}$ & $\begin{array}{l}13 \\
9 \& \\
39 \# \\
17 \& \\
-\end{array}$ \\
\hline $\begin{array}{l}1959 \\
1959 \\
1959 \\
1959 \\
1959\end{array}$ & $\begin{array}{l}03 \\
04 \\
04 \\
05 \\
06\end{array}$ & $\begin{array}{l}02 \\
01 \\
06 \\
26 \\
01\end{array}$ & $\begin{array}{l}232717 \\
181830 \\
060822 \\
155801 \\
163536.0\end{array}$ & $\begin{array}{l}36.983 \mathrm{~N} \\
39.717 \mathrm{~N} \\
39.3 \mathrm{~N} \\
36.717 \mathrm{~N} \\
32.717 \mathrm{~N}\end{array}$ & $\begin{array}{l}121.600 \mathrm{~W} \\
120.200 \mathrm{~W} \\
123.2 \mathrm{~W} \\
121.617 \mathrm{~W} \\
116.033 \mathrm{~W}\end{array}$ & $\frac{-}{\bar{E}}$ & $\begin{array}{l}324 \\
324 \\
324 \\
324 \\
292\end{array}$ & $\begin{array}{l}- \\
-\end{array}$ & $\bar{z}$ & $\begin{array}{ll}5.30 \mathrm{M}_{\mathrm{L}} & \text { BRK } \\
5.60 \mathrm{M}_{\mathrm{L}} & \text { BRK } \\
3.60 \mathrm{M}_{\mathrm{L}} & \text { BRK } \\
4.60 \mathrm{M}_{\mathrm{L}} & \text { BRK } \\
4.60 \mathrm{M}_{\mathrm{L}} & \text { PAS }\end{array}$ & $\begin{array}{l}- \\
-\end{array}$ & $\begin{array}{l}\text { VI } \\
\text { VII } \\
\text { VI } \\
\text { VI } \\
-\end{array}$ & $\begin{array}{l}32 \\
32 \\
32 \\
32 \\
-\end{array}$ & $\begin{array}{l}27 \& \\
85 \\
3 \\
12 \& \\
-\end{array}$ \\
\hline $\begin{array}{l}1959 \\
1959 \\
1959 \\
1959 \\
1959\end{array}$ & $\begin{array}{l}06 \\
06 \\
07 \\
07 \\
08\end{array}$ & $\begin{array}{l}14 \\
18 \\
01 \\
24 \\
04\end{array}$ & $\begin{array}{lll}01 & 26 & 32 \\
00 & 29 & 40 \\
23 & 49 & 23.4 \\
01 & 23 & 09 \\
07 & 36 & 59.0\end{array}$ & $\begin{array}{l}39.667 \mathrm{~N} \\
37.550 \mathrm{~N} \\
35.185 \mathrm{~N} \\
41.133 \mathrm{~N} \\
37.350 \mathrm{~N}\end{array}$ & $\begin{array}{l}120.550 \mathrm{~W} \\
118.567 \mathrm{~W} \\
119.100 \mathrm{~W} \\
125.300 \mathrm{~W} \\
118.550 \mathrm{~W}\end{array}$ & $\frac{-}{009}$ & $\begin{array}{l}324 \\
324 \\
292 \\
324 \\
292\end{array}$ & $\begin{array}{l}- \\
- \\
-\end{array}$ & $\bar{z}$ & $\begin{array}{ll}4.50 \mathrm{M}_{\mathrm{L}} & \text { BRK } \\
4.70 \mathrm{M}_{\mathrm{L}} & \text { PAS } \\
4.70 \mathrm{M}_{\mathrm{L}} & \text { PAS } \\
5.80 \mathrm{M}_{\mathrm{L}} & \text { BRK } \\
5.20 \mathrm{M}_{\mathrm{L}} & \text { PAS }\end{array}$ & $\frac{\overline{-}}{4 . \overline{\mathrm{TTH}}}$ & $\begin{array}{c}\text { V } \\
\text { V } \\
\text { V } \\
\text { IV } \\
\text { V }\end{array}$ & $\begin{array}{l}32 \\
32 \\
32 \\
32 \\
32\end{array}$ & $\begin{array}{r}12 \\
8 \\
15 \\
31\end{array}$ \\
\hline $\begin{array}{l}1959 \\
1959 \\
1959 \\
1959 \\
1959\end{array}$ & $\begin{array}{l}10 \\
10 \\
10 \\
12 \\
12\end{array}$ & $\begin{array}{l}01 \\
24 \\
31 \\
05 \\
22\end{array}$ & $\begin{array}{lll}04 & 35 & 35 \\
15 & 35 & 15.3 \\
19 & 42 & 14 \\
08 & 13 & 42 \\
02 & 38 & 57\end{array}$ & $\begin{array}{l}34.455 \mathrm{~N} \\
35.745 \mathrm{~N} \\
41.3 \mathrm{~N} \\
40.300 \mathrm{~N} \\
40.267 \mathrm{~N}\end{array}$ & $\begin{array}{l}120.522 \mathrm{~W} \\
118.023 \mathrm{~W} \\
125.5 \mathrm{~W} \\
125.417 \mathrm{~W} \\
124.517 \mathrm{~W}\end{array}$ & $\begin{array}{l}014 \\
007 \\
- \\
-\end{array}$ & $\begin{array}{l}292 \\
292 \\
324 \\
324 \\
324\end{array}$ & $\begin{array}{l}- \\
- \\
-\end{array}$ & $\bar{z}$ & $\begin{array}{ll}4.50 \mathrm{M}_{\mathrm{L}} & \text { PAS } \\
4.20 \mathrm{M}_{\mathrm{L}} & \text { PAS } \\
4.50 \mathrm{M}_{\mathrm{L}} & \text { BRK } \\
5.10 \mathrm{M}_{\mathrm{L}} & \text { BRK } \\
4.70 \mathrm{M}_{\mathrm{L}} & \text { BRK }\end{array}$ & $\begin{array}{l}- \\
z\end{array}$ & $\begin{array}{l}\mathrm{V} \\
\mathrm{VI} \\
\mathrm{V} \\
\mathrm{V}\end{array}$ & $\begin{array}{r}259 \\
32 \\
32 \\
32\end{array}$ & $\begin{array}{l}-12 \& \\
- \\
8 \&\end{array}$ \\
\hline $\begin{array}{l}1959 \\
1960 \\
1960 \\
1960 \\
1960\end{array}$ & $\begin{array}{l}12 \\
01 \\
06 \\
06 \\
07\end{array}$ & $\begin{array}{l}29 \\
20 \\
05 \\
06 \\
01\end{array}$ & $\begin{array}{lll}02 & 32 & 53 \\
03 & 25 & 53.0 \\
07 & 47 & 07.0 \\
01 & 17 & 45.5 \\
22 & 13 & 44.6\end{array}$ & $\begin{array}{l}36.900 \mathrm{~N} \\
36.783 \mathrm{~N} \\
37.517 \mathrm{~N} \\
40.84 \mathrm{~N} \\
35.147 \mathrm{~N}\end{array}$ & $\begin{array}{l}121.483 \mathrm{~W} \\
121.433 \mathrm{~W} \\
118.733 \mathrm{~W} \\
124.91 \mathrm{~W} \\
117.132 \mathrm{~W}\end{array}$ & $\begin{array}{l}- \\
\overline{016} \\
000 \\
008\end{array}$ & $\begin{array}{l}324 \\
324 \\
292 \\
480 \\
292\end{array}$ & $\bar{z}$ & $\bar{z}$ & $\begin{array}{ll}4.70 \mathrm{M}_{\mathrm{L}} & \text { BRK } \\
5.00 \mathrm{M}_{\mathrm{L}} & \text { BRK } \\
5.20 \mathrm{M}_{\mathrm{L}} & \text { PAS } \\
5.70 \mathrm{M}_{\mathrm{L}} & \text { BRK } \\
4.50 \mathrm{M}_{\mathrm{L}} & \text { PAS }\end{array}$ & $\begin{array}{c}\overline{-} \\
\overline{4} \overline{\mathrm{THH}} \\
-\end{array}$ & $\begin{array}{l}\text { VI } \\
\text { VI } \\
\text { V } \\
\text { VI } \\
-\end{array}$ & $\begin{array}{r}32 \\
33 \\
259 \\
33 \\
-\end{array}$ & $\begin{array}{l}13 \& \\
28 \& \\
12 \\
21 \& \\
-\end{array}$ \\
\hline $\begin{array}{l}1960 \\
1960 \\
1960 \\
1961 \\
1961\end{array}$ & $\begin{array}{l}08 \\
12 \\
12 \\
01 \\
02\end{array}$ & $\begin{array}{l}09 \\
27 \\
27 \\
28 \\
02\end{array}$ & $\begin{array}{l}073918 \\
103526 \\
110844 \\
081246.2 \\
000416.0\end{array}$ & $\begin{array}{l}40.317 \mathrm{~N} \\
41.517 \mathrm{~N} \\
41.500 \mathrm{~N} \\
35.778 \mathrm{~N} \\
37.450 \mathrm{~N}\end{array}$ & $\begin{array}{l}127.067 \mathrm{~W} \\
125.050 \mathrm{~W} \\
125.000 \mathrm{~W} \\
118.048 \mathrm{~W} \\
118.633 \mathrm{~W}\end{array}$ & $\begin{array}{l}\overline{-} \\
\overline{006} \\
016\end{array}$ & $\begin{array}{l}324 \\
324 \\
324 \\
292 \\
292\end{array}$ & $\begin{array}{l}- \\
- \\
-\end{array}$ & $\bar{z}$ & $\begin{array}{ll}6.20 \mathrm{M}_{\mathrm{L}} & \text { BRK } \\
5.40 \mathrm{M}_{\mathrm{L}} & \text { BRK } \\
4.70 \mathrm{M}_{\mathrm{L}} & \text { BRK } \\
5.30 \mathrm{M}_{\mathrm{L}} & \text { PAS } \\
5.30 \mathrm{M}_{\mathrm{L}} & \text { PAS }\end{array}$ & $\begin{array}{l}- \\
-\end{array}$ & $\begin{array}{l}\mathrm{V} \\
\mathrm{V} \\
\overline{\mathrm{VI}} \\
\mathrm{V}\end{array}$ & $\begin{array}{l}33 \\
33 \\
34 \\
34\end{array}$ & $\frac{17 \&}{52}$ \\
\hline $\begin{array}{l}1961 \\
1961 \\
1961 \\
1961 \\
1961\end{array}$ & $\begin{array}{l}02 \\
04 \\
04 \\
04 \\
04\end{array}$ & $\begin{array}{l}02 \\
06 \\
09 \\
09 \\
29\end{array}$ & $\begin{array}{l}000742.0 \\
040445 \\
072316 \\
072541.0 \\
091930\end{array}$ & $\begin{array}{l}37.417 \mathrm{~N} \\
40.183 \mathrm{~N} \\
36.683 \mathrm{~N} \\
36.683 \mathrm{~N} \\
40.417 \mathrm{~N}\end{array}$ & $\begin{array}{l}118.667 \mathrm{~W} \\
124.750 \mathrm{~W} \\
121.300 \mathrm{~W} \\
121.300 \mathrm{~W} \\
127.450 \mathrm{~W}\end{array}$ & $\frac{016}{-}$ & $\begin{array}{l}292 \\
324 \\
324 \\
324 \\
324\end{array}$ & $\begin{array}{l}- \\
-\end{array}$ & $\frac{-}{-}$ & $\begin{array}{ll}5.10 \mathrm{M}_{\mathrm{L}} & \text { PAS } \\
5.10 \mathrm{M}_{\mathrm{L}} & \text { BRK } \\
5.60 \mathrm{M}_{\mathrm{L}} & \text { BRK } \\
5.50 \mathrm{M}_{\mathrm{L}} & \text { BRK } \\
5.50 \mathrm{M}_{\mathrm{L}} & \text { BRK }\end{array}$ & $\bar{z}$ & $\begin{array}{l}\text { V } \\
\text { V } \\
\text { VII } \\
\text { VII } \\
-\end{array}$ & $\begin{array}{r}34 \\
259 \\
34 \\
38 \\
-\end{array}$ & $\begin{array}{l}35 \\
98 \\
358 \\
-\end{array}$ \\
\hline $\begin{array}{l}1961 \\
1961 \\
1961 \\
1961 \\
1961\end{array}$ & $\begin{array}{l}07 \\
08 \\
09 \\
10 \\
10\end{array}$ & $\begin{array}{l}31 \\
23 \\
12 \\
19 \\
20\end{array}$ & $\begin{array}{l}000708 \\
010047.8 \\
191845.5 \\
050943.9 \\
194950.5\end{array}$ & $\begin{array}{l}35.817 \mathrm{~N} \\
33.050 \mathrm{~N} \\
32.567 \mathrm{~N} \\
35.832 \mathrm{~N} \\
33.653 \mathrm{~N}\end{array}$ & $\begin{array}{l}120.367 \mathrm{~W} \\
116.238 \mathrm{~W} \\
115.452 \mathrm{~W} \\
117.762 \mathrm{~W} \\
117.993 \mathrm{~W}\end{array}$ & $\begin{array}{l}\overline{012} \\
012 \\
\overline{005}\end{array}$ & $\begin{array}{l}324 \\
292 \\
292 \\
292 \\
292\end{array}$ & $\begin{array}{l}- \\
- \\
-\end{array}$ & $\frac{-}{-}$ & $\begin{array}{ll}4.70 \mathrm{M}_{\mathrm{L}} & \text { BRK } \\
4.70 \mathrm{M}_{\mathrm{L}} & \text { PAS } \\
4.80 \mathrm{M}_{\mathrm{L}} & \text { PAS } \\
5.20 \mathrm{M}_{\mathrm{L}} & \text { PAS } \\
4.30 \mathrm{M}_{\mathrm{L}} & \text { PAS }\end{array}$ & $\begin{array}{c}\text { 4.58WYS } \\
= \\
=\end{array}$ & $\begin{array}{l}\text { V } \\
\text { V } \\
\text { IV } \\
\text { VI } \\
\text { VI }\end{array}$ & $\begin{array}{r}34 \\
34 \\
259 \\
34 \\
34\end{array}$ & $\begin{array}{c}13 \& \\
9 \& \\
10 \# \\
31 \\
3 \&\end{array}$ \\
\hline $\begin{array}{l}1961 \\
1962 \\
1962 \\
1962 \\
1962\end{array}$ & $\begin{array}{l}11 \\
02 \\
03 \\
04 \\
04\end{array}$ & $\begin{array}{l}15 \\
01 \\
05 \\
13 \\
14\end{array}$ & $\begin{array}{l}053855.5 \\
063757.0 \\
205746 \\
153851.9 \\
075314.7\end{array}$ & $\begin{array}{l}34.942 \mathrm{~N} \\
34.883 \mathrm{~N} \\
40.3 \mathrm{~N} \\
38.222 \mathrm{~N} \\
40.268 \mathrm{~N}\end{array}$ & $\begin{array}{l}118.987 \mathrm{~W} \\
120.683 \mathrm{~W} \\
125.5 \mathrm{~W} \\
119.455 \mathrm{~W} \\
125.310 \mathrm{~W}\end{array}$ & $\begin{array}{l}011 \\
016 \\
- \\
-006 \\
-\end{array}$ & $\begin{array}{l}292 \\
292 \\
324 \\
324 \\
324\end{array}$ & $\begin{array}{l}- \\
- \\
-\end{array}$ & $\bar{z}$ & $\begin{array}{ll}5.00 \mathrm{M}_{\mathrm{L}} & \text { PAS } \\
4.50 \mathrm{M}_{\mathrm{L}} & \text { PAS } \\
4.60 \mathrm{M}_{\mathrm{L}} & \text { BRK } \\
5.10 \mathrm{M}_{\mathrm{L}} & \text { BRK } \\
5.40 \mathrm{M}_{\mathrm{L}} & \text { BRK }\end{array}$ & $\begin{array}{l}- \\
- \\
-\end{array}$ & $\begin{array}{l}\text { VI } \\
\text { V } \\
\text { IV } \\
\text { V } \\
-\end{array}$ & $\begin{array}{r}34 \\
35 \\
259 \\
35 \\
-\end{array}$ & $\begin{array}{r}26 \& \\
8 \& \\
2 \& \\
19 \\
-\end{array}$ \\
\hline
\end{tabular}


CALIFORNIA-Continued

[See table 1 for hypocenter and intensity references and table 2 for definitions of magnitude source codes. \&, land area only; \#, land area only in the United States for an earthquake near a coastline; + , land area in the United States when the felt area did not extend to the coast; 0 , felt area is less than $1,000 \mathrm{~km}^{2}$. Leader (-) indicates information is not available!

\begin{tabular}{|c|c|c|c|c|c|c|c|c|c|c|c|c|c|c|}
\hline \multirow{2}{*}{\multicolumn{3}{|c|}{ Date }} & \multirow{2}{*}{$\begin{array}{l}\text { Origin } \\
\text { time (UTC) }\end{array}$} & \multicolumn{4}{|c|}{ Hypocenter } & \multicolumn{4}{|c|}{ Magnitude } & \multicolumn{2}{|c|}{ Intensity } & \multirow{3}{*}{$\begin{array}{c}\text { Felt area } \\
\left(1,000 \mathrm{~km}^{2}\right)\end{array}$} \\
\hline & & & & \multirow{2}{*}{$\begin{array}{l}\text { Latitude } \\
\left(^{\circ}\right)\end{array}$} & \multirow{2}{*}{$\begin{array}{l}\text { Longitude } \\
\left({ }^{\circ}\right)\end{array}$} & \multirow{2}{*}{$\begin{array}{c}\text { Depth } \\
\text { (km) }\end{array}$} & \multirow[t]{2}{*}{ Ref } & \multicolumn{2}{|c|}{ USGS } & \multirow[t]{2}{*}{ Other } & \multirow{2}{*}{$\begin{array}{c}\text { Moment } \\
\mathbf{M}\end{array}$} & \multirow[t]{2}{*}{ MMI } & \multirow[t]{2}{*}{ Ref } & \\
\hline Yr & Mo & $\mathrm{Da}$ & $h \mathrm{~m} s$ & & & & & $m_{b}$ & $\mathbf{M}_{\mathbf{s}}$ & & & & & \\
\hline $\begin{array}{l}1962 \\
1962 \\
1962 \\
1962 \\
1962\end{array}$ & $\begin{array}{l}04 \\
04 \\
06 \\
07 \\
08\end{array}$ & $\begin{array}{l}15 \\
27 \\
06 \\
14 \\
23\end{array}$ & $\begin{array}{lll}08 & 41 & 02.3 \\
09 & 12 & 32.1 \\
17 & 50 & 07.2 \\
19 & 43 & 46.2 \\
19 & 29 & 14.8\end{array}$ & $\begin{array}{l}36.415 \mathrm{~N} \\
33.738 \mathrm{~N} \\
39.116 \mathrm{~N} \\
40.427 \mathrm{~N} \\
41.84 \mathrm{~N}\end{array}$ & $\begin{array}{l}120.617 \mathrm{~W} \\
117.187 \mathrm{~W} \\
123.097 \mathrm{~W} \\
125.512 \mathrm{~W} \\
124.39 \mathrm{~W}\end{array}$ & $\begin{array}{l}021 \\
006 \\
010 \\
030 \\
059\end{array}$ & $\begin{array}{l}324 \\
292 \\
613 \\
324 \\
480\end{array}$ & $\begin{array}{l}- \\
- \\
-\end{array}$ & $\bar{z}$ & $\begin{array}{ll}4.70 \mathrm{M}_{\mathrm{L}} & \text { BRK } \\
4.10 \mathrm{M}_{\mathrm{L}} & \text { PAS } \\
5.20 \mathrm{M}_{\mathrm{L}} & \text { BRK } \\
5.10 \mathrm{M}_{\mathrm{L}} & \text { BRK } \\
5.60 \mathrm{M}_{\mathrm{L}} & \text { BRK }\end{array}$ & $\begin{array}{l}z \\
z\end{array}$ & $\begin{array}{c}\text { IV } \\
\text { VI } \\
\text { VII } \\
\text { V } \\
\text { VI }\end{array}$ & $\begin{array}{r}259 \\
35 \\
35 \\
35 \\
35\end{array}$ & $\begin{array}{l}\overline{12} \\
19 \& \\
7 \& \\
39 \&\end{array}$ \\
\hline $\begin{array}{l}1962 \\
1962 \\
1962 \\
1962 \\
1963\end{array}$ & $\begin{array}{l}09 \\
09 \\
10 \\
10 \\
03\end{array}$ & $\begin{array}{l}04 \\
16 \\
14 \\
29 \\
01\end{array}$ & $\begin{array}{l}171726.0 \\
053616.0 \\
101428.1 \\
024253.9 \\
002557.9\end{array}$ & $\begin{array}{l}41.01 \mathrm{~N} \\
35.755 \mathrm{~N} \\
38.680 \mathrm{~N} \\
34.325 \mathrm{~N} \\
34.932 \mathrm{~N}\end{array}$ & $\begin{array}{l}124.21 \mathrm{~W} \\
118.043 \mathrm{~W} \\
124.028 \mathrm{~W} \\
116.865 \mathrm{~W} \\
118.975 \mathrm{~W}\end{array}$ & $\begin{array}{l}045 \\
004 \\
000 \\
009 \\
014\end{array}$ & $\begin{array}{l}480 \\
292 \\
324 \\
292 \\
292\end{array}$ & $\overline{-}$ & $\bar{z}$ & $\begin{array}{ll}4.90 \mathrm{M}_{\mathrm{L}} & \text { BRK } \\
4.90 \mathrm{M}_{\mathrm{L}} & \text { PAS } \\
4.70 \mathrm{M}_{\mathrm{L}} & \text { BRK } \\
4.80 \mathrm{M}_{\mathrm{L}} & \text { PAS } \\
5.00 \mathrm{M}_{\mathrm{L}} & \text { PAS }\end{array}$ & $\begin{array}{l}z \\
z\end{array}$ & $\begin{array}{l}\text { VI } \\
\text { V } \\
\text { III } \\
\text { VI } \\
\text { V }\end{array}$ & $\begin{array}{l}35 \\
35 \\
35 \\
35 \\
36\end{array}$ & $\begin{array}{l}18 \& \\
41 \\
\overline{25} \& \\
21 \&\end{array}$ \\
\hline $\begin{array}{l}1963 \\
1963 \\
1963 \\
1963 \\
1963\end{array}$ & $\begin{array}{l}05 \\
05 \\
05 \\
06 \\
07\end{array}$ & $\begin{array}{l}22 \\
23 \\
23 \\
07 \\
08\end{array}$ & $\begin{array}{lll}22 & 41 & 04.8 \\
09 & 06 & 04.7 \\
15 & 53 & 01.8 \\
12 & 04 & 42.2 \\
04 & 19 & 08.4\end{array}$ & $\begin{array}{l}37.272 \mathrm{~N} \\
32.982 \mathrm{~N} \\
33.027 \mathrm{~N} \\
37.975 \mathrm{~N} \\
40.800 \mathrm{~N}\end{array}$ & $\begin{array}{l}122.318 \mathrm{~W} \\
115.565 \mathrm{~W} \\
115.682 \mathrm{~W} \\
122.048 \mathrm{~W} \\
125.800 \mathrm{~W}\end{array}$ & $\begin{array}{l}\overline{025} \\
000 \\
\overline{033}\end{array}$ & $\begin{array}{l}324 \\
292 \\
292 \\
324 \\
266\end{array}$ & $\frac{-}{\overline{4}}$ & $\bar{z}$ & $\begin{array}{ll}4.60 \mathrm{M}_{\mathrm{L}} & \text { BRK } \\
4.60 \mathrm{M}_{\mathrm{L}} & \text { PAS } \\
4.80 \mathrm{M}_{\mathrm{L}} & \text { PAS } \\
3.90 \mathrm{M}_{\mathrm{L}} & \text { BRK } \\
4.10 \mathrm{M}_{\mathrm{L}} & \text { BRK }\end{array}$ & $\begin{array}{c}\overline{4.60 \mathrm{WYS}} \\
- \\
-\end{array}$ & $\begin{array}{l}\text { VI } \\
\text { Felt } \\
\text { VI } \\
\text { VI } \\
-\end{array}$ & $\begin{array}{l}36 \\
36 \\
36 \\
36 \\
-\end{array}$ & $\begin{array}{l}\frac{4 \&}{10 \#} \\
4 \& \\
-\end{array}$ \\
\hline $\begin{array}{l}1963 \\
1963 \\
1963 \\
1963 \\
1963\end{array}$ & $\begin{array}{l}07 \\
09 \\
09 \\
09 \\
12\end{array}$ & $\begin{array}{l}30 \\
14 \\
14 \\
23 \\
06\end{array}$ & $\begin{array}{l}063457.3 \\
194617.0 \\
202811.2 \\
144152.6 \\
083421.5\end{array}$ & $\begin{array}{l}34.153 \mathrm{~N} \\
36.890 \mathrm{~N} \\
36.925 \mathrm{~N} \\
33.710 \mathrm{~N} \\
37.648 \mathrm{~N}\end{array}$ & $\begin{array}{l}116.210 \mathrm{~W} \\
121.597 \mathrm{~W} \\
121.618 \mathrm{~W} \\
116.925 \mathrm{~W} \\
118.397 \mathrm{~W}\end{array}$ & $\begin{array}{l}013 \\
003 \\
006 \\
017 \\
002\end{array}$ & $\begin{array}{l}292 \\
467 \\
467 \\
292 \\
292\end{array}$ & $\begin{array}{l}4.7 \\
5.4 \\
4.3 \\
5.3 \\
4.4\end{array}$ & $\bar{z}$ & $\begin{array}{ll}4.70 \mathrm{M}_{\mathrm{L}} & \text { PAS } \\
5.40 \mathrm{M}_{\mathrm{L}} & \text { BRK } \\
4.60 \mathrm{M}_{\mathrm{L}} & \text { BRK } \\
5.00 \mathrm{M}_{\mathrm{L}} & \text { PAS } \\
4.70 \mathrm{M}_{\mathrm{L}} & \text { PAS }\end{array}$ & $\begin{array}{l}- \\
-\end{array}$ & $\begin{array}{l}\text { V } \\
\text { VII } \\
\text { Felt } \\
\text { VI } \\
\text { VI }\end{array}$ & $\begin{array}{l}36 \\
36 \\
36 \\
36 \\
36\end{array}$ & $\begin{array}{l}\overline{13} \& \\
\overline{26 \#} \\
26\end{array}$ \\
\hline $\begin{array}{l}1964 \\
1964 \\
1964 \\
1964 \\
1964\end{array}$ & $\begin{array}{l}01 \\
02 \\
03 \\
06 \\
06\end{array}$ & $\begin{array}{l}06 \\
26 \\
03 \\
11 \\
21\end{array}$ & $\begin{array}{lll}23 & 47 & 12.8 \\
20 & 32 & 51.4 \\
20 & 02 & 33 \\
22 & 18 & 12 \\
15 & 32 & 51.8\end{array}$ & $\begin{array}{l}34.380 \mathrm{~N} \\
40.308 \mathrm{~N} \\
40.3 \mathrm{~N} \\
40.7 \mathrm{~N} \\
32.692 \mathrm{~N}\end{array}$ & $\begin{array}{l}116.475 \mathrm{~W} \\
124.892 \mathrm{~W} \\
125.3 \mathrm{~W} \\
127.0 \mathrm{~W} \\
117.162 \mathrm{~W}\end{array}$ & $\frac{}{\bar{Z}}$ & $\begin{array}{l}292 \\
324 \\
324 \\
324 \\
292\end{array}$ & $\begin{array}{l}- \\
- \\
5.8 \\
-\end{array}$ & $\bar{z}$ & $\begin{array}{ll}\text { 4.50M } & \text { PAS } \\
4.50 \mathrm{M}_{\mathrm{L}} & \text { BRK } \\
4.50 \mathrm{M}_{\mathrm{L}} & \text { BRK } \\
5.50 \mathrm{M}_{\mathrm{L}} & \text { BRK } \\
3.70 \mathrm{M}_{\mathrm{L}} & \text { PAS }\end{array}$ & $\begin{array}{l}\overline{-} \\
\bar{z}\end{array}$ & $\begin{array}{l}\mathrm{v} \\
\mathrm{V} \\
\overline{\mathrm{VI}}\end{array}$ & $\begin{array}{l}37 \\
37 \\
-\end{array}$ & $\begin{array}{l}12 \\
-3 \& \\
-\end{array}$ \\
\hline $\begin{array}{l}1964 \\
1964 \\
1965 \\
1965 \\
1965\end{array}$ & $\begin{array}{l}11 \\
12 \\
01 \\
02 \\
04\end{array}$ & $\begin{array}{l}16 \\
22 \\
01 \\
12 \\
15\end{array}$ & $\begin{array}{l}024641.7 \\
205433.2 \\
080418.0 \\
105018.0 \\
200833.3\end{array}$ & $\begin{array}{l}37.055 \mathrm{~N} \\
31.810 \mathrm{~N} \\
34.140 \mathrm{~N} \\
40.3 \mathrm{~N} \\
34.132 \mathrm{~N}\end{array}$ & $\begin{array}{l}121.692 \mathrm{~W} \\
117.130 \mathrm{~W} \\
117.515 \mathrm{~W} \\
124.9 \mathrm{~W} \\
117.427 \mathrm{~W}\end{array}$ & $\begin{array}{l}\overline{002} \\
006 \\
000 \\
006\end{array}$ & $\begin{array}{l}324 \\
292 \\
292 \\
324 \\
292\end{array}$ & $\begin{array}{l}5.2 \\
5.2 \\
5.3 \\
5.1\end{array}$ & $\bar{z}$ & $\begin{array}{ll}5.00 \mathrm{M}_{\mathrm{L}} & \text { BRK } \\
5.60 \mathrm{M}_{\mathrm{L}} & \text { PAS } \\
4.40 \mathrm{M}_{\mathrm{L}} & \text { PAS } \\
4.50 \mathrm{M}_{\mathrm{L}} & \text { BRK } \\
4.50 \mathrm{M}_{\mathrm{L}} & \text { PAS }\end{array}$ & $\begin{array}{l}z \\
z\end{array}$ & $\begin{array}{l}\text { VII } \\
\text { VI } \\
\text { VI } \\
\text { Felt } \\
\text { VI }\end{array}$ & $\begin{array}{l}37 \\
37 \\
75 \\
75 \\
75\end{array}$ & $\begin{array}{l}31 \& \\
23 \# \\
10 \& \\
-10 \&\end{array}$ \\
\hline $\begin{array}{l}1965 \\
1965 \\
1965 \\
1965 \\
1965\end{array}$ & $\begin{array}{l}06 \\
06 \\
07 \\
08 \\
09\end{array}$ & $\begin{array}{l}03 \\
16 \\
16 \\
26 \\
10\end{array}$ & $\begin{array}{l}162629.0 \\
024206.1 \\
074622.4 \\
133814.0 \\
212834.3\end{array}$ & $\begin{array}{l}38.3 \mathrm{~N} \\
33.055 \mathrm{~N} \\
34.485 \mathrm{~N} \\
33.233 \mathrm{~N} \\
38.010 \mathrm{~N}\end{array}$ & $\begin{array}{l}119.2 \mathrm{~W} \\
115.620 \mathrm{~W} \\
118.522 \mathrm{~W} \\
116.087 \mathrm{~W} \\
121.823 \mathrm{~W}\end{array}$ & $\begin{array}{l}033 \\
000 \\
015 \\
- \\
-\end{array}$ & $\begin{array}{l}266 \\
292 \\
292 \\
292 \\
324\end{array}$ & $\begin{array}{l}4.8 \\
4.4 \\
4.5 \\
4.5 \\
4.9\end{array}$ & $\bar{z}$ & $\begin{array}{ll}4.50 \mathrm{M}_{\mathrm{L}} & \text { PAS } \\
4.40 \mathrm{M}_{\mathrm{L}} & \text { PAS } \\
4.00 \mathrm{M}_{\mathrm{L}} & \text { PAS } \\
4.50 \mathrm{M}_{\mathrm{L}} & \text { PAS } \\
4.90 \mathrm{M}_{\mathrm{L}} & \text { BRK }\end{array}$ & $\frac{-}{-}$ & $\begin{array}{l}\text { V } \\
\text { VI } \\
\text { VI } \\
\text { Felt } \\
\text { VI }\end{array}$ & $\begin{array}{l}75 \\
75 \\
75 \\
75 \\
75\end{array}$ & $\begin{array}{r}10 \\
3 \\
8 \\
8 \&\end{array}$ \\
\hline $\begin{array}{l}1965 \\
1965 \\
1965 \\
1965 \\
1965\end{array}$ & $\begin{array}{l}09 \\
09 \\
09 \\
09 \\
09\end{array}$ & $\begin{array}{l}16 \\
19 \\
22 \\
25 \\
25\end{array}$ & $\begin{array}{lll}04 & 10 & 23.4 \\
15 & 42 & 07.8 \\
21 & 49 & 25.9 \\
17 & 43 & 44.1 \\
17 & 48 & 02.4\end{array}$ & $\begin{array}{l}40.39 \mathrm{~N} \\
35.987 \mathrm{~N} \\
37.417 \mathrm{~N} \\
34.713 \mathrm{~N} \\
34.713 \mathrm{~N}\end{array}$ & $\begin{array}{l}125.60 \mathrm{~W} \\
120.038 \mathrm{~W} \\
118.478 \mathrm{~W} \\
116.503 \mathrm{~W} \\
116.475 \mathrm{~W}\end{array}$ & $\begin{array}{l}033 \\
\frac{0}{008} \\
011 \\
005\end{array}$ & $\begin{array}{l}299 \\
324 \\
292 \\
292 \\
292\end{array}$ & $\begin{array}{l}5.6 \\
4.9 \\
-5.0 \\
-\end{array}$ & $\overline{-}$ & $\begin{array}{ll}5.00 \mathrm{M}_{\mathrm{L}} & \text { PAS } \\
4.80 \mathrm{M}_{\mathrm{L}} & \text { BRK } \\
4.50 \mathrm{M}_{\mathrm{L}} & \text { PAS } \\
5.20 \mathrm{M}_{\mathrm{L}} & \text { PAS } \\
4.90 \mathrm{M}_{\mathrm{L}} & \text { PAS }\end{array}$ & $\bar{z}$ & $\begin{array}{l}\text { IV } \\
\text { V } \\
\text { IV } \\
\text { VII } \\
\text { Felt }\end{array}$ & $\begin{array}{l}75 \\
75 \\
75 \\
75 \\
75\end{array}$ & $\begin{array}{r}-9 \\
12 \\
77 \\
-\end{array}$ \\
\hline $\begin{array}{l}1965 \\
1965 \\
1965 \\
1966 \\
1966\end{array}$ & $\begin{array}{l}09 \\
10 \\
11 \\
04 \\
05\end{array}$ & $\begin{array}{l}26 \\
17 \\
12 \\
10 \\
13\end{array}$ & $\begin{array}{lll}07 & 00 & 01.8 \\
09 & 45 & 19.0 \\
23 & 5509.8 \\
22 & 2700.9 \\
17 & 2555.9\end{array}$ & $\begin{array}{l}34.713 \mathrm{~N} \\
33.975 \mathrm{~N} \\
33.980 \mathrm{~N} \\
41.3 \mathrm{~N} \\
36.917 \mathrm{~N}\end{array}$ & $\begin{array}{l}116.027 \mathrm{~W} \\
116.775 \mathrm{~W} \\
118.392 \mathrm{~W} \\
125.6 \mathrm{~W} \\
121.567 \mathrm{~W}\end{array}$ & $\begin{array}{l}008 \\
017 \\
006 \\
033 \\
-\end{array}$ & $\begin{array}{l}292 \\
292 \\
292 \\
266 \\
324\end{array}$ & $\begin{array}{l}- \\
4.8 \\
3.7 \\
5.0 \\
4.6\end{array}$ & $\begin{array}{l}- \\
- \\
-\end{array}$ & $\begin{array}{ll}5.00 \mathrm{M}_{\mathrm{L}} & \text { PAS } \\
4.90 \mathrm{M}_{\mathrm{L}} & \text { PAS } \\
3.00 \mathrm{M}_{\mathrm{L}} & \text { PAS } \\
4.50 \mathrm{M}_{\mathrm{L}} & \text { BRK } \\
4.50 \mathrm{M}_{\mathrm{L}} & \text { BRK }\end{array}$ & $\begin{array}{c}\bar{z} \\
\bar{z} \\
3.92 \mathrm{BAK}\end{array}$ & $\begin{array}{l}\mathrm{v} \\
\mathrm{VI} \\
\mathrm{VI} \\
\frac{\mathrm{V}}{\mathrm{V}}\end{array}$ & $\begin{array}{r}259 \\
75 \\
75 \\
81\end{array}$ & $\begin{array}{l}40 \& \\
15 \\
2 \& \\
-2 \&\end{array}$ \\
\hline $\begin{array}{l}1966 \\
1966 \\
1966 \\
1966 \\
1966\end{array}$ & $\begin{array}{l}05 \\
06 \\
06 \\
06 \\
06\end{array}$ & $\begin{array}{l}24 \\
28 \\
28 \\
28 \\
29\end{array}$ & $\begin{array}{l}034955.1 \\
040856.2 \\
042613.4 \\
042836.0 \\
195325.9\end{array}$ & $\begin{array}{l}39.783 \mathrm{~N} \\
35.960 \mathrm{~N} \\
35.955 \mathrm{~N} \\
35.950 \mathrm{~N} \\
35.943 \mathrm{~N}\end{array}$ & $\begin{array}{l}121.770 \mathrm{~W} \\
120.505 \mathrm{~W} \\
120.498 \mathrm{~W} \\
120.500 \mathrm{~W} \\
120.525 \mathrm{~W}\end{array}$ & $\frac{020}{\overline{0}}$ & $\begin{array}{l}324 \\
398 \\
398 \\
292 \\
398\end{array}$ & $\begin{array}{l}4.5 \\
4.9 \\
5.3 \\
5.0\end{array}$ & $\bar{z}$ & $\begin{array}{ll}4.60 \mathrm{M}_{\mathrm{L}} & \text { BRK } \\
5.10 \mathrm{M}_{\mathrm{L}} & \text { BRK } \\
5.60 \mathrm{M}_{\mathrm{L}} & \text { KJ } \\
4.50 \mathrm{M}_{\mathrm{L}} & \text { BRK } \\
5.00 \mathrm{M}_{\mathrm{L}} & \text { BRK }\end{array}$ & $\begin{array}{c}5 . \overline{2 \mathrm{BAK}} \\
6.05 \mathrm{BAK} \\
5 . \overline{\mathrm{B} A \mathrm{BAK}}\end{array}$ & $\begin{array}{l}\text { VI } \\
\text { Felt } \\
\text { VII } \\
\frac{\text { IV }}{}\end{array}$ & $\begin{array}{l}81 \\
81 \\
81 \\
81\end{array}$ & $\frac{31}{60 \&}$ \\
\hline $\begin{array}{l}1966 \\
1966 \\
1966 \\
1966 \\
1966\end{array}$ & $\begin{array}{l}08 \\
09 \\
09 \\
09 \\
09\end{array}$ & $\begin{array}{l}07 \\
12 \\
12 \\
14 \\
14\end{array}$ & $\begin{array}{l}173627.3 \\
164102.6 \\
172011 \\
220028 \\
224028\end{array}$ & $\begin{array}{l}31.7 \mathrm{~N} \\
39.438 \mathrm{~N} \\
39.42 \mathrm{~N} \\
39.42 \mathrm{~N} \\
39.42 \mathrm{~N}\end{array}$ & $\begin{array}{l}114.5 \mathrm{~W} \\
120.160 \mathrm{~W} \\
120.15 \mathrm{~W} \\
120.15 \mathrm{~W} \\
120.15 \mathrm{~W}\end{array}$ & $\begin{array}{l}033 \\
010 \\
003 \\
003 \\
003\end{array}$ & $\begin{array}{l}266 \\
457 \\
324 \\
324 \\
324\end{array}$ & $\begin{array}{l}\overline{5.7} \\
4.8 \\
4.3 \\
4.5\end{array}$ & $\overline{-}$ & $\begin{array}{ll}6.30 \mathrm{M}_{\mathrm{L}} & \text { PAS } \\
6.00 \mathrm{M}_{\mathrm{L}} & \text { BRK } \\
5.30 \mathrm{M}_{\mathrm{L}} & \text { BRK } \\
4.60 \mathrm{M}_{\mathrm{L}} & \text { BRK } \\
4.60 \mathrm{M}_{\mathrm{L}} & \text { BRK }\end{array}$ & $\begin{array}{c}6.27 \mathrm{HHT} \\
5.90 \mathrm{KA} \\
- \\
-\end{array}$ & $\begin{array}{l}\text { VI } \\
\text { VII } \\
\text { V } \\
\text { IV } \\
\text { IV }\end{array}$ & $\begin{array}{l}81 \\
81 \\
81 \\
81 \\
81\end{array}$ & $\begin{array}{l}115 \# \\
175 \& \\
- \\
-\end{array}$ \\
\hline
\end{tabular}


CALIFORNIA-Continued

[See table 1 for hypocenter and intensity references and table 2 for definitions of magnitude source codes. \&, land area only; \#, land area only in the United States for an earthquake near a coastline; +, land area in the United States when the felt area did not extend to the coast; @, felt area is less than $1,000 \mathrm{~km}^{2}$. Leader (--) indicates information is not available]

\begin{tabular}{|c|c|c|c|c|c|c|c|c|c|c|c|c|c|c|}
\hline \multirow{2}{*}{\multicolumn{3}{|c|}{ Date }} & \multirow{2}{*}{$\begin{array}{c}\text { Origin } \\
\text { time (UTC) }\end{array}$} & \multicolumn{4}{|c|}{ Hypocenter } & \multicolumn{4}{|c|}{ Magnitude } & \multicolumn{2}{|c|}{ Intensity } & \multirow{3}{*}{$\begin{array}{l}\text { Felt area } \\
\left(1,000 \mathrm{~km}^{2}\right)\end{array}$} \\
\hline & & & & \multirow{2}{*}{$\begin{array}{l}\text { Latitude } \\
\left(^{\circ}\right)\end{array}$} & \multirow{2}{*}{$\begin{array}{l}\text { Longitude } \\
\left({ }^{\circ}\right)\end{array}$} & \multirow{2}{*}{$\begin{array}{l}\text { Depth } \\
(\mathrm{km})\end{array}$} & \multirow[t]{2}{*}{ Ref } & \multicolumn{2}{|c|}{ USGS } & \multirow[t]{2}{*}{ Other } & \multirow{2}{*}{$\begin{array}{c}\text { Moment } \\
\mathbf{M}\end{array}$} & \multirow[t]{2}{*}{ MMI } & \multirow[t]{2}{*}{ Ref } & \\
\hline $\mathrm{Yr}$ & Mo & $\mathrm{Da}$ & $h \mathrm{~m} s$ & & & & & $m_{b}$ & $\mathbf{M}_{\mathbf{s}}$ & & & & & \\
\hline $\begin{array}{l}1966 \\
1966 \\
1966 \\
1966 \\
1967\end{array}$ & $\begin{array}{l}10 \\
11 \\
11 \\
12 \\
03\end{array}$ & $\begin{array}{l}02 \\
26 \\
26 \\
17 \\
02\end{array}$ & $\begin{array}{lll}05 & 12 & 34.5 \\
04 & 30 & 58.7 \\
05 & 56 & 39.6 \\
15 & 16 & 26.2 \\
14 & 12 & 48.8\end{array}$ & $\begin{array}{l}33.968 \mathrm{~N} \\
40.3 \mathrm{~N} \\
40.3 \mathrm{~N} \\
40.38 \mathrm{~N} \\
36.503 \mathrm{~N}\end{array}$ & $\begin{array}{l}118.327 \mathrm{~W} \\
125.4 \mathrm{~W} \\
125.3 \mathrm{~W} \\
125.70 \mathrm{~W} \\
117.632 \mathrm{~W}\end{array}$ & $\begin{array}{l}002 \\
033 \\
033 \\
000 \\
010\end{array}$ & $\begin{array}{l}292 \\
266 \\
266 \\
299 \\
292\end{array}$ & $\begin{array}{l}3.9 \\
4.6 \\
4.4 \\
4.7 \\
4.7\end{array}$ & $\bar{z}$ & $\begin{array}{ll}3.50 \mathrm{M}_{\mathrm{L}} & \text { PAS } \\
4.80 \mathrm{M}_{\mathrm{L}} & \text { BRK } \\
4.70 \mathrm{M}_{\mathrm{L}} & \text { BRK } \\
4.50 \mathrm{M}_{\mathrm{L}} & \text { BRK } \\
4.40 \mathrm{M}_{\mathrm{L}} & \text { PAS }\end{array}$ & $\begin{array}{l}z \\
z\end{array}$ & $\frac{\text { VI }}{-}$ & $\frac{81}{\frac{-}{259}}$ & $\bar{z}$ \\
\hline $\begin{array}{l}1967 \\
1967 \\
1967 \\
1967 \\
1967\end{array}$ & $\begin{array}{l}05 \\
06 \\
06 \\
07 \\
09\end{array}$ & $\begin{array}{l}21 \\
15 \\
26 \\
22 \\
07\end{array}$ & $\begin{array}{l}144234.4 \\
045805.5 \\
151535 \\
092326.6 \\
123917.2\end{array}$ & $\begin{array}{l}33.507 \mathrm{~N} \\
33.997 \mathrm{~N} \\
39.3 \mathrm{~N} \\
36.535 \mathrm{~N} \\
37.035 \mathrm{~N}\end{array}$ & $\begin{array}{l}116.583 \mathrm{~W} \\
117.975 \mathrm{~W} \\
123.3 \mathrm{~W} \\
121.163 \mathrm{~W} \\
121.777 \mathrm{~W}\end{array}$ & $\begin{array}{l}019 \\
010 \\
002 \\
\overline{008}\end{array}$ & $\begin{array}{l}292 \\
292 \\
324 \\
324 \\
324\end{array}$ & $\begin{array}{l}4.7 \\
4.3 \\
4.2 \\
4.0 \\
-\end{array}$ & $\bar{z}$ & $\begin{array}{ll}4.70 \mathrm{M}_{\mathrm{L}} & \text { PAS } \\
4.10 \mathrm{M}_{\mathrm{L}} & \text { PAS } \\
3.50 \mathrm{M}_{\mathrm{L}} & \text { BRK } \\
3.80 \mathrm{M}_{\mathrm{L}} & \text { BRK } \\
4.70 \mathrm{M}_{\mathrm{L}} & \text { BRK }\end{array}$ & $\begin{array}{l}\bar{z} \\
\overline{4} \\
\overline{\overline{1 B A K}}\end{array}$ & $\begin{array}{l}\text { VI } \\
\text { VI } \\
\text { VI } \\
\text { VI } \\
\text { VI }\end{array}$ & $\begin{array}{l}40 \\
40 \\
40 \\
40 \\
40\end{array}$ & $\begin{array}{c}23 \& \\
6 \& \\
4 \\
-17 \&\end{array}$ \\
\hline $\begin{array}{l}1967 \\
1967 \\
1967 \\
1967 \\
1967\end{array}$ & $\begin{array}{l}09 \\
11 \\
12 \\
12 \\
12\end{array}$ & $\begin{array}{l}28 \\
24 \\
10 \\
10 \\
18\end{array}$ & $\begin{array}{l}153836.1 \\
135700.4 \\
120650.3 \\
123354.2 \\
172432.0\end{array}$ & $\begin{array}{l}37.230 \mathrm{~N} \\
40.4 \mathrm{~N} \\
40.5 \mathrm{~N} \\
40.5 \mathrm{~N} \\
37.010 \mathrm{~N}\end{array}$ & $\begin{array}{l}121.620 \mathrm{~W} \\
125.1 \mathrm{~W} \\
124.6 \mathrm{~W} \\
125.0 \mathrm{~W} \\
121.788 \mathrm{~W}\end{array}$ & $\begin{array}{l}008 \\
017 \\
005 \\
015 \\
013\end{array}$ & $\begin{array}{r}324 \\
74 \\
74 \\
74 \\
324\end{array}$ & $\begin{array}{l}- \\
4.6 \\
5.8 \\
4.6 \\
5.0\end{array}$ & $\bar{z}$ & $\begin{array}{cl}4.90 \mathrm{M}_{\mathrm{L}} & \text { BRK } \\
\overline{-}^{-60 \mathrm{M}_{\mathrm{L}}} & \text { BRK } \\
4.00 \mathrm{M}_{\mathrm{L}} & \text { BRK } \\
5.30 \mathrm{M}_{\mathrm{L}} & \mathrm{BRK}\end{array}$ & $\begin{array}{l}\overline{-} \\
\overline{4} \\
4.64 \mathrm{BAK}\end{array}$ & $\begin{array}{r}\frac{V I}{\text { VI }} \\
\text { Felt } \\
\text { VI }\end{array}$ & $\begin{array}{l}40 \\
40 \\
40 \\
40\end{array}$ & $\begin{array}{l}\frac{19}{15} \& \\
\frac{27}{27}\end{array}$ \\
\hline $\begin{array}{l}1967 \\
1968 \\
1968 \\
1968 \\
1968\end{array}$ & $\begin{array}{l}12 \\
04 \\
04 \\
04 \\
04\end{array}$ & $\begin{array}{l}30 \\
09 \\
09 \\
09 \\
09\end{array}$ & 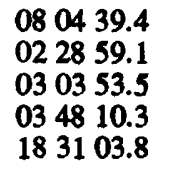 & $\begin{array}{l}40.51 \mathrm{~N} \\
33.190 \mathrm{~N} \\
33.113 \mathrm{~N} \\
33.105 \mathrm{~N} \\
33.315 \mathrm{~N}\end{array}$ & $\begin{array}{l}124.43 \mathrm{~W} \\
116.128 \mathrm{~W} \\
116.038 \mathrm{~W} \\
116.037 \mathrm{~W} \\
116.305 \mathrm{~W}\end{array}$ & $\begin{array}{l}003 \\
011 \\
005 \\
005 \\
013\end{array}$ & $\begin{array}{l}299 \\
292 \\
292 \\
292 \\
292\end{array}$ & $\begin{array}{l}4.6 \\
6.1 \\
5.1 \\
4.7 \\
4.7\end{array}$ & $\bar{z}$ & $\begin{array}{ll}4.50 \mathrm{M}_{\mathrm{L}} & \text { BRK } \\
6.40 \mathrm{M}_{\mathrm{L}} & \text { PAS } \\
5.20 \mathrm{M}_{\mathrm{L}} & \text { PAS } \\
4.70 \mathrm{M}_{\mathrm{L}} & \text { PAS } \\
4.70 \mathrm{M}_{\mathrm{L}} & \text { PAS }\end{array}$ & $\begin{array}{c}\text { 6.47HK } \\
- \\
-\end{array}$ & $\begin{array}{l}\text { V } \\
\text { VII } \\
\text { III } \\
\text { VI } \\
\text { III }\end{array}$ & $\begin{array}{l}40 \\
41 \\
41 \\
41 \\
41\end{array}$ & $\begin{array}{l}\overline{158 \#} \\
- \\
-\end{array}$ \\
\hline $\begin{array}{l}1968 \\
1968 \\
1968 \\
1968 \\
1968\end{array}$ & $\begin{array}{l}04 \\
04 \\
04 \\
05 \\
06\end{array}$ & $\begin{array}{l}16 \\
25 \\
29 \\
09 \\
03\end{array}$ & $\begin{array}{l}033029.9 \\
194945.2 \\
002138.6 \\
232746.5 \\
070555.0\end{array}$ & 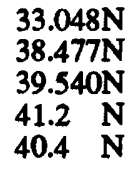 & $\begin{array}{l}115.987 \mathrm{~W} \\
122.725 \mathrm{~W} \\
122.023 \mathrm{~W} \\
125.7 \mathrm{~W} \\
124.8 \mathrm{~W}\end{array}$ & $\begin{array}{l}008 \\
008 \\
023 \\
033 \\
033\end{array}$ & $\begin{array}{r}292 \\
324 \\
324 \\
74 \\
74\end{array}$ & $\begin{array}{l}4.4 \\
4.6 \\
5.0 \\
4.7 \\
4.5\end{array}$ & $\bar{z}$ & $\begin{array}{ll}4.80 \mathrm{M}_{\mathrm{L}} & \text { PAS } \\
4.60 \mathrm{M}_{\mathrm{L}} & \text { BRK } \\
4.70 \mathrm{M}_{\mathrm{L}} & \text { BRK } \\
4.40 \mathrm{M}_{\mathrm{L}} & \text { BRK } \\
3.60 \mathrm{M}_{\mathrm{L}} & \text { BRK }\end{array}$ & $\begin{array}{c}4.70 \mathrm{TH} \\
- \\
- \\
-\end{array}$ & $\begin{array}{l}\text { V } \\
\text { VII } \\
\text { VI } \\
-\end{array}$ & $\begin{array}{l}41 \\
41 \\
41 \\
- \\
-\end{array}$ & $\begin{array}{l}\overline{13} \& \\
27 \\
-\end{array}$ \\
\hline $\begin{array}{l}1968 \\
1968 \\
1968 \\
1968 \\
1968\end{array}$ & $\begin{array}{l}06 \\
06 \\
06 \\
06 \\
07\end{array}$ & $\begin{array}{l}26 \\
26 \\
29 \\
29 \\
05\end{array}$ & $\begin{array}{lll}01 & 42 & 14.6 \\
10 & 47 & 45.0 \\
19 & 12 & 20.2 \\
19 & 13 & 57.0 \\
00 & 45 & 17.2\end{array}$ & $\begin{array}{l}40.29 \mathrm{~N} \\
40.2 \mathrm{~N} \\
34.3 \mathrm{~N} \\
34.267 \mathrm{~N} \\
34.119 \mathrm{~N}\end{array}$ & $\begin{array}{l}124.67 \mathrm{~W} \\
124.3 \mathrm{~W} \\
119.7 \mathrm{~W} \\
119.567 \mathrm{~W} \\
119.702 \mathrm{~W}\end{array}$ & $\begin{array}{l}000 \\
010 \\
002 \\
010 \\
006\end{array}$ & $\begin{array}{r}480 \\
324 \\
74 \\
292 \\
292\end{array}$ & $\begin{array}{l}5.5 \\
5.1 \\
5.0 \\
5.7\end{array}$ & $\bar{z}$ & $\begin{array}{ll}5.90 \mathrm{M}_{\mathrm{L}} & \text { BRK } \\
4.80 \mathrm{M}_{\mathrm{L}} & \text { BRK } \\
4.20 \mathrm{M}_{\mathrm{L}} & \text { PAS } \\
4.40 \mathrm{M}_{\mathrm{L}} & \text { PAS } \\
5.20 \mathrm{M}_{\mathrm{L}} & \text { PAS }\end{array}$ & $\begin{array}{l}- \\
z\end{array}$ & $\begin{array}{l}\text { VII } \\
\text { IV } \\
\text { V } \\
\text { VI } \\
\text { VI }\end{array}$ & $\begin{array}{l}41 \\
41 \\
41 \\
41 \\
41\end{array}$ & $\frac{13 \&}{-}$ \\
\hline $\begin{array}{l}1968 \\
1968 \\
1969 \\
1969 \\
1969\end{array}$ & $\begin{array}{l}07 \\
12 \\
01 \\
02 \\
02\end{array}$ & $\begin{array}{l}07 \\
17 \\
23 \\
07 \\
28\end{array}$ & $\begin{array}{lll}14 & 33 & 30.8 \\
22 & 53 & 51.2 \\
23 & 01 & 01.0 \\
21 & 25 & 48.9 \\
04 & 56 & 12.4\end{array}$ & $\begin{array}{l}34.177 \mathrm{~N} \\
33.045 \mathrm{~N} \\
33.887 \mathrm{~N} \\
40.343 \mathrm{~N} \\
34.565 \mathrm{~N}\end{array}$ & $\begin{array}{l}119.755 \mathrm{~W} \\
115.863 \mathrm{~W} \\
116.040 \mathrm{~W} \\
124.365 \mathrm{~W} \\
118.113 \mathrm{~W}\end{array}$ & $\begin{array}{l}013 \\
008 \\
018 \\
013 \\
005\end{array}$ & $\begin{array}{l}292 \\
292 \\
292 \\
324 \\
292\end{array}$ & $\begin{array}{l}4.6 \\
4.6 \\
4.9 \\
5.2 \\
4.6\end{array}$ & $\bar{z}$ & 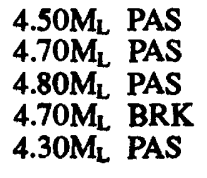 & $\begin{array}{l}- \\
- \\
-\end{array}$ & $\begin{array}{l}\text { IV } \\
\text { IV } \\
\text { V } \\
\text { VI } \\
\text { VI }\end{array}$ & $\begin{array}{r}41 \\
259 \\
42 \\
42 \\
42\end{array}$ & $\begin{array}{l}- \\
6 \# \\
13 \\
10 \& \\
12 \&\end{array}$ \\
\hline $\begin{array}{l}1969 \\
1969 \\
1969 \\
1969 \\
1969\end{array}$ & $\begin{array}{l}04 \\
05 \\
06 \\
06 \\
06\end{array}$ & $\begin{array}{l}28 \\
19 \\
07 \\
23 \\
28\end{array}$ & $\begin{array}{lll}23 & 2042.9 \\
14 & 4033.0 \\
11 & 27 & 12 \\
01 & 21 & 16.5 \\
0407 & 26.7\end{array}$ & $\begin{array}{ll}33.343 N \\
33.348 N \\
40.8 & \mathbf{N} \\
41.8 & \mathbf{N} \\
40.3 & \mathbf{N}\end{array}$ & $\begin{array}{l}116.347 \mathrm{~W} \\
116.188 \mathrm{~W} \\
125.8 \mathrm{~W} \\
125.8 \mathrm{~W} \\
124.4 \mathrm{~W}\end{array}$ & $\begin{array}{l}020 \\
009 \\
003 \\
033 \\
039\end{array}$ & $\begin{array}{r}292 \\
292 \\
324 \\
74 \\
74\end{array}$ & $\begin{array}{l}5.7 \\
4.5 \\
\overline{4.7} \\
4.5\end{array}$ & $\bar{z}$ & $\begin{array}{ll}5.80 \mathrm{M}_{\mathrm{L}} & \text { PAS } \\
4.50 \mathrm{M}_{\mathrm{L}} & \text { PAS } \\
4.00 \mathrm{M}_{\mathrm{L}} & \text { BRK } \\
4.00 \mathrm{Kn}^{\mathrm{BRK}} \\
3.90 \mathrm{M}_{\mathrm{L}} \text { BRK }\end{array}$ & $\begin{array}{c}\text { 5.75THH } \\
= \\
= \\
=\end{array}$ & 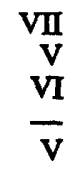 & $\begin{array}{l}42 \\
42 \\
42 \\
\frac{42}{42}\end{array}$ & $\begin{array}{l}78 \# \\
17 \# \\
- \\
6 \&\end{array}$ \\
\hline $\begin{array}{l}1969 \\
1969 \\
1969 \\
1969 \\
1969\end{array}$ & $\begin{array}{l}07 \\
10 \\
10 \\
10 \\
10\end{array}$ & $\begin{array}{l}01 \\
02 \\
02 \\
03 \\
14\end{array}$ & 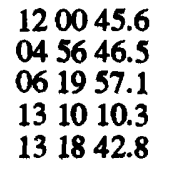 & $\begin{array}{l}40.300 \mathrm{~N} \\
38.467 \mathrm{~N} \\
38.455 \mathrm{~N} \\
37.625 \mathrm{~N} \\
32.923 \mathrm{~N}\end{array}$ & $\begin{array}{l}124.300 \mathrm{~W} \\
122.692 \mathrm{~W} \\
122.692 \mathrm{~W} \\
118.925 \mathrm{~W} \\
116.272 \mathrm{~W}\end{array}$ & $\begin{array}{l}026 \\
010 \\
010 \\
\overline{010}\end{array}$ & $\begin{array}{r}74 \\
399 \\
399 \\
292 \\
292\end{array}$ & $\begin{array}{l}4.6 \\
5.2 \\
5.1 \\
4.6 \\
4.5\end{array}$ & $\overline{4.8}$ & $\begin{array}{ll}3.90 \mathrm{M}_{\mathrm{L}} & \text { BRK } \\
5.60 \mathrm{M}_{\mathrm{L}} & \text { BRK } \\
5.70 \mathrm{M}_{\mathrm{L}} & \text { BRK } \\
4.90 \mathrm{M}_{\mathrm{L}} & \text { PAS } \\
4.50 \mathrm{M}_{\mathrm{L}} & \text { PAS }\end{array}$ & $=$ & $\begin{array}{l}\text { V } \\
\text { VIII } \\
\text { VIII } \\
\text { v } \\
\text { V }\end{array}$ & $\begin{array}{l}42 \\
42 \\
42 \\
42 \\
42\end{array}$ & $\begin{array}{l}\overline{27} \& \\
27 \& \\
= \\
-\end{array}$ \\
\hline $\begin{array}{l}1969 \\
1969 \\
1969 \\
1969 \\
1969\end{array}$ & $\begin{array}{l}10 \\
10 \\
10 \\
10 \\
10\end{array}$ & $\begin{array}{l}22 \\
23 \\
24 \\
24 \\
27\end{array}$ & $\begin{array}{lll}22 & 51 & 32.1 \\
00 & 03 & 34.4 \\
08 & 29 & 12.1 \\
20 & 26 & 42.5 \\
10 & 59 & 42.8\end{array}$ & $\begin{array}{l}34.77 \mathrm{~N} \\
34.90 \mathrm{~N} \\
33.292 \mathrm{~N} \\
33.338 \mathrm{~N} \\
36.790 \mathrm{~N}\end{array}$ & $\begin{array}{l}121.35 \mathrm{~W} \\
121.30 \mathrm{~W} \\
119.193 \mathrm{~W} \\
119.105 \mathrm{~W} \\
121.393 \mathrm{~W}\end{array}$ & $\begin{array}{l}007 \\
010 \\
010 \\
\overline{013}\end{array}$ & $\begin{array}{r}299 \\
74 \\
292 \\
292 \\
324\end{array}$ & $\begin{array}{l}5.9 \\
5.0 \\
5.1 \\
4.9 \\
4.6\end{array}$ & $\begin{array}{l}5.2 \\
5.0 \\
-\end{array}$ & $\begin{array}{ll}5.40 \mathrm{M}_{\mathrm{L}} & \text { PAS } \\
4.10 \mathrm{M}_{\mathrm{L}} & \text { PAS } \\
5.10 \mathrm{M}_{\mathrm{L}} & \text { PAS } \\
4.70 \mathrm{M}_{\mathrm{L}} & \text { PAS } \\
4.60 \mathrm{M}_{\mathrm{L}} & \text { BRK }\end{array}$ & $\frac{\bar{Z}}{\overline{4}} \overline{-}$ & $\begin{array}{c}\frac{v}{V} \\
\text { Felt } \\
\text { VI }\end{array}$ & $\begin{array}{l}\frac{42}{42} \\
42 \\
42\end{array}$ & $\bar{z}$ \\
\hline $\begin{array}{l}1969 \\
1969 \\
1969 \\
1969 \\
1969\end{array}$ & $\begin{array}{l}10 \\
10 \\
11 \\
11 \\
11\end{array}$ & $\begin{array}{l}27 \\
31 \\
04 \\
05 \\
05\end{array}$ & $\begin{array}{lll}13 & 16 & 02.3 \\
10 & 39 & 29.0 \\
00 & 40 & 44.8 \\
17 & 54 & 10.7 \\
18 & 48 & 45.1\end{array}$ & $\begin{array}{l}33.545 \mathrm{~N} \\
33.430 \mathrm{~N} \\
34.76 \mathrm{~N} \\
34.72 \mathrm{~N} \\
34.65 \mathrm{~N}\end{array}$ & $\begin{array}{l}117.807 \mathrm{~W} \\
119.097 \mathrm{~W} \\
121.37 \mathrm{~W} \\
121.28 \mathrm{~W} \\
121.31 \mathrm{~W}\end{array}$ & $\begin{array}{l}007 \\
007 \\
010 \\
011 \\
010\end{array}$ & $\begin{array}{l}292 \\
292 \\
299 \\
299 \\
299\end{array}$ & $\begin{array}{l}4.5 \\
4.9 \\
4.9 \\
5.8 \\
5.1\end{array}$ & $\bar{z}$ & $\begin{array}{ll}4.50 \mathrm{M}_{\mathrm{L}} & \text { PAS } \\
4.80 \mathrm{M}_{\mathrm{L}} & \text { PAS } \\
4.50 \mathrm{M}_{\mathrm{L}} & \text { PAS } \\
5.60 \mathrm{M}_{\mathrm{L}} & \text { PAS } \\
4.50 \mathrm{M}_{\mathrm{L}} & \text { PAS }\end{array}$ & $\begin{array}{l}- \\
- \\
-\end{array}$ & $\begin{array}{c}\text { IV } \\
\frac{V}{V} \\
\text { Felt }\end{array}$ & $\begin{array}{l}42 \\
42 \\
42 \\
42\end{array}$ & $\begin{array}{l}12 \& \\
- \\
-\end{array}$ \\
\hline
\end{tabular}


CALIFORNIA-Continued

[See table 1 for hypocenter and intensity references and table 2 for definitions of magnitude source codes. \&, land area only; \#, land area only in the United States for an earthquake near a coastline; + , land area in the United States when the felt area did not extend to the coast; @, felt area is less than $1,000 \mathrm{~km}^{2}$. Leader (-) indicates information is not available]

\begin{tabular}{|c|c|c|c|c|c|c|c|c|c|c|c|c|c|}
\hline \multirow{2}{*}{\multicolumn{3}{|c|}{$\begin{array}{cc} & \text { Origin } \\
\text { Date } & \text { time (UTC) } \\
\end{array}$}} & \multicolumn{4}{|c|}{ Hypocenter } & \multicolumn{4}{|c|}{ Magnitude } & \multicolumn{2}{|c|}{ Intensity } & \multirow{3}{*}{$\begin{array}{l}\text { Felt area } \\
\left(1,000 \mathrm{~km}^{2}\right)\end{array}$} \\
\hline & & & \multirow{2}{*}{$\begin{array}{l}\text { Latitude } \\
\left(^{\circ}\right)\end{array}$} & \multirow{2}{*}{$\begin{array}{l}\text { Longitude } \\
\left({ }^{\circ}\right)\end{array}$} & \multirow{2}{*}{$\begin{array}{l}\text { Depth } \\
(\mathrm{km})\end{array}$} & \multirow[t]{2}{*}{ Ref } & \multicolumn{2}{|c|}{ USGS } & \multirow[t]{2}{*}{ Other } & \multirow{2}{*}{$\begin{array}{c}\text { Moment } \\
\text { M }\end{array}$} & \multirow[t]{2}{*}{ MMi } & \multirow[t]{2}{*}{ Ref } & \\
\hline Yr & & & & & & & $m_{b}$ & $\mathbf{M}_{\mathbf{s}}$ & & & & & \\
\hline $\begin{array}{l}1969 \\
1969 \\
1970 \\
1970 \\
1970\end{array}$ & $\begin{array}{ll}11 & 09 \\
11 & 10 \\
01 & 03 \\
03 & 31 \\
06 & 07\end{array}$ & $\begin{array}{lll}01 & 27 & 42.0 \\
19 & 21 & 27.3 \\
02 & 51 & 58.4 \\
07 & 02 & 28.6 \\
04 & 12 & 10.3\end{array}$ & $\begin{array}{l}34.54 \mathrm{~N} \\
34.6 \mathrm{~N} \\
37.298 \mathrm{~N} \\
36.868 \mathrm{~N} \\
40.3 \mathrm{~N}\end{array}$ & $\begin{array}{l}121.40 \mathrm{~W} \\
121.5 \mathrm{~W} \\
122.088 \mathrm{~W} \\
121.418 \mathrm{~W} \\
125.9 \mathrm{~W}\end{array}$ & $\begin{array}{l}010 \\
033 \\
\overline{008} \\
033\end{array}$ & $\begin{array}{r}299 \\
74 \\
324 \\
324 \\
74\end{array}$ & $\begin{array}{l}4.7 \\
4.6 \\
4.0 \\
4.2 \\
5.0\end{array}$ & $\bar{z}$ & $\begin{array}{ll}4.10 \mathrm{M}_{\mathrm{L}} & \text { PAS } \\
4.00 \mathrm{M}_{\mathrm{L}} & \text { PAS } \\
3.70 \mathrm{M}_{\mathrm{L}} & \text { BRK } \\
4.70 \mathrm{M}_{\mathrm{L}} & \text { BRK } \\
4.70 \mathrm{M}_{\mathrm{L}} & \text { BRK }\end{array}$ & $\frac{\bar{Z}}{4.50 \mathrm{JM}}$ & $\begin{array}{l}- \\
\overline{V I} \\
\text { V } \\
\text { IV }\end{array}$ & $\begin{array}{l}- \\
- \\
43 \\
43 \\
43\end{array}$ & $\begin{array}{l}- \\
3 \\
128 \\
-\end{array}$ \\
\hline $\begin{array}{l}1970 \\
1970 \\
1970 \\
1970 \\
1970\end{array}$ & $\begin{array}{ll}06 & 12 \\
06 & 12 \\
08 & 04 \\
09 & 12 \\
09 & 13\end{array}$ & $\begin{array}{lll}03 & 30 & 04.0 \\
16 & 03 & 32.1 \\
04 & 14 & 21.4 \\
14 & 30 & 53.0 \\
21 & 1023.0\end{array}$ & $\begin{array}{l}37.802 \mathrm{~N} \\
37.805 \mathrm{~N} \\
36.647 \mathrm{~N} \\
34.270 \mathrm{~N} \\
40.2 \mathrm{~N}\end{array}$ & $\begin{array}{l}121.933 \mathrm{~W} \\
122.938 \mathrm{~W} \\
122.185 \mathrm{~W} \\
117.540 \mathrm{~W} \\
125.1 \mathrm{~W}\end{array}$ & $\begin{array}{l}009 \\
009 \\
008 \\
008 \\
033\end{array}$ & $\begin{array}{r}324 \\
324 \\
324 \\
292 \\
74\end{array}$ & $\begin{array}{l}4.6 \\
4.5 \\
5.4 \\
5.4\end{array}$ & $\bar{z}$ & $\begin{array}{ll}4.30 \mathrm{M}_{\mathrm{L}} & \text { BRK } \\
4.20 \mathrm{M}_{\mathrm{L}} & \text { BRK } \\
4.70 \mathrm{M}_{\mathrm{L}} & \text { BRK } \\
5.40 \mathrm{M}_{\mathrm{L}} & \text { PAS } \\
5.40 \mathrm{M}_{\mathrm{L}} & \text { BRK }\end{array}$ & $\frac{-}{-}$ & $\begin{array}{l}\text { VI } \\
\text { VI } \\
\text { VI } \\
\text { VII } \\
\text { V }\end{array}$ & $\begin{array}{l}43 \\
43 \\
43 \\
43 \\
43\end{array}$ & $\begin{array}{l}\frac{38}{148} \\
65 \&\end{array}$ \\
\hline $\begin{array}{l}1971 \\
1971 \\
1971 \\
1971 \\
1971\end{array}$ & $\begin{array}{ll}02 & 09 \\
02 & 09 \\
02 & 09 \\
02 & 09 \\
02 & 09\end{array}$ & $\begin{array}{l}140041.8 \\
140108.0 \\
140150.0 \\
140231.0 \\
140244.0\end{array}$ & $\begin{array}{l}34.412 \mathrm{~N} \\
34.412 \mathrm{~N} \\
34.412 \mathrm{~N} \\
34.412 \mathrm{~N} \\
34.412 \mathrm{~N}\end{array}$ & $\begin{array}{l}118.400 \mathrm{~W} \\
118.400 \mathrm{~W} \\
118.400 \mathrm{~W} \\
118.400 \mathrm{~W} \\
118.400 \mathrm{~W}\end{array}$ & $\begin{array}{l}008 \\
008 \\
008 \\
008 \\
008\end{array}$ & $\begin{array}{l}400 \\
292 \\
292 \\
292 \\
292\end{array}$ & $\begin{array}{l}6.2 \\
- \\
-\end{array}$ & $\frac{6.5}{-}$ & $\begin{array}{ll}6.40 \mathrm{M}_{\mathrm{L}} & \mathrm{KJ} \\
5.80 \mathrm{M}_{\mathrm{L}} & \text { PAS } \\
4.50 \mathrm{M}_{\mathrm{L}} & \text { PAS } \\
4.70 \mathrm{M}_{\mathrm{L}} & \text { PAS } \\
5.80 \mathrm{M}_{\mathrm{L}} & \text { PAS }\end{array}$ & $\begin{array}{c}6.62 \mathrm{HK} \\
- \\
- \\
-\end{array}$ & $\begin{array}{l}\text { XI } \\
- \\
-\end{array}$ & $\frac{44}{-}$ & $\frac{212 \#}{-}$ \\
\hline $\begin{array}{l}1971 \\
1971 \\
1971 \\
1971 \\
1971\end{array}$ & $\begin{array}{ll}02 & 09 \\
02 & 09 \\
02 & 09 \\
02 & 09 \\
02 & 09\end{array}$ & $\begin{array}{l}140745.0 \\
140838.0 \\
140853.0 \\
141021.5 \\
141028.0\end{array}$ & $\begin{array}{l}34.412 \mathrm{~N} \\
34.412 \mathrm{~N} \\
34.412 \mathrm{~N} \\
34.362 \mathrm{~N} \\
34.412 \mathrm{~N}\end{array}$ & $\begin{array}{l}118.400 \mathrm{~W} \\
118.400 \mathrm{~W} \\
118.400 \mathrm{~W} \\
118.307 \mathrm{~W} \\
118.400 \mathrm{~W}\end{array}$ & $\begin{array}{l}008 \\
008 \\
008 \\
005 \\
008\end{array}$ & $\begin{array}{l}292 \\
292 \\
292 \\
292 \\
292\end{array}$ & $\bar{z}$ & $\bar{z}$ & $\begin{array}{ll}\text { 4.50M } & \text { PAS } \\
4.50 M_{L} & \text { PAS } \\
4.60 M_{L} & \text { PAS } \\
4.70 M_{L} & \text { PAS } \\
5.30 M_{L} & \text { PAS }\end{array}$ & $\begin{array}{c}\overline{-} \\
\text { 4.92TH } \\
4.60 \mathrm{TH}\end{array}$ & $\bar{z} \frac{}{\bar{v}}$ & $\frac{-}{\overline{4}}$ & $\frac{-}{-}$ \\
\hline $\begin{array}{l}1971 \\
1971 \\
1971 \\
1971 \\
1971\end{array}$ & $\begin{array}{ll}02 & 09 \\
02 & 09 \\
02 & 09 \\
02 & 10 \\
02 & 21\end{array}$ & $\begin{array}{l}143436.1 \\
144346.7 \\
155820.7 \\
051807.2 \\
055052.6\end{array}$ & $\begin{array}{l}34.343 \mathrm{~N} \\
34.308 \mathrm{~N} \\
34.335 \mathrm{~N} \\
34.425 \mathrm{~N} \\
34.397 \mathrm{~N}\end{array}$ & $\begin{array}{l}118.637 W \\
118.453 W \\
118.330 W \\
118.413 W \\
118.438 W\end{array}$ & $\begin{array}{l}-\overline{006} \\
014 \\
006 \\
007\end{array}$ & $\begin{array}{l}292 \\
292 \\
292 \\
292 \\
292\end{array}$ & $\begin{array}{l}-\overline{4.7} \\
5.1 \\
4.7 \\
-\end{array}$ & $\bar{z}$ & $\begin{array}{ll}4.90 \mathrm{M}_{\mathrm{L}} & \text { PAS } \\
5.20 \mathrm{M}_{\mathrm{L}} & \text { PAS } \\
4.80 \mathrm{M}_{\mathrm{L}} & \text { PAS } \\
4.50 \mathrm{M}_{\mathrm{L}} & \text { PAS } \\
4.70 \mathrm{M}_{\mathrm{L}} & \text { PAS }\end{array}$ & $\begin{array}{c}4.23 \mathrm{TH} \\
- \\
- \\
-\end{array}$ & $\begin{array}{l}\text { Felt } \\
\text { IV } \\
\text { Felt } \\
\text { IV }\end{array}$ & $\begin{array}{l}-44 \\
44 \\
44 \\
44\end{array}$ & $\bar{z}$ \\
\hline $\begin{array}{l}1971 \\
1971 \\
1971 \\
1971 \\
1971\end{array}$ & $\begin{array}{ll}02 & 21 \\
02 & 27 \\
03 & 07 \\
03 & 09 \\
03 & 31\end{array}$ & $\begin{array}{lll}07 & 15 & 11.8 \\
00 & 31 & 39.9 \\
01 & 33 & 40.5 \\
15 & 35 & 16.2 \\
14 & 52 & 22.5\end{array}$ & $\begin{array}{l}34.392 \mathrm{~N} \\
40.4 \mathrm{~N} \\
34.353 \mathrm{~N} \\
36.800 \mathrm{~N} \\
34.285 \mathrm{~N}\end{array}$ & $\begin{array}{l}118.427 \mathrm{~W} \\
124.8 \mathrm{~W} \\
118.455 \mathrm{~W} \\
122.145 \mathrm{~W} \\
118.515 \mathrm{~W}\end{array}$ & $\begin{array}{l}007 \\
033 \\
003 \\
008 \\
002\end{array}$ & $\begin{array}{r}292 \\
74 \\
292 \\
324 \\
292\end{array}$ & $\begin{array}{l}\overline{5.3} \\
4.4 \\
4.8 \\
4.8\end{array}$ & $\begin{array}{l}5.1 \\
- \\
-\end{array}$ & $\begin{array}{ll}\text { 4.50M } & \text { PAS } \\
5.20 \mathrm{M}_{\mathrm{L}} & \text { BRK } \\
4.50 \mathrm{M}_{\mathrm{L}} & \text { PAS } \\
4.60 \mathrm{M}_{\mathrm{L}} & \text { BRK } \\
4.60 \mathrm{M}_{\mathrm{L}} & \text { PAS }\end{array}$ & $\frac{-}{-}$ & $\begin{array}{c}\text { IV } \\
\text { V } \\
\text { V } \\
\text { VII }\end{array}$ & $\begin{array}{l}44 \\
44 \\
44 \\
44 \\
44\end{array}$ & $\begin{array}{l}\overline{11} \& \\
\overline{9} \& \\
16 \&\end{array}$ \\
\hline $\begin{array}{l}1971 \\
1971 \\
1971 \\
1971 \\
1972\end{array}$ & $\begin{array}{ll}04 & 16 \\
09 & 12 \\
09 & 30 \\
11 & 20 \\
01 & 22\end{array}$ & $\begin{array}{l}125831.7 \\
193238.0 \\
224611.3 \\
135904.3 \\
025719.9\end{array}$ & $\begin{array}{l}36.800 \mathrm{~N} \\
41.298 \mathrm{~N} \\
33.033 \mathrm{~N} \\
40.300 \mathrm{~N} \\
37.568 \mathrm{~N}\end{array}$ & $\begin{array}{l}122.183 \mathrm{~W} \\
123.673 \mathrm{~W} \\
115.820 \mathrm{~W} \\
124.400 \mathrm{~W} \\
118.367 \mathrm{~W}\end{array}$ & $\begin{array}{l}008 \\
023 \\
008 \\
025 \\
003\end{array}$ & $\begin{array}{r}324 \\
324 \\
292 \\
74 \\
324\end{array}$ & $\begin{array}{l}4.8 \\
4.9 \\
4.9 \\
5.0 \\
4.4\end{array}$ & $\bar{z}$ & $\begin{array}{ll}4.50 \mathrm{M}_{\mathrm{L}} & \text { BRK } \\
4.60 \mathrm{M}_{\mathrm{L}} & \text { BRK } \\
5.10 \mathrm{M}_{\mathrm{L}} & \text { PAS } \\
4.90 \mathrm{M}_{\mathrm{L}} & \text { BRK } \\
4.50 \mathrm{M}_{\mathrm{L}} & \text { BRK }\end{array}$ & $\bar{z}$ & $\begin{array}{l}\mathrm{V} \\
\mathrm{V} \\
\mathrm{VI} \\
\mathrm{V} \\
\mathrm{V}\end{array}$ & $\begin{array}{l}44 \\
44 \\
44 \\
44 \\
45\end{array}$ & $\begin{array}{c}-8 \\
17 \# \\
-\end{array}$ \\
\hline $\begin{array}{l}1972 \\
1972 \\
1972 \\
1972 \\
1972\end{array}$ & $\begin{array}{ll}02 & 24 \\
02 & 27 \\
03 & 01 \\
09 & 04 \\
09 & 23\end{array}$ & $\begin{array}{l}155651.3 \\
221308.6 \\
092856.7 \\
180440.9 \\
024405.4\end{array}$ & $\begin{array}{l}36.588 \mathrm{~N} \\
36.553 \mathrm{~N} \\
40.5 \mathrm{~N} \\
36.642 \mathrm{~N} \\
41.7 \mathrm{~N}\end{array}$ & $\begin{array}{l}121.197 \mathrm{~W} \\
121.093 \mathrm{~W} \\
125.2 \mathrm{~W} \\
121.263 \mathrm{~W} \\
125.5 \mathrm{~W}\end{array}$ & $\begin{array}{l}008 \\
008 \\
033 \\
005 \\
021\end{array}$ & $\begin{array}{r}324 \\
324 \\
74 \\
324 \\
74\end{array}$ & $\begin{array}{l}4.9 \\
4.5 \\
5.4 \\
4.9 \\
4.8\end{array}$ & $\overline{5} \overline{5.9}$ & $\begin{array}{ll}5.10 \mathrm{M}_{\mathrm{L}} & \text { BRK } \\
4.70 \mathrm{M}_{\mathrm{L}} & \text { BRK } \\
5.20 \mathrm{M}_{\mathrm{L}} & \text { BRK } \\
4.70 \mathrm{M}_{\mathrm{L}} & \text { BRK } \\
4.90 \mathrm{M}_{\mathrm{L}} & \text { BRK }\end{array}$ & $\begin{array}{l}4.94 \mathrm{SOM} \\
5.21 \mathrm{JM} \\
\overline{-} \\
4.82 \mathrm{JM} \\
-\end{array}$ & $\begin{array}{l}\text { VI } \\
\text { V } \\
\text { V } \\
\text { VI } \\
-\end{array}$ & $\begin{array}{l}45 \\
45 \\
45 \\
45 \\
-\end{array}$ & $\frac{18 \&}{14 \&}$ \\
\hline $\begin{array}{l}1972 \\
1972 \\
1973 \\
1973 \\
1973\end{array}$ & $\begin{array}{ll}10 & 03 \\
11 & 14 \\
02 & 21 \\
03 & 12 \\
06 & 15\end{array}$ & $\begin{array}{l}063002.2 \\
021013.8 \\
144557.2 \\
125012.9 \\
191851.7\end{array}$ & $\begin{array}{l}36.800 \mathrm{~N} \\
40.30 \mathrm{~N} \\
34.099 \mathrm{~N} \\
40.343 \mathrm{~N} \\
41.2 \mathrm{~N}\end{array}$ & $\begin{array}{l}121.533 \mathrm{~W} \\
124.67 \mathrm{~W} \\
119.039 \mathrm{~W} \\
124.102 \mathrm{~W} \\
125.5 \mathrm{~W}\end{array}$ & $\begin{array}{l}008 \\
023 \\
017 \\
\overline{033}\end{array}$ & $\begin{array}{r}324 \\
324 \\
458 \\
401 \\
74\end{array}$ & $\begin{array}{l}4.8 \\
4.9 \\
5.7 \\
4.3 \\
5.2\end{array}$ & $\begin{array}{l}\overline{4.8} \\
5.2 \\
-\end{array}$ & $\begin{array}{ll}4.80 \mathrm{M}_{\mathrm{L}} & \text { BRK } \\
4.70 \mathrm{M}_{\mathrm{L}} & \text { BRK } \\
5.90 \mathrm{M}_{\mathrm{L}} & \text { PAS } \\
4.50 \mathrm{M}_{\mathrm{L}} & \text { BRK } \\
5.00 \mathrm{M}_{\mathrm{L}} & \text { BRK }\end{array}$ & $\begin{array}{c}4.87 \mathrm{MM} \\
5.28 \mathrm{HK} \\
-\end{array}$ & $\begin{array}{c}\text { VI } \\
\text { V } \\
\text { VII } \\
\text { V }\end{array}$ & $\begin{array}{l}45 \\
45 \\
46 \\
46 \\
-\end{array}$ & $\begin{array}{l}9 \& \\
-52 \& \\
-\end{array}$ \\
\hline $\begin{array}{l}1973 \\
1973 \\
1973 \\
1973 \\
1973\end{array}$ & $\begin{array}{ll}07 & 14 \\
08 & 06 \\
08 & 09 \\
09 & 13 \\
10 & 03\end{array}$ & $\begin{array}{l}080020.0 \\
232916.6 \\
021824.6 \\
173039.8 \\
100727.3\end{array}$ & $\begin{array}{l}34.436 \mathrm{~N} \\
33.970 \mathrm{~N} \\
40.228 \mathrm{~N} \\
32.952 \mathrm{~N} \\
37.190 \mathrm{~N}\end{array}$ & $\begin{array}{l}116.833 W \\
119.478 W \\
124.415 W \\
116.279 W \\
121.590 W\end{array}$ & $\begin{array}{l}008 \\
013 \\
003 \\
008 \\
008\end{array}$ & $\begin{array}{r}74 \\
74 \\
401 \\
74 \\
401\end{array}$ & $\begin{array}{l}- \\
4.6 \\
5.1 \\
4.5 \\
4.6\end{array}$ & $\begin{array}{l}- \\
\overline{4} \\
-\end{array}$ & $\begin{array}{ll}4.80 \mathrm{M}_{\mathrm{L}} & \text { PAS } \\
5.00 \mathrm{M}_{\mathrm{L}} & \mathrm{PAS} \\
4.90 \mathrm{M}_{\mathrm{L}} & \mathrm{BRK} \\
4.80 \mathrm{M}_{\mathrm{L}} & \text { PAS } \\
4.70 \mathrm{M}_{\mathrm{L}} & \mathrm{BRK}\end{array}$ & $\frac{\bar{z}}{\bar{z}}$ & $\begin{array}{l}\text { V } \\
\text { V } \\
\text { VI } \\
\text { V } \\
\text { V }\end{array}$ & $\begin{array}{l}46 \\
46 \\
46 \\
46 \\
46\end{array}$ & $\begin{array}{l}10 \\
8 \& \\
8 \& \\
8 \# \\
9 \&\end{array}$ \\
\hline $\begin{array}{l}1973 \\
1973 \\
1973 \\
1974 \\
1974\end{array}$ & $\begin{array}{ll}10 & 28 \\
11 & 12 \\
12 & 21 \\
01 & 06 \\
03 & 03\end{array}$ & $\begin{array}{lll}22 & 00 & 02.7 \\
18 & 17 & 13.6 \\
19 & 12 & 44.0 \\
13 & 5523.0 \\
11 & 37 & 36.8\end{array}$ & $\begin{array}{l}32.680 \mathrm{~N} \\
37.203 \mathrm{~N} \\
40.578 \mathrm{~N} \\
41.050 \mathrm{~N} \\
41.9 \mathrm{~N}\end{array}$ & $\begin{array}{l}118.077 \mathrm{~W} \\
121.982 \mathrm{~W} \\
124.662 \mathrm{~W} \\
121.483 \mathrm{~W} \\
125.4 \mathrm{~W}\end{array}$ & $\begin{array}{l}008 \\
013 \\
013 \\
001 \\
033\end{array}$ & $\begin{array}{r}74 \\
401 \\
401 \\
401 \\
47\end{array}$ & $\begin{array}{l}4.2 \\
4.2 \\
5.2 \\
4.5 \\
5.1\end{array}$ & $\overline{4} \overline{-}$ & $\begin{array}{ll}4.50 \mathrm{M}_{\mathrm{L}} & \text { PAS } \\
4.50 \mathrm{M}_{\mathrm{L}} & \text { BRK } \\
4.80 \mathrm{M}_{\mathrm{L}} & \text { BRK } \\
4.20 \mathrm{M}_{\mathrm{L}} & \text { BRK } \\
4.40 \mathrm{M}_{\mathrm{L}} & \text { BRK }\end{array}$ & $\bar{z}$ & $\begin{array}{c}\text { IV } \\
\text { V } \\
\text { V } \\
\text { VI } \\
-\end{array}$ & $\begin{array}{l}46 \\
46 \\
46 \\
47 \\
-\end{array}$ & $\begin{array}{l}-9 \& \\
3 \& \\
3 \\
-\end{array}$ \\
\hline
\end{tabular}


CALIFORNIA-Continued

[See table 1 for hypocenter and intensity references and table 2 for definitions of magnitude source codes. \&, land area only; \#, land area only in the United States for an earthquake near a coastline; +, land area in the United States when the felt area did not extend to the coast; @, felt area is less than $1,000 \mathrm{~km}^{2}$. Leader (-) indicates information is not available]

\begin{tabular}{|c|c|c|c|c|c|c|c|c|c|c|c|c|c|}
\hline \multirow{2}{*}{\multicolumn{2}{|c|}{ Date }} & \multirow{3}{*}{$\begin{array}{c}\text { Origin } \\
\text { time (UTC) }\end{array}$} & \multicolumn{4}{|c|}{ Hypocenter } & \multicolumn{4}{|c|}{ Magnitude } & \multicolumn{2}{|c|}{ Intensity } & \multirow{3}{*}{$\begin{array}{c}\text { Felt area } \\
\left(1,000 \mathrm{~km}^{2}\right)\end{array}$} \\
\hline & & & \multirow{2}{*}{$\begin{array}{l}\text { Latitude } \\
\left.{ }^{\circ}\right)\end{array}$} & \multirow{2}{*}{$\begin{array}{c}\text { Longitude } \\
\left({ }^{\circ}\right)\end{array}$} & \multirow{2}{*}{$\begin{array}{c}\text { Depth } \\
(\mathrm{km})\end{array}$} & \multirow[t]{2}{*}{ Ref } & \multicolumn{2}{|c|}{ USGS } & \multirow[t]{2}{*}{ Other } & \multirow{2}{*}{$\begin{array}{c}\text { Moment } \\
\mathbf{M}\end{array}$} & \multirow[t]{2}{*}{ MMI } & \multirow[t]{2}{*}{ Ref } & \\
\hline$\overline{Y r}$ & Mo Da & & & & & & $m_{b}$ & $\mathbf{M}_{\mathbf{s}}$ & & & & & \\
\hline $\begin{array}{l}1974 \\
1974 \\
1974 \\
1974 \\
1974\end{array}$ & $\begin{array}{ll}03 & 16 \\
03 & 21 \\
07 & 03 \\
09 & 21 \\
11 & 28\end{array}$ & $\begin{array}{l}15 \quad 5740.7 \\
21 \quad 1605.3 \\
050058.6 \\
103741.8 \\
230124.7\end{array}$ & $\begin{array}{l}40.374 \mathrm{~N} \\
38.612 \mathrm{~N} \\
40.424 \mathrm{~N} \\
33.853 \mathrm{~N} \\
36.913 \mathrm{~N}\end{array}$ & $\begin{array}{l}124.714 \mathrm{~W} \\
122.663 \mathrm{~W} \\
125.136 \mathrm{~W} \\
117.110 \mathrm{~W} \\
121.478 \mathrm{~W}\end{array}$ & $\begin{array}{l}023 \\
001 \\
012 \\
013 \\
009\end{array}$ & $\begin{array}{r}299 \\
401 \\
74 \\
402 \\
401\end{array}$ & $\begin{array}{l}4.7 \\
3.8 \\
5.4 \\
4.2 \\
5.0\end{array}$ & $\overline{\overline{5}} \overline{\frac{7}{4.5}}$ & $\begin{array}{ll}4.50 \mathrm{M}_{\mathrm{L}} & \text { BRK } \\
3.30 \mathrm{M}_{\mathrm{L}} & \text { BRK } \\
5.10 \mathrm{M}_{\mathrm{L}} & \text { BRK } \\
3.90 \mathrm{M}_{\mathrm{L}} & \text { PAS } \\
5.20 \mathrm{M}_{\mathrm{L}} & \text { BRK }\end{array}$ & $\begin{array}{l}= \\
\bar{z} \\
-\end{array}$ & $\begin{array}{l}\text { IV } \\
\text { VI } \\
\text { V } \\
\text { VI } \\
\text { VI }\end{array}$ & $\begin{array}{l}47 \\
47 \\
47 \\
47 \\
47\end{array}$ & $\overline{-}$ \\
\hline $\begin{array}{l}1974 \\
1975 \\
1975 \\
1975 \\
1975\end{array}$ & $\begin{array}{ll}12 & 06 \\
01 & 06 \\
01 & 12 \\
01 & 12 \\
01 & 13\end{array}$ & $\begin{array}{lll}12 & 13 & 08.3 \\
11 & 17 & 12.3 \\
01 & 37 & 08.2 \\
21 & 22 & 15.0 \\
11 & 21 & 50.2\end{array}$ & $\begin{array}{l}32.708 \mathrm{~N} \\
35.931 \mathrm{~N} \\
40.328 \mathrm{~N} \\
32.815 \mathrm{~N} \\
33.815 \mathrm{~N}\end{array}$ & $\begin{array}{l}115.392 \mathrm{~W} \\
120.534 \mathrm{~W} \\
124.412 \mathrm{~W} \\
117.974 \mathrm{~W} \\
118.076 \mathrm{~W}\end{array}$ & $\begin{array}{l}024 \\
010 \\
033 \\
008 \\
007\end{array}$ & $\begin{array}{r}402 \\
74 \\
401 \\
355 \\
355\end{array}$ & $\begin{array}{l}4.5 \\
4.5 \\
4.7 \\
5.1 \\
3.8\end{array}$ & $\begin{array}{l}\overline{4.1} \\
-\end{array}$ & $\begin{array}{ll}4.50 \mathrm{M}_{\mathrm{L}} & \text { PAS } \\
4.50 \mathrm{M}_{\mathrm{L}} & \text { PAS } \\
4.50 \mathrm{M}_{\mathrm{L}} & \text { BRK } \\
4.80 \mathrm{M}_{\mathrm{L}} & \text { PAS } \\
3.60 \mathrm{M}_{\mathrm{L}} & \text { PAS }\end{array}$ & $\begin{array}{l}- \\
z\end{array}$ & $\begin{array}{l}\text { V } \\
\text { V } \\
\text { VI } \\
\text { IV } \\
\text { VI }\end{array}$ & $\begin{array}{l}47 \\
48 \\
48 \\
48 \\
48\end{array}$ & $\begin{array}{l}\overline{18} \& \\
7 \& \\
-2 \&\end{array}$ \\
\hline $\begin{array}{l}1975 \\
1975 \\
1975 \\
1975 \\
1975\end{array}$ & $\begin{array}{ll}01 & 21 \\
01 & 23 \\
01 & 23 \\
01 & 28 \\
03 & 03\end{array}$ & $\begin{array}{l}164734.7 \\
170230.3 \\
232434.8 \\
135316.4 \\
153545.0\end{array}$ & $\begin{array}{l}32.944 \mathrm{~N} \\
32.949 \mathrm{~N} \\
32.993 \mathrm{~N} \\
40.415 \mathrm{~N} \\
33.925 \mathrm{~N}\end{array}$ & $\begin{array}{l}115.492 \mathrm{~W} \\
115.504 \mathrm{~W} \\
115.506 \mathrm{~W} \\
125.446 \mathrm{~W} \\
118.302 \mathrm{~W}\end{array}$ & $\begin{array}{l}002 \\
005 \\
005 \\
010 \\
012\end{array}$ & $\begin{array}{r}355 \\
355 \\
355 \\
74 \\
355\end{array}$ & $\begin{array}{l}- \\
4.9 \\
4.3 \\
4.9 \\
-\end{array}$ & $\begin{array}{l}\overline{4.6} \\
\overline{5.0}\end{array}$ & $\begin{array}{ll}3.30 \mathrm{M}_{\mathrm{L}} & \text { PAS } \\
4.90 \mathrm{M}_{\mathrm{L}} & \text { PAS } \\
4.10 \mathrm{M}_{\mathrm{L}} & \text { PAS } \\
4.80 \mathrm{M}_{\mathrm{L}} & \text { BRK } \\
3.40 \mathrm{M}_{\mathrm{L}} & \text { PAS }\end{array}$ & $\frac{-}{z}$ & $\begin{array}{l}\text { VI } \\
\text { VI } \\
\text { VI } \\
\text { V } \\
\text { VI }\end{array}$ & $\begin{array}{l}48 \\
48 \\
48 \\
48 \\
48\end{array}$ & $\begin{array}{l}\overline{14+} \\
- \\
-\end{array}$ \\
\hline $\begin{array}{l}1975 \\
1975 \\
1975 \\
1975 \\
1975\end{array}$ & $\begin{array}{ll}05 & 13 \\
06 & 01 \\
06 & 07 \\
06 & 20 \\
08 & 01\end{array}$ & $\begin{array}{lll}00 & 21 & 35.7 \\
01 & 38 & 48.8 \\
08 & 4623.2 \\
05 & 48 & 24.0 \\
16 & 27 & 17.8\end{array}$ & $\begin{array}{l}35.000 \mathrm{~N} \\
34.517 \mathrm{~N} \\
40.538 \mathrm{~N} \\
32.780 \mathrm{~N} \\
39.438 \mathrm{~N}\end{array}$ & $\begin{array}{l}119.090 \mathrm{~W} \\
116.490 \mathrm{~W} \\
124.287 \mathrm{~W} \\
115.433 \mathrm{~W} \\
121.537 \mathrm{~W}\end{array}$ & $\begin{array}{l}017 \\
001 \\
022 \\
004 \\
005\end{array}$ & $\begin{array}{l}355 \\
355 \\
401 \\
355 \\
401\end{array}$ & $\begin{array}{l}4.6 \\
5.1 \\
5.4 \\
4.3 \\
4.8\end{array}$ & $\frac{\overline{5}}{\overline{5.7}}$ & $\begin{array}{ll}4.50 \mathrm{M}_{\mathrm{L}} & \text { PAS } \\
5.00 \mathrm{M}_{\mathrm{L}} & \text { PAS } \\
5.30 \mathrm{M}_{\mathrm{L}} & \text { BRK } \\
4.20 \mathrm{M}_{\mathrm{L}} & \text { PAS } \\
4.70 \mathrm{M}_{\mathrm{L}} & \text { BRK }\end{array}$ & $\begin{array}{l}- \\
-\end{array}$ & $\begin{array}{l}\text { V } \\
\text { VII } \\
\text { VII } \\
\text { VI } \\
\text { IV }\end{array}$ & $\begin{array}{l}48 \\
38 \\
48 \\
48 \\
48\end{array}$ & $\begin{array}{l}\frac{20}{23 \&} \\
- \\
-\end{array}$ \\
\hline $\begin{array}{l}1975 \\
1975 \\
1975 \\
1975 \\
1975\end{array}$ & $\begin{array}{ll}08 & 01 \\
08 & 01 \\
08 & 01 \\
08 & 01 \\
08 & 02\end{array}$ & $\begin{array}{l}202004.8 \\
202012.9 \\
2025 \\
2029 \\
001407.7\end{array}$ & $\begin{array}{l}39.439 \mathrm{~N} \\
39.439 \mathrm{~N} \\
39.439 \mathrm{~N} \\
39.439 \mathrm{~N} \\
33.513 \mathrm{~N}\end{array}$ & $\begin{array}{l}121.528 \mathrm{~W} \\
121.528 \mathrm{~W} \\
121.528 \mathrm{~W} \\
121.528 \mathrm{~W} \\
116.559 \mathrm{~W}\end{array}$ & $\frac{008}{\overline{-}}$ & $\begin{array}{l}401 \\
401 \\
401 \\
401 \\
401\end{array}$ & $\begin{array}{l}4.4 \\
5.8 \\
- \\
4.6\end{array}$ & $\begin{array}{l}5.6 \\
- \\
-\end{array}$ & $\begin{array}{ll}4.50 \mathrm{M}_{\mathrm{L}} & \text { BRK } \\
5.70 \mathrm{M}_{\mathrm{L}} & \text { BRK } \\
4.70 \mathrm{M}_{\mathrm{L}} & \text { BRK } \\
4.60 \mathrm{M}_{\mathrm{L}} & \text { BRK } \\
4.80 \mathrm{M}_{\mathrm{L}} & \text { PAS }\end{array}$ & $\begin{array}{c}\text { 6.14HBK } \\
\overline{-} \\
4.49 \mathrm{HB}\end{array}$ & $\begin{array}{l}\text { VIII } \\
\text { Felt } \\
\text { Felt } \\
\text { III }\end{array}$ & $\begin{array}{l}-38 \\
48 \\
48 \\
48\end{array}$ & $\begin{array}{l}\overline{120} \\
\overline{-} \\
-\end{array}$ \\
\hline $\begin{array}{l}1975 \\
1975 \\
1975 \\
1975 \\
1975\end{array}$ & $\begin{array}{ll}08 & 02 \\
08 & 02 \\
08 & 03 \\
08 & 03 \\
08 & 03\end{array}$ & $\begin{array}{l}202216.3 \\
2059 \\
010305.8 \\
063516.5 \\
0638\end{array}$ & $\begin{array}{l}39.445 \mathrm{~N} \\
39.432 \mathrm{~N} \\
39.488 \mathrm{~N} \\
36.457 \mathrm{~N} \\
36.457 \mathrm{~N}\end{array}$ & $\begin{array}{l}121.463 \mathrm{~W} \\
121.466 \mathrm{~W} \\
121.518 \mathrm{~W} \\
120.340 \mathrm{~W} \\
120.340 \mathrm{~W}\end{array}$ & $\begin{array}{l}004 \\
008 \\
005 \\
005\end{array}$ & $\begin{array}{l}401 \\
401 \\
401 \\
401 \\
401\end{array}$ & $\begin{array}{l}5.3 \\
5.2 \\
5.0 \\
5.1 \\
4.1\end{array}$ & $\begin{array}{l}4.5 \\
4.7 \\
4.0 \\
-\end{array}$ & $\begin{array}{ll}5.10 \mathrm{M}_{\mathrm{L}} & \text { BRK } \\
5.20 \mathrm{M}_{\mathrm{L}} & \text { BRK } \\
4.60 \mathrm{M}_{\mathrm{L}} & \text { BRK } \\
4.90 \mathrm{M}_{\mathrm{L}} & \text { BRK } \\
4.50 \mathrm{M}_{\mathrm{L}} & \text { BRK }\end{array}$ & $\frac{-}{-}$ & $\begin{array}{c}\text { Felt } \\
\text { VI } \\
\text { Felt } \\
\text { VI } \\
-\end{array}$ & $\begin{array}{l}48 \\
48 \\
48 \\
48 \\
\end{array}$ & $\frac{-}{18}$ \\
\hline $\begin{array}{l}1975 \\
1975 \\
1975 \\
1975 \\
1975\end{array}$ & $\begin{array}{ll}08 & 06 \\
08 & 08 \\
08 & 10 \\
08 & 11 \\
08 & 15\end{array}$ & $\begin{array}{l}035029.9 \\
070050.1 \\
051640.5 \\
061136.3 \\
222751.8\end{array}$ & $\begin{array}{l}39.479 \mathrm{~N} \\
39.502 \mathrm{~N} \\
37.370 \mathrm{~N} \\
39.446 \mathrm{~N} \\
36.497 \mathrm{~N}\end{array}$ & $\begin{array}{l}121.524 \mathrm{~W} \\
121.512 \mathrm{~W} \\
119.985 \mathrm{~W} \\
121.481 \mathrm{~W} \\
120.398 \mathrm{~W}\end{array}$ & $\begin{array}{l}008 \\
008 \\
007 \\
004 \\
006\end{array}$ & $\begin{array}{l}401 \\
401 \\
401 \\
401 \\
401\end{array}$ & $\begin{array}{l}5.1 \\
5.0 \\
4.0 \\
4.8 \\
4.5\end{array}$ & $\frac{4.0}{3.8}$ & $\begin{array}{ll}4.70 \mathrm{M}_{\mathrm{L}} & \text { BRK } \\
4.90 \mathrm{M}_{\mathrm{L}} & \text { BRK } \\
4.20 \mathrm{M}_{\mathrm{L}} & \text { BRK } \\
4.30 \mathrm{M}_{\mathrm{L}} & \text { BRK } \\
4.50 \mathrm{M}_{\mathrm{L}} & \text { BRK }\end{array}$ & $\begin{array}{l}- \\
-\end{array}$ & $\begin{array}{l}\text { IV } \\
\text { IV } \\
\text { VI } \\
\text { V } \\
\text { V }\end{array}$ & $\begin{array}{l}48 \\
48 \\
48 \\
48 \\
48\end{array}$ & $\begin{array}{l}- \\
\overline{26} \\
19\end{array}$ \\
\hline $\begin{array}{l}1975 \\
1975 \\
1975 \\
1975 \\
1975\end{array}$ & $\begin{array}{ll}09 & 09 \\
09 & 13 \\
09 & 27 \\
11 & 14 \\
11 & 15\end{array}$ & $\begin{array}{lll}02 & 43 & 42.5 \\
21 & 20 & 59.8 \\
22 & 34 & 38.1 \\
09 & 29 & 49.4 \\
06 & 13 & 27.6\end{array}$ & $\begin{array}{l}40.916 \mathrm{~N} \\
36.000 \mathrm{~N} \\
39.511 \mathrm{~N} \\
40.570 \mathrm{~N} \\
34.305 \mathrm{~N}\end{array}$ & $\begin{array}{l}124.397 \mathrm{~W} \\
120.558 \mathrm{~W} \\
121.537 \mathrm{~W} \\
124.436 \mathrm{~W} \\
116.335 \mathrm{~W}\end{array}$ & $\begin{array}{l}027 \\
014 \\
008 \\
022 \\
005\end{array}$ & $\begin{array}{r}74 \\
401 \\
401 \\
401 \\
355\end{array}$ & $\begin{array}{l}4.9 \\
4.9 \\
5.3 \\
4.9 \\
4.6\end{array}$ & $\begin{array}{l}-\overline{4.3} \\
3.5 \\
4.5 \\
-\end{array}$ & $\begin{array}{ll}4.60 \mathrm{M}_{\mathrm{L}} & \text { BRK } \\
4.80 \mathrm{M}_{\mathrm{L}} & \text { BRK } \\
4.60 \mathrm{M}_{\mathrm{L}} & \text { BRK } \\
4.80 \mathrm{M}_{\mathrm{L}} & \text { BRK } \\
4.60 \mathrm{M}_{\mathrm{L}} & \text { BRK }\end{array}$ & $\begin{array}{l}z \\
z\end{array}$ & $\begin{array}{l}\text { V } \\
\text { VI } \\
\text { V } \\
\text { VI } \\
\text { IV }\end{array}$ & $\begin{array}{l}48 \\
48 \\
48 \\
48 \\
48\end{array}$ & $\begin{array}{l}\overline{16} \& \\
= \\
-\end{array}$ \\
\hline $\begin{array}{l}1976 \\
1976 \\
1976 \\
1976 \\
1976\end{array}$ & $\begin{array}{ll}01 & 01 \\
01 & 14 \\
01 & 20 \\
04 & 08 \\
06 & 20\end{array}$ & $\begin{array}{lll}17 & 20 & 12.9 \\
21 & 43 & 59.3 \\
13 & 59 & 37.2 \\
15 & 21 & 37.9 \\
10 & 15 & 24.8\end{array}$ & $\begin{array}{l}33.966 \mathrm{~N} \\
36.108 \mathrm{~N} \\
40.384 \mathrm{~N} \\
34.357 \mathrm{~N} \\
40.427 \mathrm{~N}\end{array}$ & $\begin{array}{l}117.897 \mathrm{~W} \\
120.162 \mathrm{~W} \\
125.336 \mathrm{~W} \\
118.669 \mathrm{~W} \\
120.568 \mathrm{~W}\end{array}$ & $\begin{array}{l}005 \\
005 \\
033 \\
017 \\
005\end{array}$ & $\begin{array}{r}355 \\
401 \\
74 \\
355 \\
355\end{array}$ & $\begin{array}{l}4.6 \\
5.1 \\
4.8 \\
4.7 \\
4.4\end{array}$ & $\overline{-}$ & $\begin{array}{ll}4.30 \mathrm{M}_{\mathrm{L}} & \text { PAS } \\
4.90 \mathrm{M}_{\mathrm{L}} & \text { BRK } \\
4.60 \mathrm{M}_{\mathrm{L}} & \text { BRK } \\
4.50 \mathrm{M}_{\mathrm{L}} & \text { PAS } \\
4.50 \mathrm{M}_{\mathrm{L}} & \text { BRK }\end{array}$ & $\begin{array}{l}- \\
-\end{array}$ & $\begin{array}{l}\text { VI } \\
\text { VI } \\
\text { IV } \\
\text { VI } \\
\text { V }\end{array}$ & $\begin{array}{l}49 \\
49 \\
49 \\
49 \\
49\end{array}$ & $\begin{array}{l}68 \\
\frac{34}{18} \\
-\end{array}$ \\
\hline $\begin{array}{l}1976 \\
1976 \\
1976 \\
1976 \\
1976\end{array}$ & $\begin{array}{ll}08 & 11 \\
08 & 20 \\
10 & 17 \\
11 & 04 \\
11 & 22\end{array}$ & $\begin{array}{lll}1524 & 55.4 \\
22 & 05 & 53.0 \\
05 & 38 & 11.3 \\
1041 & 37.5 \\
1755 & 11.5\end{array}$ & $\begin{array}{l}33.482 \mathrm{~N} \\
37.787 \mathrm{~N} \\
34.462 \mathrm{~N} \\
33.131 \mathrm{~N} \\
33.933 \mathrm{~N}\end{array}$ & $\begin{array}{l}116.513 \mathrm{~W} \\
121.980 \mathrm{~W} \\
118.426 \mathrm{~W} \\
115.623 \mathrm{~W} \\
118.628 \mathrm{~W}\end{array}$ & $\begin{array}{l}015 \\
004 \\
016 \\
001 \\
010\end{array}$ & $\begin{array}{l}355 \\
401 \\
355 \\
355 \\
355\end{array}$ & $\begin{array}{l}- \\
4.3 \\
4.6 \\
-\end{array}$ & $\overline{-}$ & $\begin{array}{ll}4.30 \mathrm{M}_{\mathrm{L}} & \text { PAS } \\
4.00 \mathrm{M}_{\mathrm{L}} & \text { BRK } \\
3.90 \mathrm{M}_{\mathrm{L}} & \text { PAS } \\
5.10 \mathrm{M}_{\mathrm{L}} & \text { PAS } \\
4.20 \mathrm{M}_{\mathrm{L}} & \text { PAS }\end{array}$ & $\frac{\bar{Z}}{4.93 \mathrm{HH}}$ & $\begin{array}{l}\text { VI } \\
\text { VI } \\
\text { VI } \\
\text { VI } \\
\text { VI }\end{array}$ & $\begin{array}{l}49 \\
49 \\
49 \\
49 \\
49\end{array}$ & $\begin{array}{c}\frac{15}{8} \\
25+ \\
4 \&\end{array}$ \\
\hline $\begin{array}{l}1976 \\
1976 \\
1977 \\
1977 \\
1977\end{array}$ & $\begin{array}{ll}11 & 26 \\
12 & 23 \\
01 & 08 \\
02 & 22 \\
06 & 21\end{array}$ & $\begin{array}{lll}11 & 19 & 25.2 \\
09 & 38 & 58.4 \\
09 & 38 & 07.5 \\
06 & 24 & 06.1 \\
02 & 43 & 06.6\end{array}$ & $\begin{array}{l}41.289 \mathrm{~N} \\
41.783 \mathrm{~N} \\
37.905 \mathrm{~N} \\
38.480 \mathrm{~N} \\
37.665 \mathrm{~N}\end{array}$ & $\begin{array}{l}125.709 \mathrm{~W} \\
125.953 \mathrm{~W} \\
122.183 \mathrm{~W} \\
119.283 \mathrm{~W} \\
121.670 \mathrm{~W}\end{array}$ & $\begin{array}{l}015 \\
015 \\
009 \\
022 \\
011\end{array}$ & $\begin{array}{r}74 \\
74 \\
401 \\
355 \\
401\end{array}$ & $\begin{array}{l}6.0 \\
5.5 \\
4.8 \\
5.0 \\
4.7\end{array}$ & $\begin{array}{l}6.8 \\
5.5 \\
- \\
3.5\end{array}$ & $\begin{array}{ll}6.30 \mathrm{M}_{\mathrm{L}} & \text { BRK } \\
5.10 \mathrm{M}_{\mathrm{L}} & \text { BRK } \\
4.30 \mathrm{M}_{\mathrm{L}} & \text { BRK } \\
4.80 \mathrm{M}_{\mathrm{L}} & \text { BRK } \\
4.40 \mathrm{M}_{\mathrm{L}} & \text { BRK }\end{array}$ & $\frac{\bar{Z}}{\bar{Z}}$ & $\begin{array}{l}\mathrm{V} \\
\mathbf{V} \\
\mathbf{V} \\
\mathbf{V I}\end{array}$ & $\begin{array}{l}49 \\
39 \\
39 \\
39\end{array}$ & $\begin{array}{l}6 \& \\
\frac{7}{22} \\
16 \&\end{array}$ \\
\hline
\end{tabular}


CALIFORNIA-Continued

[See table 1 for hypocenter and intensity references and table 2 for definitions of magnitude source codes. \&, land area only; \#, land area only in the United States for an earthquake near a coastline; +, land area in the United States when the felt area did not extend to the coast; @, felt area is less than $1,000 \mathrm{~km}^{2}$. Leader (--) indicates information is not available]

\begin{tabular}{|c|c|c|c|c|c|c|c|c|c|c|c|c|c|c|}
\hline & & & Origin & & Hypocente & & & & & Magnitude & & Inte & sity & \\
\hline & Date & & time (UTC) & Latitude & Longitude & Depth & Ref & & & Other & Moment & MMI & Ref & Felt area \\
\hline$Y_{r}$ & Mo [ & & $h \mathrm{~m} s$ & & o) & (m) & & $m_{b}$ & $M_{s}$ & & 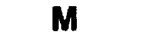 & & &, $\left.000 \mathrm{~km}^{2}\right)$ \\
\hline $\begin{array}{l}1977 \\
1977 \\
1977 \\
1977 \\
1977\end{array}$ & $\begin{array}{l}07 \\
07 \\
08 \\
10 \\
11\end{array}$ & $\begin{array}{l}12 \\
18 \\
12 \\
21 \\
14\end{array}$ & $\begin{array}{lll}01 & 43 & 28.5 \\
21 & 49 & 28.6 \\
02 & 19 & 26.1 \\
06 & 12 & 36.0 \\
02 & 05 & 48.3\end{array}$ & $\begin{array}{l}40.277 \mathrm{~N} \\
40.380 \mathrm{~N} \\
34.380 \mathrm{~N} \\
32.896 \mathrm{~N} \\
32.830 \mathrm{~N}\end{array}$ & $\begin{array}{l}123.692 \mathrm{~W} \\
125.361 \mathrm{~W} \\
118.459 \mathrm{~W} \\
115.505 \mathrm{~W} \\
115.478 \mathrm{~W}\end{array}$ & $\begin{array}{l}020 \\
015 \\
010 \\
005 \\
004\end{array}$ & $\begin{array}{r}401 \\
74 \\
355 \\
355 \\
355\end{array}$ & $\begin{array}{l}5.0 \\
4.8 \\
4.1 \\
3.7 \\
5.0\end{array}$ & $\begin{array}{l}3.8 \\
3.9 \\
- \\
-\end{array}$ & $\begin{array}{ll}4.10 \mathrm{M}_{\mathrm{L}} & \text { BRK } \\
4.70 \mathrm{M}_{\mathrm{L}} & \text { BRK } \\
4.50 \mathrm{M}_{\mathrm{L}} & \text { PAS } \\
4.10 \mathrm{M}_{\mathrm{L}} & \text { PAS } \\
4.10 \mathrm{M}_{\mathrm{L}} & \text { PAS }\end{array}$ & $\bar{z}$ & $\begin{array}{l}\mathrm{V} \\
\mathrm{VI} \\
\text { VI } \\
\text { VI }\end{array}$ & $\begin{array}{l}39 \\
39 \\
39 \\
39\end{array}$ & $\begin{array}{l}\bar{z} \\
\overline{7} \\
-\end{array}$ \\
\hline $\begin{array}{l}1977 \\
1978 \\
1978 \\
1978 \\
1978\end{array}$ & $\begin{array}{l}11 \\
03 \\
05 \\
08 \\
08\end{array}$ & $\begin{array}{l}22 \\
26 \\
23 \\
01 \\
01\end{array}$ & $\begin{array}{lll}21 & 15 & 53.9 \\
00 & 27 & 04.4 \\
21 & 42 & 02.9 \\
09 & 02 & 34.5 \\
09 & 46 & 44.6\end{array}$ & $\begin{array}{l}39.403 \mathrm{~N} \\
39.233 \mathrm{~N} \\
40.438 \mathrm{~N} \\
41.453 \mathrm{~N} \\
41.457 \mathrm{~N}\end{array}$ & $\begin{array}{l}123.273 \mathrm{~W} \\
123.233 \mathrm{~W} \\
124.850 \mathrm{~W} \\
121.875 \mathrm{~W} \\
121.870 \mathrm{~W}\end{array}$ & $\begin{array}{l}018 \\
010 \\
020 \\
002 \\
002\end{array}$ & $\begin{array}{l}401 \\
613 \\
401 \\
401 \\
401\end{array}$ & $\begin{array}{l}5.2 \\
4.9 \\
4.4 \\
4.5 \\
4.3\end{array}$ & $\bar{z}$ & $\begin{array}{ll}4.80 \mathrm{M}_{\mathrm{L}} & \text { BRK } \\
4.50 \mathrm{M}_{\mathrm{L}} & \text { BRK } \\
4.60 \mathrm{M}_{\mathrm{L}} & \text { BRK } \\
4.60 \mathrm{M}_{\mathrm{L}} & \text { BRK } \\
4.50 \mathrm{M}_{\mathrm{L}} & \text { BRK }\end{array}$ & $\bar{z}$ & $\begin{array}{c}\text { VII } \\
\text { VI } \\
\text { IV } \\
\text { V } \\
\text { Felt }\end{array}$ & $\begin{array}{r}39 \\
240 \\
240 \\
240 \\
401\end{array}$ & $\begin{array}{c}158 \\
8 \& \\
- \\
-\end{array}$ \\
\hline $\begin{array}{l}1978 \\
1978 \\
1978 \\
1978 \\
1978\end{array}$ & $\begin{array}{l}08 \\
08 \\
09 \\
09 \\
09\end{array}$ & $\begin{array}{l}13 \\
29 \\
04 \\
04 \\
25\end{array}$ & $\begin{array}{lll}22 & 54 & 52.8 \\
00 & 14 & 46.4 \\
04 & 52 & 32.3 \\
21 & 54 & 53.2 \\
02 & 10 & 51.0\end{array}$ & $\begin{array}{l}34.397 \mathrm{~N} \\
37.362 \mathrm{~N} \\
38.818 \mathrm{~N} \\
38.813 \mathrm{~N} \\
41.062 \mathrm{~N}\end{array}$ & $\begin{array}{l}119.682 \mathrm{~W} \\
121.737 \mathrm{~W} \\
119.810 \mathrm{~W} \\
119.815 \mathrm{~W} \\
125.380 \mathrm{~W}\end{array}$ & $\begin{array}{l}013 \\
008 \\
018 \\
019 \\
005\end{array}$ & $\begin{array}{l}468 \\
401 \\
401 \\
401 \\
401\end{array}$ & $\begin{array}{l}5.5 \\
3.9 \\
4.7 \\
4.6\end{array}$ & $\frac{5.6}{-}$ & $\begin{array}{ll}5.10 \mathrm{M}_{\mathrm{L}} & \text { PAS } \\
4.10 \mathrm{M}_{\mathrm{L}} & \text { BRK } \\
4.60 \mathrm{M}_{\mathrm{L}} & \text { BRK } \\
5.30 \mathrm{M}_{\mathrm{L}} & \text { BRK } \\
4.60 \mathrm{M}_{\mathrm{L}} & \text { BRK }\end{array}$ & $\begin{array}{c}\text { 5.79ED } \\
- \\
-\end{array}$ & $\begin{array}{l}\text { VII } \\
\text { VI } \\
\text { V } \\
\text { VI } \\
-\end{array}$ & $\begin{array}{r}240 \\
240 \\
240 \\
240 \\
-\end{array}$ & $\begin{array}{l}25 \& \\
13 \& \\
16 \\
45 \\
-\end{array}$ \\
\hline $\begin{array}{l}1978 \\
1978 \\
1978 \\
1978 \\
1978\end{array}$ & $\begin{array}{l}10 \\
10 \\
10 \\
10 \\
11\end{array}$ & $\begin{array}{l}04 \\
04 \\
05 \\
06 \\
20\end{array}$ & $\begin{array}{l}164248.6 \\
173903.4 \\
064130.2 \\
212634.4 \\
065509.1\end{array}$ & $\begin{array}{l}37.510 \mathrm{~N} \\
37.535 \mathrm{~N} \\
37.485 \mathrm{~N} \\
40.375 \mathrm{~N} \\
34.156 \mathrm{~N}\end{array}$ & $\begin{array}{l}118.693 W \\
118.683 W \\
118.664 W \\
124.273 W \\
116.978 W\end{array}$ & $\begin{array}{l}005 \\
007 \\
011 \\
020 \\
015\end{array}$ & $\begin{array}{l}355 \\
355 \\
355 \\
401 \\
355\end{array}$ & $\begin{array}{l}5.4 \\
5.0 \\
4.8 \\
4.0\end{array}$ & $\frac{5.1}{4.2}$ & $\begin{array}{ll}5.70 \mathrm{M}_{\mathrm{L}} & \text { PAS } \\
5.30 \mathrm{M}_{\mathrm{L}} & \text { PAS } \\
4.50 \mathrm{M}_{\mathrm{L}} & \text { PAS } \\
4.60 \mathrm{M}_{\mathrm{L}} & \text { BRK } \\
4.30 \mathrm{M}_{\mathrm{L}} & \text { PAS }\end{array}$ & $\begin{array}{c}\text { 5.45ED } \\
- \\
- \\
-\end{array}$ & $\begin{array}{c}\text { VI } \\
\text { Felt } \\
\text { V } \\
\text { VI }\end{array}$ & $\begin{array}{l}240 \\
355 \\
240 \\
240\end{array}$ & $\frac{105}{\overline{12} \&}$ \\
\hline $\begin{array}{l}1979 \\
1979 \\
1979 \\
1979 \\
1979\end{array}$ & $\begin{array}{l}01 \\
01 \\
02 \\
02 \\
03\end{array}$ & $\begin{array}{l}01 \\
24 \\
03 \\
22 \\
15\end{array}$ & $\begin{array}{lll}23 & 14 & 38.9 \\
21 & 14 & 27.2 \\
09 & 58 & 16.0 \\
15 & 57 & 28.8 \\
20 & 17 & 49.3\end{array}$ & $\begin{array}{l}33.948 \mathrm{~N} \\
37.520 \mathrm{~N} \\
40.923 \mathrm{~N} \\
40.000 \mathrm{~N} \\
34.305 \mathrm{~N}\end{array}$ & $\begin{array}{l}118.688 W \\
118.593 W \\
124.418 W \\
120.088 W \\
116.439 W\end{array}$ & $\begin{array}{l}010 \\
010 \\
022 \\
005 \\
000\end{array}$ & $\begin{array}{l}474 \\
401 \\
401 \\
401 \\
355\end{array}$ & $\begin{array}{l}5.1 \\
5.2 \\
5.0 \\
5.0\end{array}$ & $\begin{array}{l}4.7 \\
4.6 \\
4.6 \\
4.9\end{array}$ & $\begin{array}{ll}4.80 \mathrm{M}_{\mathrm{L}} & \text { PAS } \\
4.60 \mathrm{M}_{\mathrm{L}} & \text { BRK } \\
5.20 \mathrm{M}_{\mathrm{L}} & \text { BRK } \\
5.30 \mathrm{M}_{\mathrm{L}} & \text { BRK } \\
5.00 \mathrm{M}_{\mathrm{L}} & \text { PAS }\end{array}$ & $\begin{array}{l}z \\
-\end{array}$ & $\begin{array}{c}\text { VI } \\
\text { IV } \\
\text { VII } \\
\text { VI } \\
\text { Felt }\end{array}$ & $\begin{array}{l}262 \\
262 \\
262 \\
262 \\
262\end{array}$ & $\begin{array}{l}21 \& \\
28 \\
11 \& \\
46 \\
-\end{array}$ \\
\hline $\begin{array}{l}1979 \\
1979 \\
1979 \\
1979 \\
1979\end{array}$ & $\begin{array}{l}03 \\
03 \\
03 \\
04 \\
05\end{array}$ & $\begin{array}{l}15 \\
15 \\
15 \\
07 \\
08\end{array}$ & $\begin{array}{lll}21 & 07 & 16.5 \\
21 & 34 & 25.5 \\
23 & 07 & 58.0 \\
06 & 18 & 33.0 \\
05 & 11 & 07.7\end{array}$ & $\begin{array}{l}34.325 \mathrm{~N} \\
34.347 \mathrm{~N} \\
34.336 \mathrm{~N} \\
41.987 \mathrm{~N} \\
37.303 \mathrm{~N}\end{array}$ & $\begin{array}{l}116.444 \mathrm{~W} \\
116.448 \mathrm{~W} \\
116.440 \mathrm{~W} \\
126.816 \mathrm{~W} \\
121.683 \mathrm{~W}\end{array}$ & $\begin{array}{l}001 \\
000 \\
002 \\
015 \\
006\end{array}$ & $\begin{array}{r}355 \\
355 \\
355 \\
74 \\
401\end{array}$ & $\begin{array}{l}5.5 \\
4.5 \\
5.5 \\
4.3\end{array}$ & $\begin{array}{l}5.6 \\
4.4 \\
5.3 \\
4.0\end{array}$ & $\begin{array}{ll}5.30 \mathrm{M}_{\mathrm{L}} & \text { PAS } \\
4.50 \mathrm{M}_{\mathrm{L}} & \text { PAS } \\
4.90 \mathrm{M}_{\mathrm{L}} & \text { PAS } \\
5.00 \mathrm{M}_{\mathrm{L}} & \text { BRK } \\
4.80 \mathrm{M}_{\mathrm{L}} & \text { BRK }\end{array}$ & $\begin{array}{c}\text { 5.52ED } \\
-\overline{-} \\
5.62 \mathrm{ED} \\
-\end{array}$ & $\begin{array}{l}\text { VII } \\
\text { Felt } \\
\text { Felt } \\
\overline{\text { VI }}\end{array}$ & $\begin{array}{l}262 \\
262 \\
262 \\
\frac{262}{2}\end{array}$ & $\frac{77}{\overline{-}}$ \\
\hline $\begin{array}{l}1979 \\
1979 \\
1979 \\
1979 \\
1979\end{array}$ & $\begin{array}{l}06 \\
06 \\
06 \\
06 \\
08\end{array}$ & $\begin{array}{l}14 \\
29 \\
30 \\
30 \\
06\end{array}$ & $\begin{array}{l}073928.3 \\
055320.3 \\
003411.5 \\
070352.8 \\
170522.7\end{array}$ & $\begin{array}{l}35.729 \mathrm{~N} \\
34.246 \mathrm{~N} \\
34.245 \mathrm{~N} \\
34.249 \mathrm{~N} \\
37.102 \mathrm{~N}\end{array}$ & $\begin{array}{l}118.023 \mathrm{~W} \\
116.898 \mathrm{~W} \\
116.891 \mathrm{~W} \\
116.896 \mathrm{~W} \\
121.503 \mathrm{~W}\end{array}$ & $\begin{array}{l}005 \\
009 \\
010 \\
010 \\
006\end{array}$ & $\begin{array}{l}355 \\
472 \\
472 \\
472 \\
401\end{array}$ & $\begin{array}{l}4.2 \\
4.1 \\
4.6 \\
4.0 \\
5.4\end{array}$ & $\bar{z}$ & $\begin{array}{ll}4.60 \mathrm{M}_{\mathrm{L}} & \text { PAS } \\
4.60 \mathrm{M}_{\mathrm{L}} & \text { WK } \\
4.90 \mathrm{M}_{\mathrm{L}} & \text { WK } \\
4.50 \mathrm{M}_{\mathrm{L}} & \text { WK } \\
5.90 \mathrm{M}_{\mathrm{L}} & \mathrm{BRK}\end{array}$ & $\overline{\bar{Z}} \overline{\bar{Z}}$ & $\begin{array}{l}\text { VI } \\
\text { VI } \\
\text { VI } \\
\text { Felt } \\
\text { VII }\end{array}$ & $\begin{array}{l}262 \\
262 \\
262 \\
262 \\
262\end{array}$ & $\begin{array}{l}4 \\
13 \\
20 \\
638\end{array}$ \\
\hline $\begin{array}{l}1979 \\
1979 \\
1979 \\
1979 \\
1979\end{array}$ & $\begin{array}{l}10 \\
10 \\
10 \\
10 \\
10\end{array}$ & $\begin{array}{l}07 \\
08 \\
15 \\
15 \\
16\end{array}$ & $\begin{array}{lll}20 & 54 & 41.4 \\
03 & 34 & 24.0 \\
23 & 16 & 54.1 \\
23 & 19 & 28.7 \\
01 & 00 & 14.0\end{array}$ & $\begin{array}{l}38.223 \mathrm{~N} \\
38.205 \mathrm{~N} \\
32.634 \mathrm{~N} \\
32.748 \mathrm{~N} \\
32.877 \mathrm{~N}\end{array}$ & $\begin{array}{l}119.355 \mathrm{~W} \\
119.323 \mathrm{~W} \\
115.324 \mathrm{~W} \\
115.586 \mathrm{~W} \\
115.556 \mathrm{~W}\end{array}$ & $\begin{array}{l}009 \\
009 \\
010 \\
005 \\
005\end{array}$ & $\begin{array}{l}355 \\
355 \\
355 \\
355 \\
355\end{array}$ & $\frac{4.1}{\frac{5.7}{4.3}}$ & $\frac{-}{6.9}$ & $\begin{array}{ll}5.00 \mathrm{M}_{\mathrm{L}} & \text { BRK } \\
4.60 \mathrm{M}_{\mathrm{L}} & \text { BRK } \\
6.40 \mathrm{M}_{\mathrm{L}} & \text { PAS } \\
5.00 \mathrm{M}_{\mathrm{L}} & \text { PAS } \\
4.70 \mathrm{M}_{\mathrm{L}} & \text { PAS }\end{array}$ & $\begin{array}{l}\overline{-} \\
\text { 6.49ED } \\
\text { 5.03FRK } \\
-\end{array}$ & $\begin{array}{r}\text { IV } \\
\text { Felt } \\
\text { IX } \\
- \\
-\end{array}$ & $\begin{array}{r}262 \\
262 \\
262 \\
= \\
-\end{array}$ & $\frac{15}{128 \#}$ \\
\hline $\begin{array}{l}1979 \\
1979 \\
1979 \\
1979 \\
1979\end{array}$ & $\begin{array}{l}10 \\
10 \\
10 \\
10 \\
10\end{array}$ & $\begin{array}{l}16 \\
16 \\
16 \\
16 \\
16\end{array}$ & $\begin{array}{l}031047.8 \\
033935.0 \\
054911.0 \\
061949.2 \\
065523.5\end{array}$ & $\begin{array}{l}32.951 \mathrm{~N} \\
32.949 \mathrm{~N} \\
32.941 \mathrm{~N} \\
32.937 \mathrm{~N} \\
32.988 \mathrm{~N}\end{array}$ & $\begin{array}{l}115.535 \mathrm{~W} \\
115.550 \mathrm{~W} \\
115.538 \mathrm{~W} \\
115.532 \mathrm{~W} \\
115.541 \mathrm{~W}\end{array}$ & $\begin{array}{l}004 \\
005 \\
005 \\
002 \\
004\end{array}$ & $\begin{array}{l}355 \\
355 \\
355 \\
355 \\
355\end{array}$ & $\begin{array}{l}4.5 \\
4.4 \\
4.9 \\
4.8 \\
4.3\end{array}$ & $\bar{z}$ & $\begin{array}{ll}4.60 \mathrm{M}_{\mathrm{L}} & \text { PAS } \\
4.60 \mathrm{M}_{\mathrm{L}} & \text { PAS } \\
5.10 \mathrm{M}_{\mathrm{L}} & \text { PAS } \\
5.10 \mathrm{M}_{\mathrm{L}} & \text { PAS } \\
4.70 \mathrm{M}_{\mathrm{L}} & \text { PAS }\end{array}$ & $\frac{\bar{Z}}{5.16 \mathrm{ED}}$ & $\begin{array}{l}- \\
z\end{array}$ & $\begin{array}{l}\bar{z} \\
\overline{-}\end{array}$ & $\begin{array}{l}- \\
- \\
-\end{array}$ \\
\hline $\begin{array}{l}1979 \\
1979 \\
1979 \\
1979 \\
1979\end{array}$ & $\begin{array}{l}10 \\
10 \\
10 \\
10 \\
10\end{array}$ & $\begin{array}{l}16 \\
16 \\
16 \\
17 \\
24\end{array}$ & $\begin{array}{l}065843.2 \\
114656.1 \\
231632.2 \\
224533.8 \\
152350.6\end{array}$ & $\begin{array}{l}32.999 \mathrm{~N} \\
32.913 \mathrm{~N} \\
33.022 \mathrm{~N} \\
33.040 \mathrm{~N} \\
40.428 \mathrm{~N}\end{array}$ & $\begin{array}{l}115.569 \mathrm{~W} \\
115.560 \mathrm{~W} \\
115.506 \mathrm{~W} \\
115.503 \mathrm{~W} \\
124.703 \mathrm{~W}\end{array}$ & $\begin{array}{l}001 \\
005 \\
003 \\
002 \\
024\end{array}$ & $\begin{array}{l}355 \\
355 \\
355 \\
355 \\
401\end{array}$ & $\begin{array}{l}5.2 \\
4.5 \\
5.4 \\
4.8 \\
4.8\end{array}$ & $\begin{array}{l}5.7 \\
4.8 \\
-\end{array}$ & $\begin{array}{ll}5.50 \mathrm{M}_{\mathrm{L}} & \text { PAS } \\
4.80 \mathrm{M}_{\mathrm{L}} & \text { PAS } \\
5.00 \mathrm{M}_{\mathrm{L}} & \text { PAS } \\
4.70 \mathrm{M}_{\mathrm{L}} & \text { PAS } \\
4.50 \mathrm{M}_{\mathrm{L}} & \text { BRK }\end{array}$ & $\begin{array}{c}\text { 4.96ED } \\
- \\
- \\
-\end{array}$ & $\frac{\text { VI }}{\text { Felt }}$ & $\frac{262}{\overline{-}}$ & $\begin{array}{l}- \\
- \\
-\end{array}$ \\
\hline $\begin{array}{l}1979 \\
1979 \\
1980\end{array}$ & $\begin{array}{l}11 \\
12 \\
01\end{array}$ & $\begin{array}{l}08 \\
21 \\
24\end{array}$ & $\begin{array}{l}043027.9 \\
204023.4 \\
190009.5\end{array}$ & $\begin{array}{l}40.323 \mathrm{~N} \\
32.483 \mathrm{~N} \\
37.855 \mathrm{~N}\end{array}$ & $\begin{array}{l}125.173 W \\
115.194 W \\
121.816 W\end{array}$ & $\begin{array}{l}005 \\
005 \\
012\end{array}$ & $\begin{array}{l}401 \\
355 \\
466\end{array}$ & $\begin{array}{l}4.7 \\
4.5 \\
5.3\end{array}$ & $\frac{3.7}{5.9}$ & $\begin{array}{ll}4.50 \mathrm{M}_{\mathrm{L}} & \text { BRK } \\
4.80 \mathrm{M}_{\mathrm{L}} & \text { PAS } \\
5.80 \mathrm{M}_{\mathrm{L}} & \text { BRK }\end{array}$ & $\overline{\overline{7}} \overline{\mathrm{BmMU}}$ & $\underset{\text { VII }}{\overline{\text { VI }}}$ & $\begin{array}{l}\overline{262} \\
300\end{array}$ & $\begin{array}{l}\overline{10+} \\
75 \&\end{array}$ \\
\hline
\end{tabular}


CALIFORNIA-Continued

[See table 1 for hypocenter and intensity references and table 2 for definitions of magnitude source codes. \&, land area only; \#, land area only in the United States for an earthquake near a coastline; +, land area in the United States when the felt area did not extend to the coast; (-), felt area is less than $1,000 \mathrm{~km}^{2}$. Leader (--) indicates information is not available]

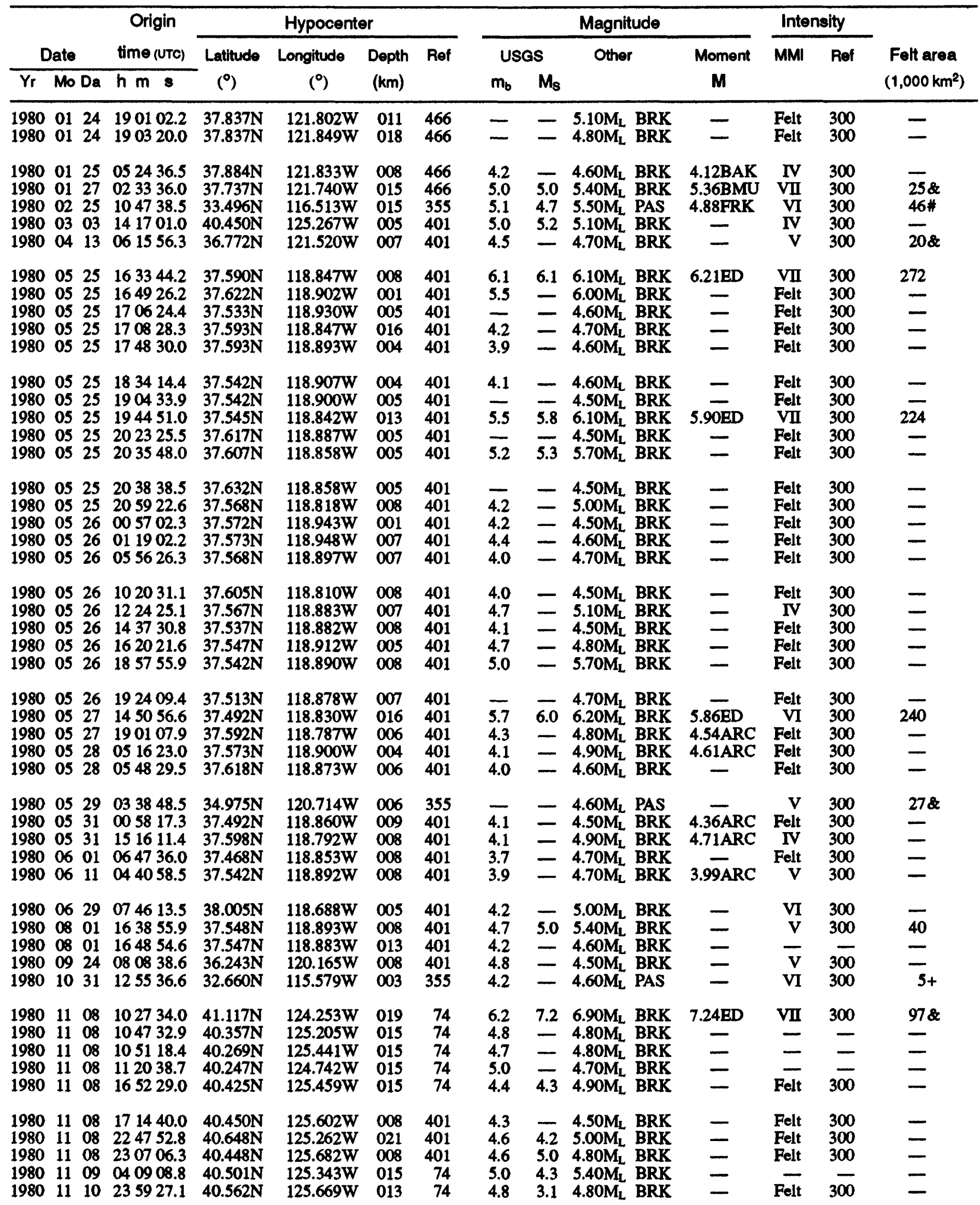


[See table 1 for hypocenter and intensity references and table 2 for definitions of magnitude source codes. \&, land area only; \#, land area only in the United States for an earthquake near a coastline; + , land area in the United States when the felt area did not extend to the coast; @, felt area is less than 1,000 km². Leader (--) indicates information is not available]

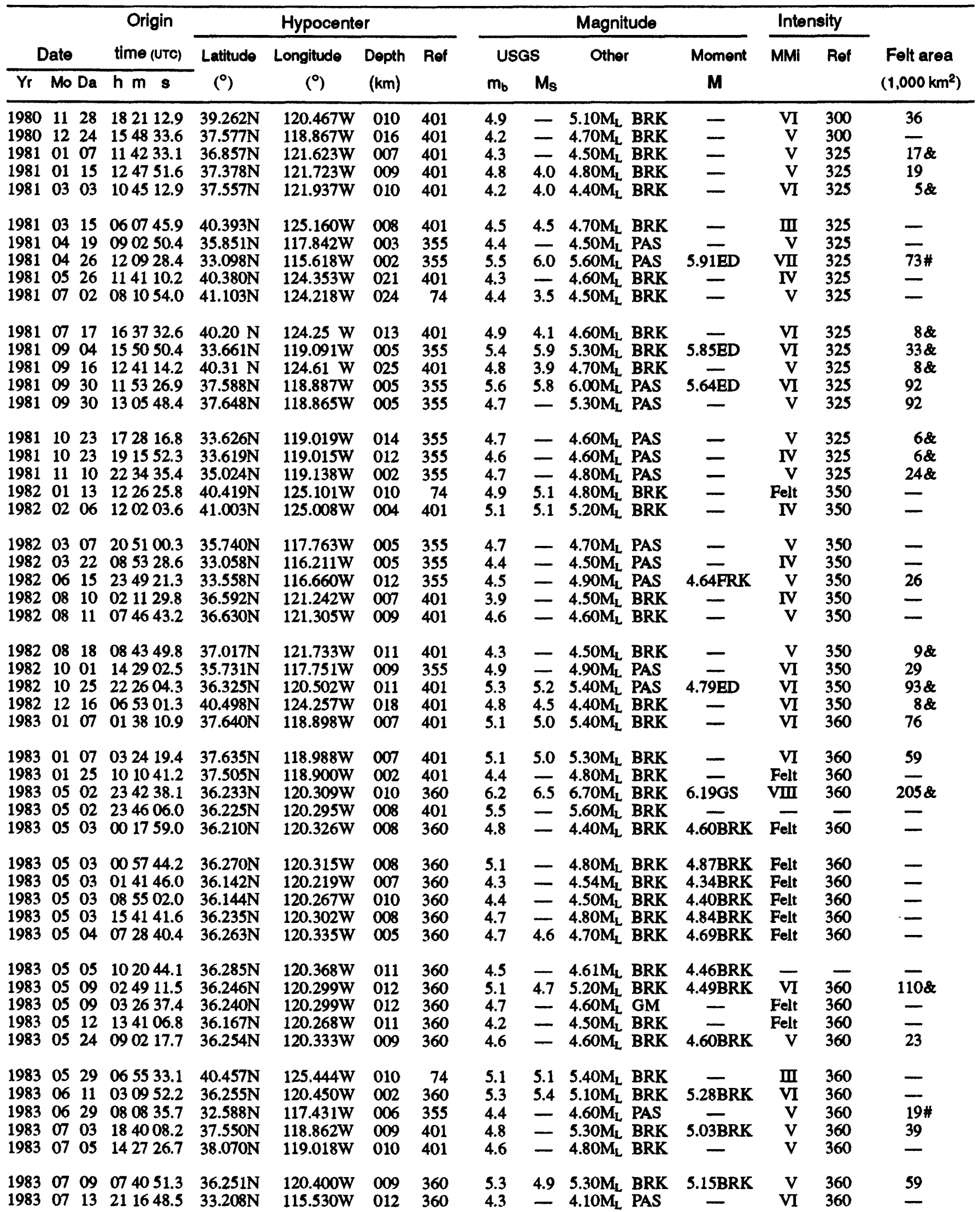


EARTHQUAKES IN CALIFORNIA

CALIFORNIA-Continued

[See table 1 for hypocenter and intensity references and table 2 for definitions of magnitude source codes. \&, land area only; \#, land area only in the United States for an earthquake near a coastline; + , land area in the United States when the felt area did not extend to the coast; 0 , felt area is less than $1,000 \mathrm{~km}^{2}$. Leader (-) indicates information is not available]

\begin{tabular}{|c|c|c|c|c|c|c|c|c|c|c|c|c|c|c|}
\hline \multirow{2}{*}{\multicolumn{3}{|c|}{ Date }} & \multirow{2}{*}{$\begin{array}{c}\text { Origin } \\
\text { time (UTC) }\end{array}$} & \multicolumn{4}{|c|}{ Hypocenter } & \multicolumn{4}{|c|}{ Magnitude } & \multicolumn{2}{|c|}{ Intensity } & \multirow{3}{*}{$\begin{array}{c}\text { Feit area } \\
\left(1,000 \mathrm{~km}^{2}\right)\end{array}$} \\
\hline & & & & \multirow{2}{*}{$\begin{array}{l}\text { Latitude } \\
\left(^{\circ}\right)\end{array}$} & \multirow{2}{*}{$\begin{array}{l}\text { Longitude } \\
\left({ }^{\circ}\right)\end{array}$} & \multirow{2}{*}{$\begin{array}{l}\text { Dopth } \\
(\mathrm{km})\end{array}$} & \multirow[t]{2}{*}{ Ref } & \multicolumn{2}{|c|}{ USGS } & \multirow[t]{2}{*}{ Other } & \multirow{2}{*}{$\begin{array}{l}\text { Moment } \\
\mathbf{M}\end{array}$} & \multirow[t]{2}{*}{ MMI } & \multirow[t]{2}{*}{ Ref } & \\
\hline Yr & Mo & $\mathrm{Da}$ & $h \mathrm{~m} s$ & & & & & $m_{b}$ & $M_{s}$ & & & & & \\
\hline $\begin{array}{l}1983 \\
1983 \\
1983\end{array}$ & $\begin{array}{l}07 \\
07 \\
07\end{array}$ & $\begin{array}{l}22 \\
22 \\
25\end{array}$ & $\begin{array}{l}023954.1 \\
034301.4 \\
223139.6\end{array}$ & $\begin{array}{l}36.241 \mathrm{~N} \\
36.222 \mathrm{~N} \\
36.229 \mathrm{~N}\end{array}$ & $\begin{array}{l}120.409 \mathrm{~W} \\
120.406 \mathrm{~W} \\
120.398 \mathrm{~W}\end{array}$ & $\begin{array}{l}007 \\
008 \\
008\end{array}$ & $\begin{array}{l}360 \\
360 \\
360\end{array}$ & $\begin{array}{l}6.0 \\
5.3 \\
5.6\end{array}$ & $\frac{5.7}{5.1}$ & $\begin{array}{ll}6.00 \mathrm{M}_{\mathrm{L}} & \text { BRK } \\
5.00 \mathrm{M}_{\mathrm{L}} & \text { BRK } \\
5.30 \mathrm{M}_{\mathrm{L}} & \text { BRK }\end{array}$ & $\begin{array}{l}\text { 5.90BRK } \\
\text { 4.88BRK } \\
\text { 5.16BRK }\end{array}$ & $\begin{array}{l}\text { VI } \\
\text { V } \\
\text { VI }\end{array}$ & $\begin{array}{l}360 \\
360 \\
360\end{array}$ & $\frac{108 \&}{60 \&}$ \\
\hline $\begin{array}{l}1983 \\
1983 \\
1983 \\
1983 \\
1983\end{array}$ & $\begin{array}{l}08 \\
08 \\
09 \\
09 \\
09\end{array}$ & $\begin{array}{l}24 \\
29 \\
09 \\
11 \\
30\end{array}$ & $\begin{array}{lll}13 & 36 & 30.5 \\
10 & 10 & 30.9 \\
09 & 16 & 13.5 \\
11 & 48 & 06.6 \\
16 & 14 & 00.9\end{array}$ & $\begin{array}{l}40.377 \mathrm{~N} \\
35.836 \mathrm{~N} \\
36.232 \mathrm{~N} \\
36.242 \mathrm{~N} \\
37.553 \mathrm{~N}\end{array}$ & $\begin{array}{l}124.832 \mathrm{~W} \\
121.345 \mathrm{~W} \\
120.265 \mathrm{~W} \\
120.383 \mathrm{~W} \\
118.840 \mathrm{~W}\end{array}$ & $\begin{array}{l}018 \\
007 \\
007 \\
010 \\
010\end{array}$ & $\begin{array}{l}401 \\
476 \\
360 \\
360 \\
401\end{array}$ & $\begin{array}{l}5.5 \\
5.3 \\
5.3 \\
5.0 \\
-\end{array}$ & $\begin{array}{l}5.8 \\
4.3 \\
5.4 \\
- \\
-\end{array}$ & $\begin{array}{ll}5.50 \mathrm{M}_{\mathrm{L}} & \text { BRK } \\
5.20 \mathrm{M}_{\mathrm{L}} & \text { BRK } \\
5.40 \mathrm{M}_{\mathrm{L}} & \text { BRK } \\
4.70 \mathrm{M}_{\mathrm{L}} & \text { PAS } \\
4.80 \mathrm{M}_{\mathrm{L}} & \text { BRK }\end{array}$ & $\begin{array}{l}\text { 5.61BRK } \\
\text { 5.31BRK } \\
5.28 \mathrm{BRK} \\
4.48 \mathrm{BRK} \\
4.49 \mathrm{BRK}\end{array}$ & $\begin{array}{l}\text { VI } \\
\text { VI } \\
\text { V } \\
\text { IV } \\
\text { IV }\end{array}$ & $\begin{array}{l}360 \\
360 \\
360 \\
360 \\
360\end{array}$ & $\begin{array}{l}10 \& \\
21 \& \\
- \\
-\end{array}$ \\
\hline $\begin{array}{l}1983 \\
1983 \\
1983 \\
1984 \\
1984\end{array}$ & $\begin{array}{l}10 \\
11 \\
12 \\
01 \\
01\end{array}$ & $\begin{array}{l}21 \\
11 \\
20 \\
23 \\
23\end{array}$ & $\begin{array}{lll}22 & 44 & 13.3 \\
12 & 07 & 43.8 \\
10 & 4105.0 \\
05 & 40 & 20.3 \\
06 & 59 & 51.3\end{array}$ & $\begin{array}{l}35.926 \mathrm{~N} \\
40.388 \mathrm{~N} \\
40.331 \mathrm{~N} \\
36.392 \mathrm{~N} \\
36.388 \mathrm{~N}\end{array}$ & $\begin{array}{l}118.334 \mathrm{~W} \\
124.902 \mathrm{~W} \\
125.117 \mathrm{~W} \\
121.878 \mathrm{~W} \\
121.872 \mathrm{~W}\end{array}$ & $\begin{array}{l}000 \\
015 \\
005 \\
008 \\
008\end{array}$ & $\begin{array}{l}355 \\
401 \\
299 \\
401 \\
401\end{array}$ & $\begin{array}{l}4.4 \\
4.9 \\
5.6 \\
5.1 \\
-\end{array}$ & $\begin{array}{l}\overline{3.8} \\
5.4 \\
4.6 \\
-\end{array}$ & $\begin{array}{ll}4.50 \mathrm{M}_{\mathrm{L}} & \text { PAS } \\
4.30 \mathrm{M}_{\mathrm{L}} & \text { BRK } \\
5.60 \mathrm{M}_{\mathrm{L}} & \text { BRK } \\
5.10 \mathrm{M}_{\mathrm{L}} & \text { BRK } \\
4.50 \mathrm{M}_{\mathrm{L}} & \text { BRK }\end{array}$ & $\begin{array}{l}-\overline{-} \\
\text { 5.90ED } \\
\text { 4.98BRK } \\
\text { 4.28BRK }\end{array}$ & $\begin{array}{r}\text { III } \\
\text { Felt } \\
V \\
V \\
\text { Felt }\end{array}$ & $\begin{array}{l}360 \\
360 \\
360 \\
370 \\
370\end{array}$ & $\begin{array}{l}- \\
\overline{32} \& \\
-\end{array}$ \\
\hline $\begin{array}{l}1984 \\
1984 \\
1984 \\
1984 \\
1984\end{array}$ & $\begin{array}{l}02 \\
02 \\
04 \\
04 \\
08\end{array}$ & $\begin{array}{l}27 \\
28 \\
24 \\
28 \\
04\end{array}$ & $\begin{array}{lll}01 & 36 & 20.6 \\
15 & 16 & 06.7 \\
21 & 15 & 19.0 \\
22 & 48 & 21.0 \\
21 & 45 & 53.2\end{array}$ & $\begin{array}{l}37.375 \mathrm{~N} \\
40.357 \mathrm{~N} \\
37.320 \mathrm{~N} \\
37.622 \mathrm{~N} \\
40.255 \mathrm{~N}\end{array}$ & $\begin{array}{l}118.598 \mathrm{~W} \\
125.897 \mathrm{~W} \\
121.698 \mathrm{~W} \\
118.897 \mathrm{~W} \\
124.578 \mathrm{~W}\end{array}$ & $\begin{array}{l}010 \\
005 \\
008 \\
003 \\
005\end{array}$ & $\begin{array}{l}401 \\
401 \\
401 \\
401 \\
401\end{array}$ & $\begin{array}{l}4.3 \\
4.9 \\
5.7 \\
4.3 \\
4.7\end{array}$ & $\begin{array}{l}\overline{4.4} \\
\overline{6.1} \\
\overline{4.6}\end{array}$ & $\begin{array}{ll}4.50 \mathrm{M}_{\mathrm{L}} & \text { BRK } \\
5.20 \mathrm{M}_{\mathrm{L}} & \text { BRK } \\
6.20 \mathrm{M}_{\mathrm{L}} & \text { BRK } \\
4.80 \mathrm{M}_{\mathrm{L}} & \text { PAS } \\
4.70 \mathrm{M}_{\mathrm{L}} & \text { BRK }\end{array}$ & $\begin{array}{c}\overline{-} \overline{16 \mathrm{GS}} \\
5 . \overline{36 \mathrm{GS}}\end{array}$ & 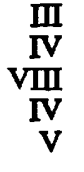 & $\begin{array}{l}370 \\
370 \\
370 \\
370 \\
370\end{array}$ & $\overline{\overline{1}} \overline{-}$ \\
\hline $\begin{array}{l}1984 \\
1984 \\
1984 \\
1984 \\
1984\end{array}$ & $\begin{array}{l}09 \\
09 \\
10 \\
10 \\
10\end{array}$ & $\begin{array}{l}10 \\
20 \\
10 \\
25 \\
26\end{array}$ & $\begin{array}{lll}03 & 14 & 10.1 \\
18 & 30 & 42.5 \\
21 & 22 & 58.9 \\
10 & 36 & 02.4 \\
17 & 20 & 43.5\end{array}$ & $\begin{array}{l}40.503 \mathrm{~N} \\
40.382 \mathrm{~N} \\
33.138 \mathrm{~N} \\
34.737 \mathrm{~N} \\
34.016 \mathrm{~N}\end{array}$ & $\begin{array}{l}126.831 \mathrm{~W} \\
125.617 \mathrm{~W} \\
116.501 \mathrm{~W} \\
120.148 \mathrm{~W} \\
118.988 \mathrm{~W}\end{array}$ & $\begin{array}{l}010 \\
005 \\
012 \\
006 \\
013\end{array}$ & $\begin{array}{r}74 \\
401 \\
370 \\
370 \\
370\end{array}$ & $\begin{array}{l}6.1 \\
4.7 \\
4.3 \\
4.5 \\
4.3\end{array}$ & $\begin{array}{l}6.7 \\
- \\
-\end{array}$ & $\begin{array}{ll}6.60 \mathrm{M}_{\mathrm{L}} & \text { BRK } \\
4.80 \mathrm{M}_{\mathrm{L}} & \text { BRK } \\
4.50 \mathrm{M}_{\mathrm{L}} & \text { PAS } \\
4.50 \mathrm{M}_{\mathrm{L}} & \text { PAS } \\
4.60 \mathrm{M}_{\mathrm{L}} & \text { PAS }\end{array}$ & $\frac{67 G S}{-}$ & $\begin{array}{l}\text { V } \\
\text { IV } \\
\text { IV } \\
\text { VI } \\
\text { V }\end{array}$ & $\begin{array}{l}370 \\
370 \\
370 \\
370 \\
370\end{array}$ & $\begin{array}{l}24 \& \\
- \\
10 \& \\
10 \&\end{array}$ \\
\hline $\begin{array}{l}1984 \\
1984 \\
1984 \\
1984 \\
1984\end{array}$ & $\begin{array}{l}11 \\
11 \\
11 \\
11 \\
11\end{array}$ & $\begin{array}{l}23 \\
23 \\
25 \\
26 \\
26\end{array}$ & $\begin{array}{lll}18 & 08 & 25.5 \\
19 & 12 & 34.6 \\
23 & 10 & 09.4 \\
16 & 21 & 47.2 \\
16 & 31 & 21.4\end{array}$ & $\begin{array}{l}37.458 \mathrm{~N} \\
37.433 \mathrm{~N} \\
37.447 \mathrm{~N} \\
37.448 \mathrm{~N} \\
37.422 \mathrm{~N}\end{array}$ & $\begin{array}{l}118.605 \mathrm{~W} \\
118.612 \mathrm{~W} \\
118.612 \mathrm{~W} \\
118.653 \mathrm{~W} \\
118.635 \mathrm{~W}\end{array}$ & $\begin{array}{l}012 \\
014 \\
005 \\
009 \\
010\end{array}$ & $\begin{array}{l}401 \\
370 \\
401 \\
401 \\
401\end{array}$ & $\begin{array}{l}5.6 \\
4.8 \\
4.3 \\
5.1 \\
-\end{array}$ & $\begin{array}{l}5.7 \\
4.7 \\
3.1 \\
4.7 \\
-\end{array}$ & $\begin{array}{ll}6.10 \mathrm{M}_{\mathrm{L}} & \text { BRK } \\
5.50 \mathrm{M}_{\mathrm{L}} & \text { BRK } \\
4.70 \mathrm{M}_{\mathrm{L}} & \text { BRK } \\
5.60 \mathrm{M}_{\mathrm{L}} & \text { BRK } \\
4.50 \mathrm{M}_{\mathrm{L}} & \mathrm{BRK}\end{array}$ & $\begin{array}{c}5.80 \mathrm{GS} \\
\overline{-14 \mathrm{GS}} \\
-\end{array}$ & $\begin{array}{r}\text { V } \\
\text { IV } \\
\text { Felt } \\
\text { V } \\
-\end{array}$ & $\begin{array}{l}370 \\
370 \\
370 \\
370 \\
-\end{array}$ & $\frac{114}{-}$ \\
\hline $\begin{array}{l}1984 \\
1984 \\
1985 \\
1985 \\
1985\end{array}$ & $\begin{array}{l}11 \\
11 \\
01 \\
02 \\
03\end{array}$ & $\begin{array}{l}28 \\
28 \\
24 \\
08 \\
25\end{array}$ & $\begin{array}{lll}16 & 23 & 26.1 \\
16 & 5737.9 \\
11 & 27 & 21.6 \\
0658 & 16.9 \\
16 & 05 & 13.6\end{array}$ & $\begin{array}{l}37.425 \mathrm{~N} \\
37.463 \mathrm{~N} \\
38.157 \mathrm{~N} \\
35.452 \mathrm{~N} \\
37.448 \mathrm{~N}\end{array}$ & $\begin{array}{l}118.630 \mathrm{~W} \\
118.582 \mathrm{~W} \\
118.853 \mathrm{~W} \\
118.898 \mathrm{~W} \\
118.545 \mathrm{~W}\end{array}$ & $\begin{array}{l}008 \\
005 \\
008 \\
011 \\
006\end{array}$ & $\begin{array}{l}401 \\
401 \\
401 \\
371 \\
371\end{array}$ & $\begin{array}{l}4.3 \\
4.2 \\
4.6 \\
4.8\end{array}$ & $\bar{z}$ & $\begin{array}{ll}4.70 \mathrm{M}_{\mathrm{L}} & \text { BRK } \\
4.60 \mathrm{M}_{\mathrm{L}} & \text { BRK } \\
5.20 \mathrm{M}_{\mathrm{L}} & \text { BRK } \\
4.60 \mathrm{M}_{\mathrm{L}} & \text { PAS } \\
5.00 \mathrm{M}_{\mathrm{L}} & \text { PAS }\end{array}$ & $\begin{array}{c}\overline{-} \\
4.82 \mathrm{BRK} \\
\text { 4.49BRK }\end{array}$ & $\begin{array}{c}\text { Felt } \\
\text { IV } \\
\text { V }\end{array}$ & $\begin{array}{l}370 \\
371 \\
371 \\
371\end{array}$ & $\begin{array}{l}- \\
25 \\
15 \\
15\end{array}$ \\
\hline $\begin{array}{l}1985 \\
1985 \\
1985 \\
1985 \\
1985\end{array}$ & $\begin{array}{l}05 \\
08 \\
08 \\
08 \\
08\end{array}$ & $\begin{array}{l}04 \\
04 \\
04 \\
22 \\
27\end{array}$ & $\begin{array}{lll}03 & 22 & 46.2 \\
11 & 29 & 16.2 \\
12 & 01 & 57.0 \\
00 & 21 & 44.1 \\
03 & 04 & 06.8\end{array}$ & $\begin{array}{l}37.469 \mathrm{~N} \\
36.122 \mathrm{~N} \\
36.130 \mathrm{~N} \\
35.883 \mathrm{~N} \\
37.412 \mathrm{~N}\end{array}$ & $\begin{array}{l}118.598 \mathrm{~W} \\
120.138 \mathrm{~W} \\
120.127 \mathrm{~W} \\
117.717 \mathrm{~W} \\
118.633 \mathrm{~W}\end{array}$ & $\begin{array}{l}006 \\
011 \\
011 \\
006 \\
006\end{array}$ & $\begin{array}{l}371 \\
401 \\
401 \\
371 \\
401\end{array}$ & $\begin{array}{l}3.7 \\
4.7 \\
5.4 \\
4.3 \\
-\end{array}$ & $\begin{array}{l}- \\
5.9 \\
-\end{array}$ & $\begin{array}{ll}4.70 \mathrm{M}_{\mathrm{L}} & \text { PAS } \\
4.70 \mathrm{M}_{\mathrm{L}} & \mathrm{BRK} \\
5.60 \mathrm{M}_{\mathrm{L}} & \mathrm{BRK} \\
4.50 \mathrm{M}_{\mathrm{L}} & \mathrm{GP} \\
4.50 \mathrm{M}_{\mathrm{L}} & \mathrm{BRK}\end{array}$ & $\begin{array}{l}\text { 4.64BRK } \\
6.09 \mathrm{GS} \\
\overline{4} \overline{4 \mathrm{BRK}}\end{array}$ & $\begin{array}{l}\text { IV } \\
\text { IV } \\
\text { VI } \\
\text { IV } \\
\text { III }\end{array}$ & $\begin{array}{l}371 \\
371 \\
371 \\
371 \\
371\end{array}$ & $\begin{array}{l}- \\
\overline{97} \& \\
-\end{array}$ \\
\hline $\begin{array}{l}1985 \\
1985 \\
1986 \\
1986 \\
1986\end{array}$ & $\begin{array}{l}10 \\
11 \\
01 \\
01 \\
02\end{array}$ & $\begin{array}{l}02 \\
28 \\
14 \\
26 \\
11\end{array}$ & $\begin{array}{lll}23 & 44 & 12.4 \\
15 & 13 & 57.2 \\
03 & 09 & 36.3 \\
19 & 20 & 51.2 \\
01 & 15 & 57.2\end{array}$ & $\begin{array}{l}34.023 \mathrm{~N} \\
36.562 \mathrm{~N} \\
36.572 \mathrm{~N} \\
36.810 \mathrm{~N} \\
41.634 \mathrm{~N}\end{array}$ & $\begin{array}{l}117.245 \mathrm{~W} \\
121.060 \mathrm{~W} \\
121.205 \mathrm{~W} \\
121.275 \mathrm{~W} \\
125.353 \mathrm{~W}\end{array}$ & $\begin{array}{l}015 \\
010 \\
007 \\
007 \\
010\end{array}$ & $\begin{array}{l}371 \\
401 \\
562 \\
562 \\
562\end{array}$ & $\begin{array}{l}4.1 \\
4.4 \\
5.0 \\
5.3 \\
5.0\end{array}$ & $\begin{array}{l}\overline{4.4} \\
\overline{5.3} \\
5.0\end{array}$ & $\begin{array}{ll}4.80 \mathrm{M}_{\mathrm{L}} & \text { PAS } \\
4.60 \mathrm{M}_{\mathrm{L}} & \text { BRK } \\
4.80 \mathrm{M}_{\mathrm{L}} & \text { BRK } \\
5.50 \mathrm{M}_{\mathrm{L}} & \text { BRK } \\
4.90 \mathrm{M}_{\mathrm{L}} & \text { BRK }\end{array}$ & $\begin{array}{l}\text { 4.61BRK } \\
\text { 4.59BRK } \\
5.48 \mathrm{BRK} \\
5.31 \mathrm{BRK}\end{array}$ & $\begin{array}{l}\text { VI } \\
\text { IV } \\
\text { IV } \\
\text { VII } \\
\text { - }\end{array}$ & $\begin{array}{l}371 \\
371 \\
562 \\
562 \\
-\end{array}$ & $\begin{array}{l}13 \\
15 \& \\
8 \& \\
36 \& \\
-\end{array}$ \\
\hline $\begin{array}{l}1986 \\
1986 \\
1986 \\
1986 \\
1986\end{array}$ & $\begin{array}{l}03 \\
05 \\
07 \\
07 \\
07\end{array}$ & $\begin{array}{l}31 \\
31 \\
08 \\
13 \\
13\end{array}$ & $\begin{array}{lll}11 & 55 & 40.1 \\
08 & 47 & 56.1 \\
09 & 2044.5 \\
13 & 47 & 08.2 \\
14 & 0133.0\end{array}$ & $\begin{array}{l}37.488 \mathrm{~N} \\
36.618 \mathrm{~N} \\
33.999 \mathrm{~N} \\
32.970 \mathrm{~N} \\
32.989 \mathrm{~N}\end{array}$ & $\begin{array}{l}121.693 \mathrm{~W} \\
121.255 \mathrm{~W} \\
116.606 \mathrm{~W} \\
117.869 \mathrm{~W} \\
117.849 \mathrm{~W}\end{array}$ & $\begin{array}{l}008 \\
004 \\
012 \\
006 \\
012\end{array}$ & $\begin{array}{l}562 \\
562 \\
597 \\
597 \\
597\end{array}$ & $\begin{array}{l}5.5 \\
4.6 \\
5.8 \\
5.6 \\
4.8\end{array}$ & $\begin{array}{l}5.5 \\
3.7 \\
6.0 \\
5.8 \\
-\end{array}$ & $\begin{array}{ll}5.70 \mathrm{M}_{\mathrm{L}} & \text { BRK } \\
4.70 \mathrm{M}_{\mathrm{L}} & \text { BRK } \\
5.60 \mathrm{M}_{\mathrm{L}} & \text { GP } \\
5.30 \mathrm{M}_{\mathrm{L}} & \text { GP } \\
4.60 \mathrm{M}_{\mathrm{L}} & \text { GP }\end{array}$ & \begin{tabular}{l} 
5.56BRK \\
$4.55 \mathrm{BRK}$ \\
$6.19 \mathrm{GS}$ \\
$5.83 \mathrm{HAV}$ \\
\multicolumn{1}{c}{}
\end{tabular} & $\begin{array}{l}\text { VI } \\
\text { IV } \\
\text { VII } \\
\text { VI } \\
-\end{array}$ & $\begin{array}{l}562 \\
562 \\
562 \\
562 \\
-\end{array}$ & $\begin{array}{l}41 \& \\
\overline{134 \#} \\
49 \# \\
-\end{array}$ \\
\hline $\begin{array}{l}1986 \\
1986 \\
1986 \\
1986 \\
1986\end{array}$ & $\begin{array}{l}07 \\
07 \\
07 \\
07 \\
07\end{array}$ & $\begin{array}{l}20 \\
20 \\
21 \\
21 \\
21\end{array}$ & $\begin{array}{l}142945.5 \\
183852.9 \\
144226.5 \\
144521.0 \\
1451 \\
14.1\end{array}$ & $\begin{array}{l}37.580 \mathrm{~N} \\
37.538 \mathrm{~N} \\
37.537 \mathrm{~N} \\
37.583 \mathrm{~N} \\
37.570 \mathrm{~N}\end{array}$ & $\begin{array}{l}118.449 \mathrm{~W} \\
118.440 \mathrm{~W} \\
118.450 \mathrm{~W} \\
118.417 \mathrm{~W} \\
118.525 \mathrm{~W}\end{array}$ & $\begin{array}{l}006 \\
009 \\
009 \\
006 \\
001\end{array}$ & $\begin{array}{l}597 \\
562 \\
562 \\
562 \\
562\end{array}$ & $\begin{array}{l}5.6 \\
3.9 \\
6.0 \\
5.1\end{array}$ & $\begin{array}{l}5.6 \\
6.2 \\
-\end{array}$ & $\begin{array}{ll}5.90 \mathrm{M}_{\mathrm{L}} & \text { BRK } \\
4.70 \mathrm{M}_{\mathrm{L}} & \text { BRK } \\
6.40 \mathrm{M}_{\mathrm{L}} & \text { BRK } \\
4.60 \mathrm{M}_{\mathrm{L}} & \text { PAS } \\
5.70 \mathrm{M}_{\mathrm{L}} & \text { BRK }\end{array}$ & $\begin{array}{c}\text { 5.38BRK } \\
4.30 \mathrm{BRK} \\
6.25 \mathrm{BRK} \\
\text { 5.04BRK }\end{array}$ & $\begin{array}{l}\text { V } \\
\text { IV } \\
\frac{\text { VI }}{\text { V }}\end{array}$ & $\begin{array}{l}562 \\
562 \\
562 \\
\overline{562}\end{array}$ & $\begin{array}{l}\overline{-} \\
\overline{258} \\
-\end{array}$ \\
\hline
\end{tabular}


CALIFORNIA-Continued

[See table 1 for hypocenter and intensity references and table 2 for definitions of magnitude source codes. \&, land area only; \#, land area only in the United States for an earthquake near a coastline; +, land area in the United States when the felt area did not extend to the coast; @, felt area is less than $1,000 \mathrm{~km}^{2}$. Leader (--) indicates information is not available!

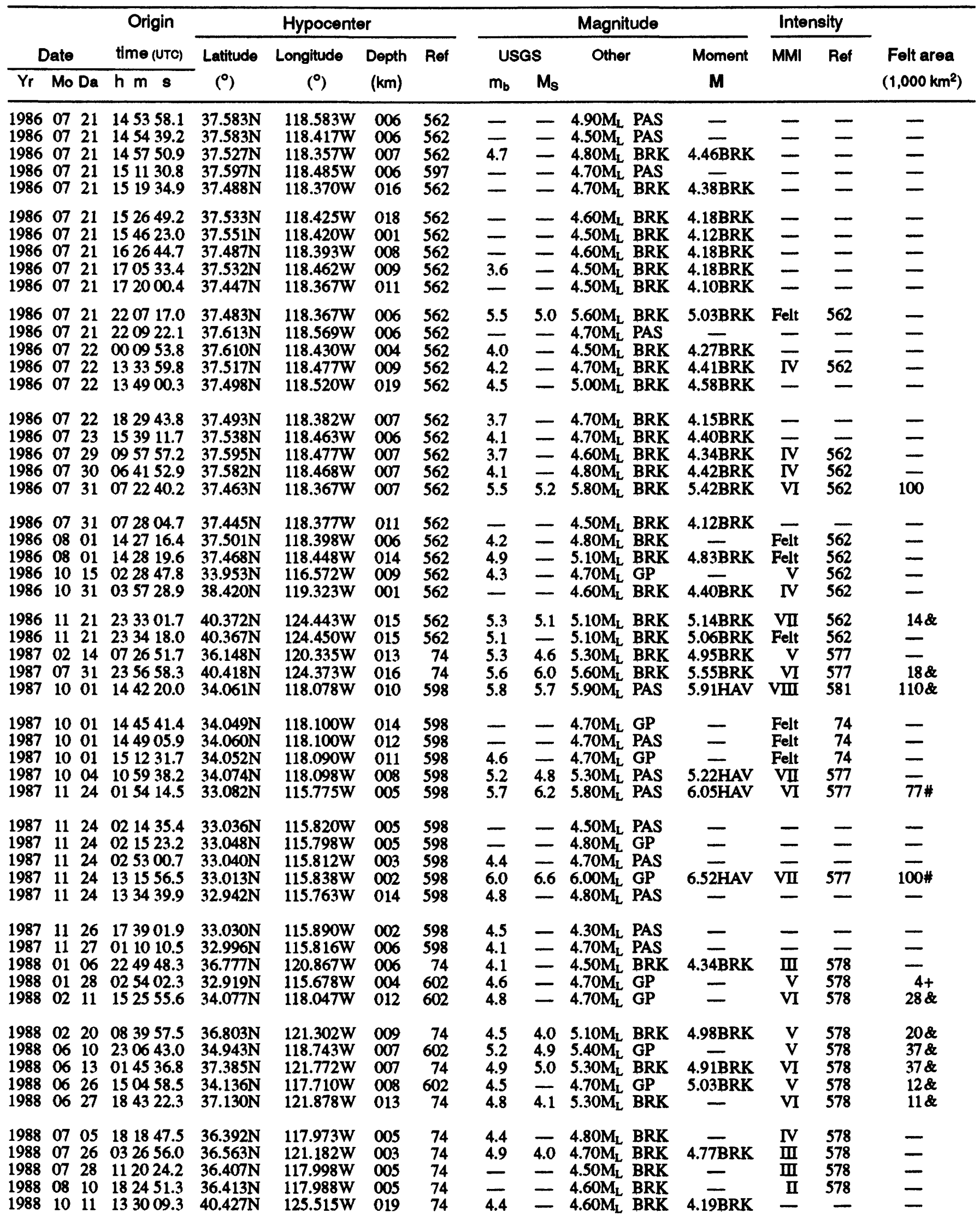


CALIFORNIA-Continued

[See table 1 for hypocenter and intensity references and table 2 for definitions of magnitude source codes. \&, land area only; \#, land area only in the United States for an earthquake near a coastline; + , land area in the United States when the felt area did not extend to the coast; $@$, felt area is less than $1,000 \mathrm{~km}^{2}$. Leader (-) indicates information is not available]

\begin{tabular}{|c|c|c|c|c|c|c|c|c|c|c|c|c|c|}
\hline \multirow{2}{*}{\multicolumn{2}{|c|}{ Date }} & \multirow{2}{*}{$\begin{array}{c}\text { Origin } \\
\text { time (UTC) }\end{array}$} & \multicolumn{4}{|c|}{ Hypocenter } & \multicolumn{4}{|c|}{ Magnitude } & \multicolumn{2}{|c|}{ Intensity } & \multirow{3}{*}{$\begin{array}{c}\text { Felt area } \\
\left(1,000 \mathrm{~km}^{2}\right)\end{array}$} \\
\hline & & & \multirow{2}{*}{$\begin{array}{l}\text { Latitude } \\
\left({ }^{\circ}\right)\end{array}$} & \multirow{2}{*}{$\begin{array}{l}\text { Longitude } \\
\left({ }^{\circ}\right)\end{array}$} & \multirow{2}{*}{$\begin{array}{c}\text { Depth } \\
(\mathrm{km})\end{array}$} & \multirow[t]{2}{*}{ Ref } & \multicolumn{2}{|c|}{ USGS } & \multirow[t]{2}{*}{ Other } & \multirow{2}{*}{$\begin{array}{c}\text { Moment } \\
M\end{array}$} & \multirow[t]{2}{*}{ MMI } & \multirow[t]{2}{*}{ Ref } & \\
\hline$\overline{Y r}$ & Mo Da & $h \mathrm{~m} s$ & & & & & $m_{b}$ & $\mathbf{M}_{\mathbf{s}}$ & & & & & \\
\hline $\begin{array}{l}1988 \\
1988 \\
1988 \\
1988 \\
1989\end{array}$ & $\begin{array}{ll}11 & 10 \\
11 & 20 \\
12 & 03 \\
12 & 16 \\
01 & 15\end{array}$ & $\begin{array}{llll}05 & 08 & 03.0 \\
05 & 39 & 28.7 \\
11 & 38 & 26.4 \\
05 & 53 & 05.0 \\
15 & 39 & 55.1\end{array}$ & $\begin{array}{l}37.373 \mathrm{~N} \\
33.507 \mathrm{~N} \\
34.149 \mathrm{~N} \\
33.979 \mathrm{~N} \\
32.948 \mathrm{~N}\end{array}$ & $\begin{array}{l}121.757 \mathrm{~W} \\
118.071 \mathrm{~W} \\
118.135 \mathrm{~W} \\
116.681 \mathrm{~W} \\
117.736 \mathrm{~W}\end{array}$ & $\begin{array}{l}007 \\
006 \\
013 \\
008 \\
006\end{array}$ & $\begin{array}{r}74 \\
74 \\
602 \\
602 \\
603\end{array}$ & $\begin{array}{l}4.5 \\
5.0 \\
4.4 \\
4.8 \\
4.5\end{array}$ & $\begin{array}{l}\frac{4.0}{4.2} \\
=\end{array}$ & $\begin{array}{l}4.80 \mathrm{M}_{\mathrm{L}} \text { BRK } \\
4.50 \mathrm{M}_{\mathrm{L}} \mathrm{GP} \\
4.90 \mathrm{M}_{\mathrm{L}} \mathrm{GP} \\
4.80 \mathrm{M}_{\mathrm{L}} \mathrm{GP} \\
4.20 \mathrm{M}_{\mathrm{L}} \mathrm{GP}\end{array}$ & $\begin{array}{c}{ }^{4.61 \mathrm{BRK}} \\
= \\
=\end{array}$ & $\begin{array}{c}\text { V } \\
\text { V } \\
\text { VI } \\
\text { V } \\
\text { IV }\end{array}$ & $\begin{array}{l}578 \\
578 \\
578 \\
468 \\
579\end{array}$ & $\begin{array}{l}14 \& \\
22 \& \\
42 \& \\
25 \& \\
-\end{array}$ \\
\hline $\begin{array}{l}1989 \\
1989 \\
1989 \\
1989 \\
1989\end{array}$ & $\begin{array}{ll}01 & 19 \\
04 & 03 \\
04 & 07 \\
06 & 04 \\
06 & 12\end{array}$ & $\begin{array}{llll}06 & 53 & 28.8 \\
17 & 46 & 34.4 \\
20 & 07 & 30.3 \\
21 & 33 & 59.7 \\
16 & 57 & 18.5\end{array}$ & $\begin{array}{l}33.919 \mathrm{~N} \\
37.422 \mathrm{~N} \\
33.619 \mathrm{~N} \\
34.597 \mathrm{~N} \\
34.022 \mathrm{~N}\end{array}$ & $\begin{array}{l}118.627 \mathrm{~W} \\
121.795 \mathrm{~W} \\
117.902 \mathrm{~W} \\
116.838 \mathrm{~W} \\
118.178 \mathrm{~W}\end{array}$ & $\begin{array}{l}012 \\
009 \\
013 \\
002 \\
016\end{array}$ & $\begin{array}{r}603 \\
74 \\
603 \\
603 \\
603\end{array}$ & $\begin{array}{l}5.2 \\
4.5 \\
5.0 \\
4.2 \\
4.3\end{array}$ & $\begin{array}{l}4.8 \\
4.3 \\
= \\
-\end{array}$ & $\begin{array}{l}5.00 \mathrm{M}_{\mathrm{L}} \text { GP } \\
4.70 \mathrm{M}_{\mathrm{L}} \mathrm{BRK} \\
4.50 \mathrm{M}_{\mathrm{L}} \mathrm{GP} \\
4.50 \mathrm{M}_{\mathrm{L}} \mathrm{GP} \\
4.40 \mathrm{M}_{\mathrm{L}} \mathrm{GP}\end{array}$ & $\begin{array}{l}\bar{z} \\
=\end{array}$ & $\begin{array}{l}\text { VI } \\
\text { VI } \\
\text { VI } \\
\text { V } \\
\text { VI }\end{array}$ & $\begin{array}{l}579 \\
579 \\
579 \\
579 \\
579\end{array}$ & $\begin{array}{c}40 \& \\
15 \& \\
14 \& \\
7 \\
14 \&\end{array}$ \\
\hline $\begin{array}{l}1989 \\
1989 \\
1989 \\
1989 \\
1989\end{array}$ & $\begin{array}{ll}08 & 08 \\
08 & 08 \\
09 & 21 \\
10 & 18 \\
10 & 18\end{array}$ & $\begin{array}{lll}08 & 13 & 27.5 \\
15 & 53 & 28.4 \\
17 & 41 & 18.0 \\
00 & 04 & 15.2 \\
00 & 25 & 04.9\end{array}$ & $\begin{array}{l}37.130 \mathrm{~N} \\
37.150 \mathrm{~N} \\
40.327 \mathrm{~N} \\
37.036 \mathrm{~N} \\
37.043 \mathrm{~N}\end{array}$ & $\begin{array}{l}121.952 \mathrm{~W} \\
121.973 \mathrm{~W} \\
124.705 \mathrm{~W} \\
121.883 \mathrm{~W} \\
121.807 \mathrm{~W}\end{array}$ & $\begin{array}{l}015 \\
015 \\
016 \\
019 \\
005\end{array}$ & $\begin{array}{l}74 \\
74 \\
74 \\
74 \\
74\end{array}$ & $\begin{array}{l}4.9 \\
4.2 \\
4.8 \\
6.5 \\
5.0\end{array}$ & $\begin{array}{l}4.5 \\
4.7 \\
7.1 \\
-\end{array}$ & $\begin{array}{l}5.40 \mathrm{M}_{\mathrm{L}} \text { BRK } \\
4.50 \mathrm{M}_{\mathrm{L}} \text { BRK } \\
4.80 \mathrm{M}_{\mathrm{L}} \text { BRK } \\
7.00 \mathrm{M}_{\mathrm{L}} \text { BRK } \\
4.80 \mathrm{M}_{\mathrm{L}} \text { BRK }\end{array}$ & $\begin{array}{c}\overline{-} \\
5.14 \mathrm{BRK} \\
7.18 \mathrm{BRK} \\
-\end{array}$ & $\begin{array}{c}\mathbf{V I I} \\
\mathbf{V} \\
\mathbf{V} \\
\mathbf{I X} \\
-\end{array}$ & $\begin{array}{l}579 \\
579 \\
579 \\
579 \\
-\end{array}$ & $\begin{array}{c}26 \& \\
\frac{8}{8} \& \\
170 \&\end{array}$ \\
\hline $\begin{array}{l}1989 \\
1989 \\
1989 \\
1989 \\
1989\end{array}$ & $\begin{array}{ll}10 & 18 \\
10 & 18 \\
10 & 18 \\
10 & 18 \\
10 & 18\end{array}$ & $\begin{array}{llll}00 & 41 & 24.7 \\
02 & 15 & 49.9 \\
04 & 5027.7 \\
05 & 18 & 34.1 \\
06 & 39 & 10.1\end{array}$ & $\begin{array}{l}37.198 \mathrm{~N} \\
36.995 \mathrm{~N} \\
37.193 \mathrm{~N} \\
36.980 \mathrm{~N} \\
36.932 \mathrm{~N}\end{array}$ & $\begin{array}{l}122.105 \mathrm{~W} \\
121.763 \mathrm{~W} \\
122.017 \mathrm{~W} \\
121.847 \mathrm{~W} \\
121.712 \mathrm{~W}\end{array}$ & $\begin{array}{l}019 \\
004 \\
014 \\
011 \\
012\end{array}$ & $\begin{array}{l}74 \\
74 \\
74 \\
74 \\
74\end{array}$ & $\begin{array}{l}4.8 \\
4.4 \\
4.6 \\
4.5 \\
4.6\end{array}$ & $\begin{array}{l}\bar{z} \\
\overline{-}\end{array}$ & $\begin{array}{l}5.10 \mathrm{M}_{\mathrm{L}} \text { BRK } \\
4.50 \mathrm{M}_{\mathrm{L}} \text { BRK } \\
4.30 \mathrm{M}_{\mathrm{L}} \text { BRK } \\
4.20 \mathrm{M}_{\mathrm{L}} \text { BRK } \\
4.30 \mathrm{M}_{\mathrm{L}} \text { BRK }\end{array}$ & $\bar{z}$ & $\begin{array}{l}\bar{z} \\
\bar{z}\end{array}$ & $\begin{array}{l}\bar{z} \\
\bar{z}\end{array}$ & $\begin{array}{l}\bar{z} \\
\bar{z}\end{array}$ \\
\hline $\begin{array}{l}1989 \\
1989 \\
1989 \\
1989 \\
1989\end{array}$ & $\begin{array}{ll}10 & 19 \\
10 & 19 \\
10 & 21 \\
10 & 21 \\
10 & 25\end{array}$ & 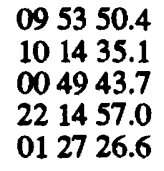 & $\begin{array}{l}36.932 \mathrm{~N} \\
36.963 \mathrm{~N} \\
37.047 \mathrm{~N} \\
37.057 \mathrm{~N} \\
37.078 \mathrm{~N}\end{array}$ & $\begin{array}{l}121.690 \mathrm{~W} \\
121.843 \mathrm{~W} \\
121.877 \mathrm{~W} \\
121.905 \mathrm{~W} \\
121.832 \mathrm{~W}\end{array}$ & $\begin{array}{l}012 \\
013 \\
014 \\
013 \\
014\end{array}$ & $\begin{array}{l}74 \\
74 \\
74 \\
74 \\
74\end{array}$ & $\begin{array}{l}4.3 \\
4.6 \\
4.4 \\
4.5 \\
4.6\end{array}$ & $\begin{array}{l}3.6 \\
\frac{4.2}{-} \\
\frac{3.6}{3.6}\end{array}$ & $\begin{array}{l}4.50 \mathrm{M}_{\mathrm{L}} \text { BRK } \\
4.60 \mathrm{M}_{\mathrm{L}} \text { BRK } \\
4.60 \mathrm{M}_{\mathrm{L}} \text { BRK } \\
4.90 \mathrm{M}_{\mathrm{L}} \text { BRK } \\
5.00 \mathrm{M}_{\mathrm{L}} \text { BRK }\end{array}$ & $\begin{array}{l}z \\
z\end{array}$ & $\begin{array}{l}\text { Felt } \\
\text { Felt } \\
\text { Felt } \\
\text { Felt } \\
\text { IV }\end{array}$ & $\begin{array}{r}74 \\
74 \\
74 \\
74 \\
579\end{array}$ & $\bar{z}$ \\
\hline $\begin{array}{l}1989 \\
1989 \\
1989 \\
1989\end{array}$ & $\begin{array}{ll}11 & 02 \\
11 & 03 \\
11 & 05 \\
12 & 28\end{array}$ & $\begin{array}{lll}05 & 50 & 11.0 \\
19 & 09 & 1.6 \\
13 & 37 & 34.3 \\
09 & 41 & 08.2\end{array}$ & $\begin{array}{l}37.057 \mathrm{~N} \\
38.567 \mathrm{~N} \\
37.058 \mathrm{~N} \\
34.192 \mathrm{~N}\end{array}$ & $\begin{array}{l}121.797 \mathrm{~W} \\
119.652 \mathrm{~W} \\
121.915 \mathrm{~W} \\
117.386 \mathrm{~W}\end{array}$ & $\begin{array}{l}012 \\
011 \\
015 \\
015\end{array}$ & $\begin{array}{r}74 \\
74 \\
74 \\
603\end{array}$ & $\frac{4.5}{=}$ & $=$ & 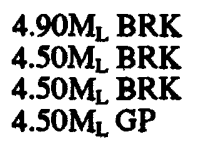 & $\bar{z}$ & $\begin{array}{r}\text { IV } \\
\text { Felt } \\
V\end{array}$ & $\begin{array}{r}579 \\
579 \\
74 \\
579\end{array}$ & $\bar{z}$ \\
\hline
\end{tabular}

[Reference (Ref.) numbers given in parentheses at the end of each description refer to sources of data in table 1. Magnitude values are described in the Introduction, and codes are defined in table 2.]

1769. July 28. Los Angeles, Calif., region. The Portola expedition felt four violent earthquakes while camped on the Santa Ana River, near the present site of Olive. Many strong aftershocks were observed through Aug. 3 as the expedition moved northwestward to near San Gabriel and then westward across Los Angeles to the Pacific. This probably was a major earthquake. (Ref. 38, 368, 493, 521.)

1800. Oct. 11. Near San Juan Bautista, San Benito County, Calif. Although the houses at San Juan Bautista were built with double walls, all were damaged from roof to foundation and were uninhabitable. Some small cracks were observed in the ground at the rancheria; another deep fissure formed in the area of the Pajaro River. A strong shock on Oct. 18 was accompanied by "such a loud noise as to deafen them." (Ref. 38, 368, 493.)

1800. Nov. 22. San Diego, Calif., region. Walls of adobe buildings were damaged in San Diego, and walls of a new church were cracked at San Juan Capistrano. (Ref. 38, 368.)

1803. May 25. San Diego, Calif. The mission church at San Diego sustained slight damage. (Ref. $56,368$.

1806. Mar. 25. Santa Barbara, Calif. An earthquake cracked the walls of a chapel at Santa Barbara. (Ref. 56, 368.)

1808. June 21. San Francisco, Calif. Adobe walls were damaged severely at the Presidio of San Francisco. One of the rooms collapsed at the 
commandant's house, and the soldiers' quarters were ruined and nearly fell. Through July 17 and 18 shocks were felt. (Ref. 38, 56, 368.)

1812. Dec. 8. Southwest San Bernardino County, Calif. The most severe loss occurred at the Missions of San Juan Capistrano and San Gabriel. The masonry church at San Juan Capistrano Mission was destroyed, killing 40 people attending services. Other buildings at the mission were left "in bad condition." The church at San Gabriel also sustained much damage, and many cracks formed in the bell tower. The upper part of the tower later fell, as did the weathervane. The living quarters of the ministers and offices of the mission also were damaged extensively. Three tremors described as "horrible" probably caused damage at San Buenaventura Mission (Ventura). Walls of buildings were impaired at Mission San Fernando Rey, but no damage was reported at San Diego or San Luis Rey Missions.

This earthquake may have ruptured the San Andreas fault at Wrightwood. The rupture may have extended at least $25 \mathrm{~km}$ to the northwest, where it is preserved in the peat bog at Pallet Creek. The fault rupture at Pallet Creek is comparable to that formed by the 1857 earthquake in the southern Coast Ranges. The main earthquake was felt from San Buenaventura Mission west to San Bernardino Valley and south to San Diego. (Ref. 38, 56, 368, 521.)

1812. Dec. 21. West of Ventura, Calif., in Santa Barbara Channel. This major earthquake caused damage in Santa Barbara, Ventura, and northern Los Angeles Counties. One fatality was reported, but many lives probably were saved by a strong foreshock about 15 minutes earlier that sent alarmed residents fleeing from buildings. The earthquakes also may have generated a tsunami because there were several reports of sea waves following the earthquakes. The sea waves reportedly did not cause loss of life or substantial loss of property.

At Santa Barbara Mission, all buildings sustained many cracks, and one chapel was flattened. The ground "opened up" in this area to such an extent "that it caused horror." At the Santa Barbara Presidio, all the buildings were left uninhabitable. The church at La Purisima Concepcion Mission (Lompoc) was ruined; some of the other buildings were "flattened to the ground;" and others required extensive repair. Part of the adobe garden wall collapsed, and that part remaining nearly fell. Damage at the Santa Ynez Mission was considerable but not so severe as at Santa Barbara and Purisima Missions. A corner of the church fell; many new houses were demolished; and many support walls were cracked. Property loss was less severe at the San Buenaventura Mission
(Ventura) and the San Fernando Rey Mission. Aftershocks were reported at Santa Barbara through April 1813. (Ref. 38, 56, 368, 521.)

1827. Sept. 24 (Sept. 23). Los Angeles, Calif. People in Los Angeles ran outdoors in panic. The 1827 report of the San Buenaventura Mission (Ventura) notes that all the buildings were in "bad condition" because of the earthquakes. The same earthquake, therefore, may have affected both Los Angeles and Ventura. (Ref. 56, 368.)

1829. September. San Francisco, Calif. Several severe shocks at San Francisco were strong enough to force open locked doors and windows. No damage has been documented for this earthquake. The intensity was taken from ref. 56. (Ref. 56, 493.)

1830. Date unknown. San Luis Obispo, Calif. All the buildings at San Luis Obispo were damaged, including the hospital and part of the rancheria, which lay in ruins. The farm and building of $\mathrm{S}$. Miguelito were destroyed, and the house of Santa Margarita sustained cracked and broken walls. (Ref. 38,368 .)

1836. June 10. San Francisco Bay area, Calif. This strong earthquake affected an area along the foothills from San Pablo to Mission San Jose. Large fissures formed in the ground in the area of maximum disturbance. The earthquake caused havoc in Monterey and Santa Clara and frightened all residents. The first shock was the most violent, and smaller aftershocks continued for a month. This earthquake may have ruptured the Hayward fault. Its intensity is similar to that of an earthquake in the same area on October 21, 1868. (Ref. 38, 56, $368,493$.

1838. June. San Francisco area, Calif. This earthquake was associated with a probable rupture on the San Andreas fault, from near Santa Clara to San Francisco (about $60 \mathrm{~km}$ ). It is suggested that the fault rupture may have extended throughout all or most of the line that was active in the 1906 earthquake. Walls were cracked at Mission Dolores (San Francisco), and some adobe walls were cracked and glassware and crockery were broken at Monterey. (Ref. 56, 368, 521.)

1840. Jan. 16. Santa Cruz, Calif. An earthquake threw down a church tower at Santa Cruz. A "tidal wave" (tsunami) was reported. Shocks continued to Jan. 18. (Ref. 56, 493.)

1841. July 3. Monterey, Calif. This earthquake left the bay and shore covered with dead and beached fish. The shaking was so severe at Monterey than an observer had to support himself against a tree to keep from falling. The main earthquake was reported felt onboard ships in the harbor and on 
inland farms. About 120 shocks were felt in Monterey in the summer of 1841 , but they were seldom severe. (Ref. 38, 56, 368.)

1851. May 15. San Francisco, Calif. A severe earthquake lasting half a minute was felt onboard ships in the harbor. It threw merchandise to the floor in San Francisco stores and sent residents rushing outdoors. No damage has been documented for this earthquake. The intensity was taken from ref. 38 and 56. (Ref. 38, 56, 368.)

1852. Nov. 29. Baja California, Mexico. A violent earthquake threw down large fragments of Chimney Rock (now called Picacho Peak) and parts of the surrounding mountains near Fort Yuma, Calif. (located across the Colorado River from what is now Yuma, Ariz.). Sand and water issued from fissures that formed all along the riverbanks and in the low alluvial plain surrounding Fort Yuma. People at the fort had difficulty standing or walking because of the shaking. Felt as far away as San Diego.

Observers on the steamer Uncle Sam, which was about $48 \mathrm{~km}$ south of Fort Yuma near Ogdens Landing (on the Colorado River), described the effects of the earthquake as follows: "The waters of the river were thrown into a sort of boiling motion with a strong rippled surface. The riverbanks on one side caved in and, on the other side, separated into a thousand cracks from which dust, sand, mud, and water were ejected. The river formed new bends, leaving portions of its old bed so suddenly that thousands of fish were left lying on the muddy bottom."

The earthquake caused significant changes in the course of the Colorado River near Pilot Knob, about $10 \mathrm{~km}$ downstream from Yuma. The schooner Capacity, which was anchored in about $3.5 \mathrm{~m}$ of water at the mouth of the Colorado River (about $32 \mathrm{~km}$ south of Fort Yuma), was left in about $0.5 \mathrm{~m}$ of water after the earthquake.

Residents at Fort Yuma observed large steam geysers at Laguna de los Volcanoes (in Baja California, about $73 \mathrm{~km}$ southwest of Fort Yuma). The steam column was estimated to be more than $300 \mathrm{~m}$ in height. Aftershocks, some of which also generated geysers, continued until September 1853. (Ref. 368, 461, 521.)

1852. Dec. 17. San Luis Obispo, Calif. Two sharp earthquakes cracked walls of two adobe houses at San Luis Obispo and threw down part of the wall of another dwelling. (Ref. 38, 56.)

1853. Feb. 1. San Luis Obispo, Calif., area. An adobe house was cracked at San Simeon, and its occupants ran outside. One report described this earthquake as a violent shock that damaged houses. (Ref. 38, 56, 368.)
1853. Oct. 23. Off the coast of Humboldt County, Calif., in Humboldt Bay. At Eureka, houses rolled and undulated like ships at sea, and many residents were thrown from their beds. Three heavy shocks were reported. (Ref. 38, 56, 368.)

1855. Jan. 25 (Jan. 24). Sierra County, Calif., area. Buildings in the area were shaken severely by this heavy shock. A large pinnacle of rocks on the Downieville (Sierra) Buttes was thrown down. At the Blue Banks Mine (probably in Sierra or Nevada County), miners at the $122-\mathrm{m}$ level ran outside the mine. Felt from Gibsonville (Sierra County) on the north to Nashville (El Dorado County) on the south and to Keystone Ranch (Yuba County) on the west. The epicenter probably was near the Nevada border. (Ref. 56, 368.)

1855. July 11 (July 10). Near San Gabriel, Los Angeles County, Calif. Bells at the San Gabriel Mission church were thrown down. Twenty-six buildings were damaged in Los Angeles, including the Star Hotel, whose walls were cracked. An adobe building sitting directly on the Raymond fault also was wrecked. Two unusually "heavy" sea waves rolled in at Point San Juan soon after the last of four shocks. (Ref. 38, 56, 368.)

1855. Aug. 27. North of San Francisco, Calif. This earthquake moved furniture at St. Ann's Valley (San Francisco) and cracked an adobe house on a ranch. It was described as violent at Petaluma and at San Francisco de Solano Mission. (Ref. 56, 368.)

1856. Jan. 2. South of San Francisco in San Mateo County, Calif. At San Francisco, masonry walls were cracked and iron shutters were warped. The shock was felt south to Monterey, where some frightened residents ran outside. (Ref. 56, 368, 610.)

1856. Feb. 15. South of San Francisco in San Mateo County, Calif. This strong earthquake threw down cornices, cracked brick walls, and knocked people from their feet in San Francisco. The water in San Francisco Bay was disturbed. Felt from Petaluma (Sonoma County) east to Stockton (San Joaquin County) and south to Monterey. (Ref. 38, 56, 368,610 .)

1856. Sept. 21 (Sept. 20). Near Santa Ysabel, San Diego County, Calif. At Santa Ysabel, plaster was shaken down and ceilings fell. The shock also stampeded cattle and terrified residents. Plaster was cracked at San Diego. (Ref. 56, 368.)

1857. Jan. 9. Near Fort Tejon, San Luis Obispo County, Calif. This earthquake occurred on the San Andreas fault, which ruptured from near Parkfield (in the Cholame Valley) almost to Wrightwood (a distance of about $300 \mathrm{~km}$ ); horizontal displacement of as much as $9 \mathrm{~m}$ was observed on the 
Carrizo Plain. It caused one fatality. A comparison of this shock to the San Francisco earthquake, which occurred on the San Andreas fault on Apr. 18, 1906, shows that the fault break in 1906 was longer but that the maximum and average displacements in 1857 were larger.

Property loss was heavy at Fort Tejon, an Army post about $7 \mathrm{~km}$ from the San Andreas fault. Two buildings were declared unsafe, three others were damaged extensively but were habitable, and still others sustained moderate damage. About $20 \mathrm{~km}$ west of Fort Tejon, trees were uprooted, and buildings were destroyed between Fort Tejon and Elizabeth Lake. One person was killed in the collapse of an adobe house at Gorman. Strong shaking lasted from 1 to 3 minutes.

Instances of seiching, fissuring, sandblows, and hydrologic changes were reported from Sacramento to the Colorado River delta. Ground fissures were observed in the beds of the Los Angeles, Santa Ana, and Santa Clara Rivers and at Santa Barbara. Sandblows occurred at Santa Barbara and in the flood plain of the Santa Clara River. One report describes sunken trees, possibly associated with liquefaction, in the area between Stockton and Sacramento.

Changes in the flow of streams or springs were observed in the areas of San Diego, Santa Barbara, Isabella, and at the south end of San Joaquin Valley. The waters of the Kern, Lake, Los Angeles, and Mokulumne Rivers overflowed their banks. Changes in the flow of water in wells were reported from the Santa Clara Valley in northern California.

Felt from Marysville south to San Diego and east to Las Vegas, Nev. (see fig. 13). Several slight to moderate foreshocks preceded the main shock by 1 to 9 hours. Many aftershocks occurred, and two (Jan. 9 and 16) were large enough to have been widely felt. (Ref. 38, 121, 368, 379, 517, 521, 599.)

1858. Nov. 26. North of San Jose in Alameda County, Calif. The earthquake was severe at San Jose, where an adobe house and the corner of a new building were knocked down. A cornice was thrown off a building in San Francisco, and part of a chimney collapsed at Mountain View. Felt to Downieville (Sierra County) on the north, Mariposa on the east, and Monterey on the south. (Ref. 38, 56, 368.)

1858. Dec. 16, 0230 UTC (Dec. 15). Near San Bernardino, Calif. This minor earthquake broke dishes and cracked walls at San Bernardino. It also was felt in Los Angeles. (Ref. 368.)

1858. Dec. 16, 10 00 UTC. Near San Bernardino, Calif. At Agua Manza, near San Bernardino, one house collapsed. The gable end of a house was knocked down by the shock at San Bernardino. (Ref. 368,521 .)
1859. Oct. 5. San Francisco, Calif. A severe local earthquake cracked walls in several brick buildings in San Francisco, downed plaster, and rang bells. (Ref. 56, 368.)

1860. Nov. 13 (Nov. 12). Near Eureka, Humboldt County, Calif. Plaster walls cracked and chimneys settled at Eureka; a house under construction shifted about $5 \mathrm{~cm}$ on its foundation. (Ref. 56, 368.)

1861. July 4 (July 3). Near Dublin, Contra Costa County, Calif. Near the present town of Dublin, at Dougherty's Ranch, the roof of the ranch kitchen was thrown off, chimneys were downed, and several men in the fields were thrown to the ground. A fissure about $20 \mathrm{~km}$ long, probably the result of surface rupture along the Calaveras fault, opened along the west side of San Ramon Valley. A new spring of water appeared. Although strong at San Francisco, the shock caused no loss to property. Felt north to Sacramento and south to Santa Cruz. (Ref. $38,56,368,493$.

1862. May 27. Near San Diego, Calif. This strong earthquake caused widespread minor damage in the Old Town and La Playa areas of San Diego. Many adobe and brick buildings sustained cracks, some of which extended completely through the walls. In some frame buildings, windows and doors were loosened in their frames and some door hinges were broken off.

Landslides occurred along the steep bluffs from La Playa to Point Loma. At La Playa, north of Point Loma, cracks formed on the beach, and water was emitted from the sand on the tidal flats. Water from the bay surged inland over the beach between 3 and $4 \mathrm{ft}$. Cracks also formed in the wet, sandy ground near the San Diego River, which "washed over its banks." This earthquake was felt north to Anaheim and Los Angeles. About 100 aftershocks occurred at San Diego to June 14, 1862. (Ref. 38, $56,368,532,610$.)

1864. Feb. 26. North of Watsonville in Santa Clara County, Calif. This earthquake cracked adobe walls at Monterey and tipped over or displaced light objects at Watsonville. Described as severe at San Francisco and Santa Cruz, the shock was felt north to Napa and south to San Luis Obispo. (Ref. $56,368$.

1864. Mar. 5. East of San Francisco in Alameda County, Calif. Walls cracked considerably, plaster cracked, and a few plate-glass windows shattered at San Francisco, where the earthquake apparently was strongest. A few buildings in San Jose also sustained cracks in plaster. Felt north to Santa Rosa (Sonoma County) and Sacramento and south to San Juan Mission (San Benito County) and Visalia (Tulare County). (Ref. 38, 368.) 


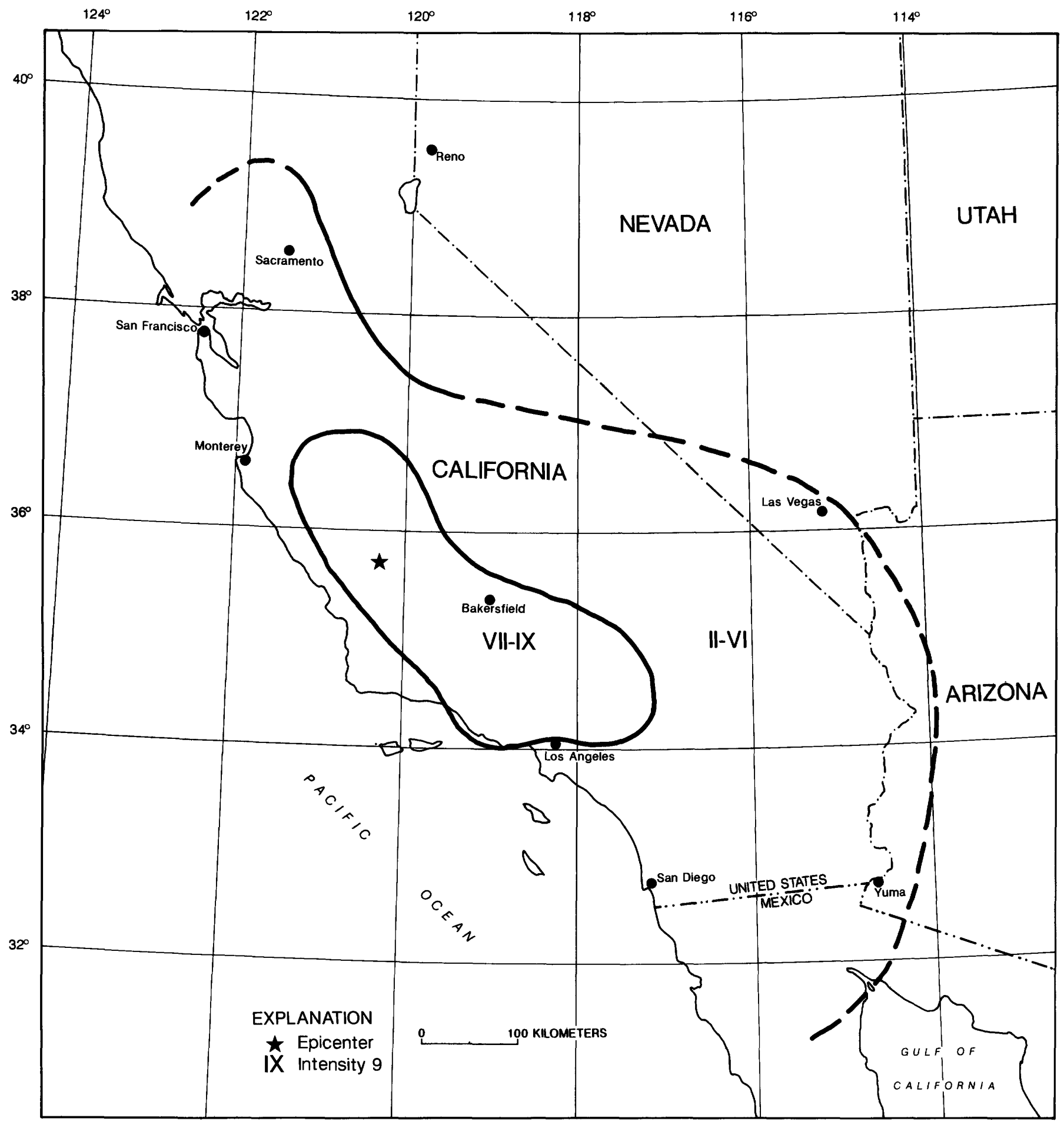

FiguRE 13.-Isoseismal map for the Fort Tejon, California, earthquake of January 9, 1857. This map is a simplified version of figure 2 in reference 379 of table 1.

1864. May 21 (May 20), Southeast of San Francisco in Alameda County, Calif. At San Francisco a few windows were broken. Felt from Monterey on the south to Sacramento and Santa Rosa (Sonoma County) on the north. (Ref. 56, 368.)
1865. Mar. 8. Bennett Valley, Sonoma County, Calif. Chimneys fell, plaster cracked, and clocks stopped running in Bennett Valley, east of Santa Rosa. The earthquake was described as severe at Santa Rosa. A foreshock was felt in the Santa Rosa-San Francisco 
area 8 hours earlier. The main shock was felt south to San Francisco and San Leandro. (Ref. 38, 56, 368.)

1865. May 24. South of San Jose, Santa Clara County, Calif. Crockery was broken at San Juan Bautista. The earthquake was "remarkably heavy" in the south San Francisco Bay counties. Felt from San Francisco on the north to Monterey on the south. (Ref. 56, 368.)

1865. Oct. 1. Near Eureka, Humboldt County, Calif. Chimneys and brickwork were damaged at Eureka. At Fort Humboldt, south of Eureka on Humboldt Bay, all fort buildings were impaired, redwood trees were uprooted, and a fissure formed along the edge of the parade ground. This shock was reported only in Humboldt and Trinity Counties. (Ref. 38, 56, 368.)

1865. Oct. 8. Santa Cruz Mountains, Santa Clara County, Calif. This strong earthquake caused severe damage in several towns, including New Almaden, Petaluma, San Francisco, San Jose, Santa Clara, and Santa Cruz. Property loss was estimated at $\$ 500,000$.

The shock knocked several houses off foundations at New Almaden and almost destroyed a large brick storehouse. At San Francisco, several poorly constructed buildings on made land were destroyed, the City Hall was ruined, and water pipes and gas pipes were broken; a crack about $2.5 \mathrm{~cm}$ wide formed in Howard Street. On the marshy lands in the Howard Street area, the ground heaved in some places and sank in others.

Almost all buildings in Santa Clara and Petaluma were affected to some extent, and every brick building was wrecked at Santa Cruz. The walls of the jail and church fell at San Jose, and several chimneys were knocked down. At Watsonville, the earth cracked in several places, and water flowed through the cracks. Ground cracks also were reported near the San Andreas fault at Mountain Charlie's on the Santa Cruz Road. The tide at Santa Cruz was affected.

This earthquake caused damage from San Juan Bautista on the south to Napa on the north. Aftershocks were reported in several towns. (Ref. 38, 56, $368,517,530,533,610$.)

1866. Mar. 26. Near Gilroy, Santa Clara County, Calif. Several chimneys were downed in Gilroy. Felt from Santa Rosa (Sonoma County) south to Monterey. (Ref. 56, 368.)

1868. Sept. 4. West of Lone Pine in Tulare County, Calif. A series of earthquakes occurred at the headwaters of the Kern River and at Lone Pine from Sept. 4 to Sept. 17. The earth shook terribly, tall trees swayed, and huge masses of boulders and earth detached from the surrounding cliffs and tumbled down along the river. At Lone Pine, 40 shocks were reported in 1 hour on Sept. 4. Foreshocks occurred on Sept. 3. (Ref. 38, 56, 368.)

1868. Oct. 21. Near Hayward, Alameda County, Calif. Because of its location in a highly populated area, this earthquake was one of the most destructive in the history of California. Property loss was extensive at towns in the San Francisco Bay area, and 30 people were killed. The total property loss was about $\$ 350,000$. This earthquake was known as the "great San Francisco earthquake" until the shock on Apr. 18, 1906. Compared to the 1906 shock, the fault trace of the 1868 earthquake-about 32 $\mathrm{km}$-was much shorter, and the amount of movement $(0.9 \mathrm{~m}$-whether it was horizontal or vertical is not clear) was much less.

Damage was most severe in Hayward and nearby towns along the Hayward fault. Slip was observed on the Hayward fault from San Leandro to Warm Springs, a distance of about $32 \mathrm{~km}$; in places, the fault trace opened $25-30 \mathrm{~cm}$. At Hayward, almost every building was damaged extensively or wrecked. San Jose, which lay in the hills several meters west of the fault trace, had many wrecked buildings and demolished chimneys. At San Leandro, the second floor of the courthouse collapsed, and other buildings were wrecked. At San Francisco, the Custom house sustained severe damage, and many cornices, awnings, and walls fell, but, as occurred later in the shock of 1906, well-constructed buildings on firm ground sustained little damage.

Damage occurred from Gilroy and Santa Cruz on the south to Santa Rosa on the north. The area shaken at MM intensity VIII or higher includes about $2,300 \mathrm{~km}^{2}$. Strong aftershocks continued into November 1868. (Ref. 38, 56, 368, 517, 521, 530,533 .)

1869. Oct. 8. Near Ukiah, Mendocino County, Calif. This earthquake downed chimneys at Ukiah and in "the Clear Lake country." Felt from Healdsburg (Sonoma County) north to Potter Valley. (Ref. $56,368$.

1870. Feb. 17. Near Santa Cruz, Calif. At Los Gatos, several chimneys were knocked down, and at Santa Cruz, chimneys were dislocated. Felt south to Monterey and north to Sacramento. (Ref. 56, 368.)

1870. Apr. 2. Near Oakland, Contra Costa County, Calif. Minor damage occurred in Oakland and San Francisco. The shock also was felt in Santa Cruz, Santa Rosa, and Stockton. (Ref. 56, 368.)

1871. Mar. 2. Near Mattole, Humboldt County, Calif. Almost every chimney was knocked to the ground at Mattole, and ground fissures were 


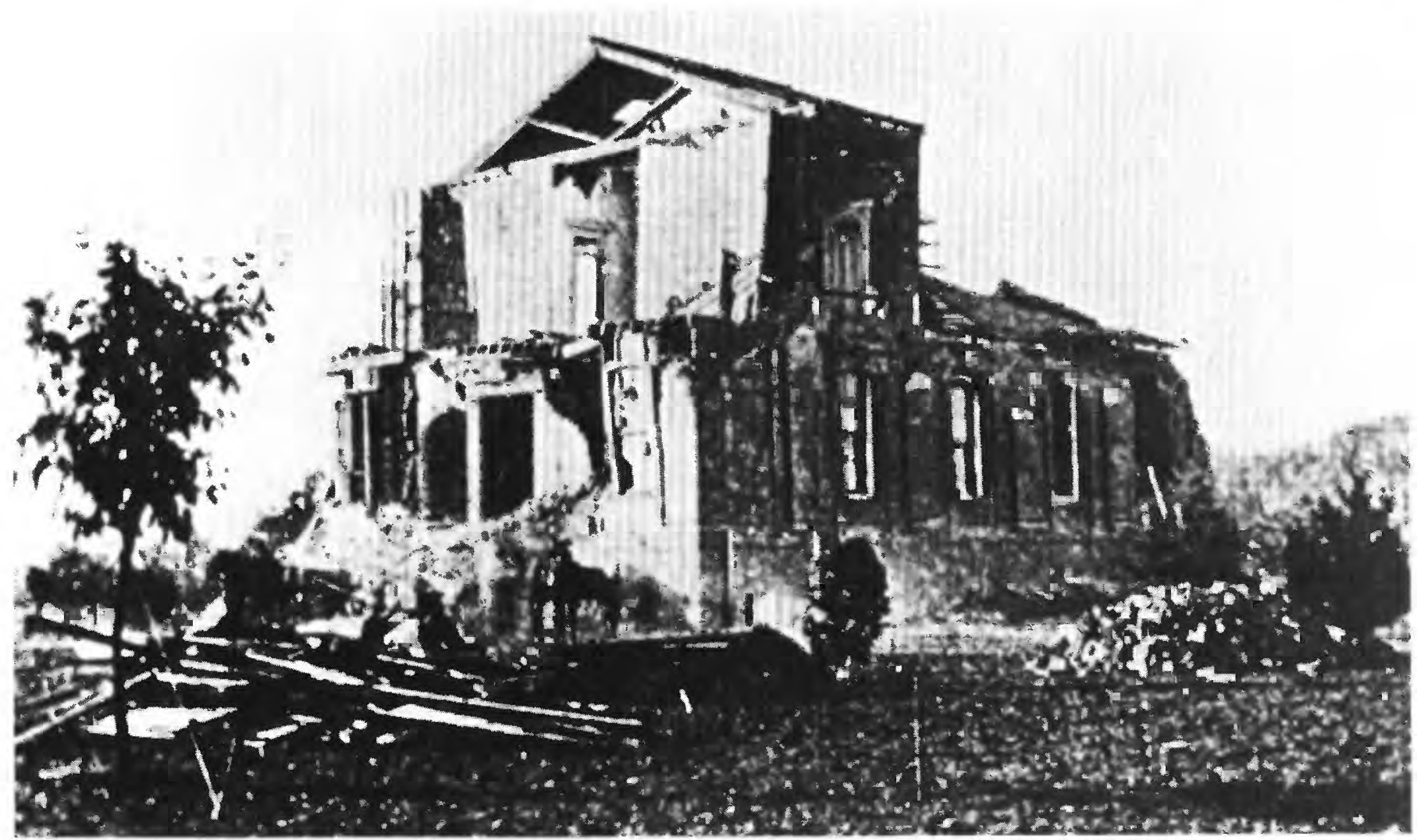

Courthouse at San Leandro, California, wrecked by the October 21, 1868, earthquake. (Photograph by University of California, Berkeley.)

observed near Mattole. Many chimneys also were ruined at Bucksport, Hydesville, Petrolia, and Rohnerville. The earthquake shook cornices from some buildings at Eureka and damaged the lightkeeper's house at Mendocino. A dozen aftershocks were reported at Mattole. (Ref. 38, 56, 368.)

1871. July 5. Near Lone Pine, Inyo County, Calif. Near Lone Pine, some wells went dry and others became muddy. People ran out of buildings at Swansea. This possible foreshock to the 1872 Owens Valley earthquake was felt northwest to Bear Valley in Mariposa County. (Ref. 38, 56, 368.)

1872. Mar. 26, 1030 UTC. Owens Valley, near Lone Pine, Inyo County, Calif. The most devastating effects of this earthquake occurred at Lone Pine, where 52 of 59 houses (mostly constructed of adobe or stone) were destroyed and 27 people were killed. A few fatalities also were reported in other parts of Owens Valley. One report states that the main buildings were thrown down in almost every town in Inyo County. About $100 \mathrm{~km}$ south of Lone Pine, at Indian Wells, adobe houses sustained cracks. Property loss has been estimated at $\$ 250,000$.

Faulting occurred on the Owens Valley fault along a line a few $\mathrm{km}$ east of the Sierra Nevada escarpment. The faulting near Lone Pine involved both dip- slip and right-lateral components of movement. The largest amount of surface deformation was observed between the towns of Lone Pine and Independence, but fault scarps formed along a length of at least 160 $\mathrm{km}$-from Haiwee Reservoir, south of Olancha, to Big Pine; cracks formed in the ground as far north as Bishop. The largest horizontal displacement of $7 \mathrm{~m}$ was measured on the fault scarps west of Lone Pine. The vertical offsets clearly were smaller, averaging about $1 \mathrm{~m}$ with the downthrown block on the east.

A comparison of this earthquake to the earthquakes of 1857 and 1906 on the San Andreas fault shows the felt area and maximum fault displacements to be comparable. However, the shocks on the San Andreas fault ruptured the fault for significantly larger distances- $-300 \mathrm{~km}$ in 1857 and 430 $\mathrm{km}$ in 1906.

This earthquake stopped clocks and awakened people at San Diego to the south, Red Bluff to the north, and Elko, Nev., to the east. MM intensity VIII or larger was observed over an area of about 25,000 $\mathrm{km}^{2}$, and MM intensity IX or larger was observed over an area of about $5,500 \mathrm{~km}^{2}$ (see fig. 14). The shock was felt over most of California and much of Nevada. Thousands of aftershocks occurred, some severe. (Ref. 38, 368, 521, 531, 533.) 


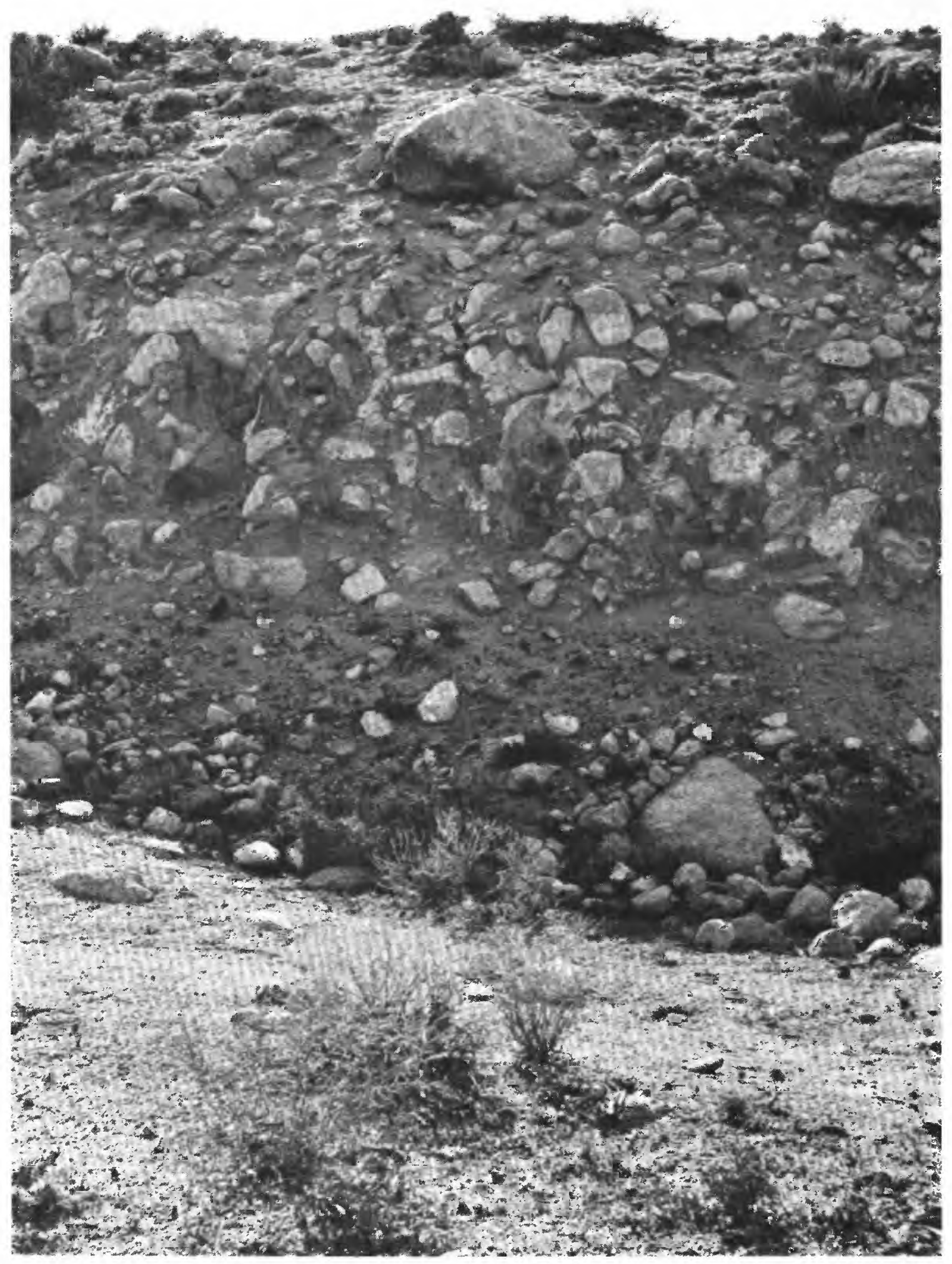

A 23-ft-high fault scarp caused by the March 26, 1872, Owens Valley, California, earthquake. (Photograph by W.D. Johnson.) 


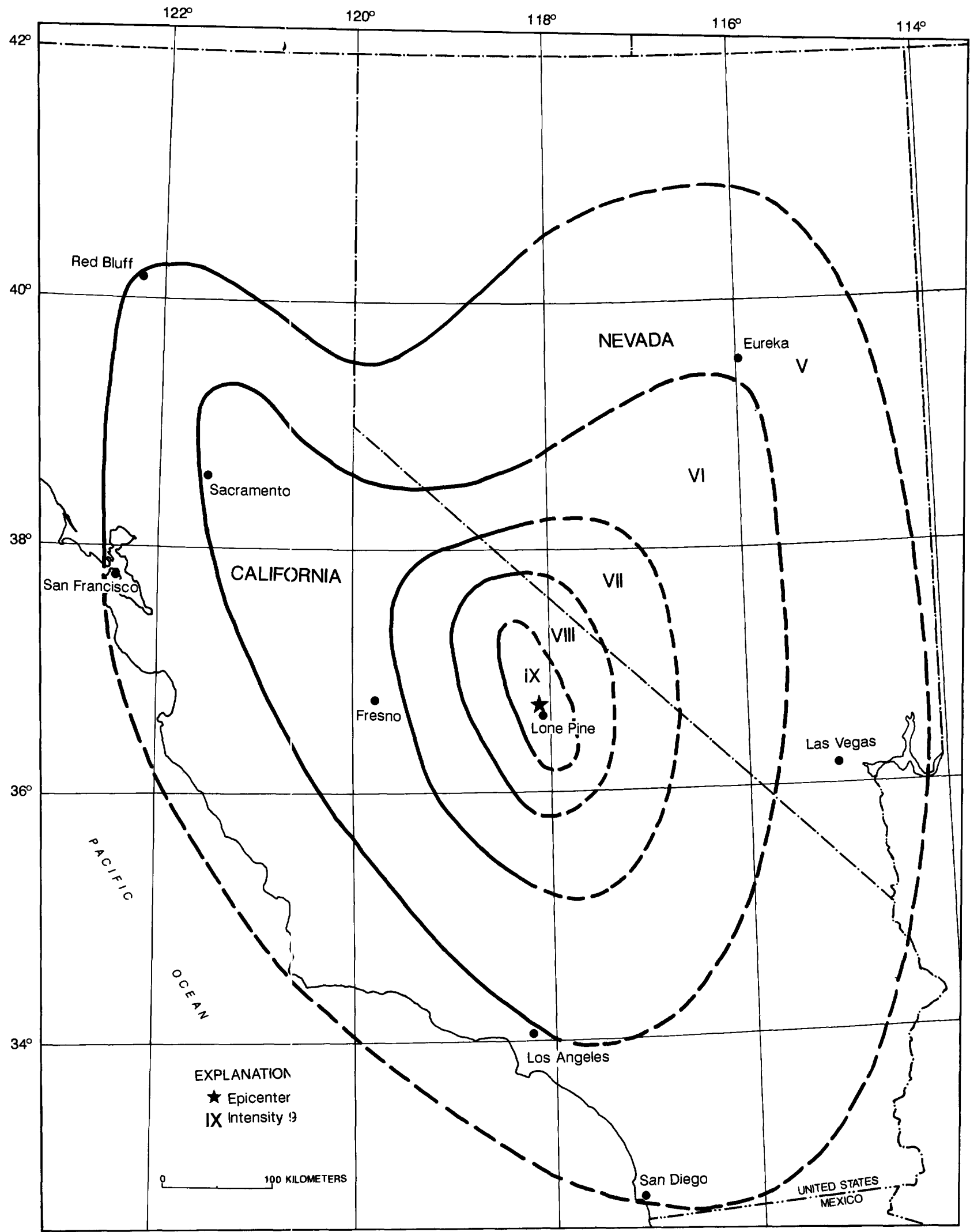

FIGURE 14.-Isoseismal map for the Owens Valley, California, earthquake of March 26, 1872. This map is a simplified version of the map in reference 368 of table 1. 
1872. Mar. 28. Near Sierraville, Sierra County, Calif. In Sierraville, bottles and chinaware in stores were broken. In Reno, Nev., the earthquake reportedly was stronger than the large Owens Valley earthquake, 2 days earlier. Also felt at Grass Valley. (Ref. 368.)

1872. Apr. 11. Near Round Valley, Inyo County, Calif. At Round Valley, stone buildings were knocked down and a frame structure was twisted on its foundation. Although this shock was felt at Susanville in Lassen County, the main shock of the Owens Valley earthquake on Mar. 26, 1872, was not reported felt there. (Ref. 368.)

1872. Apr. 18. Near Cerro Gordo, Inyo County, Calif. This rather small aftershock of the Owens Valley earthquake was reported only from Cerro Gordo. It was described as being of "unusual severity." (Ref. 56, 368.)

1872. May 3 (May 2). Imperial Valley area, Calif. A building was cracked and people ran into the streets in Arizona City (Yuma). It also was reported at San Bernardino, $300 \mathrm{~km}$ northwest of Arizona City. (Ref. 368.)

1872. May 17. Owens Valley, near Lone Pine, Calif. Furniture in a house was violently disturbed at Lone Pine, and one person was thrown from his chair. (Ref. 56, 368.)

1873. Nov. 23 (Nov. 22). Near Crescent City, Del Norte County, Calif. All chimneys were knocked down in the Smith River valley. Chimneys also fell at Crescent City, and hardly a brick building in the town escaped damage. Chimneys were damaged in many places as far north as Port Orford, Oreg., and east to Jacksonville, Oreg. Cracks in the ground were observed on the trail from Crescent City to Gasquet in Del Norte County. Felt south to San Francisco and north to Portland, Oreg. (Ref. 38, 56, 368,521 .)

1875. Jan. 24. South of Janesville in Plumas County, Calif. One chimney was downed at Janesville, and stone walls sustained cracks at Susanville (Lassen County). This shock awakened residents as far south as Sacramento. (Ref. 56, 368.)

1875. Sept. 30. Near Eureka, Humboldt County, Calif. At Eureka, most chimneys were cracked and some were knocked down. Residents of Weaverville in Trinity County were awakened. (Ref. 368.)

1876. May 29. Freestone, Sonoma County, Calif. At Freestone, some plaster was cracked and bottles and crockery were overturned. (Ref. 368.)

1878. May 9 (May 8). Near Petrolia, Humboldt County, Calif. At Petrolia, all chimneys in the city were knocked down. The earthquake also triggered large landslides along the coast in southern Humboldt County. (Ref. 368.)

1878. June 12 (June 11). Los Angeles, Calif. This earthquake shook down plaster and broke glassware at Los Angeles. Four distinct shocks occurred on June 12. (Ref. 463.)

1879. Feb. 4. Visalia, Tulare County, Calif. Two shocks occurred at Visalia, the second of which was the strongest. The second shock, which was felt over all the surrounding country, cracked walls and overturned furniture. (Ref. 56, 463.)

1880. Dec. 19. San Bernardino, Calif. Walls of the courthouse building were cracked from top to bottom. Three shocks were reported at Los Angeles. The main shock was felt south to San Diego. (Ref. $56,463$.

1881. Feb. 2 (Feb. 1). North of Imusdale, San Luis Obispo County, Calif. In Imusdale, at the north end of Cholame Valley, several chimneys were knocked down and an adobe storeroom and part of an adobe barn collapsed. Large cracks extending across the road were reported. (Ref. 56, 368.)

1881. Apr. 10. Southwest of Modesto, Stanislaus County, Calif. Minor damage to property was reported from Hollister to Stockton. Chimneys were damaged in the Modesto region. Felt from Greenville (Plumas County) on the north to Visalia (Tulare County) on the south. (Ref. $38,56,368$.)

1882. Mar. 6. East of Hollister, San Benito County, Calif. Windowpanes were broken at Salinas, and crockery was broken at Hollister. The shock, which was rather strong at Charleston and Merced, was reported from San Francisco on the north to San Luis Obispo on the south. Many aftershocks occurred. (Ref. $38,56,368$.)

1882. June 27. Santa Cruz area, Calif. Chimneys were overturned and windows were broken in the Santa Cruz Mountains. The walls of several buildings were cracked severely at San Jose, and crockery and glassware were broken at San Francisco. Reports from towns along the coast between Hollister and Petaluma (and as far east as Stockton) state that this shock was the most severe since the October 1868 earthquake. (Ref. 56, 463.)

1883. Mar. 30. Near Gilroy, Santa Clara County, Calif. This earthquake knocked down chimneys at Old Gilroy and Sargents. Plaster fell in all brick buildings at Hollister, and several large windows were broken; slight damage was sustained at Santa Cruz and Watsonville. At Gilroy, 8 to 12 aftershocks were reported, two of which were felt widely. The main shock was felt from Sacramento south to San Luis Obispo. (Ref. 38, 56, 368.) 
1883. Sept. 5. Off the coast near Santa Barbara, Calif. Plaster fell at Santa Barbara, and bottles were overturned at Los Alamos. Felt along the coast from Cayucos (near San Luis Obispo) south to Wilmington (Los Angeles County). (Ref. 38, 56, 368.)

1884. Mar. 26 (Mar. 25). Near Santa Cruz, Calif. Plaster fell, walls cracked, and windowpanes broke at Santa Cruz. Similar effects were reported at San Francisco. Felt along the coast from Monterey north to Santa Rosa (Sonoma County). (Ref. 56, 368.)

1885. Jan. 31 (Jan. 30). Near Susanville, Lassen County, Calif. Chimneys were damaged in the Honey Lake Valley towns of Buntingville and Susanville. Shocks were most severe near Janesville. Felt north to Alturas (Modoc County), south to Sacramento, and at a few towns in Nevada. More than 100 aftershocks were felt in the area to Feb. 8, 1885. (Ref. 38, 56, 368.)

1885. Mar. 31 (Mar. 30). South of Hollister, San Benito County, Calif. Chimneys were thrown down at Mulberry, and plaster fell at Hollister. Extensive fissures formed in the soft riverbanks at the junction of the Pajaro and San Benito Rivers. The shock was reported felt as far north as San Rafael. One foreshock and three aftershocks were felt at Hollister on Mar. 31. (Ref. 38, 56, 368.)

1885. Apr. 12 (Apr. 11). Southern San Benito County, Calif. This earthquake threw down two chimneys at Las Tablas, $48 \mathrm{~km}$ northwest of San Luis Obispo. Slight damage was reported at Monterey (where cracks in adobe walls were widened), Salinas, and San Luis Obispo. Felt north to Marysville (Yuba County), south to Ventura, and east to Keeler (Inyo County). Toppozada and Wong (ref. 612 ) have determined that this event was located about $50 \mathrm{~km}$ northwest of the May 2, 1983, Coalinga earthquake. Toppozada (1991, oral commun.) has also determined that the April 2, 1885 earthquake was located about $50 \mathrm{~km}$ southeast of its listed location and was near the location of this April 12 event. (Ref. $38,56,368$.)

1885. Aug. 1 (July 31). Near Cloverdale, Sonoma County, Calif. A strong local earthquake cracked walls in several buildings at Cloverdale. It was not reported in nearby towns. (Ref. 38, 368.)

1886. Apr. 14 (Apr. 13). Inyo County, Calif. This local earthquake broke considerable crockery and dishes in Lida Valley on the California-Nevada border. A dull, thunderlike noise preceded the shock. (Ref. 54.)

1887. Dec. 3. Near Halfway House, Mendocino County, Calif. At Halfway House, on the road from Mendocino to Ukiah, chimneys were downed and a stove overturned. A chimney was cracked at Christine, and clocks stopped at Ukiah. (Ref. 56, 368.)

1888. Feb. 29. North of Petaluma, Sonoma County, Calif. A sharp earthquake cracked the walls of several buildings at Petaluma. Felt north to Geyserville (Sonoma County) and south to San Francisco. (Ref. 38, 56, 368.)

1888. Apr. 14 (Apr. 13). Alturas, Modoc County, Calif. The earthquake rang a school bell and knocked down plaster at Alturas. It was reported felt only at a few towns in the area. (Ref. 368.)

1888. Apr. 29 (Apr. 28), Near Cromberg, Plumas County, Calif. Chimneys were downed at Cromberg, at the north end of Mohawk Valley. Rockslides were reported north of Downieville on the North Fork of the North Yuba River and in Rattlesnake Canyon. South of Cromberg, walls of the courthouse were cracked at Nevada City, and tops of chimneys were knocked off at Grass Valley. Felt from San Francisco northeastward into Nevada. Many aftershocks were felt during the night in the Downieville area. (Ref. 38, 56, 368.)

1888. Nov. 18. San Francisco Bay area, Calif. Several chimneys were overthrown at East Oakland and Oakland, which lie at the southern limit of the felt area. Felt from Napa south to San Lorenzo. Two slight aftershocks were felt at Oakland. (Ref. 38, 56, 368.)

1889. Apr. 15 (Apr. 14). Near Santa Cruz, Calif. This shock cracked plaster in several houses in Santa Cruz. Felt from Corral de Tierra (Monterey County) north to Martinez (Contra Costa County). (Ref. 56, 368.)

1889. May 19. West of Antioch, Contra Costa County, Calif. Many chimneys were demolished at Antioch, and two small fissures formed on Main Street. At Collinsville, one house was toppled and chimneys were knocked down. Slight loss to property also was reported at Lodi, Napa, Rio Vista, and San Francisco. Felt north to Nevada City, south to Santa Cruz, and east to Sonora (Tuolumne County). (Ref. $38,368$.

1889. June 20 (June 19). North of Susanville, Lassen County, Calif. The earthquake was most severe in the Susanville-Willow Creek area, where chimneys were thrown down, and the water in Eagle Lake was muddied. As many as 75 aftershocks occurred, 28 of which were felt within 2 hours of the main event. Felt north to Alturas (Modoc County), south to Sacramento, and east to Virginia City, Nev. (Ref. 38, 56, 368.)

1889. July 31. San Francisco Bay area, Calif. Chimneys were knocked down at Oakland and San 
Leandro, and a brick pier was cracked at the Chabot Observatory in south Oakland. Felt from Salinas (Monterey County) north to Healdsburg (Sonoma County) and east to Modesto (Stanislaus County). Aftershocks were reported felt at San Francisco. (Ref. $38,56,368$.)

1889. Aug. 28 (Aug. 27). Near Pomona, Los Angeles County, Calif. Windows and crockery were cracked and broken at Pomona, where two distinct shocks threw several people to the floor. Several towns between Los Angeles and Ontario reported minor damage, including fallen plaster and bottles knocked from store shelves. Felt mainly over an eastwest area from San Jacinto in the east to Santa Monica in the west. (Ref. 38, 56, 368.)

1890. Feb. 9. Northeast San Diego County, Calif. It is inferred from the lack of reports of damage that the epicenter was in the sparsely populated region between Los Angeles and Yuma, Ariz. Windows were broken at Pomona, and residents were awakened, but little loss was reported in the region. Telegraph dispatches from all towns along the Southern Pacific Line (from Pomona to Yuma) reported the shock was of equal intensity at each town. A possible foreshock on Feb. 6 was reported felt from San Bernardino to San Diego. (Ref. 38, 56, 368.)

1890. Apr. 24. Near Corralitos, Santa Cruz County, Calif. This severe earthquake caused extensive loss of chimneys and some damage to brick and frame buildings from San Juan Bautista to Green Valley. Chimneys were knocked over at Watsonville and Corralitos, and buildings were twisted on their foundations at Corralitos. The San Andreas fault probably ruptured in the area where it crosses the Pajaro River. Fissures were reported on or near the fault, and a railroad bridge across the Pajaro River (near the fault) shifted about $5 \mathrm{~m}$ out of alignment. Aftershocks occurred for many days. (Ref. $38,56,368$.)

1890. July 26. Near Ferndale, Humboldt County, Calif. A severe earthquake knocked down chimneys at Grizzly Bluff (near Ferndale) and at the Walker residence (near Petrolia). Although strong at Eureka and Mendocino City, the shock caused little or no damage. A spring at the Walker "place" that had stopped flowing 3 days before the shock became murky and flowed at a faster rate than before. An aftershock at 1600 UTC was reported at Ferndale, Hydesville, and Rohnerville. The main shock was reported from Crescent City and Sisson (Mt. Shasta City) in the north to Petaluma (Sonoma County) in the south. (Ref. 38, 56, 368.)

1891. Jan. 2. Near San Jose, Santa Clara County, Calif. At Mount Hamilton, several large pieces of plaster were dislodged and ceilings cracked. Plaster was knocked to the floor at Santa Cruz, and windows were broken at San Jose. Although this earthquake was strong at Gilroy, little or no damage was reported. Felt to Vallejo in the north, Monterey in the south, and Merced in the east. (Ref. 38, 56, 368.)

1891. Oct. 12 (Oct. 11). Near Napa, Calif. In Napa and Sonoma, many chimneys were overthrown and several brick buildings were cracked and thrown out of plumb. Not one house in Sonoma Valley escaped damage of some kind. A foreshock was felt at Napa and Sonoma at 0515 UTC, and many aftershocks were observed. The main shock was felt north to Lakeport and Colusa and south to Salinas. (Ref. $38,56,368$.)

1892. Feb. 24 (Feb. 23). California-Mexico border area. At the old Carrizo station in San Diego County, all adobe buildings were destroyed. In Paradise Valley, a church and schoolhouse built on stilts were knocked down and demolished; chimneys and plaster were broken in San Diego. Ground fissures were reported at McCain Valley and Jewel Valley; rockslides were observed between Campo and Carrizo and at Dulzura and Jewel Valley.

About 155 tremors were felt at Campo during the 12 hours following the main shock, and aftershocks continued there every few days into April 1892. Observers reported that 135 aftershocks were felt as far away as National City, on San Diego Bay. Felt north along the coast to Santa Barbara, east to Yuma, Ariz., and south to San Quintin, Baja California. One report stated that the tremor was felt at Visalia, Tulare County, about $700 \mathrm{~km}$ north of San Quintin. (Ref. 38, 56, 368, 521.)

1892. Apr. 19. Near Vacaville, Solano County, Calif. This earthquake caused severe damage at Allendale, Dixon, Vacaville, and Winters. Property loss was estimated at $\$ 225,000$, and one fatality was reported.

At Allendale, between Vacaville and Winters, several buildings in the area collapsed, shifted off their foundations, or were wrenched apart. Ground fissures formed near Allendale, which suggests possible faulting. At Vacaville, almost all brick structures were destroyed, many frame buildings were impaired, and chimneys were twisted or knocked to the ground. Similar damage was reported from Winters (Yolo County). Although damage in general was less serious in Dixon, many school buildings were almost ruined. MM intensity VIII or higher was observed over an area of $1,100 \mathrm{~km}^{2}$. Felt north to Redding, east to Virginia City, Nev., and south to Salinas and Fresno. (Ref. 38, 56, 368, 533, 599.) 


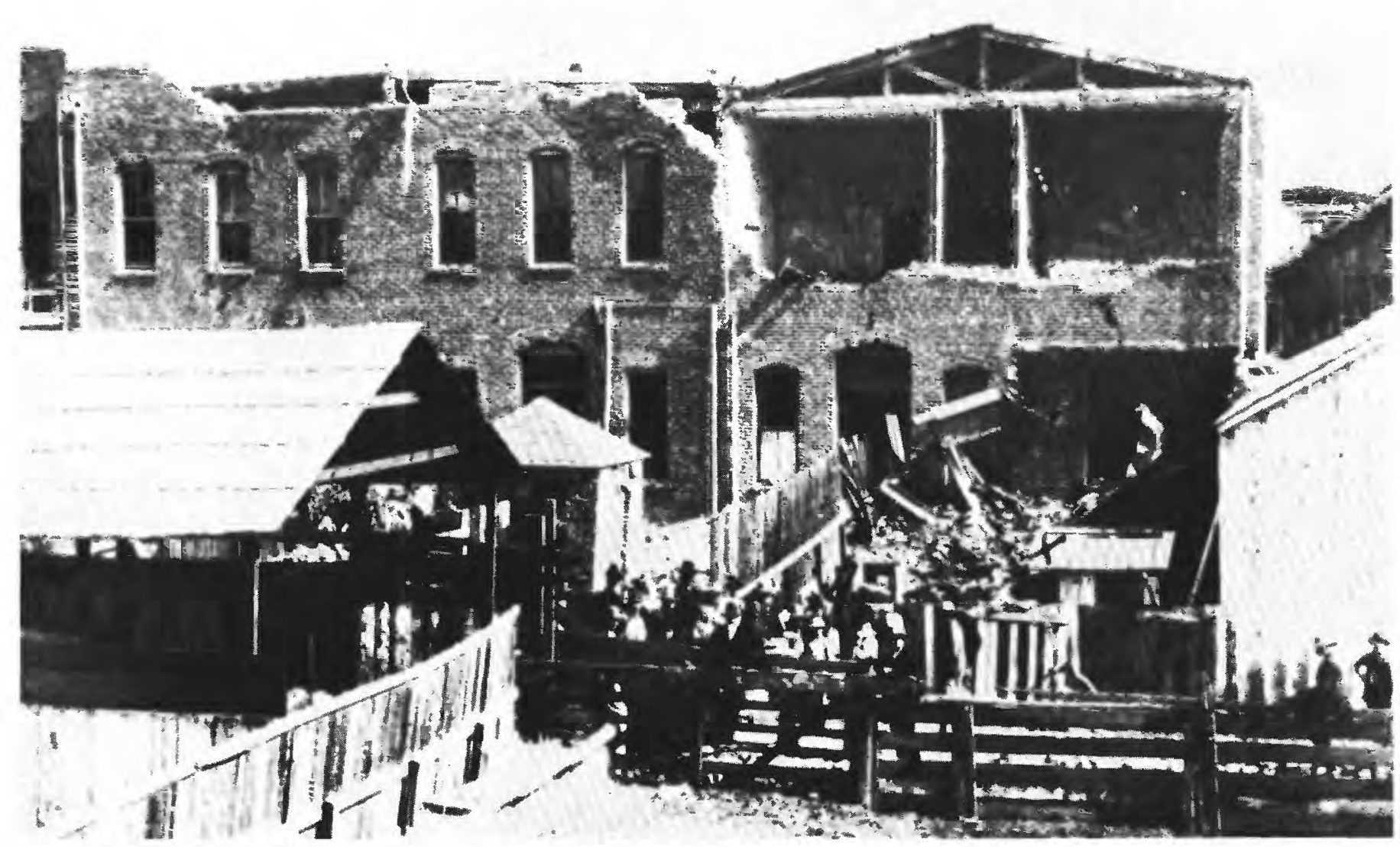

Brick walls of a hotel in Winters, California, collapsed by the April 19, 1892, earthquake. (Photograph from California Geology.)

1892. Apr. 21. Near Winters, in Solano County, Calif. Weakened structures in the communities hit by the Apr. 19 earthquake were further damaged. At Winters, where the damage was most severe, many buildings that withstood the Apr. 19 shock were leveled. Not one building on Main Street was left habitable. At Esparto, every brick chimney fell and wood-frame buildings were wrenched out of shape. Many chimneys were wrecked at Sacramento and Woodland, but additional loss was slight at Dixon and Vacaville. The area shaken at MM intensity VIII or larger was about $890 \mathrm{~km}^{2}$, but the general felt area was about the same as that of the Apr. 19 shock. (Ref. 38, 56, 368.)

1892. Apr. 30 (Apr. 29). Near Davis, Solano County, Calif. This aftershock of the Apr. 19 earthquake knocked a few loose bricks from buildings at Davisville (Davis) and sent people at Sacramento running from their houses. Felt from San Jose north to Yuba City and east to Carson City, Nev. (Ref. 56, 368.)

1892. Nov. 13. West of Hollister, San Benito County, Calif. At Hollister, a chimney was displaced and plaster fell; at Monterey, chimneys were cracked; and at Salinas, windows and dishes were broken. Three less severe aftershocks were felt at Hollister. Felt from Monterey north to Napa. (Ref. 38, 56, 368.)

1893. Apr. 4. Near Newhall, Los Angeles County, Calif. Northwest of Newhall, an old but sturdy adobe house was shaken down at the Newhall Ranch. In the area of Newhall and Pico Canyon, chimneys were wrecked, the ground was fissured, and boulders were shaken down the hillsides. Aftershocks were reported almost daily at Tapo Ranch in the Simi Hills. Felt from Santa Barbara east to San Bernardino. (Ref. 38, 56, 368.)

1893. Aug. 9. East of Santa Rosa, Sonoma County, Calif. Most of the loss to property occurred at Santa Rosa: chimneys were downed, plaster in the courthouse was cracked and broken, and windows were broken. At Petaluma, plaster was cracked and crockery was thrown from shelves. Felt north to Middletown (Lake County), south to Alameda, and east to Sacramento. (Ref. 38, 368.)

1894. July 30 (July 29). Northwest of San Bernardino, Calif. An earthquake caused minor damage from the Los Angeles basin to Mojave in 
Kern County. At Los Angeles, three distinct tremors broke a few windowpanes and knocked bottles of ink off shelves. Earlier in the evening, several foreshocks were reported at Riverside. Felt north to Mojave and south to San Diego. (Ref. 56, 368.)

1894. Sept. 30. Southern Humboldt County, Calif. This earthquake knocked down chimneys in southern Humboldt County. It was felt strongly on two schooners at sea, about $56 \mathrm{~km}$ southwest of Shelter Cove. An aftershock was felt at Eureka and Mendocino at 1758 UTC. The main shock was reported north to Crescent City, south to Ukiah, and east to Redding and Sisson (Mt. Shasta City). (Ref. 38, 368).

1894. Oct. 23. Near San Diego, Calif. Plaster was broken and brick walls were cracked in San Diego; mission bells rang in San Juan Capistrano (Orange County). A dust cloud was seen along the summit of the mountains above Buckman's Springs, and boulders were heard rolling down the canyons. Several aftershocks were felt in the area through Oct. 28. Felt north to Los Angeles and San Bernardino. (Ref. $38,56,368$.)

1896. Aug. 17. Near Independence, Inyo County, Calif. This earthquake probably occurred along or near the eastern front of the Sierra Nevada. "Crumbled adobes slid to the ground" at Independence. Loose plaster was knocked down at Hanford, and clocks stopped as far distant as Bakersfield and Merced. The shock was felt from Big Pine (Inyo County), west to Merced, and south to Bakersfield. (Ref. 56, 368.)

1897. June 20. Near Gilroy, Santa Clara County, Calif. The earthquake caused much loss to brick buildings in the Gilroy-San Felipe area. Few brick structures escaped damage at Hollister; chimneys were thrown down and fire walls fell into the street at Salinas. Damage in Gilroy was similar to that in Hollister and Salinas. A fissure was observed near Soap Lake House on the Pacheco Pass Road, and a fissure $295 \mathrm{~m}$ long formed on a ranch near San Felipe. Because the Calaveras fault crosses the Pacheco Pass Road $5 \mathrm{~km}$ northwest of San Felipe, the fissures could have resulted from rupture on the Calaveras. Felt from Woodland on the north to San Luis Obispo on the south and Visalia (Tulare County) on the east. (Ref. $38,56,368$.)

1898. Mar. 31 (Mar. 30). Southern Sonoma County, Calif. At Mare Island Naval Yard and Tubbs Island, several buildings either partly or totally collapsed. Property loss was estimated at $\$ 350,000$. The earthquake also caused severe damage at Schellville, Greenwood Estate, and along Petaluma Creek in Sonoma County, where houses were moved off their foundations. Extensive cracks developed in the ground at Greenwood Estate, Mare Island Naval Yard, and Schellville. Moderate damage occurred in San Francisco. Felt north to Chico, east to Carson City, Nev., and south to Monterey. Magnitude $6.5 \mathrm{M}_{\mathrm{S}}$ ELL. (Ref. 56, 368, 521, 615.)

1898. Apr. 15 (Apr. 14). Near Greenwood, Mendocino County, Calif. Two wood-frame houses were wrecked at Greenwood, and one was toppled at Noyo. Chimneys were knocked down in several towns in Mendocino County, including Fort Bragg, Little River, Mendocino, Pine Grove, and Ukiah. Property loss at Point Arena included cracking of the lighthouse tower. Cemetery monuments fell or were twisted on their bases at Mendocino. Landslides and fallen trees made the mountain roads east of Mendocino impassable. A foreshock was felt at Mendocino at 0645 UTC, and many aftershocks were felt there. The main shock was felt north to Eureka, east to Red Bluff and Willows, and south to San Francisco. Magnitude 6.7 $\mathrm{M}_{\mathrm{S}}$ ELL. (Ref. 38, 56, 368, 521, 615.)

1899. Apr. 16. Off the coast of Humboldt County, west of Eureka, Calif. This long-duration earthquake caused some minor impairment to a flue at a lumber mill in Eureka. It was described as one of the most severe shocks ever experienced. Felt along the coast from Crescent City on the north to Albion (Mendocino County) on the south. (Ref. 38, 56, 368,521 .)

1899. Apr. 30. Near Watsonville, Santa Cruz County, Calif. Damage from this earthquake included downed chimneys and displaced cemetery monuments in Green Valley and Watsonville, fallen plaster in Hollister, and broken crockery and windowpanes in Salinas. Felt from southern Sonoma and Napa Counties on the north to northern Monterey County on the south. (Ref. $38,56,368$.)

1899. June 2 (June 1). Near San Francisco in northern San Mateo County, Calif. In San Francisco, chimneys were knocked down, parts of several cornices fell, and windows and glassware were broken. Chimneys also were toppled in Oakland. This moderate earthquake was felt south to Santa Cruz, north to Calistoga and Sacramento, and east to Modesto (San Joaquin County). (Ref. 38, 368.)

1899. July 6. Northeast of Watsonville in Santa Clara County, Calif. Several chimneys were toppled at Watsonville; a few lamp chimneys and windows were broken in Salinas. At Pleasanton (Alameda County), about $85 \mathrm{~km}$ north of Watsonville, brick buildings were cracked, and some wooden structures were "more or less twisted." Because the intensities at sites between these two towns were not high (MM intensity $\mathrm{V}-\mathrm{VI}$ ), there may have been. two earthquakes at about the same time-one near Watsonville 
and the other near Pleasanton. Felt north to Napa, south to San Luis Obispo, and east to Merced and beyond. (Ref. 56, 368.)

1899. July 22, 0046 UTC (July 21). West of Squirrel Inn, San Bernardino County, Calif. This foreshock to the earthquake at 2032 UTC (see below) knocked off tops of chimneys at Squirrel Inn. Landslides were reported in the Cajon Pass area. Felt north to Barstow, west to Santa Monica (Los Angeles County), and south to San Diego. (Ref. 368.)

1899. July 22, 2032 UTC. Lytle Creek Canyon, San Bernardino County, Calif. North of San Bernardino, in Lytle Creek Canyon, one old adobe house was knocked down. Streams greatly increased their flow of water in the mountains north of Cucamonga and San Bernardino, and extensive landslides were observed. This severe earthquake caused loss to property from Anaheim to Barstow. Felt north to Barstow, west to Ventura, and south to San Diego. Nine aftershocks were felt in the San Bernardino area. Magnitude $5.6 \mathrm{M}_{\mathrm{S}}$ ELL (Ref. 38, 56, 368, 521.)

1899. Oct. 13 (Oct. 12). Near Santa Rosa, Sonoma County, Calif. This earthquake toppled some chimneys and knocked plaster from walls in Santa Rosa. It was felt only to Petaluma, about 23 km south of Santa Rosa. Aftershocks were reported in the area. (Ref. 38, 368.)

1899. Dec. 25. Hemet-San Jacinto, Riverside County, Calif. Property damage from this earthquake was most severe at Hemet and San Jacinto, west of Palm Springs. Six people were killed by falling adobe walls at Saboba, a few kilometers east of San Jacinto. The estimated property loss of about $\$ 50,000$ appears to be low.

Only two chimneys remained standing in Hemet, where brick buildings partly collapsed and woodframe buildings shifted off their foundations. A ground fissure about $46 \mathrm{~m}$ long extended under a house near Hemet; the house was wrenched and twisted severely. The fissure may have been surface rupture in the San Jacinto fault zone. Many brick buildings were partly wrecked at San Jacinto. At Riverside, chimneys were overthrown, and brick buildings were cracked. This severe shock was felt north to Bakersfield (Kern County), south to Jacumba (San Diego County), and northeast to Needles (San Bernardino County). It also was reported felt at Seligman, Ariz. Many aftershocks occurred on Dec. 25 and 26. Magnitude $6.6 \mathrm{M}_{\mathrm{La}}$ DMG. (Ref. 38, $56,368,384$.)

1901. Mar. 3 (Mar. 2). Near Parkfield, Monterey County, Calif. Chimneys were toppled at Bradley, Echo Valley, Parkfield, Slacks Canyon,
Stone Canyon, and Warthan Canyon. At Parkfield, three houses were twisted out of shape and one was almost wrecked. Slight loss to property also was incurred at Adelaida, El Monte, Estrella, Monterey, Paso Robles, and San Miguel. Ground cracks as much as several meters in length and $15-30 \mathrm{~cm}$ in width and vertical displacement of about $30 \mathrm{~cm}$ were observed in Stone Canyon. Felt from San Francisco on the north to San Luis Obispo on the south and Porterville (Tulare County) on the east. Magnitude 5.8 $\mathrm{M}_{\mathrm{La}}$ DMG. (Ref. 38, 56, 381.)

1902. May 19. South of Elmira, Solano County, Calif. Almost all chimneys fell at Elmira, and every flue was out of line; a few chimneys toppled at Vacaville, where many brick buildings were cracked badly. Only slight damage occurred at Fairfield, Nevada City, and Suisun City. Felt north to Colusa, south to San Francisco, and east to Ione (Amador County) and beyond. (Ref. 38, 56, 381.)

1902. July 28 (July 27). Near Los Alamos, Santa Barbara County, Calif. At Los Alamos, store buildings were damaged, chimneys were broken, and walls were cracked. Two tanks, each containing about 3,000 barrels of oil, were destroyed on the property of the Western Union Oil Company. An adobe house at the Orena Ranch, a few kilometers south of Lompoc, was affected severely and later destroyed by the July 31 aftershock. At Lompoc, chimneys toppled, one brick building was ruined, and pipelines were broken; at Santa Maria, several chimneys fell from buildings. Felt along the coast from San Luis Obispo to Ventura. Many aftershocks occurred. (Ref. $38,56,381$.)

1902. July 31; Aug. 1 (July 31). Near Los Alamos, Santa Barbara County, Calif. Two strong earthquakes affected Los Alamos and environs, and many slight shocks were felt during the day. The two events are described together because the effects for each cannot be separated. These earthquakes "completed the ruin" begun on July 28. All houses were damaged at Los Alamos, and not one chimney was left upright. The main effects were confined to an area about $24 \mathrm{~km}$ long and $6 \mathrm{~km}$ wide. Fissures formed in the ground, landslides occurred, and water began flowing in a formerly dry streambed. The shocks were felt along the coast from Cayucos (San Luis Obispo County) to Oxnard (Ventura County). (Ref. 38, 56, 380, 381.)

1902. Dec. 12. Near Los Alamos, Santa Barbara County, Calif. At Santa Maria, plaster fell in many houses and walls of a brick school were cracked. Dishes and glassware were thrown from shelves at Los Alamos. (Ref. 38, 56, 380.) 
1903. June 11. Near San Jose, Santa Clara County, Calif. One chimney fell, other chimneys lost their tops, and a brick wall was downed at San Jose. A few chimneys also toppled at Hayward, Livermore, Santa Cruz, and Watsonville. Felt north to Fort Ross (Sonoma County) and south to King City (Monterey County). Because an earthquake also was reported at San Luis Obispo, it is possible that there were two different events. Magnitude 5.4 $\mathrm{M}_{\mathrm{S}}$ ELL. (Ref. 38, 56, 381, 521.)

1903. July 24. Near Willows, Glenn County, Calif. At Willows, plaster fell from many buildings and several brick walls were cracked. Felt from Sacramento north to Greenville (Plumas County) and from Willows east to Nevada City (Yuba County). (Ref. 38, 56.)

1903. Aug. 3 (Aug. 2). Near San Jose, Santa Clara County, Calif. Few large buildings in San Jose were left undamaged, and many were damaged severely. Scores of chimneys were shaken down; brickwork on many of the larger buildings was ruined; and stone trimmings fell to the streets. At Evergreen, a few kilometers southwest of San Jose, one house was shifted on its foundation, and another house lost its chimney. Chimneys were broken to the roofline in Santa Clara. Only slight loss to property was reported at Edenvale, Mount Hamilton, Oakland, San Francisco, Santa Cruz, and Stockton. Felt from Guerneville on the north to Jamesburg on the south to Stockton on the east. Magnitude $5.3 \mathrm{M}_{\mathrm{S}}$ ELL. (Ref. 38, 381, 521.)

1903. Dec. 25. Los Angeles, Calif., region. Plaster and bricks were knocked down in Los Angeles, and bottles were thrown to the floor in a drug store. The shock also was reported at Pasadena, Riverside, San Bernardino, and Sierra Madre. (Ref. 38, 56.)

1905. July 15. Near San Bernardino, Calif. No damage could be documented for this earthquake. The intensity listed was taken from ref. 56. (Ref. 56, 380.)

1905. Sept. 3 (Sept. 2). Los Angeles, Calif., region. Plaster fell in the "Baker block" of Los Angeles, and a heavy bookcase was overturned in the City Hall. (Ref. 56, 380.)

1905. Dec. 23. Bakersfield, Kern County, Calif. Wide cracks formed in buildings in Bakersfield, and large quantities of plaster fell as a result of this strong local earthquake. Objects were thrown from shelves. Two small foreshocks occurred. (Ref. $38,56,380$.)

1906. Apr. 18. Near San Francisco, Calif. This earthquake is one of the most devastating in the history of California. The earthquake and resulting fires caused an estimated 3,000 deaths and \$524 million in property loss. Damage in San Francisco resulting only from the earthquake was estimated at $\$ 20$ million; outside the city, it was estimated at $\$ 4$ million. The sensible duration of the shaking in San Francisco was about 1 minute.

The earthquake damaged buildings and structures in all parts of the city and county of San Francisco, although over much of the area, the damage was moderate in amount and character. Most chimneys toppled or were badly broken. In the business district, which was built on ground made by filling in the cove of Yerba Buena, pavements were buckled, arched, and fissured; brick and frame houses of ordinary construction were damaged extensively or destroyed; sewers and water mains were broken; and streetcar tracks were bent into wavelike forms.

On or near the San Andreas fault, buildings were destroyed (one was torn apart), and trees were knocked to the ground. The surface of the ground was torn and heaved into furrow-like ridges. Roads crossing the faultline were impassable, and pipelines were broken. One pipeline that carried water from San Andreas Lake to San Francisco was broken, shutting off the water supply to the city. The fires that ignited soon after the onset of the earthquake quickly raged through the city because of the lack of water to control them. They destroyed a large part of San Francisco and intensified the loss at Fort Bragg and Santa Rosa.

This earthquake caused the most lengthy rupture of a fault that has been observed in the contiguous United States. The displacement of the San Andreas Fault was observed over a distance of $300 \mathrm{~km}$ from San Juan Bautista to Point Arena, where it passes out to sea. Additional displacement was observed farther north at Shelter Cove in Humbolt County, and, assuming the rupture was continuous, the total length of rupture would extend to $430 \mathrm{~km}$. The largest horizontal displacement-6.4 m-occurred near Point Reyes Station in Marin County.

In areas where dislocation of fences and roads indicated the amount of ground movement, motions of 3 to $4.5 \mathrm{~m}$ were common. Near Point Arena, in Mendocino County, a fence and a row of trees were displaced almost $5 \mathrm{~m}$. At Wright's Station, in Santa Clara County, a lateral displacement of $1.4 \mathrm{~m}$ was observed. Vertical displacement of as much as $0.9 \mathrm{~m}$ was observed near Fort Ross in Sonoma County. Vertical displacement was not detected toward the south end of the fault.

Although Santa Rosa lies about $30 \mathrm{~km}$ from the San Andreas fault, damage to property was severe, and 50 people were killed. The earthquake also was severe in the Los Banos area of the western San Joaquin Valley, where the MM intensity more than 


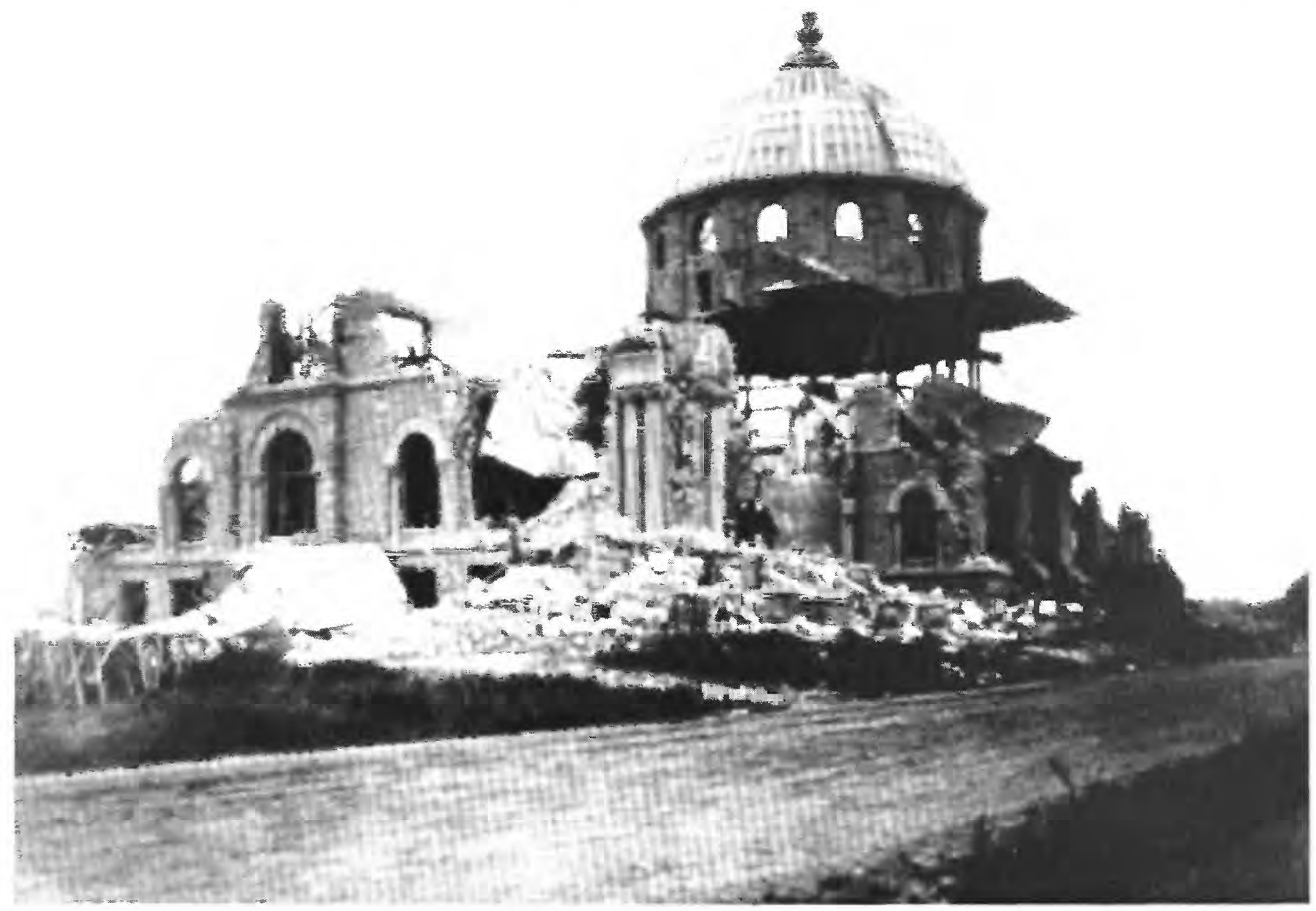

The new stone-faced brick library at Stanford University, Palo Alto, California, was destroyed by the San Francisco, California, earthquake on April 18, 1906. (Photograph by W.C. Mendenhall.)

$48 \mathrm{~km}$ from the fault zone was IX. Santa Rosa lies directly inland from the region of greatest motion on the San Andreas fault.

Trees swayed violently, and some were broken off above the ground or thrown down. The water in springs and artesian wells either increased or decreased its flow. A few sand craterlets formed in areas where water was ejected through cracks or fissures.

The region of destructive intensity extended over a distance of $600 \mathrm{~km}$. The total felt area included most of California and parts of western Nevada and southern Oregon (see fig. 15). The maximum intensity of XI was based on geologic effects, but the highest intensity based on damage was IX. Several foreshocks probably occurred, and many aftershocks were reported, some of which were severe. Magnitude 8.3 $\mathrm{M}_{\mathrm{S}}$ CFR, 8.25 $\mathrm{M}_{\mathrm{S}} \mathrm{GR}, 7.4 \mathrm{~m}_{\mathrm{b}} \mathrm{ABE}, 7.8 \mathrm{M}_{\mathrm{La}}$ DMG (Ref. 38, 56, 378, 381, 517, 533, 567, 571, $572,573$.
1906. Apr. 19 (Apr. 18). Near Brawley, Imperial County, Calif. A strong earthquake almost destroyed the Van Ness and Marlour buildings in Brawley. Walls fell into the streets, and chimneys fell to the ground. Not one brick or adobe building in the Brawley area escaped damage. Observers reported that banks along the New River collapsed. Water tanks were destroyed at Cocopar, Baja California. Slight damage was reported at Calexico, El Centro, Holtville, and Imperial. Felt north to Los Angeles, south to Baja California, and east to Yuma, Ariz. Magnitude 6+ $\mathrm{M}_{\mathrm{S}}$ CFR, $5.8 \mathrm{M}_{\mathrm{La}}$ DMG. (Ref. $38,56,381$.)

1906. Apr. 23. Near Ferndale, Humboldt County, Calif. Chimneys were toppled and loose objects were knocked down at Ferndale. The earthquake stopped clocks at Cape Mendocino, Eureka, and Trinidad Head. It was reported felt into Oregon. (Ref. $38,56$. 


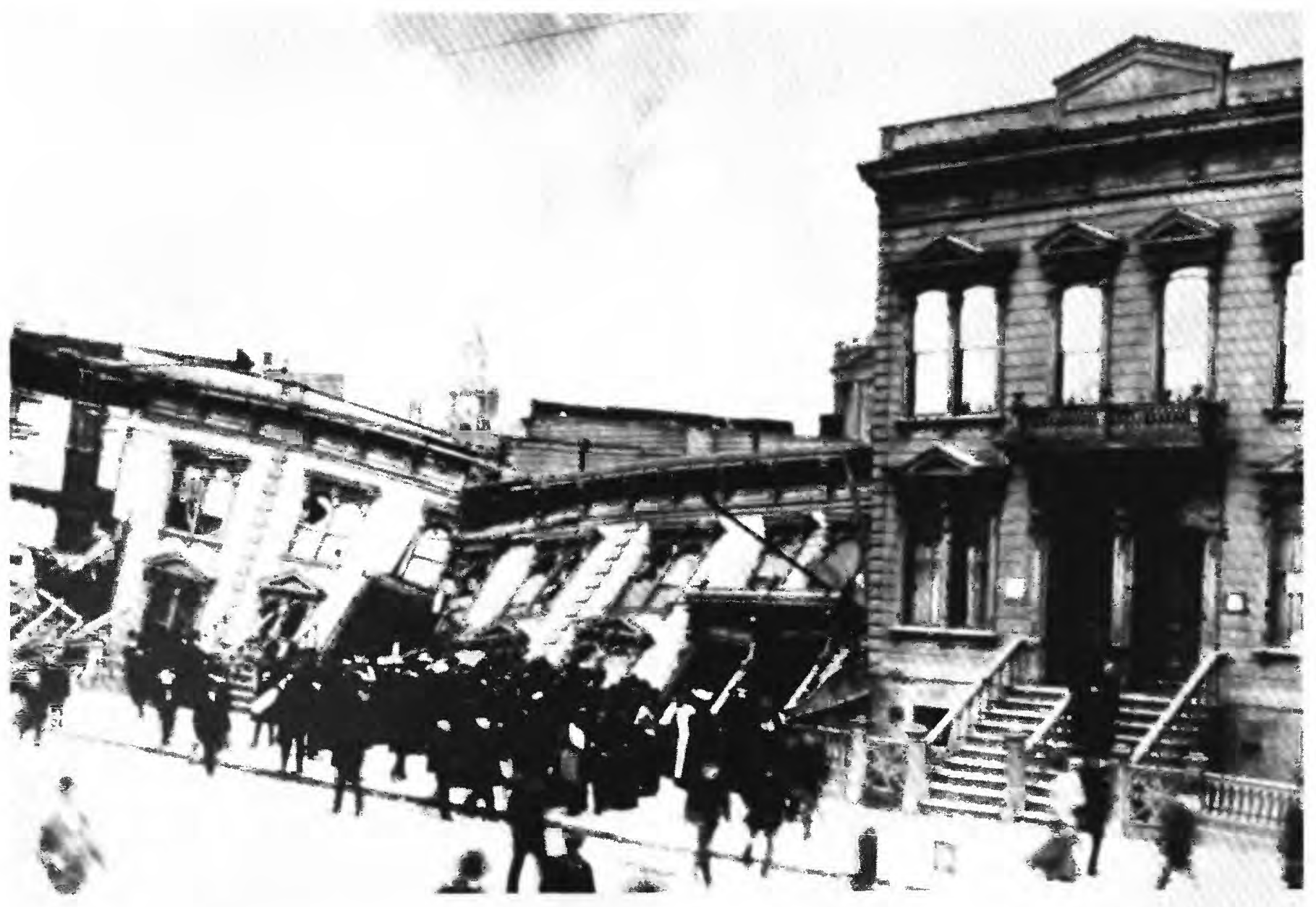

Ground slumping tilted a row of two-story buildings in San Francisco, California, during April 18, 1906, earthquake. This block of structures was destroyed by the ensuing fire.

1906. May 2 (May 1). Near Guerneville, Sonoma County, Calif. At Guerneville, much plaster cracked and articles were thrown about. (Ref. 38, 56.)

1906. May 7, 0410 and 0500 UTC (May 6). Near Upper Lake, Lake County, Calif. These earthquakes were reported to be violent at Upper Lake, about $80 \mathrm{~km}$ north of Santa Rosa. Many clocks stopped during the second shock. No damage could be documented for this earthquake. The intensity listed was taken from ref. 56. (Ref. 56, 380.)

1906. Dec. 7 (Dec. 6). Near Piedras Blancas, San Luis Obispo County, Calif. This earthquake cracked the tower at the Piedras Blancas Lighthouse. It also was reported at Cambria, San Luis Obispo, and Santa Maria. (Ref. 56, 380.)

1907. Sept. 20 (Sept. 19). Near San Bernardino, Calif. A few walls of buildings were cracked and dishes were broken at San Bernardino, and the
Shaver Building was damaged at San Jacinto. Many landslides occurred in the mountain district north of San Bernardino, and a pipeline in the Santa Anita Mountains was broken. At the Declez quarry, large rocks were thrown down the mountainside. Felt from Los Angeles south to San Diego and east to Salton. Magnitude $6 \mathrm{M}_{\mathrm{S}} \mathrm{CFR}$. (Ref. 38, 56, 381.)

1908. Jan. 27 (Jan. 26). Honey Lake region, Lassen County, Calif. Chimneys were toppled at Amedee and Milford in the Honey Lake region. Aftershocks were reported. (Ref. 38, 56, 380.)

1908. Aug. 18. Near Eureka, Humboldt County, Calif. A few chimneys toppled at Eureka; buildings walls and plate-glass windows cracked; and statues on the courthouse fell or were broken. At the Seazy Ranch, about $10 \mathrm{~km}$ north of Eureka near Freshwater, a fissure extending about $0.8 \mathrm{~km}$ in length formed in the ground. Several chimneys were thrown down in the area. (Ref. 38, 56, 380.) 


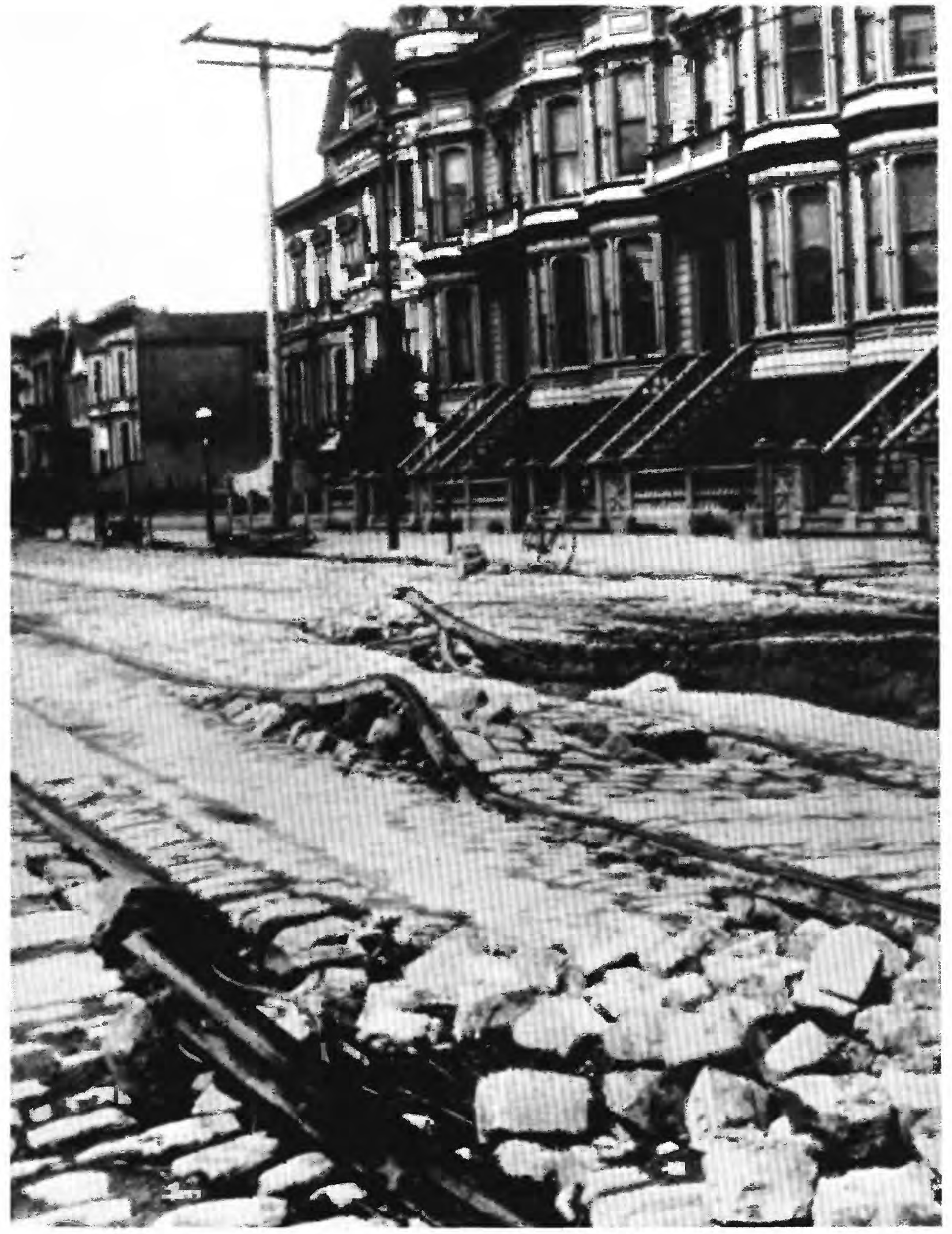

Streetcar rails on Howard Street, San Francisco, California, compressed by April 18, 1906, earthquake. (Photograph by G.K. Gilbert.) 


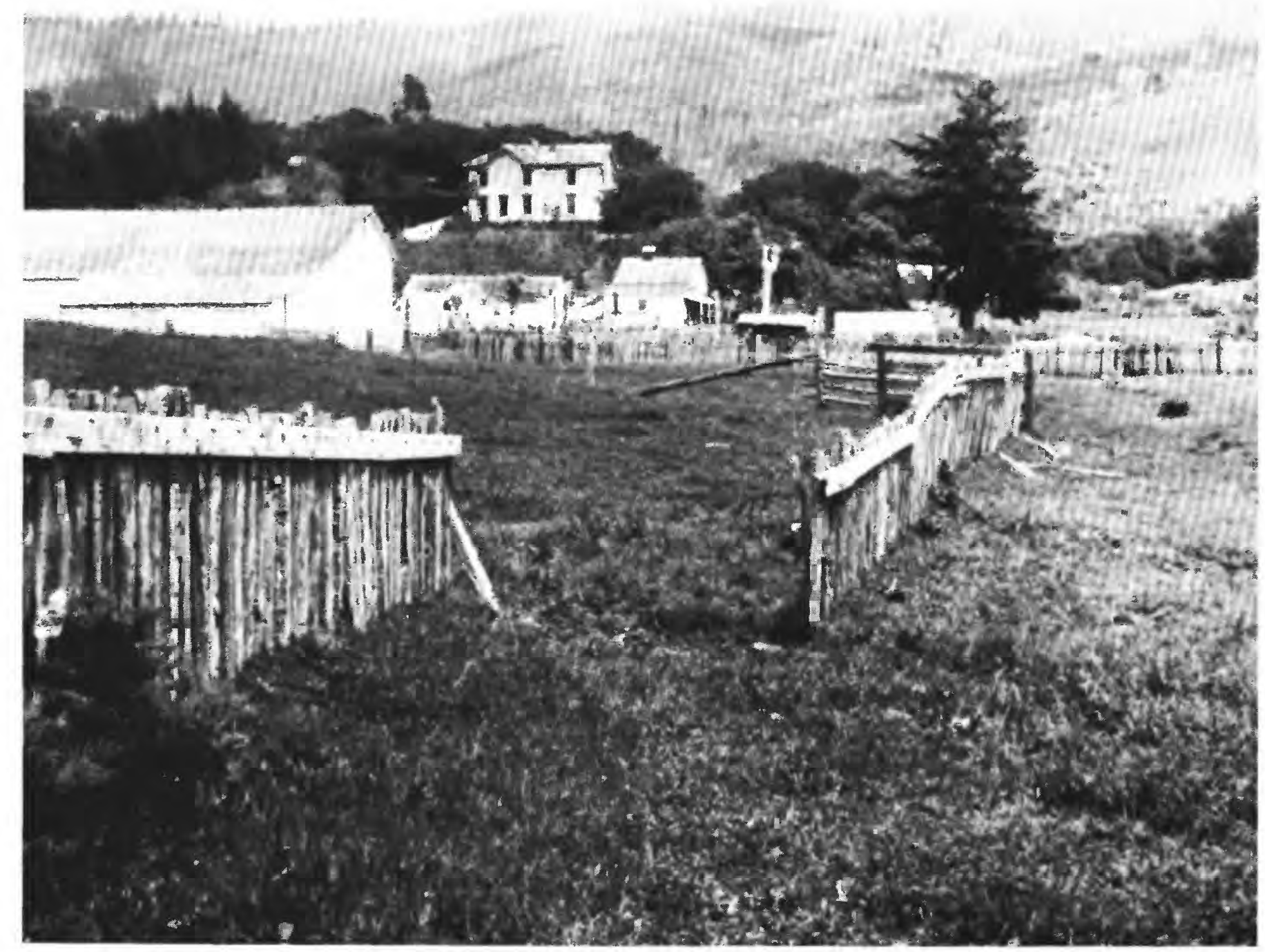

Fence offset $2.6 \mathrm{~m}$ on the main fault, $0.4 \mathrm{~km}$ northwest of Woodville, by the April 18, 1906, California, earthquake. (Photograph by G.K. Gilbert.)

1908. Nov. 4. Inyo County, Calif. This earthquake was the strongest of a series of shocks that occurred in late October and early November. The epicentral area was uninhabited except for a few prospectors, and several of them left the area because of the continuing earthquakes. This shock was felt in Tehachapi and probably to San Bernardino. No damage could be documented for this earthquake. The intensity listed was taken from ref. 382 . (Ref. 38, 56, 380,382 .)

1909. May 18 (May 17). Upper Mattole, Humboldt County, Calif. This earthquake ruined all chimneys at Upper Mattole and displaced monuments in the cemetery. The shock was reported only in
Humboldt County at Blocksburg, Eureka, and Rohnerville. (Ref. 38, 56.)

1909. June 23 (June 22). South of Downieville in Sierra County, Calif. Minor damage to chimneys, plaster, lumber flumes, and dishes occurred in parts of Sierra and Plumas Counties. The region most strongly affected by the earthquake included the area south and southeast of Downieville, where chimneys were broken and plaster was knocked down. Plaster was shaken from ceilings at Sacramento, and walls were cracked slightly. The shock also was felt at Sparks, Nev. Many aftershocks were reported in the epicentral region. (Ref. 38, 56, 380.) 


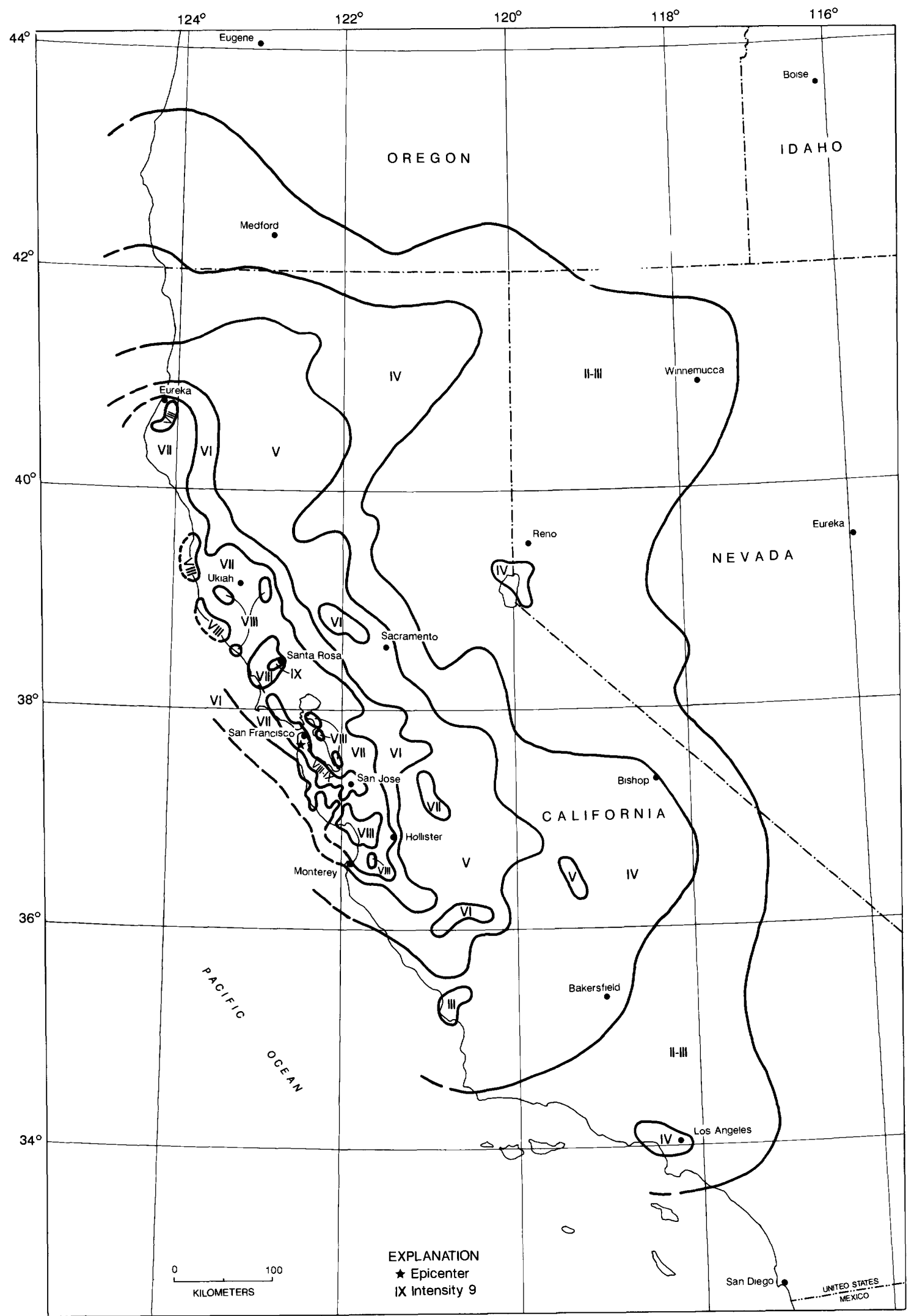

FIGURE 15.-Isoseismal map for the San Francisco, California, earthquake of April 18, 1906. Isoseismals are based on MM intensity estimates from data listed in references $381,517,571,572$, and 573 of table 1. 
1909. Oct. 29 (Oct. 28). Near Scotia, Humboldt County, Calif. The most serious loss of property, estimated at $\$ 100,000$, occurred in the FortunaRohnerville area. All chimneys were toppled at Rohnerville, and concrete construction was wrecked. At Upper Mattole, about $40 \mathrm{~km}$ south of Rohnerville, most chimneys were wrecked. At Rio Dell, every chimney was downed, houses moved on foundations, and water pipes were broken. At Eureka, Fortuna, Ferndale, and Scotia, a few buildings were damaged, and many chimneys were leveled. Felt north to Gold Beach and Grants Pass, Oreg., south to Point Arena, and east to Chico and Nevada City. It also was observed onboard a ship at sea, $40 \mathrm{~km}$ southwest of Cape Mendocino. Aftershocks continued for weeks. Magnitude 6+ $\mathrm{M}_{\mathrm{S}}$ CFR, 5.8 $\mathrm{M}_{\mathrm{S}}$ ELL. (Ref. 38, 56, $381,521,523$.

1910. Mar. 11 (Mar. 10). Near Watsonville, Santa Cruz County, Calif. Plaster fell at Santa Cruz; a marble slab was knocked out of the wall; and windows were broken. Windows and crockery broke at Watsonville. The intensity was about the same down the Pajaro Valley to Monterey Bay; aftershocks were felt over that area also. Felt north to Santa Rosa (Sonoma County), south to Priest Valley (Monterey County), and east to Modesto (Stanislaus County). Magnitude 5.5 M $\mathrm{La}_{\mathrm{La}}$ DMG. (Ref. 38, 56, 381.)

1910. May 6. Near Bishop, Inyo County, Calif. This earthquake generated rockslides in Owens River Valley and Rock Creek Canyon, northwest of Bishop, and overturned shelves at Bishop. New cracks opened in a building at Visalia, about $130 \mathrm{~km}$ southwest of the epicenter in Tulare County. The event was felt east into Nevada, northwest to Sacramento, and south to Bakersfield (Kern County). The felt area was similar to that of the shock on Sept. 30,1889, southwest of Bishop. (Ref. 56, 324, 381.)

1910. May 15. Lake Elsinore region, Riverside County, Calif. Chimneys were shaken down at Corona, Riverside, and Temescal. At Wildomar, bricks fell from chimneys and rocks rolled down the hillside. At Beaumont, a concrete-block building was cracked and plate-glass windows were broken. Felt from Barstow on the north to San Diego on the south and from Redondo Beach on the west to Palm Springs on the east. Moderate foreshocks occurred on Apr. 11 (07 57 UTC) and May 13 (06 20 UTC); slight aftershocks occurred on May 15 (20 57 UTC) and May 22 (04 15 UTC). Magnitude 6.0 $\mathrm{M}_{\mathrm{La}} \mathrm{DMG}$, 5.5 $\mathrm{M}_{\mathrm{S}}$ ELL. (Ref. 38, 56, 381, 521.)

1910. Dec. 31. East of Salinas in San Benito County, Calif. The earthquake was reported to be the "heaviest since 1906" at Salinas, where a water main and dishes were broken. The shock was "hard" at Hollister but caused little damage. It also was reported in Sonoma County, about $220 \mathrm{~km}$ to the north. (Ref. 38, 56, 324.)

1911. Mar. 11. Near Hollister, San Benito County, Calif. At Hollister, chimneys and windows were damaged, plaster cracked, and objects were thrown from walls. Effects of the earthquake were light at Salinas (Monterey County) and Campbell, Los Gatos, and San Jose (Santa Clara County). (Ref. $38,56,324$.)

1911. July 1. Near Coyote, Santa Clara County, Calif. This earthquake destroyed chimneys and cracked brick walls at Gilroy, Los Gatos, Morgan Hill, San Jose, Santa Clara, and other nearby towns. One three-story brick building at Mount Hamilton (Lick Observatory) was damaged so severely it had to be rebuilt, and chimneys on several houses were damaged. Near Coyote, three 7.5-m-high water tanks were knocked down. Slight damage to some buildings was reported at San Francisco. The shock was observed north to Woodland (Yolo County) and south to San Ardo (Monterey County). Isolated reports of this shock were received from as far east as Reno and Carson City, Nev. Several light aftershocks were felt through December 1911. Magnitude 6.2 $\mathrm{M}_{\mathrm{La}}$ DMG, 6.6 $\mathrm{M}_{\mathrm{S}}$ CFR, 6.5 $\mathrm{M}_{\mathrm{S}}$ ELL. (Ref. 38, $56,258,381,521$.)

1911. Aug. 11. Near San Jacinto, Riverside Couniy, Calif. A few walls sustained cracks and crockery was broken at San Jacinto. The shock was sharp at Redlands and San Bernardino but was slight at Los Angeles. A light foreshock occurred on Aug. 11 at 1820 UTC. (Ref. 56, 380.)

1912. Jan. 5 (Jan. 4). Near Bishop, Inyo County, Calif. Breakable goods and merchandise were damaged in some stores at Bishop, and loose articles were knocked to the floor. The shock also was reported at Bakersfield, Fresno, and Visalia, and one doubtful report was received from Blair, Nev. (Ref. $56,324$.

1912. Dec. 14. Southeast of Oxnard, Ventura County, Calif. Two sharp earthquakes overturned desks at the Ocean View School and wrecked two nearby barns. The tremors were "very marked in the country districts." (Ref. 38, 56.)

1914. Nov. 9 (Nov. 8). Santa Clara County, Calif. Near Laurel (Santa Cruz County), two chimneys were knocked down and articles were thrown from shelves. North of Laurel, two water pipes were broken at the Montezuma School. Six chimneys and several windowpanes were cracked at Los Gatos, and small amounts of plaster fell to the floor. Felt from Santa Rosa on the north to San Joaquin Valley on 
the east and to Soledad (Monterey County) on the south. (Ref. 38, 324, 381, 414.)

1915. Jan. 12 (Jan. 11). Near Los Alamos, Santa Barbara County, Calif. Some chimneys were shaken down at Los Alamos; almost every chimney was damaged to some extent; and water pipes were pulled apart at their unions. Chimneys also were downed at Careaga, Santa Ynez, and at several ranches in Santa Barbara County. Brick buildings were damaged at Lompoc, about $20 \mathrm{~km}$ southwest of Los Alamos. In the epicentral area, cracks formed in the alluvial soil and many small landslides occurred. Felt north to San Jose, east to Bakersfield, and southeast along the coast to Los Angeles. Many aftershocks occurred for about a month. (Ref. 38, 56, 381.)

1915. Feb. 22. Near Whitmore, Shasta County, Calif. A highly localized shock in the mountains near Whitmore, northwest of Lassen Peak, caused severe dislocation of the ground at a ranch about $30 \mathrm{~km}$ from the foot of Lassen Peak. The earth cracked near the ranch, and fissures formed at points along the road leading to the ranch. Water spurted from the ground in many places; bubbling springs formed; and a barn sagged where the earth sank beneath its foundation. Other unusual phenomena also were reported. This event may have been a landslide because it was not recorded on seismographs at Berkeley. (Ref. 38, 56, 324, 381.)

1915. June 23, 0359 and 0456 UTC (June 22). North of Calexico, Imperial Valley, Calif. Two destructive earthquakes wrecked buildings, overturned chimneys, and knocked down walls in the Calexico-El Centro area. The second shock, which was as strong as the first, completed the destruction of the buildings that already were weakened. This shock killed six people in Mexicali, Mexico. A foreshock occurred at 0340 UTC.

The area of heaviest property damage extended from Mexicali north to Calexico, El Centro, and Heber, where almost every brick and adobe building was damaged. Property loss, estimated at $\$ 900,000$ for both Mexico and the United States, was due as much to the poor quality of construction as to the intensity of the earthquake. Damage at El Centro, the largest city in the Imperial Valley at this time, was estimated at $\$ 600,000$. Property damage in Calexico, Heber, and Mexicali was almost as severe, but the rebuilding cost was less because the towns were much smaller than El Centro.

A few cracks formed in the alluvium parallel to the levees in the Imperial Valley, but the irrigation ditches were damaged only slightly, if at all. Many unstable banks of the Alamo and New Rivers slid into the water; cracks formed in the marshy bed of the New River northwest of El Centro. Residents about $25 \mathrm{~km}$ north of the mud volcanoes, which are west of Laguna de los Volcanes, Mexico (about $40 \mathrm{~km}$ south of Calexico), reported that columns of steam were seen rising from the vents for several days following the earthquakes and that occasional explosions were heard from that direction.

A foreshock occurred about 20 minutes before the first shock, and several aftershocks occurred through August 1915. The main earthquakes were felt north to Los Angeles and San Bernardino, east to Parker and Yuma, Ariz., and south at least to Ensenada, Mexico, and probably farther. Magnitude $5.6 \mathrm{M}_{\mathrm{La}}$ DMG (both earthquakes), 6.0 $\mathrm{M}_{\mathrm{S}}$ ELL (03 59), $5.9 \mathrm{M}_{\mathrm{S}}$ ELL (04 56). (Ref. 38, 381, 383, 521.)

1915. Oct. 8 (Oct. 7). Near Piedmont, Alameda County, Calif. This sharp earthquake knocked down a few chimneys at Piedmont, and windows were broken. A small amount of plaster fell on the campus at Berkeley. Felt from Sebastopol, Sonoma County, to Santa Clara. Three aftershocks were reported. (Ref. 38, 56, 324, 442.)

1915. Nov. 21 (Nov. 20). Baja California, Mexico. A major earthquake left large cracks in a levee at Laguna de los Volcanes, Mexico, an uninhabited area about $40 \mathrm{~km}$ south of Calexico, Calif. Two hunters near Laguna de los Volcanes, Mexico, reported that a column of steam shot up about 180-200 $\mathrm{m}$ high and was followed by a column of black mud that reached about the same height. This column alternated between steam and mud for about 1 hour. The observers, although on level ground, had trouble standing during the earthquake. Cracks were observed on both sides of the New River for a distance of about $3 \mathrm{~km}$. Felt only slightly at Los Angeles, but felt strongly at Calexico and San Diego and at Yuma, Ariz. Magnitude $7.1 \mathrm{M}_{\mathrm{S}} \mathrm{ABE}, 6.8 \mathrm{~m}_{\mathrm{b}}$ ABE. (Ref. 38, 258, 492.)

1915. Dec. 31. Off coast of Humboldt County, Calif. Felt inland only slightly at Eureka and Shively. Magnitude 6.5 $\mathrm{M}_{\mathrm{S}}$ ELL, 6.5 $\mathrm{M}_{\mathrm{S}}$ GR (Ref. 56, 258, 521.)

1916. July 5 (July 4). Near Ferndale, Humboldt County, Calif. About $3 \mathrm{~km}$ west of Ferndale, two chimneys were cracked, and a woodpile was overturned. One plate-glass window at Ferndale was broken, and vases were thrown from shelves. (Ref. $38,56,324$.)

1916. Aug. 6. Near Paicines, San Benito County, Calif. Chimneys on a hotel at Paicines were destroyed; damage was slight at Hollister. Huge boulders rolled onto the highway at Chittenden Pass in Santa Cruz County. Felt to San Francisco on the 
north, Monterey on the west, and Paso Robles on the south. (Ref. 38, 56, 324, 381.)

1916. Oct. 23, 0244 UTC (Oct. 22). Near Lebec, Kern County, Calif. The epicentral area of this earthquake was sparsely populated, and so intensity information was meager. In San Emigdio Canyon (Kern County), the top one-third of a large rock chimney was knocked off, and many rocks rolled off nearby mountains. Near Frazier Mountain (Ventura County), about $10 \mathrm{~km}$ southwest of Tejon Pass, a crack opened in the ground; on the Snedden Ranch in Lockwood Valley, an adobe house was cracked so severely that it could not be repaired. On the north side of Lockwood Valley, at the Frazier Borax mine, the shock detached the porch from a frame house. On Alamo Mountain, about $18 \mathrm{~km}$ southwest of Tejon Pass, limbs fell from pine trees and rocks fell from the canyon walls at several places. Near Gorman, a crack several meters in length and a few centimeters in width formed in the cement surface of the highway. Cracks also were reported in the highway at Bailey's Patrol Station, northwest of Quail Lake.

A strong aftershock was observed in the area 10 minutes later, and several lighter aftershocks were reported. The main earthquake was felt from Shafter (Kern County) on the north to Los Angeles on the south and from Roosevelt (Los Angeles County) on the east to Los Olivos (Santa Barbara County) on the west. Isolated felt reports, however, were received from such distant points as Fresno and San Diego. Magnitude 5.5 $\mathrm{M}_{\mathrm{S}}$ GR, $5.2 \mathrm{M}_{\mathrm{La}}$ DMG. (Ref. 38, 56, 258, 534.)

1916. Dec. 1. Near Avila, San Luis Obispo County, Calif. Some smokestacks toppled at the Union Oil Company refinery buildings in Avila. Plaster fell in several houses; much glass was broken; and merchandise fell from shelves. In Dairy Canyon, about $3 \mathrm{~km}$ north of Avila, a landslide covered the railroad tracks. One brick fell from a building in San Luis Obispo. Water in the San Luis Obispo Bay was disturbed. The shock was "severe" at Port San Luis but was slight to the southeast, at Santa Maria (Santa Barbara County). (Ref. 38, 56, 324.)

1917. May 28 (May 27). Imperial Valley, near Holtville, Calif. Walls were cracked at Brawley, and residents were panic stricken. Felt in Imperial, Riverside, and San Diego Counties and probably into northern Mexico. (Ref. 56, 380.)

1917. July 6. Owens Valley, Inyo County, Calif. This earthquake caused a break about $30.5 \mathrm{~m}$ long in the flume of the Los Angeles aqueduct south of Owens Lake. Chimneys cracked in the area, and rocks rolled down the mountain. Nine shocks occurred from July 7 to 9 . The shock was reported from Little Lake in the south to a point $18 \mathrm{~km}$ northwest of Independence in the north. (Ref. 38, 56, 324.)

1917. July 9. Lopez Canyon, San Luis Obispo County, Calif. Chimneys cracked and rocks rolled down the hillsides in Lopez Canyon, near San Luis Obispo. Four foreshocks and three aftershocks were felt in the area from July 7 at 2057 UTC to July 10 at 0045 UTC. (Ref. 38, 56, 324.)

1918. Mar. 12. Downieville, Sierra County, Calif. Chimneys were knocked over at Downieville. This sharp shock was reported only in Sierra County. An aftershock was reported 2 hours later. (Ref. 38, $56,272,324$.)

1918. Apr. 21. Near San Jacinto, Riverside County, Calif. Major damage occurred in San Jacinto, about $120 \mathrm{~km}$ southeast of Los Angeles and at Hemet, about $3 \mathrm{~km}$ south of San Jacinto. Several residents were injured, and one was killed. An earthquake of similar intensity occurred in the same area on December 25, 1899.

In the business section of San Jacinto, a town of about 1,000 population, only one new concrete building and one frame building remained standing after the earthquake. Most of the ruined buildings were of poor construction, however. Property damage at Hemet was not as severe as in San Jacinto. No buildings were wrecked, and no buildings of good construction were damaged seriously. Total property loss in the two towns was estimated at $\$ 200,000$.

Light damage to structures occurred in several towns within a $160-\mathrm{km}$ radius of San Jacinto. Concrete irrigation canals were broken in several places in the Hemet-San Jacinto area.

Many lengthwise cracks were observed in the highway between San Jacinto and Hemet, but cracks were not observed at the sides of this highway. About $1.5 \mathrm{~km}$ from the center of San Jacinto, the concrete highway was buckled, and a section about 1 $m$ wide was torn up. Cracks in the ground were noted in four areas, but all were believed to be due to the shaking, not to the surface rupture along the San Jacinto fault. Many small sand craters were observed on a farm about $1.5 \mathrm{~km}$ northwest of San Jacinto. Felt from Taft (Kern County) in the north along the coast to San Diego (and probably into Mexico) and from Needles (San Bernardino County) in the east, south to Yuma, Ariz. Many aftershocks occurred, including a strong tremor on June 6, 1918. Two moderate shocks on Apr. 22 (16 07 and 1614 UTC) shook down loose bricks and tottering walls in Hemet and San Jacinto. Magnitude 6.9 $\mathrm{M}_{\mathrm{S}}$ ELL, 6.6 $\mathrm{M}_{\mathrm{La}}$ DMG (Ref. 38, 56, 258, 381, 384, 521, 533, 599.) 


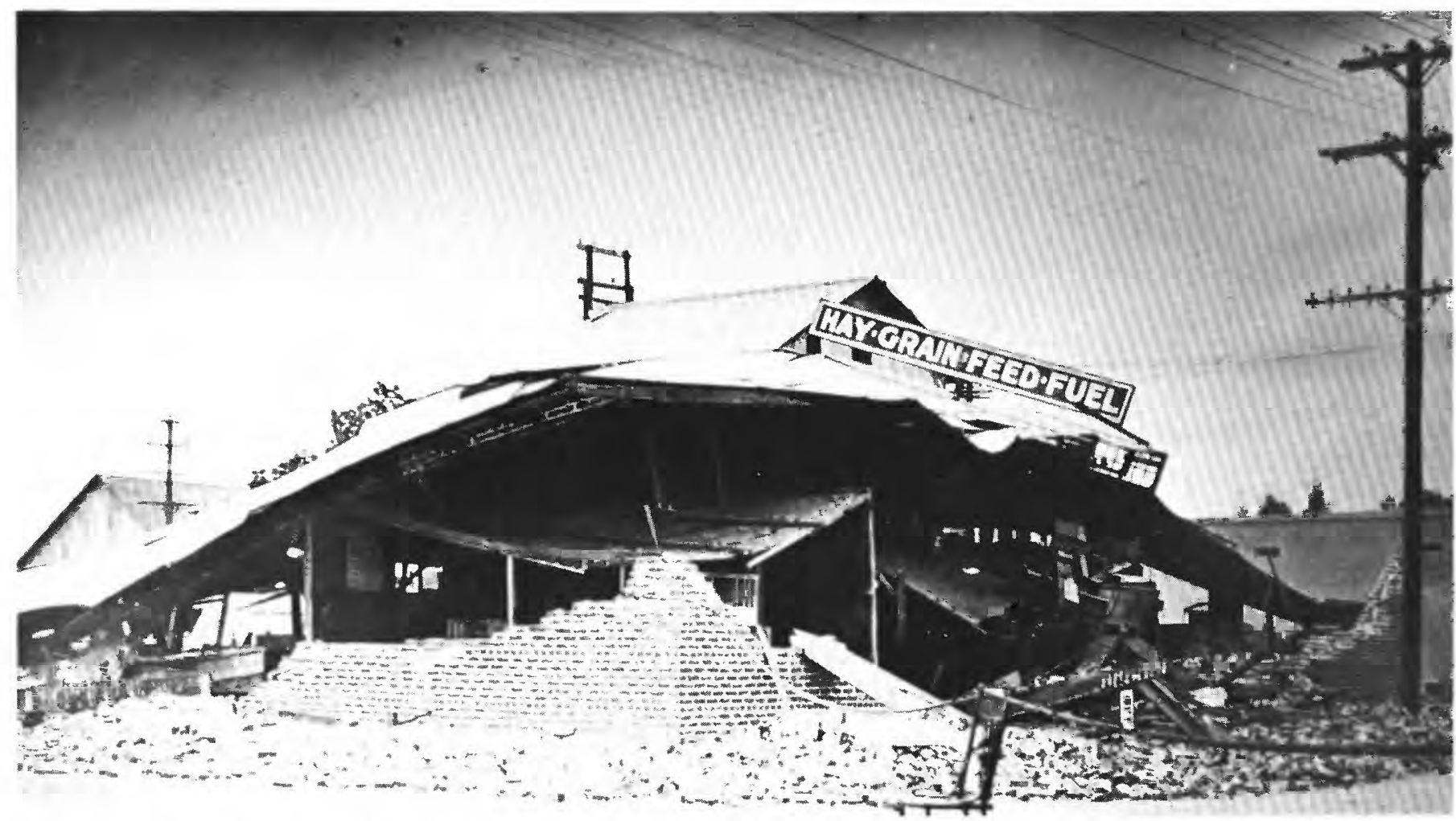

Collapsed building at San Jacinto, California, caused by the April 21, 1918, earthquake. (Photograph by University of California, Berkeley.)

1918. Apr. 22. Near Corona, Riverside County, Calif. Chimneys were cracked, and plaster was thrown down at Corona. (Ref. 38, 56, 380.)

1918. May 1 (Apr. 30). Calexico, Imperial County, Calif. Plate-glass windows were broken at Calexico, and stock was thrown from shelves at El Centro. Felt over an area that included San Diego County on the west, San Jacinto on the north, western Arizona on the east, and an unknown distance into Mexico on the south. (Ref. 56, 272.)

1918. June 6. Near Hemet, Riverside County, Calif. A strong aftershock of the event on Apr. 21, 1918, cracked plaster $7 \mathrm{~km}$ southeast of Hemet, loosened rocks on the mountainside, and cracked the ground around large trees. It was felt in Imperial, Los Angeles, Riverside, San Bernardino, and San Diego Counties. (Ref. 56, 380.)

1918. July 15 (July 14). Off the coast of northern Humboldt County, Calif. Buildings swayed alarmingly at Eureka, and most residents ran outside. The shock also was felt in Mendocino and Trinity Counties. Magnitude 6.5 $\mathrm{M}_{\mathrm{S}}$ ELL, 5.9 $\mathrm{M}_{\mathrm{La}}$ ELL. (Ref. 38, 56, 258, 521.)

1918. Nov. 19. Near Venice, Los Angeles County, Calif. At Venice, plaster was downed and dishes were thrown to the floor; at Santa Monica, chimneys were cracked. The earthquake was reported only in Los Angeles and Orange Counties. (Ref. 38, 56.)

1919. Jan. 4. East of Redding, Shasta County, Calif. In the Clover Creek area, between Fern and Whitmore, the earthquake damaged chimneys and broke dishes. A surface fracture cut across a road near Fern and caused vertical displacement of several feet. This local event was not felt at Millville, about $30 \mathrm{~km}$ distant, and was not recorded on seismographs. This event may have been a landslide similar to one that occurred at the same location on Feb. 22, 1915. (Ref. 56, 324.)

1919. Feb. 16. Near Maricopa, Kern County, Calif. The earthquake cracked buildings at Maricopa and the Grapevine pump station. At Belridge, an oil tank was split, and at Lebec, rocks rolled down the hillsides. This shock also was felt in Fresno, Los Angeles, San Luis Obispo, and Tulare Counties. Magnitude 5.7 Ukn JON. (Ref. 38, 56.)

1919. Sept. 15. Near Eureka, Humboldt County, Calif. Some chimneys were demolished at Eureka, and windows were broken. Three aftershocks occurred within about 5 hours. (Ref. 38, 56,324 .) 


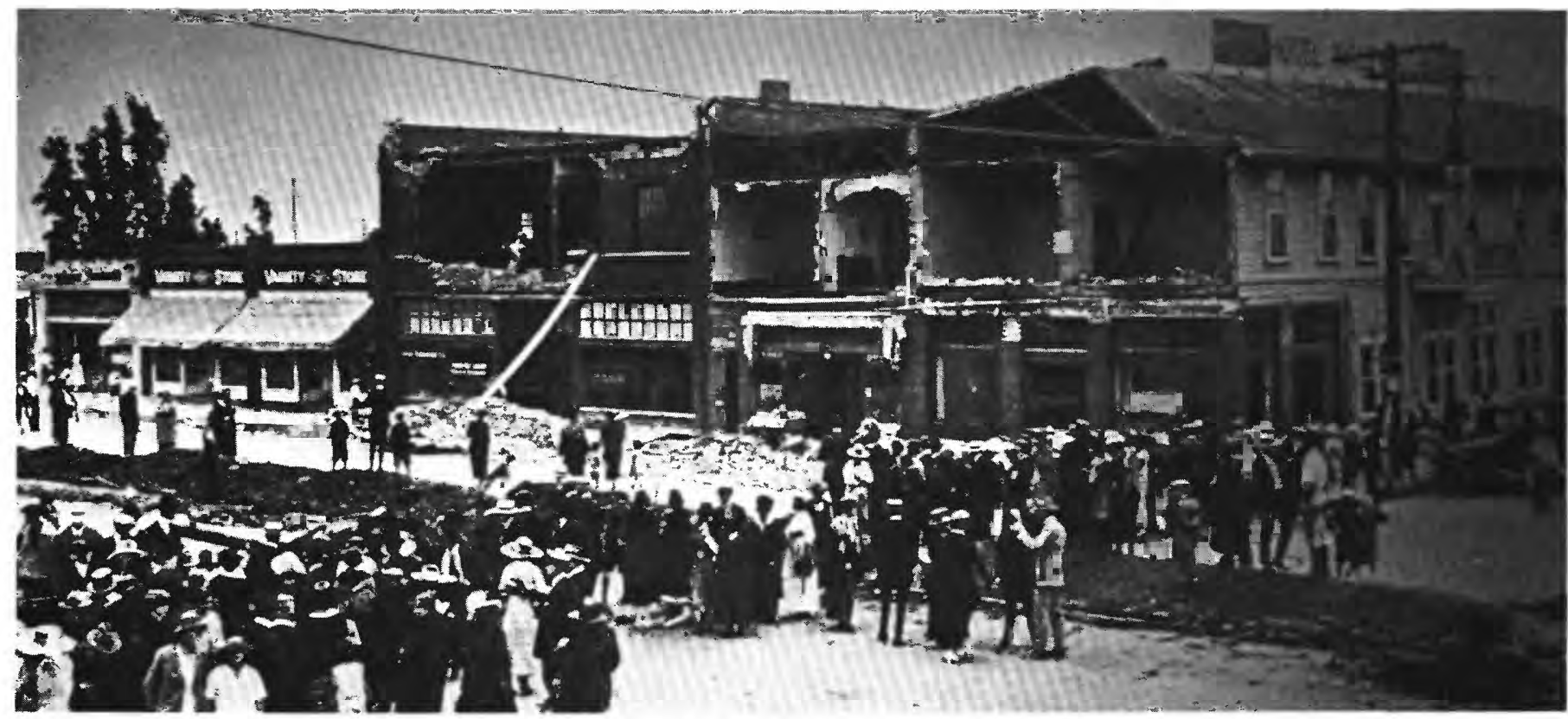

Collapsed exterior wall of Hotel Inglewood, Inglewood, California, caused by the June 22, 1920 (June 21 PST), earthquake. (Photograph by University of California, Berkeley.)

1920. Jan. 1 (Dec. 31, 1919). Near Warner Springs, San Diego County, Calif. Adobe walls were cracked at Warner Springs. The shock also was observed in Imperial, Los Angeles, and Riverside Counties. (Ref. 56, 380.)

1920. June 22 (June 21). Near Inglewood, Los Angeles County, Calif. This earthquake mainly damaged poorly built structures in the area of Inglewood, about $16 \mathrm{~km}$ south of downtown Los Angeles. Property loss was estimated at more than $\$ 100,000$. The destructive effects of the shock were most prominent along Commercial Street, where damage commonly was more severe to two-story buildings than to onestory buildings.

The Inglewood grammar school was wrecked, and walls of the Inglewood Hotel and a nearby electric substation fell into the street. Many chimneys on private houses in Inglewood were broken at the roofline. The water mains and plant of the Inglewood Water Company were damaged. At Hyde Park, $3 \mathrm{~km}$ northeast of Inglewood, about 30 percent of the chimneys were overthrown, and brick facings on the fronts of five school buildings were knocked down. In a cemetery east of Inglewood, tombstones were twisted and overturned. Many aftershocks occurred, but most were felt only in the Inglewood area. (Ref. 38, 56, $385,537$.

1920. July 16, 18 08, 2127 , and 2130 UTC. Los Angeles, Calif. A series of more than 100 earthquakes occurred in the Los Angeles area from Feb. 1 to Sept. 30, 1920. The strongest shocks were observed on July 16 . Damage from the July 16 events included cracked walls, fallen plaster from ceilings and walls, and fallen bricks from cornices and chimneys. Most of the earthquakes were felt only in the Los Angeles area. The second shock on July 16 was felt at Mount Wilson and Pasadena, and the third was felt at Pomona and Santa Ana. (Ref. 56, $380,386$.

1920. July 23 (July 22). Hot Springs, Shasta County, Calif. Chimneys and dishes were broken and dishes were shaken from shelves at Hot Springs. It was felt from Redding northeast to Fall River Mills and McArthur and along the McCloud River. Three sharp aftershocks were observed on July 23 at 1400,1600 , and 2000 UTC. (Ref. 38, 56, 324, 537.)

1922. Jan. 31. Off the coast of Humboldt County, Calif. This potentially damaging earthquake was felt from Eugene, Oreg., to San Francisco. Magnitude 7.6 $\mathrm{M}_{\mathrm{S}} \mathrm{CFR}, 7.3 \mathrm{M}_{\mathrm{S}} \mathrm{GR}, 7.3 \mathrm{~m}_{\mathrm{b}} \mathrm{ABE}$. (Ref. 56, 258, 272.)

1922. Mar. 10. Cholame Valley area, San Luis Obispo County, Calif. Houses were damaged severely along the San Andreas fault zone in Monterey and San Luis Obispo Counties. Chimneys fell at Parkfield and in southern Cholame Valley. One house was jolted from its foundation onto the 
ground, and its porch was displaced $30 \mathrm{~cm}$ away from the house. Another severely damaged house was twisted into two parts. A large water tank on a ranch at Cholame was knocked down and broken into pieces, and oil pipelines broke between Shandon and Antelope. A ground crack, $15-30 \mathrm{~cm}$ wide and about $800 \mathrm{~m}$ long, was reported in Cholame Valley. Small ground cracks also formed in the San Andreas fault zone. Felt from San Jose on the north to Los Angeles on the south and east to Springville (Tulare County). A moderate aftershock occurred on Mar. 16 at 2311 UTC. Magnitude $6.3 \mathrm{M}_{\mathrm{La}} \mathrm{DMG}, 6.3 \mathrm{M}_{\mathrm{S}}$ ELL. (Ref. 38, 56, 258, 324, 381, 521.)

1923. Jan. 22. Off the coast of Humboldt County, Calif. Houses were damaged severely at Ferndale, Petrolia, and Upper Mattole; many chimneys were downed; and water lines were broken. At Pepperwood, one house was shaken from its foundation and split apart, and another was twisted from its base. Chimneys also were knocked over at Alton, Dyerville, Fortuna, Loleta, Ocean House, and Scotia. Several landslides occurred in the canyon. Felt from Walker (Siskiyou County) south to San Francisco and beyond and east to Grass Valley (Nevada County). It also was observed on several ships at sea. Many aftershocks occurred in the Petrolia-Upper Mattole region. Magnitude 7.2 $\mathrm{M}_{\mathrm{S}} \mathrm{ABE}, 7.3 \mathrm{M}_{\mathrm{S}} \mathrm{CFR}$, 6.5 $\mathrm{M}_{\mathrm{La}}$ DMG. A small tsunami was recorded. (Ref. 38, $56,258,381,610$.)

1923. July 23 (July 22). Near Redlands, Riverside County, Calif. Many chimneys were broken in Redlands, San Bernardino, and along Base Line Road, west of Harlem Springs. The fronts of three buildings were cracked badly in Redlands, and a few fire walls were thrown down. At San Bernardino, two cornices were thrown down; parts of brick walls collapsed; and pavement buckled. Damage to walls and chimneys also occurred at Colton, Loma Linda, and Patton. Felt north to Mojave (Kern County) southeast to Calexico (Imperial County) and east to Big Bear Valley (San Bernardino County). Magnitude 6.25 $\mathrm{M}_{\mathrm{S}}$ CFR, 6.0 $\mathrm{M}_{\mathrm{La}} \mathrm{DMG}, 6.3 \mathrm{M}_{\mathrm{S}}$ ELL. (Ref. $258,381,388$.)

1923. Nov. 5. Baja California, Mexico. At Calexico, Calif., a hotel shifted several centimeters on its foundation, and other structures sustained minor damage. The intensity of the earthquake was similar at El Centro, where five pronounced shocks were reported. The earthquake also was felt at Brawley and San Diego. (Ref. 38, 56.)

1923. Nov. 7. Baja California, Mexico. This earthquake was stronger than that on Nov. 5, 1923. Additional property damage occurred at Calexico,
Calif., and one fire resulted. Felt only in Imperial and San Diego Counties. (Ref. 38, 56.)

1924. Dec. 28 (Dec. 27). Salinas, Monterey County, Calif. Considerable minor damage to plaster occurred at Salinas, where residents rushed into the streets in panic. The dining room of one house was "practically a wreck" from fallen plaster. Felt from King City (Monterey County) north to Palo Alto (Santa Clara County) and west of the Mount Hamilton Range. (Ref. 56, 324, 539.)

1925. Apr. 16 (Apr. 15). Baja California, Mexico. At Calexico, Calif., plaster was shaken from walls at the public library and residents ran outside. (Ref. 38, 56, 218.)

1925. June 29. Near Santa Barbara, Calif., in the Santa Barbara Channel. This destructive earthquake caused property damage estimated at $\$ 8$ million and killed 13 people. Most of the damage occurred at Santa Barbara and nearby towns along the coast, but the earthquake caused moderate damage at many points north of the Santa Ynez Mountains, in the Santa Ynez and Santa Maria River valleys. North of Santa Barbara, the earth dam of the Sheffield Reservoir was destroyed, but the water released caused little damage.

In Santa Barbara, few buildings on State Street escaped damage. Because parts of the main business district and the area near the seashore were built on land fill, many of the structures there were demolished, and others were so shattered that they had to be razed. In general, however, buildings of reinforced concrete were damaged little, except where workmanship was poor; frame buildings covered with stucco, sheathing, or lath also withstood the shock well. Loss to the sewage system was heavy only in areas of land fill, but the disposal plant was destroyed above the surface of the ground.

Among the most conspicuous building failures in Santa Barbara were the Arlington Hotel (a composite building of irregular shape), the Californian Hotel (a new four-story brick building), the San Marcos office building (a four-story reinforced concrete structure), the El Camino Real Hotel (a two-story brick and wood structure), and the Potter Theater building (a three-and-one-third-story brick and wood structure). Other public buildings seriously damaged included the courthouse, jail, library, schools, and churches.

Structures built on solid ground or pavement of all types withstood the earthquake well. The only severely damaged pavement was that on the boulevard paralleling the beach, where the shoulders of the pavement were displaced $20-36 \mathrm{~cm}$ horizontally. The pavement sustained cracks as wide as $40 \mathrm{~cm}$ at 


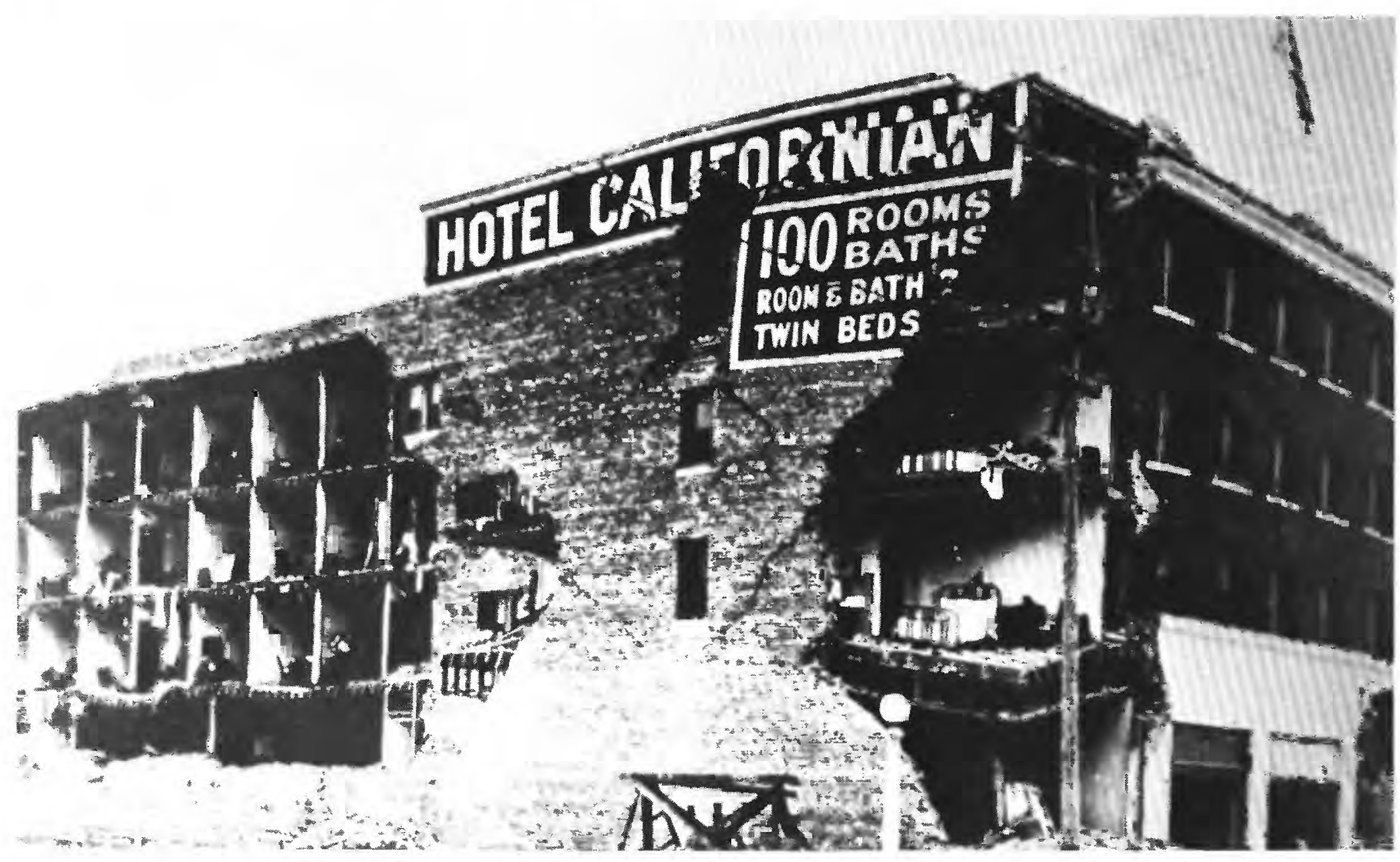

Wall of the Hotel Californian at Santa Barbara, California, downed by the June 29, 1925, earthquake. (Photograph by University of California, Berkeley.)

several points along the beachfront. Concrete curbs buckled in almost every block in Santa Barbara.

The earthquake caused damage on the Southern Pacific Company Railroad from Gaviota (mile 331 as measured from San Francisco) on the north to Ventura (mile 598) on the south. Heavy subsidence of the larger fills and slope failure of the sides of deep cuts were noted on the railroad track from Naples to Santa Barbara. Many of the bluffs in the Naples area, adjacent to the ocean, fissured and caused extensive landslides. A section of ground (about 2 acres), adjacent to the track between Naples and Santa Barbara, subsided about $30 \mathrm{~cm}$.

Felt from Paso Robles (San Luis Obispo County) on the north to Santa Ana (Orange County) on the south and to Mojave (Kern County) on the east. Several strong aftershocks occurred on July 3, and lighter shocks occurred throughout July 1925. Magnitude 6.3 $\mathrm{M}_{\mathrm{S}}$ CFR, 6.1 $\mathrm{M}_{\mathrm{La}}$ DMG. (Ref. 38, 258, $381,389,533,540,541,542$.)

1925. July 3, 1637 and 1819 UTC. Near Santa Barbara, Calif. The first aftershock of the June 29 earthquake toppled a few damaged chimneys at Santa Barbara and left cracks in walls. The second tremor was larger than the first because as surface waves were reported only for the second tremor (from Honolulu, Hawaii, and Cambridge, Mass.). The lack of information on damage from the second shock probably is due to its occurrence so soon after the first shock. (Ref. 56, 218, 380.)

1926. Feb. 18. Near Santa Barbara, Calif. Windows were broken in a Santa Barbara school, and a water main was broken in the roundhouse. Telephone equipment was damaged at Simi (Ventura County). Felt along the coast from San Luis Obispo on the northwest to south of Santa Ana, a distance of about $320 \mathrm{~km}$. Magnitude $5.0 \mathrm{M}_{\mathrm{La}}$ DMG. (Ref. 38, $56,218$.

1926. June 29. Near Santa Barbara, Calif. Some chimneys fell at Santa Barbara, and one child was killed by falling bricks. A streetcar was derailed; telephone wires became tangled; plate-glass windows were broken; and old cracks in walls enlarged. The shock was felt strongly at Buellton and Ventura and was reported as far southeast as the Los Angeles beaches. (Ref. 38, 56, 218, 533.) 


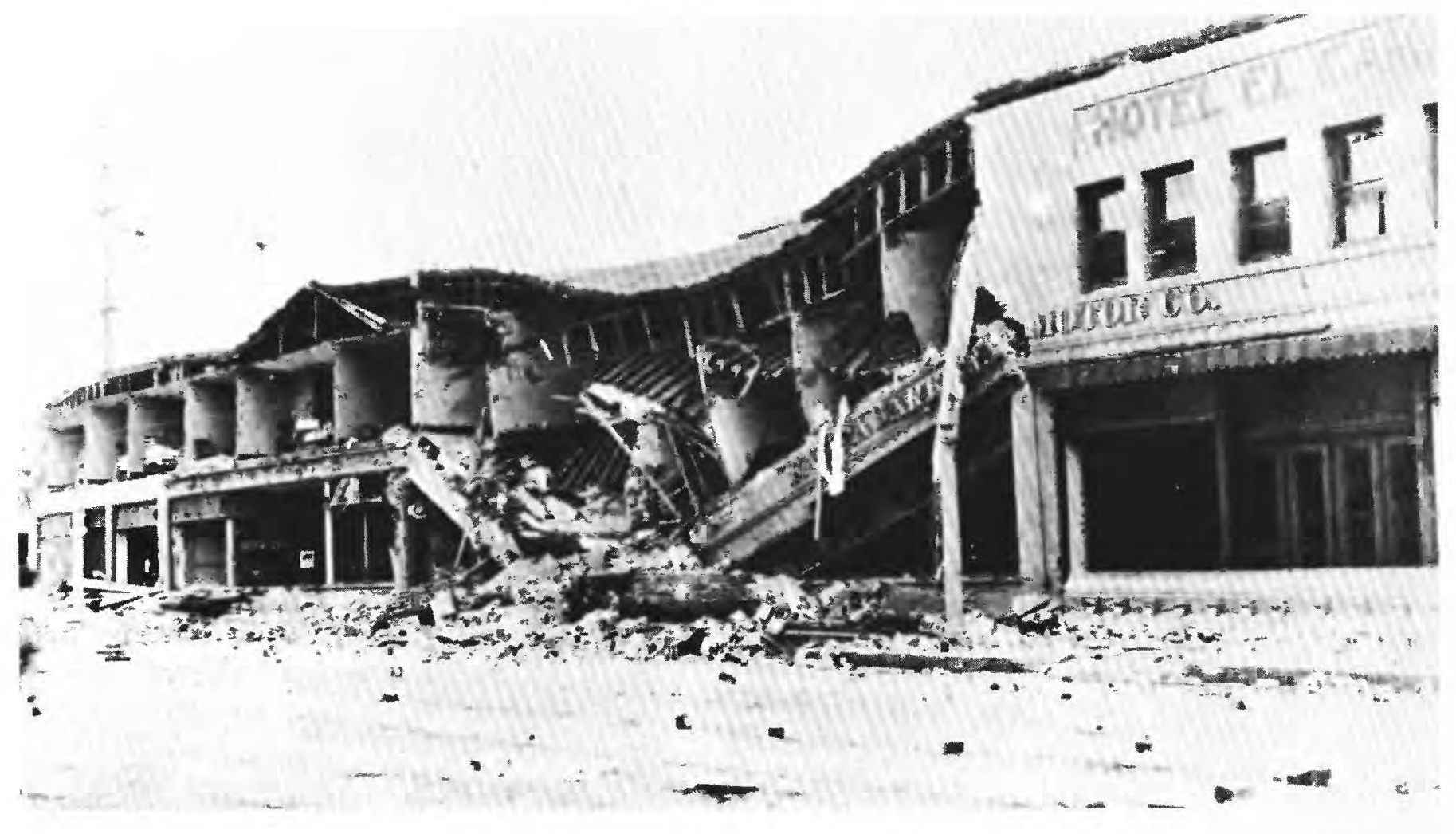

El Camino Real Hotel and garage in Santa Barbara, California, severely damaged by the June 29, 1925, earthquake.

1926. July 25. Near Idria, San Benito County, Calif. Rocks rolled down the hillside in the area of Idria and Panoche. The earthquake cracked plaster in the San Joaquin Valley. It was felt southeastward across the San Joaquin Valley to Kernville and north and northwestward to Sacramento and San Jose. (Ref. 56, 218, 390.)

1926. Oct. 22, 1235 and 1335 UTC. Off the coast of Monterey County, Calif. Two largemagnitude earthquakes caused considerable damage in the Monterey Bay region. The first shock was severe at Santa Cruz, where many chimneys were knocked down, and old brick buildings sustained damage. A few chimneys also were knocked down at Carmel and Monterey. A lamp was thrown from its base, and a lens was broken at the lighthouse on Ano Nuevo Island, northwest of Santa Cruz. Lighter effects were reported as far away as San Francisco $(120 \mathrm{~km}$ from the epicenter), where the tile surfaces of a few buildings were damaged, windows were broken, and plaster was cracked extensively.

The second shock, an hour later, was almost as widely felt as the first, and appears to have been stronger than the first earthquake at towns north of
Monterey Bay. The shaking again was heavy on Ano Nuevo Island. The shocks were felt over about the same area-north to Middletown (Lake County), south along the coast to Lompoc (Santa Barbara County), and east to Turlock (Stanislaus County). Many small aftershocks occurred. Magnitude $6.1 \mathrm{M}_{\mathrm{S}}$ CFR (both earthquakes), 6.1 $\mathrm{M}_{\mathrm{La}}$ DMG (12 35 UTC). (Ref. 56, 218, 381, 391, 521.)

1927. Jan. 1, 0816 and 0913 UTC. Imperial Valley, Calif. Two strong earthquakes began a long series of shocks, although none of the aftershocks exceeded MM intensity VI. Refer to June 23, 1915, above, for a description of a similar double earthquake occurrence at about the same location. Many buildings were damaged severely at Calexico (Imperial County), and several people were injured by collapsing roofs and walls. At Mexicali, Mexico, some buildings were destroyed and many were damaged. Water mains were broken in both towns. Slight damage to buildings also was reported at El Centro, Heber, and Imperial. Felt northwest to Orange County and east into Arizona. Hundreds of aftershocks occurred. (Ref. 38, 56, 258, 381.) 
1927. Aug. 4. Santa Monica Bay, Calif. Only minor damage occurred in the area. A water main was broken in downtown Los Angeles. The shock was reported north to Ventura, east to San Bernardino, and south to Anaheim (Orange County). Magnitude $5.0 \mathrm{M}_{\mathrm{La}}$ DMG. (Ref. $38,56,218$.)

1927. Aug. 20. Off the coast of Humboldt County, Calif. At Eureka and Arcata, chimneys were destroyed, windows and water pipes broke, and walls cracked. Plaster fell in buildings, and some doors jammed. People driving automobiles had steering problems. Cracks formed in mud and gravel in Redwood Park (Eureka), and moderate landslides occurred. Chimneys also were damaged at Fortuna, Ferndale, Freshwater, and Scotia. Felt north to the Smith River area near the Oregon-California border and south to Westport, in Mendocino County. Magnitude 5.0 M $\mathrm{La}$ DMG. (Ref. 38, 218, 324, 543.)

1927. Sept. 18 (Sept. 17). Northwest of Bishop, Inyo County, Calif. The intensity of this earthquake probably was highest near Bishop, where several chimneys were downed, many windows were cracked or broken, and parked cars moved back and forth. The shock also appears to have been strong in the sierra regions of Fresno and Madera Counties. A landslide caused damage at the powerhouse in Owens River Canyon, north of Bishop. Felt from Mono County in the north to Los Angeles County in the south and from Kings County in the west to western Nevada in the east. A slight aftershock was felt on Sept. 19 at 0523 UTC. Magnitude $5.5 \mathrm{M} \mathrm{La}$ ELL. (Ref. 56, 218, 258, 521, 535.)

1927. Nov. 4. Off the coast of Santa Barbara County, west of Lompoc, Calif. The most severe damage to property occurred in the areas west and north of Lompoc. Chimneys were wrecked at several towns, including Arroyo Grande, Berros, Guadalupe, Halcyon, Lompoc, Los Alamos, and Nipomo. On the Roberd's ranch, an earthquake fountain created between 10 and 20 sand craters; lurches and cracks were observed in the water-soaked soil. The Roberd ranch house was shifted on its foundation about 5 $\mathrm{cm}$, and small outbuildings were pushed from their foundations. The walls of a poorly built block building collapsed at White Hills. At Santa Maria and other towns in the area, chimneys were damaged, old brick walls and interior walls formed cracks, and cornices fell.

Near Surf, west of Lompoc, the Southern Pacific Railroad bridge was thrown out of alignment near its center; a concrete highway was cracked; and small rockslides and earthslides occurred. Cracks formed in the ground about $6.5 \mathrm{~km}$ north of Arlight. Felt from Morgan Hill south to Redondo Beach and from the coast east to Kernville. A tsunami was recorded on tide gages at San Francisco, La Jolla, San Diego, and Fort Point, and waves were observed at Pismo, Port San Luis $(1.5 \mathrm{~m})$, and Surf $(1.8 \mathrm{~m})$. Many aftershocks occurred. Magnitude 7.3 $\mathrm{M}_{\mathrm{S}} \mathrm{GR}, 7.3 \mathrm{~m}_{\mathrm{b}} \mathrm{ABE}$, $7.5 \mathrm{M}_{\mathrm{S}}$ CFR, 6.2 $\mathrm{M}_{\mathrm{La}}$ DMG. (Ref. 38, 56, 381, 392, 521,610 .)

1927. Nov. 19 (Nov. 18). Near Santa Maria, Santa Barbara County, Calif. Chimneys, weakened by the shock on Nov. 4, 1927, fell at Santa Maria, and windows cracked. Plaster was cracked at Betteravia. Felt from San Miguel (San Luis Obispo County) and Parkfield (Monterey County) on the north to towns in the area of Santa Barbara Channel on the south. (Ref. $38,56,218$.)

1928. Apr. 15. West of Paskenta, Tehama County, Calif. At Paskenta, one chimney was cracked and standing automobiles were moved back and forth. At a ranch near Newville, south of Paskenta, a chimney was knocked off one house. In Lyonsville, at the northeast edge of the macroseismic region, a laborer reported three tombstones fell and six graves collapsed. Felt from Rockport (Mendocino County) in the west to Stirling City (Butte County) in the east and from Jelly (Tehama County) in the north to Lakeport (Lake County) in the south. Magnitude 5.7 Ukn JON. (Ref. 393.)

1928. June 4 (June 3). Near Weaverville, Trinity County, Calif. This earthquake, which was as strong at Carrville and Trinity Center, threw chimneys down at Weaverville. It was felt west to Eureka and Scotia. Magnitude 4.5 $\mathrm{M}_{\mathrm{La}}$ DMG. (Ref. 1, 324.)

1929. July 8. Near Whittier, Los Angeles County, Calif. This earthquake was strongest in an area southeast of Whittier. Within that area, a schoolhouse and two private dwellings were damaged seriously, and several others were damaged by falling chimneys. At nearby Santa Fe Springs, the shock broke flanges on oil towers and left a few short, parallel cracks in loose ground; two oil wells were plugged by incaving.

Felt generally from Mount Wilson on the north to beyond Santa Ana on the south and from Hermosa Beach in the west to Riverside in the east. Many aftershocks occurred within a few hours of the main earthquake and continued to occur at increasingly long and irregular intervals through March 1931. Several small foreshocks were felt in the Whittier area from May 4-18, 1929. (Ref. 2, 394.)

1929. Nov. 28. Southeast of Aberdeen, Inyo County, Calif. About $8 \mathrm{~km}$ southeast of Aberdeen, concrete reservoirs were cracked and dishes were broken. A large landslide was reported about $25 \mathrm{~km}$ 
northwest of Independence at the headwaters of Goodale Creek. Five heavy shocks were reported at Fresno. The earthquake generally was felt northwest to Stockton (San Joaquin County), south to Kernville, and east to Mina, Nev. Magnitude 4.9 Ukn JON. (Ref. 2, 38.)

1930. Jan. 16, 0024 and 0034 UTC (Jan. 15). Near Summit, San Bernardino County, Calif. Two strong earthquakes knocked down chimneys and broke dishes in Fawnskin and Summit. The first shock was heavier. The tremors were felt strongly as far as Los Angeles. Magnitude 5.9 Ukn JON (00 24 UTC), 5.5 Ukn JON (00 34 UTC). (Ref. 3, 38, 258.)

1930. Feb. 26 (Feb. 25). Near Westmorland, Imperial County, Calif. At Westmorland, chimneys were knocked down, and walls were cracked. Modern buildings were undamaged. East of Westmorland, craterlets formed where mud and water were forced from the ground. Felt in Imperial, Riverside, and San Diego Counties and at a few towns in western Arizona. Several foreshocks and many aftershocks occurred. Magnitude 5.7 Ukn JON. (Ref. 3, 38.)

1930. Mar. 1. Near Brawley, Imperial County, Calif. At Brawley chimneys and overhanging cornices toppled, roofs displaced, and walls were cracked severely. Aftershocks were felt through Mar. 6, 1930. (Ref. 3, 38.)

1930. Aug. 5, 1125 UTC. Near Santa Barbara, Calif. Two local earthquakes broke windows at Santa Barbara and cracked walls at Ventura. One aftershock was observed. Magnitude $4.7 \mathrm{Ukn}$ JON. (Ref. 3, 38.)

1930. Aug. 31 (Aug. 30). Santa Monica Bay, Calif., west of the Los Angeles Basin. A cornice fell from a building at Venice, and ground cracks formed long the edge of Palisades Park bluff at Santa Monica, north of Venice. Small earthslides and rockslides were reported. At Los Angeles, minor cracks formed in buildings, plaster fell, and dishes broke. Light damage also occurred at Chatsworth, Hollywood, Owensmouth, and Pasadena. Felt throughout the Los Angeles Basin-from Kern County in the north to San Diego County in the south and east to the Palm Springs area. A tsunami of $0.6 \mathrm{~m}$ was observed at Santa Monica.

A comparison of the Jan. 1, 1979, Malibu earthquake and the 1930 Santa Monica earthquake suggests that they occurred on the Anacapa-Dume and Santa Monica faults, respectively. The epicenters of these earthquakes can be interpreted to define an 8to $10-\mathrm{km}$ north-south distance between the two faults, which suggests that the faults are unlikely to rupture simultaneously in one large event. (Ref. 3, $38,396,474,610$.)
1930. Sept. 23 (Sept. 22). Near Eureka, Humboldt County, Calif. Chimneys were toppled at Arcata, Eureka, and Fields Landing. Slight damage occurred at Capetown, Crannell, and Korbel. Felt south to Briceland, north to the Oregon border, and about $90 \mathrm{~km}$ inland from the coast. Two light aftershocks occurred on Sept. 23. Magnitude 5.1 Ukn JON. (Ref. 3, 38, 324.)

1930. Oct. 29. Near Whitmore, Shasta County, Calif. This earthquake caused damage at La Moine (50 km north of Redding) and Whitmore (40 km east of Redding). A series of 13 shocks was felt from Mineral and Viola in the east to Redding and La Moine in the west. (Ref. 3, 324.)

1931. Aug. 23. Off the coast, southwest of Cape Mendocino, Calif. This earthquake was strongest south of Eureka, along the lower course of the Eel River. At the Punta Gorda Lighthouse, the mantle on the revolving lamp was broken. Damage, if any, was superficial at other towns. Felt north to southern Oregon and south into Mendocino County. Magnitude 5.9 $\mathrm{M}_{\mathrm{x}}$ JON. (Ref. 4, 258.)

1931. Sept. 9. Off the coast of Humboldt County, northwest of Cape Mendocino, Calif. This earthquake was strongest at towns along the lower course of the Eel River. Chimneys were damaged south of Eureka, at Weott, and bricks were dislodged a few kilometers east of Weott, at Blocksburg. Branches fell from trees in wooded areas of Humboldt County. Several aftershocks were reported. Magnitude 6.3 $\mathrm{M}_{\mathrm{x}}$ JON. (Ref. 4, 324.)

1932. Feb. 26. Near Big Sur, Calif. The location given in the California list for this earthquake has been redetermined by Toppozada (1991, oral commun.) to have occurred about $70 \mathrm{~km}$ to the northnorthwest, in the coastal area south of Big Sur.

1932. June 6. Off the coast of Humboldt County, west of Eureka, Calif. This earthquake, the strongest in the region since Jan. 22, 1923, killed one person at Eureka and injured several others. Property damage was severe at Eureka, where hundreds of chimneys were damaged and many fell, plate-glass windows were shattered, and water mains were broken. Several small houses were shaken down in Arcata, and most chimneys were damaged. Hardly a chimney remained standing at Fields Landing, and a 15-cm crack formed in the highway. Many chimneys also toppled at Loleta, where a brick wall was shaken into the street.

A 70-cm ground crack developed on Cock Robin Island, at the mouth of the Eel River, and many blowholes, some as much as $2.5 \mathrm{~m}$ in diameter, were observed. Felt north to Coos Bay, Oreg., south to 


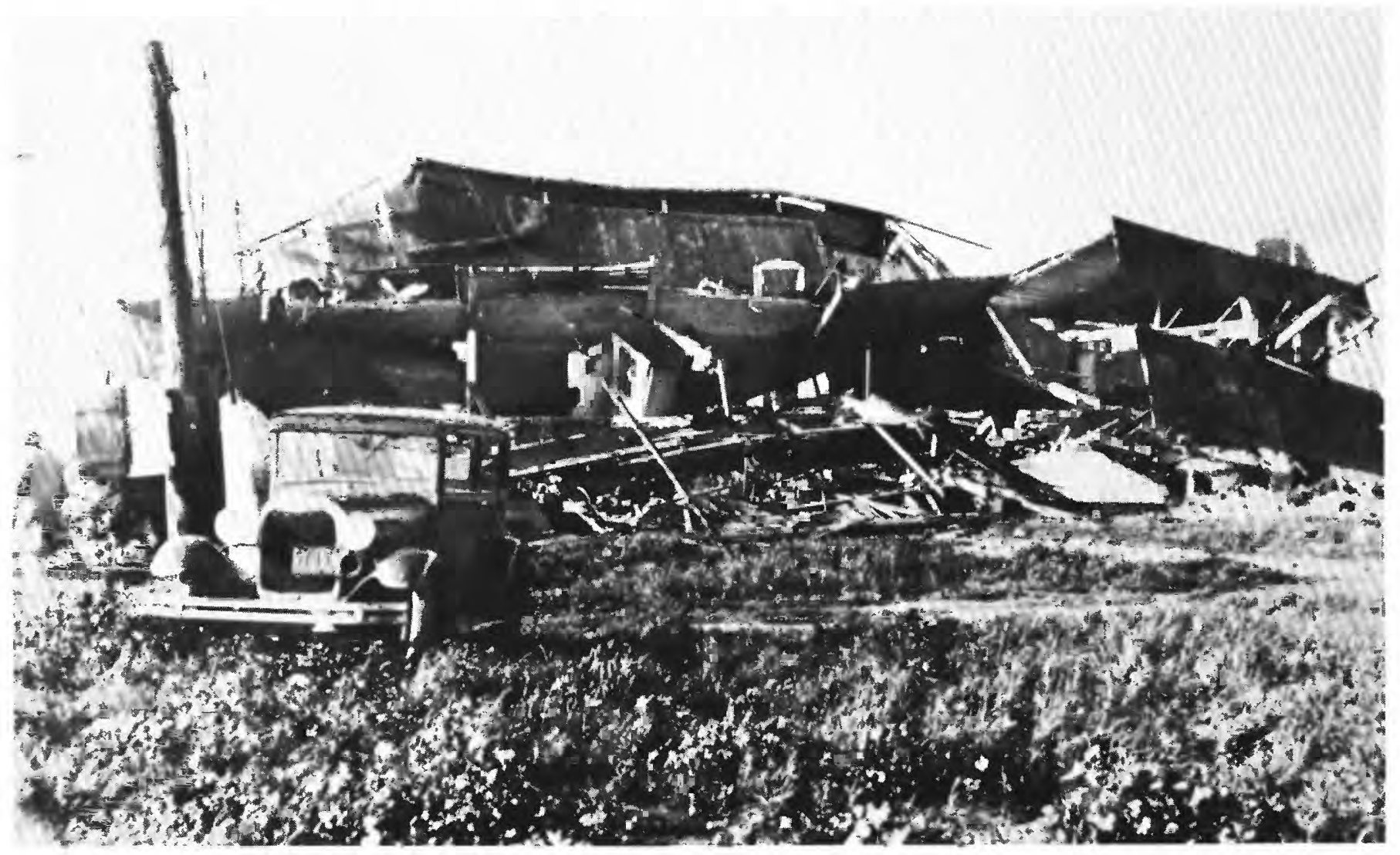

Wood-frame building in Long Beach, California, damaged by the March 11, 1933 (Mar. 10 PST), earthquake.

San Jose, and east to Nevada City (Nevada County). Several aftershocks occurred. Magnitude $5.9 \mathrm{M}_{\mathrm{La}}$ DMG. (Ref. 5, 38, 258, 381, 533, 544.)

1932. July 26 (July 25). Near Springville, Tulare County, Calif. An earthquake loosened several tons of rock from a peak on the south edge of Sequoia National Park, near Mineral King, and cracked brick chimneys at Springville (about $45 \mathrm{~km}$ southeast of Tulare). Magnitude $4.9 \mathrm{Ukn}$ JON. (Ref. 5,292 .)

1933. Mar. 11, 0154 UTC (Mar. 10). Southeast of Long Beach, near Newport Beach, Calif. Although only moderate in terms of magnitude, this earthquake caused serious damage to weak masonry structures on land fill from Los Angeles south to Laguna Beach. Property damage was estimated at $\$ 40$ million, and 115 people were killed.

Severe property damage occurred at Compton, Long Beach, and other towns in the area. Most of the spectacular structural damage was due to land fill, or deep water-soaked alluvium or sand, and to badly designed buildings. Minor disturbances of ground water, secondary cracks in the ground, and slight earth slumps occurred, but surface faulting was not observed. Along the shore between Long Beach and Newport Beach, the settling or lateral movement of road fills across marshy land caused much damage to the concrete highway surfaces and to approaches to highway bridges.

At Compton, almost every building in a three-block radius on unconsolidated material and land fill was destroyed. At Long Beach, buildings collapsed, houses were pushed from foundations, walls were knocked down, and tanks and chimneys fell through roofs. Damage to school buildings, which were among the structures most commonly and severely damaged by this earthquake, led to the State Legislature passing the Field Act, which now regulates building-construction practices in California.

This destructive earthquake was associated with the Newport-Inglewood fault. Shocks similar in magnitude and intensity to this event have occurred in this area in the past-notably July 28, 1769; Dec. 8, 1812; and July 11, 1855.

The earthquake was felt almost everywhere in the 10 southern counties of California and at some points farther to the northwest and north in the Coast 


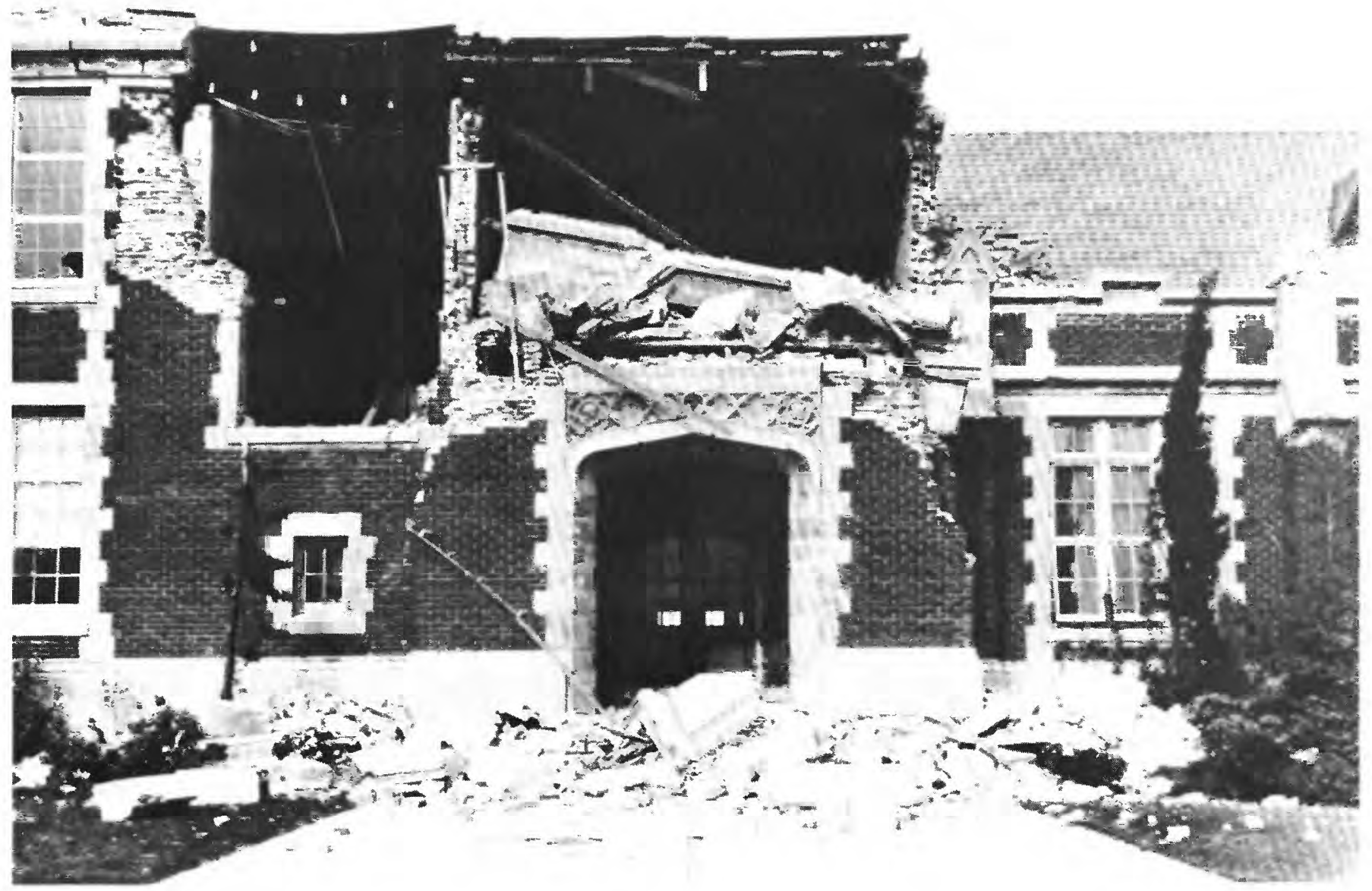

Front wall of the John Muir School, Pacific Avenue, Long Beach, California, downed by the March 11, 1933 (Mar. 10 PST), earthquake. (Photograph by W.L. Huber.)

Range, the San Joaquin Valley, the Sierra Nevada, and the Owens Valley (see fig. 16). It also was reported in northern Baja California. A sharp foreshock occurred near Huntington Beach on Mar. 9, and many aftershocks occurred through Mar. 16. For several years, minor aftershocks continued to occur, most often centering near the two ends of the disturbed segment of the Newport-Inglewood fault. Magnitude 6.25 $\mathrm{M}_{\mathrm{S}} \mathrm{GR}, 6.3 \mathrm{M}_{\mathrm{S}} \mathrm{CFR}, 6.2 \mathrm{M}_{\mathrm{La}} \mathrm{DMG}$, $6.43 \mathrm{M}_{\mathrm{L}}$ KJ. (Ref. 6, 292, 381, 397, 460, 533.)

1933. May 16. Near Niles, Alameda County, Calif. All chimneys were thrown down and some houses were damaged in the area of Overacker Station, between Niles and Irvington. North of Niles, cornices fell from buildings at Walnut Creek; chimneys fell, and the City Hall was damaged at Martinez. Rockfalls blocked the road in Niles Canyon. Felt north to Marysville (Yuba County), from the coast east to Merced, and south to Spreckels
(Monterey County). Magnitude 5.8 Ukn JON. (Ref. $38,324,536$.

1933. Oct. 2. Near Los Angeles, Calif. Widespread minor damage occurred in the business section of Los Angeles, including broken windows and dishes, cracked plaster, and damaged street lamps. Minor damage also occurred at Bell, Compton, Long Beach, and other towns in the area. Felt north along the coast to Santa Barbara, south to San Diego, and east to Victorville and San Bernardino. Magnitude $5.4 \mathrm{M}_{\mathrm{S}}$ GR. (Ref. 6, 38, 292.)

1933. Oct. 25 (Oct. 24). Near Los Angeles, Calif. At Huntington Park, some chimneys were downed, windows were broken, and brick was damaged slightly. The shock generally was felt in the suburbs of Los Angeles. (Ref. 6, 259, 292.)

1934. Apr. 23. Near Aromas, Monterey County, Calif. A local earthquake downed chimneys at Aromas and knocked merchandise from shelves. 


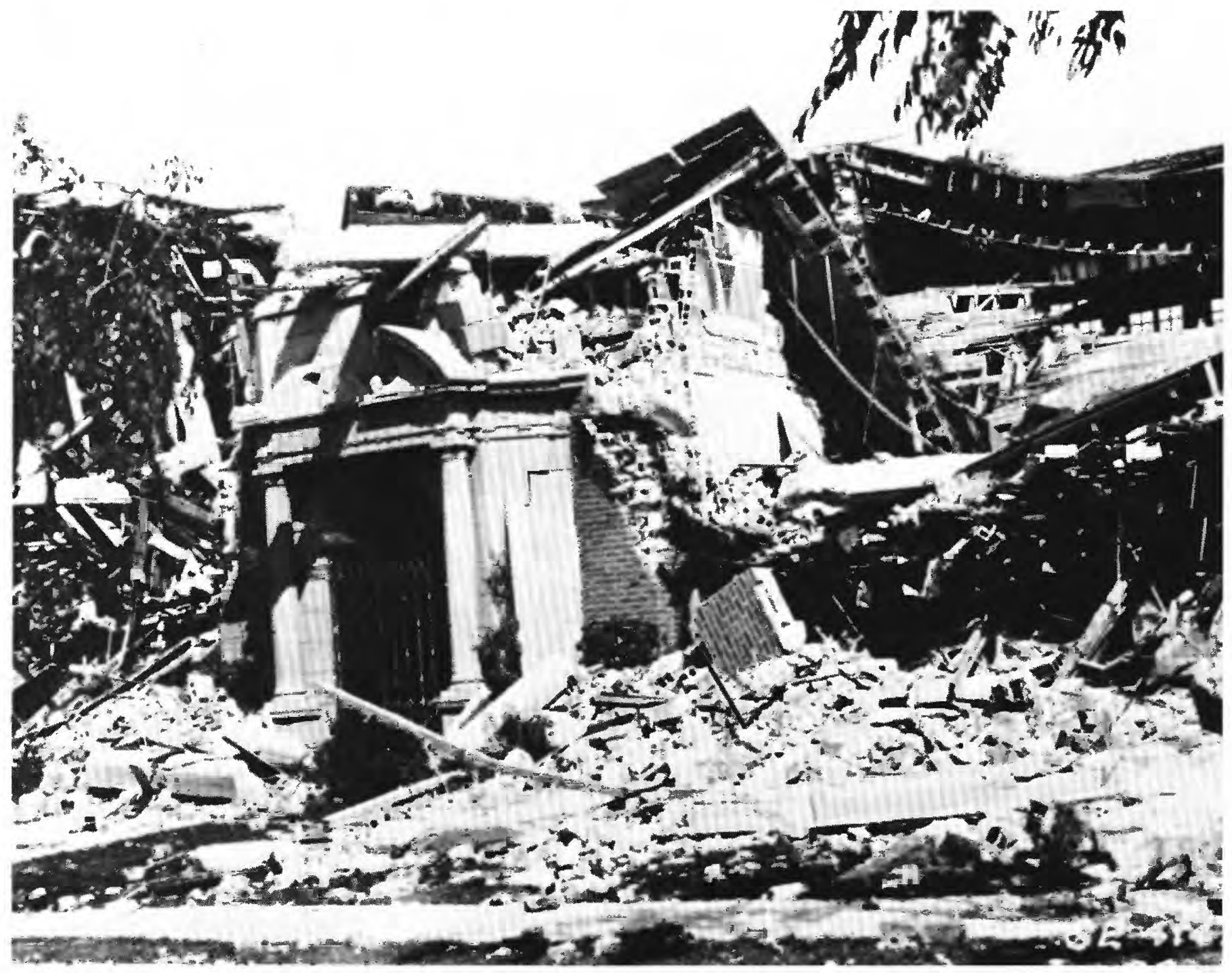

Jefferson Junior High School in Long Beach, California, destroyed by the March 11, 1933 (Mar. 10 PST), earthquake.

Felt at Hollister, Salinas, Spreckels, and Watsonville. An earlier shock was reported at 1608 UTC. (Ref. $259,324,559$.)

1934. June 5, 2252 UTC. Adelaida, San Luis Obispo County, Calif. A foreshock of the June 8 earthquakes, this local event knocked down two walls and several trees at Adelaida. It was reported only at a few towns in the area. (Ref. 7, 292.)

1934. June 8, 0430 and 0447 UTC (June 7). Near Parkfield, Monterey County, Calif. A series of earthquakes occurred in the Parkfield area from June 5 to 14. The strongest foreshock-on June 8 at 0430 UTC - caused damage in and around Parkfield and Stone Canyon. The principal earthquake, which occurred 17 minutes later, caused severe damage at Parkfield-a concrete-block house was wrecked, walls fell, and chimneys were downed. Highway bridges near Parkfield shifted slightly on their footings. Chimneys were knocked down in the area along the San Andreas fault-from Stone Canyon to the southern boundary of Monterey County.

On Middle Mountain, northwest of Parkfield, two zones of cracks about $7.5 \mathrm{~m}$ wide formed in the soil parallel to the surface traces of two faults in the San Andreas system. The largest single crack was about $17 \mathrm{~m}$ long and $46 \mathrm{~cm}$ deep. Neither vertical nor horizontal displacement, however, was observed along the cracks. Felt to Alviso in the north, Santa Ana in the south, and Kernville in the east. Magnitude 6.0 $\mathrm{M}_{\mathrm{S}}$ GR, 6.0 $\mathrm{M}_{\mathrm{S}}$ CFR, 5.6 $\mathrm{M}_{\mathrm{La}}$ DMG. (Ref. 7, 38, 292, $381,536$.

1934. Dec. 17. Near Los Alamos, Santa Barbara County, Calif. A few chimneys and plaster fell at Los Alamos, and plaster and chimneys 


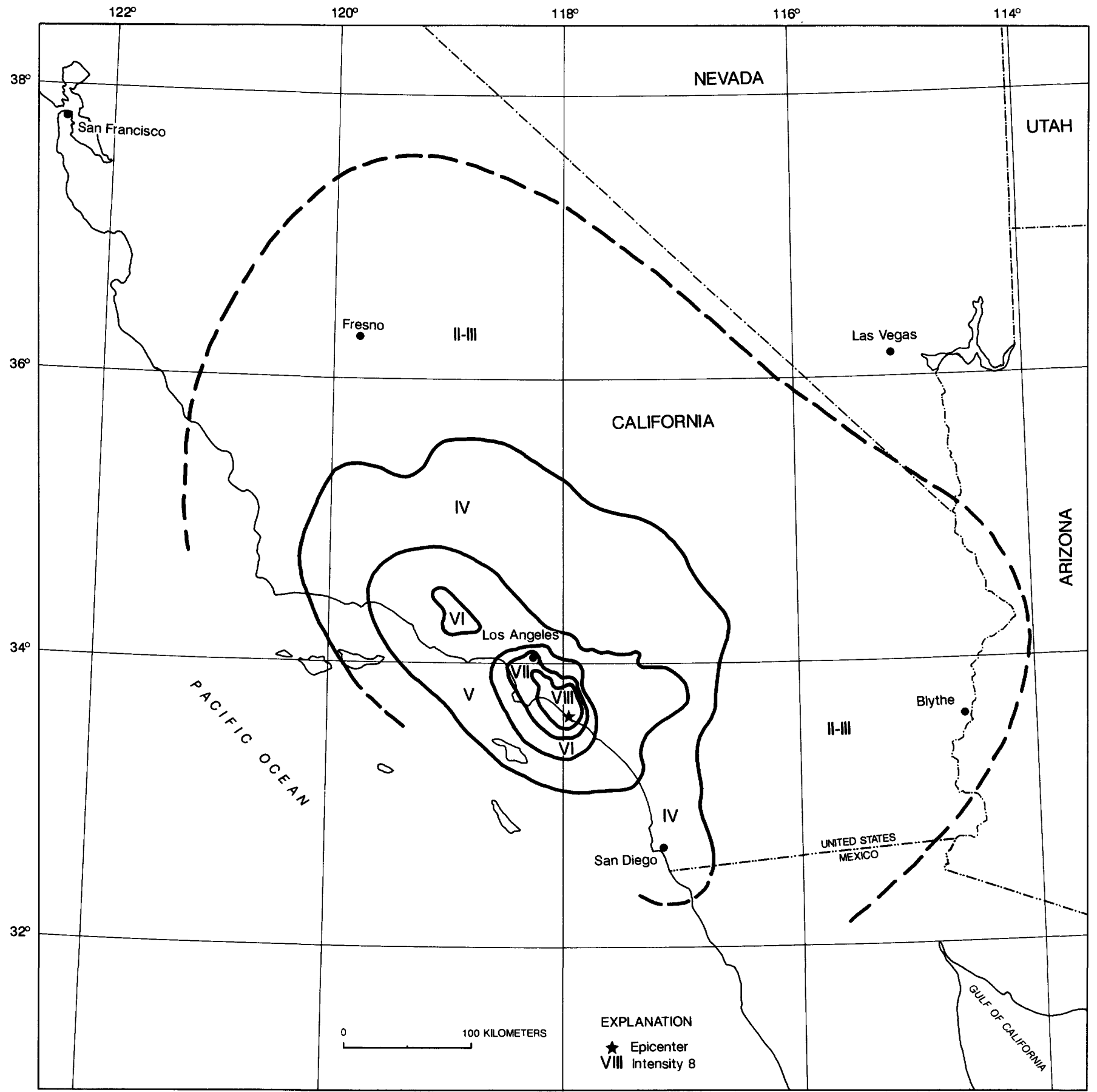

FIGURE 16.-Isoseismal map for the Long Beach, California, earthquake of March 11, 1933. Isoseismals are based on intensity estimates from data listed in references $6,259,381$, and 397 of table 1.

sustained cracks. Felt along the coast of Santa Barbara and San Luis Obispo Counties. Several aftershocks were reported at Los Alamos. Magnitude 4.8 $\mathrm{M}_{\mathrm{x}}$ JON. (Ref. 259, 292.)

1934. Dec. 30. Baja California, Mexico. Bridges were damaged, railroad tracks were twisted, and adobe houses were wrecked in the epicentral region in the Laguna Salada district south of Calexico. The strong shock cracked chimneys, walls, and windows in several California towns in the Imperial Valley. Many aftershocks occurred. Magnitude 6.7 $\mathrm{M}_{\mathrm{x}}$ JON. (Ref. 7, 38, 258.)

1934. Dec. 31. Baja California, Mexico. This earthquake was stronger than that of Dec. 30, 1934, 
and its epicenter was in the same general area. In the epicentral region, irrigation ditches were damaged, roads were buckled, and crevices opened in the ground. The shock was felt strongly throughout the Imperial Valley in southern California. At Calipatria, chimneys and walls were thrown down and windows were broken, and at Gadsden, Ariz., considerable damage to brick and masonry was reported. Slight damage occurred at several towns in California and western Arizona. The earthquake also was felt slightly at Las Vegas, Nev. Magnitude 7.1 $\mathrm{M}_{\mathrm{S}}$ CFR, 7.0 $\mathrm{M}_{\mathrm{S}}$ GR. (Ref. 7, 38, 258.)

1937. Mar. 8. San Francisco Bay region, Calif. An earthquake caused minor damage at Albany, Berkeley, El Cerrito, Elmhurst, and Oakland. Damage was heaviest in north Berkeley, where one poorly built house was condemned; several chimneys fell and many were twisted and cracked; walls were cracked; and plaster fell from walls. Felt north to Santa Rosa (Sonoma County) and south to Aptos (Santa Cruz County). (Ref. 10, 259, 324.)

1937. Mar. 25. Terwilliger Valley, San Diego County, Calif. This earthquake caused less damage than might have been expected because its origin was in a mountainous district having few residents within about $50 \mathrm{~km}$. Slight to moderate damage to chimneys, windows, plaster, or walls was reported from Anza, Garnet, Hemet, Keen Camp, Palm Springs, Ramona, and Warner Springs. Felt over most of southern California. Magnitude 6.0 M $\mathrm{M}$ CFR, $6.0 \mathrm{M}_{\mathrm{S}}$ GR, $5.9 \mathrm{M}_{\mathrm{La}}$ ELL. (Ref. 11, 259, 292, 545.)

1938. Feb. 12. Near Santa Cruz, Calif. One chimney toppled, and chimneys and windows cracked at Santa Cruz; plaster fell at Coyote and Morgan Hill. Felt north to San Rafael (Marin County) and south to Big Sur (Monterey County). (Ref. 11, 259, 324.)

1938. Aug. 31 (Aug. 30). Near Long Beach, Los Angeles County, Calif. A heavy china cabinet was overturned at Keystone (6 km north of Wilmington); beds were displaced about $20 \mathrm{~cm}$; and cracks formed in plaster. Cracks also formed in walls from top to bottom. Merchandise was knocked from shelves in stores and dishes were knocked to the floor at Long Beach and Huntington Beach. Felt along the coast between Santa Monica and Laguna Beach and inland to Mount Wilson. Several aftershocks occurred. (Ref. 11, 259, 292.)

1938. Sept. 12 (Sept. 11). South of Pepperwood, Humboldt County, Calif. One chimney fell at Pepperwood, and chimneys were cracked or twisted at Eel Rock and Ferndale. In the Redwoods, the ground was covered with branches fallen from trees. Felt along the coast north to Brookings, Oreg., east to Forbestown, Calif. (Butte County), and south to Elk, Calif. (Mendocino County). (Ref. 11, 259, 324.)

1938. Nov. 15. Near Talmage, Mendocino County, Calif. The heaviest reported damage occurred at Talmage, where one chimney fell and others were cracked. Some walls of buildings were cracked at Potter Valley. Felt generally in Lake, Mendocino, and Sonoma Counties. (Ref. 11, 259, 324.)

1938. Dec. 1. Alameda County, north of San Jose, Calif. Some chimneys were cracked at San Jose, and plaster cracked at Saratoga. Felt slightly at a few other towns in the area. (Ref. 11, 324.)

1938. Dec. 3, 1742 UTC. Inyo County, northwest of Bishop, Calif. West of Bishop, a large boulder crashed into a house and caused much damage. Other slides in the mountains reportedly occurred nearby. In the Owens River Gorge, buildings and pipelines were damaged by loosened rocks that cascaded down the mountains. Felt from Stockton (San Joaquin County), south to Bakersfield, and east into western Nevada. (Ref. 11, 292.)

1939. June 24. Southwest of Hollister, San Benito County, Calif. About $14 \mathrm{~km}$ southwest of Hollister, structural damage was sustained at the Mano ranch and the Orhwall ranch. Several chimneys fell or were twisted at the rooflines; many adobe walls were severely cracked; and several cracks in the ground were observed. The highway also was cracked in several places. At the San Benito Winery, chimneys were toppled and damage was considerable. Felt along the coast as far north as Half Moon Bay (San Mateo County), south to Nipomo (San Luis Obispo County), and inland to Tranquillity (Fresno County). (Ref. 12, 259, 324, 381.)

1939. Dec. 27. Near Long Beach, Los Angeles County, Calif. Moderate damage to property occurred at Huntington Beach and Long Beach. At Long Beach, several street lights were broken, a fireplace was shaken down, and chandeliers in stores were knocked from ceilings to the floor. At Huntington Beach, floors buckled in one house, and plaster fell from walls. Felt along the coast from Newport Beach north to Venice, inland to Altadena and San Bernardino, and southeast to Palm Springs. (Ref. 12, 292, 324.)

1939. Dec. 28. Near Cholame, San Luis Obispo County, Calif. Slight damage occurred at San Lucas, where dishes were broken and plaster was cracked. The general limits of the felt area extended from Santa Cruz south along the coast to Point Arguello and inland to Hollister, Lost Hills, and Fresno. (Ref. 12, 324.) 


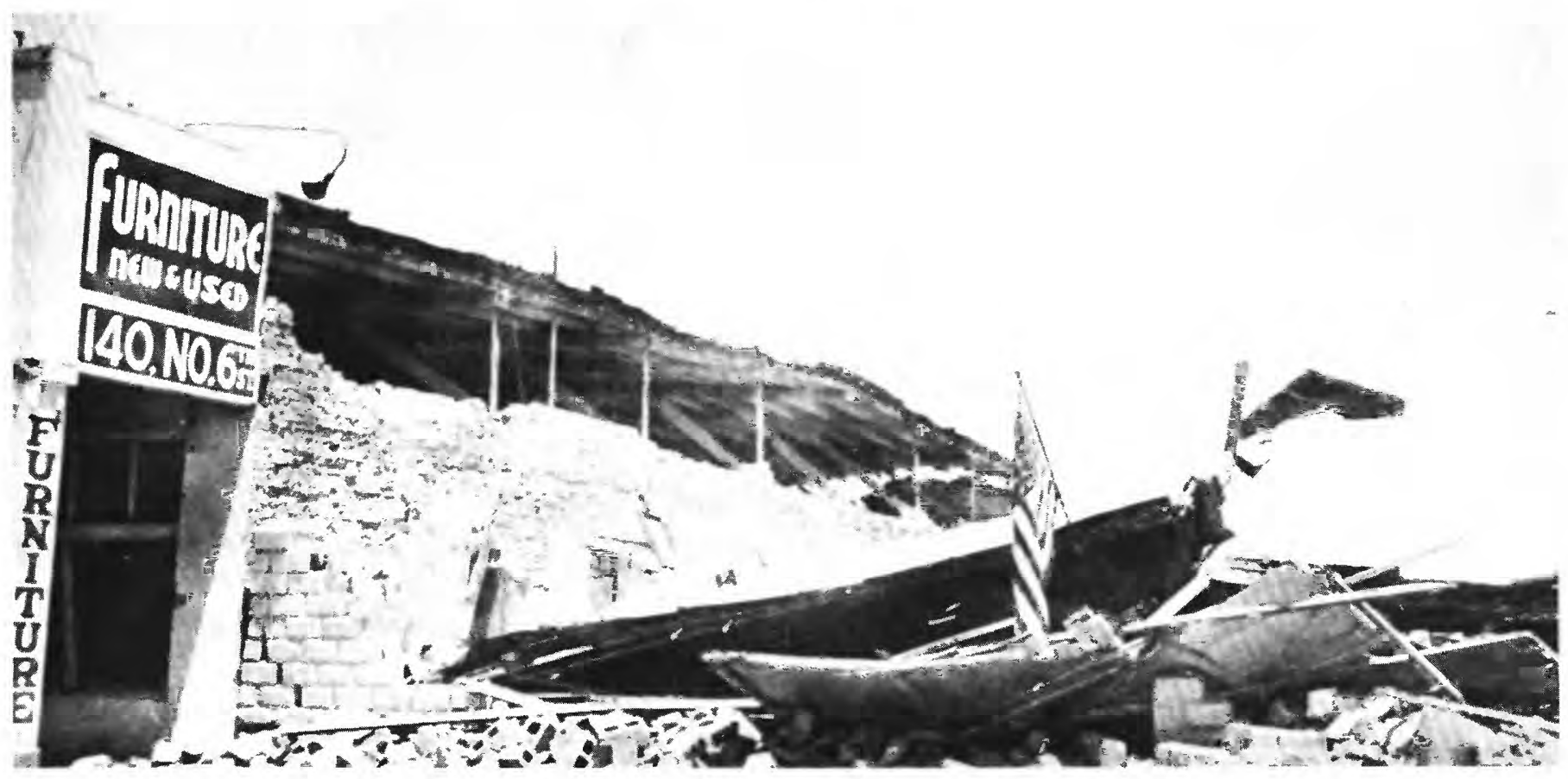

Furniture store in Brawley, California, destroyed by the Imperial Valley earthquake of May 19, 1940 (May 18 PST). (Photograph by F. Ulrich.)

1940. Feb. 8. North of Paradise, Butte County, Calif. Damage was confined to a few twisted and cracked chimneys and several broken windows and dishes in the Chico-Paradise area. The press reported that several chimneys were downed at Chico. Several landslides and dislodged rocks were reported along roads. Felt north to Dunsmuir (Siskiyou County), south to Stockton (San Joaquin County), west to the Platina area (Tehama County), and east to Reno, Nev. Magnitude $6.0 \mathrm{M}_{\mathrm{S}} \mathrm{CFR}, 6.0$ MS GR. (Ref. 13, 259, 324.)

1940. Feb. 13. Neax Branscomb, Mendocino County, Calif. Chimneys were cracked at Branscomb, and furnishings were displaced. The shock also was fairly strong in Humboldt County, at Ferndale and Scotia, and in Mendocino County, at Elk, Ukiah, and Westport. (Ref. 259, 324.)

1940. May 19, 0436 and 0551 UTC (May 18). Imperial Valley, near El Centro, Calif. The main earthquake took nine lives and caused property damage estimated at $\$ 6$ million. Damage from a strong aftershock near Brawley at 0551 UTC is included in this estimate.

The first shock damaged about 80 percent of the buildings in Imperial. Many buildings in the business district were condemned, and older residences sustained severe damage. Four people were killed in the collapse of a grocery store. Damage to a lesser extent occurred at El Centro and Holtville. Elevated water tanks at Holtville and Imperial collapsed, and a water tank at Brawley was damaged.

The downtown business area at Brawley was damaged severely by the second shock, and about 25 percent of the houses in the residential area were damaged. About half of the business structures had to be condemned. Many breaks in water mains occurred and water pipes were broken.

Damage to the structures and canals of the Imperial Irrigation District in the United States and Mexico was widespread. Breaks occurred over almost the entire length of the Ash Canal, from Holtville to the Mexico border. The Alamo Canal, the main feeder for the entire system, had eight major breaks; a section of the Solfatara Canal in Baja California was destroyed south of Cocopar. The earthquake demolished the New River flume, a 427-m-long timber structure on the West Side Main Canal south of Mexicali.

Right-lateral offset occurred along the Imperial fault. The pattern of offset indicates that the main part of the offsets occurred along a surface fracture about 20 to $25 \mathrm{~km}$ long, extending from the epicenter of the main shock southeast, about $5 \mathrm{~km}$ past Cocopar. Rupture of the northwest section of the fault may have occurred during a damaging aftershock at 0551 UTC. Where the surface fracture crosses the All American Canal east of Calexico, the largest 
displacement of $4.5 \mathrm{~m}$ occurred. At one point on the Solfatara Canal, the slip was as much as $3.7 \mathrm{~m}$.

In Baja California, the Inter-California Railroad track was displaced at Grape, and about $300 \mathrm{~m}$ of railroad track settled north of Grape. At Cocopar, the track shifted $2 \mathrm{~m}$, and at Meloland, it shifted about $46 \mathrm{~cm}$.

Many sand boils were observed near Gadsden on the Yuma Project in Baja California. Geysers spouting water several meters high also were reported. Canals, drainage channels, flumes, and bridges were damaged near Gadsden. The main earthquake was felt over much of southern California, southwest Arizona, and northern Baja California. About 48 aftershocks occurred through the end of 1940. Those on May 23 caused more damage at Brawley. Magnitude of first shock $6.9 \mathrm{~m}_{\mathrm{b}} \mathrm{ABE}, 6.7 \mathrm{M}_{\mathrm{S}} \mathrm{GR}, 7.1 \mathrm{M}_{\mathrm{S}} \mathrm{CFR}$, 6.4 $\mathrm{M}_{\mathrm{La}} \mathrm{DMG}, 7.2 \mathrm{M}_{\mathrm{S}}$ ELL, 7.2 $\mathrm{M}_{\mathrm{L}} \mathrm{KS}$. (Ref. 13, $292,381,521,533,546,547$.

1940. May 23, 1100,1730 , and 1845 UTC. Near Brawley, Imperial County, Calif. Three strong aftershocks of the Imperial Valley earthquakes (see May 19, 1940, above) cracked chimneys, walls, and plaster and broke windows and dishes at Brawley. (Ref. 259.)

1940. Oct. 11 (Oct. 10). Santa Monica Bay, Calif. This earthquake was strongest at Keystone (6 $\mathrm{km}$ north of Wilmington), where chimneys and walls were cracked, and dishes were broken. Slight damage also was reported at Long Beach, Los Angeles, Manhattan Beach, Maywood, and Redondo Beach. Felt along the coast north to Santa Barbara, south to San Diego, and east to the Big Bear Lake area (San Bernardino County). Magnitude $4.6 \mathrm{M}_{\mathrm{x}}$ JON. (Ref. 13, 259, 292.)

1940. Oct. 22. Near Scotia, Humboldt County, Calif. At Scotia, three underground pipes, windows, and plaster walls were broken. Felt over a small area of Humboldt County, extending along the coast from about Arcata to Shelter Cove and inland to the Bridgeville area. Several aftershocks were reported at Scotia. (Ref. 13, 259, 324.)

1940. Nov. 19. Off the coast of Humboldt County, west of Eureka, Calif. Loose bricks were thrown from a chimney at Upper Mattole, and furnishings were shifted. Slight damage also occurred at Garberville and Westport. Felt along the coast north to the Arcata area, south to Elk (Mendocino County), and east to the Forest Glen area (Trinity County). (Ref. 13, 259, 324.)

1940. Dec. 20. Southwest of Benbow, Humboldt County, Calif. This slight earthquake broke dishes and knocked books down at Fort Bragg and cracked plaster and overturned small objects at Benbow. Felt along the coast from Arcata south to Ukiah and east to Weaverville (Trinity County). (Ref. 13, 259,324 .)

1941. Feb. 9. Off the coast of Humboldt County, west of Ferndale, Calif. At Punta Gorda Light Station, slight cracks in plaster were observed. At Shelter Cove, the bluff slid in several places. Felt along the coast from San Francisco on the south to Port Orford, Oreg., on the north. Magnitude 6.4 $\mathrm{M}_{\mathrm{L}}$ ELL. (Ref. 14, 258, 259, 381.)

1941. July 1, 0750 UTC (June 30). Near Santa Barbara, Calif. Moderate damage to property occurred in the Carpinteria-Santa Barbara area. Property damage was estimated at $\$ 100,000$. About 25 chimneys and several walls fell at Carpinteria, and the cornice of a building was shaken to the ground. At Santa Barbara, one chimney toppled, bricks were dislodged from buildings, several buildings were cracked, and plate-glass windows were shattered. In addition, sidewalks buckled in places, and many water mains were broken. Several aftershocks occurred. The main earthquake was felt from near Stockton (San Joaquin County) in the north to Havilah (Kern County) in the south and from Coalinga (Fresno County) in the west to Mina, Nev., in the east. Magnitude $5.9 \mathrm{M}_{\mathrm{S}}$ GR, $5.9 \mathrm{M}_{\mathrm{S}}$ CFR, 5.5 $\mathrm{M}_{\mathrm{La}}$ DMG. (Ref. 14, 292, 381, 533.)

1941. Sept. 14, 1643,18 21, and 1839 UTC. Near Mammoth Lakes, Mono County, Calif. Five earthquakes occurred on this date, the three largest at the times given. Rockslides in the mountains blocked roads and trails, and one cabin was destroyed. Cracks formed in walls and chimneys at the Pineridge Shaver Ranger Station, and several chimneys were damaged at Yosemite Valley. Slight damage also was reported at Benton, Doyles, Miami Ranger Station, and Pinedale. Magnitude 5.8 $\mathrm{M}_{\mathrm{S}} \mathrm{GR}$ (first shock); 6.0 $\mathrm{M}_{\mathrm{S}} \mathrm{CFR}, 6.0 \mathrm{M}_{\mathrm{S}} \mathrm{GR}$ (third shock), 5.6 $\mathrm{M}_{\mathrm{La}}$ DMG (first and third shocks). (Ref. 259, 292,381 .)

1941. Oct. 3. Off the coast of Humboldt County, Calif. This earthquake was strongest at Eureka, where chimneys twisted and cracked, and plaster cracked and fell. Slight damage also occurred at Ferndale, Fields Landing, Korbel, Pepperwood, Punta Gorda, Rio Dell, Rockport, and Upper Mattole, where chimneys and walls cracked and plaster fell. Felt north to southwest Oregon and south, along the coast, to the San Francisco Bay area. Magnitude 6.4 $\mathrm{M}_{\mathrm{S}}$ CFR. (Ref. 259, 324.)

1941. Oct. 6 (Oct. 5). Off the coast of Humbolt County, Calif. Magnitude $5.25 \mathrm{M}_{\mathrm{S}}$ GR, $5.4 \mathrm{M}_{\mathrm{x}}$ JON. 
1941. Oct. 22, 0657 UTC (Oct. 21). Near Gardena, Los Angeles County, Calif. Property damage generally was confined to an area that included Compton, Hynes, Moneta, Gardena, Los Angeles, and the West Dominguez Oil Field, east of Gardena. Widespread minor damage-mainly cracked walls, fallen plaster, and broken windows and dishes-occurred at Gardena and Compton. At Keystone $(6 \mathrm{~km}$ north of Wilmington), chimneys were twisted, plaster and dishes were broken, and walls were cracked; at Moneta, the east and west fire walls on one brick building were knocked down. Felt along the coast from Montalvo (Ventura County) on the north to Newport Beach on the south and inland to Riverside and San Bernardino Counties. (Ref. 14, 259, 292.)

1941. Oct. 22, 1032 UTC. Near Gardena, Los Angeles County, Calif. This slight aftershock of the earthquake described above (06 57 UTC) cracked chimneys, walls, and plaster at Hondo. At Gardena and Pacific Palisades, the shock cracked plaster and overturned small objects. It also was observed by residents in a few other towns in the area. (Ref. 14, 259, 292.)

1941. Nov. 14. Near Gardena, Los Angeles County, Calif. This strong earthquake caused property damage estimated at $\$ 1.1$ million in the Gardena-Torrance area. No deaths or injuries were reported, perhaps because the earthquake occurred a little after midnight (local time) when most residents were at home sleeping. In nearby oil fields, two tanks were demolished, two were buckled, and a pipeline was broken.

Damage was most severe in Torrance, where hardly a building escaped damage. Two schools sustained heavy structural damage, and one was condemned; the fire station was abandoned because of heavy damage. The shock also moved several houses off their foundations. About 50 percent of all brick chimneys and fireplaces either were twisted, broken loose, or thrown down.

Severe damage also occurred in the city of Gardena. The walls of a two-story structure fell on and destroyed the roof of an adjoining building. The Gardena Elementary School building was condemned; the Bank of America Building was damaged severely; and the roof of a newspaper building almost collapsed. Several fire walls and many chimneys were knocked down or otherwise damaged. Felt from Carpinteria (Santa Barbara County) on the north to San Diego on the south and inland to Cabazon and San Jacinto in Riverside County. Magnitude $5.5 \mathrm{M}_{\mathrm{x}}$ JON. (Ref. 14, 259, 292, 533, 599.)
1941. Dec. 31, 0648 UTC (Dec. 30). Near Bishop, Inyo County, Calif. A moderate earthquake twisted chimneys and cracked plaster at the Adams Main Powerhouse in Owens River Gorge, near Bishop. Reports of damage were not received from other towns in the area. Felt from Georgetown (Eldorado County) on the north to Kernville on the south and from Coalinga on the west to Mina, Nev., on the east. Magnitude $5.4 \mathrm{M}_{\mathrm{S}}$ GR. (Ref. 14, 259, 292.)

1942. Oct. 21, 16 22 UTC. Near Borrego Valley, Imperial County, Calif. At Carrizo Gorge, about $19 \mathrm{~km}$ north of Jacumba, landslides broke timbers and wiring on a railroad bridge. Slight damage was observed at several towns in the area. Several aftershocks occurred, the strongest at 0150 UTC on Oct. 22. Felt from the coast east to Mobile, Ariz., and from Crucero (San Bernardino County) on the north to Baja California on the south. Magnitude 6.5 $\mathrm{M}_{\mathrm{S}}$ CFR, 6.0 $\mathrm{M}_{\mathrm{La}}$ DMG. (Ref. 15, 292, 381.)

1943. Aug. 29 (Aug. 28). Near Lake Arrowhead, San Bernardino County, Calif. The earthquake was most severe in the Big Bear Lake-Lake Arrowhead-Seven Oaks area. At Redlands, plaster fell, dishes broke, and bricks fell into a chimney. Slight damage also occurred at Lake Arrowhead. One foreshock and two aftershocks were recorded. The main shock was felt north to Mojave (Kern County), south to San Diego, and from the coast east to Ludlow. (Ref. 16, 259, 292.)

1943. Oct. 26 (Oct. 25). Near San Jose, Santa Clara County, Calif. In San Jose, a gas main was broken, plate-glass windows were shattered, and plaster was knocked off walls. A water line was broken at Sunnyvale. Slight damage to plaster occurred at several towns in the area. Felt along the coast from Santa Rosa (Sonoma County) south to San Ardo (Monterey County) and east as far as Bridgeport (Mono County), near the Nevada border. (Ref. 16, 259, 324.)

1943. Nov. 16. Near San Leandro, Alameda County, Calif. At San Leandro, chimneys were cracked, windows were broken, and plaster and knickknacks fell to the floor. One chimney pulled away from the wall of a house, leaving a $6.5-\mathrm{cm}$ crack at the eaves. Felt slightly over a small area in and around San Leandro. (Ref. 16, 259, 324.)

1944. June 12, 1045 and 1116 UTC. East of Banning, Riverside County, Calif. Plaster cracked and fell, dishes broke, and vases overturned at Banning; plaster cracked at Corona. Several light aftershocks were reported. The felt area extended from Oxnard (Ventura County), along the coast, south to San Diego and northeast to Ludlow (San Bernardino County). (Ref. 17, 259, 292.) 
1944. June 19, 0003 and 0306 UTC (June 18). Near Redondo Beach, Los Angeles County, Calif. Two sharp earthquakes caused minor damage to property in the Los Angeles area at Compton, Gardena, Huntington Park, Lynwood, and Maywood. The damage commonly included cracked chimneys and plaster and broken windows. Other damage included the dislodging of a large marble slab from the front of a store in Redondo Beach. Both shocks were felt along the coast south to San Diego and east to the Palm Springs area (Riverside County). Several light aftershocks were observed in the area. (Ref. 17, 259, 292.)

1945. Jan. 7. Near Hollister, San Benito County, Calif. This minor earthquake cracked chimneys and plaster at Hollister and broke windows and displaced stock on store shelves. It also knocked books and pictures from shelves at Paicines and San Benito. Two chimneys were damaged at the old Santa Anita ranch. Felt from San Francisco, south along the coast, to Big Sur (Monterey County) and east as far as Yosemite National Park. (Ref. 18, 259,324 .)

1945. May 17. Near Hollister, San Benito County, Calif. Buildings were damaged slightly at Hollister, where brick fell from chimneys; chimneys were cracked; several windows were broken; and plaster fell from walls. Considerable damage also was done to stock in stores. Felt north to San Francisco, east to Merced, and south to San Ardo (Monterey County). (Ref. 18, 259, 324.)

1945. May 19. Off the coast of southern Humboldt County, Calif. This earthquake was felt through most of the coastal towns, from Crescent City south to Fort Bragg and Willits. Magnitude 6.2 $\mathrm{M}_{\mathrm{S}}$ CFR, 6.0 $\mathrm{M}_{\mathrm{L}}$ BRK. (Ref. 18, 258.)

1945. Aug. 15. Near Borrego Valley, San Diego County, Calif. Some damage to cables and powerlines was reported at Fullerton, and plaster was cracked at Fall Brook and San Jacinto. Felt from Los Angeles south along the coast to San Diego and east to the Twentynine Palms area (San Bernardino County). (Ref. 18, 259, 292.)

1945. Aug. 27. Near San Jose, Santa Clara County, Calif. One chimney was shaken loose and plaster was cracked at San Jose. Felt to Saint Helena (Napa County) on the north, Big Sur on the south, and Pinecrest (Tuolumne County) on the east. (Ref. 18, 259, 324.)

1946. Jan. 13. West of Bishop, Inyo County, Calif. Near Bishop, at the Owens River Gorge, this earthquake cracked chimneys, walls, and plaster and displaced heavy furniture. Many rocks rolled down the sides of the canyon. Felt over a small area, which included Hawthorne, Nev. (Ref. 259, 292.)

1946. Mar. 15, 13 21, 1349 , and 1400 UTC. North of Walker Pass, Kern County, Calif. The main shock at 1349 UTC caused moderate damage at Onyx, about $19 \mathrm{~km}$ southwest of the epicenter. Damage to wood, brick, masonry, and concrete was reported to be conside rable. Chimneys, walls, plaster, and windows cracked; dishes broke; and plaster, books, and pictures fell. Cracks formed in the ground and concrete along the Los Angeles Aqueduct. Rockslides occurred in the canyons. Elsewhere in the region of Walker Pass and the South Fork of the Kern River, adobe houses were damaged, brick chimneys cracked, and plaster fell.

The earthquakes were felt from Commache (Calaveras County) on the north to San Diego (an isolated report) on the south and from Cambria (San Luis Obispo County) on the coast to Death Valley (see fig. 17). Several aftershocks occurred. Magnitude of second shock 6.25 $\mathrm{M}_{\mathrm{S}} \mathrm{CFR}, 6.25 \mathrm{M}_{\mathrm{S}} \mathrm{GR}, 6.1 \mathrm{M}_{\mathrm{La}}$ DMG. (Ref. 19, 259, 292, 381.)

1946. Sept. 28 (Sept. 27). Near Banning, Riverside County, Calif. Slight damage was reported at Banning, Cabazon, Cathedral City, Palm Springs, and Twentynine Palms. Felt in parts of Los Angeles, Riverside, San Bernardino, and San Diego Counties. (Ref. 19, 259, 292.)

1947. Feb. 5 (Feb. 4). East of King City, Monterey County, Calif. Buildings swayed visibly at Lonoak (Priest Valley), where plaster cracked and fell and small objects fell. Felt over a small area of Fresno, Monterey, and San Benito Counties. (Ref. 20, 259,324 .)

1947. Apr. 10, 1558 UTC. Near Manix, San Bernardino County, Calif. This moderate shock was strongest in the Newberry Springs area, about $40 \mathrm{~km}$ east of Barstow. One schoolhouse was condemned at Newberry Springs, and three adobe and brick houses were damaged severely. Minor damage, including one toppled chimney, fallen walls, cracks in chimneys and concrete, and cracked and slumped highways, were reported in the area. Also, cracks formed in the banks of the Mojave River. Felt over most of the southern half of California, a small part of southwest Nevada, and at several towns in western Arizona (see fig. 18). Several light aftershocks occurred. Magnitude 6.4 $\mathrm{M}_{\mathrm{S}} \mathrm{CFR}, 6.4 \mathrm{M}_{\mathrm{S}} \mathrm{GR}, 6.3$ $\mathrm{M}_{\mathrm{La}}$ DMG. (Ref. 20, 259, 292, 381.)

1947. May 27. Off the coast of Humboldt County, Calif. This moderate earthquake cracked plaster at Upper Mattole and shook trees and bushes strongly. Felt over a small area in Humboldt County. (Ref. 20, 259, 324.) 


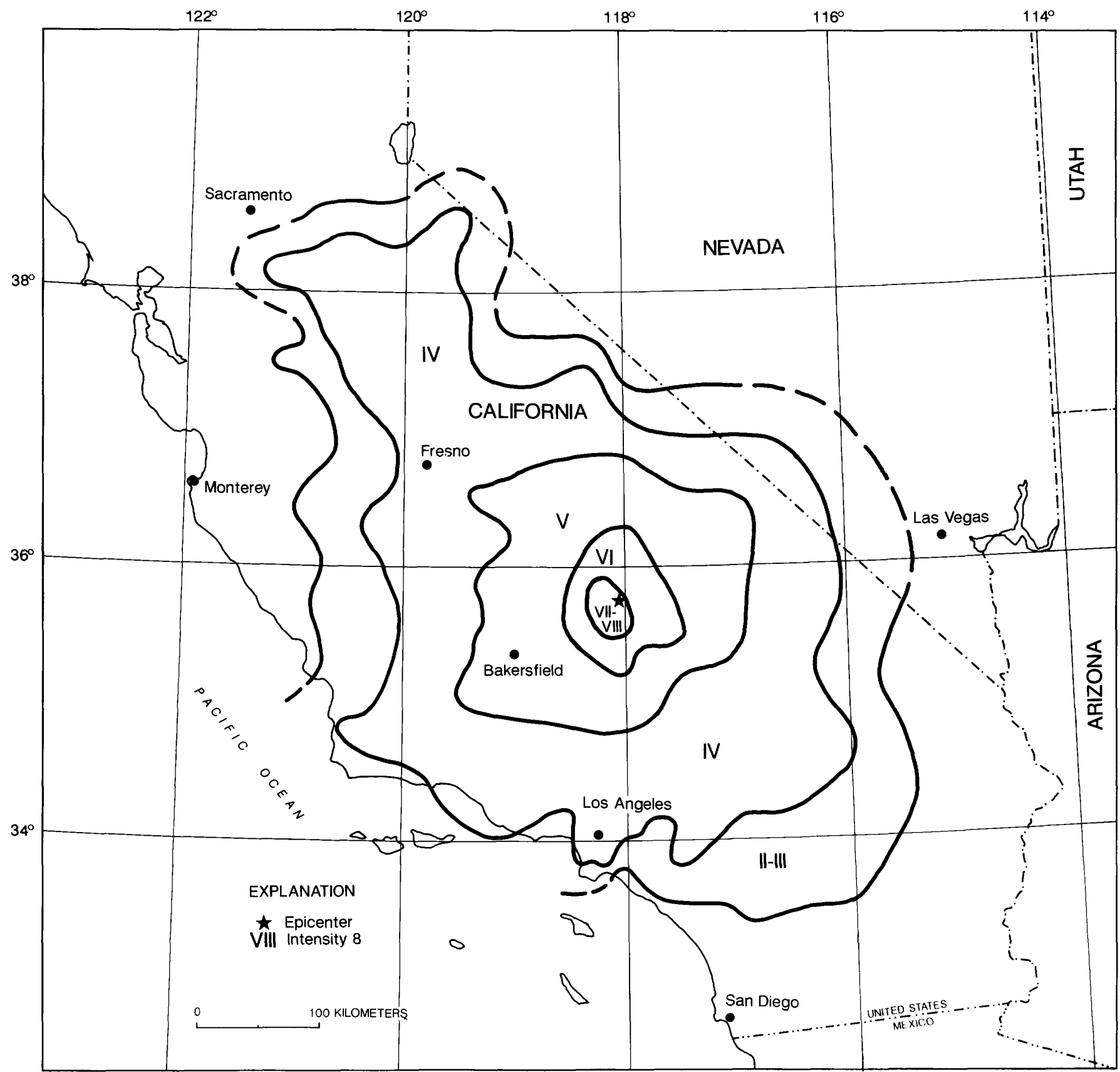

FiguRE 17.-Isoseismal map for the Kern County, California, earthquake of March 15, 1946. Isoseismals are based on intensity estimates from data listed in references 19,259 , and 381 of table 1.

1947. June 22. Near Santa Cruz, Calif. Chimneys were damaged at Corralitos and Watsonville; windows were broken at Santa Cruz; and plaster fell at Gilroy, Hollister, and Watsonville. It was reported that boulders blocked a highway near Chittenden Pass and that landslides closed Hecker Pass in Santa Clara County. Felt over most of the San Francisco Bay area. (Ref. 20, 259, 324.)

1947. Aug. 10. Near Hollister, San Benito County, Calif. The shock was strongest at Hollister, where stones were dislodged from a masonry pillar, plate-glass windows were cracked, a water main was broken, and stock was knocked from store shelves. The felt area extended from Glenwood (Santa Cruz County) southeast to Los Banos Creek (Merced County) south to San Ardo (Monterey County) and northwest to Big Sur on the coast. (Ref. 20, 259, 324.)

1947. Sept. 8, 0713 UTC (Sept. 7). Placer County, Calif., southwest of Reno, Nev. A series of small earthquakes was observed in the Reno, Nev., 


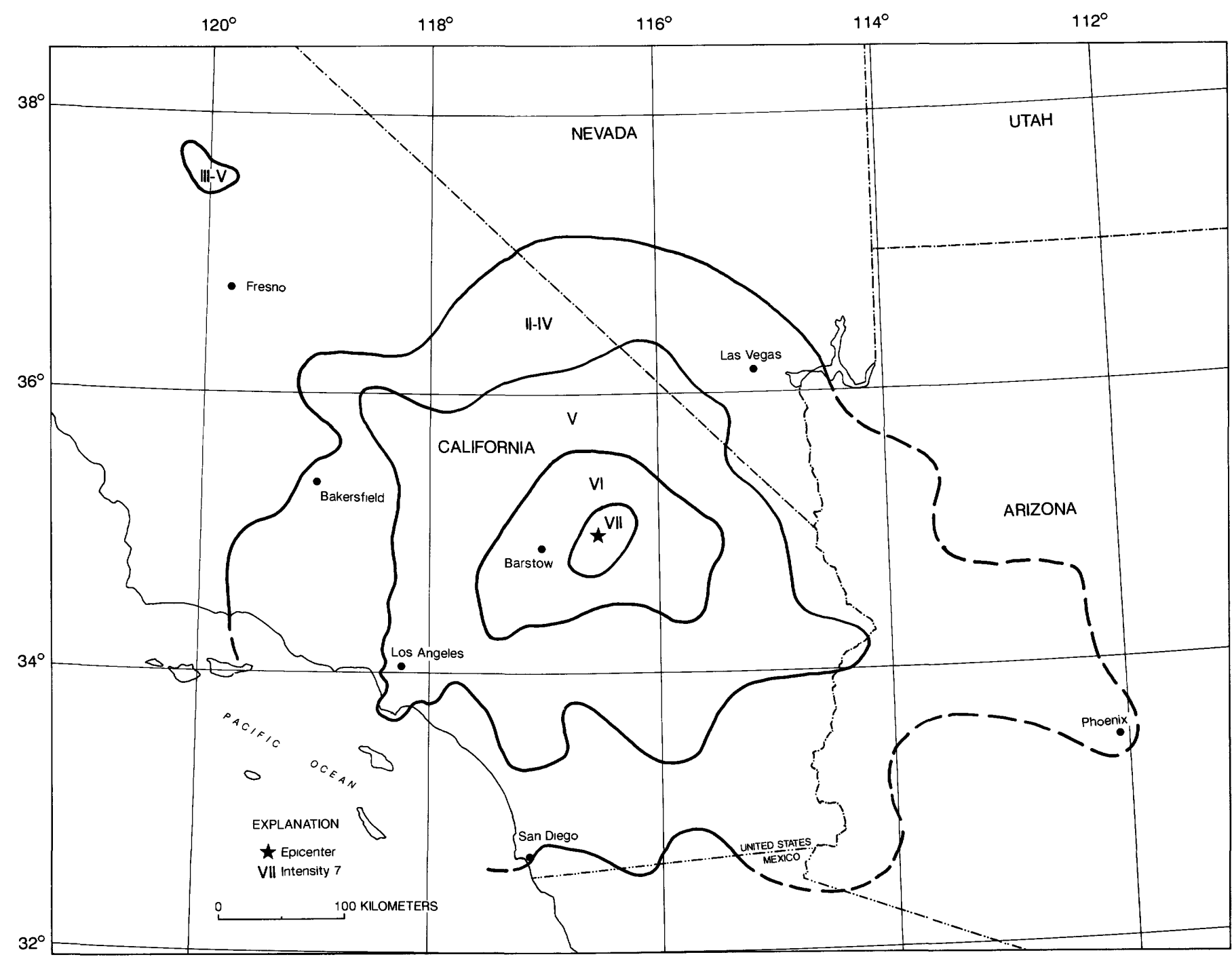

FIGURE 18.-Isoseismal map for the San Bernardino County, California, earthquake of April 10, 1947. Isoseismals are based on intensity estimates from data listed in references 20 and 259 of table 1.

area. The main shock knocked plaster from the ceiling in one house southeast of Reno and loosened mortar in the stone walls. Unusual activity in the hot springs was observed after the tremors. The earthquakes were felt over a small area of western Nevada and probably in a few towns in California. (Ref. 20, 259, 324.)

1948. Feb. 11 (Feb. 10). East of Tipton, Tulare County, Calif. A moderate earthquake cracked plaster at Ducor, Kingsburg, Lindsay, Springville, and Tipton. School buildings near Fresno, Dinuba, and Clovis were closed because of cracked walls. Felt extensively in the southern Sierra Nevadas and along the east side of the southern Joaquin Valley. (Ref. 21, 259, 324.)

1948. Feb. 20 (Feb. 19). Near Los Angeles, Calif. This shock sent many people running into the streets in the Los Angeles area. Slight damage to plaster was reported at North Hollywood. Felt over a small area of Los Angeles County. (Ref. 21, 259, 292.)

1948. Mar. 1. Northwest of San Bernardino, Calif. Slight damage to plaster and walls was reported at Burbank and Pasadena. Near Devore, cracks formed in the concrete floor of a cabin and dishes were broken. Felt over an area that included parts of Kern, Los Angeles, Orange, Riverside, San Bernardino, and San Diego Counties. (Ref. 21, 259, 292.)

1948. Apr. 16. Southeast of Oxnard, Ventura County, Calif. This earthquake was strongest at Oxnard, where plaster cracked and fell, dishes were broken, and lamps were knocked over. Felt at towns near the coast, southeast to Long Beach, and inland about $40 \mathrm{~km}$. (Ref. $21,259,292$.) 
1948. June 18. Near Ukiah, Mendocino County, Calif. Chimneys were cracked and vases overturned at Ukiah, and plaster was damaged at Lakeport and Talmage. Felt over a small area of Lake, Mendocino, and Sonoma Counties. (Ref. 21, 259, 324.)

1948. Dec. 4. Near Desert Hot Springs, Riverside County, Calif. The earthquake probably was caused by displacement on the Mission Creek fault, one of the major branches of the San Andreas fault system in southern California. The highest intensities in the area were reported from the upper Coachella Valley from Thousand Palms to White Water, which also was the most densely populated area near the epicenter.

Considerable structural damage and slight cracks in the ground were observed in Desert Hot Springs. Some minor structural damage also occurred at Palm Springs. At Willis Palms, cracks formed in the ground and cliffs, riverbanks slumped, and springs increased in flow. Landslides and cracks in the ground were reported in the Indio Hills. Felt throughout southern California and at a few towns in western Arizona, southwest Nevada, and northern Baja California (see fig. 19). About 72 aftershocks were accurately located in a zone $18 \mathrm{~km}$ long, parallel to (but $5 \mathrm{~km}$ north of) the trace of the Mission Creek fault. Magnitude 6.5 $\mathrm{M}_{\mathrm{S}} \mathrm{CFR}, 6.5 \mathrm{M}_{\mathrm{S}} \mathrm{GR}, 6.2$ $\mathrm{M}_{\mathrm{La}}$ DMG. (Ref. 21, 259, 292, 381, 548.)

1948. Dec. 29. West of Reno, Nev., in Sierra County, Calif. The town of Verdi, Nev., which lies at the sharp bend of the Truckee River, sustained the most damage to property. The west wall of the old general store at Verdi collapsed, and the building was wrecked. Chimneys on houses were toppled or twisted out of line, sections of parapet walls on a schoolhouse fell, and many windows were broken. Minor damage also occurred at Reno and at Chilcoot, Calif. Felt over a large area of central California and western Nevada. Magnitude $6.0 \mathrm{M}_{\mathrm{S}} \mathrm{CFR}, 6.0 \mathrm{M}_{\mathrm{S}}$ GR, 5.7 M $\mathrm{Ma}_{\mathrm{La}}$ DMG. (Ref. 21, 259, 324, 381.)

1949. Jan. 1 (1948. Dec. 31). Near Watsonville, Santa Cruz County, Calif. Near Chittenden, six houses standing on stilts or blocks were displaced a few centimeters on their foundations, chimneys were broken off two of the houses, and a small landslide nearby partly blocked the Old Chittenden Road. Slight damage also occurred at Hollister and Santa Cruz. Felt over a small area of the coastal region of central California from Oakland (Alameda County) south to Big Sur (Monterey County). (Ref. $21,259,324$.)

1949. Feb. 11. Southeast of Bishop, Inyo County, Calif. Slight damage, in the form of cracks in brick, walls, and plaster, was reported at Ash Mountain (Sequoia National Park), Bakersfield, Big Pine, and Olancha. Felt over a large area of southcentral California and into Nevada as far as Beatty and Goldfield. (Ref. 22, 259, 292.)

1949. Mar. 9. Northwest of Hollister, San Benito County, Calif. Although felt strongly in the area, this shock was most severe at Hollister. Three chimneys were toppled, and a brick wall separating two stores split lengthwise. One market sustained severe cracks in plaster and walls. Considerable loss also was sustained from damaged merchandise in stores. Slight damage was reported from several other towns in the area. Felt over the western half of north-central California from Santa Rosa (Sonoma County) south to Paso Robles (San Luis Obispo County). A few aftershocks were reported, the largest on Mar. 14 at 0610 UTC. Magnitude $5.8 \mathrm{M}_{\mathrm{L}} \mathrm{KJ}$. (Ref. 22, 259, 324, 460.)

1949. Mar. 24. Off the coast of southern Humboldt County, Calif. Reported felt along the California and Oregon coasts. Magnitude $6.2 \mathrm{M}_{\mathrm{S}}$ CFR, 6.2 $\mathrm{M}_{\mathrm{S}}$ GR. (Ref. 324.)

1949. June 10 (June 9). Near San Jose, Santa Clara County, Calif. A series of three earthquakes occurred, the strongest at 0306 UTC. At San Jose, a water main was split open, windows were shattered in houses, and stock tumbled from store shelves. The main shock was felt north to Petaluma (Sonoma County) and south to Big Sur (Monterey County). (Ref. 22, 259, 324.)

1949. Aug. 8. Near Richmond, Contra Costa County, Calif. Slight damage was reported at Richmond (windows broke and dishes fell from shelves) and Vallejo (plaster cracked). Felt over a small area of the San Francisco Bay region. (Ref. 22, 259, 324.)

1949. Aug. 27. Near Point Concepcion, Santa Barbara County, Calif. Slight damage occurred at Arlight and Sudden, where a chimney fell and dishes broke, and at Lompoc, where dishes broke and knickknacks fell. Felt over a small area along the coast in Santa Barbara and San Luis Obispo Counties. (Ref. $22,259,292$.)

1949. Sept. 19 (Sept. 18). Near Huntington Park, Los Angeles County, Calif. Broken windows and dishes and cracks in cement driveways were reported in Los Angeles. The shock also was felt strongly at Long Beach and San Gabriel. It was reported over a small area of central Los Angeles County. (Ref. 22, 259, 292.)

1949. Nov. 4. Baja California, Mexico. A moderate earthquake caused minor property damage at Guadalupe, Mexico. In California, slight damage also occurred at Borrego Valley, Campo, Coronado, Del 


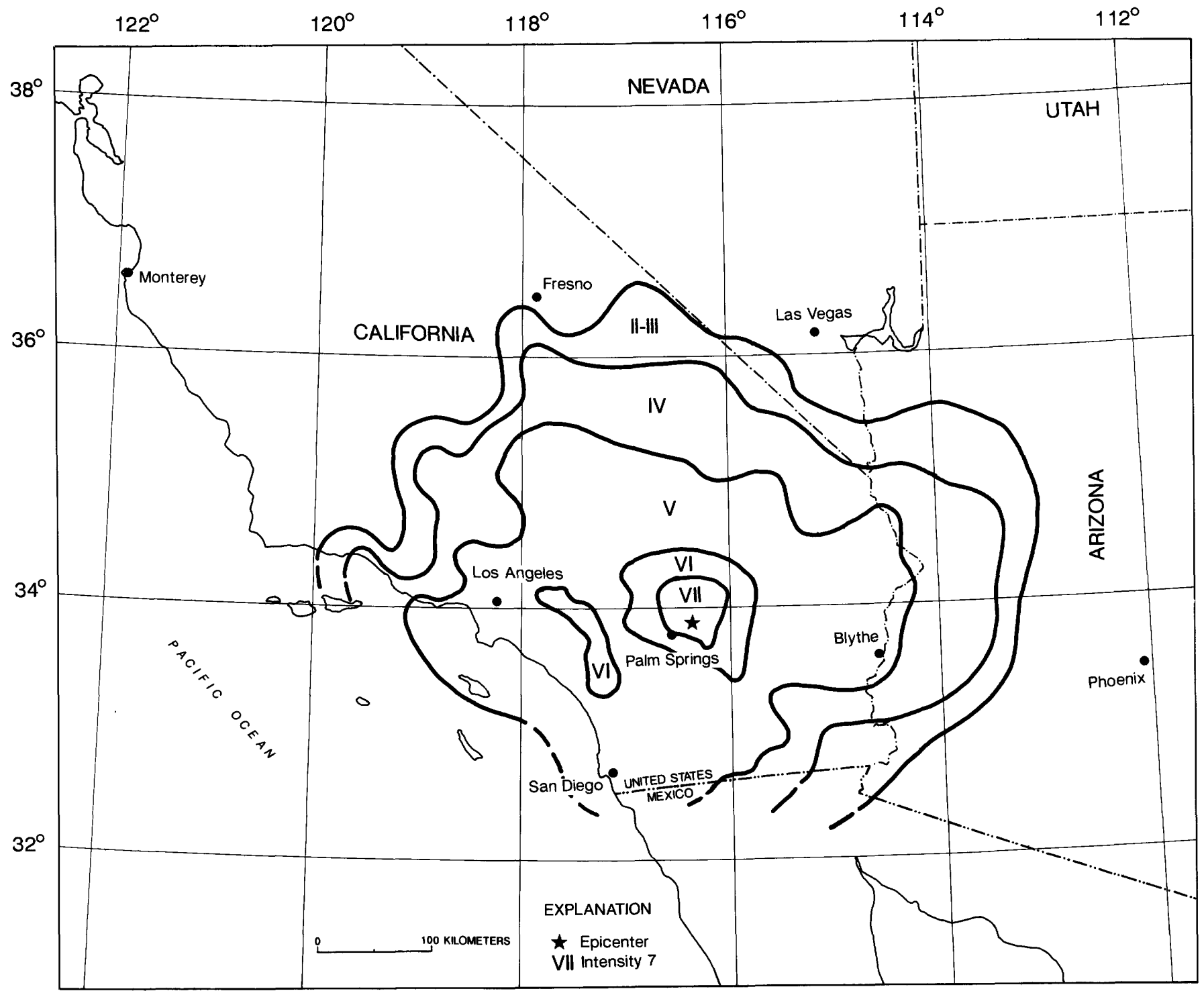

FIGURE 19.-Isoseismal map for the Riverside County, California, earthquake of December 4, 1948. Isoseismals are based on intensity estimates from data listed in references 21 and 259 of table 1.

Mar, San Diego, Santee, San Ysidro, and Spring Valley. Felt from Baja California north to Palmdale (northern Los Angeles County). (Ref. 22, 259, 292.)

1949. Nov. 5 (Nov. 4). Baja California, Mexico. An aftershock of the Nov. 4 earthquake (see above) caused slight damage to plaster and walls at Grossmont, La Jolla, and San Diego. (Ref. 22, 259, 292.)

1950. Jan. 14. Near Punta Gorda, Humboldt County, Calif. The earthquake was strongest at the Punta Gorda Light Station, where cracks formed in plaster, in the light tower, and in the concrete floor. Felt over a small area along the coast of Humboldt County in northern California. (Ref. 23, 259, 324.)
1950. Feb. 26 (Feb. 25). North of Santa Paula, Ventura County, Calif. A few bricks fell from an old chimney at Ventura, and a window was broken. Damage to plaster and fallen merchandise was reported from Oxnard and Santa Paula. The felt area extended along the coast from Lompoc (Santa Barbara County) to Van Nuys and in the east from Wheeler Ridge (Kern County) to Palmdale. (Ref. 23, 259, 292.)

1950. July 27, 1129 UTC; July 28, 1750 UTC; and July 29, 1436 UTC. Near Calipatria, Imperial County, Calif. A series of damaging earthquakes occurred in the Imperial Valley from July 27 to July 29 . The first two earthquakes caused only 
slight damage in the Brawley-Calipatria area. Damage from this series of earthquakes was estimated at $\$ 300,000$.

Near Calipatria, several concrete stand-pipes were broken and a small railroad bridge was shifted 15 to $20 \mathrm{~cm}$. Many sand boils were formed; irrigation ditch banks sloughed; and the ground settled and cracked. An old sheet piling broke when the levee settled at the North End Dam, about $3 \mathrm{~km}$ west of Calipatria. The main shock on July 29 was felt from the Mexican border to Banning, Calif., and Parker, Ariz., and from San Diego, Calif., to Yuma, Ariz. Aftershocks occurred through Aug. 14, 1950, and probably beyond. Magnitude $5.4 \mathrm{M}_{\mathrm{S}} \mathrm{GR}$ (second shock); $5.4 \mathrm{M}_{\mathrm{S}} \mathrm{GR}$ (third and main shocks). (Ref. 23, $259,292,560$.)

1950. Aug. 1. Near Calipatria, Imperial County, Calif. An aftershock of the July 29 earthquake collapsed a small part of a wall at Calipatria and knocked a few bricks from houses. In addition, ground cracks in the area of the North End Dam opened wider, and new sand boils were observed at the dam site and along the levees of the Vail Canal. Felt over a small area of southern California and at Somerton, Ariz. (Ref. 23, 259, 292.)

1950. Sept. 5. Near Idyllwild, Riverside County, Calif. Dishes were broken at Idyllwild and San Jacinto, plaster was cracked at San Jacinto, and a clock was thrown from its shelf at Idyllwild. Felt from Barstow (San Bernardino County) in the north to San Diego in the south and from the coast to Twentynine Palms in the east. (Ref. 23, 259, 292, 561.)

1950. Nov. 17 (Nov. 16). Near Hawthorne, Los Angeles County, Calif. Scattered reports of cracked plaster and broken dishes were received from southwest Los Angeles. This was the strongest of three small earthquakes that were felt in the area between 0159 and 1946 UTC on Nov. 17. The shocks were felt only in Los Angeles County. (Ref. 23, 259, 292.)

1950. Dec. 14, 1324 UTC. Near Herlong, Lassen County, Calif. This main shock of a series caused considerable structural damage at Herlong. Many structures sustained cracks from about 0.3 to $0.6 \mathrm{~cm}$ in width to as much as $24 \mathrm{~m}$ in length. Many chimneys were broken, trusses and roof rafters were split, and several buildings were displaced on their foundations. Damage to water mains, steam pipes, and sewers also was reported. Felt from Alturas (Modoc County) south to Sacramento and east to Lovelock, Nev. Several foreshocks and aftershocks were felt in the area. Magnitude 5.6 $\mathrm{M}_{\mathrm{S}} \mathrm{GR}$. (Ref. $23,259,324,561$.)
1951. Jan. 24 (Jan. 23). Near Westmorland, Imperial County, Calif. About $5.5 \mathrm{~km}$ southwest of Westmorland, in the Imperial Valley Irrigation District, $30 \mathrm{~m}$ of ground running northwest-south east settled $2.5 \mathrm{~cm}$; a water main in the Trifolium area was cracked; and canal banks were cracked. Slight damage also was reported in the nearby towns of Brawley, Calexico, Coachella, El Centro, Holtville, and Imperial. Felt over a large area of southern California and into southwest Arizona. (Ref. 24, 259, 292.)

1951. Jan. 25. Near San Leandro, Alameda County, Calif. Windows were broken or cracked and dishes fell from shelves at Oakland. Large chunks of plaster fell in the San Leandro Post Office, and dishes broke in houses. Felt over a small area of Alameda and Contra Costa Counties. (Ref. 24, 259, 324.)

1951. July 29. Pinnacles National Monument area, San Benito County, Calif. Chimneys twisted, plaster fell, and concrete pipes were damaged slightly at Pinnacles. Plaster fell in some houses in San Benito, and the highway from San Benito to Hernandez Valley was covered with boulders. Outside stucco and plaster walls were damaged slightly at Bitterwater (Lonoak). Felt from Pescadero (San Mateo County) in the north to San Luis Obispo in the south and Caruthers (Fresno County) in the east. (Ref. 24, 259, 324.)

1951. Aug. 6. Pinnacles National Monument area, San Benito County, Calif. Plaster was downed and vases were overturned south of Hollister, at the Harris Ranch. This earthquake was felt over the coastal area of west-central California. Two aftershocks were felt at 0954 and 1721 UTC. (Ref. $24,259,324$.)

1951. Oct. 8 (Oct. 7). Off Cape Mendocino, Humboldt County, Calif. Damage was considerable to a partly completed bridge over the Van Duzen River near Alton: columns were out of plumb, spans were out of position, diagonals were bent, and anchor bolts and grout pads were damaged. Cracks about $36 \mathrm{~m}$ in length formed in the pavement of both approaches to the bridge and in fill beside the approaches. Chimneys were broken or toppled at Bridgeville, Fortuna, Grizzly Bluff (a fireplace also was damaged considerably), Metropolitan, Rio Dell, Scotia, and Weott. This strong shock was felt along the coast from Orick (Humboldt County) in the north to Manchester (Mendocino County) in the south and east to Red Bluff (Tehama County) and Richvale (Butte County). Magnitude 6.0 $\mathrm{M}_{\mathrm{S}}$ CFR. (Ref. 24, $259,324,563$.)

1951. Nov. 14. South of Eureka, Humboldt County, Calif. A large window was broken in the Humboldt Times building in Eureka; windows and 
dishes were broken at Fields Landing; and plaster was cracked in a store at Fortuna. Felt over a small area in the Eel River Valley in northwest California. (Ref. 24, 259, 324, 563.)

1951. Dec. 5. Near Brawley, Imperial County, Calif. About $16 \mathrm{~km}$ north of Brawley, a crack about $30 \mathrm{~m}$ long and $4 \mathrm{~cm}$ wide formed in a gravel road; canal banks were damaged; plumbing broke in several houses; windows broke and stucco was damaged; and ceiling lights crashed to the floor. A bridge across the Highland canal also was damaged. One artesian well ejected water $3 \mathrm{~m}$ during the shock, and another well ran muddy water. Felt over a small area of the Imperial Valley in southeast California. (Ref. 24, 259, 292, 563.)

1951. Dec. 26 (Dec. 25). Near the southeast point of San Clemente Island, Calif. This Christmas Day earthquake caused slight damage to plaster, windows, or chimneys at Avalon, Long Beach (a large piece of tile also fell), San Diego, San Clemente Island, and San Pedro. The sharp shock was felt over a large area of southwest California and probably into northern Baja California. Magnitude $5.9 \mathrm{M}_{\mathrm{S}}$ CFR, 5.9 M $\mathrm{M}_{\mathrm{S}}$ GR. (Ref. 24, 259, 292.)

1952. Feb. 9. Northeast of Lone Pine, Inyo County, Calif. A series of three earthquakes occurred in the Lone Pine area. A building was cracked by the main shock at 0843 UTC, and plaster fell in several houses. A cafe sustained a large crack that extended halfway around the building and completely through the wall. Felt only at a few towns in Inyo County. (Ref. 25, 259, 292.)

1952. July 21, 1152 UTC. South of Bakersfield, Kern County, Calif. This earthquake was the largest in the conterminous United States since the San Francisco shock of 1906. It claimed 12 lives and caused property damage estimated at $\$ 60$ million. MM intensity XI was assigned to a small area on the Southern Pacific Railroad southeast of Bealville. There, the earthquake cracked reinforced-concrete tunnels having walls $46 \mathrm{~cm}$ thick; it shortened the distance between portals of two tunnels about $2.5 \mathrm{~m}$ and bent the rails into S-shaped curves. At Owens Lake (about $160 \mathrm{~km}$ from the epicenter), salt beds shifted, and brine lines were bent into S-shapes.

Many surface ruptures were observed along the lower slopes of Bear Mountain, in the White Wolf fault zone. The somewhat flat, poorly consolidated alluvium in the valley was erratically cracked and recontoured. The cracking along Bear Mountain indicated that the mountain itself moved upward and to the north. Southwest of Arvin, on the San Joaquin Valley floor, ground cracks traversed and split the concrete foundation of one house, causing partial collapse. The ground slumped; cotton rows were offset more than $30 \mathrm{~cm}$; and pavement on one highway was crumpled for more than $300 \mathrm{~m}$. East of Caliente, one large crack, about $1.5 \mathrm{~m}$ at its widest point and more than $60 \mathrm{~cm}$ deep, was observed. Fill areas in the mountainous region along U.S. Highway 466 (now State Highway 58) settled from a few centimeters to more than $30 \mathrm{~cm}$ in places, and a large part of the highway was cracked and wrinkled. Northeast of that highway, the ground was displaced vertically about $60 \mathrm{~cm}$ and horizontally about $45 \mathrm{~cm}$.

Maximum MM intensities in nearby cities did not exceed VIII. At Tehachapi, Bakersfield, and Arvin, old and poorly built masonry and adobe buildings were cracked, and some collapsed.

Property damage was heavy in Tehachapi, where both brick and adobe buildings were hit hard, and 9 people were killed. Three people were killed in other towns. Although damage was severe, the total extent of damage to property did not exceed that in Long Beach in 1933. Only a few wood-frame structures were damaged seriously in this earthquake, compared to the 1933 shock in which many such structures were thrown off foundations.

The generally moderate damage in Bakersfield was confined mainly to isolated parapet failure. Cracks formed in many brick buildings, and older school buildings were damaged somewhat. In contrast, however, the Kern General Hospital was damaged heavily. Multistory steel and concrete structures sustained minor damage, which commonly was confined to the first story. Similar kinds of damage also occurred at Arvin, which lies southeast of Bakersfield and west of Tehachapi.

Reports of long-period wave effects from the earthquake were widespread. Water splashed from swimming pools as far distant as the Los Angeles area, where damage to tall buildings was nonstructural but extensive. Water also splashed in pressure tanks on tops of buildings in San Francisco. At least one building was damaged in San Diego, and in Las Vegas, Nev., a building under construction required realignment of the structural steel.

The main shock was felt over most of California and in parts of western Arizona and western Nevada (see fig. 20). It was observed at such distant points as Stirling City, Calif., Phoenix, Ariz., and Gerlach, Nev. The California Institute of Technology at Pasadena recorded 188 aftershocks of magnitude 4.0 and higher through September 26, 1952; six aftershocks on July 21 were of magnitude 5.0 and higher. Magnitude 7.8 $\mathrm{M}_{\mathrm{S}} \mathrm{ABE}, 7.3 \mathrm{~m}_{\mathrm{b}} \mathrm{ABE}, 7.7 \mathrm{M}_{\mathrm{S}} \mathrm{CFR}, 7.7 \mathrm{M}_{\mathrm{S}}$ GR, 7.0 $\mathrm{M}_{\mathrm{La}}$ ELL, 7.21 $\mathrm{M}_{\mathrm{L}} \mathrm{KJ}$. (Ref. 25, 38, 292, $460,521,533,549$.) 


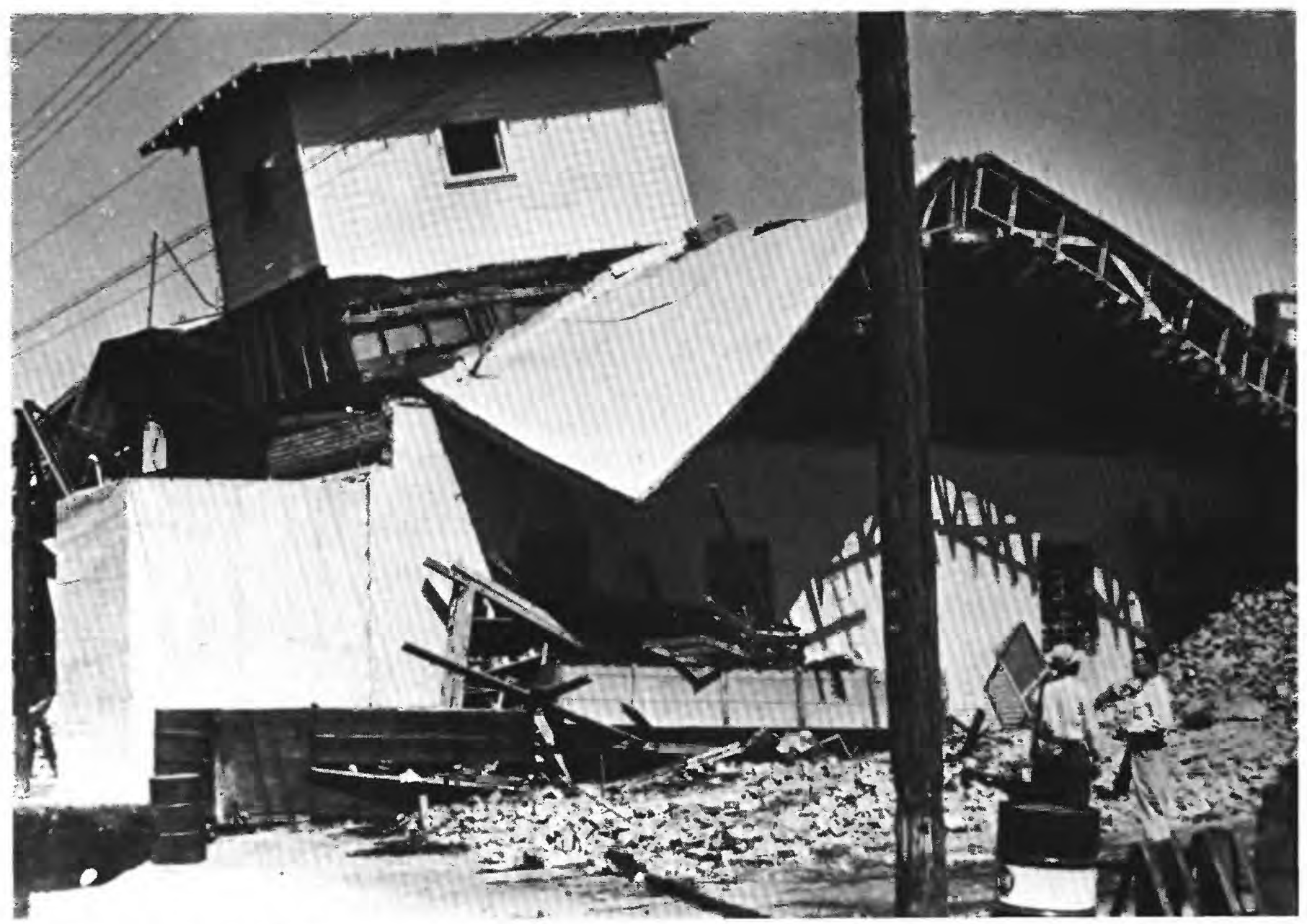

Building in Bakersfield, California, damaged by the Kern County earthquake of July 21, 1952. (Source of photograph unknown.)

1952. July 21, 1205 UTC. Kern County, Calif., aftershock. This earthquake was felt at Huasna. Magnitude 6.4 $\mathrm{M}_{\mathrm{S}}$ CFR. (Ref. 25, 292.)

1952. July 21, 1513 UTC. Kern County, Calif., aftershock. A strong aftershock (time reported as 0720 PST) caused additional damage to buildings in the Arvin area. (Ref. 25, 259, 292.)

1952. July 23, 0038 UTC (July 22). Kern County, Calif., aftershock. A large aftershock of the July 21 earthquake caused damage to an old brick building in Arvin that was damaged in the main shock. Slight damage also occurred south of Bakersfield and at Fresno. This shock was reported mainly in Kern County. Magnitude 6.1 $\mathrm{MS}_{\mathrm{S}}$ CFR. (Ref. 25, 259, 292.)

1952. July 23, 0753 (July 22), 1317 , and 18 13 UTC. Kern County, Calif., aftershocks. One house at Arvin that sustained only minor damage in the main event on July 21 was almost destroyed by the aftershock at 0753 UTC. Walls and fronts of buildings of weakened structures collapsed; gas and water mains were broken; and transformers were torn off. The second aftershock also caused serious damage to already weakened buildings at Arvin and Tehachapi, and the third caused only minor damage at Arvin. These aftershocks were reported only at a few towns in Kern County. (Ref. 25, 259, 292.)

1952. July 25, 1909 and 1943 UTC. Kern County, Calif., aftershocks. Some pipeline damage and changes in ground level occurred $8 \mathrm{~km}$ south of Bakersfield (at Fairfax) as a result of these strong aftershocks. Light damage to buildings was reported from several towns in the area. Ground cracks were enlarged in Tejon Canyon, $16 \mathrm{~km}$ southeast of Arvin. 


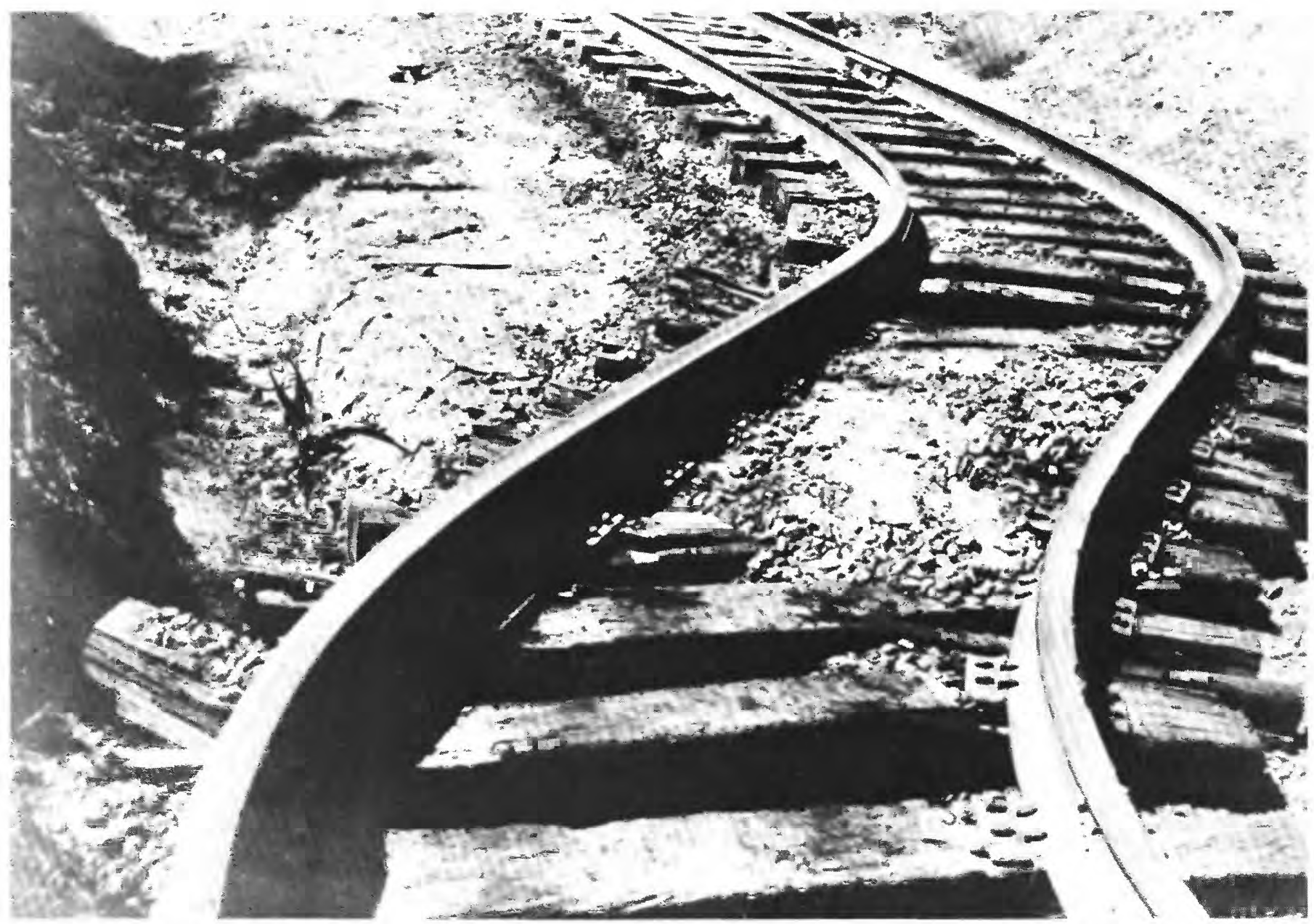

Railroad tracks near Bealville, California, bent and twisted by the July 21, 1952, earthquake.

(Photograph from the National Geophysical Data Center, NOAA.)

Landslides occurred in the Caliente Creek Canyon, Oiler Canyon Grade, State Highway 178 (between Bakersfield and Kernville), and White Wolf Ranch. Both shocks were felt over a wide area of south-central California. (Ref. 25, 259, 292.)

1952. July 29, 0703 UTC (July 28). Kern County, Calif., aftershock. Another strong aftershock of the July 21 earthquake caused severe damage to buildings that were damaged in the main earthquake. One store at Bakersfield sustained additional damage, including a collapsed parapet and a crumbled wall. Cracks formed in several large buildings, and bricks tumbled from the tops of previously damaged structures. At least 10 fires were ignited in the area. Moderate damage to chimneys and walls occurred at Edison. This aftershock was felt widely in south-central California. Magnitude 6.1 $\mathrm{M}_{\mathrm{S}}$ CFR. (Ref. 25, 259, 292.)

1952. July 31, 12 09 UTC. Kern County, Calif., aftershock. Another aftershock at Arvin jarred bricks loose and broke windows. Slight damage also occurred at Bakersfield and in the area of Taft. Felt over a small area of south-central California. (Ref. 25, 259, 292.)

1952. Aug. 13, 1739 UTC. Kern County, Calif., aftershock. The earthquake caused slight damage to brick at Arvin. The shock also was reported felt in Los Angeles. (Ref. 25, 259, 292.)

1952. Aug. 22. East of Bakersfield, Kern County, Calif. Heavy damage, estimated at $\$ 10$ million, occurred in the downtown area of Bakersfield. Two people were killed and several were injured.

Damage was confined mainly to brick buildings in a 64-block area of downtown Bakersfield. Larger, multistory, concrete and steel frame buildings sustained rather light damage. There were few complete collapses of buildings, but out of 396 structures that were damaged, 90 or more had to be razed. Many of these buildings had suffered considerable damage 


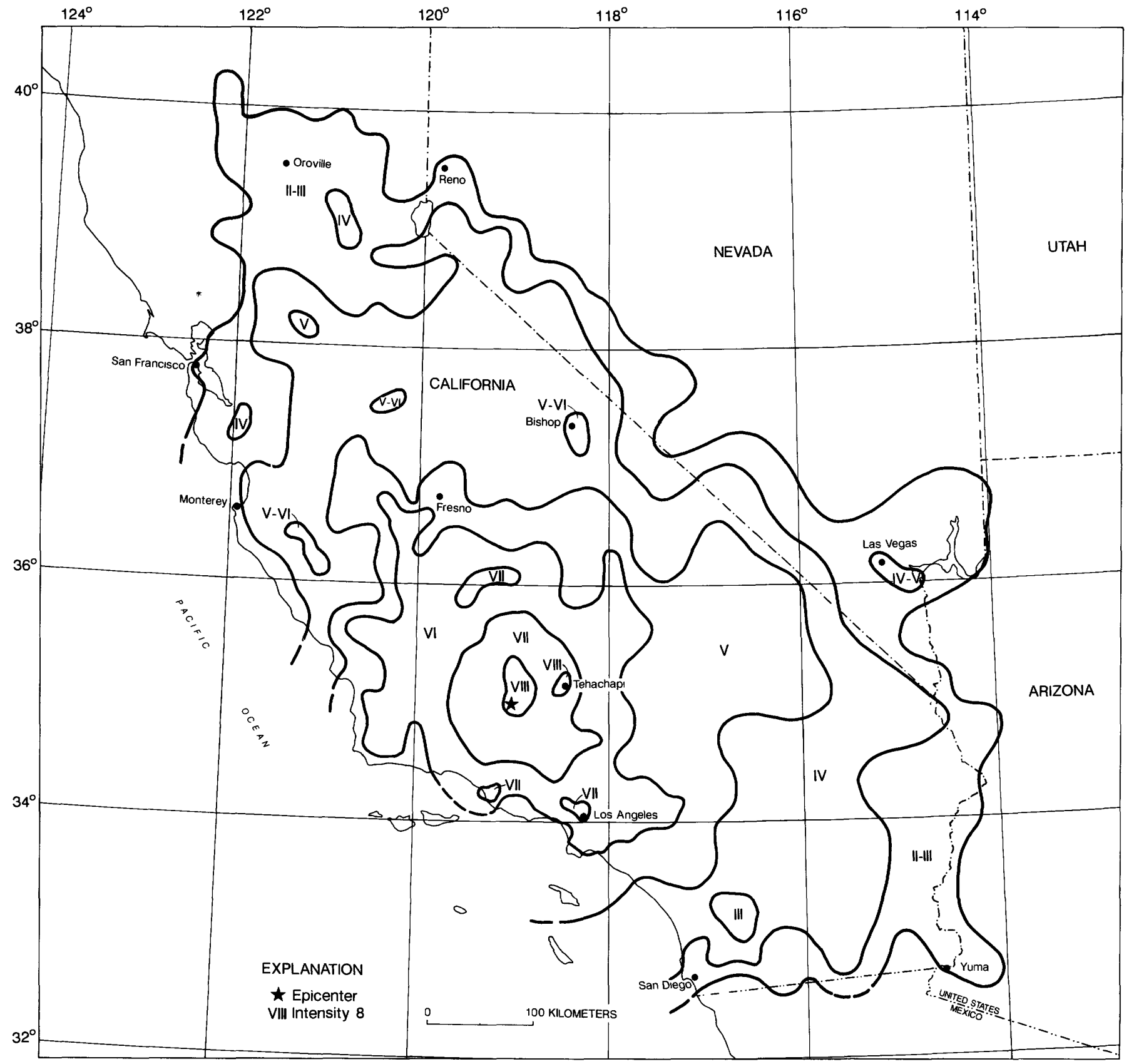

Figure 20.-Isoseismal map for the Kern County, California, earthquake of July 21, 1952. Isoseismals are based on intensity estimates from data listed in references 25,259 , and 549 of table 1.

from the earthquake on July 21,1952 , and the aftershocks. Outside the downtown area, chimneys twisted and fell, and plaster and walls cracked. Felt over a large part of south-central California-from Hollister south to Los Angeles and from the coast east to the Nevada border. (Ref. 25, 292, 549.)

1952. Aug. 23. Near Acton, Los Angeles, County, Calif. Southwest of the epicenter, slight damage was reported in Ventura County at
Camarillo, Moorpark, and Oxnard. Chimneys were cracked at Moorpark, and a water tower was damaged at Amboy (San Bernardino County). The higher intensities reported north into Kern County and at Amboy (northeast of San Bernardino) may be due to confusion with other shocks on Aug. 22. (Ref. $25,259,292$.)

1952. Sept. 22. Southwest of Petrolia, Humboldt County, Calif. At Petrolia, some houses were 
displaced as much as $5 \mathrm{~cm}$ on their foundations. Several chimneys fell and many were others were damaged. An oil well casing cracked at the Petrolia Oil Well Company, and a bunkhouse was displaced from its foundation. This shock generally was felt from the Eureka area on the north, south along the coast to Elk (Mendocino County), and east from the coast to the Ruth area (Trinity County). (Ref. 25, 259,324 .)

1952. Nov. 22 (Nov, 21). Near Bryson, Monterey County, Calif. Chimneys twisted and fell in the Bradley area and at San Luis Obispo; five or more old chimneys were knocked down at Bryson. Cracks formed in walls of buildings and in the ground in the Bryson area. Felt along the coast from San Francisco on the north to the Los Angeles area on the south and as far east as Olancha (Tulare County). Magnitude 6.0 $\mathrm{M}_{\mathrm{S}} \mathrm{CFR}, 6.0 \mathrm{M}_{\mathrm{S}} \mathrm{GR}$. (Ref. 25, 259, 476.)

1953. May 25, 0407 UTC (May 24). Near Calpella, Mendocino County, Calif. This earthquake reportedly cracked walls and twisted doors out of line at Calpella. Felt only at a few other towns in the area. (Ref. 26, 259, 324.)

1953. June 14, 0417 UTC (June 13). Brawley area, Imperial County, Calif. Damage was considerable near Brawley, where chimneys cracked and fell. At the Thistle Lateral Canal $(5 \mathrm{~km}$ south of Westmorland), the shock damaged one of the canal structures and cracked several meters of bank along the canal. The ground settled extensively at the Tokay Canal. Felt north to the Palm Springs area (Riverside County), west to San Diego, and southeast to Yuma, Ariz. Several aftershocks occurred. (Ref. 26, 259, 292.)

1954. Jan. 12. West of Wheeler Ridge, Kern County, Calif. The epicenter of this earthquake was close to that of the major shock of July 21, 1952. This shock caused minor damage at the Maricopa Seed Farm, about $25 \mathrm{~km}$ east of Maricopa. The damage included broken bracing rods in three steel-frame corrugated-iron buildings and a shattered base of one elevated water tank. Near the farm, unanchored platform pole transformers were toppled. Slight damage was reported from many towns in the area. The earthquake also was observed slightly in tall buildings in Sacramento, San Diego, and San Francisco. (Ref. 27, 259, 292.)

1954. Mar. 19, 0954 UTC. Santa Rosa Mountains, Riverside County, Calif. Minor damage reported at several towns in the area included fallen plaster from walls, broken windows and dishes, broken water pipes, cracked swimming pools, and damaged stock in stores. The main shock was felt over a large area of southern California and parts of western Arizona and southwest Nevada. Of the many aftershocks that were located, the largest occurred on Mar. 19 at 1021 UTC. Magnitude 6.2 $\mathrm{M}_{\mathrm{S}}$ CFR, 6.2 $\mathrm{M}_{\mathrm{La}}$ ELL (main shock). (Ref. 27, 259, 292, 521.)

1954. Apr. 22. East of Watsonville, Santa Cruz County, Calif. Plaster fell and walls cracked at Gilroy, and dishes broke and knickknacks fell at Aptos. Felt over a small area of the coastal region of west-central California. (Ref. 27, 259, 324.)

1954. Apr. 25. East of Watsonville, Santa Cruz County, Calif. Several poorly built houses, east of Watsonville along the Chittenden Road, were damaged severely when they were shifted on their foundations; ground cracks formed along the Pajaro River. Several chimneys toppled at Aromas and in the Interlaken District. The shock was strong enough to shatter a few windows and knock plaster from walls in the San Francisco Bay area. It was felt along the coast from Santa Rosa (Sonoma County) on the north to San Ardo (Monterey County) on the south and east as far as Fresno. (Ref. 27, 259, 324.)

1954. Aug. 26. Off the coast of Ventura County, near Anacapa Island, Calif. This slight earthquake shook plaster from the ceiling of the Ventura County Courthouse. Felt slightly at several towns in the area. (Ref. 27, 259, 292.)

1954. Nov. 10. Near Lakeport, Lake County, Calif. Slight damage in the form of cracks in windows, concrete, and plaster occurred at Nice, Potter Valley, Talmage, Willits, and Witter Springs. The earthquake was observed over a small area of Lake and Mendocino Counties. (Ref. 27, 259, 324.)

1954. Nov. 25. Off Cape Mendocino, Calif. The shock caused no damage but was felt over a large area of Humboldt and Mendocino Counties. Magnitude 6.5 MS CFR. (Ref. 27, 259, 324.)

1954. Dec. 17 (Dec. 16). East of San Leandro, Alameda County, Calif. Chimneys and a water main were broken at Oakland, and plaster fell. Cracks in plaster and walls were reported at Hayward and San Leandro. Felt over a moderate area of west-central California. (Ref. 27, 259, 324.)

1954. Dec. 21. Near Eureka-Arcata, Humboldt County, Calif. This earthquake and a strong aftershock on Dec. 30 caused property damage estimated at $\$ 2.1$ million. One person was killed when he fell into Humboldt Bay, and several people were injured by falling objects.

The shock caused only slight structural damage to reinforced concrete and concrete block and woodframe buildings in the Eureka-Arcata areas. The Eureka City Hall and the Humboldt County Courthouse in Eureka were cracked extensively. The main 
damage in both Arcata and Eureka was to chimneys, plaster, plate-glass windows, and merchandise in stores, but several old and poorly constructed brick walls bulged, and some parapet damage was sustained. Damage to structures and underground pipelines occurred in areas of unstable ground. Previous ground settling, as well as subsidence at the time of the shock, were observed in some of the damaged areas. Between Eureka and Arcata, U.S. Highway 101 was cracked and bulged in places.

Felt from southern Oregon on the north to San Francisco on the south and to Lake City (Modoc County) on the east. A strong aftershock occurred on Dec. 30. Magnitude 6.6 $\mathrm{M}_{\mathrm{S}} \mathrm{CFR}$ (main shock). (Ref. $27,259,480,533,550$.)

1954. Dec. 30. Near Eureka-Arcata, Humboldt County, Calif. An aftershock of the Dec. 21 earthquake broke plate-glass windows, cracked chimneys, and further damaged the Eureka water-supply pipeline. (Ref. 27, 259, 324, 550.)

1955. Mar. 2. Near San Ardo, Monterey County, Calif. About $1.5 \mathrm{~km}$ west of San Lucas, a brick chimney collapsed on a house. Plaster was cracked at Adelaida, Indian Valley (northeast of San Miguel), and Templeton. Felt north along the coast to Pescadero (San Mateo County), south to Lompoc (Santa Barbara County), and east to Avenal (Kings County). (Ref. 28, 259, 324.)

1955. Apr. 29. Near Lower Lake, Lake County, Calif. Chimneys, windows, and dishes were broken, and plaster and walls were cracked at Lower Lake. Felt mainly in Lake County over a small area. (Ref. 28, 324.)

1955. May 7. Near Lower Lake, Lake County, Calif. A few weak chimneys on older houses fell at Lower Lake, and plaster cracked and fell. Minor damage also was sustained at Clearlake Highlandswalls, ceilings, and floors cracked; a chimney was damaged; and much plaster fell. Felt mainly in Lake County over a small area. Several slight aftershocks were reported. (Ref. 28, 259, 324.)

1955. Sept. 5 (Sept. 4). East of San Jose, Santa Clara County, Calif. This earthquake caused extensive minor damage, estimated at $\$ 100,000$ in the San Jose area. The damage consisted mainly of toppled chimneys and broken plateglass windows. Damage was most severe in the Willow Glen District of San Jose, about $13 \mathrm{~km}$ west of the epicenter, where about 100 chimneys were damaged and many collapsed. One house was displaced on its foundation. Walls were cracked at the County Hospital and County Jail, and a brick garden wall toppled. Felt north to Santa Rosa (Sonoma County), south to San Ardo (Monterey County), and east to the La Grange area (Stanislaus County). (Ref. 28, 259,324 .)

1955. Oct. 24 (Oct. 23). Concord-Walnut Creek area, Contra Costa County, Calif. This earthquake killed one person and caused property damage estimated at $\$ 1$ million. The damage generally was minor, however, consisting mainly of cracked walls and plaster, broken windows, and loss from damaged merchandise. Damage was most severe at Walnut Creek where walls cracked, 80 plate-glass windows were broken, and much damage occurred to store merchandise. Minor damage to brick chimneys (some toppled) and walls occurred at several other towns in the region. Felt over a moderate area of west-central California. Several aftershocks occurred. Magnitude 5.4 M $\mathrm{S}$ CFR. (Ref. 28, 259, $324,533$.

1955. Nov. 2. Near San Ardo, Monterey County, Calif. This earthquake cracked plaster at Bryson, King City, and San Miguel and broke dishes at San Ardo. Felt over a small area along the coastal region of west-central California. (Ref. 28, 259, 324.)

1955. Dec. 17, 0607 UTC (Dec. 16). Near Brawley, Imperial County, Calif. This main shock of a series caused minor damage at Brawley. The ceiling in a hardware store buckled; cracks formed in walls of buildings and existing cracks were enlarged; 12 water mains broke; street lights and about 20 plate-glass windows broke; and merchandise in stores was damaged. Slight damage also was reported at Calexico, El Centro, Holtville, and Imperial. Felt over a moderate area of southern California and western Arizona. About 81 earthquakes were felt in Brawley through 1151 UTC, Dec. 19. (Ref. 28, 259, 292, 564.)

1956. Jan. 3 (Jan. 2). Near Glen Ivy, Riverside County, Calif. Broken windows, dishes, and plaster were reported from several nearby towns. Rockslides were reported in the area of Glen Ivy, in Temescal Canyon. Felt along the coast of southern California. (Ref. 29, 259, 292.)

1956. Feb. 9. Baja California, Mexico. Near El Alamo, Mexico, a new fault line, $29 \mathrm{~km}$ long, was reported, and new springs formed along the fault line. Minor damage, consisting mainly of broken windows, cracked plaster, and damaged stock in stores, was reported from Yuma, Ariz., and from several towns in southern California. Felt over a large area of southern California and western Arizona. Many aftershocks occurred through Apr. 26, 1956. (Ref. 29, 38, 259, 292.)

1956. Apr. 5 (Apr. 4). Near Saint Helena, Napa County, Calif. In the Angwin-Saint Helena Sanitarium area, residents reported many instances 
of cracks in plaster walls and concrete foundations. Tile flooring was cracked at the sanitarium. At Saint Helena, plaster fell from a ceiling and pine walls were cracked. Felt over a small part of the coastal area of north-central California. (Ref. 29, 259, 324.)

1956. Oct. 11, 1648 UTC. Off the coast of Humboldt County, Calif. With the exception of a few broken dishes at Eureka, no damage was reported. Felt over a small area of Humboldt County. Magnitude 6.0 $\mathrm{M}_{\mathrm{S}}$ CFR. (Ref. 29, 259, 324.)

1956. Nov. 16 (Nov. 15). Northwest of Parkfield, Monterey County, Calif. Plaster cracked at the Mee Ranch (Lonoak). The shock was reported felt in Fresno, Kings, Monterey, San Benito, San Luis Obispo, Santa Barbara, and Santa Cruz Counties. (Ref. 29, 259, 324.)

1957. Mar. 18. South of Oxnard, Ventura County, Calif. Minor damage at Oxnard, Port Hueneme, and Ventura consisted mainly of cracked walls, fallen plaster, and loss from breakage of stock in stores. Felt over a small area of southern California, mainly in Ventura County. (Ref. 30, 259, 324.)

1957. Mar. 22 and 23. West of Daly City, San Mateo County, Calif. These earthquakes caused property damage estimated at $\$ 1$ million and injured about 40 people. The first and strongest shock caused one death. Minor damage at several houses was reported along the ocean in the Westlake-Palisades tract, west of Daly City. Many chimneys were damaged at Daly City. In San Francisco, damage to chimneys, plaster, windows, and merchandise was widespread. The pavement along the edge of Lake Merced sloughed off into the lake, and both ends of a pedestrian bridge collapsed. Landslides blocked State Highway 1 near Mussel Rock, and the shoulder of the highway was cracked extensively.

The main shock was felt over a moderate area of west-central California, and many aftershocks were observed. The shock on Mar. 23 caused slight damage at Menlo Park and San Francisco. Magnitude 5.3 $\mathrm{M}_{\mathrm{S}}$ CFR, 5.27 $\mathrm{M}_{\mathrm{L}} \mathrm{KJ}$ (both Mar. 22). (Ref. 30, 259, 324, 460, 533, 599.)

1957. Apr. 25, 2157 UTC. Southwest end of Salton Sea, Imperial County, Calif. The most severe effects of this earthquake occurred in the Calipatria area, about $2.5 \mathrm{~km}$ north of Vail Canal, where a strip of land about $0.6 \mathrm{~km}$ wide and $1.5 \mathrm{~km}$ long was broken and cracked; water seeped from hundreds of blowholes that formed. Only slight damage was reported at other towns in the area. The main shock was felt over much of southern California and western Arizona. Many aftershocks occurred. (Ref. $30,259,292$.)
1958. Sept. 21 (Sept. 20). Southeast of Soledad, in San Benito County, Calif. This local earthquake cracked a fireplace and broke dishes in the San Benito area. A landslide and damage to two other houses were reported in the area also. (Ref. 31,324 .)

1958. Oct. 1. Southeast of Sierraville, Sierra County, Calif. This earthquake cracked chimneys at Hallelujah Junction (about $30 \mathrm{~km}$ northwest of Reno, Nev.) and broke dishes at Reno. Felt over a small area of northeast California and western Nevada. (Ref. 31, 259, 324.)

1958. Dec. 1 (Nov. 30). Baja California, Mexico. A series of moderate earthquakes in Mexico caused minor damage in California at Calexico, Nestor, Potrero, and Seeley. The damage included fallen plaster from walls and slight cracks in chimneys, walls, and windows. The main shock at 0321 UTC was felt over much of southern California and in parts of western Arizona. (Ref. 31, 259, 292.)

1958. Dec. 11. Southwest of San Francisco, Calif. Cracked plaster and windows, broken dishes, and damaged stock in stores were reported from several Bay area towns. Near Daly City, a minor landslide occurred on State Highway 1. Felt over a small area of west-central California. (Ref. 31, 259, 324.)

1959. Mar. 2. Near Gilroy, Santa Clara County, Calif. Residents of several towns near Gilroy reported slight damage, including broken windows, cracked plaster, and loss from fallen stock. One minor earthslide occurred east of Watsonville, and boulders rolled onto a highway between Gilroy and Watsonville. In San Francisco, 70 windowpanes were broken in one store. Felt along the coastal area from Jenner on the north to Big Sur on the south and inland to Gustine. (Ref. 32, 259, 324.)

1959. Apr. 1. Northeast of Loyalton, Sierra County, Calif. Most of the serious property damage occurred at Loyalton, where several chimneys fell, windows were broken, and plaster and walls were cracked. Felt over a large area of northeast California and western Nevada. (Ref. 32, 259, 324.)

1959. Apr. 6 (Apr. 5). North of Ukiah, Mendocino County, Calif. Chimneys cracked and windows broke at Redwood Valley. Felt in Lake and Mendocino Counties in northwest California. (Ref. $32,259,324$.)

1959. May 26. Near Salinas, Monterey County, Calif. Windows and dishes were broken at Salinas, and a wall was cracked. Slight damage also occurred at Camp McCallum and Hollister. Felt most strongly in the area around Monterey Bay. (Ref. 32, 259, 324.) 
1959. Oct. 24. Near Owens Peak, Kern County, Calif. A series of four earthquakes occurred in the Owens Peak area, the first of which was the strongest. Two hunters in the area reported that boulders tumbled down the mountainside and that a landslide occurred. Tops of dead trees were snapped off. Several shocks also were reported by residents of nearby Onyx. (Ref. 32, 259, 292.)

1959. Dec. 29 (Dec. 28). Northwest of Hollister, San Benito County, Calif. A few chimneys toppled at Hollister, and a large piece of timber fell from the roof of the City Hall building. Felt over the coastal area of west-central California from San Francisco south to San Miguel (San Luis Obispo County). (Ref. 32, 259, 324.)

1960. Jan. 20 (Jan. 19). South of Hollister, San Benito County, Calif. Minor damage occurred south of Hollister at a winery and a nearby ranch. At the winery, small bits of a ceiling fell, a chimney cracked at its roofline, cracks formed in pavement, and an underground pipeline broke. At the ranch, walls were cracked and an existing crack in a reservoir was enlarged. Felt along the coastal areas north of San Luis Obispo to San Francisco and inland to Merced. (Ref. 33, 259, 324.)

1960. June 6 (June 5). Off the coast of Humboldt County, Calif. Slight damage occurred at Eureka, where plaster fell in the old City Hall. At Crannell, north of Eureka, a brick chimney on one house was twisted slightly. Felt mainly in Humboldt and Trinity Counties. (Ref. 33, 259, 480.)

1961. Jan. 28. Near Walker Pass, Kern County, Calif. Slight damage was reported at Johannesburg, where the floor of a carport was cracked, and at Bodfish, where dishes were broken. At Kernville, rocks rolled down the mountainside. Felt over a large area, mainly in Kern and Tulare Counties. Many aftershocks were recorded. (Ref. 34, 259, 292.)

1961. Apr. 9, 0723 and 0725 UTC (Apr. 8). South of Hollister, San Benito County, Calif. Two strong earthquakes damaged many buildings at Hollister, but major damage was confined to the County Courthouse, the Dabo Hotel, and the Elks building. Property damage was estimated at $\$ 250,000$. South of Hollister, on Cienega Road, a 15$\mathrm{m}$-long fissure occurred near a winery, which also sustained severe damage. Some chimneys in the area were damaged or fell, and water lines were ruptured. Felt along the coast of west-central California from the San Francisco Bay area south to Creston (San Luis Obispo County). (Ref. 34, 38, 259, 324.)

1961. Oct. 19 (Oct. 18). East of Brown, Kern County, Calif. This shock shifted heavy machinery and changed the water level in one well near Brown. The only damage was reported from Mojave, where slight cracks formed in a bathroom. The earthquake was reported in a few scattered areas of northern Los Angeles and San Bernardino Counties in south-central California. (Ref. 34, 259, 292.)

1961. Oct. 20. Near Huntington Beach, Orange County, Calif. This earthquake was one of a series of nine sharp tremors, that were felt mainly in Orange County. The main shock caused slight damage, including broken windows, cracked plaster and walls, and damaged stock in stores, in several towns in the County. (Ref. 34, 259, 292.)

1961. Nov. 15 (Nov. 14). Near Wheeler Ridge, Kern County, Calif. At Wilsona, in Antelope Valley, cracks formed in the walls of a building, the bottom of swimming pool, and in the ground. Damage was not reported from other towns in the area. This shock was felt mainly in Kern County, but also was observed in scattered areas of Kings, Los Angeles, Santa Barbara, Tulare, and Ventura Counties. (Ref. 34, 259, 292.)

1962. Apr. 27. Near Perris, Riverside County, Calif. Damage was slight at Romoland where windows and dishes were broken and at Winchester where cracks formed in walls, plaster, and windows. Felt over a small area of southern California, mainly in Riverside and San Bernardino Counties. (Ref. 35, 259, 292.)

1962. June 6. Near Lakeport, Lake County, Calif. In the area near Lakeport, water in Scott Creek (which was almost dry before the shock) rose about $0.5 \mathrm{~m}$ and flowed steadily for 11 days. The water in several wells in Scott Valley rose 2-3 m and turned milky white for 3-5 days. About $16 \mathrm{~km}$ southwest of Lakeport, a geyserlike spout of water was observed in Clear Lake. Slight damage, which occurred mainly in the Lakeport-Ukiah area, included fallen bricks from fireplaces and cracks in chimneys and walls. One chimney fell at Finley. Felt mainly in Lake and Mendocino Counties. (Ref. 35, 259, 324.)

1962. Aug. 23. Off the coast of Del Norte County, Calif. Slight damage occurred, mainly in the Crescent City-Smith River areas of Del Norte County. Damage consisted of broken windows, cracks in chimneys and walls, and loss from fallen stock in stores. The tops of some redwood trees were snapped off. Felt over a moderate area of northern California and southwest Oregon. (Ref. 35, 259, 480.)

1962. Sept. 4. Northwest of Arcata, Humboldt County, Calif. Plaster fell at the Eureka County Courthouse; new cracks formed in the walls of the old high school building; and windows broke at 
a drugstore. At Orick, plaster and walls were cracked and stock fell in stores. Felt over a small area of northern California, mainly in Humboldt County. (Ref. 35, 259, 480.)

1962. Oct. 29 (Oct. 28). Near Big Bear, San Bernardino County, Calif. A window was broken and stock fell from shelves at Big Bear City, and a water main was broken at Redlands. Rocks rolled onto the highway between Bear Valley and Lucerne Valley. Felt mainly in Riverside and San Bernardino Counties. Many aftershocks were recorded. (Ref. 35, 259, 292.)

1963. May 22. West of Sunnyvale, in Santa Cruz County, Calif. One roof reportedly caved in at Sunnyvale. Felt over a small part of the coastal area of west-central California. (Ref. 36, 259, 324.)

1963. May 23, 1553 UTC. Imperial Valley, Calif. The main shock of a series on May 22-23 caused minor damage at Brawley (plate-glass window broke; plaster cracked) and Westmorland (plaster fell; windows and dishes broke). Felt mainly in the Imperial Valley of southern California. (Ref. 36, 259,292 .)

1963. June 7. West of Antioch, Contra Costa County, Calif. Slight damage occurred at Antioch, where cracks formed in the walls and foundation of a school, old cracks were enlarged, and beams and doors "twisted." Plaster was cracked at Clayton, Concord, and Cowell. Felt in the San Francisco Bay area, mainly in Contra Costa County. (Ref. 36, 259, 324.)

1963. Sept. 14, 1946 UTC. Near Watsonville, Monterey County, Calif. The earthquake caused minor damage east of Watsonville in the Chittenden-Soda Lake area. In this small area along the San Andreas fault, the second floor of a wood-frame house was displaced; a water tank shifted on its foundation; one stone chimney was shaken loose, and one was cracked; and footings of a bridge across the Pajaro River were damaged slightly. Landslides were reported in the Soda Lake and Pajaro Gap areas. Felt along the coastal area of west-central California from Bolinas to Big Sur. (Ref. 36, 259, 467.)

1963. Sept. 23. Near San Jacinto, Riverside County, Calif. Chimneys were cracked and twisted at Hemet. Plaster cracked and fell at Hemet and San Jacinto, and windows and dishes were broken. Felt over a large part of southern California-from Los Angeles east to Twentynine Palms (San Bernardino County) and south to San Diego. (Ref. 36, 259, 292.)

1963. Dec. 6. Near Toms Place, Mono County, Calif. Plaster was cracked at Bishop and in the Paradise area northwest of Bishop. Also, a 230-kV-transformer bushing was cracked at Bishop. Felt over a moderate area of east-central California and western Nevada. (Ref. 36, 259, 292.)

1964. June 21. California-Mexico border region. Plaster fell from a 2 -m-long crack in the ceiling of a building at San Diego, and new cracks formed in the beams at the post office. Slight damage also was reported at Coronado. Felt over a small area of southwest California. (Ref. 37, 259, 292.)

1964. Nov. 16 (Nov. 15). Near Corralitos, Santa Clara County, Calif. The most serious damage to property occurred in Corralitos. Several chimneys were toppled there, other chimneys were twisted, and water service was disrupted. Minor damage occurred at several towns in the Corralitos area. Felt along the coast of west-central California from Napa on the north to San Simeon (San Luis Obispo County) on the south. Magnitude $4.5 \mathrm{~m}_{\mathrm{b}}$ NUT, 4.3 $\mathrm{M}_{\mathrm{S}}$ NUT. (Ref. 37, 259, 263, 324.)

1964. Dec. 22. West of Ensenada, Mexico. The San Diego area sustained the most severe damage, which included fallen stones from a chimney, exterior crack in a fire station wall, broken windows, and fallen light fixtures. Slight damage also occurred at Boulevard, Imperial Beach, La Mesa, and Potrero. Felt along the coast from Los Angeles to Tijuana (Mexico) and inland to Calipatria (Imperial County). Magnitude $5.0 \mathrm{~m}_{\mathrm{b}}$ NUT, $5.5 \mathrm{M}_{\mathrm{S}}$ NUT. (Ref. $37,259,263,292$.)

1965. Jan. 1. Southwest of Fontana, San Bernardino County, Calif. Glassware and dishes were broken at Fontana, and the ceiling of one house was shattered. A cement sidewalk was cracked at Lytle Creek, northwest of San Bernardino. Felt over a small area, mainly in Riverside and San Bernardino Counties. (Ref. 75, 259, 292.)

1965. Apr. 15. San Bernardino Valley, Calif. The press reported broken windows and merchandise shaken from shelves throughout the San Bernardino Valley, including Chino, Colton, Fontana, Rialto, and San Bernardino. Felt in Los Angeles, Orange, Riverside, and San Bernardino Counties. (Ref. 75, 259, 292.)

1965. June 16 (June 15). Imperial Valley, Calif. This was the main shock of a series that occurred in the Imperial Valley June 15-17. Slight damage was sustained at Westmorland, where plateglass windows and dishes were broken, and at Imperial, where walls were cracked. Felt over a small area of Imperial County. (Ref. 75, 259, 292.)

1965. July 16 (July 15). Near Saugus, Los Angeles County, Calif. Stucco was cracked in several places in a house at Saugus, and one existing crack in cement was enlarged. Felt mainly in Kern and Los Angeles Counties. (Ref. 75, 259, 292.) 
1965. Sept. 10. Near Pittsburg, Contra Costa County, Calif. Windows were broken in two houses at Concord, and in two stores at Pittsburg. At Cowell, chimneys were cracked and dishes were broken. Small rockslides were observed in Mount Diablo State Park. Felt over a small part of the San Francisco Bay region, mainly in Contra Costa County. (Ref. 75, 259, 324.)

1965. Sept. 25, 1743 UTC. Southeast of Newberry, San Bernardino County, Calif. The main shock of a series of three events on Sept. 25 and 26 first increased and then decreased the flow of a spring at the Camp Cady Ranch, south of Manix Station. Gas pipes were damaged at Newberry, causing an explosion; an underground water tank was cracked at Hodge; and plaster cracked and fell at Kelso. The main shock was felt over a large area of southern California and at a few towns in southern Nevada and southwest Arizona. Two strong aftershocks were felt in the area at 1748 UTC on Sept. 25 and 0700 UTC on Sept. 26. Magnitude $4.7 \mathrm{~m}_{\mathrm{b}}$ NUT, 4.4 $\mathrm{M}_{\mathrm{S}}$ NUT. (Ref. 75, 259, 263, 292.)

1965. Oct. 17. Near Palm Springs, Riverside County, Calif. At Palm Springs, windows cracked and dishes broke, and at Cathedral City, a garden wall cracked. Felt mainly in Riverside and San Bernardino Counties. (Ref. 75, 259, 292.)

1965. Nov. 12. South of Glendale, Los Angeles County, Calif. Minor cracks formed in concrete on the 30th floor of the Occidental Center in Los Angeles, and plaster was cracked slightly in the Federal Building in Glendale. Felt only in a small area of southwest Los Angeles County. (Ref. 75, 259, 292.)

1966. May 24 (May 23). Near Chico, Butte County, Calif. This light shock generated a rockslide at Las Plumas, near Oroville, and cracked plaster northeast of Chico, at Forest Ranch. Felt over a moderate area in east-central California. Magnitude $3.8 \mathrm{~m}_{\mathrm{b}}$ NUT, $4.0 \mathrm{M}_{\mathrm{S}}$ NUT. (Ref. $81,259,263,324$. )

1966. June 28, 0426 UTC (June 27). Near Parkfield, Monterey County, Calif. This earthquake occurred in a sparsely populated region near Parkfield, so little building damage was sustained. The main damage included broken windows, cracked walls and swimming pools, and overturned tombstones in the Parkfield cemeteries.

Minor surface faulting, about $35 \mathrm{~km}$ long, occurred in a narrow zone along the San Andreas fault-from a few kilometers northwest of Parkfield almost to Cholame. About $1.5 \mathrm{~km}$ northeast of Cholame, the white dividing line on Highway 466 was offset about $10 \mathrm{~cm}$. At that same site, a small concrete bridge sustained minor cracks and the pavement buckled. Bridges on the Parkfield-Cholame Highway, which parallels and crosses the fault trace several times, sustained minor damage. It was felt generally from Santa Cruz to Oxnard and northeastward into the Sierra Nevada foothills. It was preceded by a strong shock at 0408 UTC on June 28 and followed by shocks of lower magnitude on June 28 and 29. More than 200 aftershocks occurred through December 1966. Magnitude 6.0 $\mathrm{M}_{\mathrm{S}}$ ELL, $5.7 \mathrm{M}_{\mathrm{La}}$ ELL, $5.91 \mathrm{M}_{\mathrm{L}}$ KJ. (Ref. 81, 259, 398, 521, 551.)

1966. Aug. 7. Gulf of California, Sonora, Mexico. This earthquake cracked the ground $48 \mathrm{~km}$ south of San Luis and Rio Colorado in the El Golfo de Santa Clara area of Mexico. Damage in the United States was most severe at Yuma, Ariz., where a sidewalk sagged about $10 \mathrm{~cm}$ and the facades of several buildings sustained cracks. Slight damage also occurred at Blythe, Holtville, and Winterhaven, Calif., and at Picacho and Somerton, Ariz. The earthquake was felt widely in southern California and southwest Arizona and also was reported felt at Boulder City, Nev. (Ref. 38, 81, 259, 266.)

1966. Sept. 12, 1641 UTC. Near Boca, Nevada County, Calif. Minor but extensive fractures in the ground were observed in the area northeast of Truckee, extending $16 \mathrm{~km}$ on a trend N. $30^{\circ}$ E. from Prosser Reservoir to Hoke Valley. Chimneys toppled and masonry walls were cracked at Boca, Hirschdale (near Boca Dam), Hobart Mills (near Prosser Dam), Loyalton, and Sierraville. Pipelines were ruptured at Loyalton and Boca Dam. Several bridges on Highway 80 sustained minor damage, and Boca and Prosser earthfill dams were cracked. Landslides, rockslides, and slumping occurred on area highways and on the Southern Pacific Railway roadbed. This was the main shock of a series occurring near Boca. It was felt over a large area of east-central California and northwest Nevada. Magnitude $5.2 \mathrm{~m}_{\mathrm{b}}$ NUT, 5.6 $\mathrm{M}_{\mathrm{S}}$ NUT. (Ref. 81, 259, 263, 324, $457,555$.

1966. Oct. 2 (Oct. 1). Palos Verdes Peninsula, Los Angeles County, Calif. On Palos Verdes Peninsula, two windows were broken, the curb and street were split, and the ground pulled away from the courtyard wall. Felt mainly in southwest Los Angeles County and western Orange County. (Ref. $81,259,292$.)

1967. May 21. Near Anza, Riverside County, Calif. The shock rolled rocks onto Highway 74 from Nightingale to Palm Desert. A water reservoir at Norco sustained about $\$ 40,000$ damage. Near Anza, well water appeared murky later in the day. Although this earthquake was felt mainly in Riverside, San Bernardino, and San Diego Counties, it 
was reported in scattered towns in Los Angeles and Orange Counties. (Ref. 40, 72, 292.)

1967. June 15 (June 14). Near Whittier, Los Angeles County, Calif. At Whittier, a plate-glass window broke at a market, a water pipe broke in an office building, and a wall cracked in a garage. Hairline cracks formed in a house foundation at San Gabriel, and plaster fell from a house in north Los Angeles. Felt mainly in the Whittier and San Gabriel Valley areas of Los Angeles County. (Ref. 40, 72,292 .)

1967. June 26. Near Redwood Valley, Mendocino County, Calif. Brick chimneys and dishes were damaged at Ukiah, and chimneys and walls were cracked in Redwood Valley, about $11 \mathrm{~km}$ north of Ukiah. At Coyote Dam, northeast of Ukiah, concrete spalled slightly on the curb of the intake tower access bridge. Felt over a small area of Lake and Mendocino Counties. (Ref. 40, 72, 324.)

1967. July 22. Near Paicines, San Benito County, Calif. At the Bear Valley Fire Control Station at Paicines, bricks and plaster were cracked and furniture was displaced. Felt only at a few towns in San Benito County and at Coalinga in Fresno County. (Ref. 40, 72, 324.)

1967. Sept. 7. Near Corralitos, Santa Cruz County, Calif. The only damage reported was at San Jose, about $40 \mathrm{~km}$ north of the epicenter, where cracks in a cement fence and in plaster were enlarged. Felt from Point Reyes Station (Marin County) south to King City (Monterey County). Magnitude $4.0 \mathrm{M}_{\mathrm{b}}$ NUT, $4.1 \mathrm{M}_{\mathrm{S}}$ NUT (Ref. 40, 72, 263,324 .)

1967. Sept. 28. Northeast of Morgan Hill, Santa Clara County, Calif. Rockslides were observed within $5 \mathrm{~km}$ of the epicenter. At San Jose, exterior walls of a house were cracked. Felt from Marin County south to Monterey County and east to Merced County. Magnitude $4.3 \mathrm{mb}_{\mathrm{b}}$ NUT, $4.1 \mathrm{M}_{\mathrm{S}}$ NUT. (Ref. 40, 72, 263, 324.)

1967. Dec. 10, 1206 UTC. Off the coast of Humboldt County, Calif. Minor damage occurred at several towns, including Crannell (chimney twisted), Denny (brick chimney cracked), Ferndale (windows cracked), and Scotia (plate-glass window cracked). The first shock was felt mainly in Humboldt County. Two aftershocks were observed, the strongest at 1233 UTC. Magnitude $5.6 \mathrm{~m}_{\mathrm{b}}$ NUT, 5.5 $\mathrm{M}_{\mathrm{S}}$ NUT. (Ref. $40,72,74,263$.)

1967. Dec. 18. Near Corralitos, Santa Cruz County, Calif. Minor damage occurred mainly in the area of Corralitos, Gilroy, and Watsonville. It included cracks in chimneys and plaster, broken windows, and damage to fallen stock in stores. Felt from
Marin County south to Monterey County and east to Merced County. Magnitude $4.8 \mathrm{~m}_{\mathrm{b}}$ NUT, $4.7 \mathrm{M}_{\mathrm{S}}$ NUT. (Ref. 40, 72, 263, 324.)

1968. Apr. 9, 0228 and 0348 UTC (Apr. 8). Borrego Mountain earthquake, near Ocotillo Wells, San Diego County, Calif. Along the Coyote Creek fault, surface rupture $31 \mathrm{~km}$ in length was observed. Highway 78 sustained cracks adjacent to Ocotillo Wells. Rockslides occurred in Palm Canyon, Split Mountain, and Font's Head in the Anza-Borrego Desert State Park, and huge boulders blocked the Montezuma-Borrego Highway. The walls of one house at Ocotillo Wells were split over doorways and at corners of rooms, and the bedroom was separated from the rest of the house. The main shock at 0228 UTC was felt over a large area, including southern California, southwest Arizona, and southern Nevada (see fig. 21). Several aftershocks were reported. The largest one at 0348 UTC knocked plaster to the floor in a theater at Calexico. Magnitude $7.0 \mathrm{M}_{\mathrm{S}} \mathrm{ABE}, 6.6$ $\mathrm{m}_{\mathrm{b}} \mathrm{ABE}, 6.8 \mathrm{M}_{\mathrm{S}} \mathrm{ELL}, 6.3 \mathrm{M}_{\mathrm{La}}$ ELL, $6.0 \mathrm{~m}_{\mathrm{b}}$ NUT, 6.7 $\mathrm{M}_{\mathrm{S}} \mathrm{NUT}, 6.93 \mathrm{M}_{\mathrm{L}} \mathrm{KJ}$ (main shock). (Ref. 41, 263, $292,521,552,554$.)

1968. Apr. 25. Near Santa Rosa, Sonoma County, Calif. Damage in the Santa Rosa area was limited to broken chimneys, windows, plaster, and stock in stores. One chimney toppled in the Larkfield housing tract north of Santa Rosa, about 20 percent of the chimneys were damaged visibly, and many windows were broken. Considerable damage to plaster was reported in light wood-frame buildings. Several tombstones were rotated in the cemetery north of Santa Rosa. Felt over a small area of west-central California from Mendocino County on the north to San Mateo County on the south. Magnitude 4.6 $\mathrm{M}_{\mathrm{S}}$ NUT. (Ref. 41, 263, 324.)

1968. Apr. 29 (Apr. 28). South of Willows, Glenn County, Calif. Most of the damage from this shock was to merchandise in stores in the Chico area. One water well near Tehama went dry after the earthquake. At Chico, a sliding glass door and a glass trophy shelf were broken at a high school. Felt mainly in Butte, Glenn, and Tehama Counties in northern California. (Ref. 41, 324.)

1968. June 26, 0142 UTC (June 25). Near Petrolia, Humboldt County, Calif. Several chimneys were toppled in the Honeydew-Petrolia area, and much damage to plaster and windows was reported. In Mattole Valley, a porch on a building collapsed and several fireplaces were damaged. Landslides and cracks in the ground were observed near the mouth of the Mattole River. Felt mainly in Humboldt and Mendocino Counties in northern California. Several aftershocks were felt in the area. 


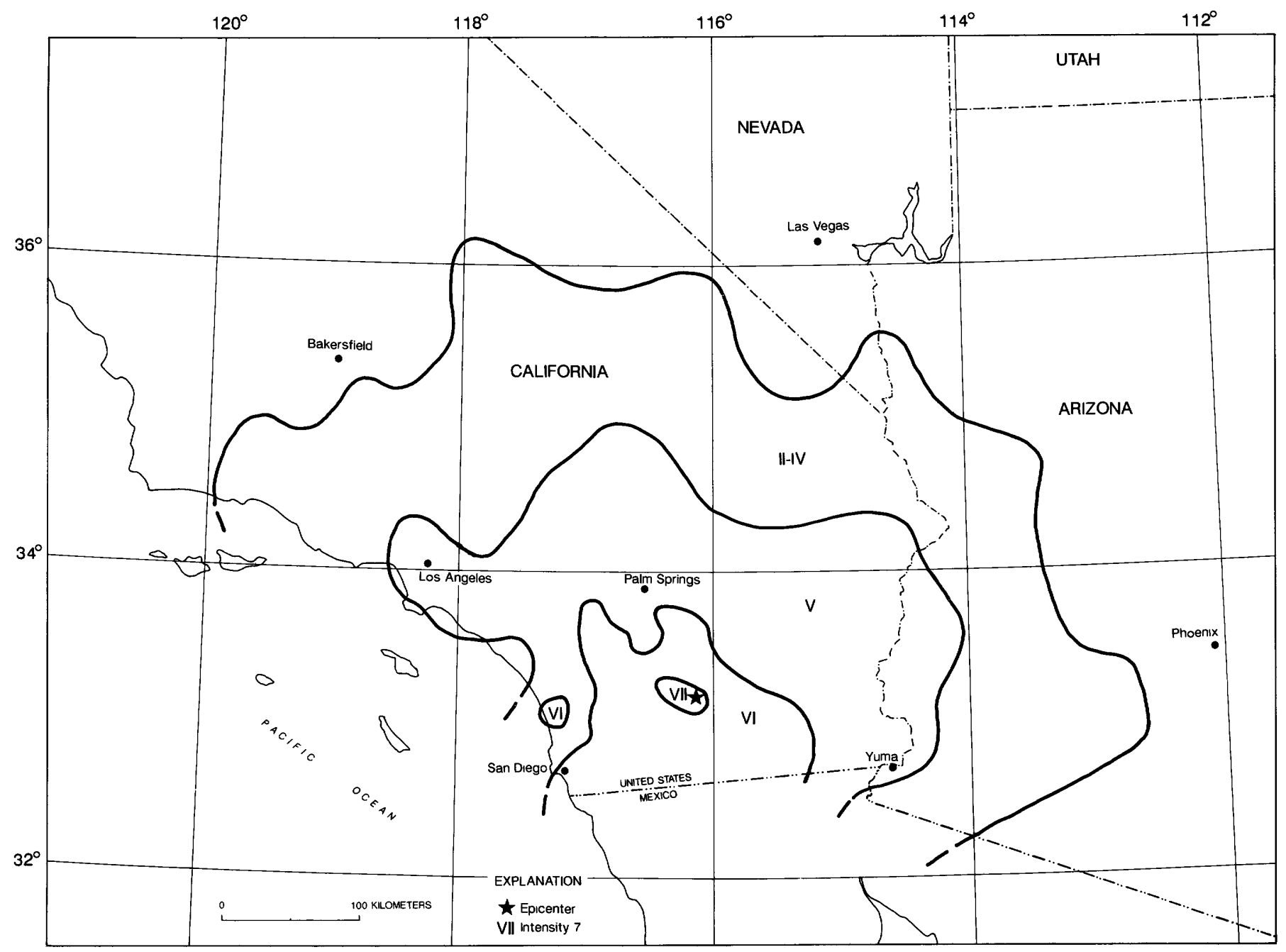

FIGURE 21.-Isoseismal map for the Borrego Mountain, California, earthquake of April 9, 1968. Isoseismals are based on intensity estimates from data listed in references 41 and 72 of table 1.

Magnitude 5.4 $\mathrm{M}_{\mathrm{S}}$ ELL, $4.9 \mathrm{~m}_{\mathrm{b}} \mathrm{NUT}, 5.6 \mathrm{M}_{\mathrm{S}}$ NUT. (Ref. 41, 263, 480, 521.)

1968. June 29. Off the coast of southern California in Santa Barbara Channel. At Goleta, a bridge at one overcrossing had spalled concrete chips and cracked tar and mortar. Much stock was damaged in the stores at Goleta. The only other town reporting the shock was Santa Barbara. Several light tremors were felt in the area from June 23 to July 31 , the strongest on July 5 at 0045 UTC (see next paragraph). (Ref. 41, 292.)

1968. July 5 (July 4). Off the coast of southern California in Santa Barbara Channel. Only minor damage was sustained at Carpinteria (light fixtures and plaster fell; one chimney cracked), Goleta (plate-glass windows broke; tile panels and lights fell), and Santa Barbara (tile panels fell). Felt over a moderate area of southwest California from
Santa Barbara and Kern Counties south to Orange County. Magnitude $5.2 \mathrm{~m}_{\mathrm{b}}$ NUT, $4.8 \mathrm{M}_{\mathrm{S}}$ NUT. (Ref. $41,72,263,292$.)

1969. Feb. 7. Off the coast of Humboldt County, Calif. Chimneys were twisted at Petrolia and landslides occurred. One tombstone toppled at Ferndale, and several small cracks formed in the walls of a school building. Slight damage to merchandise occurred in several stores in Rio Dell and Scotia. Felt over a small area of Humboldt, Mendocino, and Trinity Counties. More than 40 aftershocks were recorded. (Ref. 42, 72, 324.)

1969. Feb. 28 (Feb. 27). Near Palmdale, Los Angeles County, Calif. Slight damage occurred at Palmdale, where fluorescent lights fell and windows were broken. Felt mainly in the Palmdale area of northern Los Angeles County. (Ref. 42, 72, 292.) 
1969. Apr. 28. Near Borrego Springs, San Diego County, Calif. At Borrego Springs, large pieces of the ceiling fell at a bank, light fixtures were damaged, and brick walls were cracked. Several plate-glass windows were shattered in the town, and loss of merchandise in stores was common. Several rockslides occurred in the Santa Rosa Mountains, northeast of Borrego Springs. Felt over much of southern California and northern Baja California and at a few towns in southwest Arizona and southern Nevada. Magnitude 5.5 mb NUT, 5.1 $\mathrm{M}_{\mathrm{S}}$ NUT. (Ref. $42,72,263,292$.)

1969. June 7. Off the coast of Humboldt County, Calif. Near Capetown (between Ferndale and Petrolia), the top of a chimney fell, one chimney was cracked, and dishes were broken. The shock was felt only slightly at three other towns in Humboldt County. (Ref. 42, 72, 324.)

1969. Oct. 2, 0456 and 0619 UTC (Oct. 1). Near Santa Rosa, Sonoma County, Calif. These earthquakes caused one fatality and left severe property damage in Santa Rosa: several old brick and wood-frame buildings were damaged beyond repair; chimneys were destroyed; sidewalks buckled; and underground pipes ruptured. Other buildings in Santa Rosa that sustained substantial damage included Fremont Elementary School, Sonoma County Social Service Building, J.C. Penney Company store, and Veterans Memorial Building. Total damage was estimated at $\$ 8.35$ million.

Significant cracking or offset of roads was not observed, except for some settlement of fill at one freeway overpass. On the north side of Santa Rosa, about 36 transverse fractures were observed in the asphalt of Poppy Drive; and fresh, irregular cracks formed in the dry dirt roads in the Rural Cemetery near Poppy Drive. Many tombstones in cemeteries were thrown down or twisted. Both earthquakes were felt over a moderate area of west-central California-from Sonoma County east to Sacramento County and south to Santa Cruz County. Several aftershocks were felt in the Santa Rosa area. Magnitude $4.8 \mathrm{~m}_{\mathrm{b}} \mathrm{NUT}, 4.5 \mathrm{M}_{\mathrm{S}}$ NUT (first shock.) (Ref. 42, $72,263,399,599$.)

1969. Oct. 27, 1059 UTC. Near Hollister, San Benito County, Calif. Slight damage occurred at Hollister, where a wall joint opened slightly, plaster was cracked, and stock was damaged in stores. Felt over a small area of central California. (Ref. 42, 72, 324.)

1970. Jan. 3 (Jan. 2). Near Cupertino, Santa Clara County, Calif. At Cupertino, plaster broke and fell and bottles toppled from shelves in a store. This light shock was felt over a small area, mainly in the Cupertino region west of San Jose. (Ref. 43, $72,324$.

1970. June 12, 0330 UTC (June 11). Near Danville, Contra Costa County, Calif. This earthquake was the strongest of a series of shocks that occurred in the Danville area in May and June. One chimney fell in Danville, and a brick pillar partly crumbled at the Greenbrook Clubhouse. Also damaged in Danville were several outside brick facades, a fence, and several windows. Felt over a small area of the San Francisco Bay region, mainly in the Danville area. (Ref. 43, 72, 324.)

1970. June 12, 1603 UTC. Near Danville, Contra Costa County, Calif. At the Danville Post Office, a wooden upright support beam was damaged slightly and existing plaster cracks were enlarged. Much stock was damaged in local stores. Felt mainly in the area of Danville. (Ref. $43,72,324$.)

1970. Aug. 4 (Aug. 3). Off the coast of central California in Monterey Bay. Plaster cracked and fell at Monterey; a window broke; and a few telephone lines were knocked down. Damage was slight at Carmel Valley, where beams in one building twisted and cracked. Felt from the San Francisco Bay area south to Monterey County. Magnitude 4.4 $\mathrm{m}_{\mathrm{b}}$ NUT, $3.6 \mathrm{M}_{\mathrm{S}}$ NUT. (Ref. $43,72,263,324$. )

1970. Sept. 12. Lytle Creek area, San Bernardino County, Calif. This was the main shock of a series that centered northwest of San Bernardino near Lytle Creek. Chimneys and tombstones were twisted and overturned and cracks formed in the ground at Lytle Creek. Several roads in the area were blocked by rockslides. Felt over a large area of southern California. Magnitude 5.7 $\mathrm{M}_{\mathrm{L}} \mathrm{KJ}$. (Ref. 43, $72,292$.

1971. Feb. 9, 1400 UTC. North of San Fernando, Los Angeles County, Calif. This destructive earthquake occurred in a sparsely populated area of the San Gabriel Mountains, near San Fernando. It lasted about 60 seconds, and, in that brief span of time, took 65 lives, injured more than 2,000 , and caused property damage estimated at $\$ 505$ million.

The earthquake created a zone of discontinuous surface faulting, named the San Fernando fault zone, which partly follows the boundary between the San Gabriel Mountains and the San FernandoTujunga Valleys and partly transects the northern salient of the San Fernando Valley. This latter zone of tectonic ruptures was associated with some of the heaviest property damage sustained in the region. Within the entire length of the surface faulting, which extended roughly east-west for about $15 \mathrm{~km}$, the maximum vertical offset measured on a single 


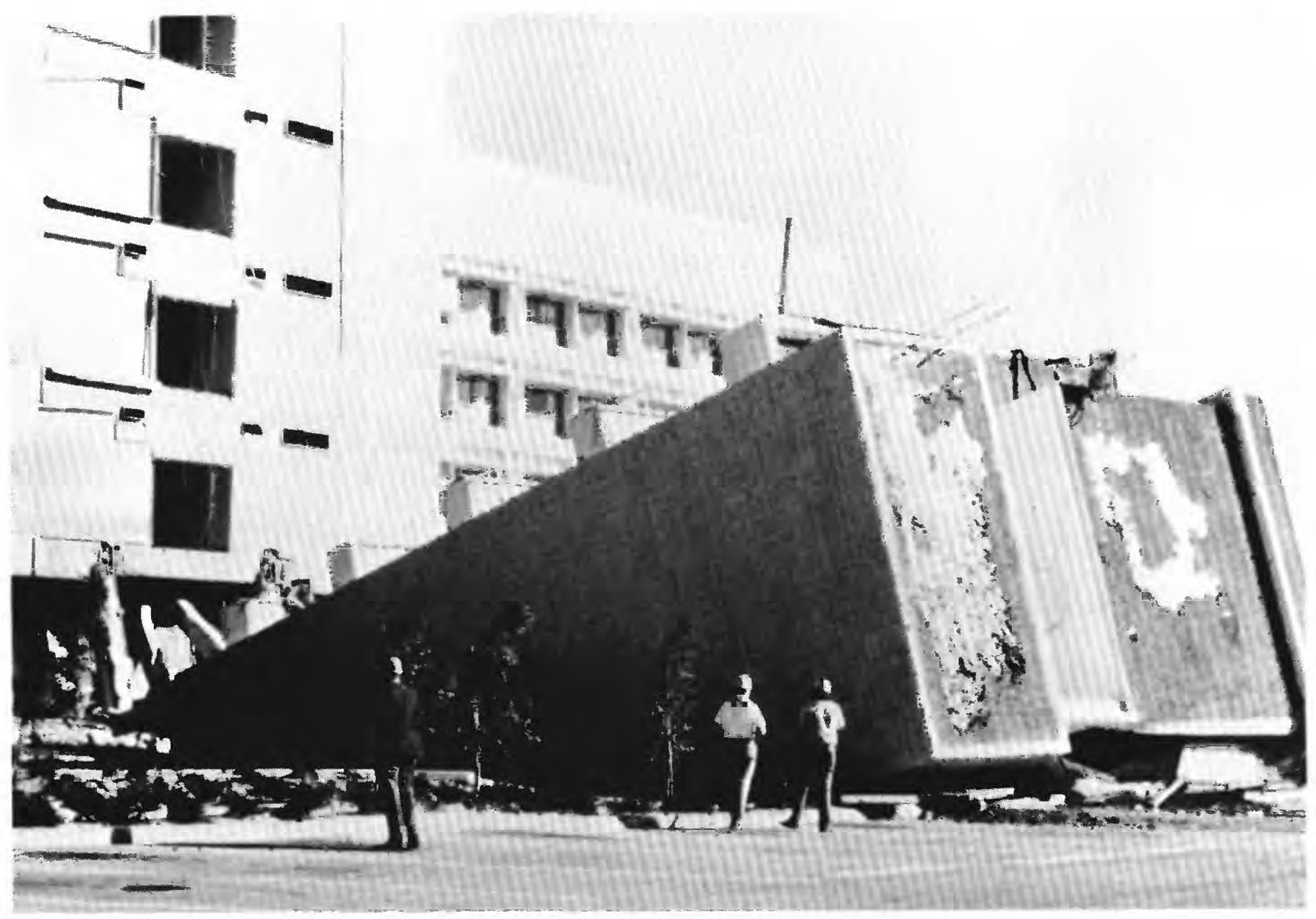

Collapsed stair tower at the west end of wing B, Olive View Hospital, Sylmar area, California, caused by the February 9,1971 , San Fernando earthquake. (Photograph from the National Geophysical Data Center, NOAA.)

scarp was about $1 \mathrm{~m}$, the maximum lateral offset about $1 \mathrm{~m}$, and the maximum shortening (thrust component) about $0.9 \mathrm{~m}$.

The most spectacular damage included the destruction of major structures at the Olive View and the Veterans Administration Hospitals and the collapse of freeway overpasses. The newly built, earthquakeresistant buildings at the Olive View Hospital in Sylmar were destroyed-four five-story wings pulled away from the main building and three stair towers toppled. Older, unreinforced masonry buildings collapsed at the Veterans Administration Hospital at San Fernando, killing 49 people. Many older buildings in the Alhambra, Beverly Hills, Burbank, and Glendale areas were damaged beyond repair, and thousands of chimneys were damaged in the region. Public utilities and facilities of all kinds were damaged, both above and below ground.

Severe ground fracturing and landslides were responsible for extensive damage in areas where faulting was not observed. The most damaging landslide occurred in the Upper Lake area of Van Norman Lakes, where highway overpasses, railroads, pipelines, and almost all structures in the path of the slide were damaged severely. Several overpasses collapsed. Two dams were damaged severely (Lower Van Norman Dam and Pacoima Dam), and three others sustained minor damage. Widespread landslides and rockfalls blocked many highways in the area.

Felt throughout southern California and into western Arizona and southern Nevada (see fig. 22). No foreshocks were recorded, but aftershocks were reported in the area for several months. Magnitude 6.5 $\mathrm{M}_{\mathrm{L}}$ BRK, 6.5 $\mathrm{M}_{\mathrm{La}}$ ELL, $6.2 \mathrm{~m}_{\mathrm{b}} \mathrm{NUT}, 6.6 \mathrm{M}_{\mathrm{S}} \mathrm{NUT}$, $6.35 \mathrm{M}_{\mathrm{L}}$ KJ. (Ref. 44, 72, 263, 400, 521, 553, 599.)

1971. Mar. 31. Near San Fernando, Los Angeles County, Calif. This was the most damaging aftershock of the Feb. 9 earthquake. The main damage was reported in the Granada Hills-Northridge-Porter Ranch area of San Fernando Valley. More than 300 


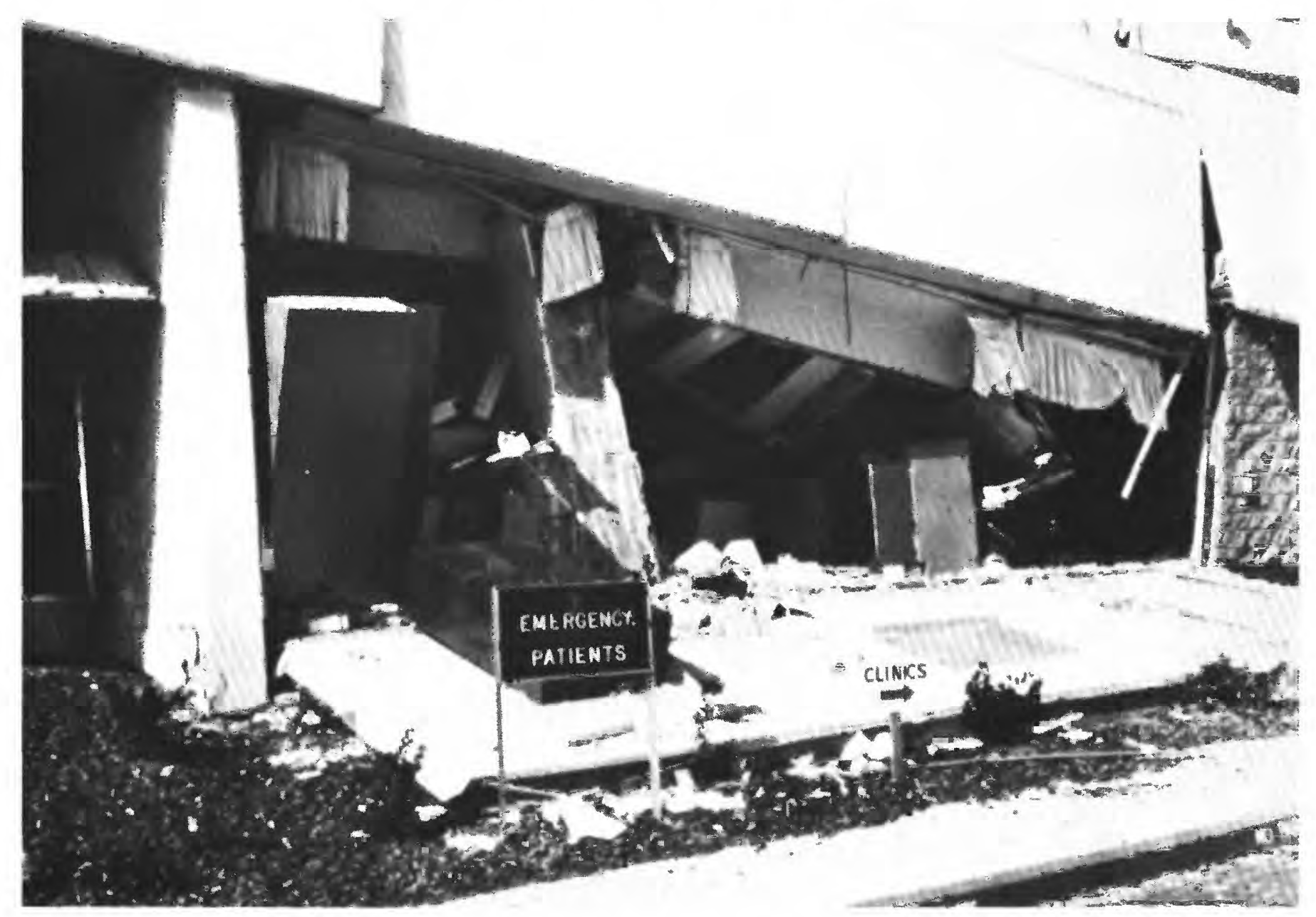

Partial collapse of first floor of Olive View Hospital Medical treatment and care unit, Sylmar area, California, caused by February 9 , 1971, San Fernando earthquake. (Photograph from the National Geophysical Data Center, NOAA.)

buildings sustained some kind of damage, including cracked foundations, shifted walls, damaged or destroyed chimneys, and broken windows. Many outdoor concrete garden walls fell, and some water lines were damaged. Felt over a large area of southern California, including Kern, Los Angeles, Orange, Riverside, San Bernardino, Santa Barbara, and Ventura Counties. Magnitude $4.2 \mathrm{~m}_{\mathrm{b}}$ NUT, $4.3 \mathrm{M}_{\mathrm{S}}$ NUT. (Ref. 44, 72, 263, 292.)

1971. Sept. 30. West of Brawley, Imperial County, Calif. Near Westmorland, dishes and food were knocked to the floor. An observer northwest of Brawley reported she was knocked to the floor and that dishes fell from shelves. Felt over a small area of southern California and at Ehrenberg, Ariz. A few small aftershocks were felt in the epicentral area. (Ref. 44, 72, 292.)

1972. Feb. 24. Southeast of Hollister, San Benito County, Calif. This was the main shock of a series that occurred on this date. Many rockfalls along the San Benito River and the renewed movement of an old landslide east of Paicines were the main ground disturbances. Much cracking of the ground was observed on or near the old landslide. Mouldings separated slightly from walls at the Bear Valley Fire Control Station. At the nearby Melendy Ranch, a brick chimney on the ranch house was damaged severely. The main shock was felt over a moderate area of west-central California. Fourteen aftershocks of magnitude 2.5 to 3.6 were recorded on Feb. 24, and many were recorded for several days thereafter. (Ref. $45,72,324$.

1972. Sept. 4. Southeast of Hollister, San Benito County, Calif. Slight damage was reported at the Bear Valley Fire Control Station, including small cracks in brick and mortar and plaster, and displacement of a vertical pipe in the ceiling, which damaged the sheetrock. Many rockfalls occurred 


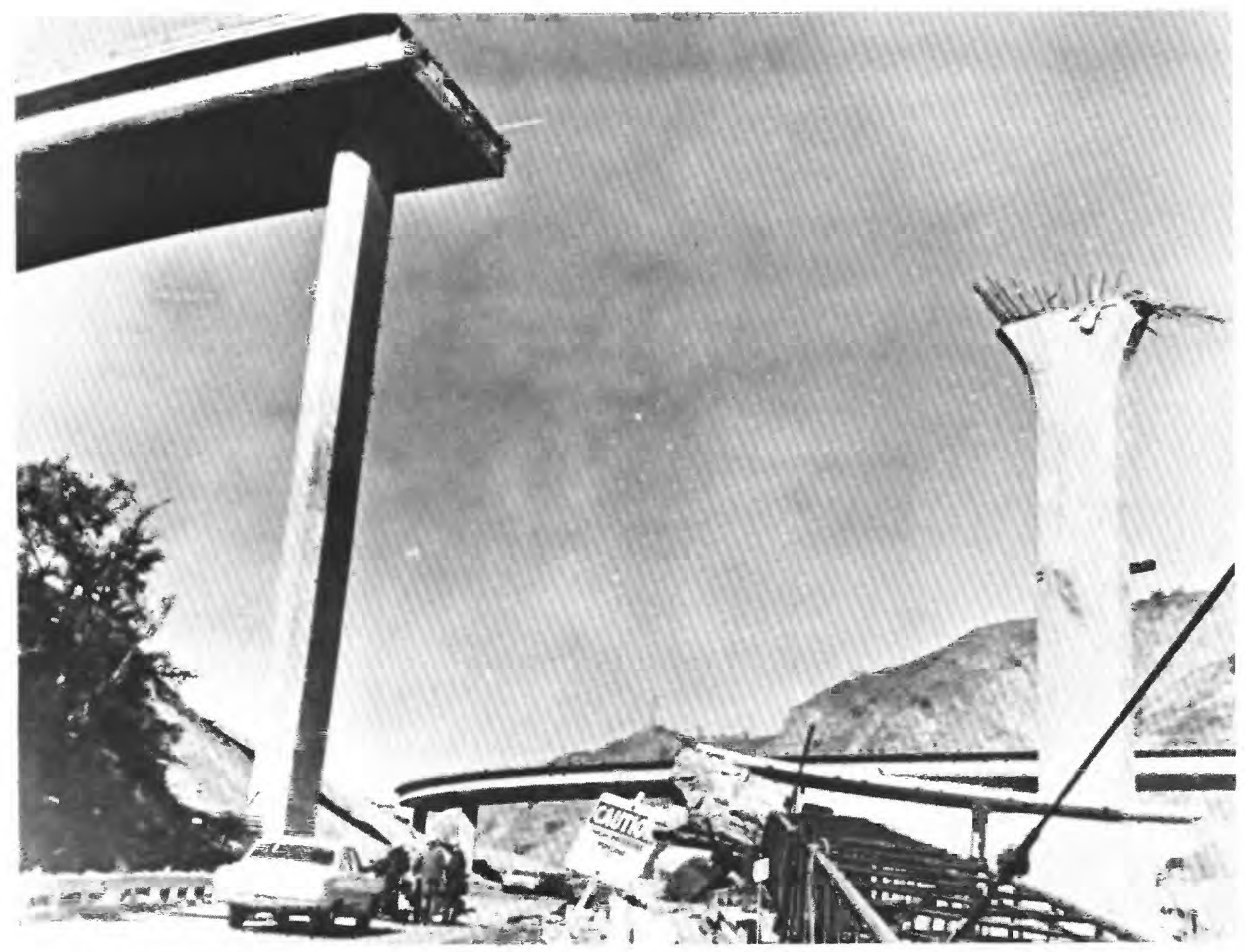

Collapsed overpass at the Route 14-Route 5 interchange, northwest of San Fernando, California, caused by the February 9 , 1971, earthquake. (Photograph by the Newhall Signal.)

along the banks of the San Benito River; landslides and cracks in the ground were observed at Paicines. Felt over a small area of west-central California. Several light aftershocks were reported. Magnitude $4.6 \mathrm{~m}_{\mathrm{b}}$ NUT, $4.3 \mathrm{M}_{\mathrm{S}}$ NUT. (Ref. $45,72,263,324$.)

1972. Oct. 3 (Oct. 2). South of San Juan Bautista in Monterey County, Calif. In the San Juan Bautista area, old asphalt road cracks were widened, and fresh cracks formed in areas previously repaired. At the San Juan Bautista Mission, the top section of an old adobe wall was thrown down. Plaster cracked and fell at ranch houses southeast of San Juan Bautista. Much merchandise was thrown from shelves in San Juan Bautista and the surrounding area. Felt over a small area of west-central California. Several foreshocks and aftershocks were recorded. Magnitude 4.1 $\mathrm{m}_{\mathrm{b}}$ NUT, $4.1 \mathrm{M}_{\mathrm{S}}$ NUT. (Ref. 45, 72, 263, 324.)
1973. Feb. 21. Off the coast of Ventura County, near Point Mugu, Calif. Property damage in the Oxnard, Point Mugu, and Port Hueneme areas was estimated at about $\$ 1$ million. The heaviest damage occurred in downtown Oxnard, where walls failed in a few unreinforced brick buildings. Many chimneys were damaged, mainly in the older part of Oxnard; false ceilings and acoustical tiles fell to the floor and many plate-glass windows were broken. Chimneys were twisted and overturned northeast of Point Mugu at the Camarillo State Hospital. Some large boulders fell onto the Pacific Coast Highway in the Point Mugu area, and a long narrow crack was observed in the highway in the Solromar area. Several hundred sand craters (as much as $2 \mathrm{~m}$ in diameter) formed in Mugu Lagoon, and water spouted from a few of them. Lurch cracks associated with clusters 


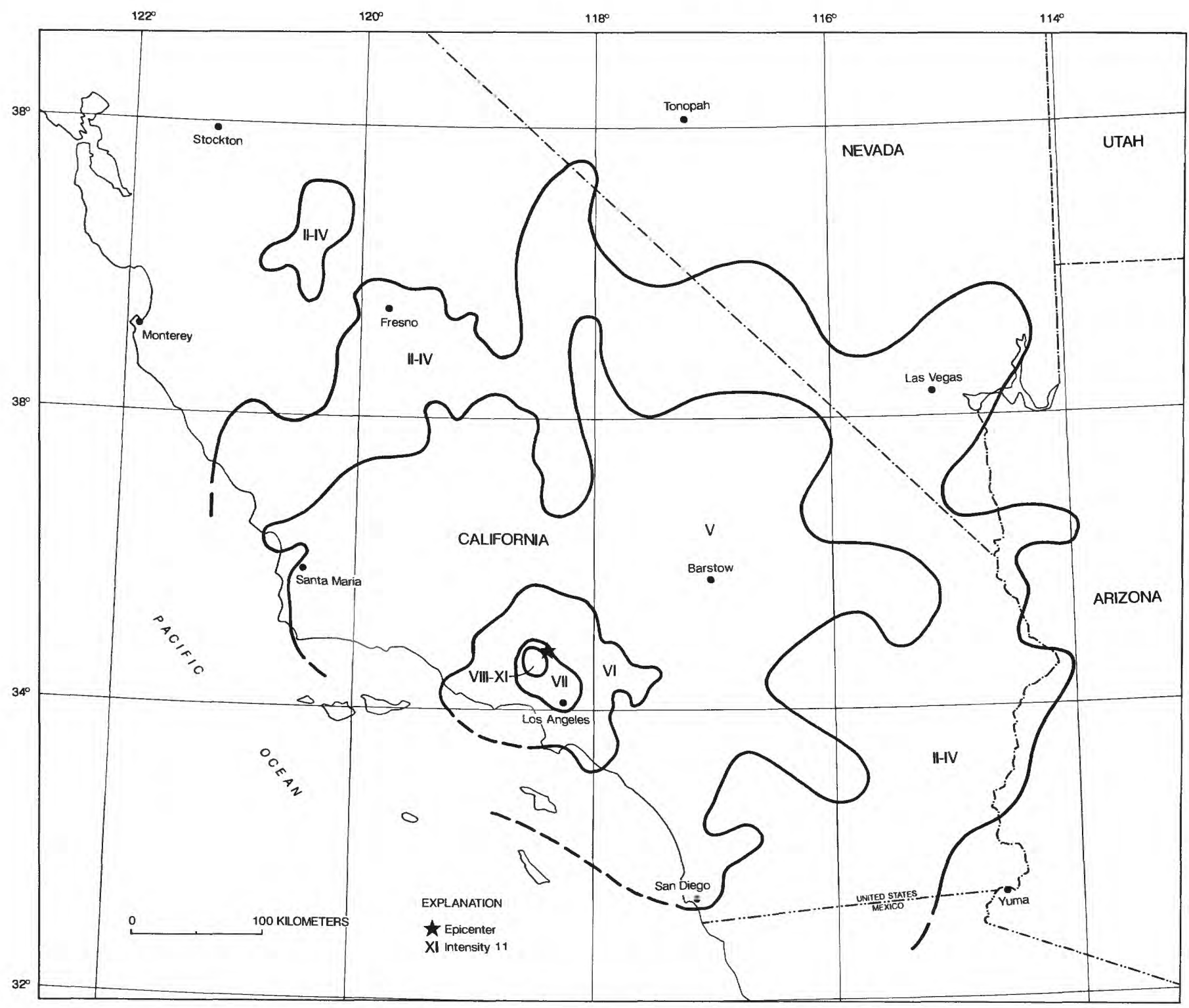

FIGURE 22.-Isoseismal map for the San Fernando, California, earthquake of February 9, 1971. Isoseismals are based on intensity estimates from data listed in references 44 and 72 of table 1.

of craters occurred as far as $8 \mathrm{~km}$ inland from the lagoon.

The main shock of this series of earthquakes was felt over a large area of southern California-from Lompoc (Santa Barbara County) northeast through the Bakersfield area to northeast Kern County, southeast to the Palm Springs area, and then southwest to the San Diego area. Many aftershocks were recorded. Magnitude 5.6 $\mathrm{M}_{\mathrm{L}}$ BRK. (Ref. 46, 72, 458.)

1973. Aug. 9 (Aug. 8). Off the coast of Humboldt County, Calif. Damage sustained in the Ferndale-Scotia area consisted mainly of broken windows, cracked and broken plaster, and loss from damaged merchandise in stores. Felt over a small area of Humboldt and Mendocino Counties. (Ref. 46, $72,401$.

1974. Jan. 6. Southwest of Nubieber in Shasta County, Calif. The earthquake knocked plaster to the floor at Bieber and ruptured a gas pipe at Nubieber. It was felt over a small area, including parts of Lassen, Modoc, Shasta, and Siskiyou Counties. (Ref. 47, 401.)

1974. Mar. 21. Near Healdsburg, Sonoma County, Calif. The floor at the Union Oil workshop at Cloverdale was cracked; boulders rolled down hillsides; and several cracks formed in the earth. Felt 
over a small area of Napa and Sonoma Counties. (Ref. 47, 107, 401.)

1974. Sept. 21. Near Sunnymead, Riverside County, Calif. Three walls were cracked at a ranch in Sunnymead, and damage was reported at San Bernardino. Felt over a small area of Riverside and San Bernardino Counties. (Ref. 47, 402.)

1974. Nov. 28. Near Hollister, San Benito County, Calif. Widespread minor damage occurred at Hollister, including fallen bricks from one chimney, broken windows, cracked water pipe, and cracks in wall and ceiling. Felt over a moderate area of westcentral California. (Ref. 47, 401.)

1975. Jan. 12, 0137 UTC (Jan. 11). Near Petrolia, Humboldt County, Calif. A small amount of chimney damage was reported at Petrolia, and one television antenna fell. Felt along the coast of Humboldt County from Trinidad on the north to Piercy (northern Mendocino County) on the south. (Ref. 48, 87, 401.)

1975. Jan. 13. Near Long Beach, Los Angeles County, Calif. Plaster cracked and fell at Long Beach. The press reported minor damage at Lakewood. The tremor was felt along the coast of Los Angeles County south to the Vista area and from the coast east to Bloomington (San Bernardino County). (Ref. 48, 87, 355.)

1975. Jan. 21. Near Calipatria, Imperial County, Calif. At Calipatria, bricks were separated and plaster fell. This earthquake was part of a series that centered in the Brawley area through early February. It was felt over a small area of Imperial County near the U.S.-Mexico border. (Ref. $48,87,355$.)

1975. Jan. 23, 1702 UTC. South of Calipatria, Imperial County, Calif. This was the main shock of a swarm that struck Imperial Valley in late January and early February. It generated surface ruptures along a 10.4-km segment of a newly recognized fault southeast of Brawley, now designated the Brawley fault. Displacement, which apparently was vertical at the surface, reached a maximum of more than $0.2 \mathrm{~m}$ at Keystone Road. The shock knocked down several light fixtures and bits of plaster from a store ceiling in Brawley; it also broke windows in houses and cracked plaster. Plaster also fell at Calipatria and a large crack formed in one wall. Residents throughout the Imperial Valley reported feeling this shock. (Ref. 48, 355, 565.)

1975. Jan. 23, 2324 UTC. Near Brawley, Imperial County, Calif. Ceiling tiles fell to the floor at two stores in Brawley, and a small crack formed in a store wall. This earthquake was reported only at Brawley. (Ref. 48,355 .)
1975. Mar. 3. Near Compton, Los Angeles County, Calif. Joints in a concrete walk were separated and plaster was cracked at Compton. Windows were broken at Gardena. (Ref. 48, 355.)

1975. June 1 (May 31). Galway Lake area, San Bernardino County, Calif. Property damage was not reported from the epicentral area, which is in the Mojave Desert and virtually uninhabited. Ground ruptures observed followed a preexisting, unmapped fault in the Galway Lake area. The ruptures began about $1 \mathrm{~km}$ north of Galway Lake and extended south toward Emerson Lake, a distance of $6.8 \mathrm{~km}$. Boulders were overturned in the area, and cracks formed in the ground. Felt mainly in San Bernardino County. (Ref. 38, 355.)

1975. June 7. Near Fortuna, Humboldt County, Calif. At Fortuna, 10 weak chimneys toppled and 20 others were damaged; sidewalks cracked and some sank. Minor damage to chimneys also occurred at Carlotta, Fernbridge, Ferndale, Hydesville, Loleta, Petrolia, Rio Dell (water main also broke), Rohnerville, Scotia, and Waddington. Landslides were observed in the Fortuna-Rio Dell areas. Felt along the coast from Crescent City south to Albion (Mendocino County) and from the coast east to Lewiston (Trinity County). (Ref. 48, 401.)

1975. June 20 (June 19). Near Calexico, Imperial County, Calif. Slight damage was reported, but not described, at Calexico. The shock was felt at a few other towns in Imperial County and in southwest Arizona. (Ref. 48, 355.)

1975. Aug. 1, 2020 UTC. Near Oroville, Butte County, Calif. Structural damage, consisting mainly of cracks in chimneys and walls, broken windows and plaster, and loosened light fixtures, occurred at several schools, hospitals, and houses in the OrovilleThermalito area. Many chimneys toppled or had to be taken down in Oroville and Palermo. Property damage was estimated at $\$ 2.5$ million.

This earthquake was associated with the first recorded surface faulting in the western foothills of the Sierra Nevada. New fractures in the ground were observed in a 3.8-km-long north- to north-northwest-trending zone. The block east of the fault moved upward relative to that on the west, as shown by about $55 \mathrm{~mm}$ of slip across the surface ruptures and $180 \mathrm{~mm}$ of vertical movement of benchmarks near the rupture zone. Felt over a large area of northern California and western Nevada. (Ref. 38, $401,566,599$.

1975. Aug. 2. Oroville area, Butte County, Calif. The University of California at Berkeley assigned MM intensity VI to this earthquake but did 
not describe the damage. Felt throughout Butte and surrounding counties. (Ref. 48, 401.)

1975. Aug. 3, 0635 UTC (Aug. 2). Near Firebaugh, Fresno County, Calif. A water line broke and plaster cracked at Firebaugh; a church was damaged at Three Rocks. Felt mainly in Fresno and surrounding counties. Magnitude 4.9 $\mathrm{M}_{\mathrm{L}}$ PAS. (Ref. 48,401 .)

1975. Aug. 10 (Aug. 9). South of Mariposa, Calif. Rockslides and broken windows were reported at Mariposa; slight damage was reported at San Joaquin in Fresno County. Felt over a small area of central California. (Ref. 48, 401.)

1975. Sept. 13. West of Avenal in Monterey County, Calif. Cracks formed in plaster and ground at Avenal. Felt along the coast of Monterey and San Luis Obispo Counties and east to Fresno County. Magnitude 5.0 $\mathrm{M}_{\mathrm{L}}$ PAS. (Ref. 48, 401.)

1975. Nov. 14. Near Eureka, Humboldt County, Calif. Damage was slight at Eureka, where windows were broken. Dishes and dolls were broken at a store in Ferndale. The press reported that the shock was felt north to Oregon and south to San Francisco. (Ref. 48, 401.)

1976. Jan. 1. Near Brea, Los Angeles County, Calif. This New Year's Day earthquake disturbed millions of residents in the Los Angeles region but caused no injuries and only slight damage. Damage occurred at Brea (windowpane broke and fireplace cracked), La Habra (water pipe broke), and Yorba Linda (walls and ceilings cracked). A strong-motion record obtained at Whittier, about $13.8 \mathrm{~km}$ west of the epicenter, recorded a maximum acceleration of $0.28 \mathrm{~g}$ (gravity). The shock also was felt in Orange, Riverside, and San Bernardino Counties. (Ref. 49, 355.)

1976. Jan. 14. North of Avenal in Fresno County, Calif. At Avenal, plaster was cracked and a light fixture was knocked from the ceiling at a school. The shock also was felt in Kings, Monterey, and San Luis Obispo Counties. Magnitude 4.6 $\mathrm{M}_{\mathrm{L}}$ PAS. (Ref. 49, 401.)

1976. Apr. 8. Near Granada Hills, Los Angeles County, Calif. Plaster was cracked at Granada Hills, and slight damage was reported, but not described, at Inglewood. Felt from Bakersfield (Kern County) to San Diego. Magnitude $4.8 \mathrm{M}_{\mathrm{L}}$ BRK. (Ref. 49, 355.)

1976. Aug. 11. Near Borrego Springs in Riverside County, Calif. In Borrego Springs, mudslides and cracks in the ground were reported in Anza-Borrego State Park. A cement curb buckled at Palm Desert. Felt in Imperial, Orange, San Bernardino, and San Diego Counties. (Ref. 49, 355.)
1976. Aug. 20. Near Danville, Contra Costa County, Calif. This earthquake was the largest of a series in the San Francisco Bay area Aug. 15-16 and 20-22. The only reported damage occurred in Danville, where plaster was cracked in some houses. Felt throughout the San Francisco Bay area. (Ref. 49,401 .)

1976. Oct. 17 (Oct. 16). Near Newhall, Los Angeles County, Calif. Slight damage was observed at Newhall, where a water main was broken, and at Tarzana, where plaster and masonry sustained cracks. The shock also was felt in Kern and Ventura Counties. Magnitude 4.1 $\mathrm{M}_{\mathrm{L}}$ BRK. (Ref. 49, 355.)

1976. Nov. 4. Northwest of Brawley, Imperial County, Calif. A series of earthquakes was recorded on this date, but only slight damage occurred. Plaster and drywall were cracked at Brawley; fences were displaced slightly, and cracks formed in plaster and tiles at El Centro; and cracks formed in plaster and ground at Westmorland. Felt over a large area of southern California (including Imperial, Riverside, and San Diego Counties) and southwest Arizona (including the towns of San Luis and Yuma). Magnitude 5.5 $\mathrm{M}_{\mathrm{L}} \mathrm{BRK}$. (Ref. 49, 355.)

1976. Nov. 22. West of Los Angeles, Calif. Windows were broken and plaster was cracked at North Hollywood; slight damage was reported at Long Beach and Los Angeles (cracks in plaster). Felt over a small area along the west coast of Los Angeles County. (Ref. 49, 355.)

1977. Jan. 8. Near Oakland, Contra Costa County, Calif. This main shock of a series of 58 tremors caused minor damage at several towns, including Berkeley (broken windows, enlarged cracks in walls); El Cerrito (dislodged chimney brick, cracks in walls); Napa (cracks in plaster and drywall); Oakland (cracks in plaster in several houses); San Francisco (chandelier knocked down, cracks in ceilings); and Walnut Creek (cracks in drywall). Felt along the coast of California from Sonoma County south to Santa Cruz County. (Ref. 39, 401.)

1977. June 21 (June 20). Southwest of French Camp in Alameda County, Calif. This earthquake cracked sidewalks slightly at French Camp and left cracks in plaster and drywall at El Granada. It was felt along the coast from Point Reyes Station (Marin County) south to Santa Cruz and from the coast east to the Sonora area (Tuolumne County). (Ref. 39, 401.)

1977. Aug. 12 (Aug. 11). Near San Fernando, Los Angeles County, Calif. One person was injured at San Fernando when a shelf of dishes fell on her. Windows were broken in Los Angeles, Northridge, 
and Van Nuys; exterior walls were cracked at Reseda; and plaster was cracked at Studio City. Water sloshed onto sides of pools at Glendale and Van Nuys. Felt along the coast of southern California from Santa Barbara south to San Pedro (Los Angeles County) and from the coast east to Palmdale and Riverside. (Ref. 39, 355.)

1977. Oct. 21 (Oct. 20). Imperial Valley, Calif. At Brawley, plaster was cracked and small objects were shifted. Felt in many towns in the Imperial Valley. (Ref. 39, 355.)

1977. Nov. 14 (Nov. 13). Near El Centro, Imperial County, Calif. At El Centro, plaster fell in the Post Office and some windows were broken; at Imperial, cracks formed in plaster walls. Slight damage (cracks in windows) also was reported at Somerton, Ariz. Felt over a small area of southern California and southwest Arizona. (Ref. 39, 355.)

1977. Nov. 22. Near Willits, Mendocino County, Calif. Moderate structural damage occurred at Willits, where 65 chimneys were damaged, and windows and walls were cracked and broken. Cracks and offsets of as much as $12 \mathrm{~mm}$ were formed in the walls of a store on Main Street; concrete columns were cracked and the ceiling dropped as much as $50 \mathrm{~mm}$. A few interior walls collapsed in older houses. An interior wall collapsed in one house, and its chimney fell apart both inside and outside the house. East of Willits, in Little Lake Valley, a chimney was cracked above the roofline, heavy furniture was displaced, toilet-tank lids flew off their bases, and a water pipe broke. Felt along the coast from Scotia (Humboldt County) in the north to Stewarts Point (Sonoma County) in the south and from the coast east to the Willows area (Glenn County). (Ref. 39, 401.)

1978. Mar. 26 (Mar. 25). Near Ukiah, Mendocino County, Calif. Near Ukiah, huge storage tanks in a new warehouse at the Parducci Winery were damaged by sloshing liquids. Loss to merchandise at stores in the Ukiah area totaled about $\$ 10,000$. Windows were broken at Comptche. Felt northwest to Westport (Mendocino County), south along the coast to Stewarts Point (Sonoma County), and from the coast east to Clearlake Highlands (Lake County). (Ref. 240, 401.)

1978. Aug. 13. Near Goleta, Santa Barbara County, Calif. This moderate earthquake injured 65 people and caused property damaged estimated at $\$ 12$ million. The most severe damage occurred at Santa Barbara and at the University of California Santa Barbara campus at Goleta. There, nine buildings sustained extensive cracks in shear walls. Plaster, ceilings, and light fixtures were damaged throughout the campus. A few old adobe or woodframe buildings were damaged severely. In the commercial district of Goleta and in the Santa Barbara area, similar but less severe property damage was sustained. In Santa Barbara, multistory, reinforcedconcrete structures sustained diagonal cracks in the shear walls of their lower stories. The roof on a restaurant being remodeled collapsed. The most common damage to residential and small commercial buildings consisted of differential settlement of foundations, failure of reinforced chimneys, cracked and fallen plaster, and breakage of glass.

Three overpasses on U.S. Highway 101 in the Goleta area sustained severe damage. The most extensive damage occurred at the Ward Memorial bridges, where the superstructures shifted relative to the abutments, causing the concrete to crack and spall in several places. West of Goleta, a freight train derailed when passing over a "kink" in the tracks, apparently caused by failure of the roadbed fill. Several rockslides occurred on U.S. Highway 101 between Goleta and Santa Barbara.

Felt over a moderate area of southern Californiafrom Morro Bay (San Luis Obispo County) on the north to Santa Ana (Orange County) on the south and from the coast east to Bakersfield (Kern County) and Lake Hughes (Los Angeles County). (Ref. 38, $240,468,599$.)

1978. Aug. 29 (Aug. 28). Near San Jose, Santa Clara County, Calif. At San Jose, acoustical ceiling tiles fell to the floor in a market. In addition, walls and ceilings sustained cracks in a house and its walls were elevated $1.7 \mathrm{~cm}$ from the floor. Felt over a small area of northern California, mainly in Alameda, Contra Costa, Santa Clara, and Santa Cruz Counties. (Ref. 240, 401.)

1978. Sept. 4, 2154 UTC. Lake Tahoe region, El Dorado County, Calif. This is the largest of a series of earthquakes that occurred in the area south of Lake Tahoe on Sept. 3 and 4. Cracks in drywall and hairline cracks in exterior walls were sustained at Mt. Aukum, Calif., and Genoa, Nev. Water splashed onto sides of lakes and pools at Mt. Aukum. Felt from Dobbins (Yuba County) and Stockton (San Joaquin County) on the west to Vernon, Nev., in the east and from Surcliffe, Nev., on the north to Mariposa, Calif., on the south. (Ref. 240, 401.)

1978. Oct. 4, 1642 UTC. Lake Crowley area, Mono County, Calif. The strongest effects from this earthquake occurred in the Bishop area. Large amounts of merchandise fell from store shelves throughout the area, and pictures were knocked from walls. Minor landslides occurred in the canyon areas near Bishop, and boulders rolled onto roads. Slight 


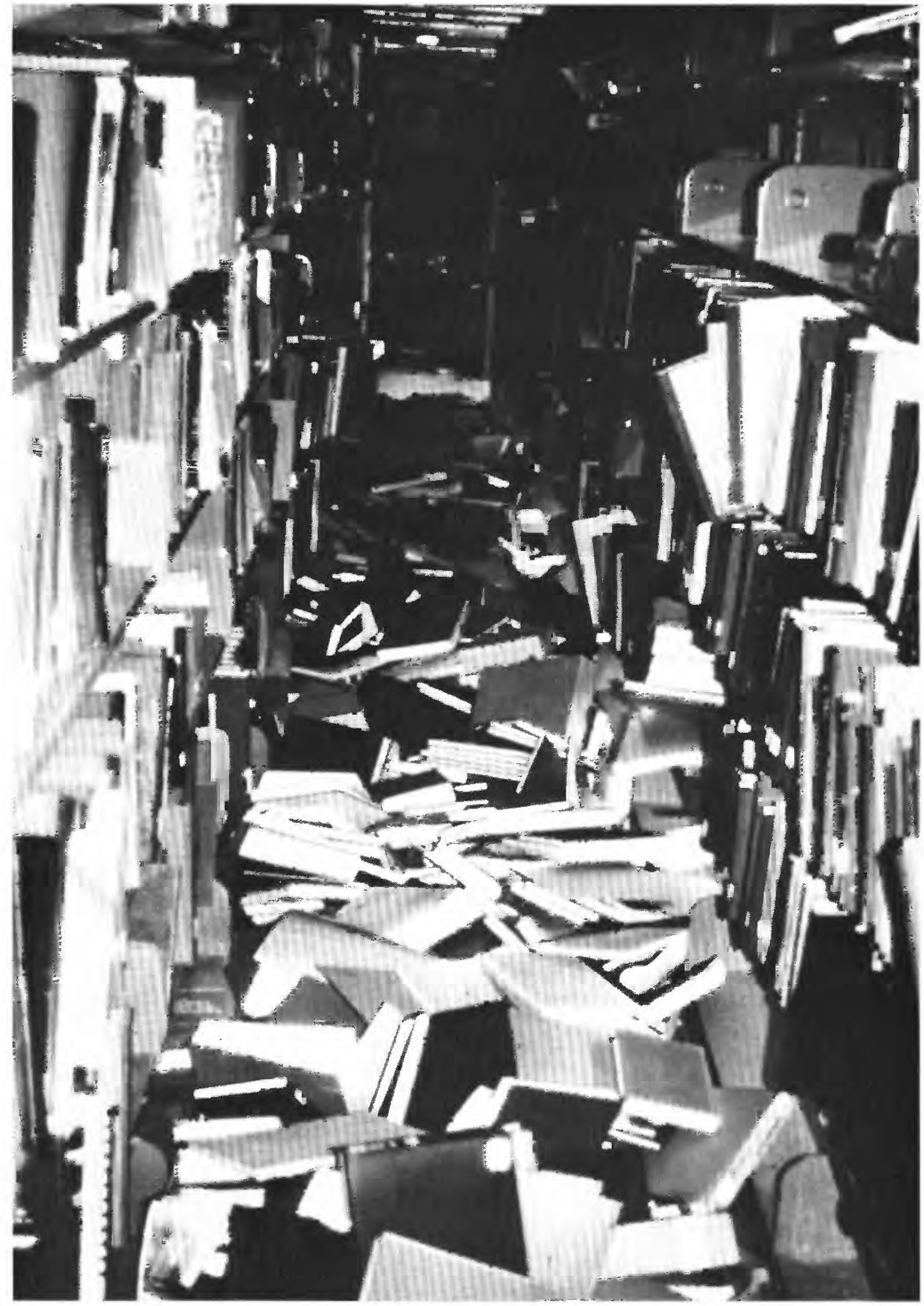

Books shaken off shelves in library of the University of California, Santa Barbara, at Goleta, California, by the August 13, 1978, earthquake. 
damage to walls, plaster, and windows occurred at Benton, Bishop, Easton, Friant, Mammoth Lakes, and Paradise Camp. Felt over a large area of northern California and into western Nevada-from Sacramento on the north to Santa Barbara on the south and east to Mina and Beatty, Nev. Magnitude 5.8 $\mathrm{M}_{\mathrm{L}}$ BRK. (Ref. 240, 355.)

1978. Nov. 20 (Nov. 19). Near Redlands, San Bernardino County, Calif. This earthquake caused slight damage at Redlands, where interior plaster walls cracked and split and hairline cracks formed in exterior walls. Felt in parts of Los Angeles, Orange, Riverside, San Bernardino, and San Diego Counties. (Ref. 240, 355.)

1979. Jan. 1. Near Malibu, Los Angeles County, Calif. Windows were reported broken in Culver City, Malibu, Santa Monica, Tustin, and Venice. Slight damage to walls (mainly cracks) occurred at Studio City and Woodland Hills. Boulders fell onto the Pacific Coast Highway in the Malibu area, and mud and boulders tumbled onto other roads in Malibu. The main earthquake was felt along the coast from Santa Barbara south to Bonita (San Diego County) and from the coast east to the area of Barstow (San Bernardino County) and Palm Springs (Riverside County). The California Institute of Technology recorded about 50 aftershocks in the next 2 hours. (Ref. 262, 474.)

1979. Feb. 3. Off the coast of Humboldt County, Calif. Many store windows were broken and stock tumbled from shelves in stores in the downtown areas of both Arcata and Eureka. At Eureka, chimneys were cracked and broken (one fell through a roof); drywall, plaster and exterior walls were cracked; three water mains were broken; and ceiling tiles and light fixtures fell in some stores. Felt in parts of Del Norte, Humboldt, Mendocino, Siskiyou, and Trinity Counties and in southwest Oregon. (Ref. 262, 401.)

1979. Feb. 22. Honey Lake Valley, Lassen County, Calif. This earthquake interrupted telephone service in the epicentral area but caused only minor property damage. Drywall was cracked at Doyle, near the Nevada border, and desks were displaced. The earthquake was felt over a large area of northeast California and western Nevada. It was preceded by a small foreshock and was followed by aftershocks through Feb. 23. (Ref. 262, 401.)

1979. Mar. 15, 2107 UTC. Near Landers, San Bernardino County, Calif. This earthquake was the strongest of a series of shocks in the area on Mar. 15. A surface rupture formed in the Homestead Valley area along the east bank of Pipes Wash and at three sites west of the Pipes Wash fault. The highest intensity was observed at Landers, where moderate damage to buildings and their contents occurred (downed chimney, cracked walls, broken windows and dishes), and electric and telephone services were disrupted for several hours. Slight damage to plaster and walls was reported from several other towns in the area. The main shock was felt in Los Angeles, Orange, Riverside, San Bernardino, and San Diego Counties and at a few towns in southwest Arizona and western Nevada. (Ref. 262, 355.)

1979. May 8 (May 7). Near San Jose, Santa Clara County, Calif. This earthquake was strongest in East San Jose, where windows were broken, plaster was cracked, pictures fell from walls, and a refrigerator fell over. Felt mainly in the coastal area around San Francisco Bay. (Ref. 262, 401.)

1979. June 14 (June 13). Near Onyx, Kern County, Calif. Minor damage consisting of large cracks in plaster walls and cracks in brick fences and sidewalks occurred at Onyx. The earthquake was felt only at a few towns in the epicentral area. (Ref. $262,355$.

1979. June 29 (June 28). Big Bear Lake, San Bernardino County, Calif. This earthquake was felt strongly in the Big Bear Lake recreation area, where large cracks formed in plaster, a foundation cracked, a plate-glass window shattered, and several burglar alarms were triggered. Felt in the Los Angeles Basin and as south as San Diego. (Ref. 262, 472.)

1979. June 30, 0034 UTC (June 29). Near Big Bear Lake, San Bernardino County, Calif. This earthquake is the largest of a series in the area that began on June 29. At Big Bear City, several windows were broken, walls were cracked, and a large section of acoustical-tile ceiling fell. At Sugarloaf, chimney bricks loosened and ceiling tile and a foundation cracked. Felt west to Long Beach, south to San Diego, and north to Yermo (San Bernardino County), but the felt area east of Big Bear City is unknown. (Ref. 262, 472.)

1979. Aug. 6. Near Gilroy, Santa Clara County, Calif. No fatalities occurred, but 16 people were injured in Hollister and Gilroy. Property damage in the two towns, estimated at $\$ 500,000$, consisted mainly of damaged chimneys, broken glassware in stores, and structural damage to five buildings in Gilroy.

Many chimneys were damaged in older houses near Gilroy's downtown area. A crack split a wall in the City Hall, and the ceiling in a court room of the Municipal Courthouse caved in. Beams and uprights were damaged at Fords Department Store and the store was closed. At Hollister, a parapet toppled and caved in the roof of a real estate office; a ceiling 
partly collapsed at a new building on San Felipe Road. The J.C. Penney Store sustained a 3-m hole in its ceiling, and extensive cracks formed in the ceiling throughout the store. A service station at nearby Casa de Fruta sustained extensive damage, including fallen bricks from the chimney, bulging of exterior walls, and separation of interior walls from ceiling or floor. The fire station at Pacheco Pass, about $16 \mathrm{~km}$ northeast of Hollister, sustained extensive damage, and the nearby lookout station was vacated because of structural damage.

Ground displacement was observed along the Calaveras fault zone from Hollister north to the Anderson Lake area, about $39 \mathrm{~km}$. The maximum horizontal displacement, 5-6 mm, occurred about $10 \mathrm{~km}$ east of Gilroy, where the Calaveras fault zone intersects Highway 152. Ground lurching, settlement, and slumping were observed in many places between Anderson Lake and Hollister. Felt from about $60 \mathrm{~km}$ north of Bakersfield, north to Sacramento, east to the area of Reno-Lake Tahoe, Nev., and west to the Pacific Ocean. During August, most of the 31 located aftershocks were clustered in the area south of the epicenter of the main shock. Magnitude $5.6 \mathrm{M} \mathrm{La}$ ELL. (Ref. 262, 401, 521.)

1979. Oct. 15, 2316 UTC. Imperial Valley area, on the Baja Calif., Mexico-California border. This major earthquake injured 91 people and caused an estimated $\$ 30$ million in property damage in the Imperial Valley area. It destroyed two houses and 11 commercial buildings and damaged 1,565 houses and 440 commercial buildings. The most severe damage (MM intensity IX) was to the Imperial County Services building in El Centro, which had to be razed. The support pillars failed on this six-story reinforced concrete-frame structure, causing partial collapse of the east part of the building. It was designed under the 1967 provisions of the California Uniform Building Code. Other property damage caused by this earthquake at El Centro, Brawley, and Calexico and at Mexicali, Mexico, is typical of MM intensity VII, which is the highest intensity assigned to any location except the Imperial County Services building.

Movement along the Imperial fault also caused damage to the irrigation system in the Imperial Valley. The All American Canal, which brings water from the Colorado River to the Imperial Valley, was damaged most severely. East of Calexico, the earthquake shook down levees on both sides of the canal. The banks settled more than $1 \mathrm{~m}$ in places.

Ground displacement on the Imperial fault extended from about $4 \mathrm{~km}$ north of the International Border to about $4 \mathrm{~km}$ south of Brawley. The maximum lateral displacement-about $55 \mathrm{~cm}$-was observed in Heber Dunes; the maximum vertical displacement-19 $\mathrm{cm}$-occurred southeast of Brawley. Ground rupture followed the same trace as that in the shock on May 18, 1940, and showed many of the same features and characteristics. Aftershock activity shifted to the north in both earthquakes, and both sustained damaging aftershocks near Brawley. There also is evidence that the Brawley fault experienced sympathetic movement in both earthquakes.

Felt over a large area, including southern California, southern Nevada, western Arizona, and an unknown area in Mexico. Many aftershocks occurred, the most damaging of which were on Oct. 16 and Dec. 21 (see descriptions below). Magnitude $7.0 \mathrm{M}_{\mathrm{L}}$ BRK, $6.0 \mathrm{M}_{\mathrm{La}}$ ELL. (Ref. 38, 262, 355, 521.)

1979. Oct. 16, 0658 UTC (Oct. 15). Imperial Valley area aftershock. Aftershocks of the major earthquake on Oct. 15 caused additional damage at Brawley and Imperial. According to the press, near midnight on Oct. 15, an aftershock at Imperial buckled the floor in one house, displaced walls, and crumbled the porch steps. (Ref. 262, 355.)

1979. Dec. 21. Imperial Valley area aftershock. Another aftershock of the major earthquake on Oct. 15 left large cracks in exterior walls at Imperial and cracked windows and stone fences. Felt over a small area of southern California and western Arizona. (Ref. 262, 355.)

1980. Jan. 24, 1900 UTC. North of Livermore Valley in Contra Costa County, Calif. This earthquake injured 44 people and caused an estimated $\$ 11.5$ million in property damage (of which $\$ 10$ million damage occurred at the Lawrence Livermore Laboratory at Livermore). The shock was associated with surface rupture along the Greenville fault. The rupture propagated more than $15 \mathrm{~km}$ to the southeast along the Marsh Creek-Greenville faults, ceasing in the area of Interstate Highway 580.

Most of the damage to property, including that at the Lawrence Livermore Laboratory, was nonstructural. It consisted mainly of fallen ceiling tiles, fallen bricks from chimneys, broken gas lines and water lines, broken windows, and displacement of mobile houses from supporting foundations. However, at the Ordway Ranch (on Vasco Road north of Livermore), a brick-and-stone fireplace was cracked and displaced from the wall, as was a smaller fireplace in another room. At Interstate 580 and Greenville Road (about 4 $\mathrm{km}$ north of the Lawrence Livermore Laboratory), pavement on the overpass settled about $30 \mathrm{~cm}$ and concrete on one abutment cracked and spalled.

Faulting was observed for a distance of about $6 \mathrm{~km}$ along the Greenville fault, beginning near the overpass 


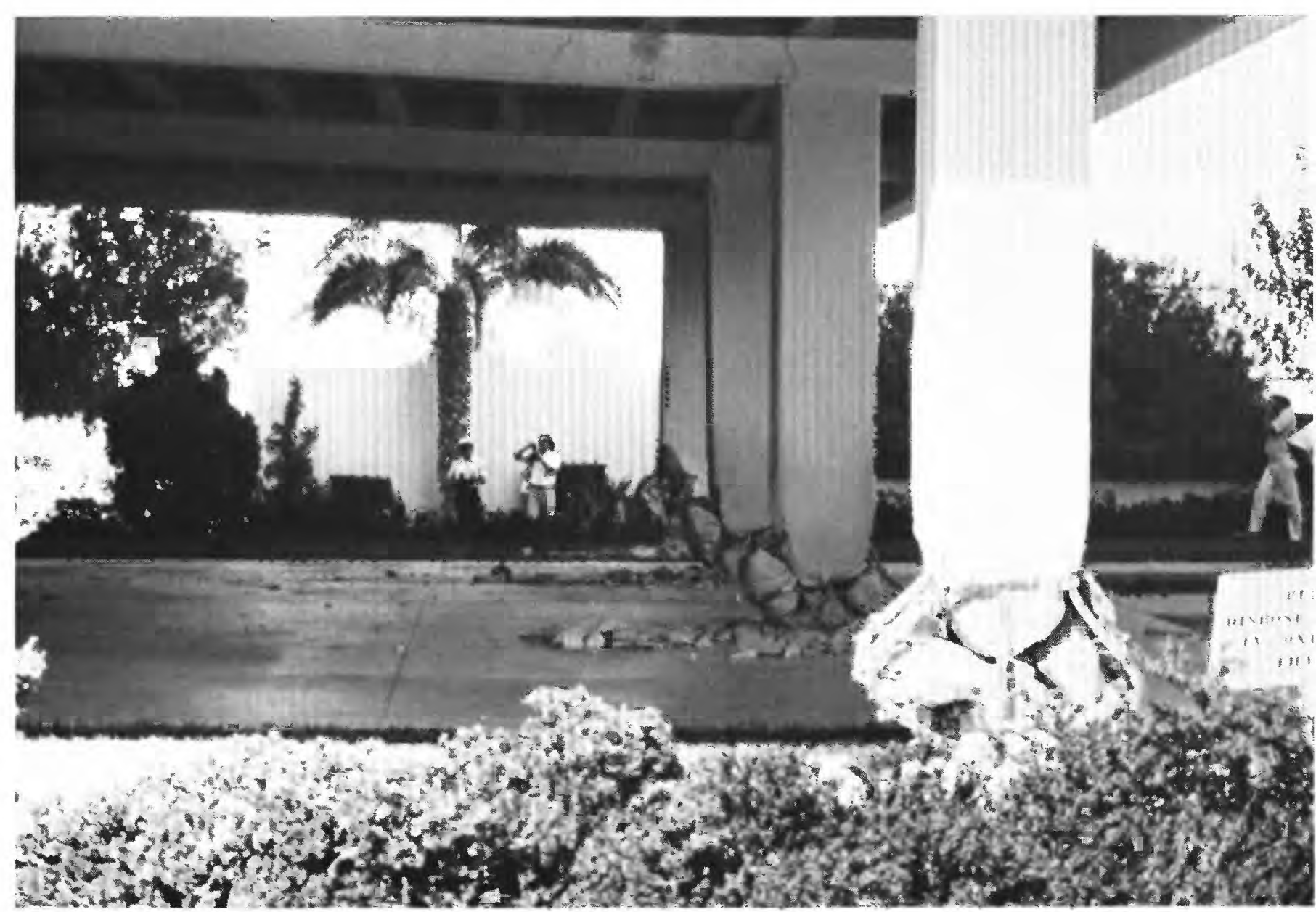

Support pillar failure on the ground level of the Imperial County Services building, El Centro, California, caused by the October 15, 1979, Imperial Valley earthquake.

at Interstate Highway 580 and Greenville Road. Where the fault crosses Vasco Road, right-lateral offset was as much as $2 \mathrm{~cm}$; on Laughlin Road and to the northwest for about $300 \mathrm{~m}$, right-lateral offset of 5 to $10 \mathrm{~mm}$ was observed. Felt over a large area of central California and at a few towns in western Nevada. A small foreshock occurred at 1858 UTC on Jan. 24, and a sequence of 59 aftershocks followed in the next 6 days. A second principal earthquake occurred on Jan. 27 (see description below). (Ref. 300, 466.)

1980. Jan. 27 (Jan. 26). Near Livermore, Alameda County, Calif. A second damaging earthquake, near the south end of the Greenville fault (about $14 \mathrm{~km}$ south of the Jan. 24 epicenter), occurred on Jan. 27. Six persons were injured at Livermore by flying glass and falling ceiling tiles and supports. It caused 1-2 $\mathrm{mm}$ of additional rightlateral movement on the Greenville fault across Laughlin Road as well as additional movement and displacements along the surface rupture of Jan. 24, north of Vasco Road.

The most severe property damage reported was in the Tassajaro Valley area and at Danville, about 17 and $28 \mathrm{~km}$, respectively, northwest of the epicenter. In Tassajaro Valley (east of Danville), about 50 houses sustained minor damage, including one fireplace damaged, walls and concrete cracked, walls separated from ceiling, windows broken, and a chimney toppled. Damage at Danville included one brick chimney broken at the roofline; a fireplace damaged; a stone wall demolished; and walls, ceilings, sidewalks, and patio cracked. Light damage was sustained at several other towns in the area. Felt over a moderate area of central California. (Ref. 300, 466.)

1980. Feb. 25. Southeast of Anza, Riverside County, Calif. Slight damage consisting of broken windows, large cracks in drywall and plaster, loosened bricks on chimneys, and a broken gas line occurred in the Anza-Idyllwild-Palm Desert area. 
Several small landslides forced the closing of State Highway 74 between Spring Crest and Palm Desert; cracks as wide as $3.8 \mathrm{~cm}$ were reported in State Highway 74. Felt over a large area of southern California and an unknown area in Mexico. It was reported from Eagle Mountain (Riverside County) and Palo Verde (Imperial County) on the east to Los Angeles and San Diego on the west. (Ref. 300, 355.)

1980. May 25, 1633 and 1944 UTC. Mammoth Lakes area, Mono County, Calif. In the Mammoth Lakes region, property damage caused by these earthquakes (plus a third strong shock on May 27,1450 UTC) to schools, other public buildings, highways, and merchandise in stores has been estimated at $\$ 1.5$ million. Nine people were injured by the two largest earthquakes, mainly from falling rocks. Landslides and rockfalls were common in this area and in Yosemite National Park.

The most severe property damage occurred at Mammoth Lakes: chimneys toppled, water mains broke, windows shattered, and plaster cracked. The 20-year-old Mammoth Elementary School was damaged severely by faulting beneath the school building. Ground cracks were abundant in fill along both paved and dirt roads. A 17-km-long zone of discontinuous surface fractures associated with the Hilton Creek fault was observed. It had a net vertical displacement of less than $50 \mathrm{~mm}$ and more than 200 $\mathrm{mm}$ of slip on single fractures.

The first earthquake was felt over a large area of California and western Nevada-from Reno and Las Vegas, Nev., to the coast at Los Angeles and San Francisco. The second shock was felt over a similar area. Hundreds of aftershocks, many of which were felt in the Mammoth Lakes area, occurred through 1980. Magnitude 6.4 $\mathrm{M}_{\mathrm{L}}$ PAS (first shock), $6.6 \mathrm{M}_{\mathrm{L}}$ PAS (second shock). (Ref. 300, 401, 599.)

1980. May 27, 1450 UTC. Owens Valley area, Mono County, Calif. It was difficult to differentiate the effects of this earthquake from those caused by the two shocks on May 25 . It was felt over a similar area, however, and four people were injured. Minor damage was reported in several towns in the area. Landslides and falling rocks were common in Yosemite National Park. Old U.S. Highway 395, east of Mammoth Lakes, was closed because of severe cracks. Felt from Eureka and Las Vegas, Nev., in the east to La Honda (San Mateo County) and Los Angeles on the coast. Many aftershocks were felt in the Mammoth Lakes area. Magnitude 6.4 $\mathrm{M}_{\mathrm{L}}$ PAS. (Ref. 300,401 .)

1980. June 29 (June 28). Owens Valley area, Mono County, Calif. Slight damage at Mono Hot Springs included cracks in plaster walls, foundation, and exterior stone walls. Felt over a small area of California and western Nevada. Magnitude $4.7 \mathrm{M}_{\mathrm{L}}$ PAS. (Ref. 300, 401.)

1980. Oct. 31. Near Calexico, Imperial County, Calif. Hairline cracks formed in plaster and drywall at Calexico. Furniture was overturned and windows were cracked. The shock was reported felt only at a few towns in the area. (Ref. 300, 355.)

1980. Nov. 8, 1027 UTC. Off the coast of Humboldt County, Calif. A major earthquake, the largest in this area in 24 years, injured six people and caused property damage estimated at $\$ 2$ million. Most of the damage occurred east of Fields Landing, where two sections of an overpass on U.S. Highway 101 collapsed onto the railroad tracks below. At Fields Landing, two houses were displaced from their foundations, one unreinforced chimney fell, and gas, water, and sewer lines were broken. This shock and most of its aftershocks occurred on a large, left-lateral, strike-slip fault that strikes about N. $50^{\circ}$ E. from the Mendocino Fracture Zone. Felt over a large area, including parts of Oregon, western Nevada, and northern California-from Eugene, Oreg., south to the San Francisco Bay area and from the coast east to Fallon, Nev. Many aftershocks occurred. Magnitude 7.2 $\mathrm{M}_{\mathrm{S}} \mathrm{ABE}$. (Ref. 74, 300, 599.)

1980. Nov. 28. East of Truckee, Nevada County, Calif. Centered in a sparsely populated mountainous area, this earthquake caused only minor damage at Georgetown and Soda Springs. At Georgetown, large cracks formed in exterior walls and drywall, bricks fell from walls, and hairline cracks occurred in plaster walls; at Soda Springs, windows were broken and merchandise was thrown from shelves. Felt from the San Francisco Bay area to Reno, Nev. (Ref. 300, 401.)

1981. Mar. 3. Near Fremont, Alameda County, Calif. A rockslide blocked the Niles Canyon Road between Fremont and Sunol. Windows were broken and burglar alarms were activated in one store. Felt from Sonoma County south along the coast to Monterey and east to Waterford (Stanislaus County). (Ref. 325, 401.)

1981. Apr. 26. Near Westmorland, Imperial County, Calif. Property damage in the CalipatriaWestmorland area was estimated at $\$ 1-3$ million. At Westmorland, 12 buildings sustained severe damage, 30 minor damage, and 70 percent of the 900 dwellings sustained damage of some kind. City officials ordered the razing of 10 downtown buildings and condemned five dwellings.

The main effects in Calipatria and Westmorland were downed chimneys, cracked and destroyed foundations, partial collapse of exterior adobe and wood 


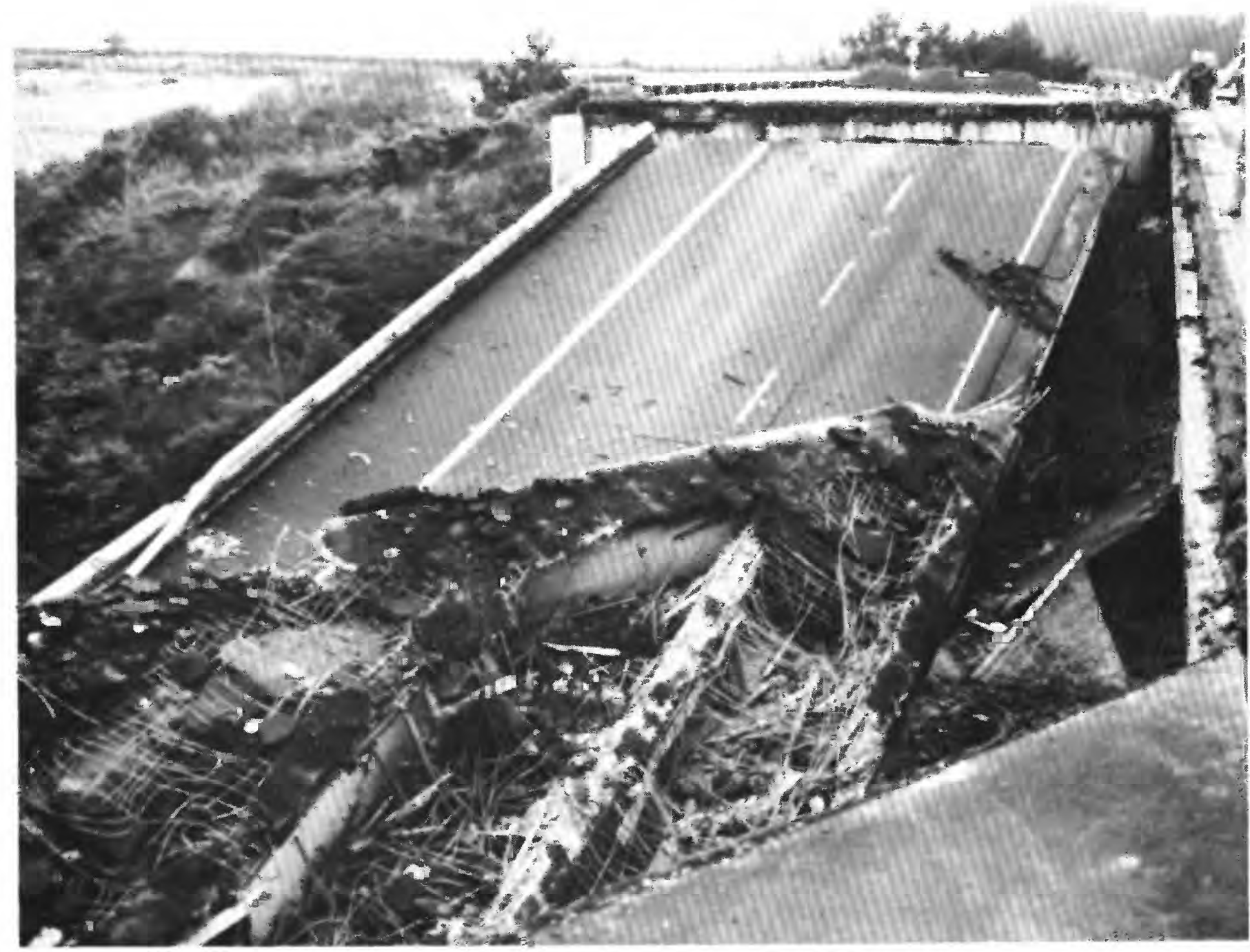

Tompkins Hill Road overpass collapsed on U.S. Highway 101, about 2 mi south of Fields Landing, California, during November 8, 1980, earthquake. (Photograph by R.T. Kilbourne, California Division of Mines and Geology.)

walls, collapse of interior walls, and broken underground pipes. In the rural areas, the main damage consisted of broken concrete-lined irrigation canals, which were not reinforced. Concrete cracked on two stretches of the Vail Canal between Calipatria and Westmorland, and the earthen embankment beneath the cracked concrete washed away. Also, the concrete on several bridges was cracked and chipped; cracks in road pavement and ground were ubiquitous. This earthquake was the largest in a swarm of at least 40 shocks in the area from Apr. 24-28. It was felt over a large part of southern California, southwest Arizona, and an unknown area in Mexico. (Ref. 325, 355.)

1981. July 17. Near Honeydew, Humboldt County, Calif. Light furniture was overturned and much glassware and dishes were broken at
Honeydew. This shock also was felt in Mendocino and Sonoma Counties. (Ref. 325, 401.)

1981. Sept. 4. Off the coast of Los Angeles County, Calif. This was the largest magnitude earthquake in the area since the San Fernando Valley shock of February 1971. Some windows were broken at Marina del Rey and exterior walls were cracked. Telephone service was interrupted briefly in some areas, burglar alarms were activated, and elevators became inoperative. Felt in southern California from San Luis Obispo to the U.S. Mexico border. (Ref. 325, 355.)

1981. Sept. 30, 1153 UTC. Near Mammoth Lakes, Mono County, Calif. This earthquake was the first, and the largest, of a swarm of shocks in the area. It knocked out electric service at the Mono County sheriff's substation in Crowley Lake and at 


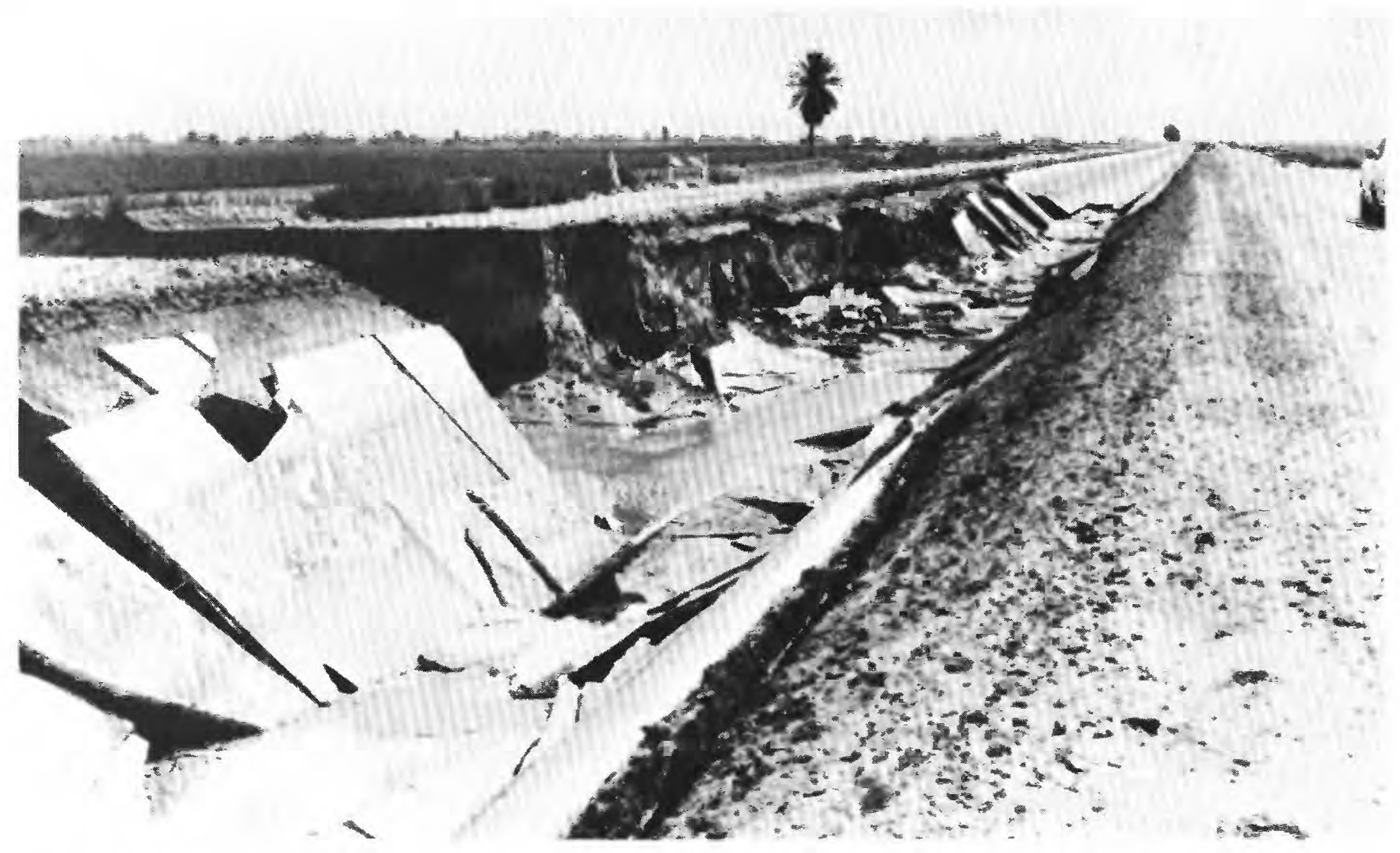

Vail Irrigation Canal, between Calipatria and Westmorland, California, damaged by the April 26, 1981, Imperial Valley earthquake. (Photograph by the Imperial Valley Press.)

the Mammoth Lakes Airport. Chimneys cracked at Mammoth Lakes, and hairline cracks formed in plaster and drywall. A shopping center under construction sustained damaged walls and broken windows. Flowing spring water was muddied at the Hot Creek Fish Hatchery, near Mammoth Lakes, and gas lines were broken. Felt over a large area of California and at a few towns in western Nevada. Magnitude 5.9 $\mathrm{M}_{\mathrm{L}}$ BRK. (Ref. 325, 355.)

1982. Oct. 1. Near Inyokern, Kern County, Calif. Many large cracks formed in interior drywalls at Barstow and Inyokern; bricks shifted in a fireplace at Ridgecrest, and hairline cracks formed in interior stucco walls. Items were thrown from store shelves in several towns. Felt in Inyo, Kern, Los Angeles, San Bernardino, and Tulare Counties. (Ref. 350,355 .)

1982. Oct. 25. Near Coalinga, Fresno County, Calif. Tiles fell from interior walls at Coalinga, and many items were thrown from store shelves. At Avenal, small amounts of plaster fell from a ceiling, and some cracks formed in interior plaster walls. A hunter reported that, about $40 \mathrm{~km}$ northwest of Coalinga, cracks formed in the wet ground. Felt from
Kern County on the south to Santa Clara County on the north and from the coast in San Luis Obispo County to Mono County on the eastern slope of the Sierra Nevada. (Ref. 350, 401.)

1982. Dec. 16 (Dec. 15). Near Fortuna, Humboldt County, Calif. Chimneys were cracked and windows were broken at Ferndale and Fortuna. Windows were broken, and items were thrown from store shelves at Rio Dell. Felt mainly in Humboldt County. (Ref. 350, 401.)

1983. Jan. 7, 0138 and 0324 UTC (Jan. 6). Mammoth Lakes area, Mono County, Calif. The first earthquake damaged a few buildings at Mammoth Lakes and knocked out electric service at Crowley Lake and Mammoth Lakes. The second one collapsed a metal hangar at the Mammoth Lakes Airport and toppled display cases. The first shock was felt from western Nevada to Merced County in the west and from El Dorado County in the north to Kern County in the south; the second one was reported from a similar but slightly smaller area. Magnitude 5.6 M $\mathrm{L}$ PAS (both shocks). (Ref. $360,401$. 


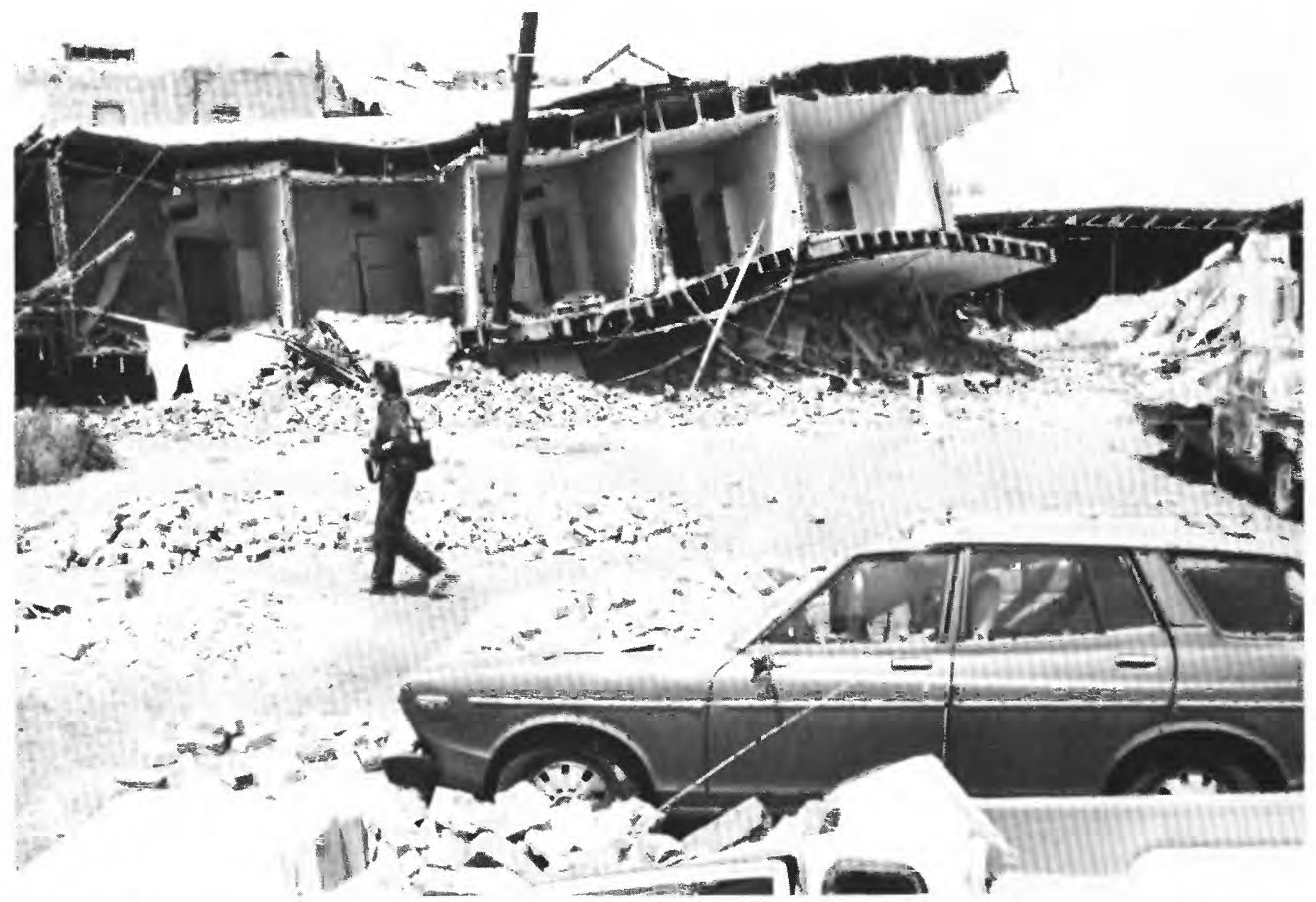

Building on 5th Street, Coalinga, California, destroyed by the May 2, 1983, earthquake. (Photograph by the Fresno Bee.)

1983. May 2, 2342 UTC. Near Coalinga, Fresno County, Calif. This earthquake caused an estimated $\$ 10$ million in property damage (according to the American Red Cross) and injured 94 people. Damage was most severe in Coalinga, where the 8-block downtown commercial district was almost completely destroyed. Here, buildings having unreinforced brick walls sustained the heaviest damage. Newer buildings, however, such as the Bank of America and the Guarantee Savings and Loan buildings, sustained only superficial damage. The most significant damage outside the Coalinga area occurred at Avenal, $31 \mathrm{~km}$ southeast of the epicenter.

A disaster assessment by the American Red Cross listed the following statistics on damage in the area: almost destroyed-309 single-family houses and 33 apartment buildings; major damage - 558 single-family houses, 94 mobile homes, and 39 apartment buildings; and minor damage-811 single-family houses, 22 mobile homes, and 70 apartment buildings. Most public buildings, including the City Hall, hospital, schools, fire house, post office, and police station, sustained only minor damage.

Only six bridges of 60 surveyed in the area sustained measurable structural damage. This damage consisted of hairline cracks and spalling at the top of the support columns, fracturing and displacement of wingwalls and parapets, and settlement of fill.

All public utilities were damaged to some degree. The water system continued to function despite many leaks in its transmission piping. Gas was shut off for several days because of broken piping and leaks, but only temporary interruptions of electric and telephone services were reported. One large section of old concrete sewer pipe west of the downtown area partly collapsed, but this system also continued to function.

In the oil fields near Coalinga, surface facilities such as pumping units, storage tanks, pipelines, and support buildings were all damaged to some degree. 


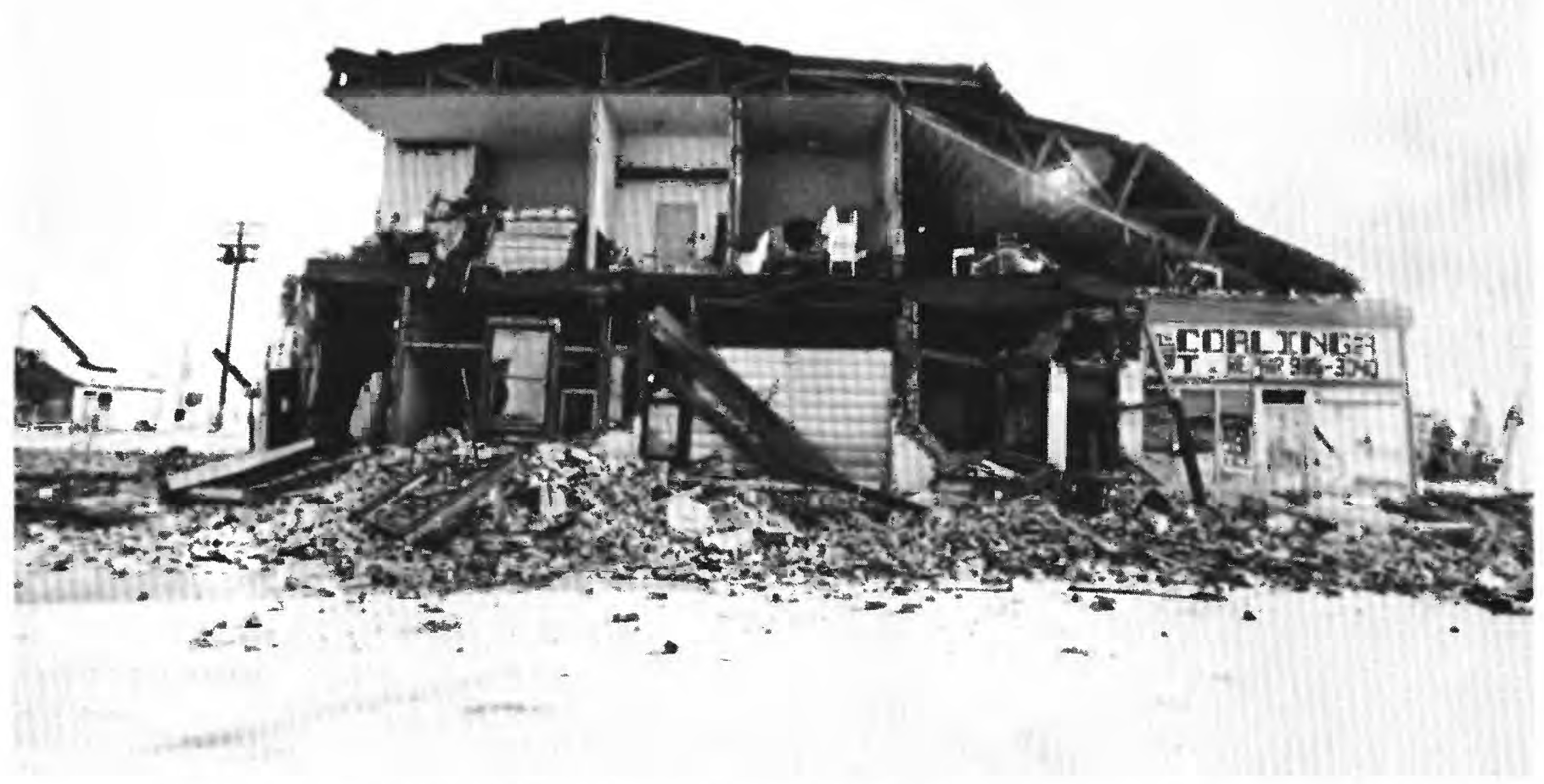

Building at 187 South 6 th Street, Coalinga, California, severely damaged by the May 2, 1983, earthquake. (Photograph by the Fresno Bee.)

One oil company administration building, about $7 \mathrm{~km}$ north of Coalinga, sustained major structural damage and its two brick chimneys were toppled. Subsurface damage, including collapsed or parted well casing, was observed only on 14 of 1,725 active wells.

This earthquake triggered thousands of rockfalls and rockslides as far as $34 \mathrm{~km}$ northwest, $15 \mathrm{~km}$ south, and $26 \mathrm{~km}$ southwest of the epicenter. Only a few slope failures occurred east of the epicenter because of the absence of steep slopes in that direction.

This damaging earthquake was caused by an $0.5-\mathrm{m}$ uplift of Anticline Ridge northeast of Coalinga, but surface faulting was not observed. Ground and aerial searches immediately after the earthquake revealed ground cracks and fissures within about 10 $\mathrm{km}$ of the instrumental epicenter, none of which appeared to represent movement on deeply rooted fault structures. About 5 weeks later, on June 11, however, an aftershock caused surface faulting about $12 \mathrm{~km}$ northwest of Coalinga (see description below.)

Felt from the Los Angeles area north to Susanville (Lassen County) and from the coast east to western Nevada (see fig. 23). Through July 31, more than 5,000 aftershocks were recorded, of which 894 had a magnitude of 2.5 or larger. Most of the larger magnitude shocks were felt in
Coalinga. Magnitude $6.1 \mathrm{M}_{\mathrm{L}}$ PAS, $6.7 \mathrm{M}_{\mathrm{L}}$ GM. (Ref. $360,593$. )

1983. May 9, 0249 (May 8). Coalinga, Fresno County, Calif., aftershock. This is one of the strongest aftershocks of the May 2 earthquake. It injured two residents at Coalinga, but additional structural damage was not observed. Felt over a moderate area in central California and at Schurtz in western Nevada. Magnitude 5.2 $\mathrm{M}_{\mathrm{L}}$ PAS, $5.3 \mathrm{M}_{\mathrm{L}}$ GM. (Ref. 360.)

1983. June 11 (June 10). Coalinga, Fresno County, Calif., aftershock. This strong aftershock of the May 2, 1983, earthquake caused surface faulting about $12 \mathrm{~km}$ northwest of Coalinga along a $3.3-\mathrm{km}$-long stretch of the previously unnamed Numez fault. Maximum reverse and right-lateral components of slip in the north segment of the fault were 64 and $20 \mathrm{~cm}$, respectively. Slip along the northern quarter of the southern segment of the fault was similar to that along the north segment. Maximum reverse and right-lateral components of slip in the southern three-fourths of the south segment were 8 and $11 \mathrm{~cm}$, respectively. Maximum net slip for the north and south segments of the fault were 65 and $13 \mathrm{~cm}$, respectively.

In the area northwest of Coalinga, along Los Gatos Creek Road, one house that was damaged heavily in 


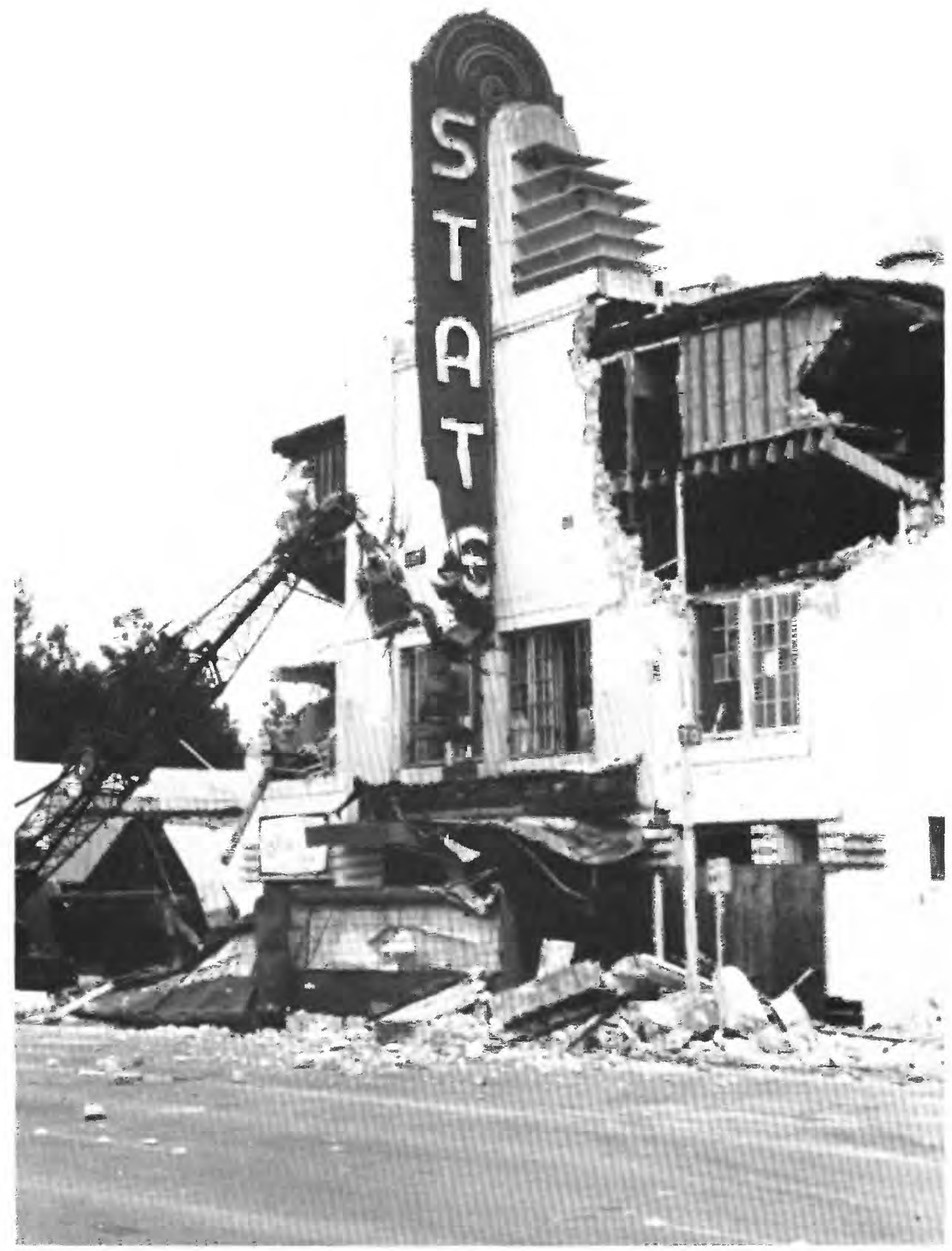

State Theater on Elm Avenue, Coalinga, California, damaged the May 2, 1983, earthquake. (Photograph by the Fresno Bee.) 


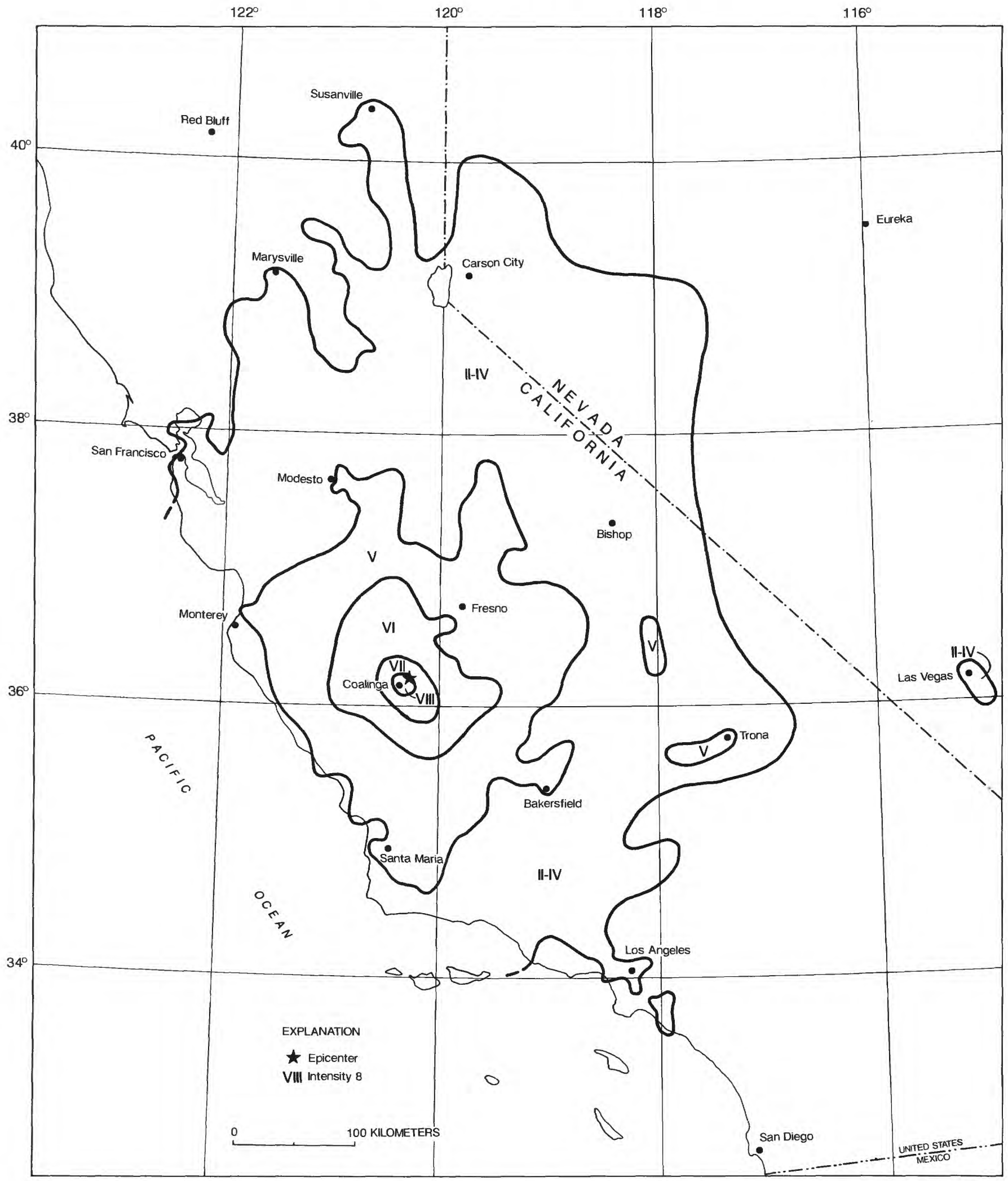

FIGURE 23.-Isoseismal map for the Coalinga, California, earthquake of May 2, 1983. This map is a simplified version of figure 13 in reference 360 of table 1 . 
the main shock on May 2 was reported destroyed. Several houses sustained minor damage, and a few mobile homes were shifted off their supports. Felt over a small area in central California. Magnitude $5.1 \mathrm{M}_{\mathrm{L}}$ PAS, $5.2 \mathrm{M}_{\mathrm{L}}$ GM. (Ref. 360 , 593.)

1983. July 13. Imperial Valley area, Calif. Slight damage occurred at Niland, a few kilometers north of Calipatria, where windows were broken, large cracks formed in walls, and plaster fell to the floor. Felt only at a few towns in the area. (Ref. 360.)

1983. July 22, 0239 UTC (July 21). Coalinga, Fresno County, Calif., aftershock. A large aftershock of the Coalinga earthquake of May 2, 1983, caused minor damage to property at Coalinga (fluorescent lights fell in store; chimneys, walls, and foundation cracked; minor landslides occurred) and injured two people. Slight damage also was observed at Lemoore Naval Air Station and Stratford. Felt over a large area in central California-from Sacramento on the north to Bakersfield and beyond on the south and from the coast east to the Nevada border. Magnitude 5.6 $\mathrm{M}_{\mathrm{L}}$ PAS, $6.0 \mathrm{M}_{\mathrm{L}}$ GM. (Ref. 360.)

1983. July 25. Coalinga, Fresno County, Calif., aftershock. Another aftershock of the Coalinga earthquake of May 2, 1983, caused minor damage and two injuries at Coalinga. Some chimneys were damaged, mobile homes were displaced from their supports, cracks formed in interior and exterior walls, and windows were broken. Damage to utilities included one ruptured gas line, two cracked water mains, and temporary interruption of telephone service. Felt over a small section of central California. Magnitude 5.4 $\mathrm{M}_{\mathrm{L}}$ PAS, $5.3 \mathrm{M}_{\mathrm{L}}$ GM. (Ref. 360.)

1983. Aug. 24. Off the coast of Humboldt County, Calif. This earthquake broke windows and overturned small objects at Scotia. Felt mainly in Humboldt, Mendocino, and Trinity Counties of northern California. Several moderate aftershocks occurred on Aug. 26, Nov. 11, and Dec. 20. (Ref. 360,401 .)

1983. Aug. 29. Northwest of San Simeon in Monterey County, Calif. About $20 \mathrm{~km}$ northwest of San Simeon, near Ragged Point, a few buildings were reported damaged, and cracks formed in chimneys. Felt mainly in Monterey, San Benito, and San Luis Obispo Counties. (Ref. 360, 476.)

1984. Apr. 24. Near Morgan Hill, Santa Clara County, Calif. The Morgan Hill earthquake injured 27 people and caused property damage estimated at $\$ 8$ million. Most of the loss occurred in Santa Clara County, where 522 private dwellings and 43 commercial buildings were damaged severely. MM intensity VIII effects were confined to a small area east of Morgan Hill on two streets: Oak Ridge
Lane and Oak Ridge Court, both in the Jackson Oaks subdivision, near Anderson Lake.

Five houses were condemned in the Jackson Oaks area of Morgan Hill, two of which fell off their concrete foundations and partly collapsed. A ranch house at Jackson Ranch (east of Anderson Lake) reportedly shifted off its foundation and collapsed. Other damage included cracks in exterior walls around garage doors and windows and a house thrown out of plumb but still on its foundation. Seventeen mobile homes were shaken off their support systems in Morgan Hill, and many partly fell. Three schools in Morgan Hill sustained damage to walls, ceiling panels, and light fixtures. Several underground water lines were broken.

Near Coyote, at the United Technologies Chemical Systems Plant, damage was estimated at $\$ 1.5$ million. Column base connections were damaged on several steel-frame buildings, and diagonal steel braces buckled. Concrete buildings at the plant had cracked walls and broken weld connections, and one wall panel was separated from its roof. At the IBM Santa Teresa Laboratories, south of Coyote, suspended ceiling panels and a light fixture fell, and an underground sprinkler pipe ruptured. This structure was designed for seismic loading and therefore did not sustain significant structural damage.

Other effects in the Morgan Hill area included slight damage to Leroy Anderson Dam, Coyote Lake Dam, Coyote Creek Bridge, and Anderson Reservoir Bridge, fallen chimneys, small landslides, and changes in flow of water in springs or wells. At Coyote Lake Dam, about $25 \mathrm{~km}$ southeast of the main shock, a large acceleration of gravity $(1.29 \mathrm{~g})$ was recorded on strong-motion instruments. This damaging earthquake was felt over a large area of California and western Nevada. (Ref. 370, 401.)

1984. Oct. 25. Near Santa Ynez, Santa Barbara County, Calif. Minor damage occurred at Santa Ynez and north of Los Olivos at the Firestone Winery. Effects at the winery included damage to several steel storage tanks and to the foundations of several large oak vats. The warehouse sustained one stress crack, and the water line to the sprinkler system ruptured. At Santa Ynez, brick fences were cracked; large cracks formed at the joints between a concrete-block wall and a wood-frame wall; and interior plaster walls were cracked. Felt over a small area of Santa Barbara and Ventura Counties. (Ref. 370.)

1985. Aug. 4, 1201 UTC. Near Avenal, Kings County, Calif. A few stores and houses were damaged at Avenal, and six residents were injured. 


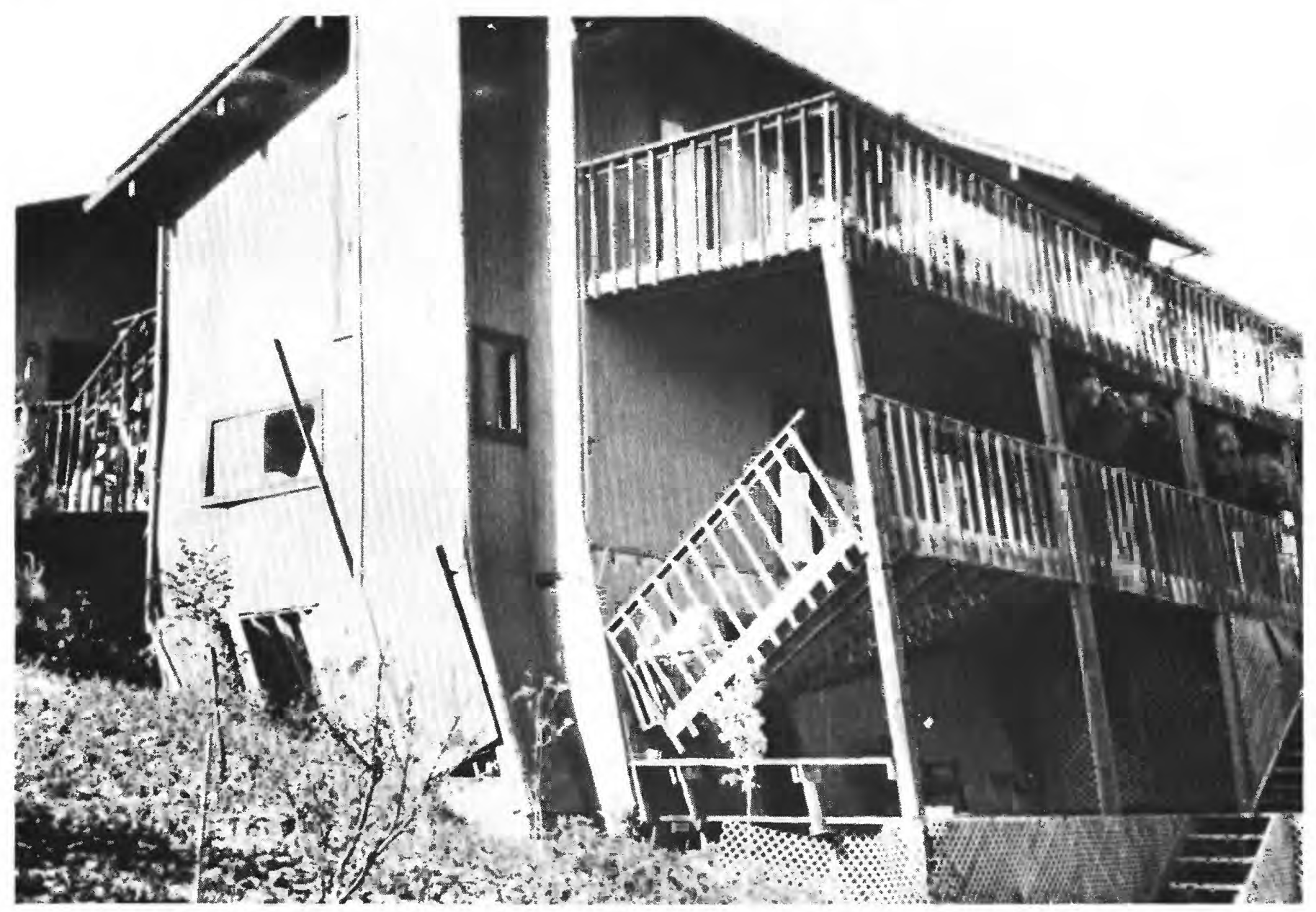

Damaged home in the Jackson Oaks subdivision of Morgan Hill, California, caused by the April 24, 1984, earthquake. (Photograph by the Morgan Hill Times.)

Damage included the partial collapse of porches at two houses, cracked and broken chimneys, cracks in sidewalks and walls, broken water mains, and broken windows and glassware. About $8 \mathrm{~km}$ northeast of Avenal, four adobe houses sustained cracks in the walls and ceilings, parts of the ceilings fell, and concrete porches were cracked. Several water lines and one gas main were broken at Kettleman City, and a cast-iron elbow on a water tank was broken. Felt over a large area of central California. More than 130 aftershocks were recorded in the next 27 hours. Magnitude 5.8 $\mathrm{M}_{\mathrm{L}}$ PAS. (Ref. 371, 401.)

1985. Oct. 2. Near Grand Terrace, San Bernardino County, Calif. Plaster fell at the Grand Terrace fire station, and minor cracks formed in its ceiling. In $x \log$ house at Lake Gregory Village, one beam was split and others were cracked. Felt in parts of Los Angeles, Orange, Riverside, San Bernardino, and San Diego Counties. (Ref. 371.)
1986. Jan. 26. Near Paicines, San Benito County, Calif. At a winery in Paicines, a huge vat full of wine was displaced $6 \mathrm{~m}$ from its foundation and shattered. Damage to wine vats at the winery was estimated at $\$ 800,000$. Damage to property in the form of broken gas pipes and ruptured water lines occurred at Hollister. At Tres Pinos, two chimneys fell at the 19th Hole Bar, and the kitchen stove was displaced. At a ranch in the Santa Ana Valley, masonry walls at the entrance to the driveway were partly collapsed. Felt generally north to San Joaquin County, south to San Luis Obispo County, and from the coast east to Fresno County. (Ref. 562.)

1986. Mar. 31. Near San Jose, Santa Clara County, Calif. This earthquake injured six residents and caused slight damage to property in Fremont, Mount Hamilton, Newark, and San Jose. The main damage was characterized by broken water lines, fallen ceiling tiles, cracks in chimneys and 
walls, and damaged stock. Known as the Mt. Lewis earthquake, it was preceded by two minor foreshocks on Mar. 24 and Mar. 31 and was followed by 22 aftershocks. The main earthquake was felt along the coast north to Sonoma County and south to San Luis Obispo County. (Ref. 562.)

1986. July 8. Near North Palm Springs, Riverside County, Calif. This strong earthquake injured 40 people in the North Palm Springs area and caused property damage estimated at $\$ 6$ million. Sixteen business structures and four houses were destroyed; 102 houses (mostly mobile homes) and 117 business structures were damaged to some degree. The earthquake disrupted electrical and telephone service, broke water lines and gas lines, and caused failure of two pumping stations in the Metropolitan Water District. North of Palm Springs, en echelon fractures formed along the Banning fault for a distance of about $9 \mathrm{~km}$ on both sides of State Highway 62. Several highways were closed temporarily by minor landslides.

Major damage to a highway bridge was observed on Interstate 10 in Coachella Valley northwest of Palm Springs. The bridge was displaced laterally, leaving a small gap between the deck and abutment. Three houses were destroyed and chimneys fell in the Whitewater Canyon area. Also sustaining damage was the Southern California Edison Devers substation, $3 \mathrm{~km}$ northwest of North Palm Springs. Many of the ceramic columns were broken at the substation, and one transformer was displaced about $4 \mathrm{~cm}$, shearing retaining bolts. Several light aftershocks were reported felt. The main shock was felt over a large area, including parts of western Arizona, southern California, and southern Nevada. (Ref. 562, 597.)

1986. July 13. Off the coast of San Diego County, Calif. This earthquake caused damage in San Diego County estimated at $\$ 700,000$ and injured one person. Damage in San Diego and nearby towns consisted mainly of broken plate-glass windows, cracked walls and plaster, and broken chimneys. Through Apr. 30, 1987, 99 aftershocks of magnitude greater than 3.0 occurred. Felt over most of southern California and reported as far away as Las Vegas, Nev., and Yuma, Ariz. (Ref. 562, 597.)

1986. July 17. North Palm Springs, Riverside County, Calif., aftershock. At Whitewater, chimneys were broken at the roofline, tombstones were toppled, foundation and interior walls were cracked, and underground pipes were broken. Rockslides were reported on Whitewater Road. Felt mainly in southern California, but also was reported at Las Vegas, Nev. (Ref. 562.)
1986. July 21, 1442 UTC. Near Chalfant, Mono County, Calif. Known as the Chalfant Valley earthquake, this shock injured two people and caused an estimated $\$ 2.7$ million damage to property in the Bishop-Chalfant area. At Bishop, a few chimneys cracked, windows broke, ceiling tile and plaster fell, and exterior walls cracked in several buildings. The brick facade on a bank on Main Street also sustained cracks. Most of the damage at Chalfant was due to mobile homes being shaken off their supports, which damaged water and gas lines. Fractures in the ground were observed in the White Mountain frontal fault zone. Many small landslides and spectacular rockfalls occurred in the epicentral area.

The shock was felt mainly in California and western Nevada but was reported in multistory buildings as far distant as Salt Lake City, Utah. A foreshock occurred on July 20 at 1429 UTC, and an aftershock occurred on July 31 at 0722 UTC (see next paragraph). Thousands of smaller aftershocks occurred through Sept. 30, 1986. Magnitude 5.9 $\mathrm{M}_{\mathrm{L}}$ PAS, 6.6 $M_{L}$ REN. (Ref. 562.)

1986. July 31 (July 30), 0722 UTC. Near Bishop, Inyo County, Calif. A strong aftershock of the Chalfant Valley earthquake (July 21, 1442 UTC) broke plate-glass windows in Bishop and toppled stock from shelves. Light fixtures were knocked down at the National Weather Service office. Felt over a small area of California and western Nevada. Magnitude 5.9 $\mathrm{M}_{\mathrm{L}}$ PAS, 5.5 $\mathrm{M}_{\mathrm{L}}$ REN. (Ref. 562.)

1986. Nov. 21, 2333 UTC. Near Petrolia, Humboldt County, Calif. The most severe damage occurred at Petrolia, where chimneys were cracked and twisted and small appliances were overturned. An old building at the Mattole Union Elementary School was knocked off its cinder-block foundation. Slight damage also occurred in several other towns in the area.

A strong aftershock occurred at 2334 UTC, but its effects could not be differentiated from those of the earthquake about 1 minute earlier. The first earthquake was felt over a moderate area of northern California, mainly in Humboldt, Mendocino, and Trinity Counties. (Ref. 562.)

1987. July 31. Near Petrolia, Humboldt County, Calif. Minor damage was reported at Petrolia, where underground pipes and windows were broken, cracks formed in chimneys and in wood foundations, and water in springs or wells was muddied. One report from Ferndale indicated that chimneys were toppled, windows were broken, and sidewalks were cracked. Felt only in Humboldt, 


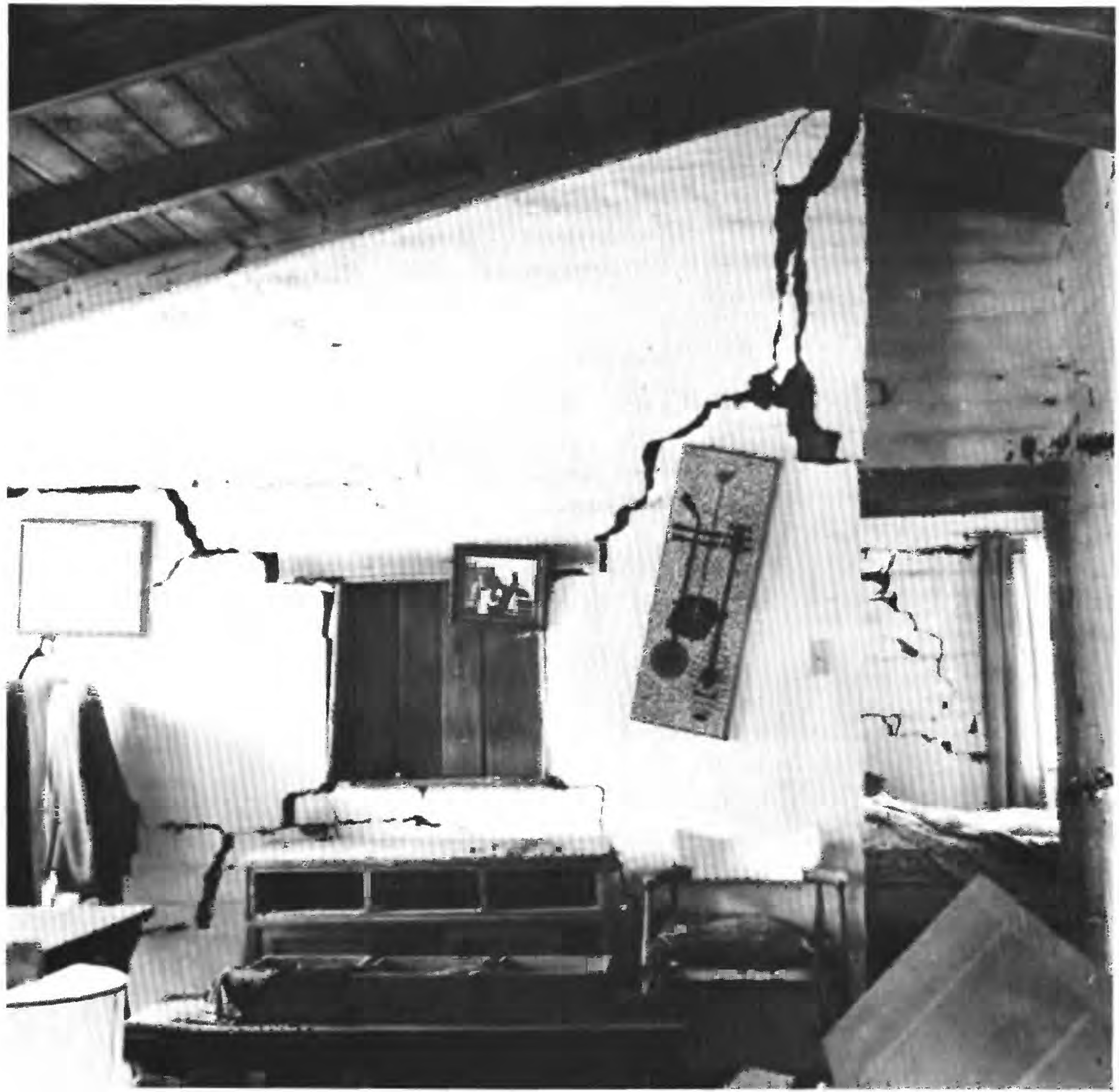

Adobe walls of a recently remodeled house in Whitewater Canyon, northwest of Palm Springs, California, cracked by the July 8 , 1986, earthquake. (Photograph by G. Borchardt, California Division of Mines and Geology.)

Mendocino, Siskiyou, and Trinity Counties in northern California. (Ref. 74, 577.)

1987. Oct. 1. Near Whittier Narrows, Los Angeles County, Calif. The Whittier Narrows earthquake caused eight fatalities, injured several hundred, and left property damage estimated at $\$ 358$ million in the east Los Angeles area, mainly at Whittier. MM intensity VII to VIII covered an area of about $500 \mathrm{~km}^{2}$-from Monrovia and Pasadena in the north to beyond Whittier in the southeast. MM intensity VI was assigned to an additional area of $1,500 \mathrm{~km}^{2}$.

Business structures in the old Whittier commercial district were the most severely damaged by the main earthquake. In the 24-square-block shopping area known as Whittier Village, 12 commercial buildings 
had to be razed, and another 20 buildings were declared unsafe. An inspection of residential houses in Los Angeles, Orange, and Ventura Counties indicated that 123 single-family houses and 1,347 apartment units were destroyed, and about 513 singlefamily houses and 2,040 apartment units sustained major damage. Property damage on the Los Angeles campus of California State University (about $10 \mathrm{~km}$ west of the epicenter) was estimated at more than $\$ 20$ million.

The most severe damage to transportation systems was to the Interstate 605-Interstate 5, a major ninespan bridge that was built in 1964. The five supporting columns sustained severe shear fractures and the overpass was closed temporarily. Minor damage also occurred on 23 other bridges in the area.

Damage and dysfunction of lifelines included the often observed failure of ceramic elements on highvoltage substation equipment, damage to large liquid-storage tanks, and saturation of the telephone system with inappropriate calls. The natural-gas transmission system was not damaged, and only one cast-iron pipe failed in the distribution system. However, about 1,400 gas leaks occurred on customer property, and many fires were ignited.

This earthquake sequence ruptured a small and previously unidentified, gently north-dipping, weststriking thrust fault beneath the uplifted Puente Hills and Elysian Park-Montebello Hills. However, tectonic slippage was not observed during a field study of the faults in the epicentral area. Geologic surface expression appeared to be limited to secondary nontectonic breaks caused by acceleration at the surface. Although many ground cracks formed along the base of the Puente Hills between Turnbull Canyon and Norwalk Boulevard, ground breakage in that area was limited to slope failures, including extensional cracks, minor landslides, and rockfalls. Ground-surface cracks also were observed at Worsham Creek oil field and Whittier Narrows golf course.

The main shock was followed by about 500 locatable aftershocks, an unusually small number for an earthquake of this magnitude. The largest aftershock, which occurred on Oct. 4 about $3 \mathrm{~km}$ northwest of the epicenter of the main shock (see description below), caused further damage to weakened buildings. (Ref. 74, 577, 580, 581, 582, 598.)

1987. Oct. 4, aftershock. Near Whittier Narrows, Los Angeles County, Calif. A strong aftershock of the Oct. 1 Whittier earthquake killed one person, injured several, and caused additional property damage in Alhambra, Los Angeles, Pico Rivera, and Whittier. Several chimneys twisted, fell, or broke at the roofline; stone fences cracked and toppled; windows broke; and large cracks formed in sidewalks and highways. The press reported that one of the two bell towers collapsed on the San Gabriel Civic Auditorium. Also felt in Orange, Riverside, San Bernardino, and San Diego Counties. (Ref. 74, 577, 598.)

1987. Nov. 24, 0154 (Nov. 23) and 1315 UTC. West of Westmorland, Imperial County, Calif. The Superstition Hills earthquakes caused an estimated $\$ 3$ million property damage in Imperial County. Epicenters of the shocks were in the western Imperial Valley on a fault system comprising the northwest-striking Superstition Hills fault and a previously unknown northeast-striking structure. The earthquake sequence consisted of foreshocks, the first main shock, and aftershocks on the northeast trend, followed by the second main shock about 11 hours later and aftershocks on the northwest trend. Significant surface ruptures occurred along the Superstition Hills fault.

Damage reported in the epicentral region at $\mathrm{El}$ Centro, Imperial, and Westmorland included fallen chimneys, broken underground pipes, broken windows, and large displacements in highways or streets. The Worthington Road Bridge across the New River needed to be replaced, owing to liquefaction damage to the approach fill on both sides of the bridge. Damage at the Desert Test Range Control Center near Westmorland, which included equipment falling through a window and small water tanks tipping against the building, was sufficient to stop operations for several days.

Damage to canal facilities in the Southern California Irrigation District was estimated to be between $\$ 600,000$ and $\$ 750,000$. The first main shock caused minor buckling of the concrete lining in canals on the west side of the valley; the second main shock collapsed thousands of feet of concrete canal lining, mainly in the northwest corner of the valley nearest the earthquake epicenters.

The shock at 0154 UTC was associated with leftlateral surface rupture on many faults in and near the Superstition Hills. A maximum surface slip of $12.5 \mathrm{~cm}$ was observed on the Elmore Desert Ranch fault. The shock at 1315 UTC ruptured the surface of the right-lateral Superstition Hills fault for a distance of $27 \mathrm{~km}$ southeastward from its epicenter. This right-lateral movement continued to increase over the following 339 days. The maximum vertical slip observed on the southernmost ruptured part of the Superstition Hills fault zone (named the Wienert fault) was $25 \mathrm{~cm}$, but that rupture also continued to increase. Both shocks were felt over most of southern 
California and in parts of western Arizona and southern Nevada. (Ref. 74, 577, 587, 588, 598, 601.)

1988. Feb. 11. Near Whittier, Los Angeles County, Calif. A small aftershock of the Whittier earthquake of Oct. 1, 1987, cracked chimneys, drywall, plaster, and windows at Los Angeles, Pasadena, Pico Rivera, and Whittier. Further, foundations of houses were cracked and exterior walls were damaged at Pico Rivera and Whittier. Also felt in Kern, Orange, Riverside, San Bernardino, and San Diego Counties. (Ref. 578, 602.)

1988. June 13 (June 12). Near San Jose, Santa Clara County, Calif. Slight damage occurred at San Jose, where open cracks formed in stone or brick fences and interior walls were cracked. Hairline cracks formed in drywall and plaster at nearby Santa Clara. Felt north along the coast to Humboldt County, east to Amador and Tulare Counties, and south to Monterey and Kern Counties. (Ref. 74, 578.)

1988. June 27. Near Santa Cruz, Calif. Slight damage occurred at Holy City (near Los Gatos, Santa Cruz County), where cracks formed in plaster, drywall, and a house foundation. Press reports described minor damage in Santa Clara County, at the Sunnyvale Town Center, where chunks of concrete fell from the parking garage, and at Los Gatos, where storefront windows were shattered. After the earthquake, the flow of water increased in a well at Holy City. Felt north along the coast to Marin County, south to Monterey County, and east to Fresno County. (Ref. $74,578$.

1988. Dec. 3. Near Whittier, Los Angeles County, Calif. Windows were broken at Whittier, and hairline cracks formed in plaster, drywall, and a house foundation. Several bricks were knocked to the ground at the San Gabriel Mission. Press reports noted that several people were injured in the San Fernando Valley and that electricity was knocked out briefly. Also felt in Kern, Orange, Riverside, San Bernardino, San Diego, and Ventura Counties. Several small aftershocks were reported. (Ref. 578,602 .)

1989. Jan. 19 (Jan. 18). Off the coast near Malibu, Los Angeles County, Calif. An earthquake off the coast of California, south of Malibu, caused minor damage in Los Angeles County at Hollywood, Lancaster, Malibu, Monterey Park, and Whittier. Typical damage included broken windows; cracks in chimneys, foundations, fences, and walls; and damaged stock in stores. An observer at Monterey Park also reported that large amounts of plaster fell from walls and that some walls fell. Rocks rolled onto the highway in Santa Monica and on Malibu Canyon Road. According to press accounts, this shock was felt from Santa Barbara south to San Diego and east to San Bernardino. Several aftershocks occurred. (Ref. 579, 603.)

1989. Apr. 3. Northeast of San Jose, Santa Clara County, Calif. At San Jose and Santa Clara, many cracks formed in drywall, plaster, and foundations of houses. One large window was knocked out of an airport control tower in San Jose. Felt north to Marin and Solano Counties, south to San Luis Obispo County, and east to Stanislaus County. (Ref. 74,579 .)

1989. Apr. 7. Near Newport Beach, Orange County, Calif. Slight damage was reported in Orange County at Corona Del Mar, Costa Mesa, and Newport Beach. Typical damage consisted of broken windows; fallen bricks from chimneys; cracked chimneys, walls, and plaster; and damaged merchandise in stores. In addition, ceiling tiles fell in several stores in Newport Beach, and part of a wall of a brick building toppled. Felt north to Los Angeles County, south to San Diego County, and east to Riverside and San Bernardino Counties. (Ref. 579, 603.)

1989. June 12. Near Bell Gardens, Los Angeles County, Calif. Minor damage reported west of Whittier, at Bell Gardens, included large cracks in exterior walls of a reinforced-concrete building and hairline cracks in plaster and drywall. Slight damage (fallen plaster and cracked ceiling) also was reported in the downtown Los Angeles area. Also felt in Orange, Riverside, San Bernardino, and Ventura Counties. An aftershock was felt about one-half hour after the main shock. (Ref. 579, 603.)

1989. Aug. 8. Near Redwood Estates, Santa Clara County, Calif. This earthquake caused one death and moderate damage to property in Santa Clara County near San Jose. Toppled and broken chimneys were reported at Cupertino (west of San Jose), Los Gatos (about $15 \mathrm{~km}$ northeast of the epicenter), and Redwood Estates (about $7.5 \mathrm{~km}$ northeast of the epicenter). Other damage observed included broken underground pipes, cracks in foundations and walls, walls separated from ceilings, and toppled water heaters and propane tanks. Light damage occurred at several other towns in Santa Clara and Santa Cruz Counties, including Ben Lomond, Brookdale, Holy City, Santa Cruz, and Saratoga. Felt to Sonoma County in the north, San Luis Obispo County in the south, and Stanislaus County in the east. (Ref. 74, 579.)

1989. Oct. 18, 00 04 UTC (Oct. 17). In the Santa Cruz Mountains in the forest of Nisene Marks State Park, about $16 \mathrm{~km}$ northeast of Santa Cruz and about $7 \mathrm{~km}$ south of Loma Prieta Mountains, Calif. This major earthquake 


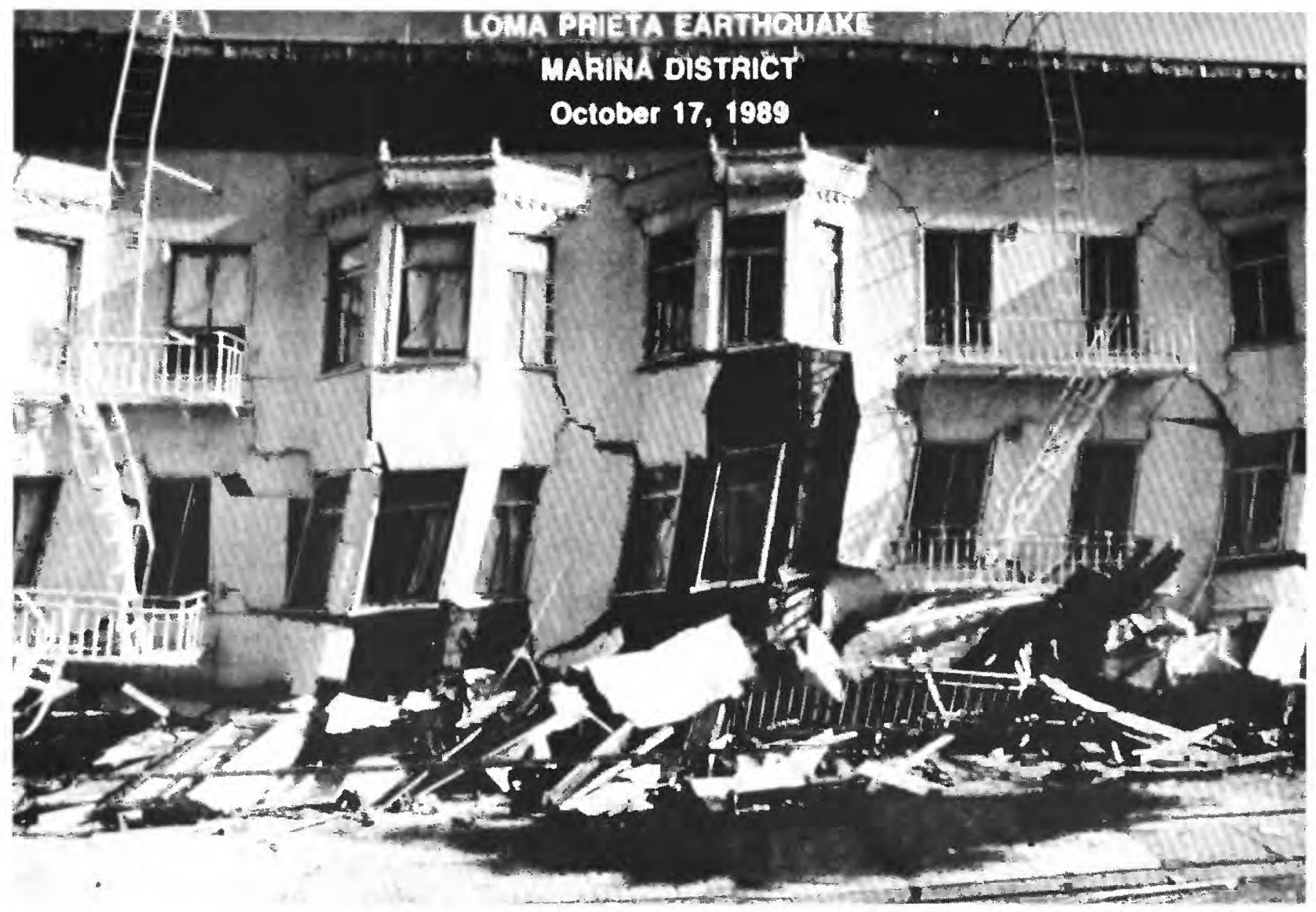

Collapsed apartment building in the Marina District, San Francisco, California, caused by the October 18, 1989 (Oct. 17 PST), Santa Cruz Mountains (Loma Prieta) earthquake. (Photograph by the Earthquake Engineering Research Institute.)

caused 63 deaths, 3,757 injuries, and an estimated $\$ 6$ billion in property damage. It was the largest earthquake to occur on the San Andreas fault since the great San Francisco earthquake in April 1906.

The most severe property damage occurred in Oakland and San Francisco, about $100 \mathrm{~km}$ north of the fault segment that slipped on the San Andreas. MM intensity IX was assigned to San Francisco's Marina District, where several houses collapsed, and to four areas in Oakland and San Francisco, where reinforced-concrete viaducts collapsed: Nimitz Freeway (Interstate 880) in Oakland, and Embarcadero Freeway, Highway 101, and Interstate 280 in San Francisco. Communities sustaining heavy damage in the epicentral area included Los Gatos, Santa Cruz, and Watsonville.

Liquefaction, as evidenced by sand boils, lateral spreading, settling, and slumping, occurred as far as $110 \mathrm{~km}$ from the epicenter. It caused severe damage to buildings in San Francisco's Marina district as well as along the coastal areas of Oakland and Alameda in the east San Francisco Bay shore area. Liquefaction also contributed significantly to the property damage in the Santa Cruz and Monterey Bay areas, which lie near the epicentral zone. Structures damaged by liquefaction include buildings, bridges, highways, pipelines, port facilities, airport runways, and levees. Subsurface soil conditions, which amplified accelerations in the San Francisco Bay area, strongly influenced structural damage patterns and probably contributed to liquefaction problems in loose, sandy fills underlain by deep, cohesive soil deposits.

Engineered buildings, including those near the epicenter, performed well during the earthquake. Hospital buildings in the region sustained only minor system and cosmetic damage, and operational interruptions did not occur. Only five schools sustained severe damage, estimated at $\$ 81$ million. 


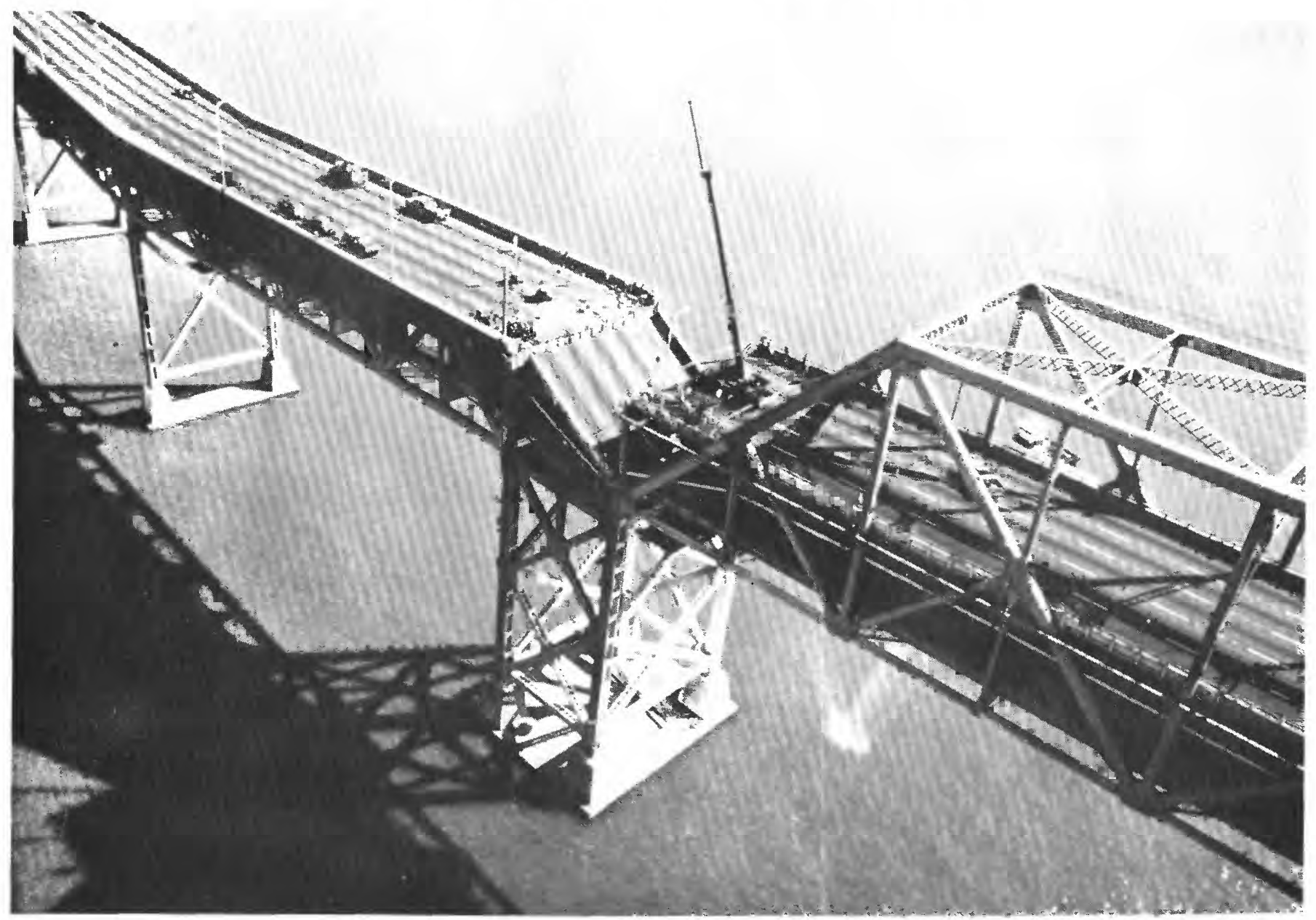

Span of the San Francisco-Oakland Bay bridge, collapsed by the October 18, 1989 (Oct. 17 PST), Santa Cruz Mountains (Loma Prieta), California, earthquake. (Photograph by E.V. Leyendecker.)

Most of the spectacular damage to buildings was sustained by unreinforced masonry buildings constructed of wood-frame roof and floor systems supported by unreinforced brick walls. These structures failed in areas near the epicenter as well as in areas far from the epicenter, at San Francisco and Monterey. The severe shaking near Santa Cruz caused heavy damage to the unreinforced masonry buildings in that area, particularly in the Santa Cruz Pacific Garden Mall, which consisted of several blocks of unreinforced masonry store buildings.

More than 80 of the 1,500 bridges in the area sustained minor damage, 10 required temporary supports, and 10 were closed owing to major structural damage. One or more spans collapsed on three bridges. The most severe damage occurred to older structures on poor ground, such as the Cypress Street Viaduct (41 deaths) and the San Francisco-Oakland Bay Bridge (one death). Damage to the transpotation system was estimated at $\$ 1.8$ billion.

Most of the more than 1,000 landslides and rockfalls occurred in the epicentral zone in the Santa Cruz Mountains. One slide, on State Highway 17, disrupted traffic for about 1 month.

The earthquake produced a pattern of northwesttrending extensional fractures in the north end of the aftershock zone northwest of the epicenter, but throughgoing right-lateral surface faulting was not found above the rupture defined by the main shock and its aftershocks. Six feet of right-lateral strike-slip 


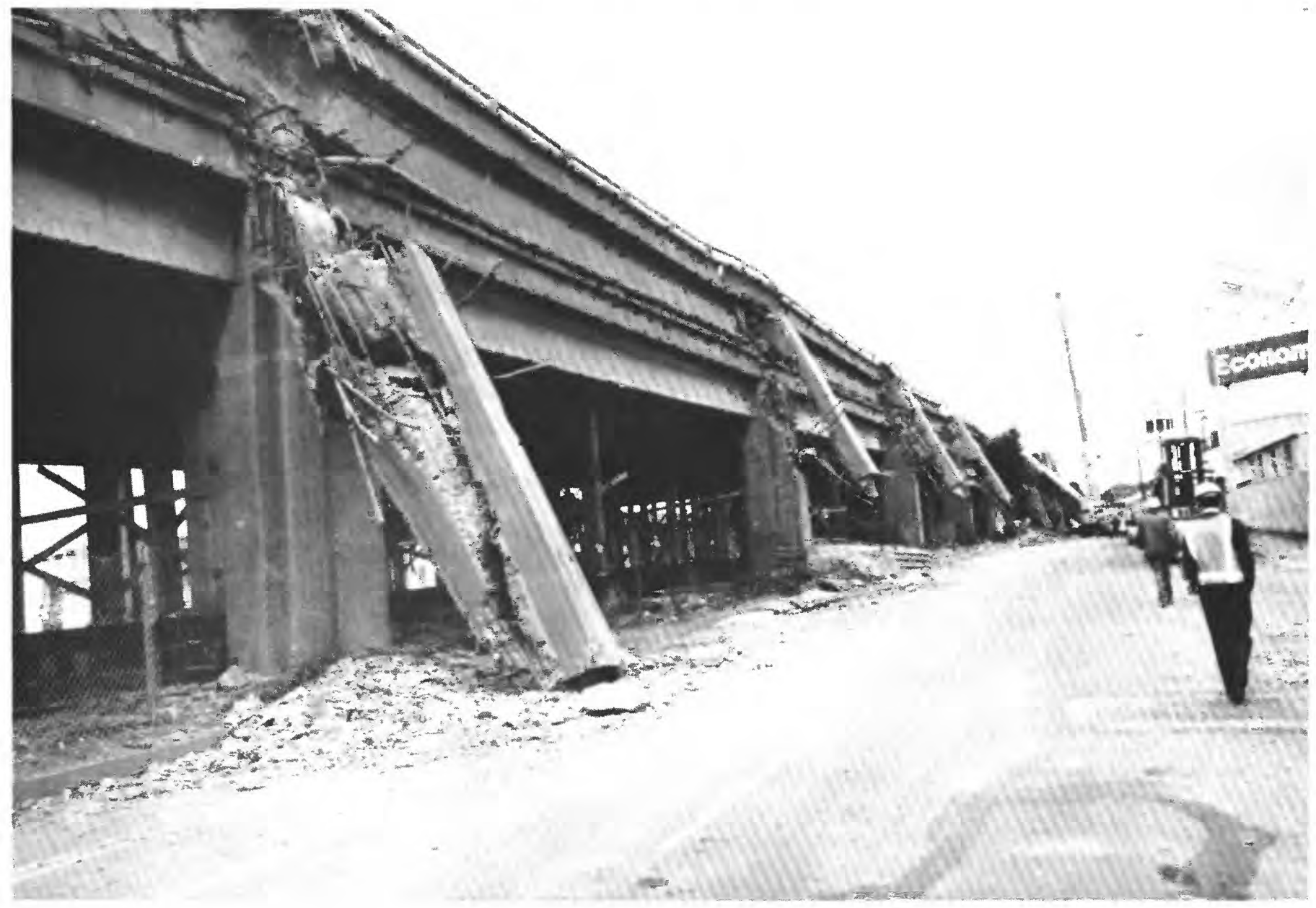

Nimitz Freeway along Cypress Street (Interstate 880) in Oakland, California, collapsed by the October 18, 1989 (Oct. 17 PST), Santa Cruz Mountains (Loma Prieta) earthquake. (Photograph by E.V. Leyendecker.)

and 4 feet of reverse-slip was inferred from geodetic data. The only surface fracturing that might be attributed to primary tectonic faulting occurred along a trace of the San Andreas near Mount Madonna Road in the Corralitos area, where en echelon cracks showed $2 \mathrm{~cm}$ of right-lateral displacement.

Extensional fractures (maximum net displacement of $92 \mathrm{~cm}$ ) were observed about $12 \mathrm{~km}$ northwest of the epicenter, in the Summit Road-Skyland Ridge area, east of State Highway 17, whereas zones of compressional deformation were found along the northeast foot of the Santa Cruz Mountains between Blossom Hill and Palo Alto. In Los Altos and Los Gatos, ground deformation appeared to be associated closely with zones of heavy structural damage and broken underground utility lines.
Other towns in the area that also experienced severe property damage include Boulder Creek, Corralitos, Hollister, Moss Landing, and several smaller communities in the Santa Cruz Mountains.

This earthquake was felt over most of central California and in part of western Nevada (see fig. 24). The rate of aftershock activity decreased rapidly with time, but the total number of aftershocks was less than that expected from a generic California earthquake of similar magnitude. Fifty-one aftershocks of magnitude 3.0 and larger occurred during the first day after the main shock, and 16 occurred during the second day. After 3 weeks, 87 magnitude 3.0 and larger aftershocks had occurred. (Ref. 74, 574, 579, $594,595,596,606,607$.) 


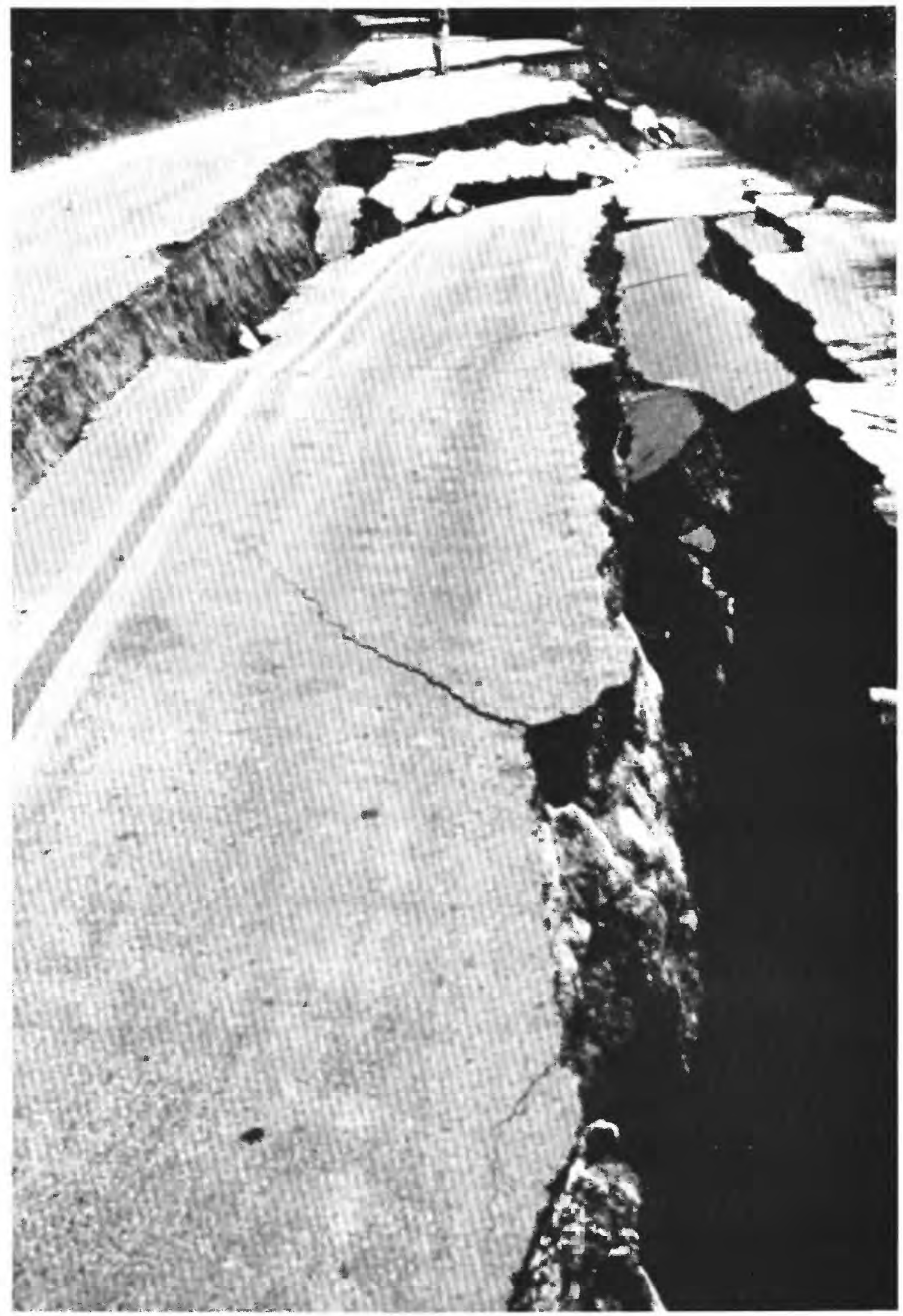

Ground failure along the San Andreas fault zone, causing slumping and cracking of Hazel Dell Road, north of Watsonville, California, during the October 18, 1989 (Oct. 17 PST), Santa Cruz Mountains (Loma Prieta) earthquake. 


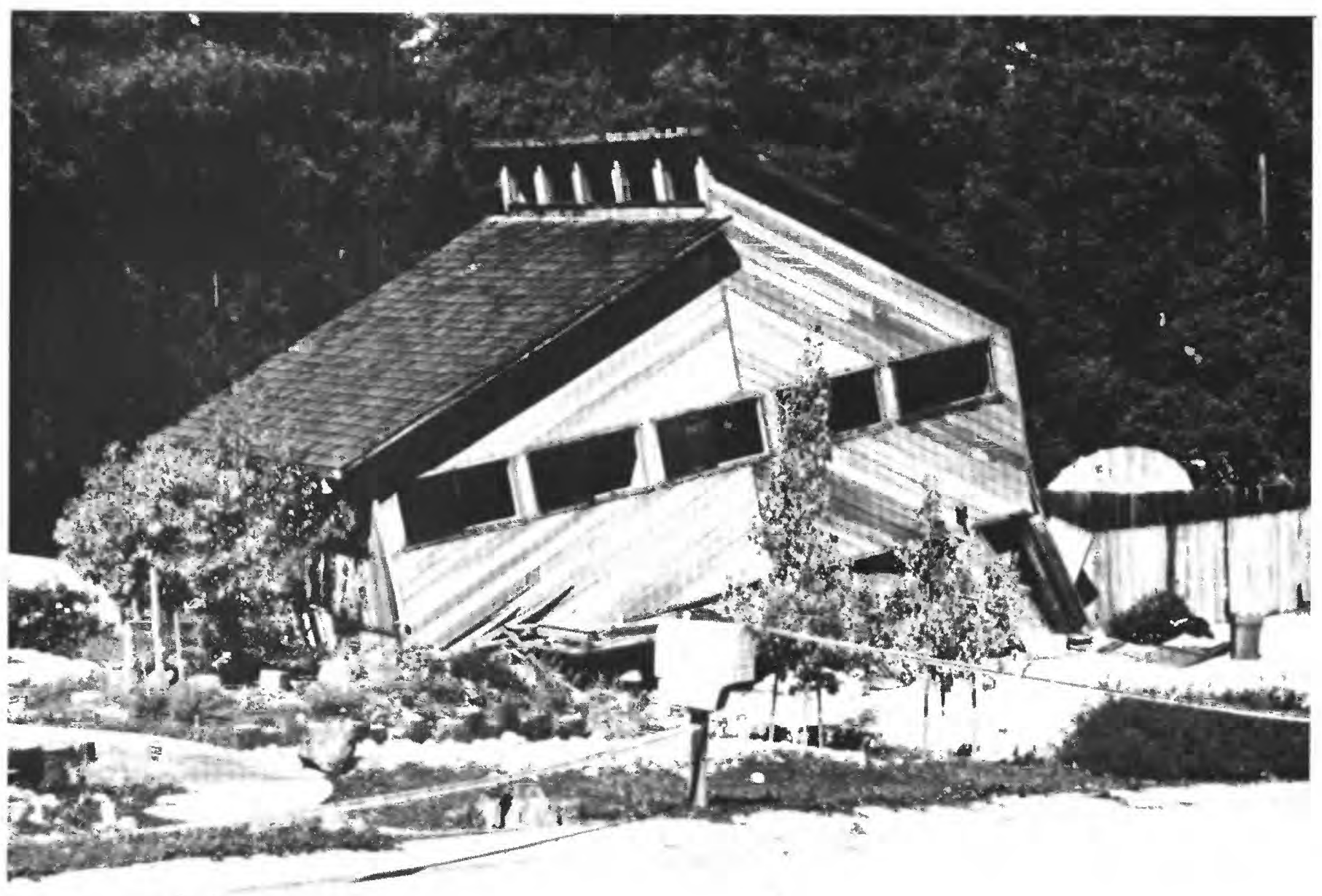

House on Rebecca Drive, Boulder Creek, California, destroyed by the October 18, 1989 (Oct. 17 PST), Santa Cruz Mountains (Loma Prieta) earthquake. 


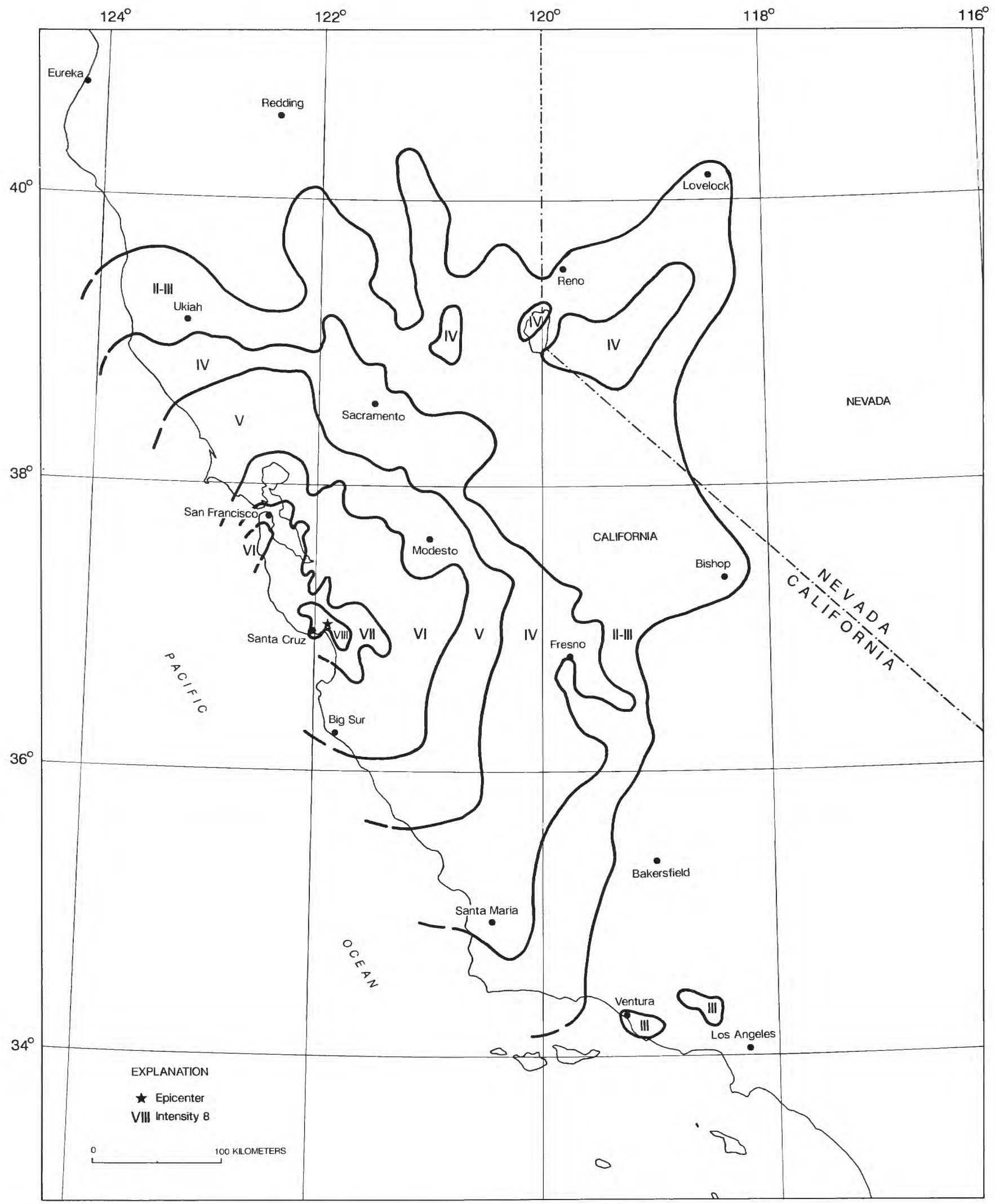

FiguRe 24.-Isoseismal map for the Santa Cruz Mountains (Loma Prieta), California, earthquake of October 18, 1989. This map is a simplified version of figure 1 in reference 574 of table 1. 


\section{COLORADO}

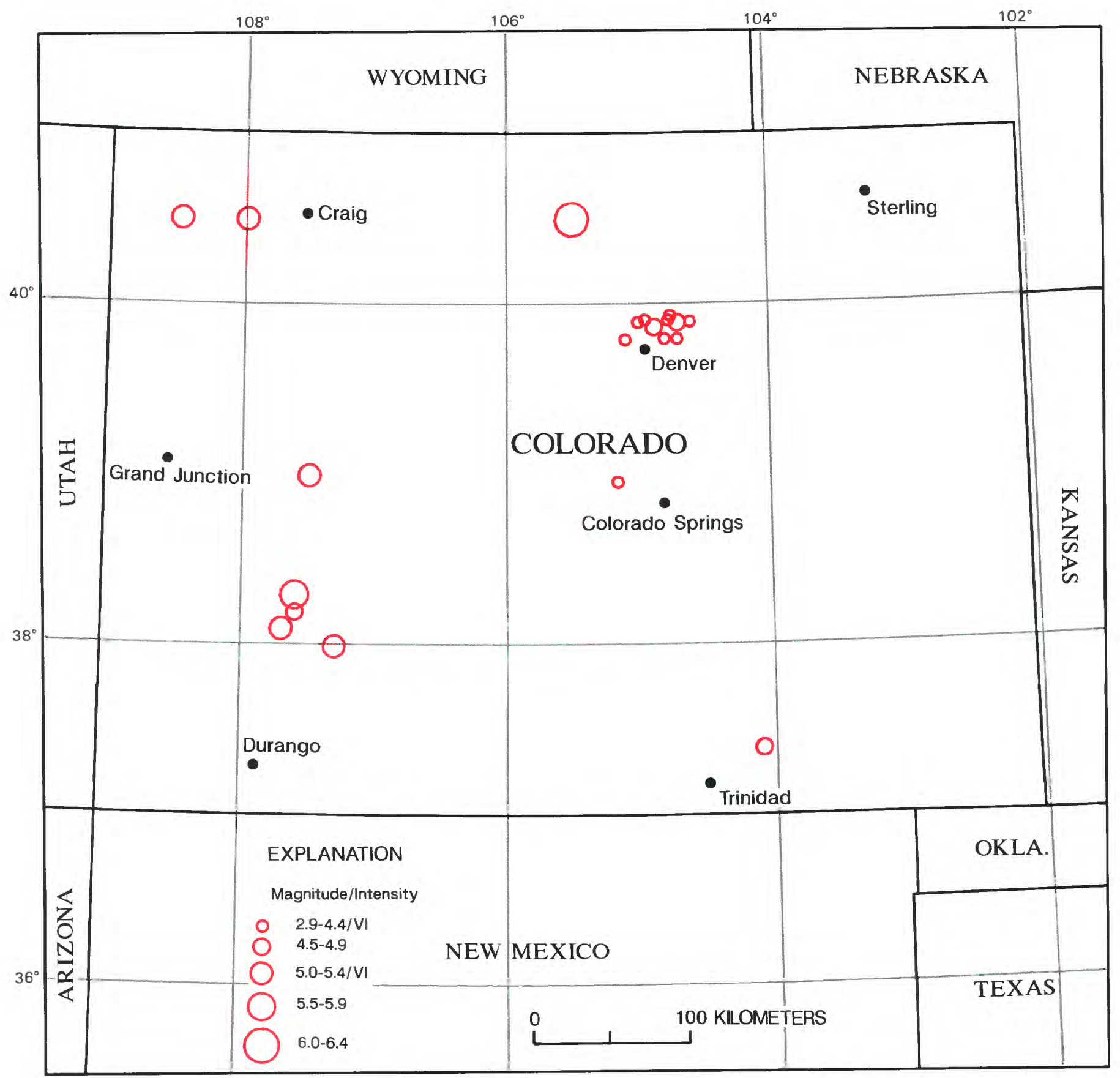

Earthquakes in Colorado with magnitudes $\geq 4.5$ or intensity $\geq$ VI. 
COLORADO

[See table 1 for hypocenter and intensity references and table 2 for definitions of magnitude source codes. Leader (--) indicates information is not available]

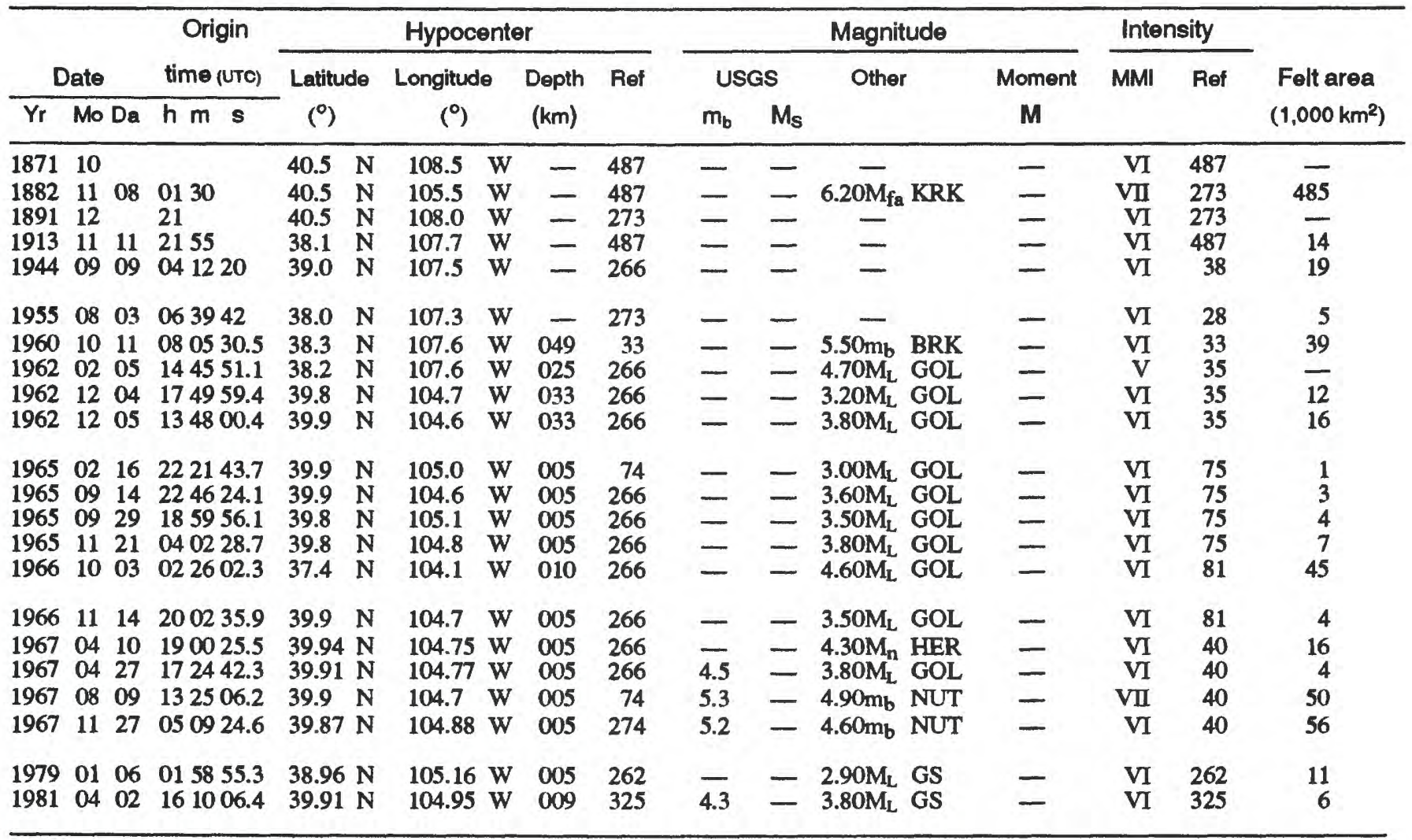

[Reference (Ref.) numbers given in parentheses at the end of each description refer to sources of data in table 1. Magnitude values are described in the Introduction, and codes are defined in table 2.]

1871. October. Lily Park, Moffat County, Colo. An avalanche of rock cascaded from the high cliffs of the canyon walls at Lily Park. The ground heaved and fell. It is possible that the date of this earthquake is Nov. 9, 1871. (Ref. 487.)

1882. Nov. 8 (Nov. 7). Near Denver, Colo. This earthquake probably was the largest event to occur in Colorado in the period of historic record. It caused minor damage in Colorado and southern Wyoming and was felt slightly in Utah and Kansas (see fig. 25 ). The location of this earthquake is very uncertain and has been postulated to have occurred in western Colorado or southern Wyoming.

In Denver, electricity was cut off after an iron bolt that connected an engine-driving pulley was broken in two at the electric power building; another bolt was bent out of shape. Buildings trembled violently and residents ran out of doors. Plaster fell and windows broke as far north as Laramie, Wyo., and plaster fell from the ceiling of a building at the University of Colorado in Boulder. Observers also reported that the walls of the railroad depot in Louisville were cracked, that timbers cracked in a house at La Porte, and that walls of one house were cracked severely and plaster fell near Thompson, Colo. An aftershock on Nov. 8 was reported to be almost as strong as the main shock in Laramie and Denver. (Ref. 273, 283, 440, 487, 488, 499.)

1891. Dec. Lily Park, Moffat County, Colo. The earth was convulsed by waves that rolled at intervals of a few seconds. Thousands of tons of boulders rolled down Fitzpatrick's Cliff. A house moved, calendars and pictures on the walls oscillated, dishes rattled, and people ran outside. A cow was knocked against a house. (Ref. 273, 487, 488.)

1913. Nov. 11. Near Ridgway, Ouray County, Colo. Damage at Ridgway included damage to the school ceiling and broken dishes. The shock was reported as strong at towns near Ridgway, including Montrose, Ouray, and Telluride; rocks rolled down cliffs at Ouray. Three shocks were reported. (Ref. 38, $487,488$. 


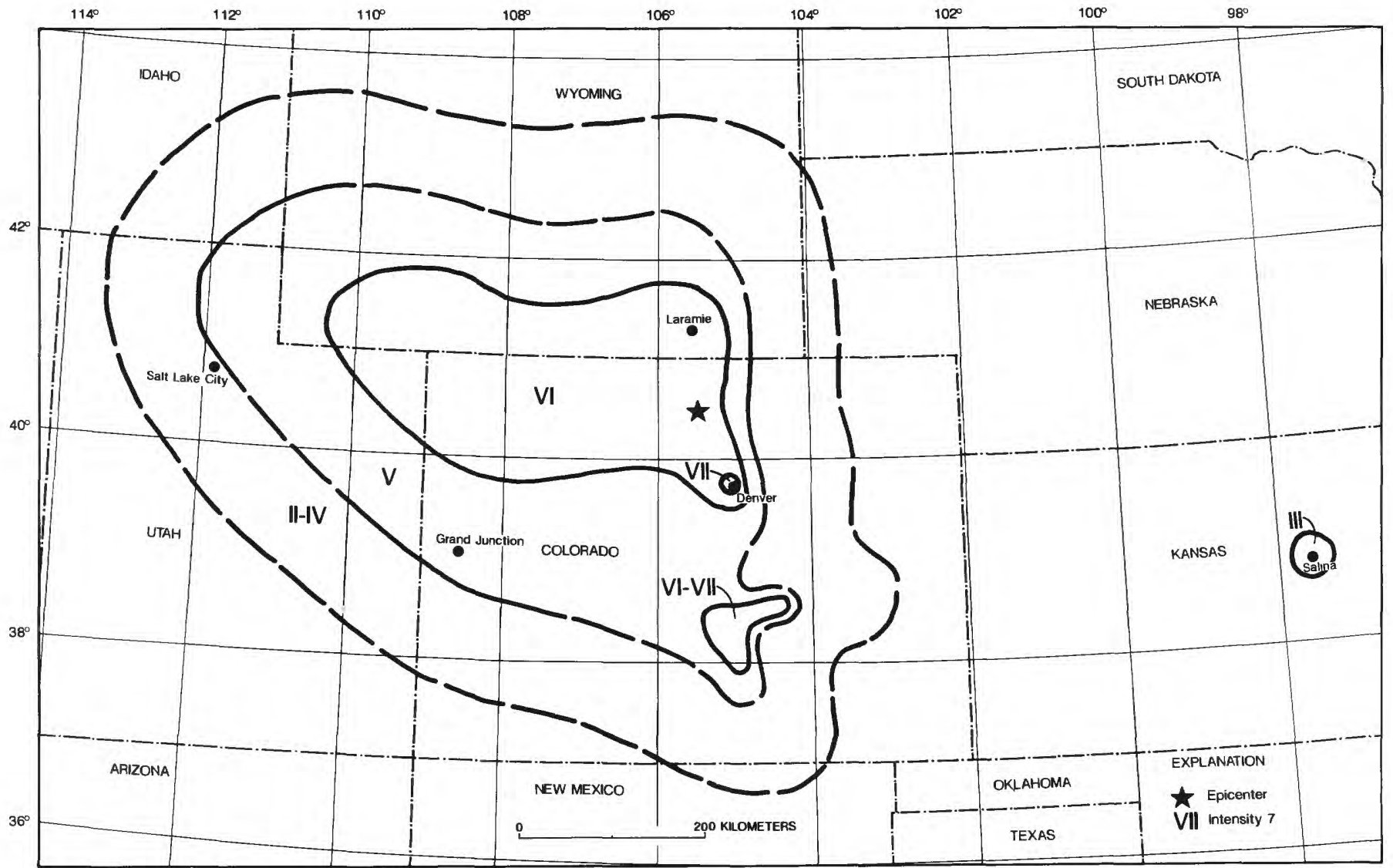

FIGURE 25.-Isoseismal map for the Colorado earthquake of November 8, 1882. This map is a simplified version of figure 1 in reference 499 of table 1.

1944. Sept. 9 (Sept. 8). Near Basalt, southwest Eagle County, Colo. Walls and chimneys cracked at Basalt; bricks fell from chimneys; and rocks rolled onto the road. A strongly built log house was moved slightly out of line at Riland. (Ref. 17,38 , $266,487,488$.)

1955. Aug. 3 (Aug. 2). Lake City, Hinsdale County, Colo. Residents at Lake City reported cracks formed in a chimney and in the ground; one chimney fell. Also felt at Ouray, west of Lake City; Silverton (San Juan County), southwest of Lake City; and Creede (Mineral County), southeast of Lake City. (Ref. 28, 273, 487.)

1960. Oct. 11. Near Montrose, Colo. At Montrose, southeast of Grand Junction, a foundation cracked in three places in a house, and all cupboards loosened from walls. Damage at nearby towns consisted of a cracked chimney at Ophir, cracked and broken windows at Placerville and Powderhorn, fallen plaster at Lake City, and cracked walls at Telluride. (Ref. 33, 487.)
1962. Dec. 4. Near Denver, Colo. At Dupont, a picture window broke and a bed moved $15 \mathrm{~cm}$ from the wall. At Irondale, windows broke at a school, electrical wall outlets were left hanging by their wires, and brick tiles loosened. (Ref. 35, 266, 487.)

1962. Dec. 5. Near Denver, Colo. This earthquake caused cracks in plaster and a wall in the Derby-Dupont area, about $13 \mathrm{~km}$ northeast of Denver. Plaster cracked in the Derby area. (Ref. 35, 266, 487.)

1965. Feb. 16. Near Denver, Colo. At Northglenn, a large crack formed in one house. At Commerce City, a washer moved from the wall and furnishings shifted. (Ref. 74, 75, 487.)

1965. Sept. 14. Near Denver, Colo. Plaster cracked and dishes and windows broke at Denver; chimneys and plaster cracked at Broomfield; and plaster cracked in the Commerce City-Derby area. Felt from $40 \mathrm{~km}$ south of Denver northwest to Boulder. (Ref. 75, 266, 487.)

1965. Sept. 29. Near Denver, Colo. Plaster and windows cracked at Commerce City, a refrigerator 


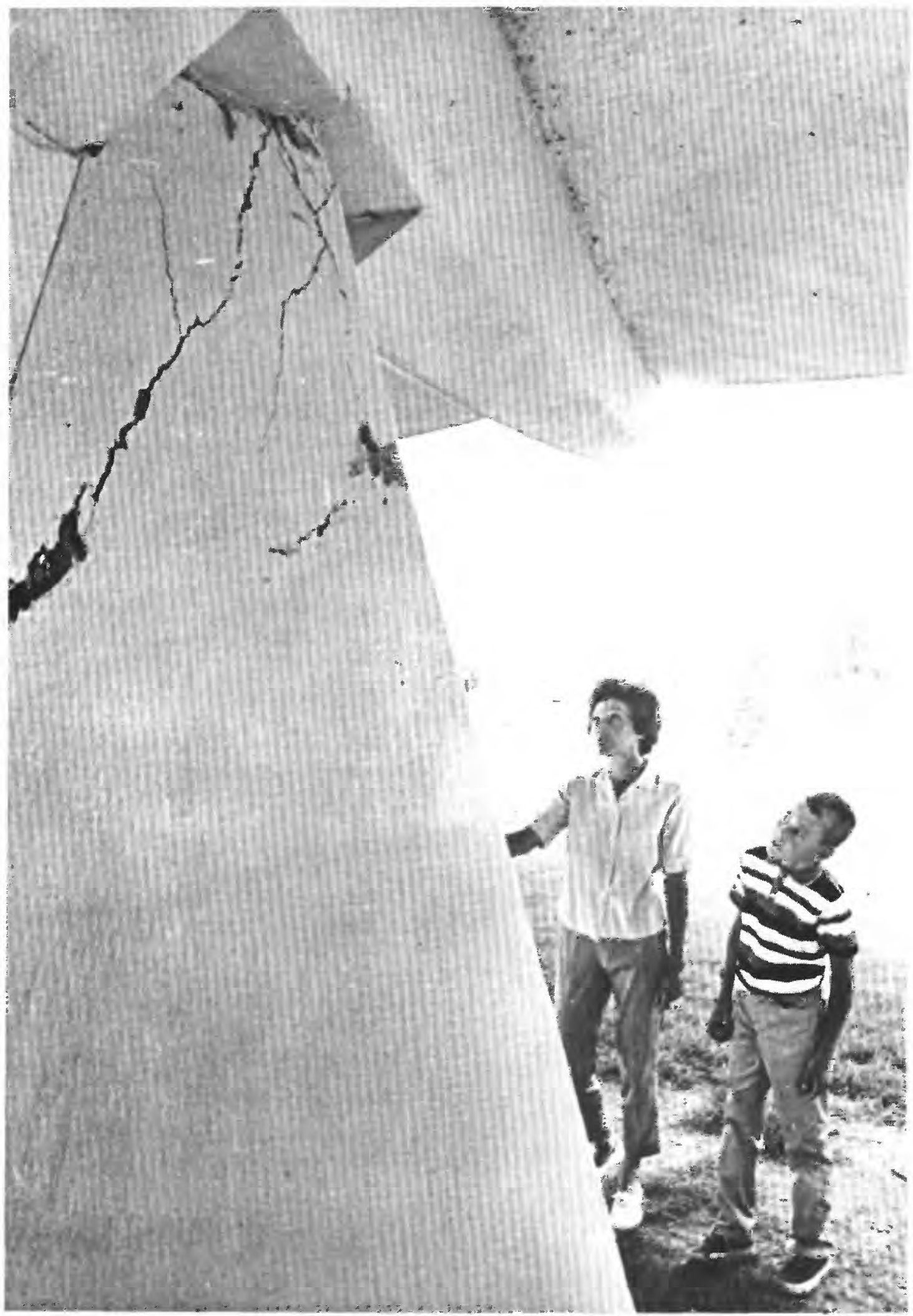

Cracks in highway overpass pillar in the Denver, Colorado, area caused by the August 9, 1967, earthquake. (Photograph by the Denver Post.) 
was moved several inches at Northglenn. The shock was felt from Denver northwest to Boulder and Nederland. (Ref. 75, 266, 487.)

1965. Nov. 21 (Nov. 20). Near Denver, Colo. The earthquake broke many windows at Northglenn and Thornton and cracked plaster at Commerce City (one window also broke), Hudson (fallen plaster), and Louisville. (Ref. 75, 266, 487.)

1966. Oct. 3 (Oct. 2). Near Trinidad, Las Animas County, Colo. Minor damage reported in Las Animas County includes cracks in houses at Aguilar and Segundo, cracks in plaster and windows at Trinchera, and broken windows and cracks in plaster at Trinidad. Felt over a large area of southeast Colorado and northeast New Mexico. Magnitude 3.6 $\mathrm{M}_{\mathrm{S}}$ NUT (Ref. 81, 263, 266, 487.)

1966. Nov. 14. Near Denver, Colo. A slight earthquake in the Denver area cracked plaster and lengthened old cracks in Commerce City and knocked merchandise from shelves in a supermarket. (Ref. 81, 266, 487.)

1967. Apr. 10 Near Denver, Colo. In the Denver-Commerce City region, plaster cracked and fell, windows broke, and a house foundation cracked. North of Denver, at the Rocky Mountain Arsenal, 118 small windows were broken; at Boulder, walls cracked at a high school. Water pipes broke at one Commerce City residence, and cracks in a parking lot were reported in the Derby area of Commerce City. Minor damage occurred in several other towns in the area, including Brighton, Golden, Lafayette, Lakewood, Thornton, and Westminster. (Ref. 40, 266, 487.)

1967. Apr. 27. Near Denver, Colo. This earthquake caused slight damage in Commerce City and Boulder. Plaster cracked and nails were forced out of walls at Commerce City, and walls and a tile ceiling were cracked at Boulder. (Ref. 40, 266, 487.)
1967. Aug. 9. Near Denver, Colo. The main damage occurred in Northglenn, a northern suburb of Denver, but minor damage occurred in many area towns. At Northglenn, concrete pillars were damaged at a church; foundations, concrete floors, and walls cracked; windows broke; and tile fell at a school. At one residence, a piano shifted about $15 \mathrm{~cm}$ and a television set overturned. Some bricks fell from a chimney in downtown Denver, damaging a car. This was the largest of a series of earthquakes in the northeast Denver area that were believed to be induced by pumping of waste fluids into a deep disposal well at the Rocky Mountain Arsenal. The Colorado School of Mines recorded more than 300 earthquakes from this zone during 1967. Felt north to Laramie, Wyo., south to Pueblo, west to Vail, and east to Sterling. Magnitude 4.4 M NUT (Ref. 40, 74, 263, 487.)

1967. Nov. 27 (Nov. 26). Near Denver, Colo. Damage occurred mainly in the suburban area of northeast Denver, at Commerce City. It consisted chiefly of cracked plaster, enlargement of existing cracks, and loss from fallen merchandise in stores. Masonry walls and basement floors in the area also were cracked. Felt north to Laramie, Wyo., south to Pueblo, west to Glenwood Springs, and east to Sterling. (Ref. 40, 263, 274, 487.)

1979. Jan. 6 (Jan. 5). Cripple Creek, Teller County, Colo. Plaster cracked and furniture shifted at Cripple Creek, west of Colorado Springs. Also felt in Florissant and the nearby area. Magnitude $3.3 \mathrm{M}_{\mathrm{n}}$ TUL. (Ref. 262, 487.)

1981. Apr. 2. Near Denver, Colo. At Thornton, cracks formed in plaster and concrete-block walls. At Commerce City, many large cracks occurred in plaster walls. Magnitude $4.5 \mathrm{M}_{\mathrm{n}}$ TUL. (Ref. $\left.325,487.\right)$ 

CONNECTICUT

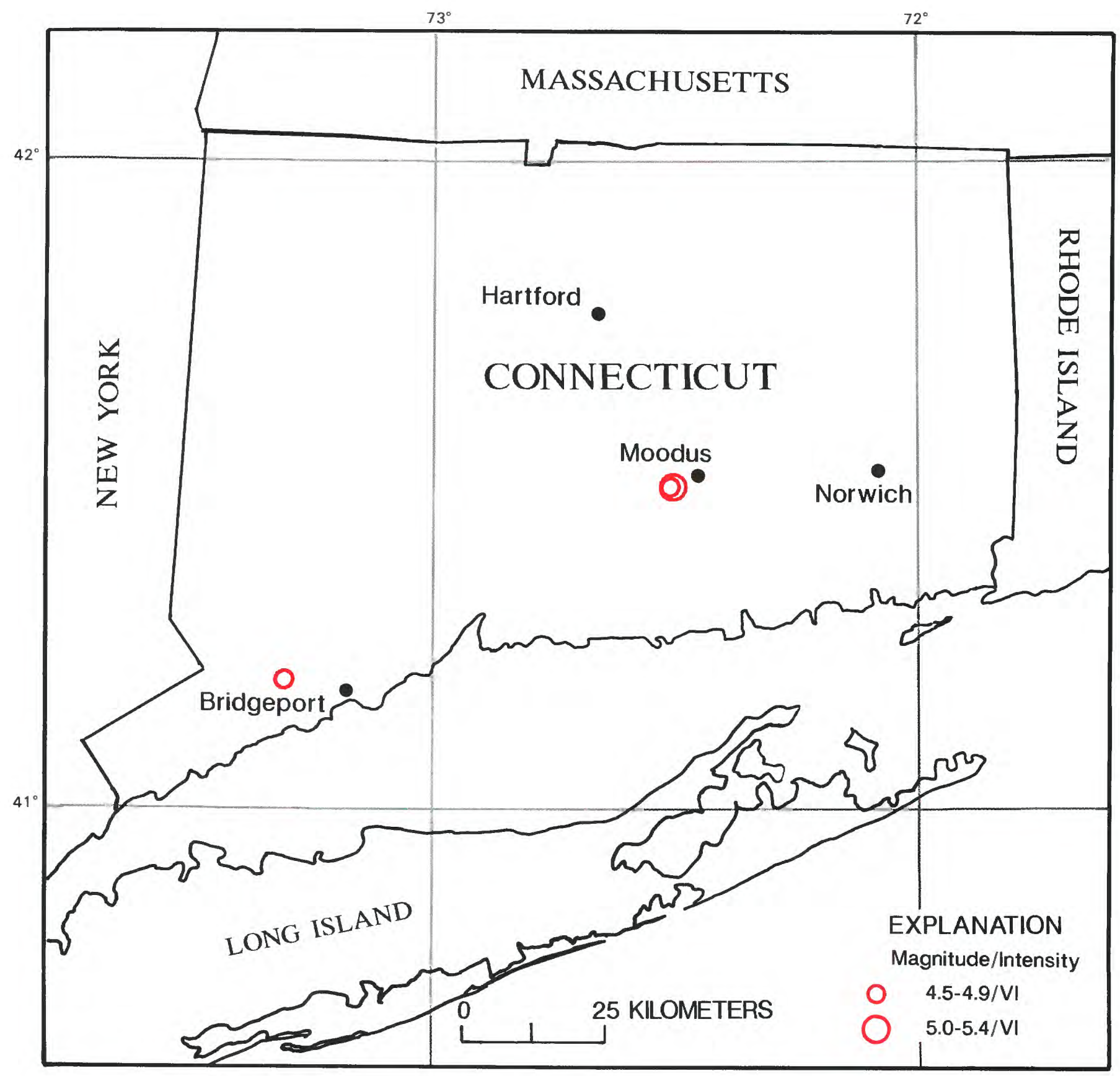

Earthquakes in Connecticut with magnitudes $\geq 4.5$ or intensity $\geq$ VI. 
CONNECTICUT

[See table 1 for hypocenter and intensity references and table 2 for definitions of magnitude source codes. Leader (--) indicates information is not available]

\begin{tabular}{|c|c|c|c|c|c|c|c|c|c|c|c|c|c|c|c|c|}
\hline \multirow{2}{*}{\multicolumn{2}{|c|}{ Date }} & \multirow{2}{*}{\multicolumn{2}{|c|}{$\begin{array}{c}\text { Origin } \\
\text { time (UTC) }\end{array}$}} & \multicolumn{6}{|c|}{ Hypocenter } & \multicolumn{4}{|c|}{ Magnitude } & \multicolumn{2}{|c|}{ Intensity } & \multirow{3}{*}{$\begin{array}{c}\text { Felt area } \\
\left(1,000 \mathrm{~km}^{2}\right)\end{array}$} \\
\hline & & & & \multirow{2}{*}{\multicolumn{2}{|c|}{$\begin{array}{l}\text { Latitude } \\
\left(^{\circ}\right)\end{array}$}} & \multirow{2}{*}{\multicolumn{2}{|c|}{$\begin{array}{l}\text { Longitude } \\
\left({ }^{\circ}\right)\end{array}$}} & \multirow{2}{*}{$\begin{array}{l}\text { Depth } \\
(\mathrm{km})\end{array}$} & \multirow[t]{2}{*}{ Ref } & \multicolumn{2}{|c|}{ USGS } & \multirow[t]{2}{*}{ Other } & \multirow{2}{*}{$\begin{array}{c}\text { Moment } \\
\mathbf{M}\end{array}$} & \multirow[t]{2}{*}{ MMI } & \multirow[t]{2}{*}{ Ref } & \\
\hline$\overline{Y r}$ & Mo Da & $\mathrm{h} \mathrm{m}$ & $s$ & & & & & & & $m_{b}$ & $M_{s}$ & & & & & \\
\hline $\begin{array}{l}1568 \\
1791 \\
1845\end{array}$ & $\begin{array}{ll}05 & 16 \\
10 & 26\end{array}$ & $\begin{array}{l}1300 \\
2315\end{array}$ & & $\begin{array}{l}41.5 \\
41.5 \\
41.2\end{array}$ & $\begin{array}{l}\mathbf{N} \\
\mathbf{N} \\
\mathbf{N}\end{array}$ & $\begin{array}{l}72.5 \\
72.5 \\
73.3\end{array}$ & $\begin{array}{l}\text { W } \\
\text { W } \\
\text { W }\end{array}$ & I & $\begin{array}{r}126 \\
78 \\
78\end{array}$ & E & & - $=$ & ${ }^{4.85 \mathrm{JOH}}$ & $\begin{array}{l}\text { VI } \\
\text { VII } \\
\text { VI }\end{array}$ & $\begin{array}{r}126 \\
78 \\
76\end{array}$ & E \\
\hline
\end{tabular}

[Reference (Ref.) numbers given in parentheses at the end of each description refer to sources of data in table 1.]

1568. Date unknown. Moodus-East Haddam, Middlesex County, Conn. Ref. 126 assigns MM intensity VI to this earthquake. The original source of the intensity data is the Massachusetts Historical Society Collection, 4th series, v. 6, 1863, by R. Williams. (Ref. 126.)

1791. May 16. Near Moodus, Middlesex County, Conn. The region around East Haddam, on the Connecticut River northeast of New Haven, has been the scene of a series of local disturbances since this country was settled. The region southeast of Middletown has been referred to in Indian tradition as Morehemoodus, or "place of noises." The first reported earthquake began on May 16 with two heavy shocks in quick succession. Stone walls were shaken down, tops of chimneys were knocked off, and latched doors were thrown open. A fissure several meters long formed in the ground. In a short time, 30 lighter shocks occurred, and more than 100 continued during the night. Reported felt at Boston, Mass., and New York City, N.Y. (Ref. 38, $76,78$.

1845. Oct. 26. Near Stamford, Fairfield County, Conn. The shock was severe in Fairfield County, Conn. At Stamford and Weston, sections of a stone fence were damaged; at Huntington, books were thrown from a table; and at Greenfield Hill, milk pans were thrown down. Also felt in New Jersey and New York. (Ref. 76, 78.) 


\section{DELAWARE}

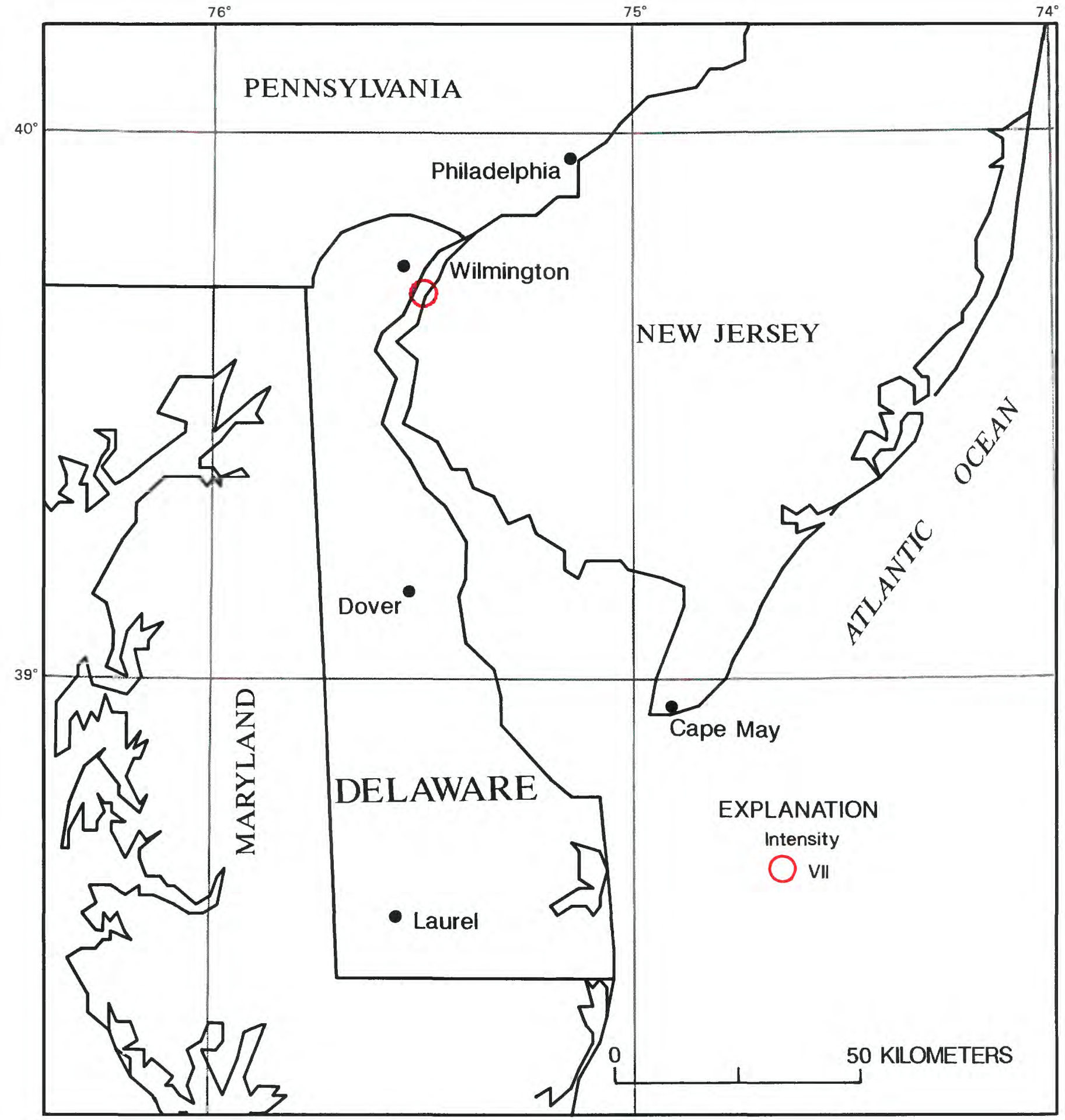

Damaging earthquake in Delaware, intensity $\geq$ VI. 


\section{DELAWARE}

[See table 1 for hypocenter and intensity references and table 2 for definitions of magnitude source codes. Leader (--) indicates information is not available]

\begin{tabular}{|c|c|c|c|c|c|c|c|c|c|c|c|c|c|c|}
\hline \multirow{2}{*}{\multicolumn{2}{|c|}{ Date }} & \multirow{2}{*}{\multicolumn{2}{|c|}{$\begin{array}{c}\text { Origin } \\
\text { time (UTC) }\end{array}$}} & \multicolumn{4}{|c|}{ Hypocenter } & \multicolumn{4}{|c|}{ Magnitude } & \multicolumn{2}{|c|}{ Intensity } & \multirow{3}{*}{$\begin{array}{c}\text { Felt area } \\
\left(1,000 \mathrm{~km}^{2}\right)\end{array}$} \\
\hline & & & & \multirow{2}{*}{$\begin{array}{l}\text { Latitude } \\
\left({ }^{\circ}\right)\end{array}$} & \multirow{2}{*}{$\begin{array}{c}\text { Longltude } \\
\left({ }^{\circ}\right)\end{array}$} & \multirow{2}{*}{$\begin{array}{l}\text { Depth } \\
(\mathbf{k m})\end{array}$} & \multirow[t]{2}{*}{ Ref } & \multicolumn{2}{|c|}{ USGS } & \multirow[t]{2}{*}{ Other } & \multirow{2}{*}{$\begin{array}{c}\text { Moment } \\
\text { M }\end{array}$} & \multirow[t]{2}{*}{ MMI } & \multirow[t]{2}{*}{ Ref } & \\
\hline$Y_{r}$ & Mo Da & $\mathrm{h} \mathrm{m}$ & 8 & & & & & $m_{b}$ & $M_{8}$ & & & & & \\
\hline 1871 & 1009 & 1440 & & $39.7 \mathrm{~N}$ & $75.5 \mathrm{~V}$ & - & 38 & - & - & - & - & VII & 38 & - \\
\hline
\end{tabular}

[Reference (Ref.) number given in parentheses at the end of each description refer to sources of data in table 1. Magnitude values are described in the Introduction, and codes are defined in table 2.]

1871. Oct. 9. New Jersey-Delaware border area. Chimneys toppled and windows broke in northern Delaware at Wilmington. Damage also was reported at New Castle (10 km south of Wilmington) and at Oxford, $\mathrm{Pa}$. (about $40 \mathrm{~km}$ west of Wilmington). Also reported felt in New Jersey. (Ref. 38.) 


\section{FLORIDA}

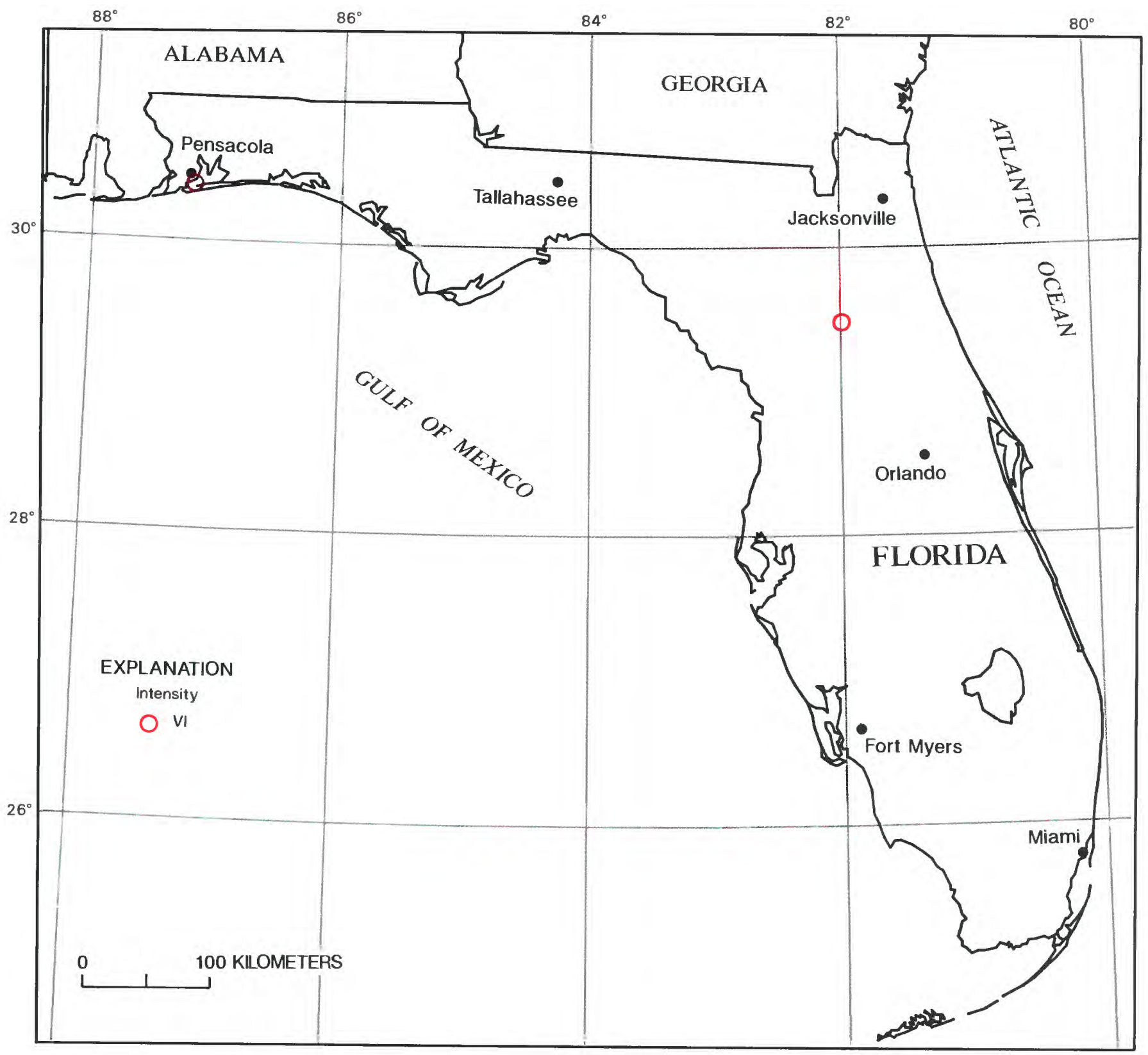

Damaging earthquakes in Florida, intensity $\geq$ VI. 
FLORIDA

[See table 1 for hypocenter and intensity references and table 2 for definitions of magnitude source codes. \&, land area only. Leader (-) indicates information is not available]

\begin{tabular}{|c|c|c|c|c|c|c|c|c|c|c|c|c|c|c|c|}
\hline \multirow{2}{*}{\multicolumn{2}{|c|}{ Date }} & \multirow{2}{*}{\multicolumn{2}{|c|}{$\begin{array}{l}\text { Origin } \\
\text { time (UTC) }\end{array}$}} & \multicolumn{5}{|c|}{ Hypocenter } & \multicolumn{4}{|c|}{ Magnitude } & \multicolumn{2}{|c|}{ Intensity } & \multirow{3}{*}{$\begin{array}{l}\text { Felt area } \\
\left(1,000 \mathrm{~km}^{2}\right)\end{array}$} \\
\hline & & & & \multirow{2}{*}{$\begin{array}{l}\text { Latitude } \\
\left({ }^{\circ}\right)\end{array}$} & \multirow{2}{*}{\multicolumn{2}{|c|}{$\begin{array}{l}\text { Longitude } \\
\left({ }^{\circ}\right)\end{array}$}} & \multirow{2}{*}{$\begin{array}{l}\text { Depth } \\
\text { (km) }\end{array}$} & \multirow[t]{2}{*}{ Ref } & \multicolumn{2}{|c|}{ USGS } & \multirow[t]{2}{*}{ Other } & \multirow{2}{*}{$\begin{array}{c}\text { Moment } \\
\text { M }\end{array}$} & \multirow[t]{2}{*}{ MMI } & \multirow[t]{2}{*}{ Rof } & \\
\hline Yr & Mo Da & $h \mathrm{~m}$ & $s$ & & & & & & $m_{b}$ & $M_{S}$ & & & & & \\
\hline $\begin{array}{l}1780 \\
1879\end{array}$ & $\begin{array}{ll}02 & 06 \\
01 & 13\end{array}$ & 0445 & & $\begin{array}{l}30.4 \mathrm{~N} \\
29.5\end{array}$ & $\begin{array}{l}87.2 \\
82.0\end{array}$ & $\begin{array}{l}\text { W } \\
\text { W }\end{array}$ & - & $\begin{array}{r}101 \\
38\end{array}$ & $=$ & & $=-$ & $=$ & $\begin{array}{l}\text { VI } \\
\text { VI }\end{array}$ & $\begin{array}{r}101 \\
38\end{array}$ & $\overline{25} \&$ \\
\hline
\end{tabular}

[Reference (Ref.) numbers given in parentheses at the end of each description refer to sources of data in table 1.]

1780. Feb. 6. Northwest Florida. Regimentals and arms racks fell from walls in many barracks; everything in the rooms was moved; doors were sprung. Chimneys were thrown together causing fires. Neighboring houses clashed together, and people buried in the ruins cried for help. This event occurred during a "fearful" storm that was accompanied by violent thunder and lightning and raging seas. Possibly a hurricane. (Ref. 101.)

1879. Jan 13 (Jan. 12). Near St. Augustine, St. Johns County, Fla. Plaster was shaken down and articles were thrown from shelves at St. Augustine and, to the south, at Daytona Beach. At Tampa, a trembling motion was preceded by a rumbling sound. Felt from a line joining Tallahassee, Fla., to Savannah, Ga., on the north to a line joining Punta Rassa and Daytona Beach, Fla., on the south. Two shocks occurred, each lasting 30 seconds. (Ref. $38,101,134$.) 


\section{GEORGIA}

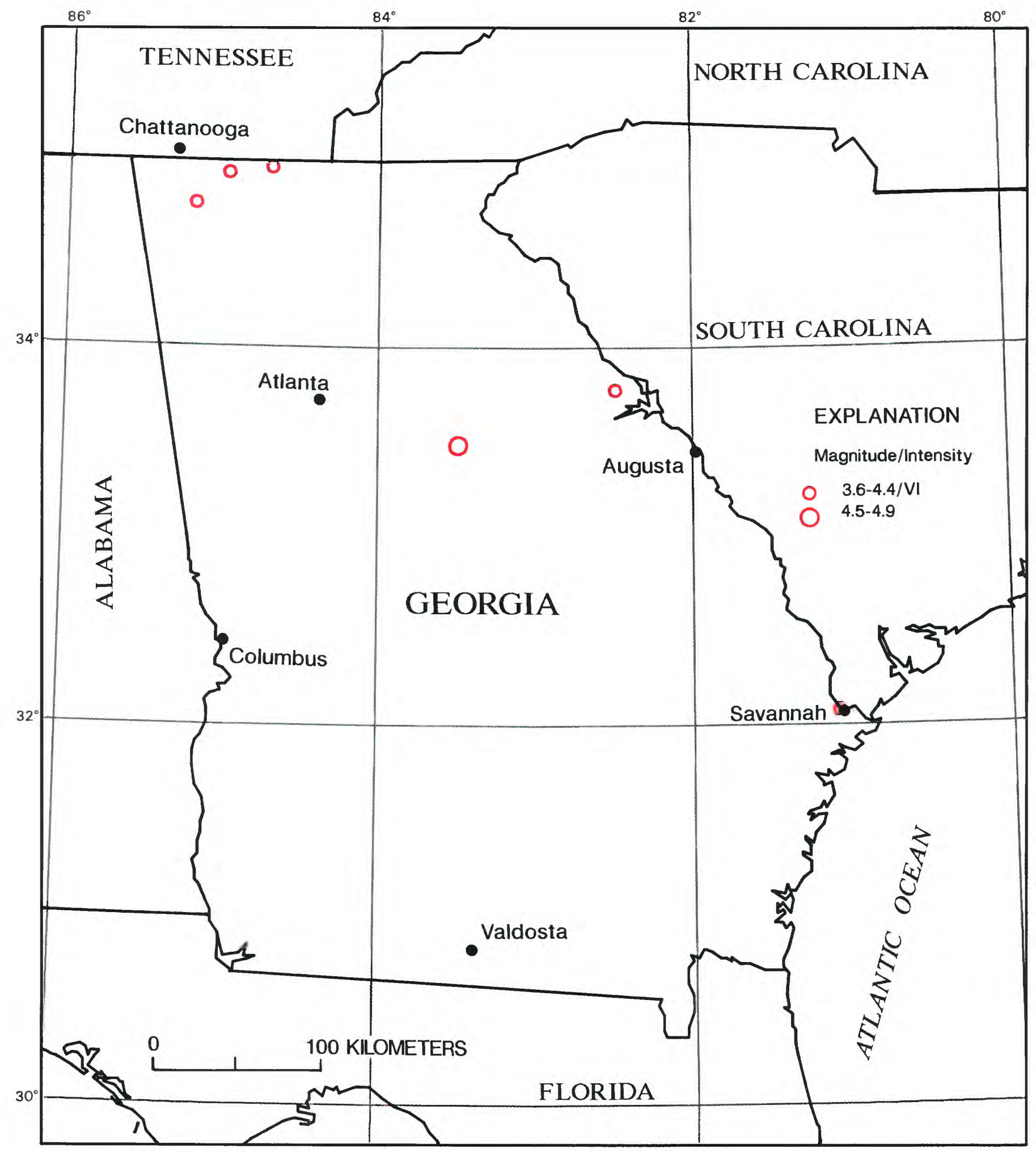

Earthquakes in Georgia with magnitudes $\geq 4.5$ or intensity $\geq$ VI. 
GEORGIA

[See table 1 for hypocenter and intensity references and table 2 for definitions of magnitude source codes. Leader ( - ) indicates information is not available]

\begin{tabular}{|c|c|c|c|c|c|c|c|c|c|c|c|c|c|}
\hline \multirow{2}{*}{\multicolumn{2}{|c|}{ Date }} & \multirow{2}{*}{$\begin{array}{c}\text { Origin } \\
\text { time (UTC) }\end{array}$} & \multicolumn{4}{|c|}{ Hypocenter } & \multicolumn{4}{|c|}{ Magnitude } & \multicolumn{2}{|c|}{ Intensity } & \multirow{3}{*}{$\begin{array}{l}\text { Folt area } \\
\left(1,000 \mathrm{~km}^{2}\right)\end{array}$} \\
\hline & & & \multirow{2}{*}{$\begin{array}{l}\text { Latitudo } \\
\left(^{\circ}\right)\end{array}$} & \multirow{2}{*}{$\begin{array}{l}\text { Longitude } \\
\left({ }^{\circ}\right)\end{array}$} & \multirow{2}{*}{$\begin{array}{l}\text { Depth } \\
(\mathrm{km})\end{array}$} & \multirow[t]{2}{*}{ Ref } & \multicolumn{2}{|c|}{ USGS } & \multirow[t]{2}{*}{ Other } & \multirow{2}{*}{$\begin{array}{c}\text { Moment } \\
\text { M }\end{array}$} & \multirow[t]{2}{*}{ MMI } & \multirow[t]{2}{*}{ Ref } & \\
\hline $\mathrm{Yr}$ & Mo Da & $h \mathrm{~m} s$ & & & & & $m_{b}$ & $M_{s}$ & & & & & \\
\hline 1875 & 1102 & 0255 & $33.8 \mathrm{~N}$ & $82.5 \mathrm{~W}$ & - & 38 & - & - & $4.30 \mathrm{M}_{\mathrm{fa}} \mathrm{SC}$ & - & VI & 38 & 65 \\
\hline 1903 & 0124 & 0115 & $32.1 \mathrm{~N}$ & $81.1 \mathrm{~W}$ & - & 38 & - & - & $4.10 \mathrm{M}_{\mathrm{fa}} \mathrm{SC}$ & - & VI & 38 & 26 \\
\hline 1914 & 0305 & 2005 & $33.5 \mathrm{~N}$ & $83.5 \mathrm{~W}$ & - & 38 & - & - & $4.50 \mathrm{M}_{\mathrm{fa}} \mathrm{SC}$ & - & v & 135 & 246 \\
\hline 1976 & 0204 & 195353.0 & $34.971 \mathrm{~N}$ & $84.702 \mathrm{~W}$ & 014 & 349 & - & - & $3.60 \mathrm{M}_{\mathrm{n}} \mathrm{DG}$ & - & VI & 49 & 8 \\
\hline 1984 & 1009 & 115426.2 & $34.775 \mathrm{~N}$ & $85.193 \mathrm{~W}$ & 015 & 370 & - & - & $4.00 \mathrm{M}_{\mathrm{n}} \mathrm{GS}$ & $4.22 \mathrm{GT}$ & VI & 370 & 8 \\
\hline 1986 & $07 \quad 11$ & 142614.8 & $34.937 \mathrm{~N}$ & $84.987 \mathrm{~W}$ & 013 & 562 & 3.7 & - & $3.80 \mathrm{M}_{\mathrm{n}}$ GS & - & VI & 562 & 13 \\
\hline
\end{tabular}

[Reference (Ref.) numbers given in parentheses at the end of each description refer to sources of data in table 1. Magnitude values are described in the Introduction, and codes are defined in table 2.]

1875. Nov. 2 (Nov. 1). Northern Georgia. The earthquake was strong enough to move a mirror back and forth from the wall and to shake doors and windows. Felt from Spartanburg and Columbia, S.C., to Atlanta and Macon, Ga., and from Gainesville (northeast of Atlanta) to Augusta, Ga. Several aftershocks were reported. (Ref. 38, 211, 473.)

1903. Jan. 24 (Jan. 23). Near Savannah, Bryan County, Ga. The highest intensity was reported at Tybee Island, Ga., east of Savannah, on the Atlantic Ocean. Houses were shaken strongly in that area. (Ref. 38.)

1976. Feb. 4. Northern Georgia, south of Conasauga, Tenn. Near Conasauga in the Lake Ocoee Dam area of Polk County, cracks formed in masonry, chimneys, and a concrete-block building.
Also felt at several towns in northern Georgia. Magnitude 3.0 $\mathrm{M}_{\mathrm{n}}$ BLA. (Ref. 49, 349.)

1984. Oct. 9. Near Ringgold, Catoosa County, Ga. The most serious damage occurred a few $\mathrm{km}$ south of Ringgold, where cracks formed in plaster and sheetrock walls, a house foundation, and exterior brick-veneer walls. One tractor plow was overturned. Light damage also was reported at Chickamauga, LaFayette, Summerville, and Trenton, Ga., and at Chattanooga, Tenn. Also felt in Alabama and Tennessee. Magnitude 4.2 $\mathrm{M}_{\mathrm{n}} \mathrm{SLM}, 3.8 \mathrm{M}_{\mathrm{D}}$ TEC. (Ref. 370.)

1986. July 11. Northwest Georgia, near Chattanooga, Tenn. Damage reported at Cohutta, Ga., near the Tennessee border, southeast of Chattanooga, consisted of cracks in a house foundation, chimneys, and outside brick walls. Slight damage also was reported at Dalton and Tunnel Hill, Ga., and Chattanooga and Turtletown, Tenn. Felt in northern Georgia, southeastern Tennessee, and southwestern North Carolina. Magnitude 3.8 $\mathrm{M}_{\mathrm{D}}$ GT. (Ref. 562.) 


\section{HAWAII}

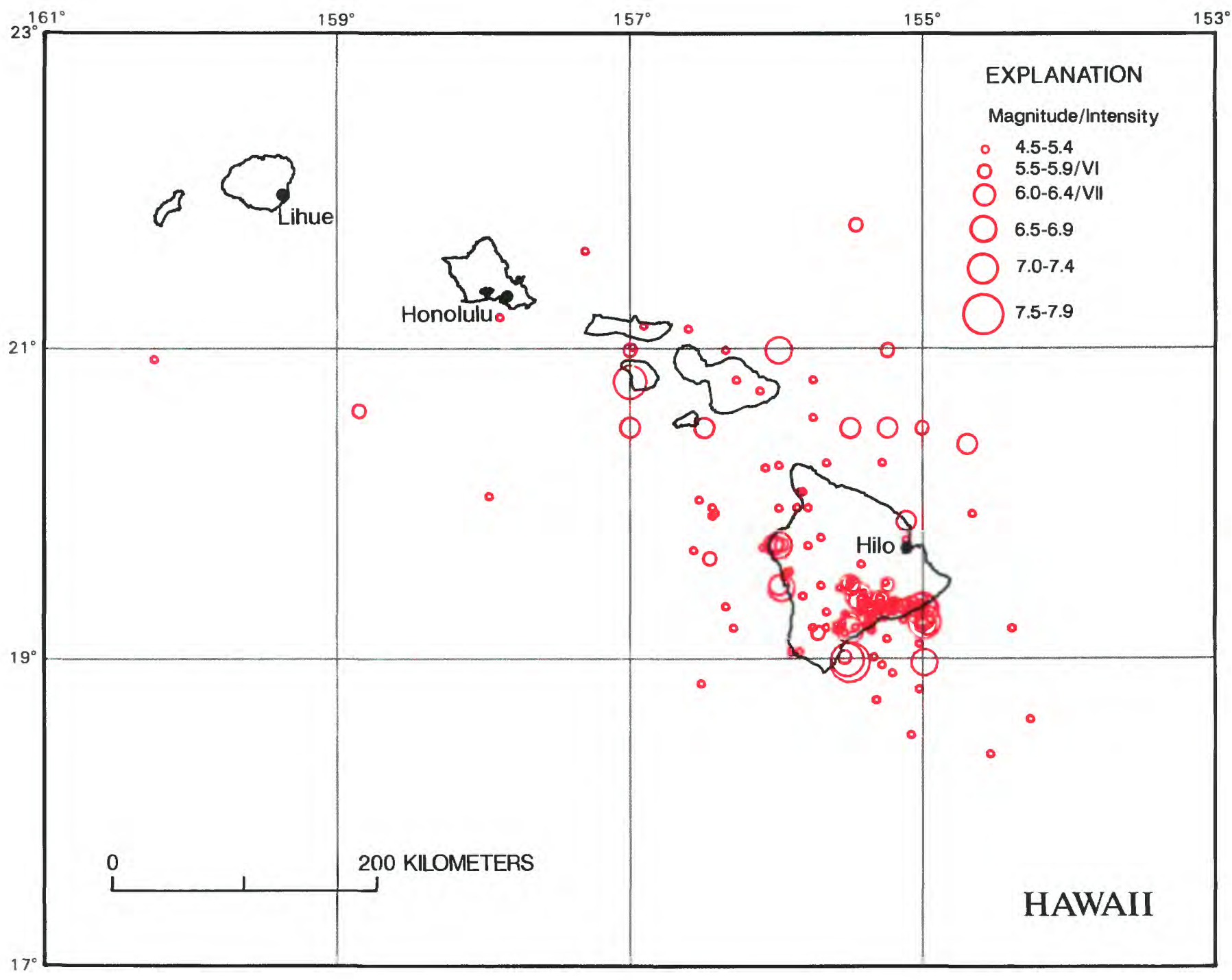

Earthquakes in Hawaii with magnitudes $\geq 4.5$ or intensity $\geq$ VI. 
HAWAII

[See table 1 for hypocenter and intensity references and table 2 for definitions of magnitude source codes. Leader (--) indicates information is not available]

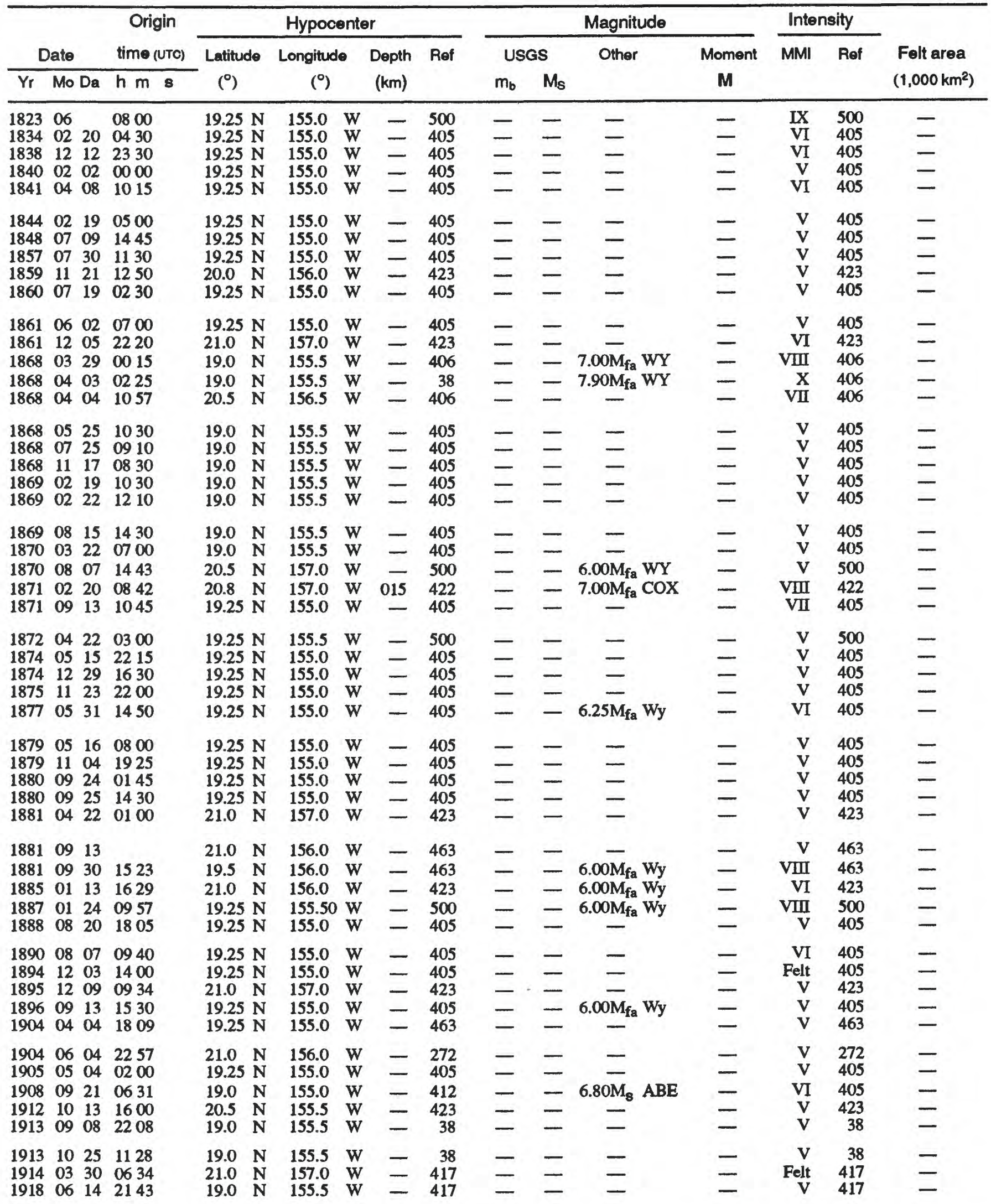


EARTHQUAKES IN HAWAII

HAWAII-Continued

[See table 1 for hypocenter and intensity references and table 2 for definitions of magnitude source codes. Leader (--) indicates information is not available]

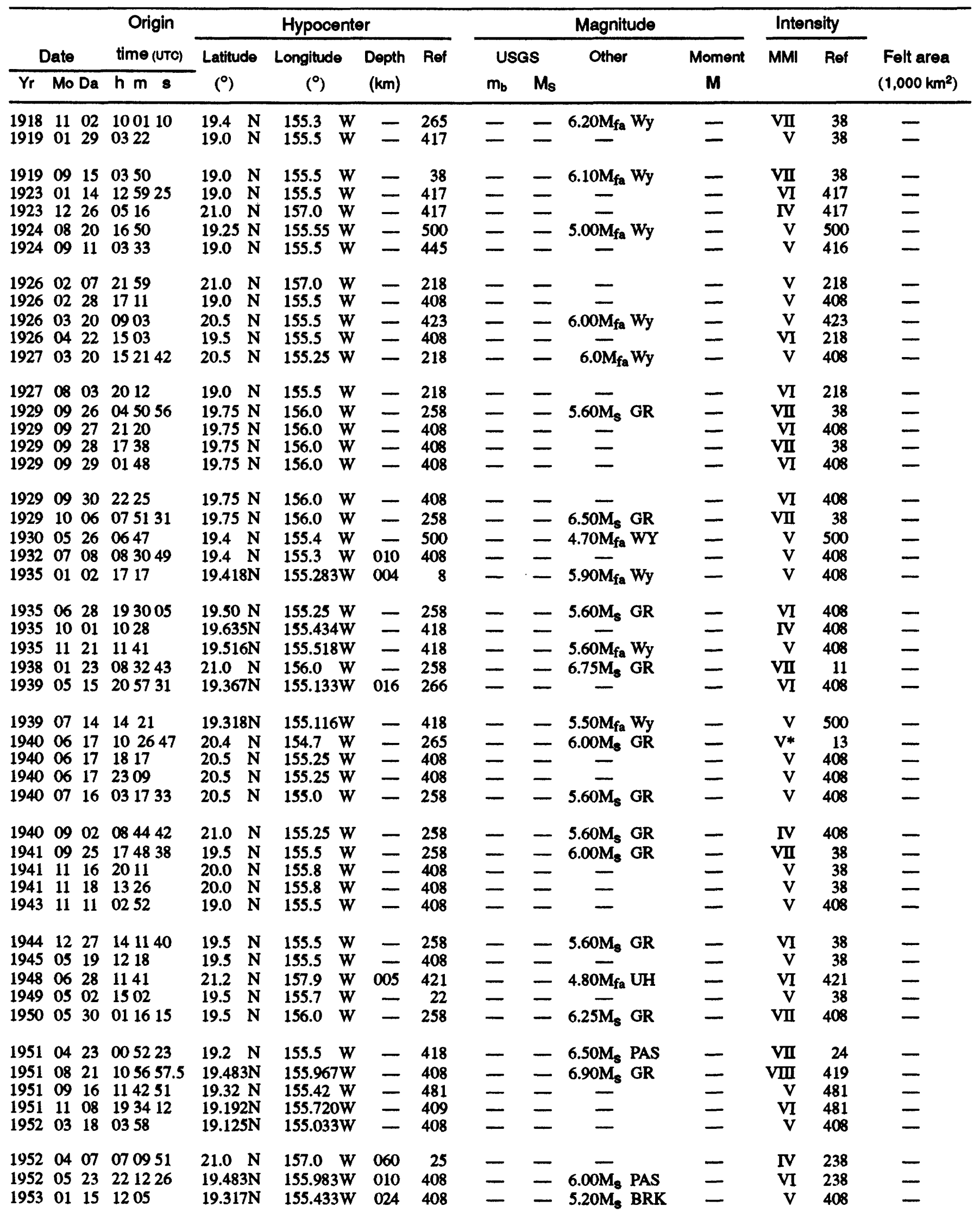


HAWAII-Continued

[See table 1 for hypocenter and intensity references and table 2 for definitions of magnitude source codes. Leader (--) indicates information is not available]

\begin{tabular}{|c|c|c|c|c|c|c|c|c|c|c|c|c|c|c|}
\hline \multirow{2}{*}{\multicolumn{3}{|c|}{ Date }} & \multirow{2}{*}{$\begin{array}{r}\begin{array}{r}\text { Origin } \\
\text { time (UTC) }\end{array} \\
\end{array}$} & \multicolumn{4}{|c|}{ Hypocenter } & \multicolumn{4}{|c|}{ Magnitude } & \multicolumn{2}{|c|}{ Intensity } & \multirow{3}{*}{$\begin{array}{l}\text { Felt area } \\
\left(1,000 \mathrm{~km}^{2}\right)\end{array}$} \\
\hline & & & & \multirow{2}{*}{$\begin{array}{l}\text { Latitude } \\
\left(^{\circ}\right)\end{array}$} & \multirow{2}{*}{$\begin{array}{l}\text { Longitude } \\
\qquad\left(^{\circ}\right)\end{array}$} & \multirow{2}{*}{$\begin{array}{l}\text { Depth } \\
(\mathbf{k m})\end{array}$} & \multirow[t]{2}{*}{ Ref } & \multicolumn{2}{|c|}{ USGS } & \multirow[t]{2}{*}{ Other } & \multirow{2}{*}{$\begin{array}{c}\text { Moment } \\
\mathbf{M}\end{array}$} & \multirow[t]{2}{*}{ MMI } & \multirow[t]{2}{*}{ Ref } & \\
\hline Yr & Mo & $\mathrm{Da}$ & $\mathrm{h} m \mathrm{~s}$ & & & & & $m_{b}$ & $\mathbf{M}_{\mathbf{s}}$ & & & & & \\
\hline $\begin{array}{l}1953 \\
1954\end{array}$ & & $\begin{array}{l}22 \\
30\end{array}$ & $\begin{array}{l}0547 \\
164002.0\end{array}$ & $\begin{array}{l}19.75 \mathrm{~N} \\
19.380 \mathrm{~N}\end{array}$ & $\begin{array}{l}155.8 \quad W \\
155.025 W\end{array}$ & $\overline{009}$ & $\begin{array}{l}408 \\
418\end{array}$ & - & - & $6.00 \overline{\mathrm{M}}_{\mathrm{s}}$ PAS & - & $\begin{array}{c}\text { V } \\
\text { VI }\end{array}$ & $\begin{array}{l}408 \\
410\end{array}$ & - \\
\hline $\begin{array}{l}1954 \\
1954 \\
1955 \\
1955 \\
1955\end{array}$ & $\begin{array}{l}03 \\
07 \\
03 \\
03 \\
04\end{array}$ & $\begin{array}{l}30 \\
03 \\
08 \\
28 \\
01\end{array}$ & $\begin{array}{ll}184156.0 \\
215235 \\
082126.1 \\
020220 \\
142425.5\end{array}$ & $\begin{array}{l}19.353 \mathrm{~N} \\
19.4 \mathrm{~N} \\
19.335 \mathrm{~N} \\
19.4 \mathrm{~N} \\
19.407 \mathrm{~N}\end{array}$ & $\begin{array}{l}155.020 \mathrm{~W} \\
155.2 \mathrm{~W} \\
154.997 \mathrm{~W} \\
155.3 \mathrm{~W} \\
155.305 \mathrm{~W}\end{array}$ & $\begin{array}{l}009 \\
012 \\
000 \\
\overline{010}\end{array}$ & $\begin{array}{l}418 \\
408 \\
482 \\
408 \\
482\end{array}$ & $\bar{z}$ & $\frac{-}{-}$ & $\begin{array}{c}6.50 \mathrm{Ukn} \text { PAS } \\
5.40 \mathrm{M}_{\mathrm{L}} \text { HVO } \\
5.40 \mathrm{M}_{\mathrm{L}} \text { HVO } \\
5.20 \overline{\mathrm{M}}_{\mathrm{L}} \text { HVO }\end{array}$ & $\begin{array}{l}- \\
-\end{array}$ & $\begin{array}{l}\text { VII } \\
\text { VI } \\
\text { IV } \\
\text { VI } \\
\text { V }\end{array}$ & $\begin{array}{r}410 \\
408 \\
408 \\
408 \\
38\end{array}$ & $\begin{array}{l}z \\
-\end{array}$ \\
\hline $\begin{array}{l}1955 \\
1955 \\
1956 \\
1956 \\
1957\end{array}$ & $\begin{array}{l}08 \\
08 \\
05 \\
10 \\
07\end{array}$ & $\begin{array}{l}07 \\
14 \\
14 \\
16 \\
04\end{array}$ & $\begin{array}{l}171744 \\
122800.0 \\
075336 \\
104455 \\
105359\end{array}$ & $\begin{array}{l}20.0 \mathrm{~N} \\
19.313 \mathrm{~N} \\
20.283 \mathrm{~N} \\
19.667 \mathrm{~N} \\
19.8 \mathrm{~N}\end{array}$ & $\begin{array}{l}155.8 \mathrm{~W} \\
155.285 \mathrm{~W} \\
155.283 \mathrm{~W} \\
156.467 \mathrm{~W} \\
155.7 \mathrm{~W}\end{array}$ & $\begin{array}{l}040 \\
029 \\
020 \\
025 \\
015\end{array}$ & $\begin{array}{l}408 \\
482 \\
418 \\
418 \\
418\end{array}$ & $\bar{z}$ & $\bar{z}$ & $\begin{array}{ll}5.70 \bar{M}_{\mathrm{L}} & \text { HVO } \\
4.50 \mathrm{M}_{\mathrm{L}} & \text { HVO } \\
5.50 \mathrm{M}_{\mathrm{L}} & \text { HVO } \\
4.50 \mathrm{M}_{\mathrm{L}} & \text { HVO }\end{array}$ & E & $\begin{array}{l}\text { V } \\
\text { VI } \\
\text { V } \\
\text { V } \\
\text { IV }\end{array}$ & $\begin{array}{l}38 \\
28 \\
38 \\
38 \\
30\end{array}$ & - \\
\hline $\begin{array}{l}1957 \\
1957 \\
1958 \\
1959 \\
1960\end{array}$ & $\begin{array}{l}07 \\
08 \\
12 \\
02 \\
01\end{array}$ & $\begin{array}{l}26 \\
18 \\
27 \\
20 \\
19\end{array}$ & $\begin{array}{l}114030 \\
104156 \\
191724 \\
060028 \\
042649.2\end{array}$ & $\begin{array}{l}20.250 \mathrm{~N} \\
21.800 \mathrm{~N} \\
19.783 \mathrm{~N} \\
19.350 \mathrm{~N} \\
19.320 \mathrm{~N}\end{array}$ & $\begin{array}{l}156.083 \mathrm{~W} \\
155.467 \mathrm{~W} \\
155.117 \mathrm{~W} \\
155.150 \mathrm{~W} \\
155.670 \mathrm{~W}\end{array}$ & $\begin{array}{l}005 \\
010 \\
045 \\
005 \\
007\end{array}$ & \begin{tabular}{r|r}
418 \\
418 \\
418 \\
32 \\
418
\end{tabular} & $\overline{-}$ & $\frac{-}{-}$ & $\begin{array}{l}4.60 \mathrm{M}_{\mathrm{L}} \text { HVO } \\
5.60 \mathrm{M}_{\mathrm{L}} \text { HVO } \\
4.50 \mathrm{M}_{\mathrm{L}} \text { HVO } \\
4.50 \mathrm{M}_{\mathrm{L}} \text { HVO } \\
4.50 \mathrm{M}_{\mathrm{L}} \text { HVO }\end{array}$ & - & $\begin{array}{l}\text { IV } \\
\text { V } \\
\text { III } \\
\text { III } \\
\text { III }\end{array}$ & $\begin{array}{l}30 \\
38 \\
31 \\
32 \\
33\end{array}$ & - \\
\hline $\begin{array}{l}1960 \\
1961 \\
1961 \\
1961 \\
1961\end{array}$ & $\begin{array}{l}12 \\
01 \\
07 \\
09 \\
09\end{array}$ & $\begin{array}{l}25 \\
21 \\
23 \\
23 \\
25\end{array}$ & $\begin{array}{lll}12 & 5627.8 \\
11 & 39 & 36.8 \\
15 & 24 & 15.5 \\
03 & 01 & 33.3 \\
05 & 28 & 53.5\end{array}$ & $\begin{array}{l}19.222 \mathrm{~N} \\
19.225 \mathrm{~N} \\
19.377 \mathrm{~N} \\
19.320 \mathrm{~N} \\
19.302 \mathrm{~N}\end{array}$ & $\begin{array}{l}155.764 \mathrm{~W} \\
155.666 \mathrm{~W} \\
155.282 \mathrm{~W} \\
155.113 \mathrm{~W} \\
154.998 \mathrm{~W}\end{array}$ & $\begin{array}{l}001 \\
010 \\
026 \\
004 \\
009\end{array}$ & $\begin{array}{l}418 \\
418 \\
418 \\
418 \\
418\end{array}$ & $\bar{z}$ & $\bar{z}$ & $\begin{array}{l}4.50 \mathrm{M}_{\mathrm{L}} \text { HVO } \\
4.60 \mathrm{M}_{\mathrm{L}} \text { HVO } \\
4.60 \mathrm{M}_{\mathrm{L}} \text { HVO } \\
4.50 \mathrm{M}_{\mathrm{L}} \text { HVO } \\
4.50 \mathrm{M}_{\mathrm{L}} \text { HVO }\end{array}$ & - & $\begin{array}{l}\text { IIII } \\
\text { V } \\
\text { v } \\
\text { V }\end{array}$ & $\begin{array}{l}33 \\
34 \\
34 \\
34 \\
34\end{array}$ & $\overline{-}$ \\
\hline $\begin{array}{l}1962 \\
1962 \\
1962 \\
1963 \\
1963\end{array}$ & $\begin{array}{l}02 \\
06 \\
07 \\
01 \\
08\end{array}$ & $\begin{array}{l}11 \\
28 \\
25 \\
08 \\
26\end{array}$ & $\begin{array}{l}020048.3 \\
042714.2 \\
034815.8 \\
193945.0 \\
184918.1\end{array}$ & $\begin{array}{l}19.224 \mathrm{~N} \\
19.399 \mathrm{~N} \\
19.540 \mathrm{~N} \\
19.390 \mathrm{~N} \\
19.375 \mathrm{~N}\end{array}$ & $\begin{array}{l}154.398 \mathrm{~W} \\
155.454 \mathrm{~W} \\
155.961 \mathrm{~W} \\
155.218 \mathrm{~W} \\
155.370 \mathrm{~W}\end{array}$ & $\begin{array}{l}003 \\
010 \\
002 \\
031 \\
006\end{array}$ & $\begin{array}{l}418 \\
418 \\
418 \\
418 \\
418\end{array}$ & $\frac{-}{4.4}$ & $\overline{-}$ & $\begin{array}{l}4.50 \mathrm{M}_{\mathrm{L}} \text { HVO } \\
6.10 \mathrm{M}_{\mathrm{L}} \text { HVO } \\
4.70 \mathrm{M}_{\mathrm{L}} \text { HVO } \\
4.60 \mathrm{M}_{\mathrm{L}} \text { HVO } \\
4.70 \mathrm{M}_{\mathrm{L}} \text { HVO }\end{array}$ & - & $\begin{array}{l}\overline{V I} \\
\mathbf{V} \\
\mathbf{V} \\
\mathbf{I V}\end{array}$ & $\begin{array}{l}-35 \\
35 \\
36 \\
36\end{array}$ & - \\
\hline $\begin{array}{l}1963 \\
1963 \\
1964 \\
1964 \\
1964\end{array}$ & $\begin{array}{l}09 \\
10 \\
09 \\
10 \\
12\end{array}$ & $\begin{array}{l}21 \\
23 \\
18 \\
11 \\
03\end{array}$ & $\begin{array}{l}162420.6 \\
202406.9 \\
102529.1 \\
100643.6 \\
082841.0\end{array}$ & $\begin{array}{l}19.428 \mathrm{~N} \\
19.376 \mathrm{~N} \\
19.315 \mathrm{~N} \\
18.856 \mathrm{~N} \\
19.405 \mathrm{~N}\end{array}$ & $\begin{array}{l}155.823 \mathrm{~W} \\
155.416 \mathrm{~W} \\
155.115 \mathrm{~W} \\
156.517 \mathrm{~W} \\
155.277 \mathrm{~W}\end{array}$ & $\begin{array}{l}006 \\
009 \\
005 \\
006 \\
025\end{array}$ & \begin{tabular}{r|}
418 \\
418 \\
37 \\
418 \\
418
\end{tabular} & $\begin{array}{l}4.6 \\
5.0 \\
4.8 \\
5.3 \\
4.7\end{array}$ & $\bar{z}$ & $\begin{array}{l}4.70 \mathrm{M}_{\mathrm{L}} \text { HVO } \\
4.60 \mathrm{M}_{\mathrm{L}} \text { HVO } \\
4.90 \mathrm{M}_{\mathrm{L}} \text { HVO } \\
5.30 \mathrm{M}_{\mathrm{L}} \text { HVO } \\
4.50 \mathrm{M}_{\mathrm{L}} \text { HVO }\end{array}$ & $\overline{-}$ & $\begin{array}{c}\text { IV } \\
\text { V } \\
\text { IV } \\
\text { V } \\
\text { V }\end{array}$ & $\begin{array}{r}36 \\
36 \\
37 \\
423 \\
37\end{array}$ & - \\
\hline $\begin{array}{l}1964 \\
1965 \\
1966 \\
1966 \\
1967\end{array}$ & $\begin{array}{l}12 \\
02 \\
08 \\
09 \\
07\end{array}$ & $\begin{array}{l}10 \\
13 \\
19 \\
05 \\
22\end{array}$ & $\begin{array}{lll}11 & 53 & 44.6 \\
23 & 0629.6 \\
15 & 2139.5 \\
16 & 3321.9 \\
07 & 34 & 10.1\end{array}$ & $\begin{array}{l}19.266 \mathrm{~N} \\
18.754 \mathrm{~N} \\
18.537 \mathrm{~N} \\
19.353 \mathrm{~N} \\
20.753 \mathrm{~N}\end{array}$ & $\begin{array}{l}155.139 \mathrm{~W} \\
155.314 \mathrm{~W} \\
155.087 \mathrm{~W} \\
155.437 \mathrm{~W} \\
156.121 \mathrm{~W}\end{array}$ & $\begin{array}{l}008 \\
015 \\
051 \\
008 \\
008\end{array}$ & \begin{tabular}{r|r}
418 \\
418 \\
418 \\
81 \\
418
\end{tabular} & $\frac{5.1}{4.7}$ & $\frac{-}{-}$ & $\begin{array}{ll}4.60 \mathrm{M}_{\mathrm{L}} & \text { HVO } \\
4.60 \mathrm{M}_{\mathrm{L}} & \text { HVO } \\
4.60 \mathrm{M}_{\mathrm{L}} & \text { HVO } \\
4.50 \mathrm{M}_{\mathrm{L}} & \text { HVO } \\
4.60 \mathrm{M}_{\mathrm{L}} & \text { HVO }\end{array}$ & $\begin{array}{l}- \\
-\end{array}$ & $\underset{\text { III }}{\text { III }}$ & $\begin{array}{l}37 \\
75 \\
81 \\
81 \\
40\end{array}$ & - \\
\hline $\begin{array}{l}1968 \\
1968 \\
1969 \\
1969 \\
1969\end{array}$ & $\begin{array}{l}02 \\
04 \\
05 \\
11 \\
11\end{array}$ & $\begin{array}{l}22 \\
28 \\
10 \\
10 \\
24\end{array}$ & $\begin{array}{lll}19 & 20 & 40.3 \\
14 & 08 & 59.0 \\
01 & 33 & 28.0 \\
05 & 12 & 12.8 \\
19 & 12 & 21.6\end{array}$ & $\begin{array}{l}19.223 \mathrm{~N} \\
19.375 \mathrm{~N} \\
19.359 \mathrm{~N} \\
19.187 \mathrm{~N} \\
19.731 \mathrm{~N}\end{array}$ & $\begin{array}{l}156.297 \mathrm{~W} \\
155.298 \mathrm{~W} \\
155.073 \mathrm{~W} \\
155.542 \mathrm{~W} \\
156.099 \mathrm{~W}\end{array}$ & $\begin{array}{l}005 \\
030 \\
013 \\
033 \\
002\end{array}$ & $\begin{array}{r}418 \\
41 \\
418 \\
418 \\
418\end{array}$ & $\begin{array}{l}\overline{4.7} \\
4.9 \\
3.7 \\
3.8\end{array}$ & $\bar{z}$ & $\begin{array}{l}4.50 \mathrm{M}_{\mathrm{L}} \text { HVO } \\
4.50 \mathrm{M}_{\mathrm{L}} \text { HVO } \\
4.30 \mathrm{M}_{\mathrm{L}} \text { HVO } \\
4.60 \mathrm{M}_{\mathrm{L}} \text { HVO } \\
4.70 \mathrm{M}_{\mathrm{L}} \text { HVO }\end{array}$ & $\begin{array}{l}- \\
-\end{array}$ & $\begin{array}{l}\text { IV } \\
\text { IV } \\
\text { III } \\
\text { III }\end{array}$ & $\begin{array}{l}-41 \\
42 \\
42 \\
42\end{array}$ & $\overline{-}$ \\
\hline $\begin{array}{l}1970 \\
1970 \\
1971 \\
1971 \\
1971\end{array}$ & $\begin{array}{l}09 \\
10 \\
08 \\
08 \\
12\end{array}$ & $\begin{array}{l}21 \\
25 \\
01 \\
16 \\
28\end{array}$ & $\begin{array}{lll}11 & 26 & 36.5 \\
19 & 55 & 29.1 \\
18 & 52 & 39.5 \\
01 & 35 & 09.1 \\
01 & 11 & 51.8\end{array}$ & $\begin{array}{l}19.332 \mathrm{~N} \\
21.146 \mathrm{~N} \\
18.402 \mathrm{~N} \\
19.367 \mathrm{~N} \\
19.262 \mathrm{~N}\end{array}$ & $\begin{array}{l}155.203 \mathrm{~W} \\
156.612 \mathrm{~W} \\
154.545 \mathrm{~W} \\
155.278 \mathrm{~W} \\
155.372 \mathrm{~W}\end{array}$ & $\begin{array}{l}011 \\
011 \\
007 \\
034 \\
005\end{array}$ & $\begin{array}{l}418 \\
418 \\
418 \\
418 \\
418\end{array}$ & $\begin{array}{l}\overline{4.5} \\
4.8 \\
4.5 \\
-\end{array}$ & $\bar{z}$ & $\begin{array}{ll}4.50 \mathrm{M}_{\mathrm{L}} & \text { HVO } \\
4.80 \mathrm{M}_{\mathrm{L}} & \text { HVO } \\
4.60 \mathrm{M}_{\mathrm{L}} & \text { HVO } \\
4.80 \mathrm{M}_{\mathrm{L}} & \text { HVO } \\
4.70 \mathrm{M}_{\mathrm{L}} & \text { HVO }\end{array}$ & $\begin{array}{l}- \\
-\end{array}$ & $\begin{array}{l}\text { IV } \\
\text { IV } \\
\text { IV } \\
\text { III }\end{array}$ & $\begin{array}{l}43 \\
43 \\
44 \\
44\end{array}$ & - \\
\hline $\begin{array}{l}1971 \\
1971 \\
1972 \\
1972 \\
1972\end{array}$ & $\begin{array}{l}12 \\
12 \\
02 \\
07 \\
09\end{array}$ & $\begin{array}{l}29 \\
29 \\
29 \\
14 \\
05\end{array}$ & $\begin{array}{l}025912.0 \\
113843.1 \\
220823.9 \\
193657.7 \\
113133.8\end{array}$ & $\begin{array}{l}19.248 \mathrm{~N} \\
19.249 \mathrm{~N} \\
19.360 \mathrm{~N} \\
19.033 \mathrm{~N} \\
19.331 \mathrm{~N}\end{array}$ & $\begin{array}{l}155.382 \mathrm{~W} \\
155.363 \mathrm{~W} \\
156.351 \mathrm{~W} \\
155.330 \mathrm{~W} \\
155.206 \mathrm{~W}\end{array}$ & $\begin{array}{l}008 \\
007 \\
008 \\
038 \\
010\end{array}$ & $\begin{array}{l}418 \\
418 \\
418 \\
418 \\
418\end{array}$ & $\frac{\overline{4}}{4.9}$ & $\bar{z}$ & $\begin{array}{l}4.60 \mathrm{M}_{\mathrm{L}} \text { HVO } \\
4.60 \mathrm{M}_{\mathrm{L}} \text { HVO } \\
4.90 \mathrm{M}_{\mathrm{L}} \text { HVO } \\
4.50 \mathrm{M}_{\mathrm{L}} \text { HVO } \\
5.00 \mathrm{M}_{\mathrm{L}} \text { HVO }\end{array}$ & $\begin{array}{l}- \\
-\end{array}$ & $\begin{array}{l}\text { IV } \\
\text { III } \\
\text { IV } \\
\text { III } \\
\text { IV }\end{array}$ & $\begin{array}{l}44 \\
44 \\
45 \\
45 \\
45\end{array}$ & $\begin{array}{l}z \\
-\end{array}$ \\
\hline
\end{tabular}


HAWAII-Continued

[See table 1 for hypocenter and intensity references and table 2 for definitions of magnitude source codes. Leader (--) indicates information is not available]

\begin{tabular}{|c|c|c|c|c|c|c|c|c|c|c|c|c|c|}
\hline \multirow{2}{*}{\multicolumn{2}{|c|}{ Date }} & \multirow{3}{*}{$\begin{array}{l}\text { Origin } \\
\text { time (UTC) } \\
h \text { m s }\end{array}$} & \multicolumn{4}{|c|}{ Hypocenter } & \multicolumn{4}{|c|}{ Magnitude } & \multicolumn{2}{|c|}{ Intensity } & \multirow{3}{*}{$\begin{array}{l}\text { Feit area } \\
\left(1,000 \mathrm{~km}^{2}\right)\end{array}$} \\
\hline & & & \multirow{2}{*}{$\begin{array}{l}\text { Latitude } \\
\left(^{\circ}\right)\end{array}$} & \multirow{2}{*}{$\begin{array}{l}\text { Longitude } \\
\left({ }^{\circ}\right)\end{array}$} & \multirow{2}{*}{$\begin{array}{l}\text { Depth } \\
(\mathbf{k m})\end{array}$} & \multirow[t]{2}{*}{ Ref } & \multicolumn{2}{|c|}{ USGS } & \multirow[t]{2}{*}{ Other } & \multirow{2}{*}{$\begin{array}{c}\text { Moment } \\
\mathbf{M}\end{array}$} & \multirow[t]{2}{*}{ MMI } & \multirow[t]{2}{*}{ Ref } & \\
\hline $\mathrm{Yr}_{\mathrm{r}}$ & Mo Da & & & & & & $m_{b}$ & $\mathbf{M}_{\mathbf{S}}$ & & & & & \\
\hline $\begin{array}{l}1972 \\
1972 \\
1973 \\
1973 \\
1973\end{array}$ & $\begin{array}{ll}12 & 23 \\
12 & 23 \\
04 & 23 \\
04 & 26 \\
10 & 09\end{array}$ & $\begin{array}{l}190452.7 \\
204307.0 \\
070753.1 \\
202630.6 \\
115345.3\end{array}$ & $\begin{array}{l}19.589 \mathrm{~N} \\
19.578 \mathrm{~N} \\
19.963 \mathrm{~N} \\
19.903 \mathrm{~N} \\
19.338 \mathrm{~N}\end{array}$ & $\begin{array}{l}155.926 \mathrm{~W} \\
155.936 \mathrm{~W} \\
154.672 \mathrm{~W} \\
155.130 \mathrm{~W} \\
155.267 \mathrm{~W}\end{array}$ & $\begin{array}{l}015 \\
015 \\
032 \\
048 \\
033\end{array}$ & $\begin{array}{l}418 \\
418 \\
418 \\
464 \\
418\end{array}$ & $\begin{array}{l}4.9 \\
\overline{4.2} \\
6.0 \\
4.8\end{array}$ & $\frac{-}{6.1}$ & $\begin{array}{ll}5.10 \mathrm{M}_{\mathrm{L}} & \text { HVO } \\
4.60 \mathrm{M}_{\mathrm{L}} & \text { HVO } \\
4.80 \mathrm{M}_{\mathrm{L}} & \text { HVO } \\
6.20 \mathrm{M}_{\mathrm{L}} & \text { HVO } \\
4.60 \mathrm{M}_{\mathrm{L}} & \text { HVO }\end{array}$ & $\begin{array}{l}z \\
z \\
=\end{array}$ & $\begin{array}{c}\text { V } \\
\text { IV } \\
\text { VIII } \\
\text { IV }\end{array}$ & $\begin{array}{l}45 \\
45 \\
46 \\
46 \\
46\end{array}$ & $\begin{array}{l}- \\
- \\
-\end{array}$ \\
\hline $\begin{array}{l}1973 \\
1974 \\
1974 \\
1974 \\
1974\end{array}$ & $\begin{array}{ll}12 & 13 \\
01 & 12 \\
06 & 19 \\
08 & 28 \\
11 & 30\end{array}$ & $\begin{array}{l}042556.1 \\
160434.2 \\
150542.6 \\
074941.0 \\
135423.8\end{array}$ & $\begin{array}{l}19.374 \mathrm{~N} \\
19.332 \mathrm{~N} \\
19.380 \mathrm{~N} \\
19.328 \mathrm{~N} \\
19.442 \mathrm{~N}\end{array}$ & $\begin{array}{l}155.292 \mathrm{~W} \\
155.120 \mathrm{~W} \\
155.422 \mathrm{~W} \\
155.205 \mathrm{~W} \\
155.416 \mathrm{~W}\end{array}$ & $\begin{array}{l}035 \\
009 \\
010 \\
010 \\
008\end{array}$ & $\begin{array}{l}418 \\
418 \\
418 \\
418 \\
418\end{array}$ & $\begin{array}{l}4.4 \\
4.8 \\
5.1 \\
4.8 \\
5.1\end{array}$ & $\overline{-}$ & $\begin{array}{ll}4.60 \mathrm{M}_{\mathrm{L}} & \text { HVO } \\
4.70 \mathrm{M}_{\mathrm{L}} & \text { HVO } \\
4.70 \mathrm{M}_{\mathrm{L}} & \text { HVO } \\
4.50 \mathrm{M}_{\mathrm{L}} & \text { HVO } \\
5.40 \mathrm{M}_{\mathrm{L}} & \text { HVO }\end{array}$ & $\begin{array}{l}- \\
-\end{array}$ & $\underset{\text { III }}{\text { II }}$ & $\begin{array}{l}46 \\
47 \\
47 \\
47 \\
47\end{array}$ & $\begin{array}{l}z \\
z\end{array}$ \\
\hline $\begin{array}{l}1974 \\
1974 \\
1974 \\
1975 \\
1975\end{array}$ & $\begin{array}{ll}12 & 16 \\
12 & 25 \\
12 & 31 \\
01 & 01 \\
01 & 01\end{array}$ & $\begin{array}{l}091729.8 \\
174749.4 \\
224048.4 \\
124111.1 \\
131859.6\end{array}$ & $\begin{array}{l}19.406 \mathrm{~N} \\
19.348 \mathrm{~N} \\
19.305 \mathrm{~N} \\
19.217 \mathrm{~N} \\
19.059 \mathrm{~N}\end{array}$ & $\begin{array}{l}155.434 \mathrm{~W} \\
155.280 \mathrm{~W} \\
155.366 \mathrm{~W} \\
155.356 \mathrm{~W} \\
155.899 \mathrm{~W}\end{array}$ & $\begin{array}{l}009 \\
032 \\
006 \\
005 \\
010\end{array}$ & $\begin{array}{r}418 \\
418 \\
418 \\
418 \\
74\end{array}$ & $\begin{array}{l}5.0 \\
4.5 \\
5.5 \\
4.7 \\
4.5\end{array}$ & $\begin{array}{l}- \\
5.2 \\
-\end{array}$ & $\begin{array}{cc}4.70 \mathrm{M}_{\mathrm{L}} & \text { HVO } \\
4.60 \mathrm{M}_{\mathrm{L}} & \text { HVO } \\
5.40 \mathrm{M}_{\mathrm{L}} & \text { HVO } \\
4.60 \mathrm{M}_{\mathrm{L}} & \text { HVO } \\
- & \end{array}$ & $\begin{array}{l}- \\
z\end{array}$ & $\begin{array}{c}\text { V } \\
\text { IV } \\
\text { V } \\
\text { IV } \\
\text { Felt }\end{array}$ & $\begin{array}{l}47 \\
47 \\
47 \\
48 \\
74\end{array}$ & $\frac{-}{-}$ \\
\hline $\begin{array}{l}1975 \\
1975 \\
1975 \\
1975 \\
1975\end{array}$ & $\begin{array}{ll}01 & 01 \\
01 & 01 \\
01 & 02 \\
01 & 03 \\
01 & 05\end{array}$ & $\begin{array}{l}132054.5 \\
134436.3 \\
132743.4 \\
173249.5 \\
013205.5\end{array}$ & $\begin{array}{l}19.475 \mathrm{~N} \\
19.071 \mathrm{~N} \\
19.232 \mathrm{~N} \\
19.207 \mathrm{~N} \\
19.247 \mathrm{~N}\end{array}$ & $\begin{array}{l}155.580 \mathrm{~W} \\
155.854 \mathrm{~W} \\
155.391 \mathrm{~W} \\
155.364 \mathrm{~W} \\
155.373 \mathrm{~W}\end{array}$ & $\begin{array}{l}010 \\
010 \\
009 \\
010 \\
007\end{array}$ & $\begin{array}{r}74 \\
74 \\
418 \\
418 \\
418\end{array}$ & $\begin{array}{l}5.1 \\
4.7 \\
4.5 \\
4.7 \\
5.1\end{array}$ & $\frac{5.3}{\overline{4.2}}$ & $\begin{array}{ll}4.90 \bar{M}_{\mathrm{L}} & \text { HVO } \\
4.90 \mathrm{M}_{\mathrm{L}} & \text { HVO } \\
4.90 \mathrm{M}_{\mathrm{L}} & \text { HVO } \\
4.90 \mathrm{M}_{\mathrm{L}} & \text { HVO }\end{array}$ & $\bar{z}$ & $\begin{array}{c}\text { Felt } \\
\text { Felt } \\
\text { V } \\
\text { V } \\
\text { V }\end{array}$ & $\begin{array}{l}74 \\
74 \\
48 \\
48 \\
48\end{array}$ & $\begin{array}{l}- \\
- \\
-\end{array}$ \\
\hline $\begin{array}{l}1975 \\
1975 \\
1975 \\
1975 \\
1975\end{array}$ & $\begin{array}{ll}03 & 30 \\
05 & 22 \\
05 & 28 \\
07 & 08 \\
11 & 06\end{array}$ & $\begin{array}{l}005626.5 \\
083258.4 \\
120208.8 \\
004742.6 \\
120528.4\end{array}$ & $\begin{array}{l}19.283 \mathrm{~N} \\
20.288 \mathrm{~N} \\
18.641 \mathrm{~N} \\
19.536 \mathrm{~N} \\
19.343 \mathrm{~N}\end{array}$ & $\begin{array}{l}155.374 \mathrm{~W} \\
155.657 \mathrm{~W} \\
154.273 \mathrm{~W} \\
155.504 \mathrm{~W} \\
155.313 \mathrm{~W}\end{array}$ & $\begin{array}{l}006 \\
012 \\
042 \\
003 \\
019\end{array}$ & $\begin{array}{l}418 \\
418 \\
418 \\
418 \\
418\end{array}$ & $\begin{array}{l}\overline{4.4} \\
4.6 \\
3.9 \\
4.4\end{array}$ & $\begin{array}{l}- \\
-\end{array}$ & $\begin{array}{l}4.60 \mathrm{M}_{\mathrm{D}} \text { HVO } \\
4.70 \mathrm{M}_{\mathrm{L}} \text { HVO } \\
5.10 \mathrm{M}_{\mathrm{D}} \text { HVO } \\
4.70 \mathrm{M}_{\mathrm{L}} \text { HVO } \\
4.50 \mathrm{M}_{\mathrm{L}} \text { HVO }\end{array}$ & $\frac{-}{z}$ & $\frac{\bar{v}}{\mathrm{IV}}$ & $\begin{array}{l}\overline{48} \\
\frac{18}{48}\end{array}$ & $\begin{array}{l}- \\
- \\
-\end{array}$ \\
\hline $\begin{array}{l}1975 \\
1975 \\
1975 \\
1975 \\
1976\end{array}$ & $\begin{array}{ll}11 & 29 \\
11 & 29 \\
11 & 29 \\
11 & 30 \\
01 & 15\end{array}$ & $\begin{array}{l}133540.7 \\
144740.1 \\
184400.2 \\
061527.4 \\
225926.2\end{array}$ & $\begin{array}{l}19.362 \mathrm{~N} \\
19.341 \mathrm{~N} \\
19.145 \mathrm{~N} \\
19.419 \mathrm{~N} \\
19.413 \mathrm{~N}\end{array}$ & $\begin{array}{l}155.039 \mathrm{~W} \\
155.004 \mathrm{~W} \\
155.252 \mathrm{~W} \\
155.373 \mathrm{~W} \\
155.295 \mathrm{~W}\end{array}$ & $\begin{array}{l}002 \\
009 \\
005 \\
012 \\
018\end{array}$ & $\begin{array}{l}418 \\
418 \\
418 \\
418 \\
418\end{array}$ & $\begin{array}{l}5.8 \\
6.0 \\
4.9 \\
\overline{4.8}\end{array}$ & $\begin{array}{l}5.1 \\
7.1 \\
- \\
-\end{array}$ & $\begin{array}{l}5.90 \mathrm{M}_{\mathrm{L}} \text { HVO } \\
7.20 \mathrm{M}_{\mathrm{S}} \text { PAS } \\
4.60 \mathrm{M}_{\mathrm{L}} \text { HVO } \\
4.50 \mathrm{M}_{\mathrm{L}} \text { HVO } \\
4.50 \mathrm{M}_{\mathrm{L}} \text { HVO }\end{array}$ & $\begin{array}{c}\overline{7.45 \mathrm{AND}} \\
- \\
-\end{array}$ & $\frac{\text { VI }}{\frac{\text { VII }}{\text { V }}}$ & $\begin{array}{l}48 \\
\frac{48}{-} \\
\frac{49}{49}\end{array}$ & $\begin{array}{l}- \\
\overline{-}\end{array}$ \\
\hline $\begin{array}{l}1976 \\
1976 \\
1976 \\
1976 \\
1976\end{array}$ & $\begin{array}{ll}01 & 29 \\
02 & 21 \\
04 & 02 \\
04 & 21 \\
05 & 24\end{array}$ & $\begin{array}{lll}20 & 19 & 56.6 \\
05 & 51 & 17.4 \\
18 & 14 & 06.7 \\
18 & 13 & 35.9 \\
09 & 24 & 07.8\end{array}$ & $\begin{array}{l}19.373 \mathrm{~N} \\
20.267 \mathrm{~N} \\
19.342 \mathrm{~N} \\
18.816 \mathrm{~N} \\
20.995 \mathrm{~N}\end{array}$ & $\begin{array}{l}154.988 \mathrm{~W} \\
155.990 \mathrm{~W} \\
155.106 \mathrm{~W} \\
155.032 \mathrm{~W} \\
156.362 \mathrm{~W}\end{array}$ & $\begin{array}{l}010 \\
021 \\
010 \\
012 \\
020\end{array}$ & $\begin{array}{l}418 \\
418 \\
418 \\
418 \\
418\end{array}$ & $\begin{array}{l}4.5 \\
4.9 \\
4.5 \\
- \\
-\end{array}$ & $\begin{array}{l}\overline{4.0} \\
- \\
-\end{array}$ & $\begin{array}{ll}4.70 \mathrm{M}_{\mathrm{L}} & \text { HVO } \\
5.00 \mathrm{M}_{\mathrm{L}} & \text { HVO } \\
4.50 \mathrm{M}_{\mathrm{L}} & \text { HVO } \\
4.50 \mathrm{M}_{\mathrm{L}} & \text { HVO } \\
4.90 \mathrm{M}_{\mathrm{D}} & \text { HVO }\end{array}$ & $\frac{-}{z}$ & $\begin{array}{l}\text { IV } \\
\text { VI } \\
\text { V } \\
\text { V }\end{array}$ & $\begin{array}{l}49 \\
49 \\
49 \\
49\end{array}$ & $\bar{z}$ \\
\hline $\begin{array}{l}1976 \\
1977 \\
1977 \\
1977 \\
1977\end{array}$ & $\begin{array}{ll}12 & 18 \\
01 & 14 \\
01 & 22 \\
02 & 04 \\
04 & 21\end{array}$ & $\begin{array}{l}140100.7 \\
232642.5 \\
223628.5 \\
012059.9 \\
044923.2\end{array}$ & $\begin{array}{l}19.330 \mathrm{~N} \\
19.329 \mathrm{~N} \\
20.940 \mathrm{~N} \\
19.347 \mathrm{~N} \\
19.326 \mathrm{~N}\end{array}$ & $\begin{array}{l}155.115 \mathrm{~W} \\
155.119 \mathrm{~W} \\
160.260 \mathrm{~W} \\
155.074 \mathrm{~W} \\
155.325 \mathrm{~W}\end{array}$ & $\begin{array}{l}010 \\
010 \\
033 \\
010 \\
012\end{array}$ & \begin{tabular}{r|r|}
418 \\
418 \\
74 \\
418 \\
418
\end{tabular} & $\begin{array}{l}5.0 \\
4.2 \\
4.7 \\
4.5 \\
-\end{array}$ & $\bar{z}$ & $\begin{array}{ll}4.80 \mathrm{M}_{\mathrm{L}} & \text { HVO } \\
4.70 \mathrm{M}_{\mathrm{L}} & \text { HVO } \\
5.10 \mathrm{M}_{\mathrm{L}} & \text { HVO } \\
4.50 \mathrm{M}_{\mathrm{L}} & \text { HVO } \\
5.00 \mathrm{M}_{\mathrm{L}} & \text { HVO }\end{array}$ & 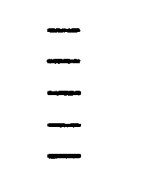 & $\frac{\mathrm{V}}{\mathrm{IV}}$ & $\begin{array}{l}49 \\
39 \\
-39 \\
39\end{array}$ & $\begin{array}{l}- \\
-\end{array}$ \\
\hline $\begin{array}{l}1977 \\
1977 \\
1979 \\
1979 \\
1979\end{array}$ & $\begin{array}{ll}06 & 06 \\
09 & 07 \\
03 & 06 \\
03 & 10 \\
03 & 22\end{array}$ & $\begin{array}{l}094219.1 \\
235106.9 \\
150758.5 \\
135514.6 \\
064659.8\end{array}$ & $\begin{array}{l}19.362 \mathrm{~N} \\
19.373 \mathrm{~N} \\
19.520 \mathrm{~N} \\
19.334 \mathrm{~N} \\
20.100 \mathrm{~N}\end{array}$ & $\begin{array}{l}155.081 \mathrm{~W} \\
155.322 \mathrm{~W} \\
155.270 \mathrm{~W} \\
155.111 \mathrm{~W} \\
155.841 \mathrm{~W}\end{array}$ & $\begin{array}{l}009 \\
031 \\
027 \\
010 \\
016\end{array}$ & $\begin{array}{l}418 \\
418 \\
418 \\
418 \\
418\end{array}$ & $\begin{array}{l}4.8 \\
5.0 \\
4.8 \\
4.6\end{array}$ & $\overline{-}$ & $\begin{array}{ll}5.10 \mathrm{M}_{\mathrm{L}} & \text { HVO } \\
4.50 \mathrm{M}_{\mathrm{L}} & \text { HVO } \\
4.70 \mathrm{M}_{\mathrm{L}} & \text { HVO } \\
4.50 \mathrm{M}_{\mathrm{L}} & \text { HVO } \\
4.50 \mathrm{M}_{\mathrm{L}} & \text { HVO }\end{array}$ & $\begin{array}{l}- \\
- \\
-\end{array}$ & 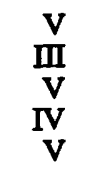 & $\begin{array}{r}39 \\
39 \\
489 \\
262 \\
262\end{array}$ & $\begin{array}{l}- \\
- \\
-\end{array}$ \\
\hline $\begin{array}{l}1979 \\
1979 \\
1979 \\
1979 \\
1980\end{array}$ & $\begin{array}{ll}03 & 28 \\
03 & 30 \\
08 & 14 \\
09 & 22 \\
01 & 20\end{array}$ & $\begin{array}{l}073009.8 \\
090639.6 \\
125142.2 \\
075937.6 \\
012848.6\end{array}$ & $\begin{array}{l}20.090 \mathrm{~N} \\
20.608 \mathrm{~N} \\
20.814 \mathrm{~N} \\
19.347 \mathrm{~N} \\
19.312 \mathrm{~N}\end{array}$ & $\begin{array}{l}155.835 \mathrm{~W} \\
158.862 \mathrm{~W} \\
156.290 \mathrm{~W} \\
155.071 \mathrm{~W} \\
155.541 \mathrm{~W}\end{array}$ & $\begin{array}{l}012 \\
010 \\
024 \\
009 \\
027\end{array}$ & $\begin{array}{l}418 \\
299 \\
418 \\
418 \\
418\end{array}$ & $\begin{array}{l}4.4 \\
4.7 \\
4.1 \\
5.7 \\
-\end{array}$ & $\frac{\overline{3.9}}{4.8}$ & $\begin{array}{ll}4.90 \mathrm{M}_{\mathrm{L}} & \text { HVO } \\
5.50 \mathrm{M}_{\mathrm{L}} & \text { HVO } \\
4.50 \mathrm{M}_{\mathrm{L}} & \text { HVO } \\
5.50 \mathrm{M}_{\mathrm{L}} & \text { HVO } \\
4.60 \mathrm{M}_{\mathrm{L}} & \text { HVO }\end{array}$ & $\bar{z}$ & $\begin{array}{l}\mathrm{V} \\
\mathrm{V} \\
\mathrm{V} \\
\mathrm{V} \\
\mathrm{V}\end{array}$ & $\begin{array}{l}262 \\
262 \\
262 \\
262 \\
300\end{array}$ & $\begin{array}{l}- \\
- \\
-\end{array}$ \\
\hline $\begin{array}{l}1981 \\
1981 \\
1981 \\
1981 \\
1981\end{array}$ & $\begin{array}{ll}01 & 12 \\
01 & 14 \\
03 & 05 \\
03 & 06 \\
08 & 10\end{array}$ & $\begin{array}{l}141810.6 \\
042016.5 \\
140939.8 \\
024336.4 \\
194035.0\end{array}$ & $\begin{array}{l}19.356 \mathrm{~N} \\
19.368 \mathrm{~N} \\
21.017 \mathrm{~N} \\
21.159 \mathrm{~N} \\
19.306 \mathrm{~N}\end{array}$ & $\begin{array}{l}155.305 \mathrm{~W} \\
155.324 \mathrm{~W} \\
156.988 \mathrm{~W} \\
156.910 \mathrm{~W} \\
155.359 \mathrm{~W}\end{array}$ & $\begin{array}{l}031 \\
029 \\
010 \\
000 \\
004\end{array}$ & $\begin{array}{r}418 \\
418 \\
74 \\
418 \\
418\end{array}$ & $\begin{array}{l}4.4 \\
4.5 \\
5.0 \\
4.5 \\
4.7\end{array}$ & $\overline{-}$ & $\begin{array}{ll}4.50 \mathrm{M}_{\mathrm{L}} & \text { HVO } \\
4.80 \mathrm{M}_{\mathrm{D}} & \text { HVO } \\
5.10 \mathrm{M}_{\mathrm{L}} & \text { HVO } \\
4.50 \mathrm{M}_{\mathrm{L}} & \text { HVO } \\
4.50 \mathrm{M}_{\mathrm{L}} & \text { HVO }\end{array}$ & $\begin{array}{l}z \\
z\end{array}$ & $\begin{array}{l}\text { V } \\
\text { V } \\
\text { VI } \\
\text { III } \\
\text { IV }\end{array}$ & $\begin{array}{l}325 \\
325 \\
325 \\
325 \\
325\end{array}$ & $\begin{array}{l}- \\
z\end{array}$ \\
\hline
\end{tabular}


HAWAII-Continued

[See table 1 for hypocenter and intensity references and table 2 for definitions of magnitude source codes. Leader (--) indicates information is not available]

\begin{tabular}{|c|c|c|c|c|c|c|c|c|c|c|c|c|c|}
\hline \multirow{2}{*}{\multicolumn{2}{|c|}{ Date }} & \multirow{2}{*}{$\begin{array}{c}\text { Origin } \\
\text { time (UTC) }\end{array}$} & \multicolumn{4}{|c|}{ Hypocenter } & \multicolumn{4}{|c|}{ Magnitude } & \multicolumn{2}{|c|}{ Intensity } & \multirow{3}{*}{$\begin{array}{c}\text { Felt area } \\
\left(1,000 \mathrm{~km}^{2}\right)\end{array}$} \\
\hline & & & \multirow{2}{*}{$\begin{array}{l}\text { Latitude } \\
\left({ }^{\circ}\right)\end{array}$} & \multirow{2}{*}{$\begin{array}{l}\text { Longitude } \\
\left({ }^{\circ}\right)\end{array}$} & \multirow{2}{*}{$\begin{array}{c}\text { Depth } \\
(\mathrm{km})\end{array}$} & \multirow[t]{2}{*}{ Ref } & \multicolumn{2}{|c|}{ USGS } & \multirow[t]{2}{*}{ Other } & \multirow{2}{*}{$\begin{array}{c}\text { Moment } \\
\text { M }\end{array}$} & \multirow[t]{2}{*}{ MMI } & \multirow[t]{2}{*}{ Ref } & \\
\hline Yr & $\mathrm{Mo} \mathrm{Da}$ & $\mathrm{h} \mathrm{m} \mathrm{s}$ & & & & & $m_{b}$ & $\mathbf{M}_{\mathbf{s}}$ & & & & & \\
\hline $\begin{array}{l}1981 \\
1982 \\
1982 \\
1982 \\
1982\end{array}$ & $\begin{array}{ll}11 & 10 \\
01 & 21 \\
01 & 21 \\
05 & 14 \\
05 & 19\end{array}$ & $\begin{array}{l}130256.6 \\
215241.2 \\
222913.9 \\
162631.7 \\
033619.8\end{array}$ & $\begin{array}{l}19.343 \mathrm{~N} \\
19.230 \mathrm{~N} \\
19.218 \mathrm{~N} \\
20.001 \mathrm{~N} \\
19.954 \mathrm{~N}\end{array}$ & $\begin{array}{l}155.211 \mathrm{~W} \\
155.592 \mathrm{~W} \\
155.552 \mathrm{~W} \\
155.864 \mathrm{~W} \\
156.436 \mathrm{~W}\end{array}$ & $\begin{array}{l}010 \\
010 \\
014 \\
020 \\
001\end{array}$ & $\begin{array}{l}418 \\
418 \\
418 \\
418 \\
418\end{array}$ & $\begin{array}{l}5.3 \\
5.4 \\
5.6 \\
4.5 \\
4.8\end{array}$ & $\begin{array}{l}\overline{4.9} \\
4.8 \\
\overline{3.7}\end{array}$ & $\begin{array}{l}4.90 \mathrm{M}_{\mathrm{D}} \text { HVO } \\
5.40 \mathrm{M}_{\mathrm{L}} \text { HVO } \\
5.40 \mathrm{M}_{\mathrm{L}} \text { HVO } \\
4.80 \mathrm{M}_{\mathrm{L}} \text { HVO } \\
4.80 \mathrm{M}_{\mathrm{L}} \text { HVO }\end{array}$ & $\begin{array}{l}\bar{z} \\
\overline{-}\end{array}$ & $\begin{array}{l}\mathbf{V} \\
\mathbf{v I} \\
\text { VI } \\
\mathrm{V} \\
\mathbf{v}\end{array}$ & $\begin{array}{l}325 \\
350 \\
350 \\
350 \\
350\end{array}$ & $\begin{array}{l}\bar{z} \\
\bar{E}\end{array}$ \\
\hline $\begin{array}{l}1983 \\
1983 \\
1983 \\
1983 \\
1984\end{array}$ & $\begin{array}{ll}03 & 08 \\
03 & 21 \\
09 & 09 \\
11 & 16 \\
06 & 09\end{array}$ & $\begin{array}{llll}16 & 41 & 03.4 \\
03 & 18 & 39.2 \\
16 & 30 & 55.3 \\
16 & 13 & 00.1 \\
03 & 34 & 10.6\end{array}$ & $\begin{array}{l}19.199 \mathrm{~N} \\
19.357 \mathrm{~N} \\
19.332 \mathrm{~N} \\
19.429 \mathrm{~N} \\
20.055 \mathrm{~N}\end{array}$ & $\begin{array}{l}155.593 \mathrm{~W} \\
155.050 \mathrm{~W} \\
155.122 \mathrm{~W} \\
155.452 \mathrm{~W} \\
157.975 \mathrm{~W}\end{array}$ & $\begin{array}{l}011 \\
007 \\
009 \\
011 \\
030\end{array}$ & $\begin{array}{l}418 \\
360 \\
360 \\
360 \\
370\end{array}$ & $\begin{array}{l}\overline{4.9} \\
5.5 \\
6.4 \\
-\end{array}$ & $\begin{array}{l}\overline{-} \\
5.0 \\
6.7\end{array}$ & $\begin{array}{l}4.50 \mathrm{M}_{\mathrm{L}} \text { HVO } \\
4.80 \mathrm{M}_{\mathrm{L}} \text { HVO } \\
5.40 \mathrm{M}_{\mathrm{L}} \text { HVO } \\
6.60 \mathrm{M}_{\mathrm{L}} \text { HVO } \\
5.00 \mathrm{M}_{\mathrm{L}} \text { HVO }\end{array}$ & $\begin{array}{l}\bar{z} \\
\overline{-}\end{array}$ & $\begin{array}{r}\text { IV } \\
\text { V } \\
\text { V } \\
\text { VIII } \\
\text { - }\end{array}$ & $\begin{array}{l}360 \\
360 \\
360 \\
360 \\
-\end{array}$ & $\begin{array}{l}\bar{z} \\
\bar{z}\end{array}$ \\
\hline $\begin{array}{l}1985 \\
1985 \\
1986 \\
1986 \\
1987\end{array}$ & $\begin{array}{ll}02 & 22 \\
12 & 12 \\
04 & 23 \\
04 & 26 \\
02 & 04\end{array}$ & $\begin{array}{l}054829.4 \\
190122.9 \\
044351.3 \\
171946.5 \\
022232.7\end{array}$ & $\begin{array}{l}19.378 \mathrm{~N} \\
20.578 \mathrm{~N} \\
19.305 \mathrm{~N} \\
20.811 \mathrm{~N} \\
20.053 \mathrm{~N}\end{array}$ & $\begin{array}{l}155.211 \mathrm{~W} \\
155.755 \mathrm{~W} \\
155.271 \mathrm{~W} \\
155.749 \mathrm{~W} \\
156.530 \mathrm{~W}\end{array}$ & $\begin{array}{l}009 \\
025 \\
031 \\
033 \\
010\end{array}$ & $\begin{array}{r}371 \\
371 \\
562 \\
562 \\
74\end{array}$ & $\begin{array}{l}5.0 \\
4.3 \\
5.1 \\
5.2\end{array}$ & $\begin{array}{l}\bar{z} \\
\overline{4.9}\end{array}$ & $\begin{array}{ll}4.80 \mathrm{M}_{\mathrm{L}} & \text { HVO } \\
4.70 \mathrm{M}_{\mathrm{L}} & \text { HVO } \\
4.50 \mathrm{M}_{\mathrm{L}} & \text { HVO } \\
4.90 \mathrm{M}_{\mathrm{L}} & \text { HVO } \\
5.20 \mathrm{M}_{\mathrm{L}} & \text { HVO }\end{array}$ & $\begin{array}{l}\bar{z} \\
\overline{5.44 \mathrm{HAV}}\end{array}$ & $\begin{array}{l}\text { V } \\
\mathbf{V} \\
\mathrm{IV} \\
\mathrm{V} \\
\mathrm{V}\end{array}$ & $\begin{array}{l}371 \\
371 \\
562 \\
562 \\
577\end{array}$ & $\begin{array}{l}\bar{z} \\
\bar{z}\end{array}$ \\
\hline $\begin{array}{l}1988 \\
1988 \\
1988 \\
1988 \\
1988\end{array}$ & $\begin{array}{ll}03 & 02 \\
03 & 25 \\
03 & 28 \\
04 & 02 \\
06 & 07\end{array}$ & $\begin{array}{l}084156.5 \\
002950.5 \\
033340.8 \\
044807.1 \\
104845.0\end{array}$ & $\begin{array}{l}19.329 \mathrm{~N} \\
19.992 \mathrm{~N} \\
19.936 \mathrm{~N} \\
19.714 \mathrm{~N} \\
19.319 \mathrm{~N}\end{array}$ & $\begin{array}{l}155.213 \mathrm{~W} \\
156.454 \mathrm{~W} \\
156.445 \mathrm{~W} \\
156.570 \mathrm{~W} \\
155.117 \mathrm{~W}\end{array}$ & $\begin{array}{l}010 \\
000 \\
001 \\
010 \\
010\end{array}$ & $\begin{array}{l}74 \\
74 \\
74 \\
74\end{array}$ & $\begin{array}{l}5.6 \\
4.7 \\
4.4\end{array}$ & $\begin{array}{l}\overline{4.5} \\
5.1 \\
-\end{array}$ & $\begin{array}{c}4.70 \mathrm{M}_{\mathrm{D}} \text { HVO } \\
5.30 \overline{\mathrm{M}}_{\mathrm{S}} \text { BRK } \\
4.70 \overline{\mathrm{M}}_{\mathrm{D}} \text { HVO }\end{array}$ & $\begin{array}{c}\bar{z} \\
5 . \overline{\mathrm{HAV}} \\
\overline{-}\end{array}$ & $\begin{array}{r}\text { V } \\
\text { IV } \\
\text { V } \\
\text { Felt } \\
\text { V }\end{array}$ & $\begin{array}{l}578 \\
578 \\
578 \\
578\end{array}$ & $\begin{array}{l}\bar{z} \\
\bar{z}\end{array}$ \\
\hline $\begin{array}{l}1988 \\
1988 \\
1989 \\
1989 \\
1989\end{array}$ & $\begin{array}{ll}07 & 04 \\
07 & 31 \\
04 & 04 \\
06 & 26 \\
12 & 05\end{array}$ & $\begin{array}{l}053809.3 \\
140433.0 \\
190957.0 \\
0327 \quad 03.9 \\
22 \quad 1658.6\end{array}$ & $\begin{array}{l}19.221 \mathrm{~N} \\
18.924 \mathrm{~N} \\
18.972 \mathrm{~N} \\
19.362 \mathrm{~N} \\
21.633 \mathrm{~N}\end{array}$ & $\begin{array}{l}155.459 \mathrm{~W} \\
155.207 \mathrm{~W} \\
155.288 \mathrm{~W} \\
155.083 \mathrm{~W} \\
157.323 \mathrm{~W}\end{array}$ & $\begin{array}{l}011 \\
017 \\
024 \\
009 \\
010\end{array}$ & $\begin{array}{l}74 \\
74 \\
74 \\
74 \\
74\end{array}$ & $\begin{array}{l}5.1 \\
4.5 \\
4.6 \\
5.8 \\
4.0\end{array}$ & $\bar{z}$ & $\begin{array}{l}5.20 \mathrm{M}_{\mathrm{L}} \text { HVO } \\
4.60 \mathrm{M}_{\mathrm{D}} \text { HVO } \\
4.40 \mathrm{M}_{\mathrm{D}} \text { HVO } \\
6.20 \mathrm{M}_{\mathrm{D}} \text { HVO } \\
4.60 \mathrm{M}_{\mathrm{L}} \text { HVO }\end{array}$ & $\frac{\bar{Z}}{{ }_{6.43 \mathrm{HAV}}}$ & $\begin{array}{l}\text { IV } \\
\text { IV } \\
\text { IV }\end{array}$ & $\begin{array}{l}578 \\
579 \\
579 \\
579\end{array}$ & $\bar{z}$ \\
\hline 1989 & 1228 & 091317.3 & $19.333 \mathrm{~N}$ & $155.212 \mathrm{~W}$ & 009 & 74 & 5.0 & 4.5 & $5.00 \mathrm{M}_{\mathrm{L}} \quad \mathrm{HVO}$ & - & V & 579 & - \\
\hline
\end{tabular}

\section{INTRODUCTION}

All earthquakes included in the hypocenter list above are of magnitude $\geq 4.5$ or Modified Mercalli intensity $\geq$ VI. Those without a computed magnitude were estimated to be $\geq 4.5$. Since the routine computation of magnitudes for Hawaiian earthquakes began in about 1956, most of the earthquakes that do not have recorded magnitudes occurred before that year.

Epicenters included in this table that are without magnitudes are estimated to be equivalent to a magnitude $\geq 4.5$. These estimates were derived by comparing pre-1956 felt or damage reports to reports from well-documented earthquakes in later years that had computed magnitudes. From that comparison, two criteria emerged that defined an estimated magnitude $\geq 4.5$ earthquake. These were: (1) reported felt on more than one island, or (2) reported as experiencing "severe" shaking. Only the earthquakes meeting one of these criteria were included in the table.

Estimates of intensity also were necessary for many of the earthquakes in the hypocenter list that had a general description of the shaking but no assigned intensity. The estimates of maximum intensity are based on the following: (1) shaking described as severe-intensity V; (2) shaking reported islandwide on Hawaii Island-intensity IV; and (3) shaking reported felt only on parts of Hawaii Island-intensity III.

Felt areas were not computed in Hawaii. Reliable estimates are not possible owing to the small land area of that State.

[Reference (Ref.) numbers given in parentheses at the end of each description refer to sources of data in table 1. Magnitude values are described in the Introduction, and codes are defined in table 2.] 
1823. June (date unknown). Kaimu, Hawaii. A strong earthquake threw down a 4-ft-wide by 6-fthigh stone wall, caused large fissures and sand blows in the ground, and damaged a native dwelling. (Ref. 500.)

1834. Feb. 20 (Feb. 19). Hilo, Hawaii. A heavy shock threw down stone walls and upset small jars. The undulating motion made it difficult to stand or walk. (Ref. 38, 405.)

1838. Dec. 12. Hilo, Hawaii. This earthquake threw down stone walls and cracked plaster. The strong motion made it difficult to walk. (Ref. 405.)

1841. Apr. 8. Hilo, Hawaii. Plaster cracked and fell to the floor in every room of a house. Water was splashed out of a bucket. (Ref. 405.)

1861. Dec. 5. Honolulu, Hawaii. Plaster fell from the ceilings of several houses, and people rushed into the streets. (Ref. 423)

1868. Mar. 29 (Mar. 28). Island of Hawaii. This is a foreshock of the Apr. 3 event. At Kahuku, a stone house was destroyed, the chimney of the Mission parsonage was thrown down, and the walls of the stone church were cracked. At Kona, stone walls were thrown down, masses of rock were thrown off the cliff at the bay, stone buildings were damaged, and the cisterns of the Kona Plantation were cracked. At Waiohinu, walls were shaken down, and the stone church was cracked from top to bottom. Also slightly felt at Hilo. (Ref. 406, 500.)

1868. Apr. 3 (Apr. 2). Near south coast of Hawaii. This major earthquake caused 77 deaths (tsunami, 46; landslide, 31). It knocked almost all wooden houses off their foundations in the Keiawa, Punaluu, and Ninole areas. In those areas, straw houses supported by posts in the ground reportedly were "torn to shreds." At Kau, the more substantial houses and every stone wall were thrown down. At Waiohinu, a large stone church collapsed within 10 seconds of the onset of shaking. The shock "ruined" the few stone buildings in Hilo and shook down almost every wall. Brooks became muddy.

At Kealakekua, strong trees were bent backward and forward "like reeds in a storm." Ground waves as much as $0.6 \mathrm{~m}$ from ground to crest were observed at Kohala. The motion was so violent at Ulupalakua that it was difficult for people to stand. Reports from Keaiwa and Kiolakaa suggest that vertical accelerations larger than $1 \mathrm{~g}$ may have occurred.

Extensive surface effects were observed in the epicentral region. Ground fissures extended from Pahala to Kilauea. At Kohuku, a fissure about $5 \mathrm{~km}$ long was reported. A volcanic eruption took place from that fissure a few days later, on Apr. 7.
Landslides, which occurred beyond Hilo as far as Waipio and Hamakua, buried 10 houses in the area. A mass of earth as much as $3 \mathrm{~km}$ wide and $9 \mathrm{~m}$ thick swept down the hillside at Kapapala, carrying with it trees, animals, and people. Thirty-one people were killed.

Along the Puna coast from Kapoho to Apua, the land subsided in places as much as $2 \mathrm{~m}$. At Kaimu, trees stood about $2.5 \mathrm{~m}$ deep in sand and water. The plain at Kalapana sank about $2 \mathrm{~m}$, and water stood as much as $1.5 \mathrm{~m}$ deep over 8 hectares (20 acres) of formerly dry land.

A tsunami that struck the Kau-Puna coast added to the devastation. The waves, which were most destructive at Honuapo, Keauhou, and Punaluu. At Keauhou (now Keauhou Landing) the water rose 12-15 $\mathrm{m}$, destroying all the houses and warehouses and drowning 46 people. At Hilo, the height of the wave was about $3 \mathrm{~m}$, and at Kealakekua, $2 \mathrm{~m}$. The tsunami also was observed on Maui and Oahu. Also felt on Lanai, Maui, Oahu, and Kauai (about $560 \mathrm{~km}$ from the epicenter). (Ref. 38, 406, 500, $570,610$.

1868. Apr. 4. Near the island of Hawaii. This earthquake was described as "almost as noticeable" as the main shock on Apr. 2. Dishes were shaken from shelves on Kauai. It was "severe" at Honolulu on Oahu. (Ref. 406, 423.)

1871. Feb. 20 (Feb. 19). Near Lanai, Hawaii. This major earthquake caused severe damage on the islands of Lanai, Molokai, and Maui, and minor damage on Hawaii and Oahu. It was felt throughout the islands.

On Lanai, in the Palawai Valley, a large part of the Pali Kaholo bluff fell into the sea, and enormous fragments broke from the towering ocean walls between Manele Bay and Kamaiki Point. Masses of the red basalt were torn from the turrets of Puupehe, located on the southeast coast of Lanai. Huge boulders were hurled from the mountainsides, and ravines were filled with debris of rocks and trees. "Several great clefts opened" on different parts of the island.

On Molokai, in the Pukoo area, the earth opened for a distance of several meters; stone houses in the area cracked in every direction. A 1.5-m-deep hole opened in the ground at Pukoo. At Kaluaaha, a small addition on the northwest corner of the old stone Mission house was thrown down and a part of the east gable end crashed down through the veranda roof below. Stone walls fell in every direction. In one place on the shore, a hole about $0.6 \mathrm{~m}$ in diameter and $5.5 \mathrm{~m}$ deep was formed by the sinking earth. 
On Maui, at Lahaina, all adobe and stone houses were cracked and some were damaged so severely that they were uninhabitable. The old Mission church was damaged, and its walls were cracked. All fence walls reportedly fell to the north. A stone building and the courthouse were damaged. The main road to Lahaina cracked open for several meters. Close to the pier, the earth cracked open for a length of $14.6 \mathrm{~m}$. Damage was much less severe on other parts of Maui-stone walls were thrown down at Kaeleku, Kapueokahi, and Wailuku; cliffs collapsed at Keanae, Koali, Muole, Pukuila, and Wailua. (Ref. 422.)

1871. Sept. 13. Hilo, Hawaii. A severe earthquake knocked down walls. (Ref. 405.)

1877. May 31. Hilo, Hawaii. A severe and longlasting earthquake damaged walls and threw objects to the floor in a house. (Ref. 405.)

1881. Sept. 30. Kona, Hawaii. Many buildings and cisterns were shattered at Kona. Stone houses were damaged severely, and several kilometers of stone-wall fencing was destroyed. Every movable object in houses was jumbled and thrown together. It was described as a severe and destructive earthquake. The shaking at Hilo also was violent. (Ref. $405,407,463$.

1885. Jan. 13. Island of Maui, Hawaii. The main earthquake, which caused some damage at Kahului, Maui, was felt severely at Honolaa and Heeia, Hawaii. Also felt at Honolulu. (Ref. 405, 423.)

1887. January 24. Kau District, Hawaii Island. Many houses were moved several inches from their foundations. Water tanks were thrown down and broken. Contents of homes and stores were thrown about and broken. Six aftershocks were felt in the following half hour. (Ref. 405, 500).

1890. Aug. 7 (Aug. 6). Hilo, Hawaii. Stone walls were knocked down. In some houses, tables and pianos were moved $15 \mathrm{~cm}$ from the wall. Small objects were thrown down in almost every room in one house. (Ref. 405.)

1908. Sept. 21 (Sept. 20). Hilo, Hawaii. An earthquake caused some damage in stores and a warehouse. It threw down vases, crockery, and pictures, and stopped clocks. It caused the water in the rivers and in Hilo harbor to rise $1.2 \mathrm{~m}$. (Ref. 405, 412,610 .)

1918. Nov. 2. Mauna Loa, Hawaii. Water tanks and stone walls were damaged at Kapapala Ranch. The earthquake was felt strongly at Hilo and Kona. (Ref. 38, 265.)

1919. Sept. 15 (Sept. 14). Kilauea, Hawaii. Chimneys fell and walls cracked in the Kau section.
Slight damage was reported at Hilo. Felt on Maui and Oahu. (Ref. 38.)

1923. Jan. 14. Island of Hawaii. This strong earthquake shook down stone walls and caused some slight damage at Hilea. It was felt on Oahu and throughout Hawaii Island. (Ref. 417.)

1926. Apr. 22. Island of Hawaii. At Hilo, one building shook about $20 \mathrm{~cm}$ off its foundation. Unstable objects were overturned. Felt widely over the island. (Ref. 218, 408.)

1927. Aug. 3. Hilo, Hawaii. Slight damage occurred at Hilo. Felt throughout the island. (Ref. $38,218$.

1929. Sept. 26 (Sept. 25), Sept. 27-30. Kona, Hawaii. The strongest shock of a series occurred on Sept. 26. This earthquake generally was felt throughout the inhabited Hawaiian Islands. Walls fell in the Kona district, and many houses were damaged. Water tanks, underground pipes, and fences throughout the Kona district were demolished. Aftershocks occurring on Sept. 27, 28, 29, and 30 added greatly to the damage to tanks, masonry, stone fences, chimneys, roadways, and weak buildings on slopes. Also felt on Maui and Oahu Islands. (Ref. 38, 258, 408, 500.)

1929. Sept. 28. Island of Hawaii. At Hilo, a church was cracked severely and a street was damaged. Strongly felt. Also see the description for events on Sept. 26, 1929. (Ref. 38, 408.)

1929. Oct. 6 (Oct. 5). Holualoa, Hawaii. Walls fell and houses were displaced on their foundations at Holualoa. Road fills were cracked and embankments overthrown at the road spurs in North Kona; telephone poles were tipped over throughout North Kona. Water tanks in Kealakekua were burst or thrown off their foundations, and some weak structures collapsed. At Puuwaawaa Ranch, unbraced foundation posts fell over, masonry in the main house basement was partly thrown down, new "avalanches" fell in the gulches of Puuwaawaa hill, boulder fences were generally collapsed, and a chimney stump was broken for the second time. Also felt on Lanai, Maui, and Oahu. (Ref. 38, 258, 408, 500.)

1935. June 28. Island of Hawaii. Some damage occurred at Hilo. Generally felt throughout the island of Hawaii. (Ref. 258, 408.)

1938. Jan. 23 (Jan. 22). North of island of Maui, Hawaii. This strong earthquake, which was felt throughout the islands, caused property damage estimated at $\$ 150,000$ on Maui. Several landslides occurred throughout the island, some chimneys fell, walls were cracked, and pipelines were damaged. Two large oil tanks were shattered at Hana. Ranches on southern Maui sustained heavy damage to water 
tanks and stone walls. Walls collapsed from Ohia to Mapulehu. The Olinda Reservoir was cracked severely, and the steel storage dam on the WailukuKahului line was damaged.

Many severe cracks formed in roadbeds throughout Maui. At Kula, cracks as wide as $3.8 \mathrm{~cm}$ formed in the Haleaka road. On Molokai, at Mapulehu, cracks 5 to $7.5 \mathrm{~cm}$ in width and as much $30.5 \mathrm{~m}$ in length formed on the east Molokai road. Minor damage also occurred on the islands of Kauai, Lanai, Oahu, and northern Hawaii. Flashes of light were observed in the sky before and during the earthquake. (Ref. 11, $258,408,500$.)

1939. May 15. Island of Hawaii. Slight damage to masonry structures and plaster occurred at Hilo. Generally felt over the island of Hawaii. (Ref. 266, 408.)

1940. June 17, 1026 UTC. North of island of Hawaii. At Kaunakakai, on Molokai, glass was cracked, vases overturned, and water spilled from containers; on Maui, sharp shocks broke medicine bottles and stopped clocks; on Hawaii, dishes fell from shelves in Hilo. Residents were awakened on the islands of Hawaii, Kauai, Lanai, Maui, Molokai, and Oahu. Loose objects rattled violently. (Ref. 38, 258,500 .)

1941. Sept. 25. Island of Hawaii. Felt sharply throughout Hawaii Island. At Pahala, roadfills cracked, shoulders along roadways failed, pipes were sprung, plaster cracked, and goods fell from shelves and broke. At the Kapapala Ranch, several stone walls were partly thrown down and two windows and many dishes were broken. Many earthslides from the walls of Halemaumau caused large dust clouds; boulders shook loose from steep slopes at the head of Wood Valley and on Hilina Pali. An old crack in a building reopened at Hilo. Also felt on Oahu at Honolulu. (Ref. 38, 258, 500.)

1944. Dec. 27. Island of Hawaii. Near Hilea, stone fences were thrown down. At Pepeekeo and Naalehu, objects toppled from shelves. Also felt on Oahu. (Ref. 38, 258, 408.)

1948. June 28. Off island of Oahu, Hawaii. This sharp earthquake caused minor damage on Oahu. Four large plate-glass windows broke in downtown Honolulu; fluorescent lamps displaced from their sockets; plaster cracked in at least 20 buildings; and one chimney cracked. At the Fort Shafter military installation, a 21-m-long crack formed in the wall of the new barracks. A landslide blocked Kamehameha Highway in Kipapa Gulch, and another landslide occurred on Moanalua Road at Red Hill. Several sidewalks in Kaimuki were cracked severely.
Also felt slightly on the islands of Hawaii, Kauai, and Molokai. (Ref. 38, 421.)

1950. May 30 (May 29). Kona, Hawaii. This widely felt earthquake damaged water tanks and stone walls in Kona. At Captain Cook, Machado's store shifted about $2.5 \mathrm{~cm}$ from its foundation and four large water tanks split open. Cracks $2.5 \mathrm{~cm}$ wide were observed along the highway from Honaunau to Captain Cook, and shoulders gave way. Residents at Hilo reported broken chinaware. (Ref. 258, 408, 500.)

1951. Apr. 23 (Apr. 22). Near Kilauea caldera, Hawaii. This strong earthquake generally was felt throughout the island of Hawaii and by many residents on Maui and Oahu. It caused slight damage on Hawaii, including broken windows and dishes and a broken water pipe. The roadbed between the Volcano House and Kilauea Overlook settled, and much damage was sustained by roads in the National Park. Small earth slips occurred in road cuts between Kilauea caldera and Hilo and north of Hilo along the Hamakua coast. Minor cracks formed in the highway at the northeast rim of Kilauea caldera. Cracks in the soil, apparently caused by lurching, formed at several places north and east of Kilauea caldera. Surface faulting was not observed. (Ref. 24, 418, 500.)

1951. Aug. 21. Near Napoopoo, Hawaii. This earthquake probably was caused by movement on the Kealakekua fault. It inflicted property damage that extended from Holualoa on the north to Honuapo on the southeast, a distance of $80 \mathrm{~km}$. Damage was most severe in the central Kona district, along a 16$\mathrm{km}$ stretch from Captain Cook to Hookena. Here, several houses, churches, and a school building were damaged severely, and about 200 water tanks were demolished or damaged beyond repair (mainly owing to damage to footings). Many stone walls were thrown down. Roads were partly blocked by small rock slides; road pavement and shoulders were cracked badly; and telephone communications and electric power were disrupted. Tombstones were shifted, rotated, or overturned in many cemeteries in the area.

The earthquake was felt strongly throughout the island of Hawaii and slightly on Maui and Oahu (at Honolulu, about $290 \mathrm{~km}$ from the epicenter). Many aftershocks, all of lower intensity, occurred through September 1951. A small tsunami was observed at Hilo, Honolulu, and the Kona Coast area (maximum at Napoopoo- $0.9 \mathrm{~m}$ ). Residents of Naalehu and Pahala reported bright flashes of white light at the time of the earthquake. (Ref. 408, 419, 481, 610.)

1951. Nov. 8. Island of Hawaii. At Kahuku Ranch headquarters, $15 \mathrm{~km}$ north of South Point, on the southern part of Hawaii Island, stone walls were 
damaged extensively and dishes were thrown from shelves. Felt throughout the island. (Ref. 409, 481.)

1952. May 23. Island of Hawaii. Slight damage reported in the central Kona district included cracks in pavement, landslides from roadcuts, damaged water tanks, broken dishes and windows, and overturned tombstones. Dishes were broken as far distant as Naalehu, about $60 \mathrm{~km}$ southeast of the epicenter. Felt throughout the island and by some residents on Maui. (Ref. 238, 408.)

1954. Mar. 30, 1640 and 1841 UTC. Island of Hawaii. The foreshock at 1640 UTC collapsed several domestic water tanks at Kalapana and Opihikao. During the stronger shock 2 hours later, extensive damage occurred in the Hilo and Puna districts. In Hilo, several chimneys and stone walls were thrown down, plaster cracked, and windows broke. Between Kalapana and Opihikao, stone fences were thrown down, people driving automobiles were disturbed, and some residents found it difficult to walk or stand. Also felt on Maui and Oahu. (Ref. 410, 418.)

1954. July 3. Island of Hawaii. This strong earthquake, widely felt over the southern part of the island, caused minor damage at Hilo. Many heavy rockfalls occurred in the area of Halape during and after the earthquake. (Ref. 408.)

1955. Mar. 28 (Mar. 27). Hawaii Volcanoes National Park. This earthquake broke water lines and cracked houses in the residential area of Hawaii Volcanoes National Park. It also opened cracks across the Mamalahoa Highway, east of park headquarters. (Ref. 408.)

1955. Aug. 14. Island of Hawaii. At a farm about $64 \mathrm{~km}$ southwest of Hilo, walls were cracked and dishes were knocked from shelves. Felt throughout the island of Hawaii and on Kauai, Maui, and Oahu. (Ref. 28, 408, 482.)

1962. June 28 (June 27). Island of Hawaii. This earthquake, which occurred near the Kaoiki fault system, caused the most severe damage to houses at Kapapala. Paint on walls was chipped, large kitchen appliances were moved several centimeters, and loose china fell from shelves. Damage reported at Hilo includes cracks in walls, plaster, and windows, and broken dishes. Landslides occurred at Kawalii Gulch near Laupahoehoe, on Kealakekua Bay, and in the remote Waipio Valley on the northern end of the island. Also felt slightly on Maui and Oahu. One report of this earthquake came from the North Kohala district, a region where earthquakes are not commonly felt. More than 1,500 aftershocks were recorded in the next 3 days, and intense aftershock activity continued for several weeks. Magnitude 5.75 $\mathrm{M}_{\mathrm{S}} \mathrm{BRK}, 5.25-5.5 \mathrm{M}_{\mathrm{S}}$ PAL. (Ref. 35, 411, 418.)

1973. Apr. 26. Near northeast coast of island of Hawaii. This damaging earthquake was felt from the east coast of Hawaii Island through the main islands of Kahoolawe, Kauai, Lanai, Molokai, and Oahu, a distance of about $595 \mathrm{~km}$. Property damage in and near Hilo was estimated at $\$ 5.75$ million, and 11 people were injured.

Damage to buildings, roads, and utilities led authorities to declare a state of emergency. Ground effects, mainly landslides but including ground cracks induced by lateral displacement and local subsidence, were severe locally. Subsidence damaged the main wharf in Hilo, and landslides damaged roads and structures over a large area.

It was reported that 17 houses in the Hilo area were shaken from their foundations and that five collapsed. One structure in downtown Hilo collapsed, and the outside walls of an apartment building-a two-story concrete-block structure-were torn loose at two ends. Tombstones and chimneys overturned in several towns; water pipes and tanks were damaged. Several schools sustained damage, and four were closed temporarily. At Papaikou, the roof on the Kalaniaonaole School dropped 7-10 cm, and its ceiling was warped. Magnitude 6.3 $\mathrm{M}_{\mathrm{S}} \mathrm{PAS}, 6.1 \mathrm{M}_{\mathrm{S}}$ BRK. (Ref. 46, 418, 423, 464.)

1975. Nov. 29, 1335 UTC. Island of Hawaii. Campers at Halape saw dust clouds rising from rockfalls that the earthquake sent crashing down the face of Puu Kapukapu. Felt strongly in Hilo and Puna. (Ref. 48, 418.)

1975. Nov. 29, 1447 UTC. Southeast coast of island of Hawaii. The largest earthquake in more than a century (since April 2, 1868) struck Hawaii on the morning of Nov. 29, killing two people, injuring several, and inflicting property damage estimated at $\$ 4.1$ million in Hawaii (including damage caused by the tsunami). It was accompanied by a damaging tsunami, massive ground movements, hundreds of aftershocks, and a brief, small-volume volcanic eruption. The earthquake was felt throughout Hawaii Island, and on Lanai, Molokai, and Oahu.

Most of the buildings in the epicentral area sustained little or no structural damage from ground shaking. Structural and nonstructural damage in Hilo, $45 \mathrm{~km}$ north of the epicenter, was slight to moderate but was more extensive than elsewhere on the island.

Structural damage on the southeast part of the island included minor cracks, floor-to-wall separations a few millimeters wide, and bowing of walls in 
Hilo at a hospital, schools, and libraries. Floor sections dropped 5 to $10 \mathrm{~mm}$ in some of the buildings; hotel, apartment, and commercial buildings sustained structural and equipment damage. A damage survey of the area revealed that slight to moderate structural damage was sustained by five churches (Hilo, 4; Opihikao, 1), 11 commercial buildings (Hilo, 10; Mountain View, 1), and 80 private dwellings (Hilo, 51; Puna, 23; Hamakua, 2; Kau, 3; and Kona, 1). Five poorly constructed or old houses were demolished (Hilo, 4; Kau, 1). Houses shifted on their foundations at Kalapana, Kurtistown, Pahoa, and Hawaiian Paradise Park. Water tanks were destroyed at Opihikao, Pahoa, and Volcano.

The summit and south flank areas of Kilauea Volcano were displaced by vertical and horizontal movements of several meters, forming many ground cracks and faults. Roads in Hawaii Volcanoes National Park were damaged by extensive ground cracking. Landslides occurred on Coast Road.

Fault displacements resulted in widespread subsidence (locally as much as $3.5 \mathrm{~m}$ near Halape), leaving coconut palms stranded in the sea and almost submerging a small nearby island. Inland, an almost continuous zone of ground cracking and faulting, having vertical offsets as much as $1.5 \mathrm{~m}$, occurred along the Hilina fault system over a $25-\mathrm{km}^{2}$ area. To the south and southwest of this zone of maximum faulting, vertical displacements of as much as 0.5-1.0 $\mathrm{m}$ were common along other faults.

A large part of the coastal area between Cape Kumukahi and Punaluu subsided during or soon after the earthquake. A leveling survey of the bench marks near the Keauhou tide gage ( $2 \mathrm{~km}$ east of Halape) indicated that the coast subsided about $3.5 \mathrm{~m}$.

The tsunami generated by this major earthquake consisted of five or more distinct waves in some places. The only locally generated tsunami in Hawaii this century to be destructive, it killed two people at Halape, where it was about $7.9 \mathrm{~m}$ high, and caused property damage estimated at $\$ 1.2$ million at Punaluu, Honuapo, Kaalualu Bay, Hilo, and KailuaKona. At Punaluu $(7.6 \mathrm{~m}), 30 \mathrm{~km}$ southwest of Halape, several wood-frame houses were flattened or washed off their foundations; nearby county park facilities were damaged severely, and buildings at the Seamountain Resort sustained heavy nonstructural damage. Tsunami damage also was severe at Honuapo $(6.6 \mathrm{~m}), 6 \mathrm{~km}$ southwest of Punaluu. The highest wave reached a maximum height of $14.6 \mathrm{~m}$ above the postsubmergence shoreline, $1.5 \mathrm{~km}$ east of Halape. The tsunami was recorded in Alaska, California,
Japan, Okinawa, Samoa, and on Johnston and Wake Islands. (Ref. 48, 418, 453, 501, 610.)

1976. Feb. 21 (Feb. 20). Island of Hawaii. This minor earthquake caused damage to walls at Kawaihae. Also felt on Maui and Oahu. (Ref. 49, 418.)

1979. Sept. 22 (Sept. 21). Island of Hawaii. In the Hilo area, several hundred houses were damaged and several businesses lost merchandise. Foundations were damaged; water lines ruptured; and windows broke. At Reeds Island, a fireplace and house foundation sustained damage. (Ref. 262, 418.)

1981. Mar. 5. Near island of Molokai, Hawaii. The pipe that carries water from Waikolu Valley to Kalaupapa sustained four breaks and some cracks. Underground water pipes cracked near Kalaupapa on Molokai. Also felt on Hawaii, Lanai, Maui, and Oahu. (Ref. 74, 325.)

1982. Jan. 21, 2152 and 2229 UTC. Island of Hawaii. The first earthquake caused widespread minor damage in the Kau area; two small landslides occurred in Laupahoehoe Gulch. At Pahala, rock walls fell and chimneys cracked. The second shock, 37 minutes later, was not as strong, but it caused a rockfall in Kaawali Gulch that injured one person. Both shocks also were felt on Maui and Oahu. (Ref. $350,418$.

1983. Nov. 16. Island of Hawaii. This earthquake, the most destructive in Hawaii since a magnitude 7.1 event occurred there in 1975, caused heavy property damage on the island of Hawaii and injured six people. The Small Business Administration reported 35 commercial buildings sustained varying degrees of damage, 317 houses had minor damage, and 39 houses had major damage. Unanchored chimneys fell. Roads, bridges, and other government facilities also were damaged.

At Volcano, many houses and garages were moved off their foundations, causing extensive damage to ceilings and walls. Highways in the area were cracked severely and were closed temporarily. Elevated water tanks were thrown down; water tanks on gravel bases were moved as much as $5 \mathrm{~cm}$, and some had their roofs damaged or knocked off by sloshing water. Three chimneys collapsed. Moderate damage on Hawaii Island also occurred at Hawaiian Volcano Observatory, Hilo, Kipapala Ranch, Kaumana, Kilauea Military Camp, and Wood Valley.

Landslides and ground cracking occurred in many areas on southern Hawaii Island. The most severe ground failures were on Crater Rim Drive, a road around Kilauea crater. The road extended near the edge of the crater wall in several places, and a 


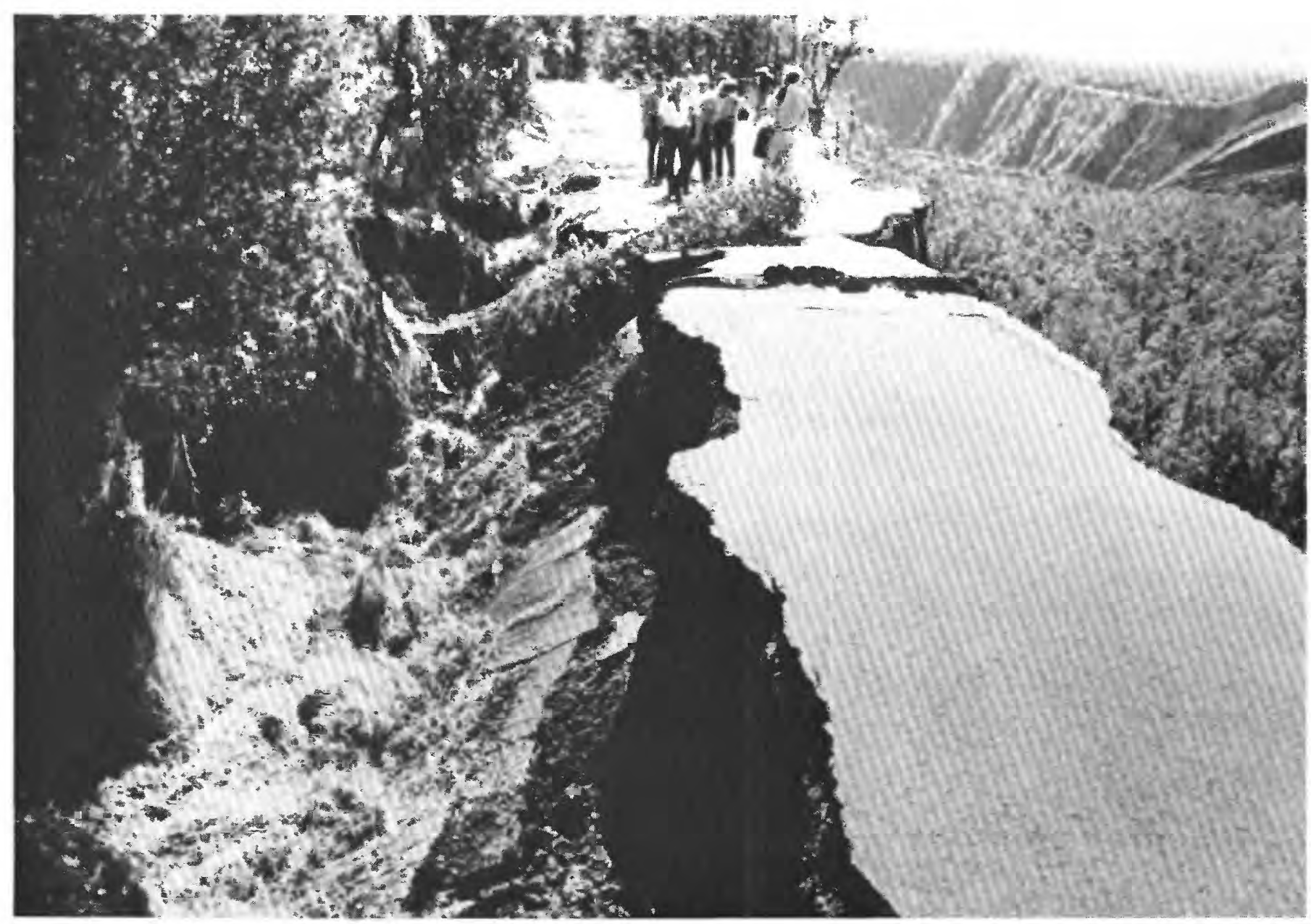

Ground failure on Crater Rim Drive, around Kilauea crater on the island of Hawaii, caused by the November 16, 1983, earthquake. (Photograph by J.D. Griggs.)

section of the road fell into the crater. In other areas, cracks as much as 1-1.5 m wide and 3-6 m deep formed in the road. Also, many sections of trails in Hawaiian Volcanoes National Park collapsed into the main caldera of Kilauea Volcano. At South Point and Kealakekua Bay, parts of the cliffs fell into the ocean. Felt on Kauai, Lanai, Maui, Molokai, and Oahu. (Ref. 360.)

1989. June 26 (June 25). Near Kalapana, Puna District, Hawaii. This strong earthquake injured five people in the Puna area and destroyed five houses. Additional structural damage to residential property in the County of Hawaii included major damage to 10 houses and minor damage to 100 houses. Many of the houses sustained cracks in walls, ceilings, and concrete pads, and slight shifting of their foundations. Total damage to property was estimated at $\$ 1$ million (Robert Y. Koyanagi, oral commun., Hawaiian Volcano Observatory, Aug. 10, 1989.)

Damage to structures in the Puna area included the collapse of at least five houses in Kaimu, Kalapana, and Royal Gardens. Ground cracks were observed across the coast road in Kalapana, and landslides were reported in Puna and along the Hamakua coast between Honokaa and Hilo. Only slight damage to houses was reported at. Hilo. Power outages occurred in the Hilo, Kau, and Puna districts.

A small tsunami was recorded on tide gauges at Hilo, Honuapo, and Kapoho. The main shock was felt on Maui, Oahu, and Hawaii Islands. Several aftershocks occurred. (Ref. 74, 579.) 


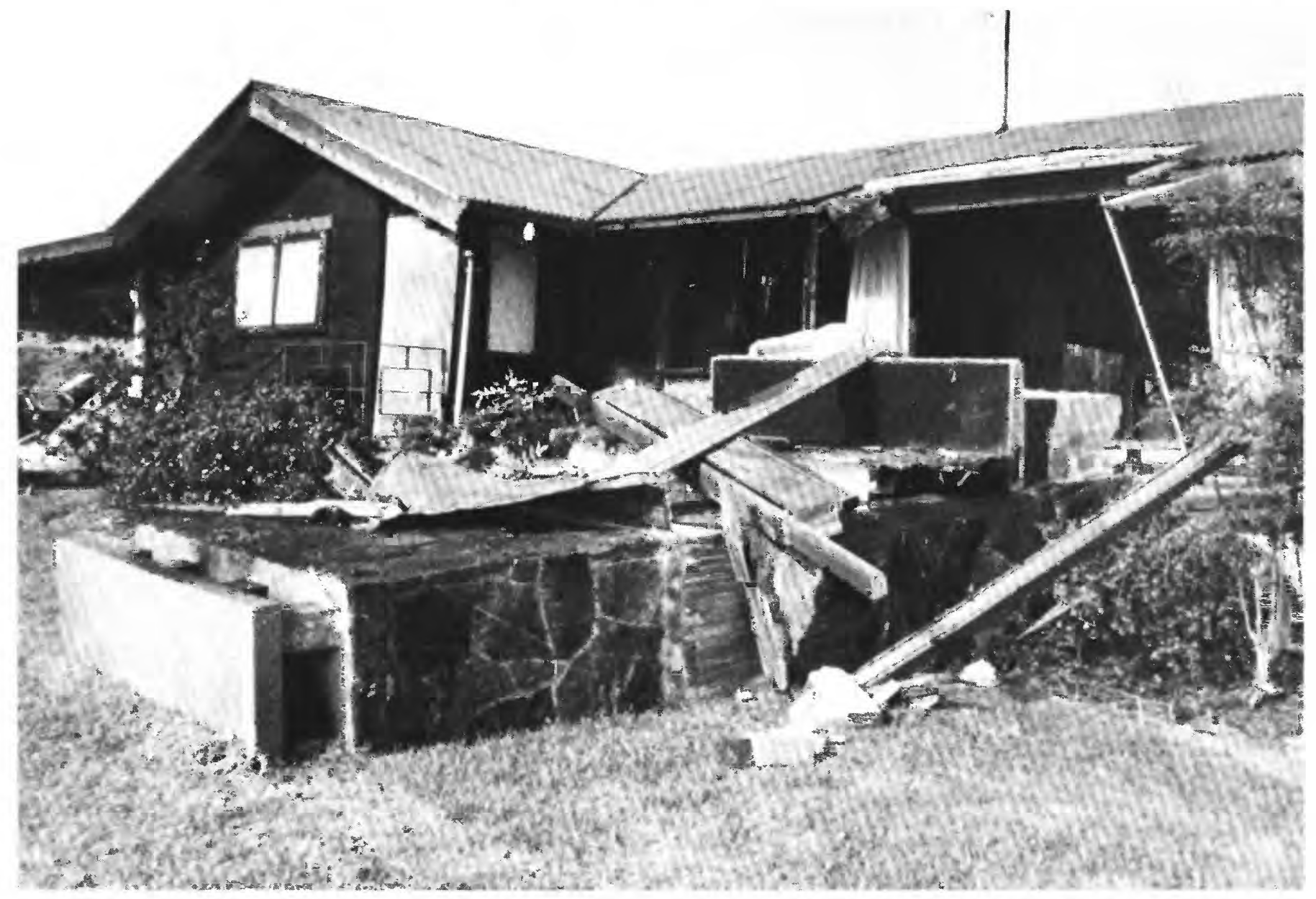

Chimney in Volcano, Hawaii, toppled by the November 16, 1983, earthquake. (Photograph by the Hawaii Tribune-Herald, Ltd.) 



\section{IDAHO}

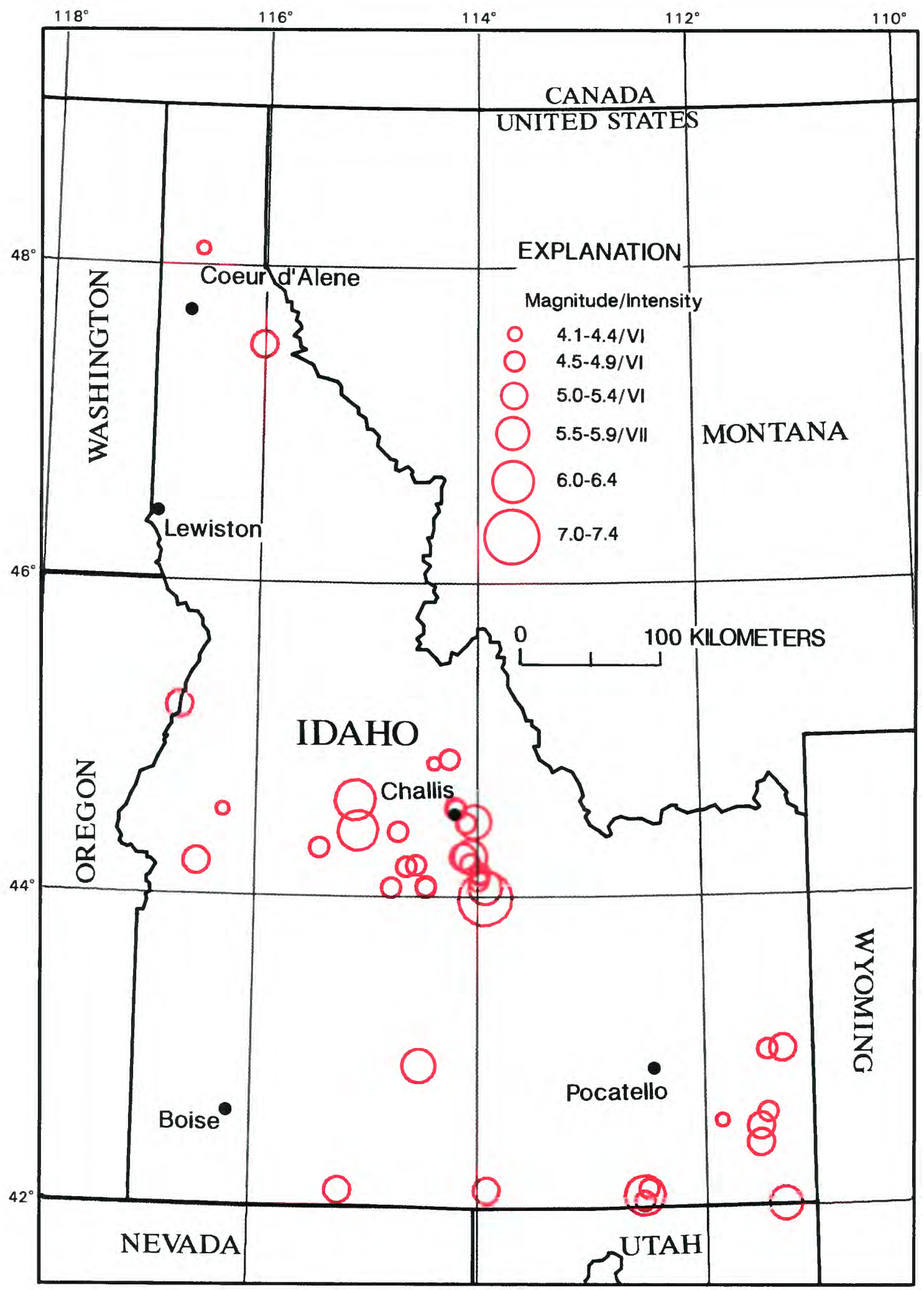

Earthquakes in Idaho with magnitudes $\geq 4.5$ or intensity $\geq$ VI. 
[See table 1 for hypocenter and intensity references and table 2 for definitions of magnitude source codes. @, felt area is less than $1,000 \mathrm{~km}^{2}$. Leader (--) indicates information is not available]

\begin{tabular}{|c|c|c|c|c|c|c|c|c|c|c|c|c|c|c|}
\hline & & & & & Hypocente & & & & & Magnitude & & Intel & sity & \\
\hline & ate & & time (UTC) & Latitude & Longitude & Depth & Ref & US & & Other & Moment & MMI & Ref & Felt area \\
\hline $\mathrm{Yr}$ & Mo & Da & $h \mathrm{~m} s$ & & & $(\mathrm{~km})$ & & $m_{b}$ & $\mathbf{M}_{\mathbf{s}}$ & & M & & & $\left(\mathrm{m}^{2}\right)$ \\
\hline 1884 & 11 & 10 & 0850 & $42.0 \mathrm{~N}$ & $111.3 \mathrm{~W}$ & - & 298 & - & - & - & - & VII & 52 & 15 \\
\hline 1905 & & 11 & 2129 & $42.9 \mathrm{~N}$ & $114.5 \mathrm{~W}$ & - & 38 & - & - & - & 一 & VII & 38 & - \\
\hline 1913 & 10 & 14 & 2300 & $45.2 \mathrm{~N}$ & $116.7 \mathrm{~W}$ & - & 56 & - & - & - & - & VI & 56 & - \\
\hline 1916 & 05 & 13 & 0226 & $44.2 \mathrm{~N}$ & $116.5 \mathrm{~W}$ & - & 498 & - & - & 5.30Ukn PAS & - & VII & 38 & 130 \\
\hline 1917 & 12 & 12 & 1050 & $43.0 \mathrm{~N}$ & $111.3 \mathrm{~W}$ & - & 38 & - & - & 5.30Ukn JON & - & V & 272 & 21 \\
\hline 1924 & 11 & 25 & 0710 & $42.5 \mathrm{~N}$ & $111.5 \mathrm{~W}$ & - & 38 & - & - & - & - & VI & 218 & 52 \\
\hline 1928 & 09 & 05 & 0536 & $42.1 \mathrm{~N}$ & $115.2 \mathrm{~W}$ & - & 54 & - & - & 5.20Ukn JON & - & 二 & - & - \\
\hline 1937 & 11 & 19 & 005020 & $42.1 \mathrm{~N}$ & $113.9 \mathrm{~W}$ & - & 54 & - & - & $5.40 \mathrm{M}_{\mathrm{x}}$ SJG & 一 & IV & 10 & $\overline{-}$ \\
\hline 1942 & 11 & 01 & 185006 & $48.1 \mathrm{~N}$ & $116.6 \mathrm{~W}$ & - & 266 & - & - & - & - & V & 15 & 65 \\
\hline 1944 & 07 & 12 & 193020.7 & $44.412 \mathrm{~N}$ & $115.063 \mathrm{~W}$ & 010 & 354 & - & - & $6.10 \mathrm{M}_{\mathrm{s}} \mathrm{GR}$ & - & VII & 17 & 153 \\
\hline 1945 & 02 & 14 & 030111.3 & $44.607 \mathrm{~N}$ & $115.087 \mathrm{~W}$ & 010 & 354 & - & - & 6.00Ukn PAS & - & VI & 18 & 128 \\
\hline 1947 & 09 & 25 & 013430 & $44.3 \mathrm{~N}$ & $115.4 \mathrm{~W}$ & - & 20 & - & - & $4.70 \mathrm{M}_{\mathrm{x}}$ JON & - & VI & 259 & - \\
\hline 1957 & 12 & 19 & 0625 & $47.5 \mathrm{~N}$ & $116.0 \mathrm{~W}$ & - & 38 & - & - & - & - & VI & 30 & @ \\
\hline 1960 & 08 & 07 & 162716.2 & $42.4 \mathrm{~N}$ & $111.5 \mathrm{~W}$ & 049 & 266 & - & - & - & - & VI & 33 & 2 \\
\hline 1963 & 01 & 27 & 152443.8 & $44.190 \mathrm{~N}$ & $114.528 \mathrm{~W}$ & 011 & 354 & - & - & $4.80 M_{n} D W$ & - & VI & 36 & 15 \\
\hline 1963 & 09 & 11 & 020843.7 & $44.177 \mathrm{~N}$ & $114.615 W$ & 008 & 354 & 4.9 & - & $4.80 M_{n}$ DW & - & VI & 36 & 9 \\
\hline 1963 & 09 & 12 & 062348.9 & $44.181 \mathrm{~N}$ & $114.621 \mathrm{~W}$ & 009 & 354 & 4.4 & - & $4.70 M_{n} D W$ & 一 & IV & 36 & - \\
\hline 1963 & 10 & 17 & 012207.7 & $44.4 \mathrm{~N}$ & $114.7 \mathrm{~W}$ & 030 & 74 & 4.7 & - & - & 一 & - & - & - \\
\hline 1969 & 04 & 26 & 104153.1 & $44.058 \mathrm{~N}$ & $114.444 W$ & 018 & 354 & 4.9 & - & $4.90 \mathrm{M}_{\mathrm{n}} \mathrm{DW}$ & - & VI & 42 & 23 \\
\hline 1969 & 09 & 19 & 133315.0 & $42.99 \mathrm{~N}$ & $111.43 \mathrm{~W}$ & 005 & 74 & 4.5 & - & $4.90 \mathrm{M}_{\mathrm{L}} \mathrm{GS}$ & - & - & - & - \\
\hline 1975 & 03 & 28 & 023106.0 & $42.06 \mathrm{~N}$ & $112.52 \mathrm{~W}$ & 005 & 298 & 6.1 & 6.0 & $6.00 \mathrm{M}_{\mathrm{L}} \mathrm{UU}$ & 6.13BAS & VIII & 48 & 160 \\
\hline 1975 & 03 & 29 & 130119.9 & $42.03 \mathrm{~N}$ & $112.52 \mathrm{~W}$ & 007 & 298 & 4.7 & - & $4.70 M_{L}$ UU & 4.93BAS & $\bar{V}$ & 48 & - \\
\hline 1977 & 11 & 27 & 092555.6 & $44.537 \mathrm{~N}$ & $116.276 \mathrm{~W}$ & 009 & 354 & 4.2 & - & $4.40 M_{n} D W$ & - & VI & 39 & 24 \\
\hline 1978 & 10 & 24 & 203059.3 & $42.55 \mathrm{~N}$ & $111.84 \mathrm{~W}$ & 007 & 240 & 4.2 & - & $4.10 \mathrm{M}_{\mathrm{L}} \mathrm{UU}$ & - & VI & 240 & 5 \\
\hline 1978 & 10 & 29 & 134645.6 & $44.866 \mathrm{~N}$ & $114.243 W$ & 012 & 354 & 4.2 & - & $4.70 M_{n} D W$ & 一 & V & 240 & 25 \\
\hline 1978 & 11 & & 065340.1 & $42.11 \mathrm{~N}$ & $112.49 \mathrm{~W}$ & 004 & 240 & 4.6 & - & $4.70 \mathrm{M}_{\mathrm{L}} \cup U$ & - & V & 240 & 18 \\
\hline 1982 & 10 & 14 & 041024.3 & $42.59 \mathrm{~N}$ & $111.43 \mathrm{~W}$ & 007 & 350 & 4.6 & - & $4.70 \mathrm{M}_{\mathrm{L}} \mathrm{UU}$ & - & VI & 350 & 13 \\
\hline 1983 & 10 & 28 & 140606.5 & $43.974 \mathrm{~N}$ & $113.916 \mathrm{~W}$ & 014 & 354 & 6.2 & 7.3 & $7.20 \mathrm{M}_{\mathrm{L}}$ BRK & 6.95ED & IX & 360 & 855 \\
\hline 1983 & 10 & 28 & 151407.7 & $44.127 \mathrm{~N}$ & $113.968 \mathrm{~W}$ & 010 & 360 & 4.3 & - & $4.60 \mathrm{M}_{\mathrm{L}} \mathrm{UU}$ & - & - & 一 & - \\
\hline 1983 & 10 & 28 & 195125.0 & $44.045 \mathrm{~N}$ & $113.918 \mathrm{~W}$ & 013 & 354 & 5.4 & 5.1 & $5.80 \mathrm{M}_{\mathrm{L}}$ UU & 5.58ED & Felt & 360 & 一 \\
\hline 1983 & 10 & 29 & 232911.8 & $44.244 \mathrm{~N}$ & $114.055 \mathrm{~W}$ & 010 & 354 & 5.4 & 5.0 & $5.80 \mathrm{M}_{\mathrm{L}}$ UU & 5.48ED & Felt & 360 & - \\
\hline 1983 & 10 & 29 & 233905.4 & $44.241 \mathrm{~N}$ & $114.109 \mathrm{~W}$ & 011 & 354 & 5.5 & 5.0 & $5.40 \mathrm{M}_{\mathrm{L}} \mathrm{UU}$ & $4.71 \mathrm{GM}$ & Felt & 360 & - \\
\hline 1983 & 10 & 30 & 012451.3 & $44.089 \mathrm{~N}$ & $113.977 \mathrm{~W}$ & 013 & 360 & 4.3 & - & $4.80 \mathrm{M}_{\mathrm{L}} \mathrm{UU}$ & $4.36 \mathrm{GM}$ & - & 一 & - \\
\hline 1983 & 10 & 30 & 015902.0 & $44.200 \mathrm{~N}$ & $114.056 \mathrm{~W}$ & 016 & 360 & 4.2 & - & $4.70 \mathrm{M}_{\mathrm{L}} \mathrm{UU}$ & $4.18 \mathrm{GM}$ & $=$ & - & - \\
\hline 1983 & 11 & 06 & 210448.7 & $44.140 \mathrm{~N}$ & $113.963 W$ & 011 & 360 & 4.3 & - & $4.60 \mathrm{M}_{\mathrm{L}} \mathrm{UU}$ & $4.16 \mathrm{GM}$ & III & 360 & 一 \\
\hline 1984 & 01 & 24 & 210757.5 & $44.047 \mathrm{~N}$ & $114.442 \mathrm{~W}$ & 010 & 370 & 4.5 & - & $4.60 \mathrm{M}_{\mathrm{L}} \quad \mathrm{MMT}$ & - & IV & 370 & 15 \\
\hline 1984 & 08 & 22 & 094630.2 & $44.467 \mathrm{~N}$ & $114.008 \mathrm{~W}$ & 010 & 370 & 5.0 & 5.1 & $5.80 \mathrm{M}_{\mathrm{L}}$ UU & - & V & 370 & 173 \\
\hline 1985 & 02 & 06 & 193419.4 & $44.551 \mathrm{~N}$ & $114.176 \mathrm{~W}$ & 010 & 371 & 4.7 & - & $4.80 \mathrm{MD} B U$ & 一 & V & 371 & 15 \\
\hline 1985 & 03 & 17 & 065617.1 & $44.553 \mathrm{~N}$ & $114.182 \mathrm{~W}$ & 010 & 371 & 4.5 & - & $4.70 \mathrm{M}_{\mathrm{D}} \mathrm{BU}$ & - & V & 371 & 15 \\
\hline 1986 & 09 & 26 & 224857.9 & $44.043 \mathrm{~N}$ & $114.756 \mathrm{~W}$ & 005 & 562 & 4.6 & - & $4.50 \mathrm{M}_{\mathrm{L}}$ GS & 一 & IV & 562 & - \\
\hline 1988 & 01 & 10 & 232219.5 & $44.840 \mathrm{~N}$ & $114.377 \mathrm{~W}$ & 005 & 74 & 4.8 & - & $4.40 \mathrm{M}_{\mathrm{L}} \mathrm{GS}$ & - & III & 578 & - \\
\hline 1988 & 07 & 14 & 173133.0 & $44.456 \mathrm{~N}$ & $114.083 W$ & 005 & 74 & 4.9 & 4.1 & - & - & IV & 578 & 110 \\
\hline 1988 & 11 & 19 & 200053.1 & $42.007 \mathrm{~N}$ & $111.477 \mathrm{~W}$ & 005 & 74 & 5.0 & - & $4.30 \mathrm{M}_{\mathrm{L}} \mathrm{UU}$ & - & Felt & 578 & - \\
\hline
\end{tabular}

[Reference (Ref.) numbers given in parentheses at the end of each description refer to sources of data in table 1. Magnitude values are described in the Introduction, and codes are defined in table 2.]

1884. Nov. 10. Paris, Franklin County, Idaho. The earthquake damaged houses considerably in
Paris, about $100 \mathrm{~km}$ southeast of Pocatello, near the Idaho-Utah-Wyoming border. It knocked down chimneys and shook stock from shelves in Richmond, Utah, about $125 \mathrm{~km}$ north of Salt Lake City. In an area north of Ogden, Utah, the tremor shook a Utah and Great Northern Railroad train. Also reported felt 
at Salt Lake City, Utah, and Franklin, Idaho. (Ref. $52,298$.

1905. Nov. 11. Near Shoshone, Lincoln County, Idaho. Cracks formed in the walls of the courthouse and schools in Shoshone, and plaster fell from ceilings in almost all the buildings. Felt from Salt Lake City, Utah, to Baker, Oreg. (Ref. 38.)

1913. Oct. 14. North-central Idaho. A tremor broke windows and dishes in the area of Idaho and Adams Counties. (Ref. 56.)

1916. May 13 (May 12). Boise, Idaho. The earthquake wrecked several brick chimneys at Boise and sent residents rushing into the street. The shock was described as "violent" at Emmett, $40 \mathrm{~km}$ north of Boise, and at Weiser, $96 \mathrm{~km}$ west of Boise. Reclamation ditches in the area were damaged. Pressure in a new gas well increased noticeably immediately after the shock. Also felt in western Montana and eastern Oregon. (Ref. 38, 272, 498.)

1924. Nov. 25. Near Wardboro, Franklin County, Idaho. A slight earthquake in Franklin County on this date broke windows at Wardboro, cracked ceilings at Montpelier, and displaced furniture at Geneva and Montpelier. (Ref. 38, 218.)

1944. July 12. Near Sheep Mountain, southwest Idaho. This earthquake apparently was most severe in the area of Fontez Creek, near Sheep Mountain, Idaho, where buildings were shaken so severely that occupants thought the structures were falling apart. A new cabin set on concrete piers was displaced on its foundation. Along Seafoam Creek, rocks and boulders were thrown down the hillside.

Cracks about $30.5 \mathrm{~m}$ long formed in the ground in the Duffield Canyon trail along Fontez Creek. Cracks 2.5 to $7.5 \mathrm{~cm}$ wide extended for several meters in a continuous break near Seafoam. A section of the Rapid River Canyon wall (near Lime Creek) fell into the river. Also felt in Montana, Oregon, and Washington (see fig. 26). Seventeen shocks were reported felt, the first of which was the strongest. (Ref. 17, 354.)

1945. Feb. 14 (Feb. 13). Idaho City, Boise County, Idaho. This tremor broke dishes at Idaho City and cracked plaster at Weiser, northwest of Boise in Washington County. Also felt in Montana, Oregon, and Washington. (Ref. 18, 354.)

1947. Sept. 25 (Sept. 24). Boise, Ada County, Idaho. Several large cracks formed in a wellconstructed brick building at Boise, but damage generally was slight. (Ref. 20, 259.)

1957. Dec. 19 (Dec. 18). Northern Idaho. Timbers fell and mine walls collapsed at the Galena Silver mine near Wallace, Shoshone County. (Ref. 30,38 .)
1960. Aug. 7. Near Soda Springs, Caribou County, Idaho. Southeast of Pocatello and about 14 $\mathrm{km}$ east of Soda Springs, cracks formed in plaster and a concrete foundation at a ranch. (Ref. 33, 266.)

1963. Jan. 27. Clayton, Custer County, Idaho. Plaster and windows cracked at Clayton, northeast of Boise. Large boulders rolled down a hill at Livingston Camp, about $22 \mathrm{~km}$ south of Clayton. Several aftershocks were felt in the area. (Ref. 36, 354.)

1963. Sept. 11 (Sept. 10). Central Idaho. Plaster fell in buildings at Redfich Lake, south of Stanley in Custer County; a windowpane was broken at a fire station in Challis National Forest. (Ref. 36, 354.)

1969. Apr. 26. Ketchum, Blaine County, Idaho. Cracks formed in concrete floors of structures in Warm Springs and Ketchum. Plaster was cracked at Livingston Mill, $20 \mathrm{~km}$ south of Clayton. (Ref. 42, 354.)

1975. Mar. 28 (Mar. 27). Eastern Idaho. In the Ridgedale area of the sparsely populated Pocatello Valley, this earthquake shifted several ranch houses on their foundations and toppled many chimneys. At Malad City, $20 \mathrm{~km}$ northeast of the epicenter, about 40 percent of the chimneys on old buildings were damaged. Total property damage was estimated at $\$ 1$ million.

Geologists observed one zone of ground fractures-about $0.6 \mathrm{~km}$ long and $5 \mathrm{~cm}$ wide-in the south-central section of the valley. The shock triggered many snow avalanches northeast of the valley. Fourteen aftershocks ranging in magnitude from 3.8 to 4.7 were located through Mar. 31. Felt in parts of Colorado, Idaho, Nevada, Utah, and Wyoming. (Ref. 48, 298.)

1977. Nov. 27. Cascade, Valley County, Idaho. Property damage was reported only at Cascade, a few kilometers east of the epicenter, near Cascade Dam. The tremor cracked foundations and sheetrock walls, separated ceiling beams, and left water muddy in wells and springs. Also felt in Oregon. (Ref. 39, 354.)

1978. Oct. 24. Southeast Idaho. Cracks formed in plaster and a concrete foundation at Thatcher in Franklin County. This earthquake was felt mainly in Bannock and Franklin Counties of southeast Idaho, and at Plymouth, Utah, south of Pocatello, Idaho. (Ref. 240.)

1982. Oct. 14 (Oct. 13). Near Soda Springs, Caribou County, Idaho. In the Soda Springs area, about $45 \mathrm{~km}$ southeast of Pocatello, bricks fell from chimneys and cracks formed in the foundation of a house and in interior drywalls. Also felt in Utah and Wyoming. (Ref. 350.)

1983. Oct. 28. Borah Peak, Custer County, Idaho. The Borah Peak earthquake is the largest ever 


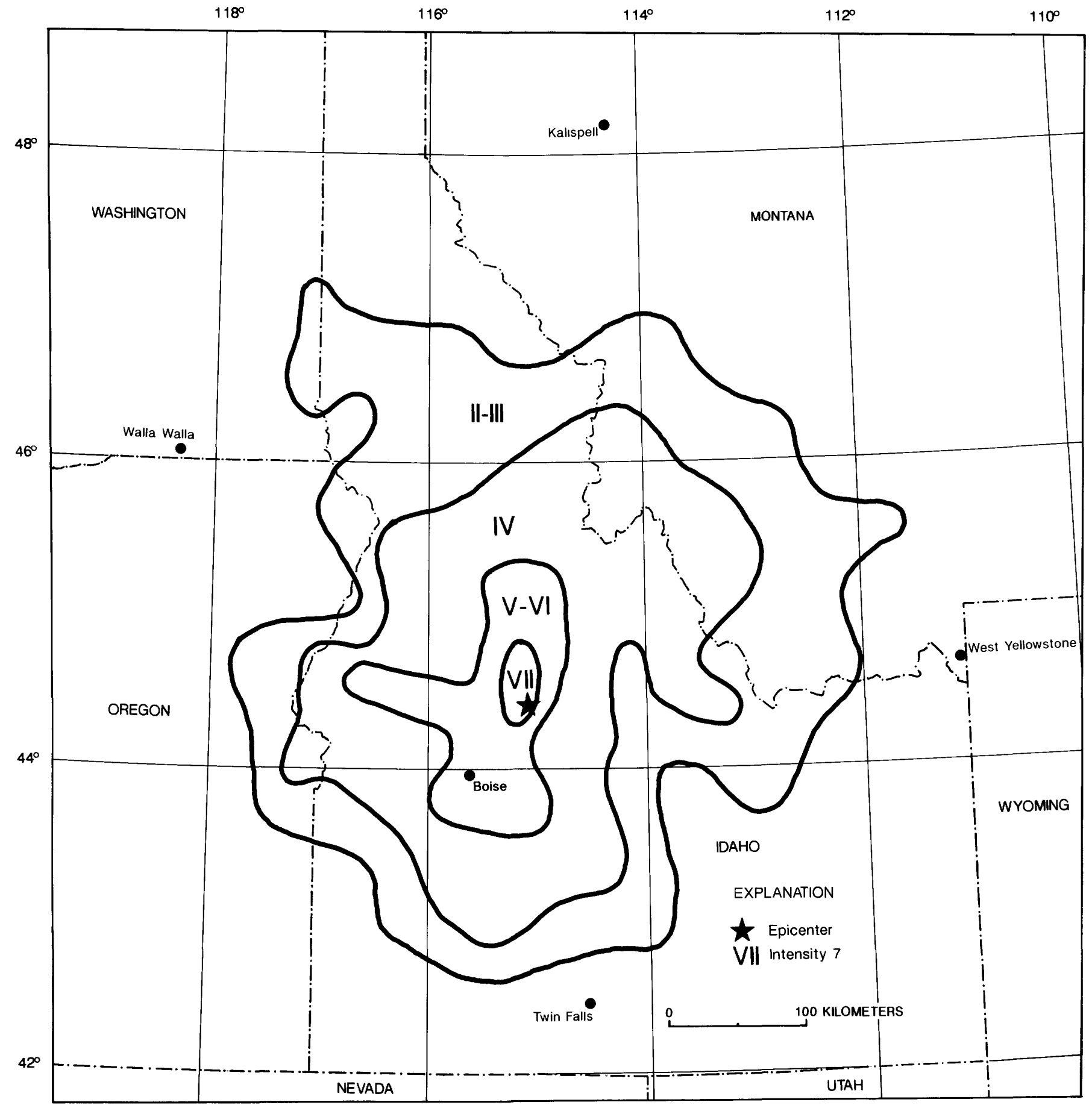

FIGURE 26.-Isoseismal map for the southwest Idaho earthquake of July 12, 1944. Isoseismals are based on intensity estimates from data listed in references 17 and 259 of table 1.

recorded in Idaho-both in terms of magnitude and in amount of property damage. It caused two deaths in Challis, about $200 \mathrm{~km}$ northeast of Boise, and an estimated $\$ 12.5$ million in damage in the Challis-Mackay area. A maximum MM intensity IX was assigned to this earthquake on the basis of surface faulting.
Vibrational damage to structures was assigned intensities in the VI to VII range (see fig. 27.)

Spectacular surface faulting was associated with this earthquake-a 34-km-long northwest-trending zone of fresh scarps and ground breakage on the southwest slope of the Lost River Range. The most 


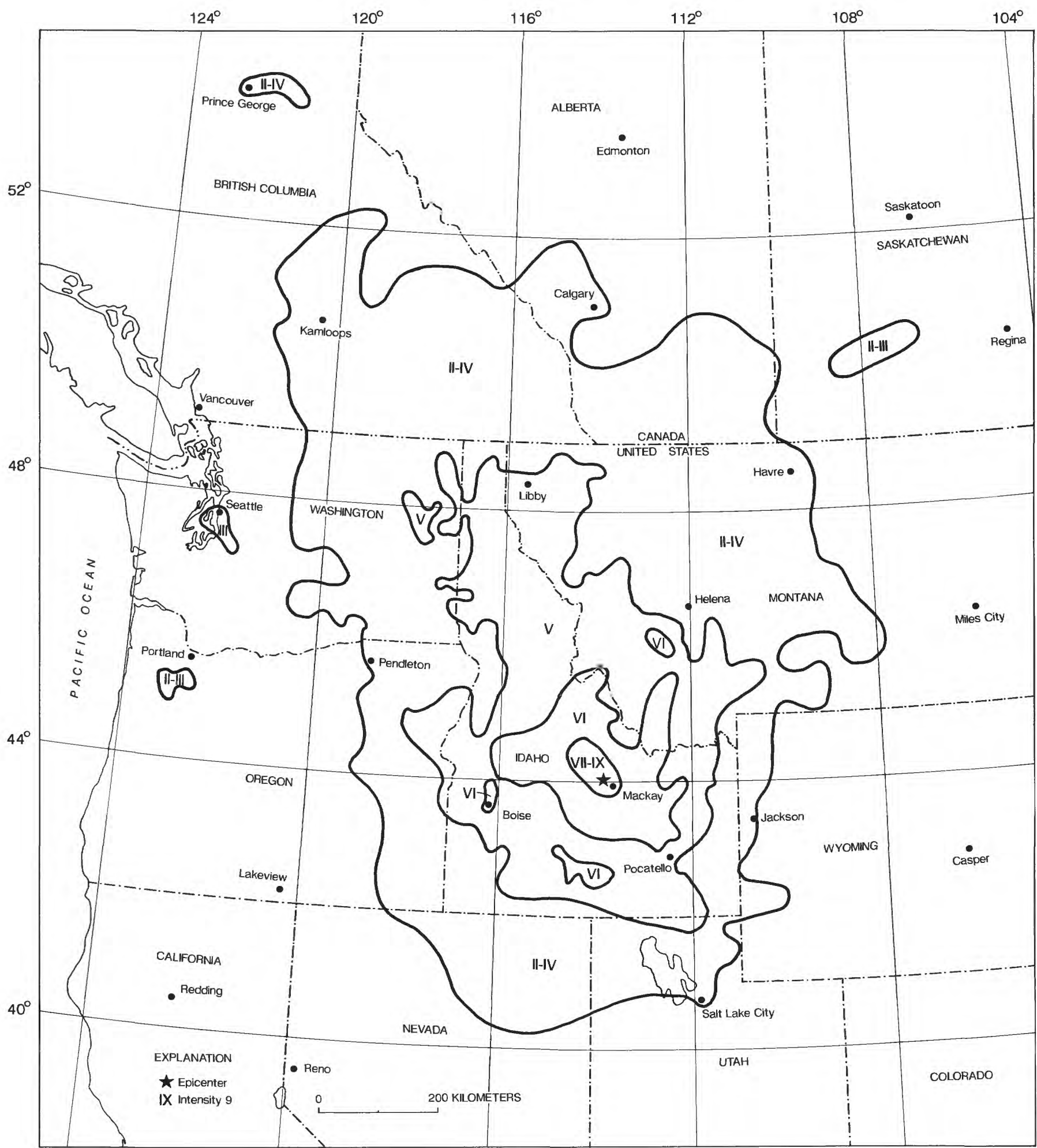

FIGURE 27.-Isoseismal map for the Borah Peak, Idaho, earthquake of October 28, 1983. This map is a simplified version of figure 28 in reference 360 of table 1 .

extensive breakage occurred along the $8-\mathrm{km}$ zone $\mid$ blocks several meters in width. The ground breakage between West Spring and Cedar Creek. Here, the ground surface was shattered into randomly tilted was as wide as $100 \mathrm{~m}$ and commonly had four to eight en echelon scarps as high as 1-2 $\mathrm{m}$. The throw 


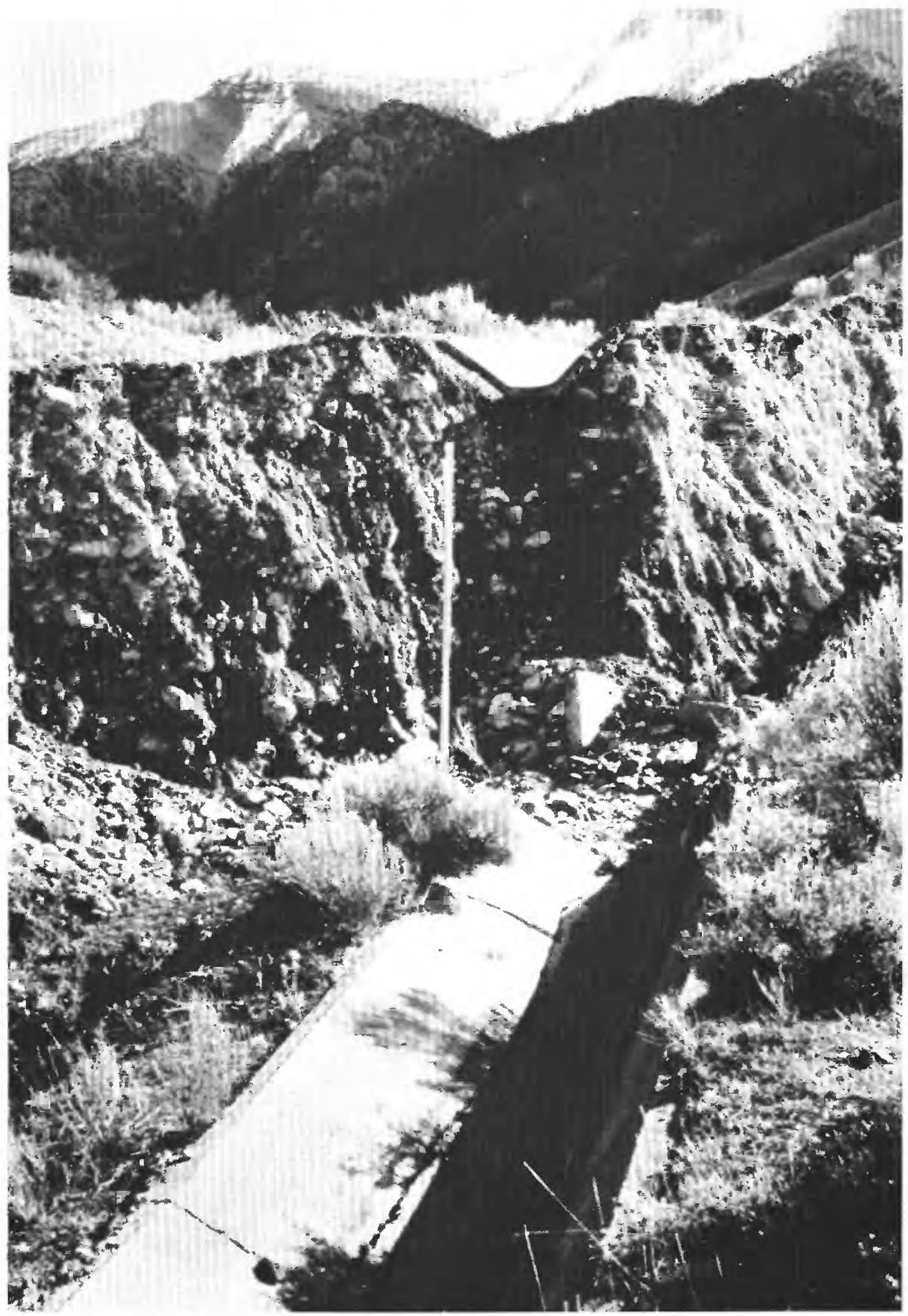

Fault scarp more than $1.8 \mathrm{~m}$ in height, northwest of Mackay, Idaho, caused by the October 28, 1983, earthquake. 


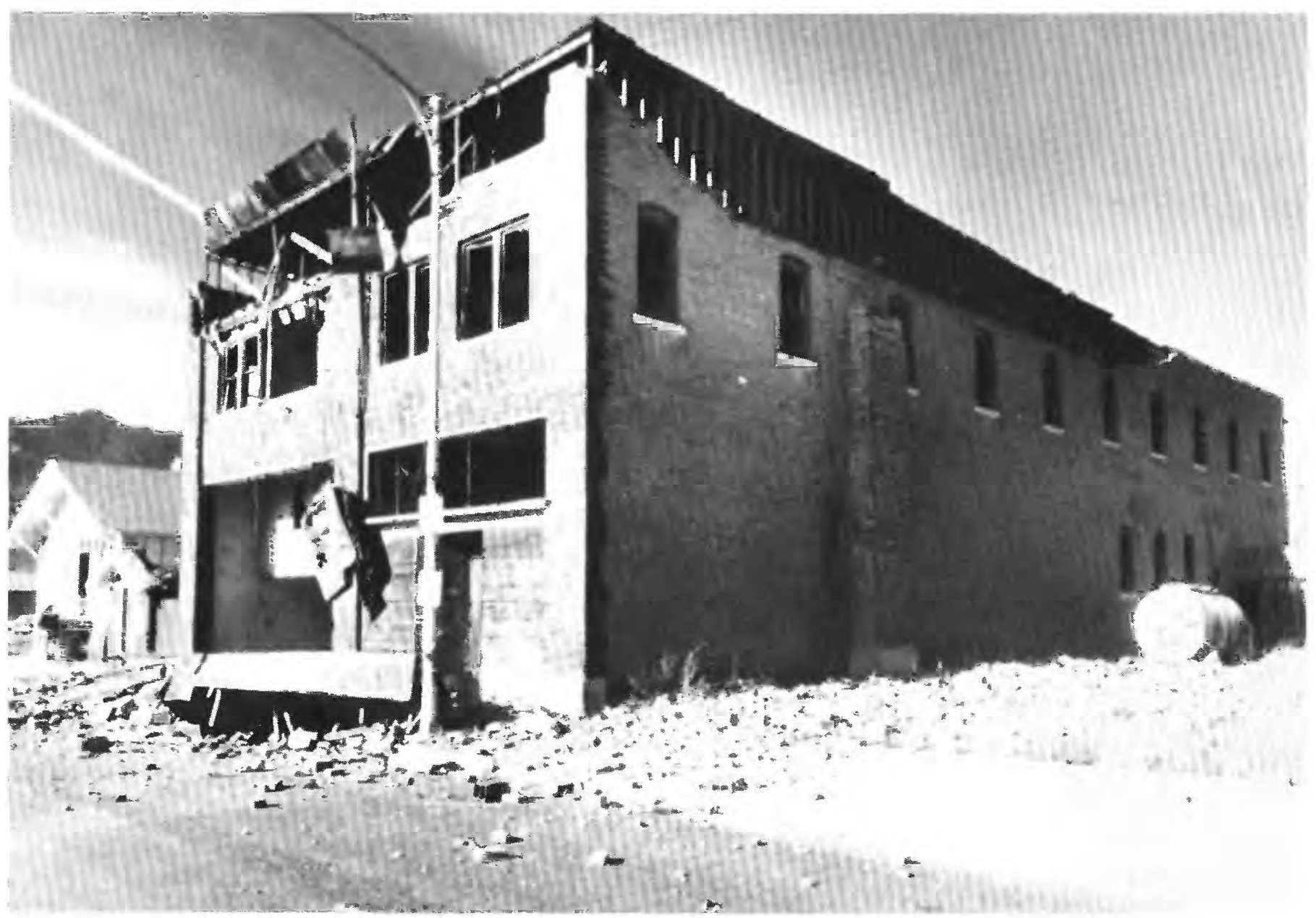

Brick walls of the old Custer Hotel, in Mackay, Idaho, damaged by the October 28, 1983, earthquake.

(Photograph by the Idaho Falls, Idaho, Post-Register.)

on the faulting ranged from $<50 \mathrm{~cm}$ on the southernmost section to $2.7 \mathrm{~m}$ south of Rock Creek at the western base of Borah Peak.

Other geologic effects included rockfalls and landslides on the steep slopes of the Lost River Range, water fountains and sand boils near the geologic feature of Chilly Buttes and the Mackay Reservoir, increase or decrease in flow of water in springs, and fluctuations in well water levels. A temporary lake was formed by the rising water table south of Dickey.

The most severe property damage occurred in the towns of Challis and Mackay, where 11 commercial buildings and 39 private houses sustained major damage and 200 houses sustained minor to moderate damage.

At Mackay, about $80 \mathrm{~km}$ southeast of Challis, most of the commercial structures on Main Street were damaged to some extent; building inspectors condemned eight of them. Damaged buildings were mainly of masonry construction, including brick, concrete block, or stone. Visible damage consisted of severe cracking or partial collapse of exterior walls, cracking of interior walls, and separation of ceilings and walls at connecting corners. About 90 percent of the residential chimneys were cracked, twisted, or collapsed.

At Challis, less damage to buildings and chimneys was sustained, but two structures were damaged extensively: the Challis High School and a vacant concrete-block building (100 years old) on Main Street. Many aftershocks occurred through 1983. Also felt in parts of Montana, Nevada, Oregon, Utah, Washington, Wyoming, and in the Provinces of Alberta, British Columbia, and Saskatchewan, Canada. (Ref. 354, 360, 608.) 



\section{ILLINOIS}

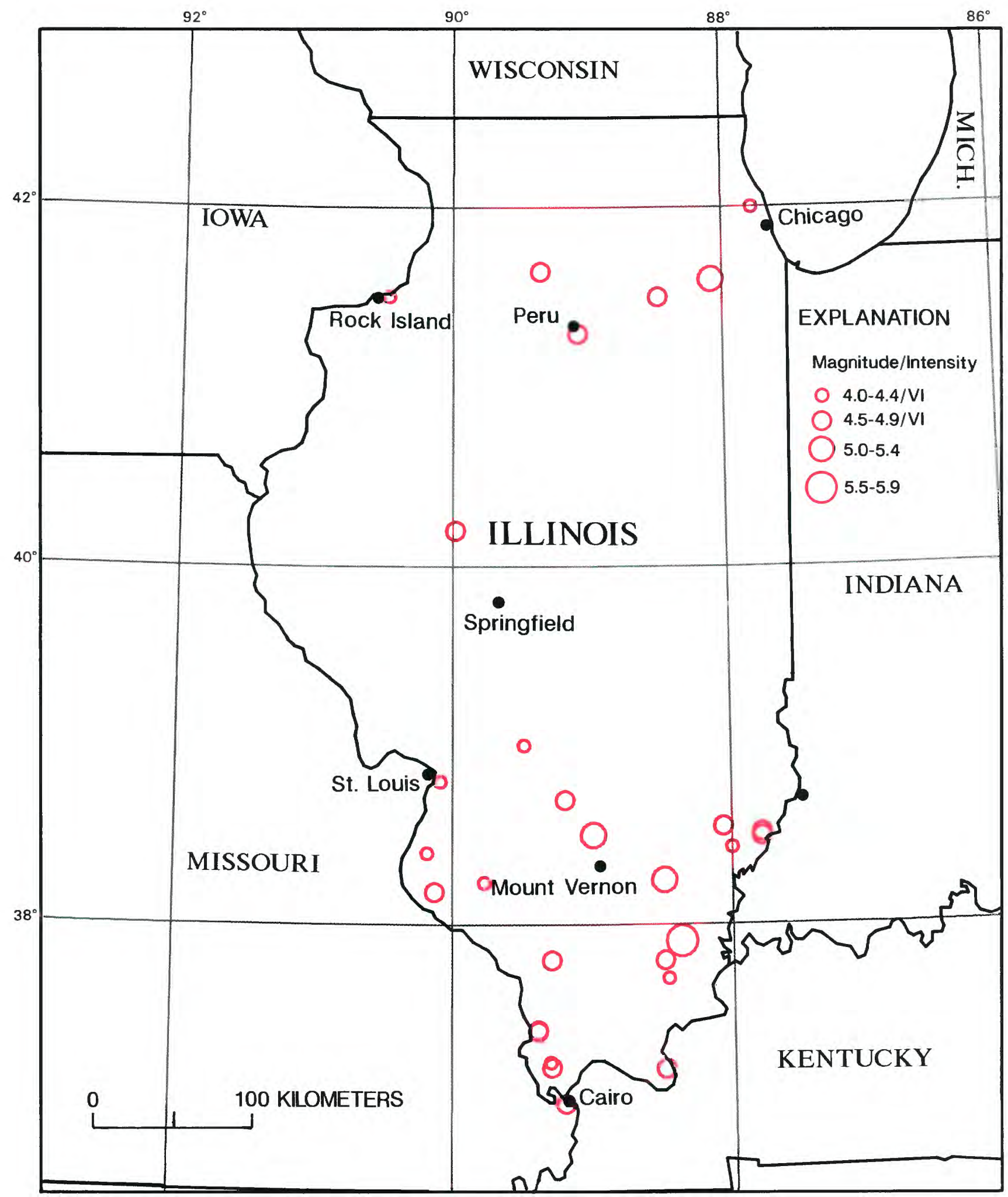

Earthquakes in Illinois with magnitudes $\geq 4.5$ or intensity $\geq$ VI. 
ILLINOIS

[See table 1 for hypocenter and intensity references and table 2 for definitions of magnitude source codes. Leader (--) indicates information is not available]

\begin{tabular}{|c|c|c|c|c|c|c|c|c|c|c|c|c|c|c|c|c|}
\hline \multirow{3}{*}{\multicolumn{4}{|c|}{\begin{tabular}{cc} 
& \multicolumn{2}{c}{ Origin } \\
Date & time (UTC) \\
$\mathrm{Yr} \quad \mathrm{Mo} \mathrm{Da}$ & $\mathrm{h} \mathrm{m} s$
\end{tabular}}} & \multicolumn{6}{|c|}{ Hypocenter } & \multicolumn{4}{|c|}{ Magnitude } & \multicolumn{2}{|c|}{ Intensity } & \multirow{3}{*}{$\begin{array}{l}\text { Felt area } \\
\left(1,000 \mathrm{~km}^{2}\right)\end{array}$} \\
\hline & & & & \multirow{2}{*}{\multicolumn{2}{|c|}{$\begin{array}{l}\text { Latitude } \\
\left({ }^{\circ}\right)\end{array}$}} & \multirow{2}{*}{\multicolumn{2}{|c|}{$\begin{array}{c}\text { Longitude } \\
\left({ }^{\circ}\right)\end{array}$}} & \multirow{2}{*}{$\begin{array}{l}\text { Depth } \\
(\mathrm{km})\end{array}$} & \multirow[t]{2}{*}{ Ref } & \multicolumn{2}{|c|}{ USGS } & \multirow[t]{2}{*}{ Other } & \multirow{2}{*}{$\begin{array}{c}\text { Moment } \\
\mathbf{M}\end{array}$} & \multirow[t]{2}{*}{ MMI } & \multirow[t]{2}{*}{ Ref } & \\
\hline & & & & & & & & & & $m_{b}$ & $\mathbf{M}_{\mathbf{s}}$ & & & & & \\
\hline 1804 & 08 & 20 & 2010 & 42.0 & $\mathbf{N}$ & 87.8 & W & - & 105 & - & - & $4.40 \mathrm{M}_{\mathrm{fa}} \mathrm{SC}$ & - & VI & 105 & 78 \\
\hline 1838 & 06 & 09 & 1445 & 38.5 & $\mathbf{N}$ & 89.0 & W & - & 113 & - & - & $5.20 \mathrm{M}_{\mathrm{fa}} \mathrm{SC}$ & - & VII & 113 & 500 \\
\hline 1857 & 10 & 08 & 1000 & 38.7 & $\mathbf{N}$ & 89.2 & W & - & 113 & - & - & $4.90 \mathrm{M}_{\mathrm{fa}} \mathrm{SC}$ & - & VII & 113 & 200 \\
\hline 1876 & 09 & 25 & 06 & 38.5 & $\mathbf{N}$ & 87.8 & W & - & 105 & - & - & $4.50 \mathrm{M}_{\mathrm{fa}} \mathrm{SC}$ & - & VI & 105 & 150 \\
\hline 1876 & 09 & 25 & 0615 & 38.5 & $\mathbf{N}$ & 87.8 & W & - & 353 & - & - & $4.80 \mathrm{M}_{\mathrm{fa}} \mathrm{SC}$ & - & VII & 529 & 150 \\
\hline 1881 & 05 & 27 & & 41.3 & $\mathbf{N}$ & 89.1 & W & - & 105 & - & - & $4.60 \mathrm{M}_{\mathrm{fa}} \mathrm{SC}$ & - & VI & 105 & 181 \\
\hline 1882 & 09 & 27 & 1020 & 39.0 & $\mathbf{N}$ & 89.5 & W & - & 113 & - & - & $4.40 \mathrm{M}_{\mathrm{fa}} \mathrm{SC}$ & - & VI & 38 & 100 \\
\hline 1883 & 04 & 12 & 0836 & 37.0 & $\mathrm{~N}$ & 89.2 & W & - & 38 & - & - & - & - & VI & 173 & - \\
\hline 1887 & 08 & 02 & 1836 & 37.2 & $\mathbf{N}$ & 88.5 & W & - & 529 & - & - & $4.90 \mathrm{M}_{\mathrm{fa}} \mathrm{SC}$ & - & VI & 529 & 450 \\
\hline 1891 & 09 & 27 & 0455 & 38.25 & $\mathbf{N}$ & 88.50 & W & - & 302 & - & - & $5.20 \mathrm{M}_{\mathrm{fa}} \mathrm{SC}$ & - & VII & 302 & 560 \\
\hline 1903 & 02 & 09 & 0021 & 37.8 & $\mathbf{N}$ & 89.3 & W & - & 105 & - & - & $4.90 \mathrm{M}_{\mathrm{fa}} \mathrm{SC}$ & - & VII & 529 & 202 \\
\hline 1905 & 08 & 22 & 0508 & 37.2 & $\mathbf{N}$ & 89.3 & W & - & 529 & - & - & $4.80 \mathrm{M}_{\mathrm{fa}} \mathrm{SC}$ & - & VI & 529 & 375 \\
\hline 1909 & 05 & 26 & 1442 & 41.6 & $\mathbf{N}$ & 88.1 & W & - & 529 & - & - & $5.10 \mathrm{M}_{\mathrm{fa}} \mathrm{SC}$ & - & VII & 38 & 430 \\
\hline 1909 & 07 & 19 & 0434 & 40.2 & $\mathrm{~N}$ & 90.0 & W & - & 38 & - & - & $4.80 \mathrm{M}_{\mathrm{fa}} \mathrm{SC}$ & - & VII & 38 & 121 \\
\hline 1912 & 01 & 02 & 1621 & 41.5 & $\mathbf{N}$ & 88.5 & W & - & 38 & - & - & $4.50 \mathrm{M}_{\mathrm{fa}} \mathrm{SC}$ & - & VI & 38 & 150 \\
\hline 1917 & 04 & 09 & 2052 & 38.1 & $\mathbf{N}$ & 90.2 & W & - & 113 & - & - & $5.10 \mathrm{M}_{\mathrm{fa}} \mathrm{SC}$ & - & VII & 529 & 408 \\
\hline 1922 & 03 & 22 & 222910 & 37.4 & $\mathrm{~N}$ & 89.4 & W & - & 529 & - & - & $4.80 \mathrm{M}_{\mathrm{fa}} \mathrm{SC}$ & - & VII & 529 & 135 \\
\hline 1922 & 03 & 23 & 0222 & 37.4 & $\mathbf{N}$ & 89.4 & W & - & 529 & - & - & $4.60 \mathrm{M}_{\mathrm{fa}} \mathrm{SC}$ & - & VI & 529 & 175 \\
\hline 1922 & 11 & 27 & 0331 & 37.8 & $\mathbf{N}$ & 88.5 & W & - & 105 & - & - & $4.80 \mathrm{M}_{\mathrm{fa}} \mathrm{SC}$ & - & VII & 529 & 130 \\
\hline 1934 & 11 & 12 & 1445 & 41.5 & $\mathbf{N}$ & 90.5 & W & - & 149 & - & - & $4.00 \mathrm{M}_{\mathrm{fa}} \mathrm{SC}$ & - & VI & 38 & 13 \\
\hline 1939 & 11 & 23 & 151452.0 & 38.180 & & 90.137 & $7 W$ & 000 & 349 & - & - & $4.60 \mathrm{M}_{\mathrm{fa}} \mathrm{SC}$ & - & V & 38 & 440 \\
\hline 1947 & 06 & 30 & 042353 & 38.4 & $\mathbf{N}$ & 90.2 & W & - & 20 & - & - & $4.20 \mathrm{M}_{\mathrm{fa}} \mathrm{SC}$ & - & VI & 38 & 40 \\
\hline 1953 & 09 & 11 & 182628 & 38.8 & $\mathbf{N}$ & 90.1 & W & - & 105 & - & - & $4.00 \mathrm{M}_{\mathrm{fa}} \mathrm{SC}$ & - & VI & 26 & 15 \\
\hline 1955 & 04 & 09 & 130123.3 & 38.232 & & 89.785 & $5 W$ & 011 & 349 & - & - & $4.30 \mathrm{M}_{\mathrm{fa}} \mathrm{SC}$ & - & VI & 28 & 54 \\
\hline 1958 & 11 & 08 & 024112.6 & 38.43 & & 88.008 & $8 W$ & 005 & 349 & - & - & $4.40 \mathrm{M}_{\mathrm{fa}} \mathrm{SC}$ & - & VI & 31 & 75 \\
\hline 1965 & 08 & 14 & 131356.9 & 37.22 & & 89.307 & $7 \mathrm{~W}$ & 001 & 349 & & - & $3.80 \mathrm{M}_{\mathrm{n}} \mathrm{SLM}$ & 3.44STT & VII & 75 & 1 \\
\hline 1968 & 11 & 09 & 170140.5 & 37.91 & & 88.373 & $3 w$ & 021 & 349 & 5.3 & - & $5.50 \mathrm{M}_{\mathrm{n}} \mathrm{SLM}$ & 5.27HRN & VII & 490 & 1473 \\
\hline 1972 & 09 & 15 & 052215.9 & 41.64 & & 89.369 & $9 W$ & 011 & 349 & 3.7 & - & $4.50 \mathrm{M}_{\mathrm{n}} \mathrm{DG}$ & $4.03 \mathrm{STT}$ & VI & 45 & 230 \\
\hline 1974 & 04 & 03 & 230502.8 & 38.54 & & 88.072 & $2 W$ & 014 & 349 & 4.5 & - & $4.70 \mathrm{M}_{n} \mathrm{DG}$ & 4.36STT & VI & 47 & 400 \\
\hline 1984 & 06 & 29 & 075829.3 & 37.70 & & $\mathbf{8 8 . 4 7 0}$ & ow & 002 & 370 & - & - & $4.10 \mathrm{M}_{\mathrm{n}} \mathrm{GS}$ & - & VI & 370 & 7 \\
\hline 1987 & 06 & 10 & 234854.8 & 38.71 & & 87.954 & $4 W$ & 010 & 74 & 4.9 & 5.1 & $5.20 \mathrm{M}_{\mathrm{n}} \mathrm{SLM}$ & $4.96 \mathrm{JOH}$ & VI & 592 & 433 \\
\hline
\end{tabular}

[Reference (Ref.) numbers given in parentheses at the end of each description refer to sources of data in table 1. Magnitude values are described in the Introduction, and codes are defined in table 2.]

1804. Aug. 20. Fort Dearborn (Chicago), Ill. The earthquake was felt at the south end of Lake Michigan and at Fort Wayne, Ind. (about $320 \mathrm{~km}$ from the epicenter). (Ref. 38, 105, 353.)

1838. June 9. Southern Illinois. Several catalogs place the epicenter of this earthquake near St. Louis, Mo., because of a report of a chimneys being thrown down at St. Louis and because it was "severely felt" at St. Charles, Mo. Although reported effects do not support an intensity of VII, that intensity is assigned because of the similarity of the distribution of intensity to that of the earthquake of Oct. 8, 1857. Felt reports recorded at common points are one-half to one unit of intensity higher for the 1857 earthquake. Also felt in Illinois, Indiana, and Kentucky. Magnitude $\mathrm{M}_{\mathrm{S}} 5.7 \mathrm{M}_{\mathrm{fa}}$ BAR. (Ref. 113, 353,529 .)

1857. Oct. 8. Southern Illinois. This severe earthquake was centered in the Mississippi River valley between St. Louis, Mo., and Centralia, Ill. At Centralia, the first of three reported shocks threw down chimneys; at St. Louis, it moved furniture, 
dislocated bricks, and felled plaster. The largest buildings rocked and articles fell from mantles. Reports indicate that the Mississippi River was in tumult. Felt in many towns in Illinois, along the Mississippi River south of Hannibal, Mo., in western Kentucky, and in parts of Indiana and Iowa. (Ref. $105,109,113,353,529$.)

1876. Sept. 25, 06 and 0615 UTC. Wabash River valley. These earthquakes were felt most strongly between Friendsville and Mt. Carmel, Ill., and Evansville, Ind. They were described as "heavy" at Friendsville. The second shock threw down chimneys at Vincennes, Ind., alarmed residents at Evansville, Ind., and caused slight damage at Louisville and Owensboro, Ky. They were felt from St. Louis, Mo., to Indianapolis, Ind., and Louisville, Ky. (Ref. $38,105,353,463,529$.)

1881. May 27. La Salle, Ill. Before daybreak, a shock in the southwest part of La Salle, about 90 $\mathrm{km}$ northeast of Peoria, formed six parallel fissures that were traceable for $183 \mathrm{~m}$ in a northwest-southeast direction. Walls, foundations, and furnaces in bottle and glass factories cracked in many places. (Ref. 105, 463.)

1882. Sept. 27. Southern Illinois. A chimney was cracked severely at Greenfield, Green County, IIl., and a crack in the wall of a building was widened considerably at Salem, Marion County. People were awakened and small objects were displaced throughout the area. The felt area extended from Mexico, Mo., to Vincennes, Ind., and Henderson, Ky., in an east-west direction, and from Springfield to Pickneyville, Ill., in a north-south direction. (Ref. 38, 113, 353, 463.)

1883. Apr. 12. Cairo, Pulaski County, Ill. A strong local earthquake rattled windows for $30 \mathrm{sec}-$ onds and awakened everyone in Cairo, in southern Illinois near the Kentucky-Missouri border. People were injured slightly in the collapse of an old frame house. (Ref. 38, 105, 173, 463.)

1887. Aug. 2. Southern Illinois. This severe shock broke windows at Cobden, Ill., cracked brick walls at Jonesboro, Ill., and Russellville, Ky., and loosened some plaster at Nashville, Tenn. Also felt in Indiana and Missouri and as far south as Huntsville, Ala. Magnitude $4.7 \mathrm{M}_{\mathrm{fa}}$ BAR. (Ref. 38, 529.)

1891. Sept. 27 (Sept. 26). Near Mount Vernon, Jefferson County, IIl. Several chimneys were toppled at Mount Vernon, and the ceiling and sidewalls of the Methodist Church were damaged. Chimney damage also was reported at Browns and Nashville, Ill., and Cloverport, Ky. Plaster was knocked down at Jerseyville, Murphysboro, and Warsaw, Ill. Also felt in all or parts of Indiana, Iowa, Kentucky, Missouri,
Ohio, and Tennessee (see fig. 28). Magnitude $5.8 \mathrm{~m}_{\mathrm{b}}$ BAR. (Ref. 302, 353, 529.)

1903. Feb. 9 (Feb. 8). Mississippi River valley. This earthquake threw down chimneys in Jackson County at Grand Tower and Murphysboro, Ill., and damaged chimneys east of Murphysboro, at Carterville and Harrisburg, Ill. It was strongly felt from Jeffersonville, Mo., to Louisville, Ky., and from Cairo, Ill., to Hannibal, Mo. (Ref. 38, 105, 353, 529.)

1905. Aug. 22 (Aug. 21). Southern Illinois. Chimneys were shaken down at Cairo, Pulaski County, Ill., and, about $40 \mathrm{~km}$ southwest, at Sikeston, Mo. Chimneys also were broken or partly collapsed at nearby Charleston, Mo., and, about 175 $\mathrm{km}$ southeast, at Clarksville, Tenn. The earthquake was felt most strongly along the Mississippi and Ohio River valleys, including parts of Arkansas, Illinois, Indiana, Kentucky, Mississippi, Missouri, and Tennessee (Ref. 109, 353, 529.)

1909. May 26. Aurora, Kane County, Ill. This earthquake has been related to the La Salle anticline in the Illinois Basin. Many chimneys fell, a stove overturned, and gas line connections broke at Aurora, west of Chicago. Several chimneys were downed at Forreston, Naperville, Streator, Triumph, and Troy Grove, and one fell at Waukegan. Brick walls cracked at Bloomington, and sidewalks cracked and many chimneys were damaged at Freeport. At Platteville, Wis., about $130 \mathrm{~km}$ northwest of Chicago, an old building was cracked; houses were jostled out of plum at Beloit, Wis., about $240 \mathrm{~km}$ northwest of Chicago. Felt from Missouri to Michigan and Minnesota to Indiana. Magnitude 5.1 $\mathrm{M}_{\mathrm{fa}}$ BAR. (Ref. 38, $105,353,529$.

1909. July 19 (July 18). Between Havana and Petersburg, Ill. Chimneys were demolished on more than 100 buildings in Menard County at Petersburg, northwest of Springfield. At a farm west of Petersburg, 20 windows broke and bricks pushed out above the doors. Fallen chimneys also were reported northwest of Springfield at Davenport, Iowa, and west of Springfield at Hannibal, Mo. Several newspaper articles describe this earthquake but do not report property damage. (Ref. 38, 105, 353, 529.)

1912. Jan. 2. Near Aurora, Freeport, Morris, and Yorkville, IIl. The highest intensity was reported at those towns in Kane, Stephenson, Grundy, and Kendall Counties, respectively. Slight damage to chimneys was reported at Batavia and Geneva, Ill., north of Aurora, in Kane County. Two distinct shocks were observed at some places. The stronger shock also was felt in parts of Indiana, Iowa, Kentucky (Fulton County), and Wisconsin. (Ref. 38, 105, 353, 529.) 


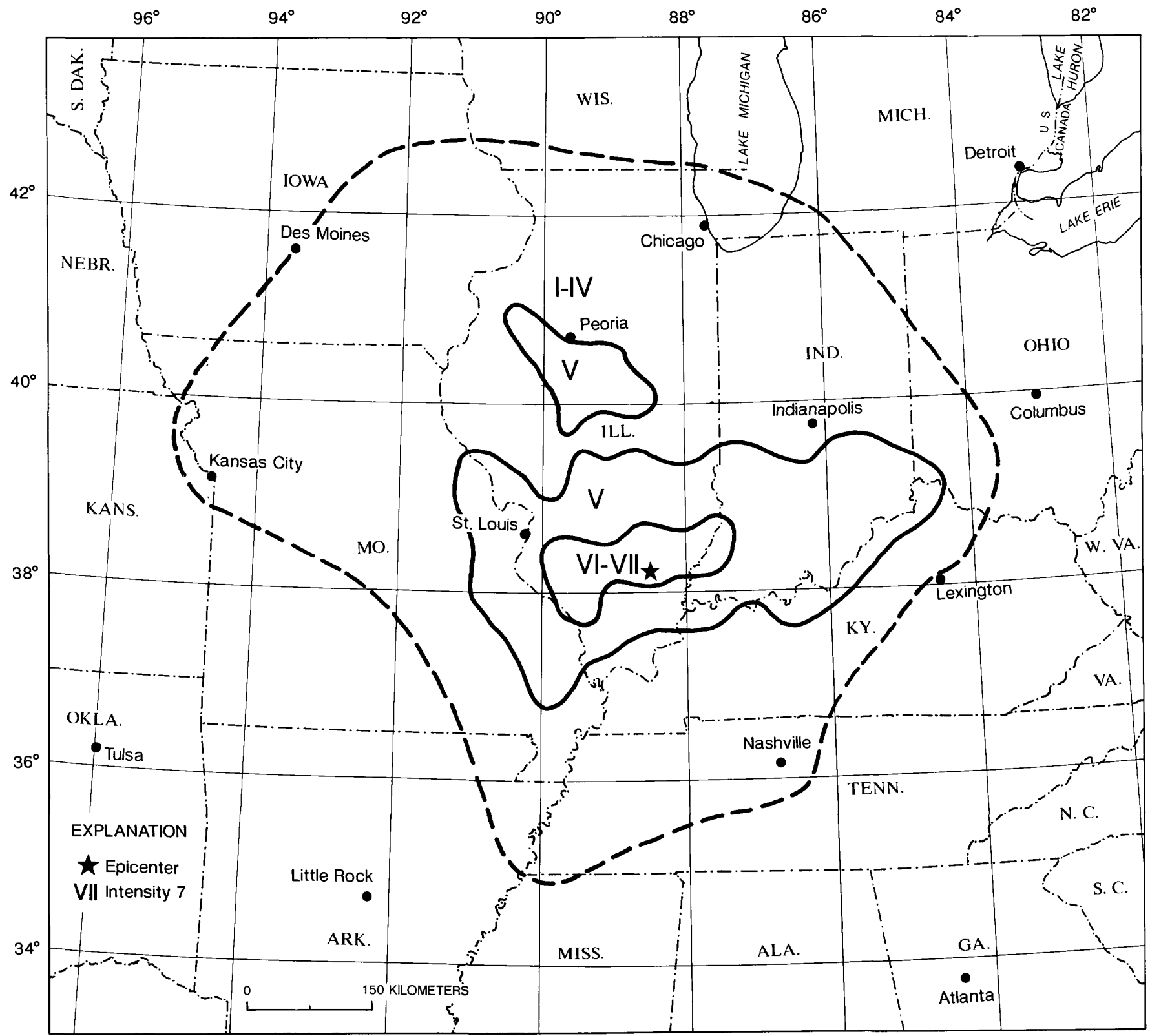

FIGURE 28.-Isoseismal map for the southern Illinois earthquake of September 27, 1891. Isoseismals are based on intensity estimates from data listed in references 302 and 529 of table 1.

1922. Mar. 22. Southern Illinois. This strong earthquake knocked down 25 chimneys at Illmo, Scott County, Mo., and sent people rushing out of stores. Dishes fell from shelves at Carbondale, Ill. Also felt in Kentucky and Tennessee. (Ref. 529.)

1922. Mar. 23 (Mar. 22). Soutlern Illinois. At Illmo, Mo., south of Cape Girardeau in Scott County, the earthquake knocked down "many more chimneys" (see above description of the main shock on Mar. 22). The shock was "violent" at Belleville, Ill., and "severe" at Jonesboro, Ill. Stovepipes were downed at Cape Girardeau, Mo., and people were knocked off their feet. Also felt at Evansville, Ind. (Ref. 529.)
1917. Apr. 9. Southern Illinois in the Mississippi River valley. At St. Louis, Mo., several chimneys were knocked down, windows were broken, and people were thrown to the pavement. At Granite City, Mo., buildings shifted on their foundations. At DeSoto, Mo., in Jefferson County, bricks fell from chimneys and the walls of several buildings were cracked. Many windows were broken and buildings rocked at Ste. Genevieve and St. Mary, Mo., south of St. Louis near the Illinois border. Heavy rumbling preceded and accompanied the earthquake in places. Felt from Kansas to Ohio and from Wisconsin to Mississippi (see fig. 29). (Ref. 38, 113, 353, 529.) 


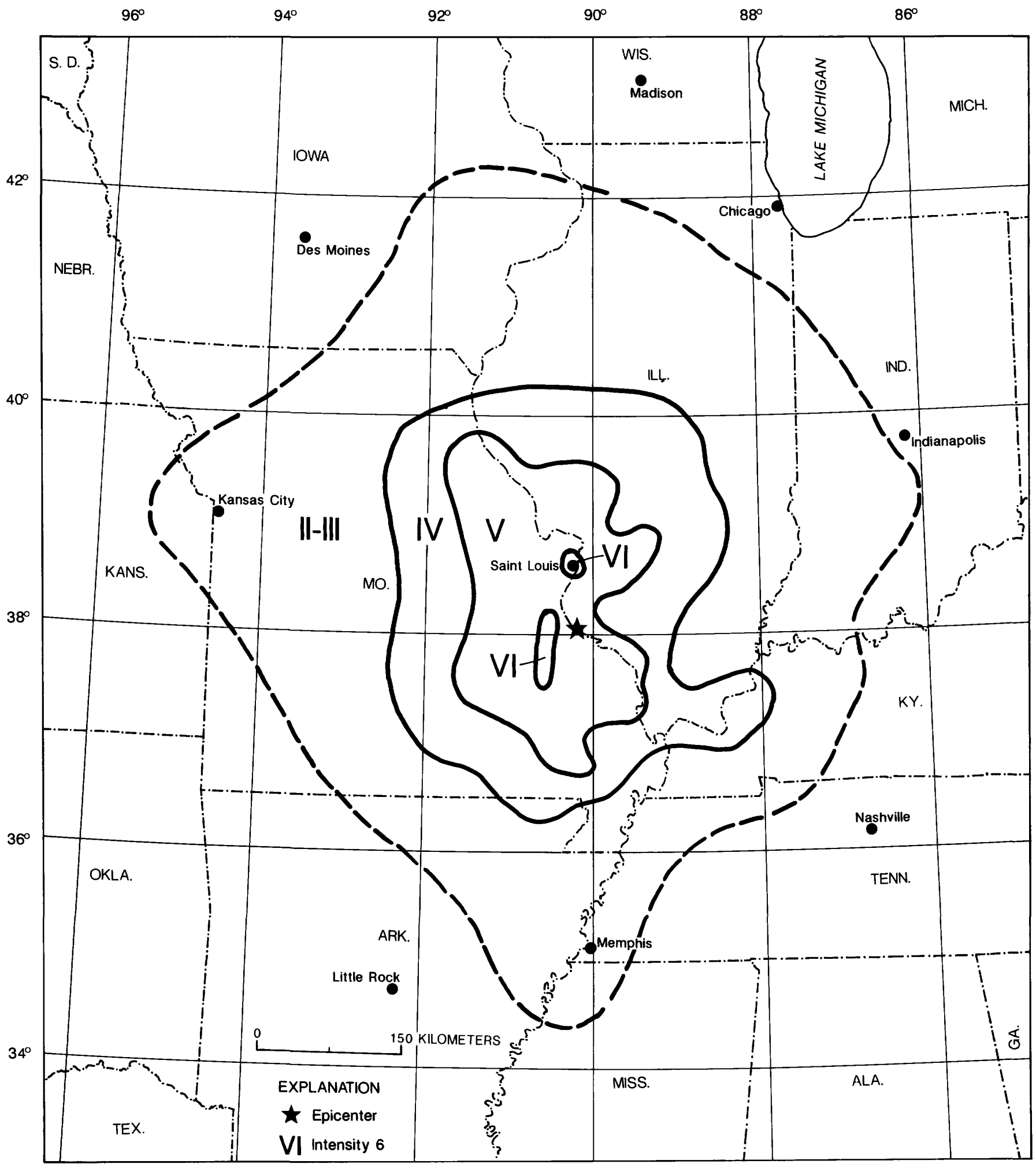

FIGURE 29.-Isoseismal map for the southern Illinois earthquake of April 9, 1917. Isoseismals are based on intensity estimates from data listed in references 272 and 529 of table 1.

1922. Nov. 27 (Nov, 26). Near Eldorado, chimney flue was demolished and stovepipes fell at Saline County, Ill. The earthquake broke several windows and downed chimneys at Eldorado. One Harrisburg, $8 \mathrm{~km}$ southwest of Eldorado. Generally felt in southern Illinois, western Indiana, northern 
Kentucky, eastern Missouri, and western Tennessee. (Ref. 105, 109, 353, 529.)

1934. Aug. 20 (Aug. 19). Mississippi River valley near the Illinois-Missouri border. In the area near Charleston, Mississippi County, Mo., chimneys were thrown down or lost bricks, windows were broken, and plaster was cracked. Destructive intensity covered an area of $600 \mathrm{~km}^{2}$, including Charleston, Mo., Arlington and Wickliffe, Ky., and Cairo and Mounds, Ill. Felt north to Alton, Ill.; east to Paducah and Marion, Ky.; south to Paris and Ripley, Tenn., and Corning and Paragould, Ark.; and west to Poplar Bluff and Greeneville, Mo. (Ref. 7, 38, 149, 149, 353.)

1934. Nov. 12. Near Rock Island, Ill. In Rock Island and Moline, Ill., and Davenport, Iowa, bricks fell from a few chimneys and pendulum clocks stopped. In Rock Island, a stucco cornice was dislodged from St. Joseph's School; some loose plaster was shaken from ceilings in the men's dormitory at Augustana College, and loose bricks were shaken from a few buildings. (Ref. 7, 38, 129, 149, 353.)

1947. June 30 (June 29). Waterloo-Dupo, Ill., area, south of St. Louis, Mo. At St. Louis, several chimneys were toppled and a sidewalk was cracked. (Ref. 20, 38, 105, 353.)

1953. Sept. 11. Southwest Illinois. At Roxana, north of East St. Louis, in Madison County, cracks formed in a concrete-block foundation and in plaster. Also felt in eastern Missouri. (Ref. 26, 105, 353.)

1955. Apr. 9. West of Sparta, Randolph County, IIl. Concrete foundations and plaster walls were cracked at Evansville, Ill. (about $20 \mathrm{~km}$ west of Sparta), and at Lemay, University City, and Webster Groves, Mo. Also felt in Kentucky and Missouri. Magnitude $4.5 \mathrm{M}_{\mathrm{fa}}$ BAR. (Ref. 28, 349, 353.)

1958. Nov. 8 (Nov. 7). Southeast Illinois, near the Indiana border. Plaster fell at Dale (Hamilton County) and Albion (Edwards County), and a basement wall cracked at Maunie (White County). Also felt in Indiana, Kentucky, and Missouri. Magnitude 4.5 $\mathrm{M}_{\mathrm{fa}}$ BAR. (Ref. 31, 38, 105, 349, 353.)

1965. Aug. 14. Southwest Illinois. This strong local earthquake at Tamms (Alexander County) downed chimneys, cracked walls, muddied water, and knocked stock from shelves. (Ref. 75, 349, 353.)

1968. Nov. 9. Southern Illinois. This was the strongest felt earthquake in southern Illinois since the 1895 Missouri event. Property damage in the area consisted mainly of fallen bricks from chimneys, broken windows, toppled television aerials, and cracked or fallen plaster. In the epicentral area, near Dale, Hamilton County, MM intensity VII was characterized by downed chimneys, cracked foundations, overturned tombstones, and scattered instances of collapsed parapets.

Most buildings that sustained damage to chimneys were 30 to 50 years old. A large two-story brick house near Dale, Ill., sustained several thousand dollars damage. About $10 \mathrm{~km}$ west of Dale, near Tuckers Corners, a concrete and brick cistern collapsed. A large amount of masonry damage occurred at the City Building at Henderson, Ky., $80 \mathrm{~km}$ east-southeast of the epicenter. Moderate damage to chimneys and walls occurred in several towns in south-central Illinois, southwest Indiana, and northwest Kentucky. Felt over all or parts of 23 States (see fig. 30): from southeast Minnesota to central Alabama and Georgia and from western North Carolina to central Kansas. People in multistory buildings in Boston, Mass. and southern Ontario, Canada, felt the earthquake. Magnitude 5.2 M NTT, $5.5 \mathrm{~m}_{\mathrm{b}}$ NUT, $5.38 \mathrm{M}$ JOH (Ref. $41,263,349,353,490$.)

1972. Sept. 15 (Sept. 14). Northern Illinois. Cracks in chimneys, tombstones, elevated water tanks, and plaster occurred at Amboy (Lee County), south of Rockford. Chimney and plaster cracks were observed at Holcomb, northeast of Amboy, in Ogle County. Also felt in Indiana, Iowa, Michigan, Minnesota, Missouri, Ohio, and Wisconsin. Magnitude 3.3 $\mathrm{M}_{\mathrm{S}}$ NTT, $4.4 \mathrm{~m}_{\mathrm{b}}$ NUT. (Ref. $\left.45,263,349,353.\right)$

1974. Apr. 3. Southeast Illinois. Minor damage, generally in the form of cracked and broken chimneys, occurred in Wabash County. At West Salem, a few chimneys and tombstones were shaken down and other chimneys were damaged. Slight damage occurred at many towns in Indiana and Illinois. Also felt in Arkansas, Iowa, Kentucky, Michigan, Missouri, Ohio, Tennessee, Virginia, and Wisconsin. Magnitude 4.7 $\mathrm{M}_{\mathrm{n}}$ SLM. (Ref. 47, 349, 353.)

1984. June 29. Southern Illinois. At Harrisburg, in Saline County, one house sustained structural damage. Also felt in western Kentucky and southeast Missouri. Magnitude $3.8 \quad \mathrm{M}_{\mathrm{n}} \quad$ SLM. (Ref. 370.)

1987. June 10. Near Olney, Richland County, Ill. Minor damage in the form of cracks in chimneys, hairline cracks in plaster and drywall, and cracks in house foundations was reported in 


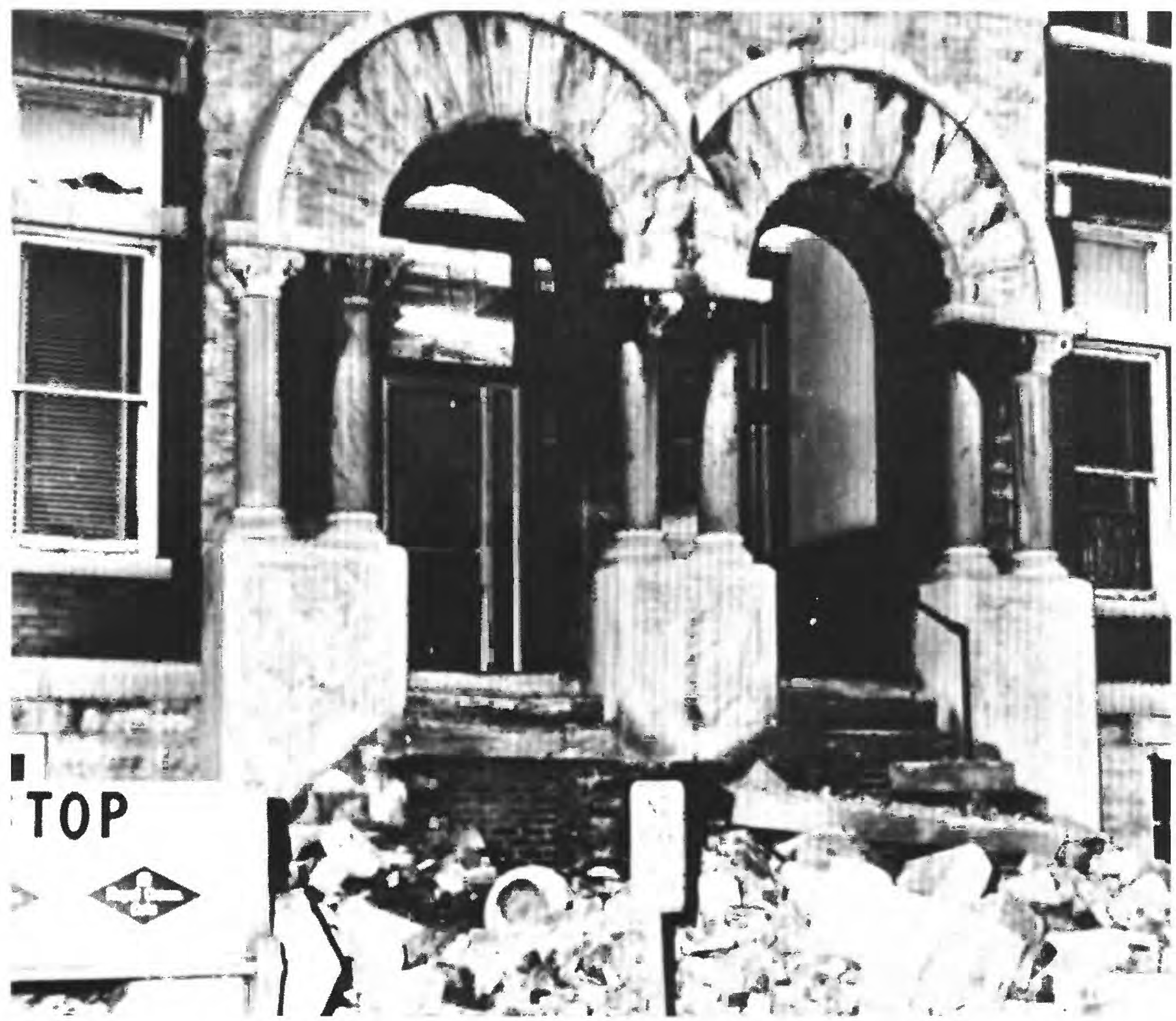

City Building in Henderson, Kentucky, damaged by the November 9, 1968, southern Illinois earthquake.

(Photograph by the Gleaner Journal.)

several towns in Illinois and Indiana. The most serious damage was observed at Olney, Ill., where chimneys toppled and bricks fell from chimneys; at West York, Ill. (about $60 \mathrm{~km}$ northeast of Olney), where chimneys were broken at their rooflines; at Evansville, Ind. (about $100 \mathrm{~km}$ southeast of Olney), where underground pipes were damaged and large cracks formed in sidewalks and streets; and at Louisville, Ky. (about $200 \mathrm{~km}$ southeast of Olney), where one downed chimney and widespread minor damage were reported.

Felt over a large area of the United States, including all or parts of 17 States-from Illinois east to Pennsylvania and West Virginia, west to Kansas and Nebraska, south to Alabama and Georgia, and north to Minnesota, Wisconsin, and southern Ontario, Canada. This was the largest earthquake in the area in 19 years-since Nov. 9, 1968. (Ref. 74, 577, 583, 592.) 


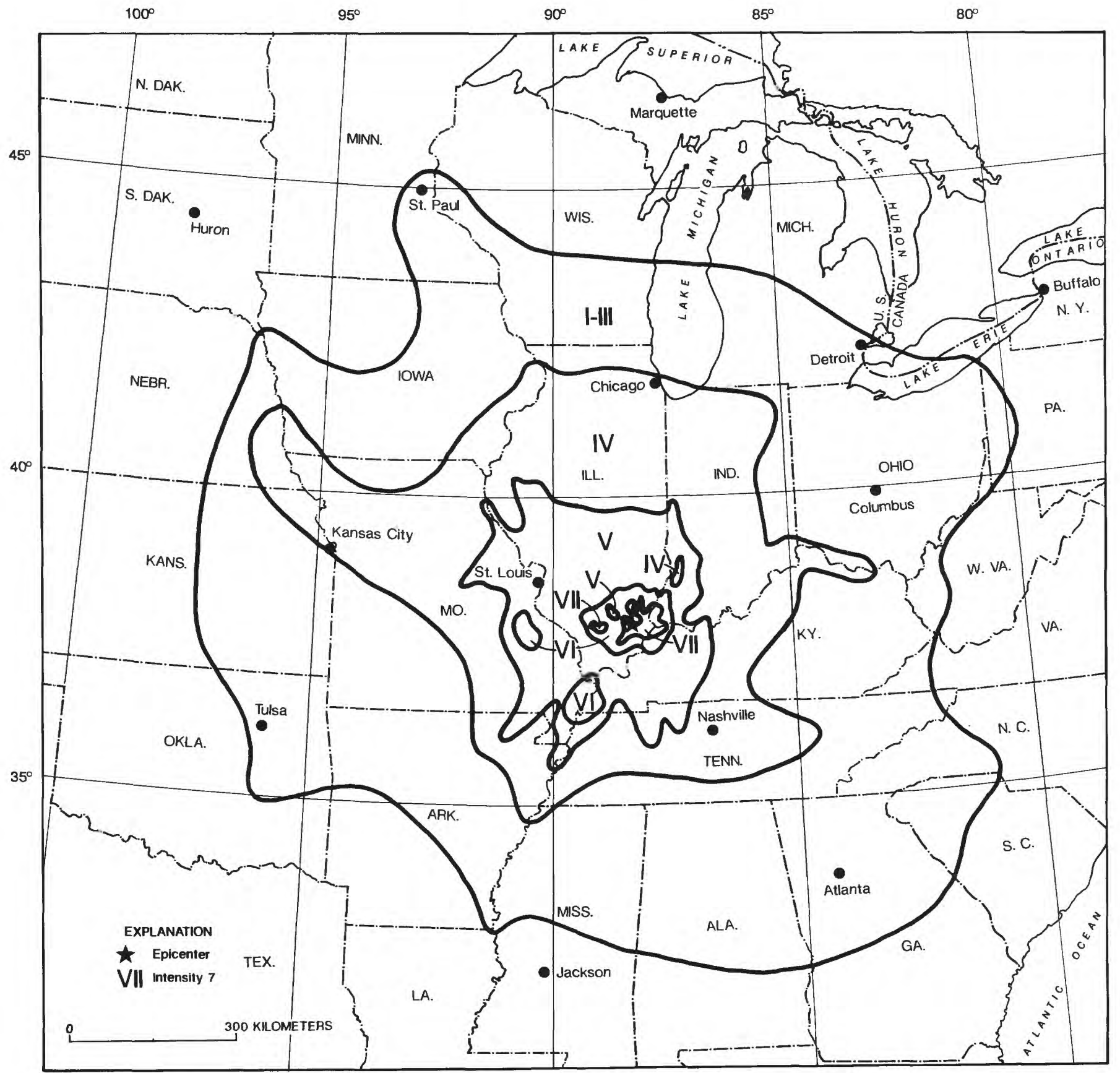

FIGURE 30.-Isoseismal map for the southern Illinois earthquake of November 9, 1968. This map is a simplified version of figure 3 in reference 490 of table 1. 


\section{INDIANA}

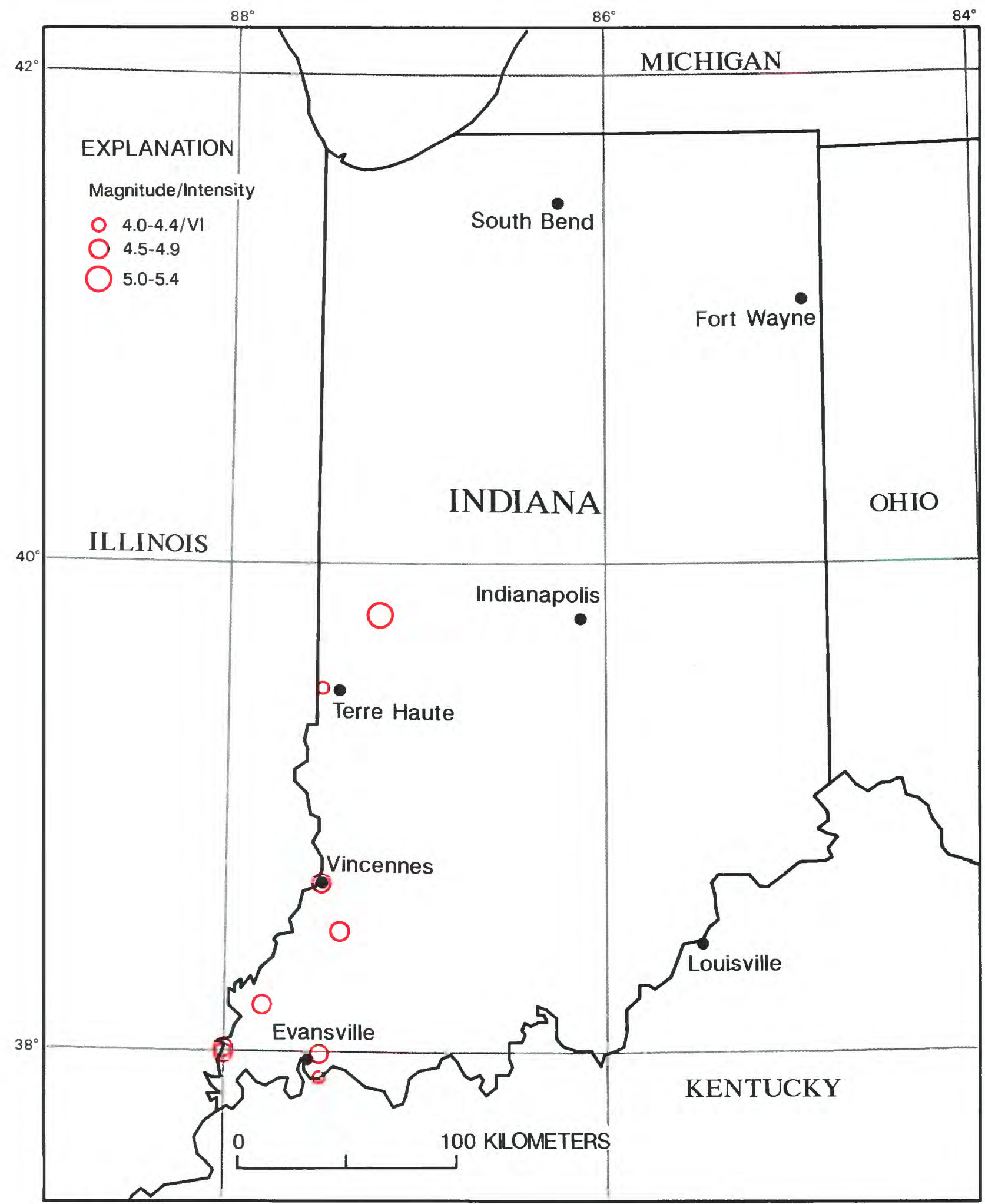

Earthquakes in Indiana with magnitudes $\geq 4.5$ or intensity $\geq$ VI. 
INDIANA

[See table 1 for hypocenter and intensity references and table 2 for definitions of magnitude source codes. Leader (-) indicates information is not available]

\begin{tabular}{|c|c|c|c|c|c|c|c|c|c|c|c|c|c|c|c|c|}
\hline \multirow{2}{*}{\multicolumn{2}{|c|}{ Date }} & \multirow{2}{*}{\multicolumn{2}{|c|}{$\begin{array}{c}\text { Origin } \\
\text { time (UTC) }\end{array}$}} & \multicolumn{6}{|c|}{ Hypocenter } & \multicolumn{4}{|c|}{ Magnitude } & \multicolumn{2}{|c|}{ Intensity } & \multirow{3}{*}{$\begin{array}{l}\text { Felt area } \\
\left(1,000 \mathrm{~km}^{2}\right)\end{array}$} \\
\hline & & & & \multirow{2}{*}{\multicolumn{2}{|c|}{$\begin{array}{l}\text { Latitude } \\
\left({ }^{\circ}\right)\end{array}$}} & \multirow{2}{*}{\multicolumn{2}{|c|}{$\begin{array}{l}\text { Longitude } \\
\left({ }^{\circ}\right)\end{array}$}} & \multirow{2}{*}{$\begin{array}{l}\text { Depth } \\
(\mathrm{km})\end{array}$} & \multirow[t]{2}{*}{ Ref } & \multicolumn{2}{|c|}{ USGS } & \multirow[t]{2}{*}{ Other } & \multirow{2}{*}{$\begin{array}{l}\text { Moment } \\
\text { M }\end{array}$} & \multirow[t]{2}{*}{ MMI } & \multirow[t]{2}{*}{ Ref } & \\
\hline Yr & Mo Da & $h \mathrm{~m}$ & s & & & & & & & $m_{b}$ & $M_{S}$ & & & & & \\
\hline 1827 & $07 \quad 05$ & 1130 & & 38.0 & $\mathbf{N}$ & 87.5 & W & - & 529 & - & - & $4.80 \mathrm{M}_{\mathrm{fa}} \mathrm{SC}$ & - & VI & 353 & 430 \\
\hline 1827 & $\begin{array}{ll}08 & 07\end{array}$ & 0430 & & 38.0 & $\mathbf{N}$ & 88.0 & W & - & 529 & - & - & $4.80 \mathrm{M}_{\mathrm{fa}} \mathrm{SG}$ & - & V & 529 & - \\
\hline 1827 & 0807 & 07 & & 38.0 & $\mathbf{N}$ & 88.0 & w & - & 105 & - & - & $4.70 \mathrm{M}_{\mathrm{fa}}$ BAR & - & v & 105 & - \\
\hline 1887 & 0206 & 2215 & & 38.7 & $\mathbf{N}$ & 87.5 & w & - & 38 & - & - & $4.60 \mathrm{M}_{\mathrm{fa}} \mathrm{SC}$ & - & VI & 529 & 170 \\
\hline 1891 & 0727 & 0228 & & 37.9 & $\mathbf{N}$ & 87.5 & W & - & 38 & - & - & $4.10 \mathrm{M}_{\mathrm{fa}} \mathrm{SC}$ & - & VI & 38 & 22 \\
\hline 1899 & 0430 & 0205 & & 38.5 & $\mathbf{N}$ & 87.4 & w & - & 529 & - & - & 4.901 & - & VIII & 38 & 179 \\
\hline 1909 & 0927 & 0945 & & 39.8 & $\mathbf{N}$ & 87.2 & W & - & 529 & - & - & $5.10 \mathrm{M}_{\mathrm{fa}} \mathrm{SC}$ & - & VII & 38 & 377 \\
\hline 1921 & 0314 & 1215 & & 39.5 & $\mathbf{N}$ & 87.5 & w & - & 113 & - & - & $4.40 \mathrm{M}_{\mathrm{fa}} \mathrm{SC}$ & - & VI & 529 & 89 \\
\hline 1925 & $04 \quad 27$ & 0405 & & 38.2 & $\mathbf{N}$ & 87.8 & w & - & 529 & - & - & $4.80 \mathrm{M}_{\mathrm{fa}} \mathrm{SC}$ & - & VI & 67 & 325 \\
\hline
\end{tabular}

[Reference (Ref.) numbers given in parentheses at the end of each description refer to sources of data in table 1. Magnitude values are described in the Introduction, and codes are defined in table 2.]

1827. July 5. Near New Harmony, Posey County, Ind. The earthquake cracked a brick store at New Harmony, Ind., and "greatly alarmed" some people. It was described as violent at New Madrid, Mo., and severe at St. Louis. It also alarmed many at Cincinnati, Ohio, and Frankfort, Ky. Magnitude 4.8 $\mathrm{M}_{\mathrm{fa}}$ BAR. (Ref. 105, 353, 529.)

1887. Feb. 6. Near Vincennes, Knox County, Ind. This shock was strongest in southwest Indiana and southeast Illinois. Plaster was shaken from walls at Vincennes, Ind., and west of Terre Haute, at Martinsville, Ill.; a cornice reportedly fell from a building at Huntington, Ind. It was felt distinctly at Evansville, Ind., but only slightly in the outskirts of St. Louis, Mo. Also reported felt at Louisville, Ky. (Ref. $38,105,353,529$.)

1891. July 27 (July 26). Evansville, Vanderburgh County, Ind. A strong local earthquake damaged a wall on a hotel, broke dishes, and overturned furniture at Evansville. The shock also was strong near Evansville at Mount Vernon and Newburgh, Ind., and at Hawesville, Henderson, and Owensboro, Ky. Magnitude $3.8 \mathrm{M}_{\mathrm{fa}}$ BAR. (Ref. $38,105,529$.)

1899. Apr. 30 (Apr. 29). Near Vincennes, Knox County, Ind. Brick walls cracked and several chimneys fell at Vincennes, and the tops of many chimneys were shaken down at Princeton in Gibson County. Toppled chimneys also were reported at
Greencastle, Putnam County, northeast of Terre Haute. The shock was "heavy" at Jeffersonville, near Louisville, Ky. Also felt in Illinois and Kentucky. Magnitude $4.6 \mathrm{M}_{\mathrm{fa}}$ BAR. (Ref. 38, 105, 353, 529.)

1909. Sept. 27. Wabash River valley, between Terre Haute and Vincennes, Ind. At Terre Haute (Vigo County), two chimneys were thrown down, plaster was cracked, and pictures were shaken from walls. At Covington, north of Terre Haute in Fountain County, a few chimneys were downed and windows were broken. Chimneys were "jarred loose" south of Vincennes at Princeton, Ind., one chimney was shaken to pieces at Olivette, Mo. (a suburb of St. Louis), and a brick wall was shaken down at St. Louis, Mo. Also reported felt in Arkansas, Illinois, Iowa, Kentucky, Ohio, and Tennessee (see fig. 31). Magnitude $4.8 \mathrm{M}_{\mathrm{fa}}$ BAR. (Ref. 38, 105, 353, 529.)

1921. Mar. 14. Near Terre Haute, Vigo County, Ind. This earthquake broke windows in many buildings and sent residents rushing into the streets at Terre Haute. Small articles were overturned at Paris, Ill., about $35 \mathrm{~km}$ northwest of Terre Haute. Magnitude 4.4 $\mathrm{M}_{\mathrm{fa}}$ BAR. (Ref. 113, 529.)

1925. Apr. 27 (Apr. 26). Wabash River valley, near Princeton, Gibson County, Ind. Chimneys were downed at Princeton and at Carmi, Ill., $100 \mathrm{~km}$ southwest; chimneys were "broken" at Louisville, Ky. Crowds fled from the theatres at Evansville, Ind. The felt area includes parts of Indiana, Illinois, Kentucky, Missouri, and Ohio. Magnitude $4.8 \mathrm{M}_{\mathrm{fa}} \mathrm{BAR}$. (Ref. $67,105,218,353,529$. 


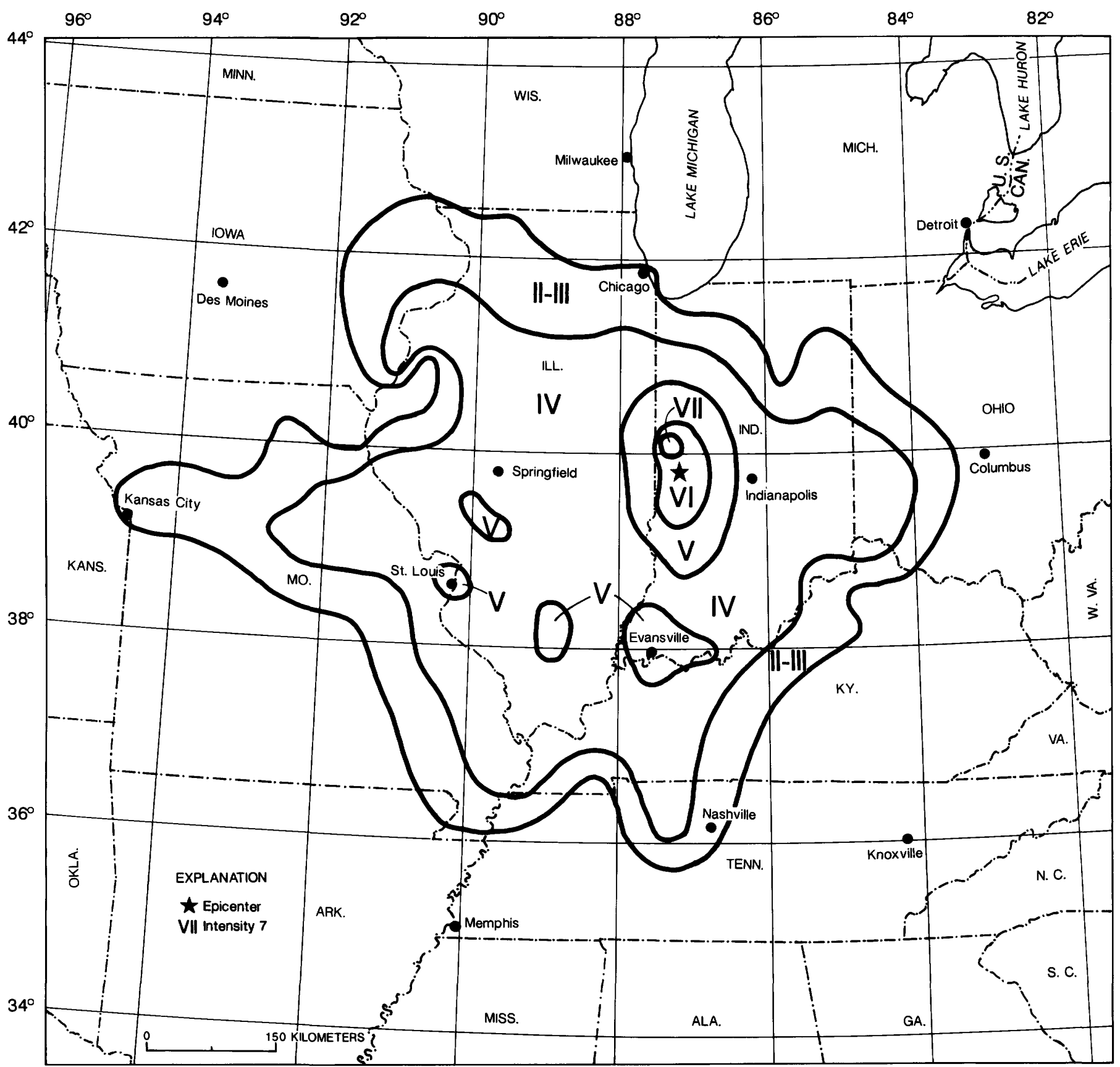

FIGURE 31.-Isoseismal map for the Wabash River valley, Indiana, earthquake of September 27, 1909. Isoseismals are based on intensity estimates from data listed in reference 529 of table 1. 



\section{KANSAS}

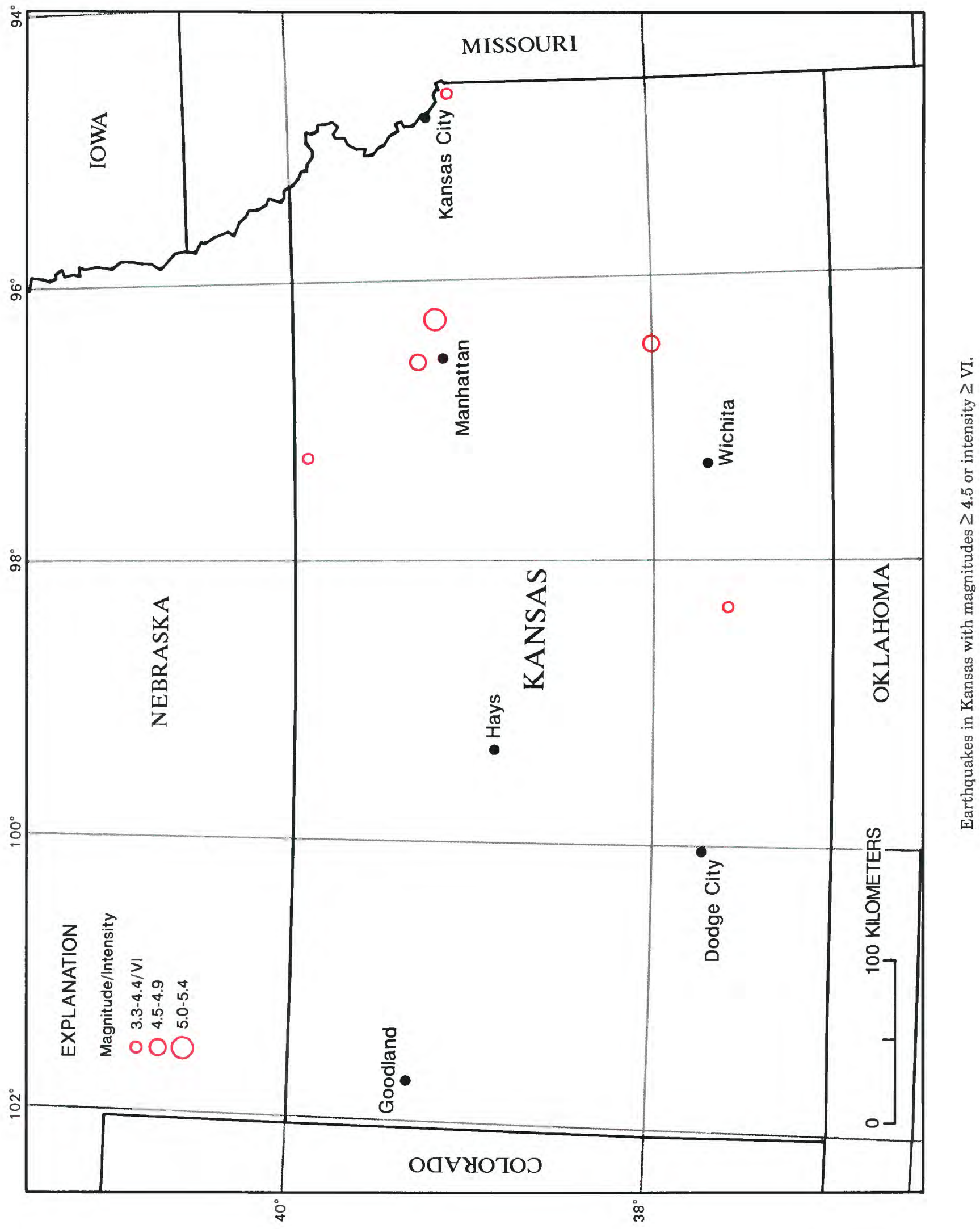


KANSAS

[See table 1 for hypocenter and intensity references and table 2 for definitions of magnitude source codes. Leader (--) indicates information is not available]

\begin{tabular}{|c|c|c|c|c|c|c|c|c|c|c|c|c|c|}
\hline \multirow{2}{*}{\multicolumn{2}{|c|}{ Date }} & \multirow{2}{*}{$\begin{array}{c}\text { Origin } \\
\text { time (UTC) }\end{array}$} & \multicolumn{4}{|c|}{ Hypocenter } & \multicolumn{4}{|c|}{ Magnitude } & \multicolumn{2}{|c|}{ Intensity } & \multirow{3}{*}{$\begin{array}{c}\text { Felt area } \\
\left(1,000 \mathrm{~km}^{2}\right)\end{array}$} \\
\hline & & & \multirow{2}{*}{$\begin{array}{l}\text { Latitude } \\
\left({ }^{\circ}\right)\end{array}$} & \multirow{2}{*}{$\begin{array}{l}\text { Longitude } \\
\left({ }^{\circ}\right)\end{array}$} & \multirow{2}{*}{$\begin{array}{l}\text { Depth } \\
(\mathrm{km})\end{array}$} & \multirow[t]{2}{*}{ Ref } & \multicolumn{2}{|c|}{ USGS } & \multirow[t]{2}{*}{ Other } & \multirow{2}{*}{$\begin{array}{l}\text { Moment } \\
\text { M }\end{array}$} & \multirow[t]{2}{*}{ MMI } & \multirow[t]{2}{*}{ Rof } & \\
\hline$\overline{Y r}$ & Mo Da & $\mathrm{h} \mathrm{m} \mathrm{s}$ & & & & & $m_{b}$ & $M_{s}$ & & & & & \\
\hline 1867 & 0424 & 2022 & $39.2 \mathrm{~N}$ & $96.3 \mathrm{~W}$ & - & 174 & - & - & $5.10 \mathrm{M}_{\mathrm{fa}}$ BAR & - & VII & 38 & 500 \\
\hline 1897 & 1202 & 0710 & $38.0 \mathrm{~N}$ & $96.5 \mathrm{~W}$ & - & 174 & - & - & $4.50 \mathrm{M}_{\mathrm{fa}}$ BAR & - & VI & 174 & 116 \\
\hline 1906 & 0108 & 0015 & $39.3 \mathrm{~N}$ & $96.6 \mathrm{~W}$ & - & 63 & - & - & $4.90 \mathrm{M}_{\mathrm{fa}}$ BAR & - & VII & 109 & 95 \\
\hline 1931 & 0809 & 061837 & $39.1 \mathrm{~N}$ & $94.7 \mathrm{~W}$ & - & 105 & - & - & $3.80 \mathrm{M}_{\mathrm{fa}}$ BAR & - & VI & 174 & 1 \\
\hline 1956 & 0106 & 115807.4 & $37.583 \mathrm{~N}$ & $98.346 \mathrm{~W}$ & 029 & 349 & - & - & $4.40 \mathrm{M}_{\mathrm{fa}} \mathrm{DG}$ & - & VI & 29 & 41 \\
\hline 1979 & 0630 & 204642.3 & $39.922 \mathrm{~N}$ & $97.287 \mathrm{~W}$ & 007 & 349 & - & - & $3.30 M_{n}$ GS & - & VI & 262 & 3 \\
\hline
\end{tabular}

[Reference (Ref.) numbers given in parentheses at the end of each description refer to sources of data in table 1. Magnitude values are described in the Introduction, and codes are defined in table 2.]

1867. Apr. 24. Manhattan, Riley County, Kans. This earthquake inflicted several minor injuries, cracked walls, and loosened stones from buildings. At Manhattan, a $0.6-\mathrm{m}$ wave was observed moving south to north on the Kansas River. Chimneys were downed in Louisville (Pottawatomie County) and Leavenworth. One side of a large building that housed a newspaper office was knocked down at Paola, south of Kansas City, in Miami County. East of Manhattan, the earth opened and ejected much water on a farm about $5 \mathrm{~km}$ south of Wamego.

Additional minor damage occurred in Iowa at Dubuque (plaster fell); in Kansas at Junction City (a well being dug was destroyed), Kansas City (plaster was shaken down), Lawrence (several stones were knocked off a church), Olathe (roof shingles were knocked to the ground), and Wamego (walls were cracked and plaster was broken); and in Missouri at Chillicothe (plaster fell from ceilings), St. Joseph (walls of new school house were cracked), and Warrensburg (plaster fell from ceiling). This earthquake is one of the important shocks that define the Midcontinent seismic trend. Also felt in Indiana and Illinois.

The felt area shown in the Kansas hypocenter list $\left(500,000 \mathrm{~km}^{2}\right)$ is based on information in the original source reference, which states that the earthquake was felt only in the territory east of the epicenter.
Information on this shock is sparse for the region west of the epicenter, and so the felt area given is only a rough estimate. (Ref. $38,63,105,174,353$.

1906. Jan. 8 (Jan. 7). Manhattan, Riley County, Kans. The earth movement at Manhattan sent residents fleeing from their houses. Only a few chimneys fell, but something was broken in almost every house. Plaster was knocked from walls in the surrounding towns of Junction City, Wamego, and Westmoreland. Also felt in Missouri and Nebraska. (Ref. 63, 109, 174, 353.)

1931. Aug. 9. Near Merriam, Johnson County, Kans. A strong local earthquake broke dishes and bounced pictures from walls at Merriam and overturned furniture at Turner. Also felt in Missouri. (Ref. 4, 105, 174, 353.)

1956. Jan. 6. South-central Kansas. Chimneys sustained slight damage at Coldwater and Medicine Lodge, Kans., near the Oklahoma border. Plaster fell to the floor at Wilmore, Kans., and Alva, Okla. Walls were reported cracked in a few towns. This earthquake was centered near the northern margin of the Anadarko Basin. Magnitude $4.3 \mathrm{M}_{\mathrm{fa}}$ BAR. (Ref. 29, $105,349,353$.

1979. June 30. Mahaska, Washington County, Kans. Large amounts of plaster cracked and fell and the foundation of a concrete-block building cracked at Mahaska, on the Kansas-Nebraska border, north of Salina. Slight damage also occurred in the towns of Haddam, Narka, and Morrowville. Also felt in Nebraska. (Ref. 262, 349.) 


\section{KENTUCKY}

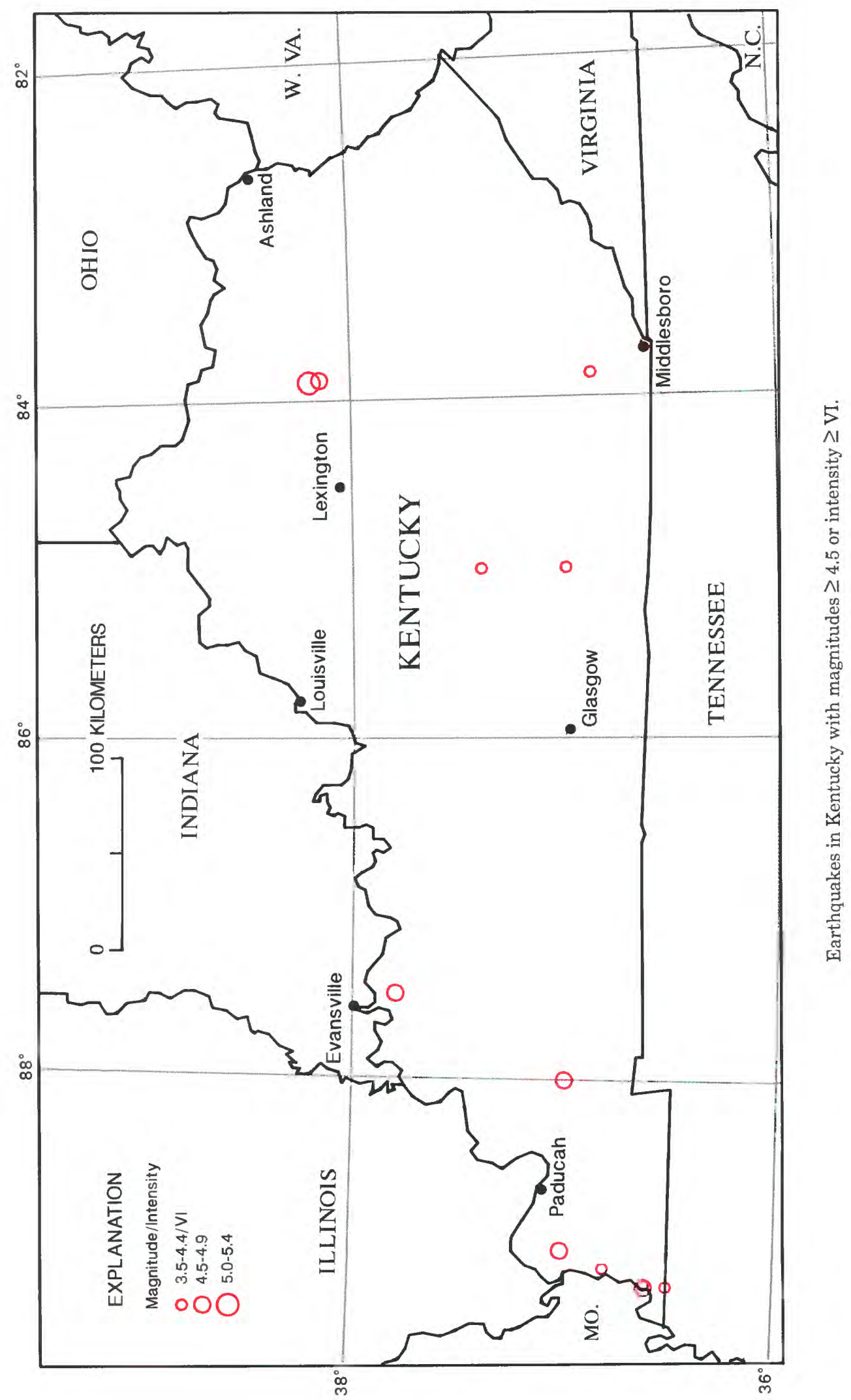


[See table 1 for hypocenter and intensity references and table 2 for definitions of magnitude source codes. (, felt area is less than $1,000 \mathrm{~km}^{2}$. Leader (-) indicates information is not available]

\begin{tabular}{|c|c|c|c|c|c|c|c|c|c|c|c|c|c|c|}
\hline \multirow{2}{*}{\multicolumn{2}{|c|}{ Date }} & \multirow{2}{*}{$\begin{array}{c}\text { Origin } \\
\text { time (UTC) }\end{array}$} & \multicolumn{5}{|c|}{ Hypocenter } & \multicolumn{4}{|c|}{ Magnitude } & \multicolumn{2}{|c|}{ Intensity } & \multirow{3}{*}{$\begin{array}{c}\text { Felt area } \\
\left(1,000 \mathrm{~km}^{2}\right)\end{array}$} \\
\hline & & & \multirow{2}{*}{$\begin{array}{l}\text { Latitude } \\
\left({ }^{\circ}\right)\end{array}$} & \multirow{2}{*}{\multicolumn{2}{|c|}{$\begin{array}{l}\text { Longitude } \\
\left({ }^{\circ}\right)\end{array}$}} & \multirow{2}{*}{$\begin{array}{l}\text { Depth } \\
(\mathrm{km})\end{array}$} & \multirow[t]{2}{*}{ Ref } & \multicolumn{2}{|c|}{ USGS } & \multirow[t]{2}{*}{ Other } & \multirow{2}{*}{$\begin{array}{c}\text { Moment } \\
\text { M }\end{array}$} & \multirow[t]{2}{*}{ MMI } & \multirow[t]{2}{*}{ Ref } & \\
\hline $\mathrm{Yr}_{r}$ & Mo Da & $\mathrm{h} \mathrm{m} \mathrm{s}$ & & & & & & $m_{b}$ & $M_{s}$ & & & & & \\
\hline 1779 & & & $37.0 \mathrm{~N}$ & 85.0 & w & - & 38 & - & - & - & - & Felt & 130 & - \\
\hline 1791 & 04 & 12 & $37.5 \mathrm{~N}$ & 85.0 & w & - & 145 & - & - & - & - & Felt & 145 & - \\
\hline 1841 & 1228 & 0550 & $36.6 \mathrm{~N}$ & 89.2 & w & - & 105 & - & - & $4.60 \mathrm{M}_{\mathrm{fa}} \mathrm{SG}$ & - & v & 38 & - \\
\hline 1850 & 0405 & 0205 & $37.0 \mathrm{~N}$ & 88.0 & W & - & 529 & - & - & $4.90 \mathrm{M}_{\mathrm{fa}} \mathrm{SG}$ & - & V & 159 & - \\
\hline 1858 & 0921 & & $36.5 \mathrm{~N}$ & 89.2 & W & - & 105 & - & - & $4.20 \mathrm{M}_{\mathrm{fa}} \mathrm{BAR}$ & - & VI & 159 & - \\
\hline 1878 & $03 \quad 12$ & 1000 & $36.8 \mathrm{~N}$ & 89.1 & w & - & 105 & - & - & $4.20 \mathrm{M}_{\mathrm{fa}} \mathrm{SC}$ & - & VI & 463 & 40 \\
\hline 1883 & 0111 & 0712 & $37.0 \mathrm{~N}$ & 89.0 & w & - & 529 & - & - & $4.60 \mathrm{M}_{\mathrm{fa}} \mathrm{SC}$ & - & VI & 529 & 185 \\
\hline 1916 & 1219 & 0542 & $36.6 \mathrm{~N}$ & 89.2 & W & - & 105 & - & - & $3.80 \mathrm{M}_{\mathrm{fa}} \mathrm{BAR}$ & - & VI & 109 & @ \\
\hline 1925 & 0902 & 1156 & $37.8 \mathrm{~N}$ & 87.5 & w & - & 353 & - & - & $4.60 \mathrm{M}_{\mathrm{fa}} \mathrm{SC}$ & - & VI & 113 & 200 \\
\hline 1954 & 0102 & 0325 & $36.6 \mathrm{~N}$ & 83.7 & w & - & 38 & - & - & $4.30 \mathrm{M}_{\mathrm{fa}} \mathrm{SC}$ & - & VI & 27 & 60 \\
\hline 1976 & $01 \quad 19$ & 062039.6 & $36.866 \mathrm{~N}$ & 83.86 & $61 \mathrm{~W}$ & 001 & 349 & 4.0 & - & $3.80 \mathrm{M}_{\mathrm{n}}$ SLM & - & VI & 49 & 15 \\
\hline 1980 & $07 \quad 27$ & 185221.4 & $38.193 \mathrm{~N}$ & 83.89 & $91 \mathrm{~W}$ & 006 & 349 & 5.1 & 4.7 & $5.00 \mathrm{M}_{\mathrm{n}} \mathrm{SLM}$ & $5.03 \mathrm{HRR}$ & VII & 300 & 667 \\
\hline 1988 & 0907 & 022809.5 & $38.143 \mathrm{~N}$ & 83.87 & $78 \mathrm{~W}$ & 010 & 74 & 4.5 & - & $4.60 \mathrm{M}_{\mathrm{n}}$ BLA & - & VI & 578 & 103 \\
\hline
\end{tabular}

[Reference (Ref.) numbers given in parentheses at the end of each description refer to sources of data in table 1. Magnitude values are described in the Introduction, and codes are defined in table 2.]

1791 or 1792. April or May. Kentucky. This earthquake is assumed to have caused damage. Furniture was agitated by the shock. Felt in northern and eastern parts of Kentucky. Few settlements existed farther west to report this event. The shock was preceded by a rumbling noise. (Ref. 38,145 .)

1858. Sept. 21. Line Shore, Fulton County, Ky. The earthquake was so severe at Line Shore, a community south of Hickman, that a woman $200 \mathrm{~m}$ from her house fell down four times before reaching her door. She thought that her house was going to collapse. (Ref. 105, 159.)

1878. Mar. 12. Columbus, northwest Hickman County, Ky. A severe earthquake in western Kentucky overturned furniture at Columbus, about $55 \mathrm{~km}$ southwest of Paducah. A section of a bank on the Mississippi River caved in. (Ref. 105, 463.)

1883. Jan. 11. Mississippi River valley. A moderate tremor shook an area from St. Louis, Mo., to Memphis, Tenn. At Paducah, Ky., several brick walls were cracked slightly, and at Hopkinsville, windows were broken. Small boats were "dashed about" on the river at Mound City, Ill. In the St. Louis area, buildings swayed gently and engine bells rang. At Clarksville, Tenn., cooking utensils and other small articles were displaced. Mag. 4.6 $\mathrm{M}_{\mathrm{fa}}$ BAR. (Ref. 105, $353,463,529$.)
1916. Dec. 19 (Dec. 18). Hickman, Fulton County, Ky. Bricks were shaken from chimneys at Hickman in southwest Kentucky. Two strong local shocks were felt. (Ref. 105, 109, 272, 353.)

1925. Sept. 2. Near Henderson, Ky. Landslides occurred at Henderson, about $15 \mathrm{~km}$ south of Evansville, Ind., and one chimney fell. Plaster was knocked from ceilings and walls at Owensboro, Ky., west of Henderson; a few bricks were displaced on chimneys at Evansville, Ind.; and a chimney toppled at Louisville, Ky., about $100 \mathrm{~km}$ northeast of Henderson. Several strong shocks were felt, but the last one was the strongest. The felt area of the main earthquake includes southern Illinois, southern Indiana, western Kentucky, southeastern Missouri, and northern Tennessee. Magnitude $4.8 \mathrm{M}_{\mathrm{fa}}$ BAR. (Ref. $105,113,353,529$.

1954. Jan. 2. (Jan. 1). Southeast Kentucky. The earthquake left cracks in foundations of houses and dislodged loose bricks in Bell County at Middlesboro. Tables slid across the floor, and residents were alarmed. Also felt in parts of North Carolina, Tennessee, and Virginia. (Ref. 27, 38, 508.)

1976. Jan. 19. Southeast Kentucky. Minor property damage occurred in Knox and Bell Counties. Damage reports from the area of maximum intensity include broken windows at Artemus; cracks in plaster and walls at Barbourville, Hinkle, Kettle Island, Pineville, and Woodbine; cracks in a brick school building at Walker; and cracks in a concrete sidewalk at Green Road. Material fell from a ceiling 


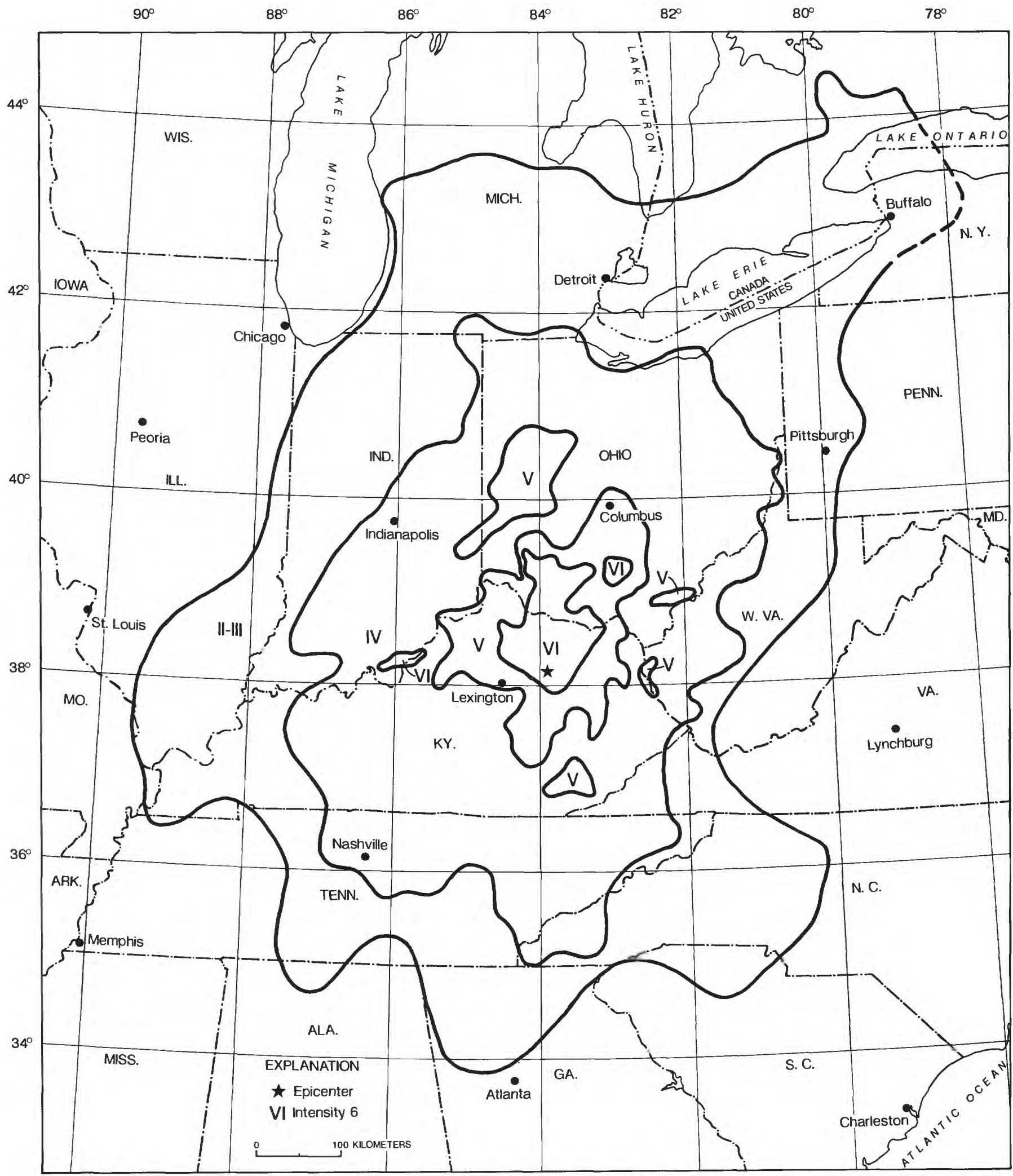

FIGURE 32.-Isoseismal map for the northern Kentucky earthquake of July 27, 1980. This is a simplified version of figure 21 in reference 300 of table 1. 


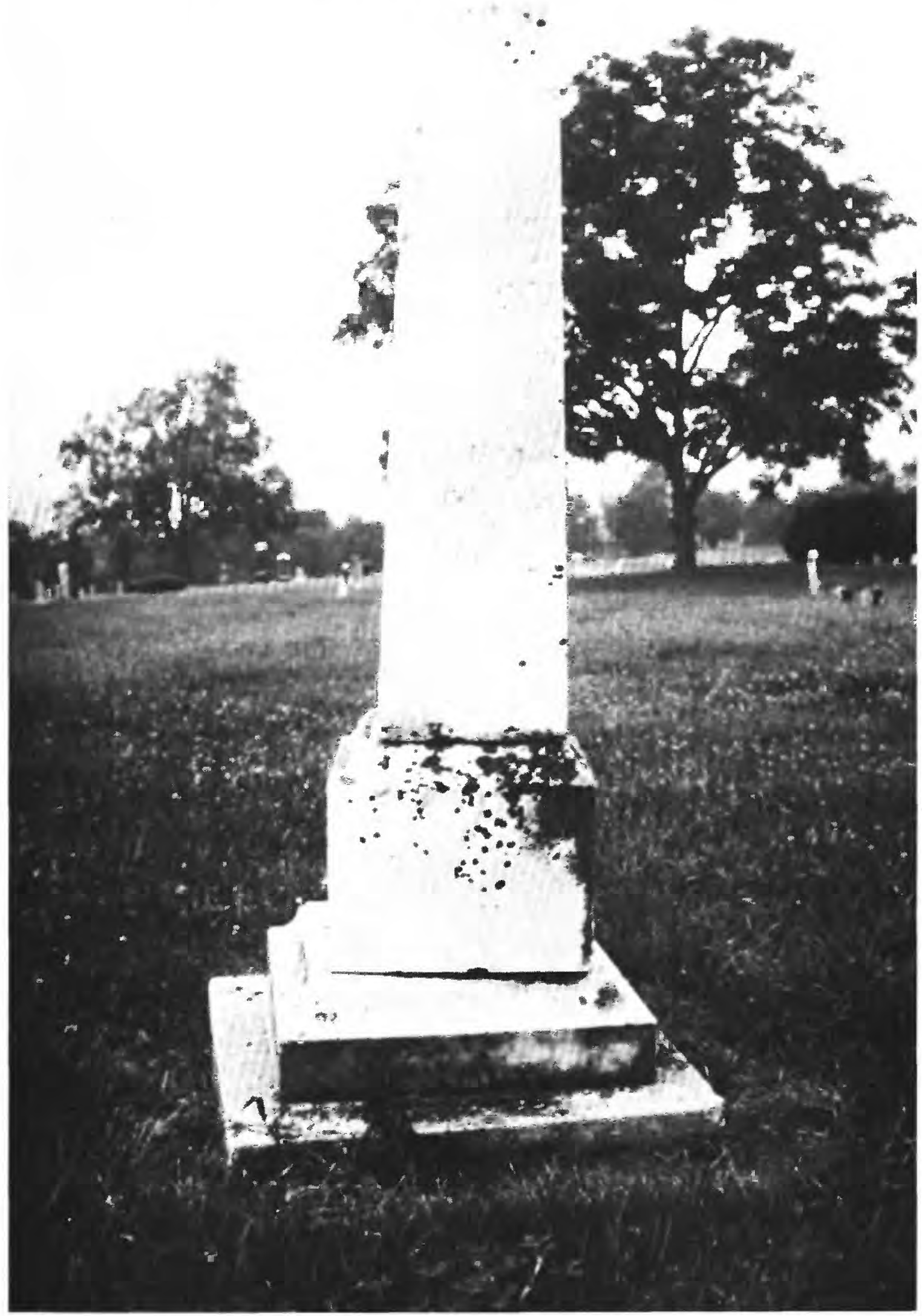

Cemetery monument in Washington, Kentucky, twisted by the July 27, 1980, Sharpsburg earthquake. (Photograph by M. Hopper.) 
at Lexington, and rocks slid on tracks at the Eastover mine. Also felt in northwest North Carolina, northeast Tennessee, western Virginia, and southwest West Virginia. (Ref. 49, 349.)

1980. July 27. Northeast Kentucky, near Sharpsburg, Batli County. This earthquake, the strongest in the history of Kentucky, was felt over all or parts of 15 States and in Ontario, Canada (see fig. 32). Damage occurred in Indiana, Kentucky, and Ohio.

Property damage was estimated at $\$ 1$ million at Maysville, about $50 \mathrm{~km}$ north of the epicenter, in Mason County, where 37 commercial structures and 269 private residences were damaged to some extent. Multistory all-brick structures in the downtown area, many of which were built in the mid-1800's, were affected the most. Broken chimneys represented the most common type of damage observed: several toppled or were broken at or near the roofline, some had bricks loosened or broken off their tops, and others sustained cracks of varying lengths and widths. This type of damage was a community-wide effect only in Maysville.

Cracks formed in the ground about $12 \mathrm{~km}$ from the epicenter. East of the epicenter, at Owingsville, ground cracks were estimated to be 6 to $10 \mathrm{~cm}$ deep and $30 \mathrm{~m}$ long. West of the epicenter, near Little Rock, ground cracks extending toward a cistern were observed on Stoner Road. Magnitude 4.7 $\mathrm{M}_{\mathrm{S}}$ NLI, 5.2 $\mathrm{M}_{\mathrm{n}}$ TUL, 5.0 $\mathrm{M}_{\mathrm{n}}$ PAL, 5.05 M JOH. (Ref. 38, 300, 340, 349.)

1988. Sept. 7 (Sept. 6). Northeast Kentucky, near Sliarpsburg, Bath County. An earthquake northeast of Lexington caused slight damage in Bath, Menifree, Montgomery, and Nicholas Counties. Cracks in chimneys and foundations occurred east of Lexington, at Jeffersonville and Means, and northeast of Lexington, at Moorefield. Large cracks formed in exterior walls at Olympia, north of Means, in Bath County. Felt over a large area in several States, including parts of Indiana, Kentucky, Ohio, Tennessee, and West Virginia. (Ref. 74, 578.) 



\section{LOUISIANA}

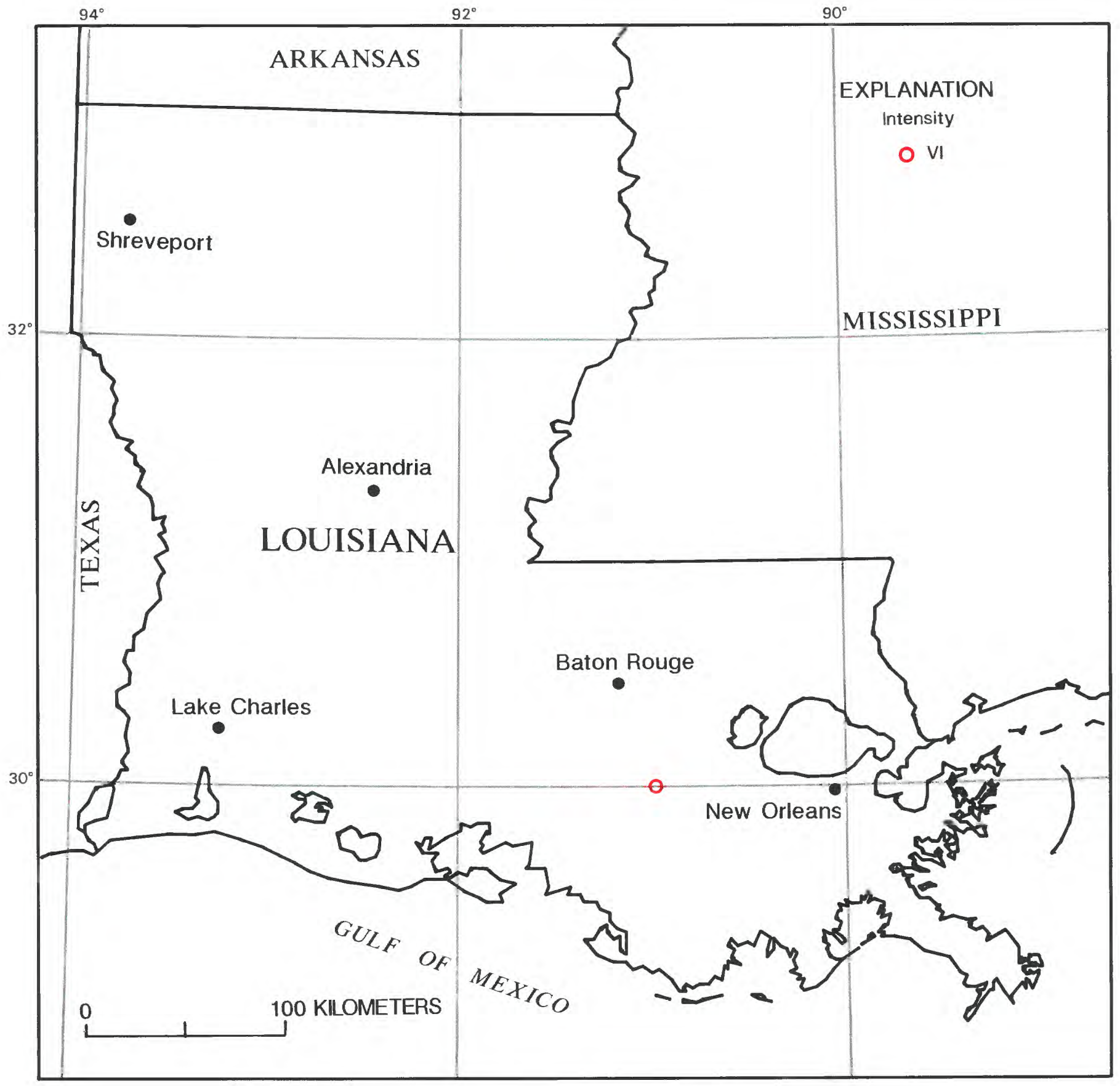

Damaging earthquake in Louisiana, intensity $\geq \mathrm{VI}$. 


\section{LOUISIANA}

[See table 1 for hypocenter and intensity references and table 2 for definitions of magnitude source codes. \&, land area only. Leader (-) indicates information is not avail $\cdot$ hle]

\begin{tabular}{|c|c|c|c|c|c|c|c|c|c|c|c|c|c|}
\hline \multirow{2}{*}{\multicolumn{2}{|c|}{ Date }} & \multirow{2}{*}{$\begin{array}{l}\text { Origin } \\
\text { time (UTC) }\end{array}$} & \multicolumn{4}{|c|}{ Hypocenter } & \multicolumn{4}{|c|}{ Magnitude } & \multicolumn{2}{|c|}{ Intensity } & \multirow{3}{*}{$\begin{array}{c}\text { Felt area } \\
\left(1,000 \mathrm{~km}^{2}\right)\end{array}$} \\
\hline & & & \multirow{2}{*}{$\begin{array}{l}\text { Latitude } \\
\left({ }^{\circ}\right)\end{array}$} & \multirow{2}{*}{$\begin{array}{l}\text { Longitude } \\
\left(^{\circ}\right)\end{array}$} & \multirow{2}{*}{$\begin{array}{l}\text { Depth } \\
(\mathrm{km})\end{array}$} & \multirow[t]{2}{*}{ Ref } & \multicolumn{2}{|c|}{ USGS } & \multirow[t]{2}{*}{ Other } & \multirow{2}{*}{$\begin{array}{l}\text { Moment } \\
\text { M }\end{array}$} & \multirow[t]{2}{*}{ MMI } & \multirow[t]{2}{*}{ Ref } & \\
\hline$\overline{Y r}$ & Mo Da & $\mathrm{hm} \mathrm{s}$ & & & & & $m_{b}$ & $M_{s}$ & & & & & \\
\hline 1930 & 1019 & 1217 & $30.0 \mathrm{~N}$ & $91.0 \mathrm{~W}$ & - & 3 & - & - & $4.20 \mathrm{M}_{\mathrm{fa}} \mathrm{BAR}$ & - & VI & 38 & $48 \&$ \\
\hline
\end{tabular}

[Reference (Ref.) numbers given in parentheses at the end of the description refer to sources of data in table 1. Magnitude values are described in the Introduction, and codes are defined in table 2.]

1930. Oct. 19. Near Napoleonville, Assump- tion Parish, La. The earthquake damaged chimneys and broke windows at Napoleonville and cracked plaster at White Castle, northwest of Napoleonville. Many people in the area rushed into the streets. Magnitude 4.4 $\mathrm{M}_{\mathrm{n}}$ SLM. (Ref. 3, 38, 105.) 


\section{MAINE}

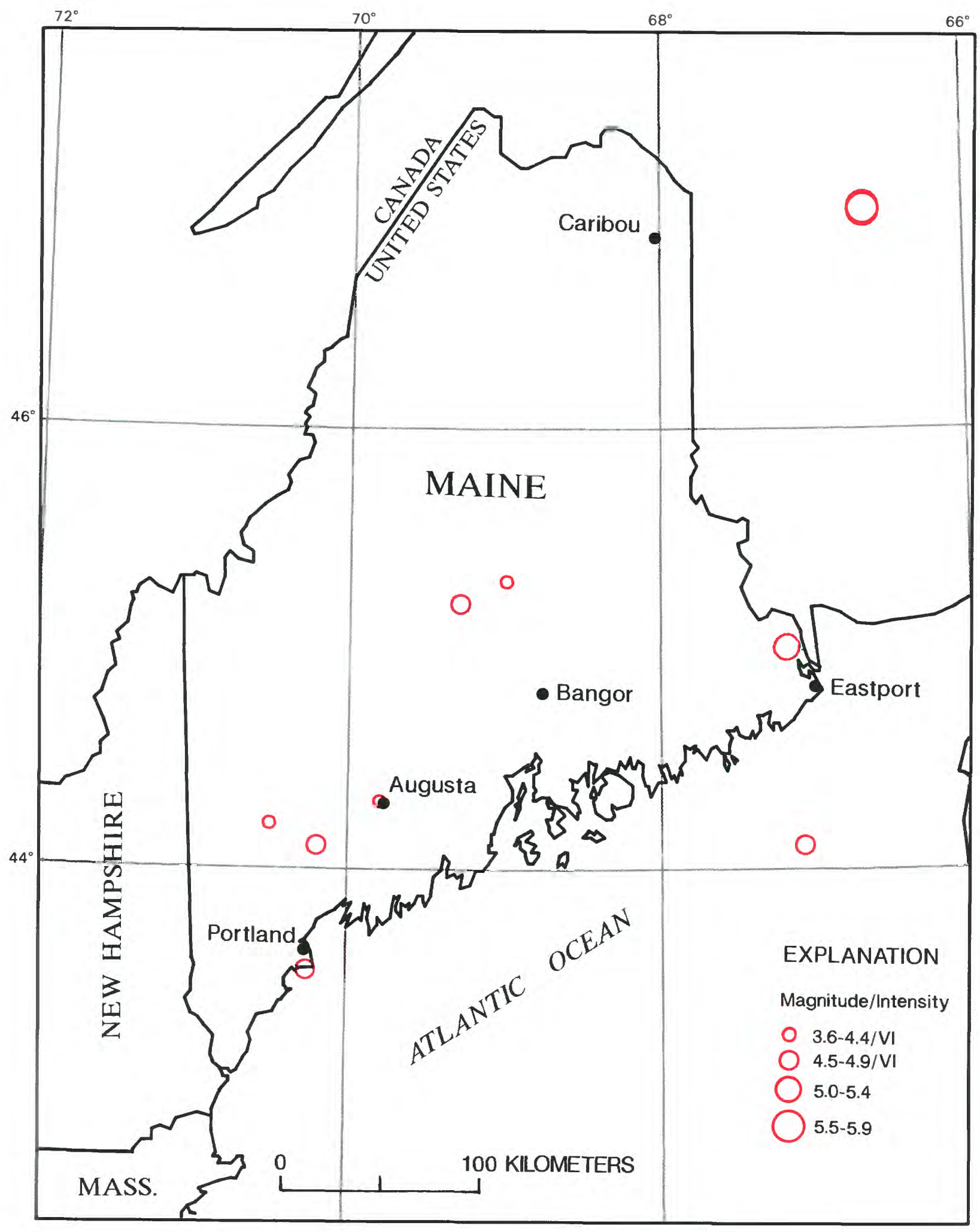

Earthquakes in Maine with magnitudes $\geq 4.5$ or intensity $\geq$ VI. 


\begin{tabular}{|c|c|c|c|c|c|c|c|c|c|c|c|c|c|}
\hline \multirow{2}{*}{\multicolumn{2}{|c|}{ Date }} & \multirow{2}{*}{$\begin{array}{c}\text { Origin } \\
\text { time (UTC) }\end{array}$} & \multicolumn{4}{|c|}{ Hypocenter } & \multicolumn{4}{|c|}{ Magnitude } & \multicolumn{2}{|c|}{ Intensity } & \multirow{3}{*}{$\begin{array}{c}\text { Felt area } \\
\left(1,000 \mathrm{~km}^{2}\right)\end{array}$} \\
\hline & & & \multirow{2}{*}{$\begin{array}{l}\text { Latitude } \\
\left({ }^{\circ}\right)\end{array}$} & \multirow{2}{*}{$\begin{array}{l}\text { Longitude } \\
\left({ }^{\circ}\right)\end{array}$} & \multirow{2}{*}{$\begin{array}{l}\text { Dopth } \\
(\mathrm{km})\end{array}$} & \multirow[t]{2}{*}{ Ref } & \multicolumn{2}{|c|}{ USGS } & \multirow[t]{2}{*}{ Other } & \multirow{2}{*}{$\begin{array}{c}\text { Moment } \\
\text { M }\end{array}$} & \multirow[t]{2}{*}{ MMI } & \multirow[t]{2}{*}{ Ref } & \\
\hline $\mathrm{Yr}$ & Mo De & $h \mathrm{~m} s$ & & & & & $m_{b}$ & $M_{S}$ & & & & & \\
\hline 1857 & 1223 & 1830 & $\begin{array}{ll}44.1 & \mathbf{N} \\
44.1 & \mathbf{N}\end{array}$ & $70.2 \quad \mathrm{~W}$ & - & 76 & - & - & - & - & VI & 78 & $11 \&$ \\
\hline $\begin{array}{l}1870 \\
1904\end{array}$ & $\begin{array}{l}0208 \\
0321\end{array}$ & 0604 & $\begin{array}{ll}44.1 & \mathrm{~N} \\
45.0 & \mathrm{~N}\end{array}$ & $\begin{array}{ll}67.1 & W \\
67.2 & W\end{array}$ & E & $\begin{array}{l}76 \\
38\end{array}$ & $\overline{-}$ & - & $5.10 \overline{\mathrm{M}}_{\mathrm{fa}} \mathrm{SC}$ & - & $\begin{array}{l}\text { VI } \\
\text { VII }\end{array}$ & $\begin{array}{l}76 \\
38\end{array}$ & $\overline{388}$ \\
\hline 1905 & $\begin{array}{lll}07 & 15\end{array}$ & 1010 & $44.3 \mathrm{~N}$ & $69.8 \mathrm{~W}$ & - & 76 & - & - & $4.40 \mathrm{M}_{\mathrm{fa}} \mathrm{SC}$ & - & VI & 126 & 100 \\
\hline 1918 & 0821 & 041154 & $44.2 \mathrm{~N}$ & 70.5 W & - & 78 & - & - & $4.20 \mathrm{M}_{\mathrm{fa}} \mathrm{SC}$ & - & VII & 76 & 9 \\
\hline 1928 & 0208 & & $45.3 \mathrm{~N}$ & $69.0 \mathrm{~W}$ & - & 1 & - & - & $3.60 \mathrm{M}_{\mathrm{fa}} \mathrm{SC}$ & - & VI & 38 & 2 \\
\hline 1947 & 1228 & 195818 & $45.2 \mathrm{~N}$ & $69.3 \mathrm{~W}$ & - & 77 & - & - & $4.50 \mathrm{M}_{\mathrm{L}} \mathrm{EPB}$ & - & V & 20 & 12 \\
\hline 1957 & 0426 & 114008.6 & $43.535 \mathrm{~N}$ & $70.255 \mathrm{~W}$ & 005 & 349 & - & - & $4.70 M_{n} S T$ & 4.40ST & VI & 30 & 82\& \\
\hline 1982 & 0109 & 125351.8 & $46.984 \mathrm{~N}$ & $66.656 \mathrm{~W}$ & 010 & 74 & 5.7 & 5.2 & $5.80 \mathrm{M}_{\mathrm{n}}$ BLA & 5.47EPB & VI & 350 & 570\& \\
\hline 1982 & $01 \quad 11$ & 214107.9 & $46.975 \mathrm{~N}$ & $66.659 \mathrm{~W}$ & 006 & 74 & 5.4 & 4.5 & $5.50 \mathrm{M}_{\mathrm{n}}$ BLA & - & VI & 350 & 260\& \\
\hline
\end{tabular}

[Reference (Ref.) numbers given in parentheses at the end of each description refer to sources of data in table 1 . Magnitude values are described in the Introduction, and codes are defined in table 2.]

1857. Dec. 23. Near Lewiston, Androscoggin County, Me. At Lewiston, chimneys were thrown down and the ceiling of the depot building was shaken down. Residents described the earthquake as the most severe ever felt in the area. Glass was broken at Augusta and Gardiner, northeast of Lewiston, and residents rushed outside. Felt from Portland north to Waterville and from the coast west to Paris. (Ref. 76, 78.)

1870. Feb. 8. Bay of Fundy. This earthquake was reported in Maine and the Maritime Provinces of Canada. Little information is available on which to base an intensity evaluation. The intensity value listed here was assigned in ref. 76. (Ref. 76.)

1904. Mar. 21. Southeast Maine. This strong earthquake overthrew chimneys in Washington County, in the area of Calais and Eastport, Me., and at St. Stephen, New Brunswick. Felt throughout most of New England and the Provinces of New Brunswick and Nova Scotia. It was observed west to the Hudson River and Montreal, Canada, and south to southern Connecticut. (Ref. 38, 76.)

1905. July 15. Southern Maine. In Gardiner, south of Augusta, several chimneys in disrepair toppled; bricks were shaken from chimneys in Androscoggin County at Auburn and Monmouth. Felt from northeastern Massachusetts through southern New Hampshire to central Maine. (Ref. 38, $76,78,126$.
1918. Aug. 21 (Aug. 20). Southern Maine. West of Augusta, at Norway and South Paris, bricks tumbled from chimneys and a few chimneys were cracked. "A hundred or more chimneys will need topping out before winter." Stovepipes were "unjointed" in houses at Norway, and bed slats dropped to the floor. A temporary change of about $10 \mathrm{~cm}$ in the level of Lake Sebago, northwest of Portland, was observed. (Ref. 38, 76, 78, 272.)

1928. Feb. 8. Milo, Piscataquis County, Me. This local event cracked plaster walls and knocked dishes from shelves at Milo, north of Bangor. It was a distinct tremor, followed at 5- to 6-minute intervals by smaller shocks. (Ref. 1, 38.)

1957. Apr. 26. Near coast of Maine. Minor damage occurred in Cumberland County at Portland and Westbrook, Me. Chimneys split and windows and dishes broke at Westbrook; plaster and walls cracked and merchandise fell from shelves at Portland. Reports of minor damage also were received from observers on ships about $32 \mathrm{~km}$ offshore. (Ref. 30, 38, 349.)

1982. Jan. 9. New Brunswick, Canada. Cracks in streets, chimneys, and foundations, the most common type of damage sustained, occurred at several towns in Aroostook County, in northern Maine. In addition, cracks formed in sidewalks, plaster, and drywall in several towns. Tombstones were displaced at Lubec, and underground pipes were put out of service at Stockholm. Moderate aftershocks occurred on Jan. 11 (see description below), Mar. 31, Apr. 11, and June 16. The main earthquake was felt in Canada from the Gaspe Peninsula in the north to Prince Edward Island in the east and west to Montreal. In the United States, it was felt in Connecticut, Maine, 
Massachusetts, New Hampshire, New York, Rhode Island, and Vermont. Magnitude 5.8 $\mathrm{M}_{\mathrm{n}}$ EPB. (Ref. $74,350$.

1982. Jan. 11. New Brunswick, Canada. This earthquake is an aftershock of the event on Jan. 9 (see description above). Minor damage, including cracks in foundations and walls, occurred in
Aroostook County, Maine, at Caribou, Haynesville, Loring Air Force Base, Presque Isle, and Saint Francis. Felt in Canada from the Saint Lawrence River south to Nova Scotia and in the States of Connecticut, Maine, Massachusetts, New Hampshire, New York, Rhode Island, and Vermont. Magnitude $5.5 \mathrm{M}_{\mathrm{n}}$ EPB. (Ref. 74, 350.) 



\section{MASSACHUSETTS}

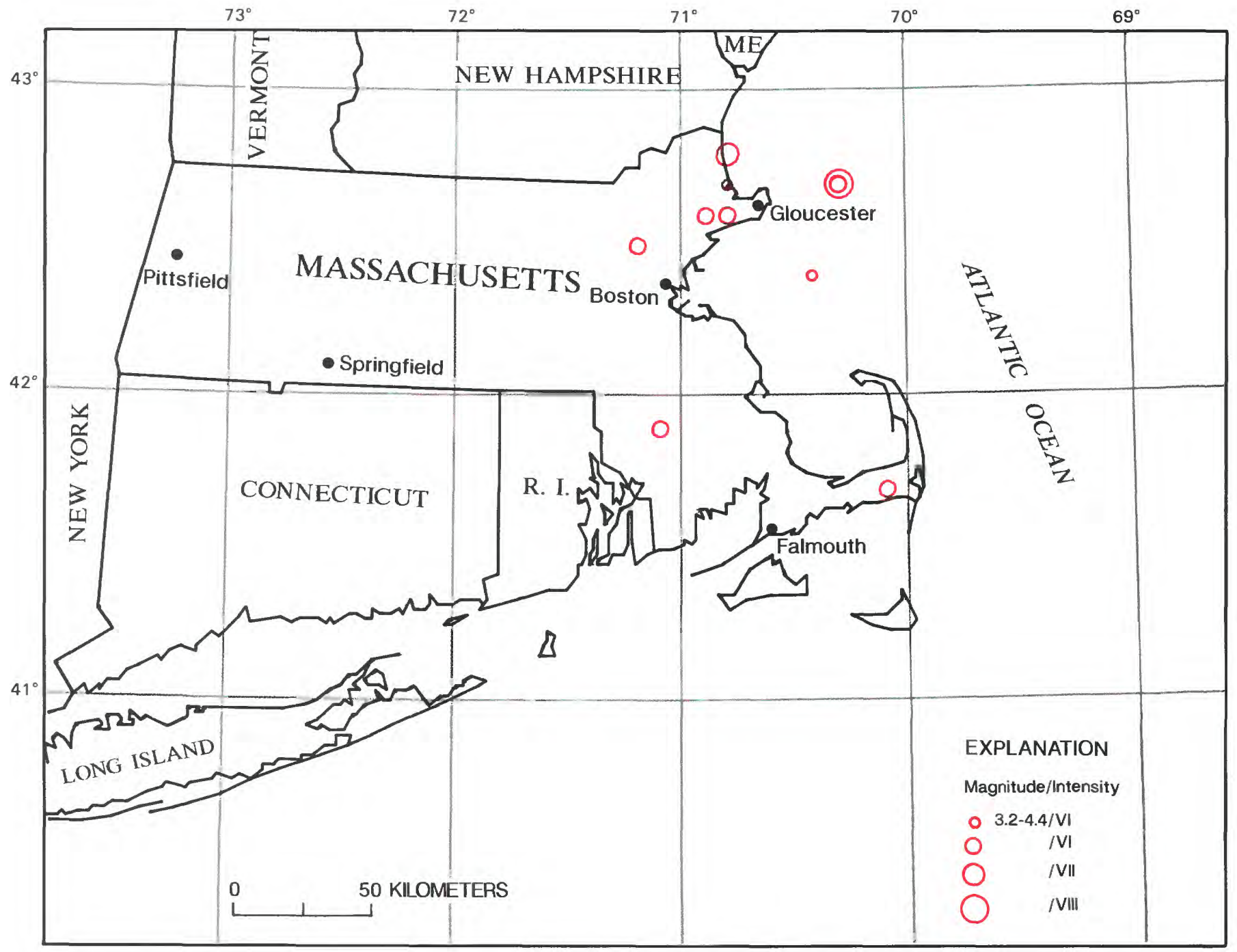

Damaging earthquakes in Massachusetts, intensity $\geq V I$. 


\begin{tabular}{|c|c|c|c|c|c|c|c|c|c|c|c|c|c|}
\hline \multirow{2}{*}{\multicolumn{2}{|c|}{ Date }} & \multirow{2}{*}{$\begin{array}{c}\text { Origin } \\
\text { time (UTC) }\end{array}$} & \multicolumn{4}{|c|}{ Hypocenter } & \multicolumn{4}{|c|}{ Magnitude } & \multicolumn{2}{|c|}{ Intensity } & \multirow{3}{*}{$\begin{array}{c}\text { Felt area } \\
\left(1,000 \mathrm{~km}^{2}\right)\end{array}$} \\
\hline & & & \multirow{2}{*}{$\begin{array}{l}\text { Latitude } \\
\left({ }^{\circ}\right)\end{array}$} & \multirow{2}{*}{$\begin{array}{l}\text { Longitude } \\
\left({ }^{\circ}\right)\end{array}$} & \multirow{2}{*}{$\begin{array}{l}\text { Depth } \\
(\mathrm{km})\end{array}$} & \multirow[t]{2}{*}{ Ref } & \multicolumn{2}{|c|}{ USGS } & \multirow[t]{2}{*}{ Other } & \multirow{2}{*}{$\begin{array}{c}\text { Moment } \\
\text { M }\end{array}$} & \multirow[t]{2}{*}{ MMI } & \multirow[t]{2}{*}{ Ref } & \\
\hline $\mathrm{Yr}_{r}$ & Mo Da & $\mathrm{h} \mathrm{m} \mathrm{s}$ & & & & & $m_{b}$ & $M_{\mathbf{S}}$ & & & & & \\
\hline 1627 & & & $42.6 \mathrm{~N}$ & $70.8 \mathrm{~W}$ & - & 126 & - & - & - & - & VI & 76 & - \\
\hline 1638 & $06 \quad 11$ & 07 & $46.5 \mathrm{~N}$ & $72.5 \mathrm{~W}$ & - & 76 & - & - & - & - & IX & 76 & - \\
\hline 1663 & 0205 & 2230 & $47.6 \mathrm{~N}$ & $70.1 \mathrm{~W}$ & - & 76 & - & - & - & - & $\mathrm{X}$ & 76 & 1900\& \\
\hline 1727 & 1110 & 0340 & $42.8 \mathrm{~N}$ & $70.8 \mathrm{~W}$ & - & 3 & - & - & - & - & VII & 7 & $296 \&$ \\
\hline 1744 & 10614 & 1515 & $42.6 \mathrm{~N}$ & 70.9 W & - & 78 & - & - & - & - & VI & 78 & 157\& \\
\hline 1755 & 1118 & 091135 & $42.7 \mathrm{~N}$ & $70.3 \mathrm{~W}$ & - & 78 & - & - & - & - & VIII & 78 & $1000 \&$ \\
\hline 1761 & 0312 & 0715 & $42.7 \mathrm{~N}$ & w & - & 7 & - & - & - & - & $\vec{v}$ & & $127 \&$ \\
\hline 1766 & 0202 & & $42.0 \mathrm{~N}$ & $68.0 \mathrm{~W}$ & - & 76 & - & - & - & - & VI & 7 & - \\
\hline 1800 & 1225 & & $41.9 \mathrm{~N}$ & $71.1 \mathrm{~W}$ & - & 76 & - & - & - & - & VI & 7 & - \\
\hline 1817 & 1005 & 1645 & $42.5 \mathrm{~N}$ & $71.2 \mathrm{~W}$ & - & 38 & - & - & - & - & vi & 78 & $55 \&$ \\
\hline 1847 & $\begin{array}{l}08 \quad 08 \\
\end{array}$ & 150 & $41.7 \mathrm{~N}$ & $70.1 \mathrm{~W}$ & - & 7 & - & - & - & - & v & 7 & 210 \\
\hline 1963 & 1016 & 153059.7 & $42.401 \mathrm{~N}$ & $70.422 \mathrm{~W}$ & 014 & 349 & - & E & $3.90 \bar{M}_{\mathrm{n}} \mathrm{S}$ & $3.40 \mathrm{ST}$ & VI & 36 & $18 \&$ \\
\hline 1963 & 1030 & 223657.9 & $42.7 \mathrm{~N}$ & $70.8 \mathrm{~W}$ & - & 36 & - & - & $3.20 \mathrm{M}_{\mathrm{L}}$ WES & $2.58 \mathrm{ST}$ & VI & 36 & $6 \&$ \\
\hline
\end{tabular}

[Reference (Ref.) numbers given in parentheses at the end of each description refer to sources of data in table 1.]

1627. Date unknown. Essex, Mass. This local earthquake at Essex, about $40 \mathrm{~km}$ northeast of Boston, was reported to be as violent as the shock of June 11, 1638 (see description below). (Ref. 59, $76,126$.

1638. June 11. Probably in the St. Lawrence River valley, Canada. Tops of chimneys were thrown down and dishes were knocked from shelves in the Salem-Lynn area, north of Boston, and at Plymouth, on the coast about $55 \mathrm{~km}$ southeast of Boston. At Plymouth, people had to hold on to posts to keep from falling. Ships near the coast reportedly were shaken. Also felt in Connecticut and Rhode Island. Aftershocks continued for 20 days. (Ref. 38, $59,76$.

1663. Feb. 5. St. Lawrence River valley region, Canada. This major earthquake caused vast landslides along the St. Maurice, Batiscan, and St. Lawrence Rivers. The earthquake was felt sharply in New England. On the shores of Massachusetts Bay, the tops of chimnevs were broken on houses and pewter was jarred from shelves. (Ref. 38, 59, 76.)

1727. Nov. 10 (Nov. 9). Northern Cape Ann region, east of Newbury, Essex County, Mass. At Newbury, many stone walls and chimney bricks were shaken down, and almost all tops of chimneys were knocked off. Considerable changes occurred in the flow of water in springs and, in some springs, changes occurred in the character of the water. "Some firm land became quagmire, and marshes were dried up." The rise and fall of the ground made it difficult to walk, and houses shook and rocked as if they would fall apart. Sand blows were reported near Spring Island. Felt from the Kennebec River in Maine to the Delaware River on the New YorkPennsylvania border and from ships at sea to the "extreme western settlements." Aftershocks occurred in the area for several months. The strongest aftershock (MM intensity V) occurred in the Newbury area on Dec. 28, 1727, and Jan. 4 ahd Feb. 10, 1728 (local dates). (Ref. 38, 59, 78.)

1744. June 14. Southern Cape Ann, Mass., region (near Salem, Essex County, Mass.). Bricks were shaken from several chimneys in Boston and other towns, and pieces of stone fence were thrown down in the country. Many persons were alarmed at Newbury and Ipswich, Mass. The shock was reported from Falmouth, Me., to New York City. Several aftershocks occurred. (Ref. 38, 78.)

1755. Nov. 18. East of Cape Ann, Mass. This earthquake caused the heaviest damage in the region around Cape Ann and Boston. At Boston, much of the damage was confined to an area of infilled land near the wharfs. There, about 100 chimneys were leveled with the roofs of houses, and many others $(1,200$ to 1,500$)$ were shattered and partly thrown down. Some chimneys, which were broken off below their tops, tilted dangerously 3 or $4 \mathrm{~cm}$; others were twisted or partly turned. The gable ends of several brick buildings (12 to 15 ) were thrown down, and the roofs of some houses were damaged by the fall of chimneys. Stone fences were thrown down throughout the countryside, particularly on a line extending 
from Boston to Montreal. New springs formed, and old springs dried up. At Scituate (on the coast southeast of Boston), Pembroke (about $15 \mathrm{~km}$ southwest of Scituate), and Lancaster (about $40 \mathrm{~km}$ west of Boston), cracks opened in the earth. Water and fine sand issued from some of the ground cracks at Pembroke.

The earthquake generated a tsunami that left vessels aground and fish on the banks after the water withdrew from St. Martin's Harbor in the West Indies. When the water flowed back into the harbor, it rose about $2 \mathrm{~m}$ higher than normal and inundated the low-lying lands.

This earthquake was reported from Halifax, Nova Scotia, south to the Chesapeake Bay in Maryland and from Lake George, N.Y., east to a ship $320 \mathrm{~km}$ east of Cape Ann. The location of the ship is thought to be near the epicenter, because the shock was felt so strongly that those onboard believed the ship had run aground. Several aftershocks occurred. (Ref. 59, 78,502 .)

1761. Mar. 12. East of Cape Ann, Mass. This earthquake is included in the list of magnitude $\geq 4.5$ or intensity $\geq$ VI events based on the large felt area documented on land from the estimated offshore location. While no damage was documented the large felt area and strong shaking indicates that this event is as large or larger than a magnitude 4.5 earthquake.

1766. Feb. 2. Off the coast of Massachusetts. This shock was felt throughout Massachusetts, Rhode Island, and other parts of New England. It was reported that the earthquake was "accompanied by a remarkable meteor." Note: Existing felt reports do not substantiate the MM intensity VI published in ref. 76 for this earthquake and the one in 1800 (see next paragraph), but an intensity of that level probably was assigned because the shocks were felt over such wide areas. (Ref. 59, 76.)
1800. Dec. 25. Eastern Massachusetts. A severe shock was felt at Boston and Concord, Mass; Newport, R.I.; and elsewhere (see 1766 description above). (Ref. 59, 76.)

1817. Oct. 5. Northeast Massachusetts. Walls were thrown down at Woburn, in Middlesex County, according to ref. 59. Ref. 78 states that the "walls" referred to by ref. 59 possibly were stone fences, characteristic of rural New England pasture land, rather than house walls. Such walls were constructed of glacial boulders piled loosely on top of each other to form a stone fence. Felt in Connecticut, Massachusetts, New Hampshire, New York, and probably Rhode Island and Vermont. (Ref. 38, 59, 76, 78.)

1847. Aug. 8. Near Harwich, Barnstable County, Mass. A section of "the plastering" was thrown down at the Harwich Baptist Church; mirrors hanging on the wall were broken at Nantucket (south of Hyannis) and Sandwich (northeast of Hyannis). In the Yarmouth area, northeast of Hyannis, glass was broken and crockery shook from shelves. Felt throughout eastern Massachusetts. (Ref. 76, 78.)

1963. Oct. 16. Near the coast of Massachusetts. Slight damage at Somerville, north of downtown Boston, consisted of fallen plaster, cracks in walls, and fallen stones from building foundations. Also felt in Maine, New Hampshire, and Rhode Island. (Ref. 36, 38, 349.)

1963. Oct. 30. Northeast of Peabody, Essex County, Mass. Slight damage occurred at Framingham, Peabody, and Swampscott. At Framingham, in Middlesex County, southwest of Boston, $3 \mathrm{~m}$ of stone foundation on a 155-year-old house collapsed. Northwest of Boston, at Peabody, cracks in wall plaster and a sidewalk occurred; and southeast of Peabody, at Swampscott, basement stairs were thrown out of alignment. Also felt in New Hampshire. (Ref. 36, 38.) 



\section{MICHIGAN}

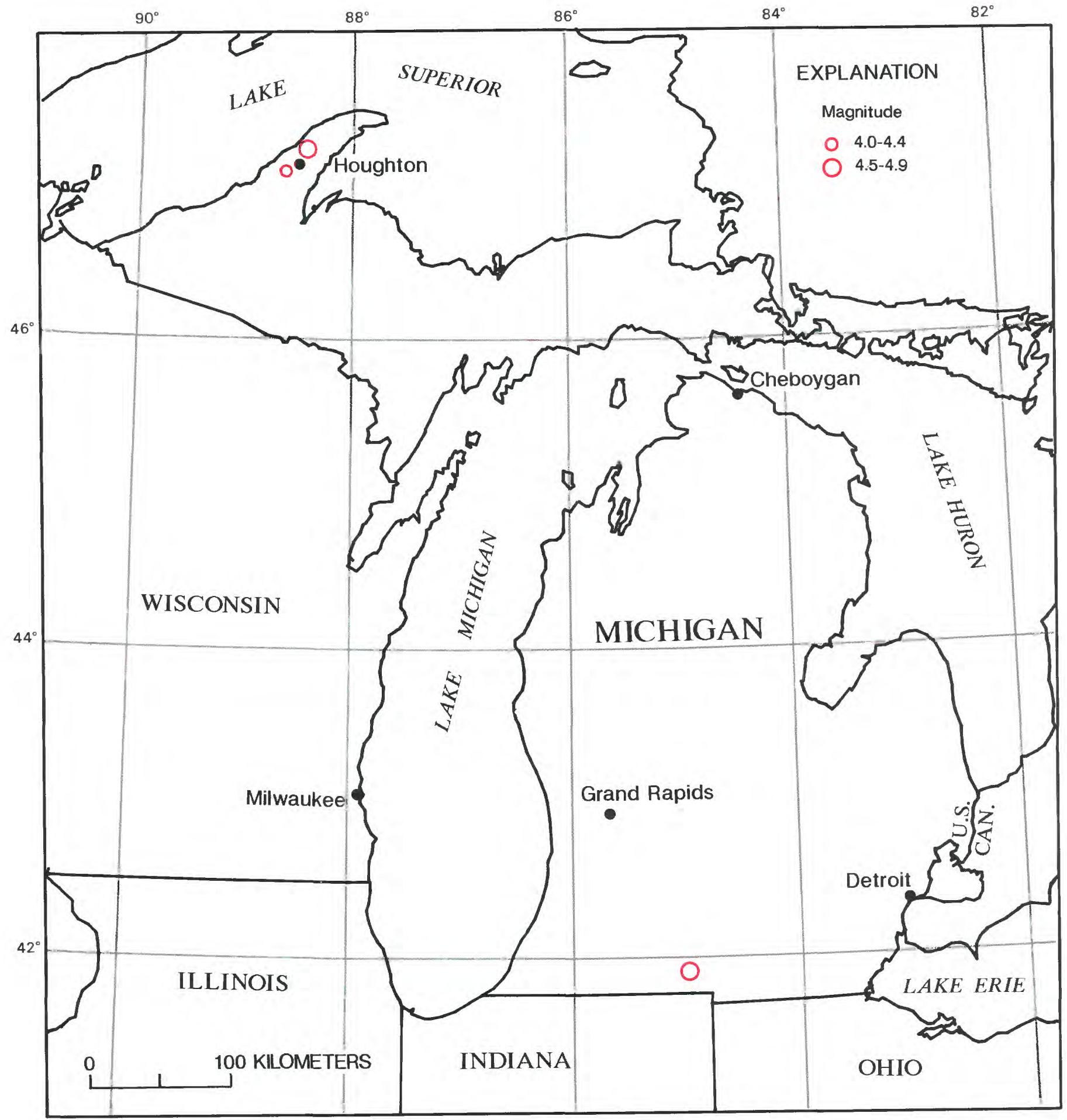

Earthquakes in Michigan with magnitudes $\geq 4.5$ or intensity $\geq$ VI. 


\section{MICHIGAN}

[See table 1 for hypocenter and intensity references and table 2 for definitions of magnitude source codes. Leader (--) indicates information is not available]

\begin{tabular}{|c|c|c|c|c|c|c|c|c|c|c|c|c|c|}
\hline \multirow{2}{*}{\multicolumn{2}{|c|}{ Date }} & \multirow{2}{*}{$\begin{array}{c}\text { Origin } \\
\text { time (UTC) }\end{array}$} & \multicolumn{4}{|c|}{ Hypocenter } & \multicolumn{4}{|c|}{ Magnitude } & \multicolumn{2}{|c|}{ Intensity } & \multirow{3}{*}{$\begin{array}{l}\text { Felt area } \\
\left(1,000 \mathrm{~km}^{2}\right)\end{array}$} \\
\hline & & & \multirow{2}{*}{$\begin{array}{l}\text { Latitude } \\
\left({ }^{\circ}\right)\end{array}$} & \multirow{2}{*}{$\begin{array}{c}\text { Longitude } \\
\left({ }^{\circ}\right)\end{array}$} & \multirow{2}{*}{$\begin{array}{l}\text { Depth } \\
(\mathrm{km})\end{array}$} & \multirow[t]{2}{*}{ Ref } & \multicolumn{2}{|c|}{ USGS } & \multirow[t]{2}{*}{ Other } & \multirow{2}{*}{$\begin{array}{c}\text { Moment } \\
\text { M }\end{array}$} & \multirow[t]{2}{*}{ MMI } & \multirow[t]{2}{*}{ Ref } & \\
\hline Yr & Mo Da & $\mathrm{h} \mathrm{m} \mathrm{s}$ & & & & & $m_{b}$ & $M_{s}$ & & & & & \\
\hline 1905 & $07 \quad 27$ & 0020 & $47.24 \mathrm{~N}$ & $88.45 \mathrm{~W}$ & - & 326 & - & - & $4.50 \mathrm{M}_{\mathrm{fa}} \mathrm{SC}$ & - & VII & 38 & 40 \\
\hline 1906 & 0526 & 1442 & $47.10 \mathrm{~N}$ & $88.64 \mathrm{~W}$ & - & 336 & - & - & $4.20 \mathrm{M}_{\mathrm{fa}} \mathrm{SC}$ & - & VIII & 38 & 2 \\
\hline 1947 & $\begin{array}{ll}08 & 10\end{array}$ & 024641.3 & $41.928 \mathrm{~N}$ & $85.004 \mathrm{~W}$ & 002 & 349 & - & - & $4.60 \mathrm{M}_{\mathrm{n}}$ BAS & - & VI & 20 & 158 \\
\hline
\end{tabular}

[Reference (Ref.) numbers given in parentheses at the end of each description refer to sources of data in table 1. Magnitude values are described in the Introduction, and codes are defined in table 2.]

1905. July 27 (July 26). Calumet, Houghton County, Mich. This earthquake was associated with unstable geological conditions induced by mining operations in the area. The shock downed many chimneys and broke plate glass windows at Calumet, in northwest Michigan. Accompanied by a terrific explosion, the shock was felt throughout the Keweenaw Peninsula in Michigan and as far east as Marquette. Magnitude 4.5 $\mathrm{M}_{\mathrm{fa}}$ BAR. (Ref. 38, $326,353$.
1906. May 26. Houghton, Mich. At the Atlantic mine, near Houghton (about $20 \mathrm{~km}$ southwest of Calumet), rails were twisted and a notable sinking of the earth was observed above the workings of the mine. These effects were not observed elsewhere. About 50 shocks were reported. Magnitude $3.6 \mathrm{M}_{\mathrm{fa}}$ BAR. (Ref. 38, 336, 353.)

1947. Aug. 10 (Aug. 9). Southern Michigan. Damage was heaviest in the area southeast of Kalamazoo at Athens, Bronson, Coldwater, Colon, Matteson Lake, Sherwood, and Union City. Chimneys were damaged, windows and plaster were broken, and brick cornices were downed. Also felt in Indiana, Illinois, Ohio, Wisconsin, and Ontario, Canada. (see fig. 33). Magnitude $4.7 \mathrm{M}_{\mathrm{fa}}$ BAR. (Ref. 20, 349, 353.) 


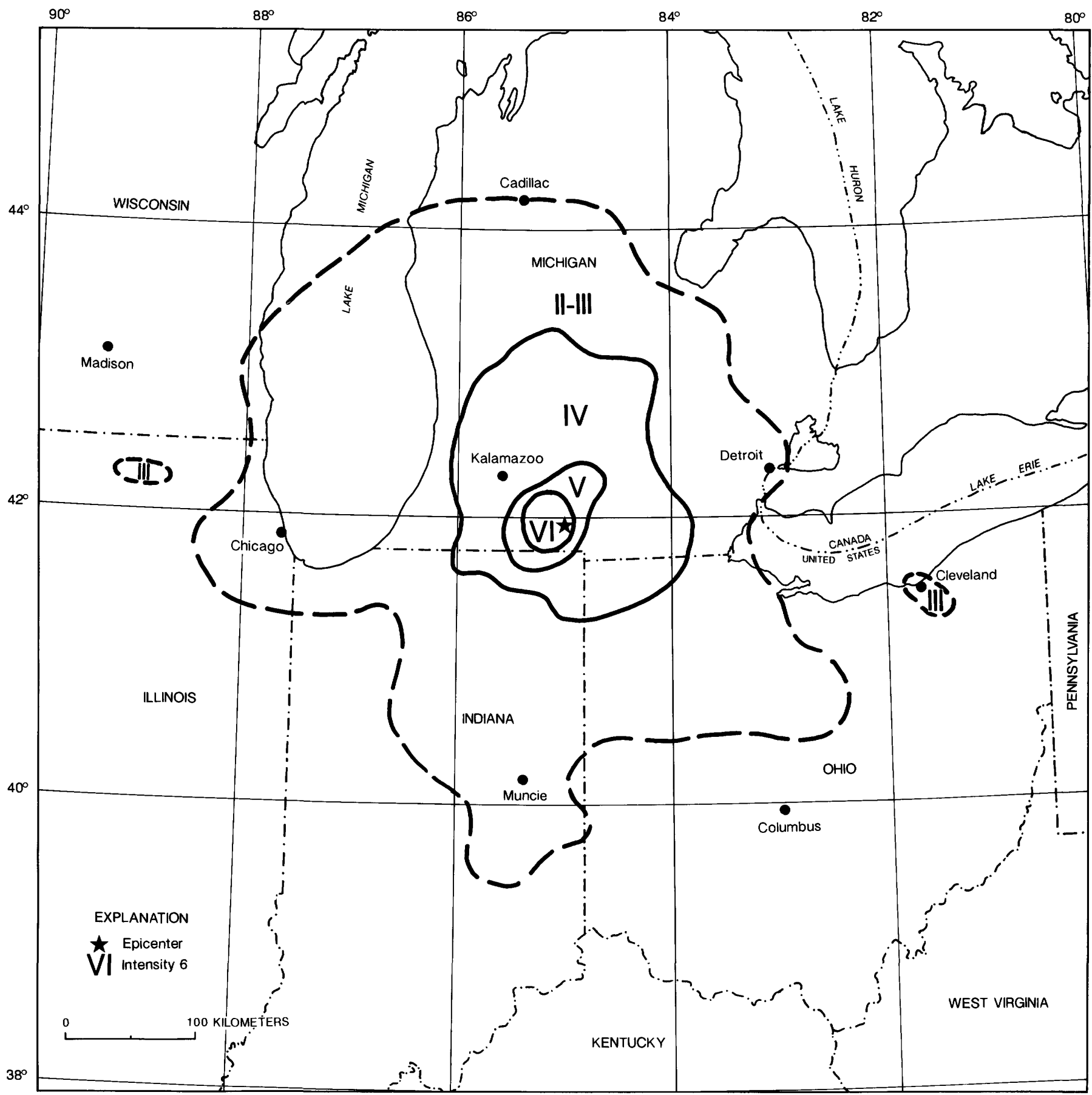

FiguRE 33.-Isoseismal map for the southern Michigan earthquake of August 10, 1947. Isoseismals are based on intensity estimates from data listed in reference 20 of table 1 . 


\section{MINNESOTA}

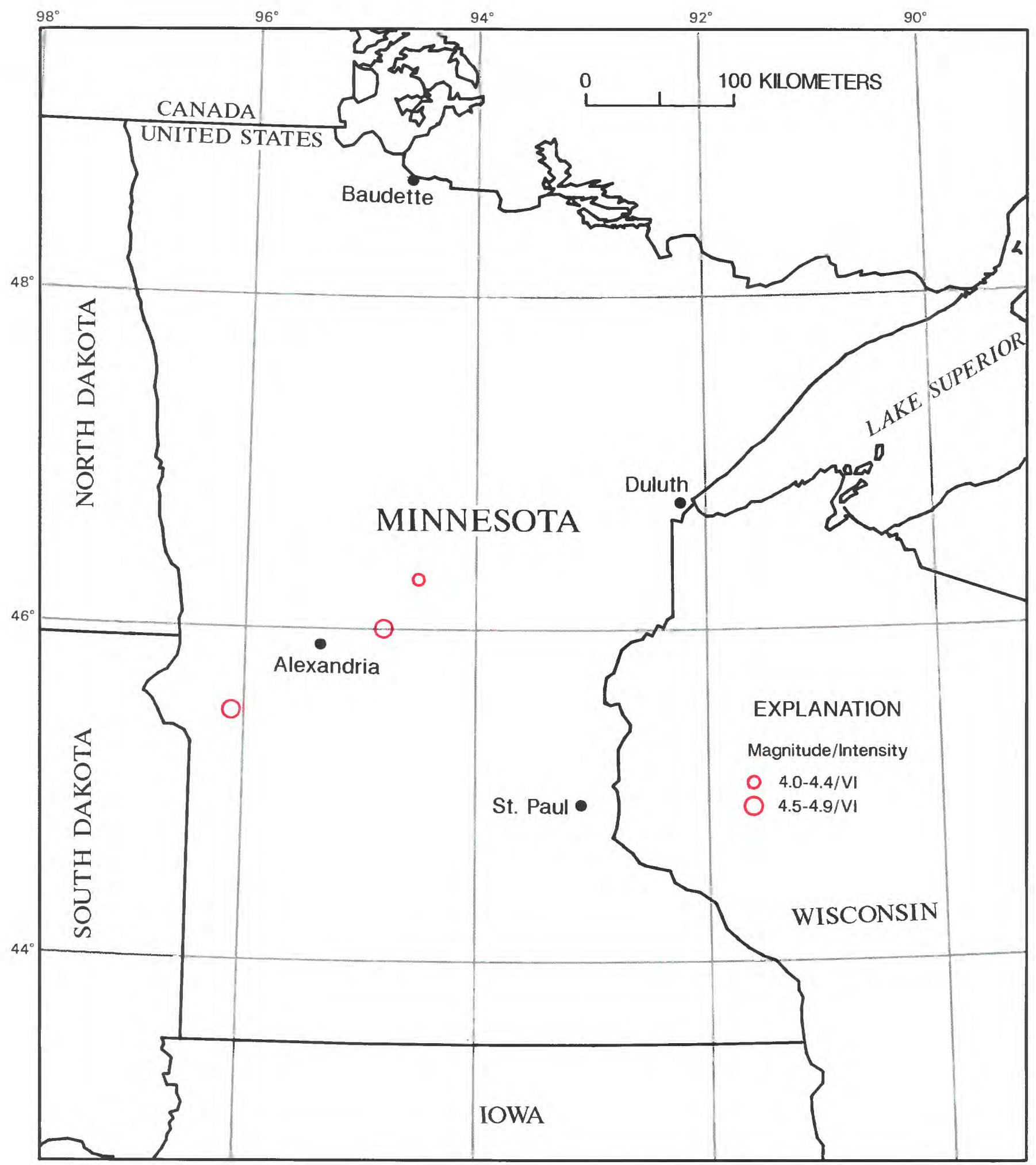

Earthquakes in Minnesota with magnitudes $\geq 4.5$ or intensity $\geq$ VI. 


\section{MINNESOTA}

[See table 1 for hypocenter and intensity references and table 2 for definitions of magnitude source codes. Leader (--) indicates information is not available]

\begin{tabular}{|c|c|c|c|c|c|c|c|c|c|c|c|c|c|}
\hline \multirow{2}{*}{\multicolumn{2}{|c|}{ Date }} & \multirow{2}{*}{$\begin{array}{c}\text { Origin } \\
\text { time (UTC) }\end{array}$} & \multicolumn{4}{|c|}{ Hypocenter } & \multicolumn{4}{|c|}{ Magnitude } & \multicolumn{2}{|c|}{ Intensity } & \multirow{3}{*}{$\begin{array}{l}\text { Felt area } \\
\left(1,000 \mathrm{~km}^{2}\right)\end{array}$} \\
\hline & & & \multirow{2}{*}{$\begin{array}{l}\text { Latitude } \\
\left({ }^{\circ}\right)\end{array}$} & \multirow{2}{*}{$\begin{array}{l}\text { Longitude } \\
\left({ }^{\circ}\right)\end{array}$} & \multirow{2}{*}{$\begin{array}{l}\text { Depth } \\
\text { (km) }\end{array}$} & \multirow[t]{2}{*}{ Ref } & \multicolumn{2}{|c|}{ USGS } & \multirow[t]{2}{*}{ Other } & \multirow{2}{*}{$\begin{array}{c}\text { Moment } \\
\mathbf{M}\end{array}$} & \multirow[t]{2}{*}{ MMI } & \multirow[t]{2}{*}{ Ref } & \\
\hline $\mathrm{Yr}_{\mathrm{r}}$ & Mo Da & $\mathrm{h} \mathrm{m} s$ & & & & & $m_{b}$ & $M_{s}$ & & & & & \\
\hline 1860 & & & $46.0 \mathrm{~N}$ & $94.8 \mathrm{~W}$ & - & 105 & - & - & - & - & VI & 272 & - \\
\hline 1917 & $\begin{array}{ll}09 & 03\end{array}$ & 2130 & $46.3 \mathrm{~N}$ & $94.5 \mathrm{~W}$ & - & 38 & - & - & $4.30 \mathrm{M}_{\mathrm{fa}} \mathrm{SC}$ & - & VI & 38 & 48 \\
\hline 1975 & 0709 & 145421.3 & $45.498 \mathrm{~N}$ & $96.100 \mathrm{~W}$ & 008 & 349 & 5.0 & - & $4.60 \mathrm{M}_{\mathrm{n}} \mathrm{DG}$ & $4.29 \mathrm{HRN}$ & VI & 48 & 75 \\
\hline
\end{tabular}

[Reference (Ref.) numbers given in parentheses at the end of each description refer to sources of data in table 1. Magnitude values are described in the Introduction, and codes are defined in table 2.

1860. Date unknown. Long Prairie, Todd County, Minn. Little is known about the effects of this earthquake. MM intensity VI is assigned to the shock because it is described as "a harder shock" than that on Sept. 3, 1917. Ref. 272 describes an earthquake on a Sunday afternoon between the years 1865 and 1870 . The above reports probably refer to an earthquake between 1860 and 1870. (Ref. 105, 272.)

1917. Sept. 3. Staples, Todd County, Minn. The earthquake was most severe at Staples, a railway village on the north edge of Todd County, where chimneys toppled and many windows were broken. The shock also cracked the wall of a brick building, the concrete floor in the city clerk's office, and concrete sidewalks. Several courses of bricks were dislodged from a chimney at Brainerd, east of Staples, and a chimney was thrown down near Lincoln, south of Staples. Also observed in Minneapolis, $190 \mathrm{~km}$ southeast of Staples. (Ref. 38, 105, $272,491$.

1975. July 9. Western Minnesota. The earthquake caused minor damage to walls and foundations of basements in Stevens County around Morris. Also felt in Iowa, North Dakota, and South Dakota. Magnitude 4.8 $\mathrm{M}_{\mathrm{n}}$ SLM. (Ref. 38, 48, 349.) 


\section{MISSISSIPPI}

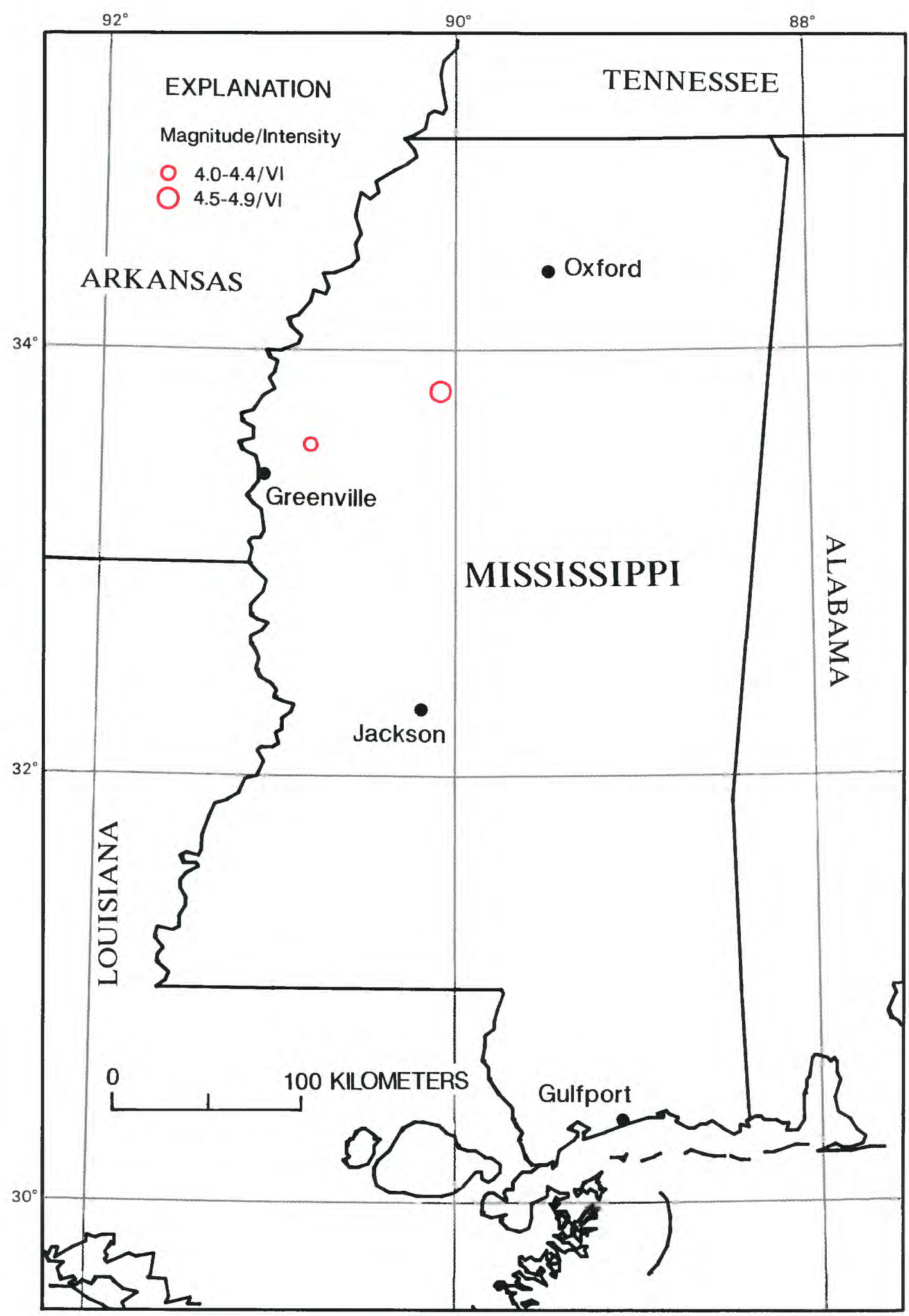

Earthquakes in Mississippi with magnitudes $\geq 4.5$ or intensity $\geq$ VI. 
MISSISSIPPI

[See table 1 for hypocenter and intensity references and table 2 for definitions of magnitude source codes. Leader (--) indicates information is not available]

\begin{tabular}{|c|c|c|c|c|c|c|c|c|c|c|c|c|c|}
\hline \multirow{2}{*}{\multicolumn{2}{|c|}{ Date }} & \multirow{2}{*}{$\begin{array}{c}\text { Origin } \\
\text { time (UTC) }\end{array}$} & \multicolumn{4}{|c|}{ Hypocenter } & \multicolumn{4}{|c|}{ Magnitude } & \multicolumn{2}{|c|}{ Intensity } & \multirow{3}{*}{$\begin{array}{l}\text { Felt area } \\
\left(1,000 \mathrm{~km}^{2}\right)\end{array}$} \\
\hline & & & \multirow{2}{*}{$\begin{array}{l}\text { Latitude } \\
\left({ }^{\circ}\right)\end{array}$} & \multirow{2}{*}{$\begin{array}{l}\text { Longitude } \\
\left({ }^{\circ}\right)\end{array}$} & \multirow{2}{*}{$\begin{array}{c}\text { Depth } \\
(\mathrm{km})\end{array}$} & \multirow[t]{2}{*}{ Ref } & \multicolumn{2}{|c|}{ USGS } & \multirow[t]{2}{*}{ Other } & \multirow{2}{*}{$\begin{array}{c}\text { Moment } \\
\mathbf{M}\end{array}$} & \multirow[t]{2}{*}{ MMI } & \multirow[t]{2}{*}{ Ref } & \\
\hline $\mathrm{Yr}_{\mathrm{r}}$ & Mo Da & $\mathrm{h} \mathrm{m} \mathrm{s}$ & & & & & $m_{b}$ & $\mathbf{M}_{\mathbf{S}}$ & & & & & \\
\hline 1931 & $\begin{array}{ll}12 & 17\end{array}$ & 0336 & $33.8 \mathrm{~N}$ & $90.1 \mathrm{~W}$ & $\overline{0}$ & $\begin{array}{r}4 \\
340\end{array}$ & $\overline{38}$ & - & $4.60 \mathrm{M}_{\mathrm{fa}} \mathrm{SC}$ & - & VI & 4 & 175 \\
\hline 1967 & 0604 & 161412.6 & & 90.836W & 006 & 349 & 3.8 & - & $4.40 M_{n}$ DG & $4.28 \mathrm{HRN}$ & VI & 40 & 54 \\
\hline
\end{tabular}

[Reference (Ref.) numbers given in parentheses at the end of each description refer to sources of data in table 1. Magnitude values are described in the Introduction, and codes are defined in table 2.]

1931. Dec. 17 (Dec. 16). Charleston, Tallahatchie County, Miss. Several chimneys were thrown down at Charleston, and the walls and foundation of the Agricultural High School were cracked. Minor damage to chimneys also occurred at Tillatoba and Water Valley; several buildings were damaged slightly and plaster fell at Belzoni. Also felt in Alabama, Arkansas, and Tennessee. (Ref. 4, 105.)

1967. June 4. Near Greenville, Washington County, Miss. A few instances of cracked plaster were reported at Greenville. One person near the epicenter observed a crack in his lawn about $0.5-1.3 \mathrm{~cm}$ wide and $12 \mathrm{~m}$ long. Also felt in Arkansas, Louisiana, and Tennessee. A slight aftershock was observed in the Greenville area on June 29. Magnitude $4.5 \mathrm{M}_{\mathrm{n}}$ BAR, 3.0 $\mathrm{M}_{\mathrm{S}}$ NUT, $4.5 \mathrm{~m}_{\mathrm{b}}$ NUT.. (Ref. $40,263,349$. ) 


\section{MISSOURI}

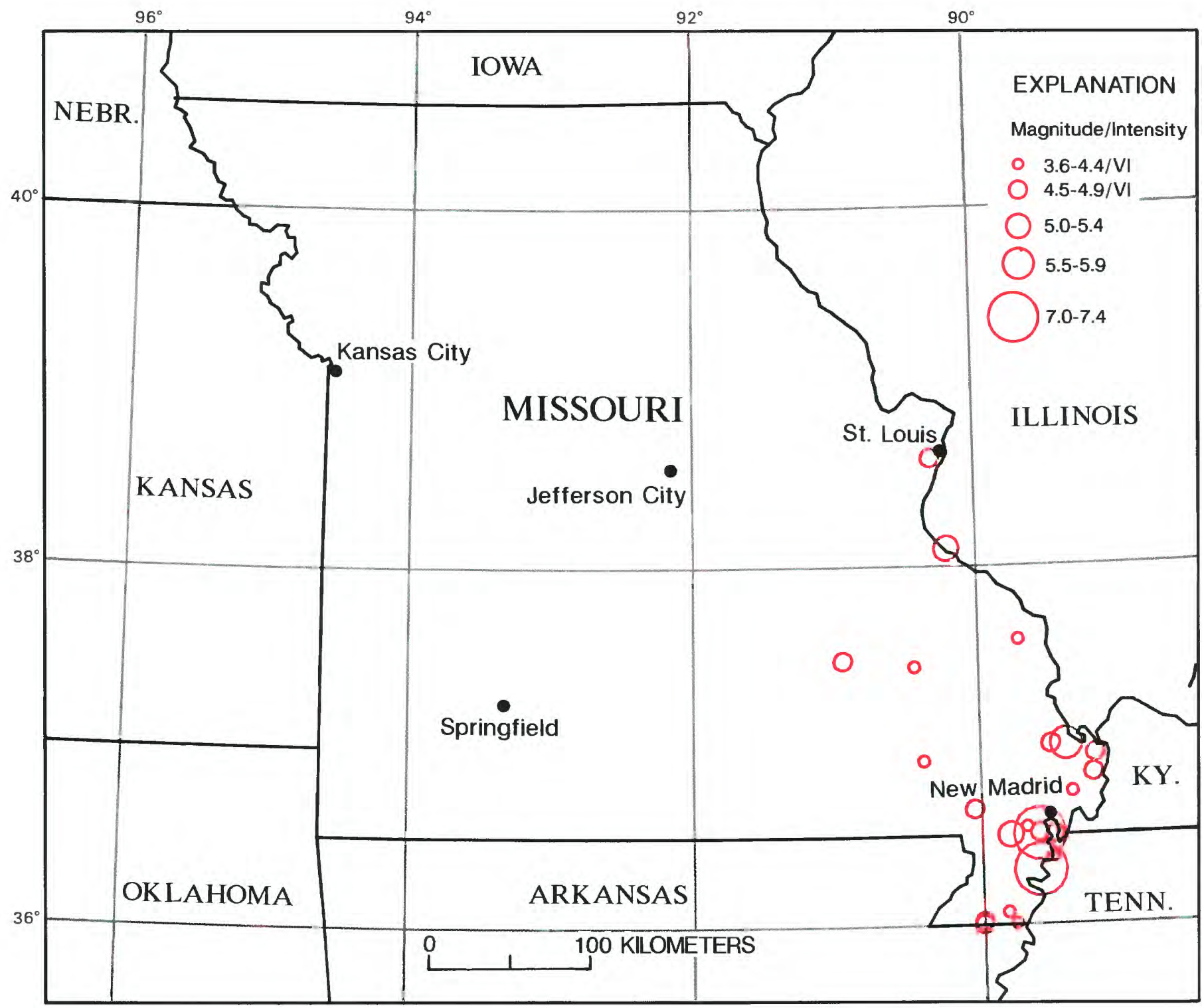

Earthquakes in Missouri with magnitudes $\geq 4.5$ or intensity $\geq$ VI. 
MISSOURI

[See table 1 for hypocenter and intensity references and table 2 for definitions of magnitude source codes. Leader (-) indicates information is not available]

\begin{tabular}{|c|c|c|c|c|c|c|c|c|c|c|c|c|c|}
\hline \multirow{2}{*}{\multicolumn{2}{|c|}{ Date }} & \multirow{2}{*}{$\begin{array}{c}\text { Origin } \\
\text { time (UTC) }\end{array}$} & \multicolumn{4}{|c|}{ Hypocenter } & \multicolumn{4}{|c|}{ Magnitude } & \multicolumn{2}{|c|}{ Intensity } & \multirow{3}{*}{$\begin{array}{c}\text { Felt area } \\
\left(1,000 \mathrm{~km}^{2}\right)\end{array}$} \\
\hline & & & \multirow{2}{*}{$\begin{array}{l}\text { Latitude } \\
\left({ }^{\circ}\right)\end{array}$} & \multirow{2}{*}{$\begin{array}{l}\text { Longitude } \\
\text { (') }\end{array}$} & \multirow{2}{*}{$\begin{array}{c}\text { Depth } \\
(\mathrm{km})\end{array}$} & \multirow[t]{2}{*}{ Ref } & \multicolumn{2}{|c|}{ USGS } & \multirow{2}{*}{ Other } & \multirow{2}{*}{$\begin{array}{c}\text { Moment } \\
\text { M }\end{array}$} & \multirow{2}{*}{ MMI } & \multirow{2}{*}{ Ref } & \\
\hline$Y_{r}$ & Mo Da & $\mathrm{h} \mathrm{m} s$ & & & & & $m_{b}$ & $M_{s}$ & & & & & \\
\hline 312 & 0123 & 1500 & $36.3 \mathrm{~N}$ & $89.6 \mathrm{~W}$ & - & 301 & - & - & $7.10 \mathrm{M}_{\mathrm{fa}} \mathrm{NU}$ & $7.55 \mathrm{NLI}$ & XI & 473 & 5000 \\
\hline 1812 & 0207 & 0945 & $36.5 \mathrm{~N}$ & 89.6 W & - & 301 & - & - & $7.40 \mathrm{M}_{\mathrm{fa}} \mathrm{NU}$ & $7.88 \mathrm{NLI}$ & XII & 473 & 5000 \\
\hline 1812 & 1109 & 22 & $36.5 \mathrm{~N}$ & $89.6 \mathrm{~W}$ & - & 143 & - & - & - & - & VI & 143 & - \\
\hline 1895 & 1031 & 1108 & $37.0 \mathrm{~N}$ & 89.4 W & - & 38 & - & - & $5.90 \mathrm{M}_{\mathrm{fa}} \mathrm{SC}$ & - & VIIII & 527 & 2037 \\
\hline 1902 & 0124 & 1048 & $38.6 \mathrm{~N}$ & $90.3 \mathrm{~W}$ & - & 38 & - & - & $4.50 \mathrm{M}_{\mathrm{fa}} \mathrm{SC}$ & - & VI & 38 & 130 \\
\hline 903 & 1104 & 1818 & $36.5 \mathrm{~N}$ & $89.5 \mathrm{~W}$ & - & 529 & - & - & $4.60 \mathrm{M}_{\mathrm{fa}} \mathrm{SC}$ & - & VI & 38 & 168 \\
\hline 1903 & 1104 & 1914 & $36.5 \mathrm{~N}$ & 89.8 W & - & 529 & - & - & $5.10 \mathrm{M}_{\mathrm{fa}} \mathrm{SC}$ & - & VII & 529 & 340 \\
\hline 1909 & 1023 & 0710 & $37.0 \mathrm{~N}$ & $89.5 \mathrm{~W}$ & - & 38 & - & - & $4.50 \mathrm{M}_{\mathrm{fa}} \mathrm{BAR}$ & - & V & 38 & 125 \\
\hline 1915 & 1207 & 1840 & $36.0 \mathrm{~N}$ & $90.0 \mathrm{~W}$ & - & 529 & - & - & $4.50 \mathrm{M}_{\mathrm{fa}} \mathrm{BAR}$ & - & v & 109 & 120 \\
\hline 1924 & 0101 & 0305 & $36.0 \mathrm{~N}$ & $90.0 \mathrm{~W}$ & - & 529 & - & - & $4.50 \mathrm{M}_{\mathrm{fa}} \mathrm{SC}$ & - & VI & 529 & 150 \\
\hline 934 & 0820 & 004727 & $36.95 \mathrm{~N}$ & $89.2 \mathrm{~W}$ & - & 38 & - & - & $4.70 \mathrm{M}_{\mathrm{fs}}$ & - & VII & 38 & 85 \\
\hline 1955 & 0125 & 072439.1 & $36.073 \mathrm{~N}$ & $89.827 \mathrm{~W}$ & 008 & 349 & - & - & $4.40 \mathrm{M}_{\mathrm{fa}} \mathrm{SC}$ & - & $\overline{V I}$ & 28 & 85 \\
\hline 1956 & 1126 & 041243.3 & $36.914 \mathrm{~N}$ & $90.387 \mathrm{~W}$ & 001 & 349 & - & - & $4.30 \mathrm{M}_{\mathrm{fa}} \mathrm{SC}$ & - & VI & 29 & 70 \\
\hline 1962 & 0202 & 064330.0 & $36.374 \mathrm{~N}$ & 89.511W & 004 & 349 & - & - & $4.30 \mathrm{M}_{\mathrm{n}}$ BAR & $4.22 \mathrm{HRN}$ & VI & 35 & 90 \\
\hline 1963 & 0303 & 173010.6 & $36.642 \mathrm{~N}$ & $90.050 \mathrm{~W}$ & 009 & 349 & - & - & $4.80 \mathrm{M}_{\mathrm{n}} \mathrm{DG}$ & 4.64HRN & VI & 38 & 208 \\
\hline 1965 & 1021 & 020439.1 & $37.479 \mathrm{~N}$ & $90.944 \mathrm{~W}$ & 007 & 34 & 5.1 & - & $4.80 M_{n}$ DG & $4.59 \mathrm{~F}$ & VI & 75 & 420 \\
\hline 1967 & 0721 & 091448.8 & $37.440 \mathrm{~N}$ & $90.443 W$ & 012 & 34 & 3.9 & - & n STT & 4.03HRN & VI & 40 & 53 \\
\hline 1974 & 0513 & 065218.7 & $36.739 \mathrm{~N}$ & $89.357 W$ & 004 & 349 & 4.3 & - & $3.60 \mathrm{M}_{\mathrm{fa}} \mathrm{SC}$ & - & VI & 47 & 2 \\
\hline 1975 & 0613 & 224027.5 & $36.543 \mathrm{~N}$ & $89.682 \mathrm{~W}$ & 009 & 34 & 4.3 & - & In DG & 3.73HRN & VI & 48 & 13 \\
\hline 1977 & 0103 & 225648.5 & $37.583 \mathrm{~N}$ & $89.714 \mathrm{~W}$ & 005 & 349 & - & - & $3.60 \mathrm{M}_{\mathrm{n}}$ DG & - & VI & 39 & 6 \\
\hline 1987 & 0929 & 00 & 36. & ow & 005 & & 4.6 & - & & - & V & & 35 \\
\hline 1989 & 0427 & 164749.8 & $36.006 \mathrm{~N}$ & $89.768 \mathrm{~W}$ & 010 & 74 & 4.6 & - & $4.30 M_{n}$ GS & - & VI & 579 & 57 \\
\hline
\end{tabular}

[Reference (Ref.) numbers given in parentheses at the end of each description refer to sources of data in table 1. Magnitude values are described in the Introduction, and codes are defined in table 2.]

1812. Jan. 23. New Madrid, Mo. This is the third principal shock of the 1811-12 sequence. The first earthquake of this series on Dec. 16, 1811, was located in northeast Arkansas. It is difficult to assign intensities to the principal shocks that occurred after 1811 because many of the published accounts describe the cumulative effects of all the earthquakes. Using the Dec. 16 earthquake as a standard, however, a comparison between it and the shock on Jan. 23 indicates that the intensities were about equal at similar locations. The meizoseismal area was characterized by general ground warping, ejections, fissuring, severe landslides, and caving of stream banks. Magnitude $8.4 \mathrm{M}_{\mathrm{Sn}}$ NLI. (Ref. 143, $301,353,473,529$.)

1812. Feb. 7. New Madrid, Mo. This is the fourth and largest earthquake of the 1811-12 series. Several destructive shocks occurred on Feb. 7, the last of which equaled or surpassed the magnitude of any previous event. The town of New Madrid was destroyed. At St. Louis, many houses were damaged severely and their chimneys were thrown down. The meizoseismal area was characterized by general ground warping, ejections, fissuring, severe landslides, and caving of stream banks. Magnitude 8.8 $\mathrm{M}_{\mathrm{Sn}}$ NLI. (Ref. 114, 143, 301, 353, 473, 529.)

1812. Nov. 9. New Madrid, Mo. This earthquake caused "much motion to furniture" at Cape Girardeau, about $75 \mathrm{~km}$ north of New Madrid. (Ref. 143.)

1895. Oct. 31. Near Charleston, Mississippi County, Mo. This is the largest earthquake to occur in the central Mississippi River valley since the 181112 series in the area of New Madrid, Mo. Structural damage and liquefaction phenomena were reported along a line from Bertrand, Mo., in the west to Cairo, Ill., in the east. Many sand blows were observed in an area southwest of Charleston, Mo., and south of Bertrand, Mo. Isolated occurrences of sand blows also were reported north and south of Charleston.

The most severe damage occurred in Charleston, Puxico, and Taylor, Mo.; Alton and Cairo, Ill.; Princeton, Ind.; and Paducah, Ky. The earthquake caused 


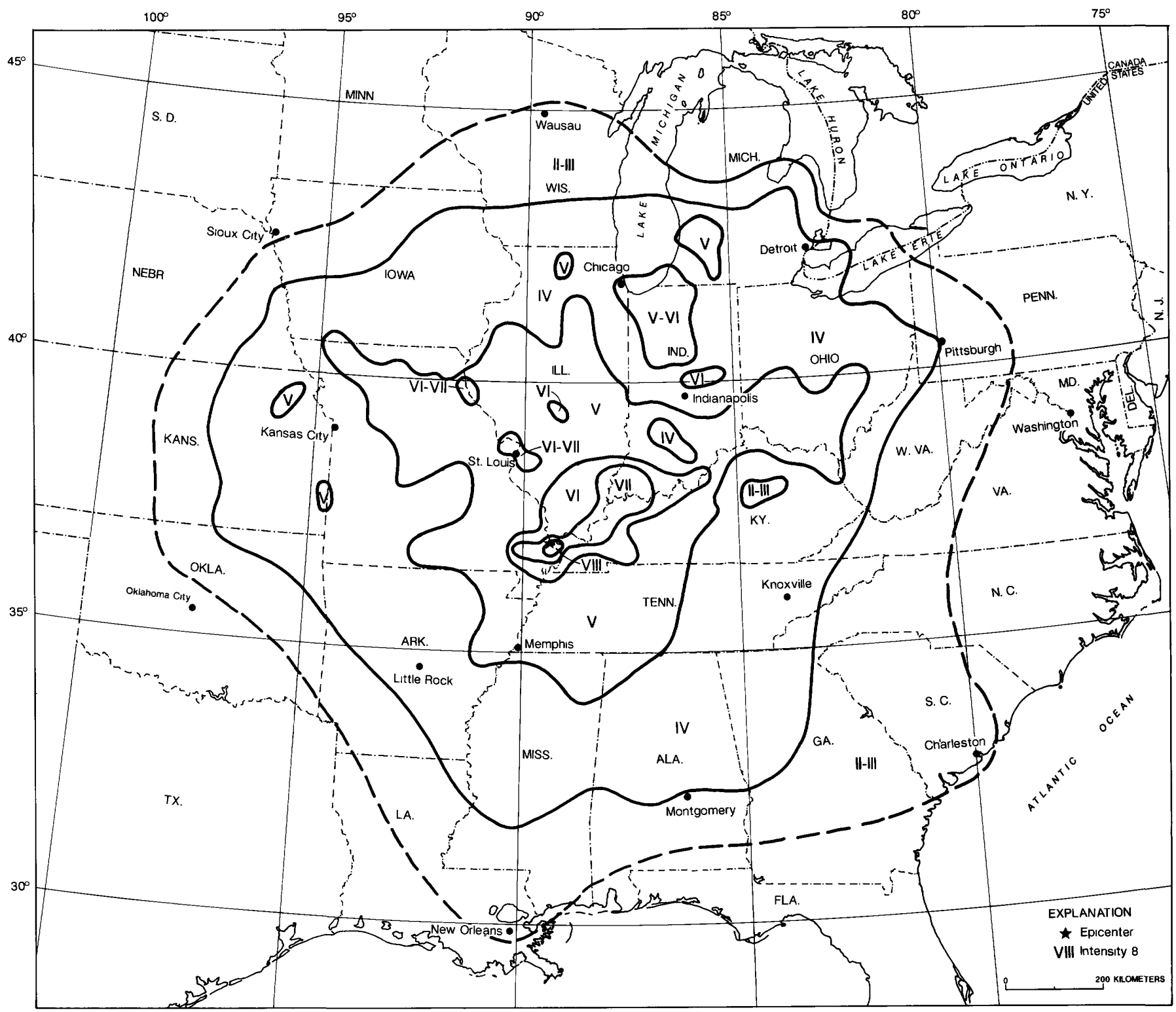

FIGURE 34.-Isoseismal map for the Charleston, Missouri, earthquake of October 31,1895 . Isoseismals are based on intensity estimates from data listed in references 527 and 529 of table 1.

extensive damage (including downed chimneys, cracked walls, shattered windows, and broken plaster) to school buildings, churches, private houses, and to almost all the buildings in the commercial section of Charleston.

East of Charleston, at Cairo, Ill., few buildings escaped at least the loss of a chimney or a broken window. The courthouse, library, and a church at Cairo sustained extensive damage, and the brick walls of many buildings in the downtown section were cracked badly. Other damage included a cracked pier on the Illinois Central Railroad bridge over the Ohio River and downed chimneys at
Gadsden, Ala.; Evansville and New Waverley, Ind.; Covington, Spottsville Depot, and Uniontown, Ky.; St. Louis, Mo.; and Memphis, Tenn. Minor damage to property occurred throughout the region.

Felt from Chatham, Ontario, Canada, to New Orleans, La., and from Washington, D.C., to Wichita, Kans., an area that includes 23 States (see fig. 34). Magnitude 6.7 $\mathrm{M}_{\mathrm{Sn}}$ NTL. (Ref. 38, 109, 113, 353, $366,527,529$.)

1902. Jan. 24. Near St. Louis, Mo. Two distinct earthquakes were felt in western Illinois, Kansas, and Missouri. The shocks were particularly severe in the western part of St. Louis. A street car conductor 
stated that the Eads Bridge swayed perceptibly. Felt from Leavenworth, Kans., and Kansas City, Mo., to Springfield, Ill. (Ref. 38, 105, 109, 353.)

1903. Nov. 4, 1818 and 1914 UTC. Near Charleston, Mississippi County, Mo. Two earthquakes occurred on this date, the second of which was the strongest. The first shock which damaged chimneys at New Madrid, Mo., was felt in Arkansas, Illinois, Kentucky, Mississippi, and Tennessee. During the second shock at 1914 UTC, chimneys were downed at Cape Girardeau and New Madrid, Mo. Minor damage also was reported at Malden, Mo., Oxford, Miss., and Quincy, Ill. This earthquake was felt over an area similar to that of the first shock but also was reported in Indiana. Magnitude $4.9 \mathrm{M}_{\mathrm{fa}}$ BAR (first shock); 4.7 $\mathrm{M}_{\mathrm{fa}} \mathrm{BAR}$ (second shock). (Ref. $38,105,109,353,529$.)

1924. Jan. 1 (1923, Dec. 31). Missouri. A few plate-glass windows were broken at Osceola (Mississippi County), Ark., and a few windowpanes were broken at Little Rock, Ark. Also felt in Illinois, Kentucky, Missouri, and Tennessee. Magnitude $4.6 \mathrm{M}_{\mathrm{fa}}$ BAR. (Ref. 529.)

1955. Jan. 25. Mississippi River valley, near Finley, Tenn. Windows were shattered and plaster walls and ceilings were cracked in southeast Missouri and western Tennessee (in Dyer County at Finley). Windows cracked northwest of Finley at Hayti, Mo. Felt from Lepanto, Ark., north to Paducah, Ky., and east to Birmingham, Ala. Also felt in southern Illinois and northern Mississippi. Magnitude $4.5 \mathrm{M}_{\mathrm{fa}}$ BAR. (Ref. 28, 349, 353.)

1956. Nov. 26 (Nov. 25). Wayne County, Mo. Minor damage in the form of shattered windows and cracked walls was reported at Grubville, Richmond Heights (suburb of St. Louis), and St. Louis; a concrete porch was cracked at Sturdivant. The felt area includes parts of Arkansas, Illinois, Kentucky, Missouri, and Tennessee. Magnitude 4.3 $\mathrm{M}_{\mathrm{fa}}$ BAR. (Ref. $29,38,349,353$.

1962. Feb. 2. New Madrid, Mo., near Catron and Marston. At Catron, two water pipes broke and walls and plaster cracked; at Marston, chimneys were damaged and windows cracked. Also felt in parts of Arkansas, Illinois, Kentucky, and Tennessee. Magnitude 3.5 $\mathrm{M}_{\mathrm{S}}$ NUT, $4.3 \mathrm{~m}_{\mathrm{b}}$ NUT. (Ref. 35, 38, 263, 349, 353.)

1963. Mar. 3. Near Poplar Bluff, Butler County, Mo. Minor damage in the form of fallen and cracked plaster; cracks in foundations, walls, sidewalks, chimneys, and windows; fallen bricks from chimneys; and damaged water lines occurred in many towns in southeast Missouri. In addition, cracks in plaster and walls occurred in several towns in Arkansas, Illinois, Kentucky, and Tennessee. Also reported felt in parts of Indiana, Kansas, and Mississippi. (see fig. 35). Magnitude 4.7 $\mathrm{M}_{\mathrm{n}} \mathrm{BAR}, 4.1 \mathrm{M}_{\mathrm{S}}$ BAR, $4.8 \mathrm{mb}_{\mathrm{b}} \mathrm{NUT}, 4.1 \mathrm{M}_{\mathrm{S}} \mathrm{NUT}, 4.66 \mathrm{M} \mathrm{JOH}$. (Ref. $38,263,349,353$.

1965. Oct. 21 (Oct. 20). Eastern Missouri. Plaster was knocked down at St. Louis, northeast of the epicenter. Minor damage also occurred at Augusta (cistern cracked), Illmo (basement floor cracked), Pacific (sewer vent line cracked), and Reynolds (bricks fell from flue). Minor cracks in plaster, walls, and windows were reported from several towns in Illinois, Iowa, and Kansas. Also felt in Kentucky, Nebraska, Oklahoma, and Tennessee. Magnitude 4.9 $\mathrm{M}_{\mathrm{b}}$ NUT, $4.1 \mathrm{M}_{\mathrm{S}}$ NUT. (Ref. 75, 263, 349, 353.)

1967. July 21. Near Poplar Bluff, Butler County, Mo. Plaster fell at Poplar Bluff, and plaster cracked north of Poplar Bluff at Elvins and Fredericktown. Felt mainly in southeast Missouri and southern Illinoi s. Magnitude $4.3 \mathrm{M}_{\mathrm{n}}$ BAR, $2.8 \mathrm{M}_{\mathrm{S}}$ BAR. (Ref. 40, 349, 353.)

1974. May 13. New Madrid, Mo., region. The City swimming pool at East Prairie, northeast of New Madrid, was "badly damaged," and plaster was cracked in several buildings. Also felt in Arkansas, Illinois, Kentucky, and Tennessee. Magnitude $4.1 \mathrm{M}_{\mathrm{n}}$ SLM. (Ref. 47, 349.)

1975. June 13. New Madrid, Mo., region. Damage was slight at Lilbourn, southwest of New Madrid, where plaster cracked and fell. Furniture overturned and broke at nearby Marston. Also felt in Arkansas, Kentucky, and Tennessee. Magnitude 4.3 $M_{n}$ SLM. (Ref. 48, 349.)

1977. Jan. 3. Cape Girardeau, Mo., region. Plaster cracked and small objects fell at Old Appleton, about $35 \mathrm{~km}$ north of Cape Girardeau. Also felt in Illinois. Many aftershocks were felt on Jan. 3. Magnitude 3.4 $\mathrm{M}_{\mathrm{n}}$ SLM. (Ref. 39, 349.)

1989. Apr. 27. Near Steele, Pemiscot County, Mo. At Steele, near the Arkansas-Missouri-Tennessee border in southeast Missouri, large cracks formed in exterior walls of a brick building and plaster and drywall sustained hairline cracks. Chimneys, plaster, and drywall were cracked at nearby Hayti, and items fell from shelves in stores. Felt in parts of Arkansas, Illinois, Kentucky, Missouri, and Tennessee. Magnitude 4.2 $\mathrm{M}_{\mathrm{n}}$ SLM. (Ref. 74, 579.) 


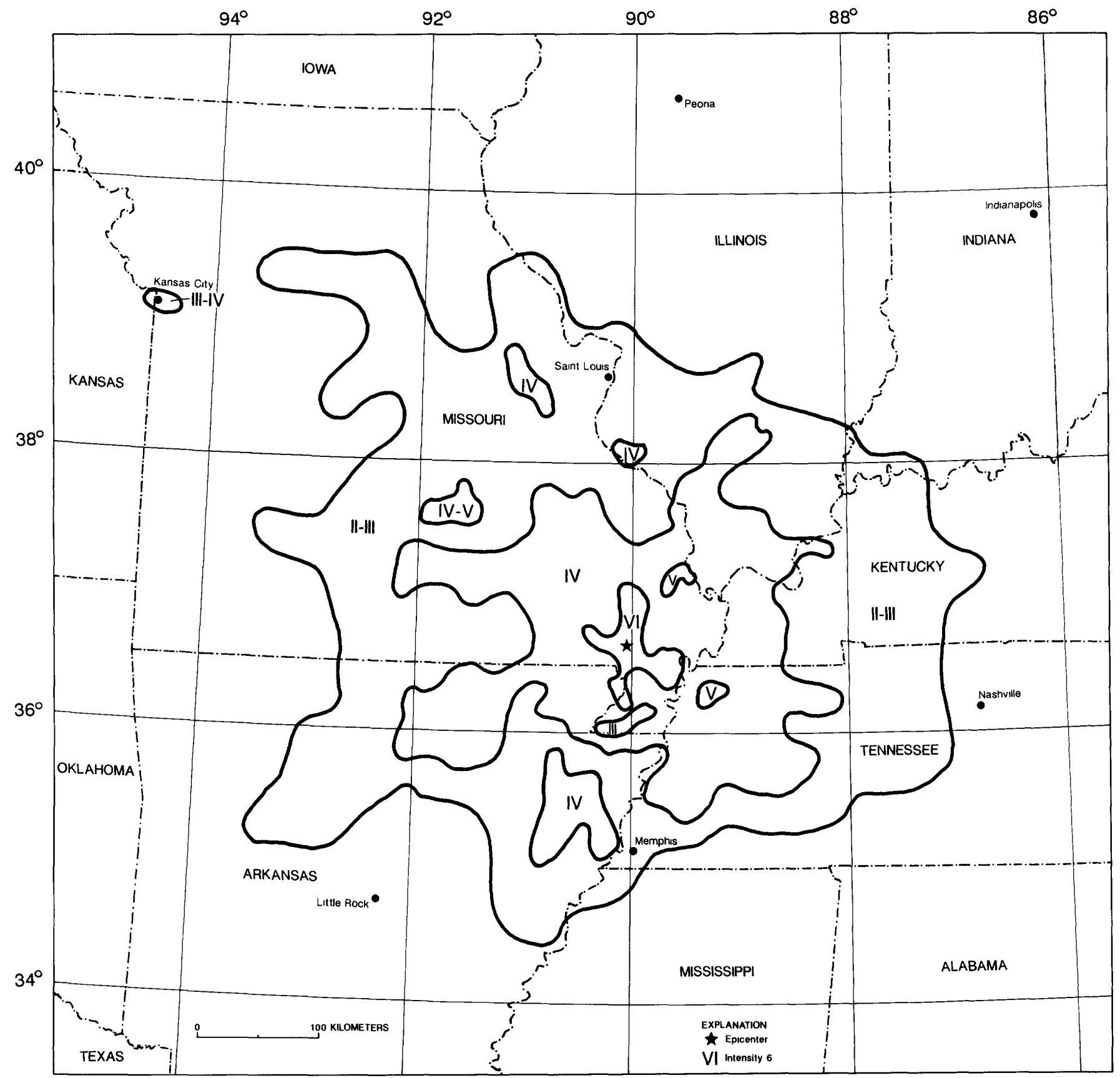

FIGURE 35.-Isoseismal map for the southeast Missouri earthquake of March 3, 1963. Isoseismals are based on intensity esimates from data listed in reference 36 of table 1 . 



\section{MONTANA}

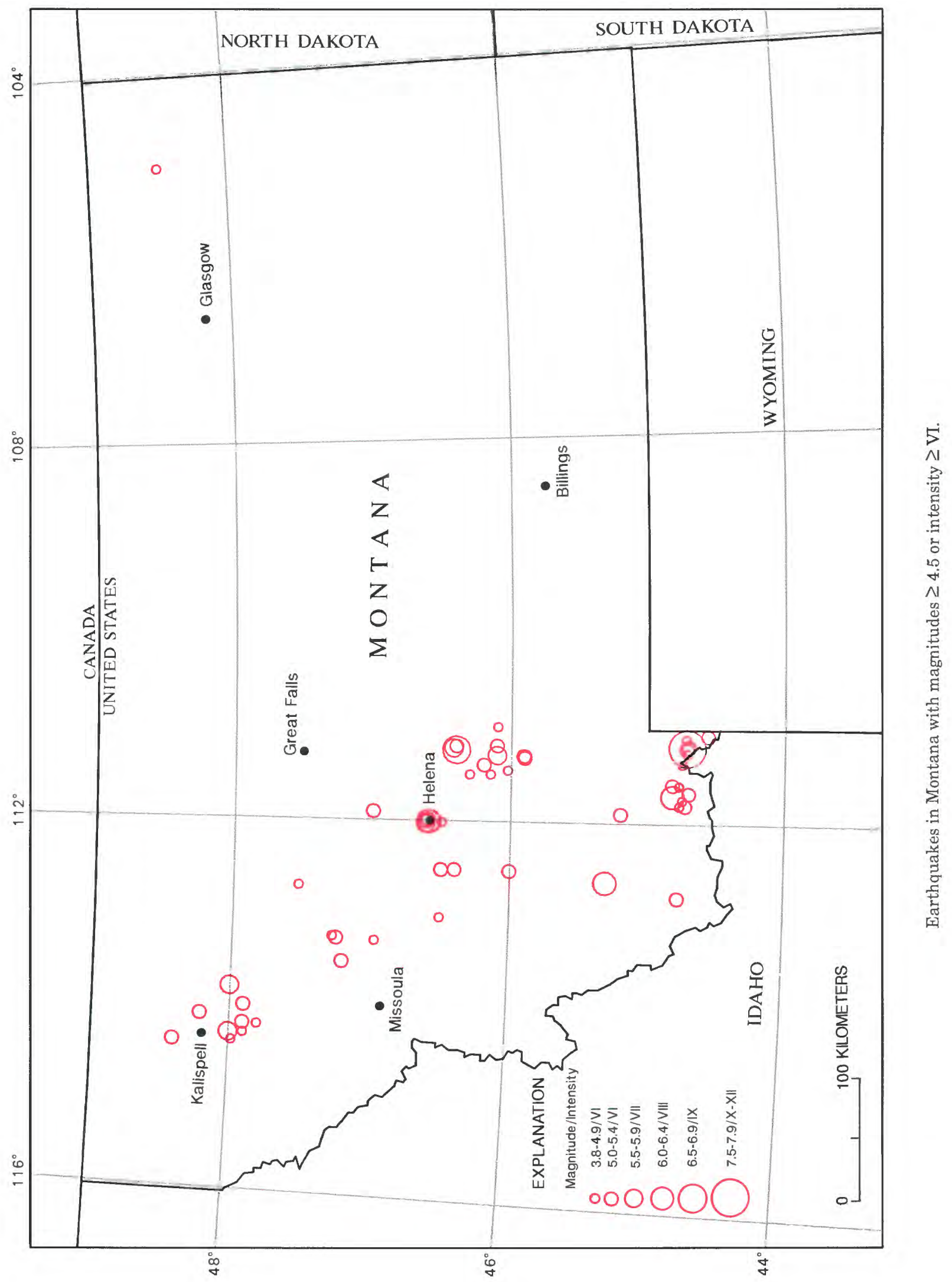


MONTANA

[See table 1 for hypocenter and intensity references and table 2 for definitions of magnitude source codes. @, felt area is less than $1,000 \mathrm{~km}^{2}$. Leader (--) indicates information is not available]

\begin{tabular}{|c|c|c|c|c|c|c|c|c|c|c|c|c|c|c|c|c|}
\hline \multirow{3}{*}{\multicolumn{4}{|c|}{$\begin{array}{cc} & \text { Origin } \\
\text { Date } & \text { time (UTC) } \\
\mathrm{Yr} \text { Mo Da h } \mathrm{m} \mathrm{s}\end{array}$}} & \multicolumn{6}{|c|}{ Hypocenter } & \multicolumn{4}{|c|}{ Magnitude } & \multicolumn{2}{|c|}{ Intensity } & \multirow{3}{*}{$\begin{array}{c}\text { Felt area } \\
\left(1,000 \mathrm{~km}^{2}\right)\end{array}$} \\
\hline & & & & \multirow{2}{*}{\multicolumn{2}{|c|}{$\begin{array}{l}\text { Latitude } \\
\left(^{\circ}\right)\end{array}$}} & \multirow{2}{*}{\multicolumn{2}{|c|}{$\begin{array}{l}\text { Longitude } \\
\left.\text { ( }^{\circ}\right)\end{array}$}} & \multirow{2}{*}{$\begin{array}{c}\text { Depth } \\
(\mathrm{km})\end{array}$} & \multirow[t]{2}{*}{ Ref } & \multicolumn{2}{|c|}{ USGS } & \multirow[t]{2}{*}{ Other } & \multirow{2}{*}{$\begin{array}{c}\text { Moment } \\
\mathbf{M}\end{array}$} & \multirow[t]{2}{*}{ MMI } & \multirow[t]{2}{*}{ Ref } & \\
\hline & & & & & & & & & & $m_{b}$ & $M_{S}$ & & & & & \\
\hline 1872 & 12 & 10 & 2330 & 46.4 & $\mathbf{N}$ & 112.5 & W & - & 38 & - & - & $5.00 \mathrm{M}_{\mathrm{fa}} \mathrm{MMT}$ & - & VI & 38 & 30 \\
\hline 1895 & 09 & 06 & 1920 & 46.0 & $\mathbf{N}$ & 112.5 & W & - & 249 & - & - & $5.00 \mathrm{M}_{\mathrm{fa}} \mathrm{MMT}$ & - & V & 249 & 30 \\
\hline 1897 & 06 & 05 & 1222 & 46.6 & $\mathbf{N}$ & 112.0 & W & - & 249 & - & - & $5.00 \mathrm{M}_{\mathrm{fa}} \mathrm{MMT}$ & - & V & 249 & 30 \\
\hline 1897 & 11 & 04 & 0929 & 45.3 & $\mathbf{N}$ & 112.6 & W & - & 38 & - & - & $6.40 \mathrm{M}_{\mathrm{fa}} \mathrm{MMT}$ & - & VI & 38 & 500 \\
\hline 1903 & 03 & 15 & 1757 & 46.6 & $\mathbf{N}$ & 112.0 & W & - & 249 & - & - & - & - & VI & 249 & - \\
\hline 1908 & 12 & 27 & & 45.2 & $\mathbf{N}$ & 111.9 & W & - & 316 & - & - & - & - & VI & 38 & - \\
\hline 1910 & 04 & 19 & 0830 & 46.0 & $\mathbf{N}$ & 112.5 & W & - & 249 & - & - & 5.40Ukn MMT & - & V & 316 & 70 \\
\hline 1925 & 06 & 28 & 012105 & 46.40 & $\mathbf{N}$ & 111.24 & W & 025 & 208 & - & - & $6.75 \mathrm{M}_{8}$ GR & 6.62DOR & VIII & 38 & 1000 \\
\hline 1925 & 06 & 28 & 020527 & 46.4 & $\mathbf{N}$ & 111.2 & W & - & 218 & - & - & - & - & VII & 312 & 518 \\
\hline 1925 & 06 & 28 & 0420 & 46.4 & $\mathbf{N}$ & 111.2 & W & - & 312 & - & - & - & - & VI & 312 & 130 \\
\hline 1925 & 07 & 10 & 1442 & 46.4 & $\mathbf{N}$ & 111.2 & W & - & 312 & - & - & - & - & V & 218 & 130 \\
\hline 1926 & 12 & 13 & 0044 & 46.1 & $\mathbf{N}$ & 111.2 & W & - & 38 & - & - & $5.40 \mathrm{M}_{\mathrm{fa}} \mathrm{MMT}$ & - & V & 38 & 78 \\
\hline 1928 & 02 & 29 & 2238 & 46.5 & $\mathbf{N}$ & 112.0 & W & - & 249 & - & - & $4.90 \mathrm{M}_{\mathrm{X}}$ REN & - & IV & 1 & - \\
\hline 1929 & 02 & 16 & 0300 & 46.1 & $\mathbf{N}$ & 111.3 & W & - & 38 & - & - & $5.60 \mathrm{M}_{\mathrm{fa}} \mathrm{MMT}$ & - & V & 38 & 161 \\
\hline 1935 & 10 & 07 & 1930 & 47.0 & $\mathbf{N}$ & 111.9 & W & - & 249 & - & - & - & - & VI & 38 & - \\
\hline 1935 & 10 & 12 & 075039 & 46.6 & $\mathbf{N}$ & 112.0 & W & 005 & 8 & - & - & $5.90 \mathrm{M}_{\mathrm{fa}} \mathrm{MMT}$ & 一 & VII & 38 & 181 \\
\hline 1935 & 10 & 15 & 2030 & 46.6 & $\mathbf{N}$ & 112.0 & W & - & 8 & - & - & - & - & VI & 249 & - \\
\hline 1935 & 10 & 19 & 044802 & 46.6 & $\mathbf{N}$ & 112.0 & W & - & 8 & - & - & $6.25 \mathrm{M}_{\mathrm{s}} \mathrm{GR}$ & - & VIII & 38 & 570 \\
\hline 1935 & 10 & 27 & 1921 & 46.6 & $\mathbf{N}$ & 112.0 & W & - & 316 & - & - & - & - & VI & 38 & - \\
\hline 1935 & 10 & 31 & 183747 & 46.6 & $\mathbf{N}$ & 112.0 & W & - & 8 & - & - & $6.00 \mathrm{M}_{\mathrm{S}} \mathrm{GR}$ & - & VIII & 38 & 315 \\
\hline 1935 & 11 & 04 & 1123 & 46.6 & $\mathbf{N}$ & 112.0 & W & - & 8 & - & - & - & - & VI & 249 & 一 \\
\hline 1935 & 11 & 22 & 0358 & 46.6 & $\mathbf{N}$ & 112.0 & W & - & 38 & - & - & $3.80 \mathrm{M}_{\mathrm{L}} \mathrm{KJ}$ & - & VI & 38 & 34 \\
\hline 1935 & 11 & 28 & 144148 & 46.6 & $\mathbf{N}$ & 112.0 & W & - & 8 & - & - & $5.00 \mathrm{M}_{\mathrm{L}} \mathrm{KJ}$ & - & VI & 38 & 233 \\
\hline 1936 & 02 & 14 & 0030 & 46.6 & $\mathbf{N}$ & 112.0 & W & - & 38 & - & - & - & - & VI & 259 & - \\
\hline 1938 & 06 & 13 & 1130 & 45.9 & $\mathbf{N}$ & 111.3 & W & - & 11 & - & - & - & 一 & VI & 249 & - \\
\hline 1938 & 06 & 14 & 0532 & 45.9 & $\mathbf{N}$ & 111.3 & W & - & 11 & - & - & - & - & VI & 249 & - \\
\hline 1939 & 05 & 12 & 0000 & 45.9 & $\mathbf{N}$ & 111.3 & W & - & 12 & - & - & - & 一 & VI & 12 & - \\
\hline 1940 & 12 & 23 & 215029 & 46.5 & $\mathbf{N}$ & 112.5 & W & - & 266 & - & - & - & - & VI & 38 & 18 \\
\hline 1943 & 06 & 25 & 042500 & 48.5 & $\mathbf{N}$ & 105.0 & W & - & 330 & - & - & $4.00 \mathrm{M}_{\mathrm{n}}$ EPB & 一 & VI & 38 & 3 \\
\hline 1945 & 09 & 23 & 095748 & 48.0 & $\mathbf{N}$ & 114.3 & W & - & 266 & - & - & $5.50 \mathrm{M}_{\mathrm{fa}} \mathrm{MMT}$ & - & VI & 18 & 95 \\
\hline 1946 & 12 & 11 & 1309 & 48.4 & $\mathbf{N}$ & 114.4 & W & - & 249 & - & - & - & - & VI & 19 & 8 \\
\hline 1947 & 03 & 14 & 1800 & 47.2 & $\mathbf{N}$ & 113.5 & W & - & 38 & - & - & - & - & VI & 38 & @ \\
\hline 1947 & 11 & 23 & 094603.3 & 44.820 & & 111.713 & $3 W$ & 005 & 576 & - & - & $6.25 \mathrm{M}_{\mathrm{s}} \mathrm{GR}$ & 6.12DOR & VIII & 20 & 340 \\
\hline 1950 & 08 & 20 & 014455 & 47.25 & $N$ & 113.25 & W & - & 266 & - & - & - & - & VI & 23 & 10 \\
\hline 1952 & 04 & 01 & 003741 & 48.0 & $\mathbf{N}$ & 113.8 & W & - & 25 & - & - & $5.50 \mathrm{M}_{\mathrm{fa}} \mathrm{MMT}$ & - & VII & 25 & 77 \\
\hline 1952 & 04 & 22 & 165442.5 & 46.2 & $\mathbf{N}$ & 111.4 & W & - & 25 & - & - & - & - & VI & 25 & 4 \\
\hline 1953 & 08 & 08 & 1650 & 47.9 & $\mathbf{N}$ & 114.0 & W & - & 249 & - & - & - & - & VI & 26 & - \\
\hline 1958 & 05 & 28 & 164554 & 46.5 & $\mathbf{N}$ & 113.0 & W & - & 31 & - & - & $4.40 \mathrm{M}_{\mathrm{L}}$ EPB & - & VI & 31 & 13 \\
\hline 1959 & 08 & 18 & 063713.5 & 44.712 & & 111.215 & & 005 & 576 & - & - & $7.70 \mathrm{M}_{\mathrm{L}}$ BOT & 7.28DSR & $\mathrm{X}$ & 38 & 1175 \\
\hline 1959 & 08 & 19 & 190627.0 & 44.818 & & 111.588 & 8W & 005 & 576 & - & - & 5.00Ukn BRK & - & V & 249 & - \\
\hline 1959 & 08 & 19 & 214557.4 & 44.77 & & 111.606 & $6 \mathrm{~W}$ & 005 & 576 & - & - & 4.70Ukn BRK & - & Felt & 32 & - \\
\hline 1959 & 08 & 20 & 191126.8 & 44.70 & & 111.678 & $8 \mathrm{~W}$ & 005 & 576 & - & - & 5.00Ukn BRK & - & V & 38 & - \\
\hline 1959 & 08 & 23 & 0840 & 44.7 & $\mathbf{N}$ & 111.2 & W & - & 32 & - & - & - & - & VI & 38 & - \\
\hline 1959 & 09 & 05 & 1204 & 44.7 & $\mathbf{N}$ & 111.2 & W & - & 32 & - & - & - & - & VI & 38 & - \\
\hline 1959 & 09 & 13 & 194934.8 & 44.72 & & 111.127 & $7 \mathrm{~W}$ & 005 & 576 & - & - & $4.40 \mathrm{M}_{\mathrm{L}} \mathrm{EPB}$ & 一 & VI & 32 & - \\
\hline 1959 & 11 & 03 & 170308 & 44.7 & $\mathbf{N}$ & 111.2 & W & - & 32 & - & - & - & - & VI & 32 & - \\
\hline 1963 & 02 & 16 & 030141.0 & 46.1 & $\mathbf{N}$ & 111.0 & W & 033 & 266 & 4.5 & - & - & - & $\mathrm{v}$ & 36 & 15 \\
\hline 1964 & 10 & 09 & 022602.4 & 47.8 & $\mathbf{N}$ & 114.2 & W & 033 & 266 & 4.6 & - & - & - & V & 37 & - \\
\hline 1964 & 10 & 14 & 160353.6 & 47.9 & $\mathbf{N}$ & 114.3 & W & 033 & 266 & 4.6 & - & - & - & Felt & 37 & - \\
\hline 1964 & 10 & 21 & 073827.0 & 44.730 & & 111.807 & $7 \mathrm{~W}$ & 005 & 576 & 5.8 & - & $5.00 \mathrm{M}_{\mathrm{s}} \mathrm{NUT}$ & 5.22DOR & V & 37 & 65 \\
\hline
\end{tabular}


MONTANA-Continued

[See table 1 for hypocenter and intensity references and table 2 for definitions of magnitude source codes. @, felt area is less than $1,000 \mathrm{~km}^{2}$. Leader (--) indicates information is not available

\begin{tabular}{|c|c|c|c|c|c|c|c|c|c|c|c|c|c|}
\hline \multirow{2}{*}{\multicolumn{2}{|c|}{ Date }} & \multirow{2}{*}{$\begin{array}{c}\text { Origin } \\
\text { time (UTC) }\end{array}$} & \multicolumn{4}{|c|}{ Hypocenter } & \multicolumn{4}{|c|}{ Magnitude } & \multicolumn{2}{|c|}{ Intensity } & \multirow{3}{*}{$\begin{array}{l}\text { Felt area } \\
\left(1,000 \mathrm{~km}^{2}\right)\end{array}$} \\
\hline & & & \multirow{2}{*}{$\begin{array}{l}\text { Latitude } \\
\text { ( })\end{array}$} & \multirow{2}{*}{$\begin{array}{l}\text { Longitude } \\
\left({ }^{\circ}\right)\end{array}$} & \multirow{2}{*}{$\begin{array}{l}\text { Depth } \\
\text { (km) }\end{array}$} & \multirow{2}{*}{ Ref } & \multicolumn{2}{|c|}{ USGS } & \multirow[t]{2}{*}{ Other } & \multirow{2}{*}{$\begin{array}{c}\text { Moment } \\
\text { M }\end{array}$} & \multirow{2}{*}{ MMI } & \multirow{2}{*}{ Ref } & \\
\hline $\mathrm{Yr}$ & Mo Da & $h \mathrm{~m} s$ & & & & & $m_{b}$ & $M_{s}$ & & & & & \\
\hline 1965 & 0106 & 020120.7 & $44.772 \mathrm{~N}$ & $112.746 \mathrm{~W}$ & 005 & 576 & 5.1 & - & $5.00 \mathrm{M}_{\mathrm{n}} \mathrm{DW}$ & - & VI & 75 & 37 \\
\hline 1965 & 1008 & 193459.8 & $44.713 \mathrm{~N}$ & $111.272 W$ & 005 & 576 & 4.9 & - & $4.70 \mathrm{mb}_{\mathrm{b}}$ ISC & - & v & 75 & - \\
\hline 1966 & 0307 & 180942.6 & $46.3 \mathrm{~N}$ & $111.5 \mathrm{~W}$ & 013 & 266 & 4.8 & - & $4.70 \mathrm{~m}_{\mathrm{b}}$ ISC & - & V & 81 & 35 \\
\hline 1969 & 0401 & 164509.1 & $47.90 \mathrm{~N}$ & $114.30 \mathrm{~W}$ & 010 & 74 & 4.7 & - & $4.30 \mathrm{M}_{\mathrm{L}} \mathrm{GS}$ & - & VII & 42 & 26 \\
\hline 1969 & 0401 & 170518.1 & $47.98 \mathrm{~N}$ & $114.38 \mathrm{~W}$ & 015 & 74 & 4.5 & - & - & - & Felt & 74 & - \\
\hline 1969 & $09 \quad 15$ & 000239.0 & $47.9 \mathrm{~N}$ & $114.2 \mathrm{~W}$ & 019 & 74 & 4.3 & - & $4.10 \mathrm{M}_{\mathrm{L}} \mathrm{GS}$ & - & VI & 42 & 5 \\
\hline 1969 & 1107 & 001129.1 & $47.9 \mathrm{~N}$ & $114.2 \mathrm{~W}$ & 0 & 74 & 4.3 & - & & - & VI & 42 & @ \\
\hline 1972 & 1102 & 034131.3 & $46.15 \mathrm{~N}$ & $111.50 \mathrm{~W}$ & 00 & 7 & 4. & - & $4.50 \mathrm{M}_{\mathrm{L}} \mathrm{GS}$ & - & $\bar{v}$ & 45 & 3 \\
\hline 1974 & 0701 & 182307.3 & $44.56 \mathrm{~N}$ & $111.09 \mathrm{~W}$ & 00 & 47 & 4. & - & $5.10 \mathrm{M}_{\mathrm{L}} \mathrm{GS}$ & - & $\overline{-\pi}$ & - & - \\
\hline 1975 & 0204 & 013252.1 & $48.21 \mathrm{~N}$ & $114.11 \mathrm{~W}$ & 008 & 48 & 4.6 & $=$ & $5.00 \mathrm{M}_{\mathrm{L}}$ GS & $=$ & $\overline{V I}$ & 48 & 50 \\
\hline 1977 & 0311 & 050938.3 & $46.024 \mathrm{~N}$ & $111.457 \mathrm{~W}$ & 005 & 46 & 4.6 & - & $4.80 \mathrm{M}_{\mathrm{L}}$ GS & - & VI & 39 & - \\
\hline 197 & & & 44.77 & $1.81 \mathrm{~W}$ & 010 & 3 & 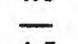 & - & $4.70 \mathrm{M}_{\mathrm{L}} \mathrm{GS}$ & - & VI & & $\overline{0}$ \\
\hline 1978 & 0423 & 232437 & $46.97 \mathrm{~N}$ & $113.27 \mathrm{~W}$ & 005 & 24 & 4.5 & - & $4.90 \mathrm{M}_{\mathrm{L}} \mathrm{GS}$ & - & v & & 94 \\
\hline 1979 & 0508 & 005844.8 & $44.75 \mathrm{~N}$ & $111.38 \mathrm{~W}$ & 005 & 26 & - & - & $4.60 \mathrm{M}_{\mathrm{L}} \mathrm{GS}$ & - & IV & 262 & - \\
\hline 1982 & 1026 & 082629.9 & $44.75 \mathrm{~N}$ & $111.75 \mathrm{~W}$ & 005 & 350 & - & - & $4.60 \mathrm{M}_{\mathrm{L}} \mathrm{GS}$ & - & IV & 350 & - \\
\hline 198 & $03 \quad 17$ & 07 & 47.52 & $22 \mathrm{~W}$ & 005 & 30 & 4 & - & MSO & - & VI & 360 & \\
\hline 1985 & 0401 & 314.2 & $47.276 \mathrm{~N}$ & $113.233 \mathrm{~W}$ & 010 & 371 & 4.8 & - & $\mathrm{AD} \mathrm{BC}$ & - & v & 371 & 68 \\
\hline
\end{tabular}

[Reference (Ref.) numbers given in parentheses at the end of each description refer to sources of data in table 1. Magnitude values are described in the Introduction, and codes are defined in table 2.]

1872. Dec. 10. Western Montana. This earthquake apparently was centered at some distance west of Helena. It was felt most strongly in Beartown (overturned a stove and broke much glass), Deer Lodge (cracked walls of the penitentiary and threw several people off their feet), and Philipsburg (cracked windows). This shock opened a crack in the plastering of a stone-block building on Main Street in Helena and shook violently the massive newspaper building. It was observed as far west as Missoula and south to Warm Springs. Two aftershocks occurred on Dec. 11. (Ref. 38, 249, 316.)

1897. Nov. 4. Near Dillon, Beaverhead County, Mont. The earthquake was severe at Dillon, where cracks formed in the courthouse and in many other buildings. Chimneys fell and plate glass was broken in Butte, north of Dillon. The shock was widely felt in Idaho, western Montana, and Utah. (Ref. 38, 249, 316.)

1903. Mar. 15. Helena, Mont. The earthquake was severe at Helena, where windows broke and rafter beams in less substantial buildings cracked.
Cracks also formed in pillars of the rotunda at the State Capitol. (Ref. 249.)

1908. Dec. 27. Crater Lake, Madison County, Mont. At Crater Lake, about $10 \mathrm{~km}$ southeast of Virginia City, several buildings were cracked, plaster was cracked, and people were panic-stricken. Dishes were shaken from shelves and tables, and the electric powerplant was put out of commission. Of the 30 or more foreshocks, two were severe enough to crack plaster. (Ref. 38, 316.)

1925. June 28, 0121 (June 27). Clarkston Valley, north of Gallatin County, Mont. The most severe damage from this strong earthquake occurred in Gallatin County at Manhattan, Three Forks, Logan, and Lombard. Because no large cities were near the epicenter, property damage did not exceed $\$ 150,000$.

At Manhattan, the community high school and the grade school were both damaged severely, but reinforced concrete buildings were undamaged. Many chimneys were toppled.

At Three Forks, walls of the schoolhouse bulged on all sides, and its foundation and basement were damaged. A church, whose walls were not tied together by an upper floor, also sustained heavy damage. Later shocks demolished the walls. Almost all masonry buildings showed cracks and damage, but 


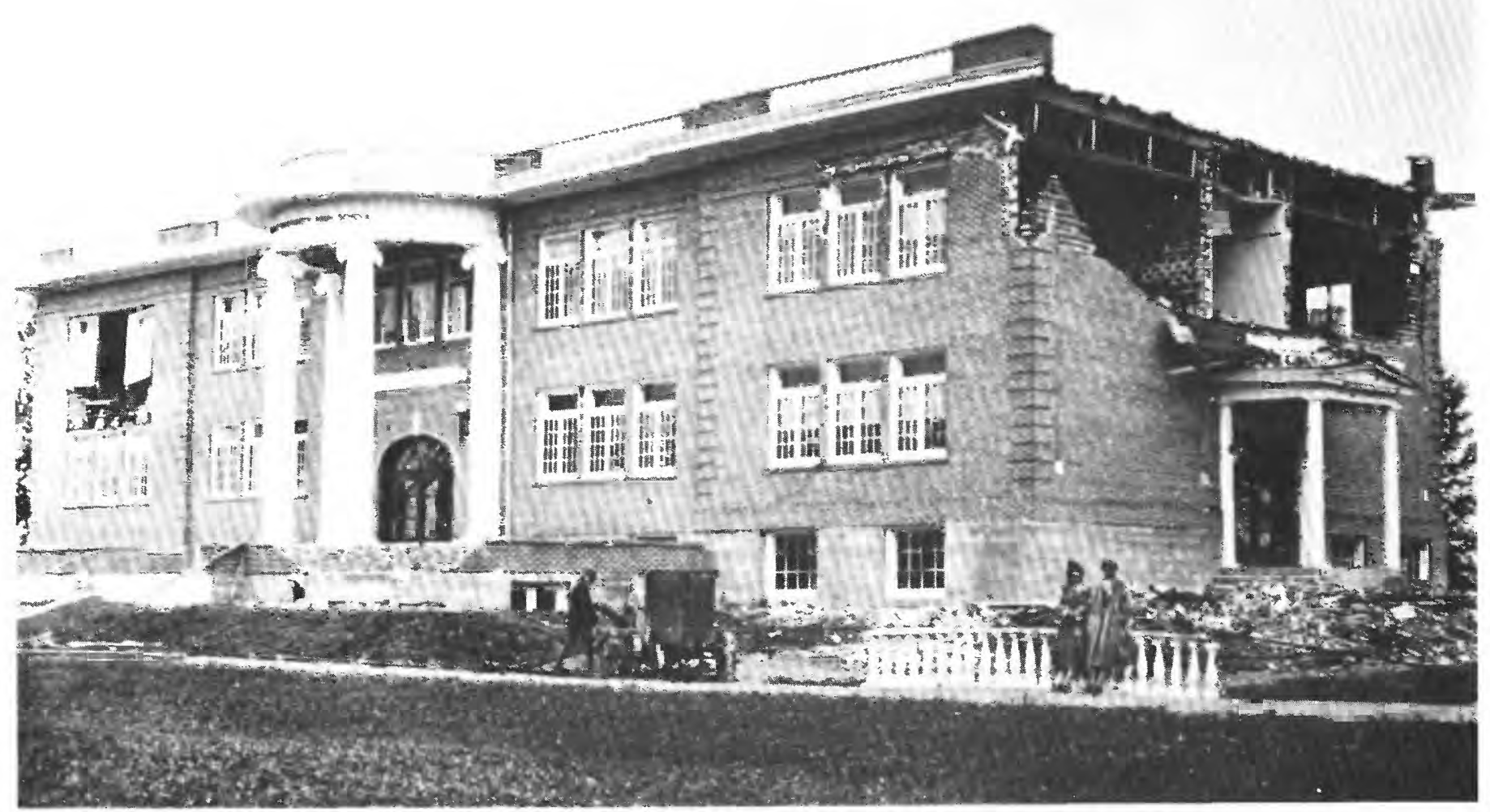

Community High School at Manhattan, Montana, damaged by the June 28, 1925 (June 27 MST), earthquake. (Photograph by J.T. Pardee.)

because most of the buildings were of frame construction, they sustained only cracks in plaster and some fallen chimneys.

At Logan, the poorly designed and constructed schoolhouse was damaged heavily. However, a large brick roundhouse sustained only a few cracks. As at Three Forks, most of the buildings at Logan were of frame construction and therefore sustained only cracks in plaster and destruction of chimneys.

At Lombard, where the Chicago, Milwaukee and St. Paul Railway crosses the Northern Pacific Railway, large boulders were dislodged to such an extent that trains were delayed on the Northern Pacific line. Also, a huge rock slide blocked the Deer Park entrance of the Lombard Tunnel on the Chicago, Milwaukee and St. Paul Railway and the canyon of Sixteenmile Creek, causing a lake to form.

Cracks occurred in graded and filled roads but not in cuts or where the natural surface had not been disturbed. Approaches to many bridges settled as much as $30 \mathrm{~cm}$. One spring formed near Josephine and began to flow, but other springs and sources of water in the neighborhood ceased to flow. Felt from the North Dakota line to Washington, and from southern Canada to southern Wyoming (see fig. 36). Aftershocks continued for several months. (Ref. 38, $168,208,218,312,316$.

1925. June 28, 0205 UTC (June 27). Near Three Forks, Gallatin County, Mont. This was described as the strongest aftershock of the damaging earthquake at 0121 UTC. Most observers reported that it was almost as strong as the main shock, but it was of shorter duration. It caused additional rock slides, threw down chimneys, and toppled previously damaged brick walls. Bricks fell from buildings at Belgrade, Logan, Manhattan, Maudlow, and Three Forks; about $5 \mathrm{~km}$ south of Manhattan, the upper part of a brick chimney was twisted. The shock was so violent at Deer Park that people could hardly stand; some Three Forks residents were thrown to the floor. The aftershock at 0420 UTC was "violent" at Anaconda. (Ref. 38, 168, 218, 312.)

1935. Oct. 7. Craig, Lewis and Clark County, Mont. This local earthquake damaged chimneys and cracked plaster and walls. Pendulum clocks stopped and objects fell from shelves. (Ref. 8, 38, 249.) 


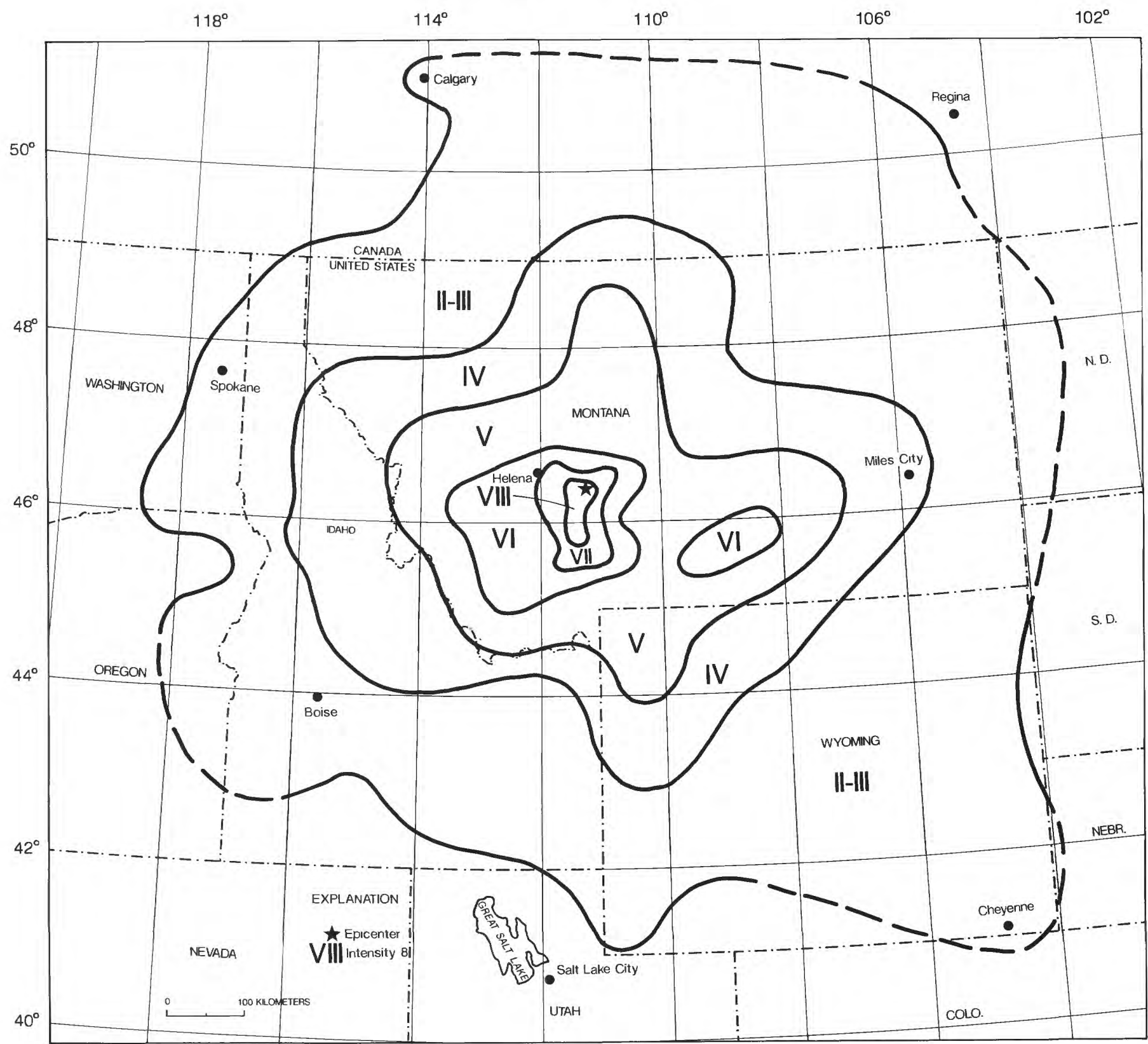

FIGURE 36.--Isoseismal map for the Gallatin County, Montana, earthquake of June 28, 1925. Isoseismals are based on intensity estimates from data listed in references 168 and 218 of table 1.

1935. Oct. 12. Helena, Mont. This was the first destructive foreshock of the 1935 series of earthquakes at Helena on Oct. 12, 18, and 31. An estimated total of $\$ 4$ million in property damage was caused by the series. The fault zone from which the tremors originated lies on the south side of Prickly Pear Valley, north and northeast of Helena. The heavy damage sustained was due to the fact that the shocks occurred almost directly beneath Helena and that each successive shock further damaged previously weakened buildings.
Damage in East Helena from the first tremor was confined to toppled chimneys, broken plaster, and cracked windows. Cracks formed in walls of buildings in Helena, but damage was generally slight. Chimneys toppled at Fort Harrison, plaster was torn from walls, and a hospital was damaged. Total property damage, about $\$ 50,000$, generally was confined to a few structures. (Ref. 8, 38, 316, 503, 533.)

1935. Oct. 15. Helena, Mont. Some damage to property occurred at Helena, and students were dismissed from schools. (Ref. 8, 38, 249.) 


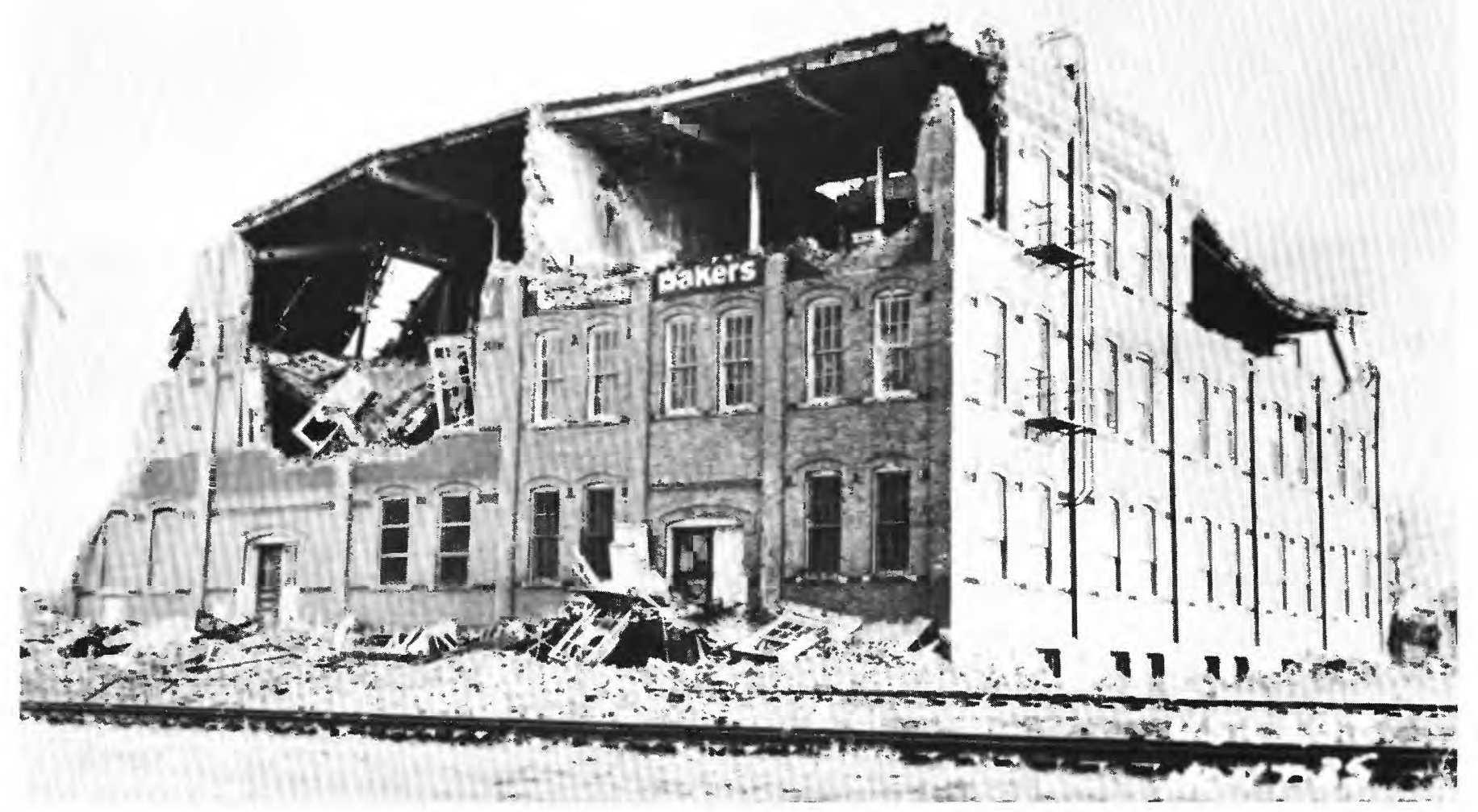

Front brick walls collapsed at the National Biscuit Company building in Helena, Montana, by the October 19, 1935 (Oct. 18 MST), earthquake. (Photograph by F. Ulrich.)

1935. Oct. 19 (Oct. 18). Helena, Mont. This is the main earthquake of the 1935 series of shocks at Helena. Two people were killed by falling bricks, several were injured, and property damage was estimated at about $\$ 3$ million. The earthquake damaged about 300 buildings, of which more than 200 lost their chimneys. Damage was most severe in old 2and 2 1/2-story brick houses on alluvial soil in northeast Helena, but severe damage also occurred in the southern business section of Helena. Downed chimneys and cracked plaster were common throughout the city, and in sections, almost all chimneys were destroyed. Gables commonly were damaged, regardless of the structural material used or the location of the building.

The most severely damaged structure in the area was the Helena High School, constructed a few months earlier, in August 1935. Many large buildings were damaged heavily, including the City Hall, Kessler Brewery, and St. Joseph's Orphanage, but damage was slight to structures on solid bedrock (e.g., the State Capitol, Federal Building, and St. Helena Cathedral). In general, wood buildings covered with wood siding and structures having a framework of reinforced concrete or steel sustained little damage. Tombstones in all the cemeteries in the area were twisted or overturned.

The ground cracks observed were shallow, narrow, surface cracks in alluvial material caused by shaking of the ground, and none represent slip along the fault plane. East of town, water flowed from the cracks that formed in the ground. The largest crack, a maximum of $13 \mathrm{~cm}$ wide, and $91 \mathrm{~m}$ long, was observed on the gravel road leading into the Stanchfield Gun Club.

Changes in the volume of flow of many wells and springs occurred. The most noted change was an increase in flow of springs or the formation of new springs. Seven Mile Creek, which was almost dry before the earthquake, was about $1.5 \mathrm{~m}$ wide and 30 to $45 \mathrm{~cm}$ deep when it was observed on Oct. 31 . Also felt in parts of Idaho, Washington, Wyoming, and in adjacent areas of Canada. (Ref. 8, 38, 316, 503.)

1935. Oct. 27. Helena, Mont. Described as strong, this aftershock of the Oct. 19 earthquake 


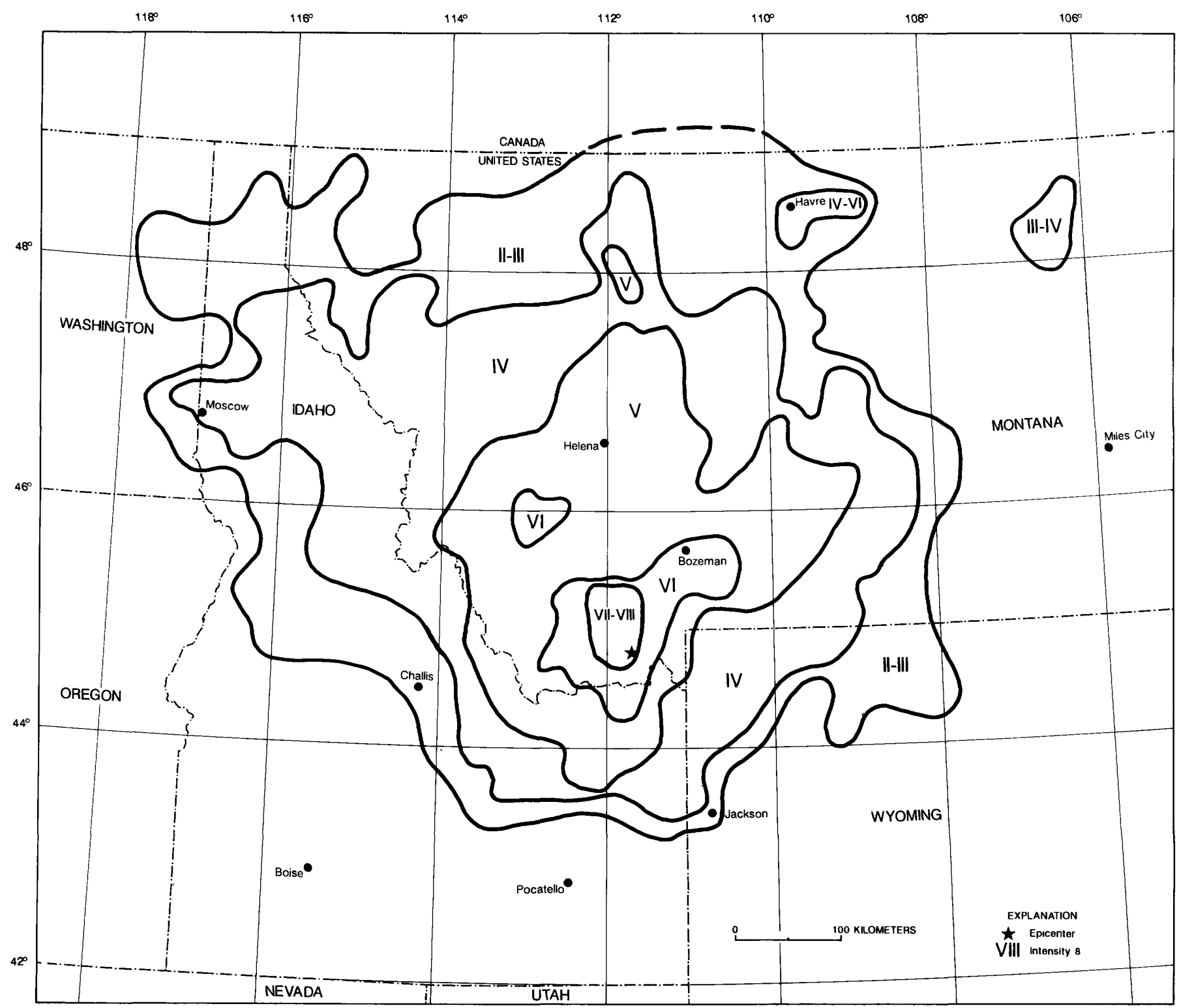

FIGURE 37.-Isoseismal map for the southwest Montana earthquake of November 23, 1947. Isoseismals are based on intensity estimates from data listed references 20 and 259 of table 1.

shook down previously weakened chimneys at Helena. (Ref. 8, 38, 316.)

1935. Oct. 31. Helena, Mont. This aftershock was almost as severe as the main tremor on Oct. 19. Two people were killed at Helena, and about $\$ 1$ million in property damage occurred, bringing the death toll from this series to four and the damage total to $\$ 4$ million. (Ref. 512 reports a total of 6 deaths and $\$ 5.5$ million damage). It intensified the damage in all the towns and damaged structures weakened by previous shocks. Most residents described it as sharper and more pronounced than the main earthquake on Oct. 19.
Many buildings previously damaged were demolished, including the new Helena High School and the Kessler Brewery. Damage was most severe in the neighborhood of the City Hall on Main Street and in the residential district on 9 th Street. On the west side of town, damage from this shock was more severe than that caused by the Oct. 19 earthquake. Damage to frame buildings was slight, except to their chimneys and brick-veneer facing.

The ground in Helena Valley again was cracked. Water was observed spurting $30 \mathrm{~cm}$ or more from cracks, and dust was emitted from others. All chimneys in this neighborhood were downed, and a bridge 
was shifted slightly. Several tombstones turned over in the Resurrection Cemetery, about $5 \mathrm{~km}$ north of Helena. Also felt in parts of Idaho, Washington, Wyoming, and Canada. Magnitude 5.5 $\mathrm{M}_{\mathrm{L}} \mathrm{KJ}$. (Ref. 8, $38,316,460,512$.)

1935. Nov. 4. Helena, Mont. A series of strong tremors, the strongest at 1123 and 1242 UTC, threw down weakened structures at Helena. Walls of the Federal Reserve Bank cracked. One additional building was designated as unsafe for habitation. (Ref. 8, 249.)

1935. Nov. 22 (Nov. 21). Helena, Mont. Buildings previously weakened were damaged slightly at Helena. (Ref. 8, 38.)

1935. Nov. 28. Helena, Mont. Brick walls damaged by earlier earthquakes were further weakened at Helena, and a few were thrown down. This event was described as the fourth strongest of the Helena series of earthquakes. (Ref. 8, 38.)

1936. Feb. 14 (Feb. 13). Helena, Mont. This strong tremor caused some minor damage and much alarm at Helena. (Ref. 9, 38, 259.)

1938. June 13. Trident, Gallatin County, Mont. This shock was strongest at Trident, where plaster cracked slightly in several houses and objects fell from shelves. (Ref. 11, 249.)

1938. June 14 (June 13). Trident, Gallatin County, Mont. Strongest at Trident, this shock cracked plaster in several houses and threw down rocks in a quarry at a cement plant. Though alarmed, residents reported that it was slightly less severe than the earthquake 10 hours earlier. (Ref. 11, 249.)

1939. May 12 (May 11). Trident, Gallatin County, Mont. Plaster cracked and people ran outdoors at Trident. (Ref. 12.)

1940. Dec. 23. Helena, Mont. At Helena, plaster cracked in a few buildings and one wall cracked. At East Helena, windows cracked. About $10 \mathrm{~km}$ north of Helena, in Scratch Gravel Hills, rocks rolled from cliffs. City officials reported an increase in the flow of water into the Hale Reservoir. (Ref. 13, 38, 266.)

1943. June 25 (June 24). Southern Sheridan County, Mont. In Froid, a well-constructed granary was cracked so severely that grain spilled out. Cracks formed in plaster and chimneys at Homestead, Redstone, and Reserve. (Ref. 38, 330.)

1945. Sept. 23. Northwest Montana. The earthquake cracked chimneys and broke windows at Elmo and cracked plaster at Bigfork, near Flathead Lake, south of Kalispell. One window was broken at Polson, south of Elmo. Also felt in Idaho and Washington. (Ref. 18, 266.)
1946. Dec. 11. Northwest Montana. This earthquake was described as sharp at Kalispell, where plaster and chimneys cracked, bricks fell, and dishes broke. Plaster also cracked at Whitefish. (Ref. 19, 249.)

1947. Mar. 14. Seeley Lake, Missoula County, Mont. Plaster cracked and fell to the floor at Seeley Lake, northeast of Missoula. (Ref. 38.)

1947. Nov. 23. Southwest Montana. Chimneys fell, twisted, or cracked in several towns in Madison County, including Alder, Cameron, Ennis, Laurin, and Virginia City. New springs formed in several areas, and creeks became muddy. Huge rocks rolled down the mountainside. Brick, masonry, and concrete structures sustained much damage. Also felt in parts of Idaho, Washington, and Wyoming (see fig. 37). (Ref. 20, 38, 576.)

1950. Aug. 20 (Aug. 19). Western Montana. Two wells became muddy in Sanders County, in the Niarada area; a minor rockslide occurred at Mud Lake Lookout. (Ref. 23, 266.)

1952. Apr. 1 (Mar. 31). Northwest Montana. Brick, masonry, and concrete sustained slight damage at Flathead Lake, south of Kalispell. Chimneys twisted and fell, and walls and chimneys cracked. (Ref. 25.)

1952. Apr. 22. Western Montana. The earthquake was strongest in the area near Townsend and Toston, southeast of Helena, in Broadwater County. Bricks fell from a chimney; plaster fell inside a camphouse; and a parked jeep was moved about 1 m. (Ref. 25.)

1953. Aug. 8. Flathead Lake area, Lake County, Mont. A sharp, jarring movement caused doors to sag on two sheds on the east shore of Flathead Lake, about $20 \mathrm{~km}$ south of Bigfork. At Yellow Bay, cracks formed in an old building. (Ref. 26, 249.)

1958. May 28. Western Montana. At Philipsburg, Granite County, bricks fell from several buildings, plaster cracked, and windows broke. In the Porters Corner area, near Philipsburg, a heavy candy case was displaced about $10 \mathrm{~cm}$. (Ref. 31.)

1959. Aug. 18 (Aug. 17). Hebgen Lake, Mont. This earthquake caused 28 fatalities and about $\$ 11$ million in damage to highways and timber. It is characterized by extensive fault scarps, subsidence and uplift, a massive landslide, and a seiche in Hebgen Lake. A maximum $\mathrm{MM}$ intensity $\mathrm{X}$ was assigned to the fault scarps in the epicentral area. The instrumental epicenter lies within the region of surface faulting. Area of perceptibility (see fig. 38), maximum intensity, and Richter magnitude all were larger for this earthquake than for any earlier earthquake on record in Montana (from May 1869). 


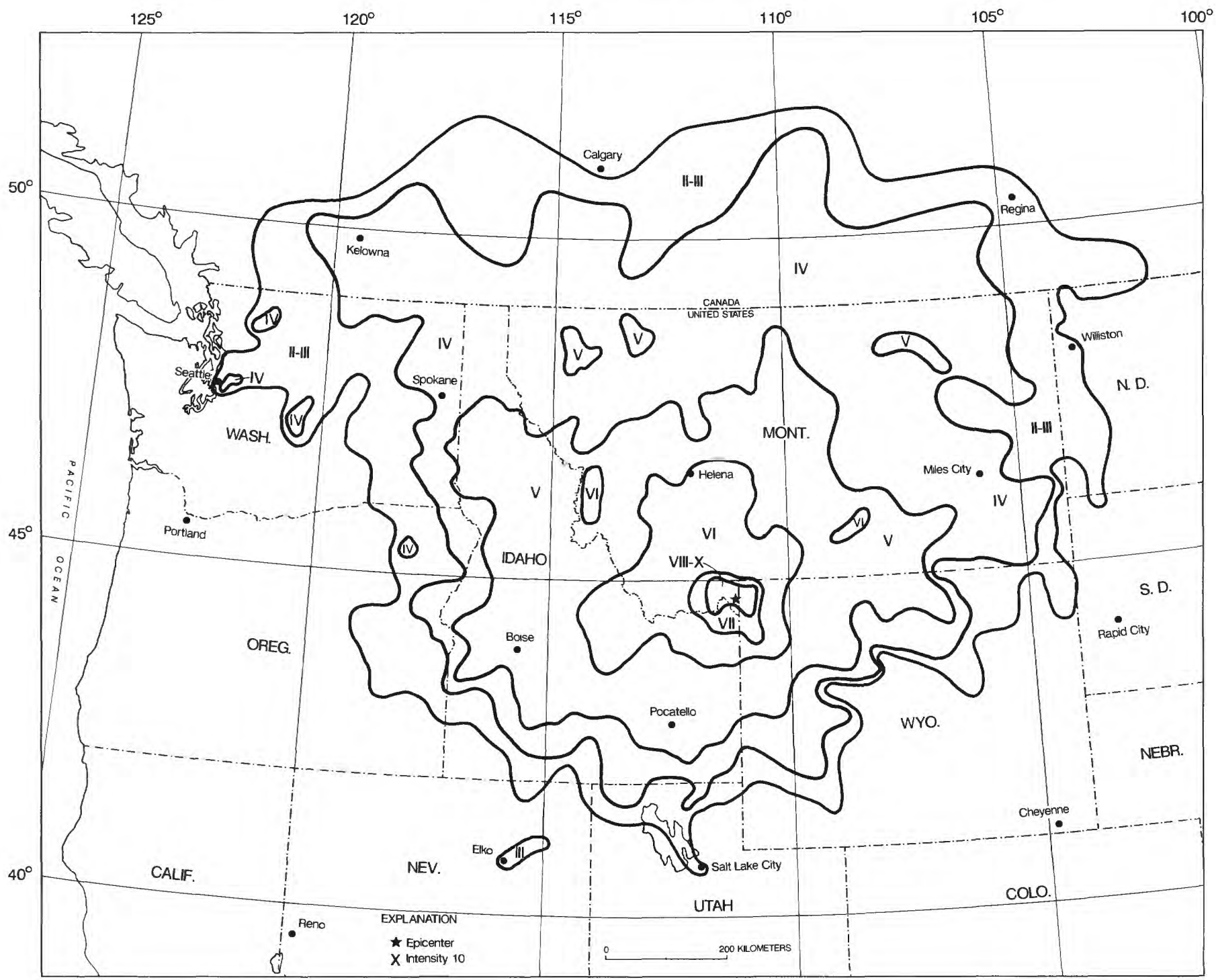

FIGURE 38.-Isoseismal map for the Hebgen Lake, Montana, earthquake of August 18, 1959. Isoseismals are based on intensity estimates from data listed in references 32,259 , and 438 of table 1.

The most spectacular and disastrous effect of the earthquake was the huge avalanche of rock, soil, and trees that cascaded from the steep south wall of the Madison River Canyon. This slide formed a barrier that blocked the gorge and stopped the flow of the Madison River and, within a few weeks, created a lake almost $53 \mathrm{~m}$ deep. The volume of material that blocked the Madison River below Hebgen Dam has been estimated at $28-33$ million $\mathrm{m}^{3}$. Most of the 28 deaths were caused by rockslides that covered the Rock Creek public campground on the Madison River, about $9.5 \mathrm{~km}$ below Hebgen Dam.

New fault scarps as high as $6 \mathrm{~m}$ formed near Hebgen Lake. The major fault scarps formed along preexisting normal faults northeast of Hebgen Lake.
Subsidence occurred over much of an area that was about $24 \mathrm{~km}$ north-south and about twice as long east-west. As result of the faulting near Hebgen Lake, the bedrock beneath the lake was permanently warped, causing the lake floor to drop and generate a seiche. Maximum subsidence was $6.7 \mathrm{~m}$ in. Hebgen Lake Basin. About $130 \mathrm{~km}^{2}$ subsided more than 3 $\mathrm{m}$, and about $500 \mathrm{~km}^{2}$ subsided more than $0.3 \mathrm{~m}$. The earth-fill dam sustained significant cracks in its concrete core and spillway, but it continued to be an effective structure.

Many summer houses in the Hebgen Lake area were damaged: houses and cabins shifted off their foundations, chimneys fell, and pipelines broke. Most small-unit masonry structures and wooden buildings 


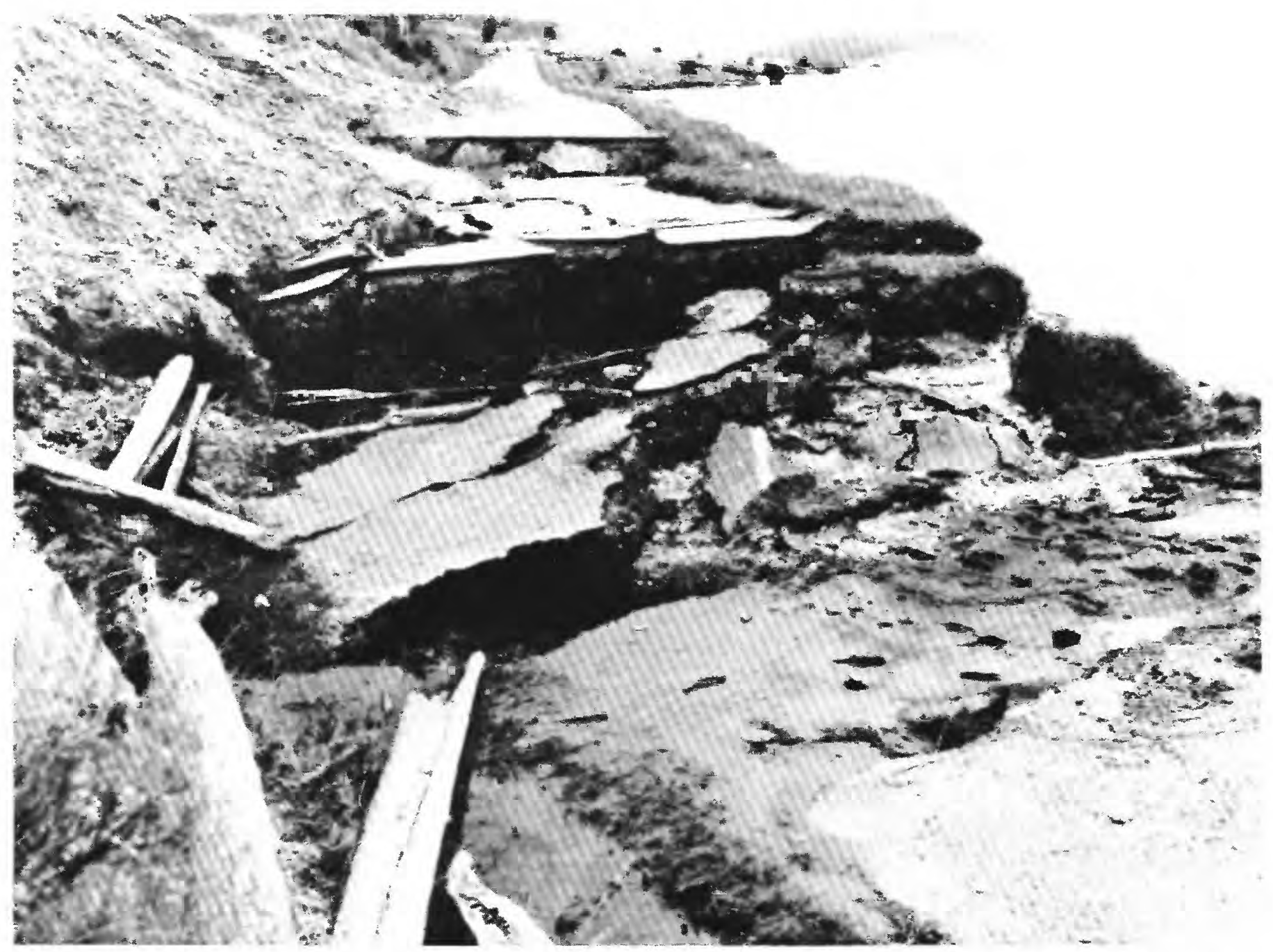

Landslide and slumping damage to State Highway 287, along the shore of Hebgen Lake, Montana, caused by the August 18,1959 (Aug. 17 MST), earthquake. (Photograph by J.R. Stacy.)

along the major fault scarps survived with little damage when subjected only to vibratory forces. Roadways were cracked and shifted extensively, and much timber was destroyed. Highway damage near Hebgen Lake was due to landslides slumping vertically and flowing laterally beneath pavements and bridges, which caused severe cracks and destruction. Three of the five reinforced bridges in the epicentral area also sustained significant damage.

High intensities were observed in the northwest section of Yellowstone National Park. Here, new geysers erupted, and massive slumping caused large cracks in the ground from which steam emitted. Many hot springs became muddy.

On the basis of vibration damage (and excluding geologic effects), damage to buildings along the fault zone was singularly unspectacular (MM intensity VIII at places, intensity VII generally). Minor damage occurred throughout southern Montana, northeast Idaho, and northwest Wyoming. Felt as far as Seattle, Wash., to the west; Banff, Canada, to the north; Dickinson, N. Dak., to the east; and Provo, Utah, to the south. This area includes nine Western States and three Canadian Provinces. Aftershocks continued for several months. Magnitude 7.5 Ukn PAS, 7.5 $\mathrm{M}_{\mathrm{S}} \mathrm{ABE}, 7.3 \mathrm{~m}_{\mathrm{b}} \mathrm{ABE}$. (Ref. 38, 281, 310, $438,504,576$.

1959. Aug. 23. Hebgen Lake, Mont, aftershock. A strong earthquake generated a rockslide above Hebgen Dam that blocked the road. (Ref. 32, 38.)

1959. Sept. 5. Hebgen Lake, Mont., aftershock. This event broke plumbing and dishes at 


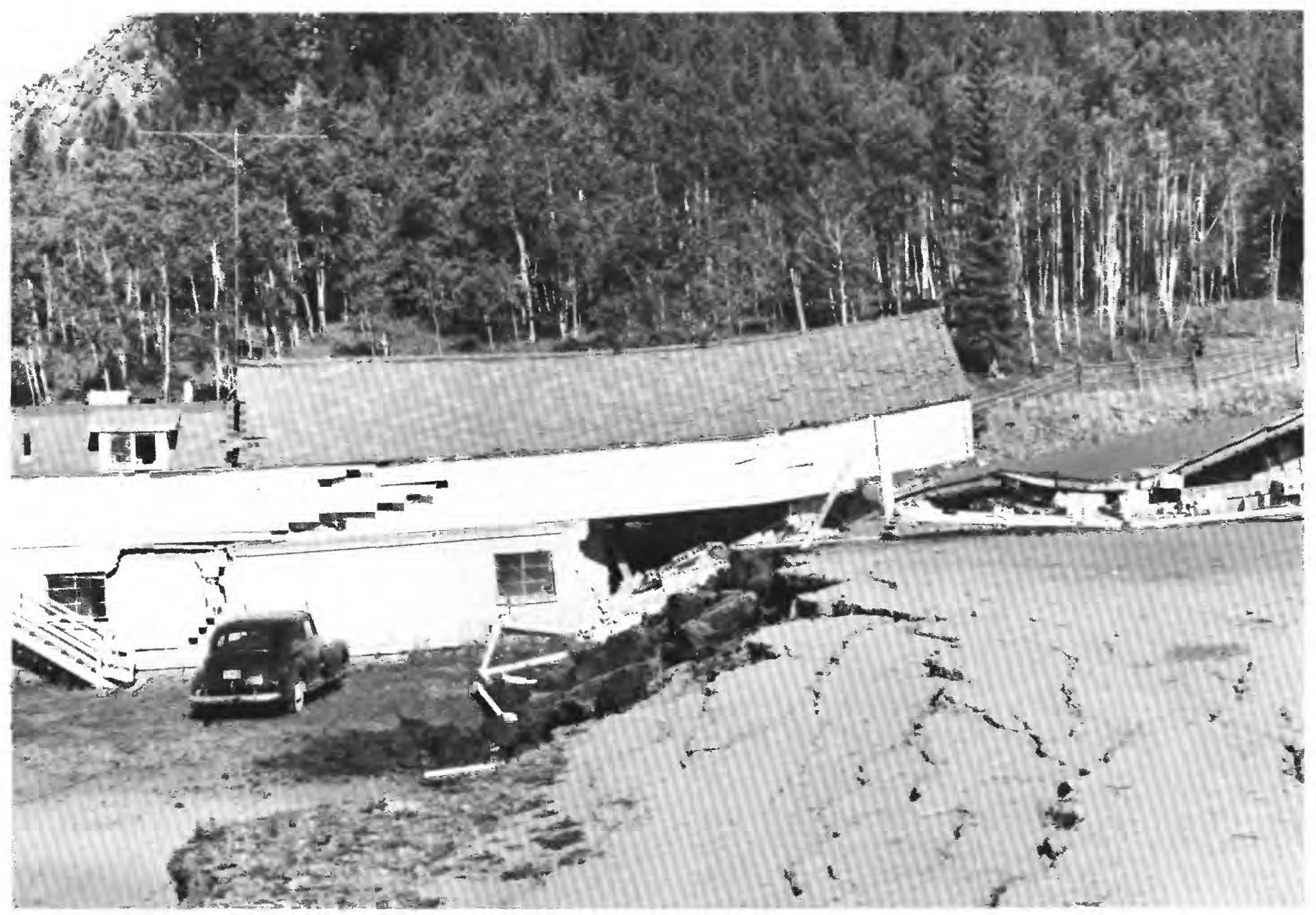

Damaged and collapsed buildings at the Blarneystone Ranch, near Hebgen Lake, Montana, caused by the August 18, 1959 (Aug. $17 \mathrm{MST}$ ), earthquake. Note the fault scarp running beneath the building. (Photograph by I.J. Witkind.)

Canyon, in Yellowstone National Park. Rockslides smashed the viewing platform in the Grand Canyon of the Yellowstone. (Ref. 32, 38.)

1959. Sept. 13. Hebgen Lake, Mont, aftershock. Grayling Creek, which flows into Hebgen Lake at Parade Rest Ranch, was muddied. Rocks fell in the Hebgen Lake area. (Ref. 32, 266, 576.)

1959. Nov. 3. Hebgen Lake, Mont., aftershock. At Duck Creek (intersection of U.S. 191 and Montana State 1), a stove was moved about $30 \mathrm{~cm}$ from the wall and a sewing machine was displaced from the window to the middle of the room. (Ref. 32.)

1965. Jan. 6 (Jan. 5). Southwest Montana. A 10.7-m flagpole was broken in three places and dishes were thrown from shelves at Dewey, about 30 $\mathrm{km}$ southwest of Butte. The shock was felt strongly to the south at Dell, Beaverhead County. Also felt in
Idaho. Magnitude $4.9 \mathrm{~m}_{\mathrm{b}}$ NUT, $4.5 \mathrm{M}_{\mathrm{S}}$ NUT. (Ref. 75, $263,354,576$.)

1969. Apr. 1. Northwest Montana. At Big Arm, on the southwest shore of Flathead Lake, the shock shifted a building several centimeters on its foundation, buckled a boathouse, damaged a dock, and muddied well water. At nearby Dayton, four chimneys were destroyed. The tremor twisted a chimney at Proctor, and caused deep wells to increase their flow of water. At Lake Mary Ronan, chimneys and concrete floors cracked and paneling on walls loosened. Also felt in Idaho. Magnitude $4.3 \mathrm{mb}_{\mathrm{b}} \mathrm{NUT}$, 3.7 $\mathrm{M}_{\mathrm{S}}$ NUT. (Ref. $42,74,263$. )

1969. Sept. 15 (Sept. 14). Northwest Montana. At Big Arm, a crack extending in both horizontal and vertical directions formed in a building. One basement wall also was cracked. (Ref. 42,74 .) 
1969. Nov. 7 (Nov. 6). Northwest Montana. Slight damage occurred at Dayton, on the west shore of Flathead Lake, where plaster fell and small objects overturned. (Ref. 42, 74.)

1975. Feb. 4 (Feb. 3). Northwest Montana. Basement walls cracked and dishes broke at Creston, about $12 \mathrm{~km}$ east of Kalispell. Cracks formed in plaster at Kalispell and, about $30 \mathrm{~km}$ northeast, at Martin City. Also felt in Alberta and British Columbia, Canada. (Ref. 48.)

1977. Mar. 11 (Mar. 10). Southwest Broadwater County, Mont. Cracks formed in plaster at Harrison, about $65 \mathrm{~km}$ southeast of Butte. Also felt at Hudson, Maudlow, Radersburg, and Trident, and southeast to Yellowstone National Park, Wyo. (Ref. 39,465 .)

1977. Oct. 19. Northwest of West Yellowstone, Mont., in Madison County. Cracks formed in plaster at West Yellowstone. Buildings shook in Lima, and vehicles rocked in Clinton. (Ref. 39.)

1983. Mar. 17. Near Lincoln, Lewis and Clark County, Mont. Damage at Lincoln included cracks in chimneys, foundations of houses, interior walls, and windows. Items were thrown from shelves at Augusta, about $65 \mathrm{~km}$ northeast of Lincoln, and furniture was displaced in the Great Falls area of Cascade County, east of Augusta. (Ref. 360.) 
NEBRASKA

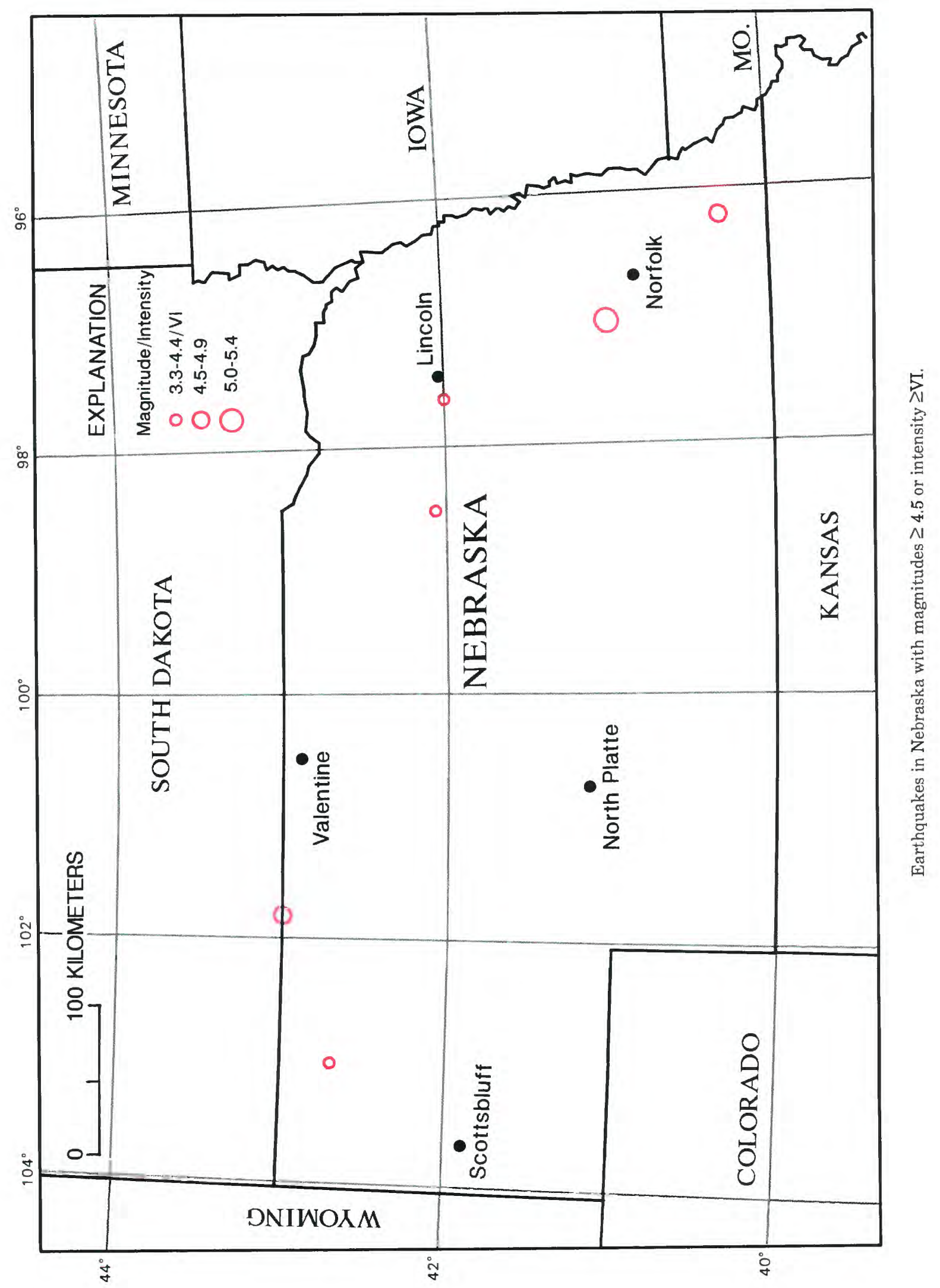




\begin{tabular}{|c|c|c|c|c|c|c|c|c|c|c|c|c|c|}
\hline \multirow{2}{*}{\multicolumn{2}{|c|}{ Date }} & \multirow{2}{*}{$\begin{array}{c}\text { Origin } \\
\text { time (UTC) }\end{array}$} & \multicolumn{4}{|c|}{ Hypocenter } & \multicolumn{4}{|c|}{ Magnitude } & \multicolumn{2}{|c|}{ Intensity } & \multirow{3}{*}{$\begin{array}{c}\text { Felt area } \\
\left(1,000 \mathrm{~km}^{2}\right)\end{array}$} \\
\hline & & & \multirow{2}{*}{$\begin{array}{l}\text { Latitude } \\
\left({ }^{\circ}\right)\end{array}$} & \multirow{2}{*}{$\begin{array}{l}\text { Longitude } \\
\left({ }^{\circ}\right)\end{array}$} & \multirow{2}{*}{$\begin{array}{l}\text { Depth } \\
\text { (km) }\end{array}$} & \multirow[t]{2}{*}{ Ref } & \multicolumn{2}{|c|}{ USGS } & \multirow[t]{2}{*}{ Other } & \multirow{2}{*}{$\begin{array}{c}\text { Moment } \\
\text { M }\end{array}$} & \multirow[t]{2}{*}{ MMI } & \multirow[t]{2}{*}{ Ref } & \\
\hline $\mathrm{Yr}$ & Mo Da & $h \mathrm{~m} s$ & & & & & $m_{b}$ & $\mathbf{M}_{\mathbf{s}}$ & & & & & \\
\hline 1877 & $11 \quad 15$ & 1745 & $41.0 \mathrm{~N}$ & $97.0 \mathrm{~W}$ & - & 105 & - & - & $5.10 \mathrm{M}$ & - & VII & 105 & 450 \\
\hline 1902 & 0728 & 1800 & $42.0 \mathrm{~N}$ & $97.6 \mathrm{~W}$ & - & 105 & - & - & $4.40 \mathrm{M}_{\mathrm{f}}$ & - & VI & 105 & 90 \\
\hline 1934 & 0730 & 0720 & $42.7 \mathrm{~N}$ & $103.0 \mathrm{~W}$ & - & 38 & - & - & $4.30 \mathrm{M}_{\mathrm{fa}} \mathrm{SC}$ & - & VI & 38 & 60 \\
\hline 1935 & 0301 & 1100 & $40.3 \mathrm{~N}$ & $96.2 \mathrm{~W}$ & - & 38 & - & - & $4.60 \mathrm{M}_{\mathrm{fa}} \mathrm{SC}$ & - & VI & 38 & 210 \\
\hline 1964 & +0328 & 100846.5 & $42.997 \mathrm{~N}$ & $101.798 \mathrm{~W}$ & 030 & 349 & 5.1 & - & $4.50 \mathrm{M}_{\mathrm{n}} \mathrm{DG}$ & - & VII & 37 & 150 \\
\hline 1975 & 50513 & 075340.0 & $42.070 \mathrm{~N}$ & $98.503 \mathrm{~W}$ & 001 & 349 & 4.3 & - & $3.30 \mathrm{M}_{\mathrm{n}} \mathrm{DG}$ & - & VI & 48 & - \\
\hline
\end{tabular}

[Reference (Ref.) numbers given in parentheses at the end of each description refer to sources of data in table 1 . Magnitude values are described in the Introduction, and codes are defined in table 2.]

1877. Nov. 15. Eastern Nebraska, between Lincoln and Columbus. This is the largest earthquake known to have originated in Nebraska. Its proposed epicenter lies on the western flank of the Keweenawan mafic belt. Damage was most severe at Columbus, in Colfax County, northwest of the epicenter, where the 30 -second shock split the courthouse walls in nine places and damaged the schoolhouse walls. Two severe shocks about $350 \mathrm{~km}$ west of Lincoln, at North Platte, cracked walls and overturned printing cases. About $200 \mathrm{~km}$ north of Lincoln, at Sioux City, Iowa, a high school sustained cracks in a wall. Felt over all or parts of Iowa, Kansas, Minnesota, Missouri, Nebraska, South Dakota, and Wisconsin. Magnitude 5.0 $\mathrm{M}_{\mathrm{fa}}$ BAR. (Ref. 38, 105, 353, 463.)

1902. July 28. Battle Creek, Madison County, Nebr. This earthquake was reported to be most strong at Battle Creek, where it lasted for 30 seconds and spilled water from buckets. At nearby Tilden, one chimney was destroyed, plaster was cracked, and dishes fell from shelves. Reported in western Iowa and as far east as Chicago, Ill. Magnitude $4.5 \mathrm{M}_{\mathrm{fa}}$ BAR. (Ref. 38, 105, 353.)

1934. July 30. Chadron, Dawes County, Nebr. Chimneys were damaged, plaster fell, and objects toppled from shelves at Chadron. Felt in western
Nebraska and in the adjacent States of South Dakota and Wyoming. Magnitude 4.5 $\mathrm{M}_{\mathrm{fa}}$ BAR. (Ref. 38, 105, 353.)

1935. Mar. 1. Tecumseh, Johnson County, Nebr. This earthquake has been attributed to slippage on the Humboldt fault along the east side of the Nemaha Ridge. Many chimneys cracked and a few collapsed at Tecumseh, and windows broke and cracks formed in plaster and stone walls. Two shocks, about 4 minutes apart, were felt; the first was the strongest. Also felt in Iowa, Kansas, and Missouri. Magnitude 3.0 $\mathrm{M}_{\mathrm{S}} \mathrm{BAR}, 4.7 \mathrm{M}_{\mathrm{fa}}$ BAR. (Ref. 38, 353.)

1964. Mar. 28. Near Merriman, northwest Cherry County, Nebr. Many cracks formed in the roadway south of Merriman, and steep slopes slumped into the Niobrara River. Merchandise in stores was broken, dishes were broken, and stucco under windows cracked. At Alliance, about $135 \mathrm{~km}$ southwest of Merriman, part of a chimney cap fell on a house; at Rushville, about $35 \mathrm{~km}$ southwest of Merriman, plaster fell and a wall cracked. Also felt in Montana, South Dakota, and Wyoming (see fig. 39). Magnitude 4.7 $\mathrm{M}_{\mathrm{n}}$ BAR. (Ref. 37, 349, 353.)

1975. May 13. Near Bartlett, Wheeler County, Nebr. The shock cracked stucco in Bartlett and knocked cans from shelves in a store. Also reported felt northeast of the epicenter at Hudson, South Dakota. Magnitude 3.5 $\mathrm{M}_{\mathrm{n}}$ SLM. (Ref. 48, 349.) 


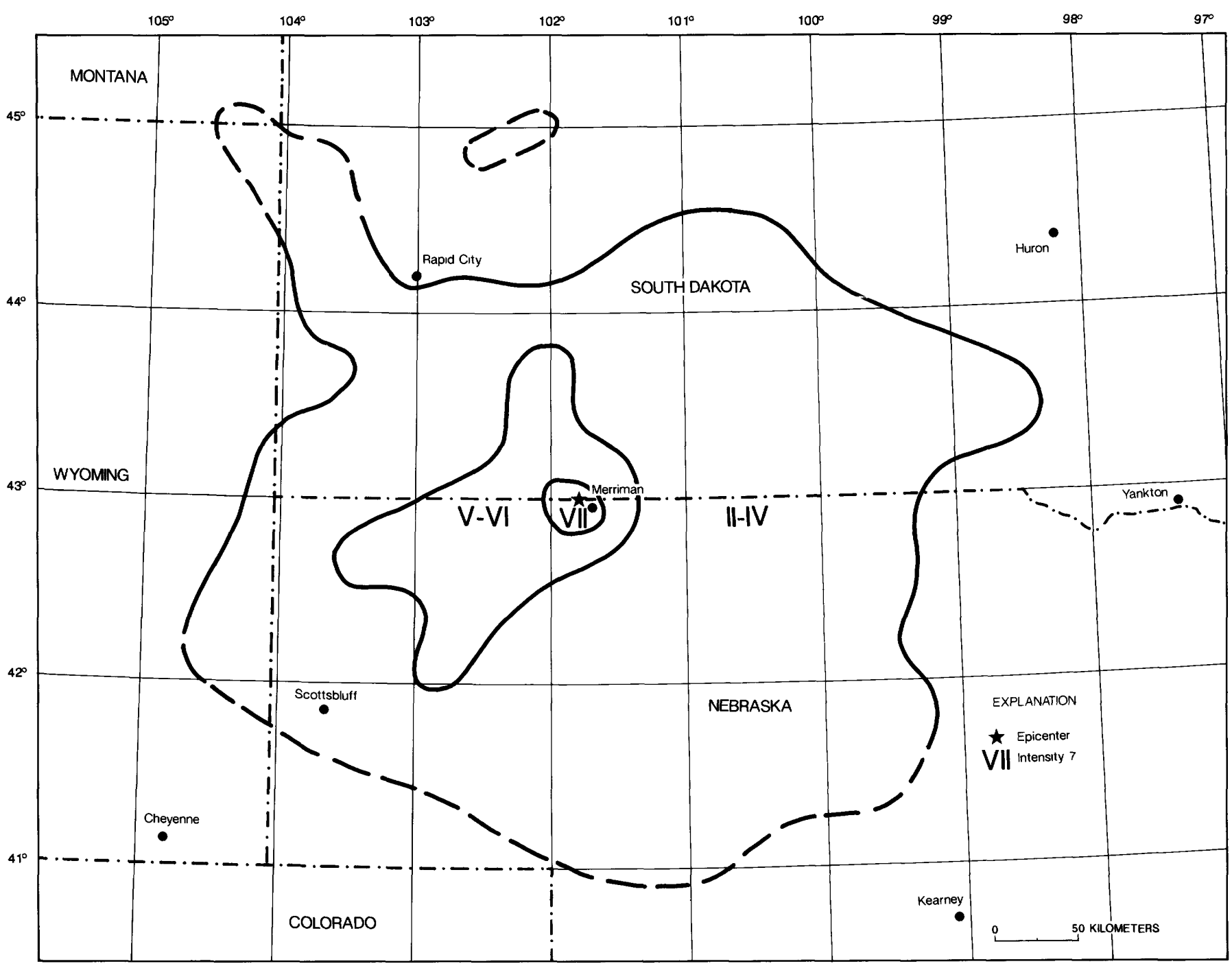

FIGURE 39.- Isoseismal map for the northwest Nebraska earthquake of March 28, 1964. Isoseismals are based on intensity estimates from data listed in reference 37 of table 1. 



\section{NEVADA}

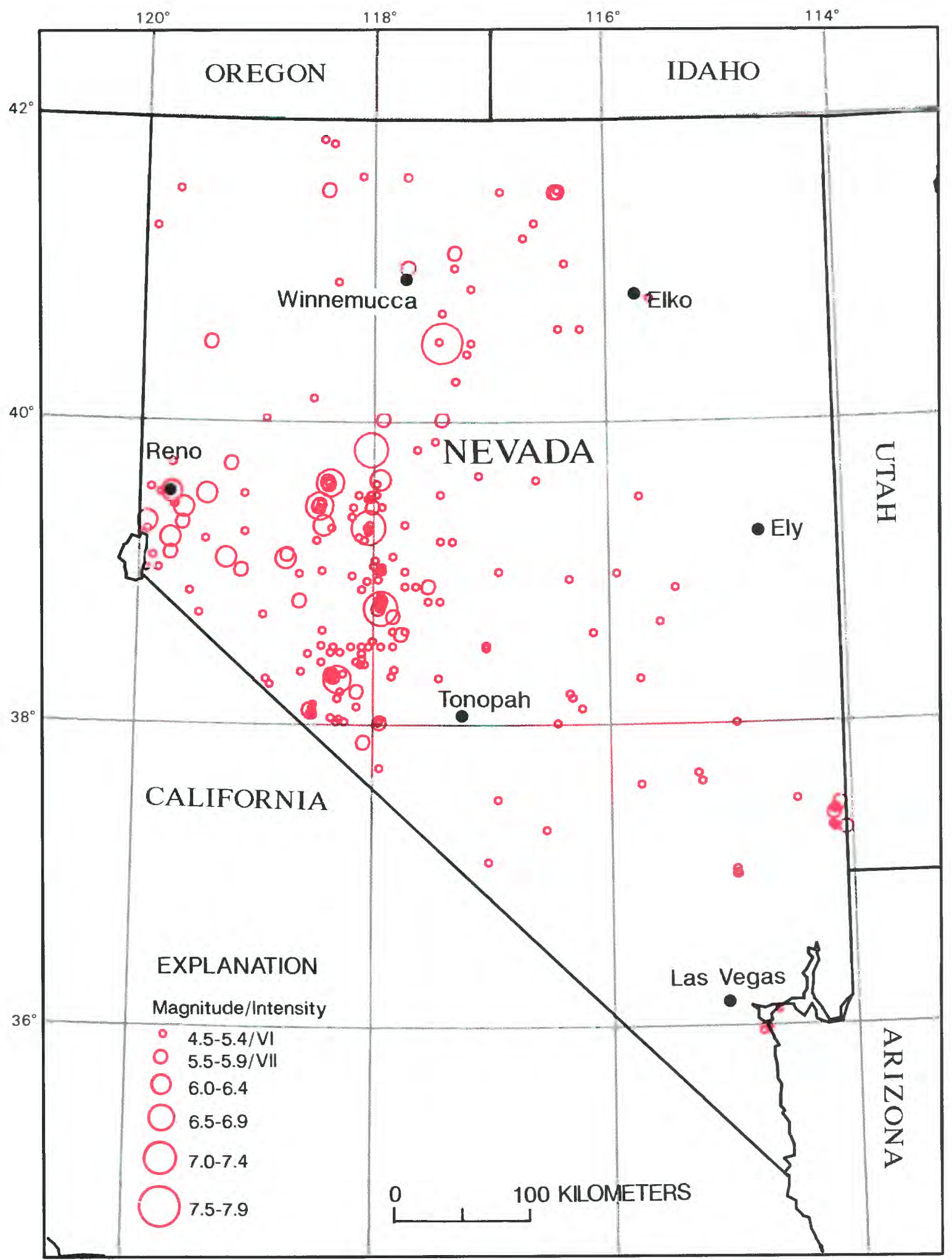

Earthquakes in Nevada with magnitudes $\geq 4.5$ or intensity $\geq$ VI. 
NEVADA

[See table 1 for hypocenter and intensity references and table 2 for definitions of magnitude source codes. @, felt area is less than $1,000 \mathrm{~km}^{2}$. Leader (-) indicates information is not available!

\begin{tabular}{|c|c|c|c|c|c|c|c|c|c|c|c|c|c|c|c|c|}
\hline \multirow{2}{*}{\multicolumn{3}{|c|}{ Date }} & \multirow{2}{*}{$\begin{array}{c}\text { Origin } \\
\text { time (UTC) }\end{array}$} & \multicolumn{6}{|c|}{ Hypocenter } & \multicolumn{4}{|c|}{ Magnitude } & \multicolumn{2}{|c|}{ Intensity } & \multirow{3}{*}{$\begin{array}{l}\text { Felt area } \\
\left(1,000 \mathrm{~km}^{2}\right)\end{array}$} \\
\hline & & & & \multirow{2}{*}{\multicolumn{2}{|c|}{$\begin{array}{l}\text { Latitude } \\
\text { ( })\end{array}$}} & \multirow{2}{*}{\multicolumn{2}{|c|}{$\begin{array}{c}\text { Longitude } \\
\text { ( })\end{array}$}} & \multirow{2}{*}{$\begin{array}{c}\text { Depth } \\
(\mathbf{k m})\end{array}$} & \multirow[t]{2}{*}{ Ref } & \multicolumn{2}{|c|}{ USGS } & \multirow[t]{2}{*}{ Other } & \multirow{2}{*}{$\begin{array}{l}\text { Moment } \\
\text { M }\end{array}$} & \multirow[t]{2}{*}{ MMI } & \multirow[t]{2}{*}{ Ref } & \\
\hline Yr & Mo & Da & $\mathrm{h} \mathrm{m} s$ & & & & & & & $m_{b}$ & $\mathbf{M}_{\mathbf{s}}$ & & & & & \\
\hline 1857 & 09 & 03 & 0305 & 39.3 & $\mathbf{N}$ & 120.0 & $\mathbf{W}$ & - & 368 & - & - & $6.00 \mathrm{M}_{\mathrm{L}} \mathrm{DMG}$ & - & V & 368 & - \\
\hline 1860 & 03 & 15 & 1900 & 39.5 & $\mathbf{N}$ & 119.5 & $\mathbf{W}$ & - & 368 & - & - & $6.30 \mathrm{M}_{\mathrm{La}} \mathrm{DMG}$ & - & VI & 368 & - \\
\hline 1868 & 05 & 30 & 0510 & 39.3 & $\mathbf{N}$ & 119.7 & $\mathbf{W}$ & - & 368 & - & - & $5.80 \mathrm{M}_{\mathrm{La}} \mathrm{DMG}$ & - & VII & 54 & 190 \\
\hline 1869 & 12 & 27 & 0155 & 39.4 & $\mathbf{N}$ & 119.7 & W & - & 368 & - & - & $6.10 \mathrm{M}_{\mathrm{La}} \mathrm{DMG}$ & - & VII & 368 & 180 \\
\hline 1869 & 12 & 27 & 1000 & 39.1 & $\mathbf{N}$ & 119.8 & W & - & 368 & - & - & $5.90 \mathrm{M}_{\mathrm{La}} \mathrm{DMG}$ & 一 & VII & 368 & 一 \\
\hline 1872 & 03 & 23 & 2141 & 40.0 & $\mathbf{N}$ & 117.5 & W & - & 54 & - & - & $5.50 \mathrm{M}_{\mathrm{fa}}$ SJG & - & VI & 38 & 31 \\
\hline 1872 & 11 & 12 & & 39.0 & $\mathbf{N}$ & 117.0 & W & - & 521 & - & - & $6.00 \mathrm{M}_{\mathrm{La}} \mathrm{ELL}$ & - & Felt & 54 & 129 \\
\hline 1873 & 11 & 05 & 1700 & 40. & $\mathbf{N}$ & 118. & W & - & 54 & - & - & $5.50 \mathrm{M}_{\mathrm{fa}} \mathrm{SJG}$ & - & Felt & 54 & - \\
\hline 1875 & 04 & 02 & 0200 & 39.5 & $\mathbf{N}$ & 115.8 & W & - & 54 & - & - & - & - & VI & 54 & - \\
\hline 1877 & 07 & 10 & 0710 & 39.3 & $\mathbf{N}$ & 120.0 & w & 一 & 54 & - & 一 & $5.00 \mathrm{M}_{\mathrm{fa}}$ SJG & - & IV & 54 & 一 \\
\hline 1887 & 06 & 03 & 1048 & 39.2 & $\mathbf{N}$ & 119.8 & W & - & 54 & - & - & $6.30 \mathrm{M}_{\mathrm{La}} \mathrm{DMG}$ & 一 & VIII & 368 & - \\
\hline 1888 & 01 & 30 & 0635 & 39.0 & $\mathbf{N}$ & 120.0 & W & - & 54 & - & - & $4.50 \mathrm{M}_{\mathrm{fa}} \mathrm{SJG}$ & - & $\mathbf{V}$ & 54 & - \\
\hline 1894 & 11 & 18 & 1049 & 39.2 & $\mathbf{N}$ & 119.5 & W & - & 54 & - & - & - & - & VI & 56 & - \\
\hline 1896 & 01 & 27 & 2101 & 39.1 & $\mathbf{N}$ & 119.8 & W & - & 54 & - & - & 一 & - & VI & 54 & - \\
\hline 1897 & 05 & 15 & 1904 & 39.3 & $\mathbf{N}$ & 119.7 & W & - & 54 & - & - & - & - & V & 54 & - \\
\hline 1901 & 07 & 26 & 2220 & 40.8 & $\mathbf{N}$ & 115.7 & $\mathbf{W}$ & - & 54 & - & - & $5.00 \mathrm{M}_{\mathrm{fa}}$ SJG & - & VII & 54 & 9 \\
\hline 1903 & & & & 39.5 & $\mathbf{N}$ & 118.1 & W & - & 54 & - & - & - & - & VI & 328 & - \\
\hline 1910 & 11 & 07 & 1720 & 37.5 & $\mathbf{N}$ & 117.0 & W & - & 54 & - & - & - & - & VI & 56 & - \\
\hline 1910 & 11 & 19 & 0225 & 38.0 & $\mathbf{N}$ & 118.0 & W & - & 54 & - & - & - & - & Felt & 56 & - \\
\hline 1910 & 11 & 21 & 2323 & 38.0 & $\mathbf{N}$ & 118.0 & W & - & 54 & - & - & 一 & 一 & VII & 56 & - \\
\hline 1910 & 11 & 22 & 0605 & 38.0 & $\mathbf{N}$ & 118.0 & W & - & 54 & - & - & - & - & Felt & 56 & 一 \\
\hline 1914 & 02 & 18 & 1817 & 39.5 & $\mathbf{N}$ & 119.8 & W & - & 54 & - & - & $6.00 \mathrm{M}_{\mathrm{fa}} \mathrm{SJG}$ & - & VI & 38 & 42 \\
\hline 1914 & 04 & 24 & 0835 & 39.5 & $\mathbf{N}$ & 119.8 & $\mathbf{W}$ & - & 54 & - & - & $6.40 \mathrm{M}_{\mathrm{fa}} \mathrm{SJG}$ & - & VII & 56 & 260 \\
\hline 1915 & 10 & 03 & 0149 & 40.5 & $\mathbf{N}$ & 117.5 & W & - & 54 & - & - & - & - & V & 327 & - \\
\hline 1915 & 10 & 03 & 065248.0 & 40.5 & $\mathbf{N}$ & 117.5 & W & - & 258 & - & - & $7.70 \mathrm{M}_{\mathrm{s}} \mathrm{AB1}$ & 7.14WAL & $\mathbf{X}$ & 56 & 788 \\
\hline 1916 & 02 & 03 & 050304 & 41.0 & $\mathbf{N}$ & 117.8 & W & - & 54 & - & - & $5.90 \mathrm{M}_{\mathrm{fa}} \mathrm{SJG}$ & - & $\mathbf{V}$ & 272 & 278 \\
\hline 1916 & 08 & 03 & 134919 & 41.5 & $\mathbf{N}$ & 116.5 & W & 一 & 54 & - & - & $5.60 \mathrm{M}_{\mathrm{x}}$ SJG & - & IV & 272 & - \\
\hline 1916 & 08 & 03 & 142238 & 41.5 & $\mathbf{N}$ & 116.5 & W & - & 54 & - & - & $5.80 \mathrm{M}_{\mathrm{X}}$ SJG & - & Felt & 56 & - \\
\hline 1916 & 08 & 04 & 041252 & 41.5 & $\mathbf{N}$ & 117.0 & W & - & 54 & - & - & $5.00 \mathrm{M}_{\mathrm{x}}$ SJG & - & Felt & 54 & - \\
\hline 1916 & 10 & 11 & 054909 & 41.5 & $\mathbf{N}$ & 116.5 & $\mathbf{W}$ & 一 & 54 & - & - & $5.00 \mathrm{M}_{\mathrm{x}}$ SJG & - & Felt & 56 & 一 \\
\hline 1917 & 03 & 28 & 111600 & 41.6 & $\mathbf{N}$ & 117.8 & W & - & 54 & - & - & 4.70Ukn JON & - & IV & 315 & - \\
\hline 1917 & 04 & 11 & 185955 & 40.0 & $\mathbf{N}$ & 118.0 & W & - & 54 & - & - & $5.10 \mathrm{M}_{\mathrm{x}}$ SJG & - & III & 272 & - \\
\hline 1925 & 08 & 21 & 111357 & 38.0 & $\mathbf{N}$ & 118.3 & W & - & 54 & - & - & $4.80 \mathrm{M}_{\mathrm{X}}$ SJG & - & $\mathbf{V}$ & 56 & - \\
\hline 1928 & 03 & 26 & 162607 & 38.3 & $\mathbf{N}$ & 117.5 & $\mathbf{W}$ & - & 54 & - & - & $4.70 \mathrm{M}_{\mathrm{x}}$ SJG & - & Felt & 54 & - \\
\hline 1928 & 04 & 17 & 103915 & 39.50 & $\mathbf{N}$ & 119.83 & $\mathbf{W}$ & - & 324 & - & - & $4.50 \mathrm{M}_{\mathrm{x}}$ SJG & 一 & IV & 1 & - \\
\hline 1929 & 09 & 10 & 2001 & 41.2 & $\mathbf{N}$ & 116.8 & W & - & 54 & - & - & $4.60 \mathrm{M}_{\mathrm{X}}$ SJG & - & IV & 54 & 一 \\
\hline 1930 & 04 & 09 & 215647 & 39.25 & $\mathbf{N}$ & 120.00 & W & - & 324 & - & - & $4.30 \mathrm{M}_{\mathrm{x}}$ SJG & - & VI & 3 & 49 \\
\hline 1930 & 04 & 12 & 125640 & 39.25 & $\mathbf{N}$ & 119.17 & $\mathbf{W}$ & - & 324 & - & - & $4.50 \mathrm{M}_{\mathrm{X}}$ SJG & - & VI & 3 & 30 \\
\hline 1932 & 12 & 21 & 061005 & 38.75 & $\mathbf{N}$ & 118.0 & W & - & 258 & - & - & $7.20 \mathrm{M}_{8} \mathrm{GR}$ & 一 & $\mathbf{x}$ & 38 & 795 \\
\hline 1932 & 12 & 22 & 074930 & 38.75 & $\mathbf{N}$ & 118.0 & W & - & 315 & - & - & 4.50Ukn SJG & - & Felt & 315 & - \\
\hline 1932 & 12 & 22 & 103415 & 38.75 & $\mathbf{N}$ & 118.0 & W & - & 315 & - & - & $4.90 \mathrm{M}_{\mathrm{x}}$ SJG & - & Felt & 315 & - \\
\hline 1932 & 12 & 23 & 2006 & 38.75 & $\mathbf{N}$ & 118.0 & W & 一 & 315 & 一 & - & 4.50Ukn SJG & 一 & - & - & - \\
\hline 1932 & 12 & 24 & 124049 & 38.8 & $\mathbf{N}$ & 118.0 & W & - & 54 & - & - & $5.00 \mathrm{M}_{\mathrm{x}} \mathrm{SJG}$ & - & Felt & 54 & - \\
\hline 1932 & 12 & 25 & 035445 & 38.8 & $\mathbf{N}$ & 118.0 & W & - & 54 & - & - & $5.50 M_{x}$ SJG & 一 & Felt & 356 & - \\
\hline 1932 & 12 & 25 & 183635 & 38.8 & $\mathbf{N}$ & 118.0 & W & 一 & 54 & - & - & $4.50 \mathrm{M}_{\mathrm{x}}$ SJG & - & Felt & 54 & 一 \\
\hline 1932 & 12 & 26 & 0503 & 38.75 & $\mathbf{N}$ & 118.0 & $\mathbf{W}$ & - & 315 & - & - & $5.30 \mathrm{M}_{\mathrm{X}}$ SJG & - & Felt & 356 & - \\
\hline 1932 & 12 & 28 & 030755 & 38.8 & $\mathbf{N}$ & 118.0 & $\mathbf{W}$ & - & 54 & - & - & $4.60 \mathrm{M}_{\mathrm{x}} \mathrm{SJG}$ & - & Felt & 54 & - \\
\hline
\end{tabular}


NEVADA-Continued

[See table 1 for hypocenter and intensity references and table 2 for definitions of magnitude source codes. @, felt area is less than $1,000 \mathrm{~km}^{2}$. Leader (--) indicates information is not available

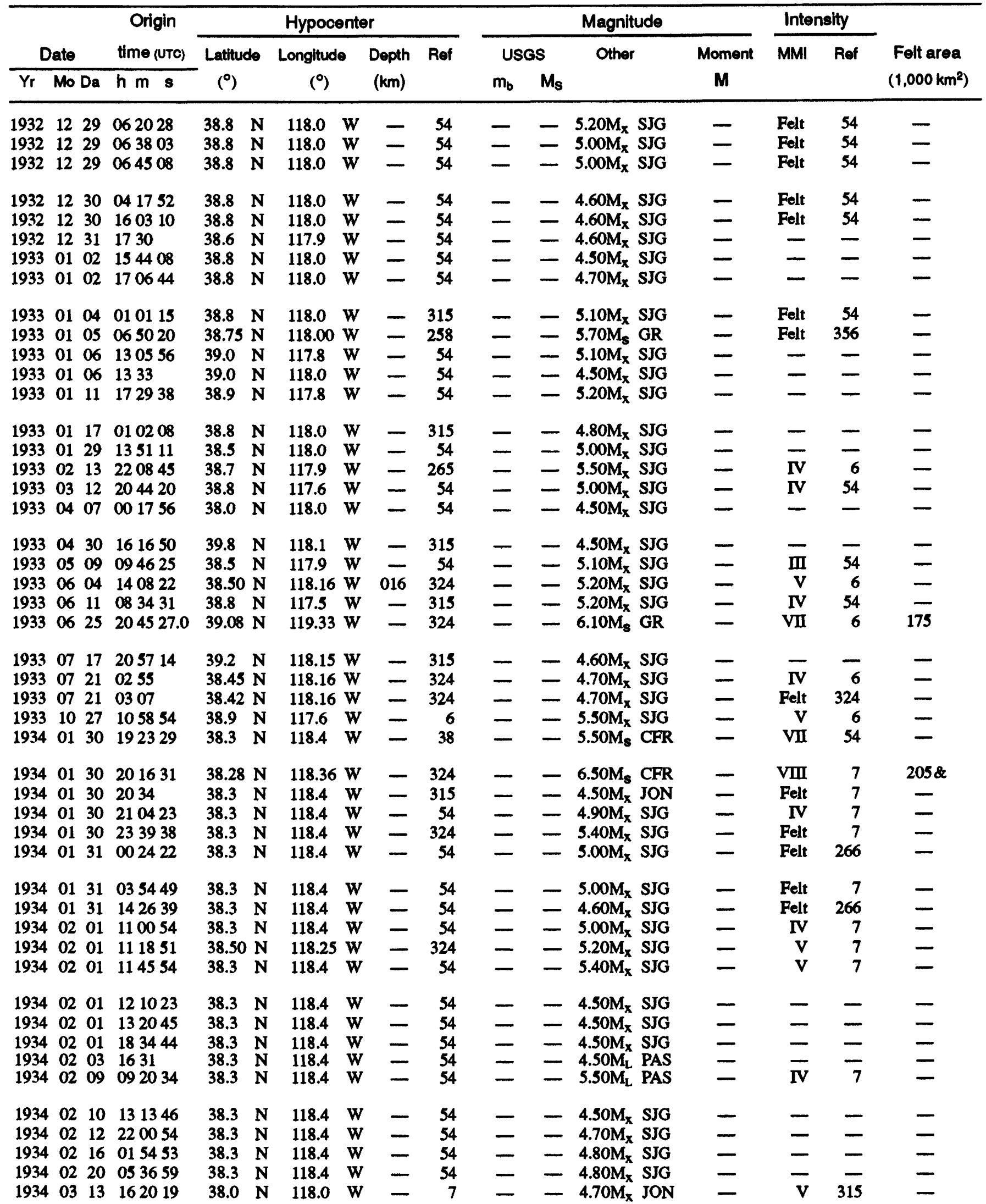


NEVADA - Continued

[See table 1 for hypocenter and intensity references and table 2 for definitions of magnitude source codes. @, felt area is less than $1,000 \mathrm{~km}^{2}$. Leader (--) indicates information is not available

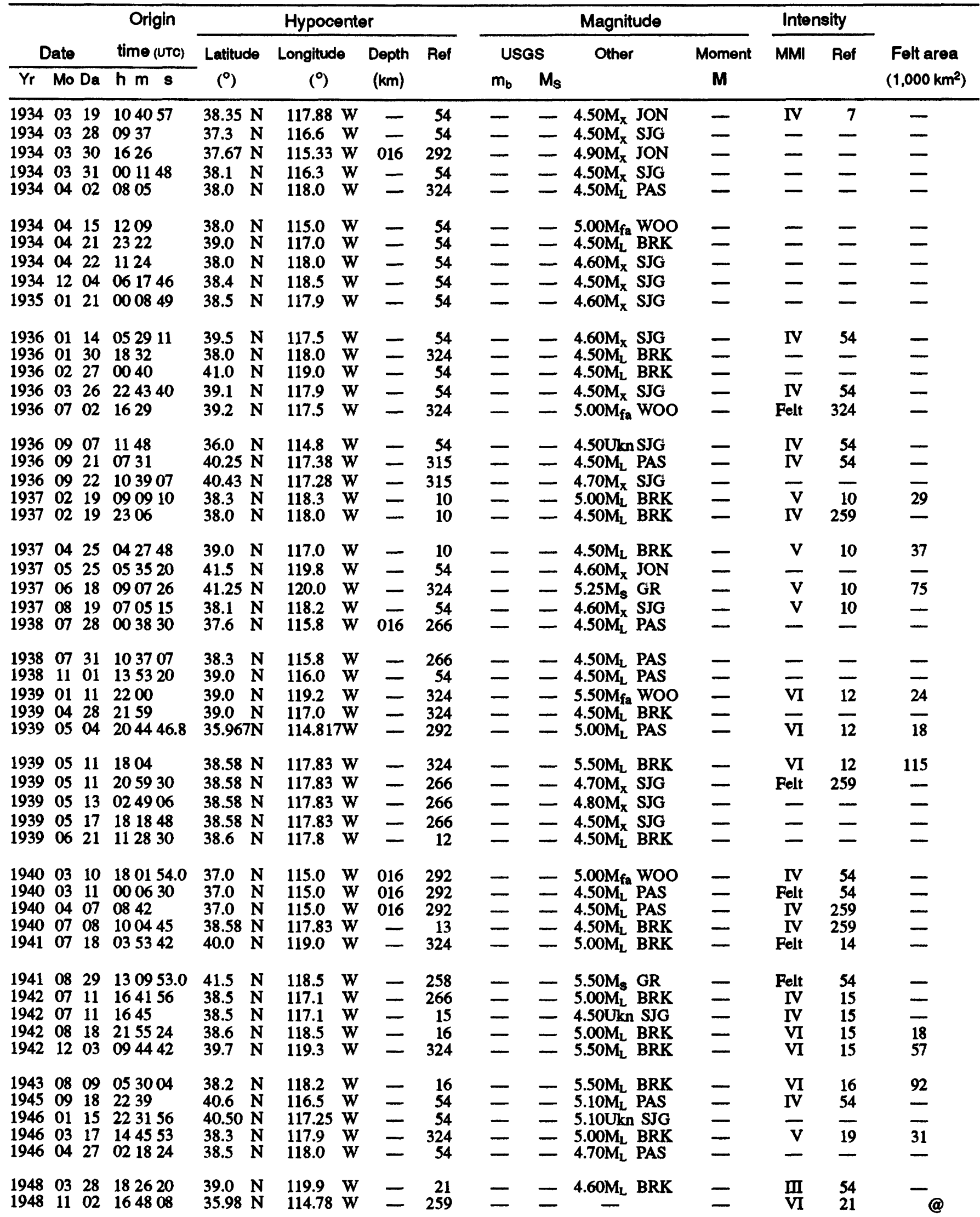


NEVADA-Continued

[See table 1 for hypocenter and intensity references and table 2 for definitions of magnitude source codes. @, felt area is less than $1,000 \mathrm{~km}^{2}$. Leader (--) indicates information is not available]

\begin{tabular}{|c|c|c|c|c|c|c|c|c|c|c|c|c|c|c|c|}
\hline \multirow{2}{*}{\multicolumn{3}{|c|}{ Date }} & \multirow{2}{*}{$\begin{array}{c}\text { Origin } \\
\text { time (UTC) }\end{array}$} & \multicolumn{5}{|c|}{ Hypocenter } & \multicolumn{4}{|c|}{ Magnitude } & \multicolumn{2}{|c|}{ Intensity } & \multirow{3}{*}{$\begin{array}{l}\text { Felt area } \\
\left(1,000 \mathrm{~km}^{2}\right)\end{array}$} \\
\hline & & & & \multirow{2}{*}{$\begin{array}{l}\text { Latitude } \\
\left({ }^{\circ}\right)\end{array}$} & \multirow{2}{*}{\multicolumn{2}{|c|}{$\begin{array}{c}\text { Longitude } \\
\left.\text { ( }{ }^{\circ}\right)\end{array}$}} & \multirow{2}{*}{$\begin{array}{c}\text { Depth } \\
(\mathrm{km})\end{array}$} & \multirow[t]{2}{*}{ Ref } & \multicolumn{2}{|c|}{ USGS } & \multirow[t]{2}{*}{ Other } & \multirow{2}{*}{$\begin{array}{c}\text { Moment } \\
\text { M }\end{array}$} & \multirow[t]{2}{*}{ MMI } & \multirow[t]{2}{*}{ Ref } & \\
\hline$Y_{r}$ & Mo & $\mathrm{Da}$ & $h \mathrm{~m} s$ & & & & & & $m_{b}$ & $M_{\mathbf{S}}$ & & & & & \\
\hline $\begin{array}{l}1949 \\
1949 \\
1950\end{array}$ & $\begin{array}{l}01 \\
07 \\
10\end{array}$ & $\begin{array}{l}02 \\
18 \\
17\end{array}$ & $\begin{array}{l}220354 \\
153105 \\
035433\end{array}$ & $\begin{array}{ll}38.7 & \mathrm{~N} \\
39.7 & \mathrm{~N} \\
39.60 & \mathrm{~N}\end{array}$ & $\begin{array}{l}119.0 \\
119.8 \\
116.69\end{array}$ & $\begin{array}{l}\mathbf{W} \\
\mathbf{W} \\
\mathbf{W}\end{array}$ & - & $\begin{array}{r}22 \\
324 \\
324\end{array}$ & E & $\overline{-}$ & $\begin{array}{ll}4.50 \mathrm{M}_{\mathrm{L}} & \text { BRK } \\
4.50 \mathrm{M}_{\mathrm{L}} & \text { BRK } \\
4.50 \mathrm{M}_{\mathrm{L}} & \mathrm{BRK}\end{array}$ & - & $\frac{\text { IV }}{-}$ & $\frac{259}{-}$ & - \\
\hline $\begin{array}{l}1950 \\
1951 \\
1951 \\
1952 \\
1952\end{array}$ & $\begin{array}{l}10 \\
01 \\
06 \\
05 \\
05\end{array}$ & $\begin{array}{l}23 \\
22 \\
16 \\
09 \\
24\end{array}$ & $\begin{array}{lll}08 & 12 & 46 \\
15 & 14 & 53 \\
05 & 52 & 56 \\
15 & 31 & 32 \\
04 & 15 & 15.0\end{array}$ & $\begin{array}{ll}39.5 & \mathbf{N} \\
39.08 & \mathbf{N} \\
37.08 & \mathbf{N} \\
39.42 & \mathbf{N} \\
36.1 & \mathbf{N}\end{array}$ & $\begin{array}{l}117.5 \\
119.95 \\
117.08 \\
119.78 \\
114.7\end{array}$ & $\begin{array}{l}\mathbf{W} \\
\mathbf{W} \\
\mathbf{W} \\
\mathbf{W} \\
\mathbf{W}\end{array}$ & $\frac{-}{016}$ & $\begin{array}{l}324 \\
324 \\
292 \\
324 \\
292\end{array}$ & $\begin{array}{l}- \\
z\end{array}$ & $\overline{-}$ & $\begin{array}{ll}4.50 \mathrm{M}_{\mathrm{L}} & \text { BRK } \\
4.80 \mathrm{M}_{\mathrm{L}} & \text { BRK } \\
4.50 \mathrm{M}_{\mathrm{L}} & \text { PAS } \\
5.10 \mathrm{M}_{\mathrm{L}} & \text { BRK } \\
4.90 \mathrm{M}_{\mathrm{L}} & \text { PAS }\end{array}$ & $\begin{array}{l}- \\
z \\
-\end{array}$ & $\begin{array}{l}\bar{v} \\
\overline{\text { VI }} \\
\text { VI }\end{array}$ & $\frac{\overline{24}}{\overline{25}}$ & $\frac{-}{8}$ \\
\hline $\begin{array}{l}1952 \\
1952 \\
1953 \\
1953 \\
1954\end{array}$ & $\begin{array}{l}11 \\
11 \\
08 \\
09 \\
07\end{array}$ & $\begin{array}{l}13 \\
18 \\
09 \\
26 \\
02\end{array}$ & $\begin{array}{l}005516 \\
040408 \\
220002 \\
033429 \\
104313\end{array}$ & $\begin{array}{ll}38.5 & \mathbf{N} \\
39.8 & \mathbf{N} \\
37.5 & \mathbf{N} \\
39.53 & \mathbf{N} \\
38.17 & \mathbf{N}\end{array}$ & $\begin{array}{l}118.5 \\
117.7 \\
114.5 \\
119.98 \\
116.37\end{array}$ & $\begin{array}{l}\mathbf{W} \\
\mathbf{W} \\
\mathbf{W} \\
\mathbf{W} \\
\mathbf{W}\end{array}$ & $\begin{array}{l}- \\
- \\
-\end{array}$ & $\begin{array}{r}324 \\
324 \\
54 \\
324 \\
324\end{array}$ & $\begin{array}{l}- \\
z\end{array}$ & $\overline{-}$ & $\begin{array}{ll}4.80 \mathrm{M}_{\mathrm{L}} & \text { BRK } \\
4.60 \mathrm{M}_{\mathrm{L}} & \text { BRK } \\
4.50 \mathrm{M}_{\mathrm{L}} & \text { REN } \\
5.30 \mathrm{M}_{\mathrm{L}} & \text { BRK } \\
4.90 \mathrm{M}_{\mathrm{L}} & \text { BRK }\end{array}$ & $\begin{array}{l}z \\
z\end{array}$ & $\begin{array}{l}- \\
\overline{\text { VI }} \\
\text { III }\end{array}$ & $\begin{array}{l}\overline{-} \\
\overline{26} \\
27\end{array}$ & $\bar{z}$ \\
\hline $\begin{array}{l}1954 \\
1954 \\
1954 \\
1954 \\
1954\end{array}$ & $\begin{array}{l}07 \\
07 \\
07 \\
07 \\
07\end{array}$ & $\begin{array}{l}06 \\
06 \\
06 \\
06 \\
06\end{array}$ & $\begin{array}{lll}11 & 13 & 20 \\
11 & 1804 \\
11 & 2655 \\
11 & 4100 \\
11 & 4900\end{array}$ & $\begin{array}{ll}39.42 & \mathrm{~N} \\
39.42 & \mathrm{~N} \\
39.42 & \mathrm{~N} \\
39.42 & \mathrm{~N} \\
39.42 & \mathrm{~N}\end{array}$ & $\begin{array}{l}118.53 \\
118.53 \\
118.53 \\
118.53 \\
118.53\end{array}$ & $\begin{array}{l}\mathbf{W} \\
\mathbf{W} \\
\mathbf{W} \\
\mathbf{W} \\
\mathbf{W}\end{array}$ & $\overline{-}$ & $\begin{array}{l}324 \\
324 \\
324 \\
324 \\
324\end{array}$ & $\bar{z}$ & $\bar{z}$ & $\begin{array}{ll}6.80 \mathrm{M}_{\mathrm{L}} & \text { BRK } \\
5.50 \mathrm{M}_{\mathrm{L}} & \text { BRK } \\
4.80 \mathrm{M}_{\mathrm{L}} & \text { BRK } \\
4.50 \mathrm{M}_{\mathrm{L}} & \text { BRK } \\
5.70 \mathrm{M}_{\mathrm{L}} & \text { BRK }\end{array}$ & $\begin{array}{c}\text { 6.20ELL } \\
- \\
- \\
-\end{array}$ & $\begin{array}{l}\text { IX } \\
\text { Felt } \\
\text { Felt } \\
\text { Felt } \\
\text { Felt }\end{array}$ & $\begin{array}{l}27 \\
27 \\
27 \\
27 \\
27\end{array}$ & $\frac{347}{-}$ \\
\hline $\begin{array}{l}1954 \\
1954 \\
1954 \\
1954 \\
1954\end{array}$ & $\begin{array}{l}07 \\
07 \\
07 \\
07 \\
07\end{array}$ & $\begin{array}{l}06 \\
06 \\
06 \\
06 \\
06\end{array}$ & $\begin{array}{lll}12 & 53 & 59 \\
13 & 15 & 11 \\
13 & 36 & 01 \\
14 & 55 & 15 \\
22 & 07 & 41\end{array}$ & $\begin{array}{ll}39.42 & \mathbf{N} \\
39.42 & \mathbf{N} \\
39.42 & \mathbf{N} \\
39.42 & \mathbf{N} \\
39.3 & \mathbf{N}\end{array}$ & $\begin{array}{l}118.53 \\
118.53 \\
118.53 \\
118.53 \\
118.5\end{array}$ & $\begin{array}{l}\mathbf{W} \\
\mathbf{W} \\
\mathbf{W} \\
\mathbf{W} \\
\mathbf{W}\end{array}$ & $\begin{array}{l}- \\
- \\
-\end{array}$ & $\begin{array}{l}324 \\
324 \\
324 \\
324 \\
324\end{array}$ & $\begin{array}{l}- \\
z\end{array}$ & $\overline{-}$ & $\begin{array}{ll}4.50 \mathrm{M}_{\mathrm{L}} & \text { BRK } \\
5.20 \mathrm{M}_{\mathrm{L}} & \text { BRK } \\
4.50 \mathrm{M}_{\mathrm{L}} & \text { BRK } \\
4.50 \mathrm{M}_{\mathrm{L}} & \text { BRK } \\
6.00 \mathrm{M}_{\mathrm{L}} & \text { BRK }\end{array}$ & $\frac{\bar{Z}}{\bar{Z}}$ & $\begin{array}{l}\text { Felt } \\
\frac{\text { Felt }}{\text { VII }}\end{array}$ & $\frac{\frac{27}{27}}{259}$ & $\begin{array}{l}- \\
z \\
-\end{array}$ \\
\hline $\begin{array}{l}1954 \\
1954 \\
1954 \\
1954 \\
1954\end{array}$ & $\begin{array}{l}07 \\
07 \\
07 \\
07 \\
07\end{array}$ & $\begin{array}{l}07 \\
08 \\
08 \\
08 \\
08\end{array}$ & $\begin{array}{l}061108 \\
021355 \\
040819 \\
125510 \\
193157\end{array}$ & $\begin{array}{ll}39.42 & \mathbf{N} \\
39.42 & \mathbf{N} \\
39.42 & \mathbf{N} \\
39.42 & \mathbf{N} \\
39.42 & \mathbf{N}\end{array}$ & $\begin{array}{l}118.53 \\
118.53 \\
118.53 \\
118.53 \\
118.53\end{array}$ & $\begin{array}{l}\mathbf{W} \\
\mathbf{W} \\
\mathbf{W} \\
\mathbf{W} \\
\mathbf{W}\end{array}$ & $\bar{z}$ & $\begin{array}{l}324 \\
324 \\
324 \\
324 \\
324\end{array}$ & $\bar{z}$ & $\begin{array}{l}- \\
- \\
-\end{array}$ & $\begin{array}{ll}4.60 \mathrm{M}_{\mathrm{L}} & \text { BRK } \\
4.80 \mathrm{M}_{\mathrm{L}} & \text { BRK } \\
4.50 \mathrm{M}_{\mathrm{L}} & \text { BRK } \\
4.70 \mathrm{M}_{\mathrm{L}} & \text { BRK } \\
5.30 \mathrm{M}_{\mathrm{L}} & \text { BRK }\end{array}$ & $\begin{array}{l}z \\
z\end{array}$ & $\frac{\overline{I V}}{\bar{V}}$ & $\begin{array}{l}\overline{27} \\
\overline{27} \\
27\end{array}$ & $\overline{-}$ \\
\hline $\begin{array}{l}1954 \\
1954 \\
1954 \\
1954 \\
1954\end{array}$ & $\begin{array}{l}07 \\
07 \\
07 \\
07 \\
07\end{array}$ & $\begin{array}{l}09 \\
10 \\
11 \\
11 \\
12\end{array}$ & $\begin{array}{l}085003 \\
012220 \\
070400 \\
095812 \\
101706\end{array}$ & $\begin{array}{ll}39.42 & \mathbf{N} \\
39.42 & \mathbf{N} \\
39.42 & \mathbf{N} \\
39.42 & \mathbf{N} \\
39.42 & \mathbf{N}\end{array}$ & $\begin{array}{l}118.53 \\
118.53 \\
118.53 \\
118.53 \\
118.53\end{array}$ & $\begin{array}{l}\mathbf{W} \\
\mathbf{W} \\
\mathbf{W} \\
\mathbf{W} \\
\mathbf{W}\end{array}$ & $\overline{-}$ & $\begin{array}{l}324 \\
324 \\
324 \\
324 \\
324\end{array}$ & $\begin{array}{l}- \\
-\end{array}$ & $\overline{-}$ & $\begin{array}{ll}4.90 \mathrm{M}_{\mathrm{L}} & \text { BRK } \\
4.60 \mathrm{M}_{\mathrm{L}} & \text { BRK } \\
4.60 \mathrm{M}_{\mathrm{L}} & \text { BRK } \\
4.60 \mathrm{M}_{\mathrm{L}} & \mathrm{BRK} \\
4.50 \mathrm{M}_{\mathrm{L}} & \mathrm{BRK}\end{array}$ & $\begin{array}{l}- \\
- \\
-\end{array}$ & $\frac{\text { IV }}{-}$ & $\begin{array}{l}27 \\
- \\
-\end{array}$ & $\begin{array}{l}- \\
- \\
-\end{array}$ \\
\hline $\begin{array}{l}1954 \\
1954 \\
1954 \\
1954 \\
1954\end{array}$ & $\begin{array}{l}07 \\
07 \\
07 \\
07 \\
08\end{array}$ & $\begin{array}{l}12 \\
20 \\
30 \\
31 \\
02\end{array}$ & $\begin{array}{lll}16 & 0525 \\
001138 \\
020010 \\
172414 \\
101853\end{array}$ & $\begin{array}{ll}39.42 & \mathbf{N} \\
38.2 & \mathbf{N} \\
39.42 & \mathbf{N} \\
39.42 & \mathbf{N} \\
39.42 & \mathbf{N}\end{array}$ & $\begin{array}{l}118.53 \\
116.4 \\
118.53 \\
118.53 \\
118.53\end{array}$ & $\begin{array}{l}\mathbf{W} \\
\mathbf{W} \\
\mathbf{W} \\
\mathbf{W} \\
\mathbf{W}\end{array}$ & $\frac{-}{-}$ & $\begin{array}{l}324 \\
324 \\
324 \\
324 \\
324\end{array}$ & $\begin{array}{l}- \\
-\end{array}$ & $\begin{array}{c}- \\
- \\
-\end{array}$ & $\begin{array}{ll}4.60 \mathrm{M}_{\mathrm{L}} & \text { BRK } \\
5.00 \mathrm{M}_{\mathrm{L}} & \text { BRK } \\
5.10 \mathrm{M}_{\mathrm{L}} & \text { BRK } \\
4.50 \mathrm{M}_{\mathrm{L}} & \text { BRK } \\
5.40 \mathrm{M}_{\mathrm{L}} & \text { BRK }\end{array}$ & $\bar{z}$ & $\frac{\text { IV }}{\frac{\text { Felt }}{V}}$ & $\frac{27}{54}$ & $\begin{array}{l}- \\
-\end{array}$ \\
\hline $\begin{array}{l}1954 \\
1954 \\
1954 \\
1954 \\
1954\end{array}$ & $\begin{array}{l}08 \\
08 \\
08 \\
08 \\
08\end{array}$ & $\begin{array}{l}03 \\
05 \\
24 \\
24 \\
25\end{array}$ & $\begin{array}{l}212454 \\
050308 \\
055132 \\
055746 \\
021713\end{array}$ & $\begin{array}{ll}39.42 & \mathbf{N} \\
39.42 & \mathrm{~N} \\
39.58 & \mathrm{~N} \\
39.58 & \mathrm{~N} \\
39.58 & \mathrm{~N}\end{array}$ & $\begin{array}{l}118.53 \\
118.53 \\
118.45 \\
118.45 \\
118.45\end{array}$ & $\begin{array}{l}\mathbf{W} \\
\mathbf{W} \\
\mathbf{W} \\
\mathbf{W} \\
\mathbf{W}\end{array}$ & $\bar{z}$ & $\begin{array}{l}324 \\
324 \\
324 \\
324 \\
324\end{array}$ & $\begin{array}{l}z \\
-\end{array}$ & $\begin{array}{l}- \\
- \\
-\end{array}$ & $\begin{array}{ll}4.70 \mathrm{M}_{\mathrm{L}} & \text { BRK } \\
4.70 \mathrm{M}_{\mathrm{L}} & \text { BRK } \\
6.80 \mathrm{M}_{\mathrm{L}} & \text { BRK } \\
5.20 \mathrm{M}_{\mathrm{L}} & \text { BRK } \\
4.80 \mathrm{M}_{\mathrm{L}} & \text { BRK }\end{array}$ & $\begin{array}{c}\overline{-} \\
\bar{Z} \\
\overline{-}\end{array}$ & $\begin{array}{l}\text { Felt } \\
\text { Felt } \\
\text { IX } \\
\text { Felt } \\
-\end{array}$ & $\begin{array}{l}54 \\
54 \\
27 \\
54 \\
-\end{array}$ & $\frac{\overline{ }}{490} \overline{-}$ \\
\hline $\begin{array}{l}1954 \\
1954 \\
1954 \\
1954 \\
1954\end{array}$ & $\begin{array}{l}08 \\
08 \\
08 \\
08 \\
08\end{array}$ & $\begin{array}{l}25 \\
26 \\
29 \\
29 \\
31\end{array}$ & $\begin{array}{llll}22 & 21 & 10 \\
12 & 56 & 15 \\
03 & 41 & 06 \\
03 & 58 & 05 \\
22 & 20 & 32\end{array}$ & $\begin{array}{ll}39.58 & \mathbf{N} \\
39.58 & \mathbf{N} \\
39.58 & \mathbf{N} \\
39.58 & \mathbf{N} \\
39.58 & \mathbf{N}\end{array}$ & $\begin{array}{l}118.45 \\
118.45 \\
118.45 \\
118.45 \\
118.45\end{array}$ & $\begin{array}{l}\mathbf{W} \\
\mathbf{W} \\
\mathbf{W} \\
\mathbf{W} \\
\mathbf{W}\end{array}$ & $\overline{-}$ & $\begin{array}{l}324 \\
324 \\
324 \\
324 \\
324\end{array}$ & $\frac{z}{z}$ & $\begin{array}{l}- \\
- \\
-\end{array}$ & $\begin{array}{ll}4.70 \mathrm{M}_{\mathrm{L}} & \text { BRK } \\
4.60 \mathrm{M}_{\mathrm{L}} & \text { BRK } \\
4.70 \mathrm{M}_{\mathrm{L}} & \text { BRK } \\
4.80 \mathrm{M}_{\mathrm{L}} & \text { BRK } \\
5.80 \mathrm{M}_{\mathrm{L}} & \text { BRK }\end{array}$ & $\frac{\bar{Z}}{\bar{E}}$ & $\overline{-}$ & $\frac{-}{E}$ & $\begin{array}{l}- \\
- \\
-\end{array}$ \\
\hline $\begin{array}{l}1954 \\
1954 \\
1954 \\
1954 \\
1954\end{array}$ & $\begin{array}{l}09 \\
09 \\
12 \\
12 \\
12\end{array}$ & $\begin{array}{l}01 \\
09 \\
16 \\
16 \\
16\end{array}$ & $\begin{array}{lll}05 & 18 & 46 \\
09 & 21 & 05 \\
11 & 07 & 11 \\
11 & 11 & 34 \\
11 & 50 & 36\end{array}$ & $\begin{array}{ll}39.58 & \mathrm{~N} \\
39.58 & \mathrm{~N} \\
39.283 \mathrm{~N} \\
39.8 \mathrm{~N} \\
39.28 \mathrm{~N}\end{array}$ & $\begin{array}{l}118.45 \\
118.45 \\
118.117 \\
118.1 \\
118.12\end{array}$ & $\begin{array}{l}W \\
W \\
7 W \\
W \\
W\end{array}$ & $\begin{array}{l}- \\
\overline{015} \\
040 \\
-\end{array}$ & $\begin{array}{l}324 \\
324 \\
358 \\
358 \\
324\end{array}$ & $\begin{array}{l}- \\
z\end{array}$ & $\bar{z}$ & $\begin{array}{ll}5.50 \mathrm{M}_{\mathrm{L}} & \text { BRK } \\
4.90 \mathrm{M}_{\mathrm{L}} & \text { BRK } \\
7.20 \mathrm{M}_{\mathrm{L}} & \text { BRK } \\
7.10 \mathrm{M}_{\mathrm{L}} & \text { BRK } \\
5.00 \mathrm{M}_{\mathrm{L}} & \text { BRK }\end{array}$ & $\begin{array}{l}- \\
\text { 7.25HHT } \\
\text { 6.90SAW } \\
-\end{array}$ & $\begin{array}{r}\text { Felt } \\
\text { Felt } \\
X \\
X \\
\end{array}$ & $\begin{array}{l}27 \\
54 \\
27 \\
27 \\
-\end{array}$ & $\frac{-}{600} \frac{-}{-}$ \\
\hline
\end{tabular}


NEVADA-Continued

[See table 1 for hypocenter and intensity references and table 2 for definitions of magnitude source codes. @, felt area is less than $1,000 \mathrm{~km}{ }^{2}$. Leader (-) indicates information is not available]

\begin{tabular}{|c|c|c|c|c|c|c|c|c|c|c|c|c|c|}
\hline & & Origin & & Нypoce & & & & & Magnitude & & Inte & sity & \\
\hline & Date & time (UTC) & Latitude & Longitude & Depth & Ref & US & & Other & Moment & MMI & Ref & Felt area \\
\hline $\mathrm{Yr}$ & Mo Da & $h \mathrm{~m} s$ & $\left({ }^{\circ}\right)$ & $\left({ }^{\circ}\right)$ & $(\mathrm{km})$ & & $m_{b}$ & $\mathbf{M}_{\mathbf{S}}$ & & $\mathbf{M}$ & & & $\left.000 \mathrm{~km}^{2}\right)$ \\
\hline $\begin{array}{l}1954 \\
1954 \\
1954 \\
1954 \\
1954\end{array}$ & $\begin{array}{ll}12 & 16 \\
12 & 16 \\
12 & 16 \\
12 & 16 \\
12 & 16\end{array}$ & $\begin{array}{lll}11 & 5730 \\
13 & 1503 \\
14 & 1657 \\
1424 & 10 \\
150942\end{array}$ & $\begin{array}{ll}39.28 & \mathrm{~N} \\
39.28 & \mathrm{~N} \\
39.28 & \mathrm{~N} \\
39.28 & \mathrm{~N} \\
39.28 & \mathrm{~N}\end{array}$ & $\begin{array}{l}118.12 \\
118.12 \\
118.12 \\
118.12 \\
118.12\end{array}$ & $\overline{-}$ & $\begin{array}{l}324 \\
324 \\
324 \\
324 \\
324\end{array}$ & $\overline{-}$ & $\begin{array}{l}- \\
- \\
-\end{array}$ & $\begin{array}{ll}5.00 M_{L} & \text { BRK } \\
5.00 M_{L} & \text { BRK } \\
5.80 M_{L} & \text { BRK } \\
5.30 M_{L} & \text { BRK } \\
5.10 M_{L} & \text { BRK }\end{array}$ & $\begin{array}{l}- \\
- \\
-\end{array}$ & $\frac{-}{I V}$ & $\frac{-}{\overline{27}}$ & - \\
\hline $\begin{array}{l}1954 \\
1954 \\
1954 \\
1954 \\
1954\end{array}$ & $\begin{array}{ll}12 & 16 \\
12 & 17 \\
12 & 17 \\
12 & 17 \\
12 & 18\end{array}$ & $\begin{array}{l}214843 \\
101526 \\
103329 \\
202706 \\
014536\end{array}$ & $\begin{array}{ll}39.28 & \mathrm{~N} \\
39.28 & \mathrm{~N} \\
39.28 & \mathrm{~N} \\
39.28 & \mathrm{~N} \\
39.28 & \mathrm{~N}\end{array}$ & $\begin{array}{l}118.12 \\
118.12 \\
118.12 \\
118.12 \\
118.12\end{array}$ & $\overline{-}$ & $\begin{array}{l}324 \\
324 \\
324 \\
324 \\
324\end{array}$ & $\overline{-}$ & $\begin{array}{l}z \\
z\end{array}$ & $\begin{array}{ll}4.50 \mathrm{M}_{\mathrm{L}} & \text { BRK } \\
4.50 \mathrm{M}_{\mathrm{L}} & \text { BRK } \\
4.70 \mathrm{M}_{\mathrm{L}} & \text { BRK } \\
5.00 \mathrm{M}_{\mathrm{L}} & \text { BRK } \\
4.70 \mathrm{M}_{\mathrm{L}} & \text { BRK }\end{array}$ & $\begin{array}{l}- \\
z \\
-\end{array}$ & $\begin{array}{l}- \\
z\end{array}$ & $\begin{array}{l}- \\
-\end{array}$ & $\begin{array}{l}- \\
-\end{array}$ \\
\hline $\begin{array}{l}1954 \\
1955 \\
1955 \\
1955 \\
1955\end{array}$ & $\begin{array}{ll}12 & 20 \\
01 & 01 \\
01 & 05 \\
01 & 09 \\
01 & 11\end{array}$ & $\begin{array}{l}173647 \\
121354 \\
082040 \\
091050 \\
102140\end{array}$ & $\begin{array}{ll}39.28 & \mathrm{~N} \\
39.0 & \mathrm{~N} \\
39 . & \mathrm{N} \\
39.0 & \mathrm{~N} \\
39.0 & \mathrm{~N}\end{array}$ & $\begin{array}{l}118.12 \\
118.0 \\
118 . \\
118.0 \\
118.0\end{array}$ & $\begin{array}{l}- \\
z \\
-\end{array}$ & $\begin{array}{r}324 \\
54 \\
54 \\
28 \\
54\end{array}$ & $\overline{-}$ & $\begin{array}{l}- \\
- \\
-\end{array}$ & $\begin{array}{cl}5.00 \mathrm{M}_{\mathrm{L}} & \text { BRK } \\
5.10 \mathrm{M}_{\mathrm{L}} & \text { BRK } \\
-\overline{\mathrm{M}}_{\mathrm{L}} & \\
5.00 \mathrm{M}_{\mathrm{L}} & \text { BRK } \\
4.70 \mathrm{M}_{\mathrm{L}} & \text { BRK }\end{array}$ & $\begin{array}{l}z \\
z\end{array}$ & $\begin{array}{c}\text { Felt } \\
\frac{\mathrm{v}}{-}\end{array}$ & $\begin{array}{l}\frac{27}{54} \\
-\end{array}$ & - \\
\hline $\begin{array}{l}1955 \\
1955 \\
1955 \\
1955 \\
1955\end{array}$ & $\begin{array}{ll}01 & 19 \\
01 & 25 \\
02 & 11 \\
02 & 19 \\
03 & 08\end{array}$ & $\begin{array}{lll}02 & 10 & 10 \\
23 & 26 & 46 \\
16 & 12 & 32 \\
23 & 50 & 07 \\
20 & 05 & 17\end{array}$ & $\begin{array}{ll}39.35 & \mathbf{N} \\
39.0 & \mathbf{N} \\
39.47 & \mathbf{N} \\
39.3 & \mathbf{N} \\
39.20 & \mathbf{N}\end{array}$ & $\begin{array}{l}118.25 \\
118.0 \\
118.10 \\
117.8 \\
118.55\end{array}$ & $\begin{array}{l}- \\
-\end{array}$ & $\begin{array}{r}324 \\
54 \\
324 \\
324 \\
324\end{array}$ & 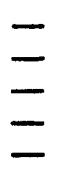 & $\begin{array}{l}- \\
z \\
-\end{array}$ & $\begin{array}{ll}4.60 \mathrm{M}_{\mathrm{L}} & \text { BRK } \\
4.70 \mathrm{M}_{\mathrm{L}} & \text { BRK } \\
4.70 \mathrm{M}_{\mathrm{L}} & \text { BRK } \\
4.80 \mathrm{M}_{\mathrm{L}} & \text { BRK } \\
4.50 \mathrm{M}_{\mathrm{L}} & \text { BRK }\end{array}$ & $\begin{array}{l}- \\
- \\
-\end{array}$ & $\begin{array}{l}\text { IV } \\
- \\
-\end{array}$ & $\frac{28}{-}$ & $\begin{array}{l}- \\
-\end{array}$ \\
\hline $\begin{array}{l}1955 \\
1955 \\
1955 \\
1955 \\
1955\end{array}$ & $\begin{array}{ll}03 & 11 \\
03 & 13 \\
03 & 14 \\
05 & 08 \\
05 & 30\end{array}$ & $\begin{array}{lll}14 & 23 & 16 \\
08 & 41 & 04 \\
18 & 23 & 47 \\
1038 & 31 \\
2128 & 26\end{array}$ & $\begin{array}{l}39.3 \mathrm{~N} \\
39.57 \mathrm{~N} \\
39.42 \mathrm{~N} \\
38.933 \mathrm{~N} \\
39.42 \mathrm{~N}\end{array}$ & $\begin{array}{l}118.1 \\
118.05 \\
118.25 \\
118.117 \\
118.00\end{array}$ & $\overline{-}$ & $\begin{array}{l}324 \\
324 \\
324 \\
324 \\
324\end{array}$ & $\overline{-}$ & $\begin{array}{l}- \\
-\end{array}$ & $\begin{array}{ll}\text { 4.50M } & \text { BRK } \\
4.60 \mathrm{M}_{\mathrm{L}} & \text { BRK } \\
4.70 \mathrm{M}_{\mathrm{L}} & \text { BRK } \\
4.50 \mathrm{M}_{\mathrm{L}} & \text { BRK } \\
4.50 \mathrm{M}_{\mathrm{L}} & \mathrm{BRK}\end{array}$ & $\begin{array}{l}z \\
z \\
-\end{array}$ & $\begin{array}{l}- \\
- \\
-\end{array}$ & $\begin{array}{l}- \\
- \\
-\end{array}$ & 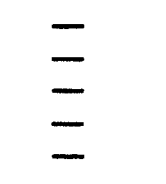 \\
\hline $\begin{array}{l}1955 \\
1955 \\
1955 \\
1955 \\
1955\end{array}$ & $\begin{array}{ll}06 & 08 \\
06 & 19 \\
06 & 19 \\
08 & 08 \\
09 & 29\end{array}$ & $\begin{array}{l}122211 \\
192000 \\
192516 \\
103535 \\
054051\end{array}$ & $\begin{array}{ll}38.88 & \mathbf{N} \\
38.97 & \mathbf{N} \\
39.0 & \mathbf{N} \\
38.33 & \mathbf{N} \\
39.22 & \mathbf{N}\end{array}$ & $\begin{array}{l}118.17 \\
118.25 \\
118.5 \\
118.67 \\
118.20\end{array}$ & $\overline{-}$ & $\begin{array}{l}324 \\
324 \\
324 \\
324 \\
324\end{array}$ & $\overline{-}$ & $\begin{array}{l}z \\
z\end{array}$ & $\begin{array}{ll}4.50 \mathrm{M}_{\mathrm{L}} & \text { BRK } \\
5.20 \mathrm{M}_{\mathrm{L}} & \text { BRK } \\
5.00 \mathrm{M}_{\mathrm{L}} & \text { BRK } \\
5.20 \mathrm{M}_{\mathrm{L}} & \text { BRK } \\
4.50 \mathrm{M}_{\mathrm{L}} & \text { BRK }\end{array}$ & $\begin{array}{l}- \\
z \\
-\end{array}$ & $\frac{\bar{z}}{\bar{v}}$ & $\frac{-}{\overline{28}}$ & $\overline{-}$ \\
\hline $\begin{array}{l}1955 \\
1955 \\
1955 \\
1955 \\
1955\end{array}$ & $\begin{array}{ll}11 & 02 \\
11 & 21 \\
12 & 22 \\
12 & 22 \\
12 & 31\end{array}$ & $\begin{array}{llll}06 & 15 & 17 \\
20 & 25 & 33 \\
12 & 05 & 07 \\
12 & 06 & 54 \\
13 & 5104\end{array}$ & $\begin{array}{l}39.50 \mathrm{~N} \\
39.42 \mathrm{~N} \\
38.98 \mathrm{~N} \\
38.98 \mathrm{~N} \\
39.00 \mathrm{~N}\end{array}$ & $\begin{array}{l}118.05 \\
118.08 \\
118.70 \\
118.70 \\
118.03\end{array}$ & $\begin{array}{l}- \\
- \\
-\end{array}$ & $\begin{array}{l}324 \\
324 \\
324 \\
324 \\
324\end{array}$ & $\frac{-}{-}$ & $\begin{array}{l}z \\
z\end{array}$ & $\begin{array}{ll}4.60 \mathrm{M}_{\mathrm{L}} & \text { BRK } \\
5.50 \mathrm{M}_{\mathrm{L}} & \text { BRK } \\
4.80 \mathrm{M}_{\mathrm{L}} & \text { BRK } \\
4.60 \mathrm{M}_{\mathrm{L}} & \text { BRK } \\
4.50 \mathrm{M}_{\mathrm{L}} & \text { BRK }\end{array}$ & $\begin{array}{l}z \\
z\end{array}$ & $\begin{array}{l}- \\
- \\
-\end{array}$ & $\begin{array}{l}- \\
-\end{array}$ & $\bar{z}$ \\
\hline $\begin{array}{l}1956 \\
1956 \\
1956 \\
1956 \\
1956\end{array}$ & $\begin{array}{ll}03 & 08 \\
07 & 06 \\
07 & 26 \\
11 & 16 \\
12 & 31\end{array}$ & $\begin{array}{lll}07 & 2620 \\
03 & 3135 \\
09 & 53 & 17 \\
08 & 26 & 10 \\
17 & 3745\end{array}$ & $\begin{array}{l}39.03 \mathrm{~N} \\
38.45 \mathrm{~N} \\
39.55 \mathrm{~N} \\
41.03 \mathrm{~N} \\
38.25 \mathrm{~N}\end{array}$ & $\begin{array}{l}118.07 \\
118.62 \\
118.45 \\
116.45 \\
118.93\end{array}$ & $\begin{array}{l}- \\
-\end{array}$ & $\begin{array}{l}324 \\
324 \\
324 \\
324 \\
324\end{array}$ & $\frac{-}{-}$ & $\begin{array}{l}\overline{-} \\
\overline{-}\end{array}$ & $\begin{array}{ll}4.60 \mathrm{M}_{\mathrm{L}} & \text { BRK } \\
4.90 \mathrm{M}_{\mathrm{L}} & \text { BRK } \\
5.10 \mathrm{M}_{\mathrm{L}} & \text { BRK } \\
4.70 \mathrm{M}_{\mathrm{L}} & \text { BRK } \\
5.00 \mathrm{M}_{\mathrm{L}} & \text { BRK }\end{array}$ & $\begin{array}{l}- \\
z \\
-\end{array}$ & $\begin{array}{c}\text { V } \\
\text { IV } \\
\text { V } \\
\text { VI }\end{array}$ & $\begin{array}{l}29 \\
29 \\
29 \\
29\end{array}$ & $\frac{-}{\overline{15}}$ \\
\hline $\begin{array}{l}1956 \\
1957 \\
1958 \\
1958 \\
1959\end{array}$ & $\begin{array}{ll}12 & 31 \\
10 & 17 \\
01 & 04 \\
04 & 19 \\
03 & 23\end{array}$ & $\begin{array}{lll}173924 \\
101409 \\
172734 \\
090102 \\
071020\end{array}$ & $\begin{array}{ll}38.28 & \mathbf{N} \\
39.28 & \mathbf{N} \\
38.9 & \mathbf{N} \\
36.0 & \mathbf{N} \\
39.60 & \mathbf{N}\end{array}$ & $\begin{array}{l}118.97 \\
118.43 \\
115.5 \\
114.8 \\
118.02\end{array}$ & $\frac{-}{-}$ & $\begin{array}{r}324 \\
324 \\
324 \\
54 \\
324\end{array}$ & $\begin{array}{l}- \\
- \\
-\end{array}$ & $\begin{array}{l}- \\
-\end{array}$ & $\begin{array}{cl}5.10 \mathrm{M}_{\mathrm{L}} & \text { BRK } \\
4.60 \mathrm{M}_{\mathrm{L}} & \text { BRK } \\
4.50 \mathrm{M}_{\mathrm{L}} & \text { BRK } \\
\overline{-} & \\
6.30 \mathrm{M}_{\mathrm{L}} & \text { BRK }\end{array}$ & $\begin{array}{l}- \\
z \\
-\end{array}$ & $\begin{array}{l}\text { VI } \\
\text { IV } \\
\overline{\text { VI }} \\
\text { VI }\end{array}$ & $\begin{array}{l}29 \\
30 \\
31 \\
32\end{array}$ & $\frac{15}{-}$ \\
\hline $\begin{array}{l}1959 \\
1959 \\
1959 \\
1960 \\
1961\end{array}$ & $\begin{array}{ll}05 & 21 \\
06 & 23 \\
06 & 23 \\
01 & 26 \\
07 & 04\end{array}$ & $\begin{array}{l}175140 \\
143500 \\
150434 \\
041736 \\
045600\end{array}$ & $\begin{array}{ll}39.47 & \mathbf{N} \\
39.08 & \mathbf{N} \\
39.1 & \mathrm{~N} \\
38.0 & \mathrm{~N} \\
40.90 & \mathrm{~N}\end{array}$ & $\begin{array}{l}118.12 \\
118.82 \\
118.8 \\
116.5 \\
118.40\end{array}$ & $\overline{-}$ & $\begin{array}{r}324 \\
324 \\
324 \\
33 \\
324\end{array}$ & $\frac{-}{-}$ & $\begin{array}{l}z \\
z\end{array}$ & $\begin{array}{ll}4.80 \mathrm{M}_{\mathrm{L}} & \text { BRK } \\
6.10 \mathrm{M}_{\mathrm{L}} & \text { BRK } \\
5.50 \mathrm{M}_{\mathrm{L}} & \text { BRK } \\
4.90 \mathrm{M}_{\mathrm{L}} & \text { BRK } \\
5.40 \mathrm{M}_{\mathrm{L}} & \text { BRK }\end{array}$ & $\begin{array}{l}z \\
z\end{array}$ & $\begin{array}{l}\bar{v} \\
\mathrm{v} \\
\bar{v}\end{array}$ & $\begin{array}{l}\overline{32} \\
\frac{32}{34}\end{array}$ & $\frac{\overline{105}}{\overline{26}}$ \\
\hline $\begin{array}{l}1961 \\
1961\end{array}$ & $\begin{array}{ll}07 & 04 \\
08 & 04\end{array}$ & $\begin{array}{l}110911 \\
165609.1\end{array}$ & $\begin{array}{l}40.13 \mathrm{~N} \\
39.2 \mathrm{~N}\end{array}$ & $\begin{array}{l}118.60 \\
117.4\end{array}$ & $\overline{012}$ & $\begin{array}{l}324 \\
324\end{array}$ & $=$ & - & $\begin{array}{ll}5.00 \mathrm{M}_{\mathrm{L}} & \text { BRK } \\
4.50 \mathrm{M}_{\mathrm{L}} & \text { BRK }\end{array}$ & - & $\underline{\text { IV }}$ & 34 & - \\
\hline
\end{tabular}


NEVADA-Continued

\begin{tabular}{|c|c|c|c|c|c|c|c|c|c|c|c|c|c|c|c|}
\hline \multirow{2}{*}{\multicolumn{3}{|c|}{ Date }} & \multirow{2}{*}{$\begin{array}{c}\text { Origin } \\
\text { time (UTC) } \\
\end{array}$} & \multicolumn{5}{|c|}{ Hypocenter } & \multicolumn{4}{|c|}{ Magnitude } & \multicolumn{2}{|c|}{ Intensity } & \multirow{3}{*}{$\begin{array}{l}\text { Felt area } \\
\left(1,000 \mathrm{~km}^{2}\right)\end{array}$} \\
\hline & & & & \multirow{2}{*}{$\begin{array}{l}\text { Latitude } \\
\left(^{\circ}\right)\end{array}$} & \multirow{2}{*}{\multicolumn{2}{|c|}{$\begin{array}{l}\text { Longitude } \\
\left({ }^{\circ}\right)\end{array}$}} & \multirow{2}{*}{$\begin{array}{l}\text { Depth } \\
(\mathrm{km})\end{array}$} & \multirow[t]{2}{*}{ Ref } & \multicolumn{2}{|c|}{ USGS } & \multirow[t]{2}{*}{ Other } & \multirow{2}{*}{$\begin{array}{c}\text { Moment } \\
\text { M }\end{array}$} & \multirow[t]{2}{*}{ MMI } & \multirow[t]{2}{*}{ Ref } & \\
\hline$Y_{r}$ & Mo & $\mathrm{Da}$ & $h \mathrm{~m} s$ & & & & & & $m_{b}$ & $M_{s}$ & & & & & \\
\hline $\begin{array}{l}1962 \\
1962 \\
1962\end{array}$ & $\begin{array}{l}04 \\
07 \\
09\end{array}$ & $\begin{array}{l}25 \\
20 \\
07\end{array}$ & $\begin{array}{lll}08 & 48 & 58.2 \\
09 & 02 & 10.0 \\
23 & 19 & 12.8\end{array}$ & $\begin{array}{ll}38.5 & \mathbf{N} \\
39.5 & \mathbf{N} \\
41.3 & \mathbf{N}\end{array}$ & $\begin{array}{ll}118.1 & V \\
118.2 & V \\
116.7 & V\end{array}$ & $\begin{array}{l}\mathbf{W} \\
\mathbf{W} \\
\mathbf{W}\end{array}$ & $\begin{array}{l}025 \\
033 \\
033\end{array}$ & $\begin{array}{r}35 \\
266 \\
266\end{array}$ & - & $\overline{-}$ & $\begin{array}{ll}4.60 \mathrm{M}_{\mathrm{L}} & \text { BRK } \\
4.70 \mathrm{M}_{\mathrm{L}} & \text { BRK } \\
4.60 \mathrm{M}_{\mathrm{L}} & \text { PAS }\end{array}$ & - & $\begin{array}{l}\mathbf{V} \\
\mathbf{v} \\
-\end{array}$ & $\begin{array}{l}35 \\
35 \\
-\end{array}$ & $\overline{35}$ \\
\hline $\begin{array}{l}1962 \\
1963 \\
1964 \\
1964 \\
1964\end{array}$ & $\begin{array}{l}12 \\
03 \\
03 \\
10 \\
10\end{array}$ & $\begin{array}{l}15 \\
25 \\
22 \\
23 \\
30\end{array}$ & $\begin{array}{l}063459.8 \\
092843.0 \\
163055.2 \\
135710.6 \\
190312.3\end{array}$ & $\begin{array}{ll}40.7 & \mathbf{N} \\
36.0 & \mathbf{N} \\
38.8 & \mathbf{N} \\
38.5 & \mathbf{N} \\
37.7 & \mathbf{N}\end{array}$ & $\begin{array}{l}117.5 \\
114.8 \\
118.7 \\
118.4 \\
118.0\end{array}$ & $\begin{array}{l}\mathbf{W} \\
\mathbf{W} \\
\mathbf{W} \\
\mathbf{W} \\
\mathbf{W}\end{array}$ & $\begin{array}{l}\overline{019} \\
016 \\
026 \\
020\end{array}$ & $\begin{array}{l}266 \\
266 \\
266 \\
266 \\
266\end{array}$ & $\begin{array}{l}-5.3 \\
4.5 \\
5.0 \\
4.4\end{array}$ & $\begin{array}{l}- \\
z\end{array}$ & $\begin{array}{ll}4.90 \mathrm{M}_{\mathrm{L}} & \text { BRK } \\
4.90 \mathrm{M}_{\mathrm{L}} & \text { PAS } \\
5.50 \mathrm{M}_{\mathrm{L}} & \text { BRK } \\
5.30 \mathrm{M}_{\mathrm{L}} & \text { BRK } \\
4.50 \mathrm{M}_{\mathrm{L}} & \text { BRK }\end{array}$ & $\frac{-}{z}$ & $\begin{array}{l}\overline{V I} \\
\mathrm{~V} \\
\mathrm{~V} \\
\mathrm{VI}\end{array}$ & $\begin{array}{l}\overline{36} \\
37 \\
37 \\
37\end{array}$ & $\begin{array}{l}\overline{45} \\
28 \\
-\end{array}$ \\
\hline $\begin{array}{l}1965 \\
1966 \\
1966 \\
1966 \\
1966\end{array}$ & $\begin{array}{l}04 \\
01 \\
04 \\
08 \\
08\end{array}$ & $\begin{array}{l}13 \\
28 \\
02 \\
16 \\
16\end{array}$ & $\begin{array}{l}131422.1 \\
180009.1 \\
124838.8 \\
180232.9 \\
195009.5\end{array}$ & $\begin{array}{ll}38.9 & \mathrm{~N} \\
41.6 & \mathrm{~N} \\
38.4 & \mathrm{~N} \\
37.46 & \mathrm{~N} \\
37.4 & \mathrm{~N}\end{array}$ & $\begin{array}{l}117.7 \\
118.2 \\
118.2 \\
114.15 \\
114.2\end{array}$ & $\begin{array}{l}\mathbf{W} \\
\mathbf{W} \\
\mathbf{W} \\
\mathbf{W} \\
\mathbf{W}\end{array}$ & $\begin{array}{l}033 \\
020 \\
009 \\
007 \\
033\end{array}$ & $\begin{array}{l}266 \\
266 \\
266 \\
298 \\
266\end{array}$ & $\begin{array}{l}4.6 \\
4.4 \\
4.0 \\
5.6 \\
4.6\end{array}$ & $\begin{array}{l}- \\
z\end{array}$ & $\begin{array}{ll}4.60 \mathrm{M}_{\mathrm{L}} & \text { BRK } \\
4.80 \mathrm{M}_{\mathrm{L}} & \text { BRK } \\
4.80 \mathrm{M}_{\mathrm{L}} & \text { BRK } \\
5.60 \mathrm{M}_{\mathrm{L}} & \text { UU } \\
4.50 \mathrm{M}_{\mathrm{L}} & \text { PAS }\end{array}$ & $\frac{\bar{Z}}{5.31 \mathrm{DOS}}$ & $\begin{array}{l}\overline{\text { III }} \\
\mathrm{VI} \\
\mathrm{V}\end{array}$ & $\begin{array}{l}-\overline{81} \\
81 \\
81 \\
-\end{array}$ & $\frac{-}{\frac{-}{66}}$ \\
\hline $\begin{array}{l}1966 \\
1966 \\
1966 \\
1966 \\
1966\end{array}$ & $\begin{array}{l}08 \\
08 \\
08 \\
08 \\
08\end{array}$ & $\begin{array}{l}17 \\
18 \\
18 \\
18 \\
19\end{array}$ & $\begin{array}{l}230758.9 \\
06 \quad 1501.3 \\
091537.5 \\
173506.4 \\
105137.9\end{array}$ & $\begin{array}{ll}37.3 & \mathbf{N} \\
37.3 & \mathbf{N} \\
37.3 & \mathbf{N} \\
37.4 & \mathbf{N} \\
37.438 \mathrm{~N}\end{array}$ & $\begin{array}{l}114.1 \\
114.2 \\
114.2 \\
114.2 \\
114.191 Y\end{array}$ & $\begin{array}{l}\mathbf{W} \\
\mathbf{W} \\
\mathbf{W} \\
\mathbf{W} \\
\mathbf{W}\end{array}$ & $\begin{array}{l}033 \\
034 \\
033 \\
033 \\
007\end{array}$ & $\begin{array}{l}266 \\
266 \\
266 \\
266 \\
298\end{array}$ & $\begin{array}{l}4.9 \\
4.2 \\
4.6 \\
5.2 \\
4.2\end{array}$ & $\begin{array}{l}- \\
z \\
-\end{array}$ & $\begin{array}{l}5.50 \mathrm{M}_{\mathrm{L}} \text { BRK } \\
4.50 \mathrm{M}_{\mathrm{L}} \text { PAS } \\
5.00 \mathrm{M}_{\mathrm{L}} \text { PAS } \\
5.00 \mathrm{M}_{\mathrm{L}} \text { PAS } \\
4.70 \mathrm{M}_{\mathrm{L}} \text { UU }\end{array}$ & $\begin{array}{l}- \\
z \\
z\end{array}$ & $\begin{array}{c}\text { Felt } \\
\text { IV } \\
\text { IV }\end{array}$ & $\begin{array}{r}81 \\
81 \\
259 \\
-\end{array}$ & $\begin{array}{c}- \\
- \\
-\end{array}$ \\
\hline $\begin{array}{l}1966 \\
1966 \\
1966 \\
1966 \\
1966\end{array}$ & $\begin{array}{l}08 \\
09 \\
09 \\
09 \\
09\end{array}$ & $\begin{array}{l}22 \\
04 \\
22 \\
22 \\
22\end{array}$ & $\begin{array}{lll}08 & 2730.2 \\
11 & 23 & 18.1 \\
18 & 5641.0 \\
18 & 57 & 36.5 \\
19 & 5939.8\end{array}$ & $\begin{array}{ll}37.3 & \mathbf{N} \\
37.4 & \mathbf{N} \\
37.3 & \mathbf{N} \\
37.4 & \mathbf{N} \\
37.3 & \mathbf{N}\end{array}$ & $\begin{array}{l}114.2 \\
114.2 \\
114.2 \\
114.2 \\
114.2\end{array}$ & $\begin{array}{l}\mathbf{W} \\
\mathbf{W} \\
\mathbf{W} \\
\mathbf{W} \\
\mathbf{W}\end{array}$ & $\begin{array}{l}033 \\
033 \\
033 \\
033 \\
033\end{array}$ & $\begin{array}{r}266 \\
266 \\
266 \\
74 \\
266\end{array}$ & $\begin{array}{l}4.3 \\
4.2 \\
4.5 \\
5.3 \\
4.4\end{array}$ & $\begin{array}{l}- \\
z\end{array}$ & $\begin{array}{ll}4.60 \mathrm{M}_{\mathrm{L}} & \text { BRK } \\
4.80 \mathrm{M}_{\mathrm{L}} & \text { BRK } \\
5.30 \mathrm{M}_{\mathrm{L}} & \text { PAS } \\
5.50 \mathrm{M}_{\mathrm{L}} & \text { PAS } \\
4.50 \mathrm{M}_{\mathrm{L}} & \text { PAS }\end{array}$ & $\begin{array}{l}z \\
z\end{array}$ & $\frac{-}{\text { Felt }}$ & $\begin{array}{l}- \\
81 \\
81 \\
-\end{array}$ & $\frac{-}{-}$ \\
\hline $\begin{array}{l}1966 \\
1966 \\
1966 \\
1966 \\
1966\end{array}$ & $\begin{array}{l}09 \\
10 \\
10 \\
10 \\
10\end{array}$ & $\begin{array}{l}23 \\
02 \\
22 \\
25 \\
26\end{array}$ & $\begin{array}{l}115609.4 \\
153941.2 \\
171626.4 \\
163932.9 \\
151738.6\end{array}$ & $\begin{array}{ll}37.3 & \mathbf{N} \\
37.3 & \mathbf{N} \\
40.6 & \mathbf{N} \\
37.3 & \mathbf{N} \\
37.3 & \mathbf{N}\end{array}$ & $\begin{array}{l}114.1 \\
114.2 \\
116.3 \\
114.2 \\
114.2\end{array}$ & $\begin{array}{l}\mathbf{W} \\
\mathbf{W} \\
\mathbf{W} \\
\mathbf{W} \\
\mathbf{W}\end{array}$ & $\begin{array}{l}033 \\
033 \\
032 \\
033 \\
033\end{array}$ & $\begin{array}{l}266 \\
266 \\
266 \\
266 \\
266\end{array}$ & $\begin{array}{l}4.5 \\
4.5 \\
4.5 \\
4.4 \\
4.3\end{array}$ & $\begin{array}{l}z \\
z\end{array}$ & $\begin{array}{ll}4.50 \mathrm{M}_{\mathrm{L}} & \text { PAS } \\
4.50 \mathrm{M}_{\mathrm{L}} & \text { BRK } \\
5.10 \mathrm{M}_{\mathrm{L}} & \text { BRK } \\
4.90 \mathrm{M}_{\mathrm{L}} & \text { BRK } \\
5.10 \mathrm{M}_{\mathrm{L}} & \text { BRK }\end{array}$ & $\bar{z}$ & $\begin{array}{l}\bar{z} \\
\bar{v}\end{array}$ & $\frac{-}{81}$ & $\begin{array}{c}- \\
-\end{array}$ \\
\hline $\begin{array}{l}1967 \\
1967 \\
1968 \\
1968 \\
1968\end{array}$ & $\begin{array}{l}02 \\
05 \\
01 \\
02 \\
02\end{array}$ & $\begin{array}{l}16 \\
07 \\
30 \\
06 \\
06\end{array}$ & $\begin{array}{l}150554.3 \\
180135.7 \\
152005.6 \\
004138.0 \\
034810.8\end{array}$ & $\begin{array}{l}37.42 \mathrm{~N} \\
37.040 \mathrm{~N} \\
41.0 \mathrm{~N} \\
38.02 \mathrm{~N} \\
38.00 \mathrm{~N}\end{array}$ & $\begin{array}{l}114.18 \\
115.012 \\
117.4 \\
118.35 \\
118.37\end{array}$ & $\begin{array}{l}W \\
W \\
W \\
W \\
W\end{array}$ & $\begin{array}{l}033 \\
015 \\
018 \\
- \\
-\end{array}$ & $\begin{array}{r}266 \\
74 \\
74 \\
324 \\
324\end{array}$ & $\begin{array}{l}4.8 \\
4.7 \\
4.5 \\
4.6 \\
4.4\end{array}$ & $\begin{array}{l}z \\
z\end{array}$ & $\begin{array}{cl}4.80 \mathrm{M}_{\mathrm{L}} & \text { BRK } \\
5.13 \mathrm{M}_{\mathrm{L}} & \mathrm{PAS} \\
-\overline{-} & \\
4.90 \mathrm{M}_{\mathrm{L}} & \mathrm{BRK} \\
4.50 \mathrm{M}_{\mathrm{L}} & \mathrm{BRK}\end{array}$ & $\begin{array}{l}- \\
z\end{array}$ & $\begin{array}{r}\overline{I V} \\
V \\
V \\
\text { Felt }\end{array}$ & $\begin{array}{l}\overline{40} \\
41 \\
41 \\
41\end{array}$ & $\begin{array}{l}- \\
9 \\
21 \\
-\end{array}$ \\
\hline $\begin{array}{l}1968 \\
1968 \\
1968 \\
1970 \\
1971\end{array}$ & $\begin{array}{l}05 \\
05 \\
07 \\
03 \\
12\end{array}$ & $\begin{array}{l}22 \\
29 \\
06 \\
28 \\
08\end{array}$ & $\begin{array}{l}132155.7 \\
114107.1 \\
140240.0 \\
093844 \\
171856.0\end{array}$ & $\begin{array}{ll}38.6 & \mathrm{~N} \\
39.07 & \mathrm{~N} \\
41.1 & \mathrm{~N} \\
38.95 & \mathrm{~N} \\
37.615 \mathrm{~N}\end{array}$ & $\begin{array}{l}116.2 \\
118.05 \\
117.4 \\
116.40 \\
115.288\end{array}$ & $\begin{array}{l}W \\
W \\
W \\
W \\
W\end{array}$ & $\begin{array}{l}013 \\
003 \\
\overline{-} \\
\overline{008}\end{array}$ & $\begin{array}{r}74 \\
324 \\
324 \\
324 \\
292\end{array}$ & $\begin{array}{l}5.1 \\
4.9 \\
5.1 \\
4.5 \\
4.8\end{array}$ & $\begin{array}{l}z \\
z\end{array}$ & $\begin{array}{ll}4.90 \mathrm{M}_{\mathrm{L}} & \text { BRK } \\
4.90 \mathrm{M}_{\mathrm{L}} & \text { BRK } \\
5.50 \mathrm{M}_{\mathrm{L}} & \text { BRK } \\
4.50 \mathrm{M}_{\mathrm{L}} & \text { BRK } \\
4.70 \mathrm{M}_{\mathrm{L}} & \text { PAS }\end{array}$ & $\begin{array}{l}z \\
z\end{array}$ & $\frac{\text { Felt }}{v}$ & $\frac{\frac{41}{41}}{44}$ & $\frac{\bar{z}}{\frac{25}{34}}$ \\
\hline $\begin{array}{l}1972 \\
1973 \\
1973 \\
1978 \\
1978\end{array}$ & $\begin{array}{l}12 \\
03 \\
03 \\
02 \\
03\end{array}$ & $\begin{array}{l}09 \\
02 \\
03 \\
14 \\
05\end{array}$ & $\begin{array}{l}024445.7 \\
112842.3 \\
030003.3 \\
043524.0 \\
224618.2\end{array}$ & $\begin{array}{l}38.676 \mathrm{~N} \\
41.831 \mathrm{~N} \\
41.810 \mathrm{~N} \\
39.63 \mathrm{~N} \\
38.94 \mathrm{~N}\end{array}$ & $\begin{array}{l}115.639 \\
118.546 \\
118.457 \\
117.18 \\
118.03\end{array}$ & $\begin{array}{l}W \\
W \\
W \\
W \\
W\end{array}$ & $\begin{array}{l}010 \\
005 \\
005 \\
005 \\
005\end{array}$ & $\begin{array}{r}74 \\
74 \\
74 \\
240 \\
240\end{array}$ & $\begin{array}{l}4.4 \\
4.2 \\
4.7 \\
4.4 \\
4.0\end{array}$ & $\begin{array}{l}- \\
z\end{array}$ & $\begin{array}{ll}4.60 \mathrm{M}_{\mathrm{L}} & \text { BRK } \\
4.60 \mathrm{M}_{\mathrm{L}} & \text { BRK } \\
4.60 \mathrm{M}_{\mathrm{L}} & \text { BRK } \\
4.80 \mathrm{M}_{\mathrm{L}} & \text { BRK } \\
4.60 \mathrm{M}_{\mathrm{L}} & \text { BRK }\end{array}$ & $\begin{array}{l}- \\
\bar{z}\end{array}$ & $\frac{\bar{Z}}{\mathrm{IV}}$ & $\begin{array}{c}\bar{Z} \\
\overline{240} \\
240\end{array}$ & $\begin{array}{c}- \\
- \\
-\end{array}$ \\
\hline $\begin{array}{l}1978 \\
1979 \\
1980 \\
1980 \\
1980\end{array}$ & $\begin{array}{l}05 \\
12 \\
04 \\
09 \\
09\end{array}$ & $\begin{array}{l}23 \\
31 \\
08 \\
04 \\
04\end{array}$ & $\begin{array}{l}054755.4 \\
082752.5 \\
001341.8 \\
13 \quad 3909.4 \\
210334.1\end{array}$ & $\begin{array}{l}40.868 \mathrm{~N} \\
38.460 \mathrm{~N} \\
39.499 \mathrm{~N} \\
38.083 \mathrm{~N} \\
38.113 \mathrm{~N}\end{array}$ & $\begin{array}{l}117.257 \\
118.428 \\
119.178 \\
118.570 \\
118.560\end{array}$ & $\begin{array}{l}W \\
W \\
W \\
W \\
W\end{array}$ & $\begin{array}{l}011 \\
008 \\
005 \\
001 \\
010\end{array}$ & $\begin{array}{r}74 \\
401 \\
74 \\
401 \\
401\end{array}$ & $\begin{array}{l}4.1 \\
4.2 \\
4.0 \\
4.9\end{array}$ & $\begin{array}{l}z \\
z\end{array}$ & $\begin{array}{ll}4.60 \mathrm{M}_{\mathrm{L}} & \text { BRK } \\
4.80 \mathrm{M}_{\mathrm{L}} & \text { BRK } \\
4.70 \mathrm{M}_{\mathrm{L}} & \text { BRK } \\
4.60 \mathrm{M}_{\mathrm{L}} & \text { BRK } \\
4.90 \mathrm{M}_{\mathrm{L}} & \text { BRK }\end{array}$ & $\begin{array}{l}- \\
- \\
-\end{array}$ & $\begin{array}{l}\text { V } \\
\text { V } \\
\text { V } \\
\text { V } \\
\text { III }\end{array}$ & $\begin{array}{l}240 \\
262 \\
300 \\
300 \\
300\end{array}$ & $\begin{array}{l}- \\
21 \\
21 \\
-\end{array}$ \\
\hline $\begin{array}{l}1980 \\
1980 \\
1980 \\
1980 \\
1980\end{array}$ & $\begin{array}{l}09 \\
09 \\
09 \\
09 \\
09\end{array}$ & $\begin{array}{l}06 \\
07 \\
07 \\
07 \\
07\end{array}$ & $\begin{array}{l}072752.3 \\
013042.8 \\
043638.2 \\
064810.6 \\
064830.6\end{array}$ & $\begin{array}{l}38.078 \mathrm{~N} \\
38.083 \mathrm{~N} \\
38.083 \mathrm{~N} \\
38.093 \mathrm{~N} \\
38.093 \mathrm{~N}\end{array}$ & $\begin{array}{l}118.572 \\
118.575 \\
118.600 \\
118.570 \\
118.570\end{array}$ & $\begin{array}{l}W \\
W \\
W \\
W\end{array}$ & $\begin{array}{l}007 \\
007 \\
010 \\
005 \\
005\end{array}$ & $\begin{array}{l}401 \\
401 \\
401 \\
401 \\
401\end{array}$ & $\begin{array}{l}4.1 \\
4.4 \\
4.9 \\
4.7\end{array}$ & $\frac{\overline{ }}{\overline{5.0}}$ & $\begin{array}{ll}4.60 \mathrm{M}_{\mathrm{L}} & \text { BRK } \\
5.10 \mathrm{M}_{\mathrm{L}} & \text { BRK } \\
5.50 \mathrm{M}_{\mathrm{L}} & \text { BRK } \\
4.70 \mathrm{M}_{\mathrm{L}} & \text { BRK } \\
5.30 \mathrm{M}_{\mathrm{L}} & \text { BRK }\end{array}$ & $\bar{z}$ & $\begin{array}{l}\text { Felt } \\
\text { Felt } \\
\text { V } \\
\text { Felt } \\
\text { Felt }\end{array}$ & $\begin{array}{l}300 \\
300 \\
300 \\
300 \\
300\end{array}$ & $\frac{-}{56}$ \\
\hline
\end{tabular}


[See table 1 for hypocenter and intensity references and table 2 for definitions of magnitude source codes. @, felt area is less than $1,000 \mathrm{~km}^{2}$. Leader (--) indicates information is not available

\begin{tabular}{|c|c|c|c|c|c|c|c|c|c|c|c|c|c|}
\hline \multirow{2}{*}{\multicolumn{2}{|c|}{ Date }} & \multirow{2}{*}{$\begin{array}{c}\text { Origin } \\
\text { time (UTC) }\end{array}$} & \multicolumn{4}{|c|}{ Hypocenter } & \multicolumn{4}{|c|}{ Magnitude } & \multicolumn{2}{|c|}{ Intensity } & \multirow{3}{*}{$\begin{array}{l}\text { Felt area } \\
\left(1,000 \mathrm{~km}^{2}\right)\end{array}$} \\
\hline & & & \multirow{2}{*}{$\begin{array}{l}\text { Latitude } \\
\left({ }^{\circ}\right)\end{array}$} & \multirow{2}{*}{$\begin{array}{l}\text { Longitude } \\
\left({ }^{\circ}\right)\end{array}$} & \multirow{2}{*}{$\begin{array}{l}\text { Depth } \\
(\mathrm{km})\end{array}$} & \multirow[t]{2}{*}{ Ref } & \multicolumn{2}{|c|}{ USGS } & \multirow[t]{2}{*}{ Other } & \multirow{2}{*}{$\begin{array}{c}\text { Moment } \\
\text { M }\end{array}$} & \multirow[t]{2}{*}{ MMI } & \multirow[t]{2}{*}{ Ref } & \\
\hline$\overline{Y r}$ & Mo Da & $h \mathrm{~m} s$ & & & & & $m_{b}$ & $\mathbf{M}_{\mathbf{S}}$ & & & & & \\
\hline $\begin{array}{l}1980 \\
1980 \\
1980 \\
1980 \\
1981\end{array}$ & $\begin{array}{ll}09 & 08 \\
09 & 16 \\
11 & 11 \\
12 & 28 \\
01 & 28\end{array}$ & $\begin{array}{l}042619.8 \\
042441.1 \\
101903.0 \\
225809.8 \\
200850.7\end{array}$ & $\begin{array}{l}38.045 \mathrm{~N} \\
38.047 \mathrm{~N} \\
38.040 \mathrm{~N} \\
38.157 \mathrm{~N} \\
38.202 \mathrm{~N}\end{array}$ & $\begin{array}{l}118.600 \mathrm{~W} \\
118.567 \mathrm{~W} \\
118.590 \mathrm{~W} \\
118.360 \mathrm{~W} \\
118.335 \mathrm{~W}\end{array}$ & $\begin{array}{l}008 \\
007 \\
005 \\
005 \\
015\end{array}$ & $\begin{array}{l}401 \\
401 \\
401 \\
401 \\
401\end{array}$ & $\begin{array}{l}\frac{\overline{4}}{4.2} \\
\frac{4.6}{4.5}\end{array}$ & $\bar{z}$ & $\begin{array}{ll}4.60 \mathrm{M}_{\mathrm{L}} & \text { BRK } \\
4.70 \mathrm{M}_{\mathrm{L}} & \text { BRK } \\
4.70 \mathrm{M}_{\mathrm{L}} & \text { BRK } \\
5.00 \mathrm{M}_{\mathrm{L}} & \text { BRK } \\
4.60 \mathrm{M}_{\mathrm{L}} & \text { BRK }\end{array}$ & $\begin{array}{l}\bar{z} \\
\bar{z}\end{array}$ & $\begin{array}{l}\text { IV } \\
\text { IV } \\
\text { III } \\
\text { IV } \\
\text { IV }\end{array}$ & $\begin{array}{l}300 \\
300 \\
300 \\
300 \\
325\end{array}$ & $\frac{7}{10}$ \\
\hline $\begin{array}{l}1981 \\
1982 \\
1982 \\
1982 \\
1982\end{array}$ & $\begin{array}{ll}04 & 28 \\
01 & 28 \\
04 & 15 \\
09 & 24 \\
12 & 28\end{array}$ & 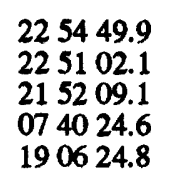 & $\begin{array}{l}38.058 \mathrm{~N} \\
38.54 \mathrm{~N} \\
38.052 \mathrm{~N} \\
37.870 \mathrm{~N} \\
38.028 \mathrm{~N}\end{array}$ & $\begin{array}{l}118.587 \mathrm{~W} \\
118.07 \mathrm{~W} \\
118.568 \mathrm{~W} \\
118.140 \mathrm{~W} \\
118.418 \mathrm{~W}\end{array}$ & $\begin{array}{l}010 \\
005 \\
007 \\
017 \\
008\end{array}$ & $\begin{array}{l}401 \\
350 \\
401 \\
401 \\
401\end{array}$ & $\begin{array}{l}4.2 \\
4.5 \\
5.0 \\
4.7\end{array}$ & $\frac{\bar{z}}{4.6}$ & $\begin{array}{ll}4.60 \mathrm{M}_{\mathrm{L}} & \text { BRK } \\
4.60 \mathrm{M}_{\mathrm{L}} & \text { BRK } \\
5.10 \mathrm{M}_{\mathrm{L}} & \text { BRK } \\
5.50 \mathrm{M}_{\mathrm{L}} & \text { BRK } \\
4.90 \mathrm{M}_{\mathrm{L}} & \text { BRK }\end{array}$ & $\bar{z}$ & $\begin{array}{l}\text { IV } \\
\text { V } \\
\text { IV } \\
\text { V } \\
\text { IV }\end{array}$ & $\begin{array}{l}325 \\
350 \\
350 \\
350 \\
350\end{array}$ & $\begin{array}{l}\overline{-} \\
45 \\
50 \\
-\end{array}$ \\
\hline $\begin{array}{l}1984 \\
1984 \\
1986 \\
1987 \\
1987\end{array}$ & $\begin{array}{ll}02 & 16 \\
02 & 17 \\
11 & 01 \\
07 & 28 \\
07 & 29\end{array}$ & $\begin{array}{lll}11 & 14 & 58.8 \\
12 & 03 & 55.8 \\
19 & 23 & 38.3 \\
18 & 55 & 11.5 \\
03 & 52 & 32.1\end{array}$ & $\begin{array}{l}39.858 \mathrm{~N} \\
38.854 \mathrm{~N} \\
38.712 \mathrm{~N} \\
38.385 \mathrm{~N} \\
38.378 \mathrm{~N}\end{array}$ & $\begin{array}{l}117.556 \mathrm{~W} \\
119.630 \mathrm{~W} \\
119.540 \mathrm{~W} \\
118.168 \mathrm{~W} \\
118.137 \mathrm{~W}\end{array}$ & $\begin{array}{l}002 \\
012 \\
017 \\
013 \\
014\end{array}$ & $\begin{array}{r}475 \\
475 \\
562 \\
74 \\
74\end{array}$ & $\begin{array}{l}4.0 \\
4.1 \\
4.4\end{array}$ & $\begin{array}{l}\bar{z} \\
\bar{z}\end{array}$ & $\begin{array}{ll}5.20 \mathrm{M}_{\mathrm{L}} & \text { BRK } \\
4.60 \mathrm{M}_{\mathrm{D}} & \text { REN } \\
4.60 \mathrm{M}_{\mathrm{L}} & \text { BRK } \\
4.60 \mathrm{M}_{\mathrm{L}} & \text { BRK } \\
4.50 \mathrm{M}_{\mathrm{L}} & \text { BRK }\end{array}$ & $\begin{array}{c}\overline{-} \\
4.37 \mathrm{BRK} \\
\overline{-}\end{array}$ & $\begin{array}{l}\text { IV } \\
\text { V } \\
\text { V } \\
\text { IV } \\
\text { Felt }\end{array}$ & $\begin{array}{r}370 \\
562 \\
577 \\
74\end{array}$ & $\frac{\bar{z}}{\frac{15}{}}$ \\
\hline 1988 & 0919 & 025631.7 & $38.461 \mathrm{~N}$ & $118.342 \mathrm{~W}$ & 009 & 74 & 4.5 & - & $5.30 \mathrm{M}_{\mathrm{L}} \quad \mathrm{BRK}$ & - & $\mathbf{v}$ & 578 & 24 \\
\hline
\end{tabular}

[Reference (Ref.) numbers given in parentheses at the end of each description refer to sources of data in table 1. Magnitude values are described in the Introduction, and codes are defined in table 2.]

1860. Mar. 15. Near Carson City, Ormsby County, Nev. Rockslides were reported between Pyramid Lake and Carson City. Merchandise was shaken from shelves at Carson City. Also reported felt in parts of California and Utah. (Ref. 358, 368.)

1868. May 30, 0510 UTC (May 29). Near Virginia City, Storey County, Nev. Brick buildings were cracked and bricks were shaken down at Virginia City. Plaster fell in almost all brick buildings. (Ref. 54, 368.)

1869. Dec. 27, 0155 UTC (Dec. 26). Near Virginia City, Storey County, Nev. Masonry walls were damaged severely in Virginia City and at Washoe City, $5 \mathrm{~km}$ west. Plaster fell at Mokelume Hill, and a stone chimney fell at Spring Gulch. Minor damage also was reported in Calaveras County in California. (Ref. 368.)

1869. Dec. 27. Western Nevada aftershock. Considerable damage occurred at Carson City, Dayton, Genoa, Steamboat, and Virginia City. Damage also was reported at Downieville and Oroville, Calif. It is difficult to separate the damage caused by this shock from that of the main shock at 0155 UTC, Dec. 27. (Ref. 38, 368.)

1872. Mar. 23. Austin, southern Lander County, Nev. Plaster fell in the Austin Courthouse.
The earthquake was described as a violent shock. (Ref. 38, 54.)

1875. Apr. 2 (Apr. 1). Near Eureka, Nev. One building was seriously damaged at Eureka. (Ref. 54.)

1887. June 3. Near Carson City, Ormsby County, Nev. The ground was reported to have been uplifted about $10 \mathrm{~m}$ north of Carson City (at Deadman's Ranch). Large fissures opened and spouted water and sand near Cradlebaugh's Bridge. The water level at Shaw's Hot Springs, near Carson City, dropped several centimeters a few weeks before the earthquake, and the water dried up after the shock. At Carson City, chimneys were reported to be in "bad condition" and brick and stone walls were cracked. At Genoa, houses were shifted on their foundations and bricks were thrown down. (Ref. 54, 368.)

1894. Nov. 18. Near Virginia City, Storey County, Nev. Some walls were damaged, plaster cracked, and windows broke at Virginia City. Many aftershocks occurred. (Ref. 54, 56.)

1896. Jan. 27. Near Carson City, Ormsby County, Nev. A large crack formed in the side of a government building in Carson City; plaster fell in the County Building. (Ref. 54,56 .)

1901. July 26. Near Elko, Nev. A brick schoolhouse and other buildings were damaged at Elko. A series of heavy shocks was reported. (Ref. 54,56.)

1903. Fall. Near Wonder, Churchill County, Nev. Interviews with long-time residents of the area establishes (with a fair degree of certainty) the date 
as the fall of 1903 for this uncataloged earthquake in Churchill County. Evidence also suggests that as much as $19 \mathrm{~km}$ of surface faulting may have occurred on the Gold King fault as result of this shock. At the Stephens dwelling, about $8 \mathrm{~km}$ northeast of Wonder, an adobe house was damaged (probably by this earthquake) and its roof required repair. (Ref. 54, 328.)

1910. Nov. 7. Southeast of Goldfield, in Nye County, Nev. A few dishes and windows were broken at Goldfield. (Ref. 54, 56.)

1910. Nov. 21. Esmeralda County, Nev. The watchman's car was thrown from the track at Tonopah Junction; windows were broken. A succession of shocks occurred at the same time as a loud, rumbling noise. (Ref. 54, 56.)

1914. Feb. 18. Near Reno, Washoe County, Nev. Plaster cracked and a few bricks were thrown from chimneys at Reno and nearby Virginia City. Windows were broken at Sparks, and the shock was severe at Verdi. Also felt in California. (Ref. $38,54,56$.

1914. Apr. 24. Near Reno, Washoe County, Nev. This earthquake probably originated northeast of Reno. Many chimneys were toppled throughout Reno, including four that fell on buildings at the University of Nevada. The shock was described as "severe" about $25 \mathrm{~km}$ east of Reno, at Hazen. Also felt in California. Magnitude $5.5 \mathrm{M}_{\mathrm{S}}$ ELL, $5.0 \mathrm{M}_{\mathrm{La}}$ DMG. (Ref. 54, 56.)

1915. Oct. 3 (Oct. 2). Pleasant Valley, Nev. This earthquake occurred along a fault on the eastern side of Pleasant Valley, which lies about $64 \mathrm{~km}$ southeast of Winnemucca, in the north-central part of Nevada. The epicentral region was almost uninhabited, and, therefore, property damage was less than might have been expected. Damage was confined mainly to an area within $80 \mathrm{~km}$ of the fault in Humboldt, Lander, and Pershing Counties, including the towns of Battle Mountain, Kennedy, Lovelock, Winnemucca, and several ranches in Pleasant Valley. Four main scarps-the China Mountain, Tobin, Pearce, and Sou Hills-developed in a right-stepping en echelon pattern. The combined length of the scarps was $59 \mathrm{~km}$, the average vertical displacement $2 \mathrm{~m}$, and the maximum displacement (which occurred on the Pearce scarp) $5.8 \mathrm{~m}$. Several northwest-striking segments of the scarps had a right-lateral component of displacement, generally less than $1 \mathrm{~m}$.

At Kennedy, two adobe houses were destroyed, mine tunnels collapsed, and concrete mine foundations were cracked. At Winnemucca, adobe buildings generally were damaged, and several multistory brick buildings lost their coping and parts of upper walls; many chimneys were demolished above the rooflines. In addition, water tanks were thrown down at Battle Mountain, Kodiak, Lovelock, and Parran. Damage occurred on several ranches at the southern end of Pleasant Valley: an adobe house was shaken down; a masonry chicken house and a hog pen were destroyed; and houses were displaced from their foundations.

One of the most striking effects of this earthquake was the large increase (and decrease) in the flow of springs and streams throughout northern Nevada. Cracks formed in unconsolidated materials for considerable distances. Felt from the State of Oregon to southern California and from the Pacific coast to beyond Salt Lake City, Utah. (see fig. 40). Two foreshocks and many aftershocks occurred. Magnitude $7.3 \mathrm{~m}_{\mathrm{b}} \mathrm{ABE}, 7.75 \mathrm{M}_{\mathrm{S}}$ GR, 7.6 $\mathrm{M}_{\mathrm{S}}$ CFR. (Ref. 56, 258, $327,589$.

1930. Apr. 9. Near Lake Tahoe, Douglas County, Nev. At the southeast end of Lake Tahoe, chimneys were damaged. Plaster was cracked at Tahoe. Also felt in California. (Ref. 3, 324.)

1930. Apr. 12. Fernley, Lyon County, Nev. Chimneys and dishes broke at Fernley; plaster cracked at Fallon, southeast of Fernley, and objects fell from shelves. Also felt in California. (Ref. 3, 324.)

1932. Dec. 21 (Dec. 20). Cedar Mountain, Nev. This major earthquake originated in an uninhabited desert region of western Nevada, near Cedar Mountain, and therefore caused minimal property loss. Two cabins, one of stone and the other of adobe, were destroyed, and ore-treating plants and mines were damaged. The main shock was strong at Fallon, Mina, Luning, Tonopah, and at many other Nevada towns. Many chimneys were downed in Mineral County, at Luning and Mina. In addition, walls fell and cracks formed in the ground at Luning.

Extensive and complicated faulting occurred over an area $63 \mathrm{~km}$ long and 6 to $14.5 \mathrm{~km}$ wide in the valley between Gabbs Valley Range and Pilot Peak on the west and Paradise Range and Cedar Mountains on the east, northeast of Mina. In this area, 60 en echelon rifts as much as $6 \mathrm{~km}$ in length and 122 $\mathrm{m}$ in width were found. The rifts consisted of zones of fissures that commonly revealed vertical displacement, and in several places showed horizontal displacement. Boulders were shaken from cliffs and hillsides in many places; large landslides occurred; and the flow of ground water either increased or decreased in some springs and wells. Felt from the Rocky Mountains to the Pacific Ocean and from San Diego to southern Oregon. (see fig. 41). One foreshock and many aftershocks occurred. Magnitude $7.3 \mathrm{M}_{\mathrm{S}}$ CFR. (Ref. 38, 258, 356.) 


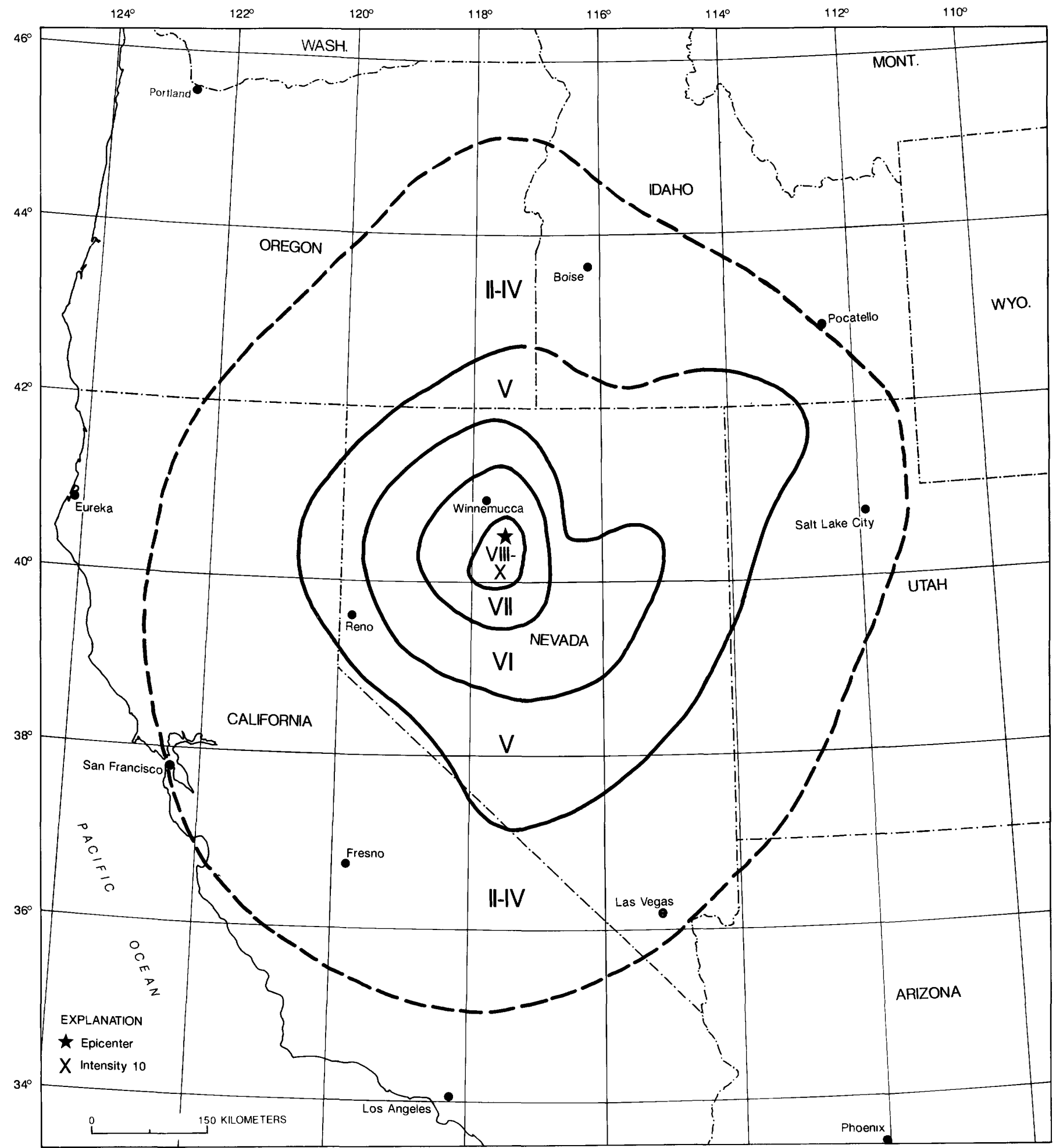

FIGURE 40.- Isoseismal map for the Pleasant Valley, Nevada, earthquake of October 3, 1915. Isoseismals are based on intensity estimates form data listed in references 272 and 327 of table 1. 


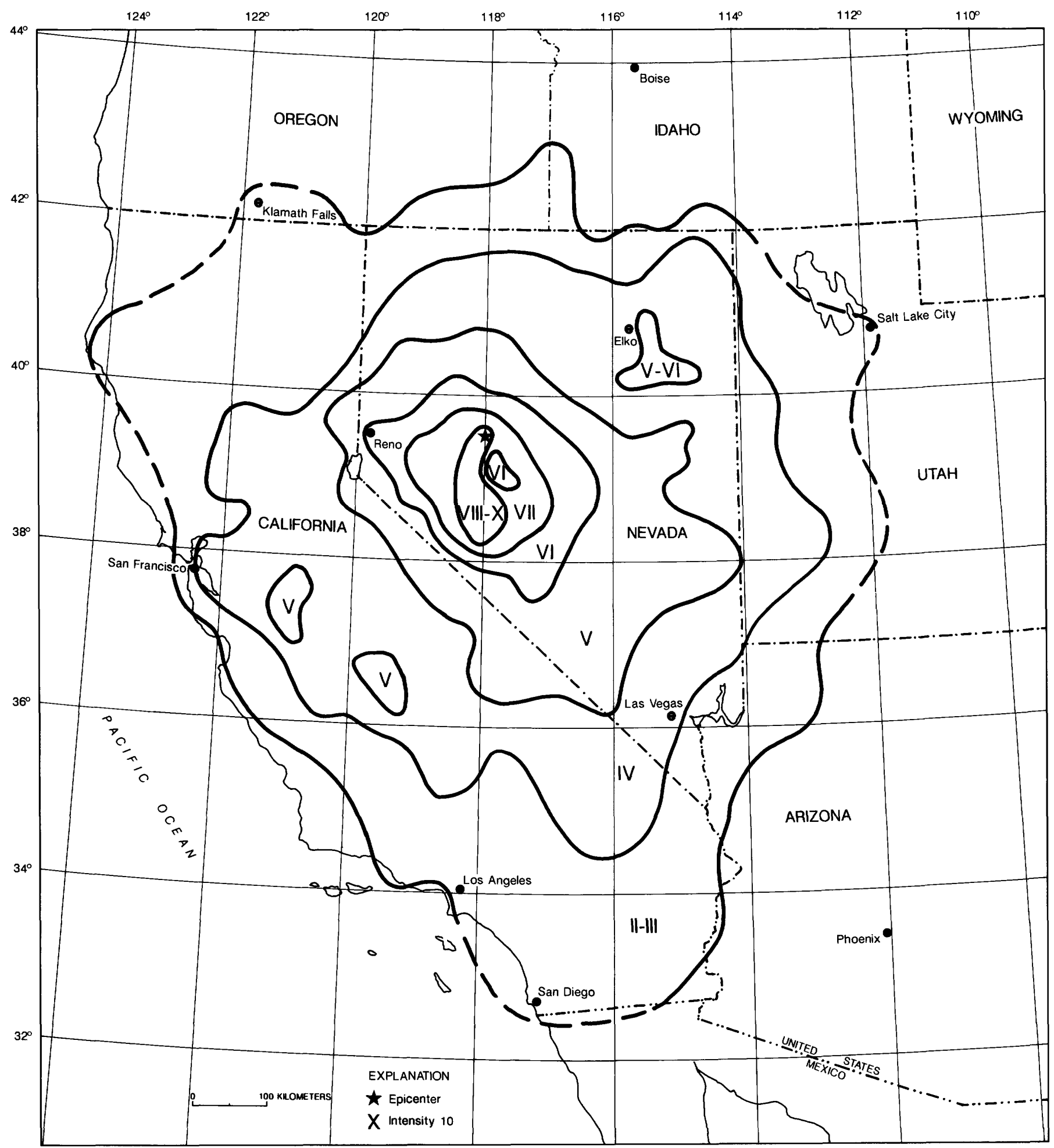

Figure 41.- Isoseismal map for the Cedar Mountain, Nevada, earthquake of December 21, 1932. Isoseismals are based on intensity estimates from data listed in references 5,259 , and 356 of table 1. 
1933. June 25. Western Nevada. Many chimneys were downed southeast of Reno, at Virginia City, and in Lyon County, at Wabuska and Yerington. A church was damaged severely at Virginia City, and a wall separated from the rest of courthouse building at Yerington. Water spurted from cracks that formed in the ground at Yerington. Minor damage occurred in many nearby towns. Also felt in California. (Ref. $6,324$.

1934. Jan. 30, 1923 UTC. Excelsior Mountains, Nev. A strong foreshock of the damaging earthquake at 2016 UTC downed chimneys and broke windows and dishes southeast of Hawthorne, at Mina (Mineral County). Also felt in California. (Ref. 38, 54, 335.)

1934. Jan. 30, 2016 UTC. Excelsior Mountains, Nev. This strong earthquake centered in an uninhabited area having few structures, and damage therefore was slight. At Mina (Mineral County), a few chimneys were broken and a small section of a brick wall fell. At Marietta, two walls of an adobe cabin collapsed; at Candelaria, a stone cabin was partly destroyed; and at the Silver Dike mine in the eastern part of the Excelsior Mountains, a rockslide destroyed a pipeline and a pumphouse. Fissures formed in alluvium; landslides occurred; and changes in the flow of springs were observed.

Fissures in alluvium, mainly related to slumping rather than to primary faulting, were found in three places: above Pepper Spring, south of Garfield Flat; on the northwest side of Teel's Marsh; and at the Endowment mine, about $5 \mathrm{~km}$ north of Marietta. The average trend of the fissures was $\mathrm{N} .10^{\circ} \mathrm{W}$., and the length about $30.5 \mathrm{~m}$. One graben was $6 \mathrm{~m}$ wide and, on its west side, was a slump hole about $0.6 \mathrm{~m}$ deep. At the Endowment mine, two fissures-one $6 \mathrm{~m}$ long and the second $30.5 \mathrm{~m}$ long-formed in the bed of the wash about $122 \mathrm{~m}$ beyond the end of the fault. An earthquake scarp about $1.4 \mathrm{~km}$ long developed on one of the faults $5 \mathrm{~km}$ north of Marietta, on the southern side of the Excelsior Mountains. This scarp had a maximum height of $12.7 \mathrm{~cm}$, and the fissures were as wide as $7.6 \mathrm{~cm}$. It appears that this was the surface expression of the movement that caused the earthquake.

Several landslides occurred, and boulders rolled down slopes throughout the Excelsior Mountains. The shock also was felt throughout central Nevada and central California as far as the west coast. Magnitude 6.3 M $\mathrm{M}_{\mathrm{S}}$ GR. (Ref. 7, 324, 335.)

1939. Jan. 11. Near Gardnerville, Douglas County, western Nevada. Damage was slight at Gardnerville, where cracks formed in plaster, and at
Hudson, where dishes broke. Also felt in California. (Ref. 12, 324.)

1939. May 4. Near Boulder City, Clark County, southern Nevada. At Boulder City, about $15 \mathrm{~km}$ southeast of Las Vegas, plaster cracked and fell. In the Hoover Dam area, large rocks rolled onto roadways and made some of them impassable. A heavy dust cloud noted south of Hoover Dam probably resulted from landslides. Plaster was cracked in several houses in Mohave City and Oatman, Ariz. Also felt in California. (Ref. 12, 292, 343.)

1939. May 11. Northeast of Mina in Nye County, Nev. At Rawhide, vases were overturned and trees and bushes were shaken strongly. Plaster was cracked at Yerington. Felt north to Lovelock, south to Beatty, east to Eureka, and west to Yosemite National Park, Calif. Magnitude 5.5 $\mathrm{M}_{\mathrm{S}}$ GR. (Ref. 12, $38,324$.

1942. Aug. 18. Near Mount Montgomery, Mineral County, Nev. Dishes fell at Mount Montgomery, in southern Mineral County, and lath on veneer board panels cracked loose. Also felt in California. (Ref. 15, 16.)

1942. Dec. 3. Near Reno, Washoe County, Nev. At Reno, plaster was cracked in several buildings, and a heavy safe was shifted several centimeters. Rocks fell onto the highway in Truckee Canyon, east of Sparks. Also felt in California. (Ref. $15,259,324$.

1943. Aug. 9 (Aug. 8). Excelsior Mountains, Nev. Plaster and walls were cracked at Dyer (Esmeralda County) and at Fallon (Churchill County), 80 $\mathrm{km}$ northeast. Also felt in California. Several aftershocks were recorded. (Ref. 16.)

1948. Nov. 2. Hoover Dam, Nev., area. Southeast of Las Vegas, rocks rolled onto the highway leading to Hoover Dam and fell from canyon walls upstream and downstream from the dam. Dust clouds from landslides also were observed. At nearby Boulder City, some minor cracking of plaster occurred. (Ref. 21, 259.)

1952. May 9. Near Carson City, Ormsby County, Nev. New cracks formed in four State buildings at Carson City. Plaster and knickknacks fell at Virginia City, about $20 \mathrm{~km}$ north. Also felt in California. (Ref. 25, 324.)

1952. May 24 (May 23). Near Boulder City, Clark County, Nev. Slight damage occurred southeast of Las Vegas, at Boulder City (cracks in plaster and foundations) and at Whitney (cracks in walls). (Ref. 25, 292.)

1953. Sept. 26 (Sept. 25). Near Reno, Washoe County, Nev. Two chimneys toppled at Reno, and plaster cracked in buildings throughout the 
northwest part of town. Much damage occurred to plaster at the University of Nevada in the Mackay School of Mines Building. Slight damage also was reported in California. (Ref. 26, 324.)

1954. July 6, 1113 UTC. Fallon-Stillwater area, Churchill County, Nev. In Fallon, the town nearest the epicenter, several old and poorly built concrete-block structures and unreinforced brick structures were damaged severely, and many brick chimneys fell. Several people were injured at the Naval Auxiliary Air Station, about $8 \mathrm{~km}$ southeast of Fallon, when the shock knocked heavy steel lockers onto them. Two areas outside Fallon that also sustained damage were the Lone Tree district to the south and the Stillwater district to the east. Ground motion and surface breakage were heavier in Stillwater.

Canals and drainage systems of the Newlands Reclamation Project near Fallon were damaged extensively. Many box-type culverts were damaged or collapsed. Failure of the Coleman Diversion Dam cut off irrigation water to most of the project.

Paved highways in the Fallon-Stillwater areas settled, cracked, and buckled in several places. One of the largest ground movements occurred in the Lone Tree area. One road dropped about $90 \mathrm{~cm}$ for a distance of several hundred meters and lurched about $90 \mathrm{~cm}$ horizontally toward a canal. In the Lone Tree and Stillwater areas, canal banks settled as much as $0.9 \mathrm{~m}$, and bottoms of canals were raised as much as $0.6 \mathrm{~m}$.

The main zone of ground fractures was observed on the east edge of Rainbow Mountain in the Stillwater Range, about $24 \mathrm{~km}$ southeast of Fallon. This earthquake and the large shock on Aug. 24 (see description below) resulted in surface evidence of faulting for about $40 \mathrm{~km}$. This break is referred to as the Rainbow Mountain fault. Vertical displacement was evident along about two-thirds of the fault; the west side uplifted everywhere with respect to the east side. Scarps as high as $30 \mathrm{~cm}$ or more formed, and small grabens developed in places. Horizontal displacement was not found. The quake was also felt in California, Idaho, Oregon, and Utah (see fig. 42). Magnitude 6.6 $\mathrm{M}_{\mathrm{S}}$ CFR, 6.3 $\mathrm{M}_{\mathrm{S}}$ ELL. (Ref. 27, 324, 575.)

1954. July 6, 2207 UTC. Fallon-Stillwater area, Churchill County, Nev., aftershock. U.S. Highway 50 settled to the extent that it had to be filled and resurfaced about $8 \mathrm{~km}$ southeast of Salt Wells. Many observed a large cloud of dust rising in that area, probably a result of landslides. Little additional damage to structures was reported from
Fallon. Also felt in California. Magnitude $6.4 \mathrm{M}_{\mathrm{S}}$ CFR. (Ref. 27, 38, 259, 324.)

1954. Aug. 24 (Aug. 23). Fallon-Stillwater area, Churchill County, Nev. Ground surface movement was increased several centimeters at the break of the main fault of July 6, 1954 (11 13 UTC), along the east edge of Rainbow Mountain. Displacement owing to this shock was much more continuous than that of July 6 , probably as a result of the larger relative movement $(76 \mathrm{~cm}$ compared to $30 \mathrm{~cm}$ on July 6). The ground breakage extended north for about $18 \mathrm{~km}$ to the region southeast of Carson Sink. Only vertical movement was observed, however. Except for the Lovelock area, where this earthquake considerably damaged the Rogers Dam, damage to buildings, roads, and irrigation facilities occurred in the same general areas as for the shock on July 6 . Also felt in California, Idaho, Oregon, and Utah (see fig. 43). Magnitude 6.8 $\mathrm{M}_{\mathrm{S}}$ CFR, 6.9 $\mathrm{M}_{\mathrm{S}}$ ELL. (Ref. 27,324 .)

1954. Aug. 31. Dixie Valley, Churchill County, Nev. A series of violent, earthquakes generated additional landslides in the Stillwater Range northeast of Stillwater. In West Lee Canyon, everything in one cabin was overturned, including the stove. Magnitude 6.3 $\mathrm{M}_{\mathrm{S}}$ CFR. (Ref. 27, 324.)

1954. Dec. 16, 1107 UTC. Dixie Valley-Fairview Peak area, Churchill County, Nev. The population was sparse in the epicentral region of this earthquake, and few man-made structures existed. Damage to structures, therefore, was minor despite the geologic and seismographic evidence of a major earthquake.

The earthquake was accompanied by offsets along many faults in four main zones of a north-trending belt $96 \mathrm{~km}$ long by $32 \mathrm{~km}$ wide. Minor geologic effects included changes in flow of springs and wells, formation of craters and water fountains, landslips and landslides, mudflows, and rockfalls.

The fault displacements mainly were along normal faults in the following areas: (1) west of Dixie Valley, (2) southeast of Dixie Valley, (3) east of Fairview Peak, and (4) east of Stingaree Valley. The maximum strike-slip component was $3.6 \mathrm{~m}$ of right-lateral movement at Fairview Peak, and the maximum vertical-slip component was $3.6 \mathrm{~m}$ at Bell Flat.

Heavy furniture was displaced at Frenchman Station, about $11 \mathrm{~km}$ west of major surface faulting, but damage to buildings was negligible. Differential settlement of about $10 \mathrm{~cm}$ that occurred under a woodframe store resulted in minor cracking of the building. Damage at Fallon, about $48 \mathrm{~km}$ west of the nearest major surface break, was limited to a few toppled chimneys. Hundreds of aftershocks occurred. The 


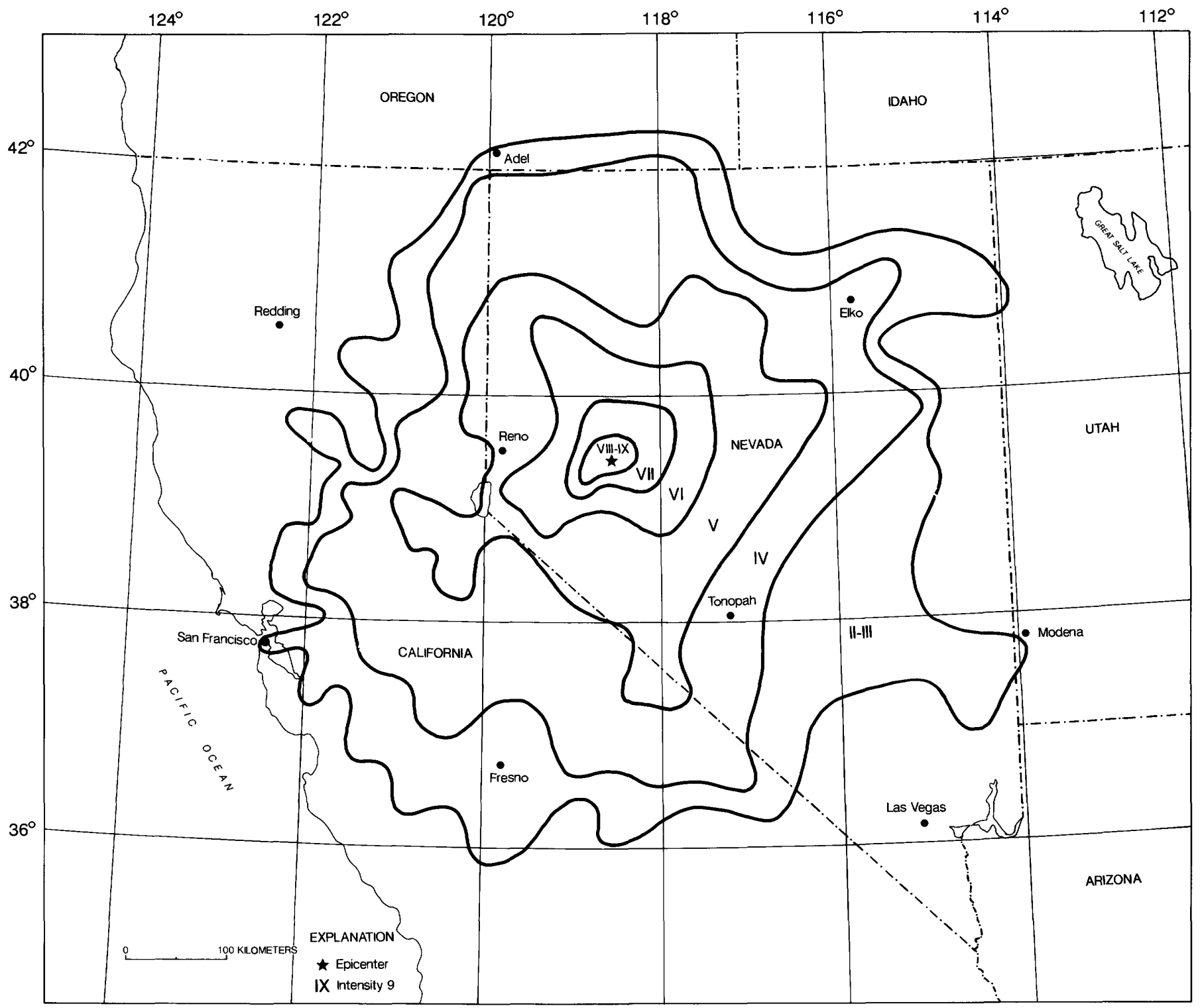

FIGURE 42.- Isoseismal map for the Fallon-Stillwater, Nevada, earthquake of July 6, 1954. Isoseismals are based on intensity estimates from data listed in references 27,259 , and 575 of table 1 .

main earthquake also was felt in Arizona, California, Idaho, Oregon, and Utah (see fig. 44). Magnitude 7.1 $\mathrm{M}_{\mathrm{S}} \mathrm{ABE}, 6.9 \mathrm{~m}_{\mathrm{b}} \mathrm{ABE}, 7.1 \mathrm{M}_{\mathrm{S}}$ CFR. (Ref. 27, 358, 505.)

1954. Dec. 16, 11 11 UTC. Dixie ValleyFairview Peak area, Churchill County, Nev., aftershock. See above description of the main earthquake at 1107 UTC on Dec. 16. Because damage from the two earthquakes cannot be separated, they are treated as one event. Magnitude $6.8 \mathrm{M}_{\mathrm{S}}$ CFR. (Ref. 27, 358.)

1956. Dec. 31, 1737 UTC. Near Hawthorne, Mineral County, Nev. Two moderate earthquakes (the second one at 1739 UTC) cracked U.S. Highway 95, south of Hawthorne, and split Highway 50, near Frenchman Station. Also felt in California. (Ref. 29, 324.)

1956. Dec. 31, 1739 UTC. Near Hawthorne, Mineral County, Nev. See above description of the earthquake at 1737 UTC, Dec. 31, 1956. (Ref. 29, 324.)

1958. Apr. 19. Boulder City area, Clark County, Nev. This minor earthquake cracked plaster, broke dishes, and overturned books and pictures at Boulder City. (Ref. 31, 54.) 


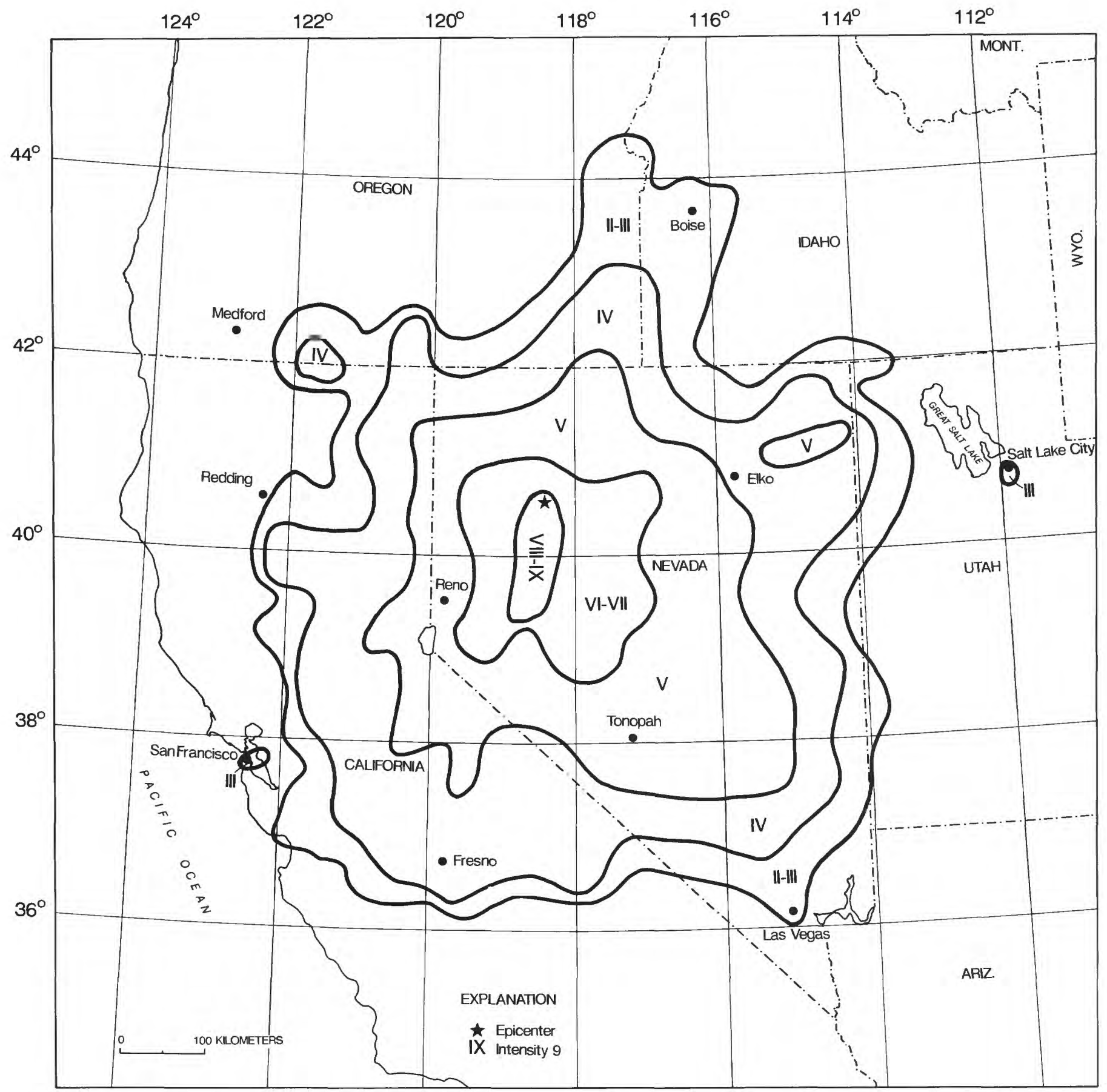

FIGURE 43.- Isoseismal map for the Fallon-Stillwater, Nevada, earthquake of August 24, 1954. Isoseismals are based on intensity estimates from data listed in references 27, 259, and 575 of table 1.

1959. Mar. 23 (Mar. 22). Dixie Valley area, Churchill County, Nev. Plaster cracked at Carson City and Frenchman Station, and the top of a new addition to the State printing office was damaged. A field investigation in Dixie Valley revealed no sign of fresh cracking on the Fairview Peak fault where it crosses U.S. Highway 50, the Dixie Valley Road northward to Horse Creek Road, nor the Horse Creek Road; fresh cracking was not noted on the extension of the Gold King fault at the south edge of Dixie Valley. Also felt in California. (Ref. 32, 259, 324.)

1959. June 23. Near Schurz, northeast Mineral County, Nev. This was the main shock of a series of four moderate earthquakes. Chimneys 


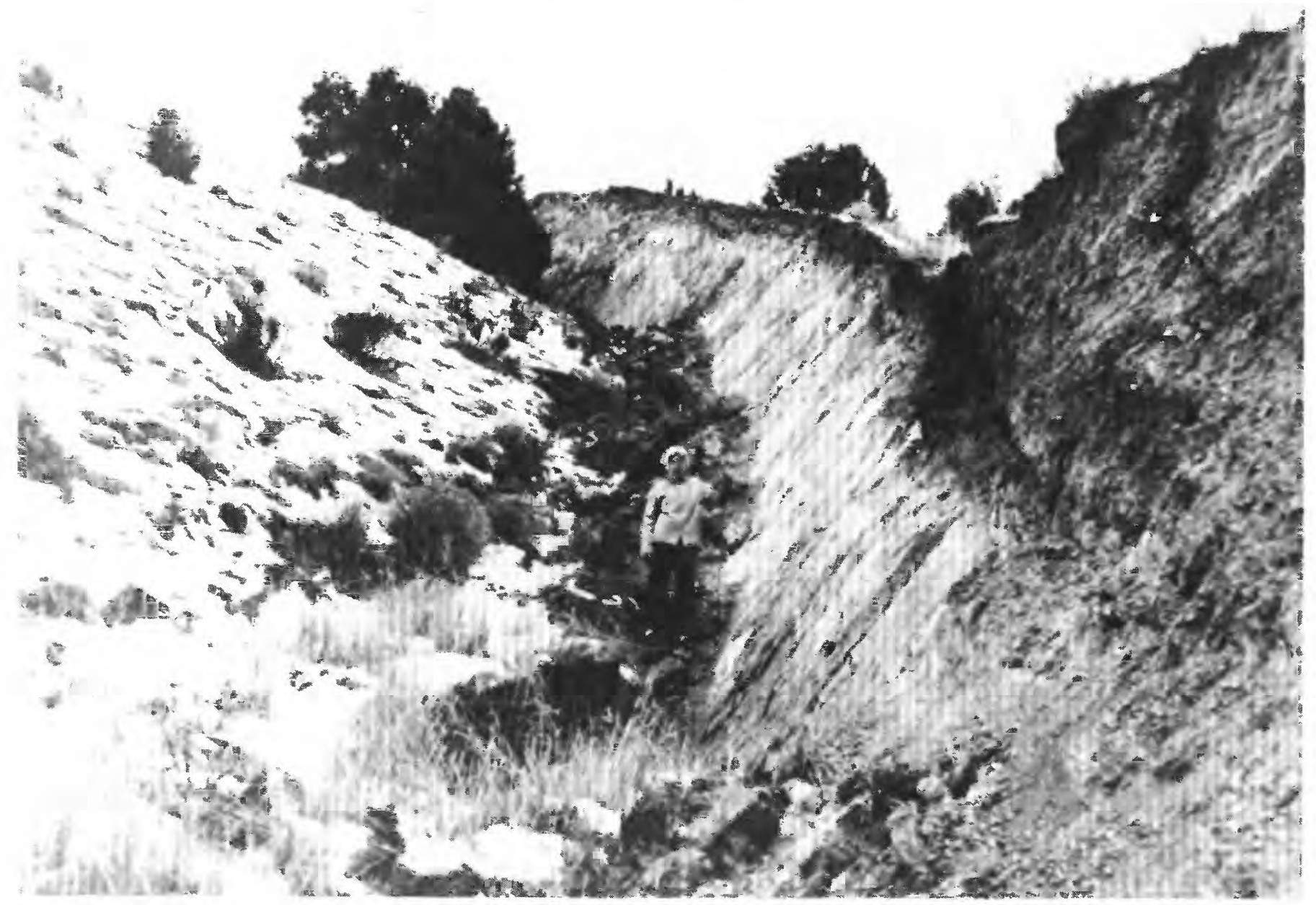

Fault scarp in the Fairview Peak area, Nevada, formed by the December 16, 1954, earthquake. (Photograph from the National Geophysical Data Center, NOAA.)

twisted and cracked at Schurz, and a garage attached to a frame house separated $5 \mathrm{~cm}$ from the house. A new roadbed, about $8 \mathrm{~km}$ west of Schurz, was cracked; plaster cracked and knickknacks fell at Genoa. Some residents reported minor rockslides in the adjacent mountains. Also felt in California. (Ref. $32,324$.

1963. Mar. 25. Near Boulder City, Clark County, Nev. Considerable minor damage-mainly cracked and fallen plaster and broken dishesoccurred at Boulder City. Several rockslides were observed in the Boulder City area. Power was interrupted for 20 minutes at Hoover powerplant and dam, and one generator possibly was misaligned. Also felt in Arizona, California, and Utah. (Ref. 36, $259,266$.

1964. Oct. 30. Dyer area, Esmeralda County, Nev. A series of earthquakes occurred along the California-Nevada border, all of which were preceded by a roaring noise. The strongest shock (at 1903 UTC) cracked a concrete-block foundation and a window at Dyer. (Ref. 37, 259, 266.)

1966. Apr. 2. South of Luning, Mineral County, Nev. This earthquake separated two parts of an old building at Luning. Small objects shifted and fell. (Ref. 81, 266.) 


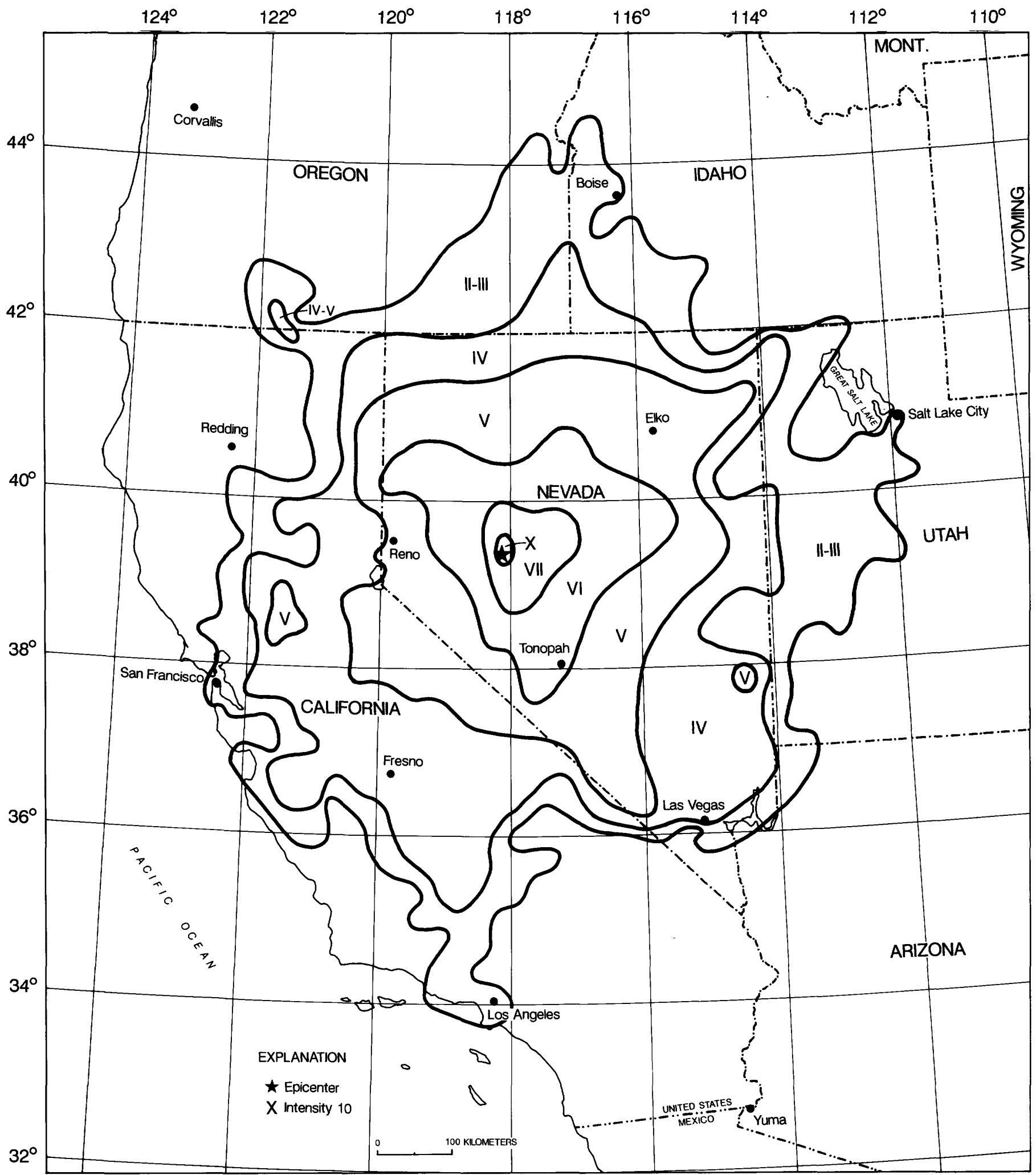

Figure 44.- Isoseismal map for the Dixie Valley-Fairview Peak, Nevada, earthquake of December 16, 1954. Isoseismals are based on intensity estimates from data listed in references 27 and 259 of table 1 . 



\section{NEW HAMPSHIRE}

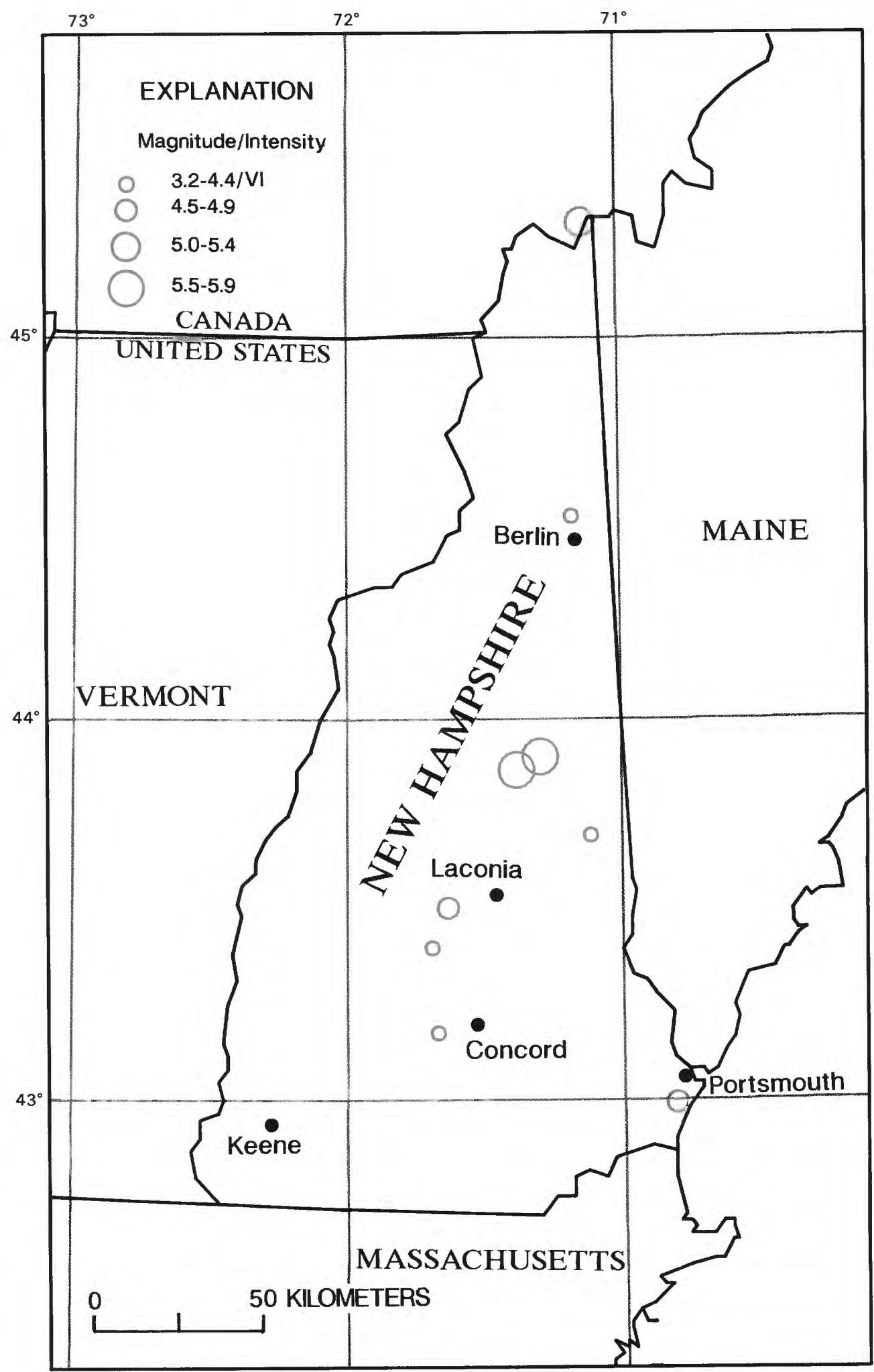

Earthquakes in New Hampshire with magnitudes $\geq 4.5$ or intensity $\geq$ VI. 
NEW HAMPSHIRE

[See table 1 for hypocenter and intensity references and table 2 for definitions of magnitude source codes. \&, land area only. Leader (--) indicates information is not available]

\begin{tabular}{|c|c|c|c|c|c|c|c|c|c|c|c|c|c|}
\hline \multirow{2}{*}{\multicolumn{2}{|c|}{ Date }} & \multirow{2}{*}{$\begin{array}{c}\text { Origin } \\
\text { time (UTC) }\end{array}$} & \multicolumn{4}{|c|}{ Hypocenter } & \multicolumn{4}{|c|}{ Magnitude } & \multicolumn{2}{|c|}{ Intensity } & \multirow{3}{*}{$\begin{array}{c}\text { Felt area } \\
\left(1,000 \mathrm{~km}^{2}\right)\end{array}$} \\
\hline & & & \multirow{2}{*}{$\begin{array}{l}\text { Latitude } \\
\left({ }^{\circ}\right)\end{array}$} & \multirow{2}{*}{$\begin{array}{l}\text { Longitude } \\
\left({ }^{\circ}\right)\end{array}$} & \multirow{2}{*}{$\begin{array}{l}\text { Depth } \\
(\mathrm{km})\end{array}$} & \multirow[t]{2}{*}{ Ref } & \multicolumn{2}{|c|}{ USGS } & \multirow[t]{2}{*}{ Other } & \multirow{2}{*}{$\begin{array}{c}\text { Moment } \\
\text { M }\end{array}$} & \multirow[t]{2}{*}{ MM } & \multirow[t]{2}{*}{ Ref } & \\
\hline $\mathrm{Yr}$ & Mo Da & $h \mathrm{~ms}$ & & & & & $m_{b}$ & $M_{s}$ & & & & & \\
\hline 1732 & 0916 & 1600 & $45.5 \mathrm{~N}$ & 73.6. W & - & 76 & - & - & - & - & $\mathbf{I X}$ & 76 & - \\
\hline 1810 & 1110 & 0215 & $43.0 \mathrm{~N}$ & $70.8 \mathrm{~W}$ & - & 78 & - & - & - & 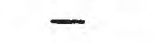 & VI & & 21\& \\
\hline 1925 & 1009 & 1355 & $43.7 \mathrm{~N}$ & $71.1 \mathrm{~W}$ & - & 76 & - & - & $4.00 \mathrm{M}_{\mathrm{fa}} \mathrm{SC}$ & - & VI & 8 & 18 \\
\hline 1940 & 1220 & 072726.2 & $43.872 \mathrm{~N}$ & $71.370 \mathrm{~W}$ & 010 & 349 & - & - & $5.50 \mathrm{M}_{\mathrm{n}} \mathrm{ST}$ & $5.25 \mathrm{ST}$ & VII & 13 & 356\& \\
\hline 1940 & 1224 & 134345.0 & $43.908 \mathrm{~N}$ & $71.283 \mathrm{~W}$ & 008 & 349 & - & - & $5.50 \mathrm{M}_{\mathrm{n}} \mathrm{ST}$ & 5.60ESM & VII & 13 & - \\
\hline 1964 & 0626 & 49.0 & $43.405 \mathrm{~N}$ & $80 \mathrm{~W}$ & 0 & 34 & - & - & $3.20 \mathrm{M}_{\mathrm{n}} \mathrm{DC}$ & - & VI & 37 & 12 \\
\hline 1973 & $06 \quad 15$ & 010905.1 & $45.307 \mathrm{~N}$ & $71.119 \mathrm{~W}$ & 012 & 349 & 4.8 & - & $5.00 \mathrm{M}_{\mathrm{n}}$ STR & 4.48HRN & VI & 46 & $182 \&$ \\
\hline 1977 & 1225 & 153554.0 & $43.185 \mathrm{~N}$ & $71.658 \mathrm{~W}$ & 012 & 349 & - & - & $3.20 M_{n}$ WES & - & VI & 39 & 3 \\
\hline 1982 & $01 \quad 19$ & 001442.7 & $43.51 \mathrm{~N}$ & $71.62 \mathrm{~W}$ & 007 & 350 & 4.5 & - & $4.50 \mathrm{M}_{\mathrm{n}}$ BLA & - & VI & 350 & 127 \\
\hline 1988 & 1020 & 130950.1 & $44.539 \mathrm{~N}$ & $71.158 \mathrm{~W}$ & 005 & 74 & - & - & $3.90 M_{n}$ GS & - & VI & 578 & 17 \\
\hline
\end{tabular}

[Reference (Ref.) numbers given in parentheses at the end of each description refer to sources of data in table 1. Magnitude values are described in the Introduction, and codes are defined in table 2.]

1732. Sept. 16. St. Lawrence River valley, Canada. An earthquake that was violent in Montreal, Canada, also damaged houses at Piscataqua, N.H., in eastern Rockingham County. In Montreal, the shock damaged 300 houses, cracked walls, knocked down chimneys, and caused one death. Felt slightly at Boston, Mass., and Annapolis, Md. (Ref. $38,59,76$.)

1810. Nov. 10 (Nov. 9). Between Portsmouth and Hampton, Rockingham County, N.H. The earthquake broke windows at Portsmouth, and caused earth noises like a heavy explosion. Felt from Concord, N.H., east to the coast and from Portland, Me., south to Charlestown, Mass. (Ref. 38, 76, 78.)

1925. Oct. 9. Eastern New Hampshire, near Lake Winnipesaukee. One chimney fell from an old colonial house at Sandwich, N.H., in Carroll County north of Laconia. Chimneys also were knocked down at Cornish, Me., about $60 \mathrm{~km}$ east of Sandwich, N.H. This severe shock broke windows in Carroll County at Ossipee, Sandwich, and Tuftonboro, N.H., and cracked a concrete sidewalk at Meredith, north of Laconia. Items were knocked from shelves and residents were frightened in many towns in the area. Felt to the north at Bethlehem (northern Grafton County), to the southwest at Keene (Cheshire County), and to the east at Portland, Me. (Cumberland County). (Ref. 38, 76, 78, 218.)

1940. Dec. 20 and Dec. 24. Near Ossipee Lake, Carroll County, N.H. The first earthquake and one of about the same intensity on Dec. 24 are described together. Their epicenters lie in the same general region west of Whittier, N.H. Although the second shock was of less duration, it was somewhat more intense than the first. However, the first shock undoubtedly weakened structures, which resulted in more severe damage from the second earthquake.

The town of Tamworth, on the edge of the Ossipee Mountains in central Carroll County, sustained the most damage. Old houses and chimneys in need of repair were most commonly damaged. Some chimneys were thrown down, and 20 others were damaged. Also, reports indicate that well water remained muddy for several days and that many cracks formed in the crusty snow. Other minor damage included cracked walls, broken water pipes, fallen plaster, and broken furniture.

At the Riverside Cemetery at Whittier, about $3 \mathrm{~km}$ south of Tamworth, five monuments were displaced. In the nearby towns of West Ossipee and Chocorua, many old chimneys in need of repair were damaged, and water in wells became muddy. At Wonalancet, 8 $\mathrm{km}$ northwest of Tamworth, the foundation of an old house (constructed of heavy timber) was damaged when it shifted about $30 \mathrm{~cm}$. Heavy furniture, including a kitchen stove, moved several centimeters across the floor.

Minor damage occurred at several towns in Maine, Massachusetts, New York, and Vermont (see fig. 45). Reports of the shock also were received from Connecticut, New Jersey, Pennsylvania, and Rhode Island, as well as from Montreal and Quebec, Canada. Several small aftershocks occurred over the next several months. Magnitude 5.6 $\mathrm{M}_{\mathrm{S}} \mathrm{GR}$ (both), $5.44 \mathrm{M} \mathrm{JOH}$ (Dec. 20), 5.62 M JOH (Dec 24). (Ref. $13,38,349,506$.

1964. June 26. Near Warner, Merrimack County, N.H. Plaster fell at Meriden, and trees and bushes were shaken lightly. Slight damage also was reported at Bradford, Merrimack County, N.H., and at Springfield, Vt. (Ref. 37, 38, 349.) 


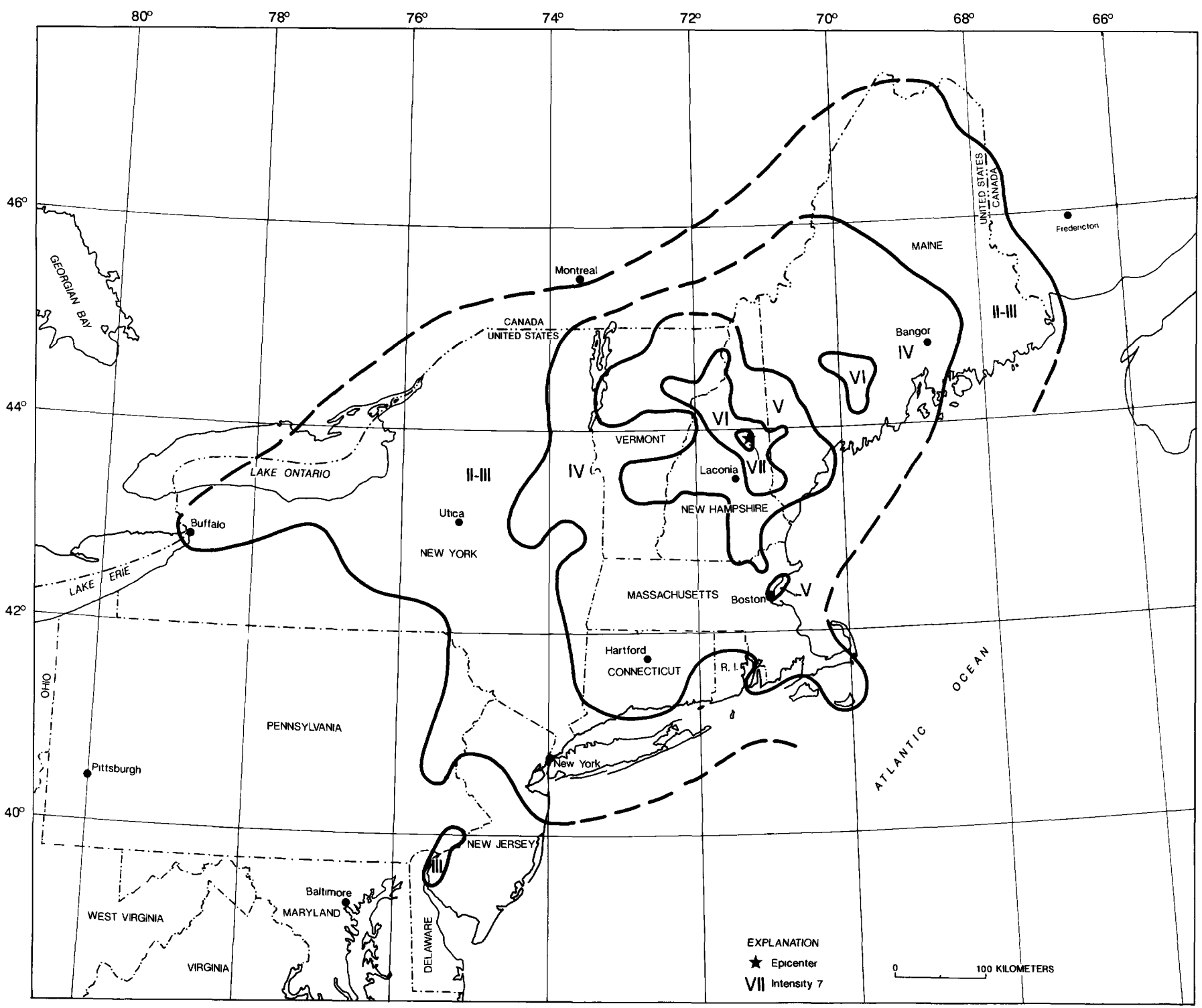

FIGURE 45.- Isoseismal map for the New Hampshire earthquake of December 20, 1940. Isoseismals are based on intensity estimates from data listed in references 13 and 506 of table 1.

1973. June 15 (June 14). Quebec-New Hampshire border area. Cracks formed in the road surface in the area about Montpelier, Vt.; chimneys and grocery stock were damaged at Woburn, Quebec. The earthquake cracked plaster and broke windows in Quebec Province and in the States of Maine, Massachusetts, New Hampshire, New York, and Vermont. Also felt in Connecticut and Rhode Island. Magnitude $4.49 \mathrm{M}$ JOH. (Ref. 46, 349.)

1977. Dec. 25. Near Concord, Merrimack County, N.H. The earthquake cracked plaster and windows in Concord. Felt over a small area of southcentral New Hampshire. Magnitude $3.1 \mathrm{M}_{\mathrm{n}}$ PAL. (Ref. 39, 349.)

1982. Jan. 19 (Jan. 18). Near Laconia, Belknap County, N.H. Damage, mainly cracks in chimneys and walls, occurred in Drury and Westford,
Mass.; Ashland, Bristol, Danbury, Laconia, and North Stratford, N.H.; and several towns in northeast Massachusetts and central Vermont. Other damage, widely reported in the area, included cracked plaster and broken merchandise and knickknacks. Also felt in Quebec, Canada and in the States of Connecticut, Maine, New York, and Rhode Island. Magnitude 4.7 $\mathrm{M}_{\mathrm{D}}$ WES. (Ref. 350.)

1988. Oct. 20. Near Berlin, Coos County, N.H. Minor damage at Berlin included cracked chimneys and house foundation, hairline cracks in interior walls, and displaced tombstones. At nearby Milan, interior walls sustained hairline cracks in plaster and drywall, and a house foundation was cracked. Felt over a small area of northwest Maine, northern New Hampshire, and northeast Vermont. (Ref. 74, 578.) 

NEW JERSEY

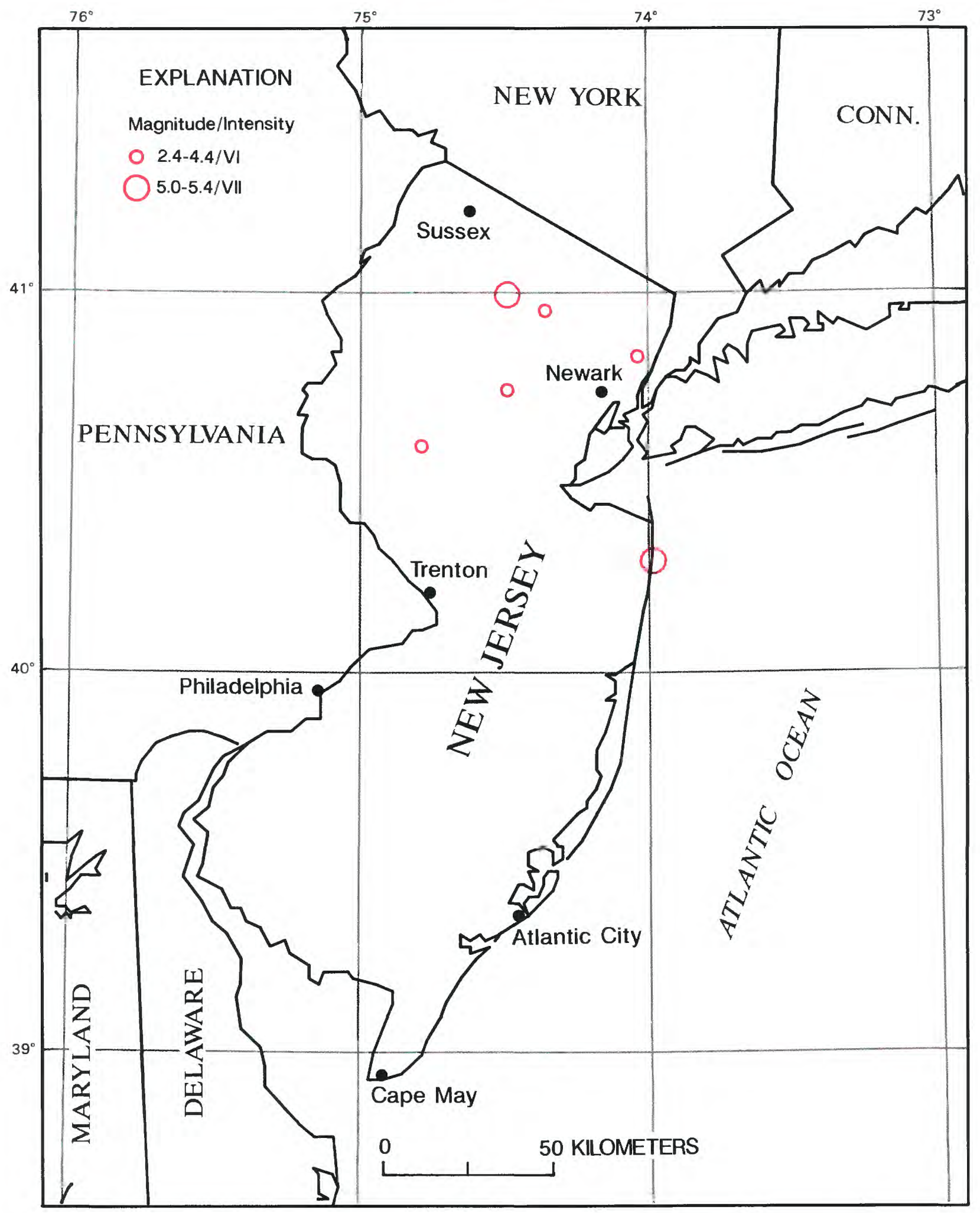

Earthquakes in New Jersey with magnitudes $\geq 4.5$ or intensity $\geq$ VI. 
[See table 1 for hypocenter and intensity references and table 2 for definitions of magnitude source codes. \&, land area only; (3, felt area is less than $1,000 \mathrm{~km}{ }^{2}$. Leader (-) indicates information is not available!

\begin{tabular}{|c|c|c|c|c|c|c|c|c|c|c|c|c|c|}
\hline \multirow{2}{*}{\multicolumn{2}{|c|}{ Date }} & \multirow{2}{*}{$\begin{array}{c}\text { Origin } \\
\text { time (UTC) }\end{array}$} & \multicolumn{4}{|c|}{ Hypocenter } & \multicolumn{4}{|c|}{ Magnitude } & \multicolumn{2}{|c|}{ Intensity } & \multirow{3}{*}{$\begin{array}{c}\text { Felt area } \\
\left(1,000 \mathrm{~km}^{2}\right)\end{array}$} \\
\hline & & & \multirow{2}{*}{$\begin{array}{l}\text { Latitude } \\
\left(^{\circ}\right)\end{array}$} & \multirow{2}{*}{$\begin{array}{l}\text { Longitude } \\
\left({ }^{\circ}\right)\end{array}$} & \multirow{2}{*}{$\begin{array}{l}\text { Depth } \\
(\mathrm{km})\end{array}$} & \multirow[t]{2}{*}{ Ref } & \multicolumn{2}{|c|}{ USGS } & \multirow[t]{2}{*}{ Other } & \multirow{2}{*}{$\begin{array}{c}\text { Moment } \\
\mathbf{M}\end{array}$} & \multirow[t]{2}{*}{ MM } & \multirow[t]{2}{*}{ Ref } & \\
\hline Yr & Mo Da & $\mathrm{h} \mathrm{m} \mathrm{s}$ & & & & & $m_{b}$ & $M_{s}$ & & & & & \\
\hline 1783 & 1130 & 0350 & $41.0 \mathrm{~N}$ & $74.5 \mathrm{~W}$ & - & 76 & - & - & $5.30 \mathrm{M}_{\mathrm{fa}} \mathrm{SET}$ & - & VI & 76 & - \\
\hline 1895 & 0901 & 1109 & $40.75 \mathrm{~N}$ & 74.5 & - & 369 & - & - & $4.10 \mathrm{M}_{\mathrm{fa}} \mathrm{SC}$ & - & VI & 38 & 30 \\
\hline 1927 & 0601 & 1220 & $40.3 \mathrm{~N}$ & $74.0 \mathrm{~W}$ & - & 38 & - & - & - & - & VII & 38 & $20 \&$ \\
\hline 1957 & 0323 & 190231 & $40.6 \mathrm{~N}$ & $74.8 \mathrm{~W}$ & 010 & 77 & - & - & $3.80 \mathrm{M}_{\mathrm{fa}}$ & - & VI & 30 & 7 \\
\hline 1976 & 0311 & 210720.4 & $40.96 \mathrm{~N}$ & $74.37 \mathrm{~W}$ & 004 & 49 & - & - & $2.40 \mathrm{M}_{\mathrm{n}}$ PAL & - & VI & 49 & @ \\
\hline 1976 & $04 \quad 13$ & 153913.6 & $40.84 \mathrm{~N}$ & $74.05 \mathrm{~W}$ & 001 & 317 & - & - & $3.10 \mathrm{M}_{\mathrm{n}}$ PAL & - & VI & 49 & @ \\
\hline
\end{tabular}

[Reference (Ref.) numbers given in parentheses at the end of each description refer to sources of data in table 1. Magnitude values are described in the Introduction, and codes are defined in table 2.]

1783. Nov. 30 (Nov. 29). West of New York City, N.Y. This earthquake was felt from New Hampshire to Pennsylvania. A foreshock at 0200 UTC on Nov. 30 and an aftershock at 0700 UTC were reported only in New York and in Philadelphia, Pa. (Ref. 76.)

1895. Sept. 1. Near High Bridge, Hunterdon County, N.J. This earthquake knocked articles from shelves and rocked buildings in several towns in New Jersey, Pennsylvania, and New York. At Asbury Park, N.J., plaster was knocked from walls. At Philadelphia, $\mathrm{Pa}$., windows were broken and crockery was overturned. Felt from Falls Church, Va., and Washington, D.C., to Connecticut. Magnitude $4.3 \mathrm{M}_{\mathrm{fa}}$ SET. (Ref. 38, 272, 369.)

1927. June 1. Near Asbury Park, Monmouth County, N.J. Several chimneys were downed, bricks fell from chimneys, and articles fell from shelves in an area from Asbury Park north to Long Branch, in northeast Monmouth County. Also, part of a ceiling fell at Long Branch, and plaster fell from walls and ceilings at Fairhaven, northwest of Long Branch. Many accounts of broken crockery and fallen plaster were reported from Westchester County, N.Y. Three shocks were felt along the New Jersey coast from Sandy Hook to Toms River. (Ref. 38, 218.)

1957. Mar. 23. Long Valley area, Morris County, N.J. At Long Valley (about $55 \mathrm{~km}$ west of Newark), walls were cracked and plaster fell to the floor. Chimneys cracked and windows and dishes broke at Lebanon (about $18 \mathrm{~km}$ southwest of Long Valley), and one chimney cracked and a well curb broke in the Hamden area ( $5 \mathrm{~km}$ southwest of Lebanon). (Ref. 30, 38, 77.)

1976. Mar. 11. Pompton Lakes area, Passaic County, N.J. Slight damage occurred about $35 \mathrm{~km}$ northwest of Newark, at Pompton Lakes (cracks in ceiling, fallen plaster), at nearby Kinnelon (cracks in plaster and windows), and north of Jersey City, at Ridgefield (cracks in ceiling). Felt in Bergen, Morris, and Passaic Counties in northeast New Jersey. (Ref. 49.)

1976. Apr. 13. Near Ridgefield, Bergen County, N.J. An earthquake slightly larger in magnitude than that on Mar. 11, 1976, centered in the same general area. Residents in Ridgefield, about 13 $\mathrm{km}$ north of Jersey City, reported that plaster was knocked to the floor. The shock was felt widely in the area. (Ref. $49,317$. 
NEW MEXICO

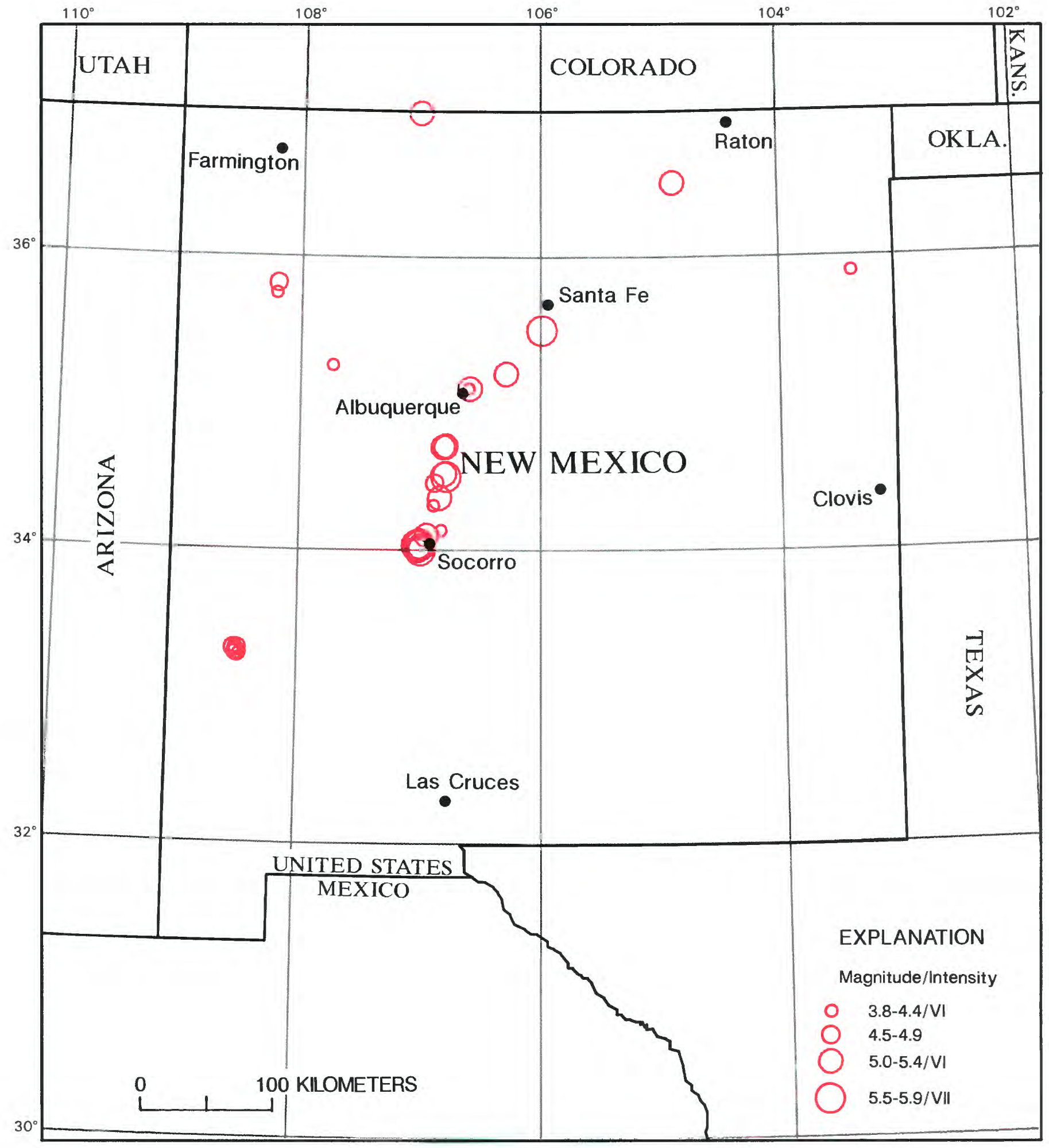

Earthquakes in New Mexico with magnitudes $\geq 4.5$ or intensity $\geq$ VI. 


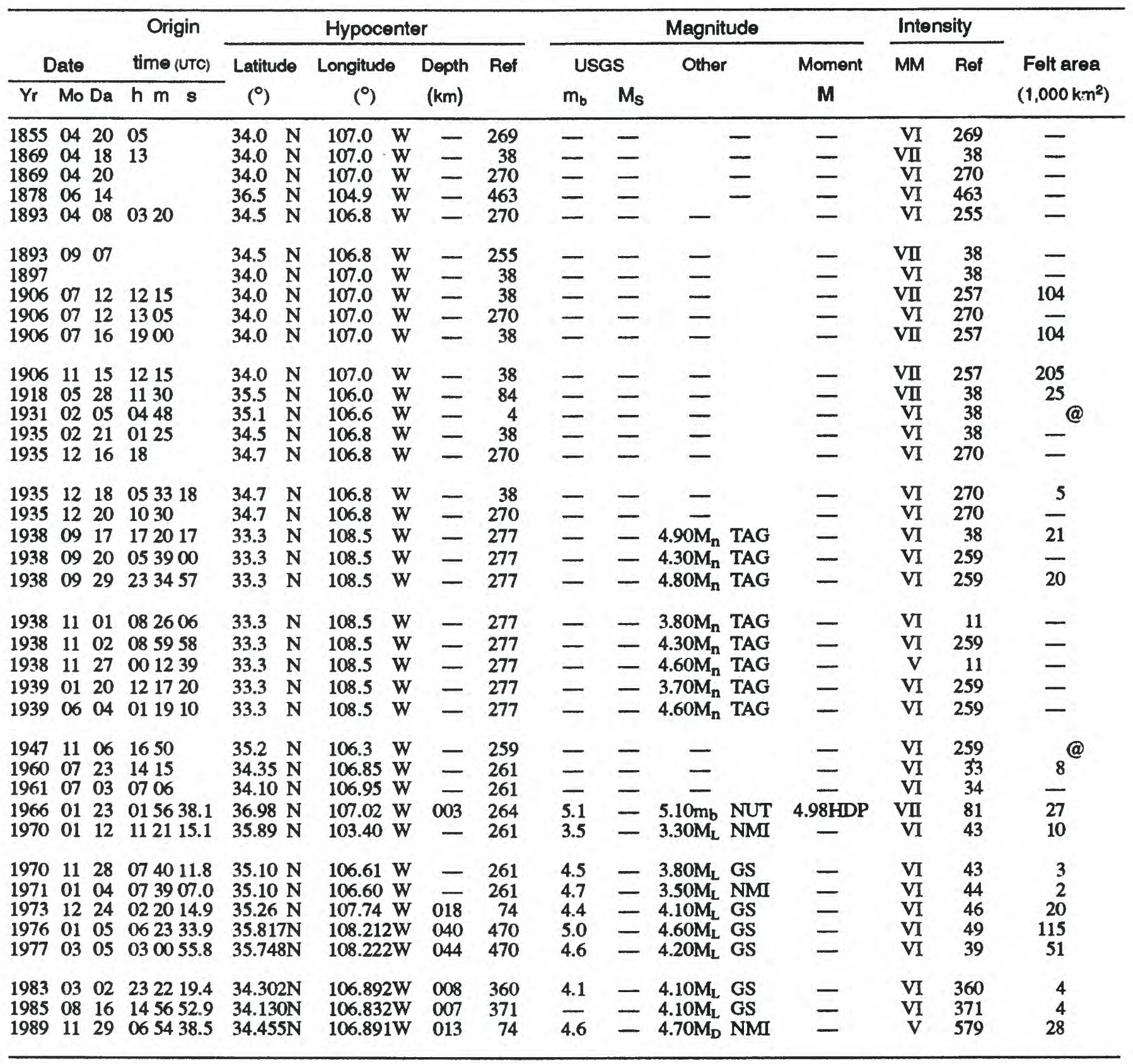

[Reference (Ref.) numbers given in parentheses at the end of each description refer to sources of data in table 1. Magnitude values are described in the Introduction, and codes are defined in table 2.]

1855. Apr. 20 (Apr. 19). Socorro area, N. Mex. The earthquake almost destroyed two houses at Socorro, about $110 \mathrm{~km}$ south of Albuquerque. The earth trembled; houses shook; and horses were frightened. (Ref. 269.)
1869. Apr. 18. Socorro area, N. Mex. Socorro Springs (at the base of Socorro Mountain) changed flow and became muddy, and its flow was diminished for many weeks. Some houses were damaged considerably at Socorro, about $110 \mathrm{~km}$ south of Albuquerque. Seven severe shocks occurred from Apr. 18-20. (Ref. 38, 270, 446.)

1869. Apr. 20. Socorro area, N. Mex. This severe aftershock cracked the walls of the church at 
Socorro, about $110 \mathrm{~km}$ south of Albuquerque. Near Socorro, the hot springs spouted water "hotter than ever and of a reddish color." (Ref. 270, 446.)

1878. June 14. Cimarron, Colfax County, N. Mex. Windowpanes were broken by an earthquake at Cimarron, about $55 \mathrm{~km}$ southwest of Raton. (Ref. 463.)

1887. May 3. Northern Sonora, Mexico. Chimneys were thrown down in Albuquerque, N. Mex. See the Arizona chapter for a complete description of this damaging earthquake. (Ref. 38, 343, 471, 494, 497.)

1893. Apr. 8 (Apr. 7). Belen, Valencia County, N. Mex. This was the strongest of a series of shocks Apr. 6-8. It damaged almost all the houses in and around Belen and threw several entirely down. The earthquake was not felt farther south than Sabinal. The effects for these earthquakes were taken from two reports, one not indicating any damage and the other as described above. The assigned intensity is lower than the above damage would indicate. (Ref. 255,270 .)

1893. Sept. 7. Los Lunas, Valencia County, N. Mex. Five shocks threw down a score of old adobe buildings already weakened by previous earthquakes in Los Lunas. At Sabinal, a spring formed in a place that always had been dry and barren. The central area of the State was subjected to tremors for a period of three months, the most severe occurring on Sept. 7. (Ref. 38, 255.)

1897. Date unknown. Socorro area, N. Mex. This violent tremor overturned chairs and small objects at Socorro, about $110 \mathrm{~km}$ south of Albuquerque. The motion was so strong that one person could not walk. (Ref. 38,270 .)

1906. July 12, 1215 UTC. Socorro area, N. Mex. About $110 \mathrm{~km}$ south of Albuquerque at Socorro, an earthquake threw down several chimneys, knocked plaster from the walls of many adobe houses and the courthouse, and hurled shelf goods, book cases, and dishes to the floor. The entire business block, extending from the plaza along the north side of Manzanares Avenue, was damaged heavily. A twostory brick house, one of the buildings most severely damaged, was abandoned because its walls were cracked badly and thrown out of plumb. Nearby, one of the walls of another cottage was damaged so severely that the occupants moved outside to a tent. Many other residences sustained damage to walls and furniture.

Many boulders rolled onto the branch railroad leading to Magdalena, west of Socorro, breaking one rail and many ties. Fissures formed in the ground near the Santa Fe depot in Socorro, and waves were seen on the ground surface. The earthquake shook residents of Carthage, Kelly, Magdalena, San Antonio, San Marcial, and other towns as far nortt. as Albuquerque and as far south as Silver City (Grant County). Tremors were felt daily from July 2, 1906, well into 1907. (Ref. 38, 257, 270.)

1906. July 12, 1305 UTC. Socorro area, $N$. Mex. This earthquake at Socorro, about $110 \mathrm{~km}$ south of Albuquerque, was described as almost as severe as the first tremor on July 12 (12 15 UTC). (Ref. 270.)

1906. July 16. Socorro area, N. Mex. Describnr as slightly stronger that the July 12 event, an earthquake caused additional damage in the form of downed chimneys, cracked houses, and damaged brick gables at Socorro, about $110 \mathrm{~km}$ south of Albuquerque. Many residents left their houses and lived in tents. The Socorro Hotel, a brick structure, was abandoned because of severe damage. The brick post office had a bulging wall, and its southeast corner was "thrown out." Three chimneys on the Socorro County Courthouse were destroyed, and two were downed at the high school. At San Marcial, the shock knocked down a few chimneys, cracked a few houses, and broke windows. At San Antonio, the earthquake caused plaster to fall, broke windows, and cracked most houses. This shock was reported felt at Raton, about $390 \mathrm{~km}$ northeast of Socorro, and Douglas, Ariz., $400 \mathrm{~km}$ southwest. (Ref. 38, 257, 270.)

1906. Nov. 15. Socorro area, N. Mex. This earthquake, which increased the property damage already sustained at Socorro, was described as the most severe shock of the year. Four rebuilt chimneys were shaken off the Socorro County Courthouse, and two others were cracked severely. Plaster fell at the courthouse, and a cornice on the northwest corner of the two-story adobe Masonic Temple was thrown onto its first floor. Several bricks fell from the front gable on one house. Plaster was shaken from walls in Santa $\mathrm{Fe}$, about $200 \mathrm{~km}$ from the epicenter. Felt over most of New Mexico and in parts of Arizona and Texas. (Ref. 38, 257, 270, 272.)

1918. May 28. Near Cerrillos, Santa Fe County, N. Mex. Many chimneys and plastered ceilings fell at Cerrillos, about $35 \mathrm{~km}$ south of Santa Fe. A "heavy" break in the surface of the earth occurred at the edge of town. Glass was shaken from several windows. People in the street were thrown off their feet. Plaster was knocked down at Santa Fe, and adobe walls were cracked at Stanley, southwest of Cerrillos. (Ref. 38, 84, 270, 272.)

1931. Feb. 5. (Feb. 4). Albuquerque, Bernalillo County, N. Mex. Bricks and adobe walls cracked at Albuquerque, chimneys cracked, and part of one chimney fell. Large rocks rolled into the 
streets from sand hills about the city. Across the river and south of town, two adobe houses were damaged severely. (Ref. 4, 38, 270.)

1935. Feb. 21 (Feb. 20). Near Bernardo, Socorro County, N. Mex. Coping on a building cracked at Bernardo, and walls and plaster cracked. Adobe and concrete buildings sustained damage. (Ref. 8, 38.)

1935. Dec. 16. Belen, Valencia County, N. Mex. Plaster fell, small cracks formed in buildings, and dishes broke at Belen. This is one of a series of 81 earthquakes that were felt from Dec. 13, 1935, to Jan. 4, 1936. (Ref. 270.)

1935. Dec. 18 (Dec. 17). Belen, Valencia County, N. Mex. Plaster fell throughout the city of Belen, objects were shaken from shelves, and several brick and adobe buildings sustained cracks. (Ref. 38, 270.)

1935. Dec. 20. Belen, Valencia County, N. Mex. A large locomotive shook on the Santa Fe tracks at Belen, and switch engines were rocked hard enough to alarm engineers and firemen. (Ref. 270.)

1938. Sept. 17. Southwest New Mexico, near the Continental Divide. This shock was strongest in Graham County, Ariz., at Clifton and Duncan, and in the southern part of Catron County, N. Mex. At Duncan, Ariz., plaster and walls cracked and bottles fell from shelves. At a Forest Service ranger station near the head of the west fork of the Gila River in New Mexico, one chimney cracked, plaster fell, and trees and bushes were shaken strongly. This was the first of a series of earthquakes that occurred through July 1939. Magnitude 5.5 $\mathrm{M}_{\mathrm{S}}$ GR. (Ref. 8, 38, 277, 343.)

1938. Sept. 20 (Sept. 19). Southwest New Mexico aftershock. An aftersliock of the Sept. 17 earthquake cracked old adobe walls and knocked others down at Duncan, Ariz., in southeast Graham County. The tremor disrupted telephone service for hours, displaced furnishings in houses, and overturned vases. The main shock also was felt in Graham County, Ariz., at Clifton, Morenci, and Safford; in northern Cochise County, at San Simon; and at several towns in New Mexico. Many small shocks were reported from Sept. 17-20. (Ref. $11,259,277,343$.)

1938. Sept. 29. Southwest New Mexico aftershock. This aftershock of the Sept. 17 earthquake was strongest at Clifton (Graham County), Ariz., where about a large amount of plaster fell from a ceiling in one house. Felt in Graham and Cochise Counties, Ariz., and at several towns in New Mexico. (Ref. 259, 277, 343.)
1938. Nov. 1. Southwest New Mexico aftershock. Another aftershock of the Sept. 17 earthquake cracked plaster and chimneys at Cliff (Grant County), N. Mex. Rocks fell in the mountains near Buckhorn, northwest of Cliff. Also felt in Arizona. (Ref. 11, 277, 343.)

1938. Nov. 2. Southwest New Mexico aftershock. Another aftershock of the Sept. 17 earthquake cracked chimneys at White Creek Ranger Station near Cliff (Grant County). Also felt in Arizona. (Ref. 259, 277, 343.)

1939. Jan. 20. Southwest New Mexico aftershock. Walls were cracked in Grant County, at Gila, by an aftershock of the tremor on Sept. 17, 1938. (Ref. 259, 277.)

1939. June 4 (June 3). Southwest New Mexico aftershock. The last damaging aftershock of the Sept. 17, 1938, earthquake cracked plaster at Duncan, Ariz., in southeast Graham County. (Ref. 259, 277.)

1947. Nov. 6. San Antonito area, Bernalillo County, N. Mex. This local earthquake cracked a fireplace and plaster at Zamora Ranch and shook dishes from shelves at San Antonito. (Ref. 259.)

1960. July 23. Near Lajoya, Socorro County, N. Mex. A weak adobe wall toppled and some adobe buildings were cracked at Lajoya. Canned goods fell from shelves and people ran outdoors at nearby Bernardo. (Ref. 33, 261.)

1961. July 3. Socorro area, N. Mex. Plaster cracked in adobe buildings at Socorro, about $110 \mathrm{~km}$ south of Albuquerque. (Ref. 34, 261.)

1966. Jan. 23 (Jan. 22). Near Dulce, Rio Arriba County, N. Mex. This earthquake affected to some extent almost every house in Dulce and damaged chimneys throughout the area. Property damage was estimated at about $\$ 200,000$. The earthquake was caused by normal faulting on a fault striking approximately north-northwest, that probably had its maximum activity in Miocene time. Distribution of aftershock epicenters suggests that the main shock triggered aftershock activity on adjacent faults. The aftershock activity continued in the area for a year.

Property damage was most severe at the Dulce Bureau of Indian Affairs School and dormitory complex and the Dulce independent schools. Much plaster fell from ceilings in the dormitories, and several brick walls sustained vertical fractures that extended from ground to roof. Brick walls in the steam-heating plant were displaced from vertical alignment as much as $3 \mathrm{~cm}$, and two boilers (each weighing 10 tons) were displaced about $0.6 \mathrm{~cm}$ at their bases. 
Also, an 18-m-high smokestack buckled, and only guy wires prevented it from falling.

Huge masses of shale and sandstone fell down the slopes from nearby Dulce Point. In addition, several small cracks formed in fill across the frozen roads in the Dulce area, but there was no evidence of ground displacement or fissuring. Also felt in southern Colorado. Magnitude 4.6 $\mathrm{M}_{\mathrm{S}}$ NUT. (Ref. 38, 81, 263, 264, 533.)

1970. Jan. 12. Amistad area, Harding County, N. Mex. Part of a ceiling fell at Amistad, and some adobe bricks in a wall crumbled at the public school gymnasium. Plaster fell at nearby Nara Visa. Also felt at Texline, Tex. (Ref. 43, 261.)

1970. Nov. 28. Near Albuquerque, Bernalillo County, N. Mex. This minor earthquake cracked plaster, a garage floor, and a concrete-block wall and broke windows. One resident reported that the roof of a barn collapsed; another said a roof air conditioner fell through a skylight. (Ref. 43, 261, 364.)

1971. Jan. 4. Near Albuquerque, Bernalillo County, N. Mex. Considerable minor damage at Albuquerque included cracked windows and plaster in buildings and fallen merchandise in markets. An old adobe building sustained both interior and exterior cracks. Slight damage also occurred at nearby Alameda and Corrales. (Ref. 44, 261.)

1973. Dec. 24 (Dec. 23). Grants area, Cibola County, N. Mex. Plaster cracked and fell, cracks formed in an exterior wall, and paneling on walls pulled apart at Grants. Walls and chimneys were cracked at Laguna, about $50 \mathrm{~km}$ southeast of Grants. (Ref. 46, 74.)
1976. Jan. 5 (Jan. 4). Near Crownpoint, McKinley County, N.Mex. Damage, which generally was minor, consisted mostly of cracks in plaster and drywall in several Colorado and New Mexico towns and at Leupp, Ariz. At Cahone, Colo., a chimney was cracked. Also felt in Utah.

This earthquake and that on Mar. 5, 1977, are two of the largest ever observed in New Mexico outside the Rio Grande rift and within the Colorado Plateau. They occurred in the southern part of the San Juan Basin (known as the Chaco slope), a region that historically has had a low level of seismicity. (Ref. 49, 470.)

1977. Mar. 5 (Mar. 4). Near Crownpoint, McKinley County, N. Mex. Fences displaced slightly at Crownpoint, and existing cracks in walls widened considerably south of Crownpoint, at Prewitt. Also felt in Arizona and Colorado. See description above for the earthquake on Jan. 5, 1976. (Ref. $39,470$.

1983. Mar. 2. Near Socorro, N. Mex. At San Acacia, plaster cracked in both exterior and interior walls of adobe buildings, dishes were broken, and stock was thrown from store shelves. The shock also was strong in Socorro County at Bosque, Lajoya, and Socorro. (Ref. 360.)

1985. Aug. 16. Near Socorro, N. Mex. At Socorro, about $110 \mathrm{~km}$ south of Albuquerque, cracks formed in sidewalks, plaster, windows, and the foundation of a brick building. Also, a few glassware items were broken. This earthquake was felt only at a few towns in the area. Magnitude $4.3 \mathrm{M}_{\mathrm{n}}$ TUL. (Ref. 371.) 



\section{NEW YORK}

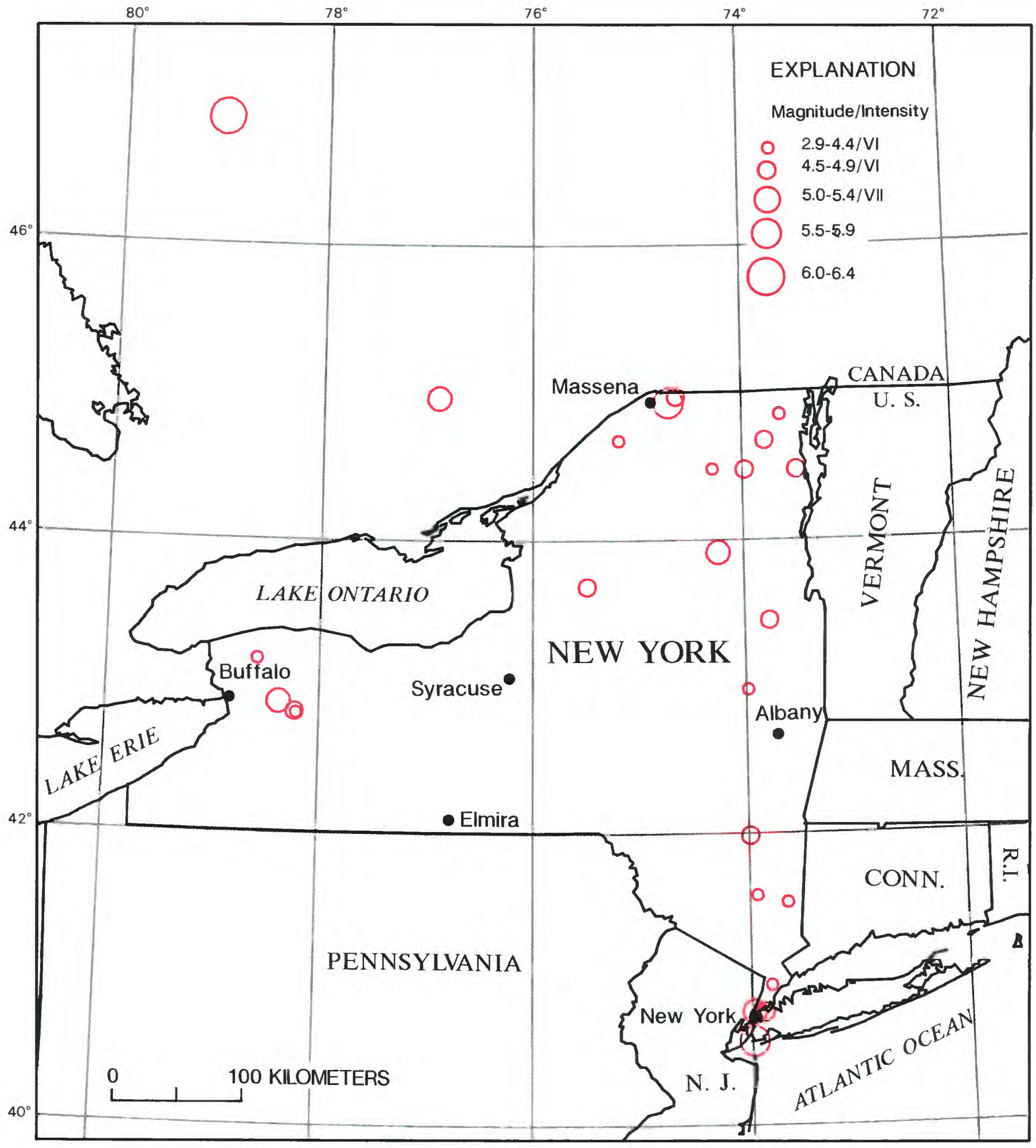

Earthquakes in New York with magnitudes $\geq 4.5$ or intensity $\geq$ VI. 


\section{NEW YORK}

[See table 1 for hypocenter and intensity references and table 2 for definitions of magnitude source codes. \&, land area only; @, felt area is less than $1,000 \mathrm{~km}{ }^{2}$. Leader (-) indicates information is not available]

\begin{tabular}{|c|c|c|c|c|c|c|c|c|c|c|c|c|c|}
\hline \multirow{2}{*}{\multicolumn{2}{|c|}{ Date }} & \multirow{2}{*}{$\begin{array}{c}\text { Origin } \\
\text { time (UTC) }\end{array}$} & \multicolumn{4}{|c|}{ Hypocenter } & \multicolumn{4}{|c|}{ Magnitude } & \multicolumn{2}{|c|}{ Intensity } & \multirow{3}{*}{$\begin{array}{c}\text { Felt area } \\
\left(1,000 \mathrm{~km}^{2}\right)\end{array}$} \\
\hline & & & \multirow{2}{*}{$\begin{array}{l}\text { Latitude } \\
\left({ }^{\circ}\right)\end{array}$} & \multirow{2}{*}{$\begin{array}{l}\text { Longitude } \\
\left({ }^{\circ}\right)\end{array}$} & \multirow{2}{*}{$\begin{array}{l}\text { Depth } \\
(\mathrm{km})\end{array}$} & \multirow[t]{2}{*}{ Ref } & \multicolumn{2}{|c|}{ USGS } & \multirow[t]{2}{*}{ Other } & \multirow{2}{*}{$\begin{array}{l}\text { Moment } \\
\text { M }\end{array}$} & \multirow[t]{2}{*}{ MM } & \multirow[t]{2}{*}{ Ref } & \\
\hline $\mathrm{Yr}$ & Mo $D r$ & $\mathrm{~h} \mathrm{~m} \mathrm{~s}$ & & & & & $m_{b}$ & $M_{S}$ & & & & & \\
\hline 1737 & 1219 & 0345 & $40.8 \mathrm{~N}$ & $74.0 \mathrm{~W}$ & - & 76 & - & - & - & - & VII & 76 & - \\
\hline 1853 & 031 & 0730 & $43.7 \mathrm{~N}$ & $75.5 \mathrm{~W}$ & - & 76 & - & - & 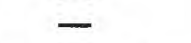 & - & VI & 76 & - \\
\hline 1855 & 020 & 0430 & $42.0 \mathrm{~N}$ & 74.0 W & - & 76 & - & - & - & - & VI & 76 & - \\
\hline 1857 & 102 & 2015 & $43.2 \mathrm{~N}$ & $78.6 \mathrm{~W}$ & - & 76 & - & - & $4.30 \mathrm{M}_{\mathrm{fa}} \mathrm{SC}$ & - & VI & 76 & 65 \\
\hline 1867 & 1218 & 0800 & $44.7 \mathrm{~N}$ & $75.2 \mathrm{~W}$ & - & 126 & - & - & $4.30 \mathrm{M}_{\mathrm{fa}} \mathrm{SC}$ & - & VI & 126 & 65 \\
\hline 1877 & 110 & 0656 & $44.5 \mathrm{~N}$ & $74.0 \mathrm{~W}$ & - & 38 & - & - & $4.90 \mathrm{M}_{\mathrm{fa}} \mathrm{SC}$ & - & VII & 38 & 200 \\
\hline 1878 & 020 & 1620 & $40.8 \mathrm{~N}$ & 73.9 W & - & 141 & - & - & - & - & VI & 463 & - \\
\hline 1884 & $08 \quad 16$ & 1907 & $40.6 \mathrm{~N}$ & $74.0 \quad \mathrm{~W}$ & - & 76 & - & - & $5.50 \mathrm{M}_{\mathrm{fa}} \mathrm{SET}$ & - & VII & 76 & 540\& \\
\hline 1897 & 0528 & 0316 & $44.5 \mathrm{~N}$ & $73.5 \mathrm{~W}$ & - & 126 & - & - & $4.70 \mathrm{M}_{\mathrm{fa}} \mathrm{SC}$ & - & VI & 76 & 225 \\
\hline 1914 & 0210 & 1831 & $44.98 \mathrm{~N}$ & $76.92 \mathrm{~W}$ & - & 76 & - & - & $5.20 \mathrm{M}_{\mathrm{fa}} \mathrm{SC}$ & - & VII & 76 & 518 \\
\hline 1916 & 020 & 0424 & $43.0 \mathrm{~N}$ & 74.0 W & - & 126 & - & - & $4.10 \mathrm{M}$ & - & VI & 272 & 21 \\
\hline 1928 & 0318 & 1520 & $44.5 \mathrm{~N}$ & $74.3 \mathrm{~W}$ & - & 38 & - & - & $4.10 \mathrm{M}_{\mathrm{L}}$ EPB & - & VI & 38 & 31 \\
\hline 1929 & $\begin{array}{ll}08 & 12\end{array}$ & 112448.7 & $42.910 \mathrm{~N}$ & $78.402 \mathrm{~W}$ & 009 & 349 & - & - & $5.20 \mathrm{M}_{\mathrm{n}} \mathrm{ST}$ & $4.69 \mathrm{ST}$ & VIII & 77 & 880 \\
\hline 1931 & 042 & 195430.6 & $43.471 \mathrm{~N}$ & $73.785 \mathrm{~W}$ & 005 & 349 & - & - & $4.70 \mathrm{M}_{\mathrm{n}} \mathrm{ST}$ & - & VII & 4 & 70 \\
\hline 1934 & 0415 & 025813.0 & $44.7 \mathrm{~N}$ & $73.8 \quad \mathrm{~W}$ & - & 77 & - & - & $4.50 \mathrm{M}_{\mathrm{L}}$ EPB & - & VI & 126 & 21 \\
\hline 1935 & 110 & 060334.2 & $46.87 \mathrm{~N}$ & $79.05 \mathrm{~W}$ & 001 & 349 & - & - & $6.20 \mathrm{M}_{8}$ GR & $5.59 \mathrm{ST}$ & VII & 77 & 2590 \\
\hline 1944 & 090 & 043845.7 & $44.958 \mathrm{~N}$ & $74.723 \mathrm{~W}$ & 012 & 349 & - & - & $5.80 M_{n} S T$ & $5.52 \mathrm{ST}$ & VIII & 17 & 1095 \\
\hline 1944 & 090 & 085106.0 & $44.999 \mathrm{~N}$ & $74.652 \mathrm{~W}$ & 001 & 349 & - & - & $4.50 \mathrm{M}_{\mathrm{n}}$ BAS & - & - & - & - \\
\hline 1966 & 010 & 132339.0 & $42.842 \mathrm{~N}$ & $78.249 \mathrm{~W}$ & 000 & 349 & 4.7 & 一 & $4.60 \mathrm{M}_{\mathrm{n}} \mathrm{STR}$ & $4.26 \mathrm{HRN}$ & VI & 81 & $26 \&$ \\
\hline 1967 & 0613 & 190855.5 & $42.837 \mathrm{~N}$ & $78.234 \mathrm{~W}$ & 001 & 349 & 3.9 & - & $4.40 \mathrm{M}_{\mathrm{n}}$ STR & $4.07 \mathrm{HRN}$ & VI & 40 & 6 \\
\hline 1974 & 0607 & 194535 . & $41.595 \mathrm{~N}$ & 73. & 003 & 34 & - & - & 2. & $2.86 \mathrm{ST}$ & VI & 47 & @ \\
\hline 1975 & 060 & 183922. & $44.874 \mathrm{~N}$ & $73.651 \mathrm{~W}$ & 011 & 349 & - & - & $3.50 M_{n} \mathrm{ST}$ & $3.28 \mathrm{ST}$ & VI & 48 & 13 \\
\hline 1983 & 022 & 195935. & 41.55 & & 007 & 360 & - & - & $2.90 \mathrm{M}_{\mathrm{n}}$ GS & - & VI & 360 & 4 \\
\hline 1983 & 100 & 101846 . & $43.938 \mathrm{~N}$ & 74.25 & 013 & 360 & 5.1 & - & $5.10 \mathrm{M}_{\mathrm{L}} \mathrm{PAL}$ & $4.88 \mathrm{SOM}$ & VI & 360 & 634 \\
\hline 1985 & 1019 & 100740.3 & $40.983 \mathrm{~N}$ & $73.829 \mathrm{~W}$ & 006 & 371 & 3.6 & - & $4.00 \mathrm{M}_{\mathrm{L}} \mathrm{PAL}$ & - & VI & 371 & 31 \\
\hline
\end{tabular}

[Reference (Ref.) numbers given in parentheses at the end of each description refer to sources of data in table 1. Magnitude values are described in the Introduction, and codes are defined in table 2.]

1737. Dec. 19 (Dec. 18). New York City area, N.Y. Several chimneys were knocked down and bells were rung in New York City. It was felt in Boston, Mass., Philadelphia, Pa., and New Castle, Del. (Ref. $38,59,76$.)

1853. Mar. 12. Near Lowville, Lewis County, N.Y. Machinery was knocked down at Lowville, about $100 \mathrm{~km}$ northeast of Syracuse. Also felt in Canada. (Ref. 59, 76.)

1855. Feb. 7 (Feb. 6). Hudson River valley area. This event was felt to the east as far as Springfield, Mass. Ref. 444 reports that this was a nontectonic event (a cryoseism) which was caused by freezing action in ice, ice-soil, and ice-rock materials. The intensity VI in the hypocenter list above was taken from ref. 76 but could not be documented with damage descriptions. (Ref. 76, 444.)

1857. Oct. 23. Near Buffalo, Niagara County, N.Y. Crocks fell from shelves at Buffalo; bells rang and walls vibrated and surged. A man sitting on a chair was thrown to the ground. Felt from Warren, $\mathrm{Pa}$., to Port Hope on Lake Ontario, and in the Montreal, Canada, region. (Ref. 38, 76.)

1867. Dec. 18. Northern New York. This earthquake was described as "quite severe" at Hammond (St. Lawrence County). The earthquake awakened residents at Ogdensburg (St. Lawrence County) and Syracuse (Onondaga County), N.Y., Burlington, Vt., and Hamilton, Ontario. Felt from Whitehall, N.Y., near the Vermont-New York border, to Belleville, Ontario, and Sackville, New Brunswick. (Ref. 38, 59, 126, 591.)

1877. Nov. 4. Northern New York. Effects of the shock were most severe along the St. Lawrence River and Lake Champlain. In that area, chimneys 


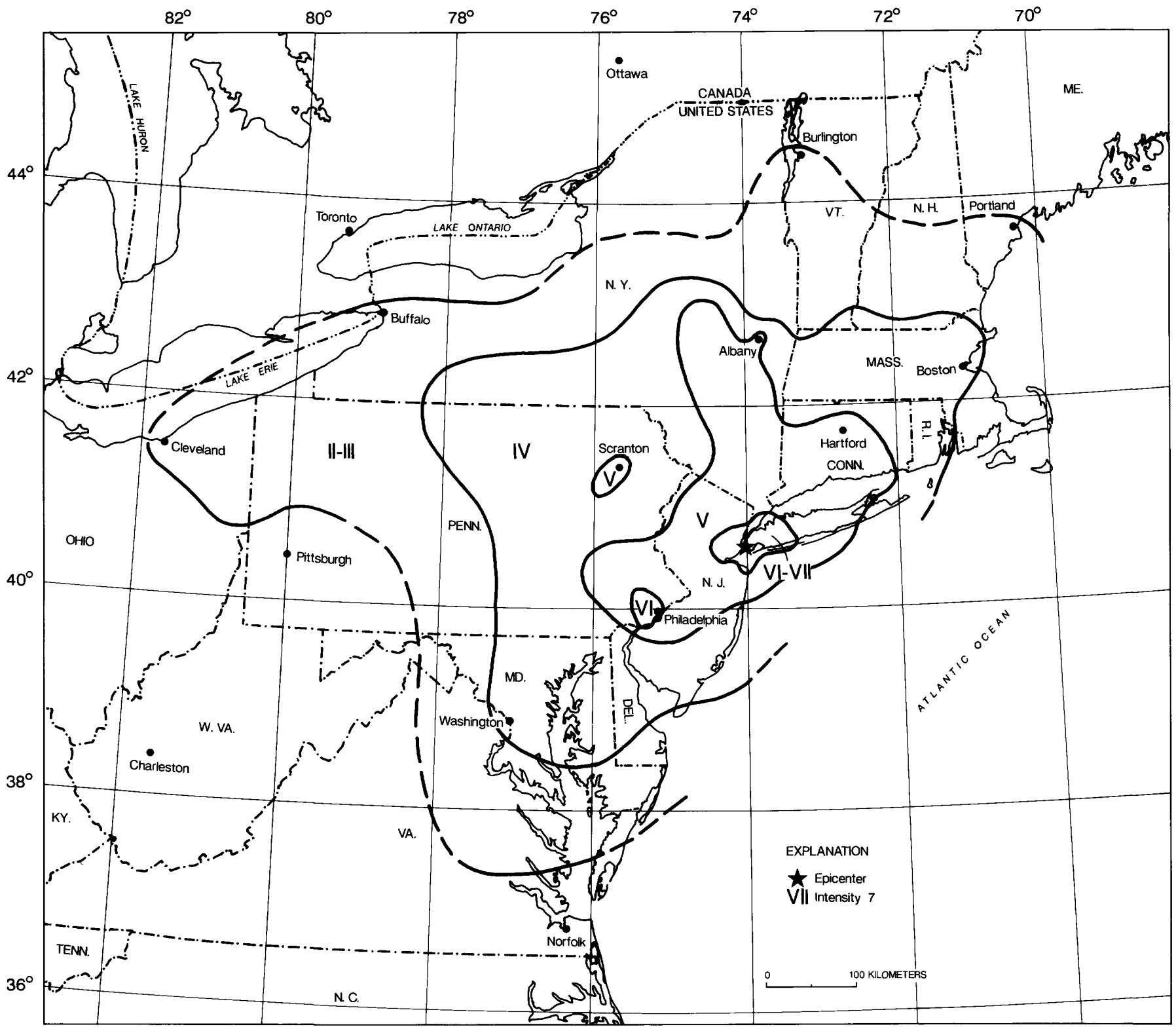

FIGURE 46.- Isoseismal map for the New York City, New York, earthquake of August 10, 1884. Isoseismals are based on intensity estimates from data listed in references $369,463,518$, and 529 of table 1.

were downed, crockery was overturned, and ceilings were cracked. As far southwest as Auburn, N.Y., windowpanes were damaged. Felt from Pembroke, Ontario, to Trois-Rivieres, Quebec, on the north; to Boston, Mass., Providence, R.I., Hartford, Conn., and Auburn, N.Y., on the south. (Ref. 38.)

1878. Feb. 5. Flushing, Queens County, N.Y. A severe shock broke windows and crockery and shook houses at Flushing. (Ref. 141, 463.)

1884. Aug. 10. Near New York City, N.Y. This severe earthquake affected an area roughly extending along the Atlantic Coast from southern Maine to central Virginia and westward to Cleveland, Ohio (see fig. 46). Chimneys were knocked down and walls were cracked in several States, including Connecticut, New Jersey, New York, and Pennsylvania. Many towns from Hartford, Conn., to West Chester, Pa., reported fallen bricks and cracked plaster.

Property damage was severe at Amityville and Jamaica, N.Y., where several chimneys were "overturned" and large cracks formed in walls. Two chimneys were thrown down and bricks were shaken from other chimneys at Stratford (Fairfield County), Conn.; water in the Housatonic River was agitated 
violently. At Bloomfield, N.J., and Chester, Pa., several chimneys were downed and crockery was broken. Chimneys also were damaged at Mount Vernon, N.Y., and Allentown, Easton, and Philadelphia, Pa. Three shocks occurred, the second of which was most violent. This earthquake also was reported felt in Vermont, Virginia, and Washington, D.C. Several slight aftershocks were reported on Aug. 11. (Ref. 38, $76,369,463$.

1897. May 28 (May 27). Northeast New York. This earthquake was reported as severe, but little damage occurred. Felt in Massachusetts, New Hampshire, New York, and Vermont. Also felt in Canada. (Ref. 38, 76, 126.)

1914. Feb. 10. Ontario, Canada. A strong earthquake near Lanark, Ontario, broke water pipes at Canton (St. Lawrence County), N.Y., caused a cave-in at Binghamton (Broome County), and cracked the road at nearby Johnson City. Objects were thrown from shelves and walls at Albany and Syracuse. Also felt in Connecticut, Massachusetts, and Pennsylvania. Magnitude 5.5 Ukn EPB. (Ref. 38, 76.)

1916. Feb. 3 (Feb. 2). Schenectady, N.Y. A distinct earthquake at Schenectady (northwest of Albany) broke windows and dishes, threw people out of bed, and shook houses. Residents within a $40-\mathrm{km}$ radius of Schenectady felt the shock. (Ref. 272.)

1928. Mar. 18. Saranac Lake, Essex County, N.Y. Dishes fell from shelves at Saranac Lake, and at Malone, about $60 \mathrm{~km}$ north, people rushed from their houses. The shock was widely felt in northeast New York and probably in adjacent Canada. (Ref. 1, 38.)

1929. Aug. 12. Attica, Wyoming County, N.Y. The earthquake was strongest in eastern Attica and the region to the east. In Attica, 250 chimneys were thrown down, several brick buildings were damaged, and a crack formed in the railroad embankment near the railroad station. East of town, almost every monument was knocked over in the Brainerd Cemetery. West of Attica Reservoir, several wells went dry and a crack formed in the bottom of one well. Several chimneys also fell a few $\mathrm{km}$ north of Attica, at Batavia, and at Warsaw, $20 \mathrm{~km}$ southeast; only slight damage occurred at other towns. It was felt from New Hampshire to Michigan and from Maryland to northern Ontario (see fig. 47). Magnitude $4.4 \mathrm{M}_{\mathrm{S}}$ NLI, 4.9 M JOH. (Ref. 2, 38, 77, 349.)

1931. Apr. 20. Lake George area, Warren County, N.Y. The most severe damage occurred at Warrensburg, a few kilometers north of Lake George, where several chimneys were thrown down and a church spire twisted. Minor damage also occurred at Glens Falls, Luzerne, and Lake George. Although widely felt, the shock was not as strong in the
Catskills to the south as it was at equal distances in other directions. (Ref. 4, 349.)

1934. Apr. 15 (Apr. 14). Adirondack Mountains area, N.Y., near Lake Champlain. The earthquake was strongest in the Lake Champlain region, near Beekmantown (where a house shifted off its foundation); a few kilometers south at Keeseville; and at Saranac Lake (northern Essex County). Also felt in Vermont and at Montreal, Canada. (Ref. 7, 77, 126.)

1935. Nov. 1. Quebec-Ontario border, Canada. Heavy damage occurred in Timiskaming area, Canada. In the United States, chimneys and plaster sustained minor damage at Cortland, N.Y., about 50 $\mathrm{km}$ south of Syracuse. Felt in eastern Maine, south to Washington, D.C., and west to Wisconsin, including 17 States and three Canadian Provinces. Magnitude 5.9 $\mathrm{M}_{\mathrm{S}}$ NLI. (Ref. 38, 77, 349.)

1944. Sept. 5 (Sept. 4). Between Massena, N.Y., and Cornwall, Ontario, Canada. This severe earthquake was felt from Canada south to Maryland and from Maine west to Indiana (see fig. 48). It caused property damage estimated at $\$ 2$ million at Massena and Cornwall. Many chimneys in that area required rebuilding, and several structures were unsafe for occupancy until repaired. Residents of St. Lawrence County reported that many water wells went dry.

At Massena, in northern St. Lawrence County, 90 percent of the chimneys were destroyed or damaged and house foundations, plumbing, and masonry were damaged severely. Similar effects were reported at Cornwall. Cracks formed in the ground at Hogansburg, and brick-masonry and concrete structures were damaged. Chimneys were downed in several towns in New York, including Fort Covington, Keeseville, Malone, Norfolk, Ogdensburg, and Waddington. Magnitude 5.6 M NLI, 5.77 M JOH. (Ref. $17,77,194,349,533$.

1966. Jan. 1. Near Attica, Wyoming County, N.Y. Chimneys and walls were damaged slightly at Attica and $10 \mathrm{~km}$ south, at Varysburg. In addition, plaster fell at the Attica State Prison, and its main smokestack was damaged. Felt in western New York, northwest Pennsylvania, and southern Ontario, Canada. (Ref. 81, 105, 349.)

1967. June 13. Near Attica, Wyoming County, N.Y. At Attica, plaster fell, chimneys cracked, and fluorescent light fixtures were damaged. At Alabama, about $30 \mathrm{~km}$ north of Attica, ceiling tile fell in a church. This shock was felt over a small area of western New York. Magnitude 3.0 $\mathrm{M}_{\mathrm{S}}$ NLI. (Ref. 38, 40,349 .) 


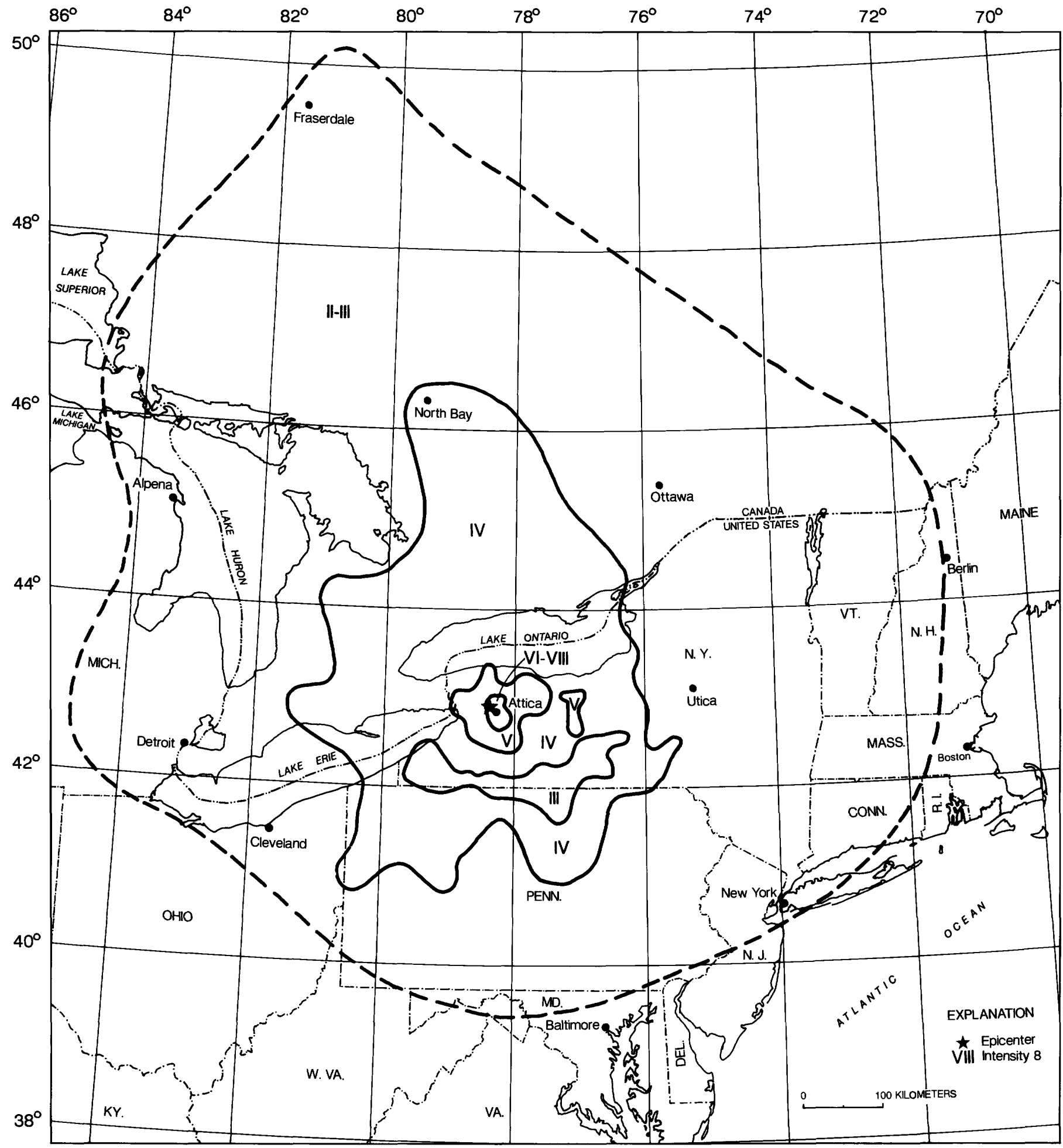

FIgURE 47.- Isoseismal map for the Attica, New York, earthquake of August 12, 1929. Isoseismals are based on intensity estimates from data listed in reference 2 of table 1. 


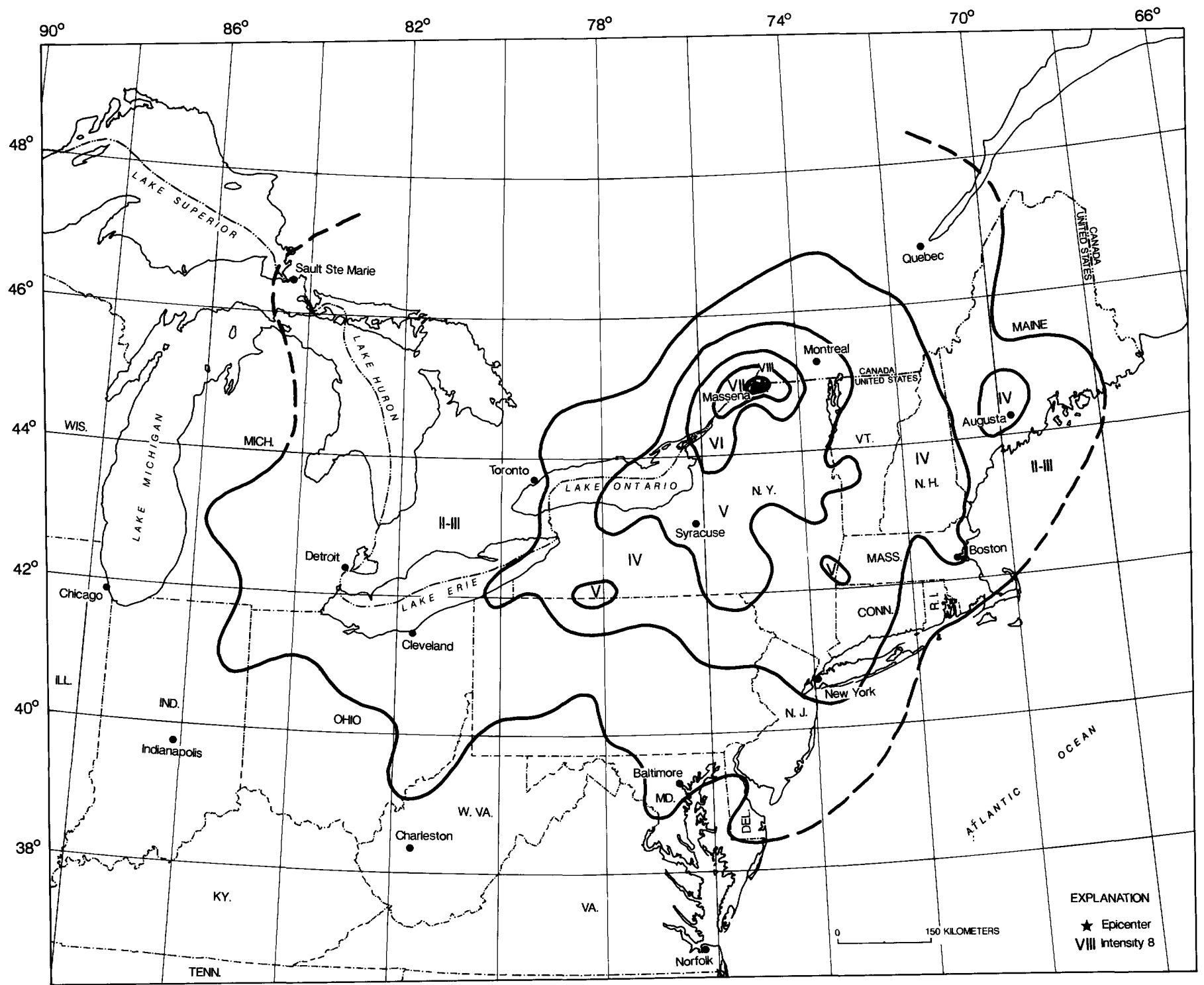

FIGURE 48.- Isoseismal map for the Massena, New York, earthquake of September 5, 1944. Isoseismals are based on intensity estimates from original U.S. Coast and Geodetic Survey questionnaires and from references 17 and 77 of table 1.

1974. June 7. Wappingers Falls area, Dutchess County, N.Y. Windows were broken in the area, and a bookcase toppled in one house. More than 100 aftershocks were recorded through June 13. Magnitude 3.8 $\mathrm{M}_{\mathrm{n}}$ PAL. (Ref. 47, 349.)

1975. June 9. Northern New York. A chimney and fireplace were cracked at Beekmantown, on Lake Champlain north of Plattsburgh. About 35 $\mathrm{km}$ east of Beekmantown, at Fairfax, Vt., slight damage also was reported. Felt in southern Quebec, Canada, and in Massachusetts, New Hampshire,
New York, and Vermont. Magnitude 4.2 $\mathrm{M}_{\mathrm{n}}$ PAL. (Ref. 38, 48, 349.)

1983. Feb. 26. Near Lagrangeville, Dutchess County, N.Y. Slight damage to property was reported at two towns in Dutchess County. At Lagrangeville, chimneys and a house foundation were cracked; at Pawling, a few buildings were damaged and a church wall was cracked. The shock was felt only in western Connecticut and southeast New York. Magnitude 3.0 $\mathrm{M}_{\mathrm{n}}$ PAL. (Ref. 360.)

1983. Oct. 7. Blue Mountain Lake area, Hamilton County, N.Y. At Blue Mountain Lake, 


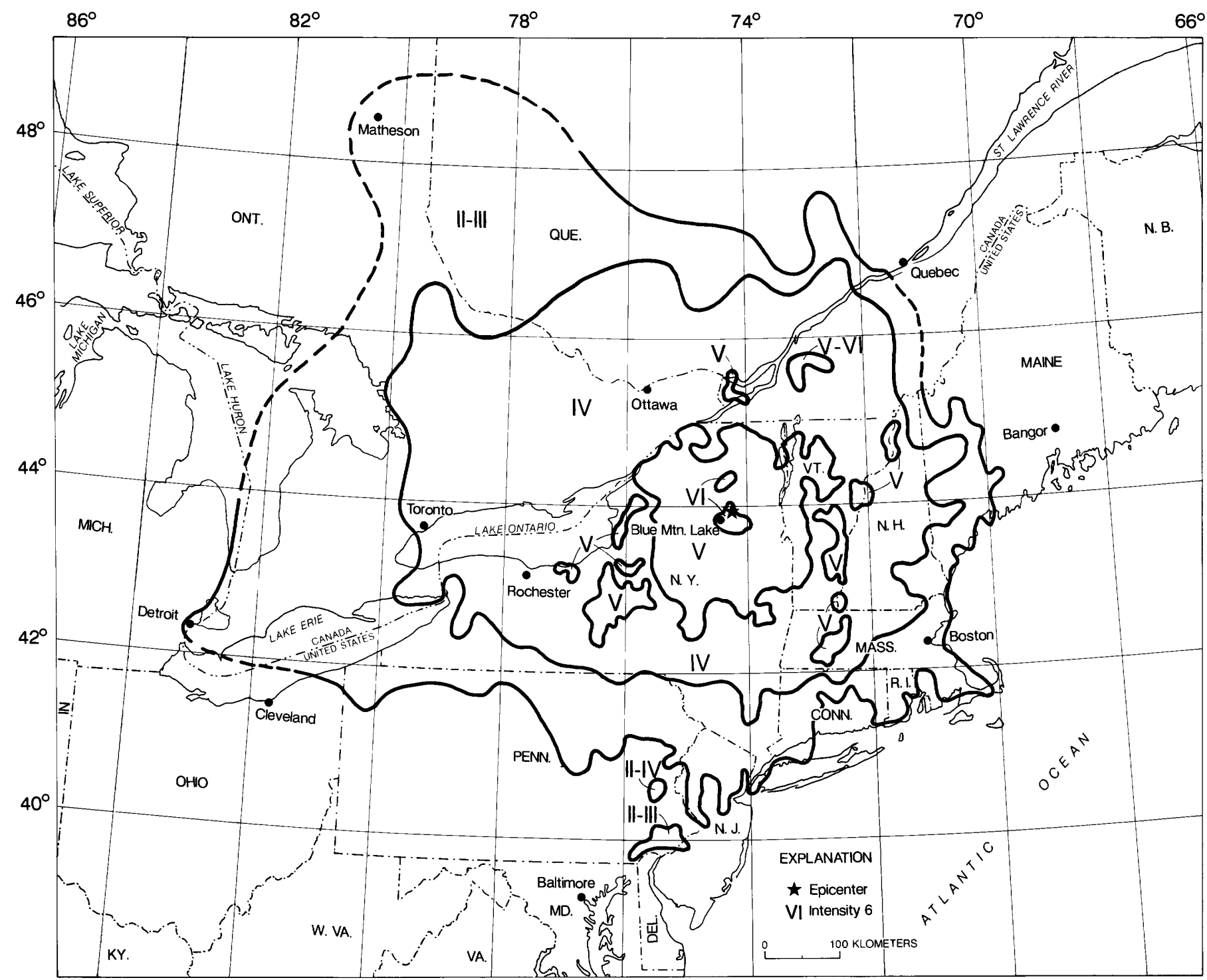

FIGURE 49.- Isoseismal map for the Blue Mountain Lake, New York, earthquake of October 7, 1983. This map is a simplified version of figure 37 in reference 360 of table 1.

one old chimney collapsed, about 20 tombstones slid or rotated, and some minor cracks formed in plaster walls. Several landslides were reported. Light damage also occurred at several other towns in the area, but the most common effects were cracked chimneys, broken dishes or glassware, and overturned or fallen objects. Although this earthquake caused only minor damage, it was felt over a wide region (see fig. 49), including two Provinces in Canada and 12 States. Magnitude 5.3 $\mathrm{M}_{\mathrm{n}}$ BLA, 5.1 $\mathrm{M}_{\mathrm{n}}$ SLM, $4.89 \mathrm{M}$ JOH. (Ref. 360.)
1985. Oct. 19. Southeast New York. Windows were broken at Newburgh, N.Y. (about $140 \mathrm{~km}$ south of Albany), and Glenville, Fairfield County, Conn. Plaster and drywall also were cracked and glassware was broken in Newburgh. Light damage was sustained at a few towns in Connecticut, New Jersey, and New York. Felt over a large area of Connecticut, Massachusetts, New Jersey, New York, and Pennsylvania. A moderate aftershock was felt in Connecticut, New Jersey, and New York on Oct. 21 (10 37 UTC). Magnitude 4.0 $\mathrm{M}_{\mathrm{D}}$ WES. (Ref. 371.) 

NORTH GAROLINA

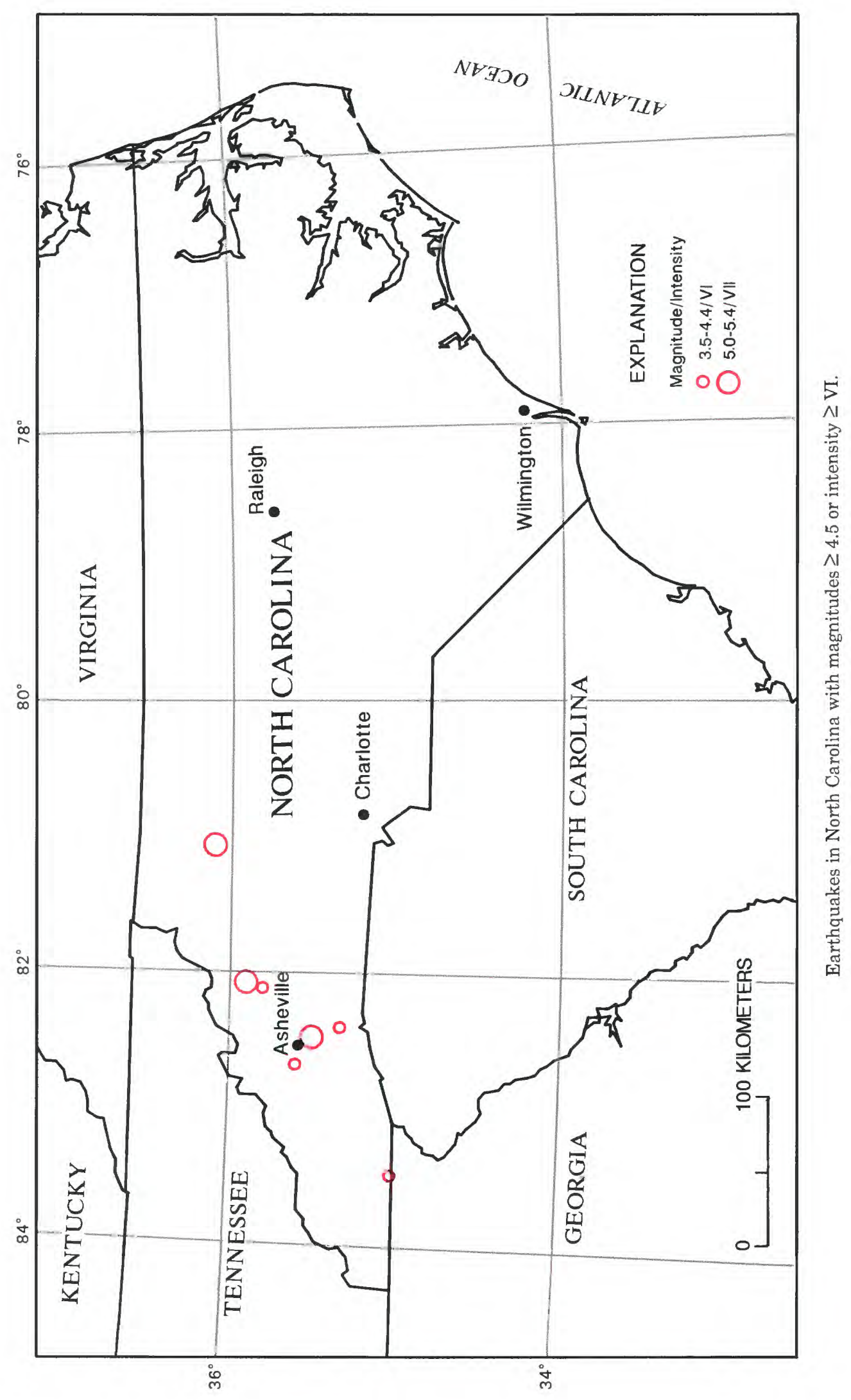


NORTH CAROLINA

[See table 1 for hypocenter and intensity references and table 2 for definitions of magnitude source codes. @, felt area is less than 1,000 $\mathrm{km}^{2}$. Leader (-) indicates information is not available]

\begin{tabular}{|c|c|c|c|c|c|c|c|c|c|c|c|c|c|}
\hline \multirow{2}{*}{\multicolumn{2}{|c|}{ Date }} & \multirow{2}{*}{$\begin{array}{c}\text { Origin } \\
\text { time (UTC) }\end{array}$} & \multicolumn{4}{|c|}{ Hypocenter } & \multicolumn{4}{|c|}{ Magnitude } & \multicolumn{2}{|c|}{ Intensity } & \multirow{3}{*}{$\begin{array}{c}\text { Felt area } \\
\left(1,000 \mathrm{~km}^{2}\right)\end{array}$} \\
\hline & & & \multirow{2}{*}{$\begin{array}{l}\text { Latitude } \\
\left({ }^{\circ}\right)\end{array}$} & \multirow{2}{*}{$\begin{array}{l}\text { Longitude } \\
\left({ }^{\circ}\right)\end{array}$} & \multirow{2}{*}{$\begin{array}{l}\text { Depth } \\
(\mathrm{km})\end{array}$} & \multirow[t]{2}{*}{ Ref } & \multicolumn{2}{|c|}{ USGS } & \multirow[t]{2}{*}{ Other } & \multirow{2}{*}{$\begin{array}{c}\text { Moment } \\
\text { M }\end{array}$} & \multirow[t]{2}{*}{ MM } & \multirow[t]{2}{*}{ Ref } & \\
\hline $\mathrm{Yr}_{\mathrm{r}}$ & $\mathrm{Mo} \mathrm{Da}$ & $\mathrm{h} \mathrm{m} \mathrm{s}$ & & & & & $m_{b}$ & $M_{S}$ & & & & & \\
\hline 1861 & $08 \quad 31$ & 1022 & $36.1 \mathrm{~N}$ & $81.1 \mathrm{~W}$ & - & 55 & - & - & $5.00 \mathrm{M}_{\mathrm{fa}} \mathrm{SC}$ & - & VI & 38 & 784 \\
\hline 1916 & $02 \quad 21$ & 2339 & $35.5 \mathrm{~N}$ & $82.5 \mathrm{~W}$ & - & 272 & - & - & $5.20 \mathrm{M}_{\mathrm{fa}} \mathrm{SC}$ & - & VII & 67 & 600 \\
\hline 1926 & 0708 & 0950 & $35.9 \mathrm{~N}$ & 82.1 W & - & 71 & - & - & - & - & VII & 68 & @ \\
\hline 1957 & $05 \quad 13$ & 142451.1 & $35.799 \mathrm{~N}$ & $82.142 W$ & 005 & 349 & - & - & $4.00 \mathrm{M}_{\mathrm{fa}} \mathrm{SC}$ & - & VI & 132 & 16 \\
\hline 1957 & 0702 & 093301 & $35.6 \mathrm{~N}$ & 82.7 W & 007 & 155 & - & - & $3.70 \mathrm{M}_{\mathrm{fa}} \mathrm{SC}$ & - & VI & 132 & 3 \\
\hline 1957 & 1124 & 200617 & $35.0 \quad \mathrm{~N}$ & $83.5 \mathrm{~W}$ & - & 30 & - & - & $3.90 \mathrm{M}_{\mathrm{fa}} \mathrm{SC}$ & - & VI & 132 & 12 \\
\hline 1981 & 0505 & 212156.7 & $35.327 \mathrm{~N}$ & $82.422 W$ & 010 & 339 & - & - & $3.50 \mathrm{M}_{\mathrm{n}}$ BLA & - & VI & 325 & 10 \\
\hline
\end{tabular}

[Reference (Ref.) numbers given in parentheses at the end of each description refer to sources of data in table 1. Magnitude values are described in the Introduction, and codes are defined in table 2.]

1861. Aug. 31. Near Wilkesboro, Wilkes County, N.C. Bricks were shaken from chimneys, doors were jarred open, and clocks stopped running at Wilkesboro, about $85 \mathrm{~km}$ west of Winston-Salem. Felt along the Atlantic Coast from Washington, D.C., southward to Charleston, S.C., and Columbus, Ga., and westward to Cincinnati, Ohio, Louisville, Ky., and Gallatin, Tenn. Magnitude $5.1 \mathrm{M}_{\mathrm{fa}}$ NUT. (Ref. $38,55,508$.

1916. Feb. 21. Near Waynesville, Haywood County, N.C. Tops of chimneys were thrown to the ground; windowpanes were broken in many houses; and people rushed into the streets at Waynesville. At Sevierville, Tenn., about $70 \mathrm{~km}$ northwest of Waynesville, bricks were shaken from chimneys. In Wear's Cove, about $16 \mathrm{~km}$ southwest of Sevierville, the flow of water in springs increased and in places water became muddy. Minor damage was reported in western Tennessee at Athens, Knoxville, Maryville, Morristown, and Newport, Tenn.; at Tryon, N.C.; and at Bristol, Va. Also reported felt in Alabama, Georgia, Kentucky, South Carolina, and West Virginia (see fig. 50). (Ref. $67,71,272,508,600$.)

1926. July 8. Southern Mitchell County, N.C. A sharp local earthquake in Mitchell County caused minor damage-one downed chimney, cracks in chimneys and foundations of houses, broken water pipes and glassware, and shifting of houses on foundations. Ground cracks also were reported. Damage was confined to an area about $1 \mathrm{~km}$ long and $275 \mathrm{~m}$ wide. Town names were not mentioned in any of the accounts on this event. (Ref. $68,71,218,508$.)
1957. May 13. Near Woodlawn, McDowell County, N.C. A sprinkler pipe was shaken loose at a factory at Woodlawn, and books fell from library shelves. Slight damage to plaster occurred at several towns in the area. Old cracks in a wall were enlarged at Busick, near Mt. Mitchell in southern Yancey County. The shock was strong in other towns in Burke and McDowell Counties and was reported felt at two towns in South Carolina. Magnitude 4.1 $\mathrm{M}_{\mathrm{fa}}$ DG. (Ref. 30, 132, 349, 508.)

1957. July 2. Buncombe County area, N.C. Minor damage reported in western North Carolina included cracks in walls and plaster at Asheville, cracks in retaining wall at Marshall, damaged chimneys and cracks in plaster at Swannanoa, and cracks in plaster at Weaverville. Also reported felt in Haywood and Madison Counties, N.C., and at Flag Pond, Tenn. (Ref. 38, 132, 155, 508.)

1957. Nov. 24. Northwest Jackson County, N.C. At Hartford, Tenn. (about $70 \mathrm{~km}$ southeast of Knoxville), slight damage to buildings included a crack in one wall and a kitchen that was separated from the rest of the house. The earthquake shifted furniture in Jackson County, at Cherokee and Sylva, N.C., about $50 \mathrm{~km}$ south of Hartford, Tenn. Also felt in South Carolina. Magnitude $4.0 \mathrm{M}_{\mathrm{fa}}$ BAR. (Ref. 30, 132.)

1981. May 5. Near Hendersonville, Henderson County, N.C. Cracks formed in windows and a concrete patio in Hendersonville, and one sidewalk shifted $5 \mathrm{~cm}$. Cracks also occurred in drywall and a house foundation a few kilometers southeast of Hendersonville, at Zirconia. Water in several wells became muddy in the Dana area. Also felt in South Carolina, Tennessee, and Virginia. Magnitude 3.3 $\mathrm{M}_{\mathrm{D}}$ TEC. (Ref. 325, 339.) 


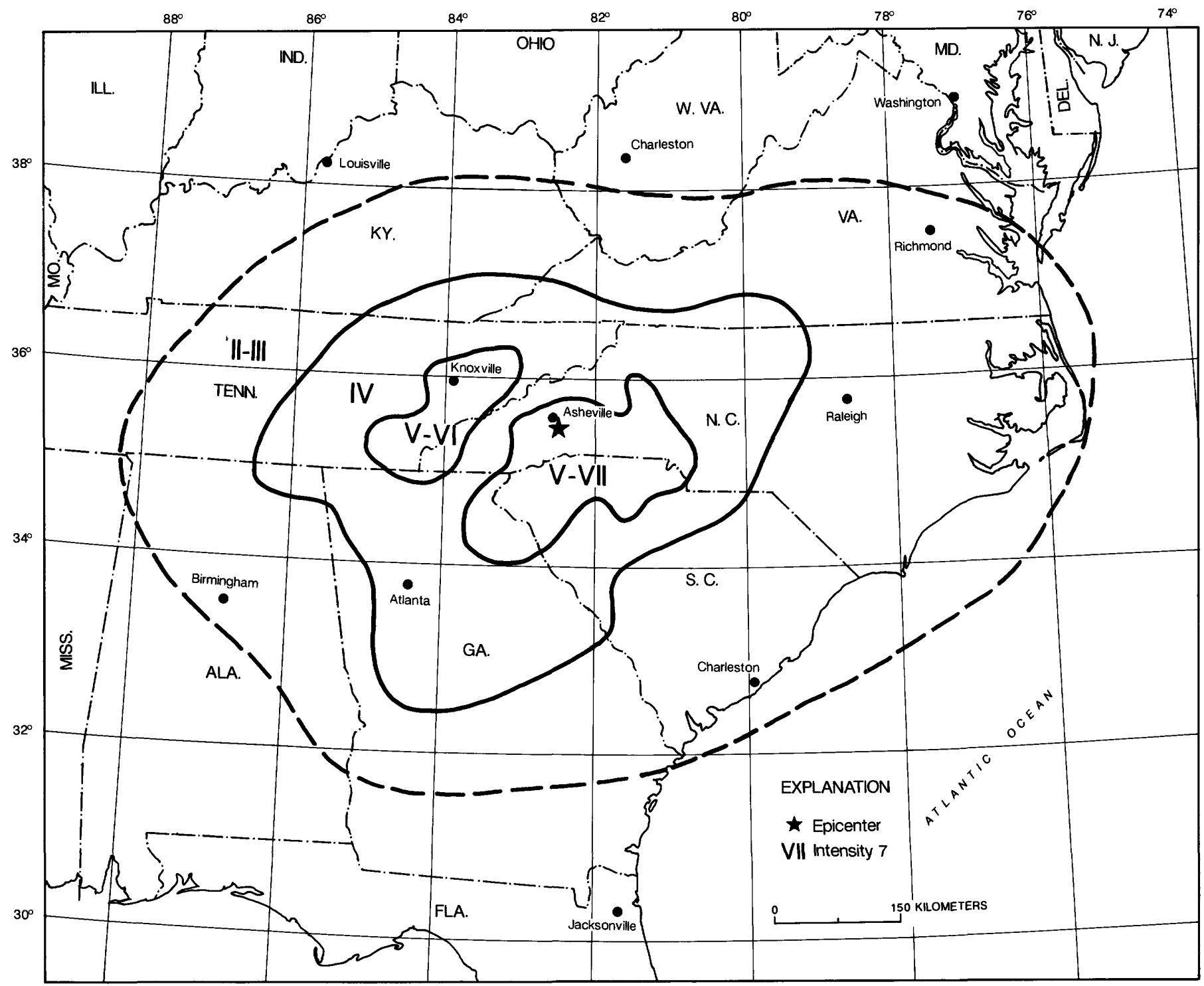

FIGURE 50.- Isoseismal map for the Waynesville, North Carolina, earthquake of February 21, 1916. Isoseismals are based on intensity estimates from data listed in reference 272 of table 1. 

NORTH DAKOTA

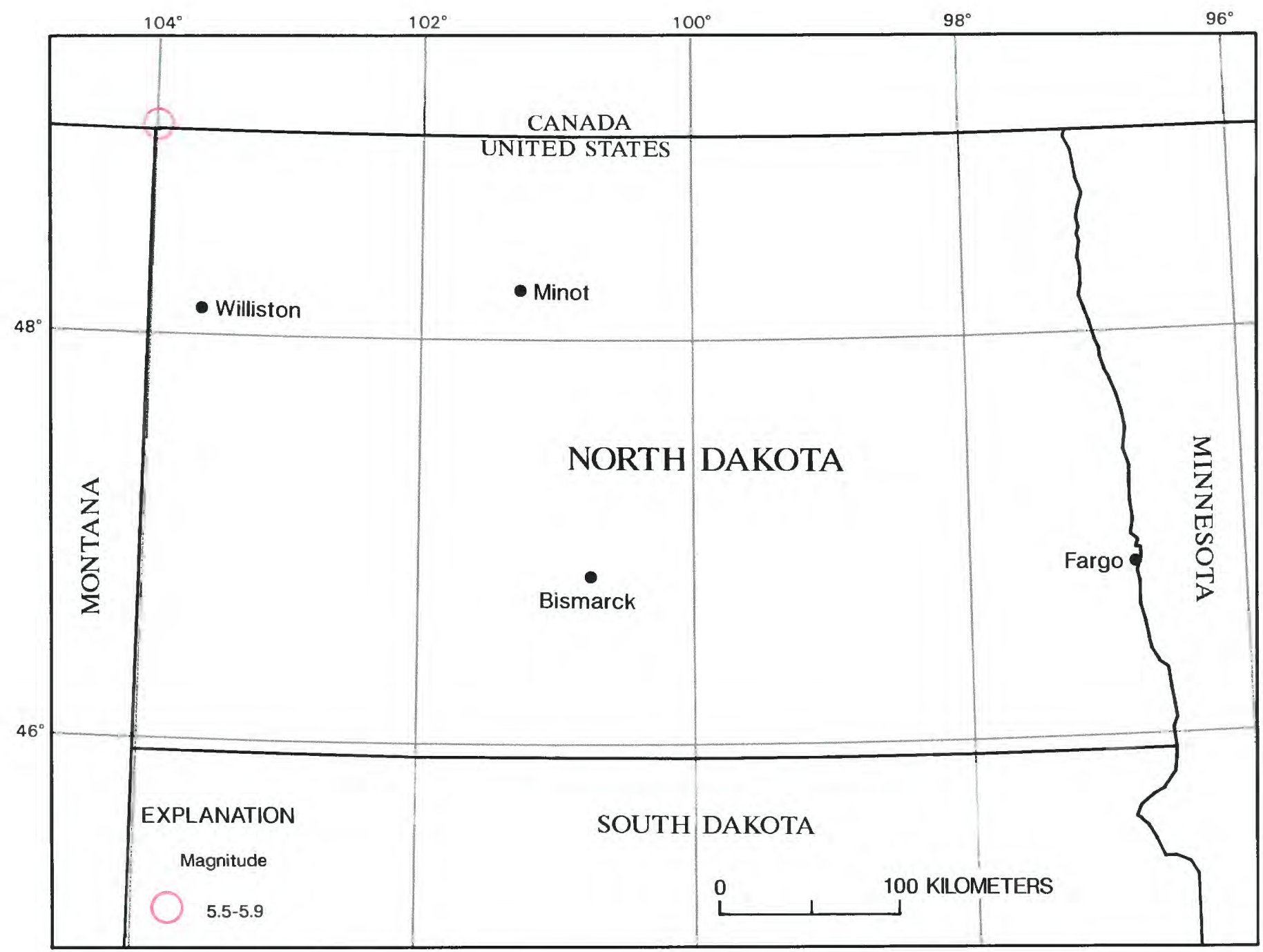

Earthquake in North Dakota with a magnitude $\geq 4.5$ or intensity $\geq$ VI. 


\section{NORTH DAKOTA}

[See table 1 for hypocenter and intensity references and table 2 for definitions of magnitude source codes. Leader (--) indicates information is not available]

\begin{tabular}{|c|c|c|c|c|c|c|c|c|c|c|c|c|c|}
\hline \multirow{2}{*}{\multicolumn{2}{|c|}{ Date }} & \multirow{2}{*}{$\begin{array}{l}\text { Origin } \\
\text { time (UTC) }\end{array}$} & \multicolumn{4}{|c|}{ Hypocenter } & \multicolumn{4}{|c|}{ Magnitude } & \multicolumn{2}{|c|}{ Intensity } & \multirow{3}{*}{$\begin{array}{c}\text { Felt area } \\
\left(1,000 \mathrm{~km}^{2}\right)\end{array}$} \\
\hline & & & \multirow{2}{*}{$\begin{array}{l}\text { Latitude } \\
\left({ }^{\circ}\right)\end{array}$} & \multirow{2}{*}{$\begin{array}{l}\text { Longitude } \\
\left(^{\circ}\right)\end{array}$} & \multirow{2}{*}{$\begin{array}{l}\text { Depth } \\
(\mathrm{km})\end{array}$} & \multirow[t]{2}{*}{ Ref } & \multicolumn{2}{|c|}{ USGS } & \multirow[t]{2}{*}{ Other } & \multirow{2}{*}{$\begin{array}{l}\text { Moment } \\
\text { M }\end{array}$} & \multirow[t]{2}{*}{ MM } & \multirow[t]{2}{*}{ Ref } & \\
\hline $\mathrm{Yr}_{\mathrm{r}}$ & Mo Da & $\mathrm{h} \mathrm{m} \mathrm{s}$ & & & & & $m_{b}$ & $\mathrm{M}_{\mathbf{S}}$ & & & & & \\
\hline 1905 & 0516 & 0415 & $49.0 \mathrm{~N}$ & $104.0 \mathrm{~W}$ & - & 413 & - & - & $5.50 M_{n}$ EPB & - & VI & 413 & 1300 \\
\hline
\end{tabular}

[Reference (Ref.) numbers given in parentheses at the end of each description refer to sources of data in table 1.]

1909. May 16 (May 15). North DakotaMontana-Saskatchewan border region. This moderate earthquake was felt widely over south-central Canada and the north-central United States, including the States of Montana and North Dakota. A retaining wall fell in Helena, southern Lewis and Clark County, Mont.; plaster was cracked at Havre (northwest of Helena, in Hill County). The shock also was "severe" in Dickinson (Stark County), N. Dak. Some windows were broken and articles fell from shelves at a few towns in Saskatchewan, Canada. (Ref. 249, 413.) 


\section{OHIO}

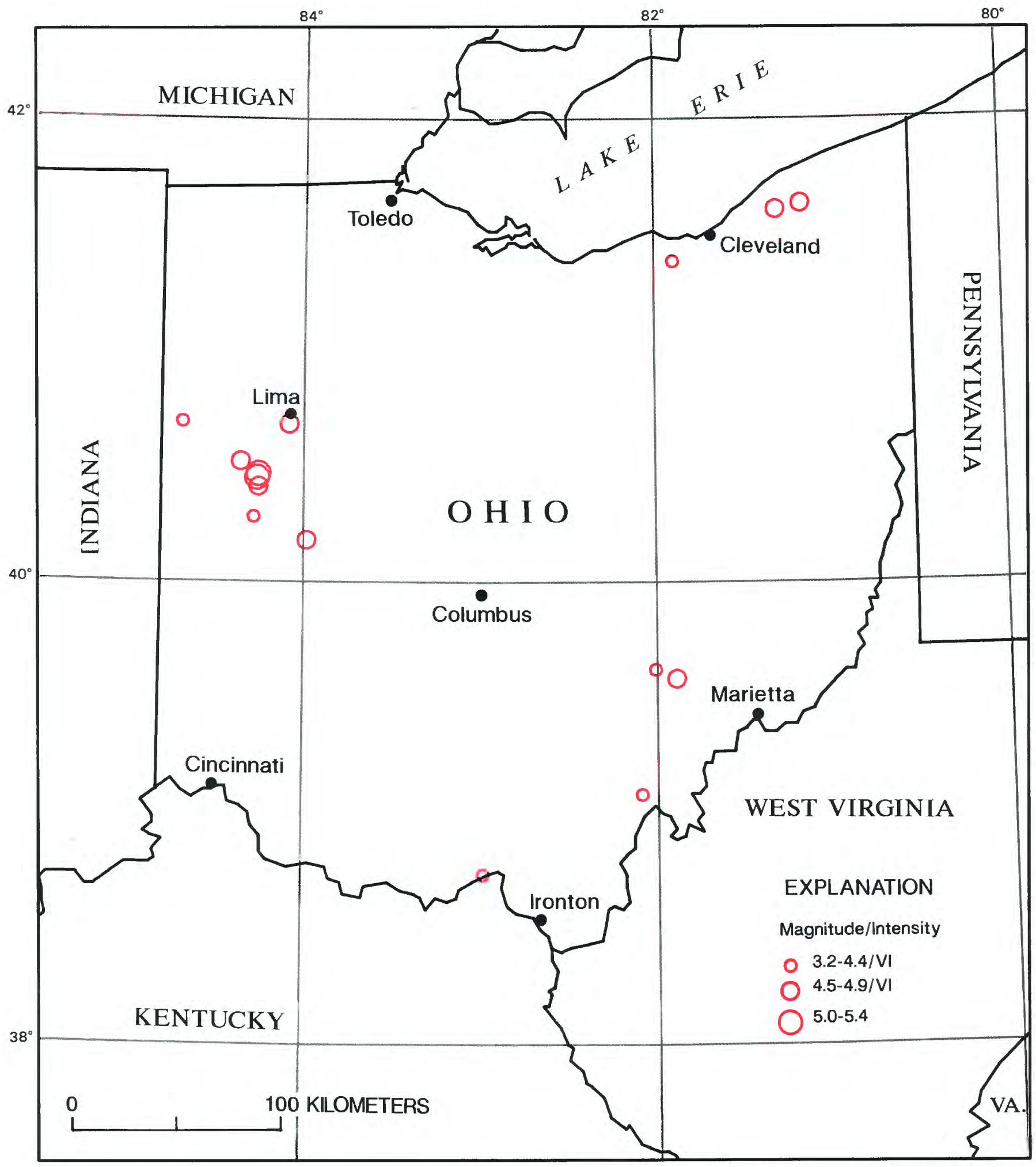

Earthquakes in Ohio with magnitudes $\geq 4.5$ or intenstiy $\geq$ VI. 
OHIO

[See table 1 for hypocenter and intensity references and table 2 for definitions of magnitude source codes. (0, felt area is less than $1,000 \mathrm{~km}^{2}$. Leader (-) indicates information is not available

\begin{tabular}{|c|c|c|c|c|c|c|c|c|c|c|c|c|c|}
\hline \multirow{2}{*}{\multicolumn{2}{|c|}{ Date }} & \multirow{2}{*}{$\begin{array}{c}\text { Origin } \\
\text { time (UTC) }\end{array}$} & \multicolumn{4}{|c|}{ Hypocenter } & \multicolumn{4}{|c|}{ Magnitude } & \multicolumn{2}{|c|}{ Intensity } & \multirow{3}{*}{$\begin{array}{l}\text { Felt area } \\
\left(1,000 \mathrm{~km}^{2}\right)\end{array}$} \\
\hline & & & \multirow{2}{*}{$\begin{array}{l}\text { Latitude } \\
\left({ }^{\circ}\right)\end{array}$} & \multirow{2}{*}{$\begin{array}{l}\text { Longitude } \\
\left({ }^{\circ}\right)\end{array}$} & \multirow{2}{*}{$\begin{array}{l}\text { Depth } \\
(\mathrm{km})\end{array}$} & \multirow[t]{2}{*}{ Ref } & \multicolumn{2}{|c|}{ USGS } & \multirow[t]{2}{*}{ Other } & \multirow{2}{*}{$\begin{array}{l}\text { Moment } \\
\text { M }\end{array}$} & \multirow[t]{2}{*}{ MM } & \multirow[t]{2}{*}{ Ref } & \\
\hline $\mathrm{Yr}_{\mathrm{r}}$ & Mo Da & $\mathrm{h} \mathrm{m} s$ & & & & & $m_{b}$ & $\mathrm{M}_{\mathbf{S}}$ & & & & & \\
\hline 1776 & & 1400 & $39.6 \mathrm{~N}$ & 81.9 W & - & 116 & - & - & - & - & VI & 60 & - \\
\hline 1875 & 0618 & 1243 & $40.2 \mathrm{~N}$ & $84.0 \mathrm{~W}$ & - & 38 & - & - & $4.70 \mathrm{M}_{\mathrm{fa}} \mathrm{SC}$ & - & VII & 38 & 100 \\
\hline 1884 & $09 \quad 19$ & 1914 & $40.7 \mathrm{~N}$ & 84.1 W & - & 38 & - & - & $4.80 \mathrm{M}_{\mathrm{fa}} \mathrm{SC}$ & - & VI & 38 & 373 \\
\hline 1900 & 0409 & 14 & $41.4 \mathrm{~N}$ & 81.9 W & - & 116 & - & - & $3.40 \mathrm{M}_{\mathrm{fa}}$ BAR & - & VI & 105 & - \\
\hline 1901 & 0517 & 0600 & $38.75 \mathrm{~N}$ & $83.0 \mathrm{~W}$ & - & 584 & - & - & $4.20 \mathrm{M}_{\mathrm{fa}} \mathrm{SC}$ & - & VI & 584 & 42 \\
\hline 1926 & 1105 & 1653 & $39.1 \mathrm{~N}$ & 82.1 W & - & 38 & - & - & $3.80 \mathrm{M}_{\mathrm{fa}}$ & - & VII & 8 & 1 \\
\hline 1930 & 0930 & 2040 & $40.3 \mathrm{~N}$ & $84.3 \quad W$ & - & 38 & - & - & $4.20 \mathrm{M}_{\mathrm{fa}}$ BAR & - & VII & 38 & - \\
\hline 1931 & 0920 & 230503.4 & $40.429 \mathrm{~N}$ & $84.270 \mathrm{~W}$ & 005 & 349 & - & - & $4.70 \mathrm{M}_{\mathrm{fa}} \mathrm{SC}$ & - & VII & 38 & 90 \\
\hline 1937 & 0302 & 144733.3 & $40.488 \mathrm{~N}$ & $84.273 W$ & 002 & 349 & - & - & $5.00 \mathrm{M}_{\mathrm{fa}} \mathrm{SC}$ & - & VII & 38 & 280 \\
\hline 1937 & 0309 & 054435.5 & $40.470 \mathrm{~N}$ & $84.280 W$ & 003 & 349 & - & - & $5.40 \mathrm{M}_{\mathrm{fa}} \mathrm{SC}$ & - & VIII & 38 & 390 \\
\hline 1943 & $03 \quad 09$ & 03252 & $\mathrm{~N}$ & W & 007 & 34 & - & - & $s$ & - & VI & 105 & 150 \\
\hline 1952 & 0620 & 093808.6 & $39.640 \mathrm{~N}$ & $82.023 W$ & 009 & 349 & - & - & $4.00 \mathrm{M}_{\mathrm{fa}} \mathrm{SC}$ & - & VI & 38 & 13 \\
\hline 1977 & $06 \quad 17$ & 153946.9 & $40.705 \mathrm{~N}$ & $84.707 \mathrm{~W}$ & 001 & 349 & - & - & $3.20 \mathrm{M}_{\mathrm{n}}$ AAM & - & VI & 39 & @ \\
\hline 1986 & 0131 & 164642.3 & $41.650 \mathrm{~N}$ & $81.162 W$ & 002 & 562 & 5.0 & - & $4.90 \mathrm{M}_{\mathrm{n}} \mathrm{SLM}$ & $4.97 \mathrm{GS}$ & VI & 562 & 322 \\
\hline 1986 & $07 \quad 12$ & 081937.9 & $40.537 \mathrm{~N}$ & $84.371 W$ & 010 & 562 & 4.5 & - & $4.60 \mathrm{M}_{\mathrm{n}}$ SLM & $4.39 \mathrm{SZ}$ & VI & 562 & 90 \\
\hline
\end{tabular}

[Reference (Ref.) numbers given in parentheses at the end of each description refer to sources of data in table 1. Magnitude values are described in the Introduction, and codes are defined in table 2.]

1776. Date unknown. Near the Muskingum River. In the summer of 1776, an earthquake frightened people and animals and overthrew furniture. Duration of the shock was estimated at 2 to $3 \mathrm{~min}$ utes. (Ref. 60, 116.)

1875. June 18. Western Ohio. This earthquake was most severe west of Columbus, at Urbana (Champaign County) and Sidney (Shelby County), where chimneys were thrown down and walls were cracked. Also felt in southern Illinois, southwest Indiana, northwest Kentucky, and eastern Missouri. (Ref. 38, 105.)

1884. Sept. 19. Near Lima, Allen County, Ohio. Slight damage occurred at Lima, where the shock was "of considerable violence and caused much excitement." Plaster was shaken from ceilings east and southeast of Columbus at Zanesville, Ohio, and Parkersburg, W. Va. Windows and dishes were broken at Defiance and Norwalk, Ohio; to the west at Fort Wayne and Muncie, Ind.; to the north at Lansing, Mich.; and to the east at Wheeling, W. Va. Furniture was displaced and buildings were heavily shaken at Urbana, Ohio, in Champaign County, and at many other towns in the region. Also felt in Iowa,
Kentucky, Pennsylvania, and western Ontario, Canada, and at Washington, D.C., by workmen on top of the unfinished Washington Monument (see fig. 51). Magnitude 4.8 $\mathrm{M}_{\mathrm{fa}} \mathrm{BAR}, 5.1 \mathrm{M}_{\mathrm{fa}}$ SG. (Ref. 38, 60, $105,463,529$.

1900. Apr. 9. Berea, southeast Cuyahoga County, Ohio. Two chimneys on one house toppled at Berea. This event was an explosion according to ref. 443. (Ref. 105, 116, 443.)

1901. May 17. Near Portsmouth, Scioto County, Ohio. The strongest effects of this earthquake were reported in Scioto County, near the Kentucky-Ohio-West Virginia border. At Portsmouth, tops of chimneys toppled, bricks tumbled from many chimneys, and windows in several houses were shattered. East of Portsmouth, at Sciotoville, many chimneys were damaged and dishes were thrown from cupboards. At Gallipolis, Gallia County, plaster in one house was shaken loose. Reported felt mainly in the area along the border of Kentucky, Ohio, and West Virginia, including Greenup and Lewis Counties, Ky., and Cabell and Kanawha Counties, W. Va., to the south; Adams and Brown Counties, Ohio, to the west; Muskingum and Washington Counties, Ohio, and Wood County, W. Va., to the northeast; and Highland County, Ohio, to the northwest. (Ref. 584.)

1926. Nov. 5. Southeast Ohio, near Pomeroy, Meigs County. This earthquake toppled chimneys 


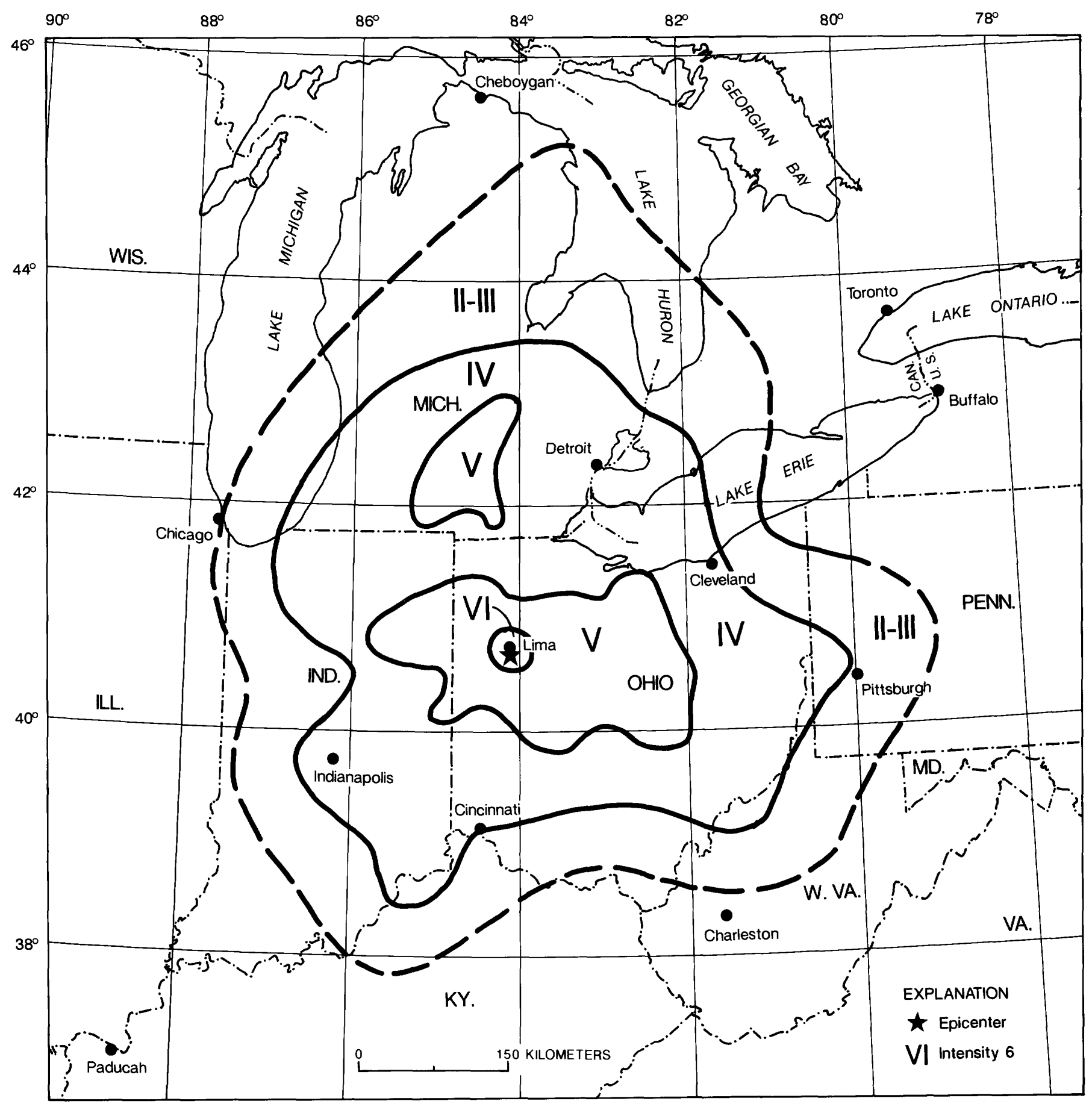

FIGURE 51.- Isoseismal map for the Lima, Ohio, earthquake of September 19, 1884. Isoseismals are based on intensity estimates from data listed in reference 463 of table 1.

at Keno and nearby Pomeroy and overturned a heating stove at Chester, west of Keno. Also, one stovepipe was knocked down at Success, and a flue was downed at Bashan. Explosive earth sounds were reported. Felt throughout Meigs County, Ohio, and at Letart, W.Va. Magnitude 3.6 $\mathrm{M}_{\mathrm{fa}}$ BAR. (Ref. $38,105,218$.)
1930. Sept. 30. Near Anna, Shelby County, Ohio. A brief but strong shock was felt over a wide area in Ohio. It was strongest at Anna, where a chimney at a schoolhouse toppled and plaster cracked and fell. Brief shocks were felt at Sidney, about $12 \mathrm{~km}$ south of Anna, on Sept. 29 at 2115 UTC and Sept. 30 at 2350 UTC. (Ref. $3,38,60$.) 
1931. Sept. 20. Anna, Shelby County, Ohio. Moderate damage occurred at Anna and in nearby towns in Shelby County. At Anna, several chimneys were downed, many chimneys were damaged, and two large cornice stones were thrown from the Lutheran Church. At Botkins, north of Anna, the roof of the public school was pulled apart and its ceiling collapsed; several chimneys also were thrown down or otherwise damaged. In southern Shelby County, at Houston and Sidney, chimneys fell and walls and windows were cracked. Felt over a large part of Ohio and in eastern Indiana and northern Kentucky. Magnitude 4.6 $\mathrm{M}_{\mathrm{fa}} \mathrm{BAR}, 3.6 \mathrm{M}_{\mathrm{S}} \mathrm{BAR}, 4.5 \mathrm{M}_{\mathrm{fa}}$ DG. (Ref. $38,105,349,353,509$.)

1937. Mar. 2. Western Ohio, near Anna and Sidney, Shelby County. Damage was heaviest to brick chimneys and buildings at Anna and Sidneymany chimneys fell, walls cracked, and plaster fell. Springs and other water wells increased their flow, but output from oil and gas wells was decreased.

At the Anna public school, walls of the building were cracked so severely that it was declared unsafe; two churches sustained minor damage. Chimneys also were damaged at nearby Botkins and Jackson Center and in southern Auglaize County, at Wapakoneta. Several tombstones were rotated in three cemeteries near Anna. Plaster fell in buildings as far away as Fort Wayne, Ind., and plaster cracked at Indianapolis. Two to five shocks were felt in many places. Also felt in the States of Indiana, Kentucky, Michigan, and West Virginia, and in Ontario, Canada. Magnitude $4.8 \mathrm{M}_{\mathrm{fa}}$ BAR, $4.7 \mathrm{M}_{\mathrm{fa}}$ DG. (Ref. 38, $349,353,509,524$.)

1937. Mar. 9 (Mar. 8). Western Ohio. An earthquake stronger than the shock on Mar. 2 centered near Anna in Shelby County. The three-story schoolhouse at Anna was cracked severely, and the churches that were damaged in the Mar. 2 shock were further damaged. Almost every chimney was broken or twisted, and house foundations and walls were cracked. A few chimneys fell at Sidney, about $12 \mathrm{~km}$ south of Anna, and plaster was damaged.

Subsurface changes caused by the two earthquakes included renewed activity of springs, conversion of ordinary wells to artesian wells, and an increase in the flow of other water wells; the output of both oil and gas wells was reduced. A spring at Huntsville (Logan County), dry for 8 years, began "spouting water" after the second shock, and the flow of artesian wells was increased at New Knoxville (about 45 $\mathrm{km}$ west of Huntsville). This shock was felt in upper stories of multistory buildings in Chicago and Milwaukee and in Toronto, Canada. Also felt in Kentucky, Michigan, Missouri, Pennsylvania, and West
Virginia. Magnitude 5.5 $\mathrm{M}_{\mathrm{S}} \mathrm{GR}, 5.0 \mathrm{M}_{\mathrm{fa}} \mathrm{BAR}, 4.7 \mathrm{M}_{\mathrm{S}}$ BAR, 4.9 $\mathrm{M}_{\mathrm{fa}}$ DG. (Ref. 38, 349, 353, 524.)

1943. Mar. 9 (Mar. 8). Lake Erie area, Ohio. An earthquake broke windows and dishes and cracked plaster in the Lake Erie area. The widely felt shock was reported in Michigan, New York, Ohio, Pennsylvania, and Canada. Magnitude $4.7 \mathrm{M}_{\mathrm{fa}}$ BAR. (Ref. 105, 349.)

1952. June 20. Near Zanesville, Muskingum County, Ohio. One old chimney fell and doors were thrown open at Zanesville. Felt throughout southeast Ohio. Magnitude 4.1 $\mathrm{M}_{\mathrm{fa}}$ BAR, 4.1 $\mathrm{M}_{\mathrm{fa}}$ DG. (Ref. 38, $105,349,353$.

1977. June 17. Northwest Ohio. The earthquake caused slight damage in several towns in Mercer County. Plaster fell at Coldwater, and cracks formed in a sidewalk and a house foundation. Cracks in sidewalks, walls, and foundations also were reported north of Coldwater, at Celina and Rockford. It was reported only in Mercer County - from Celina south to Chickasaw, west to Fort Recovery, and north to Rockford. (Ref. 39, 349, 353.)

1986. Jan. 31. Northeast Ohio. This earthquake caused minor property damage in several towns in northeast Ohio and northwest Pennsylvania; 17 people were injured in the epicentral area.

Most of the damage to houses and commercial buildings occurred in Ashtabula, Geauga, Lake, Trumbull, and Wood Counties in Ohio and Crawford and Erie Counties in Pennsylvania. It mainly included fallen ceiling tiles and plaster; cracked chimneys, foundations, and brick walls; and broken windows and underground pipes. Changes in the flow of water were observed in more than a dozen wells in Lake and Geauga Counties, east of Cleveland. The changes included variations (starting, stopping) in the flow of water and sediment deposits in water. In Leroy Township, a small pond was formed from the flow of a new artesian well. Another artesian well suddenly began feeding water to an old water trough.

Over the next 2 months, 13 aftershocks of magnitude 0.5 to 2.5 were recorded in the area, and 13 more aftershocks of about magnitude 1.0 were detected through Apr. 15, 1987. The main earthquake was felt over a large area of the Eastern United States, covering all or parts of eight States (Illinois, Indiana, Kentucky, Michigan, New York, Pennsylvania, West Virginia) and Ontario, Canada (see fig. 52). It also was reported by people on the top floors of multistory buildings in Delaware, Maryland, New Jersey, Virginia, and Wisconsin, as well as Washington, D.C. Magnitude $5.3 \mathrm{M}_{\mathrm{n}}$ EPB, $4.88 \mathrm{M}$ JOH. (Ref. 562.) 


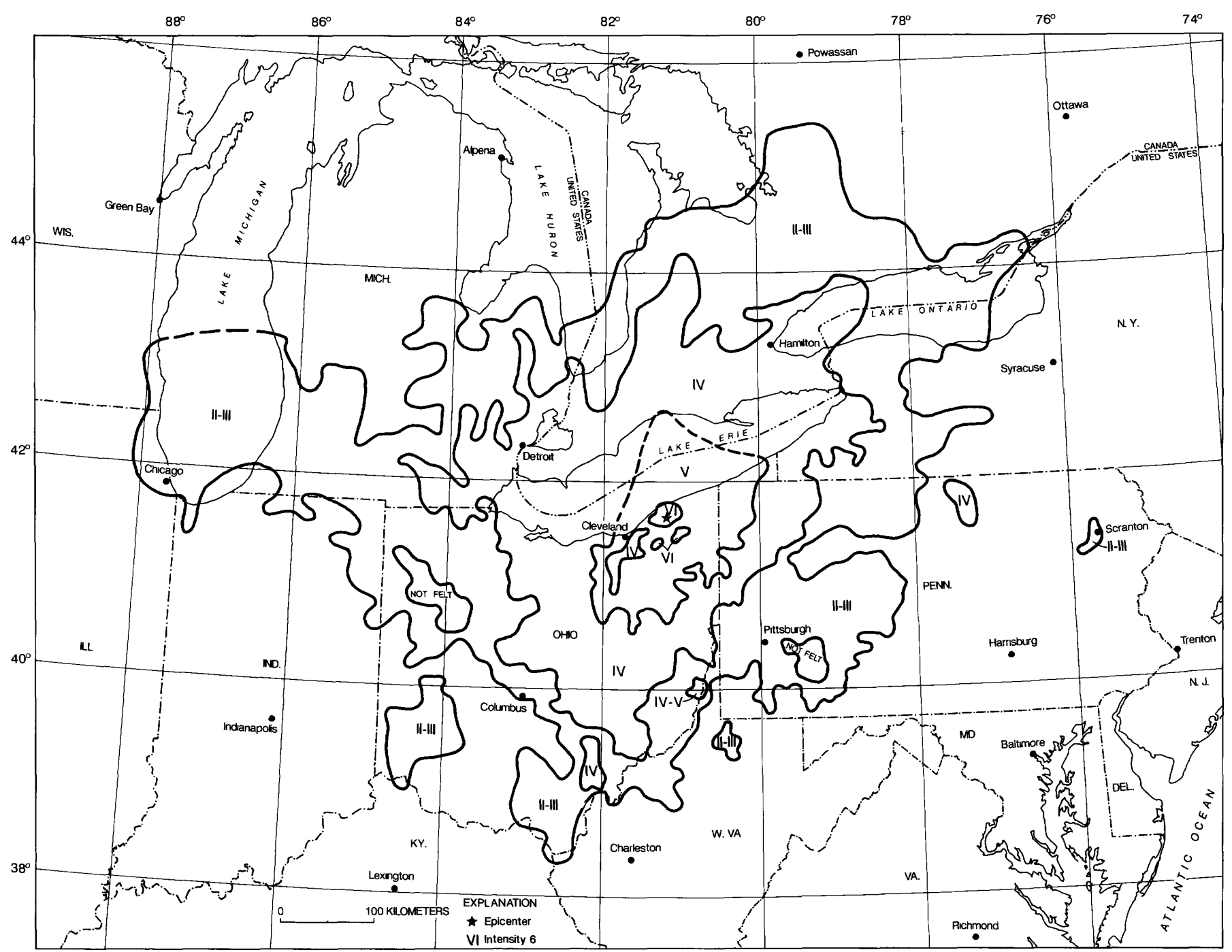

FIGURE 52.- Isoseismal map for the northeast Ohio earthquakes of January 31, 1986. This is a simplified version of figure 2 in reference 562 of table 1.

1986. July 12. Western Ohio. This earthquake occurred near Anna, in Shelby County-the same area where damaging shocks occurred in 1875, 1930-31, and 1937. It caused minor property damage in Shelby County, at Anna, and in Auglaize County, at Minster, New Bremen, and Saint Marys, Ohio.
The damage, which was much less severe than that occurring in the earlier earthquakes, consisted mainly of cracks in chimneys and walls, fallen bricks from chimneys, and broken windows. Also felt in parts of Indiana, Michigan, and West Virginia. Magnitude $4.9 \mathrm{M}_{\mathrm{n}}$ EPB. (Ref. 562.) 

OKLAHOMA

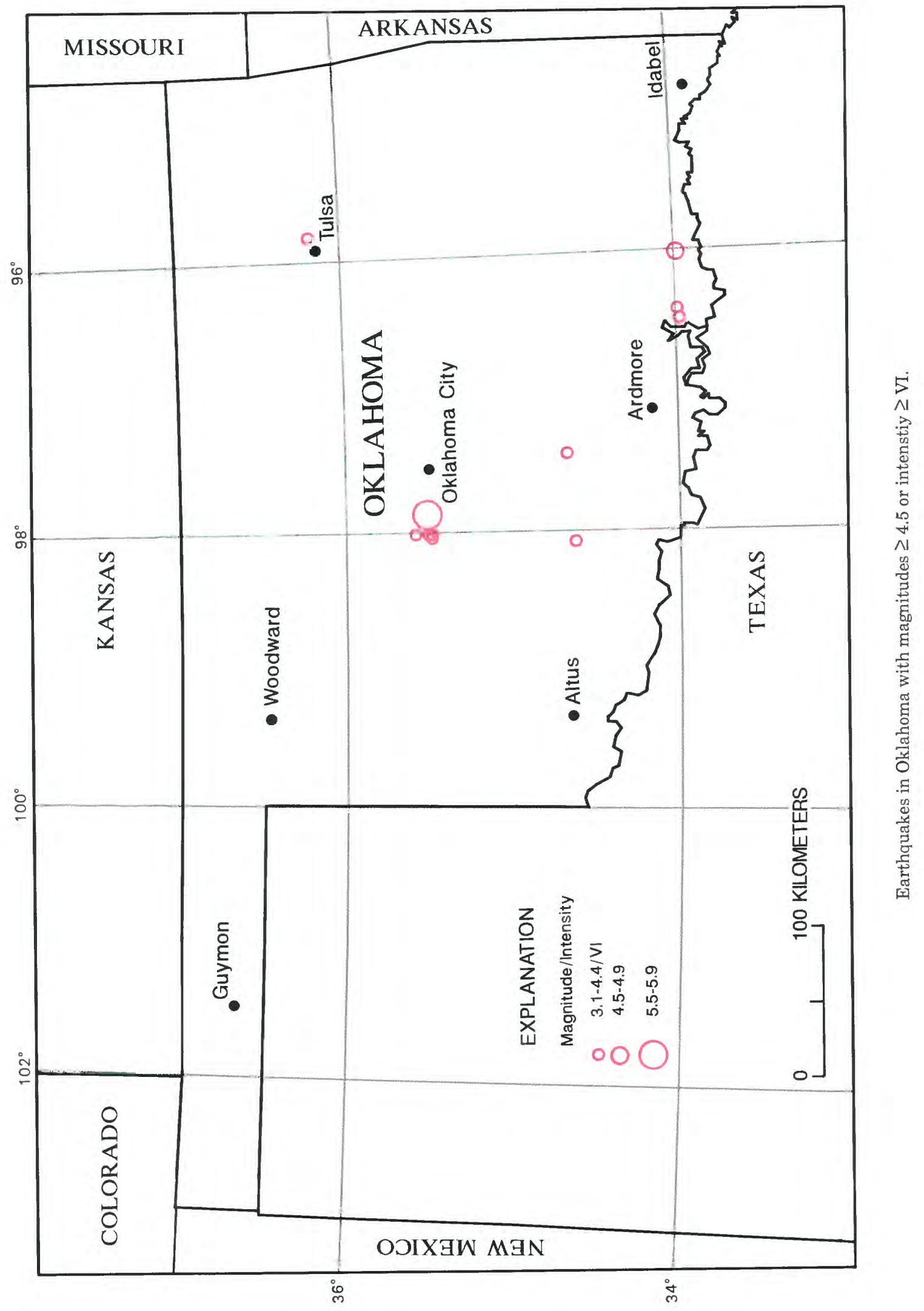


OKLAHOMA

See table 1 for hypocenter and intensity references and table 2 for definitions of magnitude source codes. @, felt area is less than $1,000 \mathrm{~km}^{2}$. Leader ( -$)$ indicates information is not available]

\begin{tabular}{|c|c|c|c|c|c|c|c|c|c|c|c|c|c|}
\hline \multirow{2}{*}{\multicolumn{2}{|c|}{ Date }} & \multirow{2}{*}{$\begin{array}{c}\text { Origin } \\
\text { time (UTC) }\end{array}$} & \multicolumn{4}{|c|}{ Hypocenter } & \multicolumn{4}{|c|}{ Magnitude } & \multicolumn{2}{|c|}{ Intensity } & \multirow{3}{*}{$\begin{array}{c}\text { Felt area } \\
\left(1,000 \mathrm{~km}^{2}\right)\end{array}$} \\
\hline & & & \multirow{2}{*}{$\begin{array}{l}\text { Latitude } \\
\left({ }^{\circ}\right)\end{array}$} & \multirow{2}{*}{$\begin{array}{l}\text { Longitude } \\
\left.\text { ( }{ }^{\circ}\right)\end{array}$} & \multirow{2}{*}{$\begin{array}{c}\text { Depth } \\
(\mathrm{km})\end{array}$} & \multirow[t]{2}{*}{ Ref } & \multicolumn{2}{|c|}{ USGS } & \multirow[t]{2}{*}{ Other } & \multirow{2}{*}{$\begin{array}{l}\text { Moment } \\
\text { M }\end{array}$} & \multirow[t]{2}{*}{ MM } & \multirow[t]{2}{*}{ Ref } & \\
\hline$\overline{\mathrm{Yr}}$ & Mo Da & $h \mathrm{~m} s$ & & & & & $m_{b}$ & $M_{S}$ & & & & & \\
\hline 1882 & 1022 & 2215 & $34.0 \mathrm{~N}$ & $96.0 \mathrm{~W}$ & - & 342 & - & - & $4.90 \mathrm{M}_{\mathrm{fa}} \mathrm{SC}$ & - & VI & 342 & 500 \\
\hline 1918 & 0911 & 0530 & $35.5 \mathrm{~N}$ & $98.0 \mathrm{~W}$ & - & 105 & - & - & $3.30 \mathrm{M}_{\mathrm{fa}} \mathrm{SC}$ & - & VI & 272 & 1 \\
\hline 1929 & 1228 & 0030 & $35.5 \mathrm{~N}$ & 98.0 W & - & 2 & - & - & $3.90 \mathrm{M}_{\mathrm{fa}} \mathrm{SC}$ & - & VI & 38 & 10 \\
\hline 1933 & $08 \quad 19$ & 1930 & $35.5 \mathrm{~N}$ & $98.0 \mathrm{~W}$ & - & 38 & - & - & $3.40 \mathrm{M}_{\mathrm{fa}} \mathrm{BAR}$ & - & VI & 38 & @ \\
\hline 1952 & 0409 & 162928.4 & $35.525 \mathrm{~N}$ & $97.850 \mathrm{~W}$ & 010 & 349 & - & - & $5.50 \mathrm{M}_{\mathrm{s}}$ GR & - & VII & 25 & 587 \\
\hline 1953 & 0317 & 1425 & $35.6 \mathrm{~N}$ & $98.0 \mathrm{~W}$ & - & 105 & - & - & $3.80 \mathrm{M}_{\mathrm{fa}} \mathrm{SC}$ & - & VI & 105 & 7 \\
\hline 1956 & 1030 & 103621 & $36.2 \mathrm{~N}$ & 95.8 W & $\overline{-}$ & 105 & - & - & $4.00 \mathrm{M}_{\mathrm{L}}$ SLM & - & VII & 29 & 12 \\
\hline 1959 & $06 \quad 17$ & 102710.6 & $34.639 \mathrm{~N}$ & $98.055 \mathrm{~W}$ & 005 & 349 & - & - & $4.20 \mathrm{M}_{\mathrm{fa}} \mathrm{SC}$ & - & VI & 32 & 37 \\
\hline 1968 & $10 \quad 14$ & 144254 & $34.0 \mathrm{~N}$ & $96.4 \mathrm{~W}$ & - & 237 & - & - & $3.50 \mathrm{M}_{\mathrm{n}} \mathrm{BAR}$ & - & VI & 41 & @ \\
\hline 1975 & 1129 & 142944.9 & $34.681 \mathrm{~N}$ & $97.421 \mathrm{~W}$ & 014 & 349 & - & - & $3.60 \mathrm{M}_{\mathrm{n}}$ TUL & - & VI & 48 & @ \\
\hline 1982 & 0503 & 075448.7 & $33.99 \mathrm{~N}$ & $96.47 \mathrm{~W}$ & 005 & 350 & - & - & $3.10 \mathrm{M}_{\mathrm{n}}$ TUL & - & VI & 350 & @ \\
\hline
\end{tabular}

[Reference (Ref.) numbers given in parentheses at the end of each description refer to sources of data in table 1. Magnitude values are described in the Introduction, and codes are defined in table 2.]

1882. Oct. 22. Southeast Oklahoma. The most severe damage was limited to the shaking of loose bricks from chimneys at Ft. Smith, Ark. (northwest of Little Rock), and the knocking of bricks from the top of a wall at Bonham, Tex. (northeast of Dallas). Even though few felt reports were received from Oklahoma residents, ref. 342 suggests that the epicenter is in southeast Oklahoma instead of northeast Texas. Felt over a wide area, including Arkansas, Kansas, Missouri, Oklahoma, and Texas. (Ref. 342, 353.)

1918. Sept. 11 (Sept. 10). El Reno, Canadian County, Okla. A series of small earthquakes was reported in the El Reno area on Sept. 10 and 11. Some plaster fell at El Reno, and clocks stopped running during the strongest shock on Sept. 11. It also broke dishes and fruit jars at Union City (on the Canadian River, south of El Reno) and was felt at the nearby town of Yukon. Magnitude $3.6 \mathrm{M}_{\mathrm{fa}} \mathrm{BAR}$. (Ref. $38,105,272$.)

1929. Dec. 28 (Dec. 27). El Reno, Canadian County, Okla. One chimney fell, plaster cracked, and people rushed from their houses at El Reno. Generally felt in El Reno, Oklahoma City, Union
City, and in the adjacent region. Magnitude $4.0 \mathrm{M}_{\mathrm{fa}}$ BAR. (Ref. 2, 38, 353.)

1933. Aug. 19. El Reno, Canadian County, Okla. This severe earthquake broke dishes and cracked chimneys and walls slightly at El Reno. It was felt less strongly at Minco and Union City, south of El Reno. (Ref. 6, 38, 353.)

1952. Apr. 9. El Reno, Canadian County, Okla. This earthquake caused moderate damage at El Reno, Oklahoma City, and Ponca City, including toppled chimneys and smokestacks, cracked and loosened bricks on buildings, and broken windows and dishes. One crack in the State Capitol at Oklahoma City was $15 \mathrm{~m}$ long. Slight damage was reported from many other towns in Oklahoma and from some towns in Kansas and Texas. The earthquake was caused by slippage along the Nemaha fault. Felt over most of Oklahoma and in Arkansas, Iowa, Kansas, Missouri, Nebraska, and Texas (see fig. 53). Magnitude 5.0 $\mathrm{M}_{\mathrm{fa}} \mathrm{DG}, 5.1 \mathrm{M}_{\mathrm{fa}}$ BAR, 5.5 $\mathrm{M}_{\mathrm{S}}$ BAR. (Ref. 25, $105,228,349,364$.

1953. Mar. 17. Concho, Canadian County, Okla. Two moderately strong earthquakes near Concho damaged the foundation of a garage slightly and cracked plaster. Felt in several nearby towns in Canadian County. Magnitude 4.2 $\mathrm{M}_{\mathrm{fa}}$ BAR. (Ref. 38, 105, 353.) 


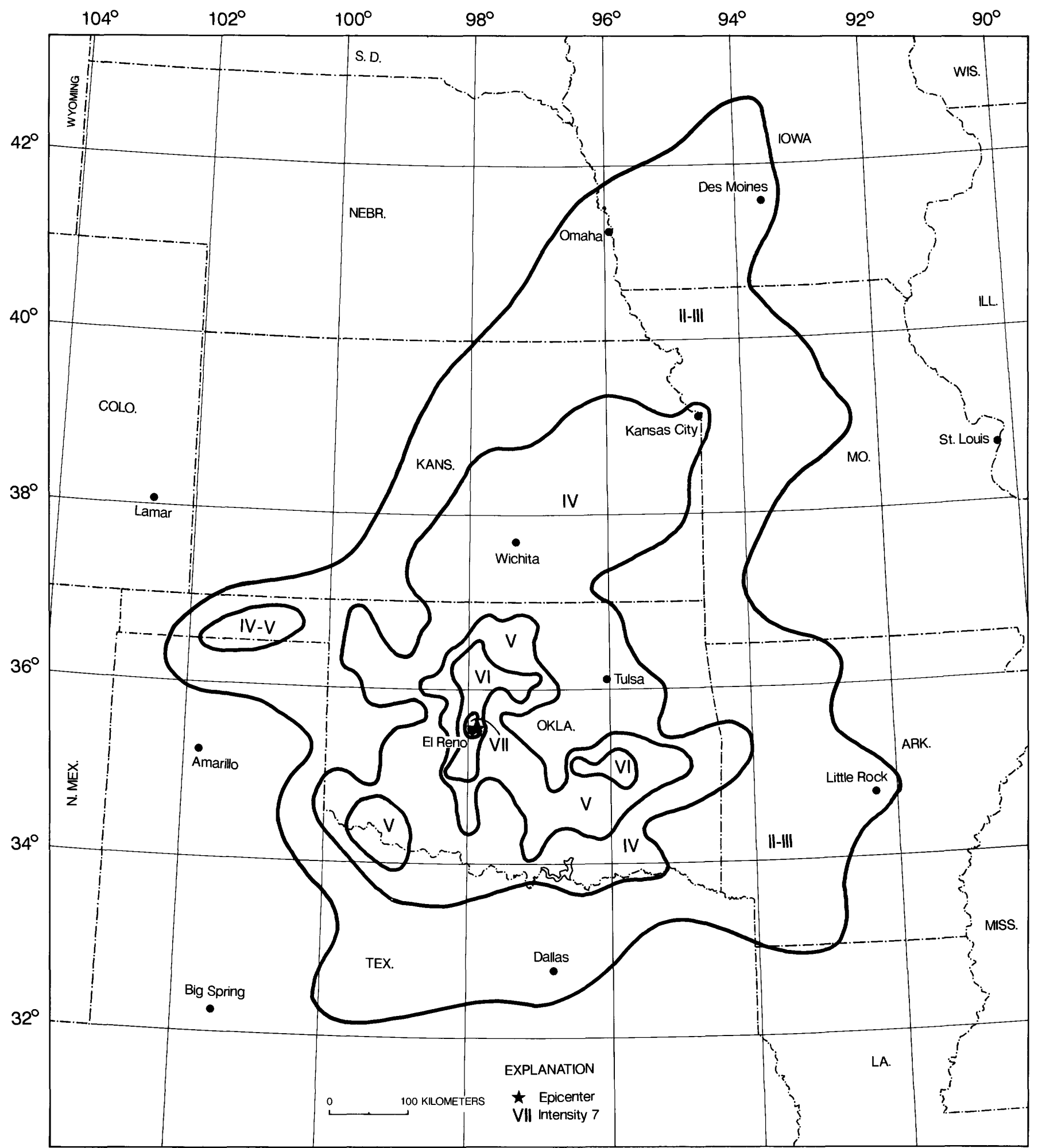

FIGURE 53.- Isoseismal map for the El Reno, Oklahoma, earthquake of April 9, 1952. Isoseismals are based on intensity estimates from data listed in reference 25 of table 1 . 
1956. Oct. 30. West of Catoosa, Rogers County, Okla. At the Foster Ranch, west of Catoosa, an oil well was shut down because of caving. Minor damage at Tulsa included cracks in plaster and in the foundation of one house. Buildings trembled violently. Magnitude 4.2 $\mathrm{M}_{\mathrm{fa}}$ BAR. (Ref. 29, 105, 353.)

1959. June 17. Southwest Oklahoma. At Duncan, southwest of Oklahoma City, in Stephens County, cracks were observed in concrete curbing and in the foundation of a house. At Lawton, about 40 $\mathrm{km}$ west of Duncan, cracks formed in pavement and in plaster on walls. Thunderous, roaring earth sounds were heard. Also felt in Texas. Magnitude 4.3 $\mathrm{M}_{\mathrm{fa}}$ BAR, 4.2 $\mathrm{M}_{\mathrm{fa}}$ DG. (Ref. 32, 349, 353.)
1968. Oct. 14. Durant, Bryan County, Okla. Felt only in the immediate area of Durant, this earthquake cracked plaster, broke windows, enlarged cracks in walls, and formed a crack more than $1 \mathrm{~m}$ long on one wall. Magnitude 3.5 $\mathrm{M}_{\mathrm{n}}$ BAR. (Ref. 41, 237.)

1975. Nov. 29. Near Foster, Garvin County, Okla. Foundations were cracked at two houses $5 \mathrm{~km}$ northwest of Foster. The shock was felt only at a few other towns in the immediate area. Magnitude 3.5 $M_{n}$ SLM. (Ref. 48, 349.)

1982. May 3. Durant, Bryan County, Okla. The movement of a concrete house slab broke water pipes at Durant; a ceiling at the house also was cracked. Felt reports were not received from other towns in the area. (Ref. 350.) 


\section{OREGON}

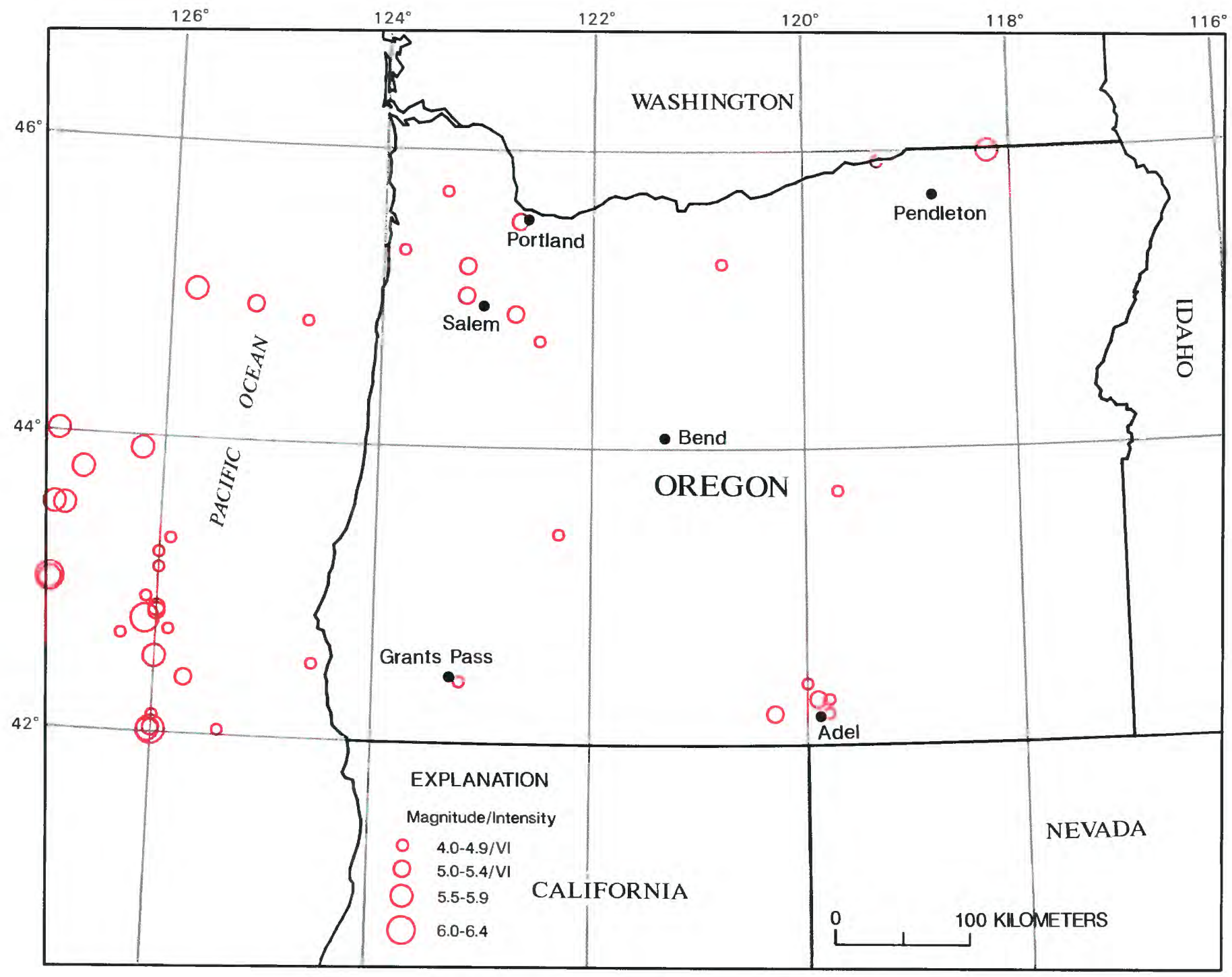

Earthquakes in Oregon with magnitudes $\geq 4.5$ or intensity $\geq$ VI. 
OREGON

[See table 1 for hypocenter and intensity references and table 2 for definitions of magnitude source codes. \&, land area only. Leader (-) indicates information is not available]

\begin{tabular}{|c|c|c|c|c|c|c|c|c|c|c|c|c|c|c|c|c|}
\hline \multirow{3}{*}{\multicolumn{4}{|c|}{$\begin{array}{cc} & \text { Origin } \\
\text { Date } & \text { time (UTC) } \\
\mathrm{Yr} \mathrm{Mo} \mathrm{Da} & \mathrm{h} m \mathrm{~s}\end{array}$}} & \multicolumn{6}{|c|}{ Hypocenter } & \multicolumn{4}{|c|}{ Magnitude } & \multicolumn{2}{|c|}{ Intensity } & \multirow{3}{*}{$\begin{array}{c}\text { Felt area } \\
\left(1,000 \mathrm{~km}^{2}\right)\end{array}$} \\
\hline & & & & \multirow{2}{*}{\multicolumn{2}{|c|}{$\begin{array}{l}\text { Latitude } \\
\left({ }^{\circ}\right)\end{array}$}} & \multirow{2}{*}{\multicolumn{2}{|c|}{$\begin{array}{l}\text { Longitude } \\
\left({ }^{\circ}\right)\end{array}$}} & \multirow{2}{*}{$\begin{array}{l}\text { Depth } \\
(\mathrm{km})\end{array}$} & \multirow[t]{2}{*}{ Ref } & \multicolumn{2}{|c|}{ USGS } & \multirow[t]{2}{*}{ Other } & \multirow{2}{*}{$\begin{array}{c}\text { Moment } \\
\mathbf{M}\end{array}$} & \multirow[t]{2}{*}{ MM } & \multirow[t]{2}{*}{ Ref } & \\
\hline & & & & & & & & & & $m_{b}$ & $M_{S}$ & & & & & \\
\hline 1892 & 02 & 04 & 0430 & 45.5 & $\mathbf{N}$ & 122.7 & W & - & 56 & - & - & 5.00Ukn EPB & - & $\mathrm{V}$ & 56 & 26\& \\
\hline 1893 & 03 & 07 & 0103 & 45.9 & $\mathrm{~N}$ & 119.3 & W & - & 53 & - & - & $4.70 \mathrm{M}_{\mathrm{La}} \mathrm{NQT}$ & - & VI & 56 & - \\
\hline 1896 & 04 & 02 & 1117 & 45.2 & $\mathbf{N}$ & 123.2 & $\mathbf{W}$ & - & 338 & - & - & 5.00Ukn EPB & - & V & 56 & - \\
\hline 1910 & 08 & 05 & 013136 & 42.0 & $\mathbf{N}$ & 127.0 & $\mathbf{W}$ & - & 258 & - & - & $6.80 \mathrm{M}_{\mathrm{s}} \mathrm{GR}$ & - & Felt & 338 & - \\
\hline 1914 & 08 & 22 & 052818 & 44.0 & $\mathbf{N}$ & 129.0 & W & - & 258 & - & - & $6.75 \mathrm{M}_{\mathrm{s}} \mathrm{GR}$ & - & - & - & - \\
\hline 1917 & 06 & 10 & 043224 & 44.0 & $\mathbf{N}$ & 129.0 & W & - & 258 & - & - & $6.50 \mathrm{M}_{\mathrm{s}} \mathrm{GR}$ & - & - & - & - \\
\hline 1923 & 01 & 11 & 0429 & 42.2 & $\mathbf{N}$ & 120.3 & $\mathbf{W}$ & - & 53 & - & - & $5.00 \mathrm{M}_{\mathrm{La}} \mathrm{DMG}$ & - & VI & 56 & 69 \\
\hline 1924 & 02 & 24 & 054510 & 44.0 & $\mathbf{N}$ & 127.0 & $\mathbf{W}$ & - & 258 & - & - & $5.75 \mathrm{M}_{\mathrm{s}} \mathrm{GR}$ & - & - & - & - \\
\hline 1926 & 06 & 05 & 195024 & 43.0 & $\mathbf{N}$ & 127.5 & $\mathbf{W}$ & - & 258 & - & - & $6.00 \mathrm{M}_{\mathrm{S}} \mathrm{GR}$ & - & - & - & - \\
\hline 1928 & 09 & 11 & 123619 & 43.5 & $\mathrm{~N}$ & 130.3 & $\mathbf{w}$ & - & 258 & - & - & $6.30 \mathrm{M}_{8} \mathrm{GR}$ & - & - & - & - \\
\hline 1930 & 07 & 19 & 0238 & 45.0 & $\mathbf{N}$ & 123.2 & $\mathbf{w}$ & - & 3 & - & - & - & - & VI & 3 & - \\
\hline 1932 & 03 & 02 & 174048 & 43.0 & $\mathbf{N}$ & 131.0 & W & - & 5 & - & - & $6.00 \mathrm{mb}_{\mathrm{b}}$ VIC & - & - & - & - \\
\hline 1932 & 06 & 20 & 092627 & 43.0 & $\mathbf{N}$ & 127.5 & W & - & 338 & - & - & $5.50 \mathrm{M}_{\mathrm{S}} \mathrm{GR}$ & - & - & - & - \\
\hline 1933 & 03 & 26 & 190553 & 43.5 & $\mathbf{N}$ & 129.0 & $\mathbf{W}$ & - & 338 & - & - & $5.50 \mathrm{M}_{\mathrm{s}} \mathrm{GR}$ & - & - & - & - \\
\hline 1936 & 04 & 30 & 105538 & 44.0 & $\mathbf{N}$ & 128.5 & W & 一 & 53 & - & - & $5.50 \mathrm{M}_{\mathrm{s}} \mathrm{GR}$ & - & - & - & - \\
\hline 1936 & 07 & 16 & 070748 & 45.966 & & 118.21 & $2 W$ & 005 & 260 & - & - & $5.75 \mathrm{M}_{\mathrm{S}} \mathrm{GR}$ & - & VII & 9 & 190 \\
\hline 1936 & 09 & 25 & 125335 & 43.0 & $\mathbf{N}$ & 129.0 & $\mathbf{W}$ & - & 338 & - & - & $6.20 \mathrm{M}_{\mathrm{s}} \mathrm{GR}$ & - & - & - & - \\
\hline 1936 & 11 & 05 & 204618 & 43.0 & $\mathbf{N}$ & 131.0 & W & - & 338 & - & - & $6.00 \mathrm{mb}_{\mathrm{b}}$ VIC & - & - & - & - \\
\hline 1937 & 11 & 10 & 071923 & 43.0 & $\mathbf{N}$ & 127.0 & W & - & 258 & - & - & $5.75 \mathrm{M}_{\mathrm{s}} \mathrm{GR}$ & - & - & - & - \\
\hline 1938 & 05 & 28 & 101401 & 42.75 & $N$ & 126.1 & W & - & 258 & - & - & $6.00 \mathrm{M}_{\mathrm{s}} \mathrm{GR}$ & - & V & 11 & - \\
\hline 1938 & 08 & 03 & 133236 & 43.9 & $\mathbf{N}$ & 126.2 & $\mathbf{W}$ & - & 338 & - & - & $5.60 \mathrm{M}_{\mathrm{s}} \mathrm{GR}$ & - & - & - & - \\
\hline 1940 & 11 & 17 & 035630 & 44.8 & $\mathbf{N}$ & 130.0 & $\mathbf{w}$ & - & 338 & - & - & $6.00 \mathrm{mb}_{\mathrm{b}} \mathrm{VIC}$ & - & - & - & - \\
\hline 1941 & 06 & 09 & 061724 & 42.8 & $\mathbf{N}$ & 126.0 & W & - & 338 & - & - & $5.25 \mathrm{M}_{\mathrm{S}} \mathrm{GR}$ & - & - & - & - \\
\hline 1941 & 06 & 09 & 084345 & 42.8 & $\mathbf{N}$ & 126.0 & W & - & 53 & - & - & $5.00 \mathrm{M}_{\mathrm{s}} \mathrm{GR}$ & - & - & - & - \\
\hline 1941 & 10 & 31 & 124100 & 43.0 & $\mathrm{~N}$ & 128.5 & W & - & 53 & - & - & $5.50 \mathrm{M}_{\mathrm{s}} \mathrm{GR}$ & - & - & - & - \\
\hline 1941 & 12 & 29 & 1837 & 45.5 & $\mathbf{N}$ & 122.7 & $\mathbf{W}$ & - & 53 & - & - & - & - & VI & 53 & 10 \\
\hline 1944 & 03 & 06 & 200908 & 44.5 & $\mathbf{N}$ & 129.0 & $\mathbf{W}$ & - & 258 & - & - & $5.75 \mathrm{M}_{\mathrm{s}} \mathrm{GR}$ & - & - & - & - \\
\hline 1944 & 03 & 06 & 231630 & 44.5 & $\mathbf{N}$ & 129.0 & $\mathbf{W}$ & - & 258 & - & - & $5.75 \mathrm{M}_{\mathrm{s}} \mathrm{GR}$ & - & - & - & - \\
\hline 1944 & 12 & 30 & 220302 & 43.75 & $\mathbf{N}$ & 126.75 & $\mathbf{W}$ & - & 258 & - & - & $5.75 \mathrm{M}_{\mathrm{s}} \mathrm{GR}$ & - & - & - & - \\
\hline 1945 & 04 & 11 & 112257 & 42.0 & $\mathbf{N}$ & 126.0 & W & - & 338 & - & - & 5.00Ukn EPB & - & - & - & - \\
\hline 1945 & 09 & 28 & 222410 & 42.0 & $\mathbf{N}$ & 126.0 & W & - & 258 & - & - & $6.00 \mathrm{M}_{\mathrm{s}} \mathrm{GR}$ & - & - & - & - \\
\hline 1947 & 09 & 22 & 021601 & 43.5 & $\mathbf{N}$ & 128.0 & W & - & 53 & - & - & $5.50 \mathrm{mb}_{\mathrm{b}} \mathrm{VIC}$ & - & - & - & - \\
\hline 1948 & 05 & 25 & 151307 & 44.0 & $\mathbf{N}$ & 127.0 & $\mathbf{W}$ & - & 258 & - & - & $5.50 \mathrm{M}_{\mathrm{s}} \mathrm{GR}$ & - & - & - & - \\
\hline 1948 & 05 & 25 & 153202 & 43.5 & $\mathrm{~N}$ & 127.0 & W & - & 338 & - & - & $5.80 \mathrm{M}_{\mathrm{L}}$ PAS & - & - & - & - \\
\hline 1949 & 03 & 28 & 194316 & 42.0 & $\mathbf{N}$ & 126.0 & W & - & 266 & - & - & 5.80Ukn EPB & - & - & - & - \\
\hline 1949 & 08 & 24 & 060714 & 43.5 & $\mathbf{N}$ & 127.0 & $\mathbf{W}$ & - & 266 & - & - & $5.50 \mathrm{M}_{\mathrm{s}} \mathrm{GR}$ & - & - & - & - \\
\hline 1950 & 08 & 24 & 174534 & 42.5 & $\mathbf{N}$ & 126.0 & W & - & 266 & - & - & 5.60Ukn EPB & - & - & - & - \\
\hline 1951 & 02 & 23 & 025642 & 44.5 & $\mathbf{N}$ & 129.5 & W & - & 338 & - & - & $5.60 \mathrm{mb}_{\mathrm{b}} \mathrm{VIC}$ & - & - & - & - \\
\hline 1951 & 06 & 16 & 234658 & 44.5 & $\mathbf{N}$ & 130.0 & $\mathbf{W}$ & - & 357 & - & - & $5.50 \mathrm{M}_{\mathrm{L}}, \mathrm{BRK}$ & - & - & - & - \\
\hline 1951 & 06 & 17 & 094015 & 44.5 & $\mathbf{N}$ & 130.0 & W & - & 258 & - & - & $6.00 \mathrm{M}_{\mathrm{L}} \mathrm{BRK}$ & - & - & - & - \\
\hline 1952 & 08 & 20 & 152459 & 43.0 & $\mathbf{N}$ & 127.0 & $\mathbf{W}$ & - & 357 & - & - & $6.00 \mathrm{M}_{\mathrm{L}}$ BRK & - & - & - & - \\
\hline 1953 & 12 & 16 & 043212 & 45.5 & $\mathbf{N}$ & 122.7 & W & - & 53 & - & - & $5.00 \mathrm{M}_{\mathrm{L}}$ EPB & - & VI & 26 & 9 \\
\hline 1955 & 08 & 23 & 153237 & 43.5 & $\mathrm{~N}$ & 128.0 & $\mathbf{W}$ & - & 266 & - & - & 6.25Ukn PAS & - & Felt & 324 & - \\
\hline 1956 & 01 & 10 & 123215 & 43.4 & $\mathbf{N}$ & 122.3 & W & - & 324 & - & - & $4.90 \mathrm{M}_{\mathrm{L}} \quad$ BRK & - & - & - & - \\
\hline 1956 & 07 & 06 & 022200 & 42.5 & $\mathrm{~N}$ & 126. & W & - & 266 & - & - & $5.00 \mathrm{M}_{\mathrm{L}}$ BRK & - & - & - & - \\
\hline 1957 & 11 & 17 & 060029 & 45.3 & $\mathbf{N}$ & 123.8 & $\mathbf{W}$ & - & 30 & - & - & $4.00 \mathrm{M}_{\mathrm{L}} \quad \mathrm{BRK}$ & - & VI & 30 & $13 \&$ \\
\hline 1958 & 03 & 12 & 120916 & 42.4 & $\mathbf{N}$ & 120.0 & $\mathbf{W}$ & - & 324 & - & - & $4.50 \mathrm{M}_{\mathrm{L}}$ BRK & - & - & - & - \\
\hline 1959 & 06 & 02 & 184900 & 43.7 & $\mathbf{N}$ & 119.7 & W & - & 324 & - & - & $4.70 \mathrm{M}_{\mathrm{L}}$ BRK & - & - & - & - \\
\hline 1959 & 08 & 21 & 002817 & 44.8 & $\mathbf{N}$ & 124.7 & W & - & 324 & - & - & $4.60 \mathrm{M}_{\mathrm{L}}$ BRK & - & - & - & - \\
\hline 1959 & 09 & 26 & 082048 & 43.7 & $\mathbf{N}$ & 128.3 & $\mathbf{W}$ & - & 324 & - & - & $6.10 \mathrm{M}_{\mathrm{L}} \mathrm{BRK}$ & - & - & - & 一 \\
\hline
\end{tabular}


OREGON-Continued

[See table 1 for hypocenter and intensity references and table 2 for definitions of magnitude source codes. \&. land area only. Leader (--) indicates information is not available]

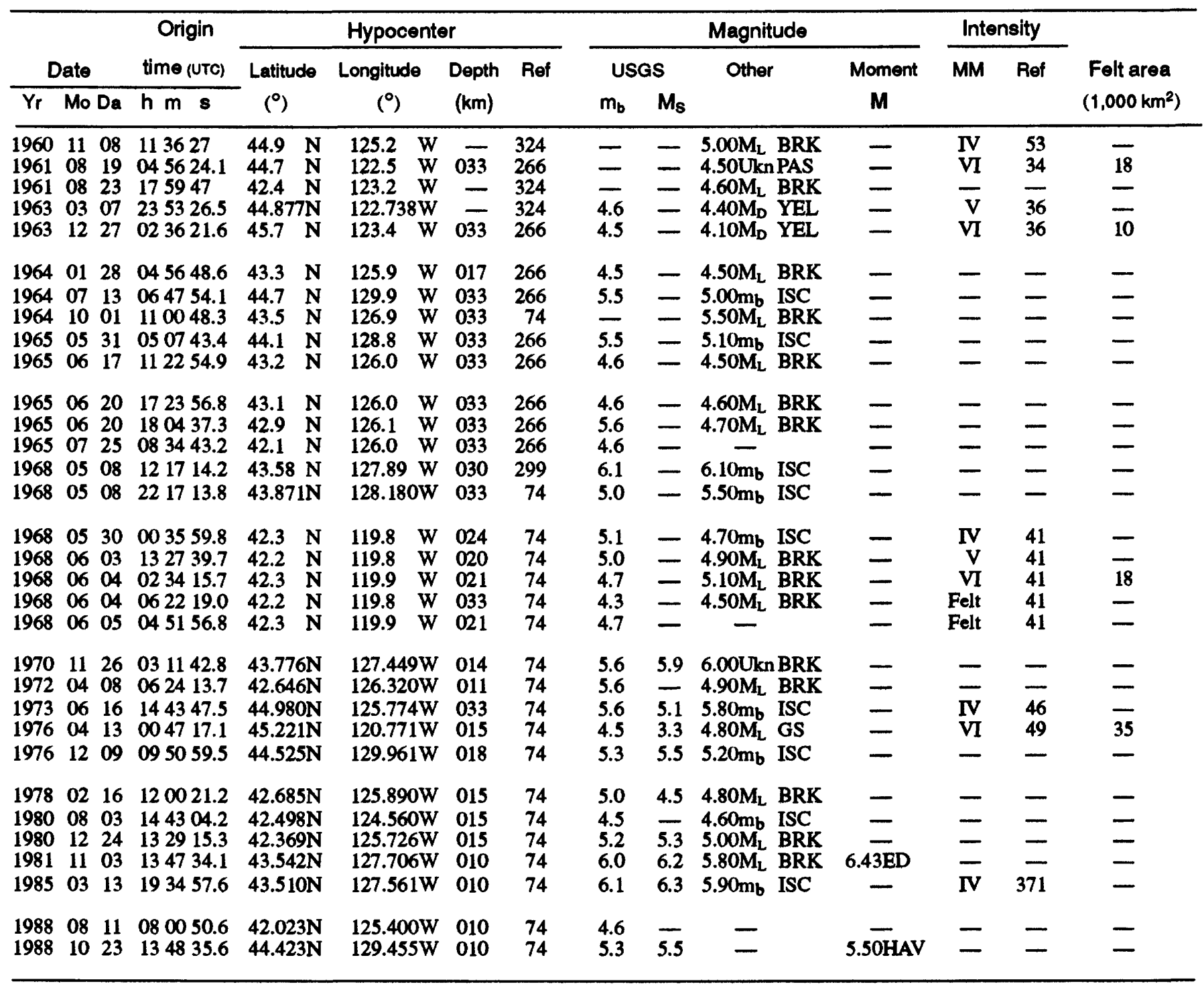

[Reference (Ref.) numbers given in parentheses at the end of each description refer to sources of data in table 1.]

1893. Mar. 7 (Mar. 6). Umatilla, Oreg. One wall of a large stone building was thrown down at Umatilla, about $75 \mathrm{~km}$ west of Milton-Freewater. Several shocks were felt by residents. (Ref. 53,56 .)

1923. Jan. 11 (Jan. 10). Lakeview, southern Lake County, Oreg. The shock was reported to be strongest in the Lakeview district. Plaster fell at Alturas (Modoc County), Calif. The intensity was taken from ref. 56 (Ref. $38,53,56$.)

1930. July 19 (July 18). Near Perrydale, Polk County, Oreg. A crack formed in the roadbed near
Perrydale, southwest of Portland. Plaster was cracked at McCoy, northwest of Salem. (Ref. 3.)

1936. July 16 (July 15). Milton-Freewater, Umatilla County, Oreg. This earthquake was strongest in the area of Milton-Freewater, where many chimneys were broken, several houses were displaced on their foundations, and cracks formed in the ground. Many capstones in area cemeteries were rotated. After the shock, water in several springs and wells started to flow again. Property damage was estimated at $\$ 100,000$. Chimneys were reported damaged at Athena and Ferndale, Oreg., and Waitsburg and Walla Walla, Wash. 


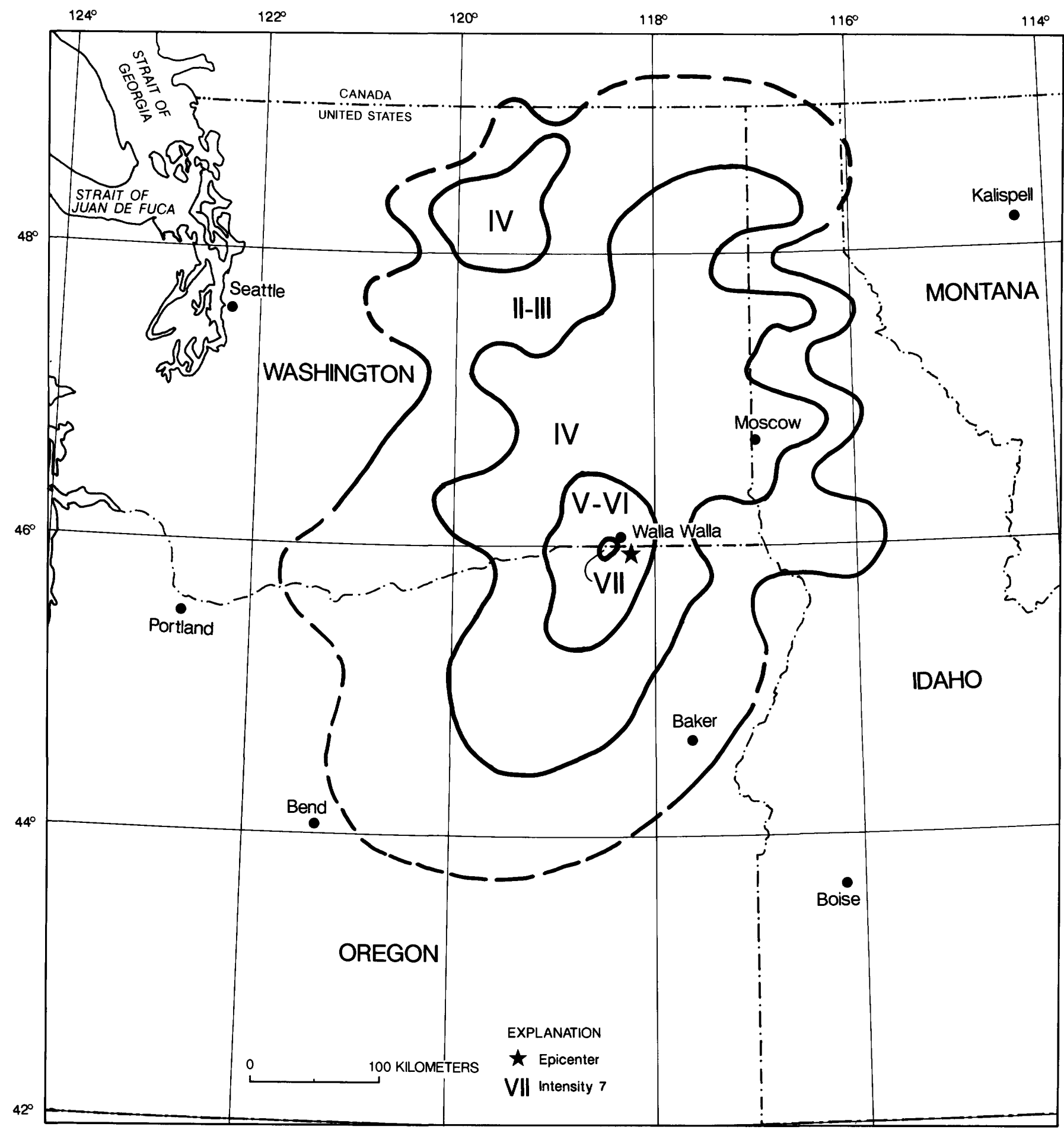

FIGURE 54.- Isoseismal map for the Milton-Freewater, Oregon, earthquake of July 16, 1936. Isoseismals are based on intensity estimates from data listed in references 9,495 , and 510 of table 1 .

At Milton-Freewater, most chimneys were damaged at the roof level. One brick house at the penitentiary was condemned as unsafe. A new house, about 6.5 $\mathrm{km}$ west of Milton-Freewater, was almost wrecked, and two concrete houses, about $11 \mathrm{~km}$ west, were almost demolished. At nearby Umapine, one concrete house collapsed and a stucco house was damaged so badly that the family moved into the yard. The 
Milton-Freewater school buildings were damaged, and repairs were estimated at $\$ 8,500$. Most wells in the area increased or decreased their flow of water.

Between the towns of Milton-Freewater and Umapine, many ground cracks as much as $0.6 \mathrm{~m}$ in width developed. The general direction of the cracks was roughly parallel to the Touchet fault. The largest earth fractures were observed about $6.5 \mathrm{~km}$ west of Sunnyside, near Glencove. On a hillside, an irregular area about $24 \mathrm{~m}$ wide was broken by cracks into blocks of several sizes and shapes. The uphill side of the area, in one cross section, dropped about $2.5 \mathrm{~m}$, and the lower side displaced to the north about 2.5 $\mathrm{m}$, pushing over and covering a 15-m length of railway fence. The largest cracks were about $0.6 \mathrm{~m}$ wide, but most were not more than $8 \mathrm{~cm}$ wide. Water was forced out of the ground in many places. Also felt in Idaho (see fig. 54). Aftershocks occurred to Nov. 17, 1936. (Ref. 9, 260, 495, 510.)

1941. Dec. 29. Portland, Multnomah County, Oreg. This minor earthquake shattered a display window in downtown Portland, cracked chimneys about $50 \mathrm{~km}$ southwest of Portland, at Yamhill, and cracked plaster near Portland at Hillsboro and Sherwood and north of Portland, at Woodland, Wash. (Ref. 14, 53.)

1953. Dec. 16 (Dec. 15). Portland, Multnomah County, Oreg. The shock cracked plaster and chimneys and damaged fireplace tile slightly at Portland. A one-story building also was cracked, and a leak developed in an apartment building. In Vancouver, Wash., commercial buildings of block and concrete were damaged slightly and plaster was cracked. (Ref. $26,53$.

1957. Nov. 17 (Nov. 16). Northwest of Salem, Marion County, Oreg. Walls and plaster cracked and furnishings shifted in West Salem. Also felt in the State of Washington. (Ref. 30.)

1961. Aug. 19 (Aug. 18). Northwest Oregon. Damage was most severe at Lebanon (Linn County), where two chimneys toppled, store windows broke, and two traffic lights and five signs fell. Plaster walls were cracked at nearby Albany. Also felt in Washington. (Ref. 34, 266.)

1963. Dec. 27 (Dec. 26). Northwest Oregon. Slight damage, mainly in the form of cracked plaster, occurred in Washington County, at North Plains and Timber, Oreg., and in northern Cowlitz County, at Toutle, Wash. Also, furnishings and small objects shifted. A car on the Tillamook-Portland Highway swayed to the opposite side of the highway before being controlled. (Ref. 36, 266.)

1968. June 4, 0234 UTC (June 3). Southern Oregon. Old chimneys fell or sustained cracks at Adel, in southern Lake County. Also, part of an old rock cellar fell, and the rest of the building was cracked. About $4 \mathrm{~km}$ northwest of Fort Bidwell, Calif., along Bidwell Creek, cracks formed in the ground, and a house foundation cracked and shifted. (Ref. 41, 74.)

1976. Apr. 13 (Apr. 12). Northern Oregon. This minor earthquake opened cracks in plaster and drywall at Dufur and Wamic, south of The Dalles, in Wasco County. Also felt in southern Washington. (Ref. 49, 74.) 

PENNSYLVANIA

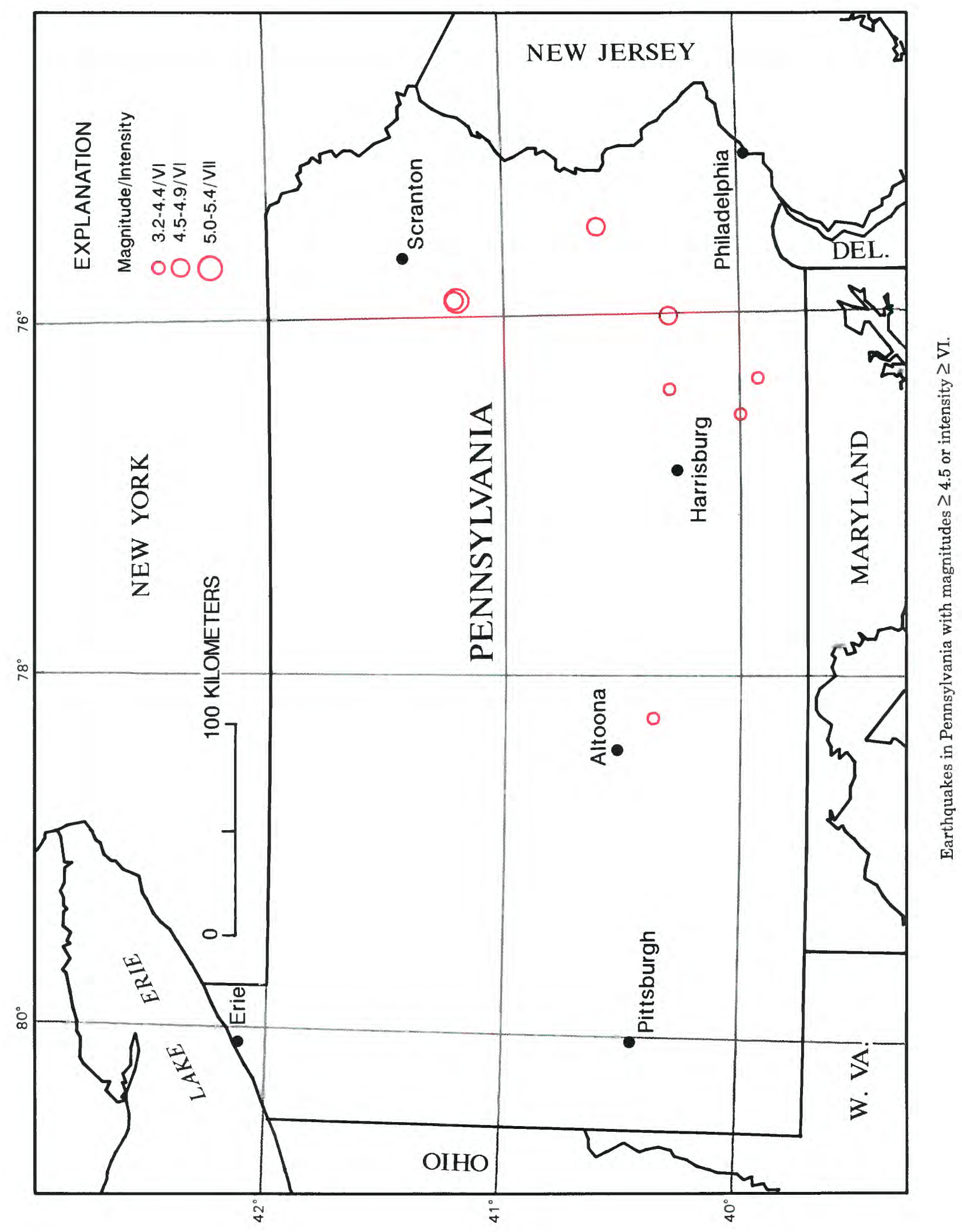


[See table 1 for hypocenter and intensity references and table 2 for definitions of magnitude source codes. @, felt area is less than $1,000 \mathrm{~km}^{2}$. Leader (--) indicates information is not available]

\begin{tabular}{|c|c|c|c|c|c|c|c|c|c|c|c|c|c|}
\hline \multirow{2}{*}{\multicolumn{2}{|c|}{ Date }} & \multirow{2}{*}{$\begin{array}{l}\text { Origin } \\
\text { time (UTC) }\end{array}$} & \multicolumn{4}{|c|}{ Hypocenter } & \multicolumn{4}{|c|}{ Magnitude } & \multicolumn{2}{|c|}{ Intensity } & \multirow{3}{*}{$\begin{array}{l}\text { Felt area } \\
\left(1,000 \mathrm{~km}^{2}\right)\end{array}$} \\
\hline & & & \multirow{2}{*}{$\begin{array}{l}\text { Latitude } \\
\left({ }^{\circ}\right)\end{array}$} & \multirow{2}{*}{$\begin{array}{l}\text { Longitude } \\
\left({ }^{\circ}\right)\end{array}$} & \multirow{2}{*}{$\begin{array}{l}\text { Depth } \\
(\mathrm{km})\end{array}$} & \multirow{2}{*}{ Ref } & \multicolumn{2}{|c|}{ USGS } & \multirow{2}{*}{ Other } & \multirow{2}{*}{$\begin{array}{l}\text { Moment } \\
\text { M }\end{array}$} & \multirow{2}{*}{ MM } & \multirow{2}{*}{ Ref } & \\
\hline $\mathrm{Yr}$ & Mo Da & $\mathrm{h} \mathrm{m} \mathrm{s}$ & & & & & $m_{b}$ & $M_{s}$ & & & & & \\
\hline 1889 & $\begin{array}{ll}03 \quad 08\end{array}$ & 2340 & $40.0 \mathrm{~N}$ & $76.55 \mathrm{~W}$ & - & 201 & - & - & $3.90 \mathrm{M}_{\mathrm{fa}} \mathrm{SC}$ & - & VI & 142 & 10 \\
\hline 1908 & 0531 & 1742 & $40.6 \mathrm{~N}$ & $75.5 \mathrm{~W}$ & - & 38 & - & - & - & - & VI & 38 & @ \\
\hline 1938 & $\begin{array}{ll}07 & 15\end{array}$ & 224612.0 & $40.37 \mathrm{~N}$ & $78.23 \mathrm{~W}$ & - & 485 & - & - & $3.30 \mathrm{M}_{\mathrm{fa}} \mathrm{DG}$ & - & VI & 38 & @ \\
\hline 1954 & 0107 & 0725 & $40.3 \mathrm{~N}$ & $76.0 \mathrm{~W}$ & - & 38 & - & - & _ & - & VI & 38 & \\
\hline 1954 & $\begin{array}{ll}02 & 21\end{array}$ & 2000 & $41.2 \mathrm{~N}$ & $75.9 \mathrm{~W}$ & $=$ & 38 & - & $=$ & - & $=$ & VII & 38 & $@$ \\
\hline 1954 & 0224 & 0355 & $41.2 \mathrm{~N}$ & $75.9 \mathrm{~W}$ & - & 38 & - & - & - & - & VI & 38 & @ \\
\hline 1964 & $05 \quad 12$ & 064510.7 & $40.298 \mathrm{~N}$ & $76.411 \mathrm{~W}$ & 001 & 349 & 4.5 & - & $3.20 M_{n}$ DG & - & VI & 37 & @ \\
\hline 1984 & 0423 & 013600.1 & $39.921 \mathrm{~N}$ & $76.355 \mathrm{~W}$ & 005 & 370 & 4.2 & - & $4.10 \mathrm{M}_{\mathrm{n}} \mathrm{GS}$ & - & VI & 370 & 49 \\
\hline
\end{tabular}

[Reference (Ref.) numbers given in parentheses at the end of each description refer to sources of data in table 1. Magnitude values are described in the Introduction, and codes are defined in table 2.]

1889. Mar. 8. Near York, Pa. At York, about $70 \mathrm{~km}$ south of Harrisburg, the earthquake knocked down chimneys, bounced articles from shelves, and threw a man off a sofa. It was reported that a "ball of fire" passed over the area at the time the shock occurred. Also felt at Harrisburg, Philadelphia, Reading, and other towns. Magnitude $4.3 \mathrm{M}_{\mathrm{fa}}$ AS. (Ref. 38, 141, 142, 201.)

1908. May 31. Allentown, Lehigh County, Pa. This local shock toppled a few chimneys and threw people down at Allentown, about $150 \mathrm{~km}$ north of Philadelphia. Because this area is known for its limestone, the shock may have been due to a rockfall in a subterranean cavern. (Ref. 38.)

1938. July 15. Southern Blair County, Pa. Dishes broke and plaster fell at Henrietta and Clover Creek, south of Altoona. Water in several wells in Clover Creek Valley became cloudy, and water in one spring stopped flowing. (Ref. 38, 349, 485.)

1954. Jan. 7. Sinking Spring, Berks County, Pa. Coal-mining operations may have caused this tremor, which inflicted minor damage in the west section of Sinking Spring, near Reading. Plaster was torn from ceilings and walls, bricks were knocked loose from several chimneys, brick walls were cracked, windows were broken, furniture was upset, and dishes tumbled from shelves. Several brick and frame buildings sustained slight damage. Light aftershocks occurred through Sept. 24, 1954. (Ref. $27,38,459$.)

1954. Feb. 21. Wilkes-Barre, Luzerne County, Pa. Effects from this local shock were confined to a five-block residential area in Wilkes-Barre, southwest of Scranton. Hundreds of houses were damaged; ceilings and cellar walls split; fences fell over; gas and water mains snapped; and sidewalks collapsed. Property damage was estimated at $\$ 1$ million. This shock may have been due to coal-mining operations. (Ref. $27,38,459,533,605$.)

1954. Feb. 24 (Feb. 23). Wilkes-Barre, Luzerne County, Pa. Effects were similar to those that occurred on Feb. 21. Ceilings and walls cracked; curbs pulled away from sidewalks; street pavements buckled; water and gas mains broke. Coal-mining operations may have caused this tremor. (Ref. 27, 38, $459,605$.

1964. May 12. Cornwall, Lebanon County, Pa. A wall was cracked and plaster fell at Cornwall, east of Harrisburg. Small landslides resulted from this local disturbance. (Ref. 37, 349.)

1984. Apr. 23 (Apr. 22). Lancaster County, Pa. This earthquake was centered near Marticville. It caused minor damage at Conestoga, where a garage shifted $1.3 \mathrm{~cm}$ off its foundation; plaster fell from a ceiling; and cracks formed in windows, concrete basement walls, and a cistern. Similar kinds of damage occurred at Lampeter, Mount Nebo, and New Providence. Also felt in Connecticut, Delaware, District of Columbia, Maryland, New Jersey, New York, Virginia, and West Virginia.

One foreshock occurred 5 days earlier and many slight aftershocks occurred. Aftershock data suggested a north-northeast fault dipping steeply east, with reverse, right-lateral slip consistent with a horizontal east-northeast axis of maximum compression. The geometry of the 1984 rupture conforms to the strike of Jurassic dikes and associated faults in the epicentral area. Magnitude 4.4 $\mathrm{M}_{\mathrm{n}}$ TUL, 4.1 $\mathrm{M}_{\mathrm{n}}$ PAL. (Ref. 370, 483.) 


\section{RHODE ISLAND}

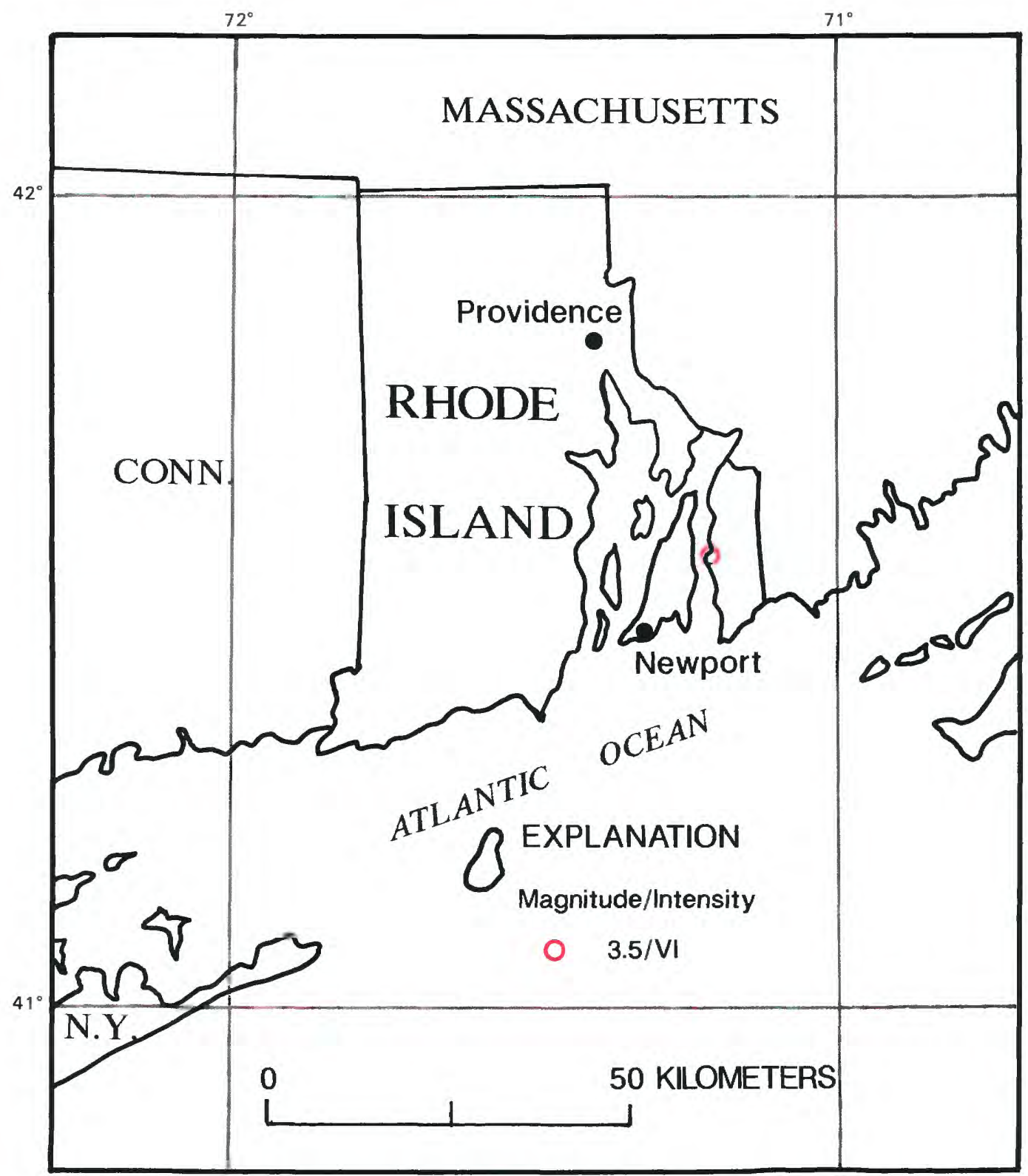

Damaging earthquake in Rhode Island, intensity $\geq$ VI. 
RHODE ISLAND

[See table 1 for hypocenter and intensity references and table 2 for definitions of magnitude source codes. \&, land area only. Leader (--) indicates information is not availablel

\begin{tabular}{|c|c|c|c|c|c|c|c|c|c|c|c|c|c|}
\hline \multirow{2}{*}{\multicolumn{2}{|c|}{ Date }} & \multirow{2}{*}{$\begin{array}{c}\text { Origin } \\
\text { time (UTC) }\end{array}$} & \multicolumn{4}{|c|}{ Hypocenter } & \multicolumn{4}{|c|}{ Magnitude } & \multicolumn{2}{|c|}{ Intensity } & \multirow{3}{*}{$\begin{array}{l}\text { Felt area } \\
\left(1,000 \mathrm{~km}^{2}\right)\end{array}$} \\
\hline & & & \multirow{2}{*}{$\begin{array}{l}\text { Latitude } \\
\left({ }^{\circ}\right)\end{array}$} & \multirow{2}{*}{$\begin{array}{l}\text { Longitude } \\
\left.\text { ( }{ }^{\circ}\right)\end{array}$} & \multirow{2}{*}{$\begin{array}{l}\text { Depth } \\
(\mathrm{km})\end{array}$} & \multirow[t]{2}{*}{ Ref } & \multicolumn{2}{|c|}{ USGS } & \multirow{2}{*}{ Other } & \multirow{2}{*}{$\begin{array}{c}\text { Moment } \\
\text { M }\end{array}$} & \multirow{2}{*}{ MM } & \multirow{2}{*}{ Ref } & \\
\hline $\mathrm{Yr}$ & Mo Da & $\mathrm{h} m \mathrm{~s}$ & & & & & $m_{b}$ & $M_{s}$ & & & & & \\
\hline 1976 & 0311 & 082932.2 & $41.56 \mathrm{~N}$ & $71.21 \mathrm{~W}$ & 000 & 49 & - & - & $3.50 \mathrm{M}_{\mathrm{n}} \mathrm{CON}$ & $2.07 \mathrm{ST}$ & VI & 49 & $23 \&$ \\
\hline
\end{tabular}

[Reference (Ref.) numbers given in parentheses at the end of each description refer to sources of data in table 1.]

1976. Mar. 11. Near Newport, in southeast Rhode Island. Plaster cracked at Rogers, Conn.; a lamp fell from a table at Newport, R.I.; and snow was knocked off a roof at Westport, Mass. Felt from Oakland, R.I., south to Newport and from Somerset, Mass., south to New Bedford and Westport. (Ref. 38,49 .) 


\section{SOUTH CAROLINA}

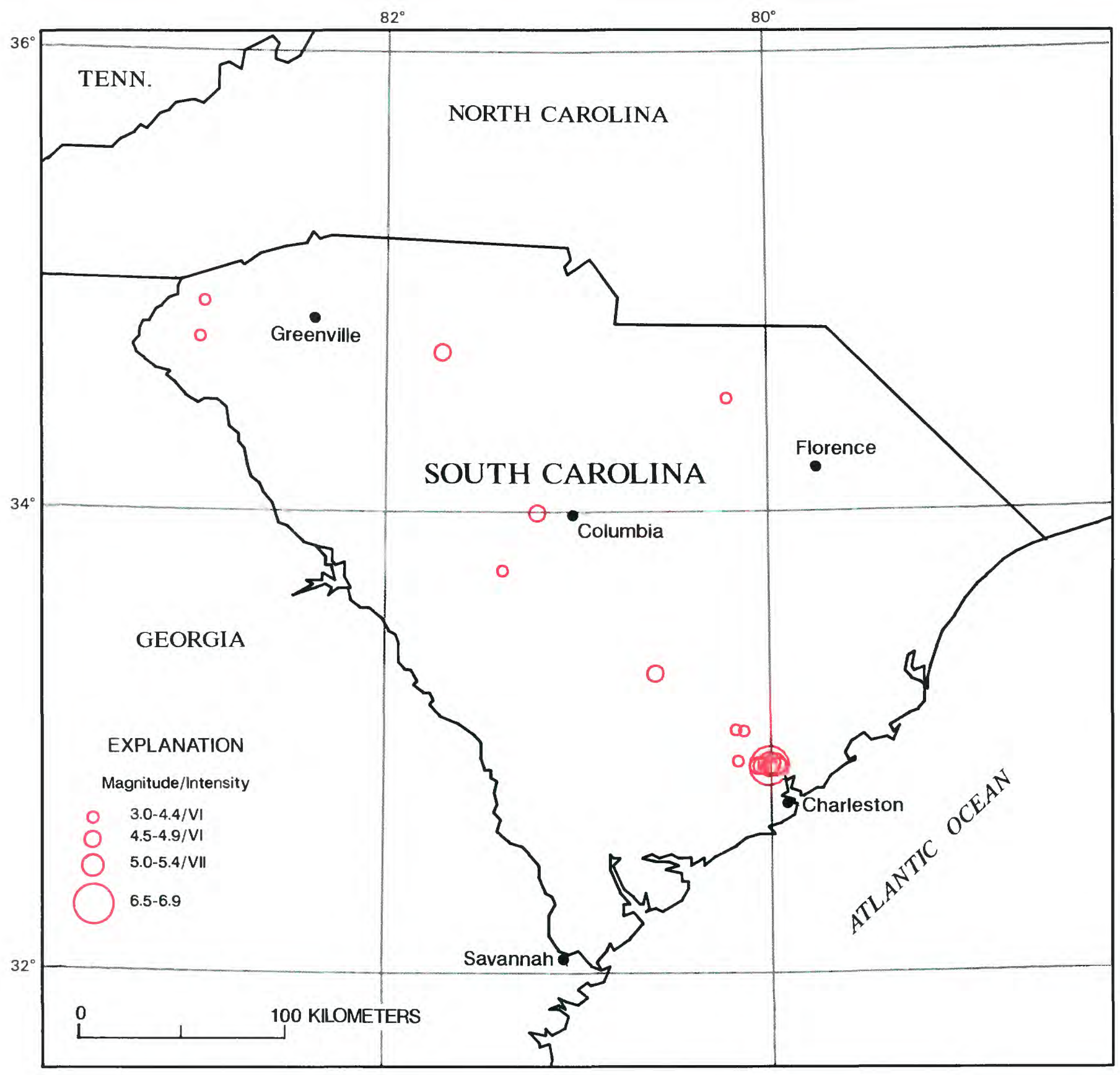

Earthquakes in South Carolina with magnitudes $\geq 4.5$ or intensity $\geq$ VI. 


\section{SOUTH CAROLINA}

[See table 1 for hypocenter and intensity references and table 2 for definitions of magnitude source codes. \&, land area only; 0 , felt area is less than $1,000 \mathrm{~km}{ }^{2}$. Leader ( - ) indicates information is not available]

\begin{tabular}{|c|c|c|c|c|c|c|c|c|c|c|c|c|c|}
\hline \multirow{2}{*}{\multicolumn{2}{|c|}{ Date }} & \multirow{2}{*}{$\begin{array}{c}\text { Origin } \\
\text { time (UTC) }\end{array}$} & \multicolumn{4}{|c|}{ Hypocenter } & \multicolumn{4}{|c|}{ Magnitude } & \multicolumn{2}{|c|}{ Intensity } & \multirow{3}{*}{$\begin{array}{l}\text { Felt area } \\
\left(1,000 \mathrm{~km}^{2}\right)\end{array}$} \\
\hline & & & \multirow{2}{*}{$\begin{array}{l}\text { Latitude } \\
\left({ }^{\circ}\right)\end{array}$} & \multirow{2}{*}{$\begin{array}{l}\text { Longitude } \\
\left({ }^{\circ}\right)\end{array}$} & \multirow{2}{*}{$\begin{array}{l}\text { Depth } \\
(\mathrm{km})\end{array}$} & \multirow[t]{2}{*}{ Ref } & \multicolumn{2}{|c|}{ USGS } & \multirow[t]{2}{*}{ Other } & \multirow{2}{*}{$\begin{array}{c}\text { Moment } \\
\text { M }\end{array}$} & \multirow[t]{2}{*}{ MM } & \multirow[t]{2}{*}{ Ref } & \\
\hline $\mathrm{Yr}_{\mathrm{r}}$ & Mo Da & $\mathrm{h} \mathrm{m} \mathrm{s}$ & & & & & $m_{b}$ & $M_{s}$ & & & & & \\
\hline 317 & 0108 & 0900 & $32.9 \mathrm{~N}$ & $80.0 \mathrm{~W}$ & - & 288 & - & - & $5.00 \mathrm{M}_{\mathrm{fa}}$ NUT & - & $\mathrm{V}$ & 288 & 516\& \\
\hline 1853 & 0520 & & $34.0 \mathrm{~N}$ & $81.2 \mathrm{~W}$ & 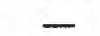 & 289 & - & - & & . & VI & & - \\
\hline 1886 & $\begin{array}{l}5 \quad 28 \\
\end{array}$ & 0845 & $32.9 \mathrm{~N}$ & $80.0 \mathrm{~W}$ & - & 289 & - & - & - & - & VI & 289 & - \\
\hline 1886 & & 0251 & $32.9 \mathrm{~N}$ & $80.0 \mathrm{~W}$ & - & 38 & - & - & $6.70 \mathrm{M}_{\mathrm{fa}} \mathrm{BOL}$ & $7.02 \mathrm{BOL}$ & $\mathrm{X}$ & 289 & 2000\& \\
\hline 1886 & 50906 & 0406 & $32.9 \mathrm{~N}$ & $80.0 \mathrm{~W}$ & - & 96 & - & - & - & - & VI & 463 & - \\
\hline 1886 & $\begin{array}{lll}5 & 09 & 17\end{array}$ & 0629 & $32.9 \mathrm{~N}$ & $80.0 \mathrm{~W}$ & - & 289 & - & - & - & - & VI & 289 & - \\
\hline 1886 & 50921 & 1015 & $32.9 \mathrm{~N}$ & $80.0 \mathrm{~W}$ & - & 96 & - & - & - & - & VI & 96 & - \\
\hline 1886 & 0927 & 1902 & $32.9 \mathrm{~N}$ & $80.0 \mathrm{~W}$ & - & 96 & - & - & - & - & VI & 289 & - \\
\hline 1886 & $\begin{array}{lll}510 & 22\end{array}$ & 1020 & $32.9 \mathrm{~N}$ & $80.0 \mathrm{~W}$ & - & 38 & - & - & - & - & VI & 38 & 78\& \\
\hline 1886 & 51022 & 1945 & $32.9 \mathrm{~N}$ & $80.0 \mathrm{~W}$ & - & 38 & - & - & - & - & VII & 38 & 78\& \\
\hline 886 & $\begin{array}{ll}11 & 05\end{array}$ & 1720 & $32.9 \mathrm{~N}$ & $80.0 \mathrm{~W}$ & - & 38 & - & - & - & - & VI & 38 & 78\& \\
\hline 1887 & $\begin{array}{ll}03 & 17\end{array}$ & 1409 & $32.9 \mathrm{~N}$ & $80.0 \mathrm{~W}$ & - & 289 & - & - & - & - & VI & & \\
\hline 1912 & 0612 & 1030 & $32.9 \mathrm{~N}$ & $80.0 \mathrm{~W}$ & - & 96 & - & - & - & - & VII & 289 & 150\& \\
\hline 1913 & 0101 & 1828 & $34.7 \mathrm{~N}$ & $81.7 \mathrm{~W}$ & - & 38 & - & - & $4.80 \mathrm{M}_{\mathrm{fa}} \mathrm{SC}$ & - & VII & 162 & 120 \\
\hline 1945 & 0726 & 103216.4 & $33.750 \mathrm{~N}$ & $81.376 \mathrm{~W}$ & 005 & 349 & - & - & $4.30 \mathrm{M}_{\mathrm{fa}} \mathrm{SC}$ & - & VI & 289 & 65 \\
\hline 1959 & 0803 & 060836.8 & $33.054 \mathrm{~N}$ & $80.126 \mathrm{~W}$ & 001 & 349 & - & - & $4.40 \mathrm{M}_{\mathrm{fa}}$ & - & VI & 32 & 60\& \\
\hline 1959 & $\begin{array}{ll}10 & 27\end{array}$ & 020728 & $34.5 \mathrm{~N}$ & $80.2 \mathrm{~W}$ & - & 38 & - & - & $3.90 \mathrm{M}_{\mathrm{fa}} \mathrm{SC}$ & - & VI & 38 & 12 \\
\hline 1971 & $07 \quad 13$ & 114226.0 & $34.76 \mathrm{~N}$ & $82.98 \mathrm{~W}$ & - & 163 & - & - & $3.70 M_{n}$ GB & - & VI & 44 & 8 \\
\hline 1972 & 0203 & 231109.7 & $33.306 \mathrm{~N}$ & $80.582 \mathrm{~W}$ & 002 & 349 & 4.5 & - & $4.50 \mathrm{M}_{\mathrm{n}} \mathrm{GB}$ & 4.34HRM & $\mathrm{v}$ & 45 & $76 \&$ \\
\hline 1974 & 1122 & 052556.7 & $32.926 \mathrm{~N}$ & $80.159 \mathrm{~W}$ & 006 & 349 & 4.7 & - & $4.30 \mathrm{M}_{\mathrm{n}} \mathrm{GB}$ & 3.95HRM & VI & 47 & 39\& \\
\hline 1977 & $\begin{array}{ll}01 & 18\end{array}$ & 182914.1 & $33.058 \mathrm{~N}$ & $80.173 W$ & 001 & 349 & - & - & In BLA & - & VI & 39 & @ \\
\hline 1979 & $08 \quad 26$ & 013145.0 & $34.916 \mathrm{~N}$ & $82.956 \mathrm{~W}$ & 001 & 349 & - & - & $3.70 \mathrm{M}_{\mathrm{n}} \mathrm{BLA}$ & - & VI & 262 & 11 \\
\hline
\end{tabular}

[Reference (Ref.) numbers given in parentheses at the end of each description refer to sources of data in table 1. Magnitude values are described in the Introduction, and codes are defined in table 2.]

1853. May 20. Lexington, S.C. Just before sunrise, a severe earthquake broke windows and knocked crockery from shelves at Lexington, southwest of Columbia. (Ref. 289.)

1886. Aug. 28. Summerville, Dorchester County, S.C. A bed was "flung forward" against the wall in one house; window glass and crockery were broken. Several distinct aftershocks occurred that day. Also felt at Augusta, Ga., and Wilmington, N.C. (Ref. 289.)

1886. Sept. 1 (Aug. 31). Charleston, S.C. This is the most damaging earthquake to occur in the Southeast United States and one of the largest historic shocks in Eastern North America. It damaged or destroyed many buildings in the old city of Charleston and killed 60 people. Hardly a structure there was undamaged, and only a few escaped serious damage. Property damage was estimated at
$\$ 5-6$ million. Structural damage was reported several hundred $\mathrm{km}$ from Charleston (including central Alabama, central Ohio, eastern Kentucky, southern Virginia, and western West Virginia), and long-period effects were observed at distances exceeding 1,000 $\mathrm{km}$ (see fig. 55.)

Effects in the epicentral region included about 80 $\mathrm{km}$ of severely damaged railroad track and more than $1,300 \mathrm{~km}^{2}$ of extensive cratering and fissuring. Damage to railroad tracks, about $6 \mathrm{~km}$ northwest of Charleston, included lateral and vertical displacement of tracks, formation of S-shaped curves, and longitudinal movement.

The formation of sand craterlets and the ejection of sand were widespread in the epicentral area, but surface faulting was not observed. Many acres of ground were overflowed with sand, and craterlets as much as $6.4 \mathrm{~m}$ across were formed. In a few locations, water from the craterlets spouted to heights of about 4.5 to $6 \mathrm{~m}$. Fissures $1 \mathrm{~m}$ wide extended parallel to canal and stream banks. A series of wide cracks opened parallel to the Ashley River, and 


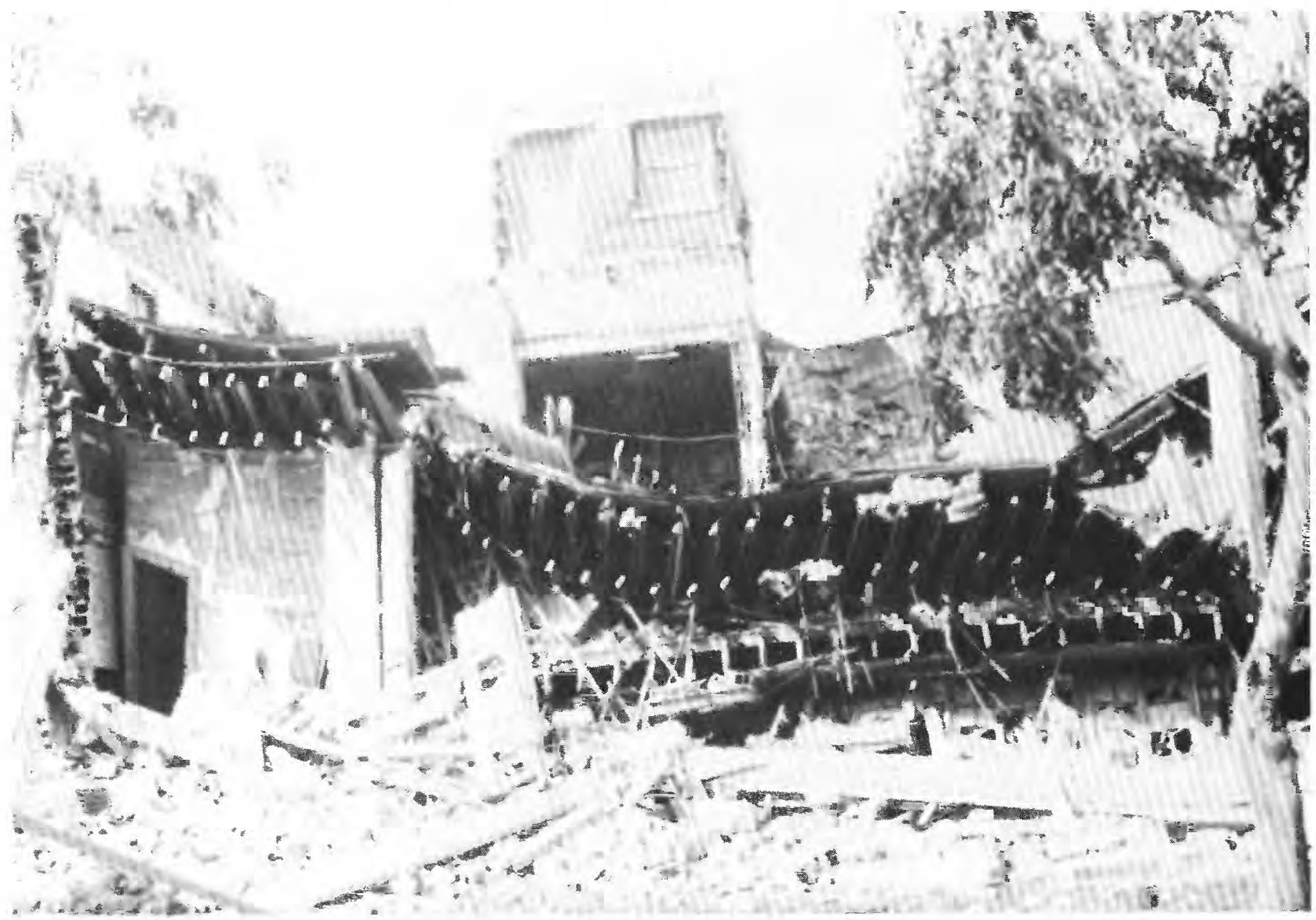

Public building in Charleston, South Carolina, severely damaged by the September 1, 1886 (Aug. 31 EST), earthquake.

several large trees were uprooted when the bank slid into the river.

At Summerville, a small town of 2,000 population, $25 \mathrm{~km}$ northwest of Charleston, many houses settled in an inclined position or were displaced as much as $5 \mathrm{~cm}$. Chimneys constructed independently of the houses commonly had the part above the roofline thrown to the ground. Many chimneys were crushed at their bases, allowing the whole chimney to sink down through the floors. The absence of overturning in piered structures and the nature of the damage to chimneys have been interpreted as evidence that the predominant motion was vertical.

The meizoseismal area of MM intensity $\mathrm{X}$ effects is an elliptical area, roughly 35 by $50 \mathrm{~km}$, trending northeast between Charleston and Jedburg and including Summerville. Middleton Place, about in the center of this ellipse, is at the southeast end of a zone (perhaps $15 \mathrm{~km}$ long) of microearthquake activity that still continues today. This seismic activity may be a continuation of the 1886 aftershock series.

The intraplate epicenter of this major shock is not unique for large earthquakes in the Eastern and Central United States. Other intraplate earthquakes include those at Cape Ann, Mass. (1755), and New Madrid, Mo. (1811-12). Earthquakes occurring along boundaries of plates (e.g., San Francisco, 1906) are well understood in terms of plate tectonics, but those occurring within plates are not similarly understood. This problem still is being studied more than 100 years after the earthquake.

This earthquake was reported from distant places such as Boston, Mass.; Milwaukee, Wis.; Chicago, Ill.; Cuba, and Bermuda. Magnitude $6.6 \mathrm{M}_{\mathrm{fa}} \mathrm{NLI}$; $7.5 \mathrm{M}_{\mathrm{Sn}}$ NLI; $7.7 \mathrm{M}_{\mathrm{Sn}}$ BOL. (Ref. 38, 140, 289, $450,526$. 


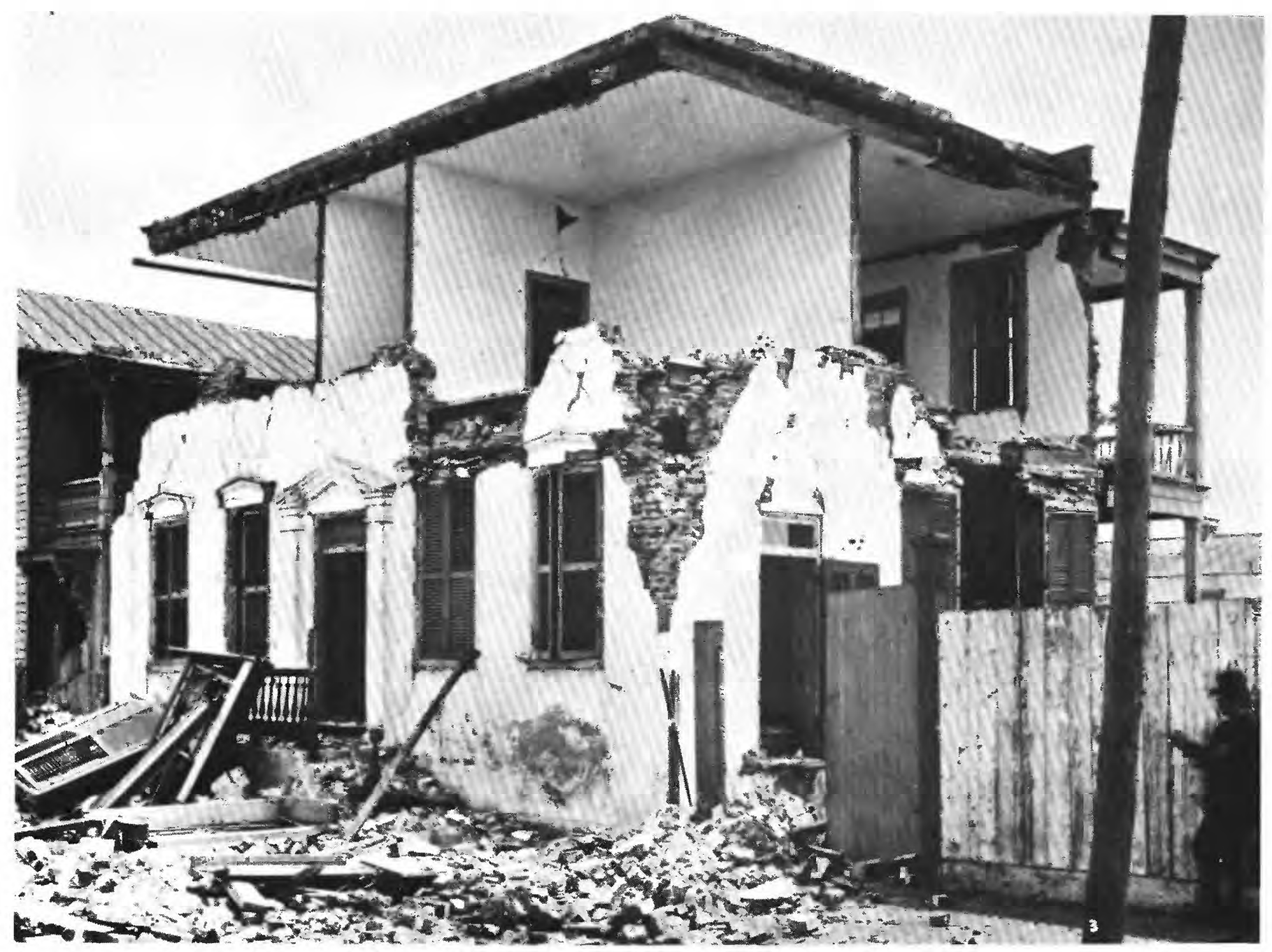

Brick house at 157 Tradd Street, Charleston, South Carolina, damaged by the September 1, 1886 (Aug. 31 EST), earthquake. (Photograph by J.K. Hillers.)

1886. Sept. 6 (Sept. 5). Charleston, S.C. This minor aftershock knocked plaster from walls at Charleston. (Ref. 96, 463.)

1886. Sept. 17. Summerville, Dorchester County, S.C. This aftershock reportedly shifted the pillars of a Summerville hotel about $6.5 \mathrm{~cm}$, cracking most of the pillars in the process. Brick fell from some chimneys and sheds. Also severely felt at Charleston. (Ref. 289.)

1886. Sept. 21. Summerville, Dorchester County, S.C. The medical college building, already weakened by previous earthquakes, was thrown down by this aftershock. Much loose plaster fell. Also felt in Georgia. (Ref. 96.)

1886. Sept. 27. Charleston, S.C. This aftershock cracked a brick building on Broad Street in Charleston. On East Bay Street, the rear wall of a store was separated about $10 \mathrm{~cm}$ from the adjoining wall. Loose plaster and bricks fell in some houses. Also felt in Georgia. (Ref. 96, 289, 463.)

1886. Oct. 22, 1020 UTC. Charleston, S.C. Two of the most severe aftershocks of the Aug. 31 earthquake occurred in Charleston on this date (also see next paragraph). The first shock cracked the west wing of the Customs House and caused the wall supporting the roof on the west to "give way" slightly. Also felt in Georgia, North Carolina, and Tennessee. (Ref. 38, 289.)

1886. Oct. 22, 1945 UTC. Summerville, Dorchester County, S.C. This was the most severe aftershock to date of the Aug. 31 earthquake. It threw down several chimneys and cracked several others at Summerville. The shock knocked bricks from several chimneys and shook down plaster at Columbia, about $160 \mathrm{~km}$ northwest of Charleston. 


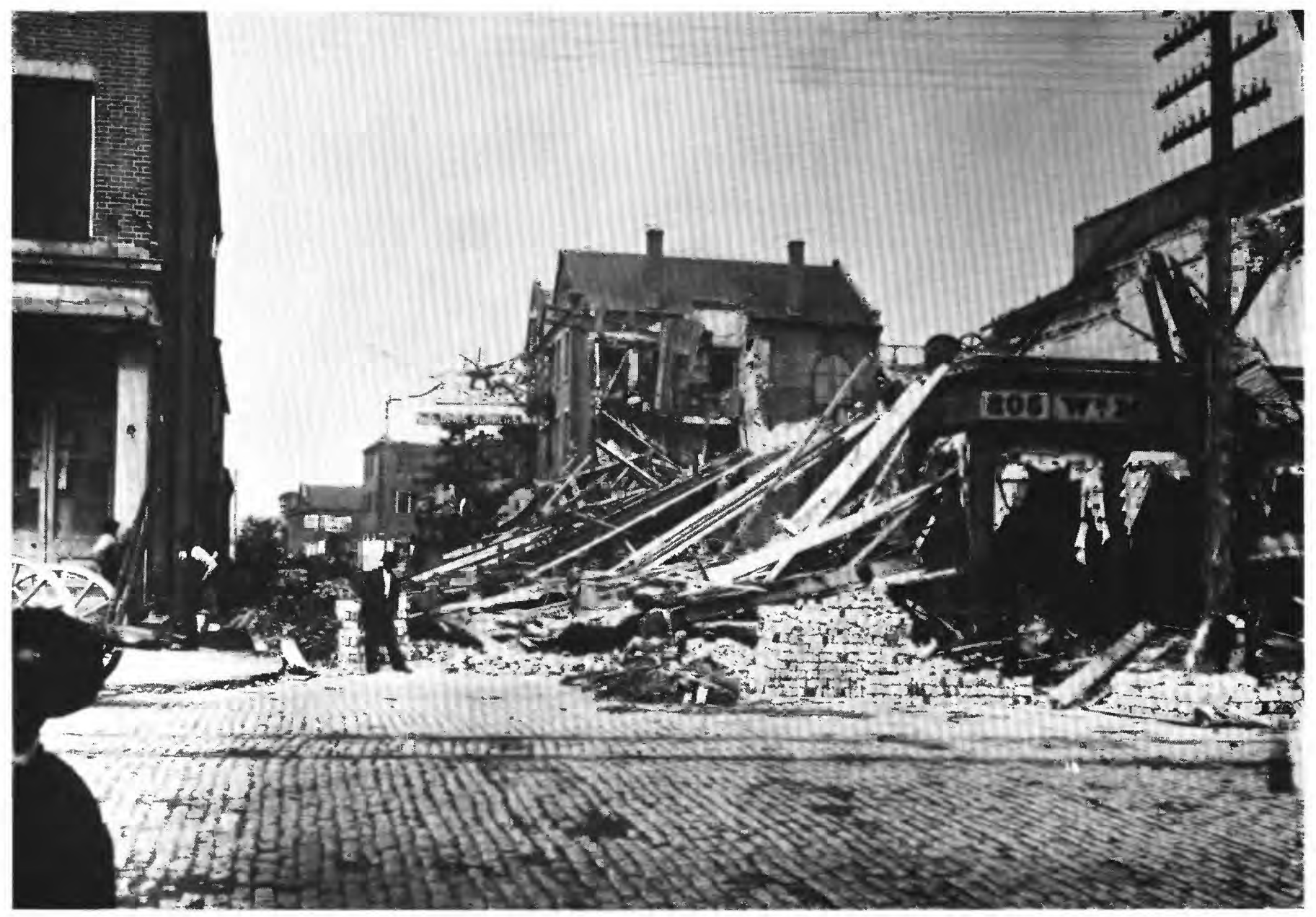

Damage on East Bay Street, Charleston, South Carolina, after the earthquake of September 1, 1886 (Aug. 31 EST).

(Photograph by J.K. Hillers.)

Also felt in the District of Columbia, Georgia, North Carolina, Ohio, and Virginia. (Ref. 38, 289.)

1886. Nov. 5. Charleston, S.C. This aftershock shattered one or two chimneys at Charleston, and knocked down the tops of a few chimneys at Summerville. Also felt in the District of Columbia, Georgia, North Carolina, and Virginia. (Ref. 38, 289.)

1887. Mar. 17. Charleston, S.C. This violent aftershock widened existing cracks in buildings and knocked down plaster in Charleston. It was described as "more severe" in Summerville. (Ref. 289.)

1912. June 12. Summerville, Dorchester County, S.C. Several chimneys were cracked in Summerville, and a few had their tops thrown down; plaster fell in several houses. The shock was strong enough to move beds several $\mathrm{cm}$ across a floor in Charleston. Also felt in Georgia, North Carolina, and South Carolina. (Ref. 38, 96, 289.)

1913. Jan. 1. Union County, S.C. This earthquake overthrew chimneys throughout the area and damaged plaster and stone walls. At Union, chimneys were thrown down in all parts of town, and plastering was damaged severely in the new courthouse. Cracks formed in the stone walls of the jail and in the brick courthouse. At Monarch, about $2.5 \mathrm{~km}$ south of Union, a house was partly shaken down. Chimneys were downed at Cross Keys, Enoree, Gaffney, Kings Mountain, Pacolet, Pauline, and West Springs. A pig was killed by bricks falling from a chimney at West Springs. Also felt in Georgia, North Carolina, and Virginia. (Ref. 38, 162, 289.)

1945. July 26. Camden, Kershaw County, S.C. Brick walls and chimneys were cracked at Camden; one chimney was damaged at Chester, about $45 \mathrm{~km}$ northwest of Camden. Felt throughout most of South Carolina and at a few towns in Georgia, North Carolina, and Tennessee. Magnitude 5.6 $\mathrm{M}_{\mathrm{S}} \mathrm{GR}$, 4.4 $\mathrm{M}_{\mathrm{fa}}$ DG. (Ref. 38, 289, 349.) 


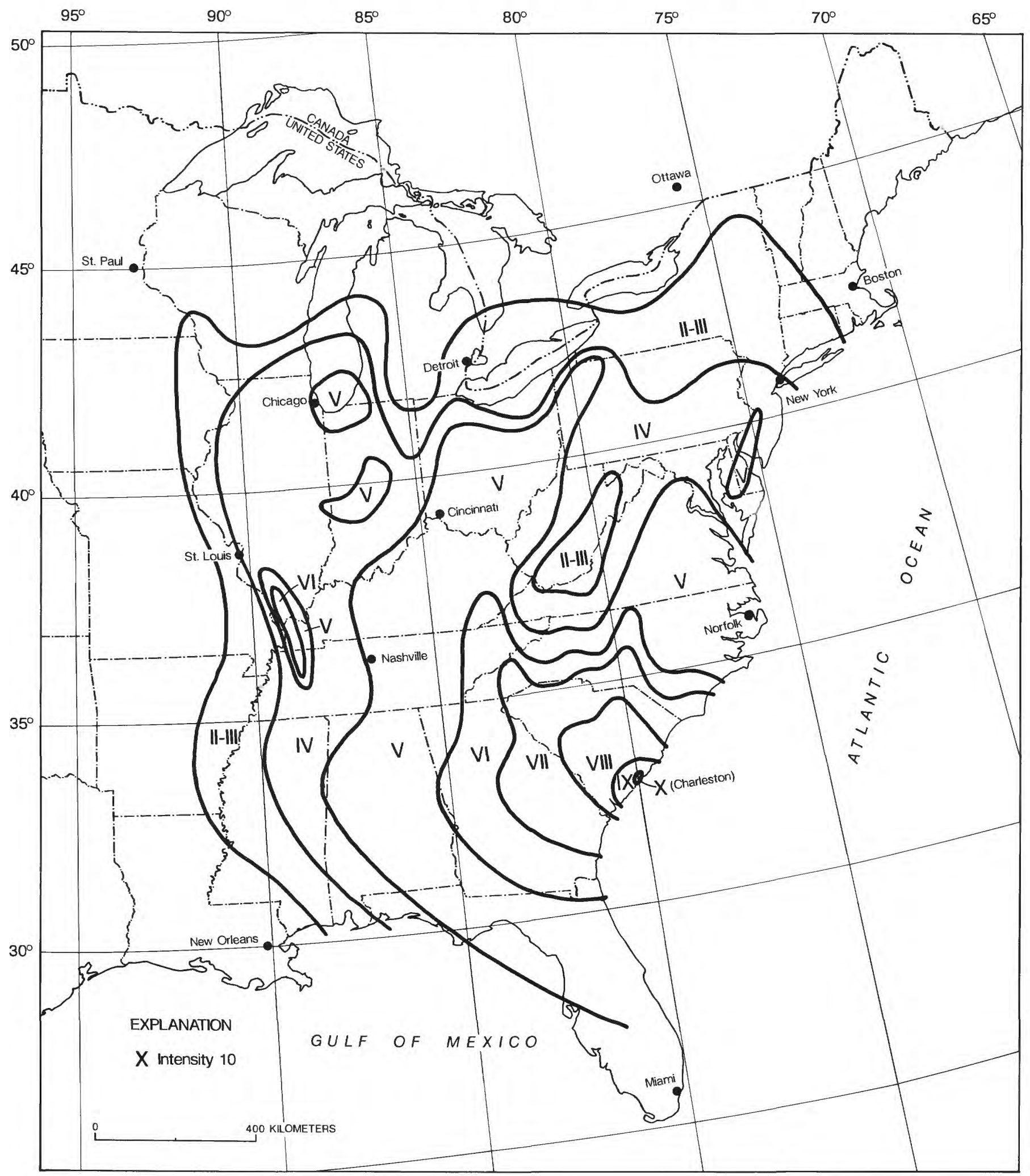

FiguRE 55.- Isoseismal map for the Charleston, South Carolina, earthquake of September 1, 1886. This map is a simplified version of figure 5 in reference 526 of table 1. 


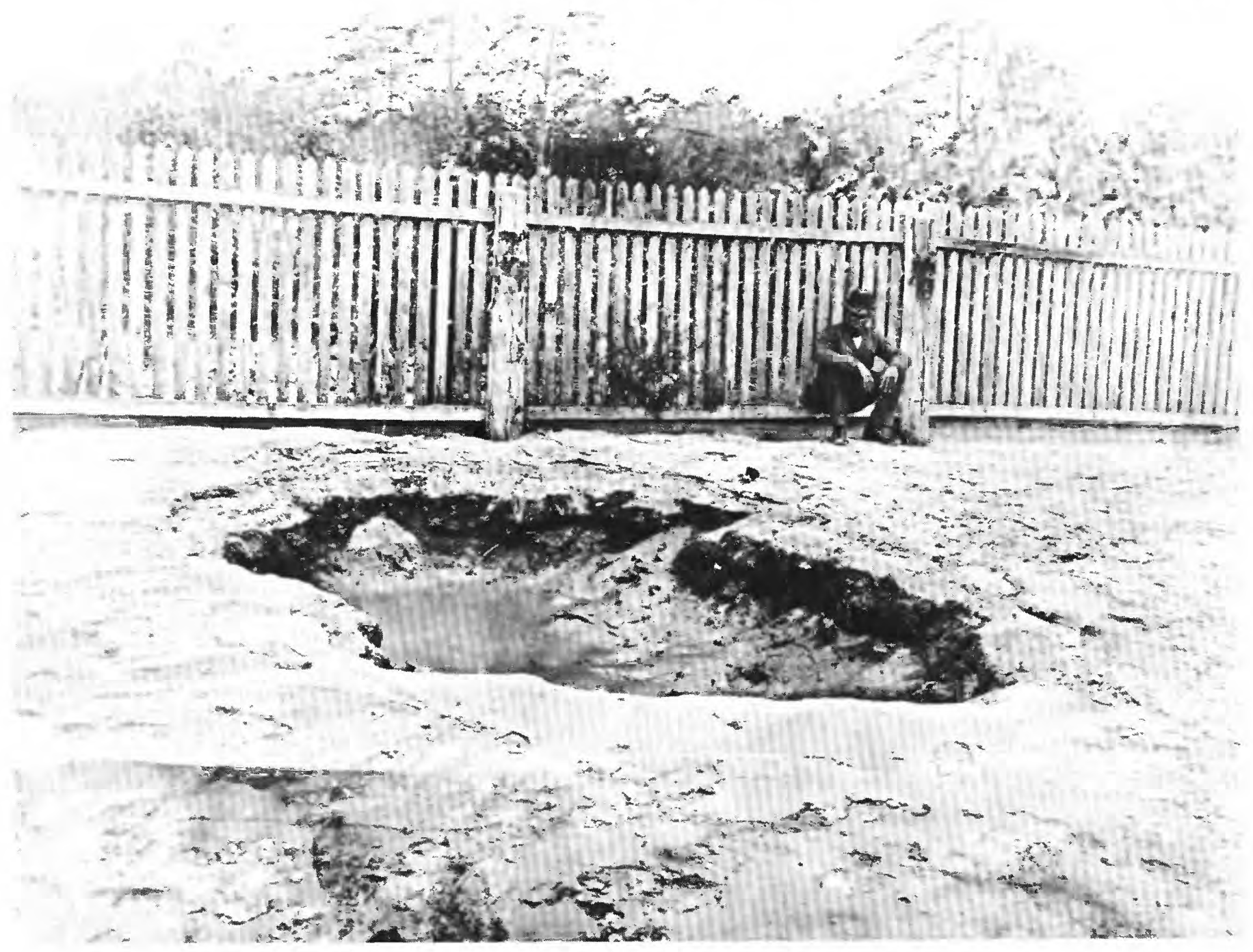

Craterlet from a sand spout at Ten Mile Hill, Berkeley County, South Carolina, formed by the September 1, 1886 (Aug. 31 EST), Charleston earthquake. (Photograph by J.K. Hillers.)

1959. Aug. 3. Charleston-Summerville, S.C. A chimney fell at Summerville, plaster was thrown to the floor, and a ceiling cracked. Damage to plaster and chimneys occurred at Charleston and on Wadmalaw Island. Also felt in Georgia. (Ref. 32, 349.)

1959. Oct. 27 (Oct. 26). Chesterfield, S.C. Plaster cracked and fell at Chesterfield, about $120 \mathrm{~km}$ northeast of Columbia, and a sheetrock wall cracked at McBee, about $30 \mathrm{~km}$ southwest of Chesterfield. Also felt in North Carolina. (Ref. 32, 38.)

1971. July 13. Near Newry, Oconee County, S.C. This earthquake cracked a chimney and displaced furniture at Newry, northwest of Clemson in northwest South Carolina. Also felt in Georgia. (Ref. $44,163$.
1974. Nov. 22. Charleston, S.C. In North Charleston, a resident in a ranch-style brick house reported cracks in the brick veneer, cracks in the driveway and sidewalk, and separation of an outside brick wall from the rest of the house. Throughout the city of Charleston, cracks formed in driveways, sidewalks, and plaster. At Summerville, damage included cracks in concrete-block footings, broken windowpanes, and enlarged cracks in a concrete patio floor. A 500-ton machine tool "jumped around" on its bed at the General Electric Plant at Ladson. Also felt in Georgia and North Carolina. Magnitude $4.3 \mathrm{M}_{\mathrm{fa}}$ NUT. (Ref. 38, 47, 263, 289, 349.)

1977. Jan. 18. Summerville, Dorchester County, S.C. Sidewalks were cracked in 
Summerville and suburban subdivisions. Felt only at a few other towns in the area. (Ref. 39, 349.)

1979. Aug. 26 (Aug. 25). Northwest South

Greenville, drywall cracked and fell and cracks formed in a concrete floor. The University of South

Carolina. At Tamassee (Oconee County), west of Georgia and North Carolina. (Ref. 262, 349.) 


\section{SOUTH DAKOTA}

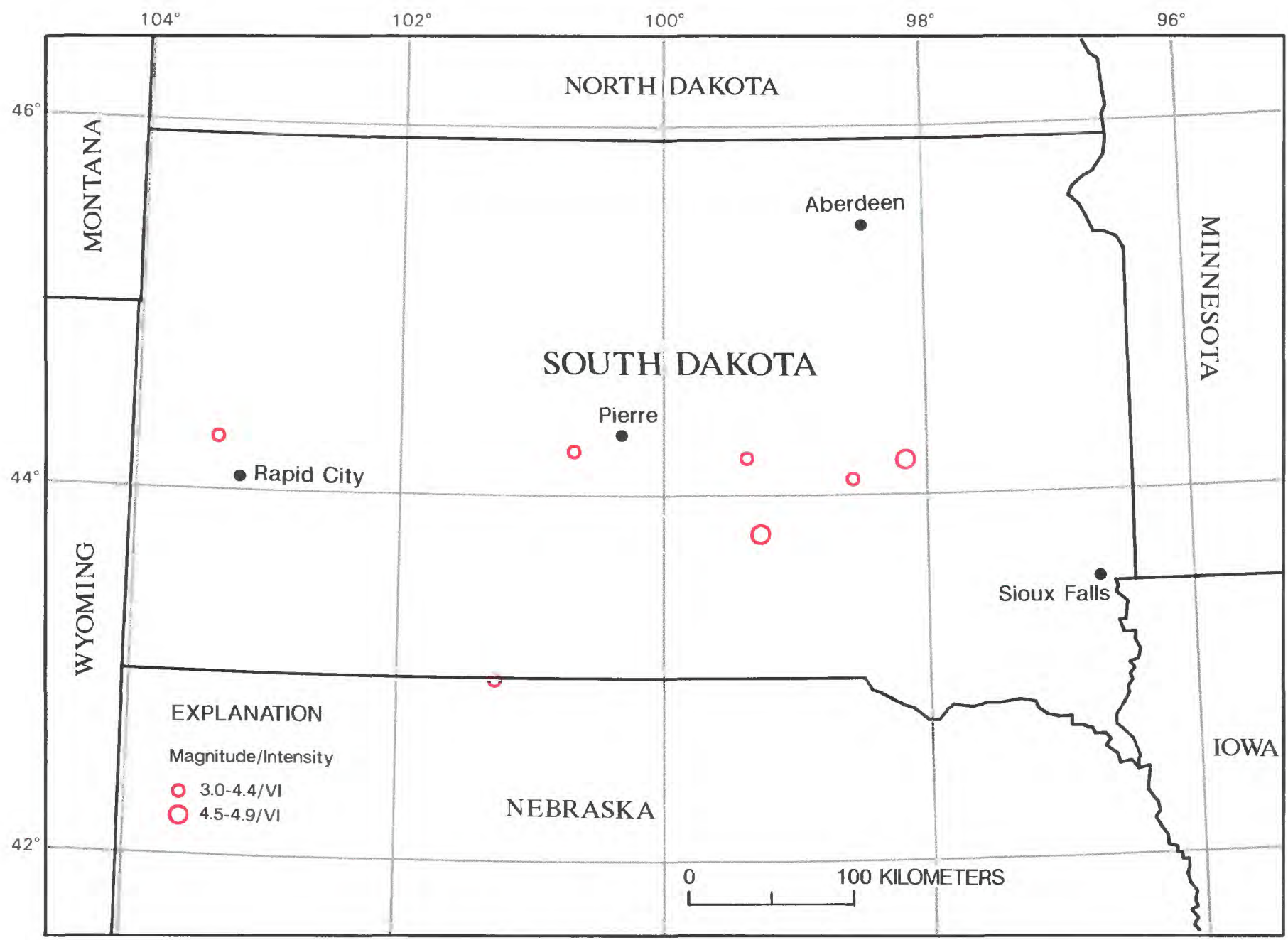

Earthquakes in South Dakota with magnitudes $\geq 4.5$ or intensity $\geq$ VI. 
[See table 1 for hypocenter and intensity references and table 2 for definitions of magnitude source codes. Leader (--) indicates information is not available]

\begin{tabular}{|c|c|c|c|c|c|c|c|c|c|c|c|c|c|}
\hline \multirow{2}{*}{\multicolumn{2}{|c|}{ Dato }} & \multirow{2}{*}{$\begin{array}{c}\text { Origin } \\
\text { time (UTC) }\end{array}$} & \multicolumn{4}{|c|}{ Hypocenter } & \multicolumn{4}{|c|}{ Magnitude } & \multicolumn{2}{|c|}{ Intensity } & \multirow{3}{*}{$\begin{array}{l}\text { Felt area } \\
\left(1,000 \mathrm{~km}^{2}\right)\end{array}$} \\
\hline & & & \multirow{2}{*}{$\begin{array}{l}\text { Latitude } \\
\left({ }^{\circ}\right)\end{array}$} & \multirow{2}{*}{$\begin{array}{l}\text { Longitude } \\
\left({ }^{\circ}\right)\end{array}$} & \multirow{2}{*}{$\begin{array}{l}\text { Depth } \\
(\mathrm{km})\end{array}$} & \multirow{2}{*}{ Ref } & \multicolumn{2}{|c|}{ USGS } & \multirow{2}{*}{ Other } & \multirow{2}{*}{$\begin{array}{c}\text { Moment } \\
\text { M }\end{array}$} & \multirow{2}{*}{ MM } & \multirow{2}{*}{ Ref } & \\
\hline$\overline{Y r}$ & Mo Da & $\mathrm{h} \mathrm{m} \mathrm{s}$ & & & & & $m_{b}$ & $M_{s}$ & & & & & \\
\hline 1906 & 0510 & 0027 & $43.0 \mathrm{~N}$ & $101.3 \mathrm{~W}$ & - & 105 & - & - & $3.70 \mathrm{M}_{\mathrm{fa}} \mathrm{SC}$ & - & VI & 105 & 45 \\
\hline 1911 & 0602 & 2234 & $44.2 \mathrm{~N}$ & $98.2 \mathrm{~W}$ & - & 38 & - & - & $4.50 \mathrm{M}_{\mathrm{fa}} \mathrm{BAR}$ & - & v & 105 & 100 \\
\hline 1922 & $\begin{array}{ll}01 & 02\end{array}$ & 1450 & $43.8 \mathrm{~N}$ & $99.3 \mathrm{~W}$ & - & 105 & - & - & - & - & VI & 105 & - \\
\hline 1946 & $07 \quad 23$ & 0645 & $44.1 \mathrm{~N}$ & $98.6 \mathrm{~W}$ & - & 105 & - & - & $4.10 \mathrm{M}_{\mathrm{fa}} \mathrm{SC}$ & - & VI & 38 & 22 \\
\hline 1961 & 1231 & 163605.8 & $44.250 \mathrm{~N}$ & $100.724 \mathrm{~W}$ & 023 & 349 & - & - & $4.20 \mathrm{M}_{\mathrm{fa}} \mathrm{SC}$ & - & VI & 34 & 34 \\
\hline 1966 & 0626 & 115943.1 & $44.296 \mathrm{~N}$ & $103.428 \mathrm{~W}$ & 002 & 349 & - & - & $3.10 \mathrm{M}_{n}$ BAR & - & VI & 38 & 3 \\
\hline 1983 & 0304 & 063218.6 & $44.214 \mathrm{~N}$ & $99.409 \mathrm{~W}$ & 005 & 360 & 4.4 & - & $4.40 \mathrm{M}_{\mathrm{L}} \mathrm{GS}$ & - & VI & 360 & 42 \\
\hline
\end{tabular}

[Reference (Ref.) numbers given in parentheses at the end of each description refer to sources of data in table 1. Magnitude values are described in the Introduction, and codes are defined in table 2.]

1906. May 10 (May 9). South DakotaNebraska border. This earthquake was reported all along the Niobrara River valley from Rushville to Valentine, Nebr., and at Rosebud, S. Dak., about 100 $\mathrm{km}$ southwest of Pierre. Plants fell from a windowsill at Cody, Nebr. Residents of towns for $100 \mathrm{~km}$ in all directions felt the shock. (Ref. 105, 353.)

1922. Jan. 2. Near Winner, Tripp County, S. Dak. Chimneys were thrown down and dishes and windows were broken at Winner, about $130 \mathrm{~km}$ south of Pierre. (Ref. 105.)

1946. July 23. Wessington, Beadle County, S. Dak. A series of earthquakes broke five water mains in the Wessington area, east of Pierre, and awakened sleepers at nearby Huron. Generally felt from Pierre east to De Smet and northward, including Redfield. Magnitude $4.2 \mathrm{M}_{\mathrm{fa}}$ BAR. (Ref. 38, 105, 353.)
1961. Dec. 31. Pierre, Hughes County, S. Dak. Cracks formed in plaster and a concrete floor at Pierre and a clothes drier was moved several centimeters. Felt from Pierre west to Midland (Haakon County) and east to Huron (Beadle County). Magnitude 4.3 $\mathrm{M}_{\mathrm{fa}}$ BAR, 4.2 $\mathrm{M}_{\mathrm{fa}}$ DG. (Ref. 34, 349, 353.)

1966. June 26. Southwest South Dakota. At Keystone (in Lawrence County, near Rapid City), well water was muddied for several hours. At Rapid City, a patio and concrete steps were cracked and objects fell from walls. Felt over a small area of southwest South Dakota. (Ref. 38, 349, 353.)

1983. Mar. 4. Near Fort Thompson, Buffalo County, S. Dak. This moderate earthquake caused minor damage at Fort Thompson (cracks in walls and ceiling), Lower Brule (cracks in reinforced concrete foundation, ceilings, and walls), and Stephan (cracks in exterior brick walls). Also felt in western Minnesota and northern Nebraska. Magnitude 4.6 $\mathrm{M}_{\mathrm{n}}$ TUL. (Ref. 360.) 
TENNESSEE

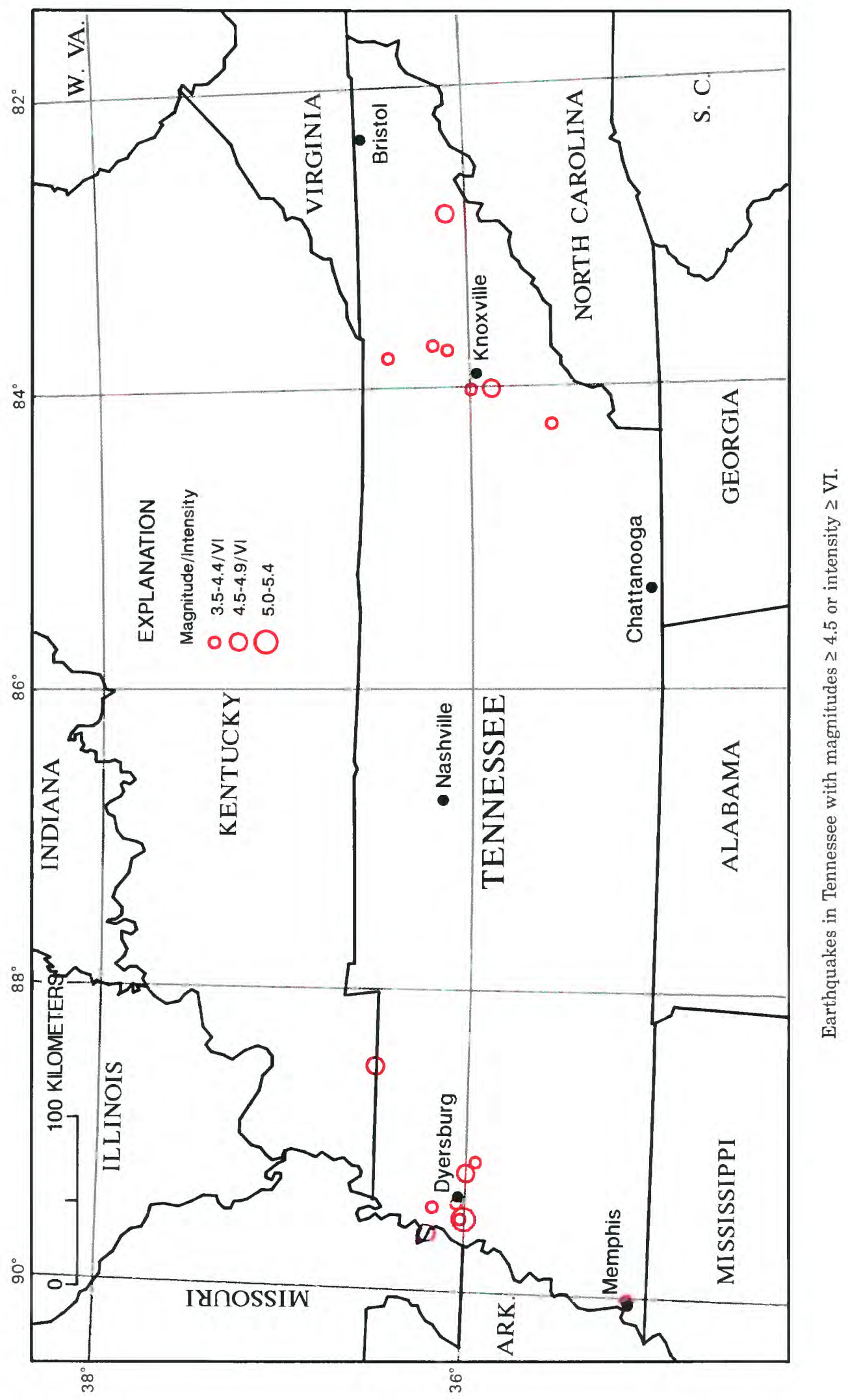


TENNESSEE

[See table 1 for hypocenter and intensity references and table 2 for definitions of magnitude source codes. @, felt area is less than 1,000 km ${ }^{2}$. Leader (--) indicates information is not available]

\begin{tabular}{|c|c|c|c|c|c|c|c|c|c|c|c|c|c|}
\hline \multirow{2}{*}{\multicolumn{2}{|c|}{ Date }} & \multirow{2}{*}{$\begin{array}{c}\text { Origin } \\
\text { time (UTC) }\end{array}$} & \multicolumn{4}{|c|}{ Hypocenter } & \multicolumn{4}{|c|}{ Magnitude } & \multicolumn{2}{|c|}{ Intensity } & \multirow{3}{*}{$\begin{array}{c}\text { Felt area } \\
\left(1,000 \mathrm{~km}^{2}\right)\end{array}$} \\
\hline & & & \multirow{2}{*}{$\begin{array}{l}\text { Latitude } \\
\left({ }^{\circ}\right)\end{array}$} & \multirow{2}{*}{$\begin{array}{l}\text { Longitude } \\
\left.\text { ( }{ }^{\circ}\right)\end{array}$} & \multirow{2}{*}{$\begin{array}{l}\text { Depth } \\
(\mathrm{km})\end{array}$} & \multirow[t]{2}{*}{ Ref } & \multicolumn{2}{|c|}{ USGS } & \multirow[t]{2}{*}{ Other } & \multirow{2}{*}{$\begin{array}{l}\text { Moment } \\
\text { M }\end{array}$} & \multirow[t]{2}{*}{ MM } & \multirow[t]{2}{*}{ Ref } & \\
\hline $\mathrm{Yr}_{\mathrm{r}}$ & Mo Da & $\mathrm{hms}$ & & & & & $m_{b}$ & $M_{S}$ & & & & & \\
\hline 1844 & 1128 & 1300 & $36.0 \mathrm{~N}$ & 84.0 W & - & 38 & - & - & $4.20 \mathrm{M}_{\mathrm{fa}} \mathrm{BAR}$ & - & VI & 38 & - \\
\hline 1865 & $08 \quad 17$ & 1500 & $36.0 \mathrm{~N}$ & 89.5 W & - & 113 & - & - & $5.00 \mathrm{M}_{\mathrm{fa}} \mathrm{S}$ & - & VII & 38 & 250 \\
\hline 1889 & $\begin{array}{ll}07 & 20\end{array}$ & 0132 & $35.1 \mathrm{~N}$ & $90.0 \mathrm{~W}$ & - & 105 & - & - & $3.80 \mathrm{M}_{\mathrm{fa}} \mathrm{BAR}$ & - & VI & 105 & @ \\
\hline 1898 & 0614 & 1526 & $36.5 \mathrm{~N}$ & 88.5 W & - & 529 & - & - & $4.50 \mathrm{M}_{\mathrm{fa}} \mathrm{SG}$ & - & V & 529 & 110 \\
\hline 1913 & 0328 & 2150 & $36.2 \mathrm{~N}$ & $83.7 \mathrm{~W}$ & - & 38 & - & - & $4.10 \mathrm{M}_{\mathrm{fa}} \mathrm{SC}$ & - & VII & 38 & 7 \\
\hline 1918 & 1016 & 0215 & $36.0 \mathrm{~N}$ & 89.2 W & - & 105 & - & - & $4.50 \mathrm{M}_{\mathrm{fa}}$ & - & V & 105 & 106 \\
\hline 1928 & 1103 & 040249.8 & $36.112 \mathrm{~N}$ & $82.828 \mathrm{~W}$ & 005 & 349 & - & - & $4.50 \mathrm{M}_{\mathrm{n}} \mathrm{DG}$ & & VI & 1 & 100 \\
\hline 1952 & $\begin{array}{ll}07 \quad 16 \\
\end{array}$ & 234810 & $36.2 \mathrm{~N}$ & 89.6 W & - & 25 & - & - & - & - & Vİ & 25 & - \\
\hline 1955 & $03 \quad 29$ & 090240 & $36.0 \mathrm{~N}$ & 89.5 W & - & 105 & - & - & $3.90 \mathrm{M}_{\mathrm{fa}} \mathrm{SC}$ & - & VI & 28 & 10 \\
\hline 1956 & 0907 & 133550.8 & $36.445 \mathrm{~N}$ & $83.787 \mathrm{~W}$ & 005 & 349 & - & - & $4.10 \mathrm{M}_{\mathrm{fa}} \mathrm{SC}$ & - & VI & 29 & 22 \\
\hline 1962 & $07 \quad 23$ & 060515.7 & 36.044 & $99 \mathrm{~W}$ & 00 & 34 & - & - & In BAR & $3.38 \mathrm{ST}$ & VI & 35 & 10 \\
\hline 1973 & 1130 & 074840.5 & $35.889 \mathrm{~N}$ & $83.993 \mathrm{~W}$ & 012 & 349 & 4.7 & - & $4.60 \mathrm{M}_{\mathrm{n}}$ BLA & 4.0OSTT & VI & 46 & 120 \\
\hline 1980 & 1202 & 085929.7 & $36.175 \mathrm{~N}$ & $89.429 \mathrm{~W}$ & 005 & 349 & & - & $3.80 M_{n}$ SLM & - & VI & 300 & 2 \\
\hline 1981 & 0807 & 115341 & & 2. W & 01 & 32 & - & - & $4.00 \mathrm{M}_{\mathrm{n}} \mathrm{SLM}$ & - & VI & 325 & 10 \\
\hline 1984 & $\begin{array}{ll}02 & 14\end{array}$ & 205430.9 & $36.125 \mathrm{~N}$ & $83.737 \mathrm{~W}$ & 010 & 370 & $=$ & - & $3.50 \mathrm{M}_{\mathrm{D}}$ TEC & - & VI & 370 & @ \\
\hline 1987 & 0327 & 072930.4 & $35.567 \mathrm{~N}$ & $84.229 W$ & 019 & 74 & 4.3 & - & $4.20 \mathrm{M}_{\mathrm{n}}$ TEC & - & VI & 577 & 23 \\
\hline
\end{tabular}

[Reference (Ref.) numbers given in parentheses at the end of each description refer to sources of data in table 1. Magnitude values are described in the Introduction, and codes are defined in table 2.]

1844. Nov. 28. Knoxville, Knox County, Tenn. Bricks were thrown from the top of a chimney at Knoxville, and one chimney in the county was thrown down. In nearby Blount County, bricks were displaced from several chimneys and houses. Also felt at Athens. (Ref. 38, 508).

1865. Aug. 17. Near Memphis, Shellby County, Tenn. At Memphis, chimneys were thrown down. At New Madrid, Mo., chimneys were damaged and waves formed on the river, that were like those made by a passing steamboat. The earth appeared to undulate. Felt from St. Louis, Mo., to Jackson, Miss. Also felt in Illinois. Magnitude 5.3 $\mathrm{M}_{\mathrm{fa}}$ BAR. (Ref. 38, 113, 353, 514.)

1889. July 20 (July 19). Memphis, Shellby County, Tenn. A severe local shock cracked walls and chimneys and frightened residents at Memphis. (Ref. 105, 353.)

1913. Mar. 28. Near Knoxville, Knox County, Tenn. This earthquake was of short duration, but caused general panic among residents. At Knoxville, bricks fell from chimneys, pictures fell to the floor, heavy furniture was overturned, and fire alarms were activated. A noticeable rise and fall of the ground was observed in a small area of Knox County. The Knox County Courthouse, a massive structure on a bluff overlooking the Tennessee River, trembled "like a leaf" for several seconds. Magnitude $4.0 \mathrm{M}_{\mathrm{fa}}$ BAR. (Ref. 38, 353, 508, 513.)

1928. Nov. 3 (Nov. 2). Southern Appalachians. This earthquake was felt strongly along the French Broad River between Asheville, N.C., and Newport, Tenn. At Newport (Cocke County), bricks were shaken from some buildings and plaster cracked. Three frame houses under construction northeast of Newport at Johnson City were shaken down; one building under construction north of Newport at Morristown was damaged; and the top of one chimney at Greenville, east of Morristown, was thrown down. Minor damage also occurred at Asheville, Bryson City, and Waynesville, N.C. Also felt in Alabama, Georgia, Kentucky, South Carolina, and Virginia. (Ref. 1, 349, 508.)

1952. July 16. Near Dyersburg, Dyer County, Tenn. Many cracks were reported in a concrete structure at Dyersburg. Also felt at Finley and Jenkinsville. (Ref. 25.)

1955. Mar. 29. Finley, Dyer County, Tenn. Plaster cracked in a house at Finley, and violent shaking and a roaring noise were reported. Also felt 
in Arkansas and Missouri. Magnitude $4.0 \mathrm{M}_{\mathrm{fa}}$ BAR. (Ref. 28, 105, 353.)

1956. Sept. 7. Knoxville, Knox County, Tenn. This earthquake caused the most severe effects at Knoxville, where one chimney was thrown down, plaster was knocked from walls, and windows were shattered. Also felt in parts of Kentucky, North Carolina, and Virginia. An aftershock was felt a few minutes after the main tremor. Magnitude $4.1 \mathrm{M}_{\mathrm{fa}}$ BAR, 4.1 $\mathrm{M}_{\mathrm{fa}}$ DG. (Ref. 29, 349, 353, 508.)

1962. July 23. Dyersburg, Dyer County, Tenn. Walls and plaster cracked at Dyersburg, and one wall was damaged slightly at Miston, northwest of Dyersburg. Also felt in Arkansas and Missouri. (Ref. 35, 349, 353.)

1973. Nov. 30. Eastern Tennessee. The earthquake caused minor damage to chimneys, walls, and windows in the Maryville-Alcoa area in Blount County, and small cracks in walls at Knoxville (about $20 \mathrm{~km}$ north of Maryville). Plaster cracked and fell and windows broke at Maryville and at Vonore, about $20 \mathrm{~km}$ southwest. Slight damage also occurred in a few towns in Georgia, Kentucky, and North Carolina. Also felt in South Carolina, Virginia, and West Virginia. Magnitude 4.6 $\mathrm{M}_{\mathrm{fa}}$ NUT. (Ref. 46, 263, 349.)

1980. Dec. 2. Northwest Tennessee. Cracks formed in a house foundation at Madie and in exterior brick walls at Ridgely in Obion County. In addition, windows were damaged at Caruthersville, Mo., and north of Dyersburg at Elbridge, Hornbeak, and Lane, Tenn. Magnitude $3.8 \mathrm{M}_{\mathrm{n}}$ TEC. (Ref. 300,349 .)

1981. Aug. 7. Western Tennessee. At Eaton, in Gibson County, southeast of Dyersburg, an earthquake cracked bathroom tile and two concrete patios. Also felt in Arkansas, Mississippi, and Missouri. Magnitude 4.0 $\mathrm{M}_{\mathrm{n}}$ BLA, $4.0 \mathrm{M}_{\mathrm{n}}$ TEC. (Ref. 325.)

1984. Feb. 14. Eastern Tennessee. A few buildings sustained damage northeast of Knoxville, at Blaine, where some windows were broken. Windows were cracked and small objects overturned at New Market (Jefferson County), northeast of Knoxville. Magnitude 3.6 $\mathrm{M}_{\mathrm{n}}$ BLA. (Ref. 370.)

1987. Mar. 27. Near Greenback, Loudon County, Tenn. In the area southwest of Knoxville, at Greenback and Friendsville, chimneys and foundations of buildings sustained cracks and hairline cracks formed in plaster and drywalls. Slight cracks in drywall and building foundations also were reported in Blount County, at Louisville and Tallassee. Felt over a moderate area of six States, including northern Georgia, southern Kentucky, western North Carolina, eastern Tennessee, southwest Virginia (Lee County), and northwest South Carolina (Pickens County). Magnitude $4.2 \mathrm{M}_{\mathrm{n}}$ TUL. (Ref. 74, 577.) 



\section{TEXAS}

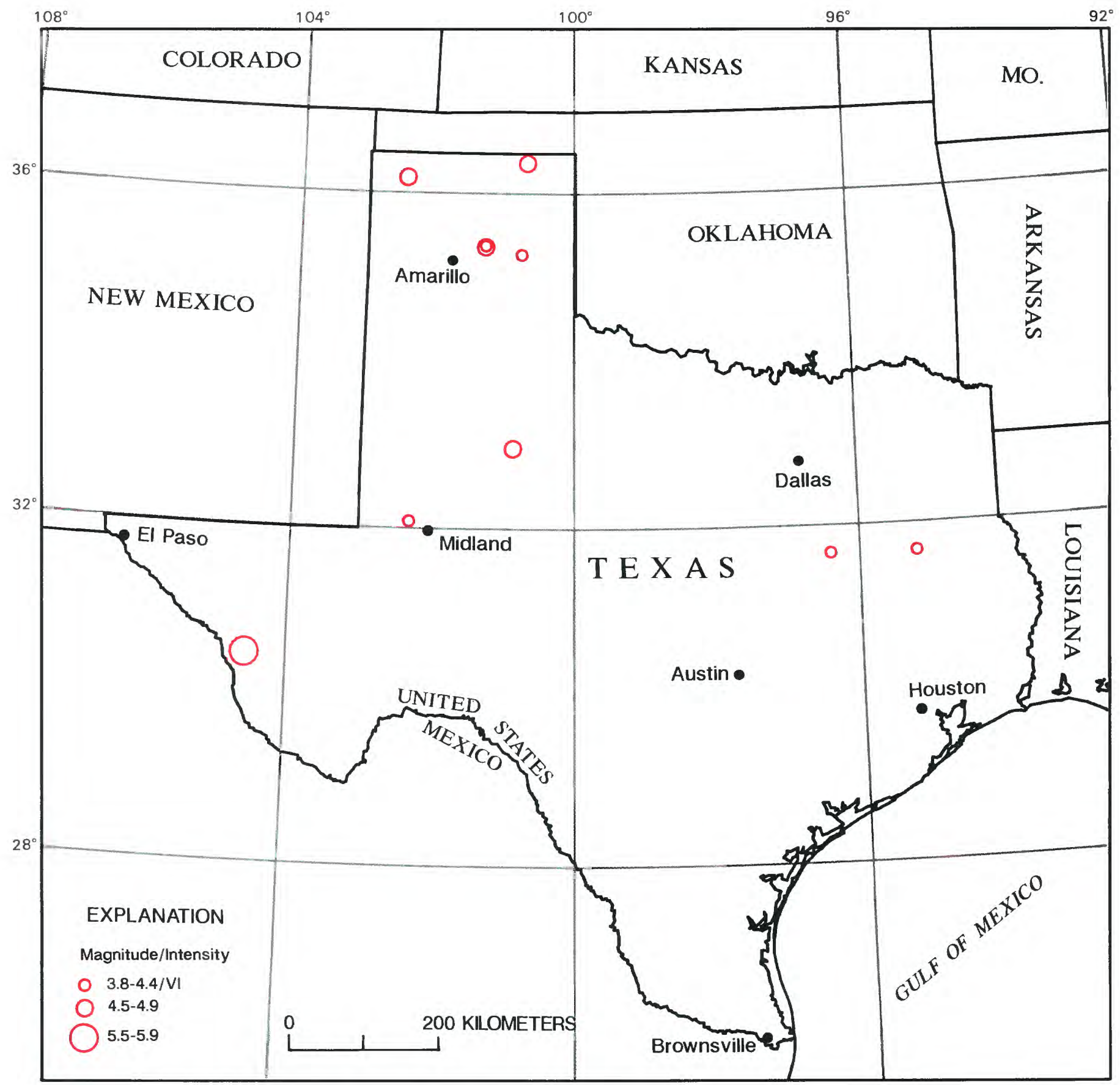

Earthquakes in Texas with magnitudes $\geq 4.5$ or intensity $\geq$ VI. 


\begin{tabular}{|c|c|c|c|c|c|c|c|c|c|c|c|c|c|}
\hline \multirow{2}{*}{\multicolumn{2}{|c|}{ Date }} & \multirow{2}{*}{$\begin{array}{c}\text { Origin } \\
\text { time (UTC) }\end{array}$} & \multicolumn{4}{|c|}{ Hypocenter } & \multicolumn{4}{|c|}{ Magnitude } & \multicolumn{2}{|c|}{ Intensity } & \multirow{3}{*}{$\begin{array}{c}\text { Felt area } \\
\left(1,000 \mathrm{~km}^{2}\right)\end{array}$} \\
\hline & & & \multirow{2}{*}{$\begin{array}{l}\text { Latitude } \\
\left({ }^{\circ}\right)\end{array}$} & \multirow{2}{*}{$\begin{array}{c}\text { Longitude } \\
\left({ }^{\circ}\right)\end{array}$} & \multirow{2}{*}{$\begin{array}{l}\text { Depth } \\
(\mathrm{km})\end{array}$} & \multirow[t]{2}{*}{ Ref } & \multicolumn{2}{|c|}{ USGS } & \multirow{2}{*}{ Other } & \multirow{2}{*}{$\begin{array}{c}\text { Moment } \\
\text { M }\end{array}$} & \multirow[t]{2}{*}{$\mathrm{MM}$} & \multirow[t]{2}{*}{ Ref } & \\
\hline $\mathrm{Yr}$ & Mo Da & $\mathrm{hms}$ & & & & & $m_{b}$ & $M_{S}$ & & & & & \\
\hline 1891 & 0108 & 0600 & $31.7 \mathrm{~N}$ & $95.2 \mathrm{~W}$ & - & 342 & - & - & $3.80 \mathrm{M}_{\mathrm{fa}} \mathrm{BAR}$ & - & VI & 342 & @ \\
\hline 1917 & $\begin{array}{ll}0328\end{array}$ & 1956 & $35.4 \mathrm{~N}$ & $101.3 \mathrm{~W}$ & - & 364 & - & - & $3.80 \mathrm{M}_{\mathrm{fa}} \mathrm{SC}$ & - & VI & 364 & 5 \\
\hline 1925 & 0730 & 1217 & $35.4 \mathrm{~N}$ & $101.3 \mathrm{~W}$ & - & 364 & - & - & $4.90 \mathrm{M}_{\mathrm{fa}} \mathrm{SC}$ & - & VI & 364 & 520 \\
\hline 1931 & $\begin{array}{ll}08 & 16\end{array}$ & 114022.3 & $30.502 \mathrm{~N}$ & $104.575 \mathrm{~W}$ & 001 & 214 & - & - & $5.80 \mathrm{M}_{\mathrm{n}}$ NTT & - & VIII & 4 & 980 \\
\hline 1932 & 0409 & 1017 & $31.7 \mathrm{~N}$ & $96.4 \mathrm{~W}$ & - & 342 & - & - & $3.90 \mathrm{M}_{\mathrm{fa}} \mathrm{SC}$ & - & VI & 342 & 8 \\
\hline 1936 & 0620 & 032403.5 & $35.310 \mathrm{~N}$ & $100.773 W$ & 005 & 349 & - & - & $4.40 \mathrm{M}_{\mathrm{fa}} \mathrm{SC}$ & - & VI & 364 & 87 \\
\hline 1948 & $03 \quad 12$ & 042906.3 & $36.221 \mathrm{~N}$ & $102.478 \mathrm{~W}$ & 005 & 349 & - & - & $4.50 \mathrm{M}_{\mathrm{fa}} \mathrm{SC}$ & - & VI & 364 & 123 \\
\hline 1966 & $\begin{array}{ll}08 & 14\end{array}$ & 152553.7 & $32.115 \mathrm{~N}$ & $102.339 \mathrm{~W}$ & 003 & 349 & 3.4 & - & $4.30 \mathrm{M}_{\mathrm{n}} \mathrm{BAR}$ & - & VI & 81 & - \\
\hline 1974 & $02 \quad 15$ & 133349.2 & $36.399 \mathrm{~N}$ & $100.688 \mathrm{~W}$ & 000 & 349 & 4.5 & - & $4.50 \mathrm{M}_{\mathrm{n}} \mathrm{DG}$ & 4.35HRN & $\mathrm{V}$ & 47 & 45 \\
\hline 1978 & 0616 & 114656.0 & $32.990 \mathrm{~N}$ & $100.875 \mathrm{~W}$ & 003 & 349 & 4.4 & - & $4.60 \mathrm{M}_{\mathrm{n}}$ SLM & $4.48 \mathrm{VOS}$ & V & 240 & 52 \\
\hline
\end{tabular}

[Reference (Ref.) numbers given in parentheses at the end of each description refer to sources of data in table 1. Magnitude values are described in the Introduction, and codes are defined in table 2.]

1887. May 3. Northern Sonora, Mexico. Chimneys were thrown down in El Paso, Tex. See Arizona section for a complete description of this earthquake. (Ref. 38, 343, 471, 494, 497.)

1891. Jan. 8. Rusk, Tex. A strong local earthquake thre w down chimneys at Rusk, southeast of Dallas, and awakened many residents. Two distinct shocks were felt. Some researchers have questioned the authenticity of this event, suggesting that it may have been a violent thunderstorm or a tornado. (Ref. $342,353$.

1917. Mar. 28. Panhandle area, Carson County, Tex. Ceiling plaster fell to the floor of the bank and the walls of many buildings were cracked at Panhandle, east of Amarillo. Distinctly felt in northern Carson County and southern Hutchinson County. Magnitude $3.8 \mathrm{M}_{\mathrm{fa}}$ BAR. (Ref. 353, 364.)

1925. July 30. Panhandle area, Carson County, Tex. Plaster fell and jars were displaced from shelves at Plemons; ground settling resulted in damage to a section of track of the Santa Fe Railroad at Cuyler. Plaster was shaken from ceilings at Guymon, Okla., north of Panhandle. Also felt in Colorado, Kansas, Missouri, and New Mexico (see fig. 56). Magnitude $4.9 \mathrm{M}_{\mathrm{fa}}$ BAR. (Ref. 218, 353, 364.)

1931. Aug. 16. Near Valentine, Jeff Davis County, Tex. In terms of magnitude and damage, this is the largest earthquake known to have occurred in Texas. The most severe damage was reported at Valentine, where all buildings except wood-frame houses were damaged severely and all brick chimneys toppled or were damaged. The schoolhouse, which consisted of one section of concrete blocks and another section of bricks, was damaged so badly that it had to be rebuilt. Small cracks formed in the schoolhouse yard.

Some walls collapsed in adobe buildings, and ceilings and partitions were damaged in wood-frame structures. Some concrete and brick walls were cracked severely. One low wall, reinforced with concrete, was broken and thrown down. Tombstones in a local cemetery were rotated. Damage to property was reported from widely scattered points in Brewster, Jeff Davis, Culberson, and Presidio Counties.

Landslides occurred in the Van Horn Mountains, southwest of Lobo; in the Chisos Mountains, in the area of Big Bend; and farther northwest, near Pilares and Porvenir. Landslides also occurred in the Guadalupe Mountains, near Carlsbad, N. Mex., and slides of rock and dirt were reported near Picacho, N. Mex. Well water and springs were muddied throughout the area. Also felt in parts of Oklahoma, New Mexico (see fig. 57), and in Chihuahua and Coahuila, Mexico. Magnitude $6.4 \mathrm{M}_{\mathrm{fa}} \mathrm{SAN}, 5.8 \mathrm{M}_{\mathrm{n}} \mathrm{NTT}, 6.4 \mathrm{M}_{\mathrm{S}}$ GR, $5.7 \mathrm{M}_{\mathrm{fa}}$ SC. (Ref. 4, 99, 124, 353, 364.)

1932. Apr. 9. Wortham, Freestone County, Tex. At Wortham, northeast of Waco, weakly mortared concrete bricks fell from at least four chimneys. Felt in Freestone, Hill, Limestone, McLennan, and Navarro Counties. Magnitude $3.6 \mathrm{M}_{\mathrm{fa}} \mathrm{BAR}, 3.9 \mathrm{M}_{\mathrm{fa}}$ CAR. (Ref. 342, 353.) 


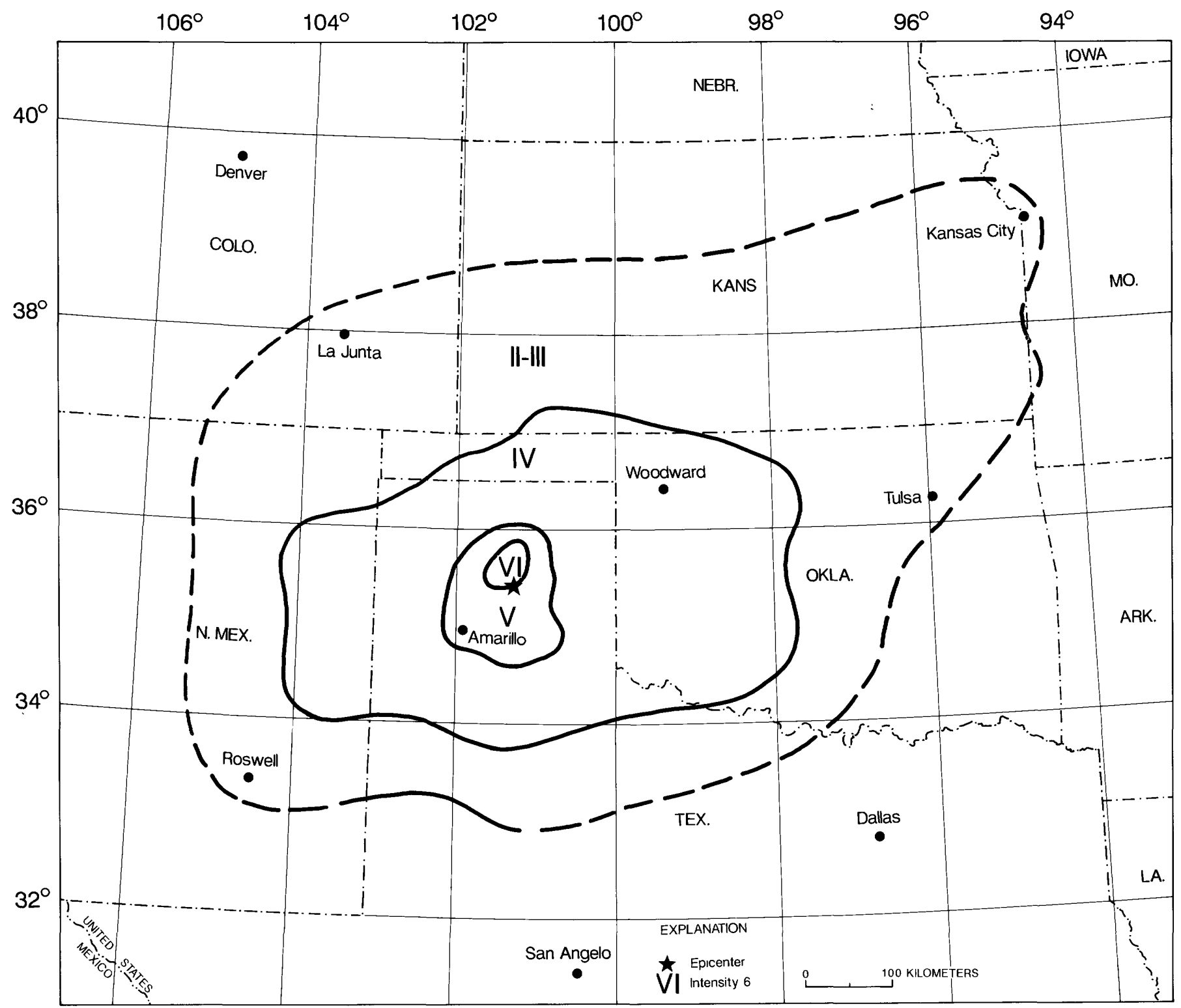

FIGURE 56.-Isoseismal map for the Texas Panhandle earthquake of July 30, 1925. Isoseismals are based on intensity estimates from data listed in references 218 and 364 of table 1.

1936. June 20 (June 19). Near Pampa, Gray County, Tex. The last shock of a series of three on this date cracked the cornice of the Pampa City Hall and enlarged an existing crack. Houses were jarred strongly at nearby Borger. Slight damage also was reported in Elkhart, Kans., and Kenton, Okla. Also felt at Richards, Colo. Magnitude $4.5 \mathrm{M}_{\mathrm{fa}} \mathrm{BAR}, 4.5$ $\mathrm{M}_{\mathrm{fa}}$ DG. (Ref. 349, 353, 364.)

1948. Mar. 12 (Mar. 11). Hartley County in northwest Texas. Cracks in walls and plaster were reported from several towns in the epicentral region, including Amarillo, Panhandle, and Perico. Slight damage to plaster also was reported in Colorado, New Mexico, and Oklahoma. Also felt in Kansas. Magnitude 4.8 $\mathrm{M}_{\mathrm{fa}}$ BAR, 4.8 $\mathrm{M}_{\mathrm{fa}}$ DG. (Ref. $349,353,364$.

1966. Aug. 14. Kermit, Winkler County, Tex. At Kermit, windows were broken and several street signs were knocked down. Plaster was cracked in one church. Also felt at Wink, Tex., and Loco Hills, N. Mex. (Ref. 81, 349, 353.) 


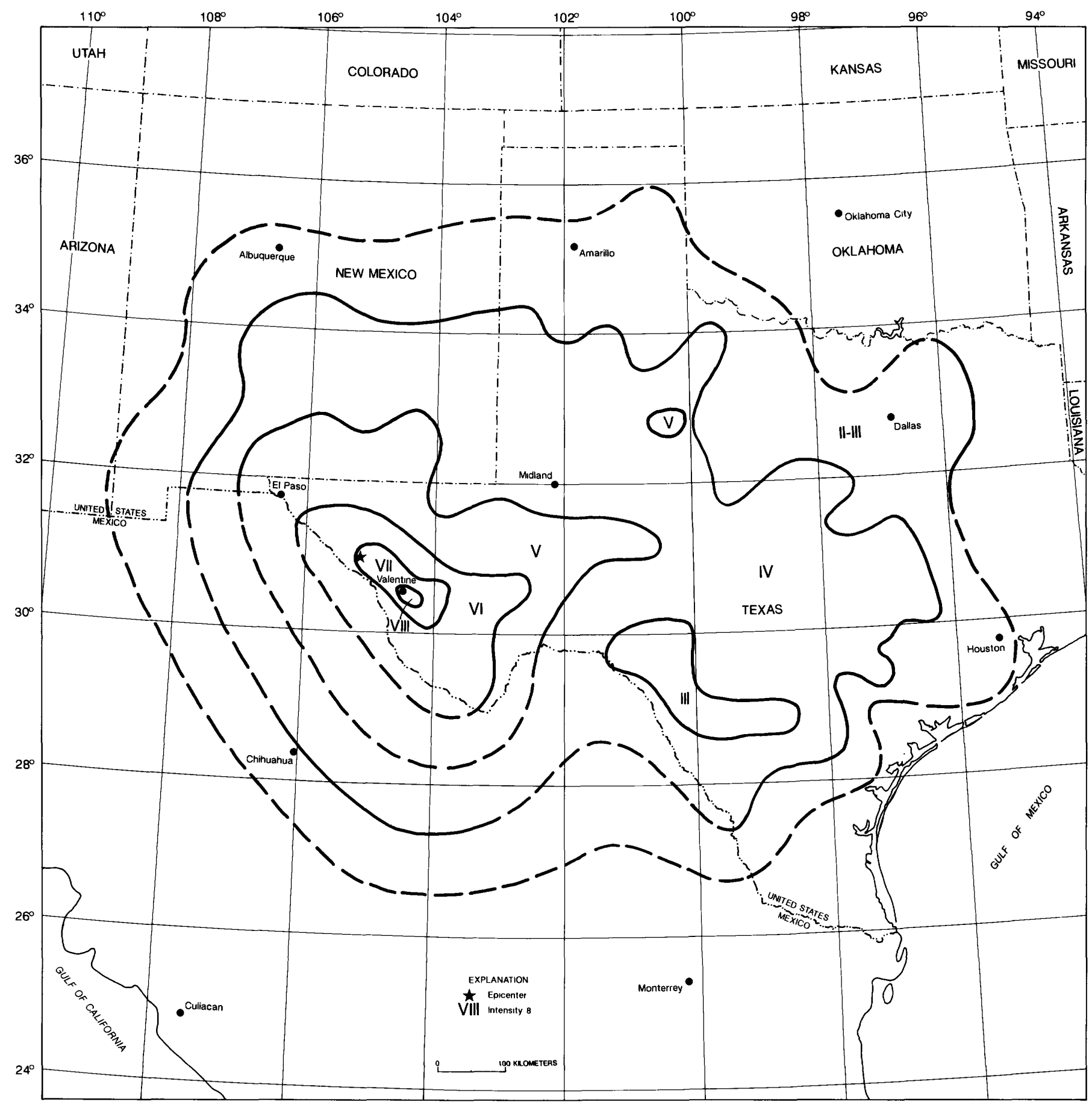

FigURE 57.-Isoseismal map for the Valentine, Texas, earthquake of August 16, 1931. Isoseismals are based on intensity estimates from data listed in references 4,124 , and 342 of table 1 . 


\section{UTAH}

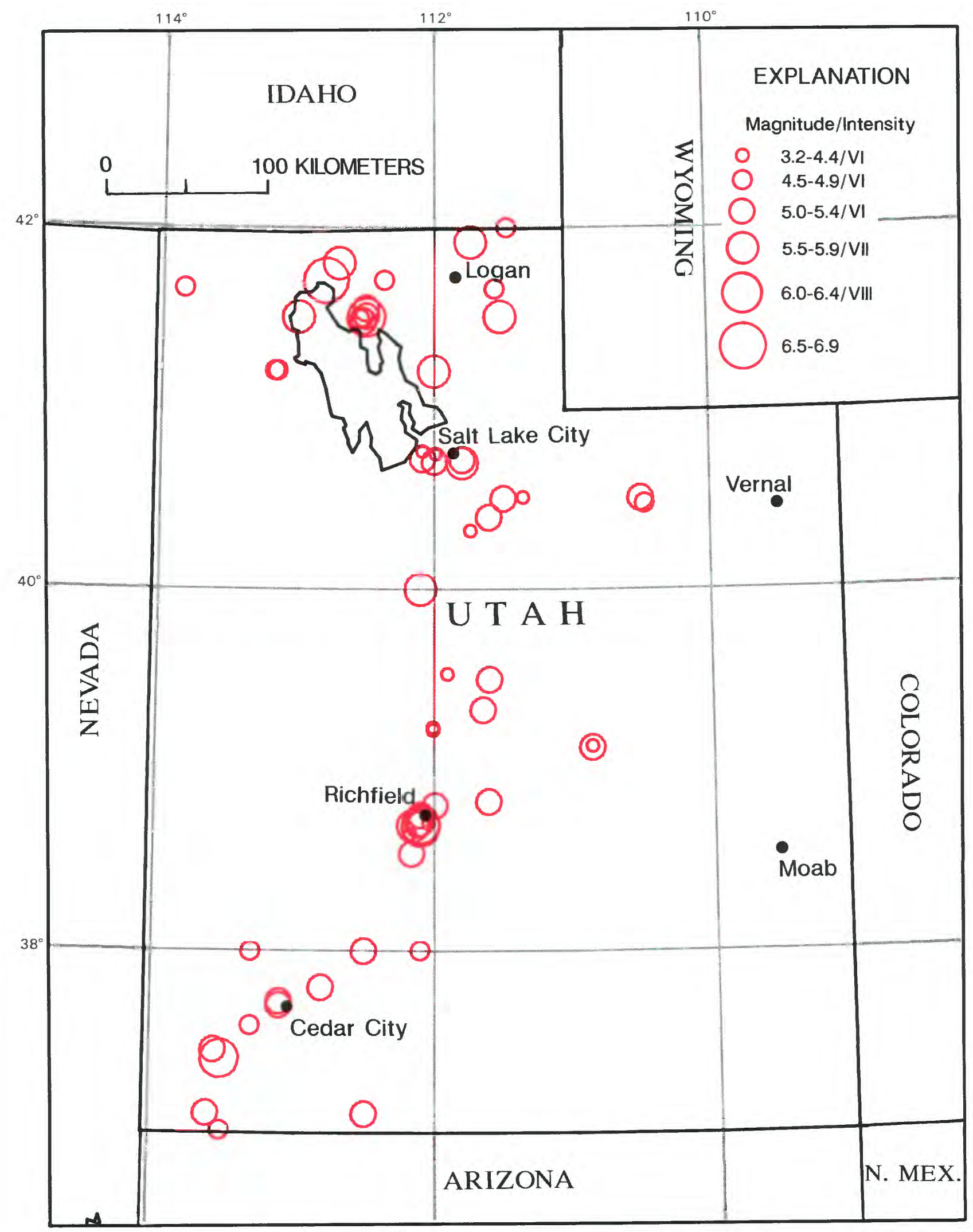

Earthquakes in Utah with magnitudes $\geq 4.5$ or intensity $\geq$ VI. 
UTAH

[See table 1 for hypocenter and intensity references and table 2 for definitions of magnitude source codes. @, felt area is less than $1,000 \mathrm{~km}^{2}$. Leader (-) indicates information is not available]

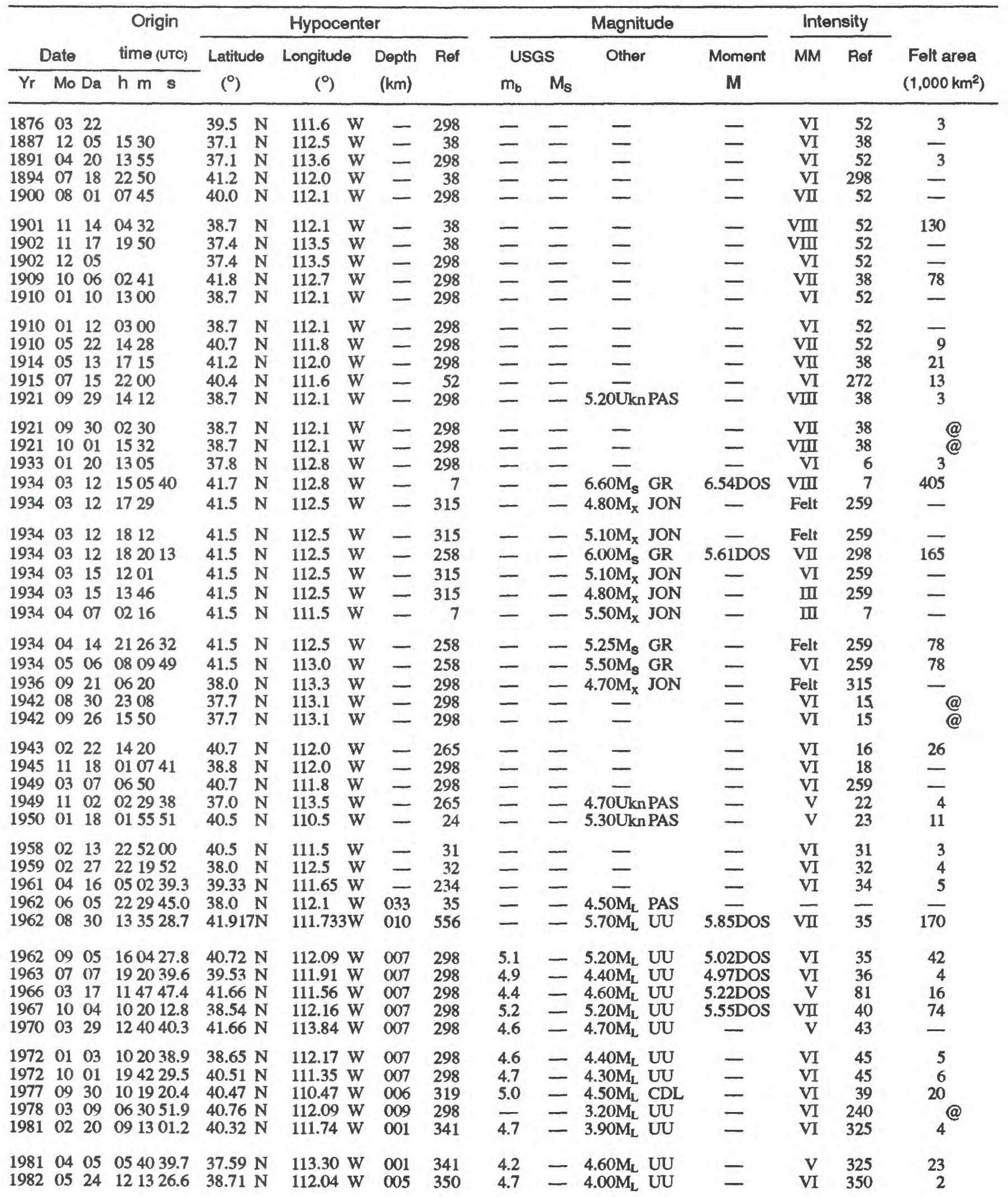


UTAH-Continued

[See table 1 for hypocenter and intensity references and table 2 for definitions of magnitude source codes. (0, felt area is less than $1,000 \mathrm{~km}^{2}$. Leader (--) indicates information is not available]

\begin{tabular}{|c|c|c|c|c|c|c|c|c|c|c|c|c|c|c|}
\hline \multirow{2}{*}{\multicolumn{3}{|c|}{ Date }} & \multirow{2}{*}{$\begin{array}{c}\text { Origin } \\
\text { time (UTC) }\end{array}$} & \multicolumn{4}{|c|}{ Hypocenter } & \multicolumn{4}{|c|}{ Magnitude } & \multicolumn{2}{|c|}{ Intensity } & \multirow{3}{*}{$\begin{array}{c}\text { Felt area } \\
\left(1,000 \mathrm{~km}^{2}\right)\end{array}$} \\
\hline & & & & \multirow{2}{*}{$\begin{array}{l}\text { Latitude } \\
\left(^{\circ}\right)\end{array}$} & \multirow{2}{*}{$\begin{array}{l}\text { Longitude } \\
\qquad\left(^{\circ}\right)\end{array}$} & \multirow{2}{*}{$\begin{array}{l}\text { Depth } \\
(\mathbf{k m})\end{array}$} & \multirow[t]{2}{*}{ Ref } & \multicolumn{2}{|c|}{ USGS } & \multirow[t]{2}{*}{ Other } & \multirow{2}{*}{$\begin{array}{c}\text { Moment } \\
\text { M }\end{array}$} & \multirow[t]{2}{*}{ MM } & \multirow[t]{2}{*}{ Ref } & \\
\hline$\overline{Y r}$ & Mo & $\mathrm{Da}$ & h m s & & & & & $m_{b}$ & $M_{s}$ & & & & & \\
\hline $\begin{array}{l}1983 \\
1986 \\
1986\end{array}$ & $\begin{array}{l}10 \\
03 \\
03\end{array}$ & $\begin{array}{l}08 \\
24 \\
25\end{array}$ & $\begin{array}{lll}115753.8 \\
224023.4 \\
025301.2\end{array}$ & $\begin{array}{l}40.748 \mathrm{~N} \\
39.236 \mathrm{~N} \\
39.223 \mathrm{~N}\end{array}$ & $\begin{array}{l}111.993 \mathrm{~W} \\
112.009 \mathrm{~W} \\
112.011 \mathrm{~W}\end{array}$ & $\begin{array}{l}006 \\
001 \\
001\end{array}$ & $\begin{array}{l}360 \\
562 \\
562\end{array}$ & $\begin{array}{l}4.5 \\
4.7 \\
4.5\end{array}$ & - & $\begin{array}{ll}4.30 \mathrm{M}_{\mathrm{L}} & \mathrm{UU} \\
4.40 \mathrm{M}_{\mathrm{L}} & \mathrm{UU} \\
3.90 \mathrm{M}_{\mathrm{L}} & \mathrm{UU}\end{array}$ & - & $\begin{array}{l}\mathrm{VI} \\
\mathrm{V} \\
\mathrm{V}\end{array}$ & $\begin{array}{l}360 \\
562 \\
562\end{array}$ & $\begin{array}{l}7 \\
3 \\
3\end{array}$ \\
\hline $\begin{array}{l}1987 \\
1987 \\
1988 \\
1988 \\
1988\end{array}$ & $\begin{array}{l}09 \\
10 \\
08 \\
08 \\
11\end{array}$ & $\begin{array}{l}25 \\
26 \\
14 \\
18 \\
19\end{array}$ & $\begin{array}{l}042758.1 \\
041600.9 \\
200303.9 \\
124453.4 \\
194237.3\end{array}$ & $\begin{array}{l}41.210 \mathrm{~N} \\
41.203 \mathrm{~N} \\
39.128 \mathrm{~N} \\
39.132 \mathrm{~N} \\
41.996 \mathrm{~N}\end{array}$ & $\begin{array}{l}113.152 \mathrm{~W} \\
113.172 \mathrm{~W} \\
110.869 \mathrm{~W} \\
110.867 \mathrm{~W} \\
111.472 \mathrm{~W}\end{array}$ & $\begin{array}{l}010 \\
009 \\
010 \\
012 \\
006\end{array}$ & $\begin{array}{l}74 \\
74 \\
74 \\
74 \\
74\end{array}$ & $\begin{array}{l}4.7 \\
4.3 \\
5.5 \\
4.5 \\
4.9\end{array}$ & $\frac{4.6}{-}$ & $\begin{array}{l}4.70 \mathrm{M}_{\mathrm{L}} \mathrm{UU} \\
4.80 \mathrm{M}_{\mathrm{L}} \mathrm{UU} \\
5.30 \mathrm{M}_{\mathrm{L}} \mathrm{UU} \\
4.40 \mathrm{M}_{\mathrm{L}} \mathrm{UU} \\
4.80 \mathrm{M}_{\mathrm{L}} \mathrm{UU}\end{array}$ & $\begin{array}{l}- \\
-\end{array}$ & $\begin{array}{l}\text { V } \\
\text { IV } \\
\text { VI } \\
\text { V } \\
\text { VI }\end{array}$ & $\begin{array}{l}\mathbf{5 7 7} \\
\mathbf{5 7 7} \\
\mathbf{5 7 8} \\
\mathbf{5 7 8} \\
\mathbf{5 7 8}\end{array}$ & $\begin{array}{r}42 \\
37 \\
110 \\
18 \\
28\end{array}$ \\
\hline $\begin{array}{l}1989 \\
1989 \\
1989\end{array}$ & $\begin{array}{l}01 \\
07 \\
07\end{array}$ & $\begin{array}{l}30 \\
03 \\
05\end{array}$ & $\begin{array}{l}040622.7 \\
224428.6 \\
225156.3\end{array}$ & $\begin{array}{l}38.824 \mathrm{~N} \\
41.706 \mathrm{~N} \\
41.707 \mathrm{~N}\end{array}$ & $\begin{array}{l}111.614 \mathrm{~W} \\
112.373 \mathrm{~W} \\
112.371 \mathrm{~W}\end{array}$ & $\begin{array}{l}010 \\
007 \\
010\end{array}$ & $\begin{array}{l}74 \\
74 \\
74\end{array}$ & $\begin{array}{l}5.0 \\
4.5 \\
4.2\end{array}$ & $\frac{4.8}{-}$ & $\begin{array}{l}5.40 \mathrm{M}_{\mathrm{L}} \text { UU } \\
4.80 \mathrm{M}_{\mathrm{L}} \text { UU } \\
4.60 \mathrm{M}_{\mathrm{L}} \text { UU }\end{array}$ & $\begin{array}{c}5.31 \mathrm{HAV} \\
-\end{array}$ & $\begin{array}{l}\text { VI } \\
\text { V } \\
\text { IV }\end{array}$ & $\begin{array}{l}579 \\
579 \\
579\end{array}$ & $\begin{array}{r}148 \\
4 \\
4\end{array}$ \\
\hline
\end{tabular}

[Reference (Ref.) numbers given in parentheses at the end of each description refer to sources of data in table 1. Magnitude values are described in the Introduction, and codes are defined in table 2.]

1876. Mar. 22. Moroni, northern Sanpete County, Utah. A local earthquake on the Thousand Lake fault cracked two buildings and sent Moroni residents running from their houses. (Ref. 52, 298.)

1887. Dec. 5. Kanab, Kane County, Utah. A local earthquake on the Sevier fault caused minor damage at Kanab, near the Arizona border. Bricks fell from chimneys, a few houses were cracked, and people who were walking were thrown to the ground. Rocks fell from nearby cliffs, causing large clouds of dust. (Ref. 38,52 .)

1891. Apr. 20. St. George, southern Washington County, Utah. One chimney was thrown from a house, plaster was shaken from the walls, and dishes were broken at St. George, near the Arizona border west of Kanab. (Ref. 52, 298.)

1894. July 18. Northern Utah. Walls were cracked and dishes were shaken from tables at Ogden, north of Salt Lake City, in Weber County. Three distinct shocks were reported. (Ref. 38, 52, 298.)

1900. Aug. 1. Near Goshen, Utah County, Utah. At Goshen, southwest of Provo, dishes and a chimney were broken and plaster fell from walls. At nearby Santaquin, an adobe house was split in two and people were thrown from their beds. The mine shaft at the Mammoth mine at Santaquin was thrown out of line and the cage could not be lowered. (Ref. 52, 298.)
1901. Nov. 14 (Nov. 13). Southern Utah. An earthquake on the Tushar fault caused much damage to brick buildings and chimneys from Parowan (Iron County) on the south to Richfield (Sevier County) on the north. Extensive rockslides occurred between Beaver and Marysvale, in Piute County. Masses of fallen rock in Bullion and Cottonwood Canyons made the roads almost impassable. Water and sand were ejected from cracks that formed in the ground about $5 \mathrm{~km}$ east of Richfield; creeks in the area increased their flow. Many towns sustained minor damage. Aftershocks continued for several weeks. Also felt at Salt Lake City, $220 \mathrm{~km}$ to the north. (Ref. 38, 52.)

1902. Nov. 17. Pine Valley, Washington County, Utah. Every chimney was destroyed in Pine Valley, north of St. George, and rockslides occurred in the nearby mountains. At Santa Clara, south of Pine Valley, almost every chimney was knocked down. Buildings were damaged considerably at St. George; chimneys and plaster fell at Pinto. The earthquake reportedly was felt as far north as Salt Lake City. (Ref. 38, 52.)

1902. Dec. 5. Pine Valley, Washington County, Utah. Many aftershocks occurred at Pine Valley, keeping chimneys in "disrepair." Students were dismissed from school because of the shocks. (Ref. 52, 298.)

1909. Oct. 6 (Oct. 5). Hansel Valley, Utah. The earthquake was felt by people on a northbound train approaching Logan in Cache County. Waves reportedly rolled over a bathhouse pier at Saltair, a few kilometers west of Salt Lake City. Windows were cracked at Salt Lake City. Felt from Lehi, Utah (about $25 \mathrm{~km}$ northwest of Provo), north to Malad 
City, Idaho, a distance of about $200 \mathrm{~km}$. West of Logan, in the Garland-Tremonton area, 30 to 60 earthquakes were reported to December 1909. A few were strong enough to throw down chimneys. (Ref. $38,52,298$.)

1910. Jan. 10. Richfield, Sevier County, Utah. A local earthquake on the Tushar fault broke windowpanes at Richfield. School was dismissed at nearby Elsinore, where six severe shocks were observed. (Ref. 38, 52, 298.)

1910. Jan. 12 (Jan. 11). Elsinore, Sevier County, Utah. Located on the Tushar fault, this local earthquake shook down chimneys and destroyed merchandise on Elsinore store shelves. (Ref. 52, 298.)

1910. May 22. Salt Lake City, Utah. Many chimneys were demolished and several buildings were damaged in Salt Lake City, but well-constructed buildings were damaged little. Slight damage was reported at Bingham and Garfield. This local shock centered on the Wasatch fault. (Ref. 52, 298.)

1914. May 13. Ogden, Weber County, Utah. Some chimneys toppled, walls cracked, and plateglass windows broke at Ogden. Dishes were broken at Salt Lake City. (Ref. 38, 52, 298.)

1915. July 15. Provo, Utah County, Utah. An earthquake that was centered on the Wasatch fault cracked a few ceilings at Provo and shook dishes from shelves. (Ref. 52, 272.)

1921. Sept. 29. Elsinore, Sevier County, Utah. Elsinore is a small town in Sevier Valley, 240 $\mathrm{km}$ south of Salt Lake City. After about 2 weeks of light foreshocks at Elsinore, three damaging local earthquakes occurred: two on Sept. 29 (local time) and one on Sept. 30. The first strong earthquake threw down scores of chimneys, or broke them at roof level, and fractured and displaced walls. A new two-story brick schoolhouse was damaged when a fire wall fell and several pilasters on the front of the building were dislodged. Gables of houses were "thrown out." The front of one store building was cracked severely (it collapsed in the strong shock on Oct. 1.). Considerable damage also occurred at the nearby towns of Joseph, Monroe, and Richfield. The shocks continued from Sept. 12 to Dec. 20, 1921. (Ref. 38, 52, 298, 311.)

1921. Sept. 30 (Sept. 29). Elsinore, Sevier County, Utah. The second damaging earthquake of this series of shocks toppled many chimneys and increased the damage caused by the previous earthquake (see paragraph above). At Monroe, about 8 $\mathrm{km}$ south of Elsinore, this earthquake was more destructive than the first shock on Sept. 29 at 1412 UTC. Felt slightly at Joseph and Richfield. (Ref. 38, 298,311 .)
1921. Oct. 1. Elsinore, Sevier County, Utah. The third destructive shock of this series of earthquakes at Elsinore downed chimneys that remained upright and caused additional structural damage. Heavy damage was sustained by buildings made of stone or "sun-dried brick" [adobe(?)]. Ten houses were wrecked and made uninhabitable. The store building that was cracked severely in the first shock on Sept. 29 collapsed during this earthquake. Large rockfalls occurred on both sides of Sevier Valley. Warm springs were discolored for hours. Additional damage was reported at Monroe, but the shock was only lightly felt at Joseph and Richfield. (Ref. 38, 52, 298, 311.)

1933. Jan. 20. Parowan, Iron County, Utali. A local earthquake on the Hurricane fault cracked plaster at Parowan and caused slight damage to brick, masonry, and concrete. Plaster also was cracked a few kilometers north, at Minersville. (Ref. 6, 52, 298.)

1934. Mar. 12, 1505 UTC. Near Kosmo, Box Elder County, Utah. This earthquake occurred in Hansel Valley, a sparsely populated area. Two people were killed. Property damage was limited mainly to downed chimneys and cracked walls in poorly constructed brick buildings. Chimneys were downed in several towns in the County, including Hooper, Kelton, Kosmo, Locomotive Springs, Monument, and Snowville. Large rockslides occurred at Aragonite, Lakeside, Monument Rock, and Snowville. An outstanding feature of this earthquake was the large quantity of water emitted from the craterlets and fissures that developed in the area.

Most of the ground cracks that formed in the epicentral area occurred in poorly consolidated rocks on the salt flats. These cracks were traced for a distance of more than $8 \mathrm{~km}$. Four distinct fractures, about $1 \mathrm{~km}$ apart, developed across a road about 5 $\mathrm{km}$ north of Kosmo. The vertical displacement along the fractures ranged from about 7.5 to $25.5 \mathrm{~cm}$. Precise leveling surveys later revealed that areas of land sank as much as $39 \mathrm{~cm}$. Horizontal displacement was not observed.

Many springs formed in the epicentral area. Most of them developed along well-defined fractures in the salt flats where water flowed along the fissure. Notable exceptions, however, were the springs that formed in Monument Rock where water flowed at individual centers and formed mud cones. In places, large holes developed around the springs by the caving of the soft material through which the water flowed. Two such holes on the salt flats northeast of Monument Rock ranged in diameter from 2.5 to 3 $\mathrm{m}$, and one was $11 \mathrm{~m}$ deep. Also felt in Idaho, Montana, Nevada, and Wyoming (see fig. 58). (Ref. $7,52,258,533$.) 


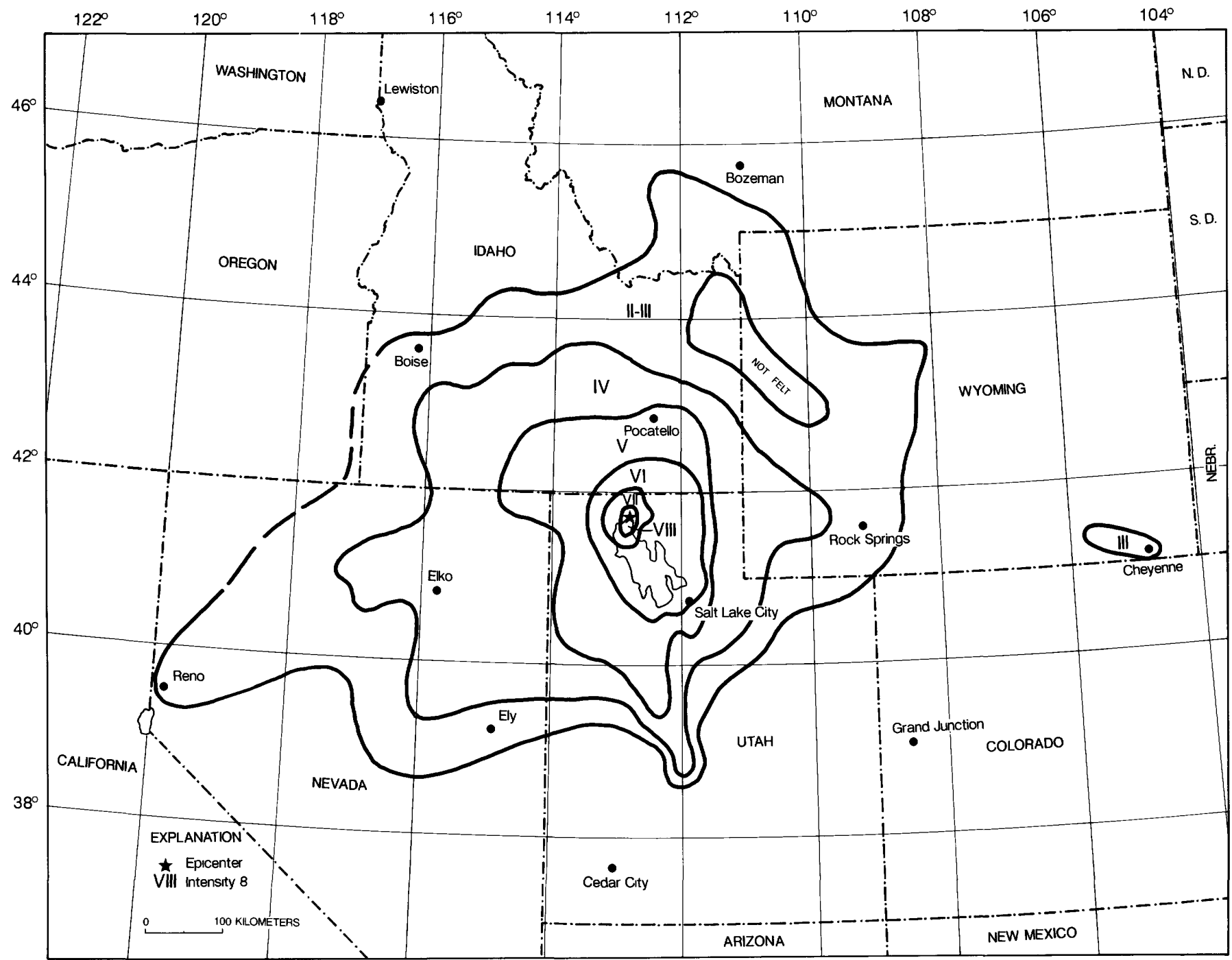

FiguRE 58.-Isoseismal map for the Kosmo (Hansel Valley), Utah, earthquake of March 12, 1934. Isoseismals are based on intensity estimates from data listed in references 7 and 259 of table 1.

1934. Mar. 12, 1820 UTC. Near Kosmo, Box Elder County, Utah. See description in the paragraph above for the effects of this earthquake because damage for the two shocks could not be separated. This shock was reported to be slightly less severe than the first, but it was felt over a similar area of Idaho, Nevada, Utah, and Wyoming. (Ref. 258, 298.)

1934. Mar. 15, 1201 UTC. Near Kosmo, Box Elder County, Utah. This earthquake damaged chimneys and overturned small objects in Locomotive Springs. It also was felt in Idaho. (Ref. 259, 298, 315.)

1934. May 6. Near Kosmo, Box Elder County, Utah. This strong earthquake apparently is an aftershock of the Mar. 12 event. A few windows were broken at Salt Lake City, and plaster was cracked at Preston, Idaho. (Ref. 52, 258, 259.)

1942. Aug. 30. Cedar City, Iron County, Utah. A local earthquake on the Hurricane fault shook bricks from a chimney in the northeast part of Cedar City. It knocked plaster from the walls of one house and cracked plaster at another. (Ref. 15, 259,298 .)

1942. Sept. 26. Cedar City, Iron County, Utah. A local earthquake on the Hurricane fault caused minor damage at Cedar City, including cracked walls, cracked plaster, and one broken plateglass window. (Ref. 15, 298.)

1943. Feb. 22. Near Salt Lake City, Utah. This earthquake, believed to be centered on the Wasatch fault, shook plaster from the wall and ceiling at the 
Bingham High School and knocked down a chimney at Camp Kearns Air Force Training Center. The shock also cracked chimneys and broke windows and dishes at Magna and cracked plaster and windows at Salt Lake City. (Ref. 16, 259, 265.)

1945. Nov. 18 (Nov. 17). South-central Utah. Slight damage occurred in Sevier County, at Glenwood and nearby Richfield. Chimneys and plaster were cracked and many residents were frightened. This local shock occurred on the Sevier fault. (Ref. 18, 298.)

1949. Mar. 7 (Mar. 6). Salt Lake City, Utah. A sharp local earthquake broke a pipeline, cracked walls in some houses, and broke windows at Salt Lake City. This shock, located on the Wasatch fault, also displaced furniture and tumbled dishes from shelves. (Ref. 22, 259, 298.)

1958. Feb. 13. North-central Utah. Plaster fell from ceilings and walls cracked in several buildings in Utah County, at Provo. Plaster also fell from a wall in the student service center at Brigham Young University. Felt mainly in the region east of Utah Lake. (Ref. 31, 259.)

1959. Feb. 27. Panguitch, western Garfield County, Utah. A minor earthquake knocked plaster from ceilings, cracked walls, and broke dishes and windows at Panguitch. Felt in several towns in southwest Utah. (Ref. 32, 259.)

1961. Apr. 16 (Apr. 15). Near Ephraim, Sanpete County, Utah. This earthquake caused minor damage at Ephraim and nearby towns to the north. Two chimneys fell, plaster was cracked, and bottles were knocked from shelves at Ephraim; chimneys were cracked at Chester; and plaster was cracked at Mount Pleasant and Spring City. (Ref. 34, 234.)

1962. Aug. 30. Cache Valley, Utah. The earthquake severely damaged many old, unreinforced brick buildings and other old structures on the east side of Cache Valley, Utah (from Logan to Lewiston). In Richmond ( $24 \mathrm{~km}$ north of Logan), where the most severe property damage occurred, 75 percent of the older brick chimneys collapsed and tombstones were overturned. One large church was damaged beyond repair. The walls of many houses were damaged badly, and building officials declared several houses were unsafe for occupancy. Heavy damage also occurred at Franklin, Lewiston, Logan, and Preston. Total property damage was estimated at $\$ 1$ million.

Mudslides were noted west of Lewiston, along the Bear River, and at Cornish. At Fairview, Idaho, two large areas of land broke loose and slid down a hill. Felt in parts of Colorado, Idaho, Nevada, Utah, and Wyoming (see fig. 59). (Ref. 35, 298, 556.)
1962. Sept. 5. Near Salt Lake City, Utah. Property damage occurred mainly in the area from Provo to Salt Lake City. It consisted of damaged chimneys, broken windows, minor cracks in walls and plaster, and loss from fallen stock in stores. In Salt Lake City, an outside wall of an older house fell through the ceiling; sections of interior walls and ceiling plaster fell at two other houses. Some large buildings sustained cracks in interior walls. Most of the damage to schools was superficial, consisting mainly of plaster cracks and loosened acoustical tile. Other towns reporting slight damage from this earthquake were Draper, Lark, Magna, Morgan, and Provo. (Ref. 35, 298.)

1963. July 7. Central Utah. A minor earthquake, felt over a small area of Juab County, caused slight damage at Levan and Nephi. Plaster fell and chimneys were cracked at Levan; plaster, walls, and chimneys were cracked at Nephi. (Ref. 36, 298.)

1967. Oct. 4. Central Utah. This earthquake caused minor damage in the Marysvale-Koosharem area. At Marysvale, in northern Piute County, brick and stone houses sustained many cracks in ceilings and walls; a cupboard in one house pulled away from the wall. Well water was muddied about $1.6 \mathrm{~km}$ north of Marysvale. Chimneys partly collapsed at Joseph, $20 \mathrm{~km}$ north of Marysvale, and root cellars caved in at Circleville, $30 \mathrm{~km}$ south of Marysvale. Rockslides were reported at Junction City, in the canyon near Joseph, and $8 \mathrm{~km}$ south of Sevier. Also felt in northern Arizona. Magnitude $5.1 \mathrm{~m}_{\mathrm{b}} \mathrm{NUT}, 4.7 \mathrm{M}_{\mathrm{S}}$ NUT. (Ref. 40, 263, 298.)

1972. Jan. 3. Elsinore, Sevier County, Utah. An earthquake toppled chimneys and cracked walls at Elsinore and damaged the contents of houses considerably. Plaster was cracked at Central and Sevier. (Ref. 45, 72, 298.)

1972. Oct. 1. Midway, Wasatch County, Utah. This earthquake shook bricks from chimneys at Midway and cracked plaster at two schools. Slight damage (mainly cracks in plaster) also occurred at Salt Lake City, about $40 \mathrm{~km}$ northwest and, at Wallsburg about $15 \mathrm{~km}$ south. (Ref. 45, 72, 298.)

1977. Sept. 30. Northeast Utah. Damage was most severe at Mountain Home, east of Provo, in Duchesne County, where a septic system drain was broken and old mortar on a log house was cracked. There was an unconfirmed report of cracks in a stone fence and in interior plaster at Fruita and Grand Junction, Colo., more than $200 \mathrm{~km}$ southeast of the epicenter.

A few indications of minor earth movement were found in the epicentral area two days after the shock: one possible rockfall in Rock Creek Canyon and 


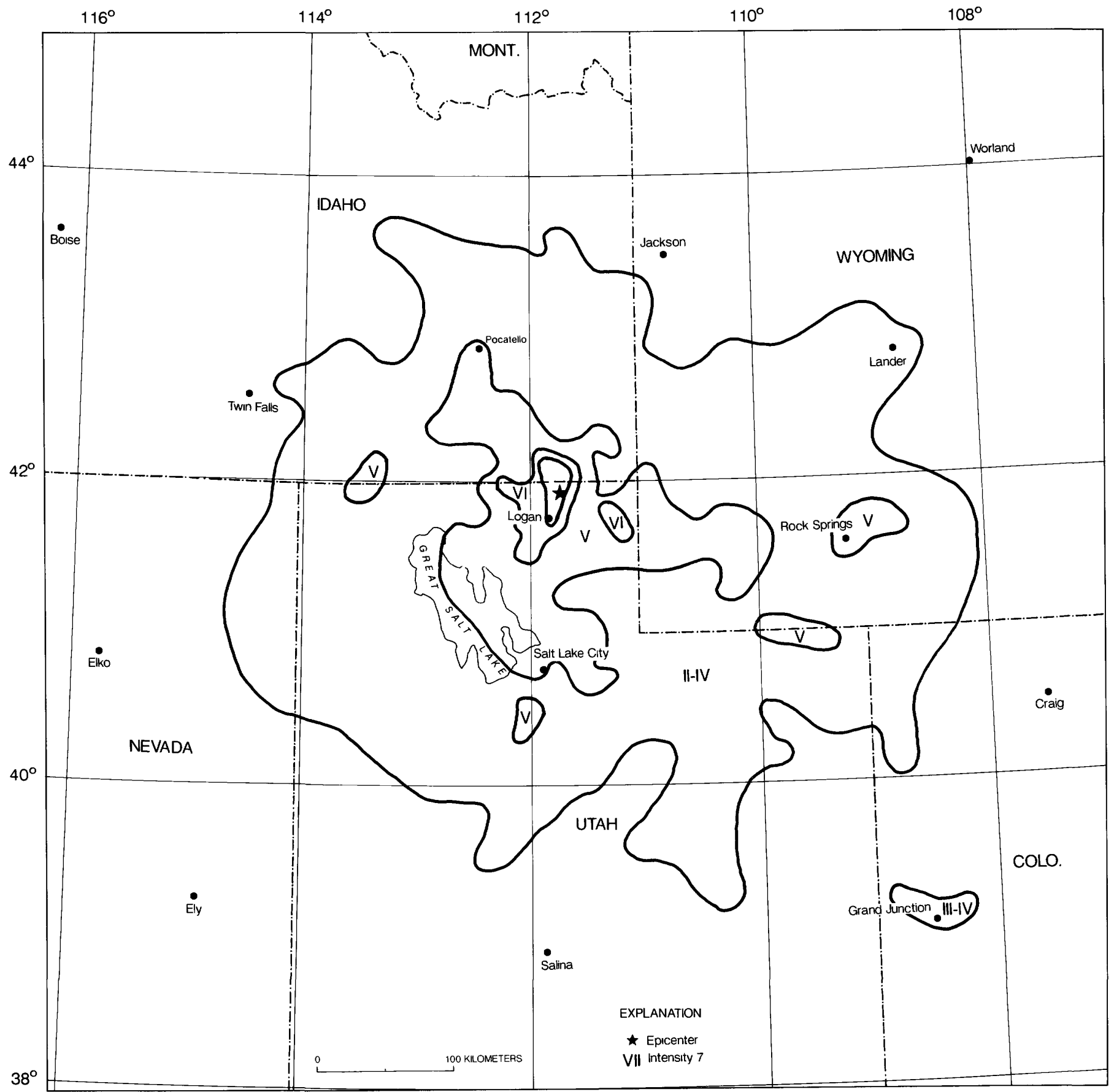

FIGURE 59.-Isoseismal map for the Cache Valley, Utah, earthquake of August 30, 1962. Isoseismals are based on intensity estimates from data listed in references 35 and 259 of table 1.

slumping of a rock slab in Farnsworth Canal, near Moon Lake. This earthquake occurred along a lineament that strikes $\mathrm{N}$. $70^{\circ} \mathrm{E}$. along the south flank of the Uinta Mountains and extends into the basin to the south. A lighter shock on Oct. 11 occurred along the same fault zone. Also felt in Idaho and Wyoming. Magnitude 5.1 $\mathrm{M}_{\mathrm{L}}$ GS. (Ref. 39,319 .)
1978. Mar. 9 (Mar. 8). Magna, Salt Lake County, Utah. This minor earthquake broke windows and cracked exterior walls, plaster, and drywall at Magna, west of Salt Lake City. (Ref. 240, 298.)

1981. Feb. 20. Orem, Utah County, Utah. A house foundation cracked at Orem, north of Provo, and hairline cracks formed in plaster and drywall. At 
Provo, about $100 \mathrm{~km}$ south of Salt Lake City, a hairline crack in a cinder-block wall was enlarged. (Ref. 325,341 .)

1982. May 24. Southwest Utah. This earthquake was observed mainly in Sevier Valley, between Aurora and Joseph. The heaviest damage occurred at Annabella, about $25 \mathrm{~km}$ south of Aurora, where bricks fell from chimneys, stone or brick fences fell or were cracked, exterior walls of houses were cracked, and a garage roof was shifted $2.5 \mathrm{~cm}$. Slight damage also occurred in the nearby towns of Elsinore, Glenwood, Koosharem, and Monroe. (Ref. 350.)

1983. Oct. 8. Northern Utah. At West Valley City (in the western suburbs of Salt Lake City), one chimney toppled, cracks formed in chimneys and plaster walls, and glassware was broken. Bricks fell from a chimney south of Salt Lake City, at Granger, and hairline cracks formed in walls of buildings at Layton and Sandy. (Ref. 360.)

1988. Aug. 14. Near Clawson, Emery County, Utah. This earthquake was felt over a wide area but caused only minor damage to property. Bricks fell from chimneys at Clawson, Elmo, Orangeville, and Sunnyside, and chimneys were cracked at Ferron and Wellington. Other reported damage included broken windows, and cracks in plaster, drywall, exterior brick walls, and foundations.

This earthquake caused hundreds of rockfalls within $40 \mathrm{~km}$ of its epicenter. Ground cracks due to liquefaction of saturated alluvium were reported 4 $\mathrm{km}$ from the epicenter, and similar ground cracks and a sand boil were observed in saturated alluvium $1.9 \mathrm{~km}$ from the epicenter. Flow of water into a spring in Tie Fork Canyon, $48 \mathrm{~km}$ from the epicenter, increased after the earthquake.

Two foreshocks occurred on Aug. 14 at 1858 and 1907 UTC, and the largest aftershock caused one rockfall on Aug. 18. More than 100 aftershocks were located through Sept. 30. Felt to Brigham City, 280 $\mathrm{km}$ to the northwest of the epicenter; Delta, $156 \mathrm{~km}$ to the west; Albuquerque, N. Mex., $567 \mathrm{~km}$ to the south; Bluff, $233 \mathrm{~km}$ to the southeast; and Golden, Colo., $475 \mathrm{~km}$ to the east. (Ref. $74,578,590$.)

1988. Nov. 19. Northeast of Logan, Cache County, Utah. Slight damage reported at Garden City included hairline cracks in plaster and cracks in a fireplace and in the foundation of a reinforced concrete building. Some windows also were broken in the area. An observer at Logan reported broken pipelines and "some structural damage." Felt over a small area of northern Utah and southeast Idaho. (Ref. 74, 578.)

1989. Jan. 30 (Jan. 29). Near Salina, Sevier County, Utah. Slight damage in the form of cracks in chimneys, walls, windows, and foundations occurred at Ferron (Emery County), Koosharem (Sevier County), and Wales (Sanpete County). In addition, underground pipes were broken at Salina, and large amounts of plaster fell from a ceiling at Wales. Felt in northern Arizona, western Colorado, eastern Utah, and southwest Wyoming. Several small aftershocks were reported. (Ref. 74, 579.) 
VERMONT

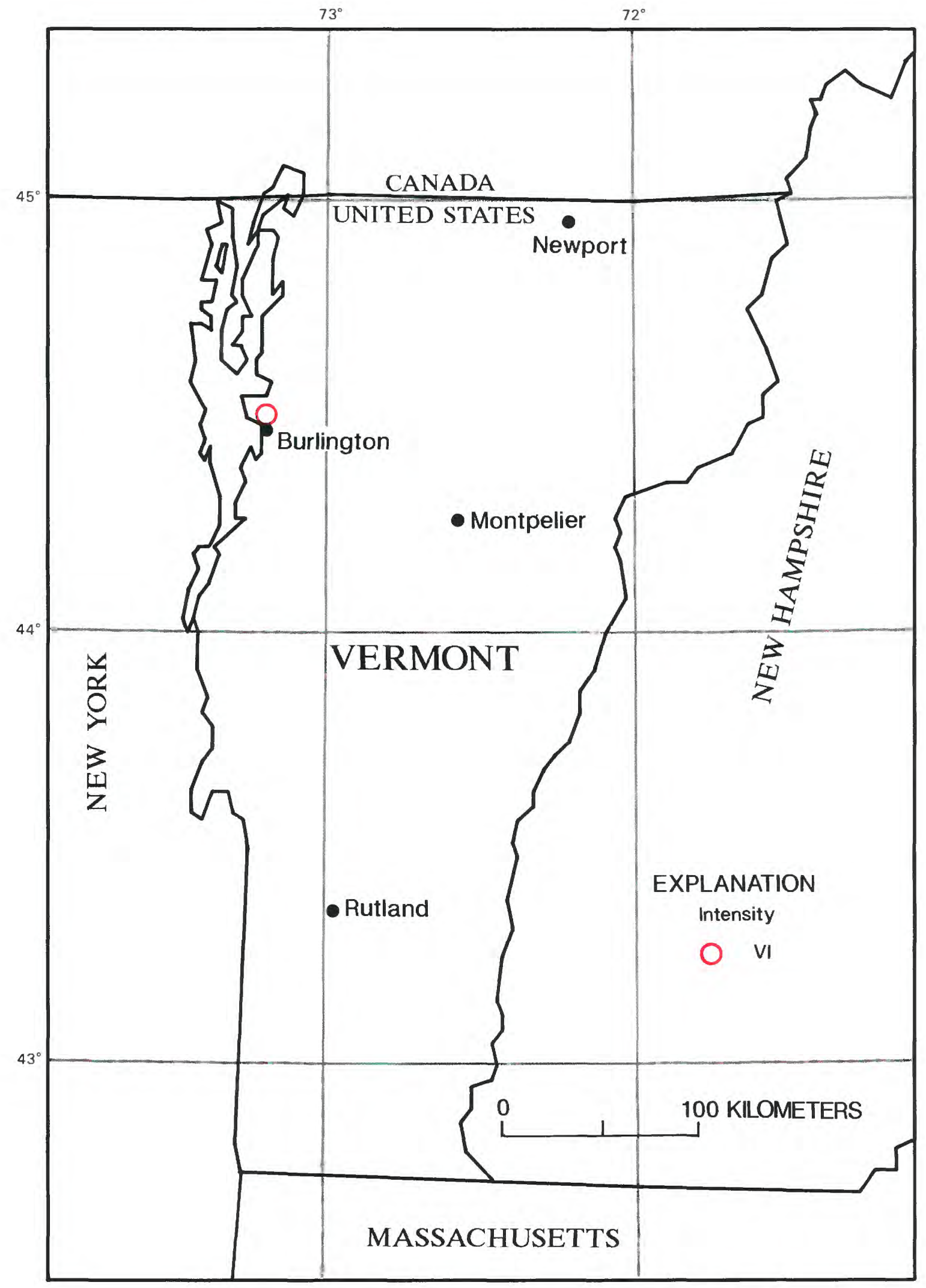

Damaging earthquake in Vermont, intensity $\geq$ VI. 
VERMONT

[See table 1 for hypocenter and intensity references and table 2 for definitions of magnitude source codes. @, felt area is less than $1,000 \mathrm{~km}^{2}$. Leader (-) indicates information is not available]

\begin{tabular}{|c|c|c|c|c|c|c|c|c|c|c|c|c|c|}
\hline \multirow{2}{*}{\multicolumn{2}{|c|}{ Date }} & \multirow{2}{*}{$\begin{array}{l}\text { Origin } \\
\text { time (UTC) }\end{array}$} & \multicolumn{4}{|c|}{ Hypocenter } & \multicolumn{4}{|c|}{ Magnitude } & \multicolumn{2}{|c|}{ Intensity } & \multirow{3}{*}{$\begin{array}{c}\text { Felt area } \\
\left(1,000 \mathrm{~km}^{2}\right)\end{array}$} \\
\hline & & & \multirow{2}{*}{$\begin{array}{l}\text { Latitude } \\
\left(^{\circ}\right)\end{array}$} & \multirow{2}{*}{$\begin{array}{c}\text { Longitude } \\
\left.\text { ( }{ }^{\circ}\right)\end{array}$} & \multirow{2}{*}{$\begin{array}{l}\text { Depth } \\
(\mathrm{km})\end{array}$} & \multirow[t]{2}{*}{ Ref } & \multicolumn{2}{|c|}{ USGS } & \multirow{2}{*}{ Other } & \multirow{2}{*}{$\begin{array}{c}\text { Moment } \\
\text { M }\end{array}$} & \multirow{2}{*}{ MM } & \multirow[t]{2}{*}{ Ref } & \\
\hline$\overline{Y_{r}}$ & Mo Da & $\mathrm{h} \mathrm{m} \mathrm{s}$ & & & & & $m_{b}$ & $\mathbf{M}_{\mathbf{s}}$ & & & & & \\
\hline 1952 & 0130 & 0400 & $44.5 \mathrm{~N}$ & $73.2 \mathrm{~W}$ & - & 77 & - & - & - & - & VI & 25 & @ \\
\hline
\end{tabular}

[Reference (Ref.) numbers given in parentheses at the end of each description refer to sources of data in table 1.1

1952. Jan. 30 (Jan. 29). Burlington, Chitlong and $3.5 \mathrm{~m}$ apart were observed in the north end of Burlington. Minor damage resulting from this local shock included cracks in pavetenden County, Vt. Ground cracks about $3 \mathrm{~km}$ ment, basement walls, and a gas main. (Ref. $25,38,77$. 


\section{VIRGINIA}

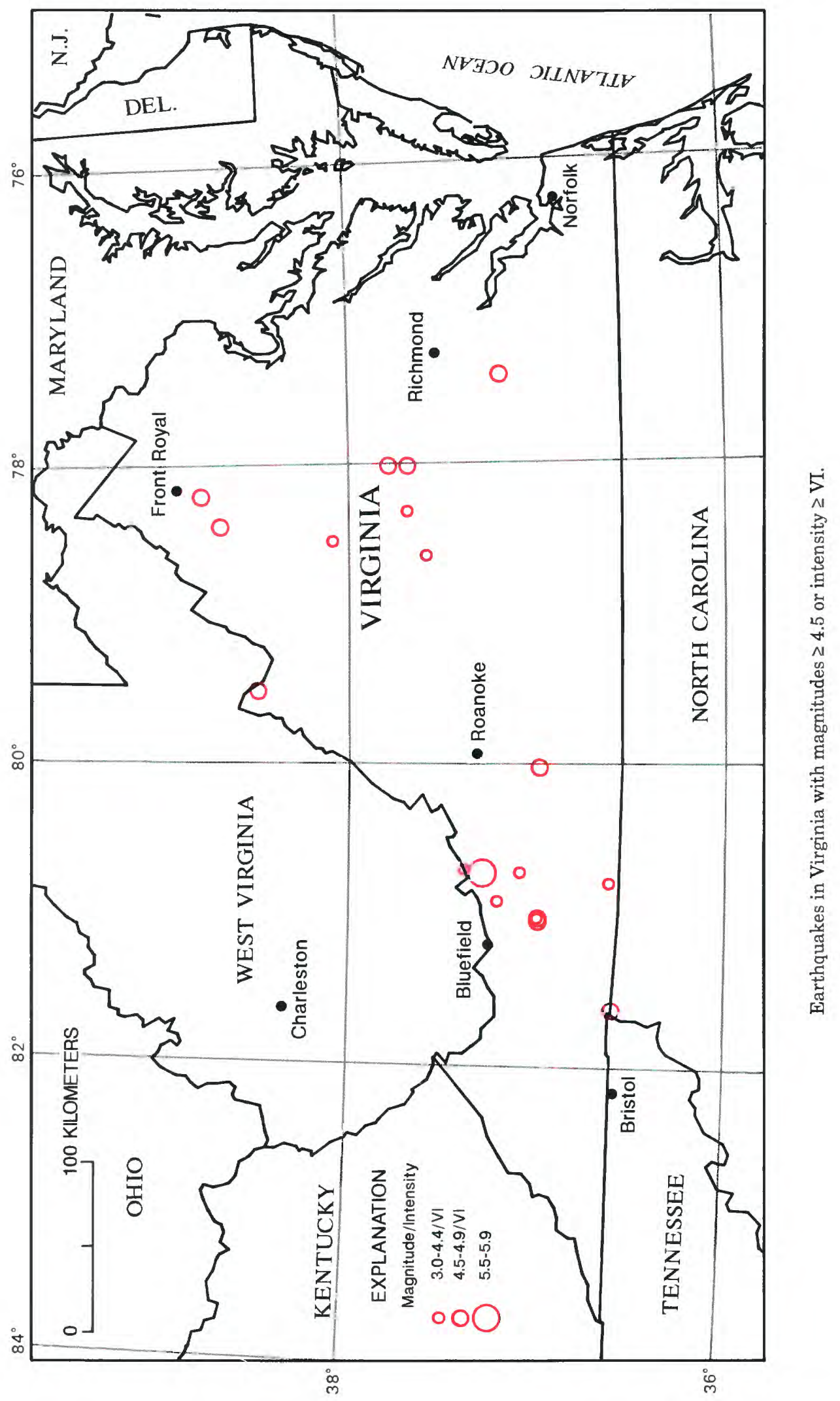


VIRGINIA

[See table 1 for hypocenter and intensity references and table 2 for definitions of magnitude source codes. Leader (-) indicates information is not available]

\begin{tabular}{|c|c|c|c|c|c|c|c|c|c|c|c|c|c|c|c|c|}
\hline \multirow{3}{*}{\multicolumn{4}{|c|}{$\begin{array}{cc} & \text { Origin } \\
\text { Date } & \text { time (UTC) } \\
Y_{r} \text { Mo Da h } \mathrm{m} \mathrm{s}\end{array}$}} & \multicolumn{6}{|c|}{ Hypocenter } & \multicolumn{4}{|c|}{ Magnitude } & \multicolumn{2}{|c|}{ Intensity } & \multirow{3}{*}{$\begin{array}{c}\text { Felt area } \\
\left(1,000 \mathrm{~km}^{2}\right)\end{array}$} \\
\hline & & & & \multirow{2}{*}{\multicolumn{2}{|c|}{$\begin{array}{l}\text { Latitude } \\
\left({ }^{\circ}\right)\end{array}$}} & \multirow{2}{*}{\multicolumn{2}{|c|}{$\begin{array}{c}\text { Longikude } \\
\left({ }^{\circ}\right)\end{array}$}} & \multirow{2}{*}{$\begin{array}{l}\text { Depth } \\
(\mathrm{km})\end{array}$} & \multirow[t]{2}{*}{ Ref } & \multicolumn{2}{|c|}{ USGS } & \multirow[t]{2}{*}{ Other } & \multirow{2}{*}{$\begin{array}{c}\text { Moment } \\
\text { M }\end{array}$} & \multirow[t]{2}{*}{ MM } & \multirow[t]{2}{*}{ Ref } & \\
\hline & & & & & & & & & & $m_{b}$ & $\mathrm{M}_{\mathbf{s}}$ & & & & & \\
\hline 1774 & 02 & 21 & 1911 & 37.2 & $\mathbf{N}$ & 77.4 & w & - & 314 & - & - & $4.50 \mathrm{M}_{\mathrm{fa}} \mathrm{SC}$ & - & VI & 55 & 127 \\
\hline 1828 & 03 & 10 & 03 & 37.0 & $\mathbf{N}$ & 80.0 & W & - & 55 & - & - & $4.60 \mathrm{M}_{\mathrm{fa}} \mathrm{SC}$ & - & V & 55 & 490 \\
\hline 1833 & 08 & 27 & 11 & 37.7 & $\mathbf{N}$ & 78.0 & W & - & 179 & - & - & $4.50 \mathrm{M}_{\mathrm{fa}} \mathrm{NUT}$ & - & V & 167 & 135 \\
\hline 1852 & 04 & 29 & 18 & 36.6 & $\mathbf{N}$ & 81.6 & W & 一 & 86 & - & - & $4.80 \mathrm{M}_{\mathrm{fa}} \mathrm{SC}$ & - & VI & 55 & 359 \\
\hline 1852 & 11 & 02 & 2335 & 37.6 & $\mathbf{N}$ & 78.6 & W & - & 55 & - & - & $4.30 \mathrm{M}_{\mathrm{fa}} \mathrm{SC}$ & - & VI & 167 & 66 \\
\hline 1853 & 05 & 02 & 1420 & 38.5 & $\mathbf{N}$ & 79.5 & W & - & 167 & - & - & $4.60 \mathrm{M}_{\mathrm{fa}} \mathrm{NUT}$ & - & V & 38 & 159 \\
\hline 1875 & 12 & 23 & 0445 & 37.8 & $\mathbf{N}$ & 78.0 & W & - & 365 & - & - & $4.80 \mathrm{M}_{\mathrm{fa}} \mathrm{SC}$ & - & VII & 365 & 120 \\
\hline 1897 & 05 & 03 & 1718 & 37.1 & $\mathbf{N}$ & 80.7 & W & - & 55 & - & - & $4.30 \mathrm{M}_{\mathrm{fa}} \mathrm{SC}$ & - & VI & 167 & 64 \\
\hline 1897 & 05 & 31 & 1858 & 37.3 & $\mathbf{N}$ & 80.7 & W & - & 55 & - & - & $5.60 \mathrm{M}_{\mathrm{fa}} \mathrm{SC}$ & - & VIII & 190 & 690 \\
\hline 1898 & 02 & 05 & 20 & 37.0 & $\mathbf{N}$ & 81.0 & w & - & 86 & - & - & $4.40 \mathrm{M}_{\mathrm{fa}} \mathrm{SC}$ & - & VI & 38 & 88 \\
\hline 1898 & 112 & 25 & 20 & 37.0 & $\mathbf{N}$ & 81.0 & W & - & 86 & - & - & $4.50 \mathrm{M}_{\mathrm{fa}} \mathrm{NUT}$ & - & V & 38 & 132 \\
\hline 1899 & 02 & 13 & 0930 & 37.0 & $\mathbf{N}$ & 81.0 & W & - & 55 & - & - & $4.50 \mathrm{M}_{\mathrm{fa}} \mathrm{SC}$ & - & V & 55 & 265 \\
\hline 1907 & 02 & 11 & 1322 & 37.7 & $\mathbf{N}$ & 78.3 & W & - & 55 & - & - & $4.00 \mathrm{M}_{\mathrm{fa}} \mathrm{SC}$ & - & VI & 55 & 14 \\
\hline 1918 & 04 & 10 & 010848 & 38.7 & $\mathbf{N}$ & 78.4 & W & - & 55 & - & - & $4.60 \mathrm{M}_{\mathrm{fa}} \mathrm{SC}$ & - & VI & 55 & 184 \\
\hline 1919 & 09 & 06 & 014545 & 38.8 & $\mathbf{N}$ & 78.2 & W & - & 55 & - & - & - & - & VI & 55 & - \\
\hline 1929 & 12 & 26 & 0256 & 38.1 & $\mathbf{N}$ & 78.5 & W & - & 189 & - & - & $3.70 \mathrm{M}_{\mathrm{fa}} \mathrm{SC}$ & - & VI & 38 & 3 \\
\hline 1959 & 04 & 23 & 205839.5 & 37.395 & & 80.682 & $2 W$ & 001 & 349 & - & - & $3.90 \mathrm{M}_{\mathrm{fa}} \mathrm{SC}$ & 一 & VI & 105 & 8 \\
\hline 1975 & 11 & 11 & 081037.6 & 37.217 & & 80.892 & $2 W$ & 001 & 349 & - & - & $3.20 \mathrm{M}_{\mathrm{n}} \mathrm{SLM}$ & - & VI & 48 & 1 \\
\hline 1976 & 09 & 13 & 185438.0 & 36.624 & & 80.768 & $8 W$ & 009 & 349 & - & - & $3.30 \mathrm{M}_{\mathrm{n}}$ BLA & - & VI & 49 & 17 \\
\hline
\end{tabular}

[Reference (Ref.) numbers given in parentheses at the end of each description refer to sources of data in table 1. Magnitude values are described in the Introduction, and codes are defined in table 2.]

1774. Feb. 21. Near Petersburg, Prince George County, Va. A sharp earthquake that was felt over much of Virginia displaced houses "considerably off their foundations" at Blandford and Petersburg. Although the shock was severe at Richmond and terrified residents about $80 \mathrm{~km}$ north of Richmond at Fredericksburg, it caused no damage at those towns. Several "smart shocks" were reported in parts of Virginia from Feb. 20th to the 22nd. The main tremor rang bells at Salem (now WinstonSalem), N.C. Magnitude $4.5 \mathrm{M}_{\mathrm{fa}}$ NUT. (Ref. 55, 167, 314.)

1833. Aug. 27. Central Virginia. A rather strong shock agitated walls of buildings at Lynchburg (west of Richmond, in southern Amherst County) and rattled windows violently. Fences along the road were shaken near the Louisa County Courthouse, northwest of Richmond. It was described as "severe" at Charlottesville, about $85 \mathrm{~km}$ northeast of Lynchburg. Two miners were killed in a panic caused by the tremor at a mine near Richmond. Ref. 179 suggests a MM intensity VI at the epicenter, although no damage was documented. Also felt in the District of Columbia, Maryland, and North Carolina. Magnitude $4.5 \mathrm{M}_{\mathrm{fa}}$ NUT. (Ref. 38, 167, 179.)

1852. Apr. 29. Near Wytheville, Wythe County, Va. A severe earthquake that was observed over a large area threw down a chimney near Wytheville, in southwest Virginia, and shook down tops of chimneys at Buckingham Courthouse, about $55 \mathrm{~km}$ south of Charlottesville. Houses were shaken violently at Staunton, about $65 \mathrm{~km}$ west of Charlottesville. A brick was shaken from a chimney as far south as Davie County, N.C. Also felt in the District of Columbia, Maryland, New York, Ohio, and Pennsylvania. Magnitude $4.9 \mathrm{M}_{\mathrm{fa}}$ NUT. (Ref. $38,55,86$, 167, 508.)

1852. Nov. 2. Central Virginia. Chimney damage occurred at Buckingham, about $55 \mathrm{~km}$ south of Charlottesville. This earthquake was reported to be "quite strong" at Fredericksburg, Richmond, and Scottsville. At Scottsville, where every house in the village was shaken, water in the canal was "troubled," and boats were tossed to and fro. Magnitude $4.3 \mathrm{M}_{\mathrm{fa}}$ NUT. (Ref. 55, 167.) 
1875. Dec. 23 (Dec. 22). Central Virginia. The highest intensities from this earthquake occurred mainly at towns near the James River waterfront in Goochland and Powhatan Counties, and in Louisa County. In Richmond (Henrico County), the most severe damage was sustained in the downtown business and residential areas adjacent to the James River or on islands in the river. Damage included bricks knocked from chimneys, fallen plaster, an overturned stove, and several broken windows. Waves "suddenly rose several feet" at the James River dock at Richmond, causing boats to "part their cables" and drift below the wharf. At Manakin, about $20 \mathrm{~km}$ west of Richmond, shingles were shaken from a roof and many lamps and chimneys were broken. Several small aftershocks were reported through Jan. 2, 1876. Felt from Baltimore, Md., to Greensboro, N.C., and from the Atlantic Coast westward to Greenbrier and White Sulphur Springs, W.Va. Magnitude $4.5 \mathrm{M}_{\mathrm{fa}}$ NUT. (Ref. 55, 167, 365.)

1897. May 3. Southwest Virginia. This earthquake was most severe at Radford (about $65 \mathrm{~km}$ west of Roanoke), where a few chimneys were wrecked and plaster fell from walls. Chimneys were damaged at nearby Pulaski and at Roanoke. Felt in most of southwest Virginia and as far south as WinstonSalem, N.C. Magnitude $4.3 \mathrm{M}_{\mathrm{fa}}$ NUT. (Ref. 38, 55, 167, 272, 508.)

1897. May 31. Giles County, Va. This earthquake was the largest in intensity and areal extent in Virginia in historical times. MM intensity VII to VIII extended over an elliptical area-from near Lynchburg, Va., west to Bluefield, W.Va., and from Giles County south to Bristol, Tenn. (see fig. 60). The MM intensity VIII assigned to this earthquake is based on "many downed chimneys" and "changes in the flow of springs."

The shock was felt severely at Narrows, about 3 $\mathrm{km}$ west of Pearisburg. Here, the surface rolled in an undulating motion, water in springs became muddy, and water in some springs ceased to flow. The flow of water in springs also was disturbed in the area of Pearisburg, about $70 \mathrm{~km}$ west of Roanoke, and Sugar Run.

The shock was strong at Pearisburg, where walls of old brick houses were cracked and many chimneys were thrown down or badly damaged. Many chimneys also were shaken down at Bedford, Pulaski, Radford, and Roanoke, Va., and Bristol, Tenn.; many chimneys were damaged at Christiansburg, Dublin, Floyd, Houston, Lexington, Lynchburg, Rocky Mount, Salem, Tazewell, and Wytheville, Va.; Charlotte, Oxford, Raleigh, and Winston, N.C.; Knoxville, Tenn.; and Bluefield, W.Va. Felt from Georgia to
Pennsylvania and from the Atlantic Coast westward to Indiana and Kentucky. Aftershocks continued through June 6, 1897. Magnitude $5.8 \mathrm{M}_{\mathrm{fa}}$ NUT. (Ref. 55, 167, 190, 525.)

1898. Feb. 5. Pulaski, Va. Bricks were thrown from chimneys, furniture was shifted in a few houses, and residents rushed into the streets at Pulaski, about $70 \mathrm{~km}$ southwest of Roanoke. Felt throughout southwest Virginia and south to Raleigh, N.C. (Ref. 38, 86, 167, 508.)

1907. Feb. 11. Near Arvonia, Buckingham County, Va. Chimneys were cracked at Ashby, about $20 \mathrm{~km}$ southeast of Arvonia, and a window was broken at a store at Buckingham, $25 \mathrm{~km}$ southwest of Arvonia. A "terrific" shock sent people rushing outdoors at Arvonia and displaced furniture. Felt strongly from Powhatan to Albemarle County. (Ref. $55,167,189$.

1918. Apr. 10 (Apr. 9). Luray, Page County, Va. In the Shenandoah Valley, at Luray, windows were broken and plaster was cracked severely. Ceilings of houses were cracked badly a few kilometers north of Luray, at Edinburg; windows were broken at Harrisonburg and Staunton, Va., and Washington, D.C. (at Georgetown University). In addition, a new spring formed in Page County, near Hamburg, almost in the middle of a road. A minor aftershock was reported in the area about 5 hours later. Also felt in Maryland, Pennsylvania, and West Virginia. (Ref. 38, $55,186,189,272$.)

1919. Sept. 6 (Sept. 5). Near Front Royal, Warren County, Va. This earthquake affected towns mainly in Warren and Rappahannock Counties. At Arco, in the Blue Ridge Mountains south of Front Royal, chimneys were damaged, plaster fell from walls, and springs and streams were muddied. Reports from the adjacent northern part of Rappahannock County state that similar shocks were felt and that streams were "rendered turbid." Also felt in parts of Maryland and West Virginia. Several aftershocks occurred. (Ref. 55, 187, 272.)

1929. Dec. 26 (Dec. 25). Charlottesville, Albemarle County, Va. A moderate tremor at Charlottesville shook bricks from chimneys in some places. Also felt in other parts of Albemarle County. (Ref. 38, 189.)

1959. Apr. 23. Giles County, Va. The earthquake was strongest in Giles County, at Eggleston and Pembroke. Residents there reported several damaged chimneys and articles shaken from shelves and walls. One chimney toppled at the Norfolk and Western Station in Eggleston. Also felt in West Virginia. Magnitude 3.8 $\mathrm{M}_{\mathrm{fa}}$ DG. (Ref. 105, 349, 508.)

1975. Nov. 11. Southwest Virginia. Windows were broken in the Blacksburg area of Montgomery 


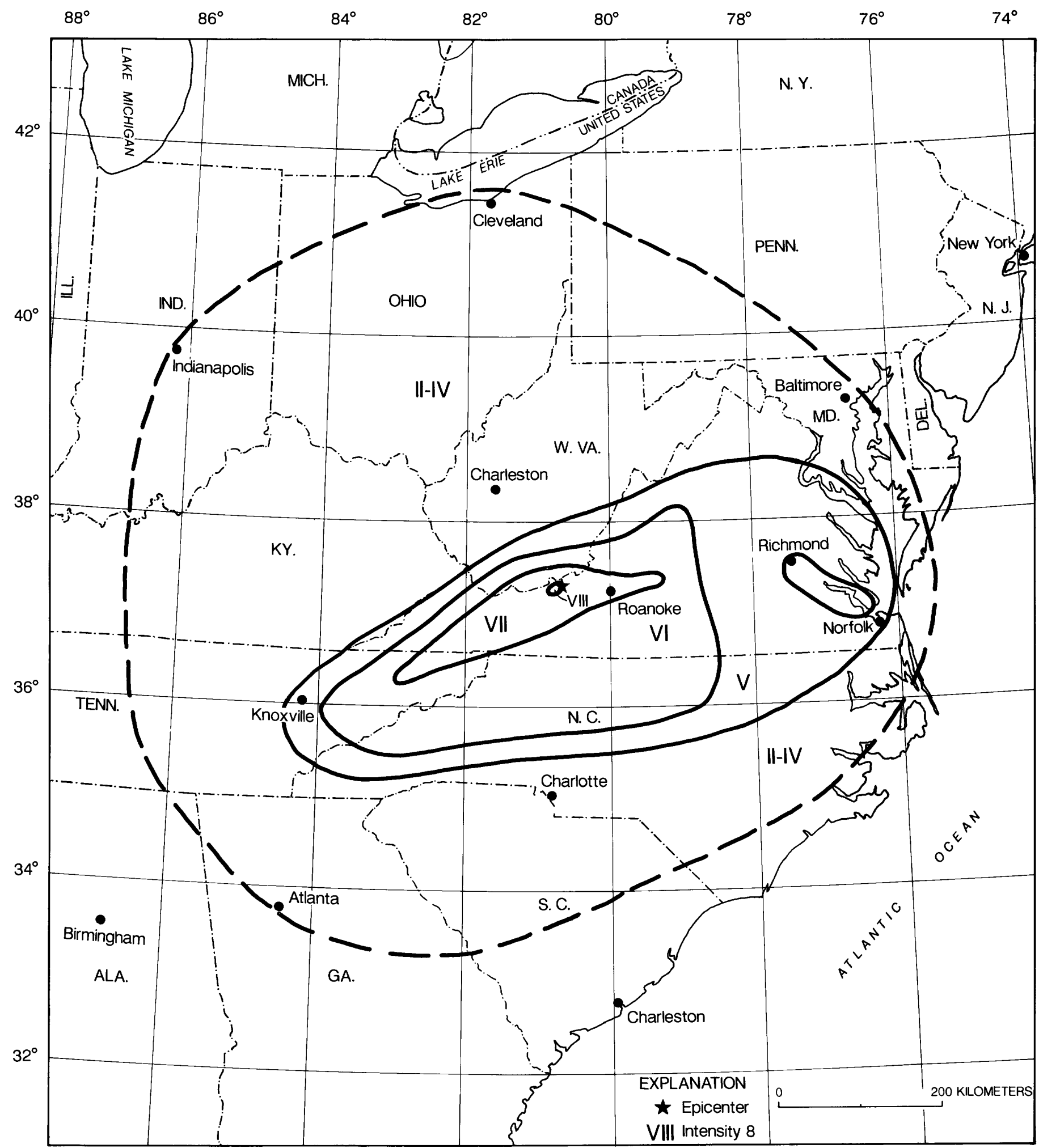

FIGURE 60.-Isoseismal map for the Giles County, Virginia, earthquake of May 31, 1897. This map is a simplified version of figure 15 in reference 525 of table 1 .

County, and plaster was cracked at Poplar Hill (south of Pearisburg, in Giles County). Also felt in Pulaski County. (Ref. 48, 349.)

1976. Sept. 13. Southwest Virginia. Bricks fell from chimneys and pictures fell from walls in Surry
County at Mount Airy, N.C. At the nearby town of Toast, N.C., cracks formed in masonry and plaster. The earthquake was observed in many towns in North Carolina and Virginia and in a few towns in South Carolina and West Virginia. (Ref. 49, 349.) 


\section{WASHINGTON}

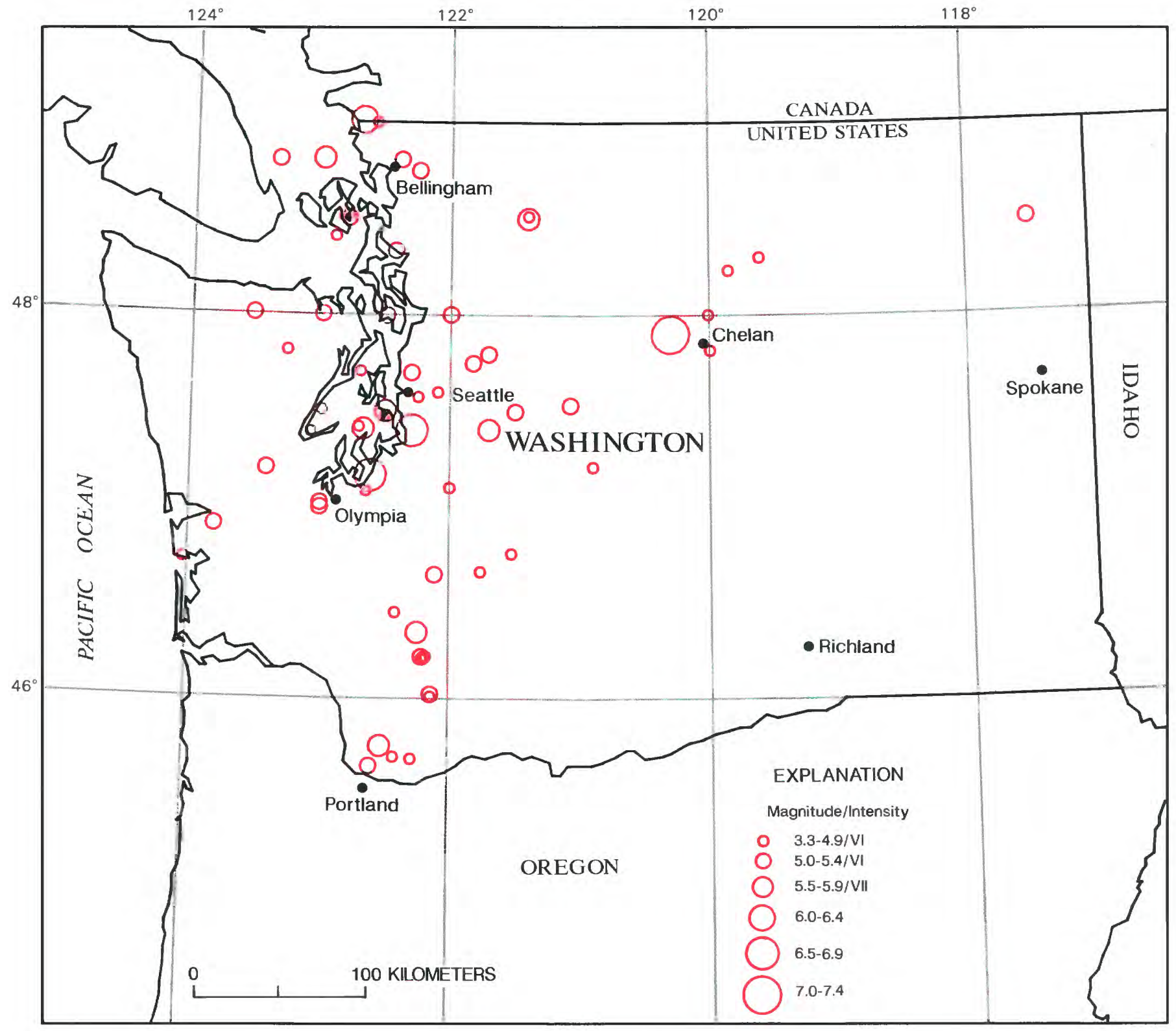

Earthquakes in Washington with magnitudes $\geq 4.5$ or intensity $\geq$ VI. 
WASHINGTON

[See table 1 for hypocenter and intensity references and table 2 for definitions of magnitude source codes. \&, land area only; \#, land area only in the United States for an earthquake near a coastline; +, land area in the United States when the felt area did not extend to the coast; (i), felt area is less than $1,000 \mathrm{~km}^{2}$. Leader (-) indicates information is not available]

\begin{tabular}{|c|c|c|c|c|c|c|c|c|c|c|c|c|c|c|c|}
\hline & & & Origin & & Hypoce & ente & & & & & Magnitude & & Inte & nsity & \\
\hline & Date & & time (UTC) & Latitude & Longitude & & Depth & Ref & & & Other & Moment & MM & Ref & Felt area \\
\hline $\mathrm{Yr}$ & Mo L & Da & $\mathrm{h} m \mathrm{~s}$ & $\left({ }^{\circ}\right)$ & & & $(\mathrm{km})$ & & $m_{b}$ & $\mathrm{M}_{\mathrm{s}}$ & & M & & & $\left.1,000 \mathrm{~km}^{2}\right)$ \\
\hline 1872 & 12 & 15 & 0540 & $47.9 \mathrm{~N}$ & 120.3 & W & - & 373 & - & - & $7.00 \mathrm{M}_{\mathrm{La}} \mathrm{SC}$ & - & IX & 373 & 650 \\
\hline 1877 & 10 & 12 & 2153 & $45.75 \mathrm{~N}$ & 122.5 & W & - & 484 & - & - & - & - & VII & 38 & 40 \\
\hline 1880 & 08 & 22 & 2125 & $48.0 \mathrm{~N}$ & 123.0 & W & - & 38 & - & - & - & - & VI & 375 & - \\
\hline 1880 & 12 & 13 & 0440 & $47.5 \mathrm{~N}$ & 122.5 & W & - & 38 & - & - & - & - & V & 136 & - \\
\hline 1882 & 05 & 01 & 0650 & $47.0 \mathrm{~N}$ & 123.0 & W & - & 463 & - & - & - & - & VI & 463 & - \\
\hline 1891 & 03 & 08 & 0340 & $47.5 \mathrm{~N}$ & 121.5 & W & - & 616 & - & - & $5.00 \mathrm{M}_{\mathrm{La}} \mathrm{JLQ}$ & - & V & 616 & 36 \\
\hline 1891 & 11 & 29 & 2321 & $48.0 \mathrm{~N}$ & 123.5 & W & - & 56 & - & - & - & - & VI & 56 & 10 \\
\hline 1892 & 04 & 17 & 2250 & $47.0 \mathrm{~N}$ & 123.0 & W & - & 38 & - & - & - & - & VI & 56 & - \\
\hline 1896 & 01 & 04 & 0615 & $48.5 \mathrm{~N}$ & 122.8 & W & - & 56 & - & - & - & - & V & 56 & - \\
\hline 1904 & 03 & 17 & 0420 & $48.5 \mathrm{~N}$ & 122.8 & W & - & 38 & - & - & $5.30 \mathrm{M}_{\mathrm{La}} \mathrm{NQT}$ & - & V & 56 & 52 \\
\hline 1906 & 01 & 03 & 1342 & $48.5 \mathrm{~N}$ & 117.5 & W & - & 375 & - & - & - & - & VI & 375 & 18 \\
\hline 1909 & 01 & 11 & 2344 & $49.0 \mathrm{~N}$ & 122.7 & W & - & 38 & - & - & $6.00 \mathrm{M}_{\mathrm{L}} \mathrm{NQT}$ & - & VII & 38 & $65 \&$ \\
\hline 1915 & 08 & 18 & 1404 & $48.5 \mathrm{~N}$ & 121.4 & W & - & 38 & - & - & $5.60 \mathrm{M}_{\mathrm{L}} \mathrm{NQT}$ & - & V & 56 & 78 \\
\hline 1920 & 01 & 24 & 070916 & $48.8 \mathrm{~N}$ & 123.0 & W & - & 374 & - & - & $5.50 \mathrm{M}_{\mathrm{La}} \mathrm{NQT}$ & - & VI & 56 & 70 \\
\hline 1923 & 02 & 12 & 1819 & $48.8 \mathrm{~N}$ & 122.4 & $\mathbf{W}$ & - & 56 & - & - & - & - & VI & 56 & - \\
\hline 1928 & 02 & 02 & 1252 & $47.8 \mathrm{~N}$ & 121.7 & W & - & 1 & - & - & - & - & VI & 38 & - \\
\hline 1931 & 04 & 18 & 0400 & $48.75 \mathrm{~N}$ & 122.25 & W & - & 4 & - & - & - & - & VI & 38 & $8+$ \\
\hline 1931 & 12 & 31 & 1525 & $47.5 \mathrm{~N}$ & 123.0 & W & - & 4 & - & - & $4.80 \mathrm{M}_{\mathrm{La}} \mathrm{SC}$ & - & VI & 4 & 25 \\
\hline 1932 & 07 & 18 & 060155 & $47.75 \mathrm{~N}$ & 121.83 & W & - & 496 & - & - & $5.20 \mathrm{M}_{\mathrm{LQ}} \mathrm{NQT}$ & - & VI & 496 & 31 \\
\hline 1932 & 08 & 06 & 2216 & $47.7 \mathrm{~N}$ & 122.3 & W & - & 38 & - & - & - & - & VI & 5 & 13 \\
\hline 1939 & 11 & 13 & 074554 & $47.5 \mathrm{~N}$ & 122.5 & W & - & 258 & - & - & $5.75 \mathrm{M}_{\mathrm{s}}$ GR & - & VII & 12 & 159\# \\
\hline 1940 & 10 & 27 & 222918.0 & $47.2 \mathrm{~N}$ & 123.4 & W & - & 14 & - & - & $5.10 \mathrm{M}_{\mathrm{La}} \mathrm{ROG}$ & - & V & 13 & $31+$ \\
\hline 1941 & 04 & 07 & 0925 & $48.3 \mathrm{~N}$ & 119.6 & W & - & 38 & - & - & $4.50 \mathrm{M}_{\mathrm{La}} \mathrm{ROG}$ & - & VI & 14 & 14 \\
\hline 1943 & 112 & 29 & 0043 & $48.4 \mathrm{~N}$ & 122.9 & W & - & 38 & - & - & $4.80 \mathrm{M}_{\mathrm{La}} R O G$ & - & VI & 16 & $27+$ \\
\hline 1944 & 12 & 07 & 0448 & $46.9 \mathrm{~N}$ & 123.8 & W & - & 259 & - & - & - & - & VI & 259 & @ \\
\hline 1945 & 04 & 29 & 201617.0 & $47.4 \mathrm{~N}$ & 121.7 & W & - & 20 & - & - & $5.50 \mathrm{M}_{\mathrm{s}} \mathrm{GR}$ & - & VII & 18 & 130 \\
\hline 1946 & 02 & 15 & 031748.3 & $47.400 \mathrm{~N}$ & 122.666 & $5 W$ & 018 & 260 & - & - & $5.75 \mathrm{M}_{\mathrm{S}} \mathrm{GR}$ & - & VII & 19 & $168 \&$ \\
\hline 1946 & 062 & 23 & 171319 & $49.76 \mathrm{~N}$ & 125.34 & W & 030 & 617 & - & - & $7.30 \mathrm{M}_{\mathrm{S}} \mathrm{GR}$ & - & VIII & 19 & $142 \#$ \\
\hline 1949 & 04 & 13 & 195542.0 & $47.167 \mathrm{~N}$ & 122.617 & jw & 070 & 376 & - & - & $6.90 \mathrm{mb} \mathrm{ABE}$ & $6.73 \mathrm{BL}$ & VIII & 22 & 295\# \\
\hline 1950 & 04 & 14 & 110348.0 & $48.0 \mathrm{~N}$ & 122.5 & W & - & 23 & - & - & - & - & $\overrightarrow{V I}$ & 23 & $67 \#$ \\
\hline 1954 & 05 & 15 & 130214.0 & $48.0 \mathrm{~N}$ & 122.0 & W & - & 266 & - & - & $5.00 \mathrm{M}_{\mathrm{L}}$ EPB & - & VI & 27 & 60 \\
\hline 1957 & 012 & 26 & 011606.0 & $48.333 \mathrm{~N}$ & 122.433 & & - & 30 & - & - & $5.00 \mathrm{M}_{\mathrm{L}} \mathrm{EPB}$ & - & VI & 30 & $30+$ \\
\hline 1957 & 02 & 11 & 170457.0 & $47.533 \mathrm{~N}$ & 121.066 & & - & 30 & - & - & - & - & VI & 30 & $10+$ \\
\hline 1958 & 04 & 12 & 223711 & $48.0 \mathrm{~N}$ & 120.0 & W & - & 31 & - & - & $4.10 \mathrm{M}_{\mathrm{L}}$ EPB & - & VI & 31 & 20 \\
\hline 1958 & 10 & 07 & 050752 & $46.716 \mathrm{~N}$ & 124.033 & $3 W$ & - & 31 & - & - & $3.30 \mathrm{M}_{\mathrm{L}} \mathrm{EPB}$ & - & VI & 31 & $5 \#$ \\
\hline 1959 & 08 & 04 & 235330 & $45.683 \mathrm{~N}$ & 122.267 & & - & 32 & - & - & $4.70 \mathrm{M}_{\mathrm{L}}$ EPB & - & V & 32 & 2 \\
\hline 1959 & 08 & 06 & 034435 & $47.817 \mathrm{~N}$ & 120.000 & & - & 32 & - & - & $4.40 \mathrm{M}_{\mathrm{L}}$ EPB & - & VI & 32 & 88 \\
\hline 1959 & 112 & 23 & 181525 & $46.667 \mathrm{~N}$ & 121.750 & W & - & 32 & - & - & $4.80 \mathrm{M}_{\mathrm{L}}$ EPB & - & V & 32 & 3 \\
\hline 1960 & 04 & 11 & 064735 & $47.567 \mathrm{~N}$ & 122.250 & WW & - & 33 & - & - & $3.60 \mathrm{M}_{\mathrm{D}}$ YEL & - & VI & 33 & 2 \\
\hline 1960 & 09 & 10 & 150632.5 & $47.7 \mathrm{~N}$ & 122.7 & W & 025 & 266 & - & - & $4.60 \mathrm{M}_{\mathrm{D}} \mathrm{YEL}$ & - & VI & 33 & 41 \\
\hline 1961 & 09 & 16 & 032455.8 & $46.011 \mathrm{~N}$ & 122.128 & & 007 & 618 & - & - & $4.80 \mathrm{M}_{\mathrm{L}} \mathrm{GW}$ & - & V & 34 & 18 \\
\hline 1961 & 09 & 17 & 155555.9 & $46.023 \mathrm{~N}$ & 122.122 & $2 \mathrm{~W}$ & 007 & 618 & - & - & $5.10 \mathrm{M}_{\mathrm{L}} \mathrm{GW}$ & - & VI & 34 & 28 \\
\hline 1961 & 11 & 07 & 012908.4 & $45.7 \mathrm{~N}$ & 122.4 & W & 033 & 266 & - & - & 4.50M $\mathrm{Y}$ YEL & - & VI & 34 & $28 \&$ \\
\hline 1962 & 11 & 06 & 033643.6 & $45.642 \mathrm{~N}$ & 122.588 & $3 \mathrm{~W}$ & 016 & 619 & - & - & $5.20 \mathrm{M}_{\mathrm{L}} \mathrm{YEL}$ & $5.20 \mathrm{YP}$ & VII & 38 & $70 \&$ \\
\hline 1962 & 123 & 31 & 204935.3 & $47.1 \mathrm{~N}$ & 122.0 & W & 033 & 266 & - & 一 & $4.70 \mathrm{M}_{\mathrm{D}} \mathrm{YEL}$ & - & VI & 35 & 36 \\
\hline 1963 & 012 & 24 & 214311.8 & $47.6 \mathrm{~N}$ & 122.1 & W & 017 & 266 & - & - & 4.50M $\mathrm{YEL}$ & - & VI & 36 & 26 \\
\hline 1964 & 07 & 02 & 170342.4 & $47.7 \mathrm{~N}$ & 128.3 & $\mathbf{W}$ & 033 & 266 & 4.9 & - & 5.50Ukn BRK & - & - & - & - \\
\hline 1964 & 07 & 14 & 155004.8 & $49.0 \mathrm{~N}$ & 122.6 & W & 013 & 266 & 4.6 & - & $4.50 \mathrm{M}_{\mathrm{D}} \mathrm{YEL}$ & - & VI & 37 & 19 \\
\hline 1965 & 042 & 29 & 152843.7 & $47.4 \mathrm{~N}$ & 122.3 & W & 059 & 266 & 6.5 & 6.5 & 6.60UknPAS & 6.71LB & VIII & 377 & $337 \&$ \\
\hline 1969 & 11 & 10 & 073840.8 & $48.516 \mathrm{~N}$ & 121.400 & & - & 42 & 4.3 & - & $4.70 \mathrm{ML}_{\mathrm{L}} \mathrm{GS}$ & - & V & 42 & 18 \\
\hline
\end{tabular}


WASHINGTON-Continued

[See table 1 for hypocenter and intensity references and table 2 for definitions of magnitude source codes. \&, land area only; \#, land area only in the United States for an earthquake near a coastline; +, land area in the United States when the felt area did not extend to the coast; @, felt area is less than $1,000 \mathrm{~km}^{2}$. Leader (-.) indicates information is not available]

\begin{tabular}{|c|c|c|c|c|c|c|c|c|c|c|c|c|c|c|}
\hline \multirow{2}{*}{\multicolumn{3}{|c|}{ Date }} & \multirow{2}{*}{$\begin{array}{c}\text { Origin } \\
\text { time }\{\text { (UTC) }\end{array}$} & \multicolumn{4}{|c|}{ Hypocenter } & \multicolumn{4}{|c|}{ Magnitude } & \multicolumn{2}{|c|}{ Intensity } & \multirow{3}{*}{$\begin{array}{c}\text { Felt area } \\
\left(1,000 \mathrm{~km}^{2}\right)\end{array}$} \\
\hline & & & & \multirow{2}{*}{$\begin{array}{l}\text { Latitude } \\
\left({ }^{\circ}\right)\end{array}$} & \multirow{2}{*}{$\begin{array}{l}\text { Longitude } \\
\left({ }^{\circ}\right)\end{array}$} & \multirow{2}{*}{$\begin{array}{l}\text { Depth } \\
(\mathbf{k m})\end{array}$} & \multirow[t]{2}{*}{ Ref } & \multicolumn{2}{|c|}{ USGS } & \multirow[t]{2}{*}{ Other } & \multirow{2}{*}{$\begin{array}{c}\text { Moment } \\
\mathbf{M}\end{array}$} & \multirow[t]{2}{*}{ MM } & \multirow[t]{2}{*}{ Ref } & \\
\hline Yr & Mo & $\mathrm{Da}$ & $h \mathrm{~ms}$ & & & & & $m_{b}$ & $M_{s}$ & & & & & \\
\hline $\begin{array}{l}1974 \\
1975 \\
1976 \\
1976 \\
1978\end{array}$ & $\begin{array}{l}04 \\
04 \\
05 \\
09 \\
03\end{array}$ & $\begin{array}{l}20 \\
23 \\
16 \\
08 \\
11\end{array}$ & $\begin{array}{lll}03 & 00 & 09.3 \\
01 & 03 & 42.4 \\
08 & 35 & 14.8 \\
08 & 21 & 01.6 \\
15 & 52 & 11.2\end{array}$ & $\begin{array}{l}46.759 \mathrm{~N} \\
47.076 \mathrm{~N} \\
48.80 \mathrm{~N} \\
47.382 \mathrm{~N} \\
47.42 \mathrm{~N}\end{array}$ & $\begin{array}{l}121.523 \mathrm{~W} \\
122.647 \mathrm{~W} \\
123.36 \mathrm{~W} \\
123.084 \mathrm{~W} \\
122.71 \mathrm{~W}\end{array}$ & $\begin{array}{l}005 \\
046 \\
062 \\
048 \\
025\end{array}$ & $\begin{array}{r}74 \\
74 \\
74 \\
74 \\
240\end{array}$ & $\begin{array}{l}4.8 \\
4.0 \\
5.1 \\
4.6 \\
4.3\end{array}$ & $\begin{array}{l}- \\
\overline{3.9} \\
3.2\end{array}$ & $\begin{array}{l}4.90 \mathrm{M}_{\mathrm{L}} \text { GS } \\
3.80 \mathrm{M}_{\mathrm{L}} \text { GS } \\
5.40 \mathrm{M}_{\mathrm{L}} \text { EPB } \\
4.80 \mathrm{M}_{\mathrm{L}} \text { GS } \\
4.80 \mathrm{M}_{\mathrm{L}} \text { WAS }\end{array}$ & $\frac{-}{-}$ & $\begin{array}{l}\text { V } \\
\text { VI } \\
\text { VI } \\
\text { VI } \\
\text { VI }\end{array}$ & $\begin{array}{r}47 \\
48 \\
49 \\
49 \\
240\end{array}$ & $\begin{array}{l}- \\
\overline{77} \\
20+ \\
20\end{array}$ \\
\hline $\begin{array}{l}1979 \\
1980 \\
1980 \\
1980 \\
1980\end{array}$ & $\begin{array}{l}03 \\
03 \\
03 \\
03 \\
04\end{array}$ & $\begin{array}{l}11 \\
27 \\
30 \\
31 \\
01\end{array}$ & $\begin{array}{l}143932.8 \\
220005.6 \\
175510.0 \\
113409.8 \\
042430.5\end{array}$ & $\begin{array}{l}46.45 \mathrm{~N} \\
46.219 \mathrm{~N} \\
46.208 \mathrm{~N} \\
46.210 \mathrm{~N} \\
46.222 \mathrm{~N}\end{array}$ & $\begin{array}{l}122.40 \mathrm{~W} \\
122.197 \mathrm{~W} \\
122.183 \mathrm{~W} \\
122.194 \mathrm{~W} \\
122.184 \mathrm{~W}\end{array}$ & $\begin{array}{l}016 \\
004 \\
000 \\
000 \\
004\end{array}$ & $\begin{array}{r}262 \\
74 \\
620 \\
620 \\
74\end{array}$ & $\begin{array}{l}3.8 \\
4.6 \\
4.5 \\
4.6 \\
5.0\end{array}$ & $\overline{-}$ & $\begin{array}{l}3.80 \mathrm{M}_{\mathrm{L}} \mathrm{GS} \\
4.50 \mathrm{M}_{\mathrm{L}} \mathrm{GS} \\
4.60 \mathrm{M}_{\mathrm{D}} \mathrm{QAM} \\
4.60 \mathrm{M}_{\mathrm{D}} \mathrm{QAM} \\
4.70 \mathrm{M}_{\mathrm{L}} \mathrm{GS}\end{array}$ & $\overline{-}$ & $\begin{array}{l}\text { VI } \\
- \\
-\end{array}$ & ${ }^{262}$ & $\frac{10}{-}$ \\
\hline $\begin{array}{l}1980 \\
1980 \\
1980 \\
1980 \\
1980\end{array}$ & $\begin{array}{l}04 \\
04 \\
04 \\
04 \\
04\end{array}$ & $\begin{array}{l}01 \\
01 \\
01 \\
02 \\
03\end{array}$ & $\begin{array}{l}123046.7 \\
085425.4 \\
231438.6 \\
093713.1 \\
0243 \quad 19.4\end{array}$ & $\begin{array}{l}46.208 \mathrm{~N} \\
46.218 \mathrm{~N} \\
46.206 \mathrm{~N} \\
46.220 \mathrm{~N} \\
46.208 \mathrm{~N}\end{array}$ & $\begin{array}{l}122.182 \mathrm{~W} \\
122.175 \mathrm{~W} \\
122.194 \mathrm{~W} \\
122.184 \mathrm{~W} \\
122.189 \mathrm{~W}\end{array}$ & $\begin{array}{l}001 \\
004 \\
005 \\
003 \\
000\end{array}$ & $\begin{array}{r}620 \\
74 \\
74 \\
74 \\
620\end{array}$ & $\begin{array}{l}4.8 \\
4.5 \\
4.5 \\
4.8 \\
4.5\end{array}$ & $\bar{z}$ & $\begin{array}{l}4.90 \mathrm{M}_{\mathrm{D}} \mathrm{QAM} \\
4.50 \mathrm{M}_{\mathrm{L}} \mathrm{GS} \\
4.60 \mathrm{M}_{\mathrm{L}} \mathrm{GS} \\
4.70 \mathrm{M}_{\mathrm{L}} \mathrm{GS} \\
4.80 \mathrm{M}_{\mathrm{D}} \mathrm{QAM}\end{array}$ & $\bar{z}$ & $\bar{z}$ & $\overline{-}$ & $\begin{array}{l}- \\
-\end{array}$ \\
\hline $\begin{array}{l}1980 \\
1980 \\
1980 \\
1980 \\
1980\end{array}$ & $\begin{array}{l}04 \\
04 \\
04 \\
04 \\
04\end{array}$ & $\begin{array}{l}03 \\
03 \\
04 \\
05 \\
06\end{array}$ & $\begin{array}{l}093527.2 \\
235752.3 \\
134505.8 \\
164205.7 \\
065804.5\end{array}$ & $\begin{array}{l}46.234 \mathrm{~N} \\
46.232 \mathrm{~N} \\
46.212 \mathrm{~N} \\
46.230 \mathrm{~N} \\
46.230 \mathrm{~N}\end{array}$ & $\begin{array}{l}122.173 \mathrm{~W} \\
122.218 \mathrm{~W} \\
122.176 \mathrm{~W} \\
122.194 \mathrm{~W} \\
122.194 \mathrm{~W}\end{array}$ & $\begin{array}{l}001 \\
002 \\
004 \\
002 \\
002\end{array}$ & $\begin{array}{l}74 \\
74 \\
74 \\
74 \\
74\end{array}$ & $\begin{array}{l}-5.0 \\
4.5 \\
4.9 \\
4.7\end{array}$ & $\begin{array}{l}- \\
\overline{3.8} \\
4.5 \\
3.8\end{array}$ & $\begin{array}{ll}4.80 \mathrm{~m}_{\mathrm{b}} & \mathrm{GS} \\
4.50 \mathrm{M}_{\mathrm{L}} & \mathrm{GS} \\
4.50 \mathrm{M}_{\mathrm{L}} & \mathrm{GS} \\
4.50 \mathrm{M}_{\mathrm{L}} & \mathrm{GS} \\
4.70 \mathrm{M}_{\mathrm{L}} & \mathrm{GS}\end{array}$ & $\overline{-}$ & $\bar{z}$ & $\begin{array}{l}- \\
- \\
-\end{array}$ & $\begin{array}{l}- \\
- \\
-\end{array}$ \\
\hline $\begin{array}{l}1980 \\
1980 \\
1980 \\
1980 \\
1980\end{array}$ & $\begin{array}{l}04 \\
04 \\
04 \\
04 \\
04\end{array}$ & $\begin{array}{l}07 \\
07 \\
08 \\
09 \\
09\end{array}$ & $\begin{array}{lll}06 & 45 & 19.2 \\
15 & 05 & 32.7 \\
0607 & 04.6 \\
10 & 13 & 20.3 \\
18 & 19 & 27.3\end{array}$ & $\begin{array}{l}46.225 \mathrm{~N} \\
46.234 \mathrm{~N} \\
46.218 \mathrm{~N} \\
46.220 \mathrm{~N} \\
46.20 \mathrm{~N}\end{array}$ & $\begin{array}{l}122.177 \mathrm{~W} \\
122.206 \mathrm{~W} \\
122.186 \mathrm{~W} \\
122.154 \mathrm{~W} \\
122.20 \mathrm{~W}\end{array}$ & $\begin{array}{l}004 \\
005 \\
003 \\
004 \\
005\end{array}$ & $\begin{array}{r}74 \\
74 \\
74 \\
74 \\
300\end{array}$ & $\begin{array}{l}4.6 \\
4.9 \\
4.7 \\
4.9 \\
4.8\end{array}$ & $\bar{z}$ & $\begin{array}{ll}4.50 M_{L} & G S \\
4.70 M_{L} & G S \\
4.50 M_{L} & G S \\
4.50 M_{L} & G S \\
4.50 M_{L} & G S\end{array}$ & $\bar{z}$ & $\bar{z}$ & $\begin{array}{l}- \\
z \\
-\end{array}$ & $\begin{array}{l}- \\
-\end{array}$ \\
\hline $\begin{array}{l}1980 \\
1980 \\
1980 \\
1980 \\
1980\end{array}$ & $\begin{array}{l}04 \\
04 \\
04 \\
04 \\
04\end{array}$ & $\begin{array}{l}10 \\
10 \\
11 \\
13 \\
14\end{array}$ & $\begin{array}{lll}00 & 44 & 15.7 \\
14 & 16 & 15.3 \\
23 & 52 & 00.0 \\
08 & 36 & 18.8 \\
06 & 59 & 22.3\end{array}$ & $\begin{array}{l}46.228 \mathrm{~N} \\
46.217 \mathrm{~N} \\
46.222 \mathrm{~N} \\
46.222 \mathrm{~N} \\
46.223 \mathrm{~N}\end{array}$ & $\begin{array}{l}122.182 \mathrm{~W} \\
122.177 \mathrm{~W} \\
122.164 \mathrm{~W} \\
122.176 \mathrm{~W} \\
122.188 \mathrm{~W}\end{array}$ & $\begin{array}{l}004 \\
002 \\
003 \\
001 \\
004\end{array}$ & $\begin{array}{l}74 \\
74 \\
74 \\
74 \\
74\end{array}$ & $\begin{array}{l}4.7 \\
4.7 \\
4.4 \\
4.7 \\
4.7\end{array}$ & $\begin{array}{l}\overline{3.3} \\
- \\
-\end{array}$ & $\begin{array}{l}4.60 \mathrm{M}_{\mathrm{L}} \mathrm{GS} \\
4.50 \mathrm{M}_{\mathrm{L}} \mathrm{GS} \\
4.80 \mathrm{M}_{\mathrm{L}} \mathrm{GS} \\
4.50 \mathrm{M}_{\mathrm{L}} \mathrm{GS} \\
4.50 \mathrm{M}_{\mathrm{L}} \mathrm{GS}\end{array}$ & $\overline{-}$ & $\bar{z}$ & - & - \\
\hline $\begin{array}{l}1980 \\
1980 \\
1980 \\
1980 \\
1980\end{array}$ & $\begin{array}{l}04 \\
04 \\
04 \\
04 \\
04\end{array}$ & $\begin{array}{l}14 \\
15 \\
15 \\
16 \\
16\end{array}$ & $\begin{array}{l}134904.1 \\
065822.3 \\
175454.3 \\
152205.6 \\
154023.5\end{array}$ & $\begin{array}{l}46.209 \mathrm{~N} \\
46.211 \mathrm{~N} \\
46.220 \mathrm{~N} \\
46.225 \mathrm{~N} \\
46.223 \mathrm{~N}\end{array}$ & $\begin{array}{l}122.190 \mathrm{~W} \\
122.201 \mathrm{~W} \\
122.176 \mathrm{~W} \\
122.175 \mathrm{~W} \\
122.167 \mathrm{~W}\end{array}$ & $\begin{array}{l}003 \\
002 \\
003 \\
003 \\
004\end{array}$ & $\begin{array}{r}74 \\
620 \\
74 \\
74 \\
74\end{array}$ & $\begin{array}{l}4.8 \\
4.5 \\
4.9 \\
4.9 \\
4.8\end{array}$ & $\frac{5.3}{3.6}$ & $\begin{array}{l}4.70 \mathrm{M}_{\mathrm{L}} \text { GS } \\
4.70 \mathrm{M}_{\mathrm{D}} \mathrm{QAM} \\
4.70 \mathrm{M}_{\mathrm{L}} \mathrm{GS} \\
4.90 \mathrm{M}_{\mathrm{L}} \mathrm{GS} \\
4.50 \mathrm{M}_{\mathrm{L}} \mathrm{GS}\end{array}$ & $\begin{array}{l}z \\
z\end{array}$ & $\begin{array}{l}- \\
- \\
-\end{array}$ & $\begin{array}{l}- \\
-\end{array}$ & $\begin{array}{l}- \\
- \\
-\end{array}$ \\
\hline $\begin{array}{l}1980 \\
1980 \\
1980 \\
1980 \\
1980\end{array}$ & $\begin{array}{l}04 \\
04 \\
04 \\
04 \\
04\end{array}$ & $\begin{array}{l}17 \\
18 \\
18 \\
18 \\
19\end{array}$ & $\begin{array}{lll}17 & 43 & 22.6 \\
00 & 53 & 40.4 \\
21 & 16 & 02.2 \\
22 & 27 & 14.5 \\
22 & 28 & 28.2\end{array}$ & $\begin{array}{l}46.221 \mathrm{~N} \\
46.213 \mathrm{~N} \\
46.220 \mathrm{~N} \\
46.208 \mathrm{~N} \\
46.226 \mathrm{~N}\end{array}$ & $\begin{array}{l}122.184 \mathrm{~W} \\
122.183 \mathrm{~W} \\
122.185 \mathrm{~W} \\
122.178 \mathrm{~W} \\
122.177 \mathrm{~W}\end{array}$ & $\begin{array}{l}\mathbf{0 0 3} \\
\mathbf{0 0 0} \\
004 \\
\mathbf{0 0 2} \\
\mathbf{0 0 3}\end{array}$ & $\begin{array}{r}74 \\
620 \\
74 \\
620 \\
74\end{array}$ & $\begin{array}{l}4.7 \\
4.7 \\
4.8 \\
4.5 \\
4.7\end{array}$ & $\frac{3.6}{E}$ & $\begin{array}{l}4.60 \mathrm{M}_{\mathrm{L}} \mathrm{GS} \\
4.70 \mathrm{M}_{\mathrm{D}} \mathrm{QAM} \\
4.70 \mathrm{M}_{\mathrm{L}} \text { GS } \\
4.60 \mathrm{M}_{\mathrm{D}} \mathrm{QAM} \\
4.60 \mathrm{M}_{\mathrm{L}} \text { GS }\end{array}$ & $\begin{array}{l}z \\
z\end{array}$ & $\bar{z}$ & $\begin{array}{l}- \\
- \\
-\end{array}$ & E \\
\hline $\begin{array}{l}1980 \\
1980 \\
1980 \\
1980 \\
1980\end{array}$ & $\begin{array}{l}04 \\
04 \\
04 \\
04 \\
04\end{array}$ & $\begin{array}{l}20 \\
21 \\
22 \\
23 \\
24\end{array}$ & $\begin{array}{lll}19 & 19 & 33.0 \\
15 & 13 & 55.5 \\
19 & 28 & 18.8 \\
12 & 30 & 53.1 \\
17 & 34 & 10.4\end{array}$ & $\begin{array}{l}46.215 \mathrm{~N} \\
46.113 \mathrm{~N} \\
46.217 \mathrm{~N} \\
46.260 \mathrm{~N} \\
46.220 \mathrm{~N}\end{array}$ & $\begin{array}{l}122.185 \mathrm{~W} \\
122.169 \mathrm{~W} \\
122.181 \mathrm{~W} \\
122.012 \mathrm{~W} \\
122.185 \mathrm{~W}\end{array}$ & $\begin{array}{l}005 \\
010 \\
004 \\
005 \\
004\end{array}$ & $\begin{array}{l}74 \\
74 \\
74 \\
74 \\
74\end{array}$ & $\begin{array}{l}4.8 \\
4.5 \\
4.6 \\
4.5 \\
4.7\end{array}$ & $\frac{3.9}{-}$ & $\begin{array}{l}4.80 \mathrm{M}_{\mathrm{L}} \mathrm{GS} \\
4.50 \mathrm{M}_{\mathrm{L}} \mathrm{GS} \\
4.60 \mathrm{M}_{\mathrm{L}} \mathrm{GS} \\
4.70 \mathrm{M}_{\mathrm{L}} \mathrm{GS} \\
4.80 \mathrm{M}_{\mathrm{L}} \mathrm{GS}\end{array}$ & $\begin{array}{l}- \\
-\end{array}$ & $\frac{-}{-}$ & $\begin{array}{l}- \\
-\end{array}$ & $\begin{array}{l}- \\
-\end{array}$ \\
\hline $\begin{array}{l}1980 \\
1980 \\
1980 \\
1980 \\
1980\end{array}$ & $\begin{array}{l}04 \\
04 \\
04 \\
04 \\
04\end{array}$ & $\begin{array}{l}25 \\
27 \\
28 \\
29 \\
29\end{array}$ & $\begin{array}{l}232027.9 \\
072621.3 \\
034933.5 \\
042430.2 \\
062238.9\end{array}$ & $\begin{array}{l}46.257 \mathrm{~N} \\
46.220 \mathrm{~N} \\
46.220 \mathrm{~N} \\
46.222 \mathrm{~N} \\
46.232 \mathrm{~N}\end{array}$ & $\begin{array}{l}122.180 \mathrm{~W} \\
122.184 \mathrm{~W} \\
122.180 \mathrm{~W} \\
122.170 \mathrm{~W} \\
122.192 \mathrm{~W}\end{array}$ & $\begin{array}{l}005 \\
004 \\
004 \\
002 \\
002\end{array}$ & $\begin{array}{l}74 \\
74 \\
74 \\
74 \\
74\end{array}$ & $\begin{array}{l}5.0 \\
4.5 \\
4.4 \\
4.7 \\
4.7\end{array}$ & $\frac{-}{3.6}$ & $\begin{array}{c}-\overline{4.60 \mathrm{M}_{\mathrm{L}}} \mathrm{GS} \\
4.60 \mathrm{M}_{\mathrm{L}} \mathrm{GS} \\
4.60 \mathrm{M}_{\mathrm{L}} \mathrm{GS} \\
4.50 \mathrm{M}_{\mathrm{L}} \mathrm{GS}\end{array}$ & $\begin{array}{l}= \\
\overline{-}\end{array}$ & $\overline{-}$ & $\begin{array}{l}- \\
-\end{array}$ & $\begin{array}{l}- \\
-\end{array}$ \\
\hline $\begin{array}{l}1980 \\
1980 \\
1980\end{array}$ & $\begin{array}{l}04 \\
05 \\
05\end{array}$ & $\begin{array}{l}30 \\
02 \\
03\end{array}$ & $\begin{array}{l}050902.7 \\
130229.6 \\
050046.0\end{array}$ & $\begin{array}{l}46.220 \mathrm{~N} \\
46.232 \mathrm{~N} \\
46.207 \mathrm{~N}\end{array}$ & $\begin{array}{l}122.174 \mathrm{~W} \\
122.196 \mathrm{~W} \\
122.181 \mathrm{~W}\end{array}$ & $\begin{array}{l}003 \\
001 \\
010\end{array}$ & $\begin{array}{l}74 \\
74 \\
74\end{array}$ & $\begin{array}{l}4.8 \\
4.6 \\
4.4\end{array}$ & $\begin{array}{l}3.8 \\
3.6 \\
4.2\end{array}$ & $\begin{array}{l}4.70 \mathrm{M}_{\mathrm{L}} \mathrm{GS} \\
4.50 \mathrm{M}_{\mathrm{L}} \text { GS } \\
4.50 \mathrm{M}_{\mathrm{L}} \text { GS }\end{array}$ & I & E & I & Z \\
\hline
\end{tabular}


WASHINGTON_Continued

[See table 1 for hypocenter and intensity references and table 2 for definitions of magnitude source codes. \&, ]and area only; \#, land area only in the United States for an earthquake near a coastline; +, land area in the United States when the felt area did not extend to the coast; @, felt area is less than $1,000 \mathrm{~km}{ }^{2}$. Leader $1--1$ indicates information is not available]

\begin{tabular}{|c|c|c|c|c|c|c|c|c|c|c|c|c|c|}
\hline \multirow{2}{*}{\multicolumn{2}{|c|}{ Date }} & \multirow{2}{*}{$\begin{array}{c}\text { Origin } \\
\text { time (UTC) }\end{array}$} & \multicolumn{4}{|c|}{ Hypocenter } & \multicolumn{4}{|c|}{ Magnitude } & \multicolumn{2}{|c|}{ Intensity } & \multirow{3}{*}{$\begin{array}{c}\text { Felt area } \\
\left(1,000 \mathrm{~km}^{2}\right)\end{array}$} \\
\hline & & & \multirow{2}{*}{$\begin{array}{l}\text { Latitude } \\
\left({ }^{\circ}\right)\end{array}$} & \multirow{2}{*}{$\begin{array}{l}\text { Longitude } \\
\left({ }^{\circ}\right)\end{array}$} & \multirow{2}{*}{$\begin{array}{l}\text { Depth } \\
(\mathrm{km})\end{array}$} & \multirow[t]{2}{*}{ Ref } & \multicolumn{2}{|c|}{ USGS } & \multirow[t]{2}{*}{ Other } & \multirow{2}{*}{$\begin{array}{c}\text { Moment } \\
\mathbf{M}\end{array}$} & \multirow[t]{2}{*}{ MM } & \multirow[t]{2}{*}{ Ref } & \\
\hline$\overline{Y r}$ & Mo Da & $h \mathrm{~m} s$ & & & & & $m_{b}$ & $M_{s}$ & & & & & \\
\hline $\begin{array}{l}1980 \\
1980\end{array}$ & $\begin{array}{ll}05 & 04 \\
05 & 05\end{array}$ & $\begin{array}{l}115827.4 \\
054304.1\end{array}$ & $\begin{array}{l}46.226 \mathrm{~N} \\
46.216 \mathrm{~N}\end{array}$ & $\begin{array}{l}122.183 \mathrm{~W} \\
122.171 \mathrm{~W}\end{array}$ & $\begin{array}{l}002 \\
003\end{array}$ & $\begin{array}{l}74 \\
74\end{array}$ & $\begin{array}{l}4.6 \\
4.6\end{array}$ & $\begin{array}{l}4.0 \\
3.7\end{array}$ & $\begin{array}{l}4.60 \mathrm{M}_{L} \text { GS } \\
4.60 \mathrm{M}_{\mathrm{L}} \mathrm{GS}\end{array}$ & $\overline{-}$ & - & - & $\overline{-}$ \\
\hline $\begin{array}{l}1980 \\
1980 \\
1980 \\
1980 \\
1980\end{array}$ & $\begin{array}{ll}05 & 06 \\
05 & 07 \\
05 & 08 \\
05 & 09 \\
05 & 09\end{array}$ & $\begin{array}{l}170450.9 \\
110918.0 \\
074846.1 \\
070101.3 \\
180626.5\end{array}$ & $\begin{array}{l}46.357 \mathrm{~N} \\
46.221 \mathrm{~N} \\
46.227 \mathrm{~N} \\
46.224 \mathrm{~N} \\
46.221 \mathrm{~N}\end{array}$ & $\begin{array}{l}122.076 \mathrm{~W} \\
122.188 \mathrm{~W} \\
122.169 \mathrm{~W} \\
122.174 \mathrm{~W} \\
122.170 \mathrm{~W}\end{array}$ & $\begin{array}{l}001 \\
001 \\
001 \\
002 \\
002\end{array}$ & $\begin{array}{l}74 \\
74 \\
74 \\
74 \\
74\end{array}$ & $\begin{array}{l}4.7 \\
4.7 \\
5.0 \\
4.8 \\
4.4\end{array}$ & $\frac{\frac{4.2}{4.0}}{3.7}$ & $\begin{array}{l}4.70 \mathrm{M}_{\mathrm{L}} \text { GS } \\
4.60 \mathrm{M}_{\mathrm{L}} \text { GS } \\
4.70 \mathrm{M}_{\mathrm{L}} \text { GS } \\
4.60 \mathrm{M}_{\mathrm{L}} \text { GS } \\
4.60 \mathrm{M}_{\mathrm{L}} \text { GS }\end{array}$ & $\begin{array}{l}\bar{z} \\
=\end{array}$ & $\begin{array}{l}\bar{z} \\
\bar{z}\end{array}$ & $\begin{array}{l}\bar{z} \\
\bar{z}\end{array}$ & $\begin{array}{l}\bar{z} \\
\bar{z}\end{array}$ \\
\hline $\begin{array}{l}1980 \\
1980 \\
1980 \\
1980 \\
1980\end{array}$ & $\begin{array}{ll}05 & 11 \\
05 & 12 \\
05 & 12 \\
05 & 14 \\
05 & 15\end{array}$ & 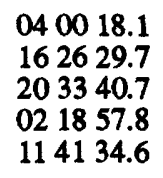 & $\begin{array}{l}46.220 \mathrm{~N} \\
46.220 \mathrm{~N} \\
46.251 \mathrm{~N} \\
46.224 \mathrm{~N} \\
46.213 \mathrm{~N}\end{array}$ & $\begin{array}{l}122.173 \mathrm{~W} \\
122.179 \mathrm{~W} \\
122.309 \mathrm{~W} \\
122.169 \mathrm{~W} \\
122.195 \mathrm{~W}\end{array}$ & $\begin{array}{l}001 \\
002 \\
010 \\
002 \\
002\end{array}$ & $\begin{array}{l}74 \\
74 \\
74 \\
74 \\
74\end{array}$ & $\begin{array}{l}4.7 \\
4.8 \\
4.4 \\
4.6 \\
4.9\end{array}$ & $\frac{\overline{4.4}}{3.6}$ & $\begin{array}{l}4.50 \mathrm{M}_{\mathrm{L}} \text { GS } \\
4.90 \mathrm{M}_{\mathrm{L}} \mathrm{GS} \\
4.60 \mathrm{M}_{\mathrm{L}} \mathrm{GS} \\
4.50 \mathrm{M}_{\mathrm{L}} \mathrm{GS} \\
4.60 \mathrm{M}_{\mathrm{L}} \mathrm{GS}\end{array}$ & $\begin{array}{l}\bar{z} \\
\overline{-}\end{array}$ & $\begin{array}{l}\bar{z} \\
\bar{z}\end{array}$ & $\begin{array}{l}\bar{z} \\
\bar{z}\end{array}$ & $\begin{array}{l}\bar{z} \\
\bar{z}\end{array}$ \\
\hline $\begin{array}{l}1980 \\
1980 \\
1981 \\
1981 \\
1989\end{array}$ & $\begin{array}{ll}05 & 16 \\
05 & 18 \\
02 & 14 \\
02 & 18 \\
03 & 05\end{array}$ & $\begin{array}{lll}12 & 34 & 54.1 \\
15 & 32 & 11.4 \\
06 & 09 & 27.2 \\
06 & 09 & 38.7 \\
06 & 42 & 00.6\end{array}$ & $\begin{array}{l}46.222 \mathrm{~N} \\
46.214 \mathrm{~N} \\
46.351 \mathrm{~N} \\
47.214 \mathrm{~N} \\
47.813 \mathrm{~N}\end{array}$ & $\begin{array}{l}122.168 \mathrm{~W} \\
122.194 \mathrm{~W} \\
122.238 \mathrm{~W} \\
120.905 \mathrm{~W} \\
123.257 \mathrm{~W}\end{array}$ & $\begin{array}{l}002 \\
004 \\
007 \\
000 \\
046\end{array}$ & $\begin{array}{l}74 \\
74 \\
74 \\
74 \\
74\end{array}$ & $\begin{array}{l}4.6 \\
4.7 \\
5.1 \\
4.6\end{array}$ & $\begin{array}{l}3.7 \\
5.2 \\
4.8 \\
-\end{array}$ & $\begin{array}{l}4.70 \mathrm{M}_{\mathrm{L}} \text { GS } \\
5.00 \mathrm{M}_{\mathrm{L}} \mathrm{GS} \\
5.50 \mathrm{M}_{\mathrm{L}} \mathrm{GS} \\
4.20 \mathrm{M}_{\mathrm{L}} \text { GS } \\
4.50 \mathrm{M}_{\mathrm{D}} \text { WAS }\end{array}$ & $\begin{array}{c}\overline{-} \\
5.48 \mathrm{BUR} \\
\overline{-}\end{array}$ & $\begin{array}{l}\overline{I V} \\
\text { VI } \\
\text { VI } \\
\text { V }\end{array}$ & $\begin{array}{l}3 \overline{300} \\
325 \\
325 \\
579\end{array}$ & $\begin{array}{c}-\overline{349 \#} \\
104 \& \\
9 \\
-\end{array}$ \\
\hline $\begin{array}{l}1989 \\
1989\end{array}$ & $\begin{array}{ll}05 & 09 \\
12 & 24\end{array}$ & $\begin{array}{l}182845.5 \\
084558.9\end{array}$ & $\begin{array}{l}48.230 \mathrm{~N} \\
46.650 \mathrm{~N}\end{array}$ & $\begin{array}{l}119.853 \mathrm{~W} \\
122.116 \mathrm{~W}\end{array}$ & $\begin{array}{l}016 \\
019\end{array}$ & $\begin{array}{l}74 \\
74\end{array}$ & $\overline{4.3}$ & $=$ & $\begin{array}{l}4.40 \mathrm{M}_{\mathrm{L}} \text { WAS } \\
5.10 \mathrm{M}_{\mathrm{L}} \text { WAS }\end{array}$ & $=$ & $\begin{array}{l}\text { VI } \\
\text { V }\end{array}$ & $\begin{array}{l}579 \\
579\end{array}$ & $\begin{array}{l}22 \\
30\end{array}$ \\
\hline
\end{tabular}

[Reference (Ref.) numbers given in parentheses at the end of each description refer to sources of data in table 1. Magnitude values are described in the Introduction, and codes are defined in table 2.]

1872. Dec. 15 (Dec. 14). Near Lake Chelan, Wash. This earthquake evidently had a large magnitude, because it was felt from British Columbia, Canada, to Oregon and from the Pacific Ocean to Montana (see fig. 61). It occurred in a wilderness area, which in 1872 had only a few inhabitantslocal Indian tribes, trappers, traders, and military men. Because there were few man-made structures in the epicentral area near Lake Chelan, most of the information available is about ground effects, including huge landslides, massive fissures in the ground, and a 9-m-high geyser.

Extensive landslides occurred in the slide-prone areas of the Columbia River. One massive slide, at Ribbon Cliff (between Entiat and Winesap), blocked the Columbia River for several hours. A field reconnaissance to the Ribbon Cliff landslide area in August 1976 showed remnants of a large landslide mass along the west edge of Lake Entiat (Columbia River Reservoir), below Ribbon Cliffs and about $3 \mathrm{~km}$ north of Entiat. Although the most spectacular landslides occurred in the Chelan-Wenatchee area, landsliding was reported throughout the Cascades.

Most of the ground fissures occurred at the east end of Lake Chelan in the area of the Indian camp; in the Chelan Landing-Chelan Falls area; on a mountain about $19 \mathrm{~km}$ west of the Indian camp area; on the east side of the Columbia River (where three springs formed); and near the top of a ridge on a hogback on the east side of the Columbia River. These fissures formed in several localities of differing physiographic environments. Slope failure or settlement or slumping in water-saturated unconsolidated sediments may have produced the fissures in areas on steep slopes or near bodies of water. Sulfurous water was emitted from the large fissures that formed in the Indian camp area. At Chelan Falls, "a great hole opened in the earth" from which water spouted as much as $9 \mathrm{~m}$ in the air. The geyser activity continued for several days, and, after diminishing, left permanent springs.

In the epicentral area, one log building on unconsolidated river deposits near the mouth of the Wenatchee River was damaged. The upper logs and roof of the cabin were displaced, and the kitchen was separated from the main building. People there were 


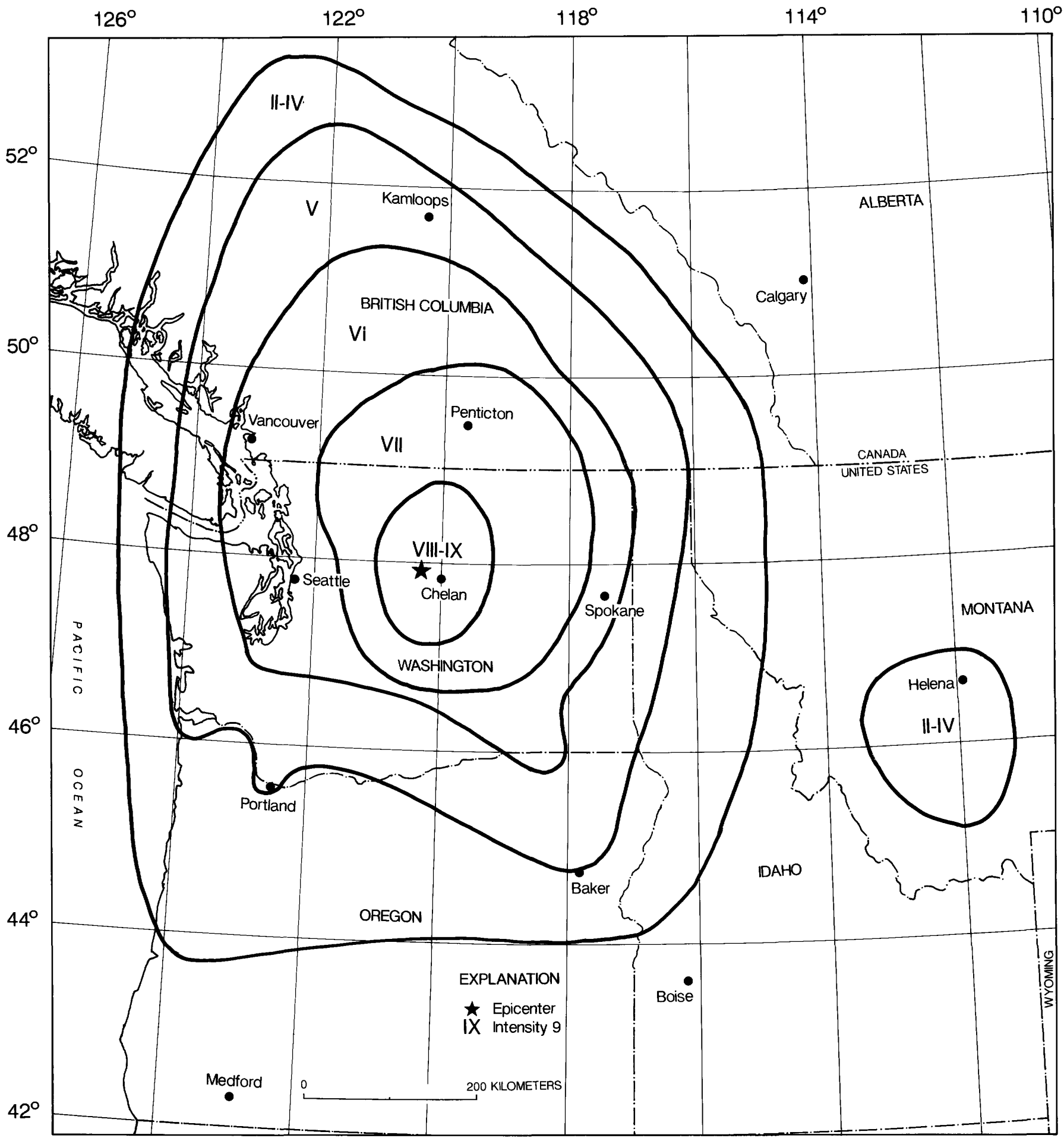

FiguRE 61.-Isoseismal map for the central Washington earthquake of December 15, 1872. This is a simplified version of an unpublished map by M.G. Hopper and S.T. Algermissen.

thrown to the floor; waves were observed in the ground; and loud detonations were heard. About 3 $\mathrm{km}$ above the Ribbon Cliff slide area, the logs on another log cabin caved in.
Damaging intensities in Washington (MM intensity VI) extended on the west throughout the now densely populated Puget Sound basin and on the southeast to beyond the Hanford nuclear reactor site. The 
earthquake also was reported in Idaho, Montana, Oregon, and Canada. Many aftershocks were observed in the area over the next 2 years. (Ref. 373, 375,518 .)

1877. Oct. 12. North of Portland, Oreg. in Clark County, Washington. Two earthquakes were reported felt in northern Oregon. The first light shock occurred east of Portland, at Cascade Locks (Hood River County) at about 0900 (local time). The second shock, about 5 hours later, was sufficiently violent at Portland to overthrow two chimneys and break windows. At the county jail, a stove was knocked from its "moorings" and thrown over. The second shock also was felt at Cascade Locks, Hubbard, and Marshfield (18 km southeast of Portland). One report indicated that the earthquake was felt on Puget Sound and at points down the Columbia River. (Ref. 38, 53, 463, 484.)

1880. Aug. 22. Puget Sound area, Washington. In Victoria, B.C., Canada, loose bricks fell and plaster was cracked. Also felt at Port Townsend and Seattle and on southern Vancouver Island. (Ref. 38, 375.)

1882. May 1 (Apr. 30). Near Olympia, Washington. This severe earthquake threw down two chimneys, broke crockery, and stopped clocks on southern Puget Sound, at Olympia (Thurston County). Large trees swayed back and forth, the ground moved in a wavy motion, and people were thrown from their feet. Also felt at Fort Canby, Wash., Portland, Oreg., and Victoria, B.C., Canada. (Ref. 56, 463.)

1891. Nov. 29. Near Port Angeles, Wash. One building in Seattle swayed so much that an elevator became jammed. On the east side of Seattle, Lake Washington was disturbed and the water "rolled onto the beach 2 feet above the mark of the highest water and 8 feet above the present stage." At Port Townsend, northwest of Seattle, residents rushed from buildings, and windowpanes were broken in a hotel at Pysht (Clallam County). Also felt in Snohomish and Bellingham Bay. (Ref. 38, 56.)

1892. Apr. 17. Near Olympia, Washington. The shock was described as "severe" at Olympia, but "sharp" in the areas a few kilometers northeast, at Tacoma, Wash., and at Portland, Oreg., about $175 \mathrm{~km}$ south. People rushed into the street when buildings began to tremble in Portland. (Ref. 38, 56.)

1906. Jan. 3. Northeast Washington. At Nelson, B.C., Canada, plaster was knocked down, articles fell from shelves, and hanging pictures were displaced. Two shocks were felt from the south boundary of Spokane County, Wash., to a point north of Bradshaw, B.C., Canada. (Ref. 38, 375.)
1909. Jan. 11. British Columbia-Washington border. This earthquake caused minor damage in Whatcom and Skagit Counties. The shock cracked a sidewalk and damaged piers slightly at Anacortes, cracked walls near the Canadian border at Blaine, and cracked plaster and twisted sidewalks at Bellingham. It was "violent" at Crescent, Dungeness, Neah Bay, and Port Angeles. Crockery was broken at Victoria, B.C., Canada. Felt throughout much of British Columbia, Canada, and as far south as Portland, Oreg. (Ref. 38, 56, 375.)

1920. Jan. 24. (Jan. 23). Strait of Georgia, Northwest Washington. Windows were broken in Whatcom County, at Bellingham, and several brick walls were cracked at Bellingham and at Anacortes, a few kilometers south. Some damage to houses was reported in Canada, on Vancouver Island, and chimneys were cracked at New Westminster. Boulders were shaken off Crown Mountain, near Vancouver, and some windows were broken in the city. (Ref. 38, $56,374$.

1923. Feb. 12. Near Bellingham, Whatcom County, Wash. Plaster cracked at Bellingham, in western Whatcom County. Also felt at Marietta, Wash., and Victoria, B.C., Canada. (Ref. 56, 375.)

1928. Feb. 2. Startup, southern Snohomish County, Wash. Plaster and wallpaper were cracked and residents were alarmed at Startup, northeast of Seattle. A strong shake and roaring noises were reported. (Ref. 1, 38.)

1931. Apr. 18 (Apr. 17). Near Bellingham, Whatcom County, Wash. A few bricks fell from chimneys at Acme (about $25 \mathrm{~km}$ southeast of Bellingham), and wallpaper was cracked at nearby Lawrence. Felt at several towns in the area, including Vancouver and Victoria, B.C., Canada. (Ref. 4, 38.)

1931. Dec. 31. Puget Sound area, Wash. At Lilliwaup, north of Olympia, in Mason County, a new concrete wall of a store was cracked and a basin hanging on a nail was knocked down. Articles were thrown from store shelves in several towns in the area. (Ref. 4, 38.)

1932. July 18 (July 17). Near the forks of Tolt River, King County, Wash. The epicenter of this earthquake was in the wilderness east of Seattle, along the western base of the Cascade Range; there were few inhabitants and few buildings or other structures for the earthquake to affect. Fissures or other displacements of the ground were not observed in this region.

Near the epicenter, on the South Fork, Tolt River, a tremendous detonation was heard and standing was almost impossible. One chimney was thrown down at 
Tolt. Some buildings were damaged north of Seattle, at Lowell and Everett; and windows were broken and standing was difficult at Farrell's Ranch, northeast of Seattle and about $6 \mathrm{~km}$ east of Duvall. At cemeteries in Monroe and South Everett, several vases, small pots, and unattached statues were toppled. Felt to the east as far as Coulee City and Conconully, to the south as far as Chehalis and Centralia, and to the north as far as Bellingham, Wash., and Vancouver, B.C., Canada. The western felt limit is unknown. (Ref. 375, 496.)

1932. Aug. 6. Seattle, King County, Wash. A strong local shock demolished a few chimneys and severely damaged others at Seattle. Several slight shocks were felt in the area through early October. (Ref. 5, 38.)

1939. Nov. 13 (Nov. 12). Southern Puget Sound area, Washington. Slight damage to chimneys, plaster, and windows occurred in towns throughout the area. Chimneys were twisted or fallen south and west of Olympia at Brooklyn, Centralia, Elma, Fairfax, and Oakville, and east of Tacoma at Auburn. At Tacoma, many buildings sustained cracks; some chimneys were damaged; and pavement was cracked. Felt north to Canada and south to Oregon (see fig. 62). Four small aftershocks occurred in the next 9 days. (Ref. 12, 38, 258.)

1941. Apr. 7. Near Okanogan, Okanogan County, north-central Washington. An observer at Mazama, Okanogan County, reported that a stove was displaced and furniture was overturned. MM intensity $\mathrm{V}$ was assigned to effects at three other towns in Okanogan County: Nespelem, Okanogan, and Omak. (Ref. 14, 38.)

1943. Nov. 29 (Nov. 28). Puget Sound area, Wash. Chimneys were cracked and furniture was displaced west of Anacortes, at Richardson, San Juan County. Also felt at several other towns in the region. (Ref. 16, 38, 375.)

1944. Dec. 7 (Dec. 6). Near Aberdeen, Grays Harbor County, Wash. At Grays Harbor Junior College, at Hoquiam, a large brick chimney on top of a building rotated about $46 \mathrm{~cm}$ out of alignment and had to be replaced. (Ref. 17, 259.)

1945. Apr. 29. Near North Bend, King County, Wash. At North Bend, chimneys and plaster were cracked, and dishes, windows, and the town water main were broken. Near North Bend, at the Mount Si Ranger Station, tons of rock and earth cascaded down the cliffs. Bricks were dislodged from a dozen or more houses in the Cle Elum area of Kittitas County. Minor damage occurred at several towns in the region. Felt over most of Washington and in western Idaho and northern Oregon. (Ref. 18, 20.)
1946. Feb. 15 (Feb. 14). Puget Sound area, Wash. Although property damage at Seattle generally was moderate, it was marked by a few instances of spectacular damage (total estimate about $\$ 250,000$ ). Structures that were most severely damaged included the Sears-Roebuck Building, Fisher flour mills and grain elevator on Harbor Island, Fry \& Company packing plant, Seattle Port of Embarkation Building No. 14, and Smith Tower. Several old brick chimneys at Tacoma broke off at the roofline, and about $24 \mathrm{~m}$ of fire wall was knocked off the Olympia Hotel in Olympia, southwest of Tacoma. Light damage occurred at several nearby towns. Also felt in Canada and northwest Oregon. Three small aftershocks occurred through Feb. 22. (Ref. 19, 260.)

1946. June 23. Strait of Georgia, B.C., Canada. Heavy damage occurred in the epicentral area. The Canadian Hydrographic Department reported the bottom of Deep Bay in the Strait of Georgia sank from about 3 to $25 \mathrm{~m}$. A 3-m vertical ground shift occurred on Read Island, and ground settlements as much as $30.5 \mathrm{~m}$ were observed at other points.

In the United States, some chimneys fell at East Sound, San Juan County, Wash.; a concrete mill was damaged at Port Angeles; and buildings were damaged slightly at Northport, Port Townsend, and to the south as far as Olympia. At Seattle, plaster fell in the County-City Building, and a few bricks fell from the Sears-Roebuck Building. Also felt at several towns in Oregon. (Ref. 19, 38, 258.)

1949. Apr. 13. Puget Sound area, Wash. This is the largest known earthquake in the history of Washington. Its epicenter lies between Olympia and Tacoma, along the southern edge of Puget Sound. Property damage in Olympia, Seattle, and Tacoma was estimated at $\$ 25$ million; eight people were killed; and many were injured. Several structures were condemned, including two schools and a church at Centralia, south of Olympia; a junior high school at Auburn, northeast of Tacoma; and a library at Chehalis, near Centralia. School buildings in widely separated towns were damaged seriously. Water spouted from cracks that formed in the ground at Centralia, Longview, and Seattle. One new spring developed on a farm at Forest. Downed chimneys and walls were reported from towns throughout the area.

At Olympia, almost all large buildings were damaged to some extent, including eight structures on the Capitol grounds. Many chimneys and two large smokestacks fell. Public utilities sustained serious damage-water and gas mains were broken, and electric and telegraph services were interrupted.

At Seattle, houses on filled ground were demolished, many old brick buildings were damaged, and 


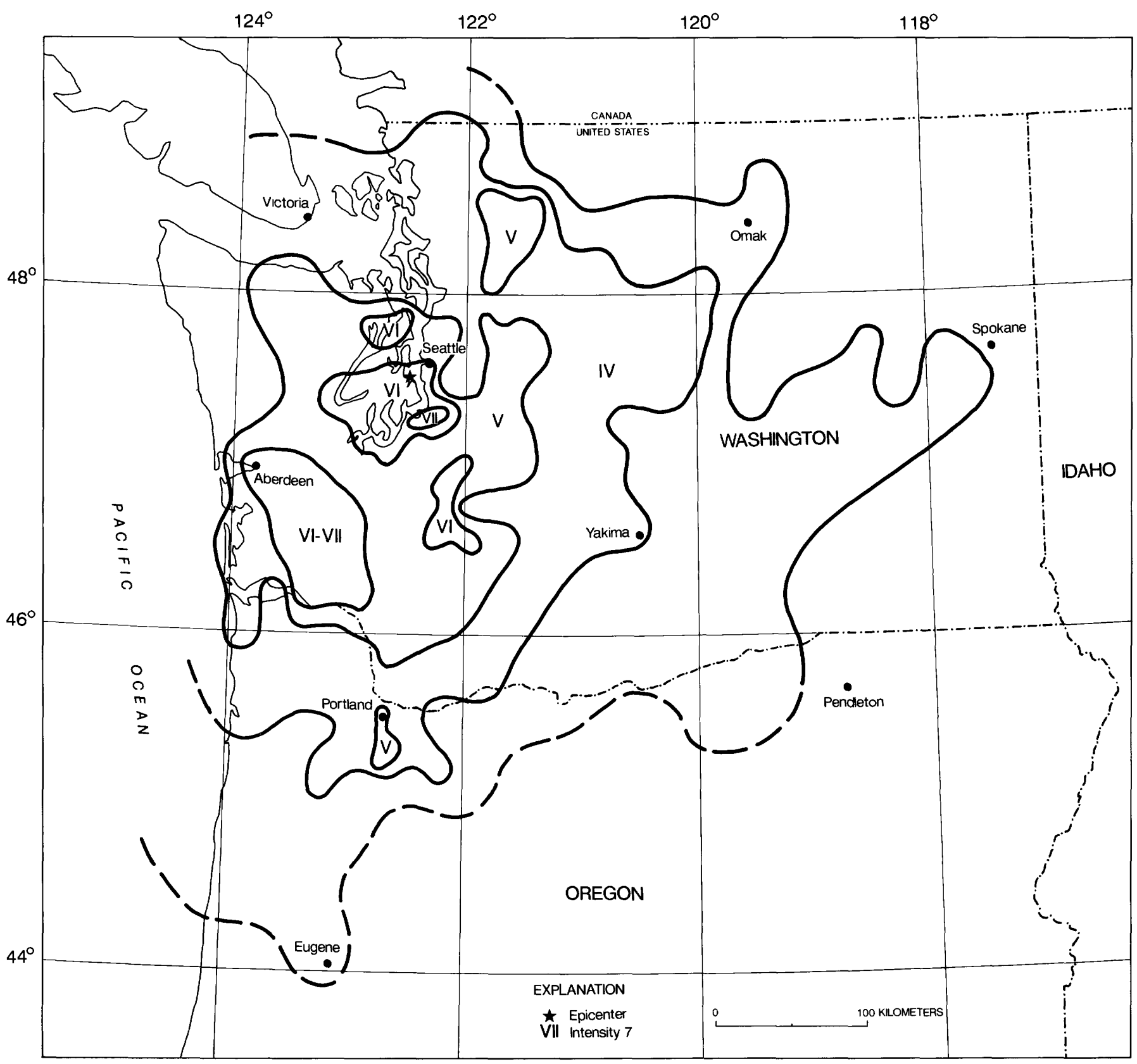

FIGURE 62.-Isoseismal map for the northwest Washington earthquake of November 13, 1939 . Isoseismals are based on intensity estimates from data listed in references 12 and 259 of table 1.

chimneys toppled. One wooden water tank and the top of a radio tower collapsed.

About $50 \mathrm{~km}$ northeast of Olympia, at Tacoma, many chimneys were knocked to the ground and many buildings were damaged. Near Tacoma, a huge section of a 73-m cliff toppled into Puget Sound shortly after the earthquake; south of Tacoma, railroad bridges were thrown out of alignment. A 23-ton cable saddle was thrown from the top of the Tacoma
Narrows bridge tower, causing considerable damage. Also felt in Idaho, Montana, Oregon, and in British Columbia, Canada (see fig. 63). Only one small aftershock occurred during the next six months. Magnitude 6.5 $\mathrm{M}_{\mathrm{S}} \mathrm{ABE}, 7.1 \mathrm{Ukn}$ PAS, 7.0 $\mathrm{M}_{\mathrm{S}} \mathrm{GR}$, $6.5 \mathrm{M}_{\mathrm{L}} \mathrm{KJ}$. (Ref. 22, 38, 376, 460, 533.)

1950. Apr. 14. Near Langley, Island County, Wash. Plaster cracked and fell and small objects overturned at Langley. A few cracks formed in 


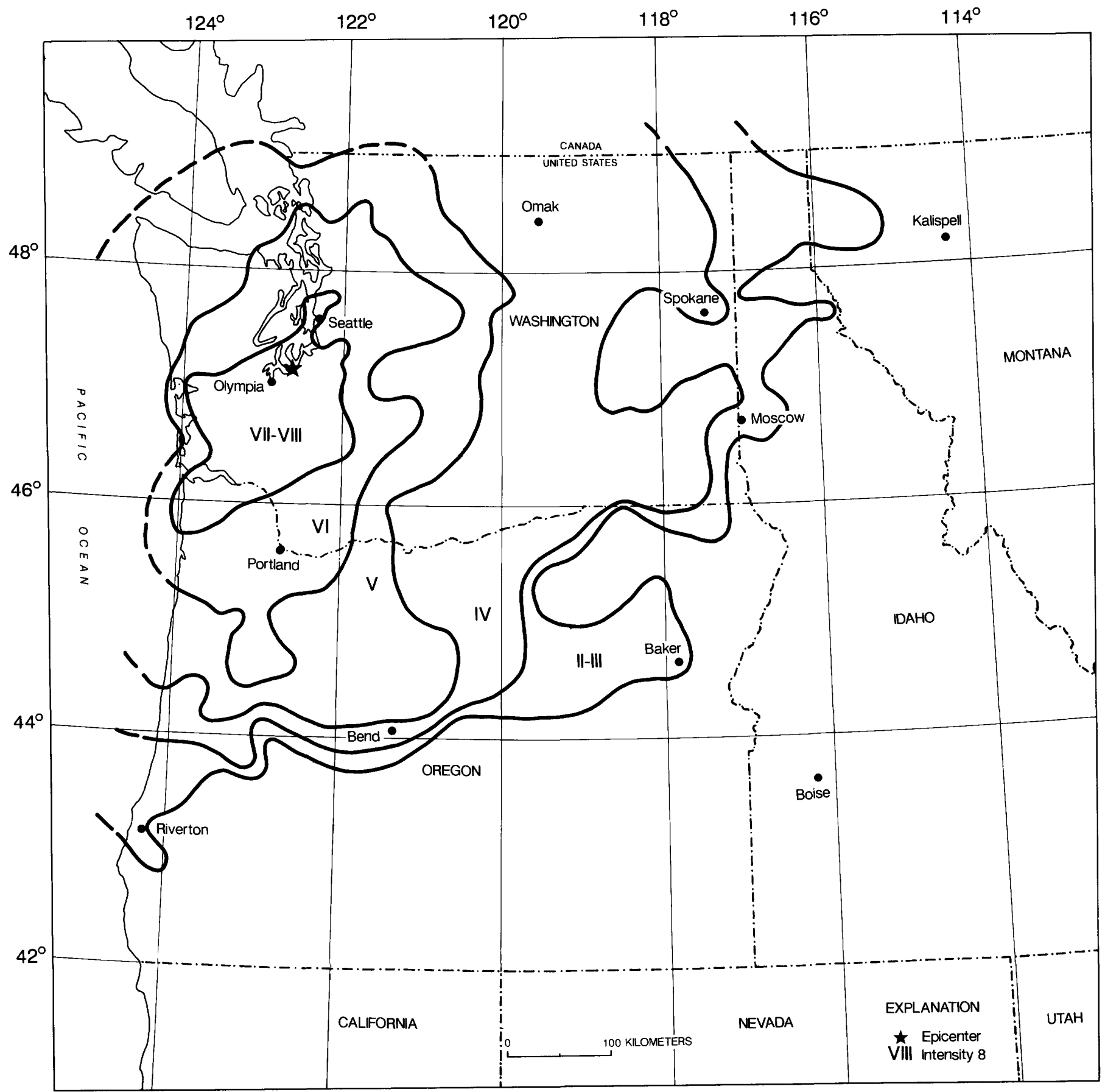

FIGURE 63.-Isoseismal map for the Puget Sound, Washington, earthquake of April 13, 1949. Isoseismals are based on intensity estimates from data listed in references 22 and 259 of table 1.

plaster northwest of Langley, at Port Townsend, and pendulum clocks stopped. Felt over a small area of northwest Washington. (Ref. 23, 38, 375.)

1954. May 15. Near Lake Stevens, Snohomish County, Wash. A moderate earthquake was felt throughout northwest Washington. Small cracks formed in walls at Belfair (northwest Mason County) and Lake Stevens; plaster cracked at Seattle (King County), and dishes broke at Skykomish (northeast King County). (Ref. 27, 38, 266.)

1957. Jan. 26 (Jan. 25). Northwest Washington. This earthquake was strongest at Clear Lake 
(southeast of Bellingham, in Skagit County), where plaster cracked and fell at a school and a knickknack shelf was displaced. Felt over most of northwest Washington. (Ref. 30, 38.)

1957. Feb. 11. Near North Bend, King County, Wash. One chimney was cracked and knickknacks fell at Fall City; plaster was damaged at North Bend and Snoqualmie. Observers over a small area of northwest Washington reported this earthquake. (Ref. 30.)

1958. Apr. 12. North-central Washington. Windows and dishes were broken in some houses at Chelan and at Pateros and Winthrop, north of Chelan in Okanogan County. Rocks fell on roadways near Chelan. Moderate to loud earth noises were heard in most areas. (Ref. 31, 38.)

1958. Oct. 7 (Oct. 6). Willapa Bay, Pacific County, Wash. At a motel in Tokeland (at the north end of Willapa Bay), several cracks formed in a patio. Felt over a small area of Grays Harbor and Pacific Counties in western Washington. (Ref. 31, 38.)

1959. Aug. 6 (Aug. 5). Near Chelan, Wash. This minor shock, which was observed over most of the State, caused slight damage in a few towns. At Chelan, about $180 \mathrm{~km}$ east of Seattle, bricks and part of a chimney fell; at Orondo, southwest of Chelan, chimneys were cracked; and at nearby Waterville, chimneys were cracked and bricks fell from several buildings. Rocks slid down the steep hills near Chelan and Waterville. (Ref. 32, 38.)

1960. Apr. 11 (Apr. 10). Near Seattle, King County, Wash. At Seattle, a concrete wall was cracked and a wood retaining wall was damaged. Felt over a small area, mainly in western King County. (Ref. 33, 38.)

1960. Sept. 10. Puget Sound area, Washington. A light earthquake cracked many concrete floors and walls in Bremerton, west of Seattle, and caused considerable plaster damage in Seattle. Felt over most of northwest Washington. (Ref. 33, 38, 266.)

1961. Sept. 17. Clark County, Washington near the Oregon-Washington border. Slight damage occurred at a few towns along the Columbia River. In southern Skamania County, chimneys and concrete foundations cracked at Stevenson, and an old house shifted about $2.5 \mathrm{~cm}$ on its foundation at North Bonneville. At Latourell Falls, Oreg., a few cracks formed in a concrete-block foundation. Felt over a small area of southwest Washington and northwest Oregon. (Ref. 34, 38, 266, 618.)

1961. Nov. 7 (Nov. 6). Clark County, Washington near the north Oregon-Washington border. Minor damage occurred in the Portland, Oreg., area. Part of a chimney fell and interior lights were broken at Portland; a concrete-block foundation of a service station was damaged at Glenwood, west of Portland; and windows were broken at Vernonia, north of Glenwood. Felt over a large area of southwest Washington and northwest Oregon. (Ref. 34, 38, 266.)

1962. Nov. 6 (Nov. 5). Clark County, Washington near the Oregon-Washington border. At Portland, Oreg., many chimneys were cracked severely, shaken down, or broken off. Tile ceilings were downed in several buildings; large cracks formed in walls; and plaster was knocked to the floor. Minor damage, consisting mainly of cracked plaster and chimneys and broken windows, occurred in many towns in both Oregon and Washington. Felt over a large area of southwest Washington and northwest Oregon. Magnitude 5 1/4-5 1/2 Ukn PAL, $4.9 \mathrm{M}_{\mathrm{D}}$ YP. (Ref.35, 38, 266, 619.)

1962. Dec. 31. Near Berkley, Pierce County, Wash. Residents in several towns (mainly in Pierce County) reported cracks in chimneys and plaster. Chimneys were cracked southeast of Tacoma, at Orting and Wilkeson, and walls were cracked at Buckley. Several rocks fell from a cut bank onto the road near Buckley. Cracks in chimneys also were reported in northern Yakima County, at Naches. (Ref. 35, 38, 266.)

1963. Jan. 24. Near Maple Valley, King County, Wash. At Maple Valley and Tacoma, cracks formed in plaster and walls and objects fell to the floor. Felt in many towns in western Washington. (Ref. 36, 38, 266.)

1964. July 14. Near Bellingham, Whatcom County, Wash. Slight damage, consisting mainly of cracks in plaster and walls, occurred at Bellingham, Custer, Nooksack, and Sumas. At Lumni Island, southwest of Bellingham, a fireplace chimney was split and mortar fell to the floor. In British Columbia, Canada, plaster cracked and fell at Abbotsford, and a ceiling cracked at White Rock. Loud earth noises were heard in several areas. Magnitude 3.7 $\mathrm{M}_{\mathrm{S}}$ NUT. (Ref. 37, 38, 263, 266.)

1965. Apr. 29. Puget Sound area, Wash. This was the second largest earthquake in the history of Washington. It caused about $\$ 12.5$ million in property damage and killed seven people. This shock was characterized by a rather large area of $M M$ intensity VII and small pockets of MM intensity VIII in Seattle and suburbs and southeast of Seattle, in Issaquah. Pockets of high earthquake intensity, as typified by damage such as fallen chimneys, almost always were associated with variations in the local geology.

Chimneys were damaged extensively in West Seattle, and two schools were damaged severely. The 


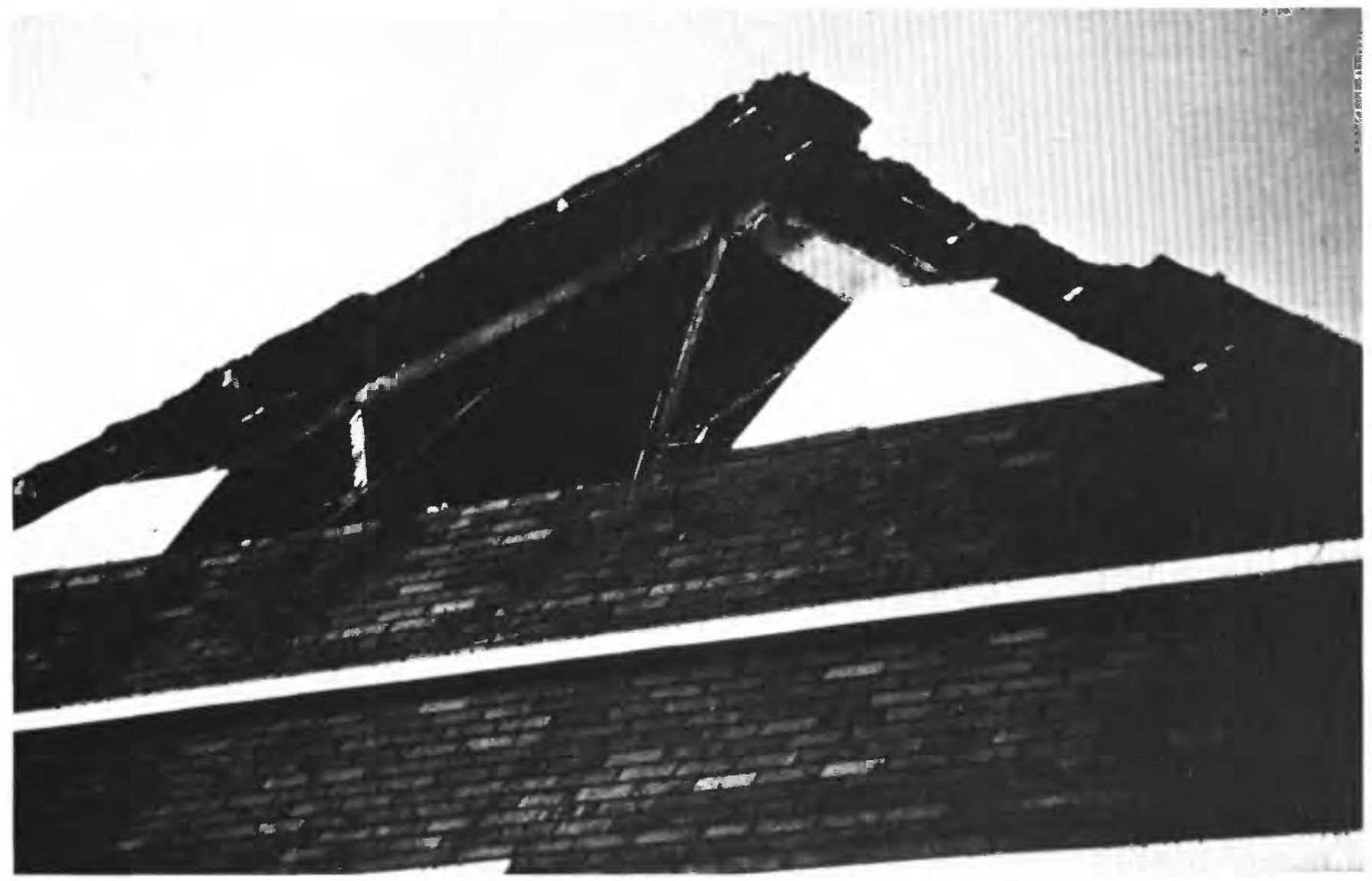

Roof of house in Seattle, Washington, damaged by the April 29, 1965, earthquake.

(Photograph from the National Geophysical Data Center, NOAA.)

low-lying and filled areas along the Duwamish River and its mouth settled, causing considerable building damage. Harbor Island, at the mouth of the Duwamish River, was severely damaged. Slumping occurred along a steep slope near Admiral Way. A brick garage partly collapsed at Issaquah; one school was damaged extensively; and chimneys in the area sustained heavy damage. Many instances of parapet and gable failure occurred. Damage to utilities in the area was not severe.

In general, damage patterns repeated those observed in the April 1949 shock, although the 1949 event was more destructive. Buildings apparently damaged in 1949 often sustained additional damage in 1965. An example is the Alki Beach section of West Seattle, where almost every chimney was knocked down in 1965. Similar damage occurred there in the 1949 earthquake.

Buildings having unreinforced brick-bearing walls with sand-lime mortar were damaged most severely. Multistory buildings, however, generally had slight or no damage. Performance of wood frame dwellings almost always was excellent, and, where damage occurred, it was confined mainly to cracks in plaster or to failure of unreinforced brick chimneys at or above the roofline. Also felt in Idaho, Montana, Oregon, and in British Columbia, Canada. Little aftershock activity was observed. Magnitude $6.2 \mathrm{~m}_{\mathrm{b}}$ NUT, 6.4 $\mathrm{M}_{\mathrm{S}}$ NUT. (Ref. 38, 75, 263, 266, 377, 533.)

1975. Apr. 23 (Apr. 22). Puget Sound area, Wash. Slight damage occurred at Sumner, a few $\mathrm{km}$ east of Tacoma in Pierce County, where tar on a roof was cracked. Felt throughout the southern Puget Sound area. (Ref. 38, 48, 74.)

1976. May 16. Vancouver Island region, Canada. Minor damage occurred at Deming, Whatcom County, Wash., and Lake Cowichan and Victoria, B.C. At Deming, cracks formed in plaster and drywall; at Victoria, one chimney was damaged; and at Lake Cowichan, a water line was broken. Felt over a large area of British Columbia and northwest Washington. (Ref. 38, 49, 74.)

1976. Sept. 8. Puget Sound area, Washington. Broken glassware and other minor damage were 
reported at Tacoma, in Pierce County. Felt throughout the Puget Sound area. (Ref. 38, 49, 74.)

1978. Mar. 11. Puget Sound area, Wash. This minor earthquake cracked a rock-and-mortar wall at Crystal Mountain Ski Resort (in Mount Rainier National Park) and displaced open roof beams 1-2 $\mathrm{cm}$. Felt over a moderate area of northwest Washington. (Ref. 38, 240.)

1979. Mar. 11. Southwest Washington. Minor damage in Cowlitz County, at Ariel, included cracks in chimneys, exterior cinder-block walls, brick walls, and sidewalks. A few windows were cracked at Castle Rock, northwest of Ariel. Felt over a small area of southwest Washington and northern Oregon. Magnitude $3.8 \mathrm{M}_{\mathrm{L}}$ GS. (Ref. 38, 262.)

1980. May 18. Mount St. Helens, Wash., earthquake and volcanic eruption. An earthquake occurred at 1532 UTC, only seconds before the explosion that began the eruption of Mount St. Helens volcano. This eruption and blast blew off the top of the volcano, reducing its elevation by $396 \mathrm{~m}$, killed 31 people, and caused an estimated property damage of between $\$ 0.5$ billion and $\$ 2$ billion. About 53 earthquakes having magnitudes larger than 4.5 were located near Mount St. Helens before the main event and eruption. No earthquakes having magnitudes higher than 4.5 were located following the eruption. The sound from the explosion and the vibratory effects from the earthquake were inseparable, resulting in a felt area that was based on both (Ref. 74, 300, 609.)

1981. Feb. 14 (Feb. 13). Southwest Washington. Several towns in the area reported cracks in foundations, plaster, drywall, and windows. Damage to chimneys (broken at roofline, twisted, cracked) occurred in Lewis County, at Mossyrock (near the epicenter); in Pierce County, at Graham and Kapowsin; and at Grays River, in Wahkiakum County (west of the epicenter). In Cowlitz County, about $50 \mathrm{~km}$ southwest of the epicenter, sidewalks were cracked at Ariel, and plaster fell at Hazel Dell. Felt over a large area of western Washington and northwest Oregon. Several aftershocks were observed in the area. Magnitude 5.2 $\mathrm{M}_{\mathrm{D}}$ WAS. (Ref. $74,325$.

1981. Feb. 18 (Feb. 17). Central Washington. Bricks fell from chimneys in the Cle Elum area of Kittitas County. Hairline cracks in drywall as well as broken windows were reported at Puyallup, about $100 \mathrm{~km}$ west of the epicenter, near Tacoma. Minor damage to plaster, drywall, windows, and glassware was reported from several towns in the Cle Elum area. A sharp crack or explosive-like sound was reported by some residents. Magnitude $4.2 \mathrm{M}_{\mathrm{D}}$ WAS. (Ref. 74, 325.)

1989. May 9. Near Okanogan, Wash. This earthquake cracked chimneys, walls, and plaster at Okanogan, about $180 \mathrm{~km}$ northwest of Spokane. Objects fell from shelves and tables in several towns in north-central Washington. Felt in northern Okanogan County near the United States-Canadian border, west to Chelan County, south to Grant County, and east to Ferry and Lincoln Counties. (Ref. 74, 579.) 


\section{WEST VIRGINIA}

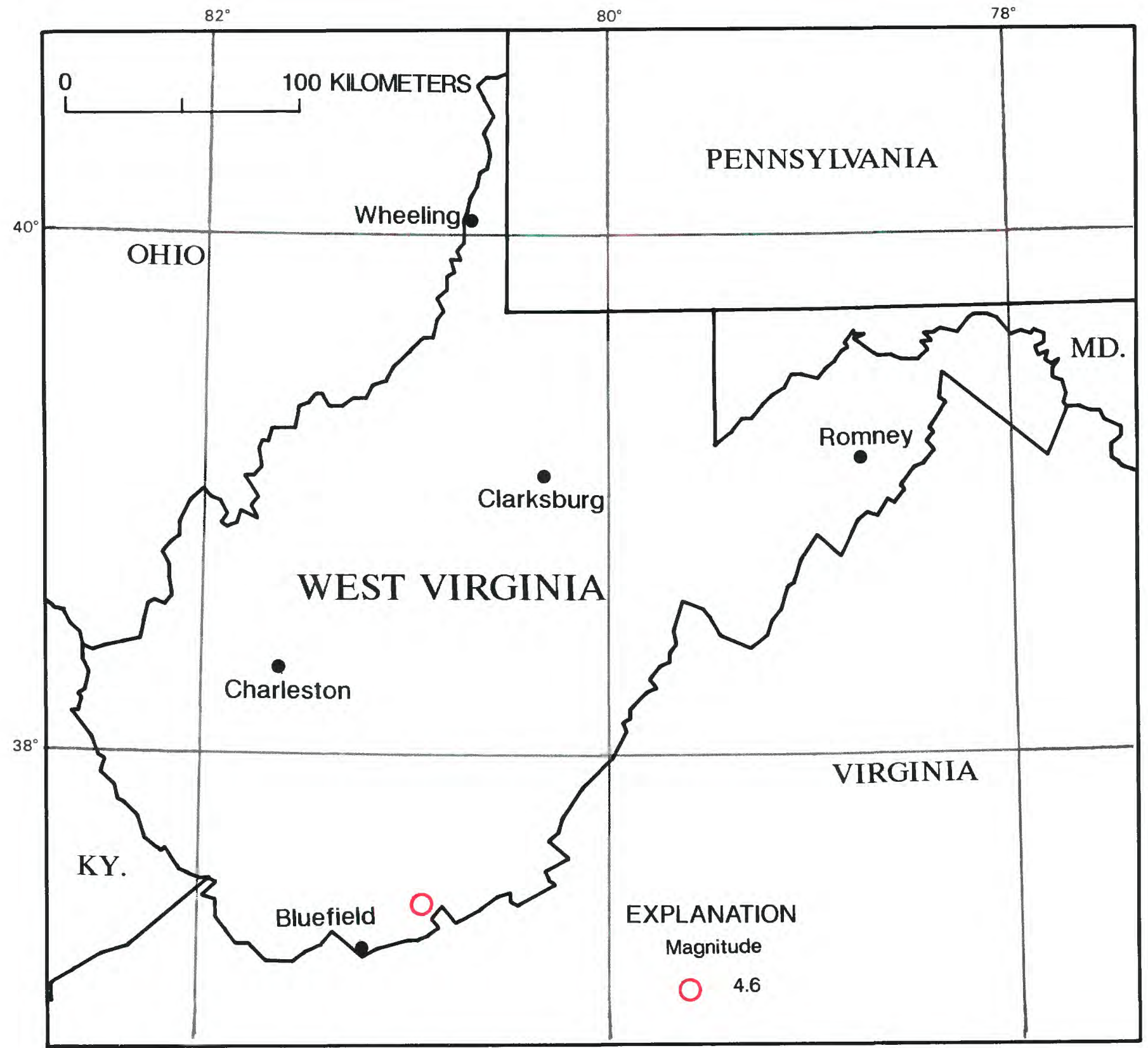

Earthquake in West Virginia with a magnitude $\geq 4.5$ and intensity $\geq$ VI. 
WEST VIRGINIA

[See table 1 for hypocenter and intensity references and table 2 for definitions of magnitude source codes. Leader (-) indicates information is not available]

\begin{tabular}{|c|c|c|c|c|c|c|c|c|c|c|c|c|c|}
\hline \multirow{2}{*}{\multicolumn{2}{|c|}{ Date }} & \multirow{2}{*}{$\begin{array}{l}\text { Origin } \\
\text { time (urc }\end{array}$} & \multicolumn{4}{|c|}{ Hypocenter } & \multicolumn{4}{|c|}{ Magnitude } & \multicolumn{2}{|c|}{ Intensity } & \multirow{3}{*}{$\begin{array}{c}\text { Felt area } \\
\left(1,000 \mathrm{~km}^{2}\right)\end{array}$} \\
\hline & & & \multirow{2}{*}{$\begin{array}{l}\text { Latitude } \\
\left({ }^{\circ}\right)\end{array}$} & \multirow{2}{*}{$\begin{array}{l}\text { Longitude } \\
\left({ }^{\circ}\right)\end{array}$} & \multirow{2}{*}{$\begin{array}{l}\text { Depth } \\
(\mathrm{km})\end{array}$} & \multirow[t]{2}{*}{ Ref } & \multicolumn{2}{|c|}{ USGS } & \multirow[t]{2}{*}{ Other } & \multirow{2}{*}{$\begin{array}{c}\text { Moment } \\
\text { M }\end{array}$} & \multirow[t]{2}{*}{ MM } & \multirow[t]{2}{*}{ Ref } & \\
\hline $\mathrm{Yr}$ & Mo Da & $\mathrm{h} \mathrm{ms}$ & & & & & $m_{b}$ & $\mathbf{M}_{\mathbf{s}}$ & & & & & \\
\hline 1969 & 1120 & 01000 & $37.449 \mathrm{~N}$ & $80.932 \mathrm{~W}$ & 003 & 349 & 4.3 & - & $4.60 M_{n} G B$ & 4.53HRN & VI & 42 & 300 \\
\hline
\end{tabular}

[Reference (Ref.) numbers given in parentheses at the end of each description refer to sources of data in table 1. Magnitude values are described in the Introduction, and codes are defined in table 2.]

1969. Nov. 20 (Nov. 19). Southern West Virginia. Minor damage occurred in Giles County, Va., at Glen Lyn and Rich Creek, and at three towns in southern West Virginia. At Glen Lyn, a few bricks were knocked from a chimney, windows were broken, and plaster was broken from most of the walls in an old house. At Rich Creek, plaster cracked and fell and windows were broken. A cornice reportedly was shaken from one building in Henry County, at Collinsville, Va. Windows also were broken in southern Mercer County, W.Va., at Lerona, Oakvale, and Elgood. Felt over all or parts of nine States: Georgia, Kentucky, Maryland, North Carolina, Ohio, South Carolina, Tennessee, Virginia, and West Virginia. Magnitude $4.7 \mathrm{M}_{\mathrm{fa}} \mathrm{NUT}$, $4.54 \mathrm{M} \mathrm{JOH}$. (Ref. $38,263,42,349,353,508$.) 


\section{WYOMING}

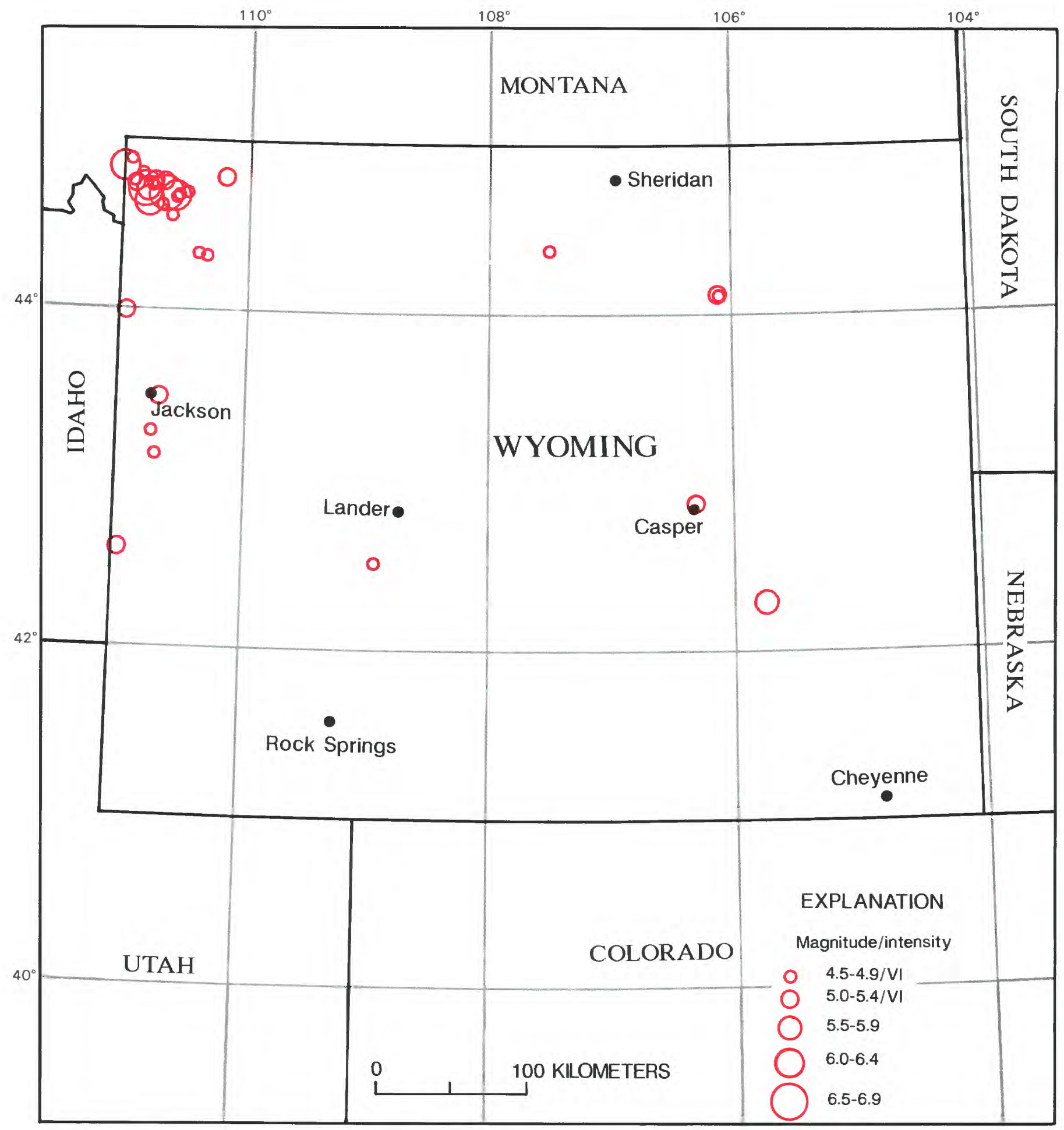

Earthquakes in Wyoming with magnitudes $\geq 4.5$ or intensity $\geq$ VI. 
WYOMING

[See table 1 for hypocenter and intensity references and table 2 for defimitions of magnitude source codes. (‥ felt area is less than $1,000 \mathrm{~km}{ }^{2}$. Leader (--) indicates infornation is not available!

\begin{tabular}{|c|c|c|c|c|c|c|c|c|c|c|c|c|c|}
\hline \multirow{2}{*}{\multicolumn{2}{|c|}{ Date }} & \multirow{2}{*}{$\begin{array}{c}\text { Origin } \\
\text { time (UTC) }\end{array}$} & \multicolumn{4}{|c|}{ Hypocenter } & \multicolumn{4}{|c|}{ Magnitude } & \multicolumn{2}{|c|}{ Intensity } & \multirow{3}{*}{$\begin{array}{c}\text { Felt area } \\
\left(1,000 \mathrm{~km}^{2}\right)\end{array}$} \\
\hline & & & \multirow{2}{*}{$\begin{array}{l}\text { Latitude } \\
\left(^{\circ}\right)\end{array}$} & \multirow{2}{*}{$\begin{array}{l}\text { Longitude } \\
\left.\text { ( }{ }^{\circ}\right)\end{array}$} & \multirow{2}{*}{$\begin{array}{l}\text { Depth } \\
(\mathrm{km})\end{array}$} & \multirow[t]{2}{*}{ Ref } & \multicolumn{2}{|c|}{ USGS } & \multirow[t]{2}{*}{ Other } & \multirow{2}{*}{$\begin{array}{c}\text { Moment } \\
\text { M }\end{array}$} & \multirow[t]{2}{*}{ MM } & \multirow[t]{2}{*}{ Ref } & \\
\hline $\mathrm{Yr}$ & Mo $\mathrm{Da}$ & $\mathrm{h} \mathrm{m} \mathrm{s}$ & & & & & $m_{b}$ & $\mathrm{M}_{\mathrm{S}}$ & & & & & \\
\hline 1897 & 1114 & 1330 & $42.9 \mathrm{~N}$ & $106.3 \mathrm{~W}$ & - & 38 & - & - & - & - & VI & 38 & - \\
\hline 1925 & $11 \quad 18$ & 0145 & $44.4 \mathrm{~N}$ & $107.5 \mathrm{~W}$ & - & 280 & - & - & - & - & $\mathrm{v}$ & 280 & 30 \\
\hline 1930 & 0612 & 0915 & $42.6 \mathrm{~N}$ & $111.0 \mathrm{~W}$ & - & 3 & - & - & - & - & VI & 3 & - \\
\hline 1932 & 0126 & 1013 & $43.5 \mathrm{~N}$ & $110.7 \mathrm{~W}$ & - & 5 & - & - & - & - & VI & 279 & 3 \\
\hline 1936 & 0115 & 0440 & $44.0 \mathrm{~N}$ & $111.0 \mathrm{~W}$ & - & 38 & - & - & - & - & VI & 38 & 3 \\
\hline 1959 & $08 \quad 18$ & 075616.8 & $44.699 \mathrm{~N}$ & $110.705 \mathrm{~W}$ & 005 & 576 & - & - & 6.50Ukn BRK & - & Felt & 32 & - \\
\hline 1959 & $08 \quad 18$ & 084147.5 & $44.854 \mathrm{~N}$ & $111.049 \mathrm{~W}$ & 005 & 576 & - & - & $6.00 \mathrm{Uk}$ & - & VI & 32 & - \\
\hline 1959 & 0818 & 110348.1 & $44.719 \mathrm{~N}$ & $110.850 \mathrm{~W}$ & 005 & 576 & - & - & $5.60 \mathrm{Ukn}$ BRK & - & Felt & 32 & - \\
\hline 1959 & $08 \quad 18$ & 152605.9 & $44.721 \mathrm{~N}$ & $110.871 \mathrm{~W}$ & 005 & 576 & - & - & $6.50 \mathrm{Ukn}$ PAS & 28Ds & . & 32 & - \\
\hline 1959 & 0819 & 040401.7 & $44.648 \mathrm{~N}$ & $110.840 \mathrm{~W}$ & 005 & 576 & - & - & $6.00 \mathrm{Ukn} \mathrm{BRK}$ & 5.98DOR & $\mathrm{v}$ & 8 & - \\
\hline 1959 & $08 \quad 19$ & 194347.2 & $44.756 \mathrm{~N}$ & $110.960 \mathrm{~W}$ & 005 & 576 & - & - & $5.00 \mathrm{Ukn}$ BRK & - & Felt & 32 & - \\
\hline 1963 & 0308 & & $44.8 \mathrm{~N}$ & $110.2 \mathrm{~W}$ & 033 & 266 & 3.8 & - & 年 & - & $\pi$ & & @ \\
\hline 1963 & 0924 & 063552.1 & $44.9 \mathrm{~N}$ & $111.0 \mathrm{~W}$ & 033 & 266 & 4.7 & - & - & - & $\mathrm{v}$ & 36 & - \\
\hline 1973 & 0330 & 003256.1 & $44.34 \mathrm{~N}$ & $110.34 \mathrm{~W}$ & 001 & 307 & 4.6 & - & $4.60 \mathrm{M}_{\mathrm{D}} \mathrm{GM}$ & - & Felt & 46 & - \\
\hline 1973 & 0331 & 203331.8 & $44.35 \mathrm{~N}$ & $110.41 \mathrm{~W}$ & 001 & 307 & 5.1 & - & $4.70 \mathrm{M}_{\mathrm{D}} \mathrm{GM}$ & - & Felt & 46 & - \\
\hline 1974 & 0609 & 0 & 44.77 & $\mathrm{v}$ & 005 & 30 & - & - & $4.90 \mathrm{~N}$ & - & II & 7 & - \\
\hline 1974 & 0830 & 4158.8 & $44.63 \mathrm{~N}$ & $110.73 \mathrm{~W}$ & 000 & 307 & 4.5 & - & $4.50 \mathrm{M}_{\mathrm{L}} \mathrm{C}$ & - & $\mathrm{v}$ & 7 & - \\
\hline 1974 & 1022 & 084307.1 & $44.74 \mathrm{~N}$ & $110.81 \mathrm{~W}$ & 005 & 47 & 4.6 & - & - & - & IV & 47 & - \\
\hline 1975 & 0630 & 184757.1 & $44.68 \mathrm{~N}$ & $110.61 \mathrm{~W}$ & 003 & 30 & 46 & - & $4.80 \mathrm{M}_{\mathrm{L}}$ GS & - & - & $\pi$ & - \\
\hline 1975 & 0630 & 185412.7 & $44.68 \mathrm{~N}$ & $110.62 \mathrm{~W}$ & 002 & 307 & 5.6 & 5.9 & $6.40 \mathrm{M}_{\mathrm{L}}$ GS & - & VII & 48 & 50 \\
\hline 1975 & 0630 & 190027.4 & $44.77 \mathrm{~N}$ & $110.72 \mathrm{~W}$ & 005 & 48 & 5.1 & - & $5.30 \mathrm{M}_{\mathrm{L}}$ GS & - & - & - & - \\
\hline 1075 & & 195633.7 & $.71 \mathrm{~N}$ & $w$ & oc & 48 & 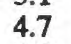 & t & 4. & 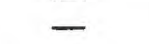 & 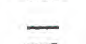 & & 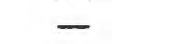 \\
\hline 1975 & 0630 & 202056.6 & $44.69 \mathrm{~N}$ & $110.59 \mathrm{~W}$ & 00 & 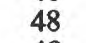 & & - & 4.6 & - & III & & \\
\hline 1976 & 1208 & 144059.1 & $44.76 \mathrm{~N}$ & $110.79 \mathrm{~W}$ & 005 & 49 & 5. & - & $4.60 \mathrm{M}_{\mathrm{L}} \mathrm{G}$ & - & $\mathrm{V}$ & 49 & 5 \\
\hline 1976 & 1209 & 223623.7 & $44.77 \mathrm{~N}$ & $110.80 \mathrm{~W}$ & 005 & 49 & 4.5 & - & $5.10 \mathrm{M}_{\mathrm{L}} \mathrm{GS}$ & - & v & 49 & 17 \\
\hline 1976 & & & & & 005 & & & 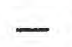 & & & $\mathrm{v}$ & 49 & @ \\
\hline 1980 & 0222 & 101827.7 & $44.81 \mathrm{~N}$ & $110.90 \mathrm{~W}$ & 000 & 30 & 4 & - & $4.70 \mathrm{M}_{\mathrm{L}} \mathrm{GS}$ & - & IV & & - \\
\hline 1983 & 0206 & 202516.5 & $44.571 \mathrm{~N}$ & $3 w$ & $\alpha$ & 36 & 4. & - & $4.50 \mathrm{M}_{\mathrm{L}} \mathrm{GS}$ & - & $\mathrm{v}$ & & 5 \\
\hline 1983 & 1220 & 225223.7 & $43.294 \mathrm{~N}$ & $110.767 \mathrm{~W}$ & 005 & 360 & 4.5 & - & - & - & IV & 360 & - \\
\hline 1984 & 0529 & 201829.6 & $44.134 \mathrm{~N}$ & $106.099 \mathrm{~W}$ & 015 & 522 & 5.0 & - & $4.80 \mathrm{M}_{\mathrm{n}}$ GDW & - & v & $370^{\circ}$ & 56 \\
\hline 1984 & 0908 & 005931.1 & $44.138 \mathrm{~N}$ & $10 \mathrm{~W}$ & 015 & 522 & 5 . & - & $5.00 \mathrm{M}_{\mathrm{n}} \mathrm{GDW}$ & $4.94 \mathrm{JOH}$ & $\mathrm{v}$ & 370 & 68 \\
\hline 1984 & 1018 & 153022.0 & $42.317 \mathrm{~N}$ & $105.735 \mathrm{~W}$ & 022 & 52 & 5. & 5.1 & $5.50 \mathrm{M}_{\mathrm{n}}$ GDW & - & VI & 370 & 287 \\
\hline 1984 & 1103 & 093008.4 & $42.534 \mathrm{~N}$ & $108.919 \mathrm{~W}$ & 005 & 522 & 5.0 & 4.1 & $4.50 \mathrm{M}_{\mathrm{n}}$ GDW & - & VI & 370 & 15 \\
\hline 1985 & 0907 & 034729.2 & $43.156 \mathrm{~N}$ & $110.724 \mathrm{~W}$ & 005 & 371 & - & - & $4.60 \mathrm{M}_{\mathrm{L}} \mathrm{GS}$ & - & $\mathrm{v}$ & 371 & 22 \\
\hline
\end{tabular}

[Reference (Ref.) numbers given in parentheses at the end of each description refer to sources of data in table 1. Magnitude values are described in the Introduction, and codes are defined in table 2.]

1897. Nov. 14. Casper, Natrona County, Wyo. The northeast corner of the Grand Central Hotel at Casper sustained a 5 - to 10 -cm-wide crack that extended from the third to the first floor. The ceiling in the hotel lobby also was cracked. An almost deafening noise preceded the shaking. (Ref. 38, 359.)

1930. June 12. Grover, northern Lincoln County, Wyo. A minor earthquake cracked a brick building in Grover and a concrete swimming pool north of Grover. Cracks also formed in plaster, and clocks stopped running. Slight aftershocks continued until Nov. 16, 1930. (Ref. 3.)

1932. Jan. 26. Western Wyoming. At Jackson, in southern Teton County, an earthquake cracked floors and foundations, broke plaster, and tore wallpaper. Slight damage also occurred in the nearby towns of Grovont, Kelly, and Moran. One light foreshock was observed in the area on Jan. 25, and several minor aftershocks occurred through Jan. 28. (Ref. 5, 38, 279.) 


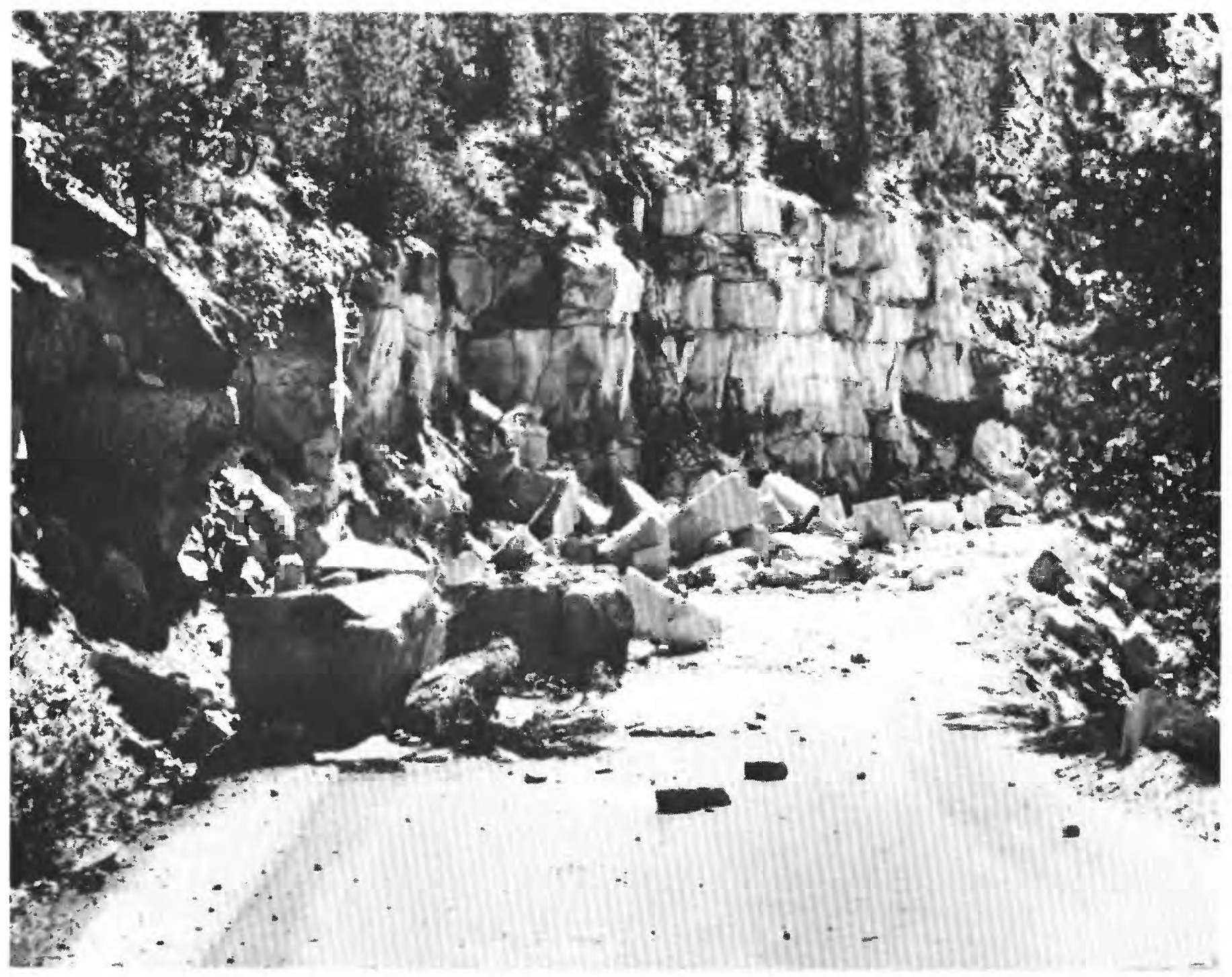

Rockslide on Virginia Cascade Road in Yellowstone National Park, Wyoming, caused by the earthquake of June 30, 1975.

1936. Jan. 15 (Jan. 14). Yellowstone National Park, Wyo. An earthquake caused extensive cracks in two chimneys at the south entrance to the park, in northwest Wyoming. (Ref. 9, 38.)

1959. Aug. 18, 0841 UTC. Yellowstone National Park, Wyo. An aftershock of the August 17, 1959, earthquake at Hegben Lake, Mont., broke a chimney at the Old Faithful Ranger Station in northwest Wyoming. Also felt at Fairfield, Idaho. (Ref. 32, 38,576 .)

1963. Mar. 8. Yellowstone National Park, Wyo. At the winter caretaker' $s$ house in Canyon, large cracks separated the walls and ceilings in several rooms, and plaster cracked and fell. Aftershocks continued until Mar. 12. (Ref. 36, 266.)
1975. June 30. Yellowstone National Park, Wyo. This widely felt earthquake downed one chimney in the park at Norris Junction and formed cracks $90 \mathrm{~m}$ long in a parking lot. Rockfalls and landslides closed or hindered traffic on many roads in the park. Two new geysers formed; the Gibbon River was muddied; and the earth settled and cracked in the backcountry. Several aftershocks occurred through early July. Also felt in Montana, Idaho, South Dakota, Nebraska, Nevada, Utah, and Washington. Magnitude 6.1 Ukn BRK. (Ref. 38, 48, 307.)

1984. Oct. 18. Eastern Wyoming. Although this earthquake was felt over a large part of eight States (see fig. 64), only minor property damage occurred. The damage was characterized by cracked chimneys and 


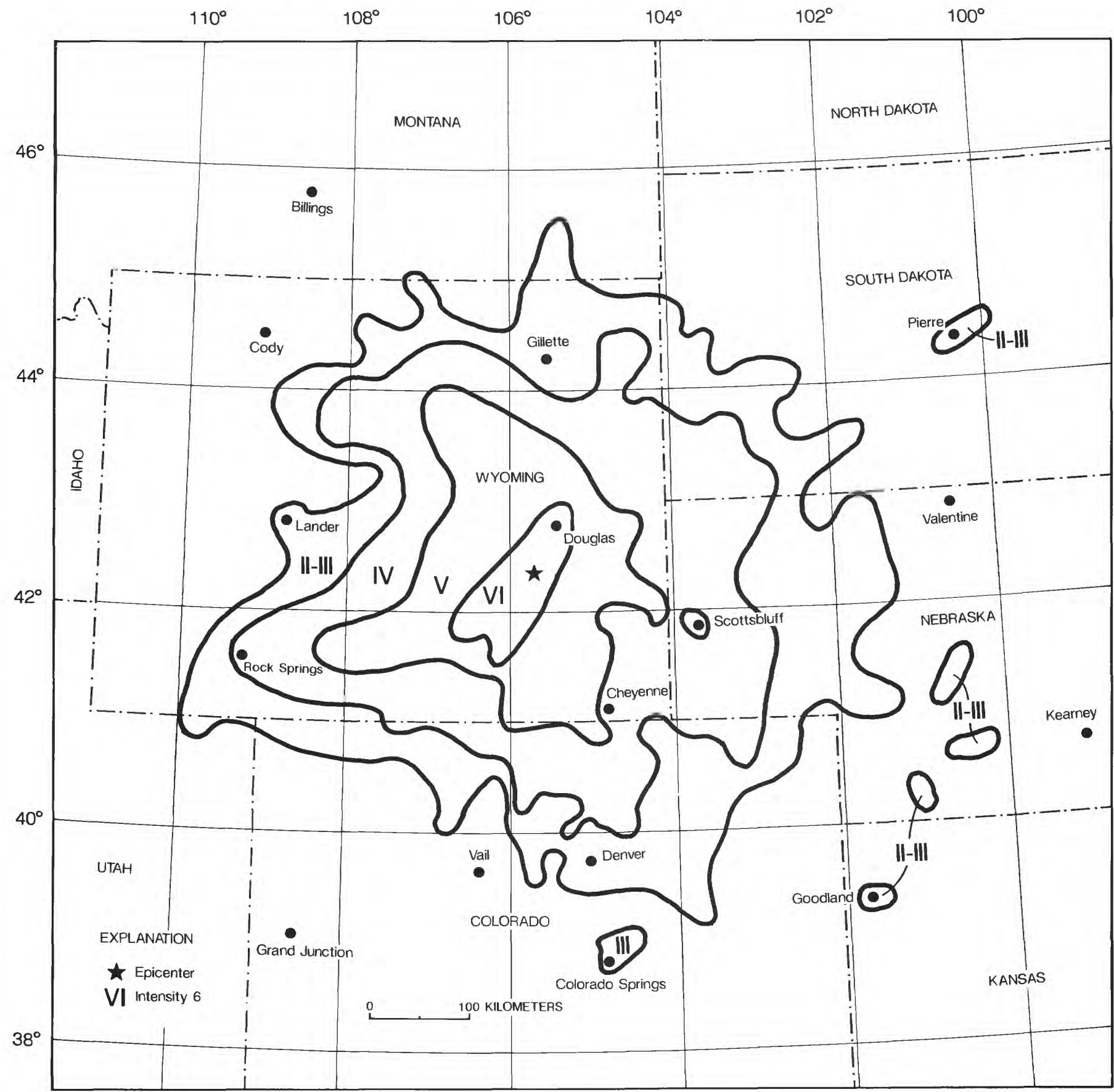

FIGURE 64.-Isoseismal map for the eastern Wyoming earthquake of October 18, 1984. This is a simplified version of figure 28 in reference 370 of table 1 .

foundations and cracked brick and cinder-block walls at several towns, including Casper, Douglas, Guernsey, Hanna, Lusk, McFadden, Medicine Bow, Rock River, and Shirley Basin. Underground pipes were broken in Natrona County, at Casper, and in Carbon County, at Shirley Basin, about $40 \mathrm{~km}$ south of Casper. In addition, slight damage was reported at a few towns in Colorado, Nebraska, and South Dakota.

An unusual report of structural damage to two five-story buildings was received from Golden, Colo., about $300 \mathrm{~km}$ south of the epicenter. This damage, which consisted of foundation failure, cracks in walls, 
and a gas leak, may not have been caused by the $\mid$ walls, foundations, and windows; and glass was broearthquake. Felt in Colorado, Kansas, Montana, ken at the local hospital. About $30 \mathrm{~km}$ south of Nebraska, South Dakota, Utah, and Wyoming. Mag- Lander, at South Pass City, cracks in windows and nitude 5.5 $\mathrm{M}_{\mathrm{L}}$ GS, 5.3 $\mathrm{M}_{\mathrm{n}}$ TUL. (Ref. 370, 487, 522.) damage to glassware occurred, and residents had dif-

1984. Nov. 3. Western Wyoming. At Lander, ficulty walking and standing. Magnitude $4.9 \mathrm{M}_{\mathrm{L}} \mathrm{BU}$, Fremont County, 50 buildings sustained cracks in $5.1 \mathrm{M}_{\mathrm{L}}$ GS. (Ref. 370, 522.) 



\section{TABLES 1-3}


TABLE 1.-Hypocenter and intensity references

[Please note that numbers in this table are not sequential because these references were selected from a master list compiled for other USGS publications]

1. Heck, N.H., and Bodle, R.R., 1930, United States earthquakes 1928: U.S. Coast and Geodetic Survey, Serial 483, 28 p.

2. Heck, N.H., and Bodle, R.R., 1931, United States earthquakes 1929: U.S. Coast and Geodetic Survey, Serial 511, 55 p.

3. Neumann, Frank, and Bodle, R.R., 1932, United States earthquakes 1930: U.S. Coast and Geodetic Survey, Serial 539, $25 \mathrm{p}$.

4. Neumann, Frank, 1932, United States earthquakes 1931: U.S. Coast and Geodetic Survey, Serial 553, 26 p.

5. Neumann, Frank, 1934, United States earthquakes 1932: U.S. Coast and Geodetic Survey, Serial 563, 21 p.

6. Neumann, Frank, 1935, United States earthquakes 1933: U.S. Coast and Geodetic Survey, Serial 579, 82 p.

7. Neumann, Frank, 1936, United States earthquakes 1934: U.S. Coast and Geodetic Survey, Serial 593, 99 p.

8. Neumann, Frank, 1937, United States earthquakes 1935: U.S. Coast and Geodetic Survey, Serial 600,90 p.

9. Neumann, Frank, 1938, United States earthquakes 1936: U.S. Coast and Geodetic Survey, Serial 610, 45 p.

10. Neumann, Frank, 1940, United States earthquakes 1937: U.S. Coast and Geodetic Survey, Serial 619, 55 p.

11. Neumann, Frank, 1940, United States earthquakes 1938: U.S. Coast and Geodetic Survey, Serial 629, 59 p.

12. Bodle, R.R., 1941, United States earthquakes 1939: U.S. Coast and Geodetic Survey, Serial 637, 69 p.

13. Neumann, Frank, 1942, United States earthquakes 1940: U.S. Coast and Geodetic Survey, Serial 647, 74 p.

14. Neumann, Frank, 1943, United States earthquakes 1941: U.S. Coast and Geodetic Survey, Serial 655, 41 p.

15. Bodle, R.R., 1944, United States earthquakes 1942: U.S. Coast and Geodetic Survey, Serial 662, 44 p.

16. Bodle, R.R., 1945, United States earthquakes 1943: U.S. Coast and Geodetic Survey, Serial 672, 47 p.

17. Bodle, R.R., 1946, United States earthquakes 1944: U.S. Coast and Geodetic Survey, Serial 682, 43 p.

18. Bodle, R.R., and Murphy, L.M., 1947, United States earthquakes 1945: U.S. Coast and Geodetic Survey, Serial 699 , 38 p.

19. Bodle, R.R., and Murphy, L.M., 1948, United States earthquakes 1946: U.S. Coast and Geodetic Survey, Serial 714 , 48 p.

20. Murphy, L.M., 1950, United States earthquakes 1947: U.S. Coast and Geodetic Survey, Serial 730, 62 p.

21. Murphy, L.M., and Ulrich, F.P., 1951, United States earthquakes 1948: U.S. Coast and Geodetic Survey, Serial 746, 50 p.

22. Murphy, L.M., and Ulrich, F.P., 1951, United States earthquakes 1949: U.S. Coast and Geodetic Survey, Serial 748,64 p.

23. Murphy, L.M., and Ulrich, F.P., 1952, United States earthquakes 1950: U.S. Coast and Geodetic Survey, Serial 755 , 47 p.

24. Murphy, L.M., and Cloud, W.K., 1953, United States earthquakes 1951: U.S. Coast and Geodetic Survey, Serial 762,50 p.

25. Murphy, L.M., and Cloud, W.K., 1954, United States earthquakes 1952: U.S. Coast and Geodetic Survey, Serial 773, 112 p.

26. Murphy, L.M., and Cloud, W.K., 1955, United States earthquakes 1953: U.S. Coast and Geodetic Survey, Serial 785, 51 p.

27. Murphy, L.M., and Cloud, W.K., 1956, United States earthquakes 1954: U.S. Coast and Geodetic Survey, Serial 793, 110 p.

28. Murphy, L.M., and Cloud, W.K., 1957, United States earthquakes 1955: U.S. Coast and Geodetic Survey, 83 p.

29. Brazee, R.J., and Cloud, W.K., 1958, United States earthquakes 1956: U.S. Coast and Geodetic Survey, 78 p.

30. Brazee, R.J., and Cloud, W.K., 1959, United States earthquakes 1957: U.S. Coast and Geodetic Survey, 108 p.

31. Brazee, R.J., and Cloud, W.K., 1960, United States earthquakes 1958: U.S. Coast and Geodetic Survey, 76 p.

32. Eppley, R.A., and Cloud, W.K., 1961, United States earthquakes 1959: U.S. Coast and Geodetic Survey, 115 p.

33. Talley, H.C., and Cloud, W.K., 1962, United States earthquakes 1960: U.S. Coast and Geodetic Survey, 90 p.

34. Lander, J.F., and Cloud, W.K., 1963, United States earthquakes 1961: U.S. Coast and Geodetic Survey, 106 p.

35. Lander, J.F., and Cloud, W.K., 1964, United States earthquakes 1962: U.S. Coast and Geodetic Survey, 114 p.

36. Cloud, W.K., and von Hake, C.A., 1965, United States earthquakes 1963: U.S. Coast and Geodetic Survey, 69 p.

37. von Hake, C.A., and Cloud, W.K., 1966, United States earthquakes 1964: U.S. Coast and Geodetic Survey, 91 p.

38. Coffman, J.L., von Hake, C.A., and Stover, C.W., 1982, Earthquake history of the United States: U.S. National Oceanic and Atmospheric Administration and U.S. Geological Survey, Publication No. 41-1, revised edition, [through 1980], 258 p.

39. Coffman, J.L., and Stover, C.W., 1979, United States earthquakes 1977: U.S. National Oceanic and Atmospheric Administration and U.S. Geological Survey, $81 \mathrm{p}$.

40. von Hake, C.A., and Cloud, W.K., 1969, United States earthquakes 1967: U.S. Coast and Geodetic Survey, 90 p.

41. Coffman, J.L., and Cloud, W.K., 1970, United States earthquakes 1968: U.S. Environmental Science Services Administration, $111 \mathrm{p}$.

42. von Hake, C.A., and Cloud, W.K., 1971, United States earthquakes 1969: U.S. National Oceanic and Atmospheric Administration, $80 \mathrm{p}$.

43. Coffman, J.L., and von Hake, C.A., 1972, United States earthquakes 1970: U.S. National Oceanic and Atmospheric Administration, $81 \mathrm{p}$.

44. Coffman, J.L., and von Hake, C.A., 1973, United States earthquakes 1971: U.S. National Oceanic and Atmospheric Administration, $174 \mathrm{p}$.

45. Coffman, J.L., and von Hake, C.A., 1974, United States earthquakes 1972: U.S. National Oceanic and Atmospheric Administration, $119 \mathrm{p}$.

46. Coffman, J.L., von Hake, C.A., Spence, W., Carver, D.L., Covington, P.A., Dunphy, G.J., Irby, W.L., Person, W.J., and Stover, C.W., 1975, United States earthquakes 1973: U.S. National Oceanic and Atmospheric Administration and U.S. Geological Survey, $112 \mathrm{p}$.

47. Coffman, J.L., and Stover, C.W., 1976, United States earthquakes 1974: U.S. National Oceanic and Atmospheric Administration and U.S. Geological Survey, $135 \mathrm{p}$. 
TABLE 1.-Hypocenter and intensity references-Continued

[Please note that numbers in this table are not sequential because these references were selected from a master list compiled for other USGS publications]

48. Coffman, J.L., and Stover, C.W., 1977, United States earthquakes 1975: U.S. National Oceanic and Atmospheric Administration and U.S. Geological Survey, $136 \mathrm{p}$.

49. Coffman, J.L., and Stover, C.W., 1978, United States earthquakes 1976: U.S. National Oceanic and Atmospheric Administration and U.S. Geological Survey, $94 \mathrm{p}$.

52. Williams, J.S., and Tapper, M.S., 1953, Earthquake history of Utah, 1850 to 1949: Seismological Society of America Bulletin, v. 43, no. 3, p. 191-218.

53. Berg, J.W., and Baker, C.D., 1963, Oregon earthquakes, 1841 through 1958: Seismological Society of America Bulletin, v. 53, no. 1, p. 95-108.

54. Slemmons, D.B., Jones, A.E., and Gimlett, J.I., 1965, Catalog of Nevada earthquakes, 1852-1960: Seismological Society of America Bulletin, v. 55, no. 2, p. 519-565.

55. MacCarthy, G.R., 1964, A descriptive list of Virginia earthquakes through 1960: Elisha Mitchell Scientific Society Journal, v. 80, no. 2, p. 94-114.

56. Townley, S.D., and Allen, M.W., 1939, Descriptive catalog of earthquakes of the Pacific Coast of the United States, 1769 to 1928: Seismological Society of America Bulletin, v. 29, no. 1, p. 1-297.

59. Brigham, W.T., 1871, Historical notes on the earthquakes of New England, 1638-1869: Memoirs Boston Society of Natural History, v. 2., p. 1-28.

60. Bradley, E.A., and Bennett, T.J., 1965, Earthquake history of Ohio: Seismological Society of America Bulletin, v. 55, no. 4, p. 745-752.

63. Merriam, D.G., 1956, History of earthquakes in Kansas: Seismological Society of America Bulletin, v. 46, no. 2, p. $87-96$.

67. Moneymaker, B.C., 1957, Earthquakes in Tennessee and nearby sections of neighboring States 1901 to 1925 : Tennessee Academy of Science Journal, v. 32, no. 2, p. 91-105.

68. Moneymaker, B.C., 1958, Earthquakes in Tennessee and nearby sections of neighboring States 1926 to 1950: Tennessee Academy of Science Journal, v. 33, no. 3, p. 224-239.

71. MacCarthy, G.R., 1957, An annotated list of the North Carolina earthquakes: Elisha Mitchell Scientific Society Journal, v. 73 , no. 1 , p. 84-100.

72. U.S. Coast and Geodetic Survey, Abstracts of earthquake reports for the United States, 1967 through 1973.

74. U.S. Geological Survey, Preliminary determination of epicenters report and monthly listing, January 1961-1989 [formerly by U.S. Coast and Geodetic Survey, U.S. Environmental Science Services Administration, and U.S. National Oceanic and Atmospheric Administration].

75. von Hake, C.A., and Cloud, W.K., 1967, United States earthquakes 1965: U.S. Coast and Geodetic Survey, 91 p.

76. Smith, W.E.T., 1962, Earthquakes of Eastern Canada and adjacent areas, 1534-1927: Publications of the Dominion Observatory, Ottawa, v. 26, no. 5, p. 271-301.

77. Smith, W.E.T., 1966, Earthquakes of Eastern Canada and adjacent areas, 1928-1959: Publications of the Dominion Observatory, Ottawa, v. 32, no. 3, p. 87-121.

78. Weston Geophysical Research, Inc., Weston, Mass., 1976, Historical seismicity of New England, prepared for Boston Edison Company, Preliminary Safety Analysis Report, Docket No. 50-471, 641 p.

81. von Hake, C.A., and Cloud, W.K., 1968, United States earthquakes 1966: U.S. Coast and Geodetic Survey, 110 p.

84. Woollard, G.P., 1968, A catalogue of earthquakes in the United States prior to 1925 based on unpublished data compiled by Harry Fielding Reid and unpublished sources prior to 1930: Hawaii Institute of Geophysics, University of Hawaii, Data Report No. 10, 163 p.

86. Bollinger, G.A., 1975, A catalogue of Southeastern United States earthquakes 1754 through 1974: Virginia Polytechnic Institute and State University, Department of Geological Sciences, Research Bulletin 101, 68 p.

96. Taber, S., 1914, Seismic activity in the Atlantic coastal plain near Charleston, South Carolina: Seismological Society of America Bulletin, v. 4, no. 3, p. 108-160.

99. Dumas, D.B., Dorman, J.H., and Latham, G.V., 1980, A reevaluation of the August 16, 1931 Texas earthquake: Seismological Society of America Bulletin, v. 70, no. 4, p. 1171-1180.

101. Campbell, R.L., 1975, Historical sketches of colonial Florida: A facsimile reproduction of the 1892 edition, A University of Florida Book, The University of Florida Press, Gainsville, Fla.

105. Docekal, Jerry, 1970, Earthquakes of the stable interior, with emphasis on the midcontinent, v. 2: Lincoln, Neb., University of Nebraska, Ph.D. dissertation, [available from Ann Arbor, Mich., University Microfilms Ltd.], 332 p.

109. Heinrich, R.R., 1941, A contribution to the seismic history of Missouri: Seismological Society of America Bulletin, v. 31, no. 3, p. 187-224.

113. Nuttli, O.W., 1974, Magnitude-recurrence relation for central Mississippi valley earthquakes: Seismological Society of America Bulletin, v. 64 , no. 4, p. 1189-1207.

114. Nuttli, O.W., 1973, The Mississippi valley earthquakes of 1811 and 1812: Intensities, ground motion, and magnitudes: Seismological Society of America Bulletin, v. 63, no. 1, p. 227-248.

116. Varma, M.M., 1975, Seismicity of the eastern half of the United States (exclusive of New England): Bloomington, Ind., University of Indiana, $\mathrm{Ph} . \mathrm{D}$. dissertation, $176 \mathrm{p}$. 
TABLE 1.-Hypocenter and intensity references-Continued

[Please note that numbers in this table are not sequential because these references were selected from a master list compiled for other USGS publications]

121. Wood, H.O., 1955, The 1857 earthquake in California: Seismological Society of America Bulletin, v. 45, no. 1, p. 47-68.

124. Sellards, E.H., 1932, The Valentine, Texas, earthquake: The University of Texas Bulletin 3201, Contributions to Geology, p. 113-137.

126. Chiburis, E.F., 1979, Seismicity, recurrence rates, and the regionalization of the Northeast United States and adjacent Southeastern Canada: Weston, Mass., Weston Observatory, [for the U.S. Nuclear Regulatory Commission], NUREG/ CR-2309, $76 \mathrm{p}$.

129. Fryxell, F.M., 1940, The earthquakes of 1934 and 1935 in northwestern Illinois and adjacent parts of Iowa: Seismological Society of America Bulletin, v. 30, no. 3, p. 213-218.

132. Moneymaker, B.C., 1972, Earthquakes in Tennessee and nearby sections of neighboring States, 1951-1970: Tennessee Academy of Science Journal, v. 47, no. 4, p. 124-132.

134. Rockwood, C.G., 1880, Notices of recent earthquakes: American Journal of Science, v. 19, no. 112, p. $295-299$.

135. Taber, Stephen, 1915, Earthquakes in South Carolina during 1914: Seismological Society of America Bulletin, v. 5, no. 2, p. 96-99.

136. Rockwood, C.G., 1881, Notices of recent American earthquakes: American Journal of Science, v. 21, no. 123, p. 198-202.

140. Dutton, C.E., 1889, The Charleston earthquake of August 31, 1886, in Ninth Annual Report of the United States Geological Survey to the Secretary of the Interior 1887-1888, p. 209-628.

141. Pomeroy, P.W., and Fakundiny, R.H., 1976, Unpublished list of earthquakes used to compile the Seismic Activity and Geologic Structure in New York and Adjacent Areas map, New York State Museum and Science Service Map and Chart Series Number 27, 2 sheets.

142. Philadelphia Electric Company, 1970, Preliminary Safety Analysis Report, Limerick Generating Station, Units 1 and 2, Nuclear Regulatory Commission, Public Documents Room, p. 25-36.

143. Fuller, M.L., 1912, The New Madrid earthquake: U.S. Geological Survey Bulletin 494, 119 p.

145. Shaler, N.S., 1869, Earthquakes of the Western United States: Atlantic Monthly, v. 24, no. 15, p. 549-659.

149. Bradford, D.C., and Dahm, C.G., 1935, The Rodney, Missouri, earthquake of August 20, 1934: Seismological Society of America Bulletin, v. 25, no. 2, p. 154-160.

155. MacCarthy, G.R., and Sinha, E.Z., 1958, North Carolina earthquakes 1957: Elisha Mitchell Scientific Society Journal, v. 74, no. 2, p. 117-121.

159. Collins, R.H., 1874, History of Kentucky: By the late Lewis Collins (revised): Collins and Co., v. 1, 683 p.

162. Taber, Stephen, 1913, The South Carolina earthquake of January 1, 1913: Seismological Society of America Bulletin, v. 3 , p. 6-13.

163. Bollinger, G.A., 1972, Historical and recent seismic activity in South Carolina: Seismological Society of America Bulletin, v. 62 , no. 3, p. 851-864.

167. Hopper, M.G., and Bollinger, G.A., 1971, The earthquake history of Virginia 1774-1900: Blacksburg, Va., Virginia Polytechnic Institute and State University, Department of Geological Sciences, $87 \mathrm{p}$.

168. Willson, F.F., 1926, The Montana earthquake of June 27, 1925, damage in Gallatin County: Seismological Society of America Bulletin, v. 16, no. 3, p. 164-169.

173. Nuttli, O.W., and Herrmann, R.B., 1978, Credible earthquakes for the Central United States, state-of-the-art for assessing earthquake hazards in the United States: U.S. Army, Chief of Engineers Report 12, p. 1-99.

174. DuBois, S.M., and Wilson, F.W., 1978, A revised and augmented list of earthquake intensities for Kansas, 1867-1977: Kansas Geological Survey, Lawrence, Kans., The University of Kansas, Environmental Geology Series 2, 56 p.

179. MacCarthy, G.R., 1958, A note on the Virginia earthquake of 1833: Seismological Society of America Bulletin, v. 48, no. 2, p. $177-180$.

186. Watson, T.L., 1918, The Virginia earthquake of April 9, 1918: Seismological Society of America Bulletin, v. 8, no. 4, p. 105-116.

187. Watson, T.L., 1919, Earthquake in Warren and Rappahannock Counties, Virginia, September 5, 1919: Seismological Society of America Bulletin, v. 9, no. 4, p. 128-134.

189. Bollinger, G.A., and Hopper, M.G., 1972, The earthquake history of Virginia 1900-1970: Blacksburg, Va., Virginia Polytechnic Institute and State University, Department of Geological Sciences, 85 p.

190. Pakiser, L.C., 1976, Review of intensity of Giles County 1897 earthquake: U.S. Geological Survey unpublished memorandum.

194. Berkey, C.P., 1945, A geological study of the Massena-Cornwall earthquake of September 5, 1944, and its bearing on the proposed St. Lawrence River Project: United States Engineer Office, Corp of Engineers, New York, p. 1-18.

201. Armbruster, J.G., and Seeber, Leonardo, 1987, The 23 April Martic earthquake and the Lancaster seismic zone in eastern Pennsylvania: Seismological Society of America Bulletin, v. 77, no. 3, p. 877-890.

208. Byerly, Perry, 1926, The Montana earthquake of June 28, 1925, G.M.C.T.: Seismological Society of America Bulletin, v. 16 , no. 4 , p. $209-265$.

211. Rockwood, C.G., 1876, Notices of recent American earthquakes-no. 6: American Journal of Science, v. 12, third series, p. 25-30. 
TABLE 1.-Hypocenter and intensity references-Continued

[Please note that numbers in this table are not sequential because these references were selected from a master list compiled for other USGS publications]

214. Gordon, D.W., 1988, Revised instrumental hypocenters and correlation of earthquake locations and tectonics in the Central United States: U.S. Geological Survey Professional Paper 1364, 69p.

218. U.S. Coast and Geodetic Survey, Seismological Reports, July 1924 through December 1927.

228. Miller, H.J., 1956, The Oklahoma earthquake of April 9, 1952: Seismological Society of America Bulletin, v. 46, no. 4, p. 269-279.

234. Cook, K.L., and Smith, R.B., 1967, Seismicity in Utah, 1850 through June 1965: Seismological Society of America Bulletin, v. 57, no. 4, p. $689-718$.

237. Lawson, J.E., Luza, K.V., DuBois, R.L., and Foster, P.H., 1979, Inventory, detection, and catalog of Oklahoma earthquakes: Oklahoma Geological Survey, [text to accompany Map GM-19], $15 \mathrm{p}$.

238. Seismological Society of America, 1952, Seismological Notes: Seismological Society of America Bulletin, v. 42, no. 3, p. 271-281.

240. Stover, C.W., and von Hake, C.A., 1980, United States earthquakes 1978: U.S. Geological Survey and U.S. National Oceanic and Atmospheric Administration, $112 \mathrm{p}$.

249. Qamar, A.I., and Stickney, M.C., 1983, Montana earthquakes 1869-1979: Butte, Mont., Montana School of Mines, Montana Bureau of Mines and Geology Memoir No. 51, 79 p.

255. Holden, E.S., 1898, A catalogue of earthquakes on the Pacific Coast 1769 to 1897: Smithsonian Miscellaneous Collections No. 1087,253 p.

257. Reid, H.F., 1911, Remarkable earthquakes in central New Mexico in 1906 and 1907: Seismological Society of America Bulletin, v. 1, no. 1, p. 10-16.

258. Gutenberg, Beno, and Richter, C.F., 1954, Seismicity of the Earth and associated phenomena: New York, Hafner Publishing Company, Inc., $310 \mathrm{p}$.

259. U.S. Coast and Geodetic Survey, Abstracts of earthquake reports for the Pacific Coast and the Western mountain region, January 1, 1934, to December 31, 1966, and 1933 abstracts from the San Francisco office, U.S. Department of Commerce.

260. Stover, C.W., 1990, U.S. Geological Survey, recomputed hypocenters (unpublished data).

261. Sanford, A.R., Olsen, H.N., and Jaksha, L.H., 1981, Earthquakes in New Mexico, 1849-1977: New Mexico Bureau of Mines and Mineral Resources Circular 171, $20 \mathrm{p}$.

262. Stover, C.W., and von Hake, C.A., 1981, United States earthquakes 1979: U.S. Geological Survey and U.S. National Oceanic and Atmospheric Administration, $170 \mathrm{p}$.

264. Herrmann, R.B., Dewey, J.W., and Park, Sam-Kuen, 1980, The Dulce, New Mexico, earthquake of 23 January 1966: Seismological Society of America Bulletin, v. 70, no. 6, p. 2171-2183.

265. International Seismological Summary, 1913-1963, Kew Observatory, Kew, England.

266. U.S. Coast and Geodetic Survey, Seismological Bulletin MSI 1 through MSI 316, January 1934 through May 1967.

269. Hammond, J.F., 1966, A surgeon's report on Socorro, N. Mex., 1852: Santa Fe Stagecoach Press, 47 p.

270. Northrop, S.A., Unpublished notes, newspaper clippings, and questionnaires, New Mexico University, Albuquerque, N. Mex.

272. Monthly Weather Review, July 1891 to June 1924, v. 19 to 68, U.S. Department of Agriculture, Washington, D.C.

273. Hadsell, F.A., 1968, History of earthquake activity in Colorado: in Geophysical and geological studies of the relationship between the Denver earthquakes and the Rocky Mountain Arsenal well, Colorado School of Mines Quarterly, v. 63, no. 1, p. $57-72$.

274. Herrmann, R.B., Park, Sam-Kuen, and Wang, Chien-Ying, 1981, The Denver earthquakes of 1967-1968: Seismological Society of America Bulletin, v. 71, no. 3, p. 731-745.

277. Taggart, James, and Baldwin, Frank, 1982, Earthquake sequence of 1938-1939 in Mogollen Mountains, New Mexico: New Mexico Geology, v. 4, no. 4, p. 49-52.

279. Fryxell, Fritiof, 1933, Earthquake shocks in Jackson Hole, Wyoming: Seismological Society of America Bulletin, v. 23, no. 4, p. $167-168$.

280. Pardee, J.T., 1927, Earthquake in the Bighorn Mountains, Wyoming, November 17, 1925: Seismological Society of America Bulletin, v. 17, no. 3, p. 129-136.

281. Tocher, Don, 1962, The Hebgen Lake, Montana, earthquake of August 17, 1959, MST: Seismological Society of America Bulletin, v. 52, no. 2, p. 153-162.

283. Dames and Moore, 1981, Geologic and seismologic investigations for Rocky Flats Plant, report prepared for U.S. Department of Energy, v. 1, chapter 8.0, p. 8.0-1 to 8.0-18.

288. Bollinger, G.A., and Visvanathan, T.R., 1977, The seismicity of South Carolina prior to 1886: U.S. Geological Survey Professional Paper 1028-C, p. 33-42.

289. Visvanathan, T.R., 1980, Earthquakes in South Carolina 1698-1975: South Carolina Geological Survey Bulletin 40, 61 p.

292. Hileman, J.A., Allen, C.R., and Nordquist, J.M., 1973, Seismicity of the southern California region, 1 January 1932 to 31 December 1972: California Institute of Technology, Pasadena, $395 \mathrm{p}$. 
TABLE 1-Hypocenter and intensity references-Continued

[Please note that numbers in this table are not sequential because these references were selected from a master list compiled for other USGS publications]

294. Seismological Society of America, 1951, Seismological Notes: Seismological Society of America Bulletin, v. 41, p. 243-254 and p. 389-404.

298. Arabasz, W.J., Smith, R.B., and Richins, W.D., 1979, Earthquake studies in Utah, 1850 to 1978: University of Utah, Department of Geology and Geophysics, $552 \mathrm{p}$.

299. Bulletin of the International Seismological Centre, 1964 to 1989, Newbury, Berkshire, United Kingdom.

300. Stover, C.W., and von Hake, C.A., 1982, United States earthquakes 1980: U.S. Geological Survey and U.S. National Oceanic and Atmospheric Administration, $182 \mathrm{p}$.

301. Nuttli, O.W., 1979, Seismicity of the Central United States, Geology in the siting of nuclear power plants: Geological Society of America, Reviews in Engineering Geology, v. 4, p. 67-107.

302. Street, R.L., 1980, The southern Illinois earthquake of September 27, 1981: Seismological Society of America Bulletin, v. 70, no. 3, p. 915-920.

307. Pitt, A.M., 1980, Catalog of earthquakes in the Yellowstone Park-Hebgen Lake region from November 1972 to December 1975: U.S. Geological Survey Open-File Report 80-2006, 32 p.

308. Pitt, A.M., 1982, Catalog of earthquakes in the Yellowstone Park-Hebgen Lake region from January 1980 to September 1981: U.S. Geological Survey, unpublished data.

310. Ryall, Alan, 1962, The Hebgen Lake, Montana, earthquake of August 18, 1959: P-waves: Seismological Society of America Bulletin, v. 52, no. 2, p. 235-271.

311. Pack, F.J., 1921, The Elsinore earthquakes in central Utah, September 29 and October 1, 1921: Seismological Society of America Bulletin, v. 11, no. 3, p. 155-165.

312. Pardee, J.T., 1927, The Montana earthquake of June 27, 1925, in Shorter contributions to general geology 1926: U.S. Geological Survey Professional Paper 147, p. 7-23.

314. Jefferson, Thomas, 1774, Unpublished memorandum book for 1774: Massachusetts Historical Society.

315. Jones, A.E., 1975, Recording of earthquakes at Reno, 1916-1951: Reno, University of Nevada, Mackay School of MinesNevada Bureau of Mines, Seismological Laboratory Bulletin, 199 p.

316. Scott, H.W., 1936, The Montana earthquakes of 1935: Butte, Mont., Montana School of Mines, Montana Bureau of Mines and Geology Memoir No. 16, 57 p.

317. Dewey, J.W., and Gordon, D.W., 1988, U.S. Geological Survey, unpublished data.

319. Carver, David, Richins, W.D., and Langer, C.J., 1983, Details of the aftershock process following the 30 September 1977, Unita Basin, Utah, earthquake: Seismological Society of America Bulletin, v. 73, no. 2, p. 435-448.

324. Bolt, B.A., and Miller, R.D., 1975, Catalogue of earthquakes in northern California and adjoining areas, 1 January 1910-31 December 1972: Berkeley, Calif., University of California, Seismographic Stations, 567 p.

325. Stover, C.W., 1984, United States earthquakes 1981: U.S. Geological Survey Special Publication, 136 p.

326. Frantti, G.E., 1983, Seismicity investigations of the southern Lake Superior Precambrian Province, [for the U.S. Nuclear Regulatory Commission], $59 \mathrm{p}$.

327. Jones, J.C., 1915, The Pleasant Valley, Nevada, earthquake of October 2, 1915: Seismological Society of America Bulletin, v. 5 , no. 4 , p. $190-205$.

328. Slemmons, D.B., Steinbrugge, K.V., Tocher, Don, Oakeshott, G.B., and Granella, V.P., 1959, Wonder, Nevada, earthquake of 1903: Seismological Society of America Bulletin, v. 49, no. 3, p. 251-265.

330. Nuttli, O.W., 1987, The effects of earthquakes in the Central United States: Central United States Earthquake Consortium, Monograph Series, v. 1, $33 \mathrm{p}$.

335. Callaghan, Eugene, and Gianella, V.P., 1935, The earthquake of January 30, 1934, at Excelsior Mountains, Nevada: Seismological Society of America Bulletin, v. 25, no. 2, p. 161-168.

336. Sleep, N.H., 1981, The events of February 4, 1883, Michigan, Indiana, and Illinois: Seismological Society of America, Eastern Section, Earthquake Notes, v. 52, no. 4, p. 3-9.

338. Couch, Richard, Victor, Linda, Keeling, Kenneth, 1974, Coastal and offshore earthquakes of the Pacific Northwest between $39^{\circ}$ and $49^{\circ} 10 \mathrm{~N}$. latitude and $123^{\circ}$ and $131^{\circ} \mathrm{W}$. longitude: Corvallis, Oregon State University, School of Oceanography, $67 \mathrm{p}$.

339. Sibol, M.S., and Bollinger, G.A., 1984, Hypocenter listing from Southeastern U.S. seismic network bulletins no. 1-12: Blacksburg, Virginia Polytechnic Institute and State University, Southeastern U.S. Seismic Network Bulletin, no. 12A, $44 \mathrm{p}$.

340. Herrmann, R.B., Langston, C.A., and Zollweg, J.E., 1982, The Sharpsburg, Kentucky, earthquake of 27 July 1980 : Seismological Society of America Bulletin, v. 72, no. 4, p. 1219-1239.

341. Richins, W.D., Arabaz, W.J., Hathaway, G.M., McPherson, Erwin, Oehmich, P.J., and Sells, L.L., 1984, Earthquake data for the Utah region, January 1, 1981 to December 31, 1983: Salt Lake City, University of Utah, Seismograph Stations, $111 \mathrm{p}$.

342. Carlson, S.M., 1984, Investigations of recent and historical seismicity in east Texas: The University of Texas at Austin, Master of Arts thesis, $197 \mathrm{p}$. 
TABLE 1.-Hypocenter and intensity references-Continued

[Please note that numbers in this table are not sequential because these references were selected from a master list compiled for other USGS publications]

343. DuBois, S.M., Smith A.W., Nye, N.K., and Nowak, T.A., Jr., 1982, Arizona earthquakes, 1776-1980: Tucson, University of Arizona, Bureau of Geology and Mineral Technology, State of Arizona, Bulletin 193, 456 p.

345. Street, Ronald, 1982, A contribution to the documentation of the 1811-1812 Mississippi valley earthquake sequence: Seismological Society of America, Eastern Section, Earthquake Notes, v. 53, no. 2, p. 39-51.

349. Dewey, J.W., and Gordon, D.W., 1984, Map showing recomputed hypocenters of earthquakes in the Eastern and Central United States and adjacent Canada, 1925-1980: U.S. Geological Survey, Miscellaneous Field Studies Map MF-1699, [pamphlet], $39 \mathrm{p}$.

350. Stover, C.W., 1985, United States earthquakes 1982: U.S. Geological Survey Bulletin 1655, 142 p.

353. Barstow, N.L., Brill, K.G., Nuttli, O.W., and Pomeroy, P.W., 1981, An approach to seismic zonation for siting nuclear electric power generating facilities in the Eastern United States: Roundout Associates, Inc., Stone Ridge, N.J., [for U.S. Nuclear Regulatory Commission], NUCREG/CR-1577, 315 p.

354. Dewey, J.W., 1987, Instrumental seismicity of central Idaho: Seismological Society of America Bulletin, v. 77, no. 3, p. 819-836.

355. Hutton, L.K., Allen, C.R., and Johnson, C.E., 1985, Seismicity of southern California; Earthquakes of $\mathrm{M}_{\mathrm{L}} 3.0$ and greater, 1975 through 1983: California Institute of Technology, Division of Geological and Planetary Sciences Contribution No. 4207, $142 \mathrm{p}$.

356. Gianella, V.P., and Callaghan, Eugene, 1934, The Cedar Mountain, Nevada, earthquake of December 20, 1932: Seismological Society of America Bulletin, v. 24, no. 4, p. 345-384.

357. Tocher, Don, 1956, Earthquakes off the North Pacific Coast of the United States: Seismological Society of America Bulletin: v. 46 , no. 3 , p. 165-173.

358. Romney, Carl, 1957, Seismic waves from the Dixie Valley-Fairview Peak earthquakes: Seismological Society of America Bulletin, v. 47, no. 4, p. 301-319.

359. Mokler, A.J., 1923, History of Natrona County, Wyoming, 1888-1922: Chicago, Lakeside Press, R.R. Donnelley and Sons Company, p. 72-74.

360. Stover, C.W., 1986, United States earthquakes 1983: U.S. Geological Survey Bulletin 1698, 197 p.

364. Davis, S.D., 1985, Investigations of natural and induced seismicity in the Texas Panhandle: The University of Texas at Austin, Master of Arts thesis, $230 \mathrm{p}$.

365. Oaks, S.D., and Bollinger, G.A., 1986, The epicenter of the $\mathrm{m}_{\mathrm{b}} 5$, December 22, 1875, Virginia earthquake: New findings from documentary sources: Earthquake Notes, Eastern Section, Seismological Society of America, v. 57, no. 3, p. 65-75.

366. Street, R.L., Couch, D., and Konkler, J., 1986, The Charleston, Missouri earthquake of October 31, 1895: Earthquake Notes, Eastern Section, Seismological Society of America, v. 57, no. 2, p. 41-51.

368. Toppozada, T.R., Real, C.R., and Parke, D.L., 1981, Preparation of isoseismal maps and summaries of reported effects for pre-1900 California earthquakes: California Division of Mines and Geology, Open-File Report 81-11SAC, 182 p.

369. Street, R.L., 1989, Personal communication, letter dated July 5, 1989, and copies of press reports from 1884 newspapers.

370. Stover, C.W., 1988, United States earthquakes 1984: U.S. Geological Survey Bulletin 1862, 284 p.

371. Stover, C.W., and Brewer, L.R., 1990, United States earthquakes 1985: U.S. Geological Survey Bulletin 1954,170 p.

373. Hopper, M.G., Algermissen, S.T., Perkins, D.M., Brockman, S.R., and Arnold, E.P., 1988, The December 14, 1872, earthquake in the Pacific Northwest: U.S. Geological Survey unpublished data.

374. Seismological Society of America, 1920, Seismological notes: Seismological Society of America Bulletin, v. 10, no. 1, p. 45-50.

375. Milne, W.G., 1956, Seismic activity in Canada, west of the 113th meridian 1841-1951: Publication of the Dominion Observatory, Ottawa, v. 18, no. 7, p. 119-146.

376. Nuttli, O.W., 1952, The western Washington earthquake of April 13, 1949: Seismological Society of America Bulletin, v. 42 , no. 1, p. 21-28.

377. Algermissen, S.T., Harding, S.T., Steinbrugge, K.V., and Cloud, W.K., 1965, The Puget Sound, Washington, earthquake of April 29, 1965: U.S. Department of Commerce, Coast and Geodetic Survey, 51 p.

378. Bolt, Bruce A., 1968, The focus of the 1906 California earthquake: Seismological Society of America Bulletin, v. 58, no. 1 , p. $457-471$.

379. Agnew, D.C., and Sieh, K.E., 1978, A documentary study of the felt effects of the great California earthquake of 1857: Seismological Society of America Bulletin, v. 68, no. 6, p. 1717-1729.

380. Toppozada, T.R., Parke, D.L., and Higgins, C.T., 1978, Seismicity of California 1900-1931: California Division of Mines and Geology, Special Report 135, $39 \mathrm{p}$.

381. Toppozada, T.R., Parke, D.L., Jensen, Laurel, and Campbell, Gordon, 1982, Areas damaged by California earthquakes 1900-1949: California Division of Mines and Geology, Open File Report 82-17SAC, 65 p.

382. Richter, C.F., 1958, Elementary seismology: San Francisco and London, W.H. Freeman and Company, 768 p.

383. Beal, C.H., 1915, The earthquake in the Imperial Valley, California, June 22, 1915: Seismological Society of America Bulletin, v. 5, no. 3, p. 130-149. 
TABLE 1.-Hypocenter and intensity references-Continued

[Please note that numbers in this table are not sequential because these references were selected from a master list compiled for other USGS publications]

384. Townley, S.D., 1918, The San Jacinto earthquake of April 21, 1918: Seismological Society of American Bulletin, v. 8, no. 2-3, p. 45-62.

385. Taber, Stephen, 1920, The Inglewood earthquake in southern California, June 21, 1920: Seismological Society of American Bulletin, v. 10, no. 3, p. 129-145.

386. Taber, Stephen, 1921, The Los Angeles earthquakes of July 1920: Seismological Society of America Bulletin, v. 11, no. 1, p. 63-79.

388. Laughlin, Homer, Arnold, Ralph, Kew, W.S.W., 1923, Southern California earthquake of July 22, 1923: Seismological Society of America Bulletin, v. 13, no. 3, p. 105-106.

389. Willis, Bailey, 1925, A study of the Santa Barbara earthquake of June 29, 1925: Seismological Society of America Bulletin, v. 15, no. 4, p. 255-278.

390. Byerly, Perry, 1927, The Idria (California) earthquake of July 25, 1926: Seismological Society of America Bulletin, v. 17 , no. 4, p. 203-206.

391. Mitchell, G.D., 1928, The Santa Cruz earthquakes of October 1926: Seismological Society of America Bulletin, v. 18, no. 3, p. 153-213.

392. Byerly, Perry, 1930, The California earthquake of November 4, 1927: Seismological Society of America Bulletin, v. 20, no. 2, p. 53-66.

393. Dyk, Karl, 1936, The California earthquake of April 15, 1928: Seismological Society of America Bulletin, v. 26, no. 3, p. 239-244.

394. Wood, H.O., and Richter, C.F., 1931, Recent earthquakes near Whittier, California: Seismological Society of America Bulletin, v. 21, no. 3, p. 183-203.

395. Richter, C.F., 1935, An instrumental earthquake magnitude scale: Seismological Society of America Bulletin, v. 25, no. 1, p. 1-32.

396. Gutenberg, B., Richter, C.F., and Wood, H.O., 1932, The earthquake in Santa Monica Bay, California, on August 30, 1930: Seismological Society of America Bulletin, v. 22, no. 2, p. 138-154.

397. Wood, H.O., 1933, Preliminary report on the Long Beach earthquake: Seismological Society of America Bulletin, v. 23 , no. 2, p. 43-56.

398. McEvilly, T.V., Bakun, W.H., and Casady, K.B., 1967, The Parkfield, California, earthquake of 1966: Seismological Society of America Bulletin, v. 57, no. 6, p. 1221-1244.

399. Cloud, W.K., Hill, D.M., Huffman, M.E., Jennings, C.W., McEvilly, T.V., Nason, R.D., Steinbrugge, K.V., Tocher, D., Unger, J.D., and Youd, T.L., 1970, The Santa Rosa earthquakes of October 1969: California Division of Mines and Geology, Mineral Information Service, v. 23, no. 3, $63 \mathrm{p}$.

400. Allen, C.R., Hanks, T.C., and Whitcomb, J.H., 1973, San Fernando earthquake: Seismological studies and their tectonic implications, in San Fernando, California, earthquake of February 9, 1971: U.S. Department of Commerce, v. III, p. 13-21.

401. Bulletin of the Seismographic Stations, 1973 to 1989, University of California-Berkeley.

402. Fuis, G.S., Friedman, M.E., and Hileman, J.A., 1977, Preliminary catalog of earthquakes in southern California, July 1974-September 1976: U.S. Geological Survey, Open-File Report 77-181, 107 p.

403. Reid, H.F., 1912, List of strong shocks in the United States and dependencies, in Report of the eightieth meeting of the British Association for the Advancement of Science, Seismological Investigations, p. 41-45.

404. Gutenberg, Beno, 1956, Great earthquakes 1896-1903: Transactions American Geophysical Union, v. 37, no. 5, p. 608-614.

405. Lyman, S.J., and family, An unpublished record of earthquakes felt at Hilo, Hawaii, from June 1833 to June 1916.

406. Wood, H.O., 1914, On the earthquakes of 1868 in Hawaii: Seismological Society of America Bulletin, v. 4, no. 4, p. 169-203.

407. Rockwood, C.G., 1882, Notes on American earthquakes-no. 11: American Journal of Science, v. 23, 3rd series, no. 133-138, p. 257-161.

408. The Volcano Letter, No. 1-No. 529, 1925-1955, Hawaiian Volcano Observatory, Volcano House, Hawaii.

409. Seismological Society of America, 1952, Seismological notes: Seismological Society of America Bulletin, v. 42, no. 1, p. 95-108.

410. Seismological Society of America, 1954, Seismological notes: Seismological Society of America Bulletin, v. 44, no. 3 , p. 529-542.

411. Koyanagi, R.Y., Krivoy, H.L., and Okamura, A.T., 1966, The 1962 Kaoiki, Hawaii, earthquake and its aftershocks: Seismological Society of America Bulletin, v. 56, no. 6, p. 1317-1335.

412. Abe, Katsuyuki, and Noguchi, Shin 'ichi, 1983, Revision of magnitudes of large shallow earthquakes, 1897-1912: Physics of the Earth and Planetary Interiors, v. 33, p. 1-11.

413. Horner, R.B., and Hasegawa, H.S., 1978, The seismotectonics of southern Saskatchewan: Canadian Journal of Earth Sciences, p. 1341-1355.

414. Beal, C.H., 1914, The earthquake in the Santa Cruz Mountains, California, November 8, 1914: Seismological Society of America Bulletin, v. 4, no. 4, p. 215-219.

417. Weekly Report and Weekly Bulletin of the Hawaiian Volcano Observatory, July 1913-December 1924, Honolulu, Hawaii. 
TABLE 1.-Hypocenter and intensity references-Continued

[Please note that numbers in this table are not sequential because these references were selected from a master list compiled for other USGS publications]

418. Koyanagi, R.Y., 1988, Unpublished list of Hawaiian earthquakes, U.S. Geological Survey, Hawaiian Volcano Observatory, Hawaii.

419. MacDonald, G.A., and Wentworth, C.K., 1952, The Kona earthquake of August, 21, 1951, and its aftershocks: University of Hawaii, Pacific Science, v. 6, no. 4, p. 269--287.

420. Tarr, R.S., and Martin, Lawrence, 1912, Earthquakes at Yakutat Bay, Alaska, in September 1899: U.S. Geological Survey Professional Paper 69, 135 p.

421. Cox, D.C., 1986, The Oahu earthquake of June 1948, associated shocks, and the hypothetical Diamond Head fault: University of Hawaii, Environmental Center, SR:0036, $32 \mathrm{p}$.

422. Cox, D.C., 1985, The Lanai earthquake of February 1871: University of Hawaii, Environmental Center, SR:0034, 50 p.

423. Cox, D.C., 1986, Earthquakes felt on Oahu, Hawaii, and their intensities: University of Hawaii, Environmental Center, SR:0038, $120 \mathrm{p}$.

424. Stover, C.W., Reagor, B.G., and Wetmiller, R.J., 1980, Intensities and isoseismal map for the St. Elias earthquake of February 28, 1979: Seismological Society of America Bulletin, v. 70, no. 5, p. 1635-1649.

425. Abe, Katsuyuki, 1983, Determination of magnitude for large shallow earthquakes 1898-1917: Physics of the Earth and Planetary Interiors, v. 32, p. 45-59.

426. Milne, John, 1912, Seismological investigations, List of earthquakes 1899-1903 inclusive, in Report of the eightieth Meeting of the British Association for the Advancement of Science, Seismological Investigations, p. 57-65.

427. Taggart, J.N., 1986, Unpublished list of earthquakes in Alaska, U.S. Geological Survey, Denver, Colo.

428. Abe, Katsuyuki, 1981, Magnitudes of large shallow earthquakes from 1904 to 1980: Physics of the Earth and Planetary Interiors, v. 27, p. 72-92.

432. Sykes, L.R., 1971, Aftershock zones of great earthquakes, seismicity gaps, and earthquake prediction for Alaska and the Aleutians: Journal of Geophysical Research, v. 76, no. 32, p. 8021-8041.

434. Seismological Society of America, 1923, Seismological notes: Seismological Society of America Bulletin, v. 13 , no. 2 , p. 82.

435. Seismological Society of America, 1927, Seismological notes: Seismological Society of America Bulletin, v. 17, no. 3, p. 193-200.

436. Dall, W.H., 1870, Alaska and its resources: London, Sampson, Lawson, and Marston, 627 p.

437. Fenner, C.N., 1925, Earth movements accompanying the Katmai eruption: The Journal of Geology, v. 33, no. 1, p. 116-139.

438. Steinbrugge, K.V., and Cloud, W.K., 1962, Epicentral intensities and damage in the Hebgen Lake, Montana, earthquake of August 17, 1959: Seismological Society of America Bulletin, v. 52, no. 2, p. 181-234.

440. Kirkham, R.M., and Rogers, W.P., 1986, An interpretation of the November 7, 1882, Colorado earthquakes: Colorado Geological Survey, Open-File Report 86-8, 36 p.

442. Davis, E.F., 1915, The earthquakes of October 7, 1915, in central California: Seismological Society of America Bulletin, v. 5, no. 4, p. 230-235.

443. Berea Advertiser, April 13, 1900, Berea, Ohio.

444. LaCroix, A.V., 1980, A short note on Cryoseisms: Seismological Society of America Bulletin, Eastern Section, Earthquake Notes, v. 51, no. 1 , p. 15-20.

445. Monthly Bulletin of the Hawaiian Volcano Observatory, February 1918--July 1929, Honolulu, Hawaii.

446. Weekly New Mexican, May 4, 1869, Sante Fe, N. Mex.

447. Tobin, D.G., and Sykes, L.R., 1966, Relationship of hypocenters of earthquakes to the geology of Alaska: Journal of Geophysical Research, v. 71, no. 6, p. 1659-1667.

448. Tobin, D.G., and Sykes, L.R., 1968, Seismicity and tectonics of the northeast Pacific Ocean: Journal of Geophysical Research, v. 73, no. 12, p. 3821-3845.

449. Rothé, J.P., 1969, The seismicity of the Earth, 1953-1965: Paris, United Nations Educational, Scientific, and Cultural Organization (UNESCO), $336 \mathrm{p}$.

450. Rankin, D.W., ed., 1977, Studies related to the Charleston, South Carolina, earthquake of 1886-A preliminary report: U.S. Geological Survey Professional Paper 1028, 204 p.

451. Sherburne, R.W., Algermissen, S.T., and Harding, S.T., 1969, The hypocenter, origin time, and magnitude of the Prince William Sound earthquake of March 28, 1964, in Leipold, L.E., ed., The Prince William Sound, Alaska, earthquake of 1964 and aftershocks: U.S. Department of Commerce, Coast and Geodetic Survey, p. 49-69.

452. Cloud, W.K., and Knudsen, C.F., 1967, The Fairbanks, Alaska, earthquakes of June 21, 1967: U.S. Department of Commerce, Environmental Science Services Administration, Preliminary Engineering Seismological Report, p. 33-60.

453. Tilling, R.I., Koyanagi, R.Y., Lipman, P.W., Lockwood, J.P., Moore, J.G., and Swanson, D.W., 1976, Earthquake and related catastrophic events, Island of Hawaii, November 29, 1975: A preliminary report: U.S. Geological Survey Circular 740, $33 p$.

454. Engdahl, E.R., 1977, Amchitka data file, 1969-1973 from seismicity and plate subduction in the central Aleutians, in Island arcs, deep sea trenches and back-arc basins: Maurice Ewing Series, v. 1, p. 259-271.

455. Stephens C.D., Lahr, J.C., Fogleman, K.A., and Horner, R.B., 1980, The St. Elias, Alaska, earthquake of February 28 , 1979: Regional recording of aftershocks and short-term, preearthquake seismicity: Seismological Society of America Bulletin, v. 70, no. 5, p. 1607-1633. 


\section{TABLE 1.-Hypocenter and intensity references-Continued}

[Please note that numbers in this table are not sequential because these references were selected from a master list compiled for other USGS publications]

456. Davies, J., Sykes, L., House, L., and Jacob, K., 1981, Shumagin seismic gap, Alaska Peninsula: History of great earthquakes, tectonic setting, and evidence for high seismic potential: Journal of Geophysical Research, v. 86, no. B5, p. 3821-3855.

457. Ryall, A., VanWormer, J.D., and Jones, A.E., 1968, Triggering of microearthquakes by earth tides, and other features of the Truckee, California, earthquake sequence of September 1966: Seismological Society of America Bulletin, v. 58, no. 1 , p. 215-248.

458. Stiermann, D.J., and Ellsworth, W.L., 1976, Aftershocks of the February 21, 1973, Point Mugu, California, earthquakes: Seismological Society of America Bulletin, v. 66, no. 6, p. 1931-1952.

459. Abdypoor, Gladees, and Bischke, R.E., 1982, Earthquakes felt in the State of Pennsylvania; with emphasis on earthquakes felt in Philadelphia, Pennsylvania, and surrounding areas: Philadelphia, Temple University, Department of Geology unpublished manuscript, $351 \mathrm{p}$.

461. Balderman, M.A., Johnson, C.A., Miller, D.G., and Schmidt, D.L., 1978, The 1852 Fort Yuma earthquake: Seismological Society of America Bulletin, v. 68, no. 3, p. 699-709.

463. Monthly Weather Review, Annual report of the Chief Signal Officer to the Secretary of War, June 1872 to June 1891 , v. 119, U.S. War Department, Washington, D.C.

464. Unger, J.D., and Ward, P.L., 1979, A large, deep Hawaiian earthquake-The Honomu, Hawaii, event of April 26, 1973: Seismological Society of America Bulletin, v. 69, no. 6, p. 1771-1781.

465. Qamar, A., and Hawley, B., 1979, Seismic activity near the Three Forks Basin, Montana: Seismological Society of America Bulletin, v. 69 , no. 6, p. 1917-1929.

466. Bolt, B.A., McEvilly, T.V., and Uhrhammer, R.A., 1981, The Livermore Valley, California, sequence of January 1980: Seismological Society of America Bulletin, v. 71, no. 2, p. 451-463.

467. Evans, D.G., and McEvilly, T.V., 1982, A note on relocating the 1963 Watsonville earthquakes: Seismological Society of America Bulletin, v. 72 , no. 4, p. 1309-1316.

468. Corbett, E.J., and Johnson, C.E., 1982, The Santa Barbara, California, earthquake of 13 August 1978: Seismological Society of America Bulletin, v. 72, no. 6, p. 2201-2226.

470. Wong, I.G., Cash, D.J., and Jaksha, L.H., 1984, The Crownpoint. New Mexico, earthquakes of 1976 and 1977 : Seismological Society of America Bulletin, v. 74, no. 6, p. 2435-2449.

471. Sbar, M.L., and DuBois, S.M., 1984, Attenutation of intensity for the 1887 Northern Sonora, Mexico, earthquake: Seismological Society of America Bulletin, v. 74, no. 6, p. 2613-2628.

472. Webb, T.H., and Kanamori, Hiroo, 1985, Earthquake focal mechanisms in the Eastern Transverse Ranges and San Emigdio Mountains, southern California and evidence for a regional decollement: Seismological Society of America Bulletin, v. 75, no. 3, p. 737-757.

473. Gupta, I.N., and Nuttli, O.W., 1976, Spatial attenuation of intensities for Central U.S. earthquakes: Seismological Society of America Bulletin, v. 66, no. 3, p. 743-751.

474. Hauksson, Egill, and Saldivar, G.V., 1986, The 1930 Santa Monica and the 1979 Malibu, California, earthquakes: Seismological Society of America Bulletin, v. 76, no. 6, p. 1542-1559.

475. Aburto, A.A., Savage, M.K., DePolo, D.M., and Delaplain, D.A., 1988, Bulletin of the Seismological Laboratory, January 1983 to May 1984: Reno, Nevada, University of Nevada-Reno, McKay School of Mines, 74 p.

476. Dehlinger, Peter, and Bolt, B.A., 1987, Earthquakes and associated tectonics in a part of coastal central California: Seismological Society of America Bulletin, v. 77, no. 6, p. 2056-2073.

477. Boyd, T.M., and Lerner-Lam, A.L., 1988, Spatial distribution of turn-of-the-century seismicity along the Alaska-Aleutian arc: Seismological Society of America Bulletin, v. 78, no. 2, p. 636-650.

479. Engdahl, E.R., Billington, S., and Kisslinger, C., 1989, Teleseismically recorded seismicity before and after the May 7 , 1986, Andreanof Islands, Alaska, earthquake: Journal of Geophysical Research, v. 94, no. B11, p. 15,48115,498.

480. Nowroozi, A.A., 1973, Seismicity of the Mendocino Escarpment and the aftershock sequence of June 26, 1968: Ocean seismic measurements: Seismological Society of America Bulletin, v. 63, no. 2, p. 441-456.

481. MacDonald, G.A., and Wentworth, C.K., 1954, Hawaiian volcanoes during 1951: U.S. Geological Survey Bulletin 996-D, $216 \mathrm{p}$.

482. MacDonald, G.A., and Eaton, J.P., 1964, Hawaiian volcanoes during 1955: U.S. Geological Survey Bulletin 1171 , p. 139.

483. Armbruster, J.G., and Scharnberger, C.K., 1986, Lancaster earthquakes, in Journal of the Lancaster County Historical Society, v. 90, no. 2, p. 78-86.

484. Thenhaus, P.C., 1978, A study of the October 12, 1877 Oregon earthquakes: U.S. Geologcial Survey Open-File Report 78-234, $17 \mathrm{p}$.

485. Landsberg, H., 1938, The Clover Creek earthquake of July 15, 1938: Seismological Society of America Bulletin, v. 28 , no. 4, p. $237-241$.

487. Kirkham, R.M., and Rogers, W.P., 1985, Colorado earthquake data and interpretations 1867 to 1985: Colorado Geological Survey Bulletin 46, 106 p.

488. Oaks, S.D., and Kirkham, R.M., 1986, Results of a search for felt reports for selected Colorado earthquakes: Colorado Geological Survey Information Series 23, 89 p. 
TABLE 1.-Hypocenter and intensity references-Continued

[Please note that numbers in this table are not sequential because these references were selected from a master list compiled for other USGS publications]

489. Stover, C.W., 1989, Reassigned intensities based on a review of the earthquake questionnaires collected by the Hawaiian Volcano Observatory, Hawaii.

490. Gordon, D.W., Bennett, T.J., Herrmann, R.B., and Rogers, A.M., 1970, The south-central Illinois earthquake of November 9, 1968: Macroseismic studies: Seismological Society of America Bulletin, v. 60, no. 3, p. 953-971.

491. Seismological Society of America, 1917, Seismological notes, Seismological Society of America Bulletin, v. 7, no. 4, p. 138-142.

492. Seismological Society of America, 1916, The earthquake at Volcano Lake, Mexico, November 20, 1915: Seismological Society of America Bulletin, v. 6, no. 2-3, p. 181-184.

493. Wood, H.O., 1916, California earthquakes: Seismological Society of America Bulletin, v. 6, no. 2-3, p. 55-180.

494. Rockwood, C.G., 1888, Notes on American earthquakes: American Journal of Science, v. 35, 3rd series, p. 110.

495. Woodward-Clyde Consultants, 1980, Seismological review of the July 16, 1936, Milton-Freewater earthquake source region: San Francisco, Calif., Contract No. 52028 C.0.11, Task No. WCC 1 for Washington Public Power Supply . System, $58 \mathrm{p}$.

496. Bradford, D.C., and Waters, A.C., 1934, The Tolt River earthquake and its bearing on the structure of the Cascade Ranges: Seismological Society of America Bulletin, v. 24, no. 1, p. 51-62.

497. MacDonald, Bernard, 1918, Remarks on the Sonora earthquake-its behavior at Tepic, Sonora, etc.: Seismological Society of America Bulletin, v. 8, no. 1, p. 74-78.

498. The News, Cambridge, Idaho, May 19, 1916, and June 2, 1916.

499. Rogers, W.P., and Kirkham, R.M., 1986, Contributions to Colorado seismicity and tectonics-A 1986 update: Colorado Geological Survey Special Publication 28, p. 122-132.

500. Wyss, Max, and Koyanagi, R.Y., in press, Isoseismal maps, macroseismic epicenters and estimated magnitudes of historical earthquakes in the Hawaiian Islands, U.S. Geological Survey Bulletin.

501. Rojahn, C., and Morrill, B.J., 1977, The Island of Hawaii earthquakes of November 29, 1975: Strong-motion data and damage reconnaissance report: Seismological Society of America Bulletin, v. 67, no. 2, p. 493-515.

502. Brasch, F.E., 1916, An earthquake in New England during the colonial period (1755): Seismological Society of America Bulletin, v. 6, no. 1, p. 26-42.

503. Engle, H.M., 1936, The Montana earthquakes of October 1935: Structural lessons: Seismological Society of America Bulletin, v. 26, no. 2, p. 99-109.

504. Witkind, I.J., Myers, W.B., Hadley, J.B., Hamilton, W., and Fraser, G.D., 1962, Geologic features of the earthquake at Hebgen Lake, Montana, August 17, 1959: Seismological Society of America Bulletin, v. 52, no. 2, p. 163-180.

505. Slemmons, D.B., 1957, Geological effects of the Dixie Valley-Fairview Peak, Nevada, earthquake of December 16, 1954: Seismological Society of American Bulletin, v. 47, no. 4, p. 353-375.

506. Devlin, J.J., Langguth, L.C., and Arringdale, R.L., 1942, Macroseismic study of the New Hampshire earthquakes of December 1940: Seismological Society of America Bulletin, v. 32, no. 2, p. 67-73.

508. Reinbold, D.J., and Johnston, A.C., 1987, Historical seismicity in the southern Appalachian seismic zone: U.S. Geological Survey Open-File Report 87-433, 858 p.

509. Westland, A.J., and Heinrich, R.R., 1940, A macroseismic study of the Ohio earthquakes of March 1937: Seismological Society of America Bulletin, v. 30, no. 3, p. 251-160.

510. Brown, B.H., 1937, The State-line earthquake at Milton and Walla Walla: Seismological Society of America Bulletin, v. 27, no. 3, p. 205-209.

511. Haar, L.C., Fletcher, J.B., and Mueller, C.S., 1984, The 1982 Enola, Arkansas, swarm and scaling of ground motion in the Eastern United States: Seismological Society of America Bulletin, v. 74, no. 6, p. 2463-2482.

512. Eickelberg, E.W., 1936, Review of earthquakes for the past year: Seismological Society of America, Eastern Section, Earthquake Notes, v. 8, nos. 1-2, p. 95.

513. Gordon, C.H., 1913, Earthquakes in east Tennessee: Seismological Society of America Bulletin, v. 3 p. $191-194$.

514. Rockwood, C.G., 1865, Notes on American earthquakes: American Journal of Science and Arts, v. 40, 2nd series, p. 362-366.

515. Kisslinger, J.B., 1983, Some volcanoes, volcanic eruptions, and earthquakes in the former Russian America: Pacific Northwest Quarterly, v. 74, no. 2, p. 59-68.

516. Cox, D.C., 1984, Probable Aleutian source of the tsunami observed in August 1872 in Hawaii, Oregon, and California: Science of Tsunami Hazards, The International Journal of the Tsunami Society, v. 2, no. 2, p. 79-94.

517. Lawson, A.C., 1908, The California earthquake of April 18, 1906: Report of the State Investigation Commission, Carnegie Institution of Washington, v. 1, 451 p.

518. Rockwood, C.G., 1885, Notes on American earthquakes: American Journal of Science, 3rd series, v. 29, p. $425-437$.

520. Kisslinger, J.B., 1980, Unpublished translations of Russian reports from the Alaskan regions.

521. Ellsworth, W.L., 1990, Earthquake history, 1769-1989, in R.E. Wallace, ed., The San Andreas fault system: U.S. Geological Survey Professional Paper 1515, p. 153-187.

522. Gordon, D.W., 1989, Personal communication, U.S. Geological Survey. 


\section{TABLE 1.-Hypocenter and intensity references-Continued}

[Please note that numbers in this table are not sequential because these references were selected from a master list compiled for other USGS publications]

523. Allen, M.W., 1925, Some remarks concerning Pacific Coast earthquakes: Seismological Society of America Bulletin, v. 15, no. 2 , p. $135-136$.

524. Westland, A.J., S.J., and Heinrich, R.R., 1940, A macroseismic study of the Ohio earthquakes of March 1937: Seismological Society of America Bulletin, v. 30, no. 3, p. 251-260.

525. Law Engineering Testing Company, 1975, Report on evaluation of intensity of Giles County, Virginia, earthquake of May 31, 1897: Marietta, Ga., Law Engineering Testing Company and Burns and Roe, Inc., 94 p.

526. Bollinger, G.A., 1977, Reinterpretation of the intensity data for the 1886 Charleston, South Carolina, earthquake, in Studies related to the Charleston, South Carolina, earthquake of 1886A preliminary report: U.S. Geological Survey Professional Paper 1028, p. 17-32.

527. Hopper, M.G., and Algermissen, S.T., 1980, An evaluation of the effects of the October 31, 1895, Charleston, Missouri, earthquake: U.S. Geological Survey Open-File Report 80-778, 44 p.

528. Espinosa, A.F., Brockman, S.R., and Michael, J.A., 1986, Modified Mercalli intensity distribution for the most significant earthquakes in Alaska 1899-1981: U.S. Geological Survey Open-File Report 86-0203, map, scale 1:12,500,000.

529. Street, R.L., and Green, R.F., 1984, The historical seismicity of Central United States, 1811-1928: University of Kentucky Research Foundation, U.S. Geological Survey Contract No. 14-08-0001, 550 p.

530. Huber, W.L., 1930, San Francisco earthquakes of 1865 and 1868: Seismological Society of America Bulletin, v. 20, no. 4, p. 261-272.

531. Bateman, P.C., 1961, Willard D. Johnson and the strike-slip component of fault movement in the Owens Valley, California, earthquake of 1872: Seismological Society of America Bulletin, v. 51, no. 4, p. 483-493.

532. Legg, Mark, and Agnew, D.C., 1979, The 1862 earthquake in San Diego, in Earthquakes and other perils: Geological Society of America Field Trip Guidebook, p. 139-141.

533. Coffman, J.L., 1969, Earthquake investigations in the United States: U.S. Department of Commerce, ESSA, Coast and Geodetic Survey Special Publication No. 282 [revised 1969 edition], p. 51-52.

534. Branner, J.C., 1917, The Tejon Pass earthquake of October 22, 1916: Seismological Society of America Bulletin, v. 7, no. 9, p. $51-60$.

535. Blackweider, Eliot, 1929, A recent earthquake in the Sierra Nevada: Seismological Society of America Bulletin, v. 19, no. 1, p. 52-53.

536. Byerly, Perry, and Wilson, J.T., 1935, The central California earthquakes of May 16, 1933, and June 7, 1934: Seismological Society of America Bulletin, v. 25, no. 3, p. 223-246.

537. Palmer, A.H., 1921, California earthquakes during 1920: Seismological Society of America Bulletin, v. 11, no. 1, p. 9-10.

539. Kemnitzer, L.E., 1924, The Salinas earthquake of December 27, 1924: Seismological Society of America Bulletin, v. 14, no. 4 , p. 230-232.

540. Byerly, Perry, 1925, Notes on the intensity of the Santa Barbara earthquake between Santa Barbara and San Luis Obispo: Seismological Society of America Bulletin, v. 15, no. 1, p. 279-281.

541. Dewell, H.D., and Willis, B., 1925, Earthquake damage to buildings: Seismological Society of America Bulletin, v. 15, no. 4, p. 282-304.

542. Kirkbride, W.H., 1927, The earthquake at Santa Barbara, California, June 29, 1925, as it affected the railroad of the Southern Pacific Company: Seismological Society of America Bulletin, v. 17, no. 1, p. 1-7.

543. Byerly, Perry, 1927, The Eureka (California) earthquake of August 20, 1927: Seismological Society of America Bulletin, v. 17 , no. 4 , p. $213-217$.

544. Sparks, N.R., 1936, The Eureka earthquake of June 6, 1932: Seismological Society of America Bulletin, v. 26, no. 1, p. $13-18$.

545. Wood, H.O., 1937, The Terwilliger Valley earthquake of March 25, 1937: Seismological Society of America Bulletin, v. 27, no. 4 , p. $305-312$.

546. Sylvester, A., 1979, Earthquake damage in Imperial Valley, California, May 18, 1940, as reported by T.A. Clark: Seismological Society of America Bulletin, v. 69, no. 2, p. 547-568.

547. Trifunac, M., and Brune, Jim, 1970, Complexity of energy release during the Imperial Valley, California, earthquake of 1940: Seismological Society of America Bulletin, v. 60, no. 1, p. 137-160.

548. Richter, C.F., Allen, C.R., and Nordquist, J.M., 1958, The Desert Hot Springs earthquakes and their tectonic environment: Seismological Society of America Bulletin, v. 48, no. 4, p. 315-337.

549. Steinbrugge, K.V., and Moran, D.F., 1954, An engineering study of the southern California earthquake of July 21 , 1952, and aftershocks: Seismological Society of America Bulletin, v. 44, no. 2B, p. $199-462$.

550. Steinbrugge, K.V., and Moran, D.F., 1957, An engineering study of the Eureka, California, earthquake of December 21, 1954: Seismological Society of America Bulletin, v. 47, no. 1, p. 129-153.

551. Cloud, W.K., 1967, Intensity map and structural damage, Parkfield, California, earthquake of June 27, 1966: Seismological Society of America Bulletin, v. 57, no. 6, p. 1161-1178.

552. Cloud, W.K., and Scott, N.H., 1968, The Borrego Mountain, California, earthquake of 9 April 1968, A preliminary engineering seismology report: Seismological Society of America Bulletin, v. 58, no. 3, p. 1187-1191.

553. Murphy, L.M. (scientific coordinator), 1973, San Fernando, California, earthquake of February 9, 1971: Washington, D.C., U.S. National Oceanic and Atmospheric Administration, v. 13. 
TABLE 1.-Hypocenter and intensity references-Continued

[Please note that numbers in this table are not sequential because these references were selected from a master list compiled for other USGS publications]

554. Clark, M.M., 1972, Surface rupture along the Coyote Creek fault, in The Borrego Mountain earthquake of April 9, 1968: U.S. Geological Survey Professional Paper 787, p. 55-86.

555. Kachadorian, R., Yerkes, R.F., and Waananen, A.O., 1967, Effects of the Truckee, California, earthquake of September 12, 1966: U.S. Geological Survey Circular 537, 14 p.

556. Westaway, Rob, and Smith, R.B., 1989, Source parameters of the Cache Valley (Logan), Utah, earthquake of 30 August 1962: Seismological Society of America Bulletin, v. 79, no. 5, p. 1410-1425.

557. Hopper, M.G., ed., 1985, Estimation of earthquake effects associated with large earthquakes in the New Madrid seismic zone: U.S. Geological Survey Open-File Report 85-457, 186 p.

558. McKeown, F.A., and Pakiser, L.C., eds., 1982, Investigations of the New Madrid, Missouri, earthquake region: U.S. Geological Survey Professional Paper 1236, $201 \mathrm{p}$.

559. Seismological Society of America, 1934, Seismological notes, Seismological Society of America Bulletin, v. 24, no. 2 , p. 329.

560. Seismological Society of America, 1950, Seismological notes, Seismological Society of America Bulletin, v. 40, no. 4 , p. 314.

561. Seismological Society of America, 1951, Seismological notes, Seismological Society of America Bulletin, v. 41, no. 1, p. 63-80.

562. Stover, C.W., and Brewer, L.R., in press, United States earthquakes 1986: U.S. Geological Survey Bulletin.

563. Seismological Society of America, 1952, Seismological notes, Seismological Society of America Bulletin, v. 42, no. 1, p. $100-106$.

564. Seismological Society of America, 1956, Seismological notes, Seismological Society of America Bulletin, v. 46, no.2, p. 150.

565. Sharp, R.V., 1976, Surface faulting in Imperial Valley during the earthquake swarm of January-February 1975: Seismological Society of America Bulletin, v. 66, no. 4, p. 1145-1154.

566. Clark, M.M., Sharp, R.V., Castle, R.O., and Harsh, P.W., 1976, Surface faulting near Lake Oroville, California, in August 1975: Seismological Society of America Bulletin, v. 66, no. 1, p. 1101-1110.

567. Hansen, Gladys, and Condon, Emmet, 1989, Denial of disaster, the untold story and photographs of the San Francisco earthquake and fire of 1906: San Francisco, Cameron and Company, $160 \mathrm{p}$.

569. Nuttli, O.W., 1983, Average seismic source-parameter relations for mid-plate earthquakes: Seismological Society of America Bulletin, v. 73, no. 2, p. 519-535.

570. Coan, T., 1869, Notes on the recent volcanic disturbances of Hawaii: American Journal of Science, 2nd series, v. 47, nos. $139,140,141$, p. 89-98.

571. Nason, Robert, 1980, Damage in San Mateo County, California, in the earthquake of 18 April 1906: U.S. Geological Survey Open-File Report 80-176, $51 \mathrm{p}$.

572. Nason, Robert, 1980, Damage in Santa Clara and Santa Cruz Counties, California, caused by the earthquake of 18 April 1906: U.S. Geological Survey Open-File Report 80-1076, 66 p.

573. Nason, Robert, 1982, Damage in Alameda and Contra Costa Counties, California, in the earthquake of 18 April 1906: U.S. Geological Survey Open-File Report 82-63, 46 p.

574. Stover, C.W., Reagor, B.G., Baldwin, F.W., and Brewer, L.R., 1990, Preliminary isoseismal map for the Santa Cruz (Loma Prieta), California, earthquake of October 18, 1989, UTC: U.S. Geological Survey Open-File Report 90-18, 24 p.

575. Steinbrugge, K.V., and Moran, D.F., 1956, Damage caused by the earthquakes of July 6 and August 23, 1954: Seismological Society of America Bulletin, v. 46, no. 1, p. 15-33.

576. Dewey, J.W., 1990, Unpublished data, U.S. Geological Survey.

577. Stover, C.W., 1992, Unpublished intensity data for 1987, U.S. Geological Survey.

578. Stover, C.W., 1992, Unpublished intensity data for 1988, U.S. Geological Survey.

579. Stover, C.W., 1992, Unpublished intensity data for 1989, U.S. Geological Survey.

580. Earthquake Engineering Research Institute, 1988, The Whittier Narrows earthquake of October 1, 1987: Earthquake Spectra, v. 4, nos. 12, 409 p.

581. Hauksson, E., Jones, L.M., Davis, T.L., Hutton, K., Brady, G.A., Reasenberg, P.A., Michael, A.J., Yerkes, R.F., Williams, P., Reagor, G., Stover, C.W., Bent, A.L., Shakal, A.K., Etheredge, E., Porcella, R.L., Bufe, C.G., Johnston, M.J.S., and Cranswick E., 1988, The 1987 Whittier Narrows earthquake in the Los Angeles metropolitan area, California: Science, v. 239 , p. $1409-1412$.

582. Hauksson, E., and Jones, L.M., 1989, The 1987 Whittier Narrows earthquake sequence in Los Angeles, southern California, seismological and tectonic analysis: Journal of Geophysical Research, v. 94, no. B7, p. 9579-9589.

583. Langer, C.J., Powers, P.S., Johnston, A.C., and Bollinger, G.A., 1987, The Olney, Illinois, earthquake of 10 June 1987 , A preliminary report: U.S. Geological Survey Open-File Report 87-623, p. 1-23.

584. Valley Sentinel, Portsmouth, Ohio, Wednesday, May 22, 1901, v. 6, p. 1.

587. Seismological Society of America, 1989, Special issue on the Elmore Ranch and Superstition Hills, California, earthquakes of 24 November 1987: Seismological Society of America Bulletin, v. 79, no. 2, 550 p.

588. Finch, M.O., 1988, Damage to irrigation facilities in Imperial Valley, in California Geology: California Division of Mines and Geology, v. 41 , no. 4 , p. 85-90.

589. Wallace, R.E., 1984, Fault scarps formed during the earthquakes of October 2, 1915, in Pleasant Valley, Nevada, and some tectonic implications, in Faulting related to the 1915 earthquakes in Pleasant Valley, Nevada: U.S. Geological Survey Professional Paper 1274-A, p. A1-A33. 
TABLE 1.-Hypocenter and intensity references-Continued

[Please note that numbers in this table are not sequential because these references were selected from a master list compiled for other USGS publications]

590. Case, W.F., 1988, Geologic effects of the 14 and 18 August 1988, earthquakes in Emery County, Utah: Utah Geological and Mineral Survey, Survey Notes, v. 22, no. 1 and 2, p. 8-15.

591. Silliman, B., and Dana, J.D., 1868, Earthquake in western New York, Vermont, and lower Canada: American Journal of Science and Arts, v. 45, nos. 133, 134, 135, p. 135.

592. Reagor, Glen, and Brewer, L.R., 1987, Preliminary isoseismal map and intensity distribution for the southeastern Illinois earthquake of June 10, 1987: U.S. Geological Survey Open-File Report 87-578, 3 p.

593. Rymer, M.J., and Ellsworth, W.L., eds., 1990, The Coalinga, California, earthquake of May 2, 1983: U.S. Geological Survey Professional Paper 1487, $417 \mathrm{p}$.

594. Benuska, Lee (technical ed.), 1990, Loma Prieta earthquake reconnaissance report, in Earthquake Spectra: Earthquake Engineering Research Institute, Supplement to v. 6, 448 p.

595. Astanek, A., Bertero, V.V., Bolt, B.A., Mahin, S.A., Moehle, J.P., and Seed, R.B., 1989, Preliminary report on the seismological and engineering aspects of the October 17, 1989, Santa Cruz (Loma Prieta), earthquake: University of California at Berkeley, Earthquake Engineering Research Center, Report No. UCB/EERC-89/14, 58 p.

596. U.S. Geological Survey, 1990, The Loma Prieta, California, earthquake: An anticipated event: Science, v. 247, p. $286-293$.

597. Given, D.D., Hutton, L.K., and Jones, L.M., 1987, The southern California network bulletin, July-December 1986: U.S. Geological Survey Open-File Report 87-448, p. 24-27.

598. Given, D.D., Wald, L.A., Jones, L.M., and Hutton, L.K., 1989, The southern California network bulletin, July-December 1987: U.S. Geological Survey Open-File Report 89-323, p. A1-A6.

599. Sherburne, R.W., 1981, Seismology program: California Geology, v. 34, no. 1, p. 3-6.

600. Taber, Stephen, 1916, The earthquake in the southern Appalachians, February 21, 1916: Seismological Society of America Bulletin, v. 6, no. 4, p. 218-226.

601. Meehan, J., Hart, E., and Schiff, A., 1988, Superstition Hills earthquake-November 23 and 24, 1987, Imperial County, California: Earthquake Engineering Research Institute Newsletter, v. 22, no. 2, p. 1-8.

602. Wald, L.A., Given, D.D., Mori, J., Jones, L.M., and Hutton, L.K., 1990, The southern California network bulletin, January-December 1988: U.S. Geological Survey Open-File Report 90-499, 45 p.

603. Wald, L.A., Given, D.D., Jones, L.M., and Hutton, L.K., 1990, The southern California network bulletin, JanuaryDecember 1989: U.S. Geological Survey Open-File Report 90-483, 22 p.

605. Retsel, Frank, Torrance, W.T., and Weaver, H.F., 1954, Report on investigation of an earth's disturbance at Wilkes-Barre, Luzerne County, Pennsylvania, U.S. Department of Interior, Bureau of Mines, Region 8, appendix 1, p. 2.5-33 to 2.5-43.

606. Housner, G.W., and Thiel, C.C., Jr., 1990, Competing against time: Report of the Governor's Board of Inquiry on the 1989 Loma Prieta earthquake: Earthquake Spectra, Earthquake Engineering Research Institute, v. 6, no. 4, p. 681-711.

607. Plafker, G., and Galloway, J.P., eds., 1989, Lessons learned from the Lome Prieta, California, earthquake of October 17 , 1989: U.S. Geological Survey Circular 1045, 48 p.

608. Stein, R.S., and Bucknam, R.C., eds., 1985, Proceedings of Workshop XXVIII, on the Borah Peak, Idaho, earthquake, volume A: U.S. Geological Survey Open-File Report 85-290, 686 p.

609. Lipman, P.W., and Mullineaux, D.R., 1981 (1982), The 1980 eruption of Mount St. Helens, Washington: U.S. Geological Survey Professional Paper 1250, 844 p.

610. Lander, J.F., and Lockridge, P.A., 1989, United States Tsunamis (including United States Possessions) 1690-1988: U.S. Department of Commerce, National Oceanic and Atmospheric Administration, Publication 41-2, 265 p.

611. Steinbrugge, K.V., 1967, Introduction to the earthquake engineering of the 1964 Prince William Sound, Alaska, earthquake, in The Prince William Sound, Alaska earthquake of 1964 and aftershocks: U.S. Department of Commerce, NOAA, Coast and Geodetic Survey, Publication 10-3, v. 2, part A, p. 1-6.

612. Toppozada, T.R., and Wong, I.G., 1990, $\mathrm{M} \geq 5.5$ earthquakes within $100 \mathrm{~km}$ of Parkfield, California [abs.], Seismological Society of America, Eastern Section, Seismological Research Letters, v. 61, no. 1, p. 42.

613. Toppozada, T.R., and Cramer, C.H., 1978, Ukiah earthquake, 25 March 1978, Seismicity possibly induced by Lake Mendocino: California Geology, v. 31, no. 12, p. 275-281.

615. Perrine, C.D., 1899, Earthquakes in California in 1898: U.S. Geological Survey Bulletin 161, 29 p.

616. Johnson, T.E., Ludwin, R.S., and Qamar, A.I., in press, The central Cascades earthquake of March 7, 1891: Washington Geology.

617. Rogers, G.C., and Hasegawa, H.S., 1978, A second look at the British Columbia earthuake of June 23, 1946: Seismological Society of America Bulletin, v. 68, no. 3, p. 653-675.

618. Grant, W.C., and Weaver, C.S., 1986, Earthquakes near Swift Reservoir, Washington, 1958-1963: Seismological Society of America Bulletin, v. 76 , no. 6 , p. $1573-1587$.

619. Yelin, T.S., and Patton, H.J., 1991, Seismotectonics of the Portland, Oregon, region: Seismological Society of America Bulletin, v. 81, no. 1, p. 109-130.

620. Qamar, A., Rathburn, A., Ludwin, R., Crosson, R.S., and Malone, S.D., 1986, Earthquake hypocenters in Washington and Northern Oregon-1980: Washington Division of Geology and Earth Resources, Information Circular 82, $64 \mathrm{p}$. 
TABLE 2.-Magnitude references

AAM

ABE

AB I

AB2

AB3

ADK

AND

ARC

AS

BAK

BAR

BAS

BL

BLA

BLT

BMU

BOL

BOT

BRK

BU

BUR

CAR

CDL

CFR

CON

$\operatorname{cox}$

CWR

DDA
University of Michigan, Ann Arbor, Mich.

Abe, Katsuyuki, 1981, Magnitude of large shallow earthquakes from 1904 to 1980: Physics of the Earth and Planetary Interiors, v. 27. p. 72-92.

Abe. Katsuyuki. and Noguchi. Shin'ichi. 1983, Determination of magnitude for large shallow earthquakes, 1898-1917: Physics of the Earth and Planetary Interiors, v. 32, p. 45-59.

Abe, Katsuyuki, and Noguchi. Shin'ichi. 1983. Revision of magnitudes of large shallow earthquakes, 1897-1912: Physics of the Earth and Planetary Interiors, v. 33, p. 1-11.

Abe, Katsuyuki, 1984, Complements to "Magnitudes of large shallow earthquakes from 1904 to 1980:" Physics of the Earth and Planetary Interiors, v. 34. p. 17-23.

Adak Observatory, U.S. National Oceanic and Atmospheric Administration. Adak Island, Aleutian Islands, Alaska.

Ando, Masataka, 1979, The Hawaii earthquake of November 29, 1975: Low dip angle due to forceful injection of magma: Journal of Geophysical Research. v. 84. no. B13, p. 7616-7626.

Archuleta, R.J., Cranswick. E., Mueller. C.. and Spudich. P.. 1982. Source parameters of the 1980 Mammoth Lakes, California, earthquake sequence: Journal of Geophysical Research. v. 87, no. B6, p. 4595-4607.

Armbruster, J.C.. and Seeber. Leonardo, 1987. The 23 April 1984 Martic earthquake and the Lancaster seismic zone in eastern Pennsylvania: Seismological Society of America Bulletin, v. 77, no. 3, p. 877-890.

Bakun, W.H., 1984. Seismic moments, local magnitudes, and coda-duration magnitudes for earthquakes in central California: Seismological Society of America Bulletin, v. 74, no. 2, p. $439-458$.

Barstow, N.L.. Brill, K.G., Nuttli, O.W., and Pomeroy, P.W.. 1981, An approach to seismic zonation for siting nuclear electric power generating facilities in the Eastern United States: Stone Ridge, N.J., Roundout Associates, Inc., report prepared for U.S. Nuclear Regulatory Commission, NUCREG/CR-1577, 315 p.

Basham, P.W., Weichert. D.H., and Berry, M.J., 1979, Regional assessment of seismic risk in Eastern Canada: Seismological Society of America, v. 69, no. 5. p. 1567-1602.

Baker, G.E., and Langston, C.A., 1987, Source parameters of the 19.49 magnitude 7.1 South Puget Sound, Washington, earthquake as determined from long-period body waves and strong ground motions: Seismological Society of America Bulletin, v. 77, no. 5, p. 1530-1557.

Seismological Observatory, Virginia Polytechnic Institute and State University, Blacksburg, Va.

Bolt, B.A., 1978, The local magnitude $M_{L}$ of the Kern County earthquake of July 21, 1952: Seismological Society of America Bulletin. v. 68, no. 2. p. 513-515.

Bolt, B.A., McEvilly, T.V., and Uhrhammer, R.A., 1981, The Livermore Valley, California, sequence of January 1980: Seismological Society of America Bulletin, v. 71. no. 2. p. 451-463.

Bollinger, G.A., 1986, Historical seismicity of South Carolina [abs.]: Seismological Society of America. Eastern Section, Earthquake Notes. v. 57, no. 1. p. 15-16.

Bolt, B.A., 1984, The magnitude of the Hebgen Lake 1959 and Idaho 1983 earthquakes [abs.]: Seismological Society of America, Eastern Section, Earthquakes Notes, v. 55, no. 1, p. 13.

Seismographic Stations. University of California. Berkeley, Calif.

Montana Bureau of Mines and Geology, Butte. Mont.

Burger, R.W., and Langston, C.A., 1985, Source mechanism of the May 18,1980. Mount St. Helens eruption from regional surface waves: Journal of Geophysical Research, v. 90. no. B9. p. 7653-7664.

Carlson, S.M., 1984, Investigations of recent and historical seismicity in east Texas: The University of Texas at Austin, Master of Arts thesis, $197 \mathrm{p}$.

Carver, D., Richins. W.D., and Langer, C.J., 1983, Details of the aftershock process following the 30 September 1977 Uinta Basin, Utah, earthquakes: Seismological Society of America Bulletin. v. 73, no. 2. p. 435-448.

Richter, C.F., 1958, Elementary Seismology: San Francisco, W.H. Freeman and Company, $768 \mathrm{p}$.

University of Connecticut seismograph station. Groton, Conn.

Cox, D.C.. 1985, The Lanai earthquake of February 1871: Environmental Center,University of Hawaii, SR:0034, 50 p.

California Department of Water Resources, Sacramento, Calif.

Duda, S.J., 1965, Secular seismic energy release in the Circum-Pacific Belt: Tectonophysics, v. 2, no. 5. p. $409-452$. 
TABLE 2-Magnitude references-Continued

DG

DMG

DOR

DOS

DSR

DW

EBE

ED

ELL

EPB

ESM

FRK

GB

GDW

GM

GOL

GP

GR

GS

GT

HAV

HB

HBK

HDP

HER

$\mathrm{HH}$

HHT

Dewey, J.W., and Gordon, D.W., 1984. Map showing recomputed hypocenters of earthquakes in the Eastern and Central United States and adjacent Canada, 1925-1980: U.S. Geological Survey Miscellaneous Field Studies Map MF-1699, [pamphlet], 39 p.

California Division of Mines and Geology, Sacramento, Calif. [see ref. 368, 380, 381 in table 1].

Doser, D.I., 1989, Source parameters of Montana earthquakes (1925-1964) and tectonic deformation in the northern intermountain seismic belt: Seismological Society of America Bulletin, v. 79, no. 1, p. 31-50.

Doser, D.I., and Smith, R.B., 1982, Seismic moment rates in the Utah region: Seismological Society of America Bulletin, v. 72, no. 2, p. 525-551.

Doser, D.I., 1985, Source parameters and faulting processes of the 1959 Hebgen Lake, Montana, earthquake sequence: Journal of Geophysical Research, v. 90, no. B6, p. 4537-4555.

Dewey, J.W., 1987, Instrumental seismicity of central Idaho: Seismological Society of America Bulletin, v. 77, no. 3, p. 819-836.

Eberhart-Phillips. D., Richardson, R.M., Sbar. M.L.. and Herrmann, R.B., 1981. Analysis of the 4 February 1976 Chino Valley, Arizona, earthquake: Seismological Society of America Bulletin, v. 71, no. 3, p. 787-801.

Ekstrom. G.. and Dziewonski, A.M.. 1985, Centroid-moment tensor solutions for 35 earthquakes in Western North America (1977-1983): Seismological Society of America Bulletin, v. 75, no. 1, p. 23-39.

Ellsworth, W.L, 1990, Historical seismicity, in Wallace. R.E.. ed., The San Andreas fault system: U.S. Geological Survey Professional Paper 1515, p 153-181.

Seismological Service, Geological Survey of Canada [formerly Earth Physics Branch], Ottawa, Ont., Canada.

Ebel, J.E., Somerville, P.G.. and McIver, J.D.. 1986, A study of the source parameters of some large earthquakes of northeastern North America: Journal of Geophysical Research, v. 91, no. B8, p. 8231-8247.

Frankel, Arthur, 1984, Source parameters of the $M_{L}$ about 5 earthquake near Anza. California, and a comparison with an Imperial Valley aftershock: Seismological Society of America Bulletin, v. 74, no. 5, p. 1509-1527.

Bollinger, G.A., 1979, Attenuation of the $L_{g}$ phase and the determination of Mb in the Southeastern United States: Seismological Society of America Bulletin, v. 69, no. 1, p. 45-63. Gordon, D.W., 1990, U.S. Geological Survey unpublished data.

U.S. Geological Survey, Menlo Park, Calif.

Geophysical Observatory, Colorado School of Mines, Golden. Colo.

U.S. Geological Survey, Pasadena, Calif.

Gutenberg, Beno, and Richter, C.F., 1954, Seismicity of the Earth and associated phenomena: New York, Hafner Publishing Inc., 310 p.

U.S. Geological Survey. Golden, Colo.

Georgia Institute of Technology, Atlanta, Ga.

Harvard University, Cambridge, Mass.

Hartzell, S.H., and Brune, J.N., 1979, The Horse Canyon earthquake of August 2, 1975-two stage stress-release processs in a strike-slip earthquake: Seismological Society of America Bulletin, v. 69, no. 4, p. 1161-1173.

Hart, R.S., Butler, R., and Kanamori, H., 1977, Surface-wave constraints on the August 1, 1975, Oroville earthquake: Seismological Society of America Bulletin, v. 67, no. 1, p. 1-7.

Herrmann, R.B.. Dewey. J.W., and Park. S-K.. 1980, The Dulce, New Mexico, earthquake of 23 January 1966: Seismological Society of America Bulletin, v. 70, no. 6, p. 2171-2183.

Herrmann, R.B., Park, S-K., and Wang, C-Y., 1981, The Denver earthquakes of 1967-1968: Seismological Society of America Bulletin, v. 71, no. 3, p. 731-745.

Heaton, T.H., and Helmberger. D.V., 1978, Predictability of strong ground motion in the Imperial Valley: Modeling the M 4.9, November 4, 1976, Brawley earthquake: Seismological Society of America Bulletin, v. 68, no. 1, p. 31-48.

Hanks, T.C., Hileman, J.A., and Thatcher, W., 1975, Seismic moments of the larger earthquakes of the southern California region: Geological Society of America Bulletin, v. 86, p. 1131-1139. 
TABLE 2-Magnitude references-Continued

HK

HLS

HRM

HRN

HRR

HVO

1SC

JDH

JLM

JLQ

JM

$\mathrm{JOH}$

JON

KA

KAN

KIR

$\mathrm{KJ}$

KRK

LB

MAT

MH

MMT

MOS

MSO

NLl

NM1

NQT

NS
Hanks. T.C.. and Kanamori. H.. 1979. A moment magnitude scale: Journal of Geophysical Research, v. 84, no. B5, p. 2348-2350.

Hasegawa, H.W., Lahr, J.C.. and Stephens. C.D., 1980. Fault parameters of the St. Elias, Alaska, earthquake of February 28, 1979: Seismological Society of America Bulletin, v. 70 , no. 5, p. 1651-1660.

Herrmann, R.B., 1986. Surface-wave studies of some South Carolina earthquakes: Seismological Society of America Bulletin, v. 76, no. 1, p. 111-121.

Herrmann, R.B., 1979, Surface wave focal mechanisms for Eastern North America earthquakes with tectonic implications: Journal of Geophysical Research, v. 84, no. B7, p. 3543-3552.

Herrmann, R.B., Langston, C.A., and Zollweg.J.E., 1982. The Sharpsburg, Kentucky, earthquake of 27 July 1980: Seismological Society of America Bulletin, v. 72, no. 4, p. 1219-1239.

Hawaiian Volcano Observatory, U.S. Geological Survey, Hawaii National Park, Hawaii.

Bulletin of the International Seismological Centre, 1954-1989, Newbury, Berkshire, United Kingdom.

Jordan, J.N., Dunphy, G.J., and Harding. S.T., 1967, The Fairbanks, Alaska, earthquakes of June 21, 1967: U.S. Department of Commerce, Coast and Geodetic Survey, Preliminary Seismological Report, p. 1-33.

Jones, F.B., Long, L.T., and McKee, J.H., 1977, Study of the attenuation and azimuthal dependence of seismic wave propagation in the Southeastern United States: Seismological Society of America Bulletin, v. 67, no. 6, p. 1503-1513.

Johnson, T.E., Ludwin. R.S., and Qamar, A.I., in press, The central Cascades earthquake of March 7, 1891: Washington Geology.

Johnson, L.R., and McEvilly, T.V., 1974, Near-field observations and source parameters of central California earthquakes: Seismological Society of America Bulletin, v. 64, no. 6, p. 1855-1886.

Johnston, A.C., in press. The stable-continental-region earthquake data base, in Coppersmith, K.J., and others, eds., Methods for assessing maximum earthquakes in the Central and Eastern United States: Palo Alto, Calif., Electric Power Research Institute Project RP-2556-12.

Jones, A.E., 1975, Recording of earthquakes at Reno, 1916-1951: Seismological Laboratory Bulletin, Mackay School of Mines-Nevada Bureau of Mines, University of Nevada-Reno, 199 p.

Kanamori, Hiroo, and Anderson, D.L.. 1975. Theoretical basis of some empirical relations in seismology: Seismological Society of America Bulletin, v. 65, no. 5, p. 1073-1095.

Kanamori, Hiroo, 1977, The energy release in great earthquakes: Journal of Geophysical Research, v. 82, no. 20, p. 2981-2987.

Kiruna Seismograph Station, University of Uppsala, Sweden.

Kanamori, Hiroo, and Jennings, P.C., 1978, Determination of local magnitude, $M_{L}$, from strong-motion accelerograms: Seismological Society of America Bulletin, v. 68, no. 2, p. 471-185.

Kirkham, R.M., and Rogers, W.P., 1986, An interpretation of the November 7, 1882, Colorado, earthquake: Colorado Geological Survey, Open-File Report 86-8, 36 p.

Langston, C.A., and Blum, D.E.. 1977. The April 29. 1965. Puget Sound earthquake and the crustal and upper mantle structure of western Washington: Seismological Society of America Bulletin. v. 67, no. 3, p. 693-711.

Matsushiro Seismological Observatory, Japan Meteorological Agency, Matsushiro, Japan.

Mori, Juim, and Hartzell, Stephen, 1990, Source inversion of the 1988 Upland, California, earthquake-Determination of a fault plane for a small event: Seismological Society of America Bulletin, v. 80, no. 3, p. 507-518.

Montana College of Mineral Sciences and Technology, Butte, Mont.

Institute of Physics of the Earth of the U.S.S.R., Moscow.

University of Montana, Missoula, Mont.

Nuttli, O.W., 1983, Average seismic source-parameter relations for mid-plate earthquakes: Seismological Society of America Bulletin, v. 73, no. 2, p. 519-535.

New Mexico Institute of Mining and Technology, Socorro. N. Mex.

Noson, L.L., Qamar, A., and Thorsen, G.W., 1988, Washington State earthquake hazards: Washington Division of Geology and Earth Resources. Information Circular 85, p. 22.

Natali, S.G., and Sbar, M.L., 1982, Seismicity in the epicentral region of the 1887 northeastern Sonoran earthquake, Mexico: Seismological Society of America Bulletin. v. 72, no. 1, p. 181-196. 
TABLE 2,-Magnitude references-Continued

Nuttli, O.W., 1974, Magnitude-recurrence relation for central Mississippi valley earthquakes: Seismological Society of America Bulletin, v. 64, no. 4, p. 1189-1207.

Nuttli, O.W., 1987, The effects of earthquakes in the Central United States: Central United States Earthquake Consortium, Monograph Series, v. 1,33 p.

Nuttli, O.W. 1979, Seismicity of the Central United States, geology in the siting of nuclear power plants: Geological Society of America, Reviews in Engineering Geology, v. 4, p. 67-107.

Nuttli, O.W., 1973, The Mississippi valley earthquakes of 1811 and 1812: Intensities, ground motion, and magnitudes: Seismological Society of America Bulletin, v. 63, no. 1, p. 227-248.

Nuttli, O.W., Bollinger, G.A., and Griffiths, D.W., 1979, On the relation between Modified Mercalli intensity and body-wave magnitude: Seismological Society of America Bulletin, v. 69 , no. 3, p. 893-909.

Lamont-Doherty Geological Observatory, Columbia University, Palisades, N.Y.

Seismological Laboratory, California Institute of Technology, Pasadena, Calif.

Alaska Tsunami Warning Center, U.S. National Oceanic and Atmospheric Administration, Palmer, Alaska.

Plafker, G., and Thatcher, W., 1982, Geological and geophysical evaluation of the great 1899-1900 Yakutat Bay, Alaska. earthquakes [abs.], in Coppersmith, K.J., and Schwartz, D.P., eds.: Chapman conference on fault behavior and the earthquake generation process, American Geophysical Union, p. 7.

Qamar, A., Rathburn, A., Ludwin R.. Crosson. R.S., and Malone, S.D., 1986, Earthquake hypocenters in Washington and Northern Oregon-1980: Washington Division of Geology and Earth Resources, Innformation Circular 82, 64 p.

Mackay School of Mines, University of Nevada-Reno, Reno, Nev.

Richter, C.F., 1935, An instrumental earthquake magnitude scale: Seismological Society of America Bulletin, v. 25, no. 1, p. 1-32.

Ruff, Larry, Kanamori, Hiroo, and Sykes, Lynn, 1985, The 1957 great Aleutian earthquake [abs.]: EOS, Transactions, American Geophysical Union, v. 66, no. 18, p. 298.

Rogers, G.C.. 1983, Seismotectonics of British Columbia: University of British Columbia, Canada, PhD. thesis, $247 \mathrm{p}$.

Rothé, J.P., 1969, The seismicity of the Earth: Paris, United Nations Educational, Scientific, and Cultural Organization, $336 \mathrm{p}$.

Sanford, A.R., and Toppozada, T.R., 1974, Seismicity of proposed waste disposal site in southeastern New Mexico: New Mexico Bureau of Mines and Mineral Resources. Circular 143, $15 \mathrm{p}$.

Scholz, C.H., Aviles, C.A., and Wesnousky, S.G., 1986, Scaling differences between large interplate and intraplate earthquakes: Seismological Society of America Bulletin, v. 76, no. 1, p. 65-70.

Stover, C.W., and Coffman, J.L., This publication.

Street, R.L., 1982, A contribution to the documentation of the 1811-1812 Mississippi valley earthquake sequence: Seismological Society of America, Eastern Section, Earthquake Notes, v. 51 , no. 2, p. 39-52.

Street, R.L., 1989, Personal communication. letter dated July 5, 1989.

Street, R.L., and Green, R.F., 1984, The historical seismicity of Central United States, 1811-1928, University of Kentucky Research Foundation, Lexington, Ky., 550 p.

Slemmons, D.B., Jones, A.E.. and Gimlet, J.I., 1965, Catalog of Nevada earthquakes, 1852-1960: Seismological Society of America Bulletin, v. 55, no. 2, p. 519-565.

Saint Louis University, Saint Louis, Mo.

Somerville, P.G., McLaren, J.P., LaFevre. L.V., Berger, R.W., and Helmberger. H.V.. 1987, Comparison of source scaling relations of Eastern and Western North American earthquakes: Seismological Society of America Bulletin, v. 77, no. 2, p. 322-346.

Schell, M.M., and Ruff, L.J., 1986, Southeastern Alaska tectonics: source process of the large 1972 Sitka earthquake [abs.]: EOS, Transactions, American Geophysical Union, v. 67, p. 304-305.

Street, R.L., 1984, Some recent Lg-phase displacement spectral densities and their implication with respect to the prediction of ground motions in Eastern North America: Seismological Society of America Bulletin, v. 74, no. 2, p. 757-762.

Street, R.L., and Turcotte, F.T., 1977, A study of northeastern North America spectral moments, magnitudes, and intensities: Seismological Society of America Bulletin, v. 67, no. 3, p. 599-614. 
STR

WAL

Street, R.L., 1976, Scaling Northeastern United States/Southeasten Canadian earthquakes by their Lg waves: Seismological Society of America Bulletin, v. 66, no. 5, p. 1525-1537.

Street, R.L., Herrmann, R.B., and Nuttli, O.W., 1975, Spectral characteristics of the $L_{g}$ wave generated by Central United States earthquakes: Geophysical Journal of Royal Astronomical Society, v. 41, p. 51-63.

Schwartz, S.Y., and Christensen, D.H., 1988. The 12 July 1986 St. Marys, Ohio, earthquake and recent seismicity in the Anna, Ohio, seismogenic zone: Seismological Society of America, Eastern Section, Seismological Research Letters, v. 59, no. 2, p. 57-62.

Taggart, James. and Baldwin. Frank, 1982. Earthquake sequence of 1938-1939 in Mogollen Mountains, New Mexico: New Mexico Geology, v. 4, no. 4, p. 49-52.

Center for Earthquake Research and Information, Memphis State University, Memphis, Tenn.

Thatcher, W., and Hanks, T.C., 1973, Source parameters of southern California earthquakes: Journal of Geophysical Research, v. 78, no. 35, p. 8547-8576.

Thatcher, W., Hileman, J.A., and Hanks, T.C., 1975, Seismic slip distribution along the San Jacinto fault zone, southem California, and its implications: Geological Society of America Bulletin, v. 86, p. 1140-1146.

Oklahoma Geophysical Observatory, Oklahoma Geological Survey, Leonard, Okla.

Thatcher, W., 1975, Strain accumulation and release mechanism of the 1906 San Francisco earthquake: Journal of Geophysical Research, v. 80, no. 35, p. 4862-4880.

University of Hawaii, Honolulu, Hawaii.

Uppsala Seismograph Station, University of Uppsala, Sweden.

Seismograph Stations, University of Utah, Salt Lake City, Utah.

Victoria Geophysical Observatory, Victoria. B.C., Canada.

Voss, J.A., and Herrmann, R.B., 1980. A surface-wave study of the June 16, 1978. Texas earthquake: Seismological Society of America, Eastern Section, Earthquake Notes, v. 51, no. 1, p. 3-14.

Wallace, R.E., 1984, Fault scarps formed during the earthquakes of October 2, 1915, in Pleasant Valley, Nevada, and some tectonic implications, in Faulting related to the 1915 earthquakes in Pleasant Valley, Nevada: U.S. Geological Survey Professional Paper 1274-A, p. A1-A33.

University of Washington, Seattle, Wash.

Weston Observatory, Boston College, Weston, Mass.

Webb, T.H., and Kanamori, Hiroo, 1985, Earthquake focal mechanism in the Eastern Transverse Ranges and San Emigdio Mountains, southern California, and evidence for a regional decollement: Seismological Society of America Bulletin, v. 75, no. 3, p. 737-757.

Wood, H.O., 1947, Earthquakes in southern California with geologic relations: Seismological Society of America Bulletin, v 37, no. 3, p. 217-258.

Wyss, Max, and Koyanagi, Robert, in press, Isoseismal maps, macroseismic epicenters and estimated magnitudes of historical earthquakes in the Hawaiian Islands: U.S. Geological Survey Bulletin.

Wyss, Max, and Brune, J.N., 1968, Seismic moment, stress, and source dimensions for earthquakes in the California-Nevada region: Journal of Geophysical Research, v. 73, no., 14, p. 4681-1694.

Yelin, Tom, 1991, University of Washington, personal communication.

Yelin, T.S., and Patton, H.J., 1991, Seismotectonics of the Portland, Oregon, region: Seismological Society of America Bulletin, v. 81, no. 1, p. 109-130. 
TABIE 3.-Deaths from earthquakes in the United States

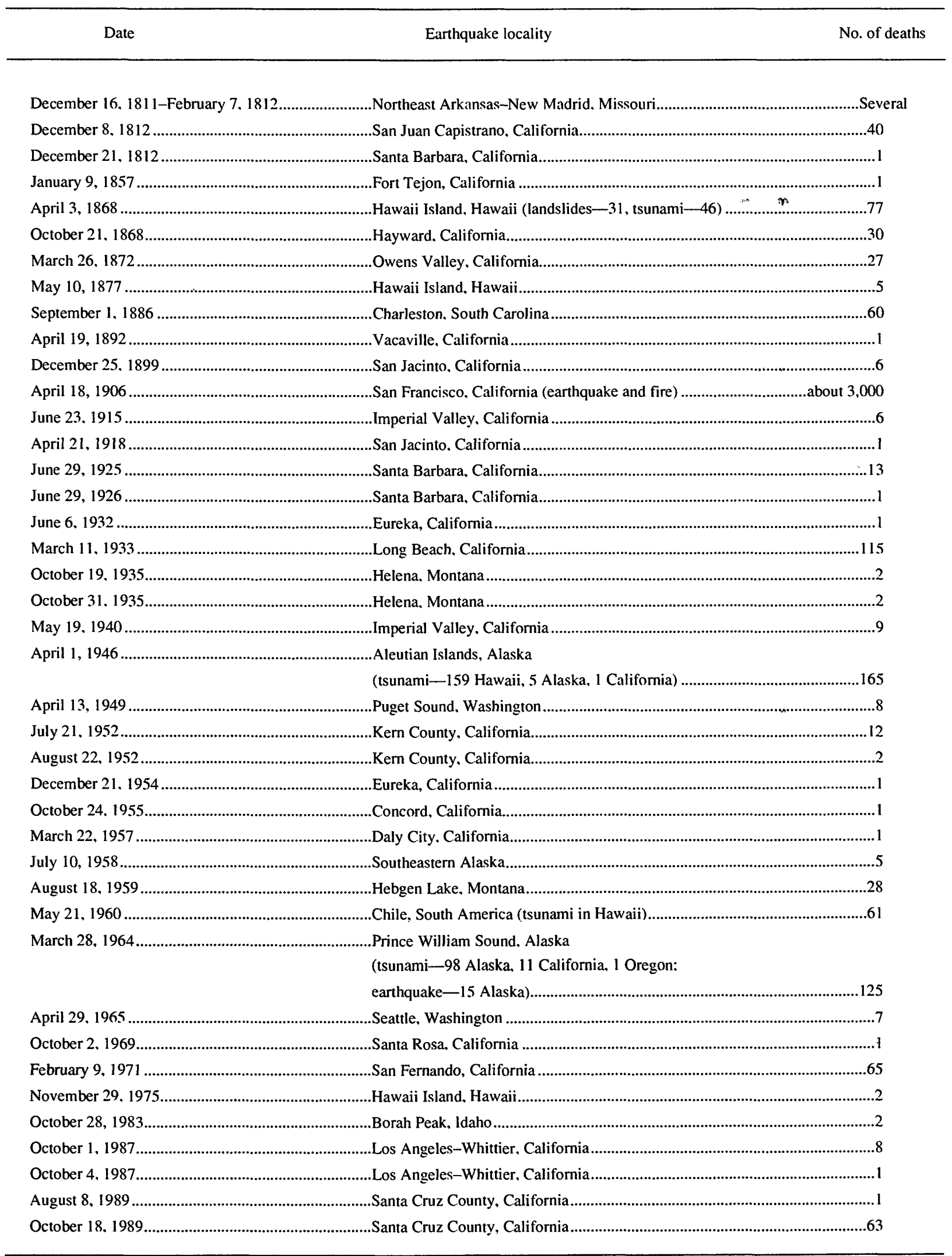

